
$\mathrm{A} / \mathrm{N} 14243$

WCMC Reports 98 


\section{CHECKLIST OF CITES SPECIES \\ LISTA DE LAS ESPECIES CITES \\ LISTE DES ESPECES CITES}

A reference to the appendices to the

Convention on International Trade in Endangered Species

of Wild Fauna and Flora

Una referencia a las especies incluidas en los Apéndices de la Convención sobre el Comercio Internacional de Especies Amenazadas de Fauna y Flora Silvestres

Référence aux annexes de la

Convention sur le commerce international des espèces de faune et de flore sauvages menacées d'extinction

Compiled by the/Recopilada por el/Compilée par le

World Conservation Monitoring Centre

Centro Mundial de Monitoreo de la Conservación

Centre mondial de surveillance continue de la conservation de la nature

With financial support from the

Secretariat of the Convention on International Trade in

Endangered Species of Wild Fauna and Flora

the European Commission

and the

Joint Nature Conservation Committee

Con la asistencia financiera de la

Secretaría de la Convención sobre el Comercio Internacional

de Especies Amenazadas de Fauna y Flora Silvestres, de la Comisión Europea

y del

Joint Nature Conservation Committee

Avec l'appui financier du

Secrétariat de la Convention sur le commerce international des espèces de faune et de flore sauvages menacées d'extinction,

de la Commission Européenne

et du

Joint Nature Conservation Committee 
The World Conservation Monitoring Centre, based in Cambridge, UK, is a joint-venture between the three partners in the World Conservation Strategy and its successor Caring For The Earth: IUCN - The World Conservation Union, UNEP - United Nations Environment Programme, and WWF - World Wide Fund for Nature. The Centre provides information services on the conservation and sustainable use of species and ecosystems and supports others in the development of their own information systems.

El Centro Mundial de Monitoreo de la Conservación, Cambridge, Reino Unido, fue fundado por las tres organizaciones que participaron en la publicación de la Estrategia Mundial para la Conservación y, posteriormente, de Cuidar la Tierra, a saber, la Unión Mundial para la Naturaleza - UICN, el Programa de las Naciones Unidas para el Medio Ambiente - PNUMA y el Fondo Mundial para la Naturaleza - WWF. El Centro ofrece servicios de información sobre la conservación y el uso sostenible de especies y ecosistemas y presta apoyo a terceros en el establecimiento de sus propios sistemas de información.

Le Centre mondial de surveillance continue de la conservation de la nature (Cambridge, Royaume-Uni) est une entreprise commune des trois partenaires de la Stratégie mondiale de la conservation et de Sauvez la Planète: l'Union mondiale pour la nature (UICN), le Programme des Nations Unies pour l'environnement (PNUE) et le Fonds mondial pour la nature (WWF). Le Centre fournit un service d'information sur la conservation et l'exploitation durable des espèces et des écosystèmes et assiste d'autres organismes dans l'élaboration de leurs propres systèmes d'information. 


\section{CONTENTS}

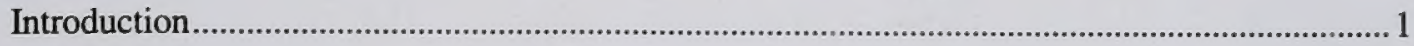

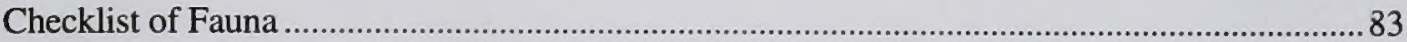

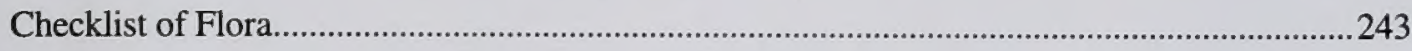

\section{INDICE}

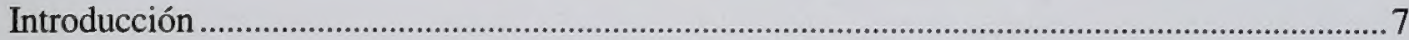

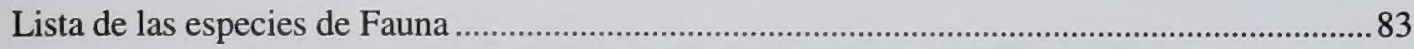

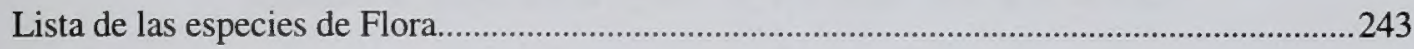

\section{TABLE DES MATIERES}

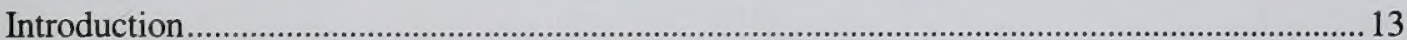

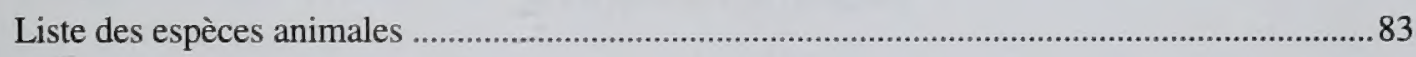

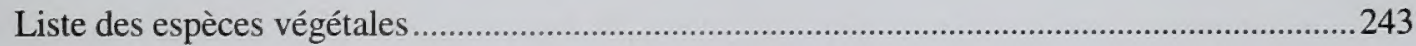


Digitized by the Internet Archive in 2010 with funding from UNEP-WCMC, Cambridge 


\title{
CHECKLIST OF CITES SPECIES
}

\author{
A reference to the appendices to the \\ Convention on International Trade in Endangered Species \\ of Wild Fauna and Flora
}

INTRODUCTION 
Compiled by:

Prepared for:

With financial support from:

Published by:

Copyright:

ISBN:

Cover design by:

Printed by:

Available from:
World Conservation Monitoring Centre, Cambridge, UK

\section{CITES Secretariat}

CITES Secretariat, European Commission and Joint Nature Conservation Committee
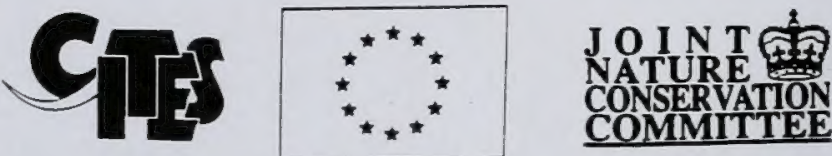

CITES Secretariat/World Conservation Monitoring Centre

1998 CITES Secretariat/World Conservation Monitoring Centre

Reproduction of this publication for educational or other noncommercial purposes is authorized without prior permission from the copyright holders.

Reproduction for resale or other commercial purposes by any means - photographic, electronic or mechanical, including photocopying, recording, taping or information storage and retrieval systems - is prohibited without the prior written permission of the copyright holders.

The geographical designations employed in this book do not imply the expression of any opinion whatsoever on the part of the compilers or the CITES Secretariat concerning the legal status of any country, territory, or area, or concerning the delimitation of its frontiers or boundaries.

1899628088

Michael Edwards

Unwin Brothers, Martins Printing Group, Old Woking, Surrey.

CITES Secretariat

15, Chemin des Anémones

CH-1219 Châtelaine-Genève

Switzerland

tel: $\quad(+4122) 9178139 / 40$

fax: $\quad(+4122) 7973417$

E-mail:cites@unep.ch 


\section{CONTENTS}

Foreword 4

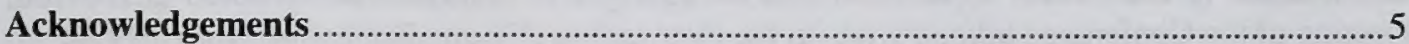

User Guide

\section{CITES Appendices I and II}

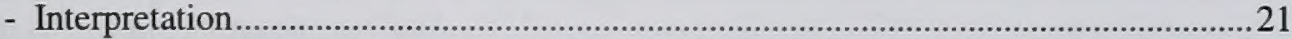

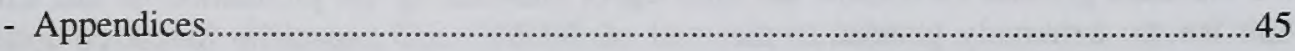

\section{CITES Appendix III}

- Interpretation

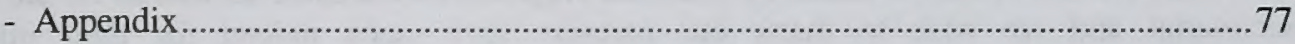

\section{Checklist of Fauna}

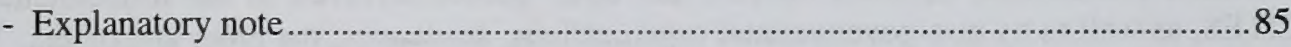

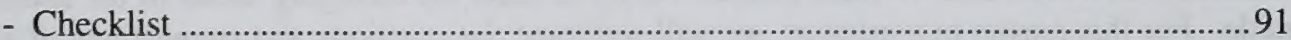

\section{Checklist of Flora}

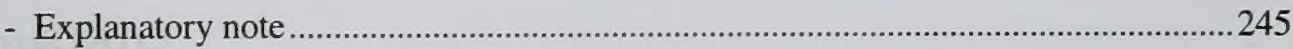

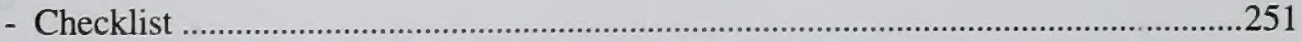




\section{Foreword}

This latest edition of the Checklist of CITES Species has been revised to take into account the amendments to the CITES appendices and the changes in nomenclature adopted at the 10th meeting of the Conference of the Parties to the Convention (Harare, Zimbabwe, June 1997).

Another important development is that many of the common names in French and Spanish, which were missing from the previous edition, have now been included. For its assistance in filling the gaps in the French names, we wholeheartedly thank the National Museum of Natural History, in Paris, France.

We are most grateful to WCMC for once again undertaking the production of this Checklist. And we are extremely grateful to the staff of WCMC, and especially to the compiler, Tim Inskipp, for the enormous amount of work that has gone into the research for and production of this book.

The costs of preparation and production have been borne by the European Commission, the Joint Nature Conservation Committee (United Kingdom) and WCMC as well as by the CITES Secretariat and we wish to thank our partners very much indeed for their important contributions.

We know that this Checklist is widely used and appreciated by Management Authorities, Scientific Authorities, Customs officers and other people involved in the implementation of CITES, as well as by non-governmental organizations, academics, the press and others. We are conscious, however, that we must continue to try to meet the changing needs of the users of this guide. For this reason in particular, we will welcome the reactions and suggestions of all users on ways to improve it in the future.

CITES Secretariat

Geneva

1998 


\section{Acknowledgements}

This checklist was prepared on behalf of the CITES Secretariat by the World Conservation Monitoring Centre. The compiler, Tim Inskipp, would like to thank the following WCMC staff for their invaluable assistance with various aspects of the checklist: project development and management - Tim Johnson; technical - Martin Sneary; plant data - Harriet Gillett and Amy MacKinven; general assistance - Julie Reay and Rose Warwick

For their assistance with the provision of additional French and Spanish common names, the compiler is most grateful to the staff of the CITES Secretariat, Jaques Berney, and the Muséum National d'Histoire Naturelle in Paris.

For their advice and assistance throughout, the compiler would like to thank the CITES Secretariat.

WCMC wishes to acknowledge the financial support of the Joint Nature Conservation Committee, UK in the production of this checklist, and for its past support of the four-volume series of JNCC checklists of CITES-listed species.

WCMC also gratefully acknowledges the financial contribution from the European Commission which has supported the production of this checklist. 


\section{USER GUIDE}

This checklist has been drawn up to provide alphabetical lists of the species of fauna and flora listed in Appendices I, II and III of CITES. It is hoped that these will act as an aid to Management and Scientific Authorities, Customs officials, and all others involved in implementing and enforcing the Convention.

The fauna and flora are treated in separate lists because the format and information content of each list is different.

For each group there is an explanatory note describing the information content, the layout of the different individual entries, and the taxonomic sources used in the compilation of the list. This is followed by the current CITES appendices (I and II together and III separately) with the official Interpretations thereof, and finally the alphabetical checklist.

The names used in the CITES appendices are scientific names. In this checklist common names are included only for reference. If the user looks for a common name of a species included in the CITES Appendices and does not find it, he/she should look under its scientific name or under another common name. 


\section{LISTA DE LAS ESPECIES CITES}

Una referencia a las especies incluidas en los Apéndices de la Convención sobre el Comercio Internacional de Especies Amenazadas de Fauna y Flora Silvestres

\section{INTRODUCCION}


Recopilado por:

Preparado para:

Con la asistencia financiera de:

Publicado por:

Copyright:

ISBN:

Diseño de portada:

Impreso por:

Distribuido por:
El Centro Mundial de Monitoreo de la Conservación, Cambridge, Reino Unido

La Secretaría de la CITES

La Secretaría de la CITES, la Comisión Europea y el Joint Nature Conservation Committee
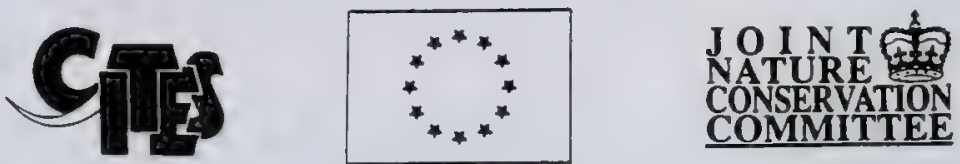

La Secretaría de la CITES/El Centro Mundial de Monitoreo de la Conservación

1998 La Secretaría de la CITES/El Centro Mundial de Monitoreo de la Conservación

Esta publicación puede reproducirse, con fines didácticos o con otros propósitos no comerciales, sin la previa autorización de los titulares del copyright.

No está permitida la reproducción de esta publicación, para la reventa o cualquier otro fin comercial, por cualquier medio, ya sea fotográfico, electrónico o mecánico, inclusive fotocopia, grabación, mecanografía, o cualquier sistema de almacenamiento o recuperación de información, sin la previa autorización por escrito de los titulares del copyright.

Las denominaciones geográficas empleadas en esta publicación no implican juicio alguno por parte de sus autores o la Secretaría CITES sobre la condición jurídica de ninguno de los países, zonas o territorios, ni respecto de la delimitación de sus fronteras o límites.

1899628088

Michael Edwards

Unwin Brothers, Martins Printing Group, Old Woking, Surrey.

Secretaría de la CITES

15, Chemin des Anémones

CH-1219 Châtelaine-Ginebra

Suiza

tel: $\quad(+4122) 9178139 / 40$

fax: $\quad(+4122) 7973417$

E-mail: cites@unep.ch 


\section{INDICE}

Prólogo

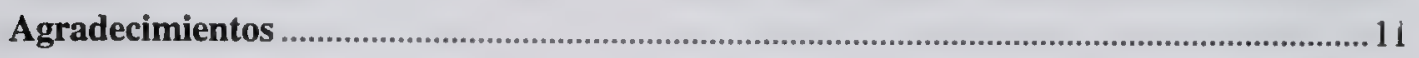

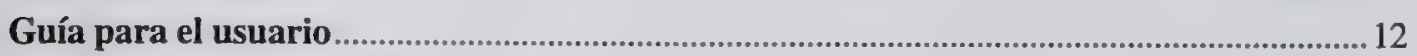

Apéndices I y II de la CITES

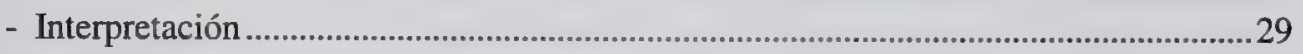

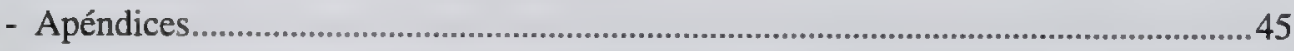

Apéndice III de la CITES

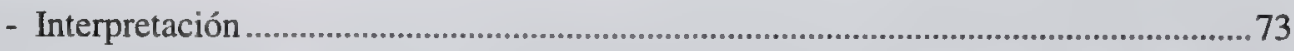

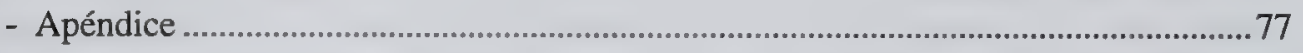

Lista de las especies de Fauna

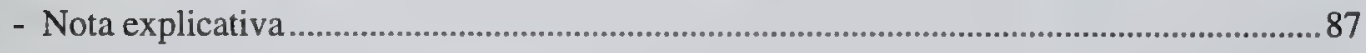

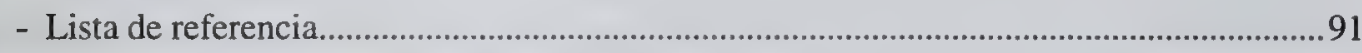

Lista de las especies de Flora

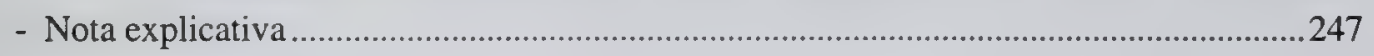

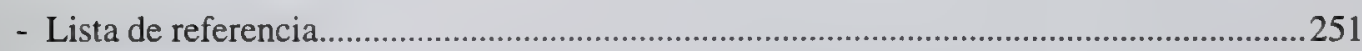




\section{Prólogo}

Esta última versión de la Lista de las especies CITES se ha revisado a fin de reflejar las enmiendas a los Apéndices de la CITES y las modificaciones en la nomenclatura aprobadas en la décima reunión de la Conferencia de las Partes en la Convención (Harare, Zimbabwe, junio de 1997).

Cabe destacar que en esta versión se han incluido muchos de los nombres comunes en español y francés que no figuraban en la versión precedente. En este sentido, agradecemos encarecidamente al Museo Nacional de Historia Natural, en París, Francia, por la asistencia prestada para colmar las lagunas en lo que concierne a los nombres comunes en francés.

Expresamos nuestro agradecimiento al WCMC por haber acometido nuevamente la tarea de producir esta Lista. Damos las gracias al personal del WCMC y, en particular, a Tim Inskipp, encargado de la compilación, por la ingente tarea que ha realizado en cuanto a la investigación y producción de esta obra.

Los gastos de preparación y producción de esta obra fueron sufragados por la Comisión Europea, el Joint Nature Conservation Committee (Reino Unido) y el WCMC, así como por la Secretaría de la CITES. Deseamos dar las gracias a nuestros colaboradores por sus importantes contribuciones.

Sabemos que esta Lista es ampliamente utilizada y apreciada por las Autoridades Administrativas, las Autoridades Científicas, los funcionarios de aduanas y otras personas concernidas en la aplicación de la Convención, así como por las organizaciones no gubernamentales, los académicos, los medios de comunicación y otros interesados. No obstante, somos conscientes de que debemos seguir tratando de satisfacer las necesidades cambiantes de los usuarios de esta guía y, en este sentido, acogemos con beneplácito los comentarios y sugerencias de todos los usuarios sobre la posibilidad de mejorarla en el futuro.

Secretaría de la CITES

Ginebra

1998 


\section{Agradecimientos}

La presente lista fue preparada por el Centro Mundial de Monitoreo de la Conservación en nombre de la Secretaria de la CITES. Tim Inskipp, encargado de la compilación, desea dar las gracias a los siguientes miembros del personal del WCMC por su valiosa asistencia en diversos aspectos de la lista: a Tim Johnson por la gestión y desarrollo del proyecto; a Martin Sneary por la asistencia técnica; a Harriet Gillett y Amy MacKinven por la documentación sobre las plantas; y a Julie Reay y Rose Warwick por su asistencia general.

Por su ayuda en lo que concierne a la aportación de nuevos nombres comunes en español y francés, los autores de esta obra expresan su agradecimiento a Jaques Berney, de la Secretaría de la CITES, y al Museo Nacional de Historia Natural en París.

El autor desea dar las gracias a la Secretaría de la CITES por su asesoramiento y asistencia a lo largo de todo el proceso de preparación de la obra.

El WCMC desea dejar constancia del apoyo financiero del Joint Nature Conservation Committee, Reino Unido, para la producción de esta lista, así como por su apoyo prestado en el pasado para la preparación de los cuatro volúmenes de las listas de las especies incluidas en la CITES.

El WCMC agradece encarecidamente la contribución financiera de la Comisión Europea, sin la cual esta publicación no habría visto la luz. 


\section{GUIA DEL USUARIO}

La presente lista se ha preparado con objeto de ofrecer una ordenación alfabética de las especies de fauna y flora incluidas en los Apéndices I, II y III de la CITES. Albergamos la esperanza de que será de gran utilidad para las Autoridades Administrativas y Científicas, los funcionarios de aduanas y todas las demás personas encargadas de aplicar la Convención.

La fauna y la flora se presentan en listas separadas debido a que el formato y el contenido informático de cada lista es diferente.

Cada lista consta de una nota explicativa en la que se describe el contenido informático, la disposición de cada una de las entradas y las fuentes taxonómicas utilizadas en la compilación de la lista; de los actuales Apéndices de la CITES (I y II conjuntamente, y III por separado) con las interpretaciones oficiales de los mismos; y de la lista de referencia por orden alfabético.

En los Apéndices de la CITES se utilizan los nombres científicos. En esta lista se incluyen los nombres comunes únicamente como referencia. En consecuencia, si una especie incluida en los Apéndices de la CITES no figura bajo un nombre común, el usuario deberá buscarla bajo su nombre científico u otro nombre común. 


\title{
LISTE DES ESPECES CITES
}

\author{
Référence aux annexes de la \\ Convention sur le commerce international des espèces de faune et de flore \\ sauvages menacées d'extinction
}

\section{INTRODUCTION}


Compilation:

Pour:

Appui financier:

Publication:

Copyright:

ISBN:

Couverture:

Imprimerie:

Disponible au:
Centre mondial de surveillance continue de la conservation de la nature, Cambridge, R-U.

\section{le Secrétariat CITES}

Secrétariat CITES, Commission européenne et Joint Nature Conservation Committee
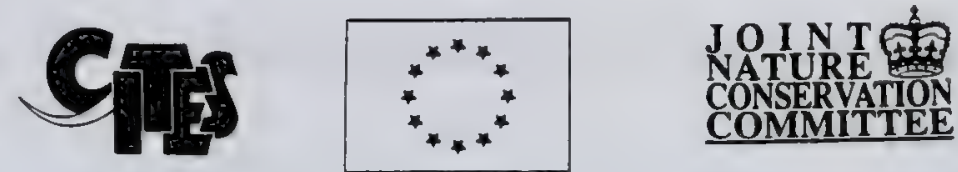

Secrétariat CITES et Centre mondial de surveillance continue de la conservation de la nature

1998 Secrétariat CITES/Centre mondial de surveillance continue de la conservation de la nature

La reproduction de cette publication à des fins non commerciales, éducatives ou autres, est autorisée sans l'autorisation préalable des détenteurs du copyright.

La reproduction destinée à la revente ou à d'autres fins commerciales par quelque moyen que ce soit - photographique, électronique ou mécanique, y compris la photocopie, l'enregistrement sur disque ou sur bande, ou par des systèmes de stockage et de recherche documentaires - est interdite sans l'autorisation écrite préalable des détenteurs du copyright.

Les appellations géographiques employées dans cette publication n'impliquent de la part des compilateurs et du Secrétariat CITES aucune prise de position quant au statut juridique des pays, territoires ou zones, ni quant à leurs frontières ou limites.

\section{8}

\section{Michael Edwards}

Unwin Brothers, Martins Printing Group, Old Woking, Surrey.

\author{
Secrétariat CITES \\ 15, chemin des Anémones \\ 1219 - Châtelaine-Genève \\ Suisse \\ $\mathrm{N}^{0}$ de téléphone: $(+4122) 9178139 / 40$ \\ $\mathrm{N}^{\circ}$ de fax: $\quad(+4122) 7973417$ \\ E-mail: cites@unep.ch
}




\section{TABLE DES MATIERES}

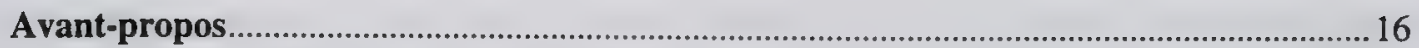

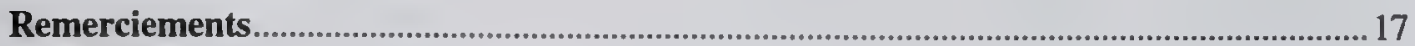

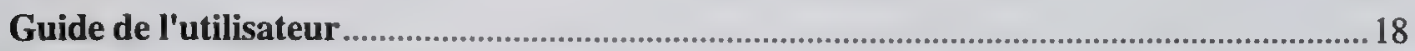

\section{Annexes I et II de la CITES}

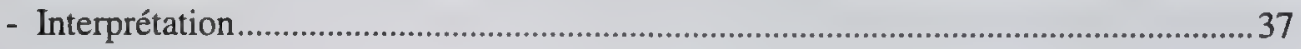

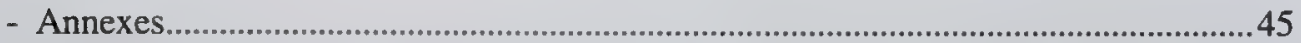

Annexe III de la CITES

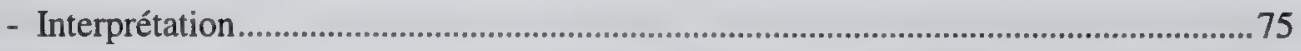

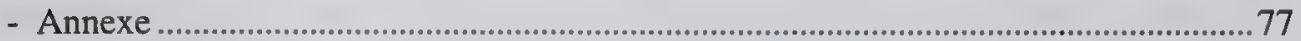

Liste des espèces animales

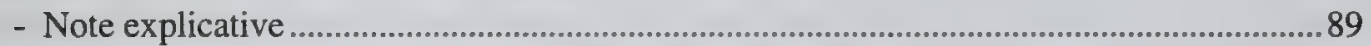

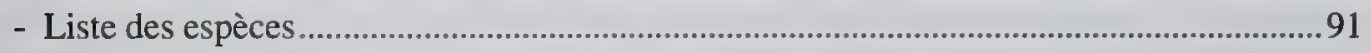

Liste des espèces végétales

- Note explicative

- Liste des espèces. 


\section{Avant-propos}

Cette nouvelle édition de la Liste des espèces CITES a été révisée pour tenir compte des amendements aux annexes CITES adoptés à la $10^{\mathrm{e}}$ session de la Conférence des Parties à la Convention. Elle tient également compte des changements de nomenclature adoptés par la Conférence des Parties (Harare, Zimbabwe, juin 1997).

Autre point important: bon nombre des noms communs en français et en espagnol qui manquaient dans l'édition précédente sont à présent inclus. Nos vifs remerciements vont au Muséum national d'histoire naturelle, Paris, France, qui nous a aidé à combler les lacunes dans les noms communs en français.

Nous sommes très reconnaissants au WCMC qui s'est à nouveau chargé de la production de la Liste des espèces CITES, à son personnel, et en particulier au compilateur, Tim Inskipp, pour leur travail considérable de recherche et de production de cet ouvrage.

Les frais de préparation et de production ont été pris en charge par la Commission européenne, le Joint Nature Conservation Committee (Royaume-Uni) et le WCMC, ainsi que par le Secrétariat CITES. Nous tenons à remercier très vivement tous nos partenaires pour leur importante contribution.

Nous savons que la Liste des espèces CITES est largement utilisée et très appréciée par les organes de gestion, les autorités scientifiques, les douanes et les autres personnes impliquées dans la mise en oeuvre de la CITES, ainsi que par les organisations non gouvernementales, les scientifiques, la presse et autres entités. Nous sommes par ailleurs conscients que nous devons continuer de nous adapter aux besoins nouveaux des utilisateurs de ce guide. C'est pour cette raison, entre autres, que nous serions heureux de connaître les réactions et les suggestions de tous les utilisateurs de cet ouvrage sur la manière dont nous pourrions l'améliorer à l'avenir.

CITES Secrétariat

Genève

1998 


\section{Remerciements}

La Liste des espèces CITES a été préparée au nom du Secrétariat CITES par le Centre mondial de surveillance continue de la nature. Son compilateur, Tim Inskipp, tient à remercier les membres suivants du personnel du WCMC pour leur précieuse assistance à différents niveaux: Tim Johnson pour l'élaboration et la gestion des projets, Martin Sneary pour son assistance technique, Harriet Gillett et Amy MacKinven pour les données sur les plantes, Julie Reay et Rose Warwick pour leur assistance générale.

Les compilateurs sont très reconnaissants au personnel du Secrétariat CITES, à Jaques Berney et au Muséum national d'histoire naturelle de Paris qui leur ont foumi certains noms communs en français et en espagnol.

Le compilateur remercie le Secrétariat CITES pour les conseils et l'assistance qu'il lui a prodigués tout au long de la préparation de l'ouvrage.

Le WCMC remercie le Joint Nature Conservation Committee, Royaume-Uni, pour son appui financier à la production de la Liste des espèces CITES et pour son appui passé aux listes des espèces CITES du JNCC.

Le WCMC exprime également sa reconnaissance à la Commission européenne pour sa contribution financière à la production de la Liste des espèces CITES. 


\section{GUIDE DE L'UTILISATEUR}

La liste des espèces CITES comprend des listes alphabétiques des espèces animales et végétales inscrites aux Annexes I, II et III de la CITES. Les compilateurs espèrent qu'elles seront utiles aux organes de gestion et aux autorités scientifiques, ainsi qu'aux douaniers et à toute personne ou service chargé de faire appliquer la Convention et de lutter contre la fraude.

La faune et la flore sont traitées séparément car la présentation et le type de renseignements donnés dans chaque liste sont différents.

Chaque liste est précédée par une note explicative décrivant le type de renseignements communiqués, la disposition des différentes entrées et les ouvrages de référence utilisés. Chaque note est suivie des annexes CITES actuelles (I et II ensemble et III séparément) et leur interprétation officielle. Enfin, la liste des espèces est donnée par ordre alphabétique.

Les noms utilisés dans les annexes CITES sont les noms scientifiques. Dans la liste, les noms communs sont donnés à titre de référence. Si l'utilisateur n'y trouve pas le nom commun d'une espèce, cela ne signifie pas qu'elle n'est pas inscrite aux annexes CITES; il devrait la chercher sous son nom scientifique ou sous un autre nom commun. 
APPENDICES I AND II

APPENDICES I Y II

ANNEXES I ET II 



\section{APPENDICES I AND II}

as adopted by the Conference of the Parties, valid from 18 September 1997

\section{INTERPRETATION}

1. Species included in these appendices are referred to:

a) by the name of the species; or

b) as being all of the species included in a higher taxon or designated part thereof.

2. The abbreviation "spp." is used to denote all species of a higher taxon.

3. Other references to taxa higher than species are for the purposes of information or classification only.

4. The following abbreviations are used for plant taxa below the level of species:

a) "ssp." is used to denote subspecies;

b) "var(s)." is used to denote variety (varieties); and

c) "fa." is used to denote forma.

5. The abbreviation "p.e." is used to denote species that are possibly extinct.

6. An asterisk $\left({ }^{*}\right)$ placed against the name of a species or higher taxon indicates that one or more geographically separate populations, subspecies or species of that species or taxon are included in Appendix I and are excluded from Appendix II.

7. Two asterisks $(* *)$ placed against the name of a species or higher taxon indicate that one or more geographically separate populations, subspecies or species of that species or taxon are included in Appendix II and are excluded from Appendix I.

8. The symbol (-) followed by a number placed against the name of a species or higher taxon denotes that designated geographically separate populations, species, groups of species or families of that species or taxon are excluded from the appendix concerned, as follows:

$-101 \quad$ Population of West Greenland

-102 Populations of Bhutan, India, Nepal and Pakistan

-103 Populations of Botswana, Namibia and Zimbabwe

-104 Population of Australia

-105 Populations of Pecari tajacu of Mexico and the United States of America

-106 - Argentina: the population of the Province of Jujuy and the semi-captive populations of the Provinces of Jujuy, Salta, Catamarca, La Rioja and San Juan

- Bolivia: the populations of the Conservation Units of Mauri-Desaguadero, Ulla Ulla and Lípez-Chichas, with a zero annual export quota

- Chile: part of the population of Parinacota Province, 1a. Region of Tarapacá

- Peru: the whole population

Populations of Afghanistan, Bhutan, India, Myanmar, Nepal and Pakistan

-108 Cathartidae

-109 Melopsittacus undulatus, Nymphicus hollandicus and Psittacula krameri

-110 Population of Argentina

-111 Population of Ecuador, subject to a zero annual export quota until an annual export quota has been approved by the CITES Secretariat and the IUCN/SSC Crocodile Specialist Group

-112 Populations of Botswana, Ethiopia, Kenya, Madagascar, Malawi, Mozambique, South Africa, Uganda, the United Republic of Tanzania, Zamoia and Zimbabwe

Apart from ranched specimens, the United Republic of Tanzania will authorize the export of no more than 1100 wild specimens (including 100 hunting trophies) in 1998, 1999 and 2000 
-114 Population of Chile

-115 All species that are not succulent

-116 Aloe vera; also referenced as Aloe barbadensis.

9. The symbol (+) followed by a number placed against the name of a species, subspecies or higher taxon denotes that only designated geographically separate populations of that species, subspecies or taxon are included in the appendix concerned, as follows:

+201 Populations of Bhutan, India, Nepal and Pakistan

+202 Populations of Bhutan, China, Mexico and Mongolia

+203 Populations of Cameroon and Nigeria

+204 Population of Asia

+205 Populations of Central and North America

+206 Populations of Bangladesh, India and Thailand

+207 Population of India

+208 Populations of Botswana, Namibia and Zimbabwe

+209 Population of Australia

+210 Population of South Africa

+211 - Argentina: the population of the Province of Jujuy and the semi-captive populations of the Provinces of Jujuy, Salta, Catamarca, La Rioja and San Juan

- Bolivia: the populations of the Conservation Units of Mauri-Desaguadero, Ulla Ulla and Lipez-Chichas, with a zero annual export quota

- Chile: part of the population of Parinacota Province, la. Region of Tarapacá

- Peru: the whole population

+212 Populations of Afghanistan, Bhutan, India, Myanmar, Nepal and Pakistan

+213 Population of Mexico

+214 Populations of Algeria, Burkina Faso, Cameroon, the Central African Republic, Chad, Mali, Mauritania, Morocco, the Niger, Nigeria, Senegal and the Sudan

+215 Population of Seychelles

+216 Population of Europe, except the area which formerly constituted the Union of Soviet Socialist Republics

+217 Population of Chile.

10. The symbol $(\Rightarrow$ ) followed by a number placed against the name of a species, subspecies or higher taxon denotes that the name of that species, subspecies or taxon shall be interpreted as follows:

$=301 \quad$ Also referenced as Phalanger maculatus

$=302$ Includes family Tupaiidae

$=303$ Formerly included in family Lemuridae

$=304$ Formerly included as subspecies of Callithrix jacchus

$=305 \quad$ Includes generic synonym Leontideus

$=306$ Formerly included in species Saguinus oedipus

$=307$ Formerly included in Alouatta palliata

$=308$ Formerly included as Alouatta palliata (villosa)

$=309$ Includes synonym Cercopithecus roloway

$=310 \quad$ Formerly included in genus Papio

$=311$ Includes generic synonym Simias

$=312$ Includes synonym Colobus badius kirkii

$=313$ Includes synonym Colobus badius rufomitratus

$=314$ Includes generic synonym Rhinopithecus

$=315$ Also referenced as Presbytis entellus

$=316$ Also referenced as Presbytis geei and Semnopithecus geei

$=317$ Also referenced as Presbytis pileata and Semnopithecus pileatus

$=318$ Includes synonyms Bradypus boliviensis and Bradypus griseus

$=319$ Includes synonym Priodontes giganteus

$=320$ Includes synonym Physeter macrocephalus

$=321$ Includes synonym Eschrichrius glaucus

$=322$ Formerly included in genus Balaena

$=323$ Formerly included in genus Dusicyon 
Includes synonym Dusicyon fulvipes

$=326$

$=327$

$=328$

$=329$

$=330$

$=331$

$=332$

$=333$

$=334$

$=335$

$=336$

$=337$

$=338$

$=339$

$=340$

$=341$

$=342$

$=343$

$=344$

$=345$

$=346$

$=347$

$=348$

$=349$

$=350$

$=351$

$=352$

$=353$

$=354$

$=355$

$=356$

$=357$

$=358$

$=359$

$=360$

$=361$

$=362$

$=363$

$=364$

$=365 \mathrm{a}$

$=365 \mathrm{~b}$

$=366$

$=367$

$=368$

$=369$

$=370$

$=371$

$=372$

$=373$

$=374$

$=375$

$=376$

$=377$

Includes generic synonym Fennecus

Also referenced as Selenarctos thibetanus

Also referenced as Aonyx microdon or Paraonyx microdon

Formerly included in genus Lutra

Formerly included in genus Lutra; includes synonyms Lutra annectens, Lutra enudris,

Lutra incarum and Lutra platensis

Includes synonym Eupleres major

Also referenced as Hyaena brunnea

Also referenced as Felis caracal and Lynx caracal

Formerly included in genus Felis

Also referenced as Felis pardina or Felis lynx pardina

Formerly included in genus Panthera

Also referenced as Equus asinus

Formerly included in species Equus hemionus

Also referenced as Equus caballus przewalskii

Also referenced as Choeropsis liberiensis

Also referenced as Cervus porcinus annamiticus

Also referenced as Cervus porcinus calamianensis

Also referenced as Cervus porcinus kuhlii

Also referenced as Cervus dama mesopotamicus

Includes synonym Bos frontalis

Includes synonym Bos grunniens

Includes generic synonym Novibos

Includes generic synonym Anoa

Also referenced as Damaliscus dorcas dorcas or Damaliscus pygargus dorcas

Formerly included in species Naemorhedus goral

Also referenced as Capricornis sumatraensis

Includes synonym Oryx tao

Includes synonym Ovis aries ophion

Formerly included as Ovis vignei (see also Decisions of the Conference of the Parties directed to the Parties regarding the inclusion of Ovis vignei vignei in Appendix I)

Also referenced as Rupicapra rupicapra ornata

Also referenced as Pterocnemia pennata

Also referenced as Sula abbotti

Also referenced as Ciconia ciconia boyciana

Includes synonyms Anas chlorotis and Anas nesiotis

Also referenced as Anas platyrhynchos laysanensis

Probably a hybrid between Anas platyrhynchos and Anas superciliosa

Also referenced as Aquila heliaca adalberti

Also referenced as Chondrohierax wilsonii

Also referenced as Falco peregrinus babylonicus and Falco peregrinus pelegrinoides

Also referenced as Crax mitu mitu

Formerly included in genus Aburria

Formerly included in genus Aburria; also referenced as Pipile pipile pipile

Formerly included in species Crossoptilon crossoptilon

Formerly included in species Polyplectron malacense

Includes synonym Rheinardia nigrescens

Also referenced as Tricholimnas sylvestris

Also referenced as Choriotis nigriceps

Also referenced as Houbaropsis bengalensis

Also referenced as Amazona dufresniana rhodocorytha

Often traded under the incorrect designation Ara caninde

Also referenced as Cyanoramphus novaezelandiae cookii

Also referenced as Opopsitta diophthalma coxeni

Also referenced as Pezoporus occidentalis

Formerly included in species Psephotus chrysopterygius

Also referenced as Psittacula krameri echo

$=379$

$=380$

Formerly included in genus Gallirex; also referenced as Tauraco porphyreolophus

Also referenced as Otus gurneyi 
$=382$ Formerly included in genus Glaucis

$=383$ Includes generic synonym Ptilolaemus

$=384$ Formerly included in genus Rhinoplax

$=385$

Also referenced as Pitta brachyura nympha

$=387$

Also referenced as Muscicapa ruecki or Niltava ruecki

$=388$

Also referenced as Dasyornis brachypterus longirostris

$=389$

Also referenced as Meliphaga cassidix

$=390$

$=391$

$=392$

$=393$

$=394$

$=395$

$=396$

$=397$

$=398$

$=399$

$=400$

$=401$

$=402$

$=403$

$=404$

$=405$

$=406$

$=407$

$=408$

$=409$

$=410$

$=411$

$=412$

$=413$

$=414$

$=415$

$=416$

$=417$

$=418$

$=419$

$=420$

$=421$

$=422$

$=423$

$=424$

$=425$

Includes generic synonym Xanthopsar

Formerly included in genus Spinus

Also referenced in genus Damonia

Formerly included as Kachuga tecta tecta

Includes generic synonyms Nicoria and Geoemyda (part)

Also referenced as Geochelone elephantopus; also referenced in genus Testudo

Also referenced in genus Testudo

Also referenced in genus Aspideretes

Formerly included in Podocnemis spp.

Includes Alligatoridae, Crocodylidae and Gavialidae

Also referenced as Crocodylus mindorensis

Also referenced in genus Nactus

Includes generic synonym Rhoptropella

Formerly included in Chamaeleo spp.

Includes generic synonyms Calumma and Furcifer

Includes families Bolyeriidae and Tropidophiidae as subfamilies

Also referenced as Constrictor constrictor occidentalis

Includes synonym Python molurus pimbura

Includes synonym Sanzinia manditra

Includes synonym $\boldsymbol{P}$ seudoboa cloelia

Also referenced as Hydrodynastes gigas

Includes synonyms Naja atra, Naja kaouthia, Naja oxiana, Naja philippinensis,

Naja samarensis, Naja sputatrix and Naja sumatrana

Includes generic synonym Megalobatrachus

Formerly included in Nectophrynoides spp.

Formerly included in Dendrobates spp.

Also referenced in genus Rana

Sensu D'Abrera

Includes synonyms Pandinus africanus and Heterometrus roeseli

Includes generic synonym Aphonopelma

Also referenced as Conchodromus dromas

Also referenced in genera Dysnomia and Plagiola

Includes generic synonym Proptera

Also referenced in genus Carunculina

Also referenced as Megalonaias nickliniana

Also referenced as Cyrtonaias tampicoensis tecomatensis and

Lampsilis tampicoensis tecomatensis

Includes generic synonym Micromya

Includes generic synonym Papuina

$=426$

$=427$

$=428$

$=429$

$=430$

$=431$

$=432$

$=433$

$=434$

$=435$

$=436$

Includes only the family Helioporidae with one species Heliopora coerulea Also referenced as Podophyllum emodi and Sinopodophyllum hexandrum Includes generic synonyms Neogomesia and Roseocactus

Also referenced in genus Echinocactus

Also referenced in genus Mammillaria; includes synonym Coryphantha densispina Also referenced as Lobeira macdougallii and Nopalxochia macdougallii

Also referenced as Echinocereus lindsayi

Also referenced in genera Cereus and Wilcoxia

Also referenced in genus Coryphantha; includes synonym Escobaria nellieae

Also referenced in genus Coryphantha; includes Escobaria leei as a subspecies

Includes synonym Solisia pectinata 
Also referenced as Backebergia militaris, Cephalocereus militaris and Mitrocereus militaris; includes synonym Pachycereus chrysomallus

$=438 \quad$ Includes Pediocactus bradyi ssp. despainii and Pediocactus bradyi ssp. winkleri and synonyms Pediocactus despainii and Pediocactus winkleri

$=439 \quad$ Also referenced in genus Toumeya

$=440$ Also referenced in genera Navajoa, Toumeya and Utahia;

includes Pediocactus peeblesianus var. fickeisenii

$=441$ Also referenced in genera Echinocactus and Utahia

$=442$ Includes generic synonym Encephalocarpus

$=443$

$=444$

Includes synonyms Ancistrocactus tobuschii and Ferocactus tobuschii

Also referenced in genera Echinomastus and Neolloydia;

includes synonyms Echinomastus acunensis and Echinomastus krausei

$=445$ Includes synonyms Ferocactus glaucus, Sclerocactus brevispinus, Sclerocactus wetlandicus and Sclerocactus wetlandicus ssp. ilseae

$=446$ Also referenced in genera Echinocactus, Echinomastus and Neolloydia

$=447$

$=448$

$=449$

$=450$

$=451$

$=452$

$=453$

$=454$

$=455$

$=456$

$=457$

$=458$

$=459$

$=460$

$=461$

$=462$

$=463$

$=464$

$=465$

$=466$

$=467$

$=468$

$=469$

Also referenced in genera Coloradoa, Echinocactus, Ferocactus and Pediocactus

Also referenced in genera Echinocactus, Mammillaria, Pediocactus and Toumeya

Also referenced in genera Echinocactus and Ferocactus

Also referenced in genus Pediocactus

Includes generic synonyms Gymnocactus, Normanbokea and Rapicactus

Also referenced as Saussurea lappa

Also referenced as Euphorbia decaryi var. capsaintemariensis

Includes Euphorbia cremersii fa. viridifolia and Euphorbia cremersii var, rakotozafyi

Includes Euphorbia cylindrifolia ssp. tuberifera

Includes Euphorbia decaryi vars. ampanihyensis, robinsonii and spirosticha

Includes Euphorbia moratii vars. antsingiensis, bemarahensis and multiflora

Also referenced as Euphorbia capsaintemariensis var. tulearensis

Also referenced as Engelhardia pterocarpa

Includes Alve compressa var. rugosquamosa and Aloe compressa var. schistophila

Includes Aloe haworthioides var. aurantiaca

Includes Aloe laeta var. maniaensis

Includes families Apostasiaceae and Cypripediaceae as subfamilies Apostasioideae and Cypripedioideae

Anacampseros australiana and A. kurtzii are also referenced in genus Grahamia

Formerly included in Anacampseros spp.

Also referenced as Sarracenia rubra ssp. alabamensis

Also referenced as Sarracenia rubra ssp. jonesii

Formerly included in ZAMIACEAE spp.

$=470$

$=471$

Includes synonym Stangeria paradoxa

Also referenced as Taxus baccata ssp. wallichiana

Includes synonym Welwitschia bainesii.

11. The symbol $\left({ }^{\circ}\right)$ followed by a number placed against the name of a species or higher taxon shall be interpreted as follows:

${ }^{\circ} 601$ A zero annual export quota has been established. All specimens shall be deemed to be specimens of species included in Appendix I and the trade in them shall be regulated accordingly

${ }^{\circ} 602$ Specimens of the domesticated form are not subject to the provisions of the Convention

${ }^{\circ} 603$ Annual export quotas for live specimens and hunting trophies are granted as follows:

$\begin{array}{lr}\text { Botswana: } & 5 \\ \text { Namibia: } & 150 \\ \text { Zimbabwe: } & 50\end{array}$

The trade in such specimens is subject to the provisions of Article III of the Convention

${ }^{\circ} 604$ For the exclusive purpose of allowing: 1) export of hunting trophies for non-commercial purposes; 2) export of live animals to appropriate and acceptable destinations (Namibia: for non- 
commercial purposes only); 3) export of hides (Zimbabwe only); 4) export of leather goods and ivory carvings for non-commercial purposes (Zimbabwe only). No international trade in ivory is permitted before 18 months after the transfer to Appendix II comes into effect (i.e. 18 March 1999). Thereafter, under experimental quotas for raw ivory not exceeding 25.3 tonnes (Botswana), 13.8 tonnes (Namibia) and 20 tonnes (Zimbabwe), raw ivory may be exported to Japan subject to the conditions established in Decision of the Conference of the Parties regarding ivory No. 10.1. All other specimens shall be deemed to be specimens of species included in Appendix I and the trade in them shall be regulated accordingly

${ }^{\circ} 605$ For the exclusive purpose of allowing international trade in live animals to appropriate and acceptable destinations and hunting trophies. All other specimens shall be deemed to be specimens of species included in Appendix I and the trade in them shall be regulated accordingly

${ }^{\circ} 606$ For the exclusive purpose of allowing international trade in wool sheared from live vicuñas of the populations included in Appendix II (see +211 ) and in the stock extant at the time of the ninth meeting of the Conference of the Parties (November 1994) in Peru of $3249 \mathrm{~kg}$ of wool, and in cloth and items made thereof, including luxury handicrafts and knitted articles. The reverse side of the cloth must bear the logotype adopted by the range States of the species, which are signatories to the Convenio para la Conservación y Manejo de la Vicuña, and the selvages the words "VICUÑA-COUNTRY OF ORIGIN", depending on the country of origin. All other specimens shall be deemed to be specimens of species included in Appendix I and the trade in them shall be regulated accordingly

${ }^{\circ} 607$ Fossils are not subject to the provisions of the Convention

${ }^{\circ} 608$ Artificially propagated specimens of the following hybrids and/or cultivars are not subject to the provisions of the Convention:

\author{
Hatiora $\mathrm{x}$ graeseri \\ Schlumbergera $x$ buckleyi \\ Schlumbergera russelliana $\times$ Schlumbergera iruncata \\ Schlumbergera orssichiana $\times$ Schlumbergera truncata \\ Schlumbergera opuntioides $\mathrm{x}$ Schlumbergera truncata \\ Schlumbergera truncata (cultivars) \\ Gymnocalycium mihanovichii (cultivars) forms lacking chlorophyll, grafted on the following \\ grafting stocks: Harrisia 'Jusbertii', Hylocereus trigonus or Hylocereus undatus \\ Opuntia microdasys (cultivars)
}

${ }^{\circ} 609$ Artificially propagated specimens of cultivars of Euphorbia trigona are not subject to the provisions of the Convention

${ }^{\circ} 610$ Seedling or tissue cultures obtained in vitro, in solid or liquid media, transported in sterile containers are not subject to the provisions of the Convention

${ }^{\circ} 611$ Artificially propagated specimens of cultivars of $C y c l a m e n$ persicum are not subject to the provisions of the Convention. However, the exemption does not apply to such specimens traded as dormant tubers.

12. In accordance with Article I, paragraph b, sub-paragraph (iii), of the Convention, the symbol (\#) followed by a number placed against the name of a species or higher taxon included in Appendix II designates parts or derivatives which are specified in relation thereto for the purposes of the Convention as follows:

\#1 Designates all parts and derivatives, except:

a) seeds, spores and pollen (including pollinia);

b) seedling or tissue cultures obtained in vitro, in solid or liquid media, transported in sterile containers; and

c) cut flowers of artificially propagated plants

Designates all parts and derivatives, except:

a) seeds and pollen; 
b) seedling or tissue cultures obtained in virro, in solid or liquid media, transported in sterile containers;

c) cut flowers of artificially propagated plants; and

d) chemical derivatives

\#3 Designates whole and sliced roots and parts of roots, excluding manufactured parts or derivatives such as powders, pills, extracts, tonics, teas and confectionery

\#4 Designates all parts and derivatives, except:

a) seeds, except those from Mexican cacti originating in Mexico, and pollen;

b) seedling or tissue cultures obtained in vitro, in solid or liquid media, transported in sterile containers;

c) cut flowers of artificially propagated plants;

d) fruits and parts and derivatives thereof of naturalized or artificially propagated plants; and

e) separate stem joints (pads) and parts and derivatives thereof of naturalized or artificially propagated plants of the genus Opuntia subgenus Opuntia

Designates all parts and derivatives, except:

a) seeds and pollen (including pollinia);

b) seedling or tissue cultures obtained in vitro, in solid or liquid media, transported in sterile containers;

c) cut flowers of artificially propagated plants; and

d) fruits and parts and derivatives there of artificially propagated plants of the genus Vanilla

Designates all parts and derivatives, except:

a) seeds and pollen;

b) seedling or tissue cultures obtained in vitro, in solid or liquid media, transported in sterile containers;

c) cut flowers of artificially propagated plants; and

d) finished pharmaceutical products.

13. As none of the species or higher taxa of FLORA included in Appendix I is annotated to the effect that its hybrids shall be treated in accordance with the provisions of Article III of the Convention, this means that artificially propagated hybrids produced from one or more of these species or taxa may be traded with a certificate of artificial propagation, and that seeds and pollen (including pollinia), cut flowers, seedling or tissue cultures obtained in vitro, in solid or liquid media, transported in sterile containers of these hybrids are not subject to the provisions of the Convention. 



\section{APENDICES I Y II}

adoptados por la Conferencia de las Partes y vigentes a partir del 18 de septiembre de 1997

\section{INTERPRETACION}

1. Las especies que figuran en estos Apéndices están indicadas:

a) conforme al nombre de las especies; o

b) como si todas las especies estuviesen incluidas en un taxón superior o en una parte de él designada.

2. La abreviatura "spp." se utiliza para denotar todas las especies de un taxón superior.

3. Otras referencias a los taxa superiores de la especie se indican únicamente para fines de información o de clasificación.

4. Las abreviaturas siguientes se utilizan para taxa de plantas por debajo del nivel de especie:

a) "spp." para denotar las subespecies;

b) "var(s)." para denotar la variedad (variedades); y

c) "fa." para denotar la forma.

5. La abreviatura "p.e." se utiliza para denotar especies posiblemente extinguidas.

6. Un asterisco $\left(^{*}\right)$ colocado junto al nombre de una especie o de un taxón superior significa que una o más de las poblaciones geográficamente aisladas, subespecies o especies de dicha especie o de dicho taxón están incluidas en el Apéndice I y excluidas del Apéndice II.

7. Dos asteriscos $(* *)$ colocados junto al nombre de una especie o de un taxón superior indican que una o más de las poblaciones geográficamente aisladas, subespecies o especies de dicha especie o de dicho taxón están incluidas en el Apéndice II y excluidas del Apéndice I.

8. El símbolo (-) seguido de un número colocado junto al nombre de una especie o de un taxón superior significa que algunas poblaciones geográficamente aisladas, especies, grupos de especies o familias de dicha especie o de dicho taxón están excluidas del Apéndice respectivo, como sigue:

-101 Población de Groenlandia Occidental

-102 Poblaciones de Bhután, India, Nepal y Pakistán

-103 Poblaciones de Botswana, Namibia y Zimbabwe

-104 Población de Australia

-105 Poblaciones de Pecari tajacu de México y Estados Unidos de América

-106 - Argentina: la población de la provincia de Jujuy y las poblaciones en semicautividad de las provincias de Jujuy, Salta, Catamarca, La Rioja y San Juan

- Bolivia: las poblaciones de las unidades de conservación de Mauri-Desaguadero, Ulla Ulla y Lípez-Chichas, con un cupo anual de exportación nulo

- Chile: una parte de la población de la provincia de Parinacota, 1a. Región de Tarapacá

- Perú: toda la población

-107 Poblaciones de Afghanistán, Bhután, India, Myanmar, Nepal y Pakistán

-108 Cathartidae

-109 Melopsittacus undulatus, Nymphicus hollandicus y Psittacula krameri

-110 Población de Argentina

-111 Población de Ecuador, sujeto a un cupo de exportación nulo hasta que la Secretaría de la CITES y el Grupo de Especialistas de Cocodrilos de la SSC/UICN hayan aprobado un cupo de exportación anual

-112 Poblaciones de Botswana, Etiopía, Kenya, Madagascar, Malawi, Mozambique, República Unida de Tanzanía, Sudáfrica, Uganda, Zambia y Zimbabwe 
Además de los especímenes criados en granjas, la República Unida de Tanzanía autorizará la exportación de no más de 1.100 especímenes silvestres (incluidos 100 trofeos de caza) en 1998 , 1999 y 2000

-113 Poblaciones de Australia, Indonesia y Papúa Nueva Guinea

-114 Población de Chile

-115 Todas las especies no suculentas

-116 Aloe vera; también denominada Aloe barbadensis.

9. El símbolo (+) seguido de un número colocado junto al nombre de una especie, subespecie o un taxón superior significa que solamente algunas poblaciones geográficamente aisladas de dicha especie, subespecie o taxón están incluidas en el Apéndice respectivo, como sigue:

+201 Poblaciones de Bhután, India, Nepal y Pakistán

+202 Poblaciones de Buthán, China, México y Mongolia

+203 Poblaciones de Camerún y Nigeria

+204 Población de Asia

+205 Poblaciones de América Central y del Norte

+206 Poblaciones de Bangladesh. India y Tailandia

+207 Población de India

+208 Poblaciones de Botswana, Namibia y Zimbabwe

+209 Población de Australia

+210 Población de Sudáfrica

+211 - Argentina: la población de la provincia de Jujuy y las poblaciones en semicautividad de las provincias de Jujuy, Salta, Catamarca, La Rioja y San Juan

- Bolivia: las poblaciones de las unidades de conservación de Mauri-Desaguadero, Ulla Ulla y Lípez-Chichas, con un cupo anual de exportación nulo

- Chile: una parte de la población de la provincia de Parinacota, la. Región de Tarapacá

- Perú: toda la población

+212 Poblaciones de Afghanistán, Bhután, India, Myanmar, Nepal y Pakistán

+213 Población de México

+214 Poblaciones de Argelia, Burkina Faso, Camerún, Chad, Malí, Marruecos, Mauritania, Niger, Nigeria, República Centroafricana, Senegal y Sudán.

+215 Población de Seychelles

+216 Población de Europa, excepto la región que constituía anteriormente la Unión de Repúblicas Socialistas Soviéticas

+217 Población de Chile.

10. El símbolo (=) seguido de un número colocado junto al nombre de una especie o de un taxón superior significa que la denominación de dicha especic o de dicho taxón debe ser interpretada como sigue:

$=301$ También denominada Phalanger maculatus

$=302$ Incluye la familia Tupaiidae

$=303$ Anteriormente incluida en la familia Lemuridae

$=304$ Anteriormente incluida como una subespecie de Callithrix jacchus

$=305$ Incluye el sinónimo genérico Leontideus

$=306$ Anteriormente incluida en la especie Saguinus oedipus

$=307$ Anteriormente incluida en Alouatta palliata

$=308$ Anteriormente incluida como Alouatta palliata (villosa)

$=309$ Incluye el sinónimo Cercopithecus roloway

$=310$ Anteriormente incluida en el género Papio

=311 Incluye el sinónimo genérico Simias

=312 Incluye el sinónimo Colobus badius kirkii

$=313$ Incluye el sinónimo Colobus badius rufomitratus

=314 Incluye el sinónimo genérico Rhinopithecus

=315 También denominada Presbytis entellus

=316 También denominada Presbytis geei y Semnopithecus geei

=317 También denominada Presbytis pileata y Semnopithecus pileatus

$=318$ Incluye los sinónimos Bradypus boliviensis y Bradypus griseus

$=319$ Incluye el sinónimo Priodontes giganteus

$=320$ Incluye el sinónimo Physeter macrocephalus 
Incluye el sinónimo Eschrichtius glaucus

Anteriormente incluida en el género Balaena

Anteriormente incluida en el género Dusicyon

Incluye el sinónimo Dusicyon fulvipes

Incluye el sinónimo genérico Fennecus

También denominada Selenarctos thibetanus

También denominada Aonyx microdon o Paraonyx microdon

Anteriormente incluida en el género Lutra

Anteriormente incluida en el género Lutra; incluye los sinónimos Lutra annectens,

Lutra enudris, Lutra incarum y Lutra platensis

Incluye el sinónimo Eupleres major

También denominada Hyaena brunnea

También denominada Felis caracal y Lynx caracal

Anteriormente incluida en el género Felis

También denominada Felis pardina o Felis lynx pardina

Anteriormente incluida en el género Panthera

También denominada Equus asinus

Anteriormente incluida en la especie Equus hemionus

También denominada Equus caballus przewalskii

También denominada Choeropsis liberiensis

También denominada Cervus porcinus annamiticus

También denominada Cervus porcinus calamianensis

También denominada Cervus porcinus kuhlii

También denominada Cervus dama mesopotamicus

Incluye el sinónimo Bos frontalis

Incluye el sinónimo Bos grunniens

Incluye el sinónimo genérico Novibos

Incluye el sinónimo genérico Anoa

También denominada Damaliscus dorcas dorcas o Damaliscus pygargus dorcas

Anteriormente incluida en la especie Naemorhedus goral

También denominada Capricornis sumatraensis

Incluye el sinónimo Oryx tao

Incluye el sinónimo Ovis aries ophion

Anteriormente incluida como Ovis vignei (véanse las decisiones de la Conferencia de las Partes dirigidas a las Partes, relativas a la inclusión de Ovis vignei en el Apéndice I)

También denominada Rupicapra rupicapra ornata

También denominada Pterocnemia pennata

También denominada Sula abbotti

También denominada Ciconia ciconia boyciana

Incluye los sinónimos Anas chlorotis y Anas nesiotis

También denominada Anas platyrhynchos laysanensis

Probablemente un híbrido entre Anas platyrhynchos y Anas superciliosa

También denominada Aquila heliaca adalberti

También denominada Chondrohierax wilsonii

También denominada Falco peregrinus babylonicus y Falco peregrinus pelegrinoides

También denominada Crax mitu mitu

Anteriormente incluida en el género Aburria

Anteriormente incluida en el género Aburria; también denominada Pipile pipile pipile

Anteriormente incluida en la especie Crossoptilon crossoptilon

Anteriormente incluida en la especie Polyplectron malacense

Incluye el sinónimo Rheinardia nigrescens

También denominada Tricholimnas sylvestris

También denominada Choriotis nigriceps

También denominada Houbaropsis bengalensis

También denominada Amazona dufresniana rhodocorytha

A menudo comercializada bajo el nombre incorrecto de Ara caninde

También denominada Cyanoramphus novaezelandiae cookii

También denominada Opopsitta diophthalma coxeni

También denominada Pezoporus occidentalis

Anteriormente incluida en la especie Psephotus chrysopterygius 
También denominada Psittacula krameri echo Anteriormente incluida en el género Gallirex; también denominada Tauraco porphyreolophus También denominada Otus gurneyi

También denominada Ninox novaeseelandiae royana

Anteriormente incluida en el género Glaucis Incluye el sinónimo genérico Ptilolaemus

Anteriormente incluida en el género Rhinoplax También denominada Pitta brachyura nympha También denominada Muscicapa ruecki o Niltava ruecki

También denominada Dasyornis brachypterus longirostris

Tambiên denominada Meliphaga cassidix Incluye el sinónimo genérico Xanthopsar Anteriormente incluida en el género Spinus Mencionada asimismo en el género Damonia Anteriormente incluida como Kachuga tecta tecta Incluye los sinónimos genéricos Nicoria y Geoemyda (en parte)

También denominada Geochelone elephantopus; mencionada asimismo en el género Testudo Mencionada asimismo en el género Testudo

Mencionada asimismo en el género Aspideretes Anteriormente incluida en Podocnemis spp. Incluye Alligatoridae, Crocodylidae y Gavialidae También denominada Crocodylus mindorensis

Mencionada asimismo en el género Nactus Incluye el sinónimo genérico Rhoptropella Anteriormente incluida en Chamaeleo spp. Incluye los sinónimos genéricos Calumma y Furcifer Incluye las familias Bolyeriidae y Tropidophiidae como subfamilias También denominada Constrictor constrictor occidentalis Incluye el sinónimo Python molurus pimbura Incluye el sinómimo Sanzinia manditra Incluye el sinónimo Pseudoboa cloelia También denominada Hydrodynastes gigas Incluye los sinónimos Naja atra, Naja kaouthia, Naja oxiana, Naja philippinensis, Naja samarensis, Naja sputatrix y Naja sumatrana Incluye el sinónimo genérico Megalobatrachus Anteriormente incluida en Nectophrynoides spp. Anteriormente incluida en Dendrobates spp. Mencionada asimismo en el género Rana Sensu D'Abrera Incluye los sinónimos Pandinus africanus y Heterometrus roeseli Incluye el sinónimo genérico Aphonopelma También denominada Conchodromus dromas Mencionada asimismo en los géneros Dysnomia y Plagiola Incluye el sinónimo genérico Proptera Mencionada asimismo en el género Carunculina También denominada Megalonaias nickliniana También denominada Cyrtonaias tampicoensis tecomatensis y Lampsilis tampicoensis tecomatensis Incluye el sinónimo genérico Micromya Incluye el sinónimo genérico Papuina Incluye solamente la familia Helioporidae con una especie Heliopora coerulea También denominada Podophyllum emodi y Sinopodophyllum hexandrum Incluye los sinónimos genéricos Neogomesia y Roseocactus Mencionada asimismo en el género Echinocactus

Mencionada asimismo en el género Mammillaria; incluye el sinónimo Coryphantha densispina También denominada Lobeira macdougallii y Nopalxochia macdougallii

También denominada Echinocereus lindsayi Mencionada asimismo en el género Cereus y Wilcoxia $=435$ Mencionada asimismo en el género Coryphantha; incluye Escobaria leei como una subespecie 
=437 También denominada Backebergia militaris, Cephalocereus militaris y Mitrocereus militaris; incluye el sinónimo Pachycereus chrysomallus

$=438$ Incluye Pediocactus bradyi spp. despainii y Pediocactus bradyi spp. winkleri y los sinónimos Pediocactus despainii y Pediocactus winkleri

$=439$ Mencionada asimismo en el género Toumeya

$=440$ Mencionada asimismo en el género Navajoa, Toumeya y Utahia; incluye Pediocactus peeblesianus var. fickeisenii

$=441$ Mencionada asimismo en el género Echinocactus y Utahia

$=442$ Incluye el sinónimo genérico Encephalocarpus

$=443$ Incluye el sinónimo Ancistrocactus tobuschii y Ferocactus tobuschii

=444 Mencionada asimismo en los géneros Echinomastus y Neolloydia; incluye los sinónimos Echinomastus acunensis y Echinomastus krausei

$=445$ Incluye los sinónimos Ferocactus glaucus, Sclerocactus brevispinus, Sclerocactus wetlandicus y Sclerocactus wetlandicus spp. ilseae Mencionada asimismo en los géneros Echinocactus, Echinomastus y Neolloydia Mencionada asimismo en los géneros Coloradoa, Echinocactus, Ferocacius y Pediocactus Mencionada asimismo en los géneros Echinocactus, Mammillaria, Pediocactus y Toumeya Mencionada asimismo en los géneros Echinocactus y Ferocactus

$=451$

$=452$

$=453$

$=454$

$=455$

$=456$

$=457$

$=458$

$=459$

$=460$

$=461$

$=462$ Mencionada asimismo en el género Pediocactus

Incluye los sinónimos genéricos Gymnocactus, Normanbokea y Rapicactus

También denominada Saussurea lappa

También denominada Euphorbia decaryi var. capsaintemariensis

Incluye Euphorbia cremersii fa. viridifolia y Euphorbia cremersii fa. rakotozafyi

Incluye Euphorbia cylindrifolia ssp, tuberifera

Incluye Euphorbia decaryi var. ampanihyensis, robinsonii y spirosticha

Incluye Euphorbia moratii var. antsingiensis, bemarahensis y multifora

También denominada Euphorbia capsaintemariensis var. tulearensis

También denominada Engelhardia pterocarpa

Incluye Aloe compressa var. rugosquamosa y Aloe compressa var. schistophila

Incluye Aloe haworthioides var. aurantiaca

Incluye Aloe laeta var. maniaensis

=463 Incluye las familias Apostasiaceae y Cypripediaceae como las subfamilias Apostasioideae y Cypripedioideae

$=464$

$=465$

$=466$

$=467$

$=468$

$=469$

$=470$

Anacampseros australiana y A. kurtzii aparecen mencionadas asimismo en el género Grahamia

Anteriormente incluida en Anacampseros spp.

También denominada Sarracenia rubra ssp. alabamensis

También denominada Sarracenia rubra ssp. jonesii

Anteriormente incluida en ZAMIACEAE spp.

Incluye el sinónimo Stangeria paradoxa

$=471$

También denominada Taxus baccata ssp. wallichiana

Incluye el sinónimo Welwitschia bainesii.

11. El símbolo $\left({ }^{\circ}\right)$ seguido de un número colocado junto al nombre de una especie o de un taxón superior debe ser interpretado como sigue:

${ }^{\circ} 601$ Se ha establecido un cupo de exportación nulo. Todos los especímenes deben considerarse como especímenes de especies incluidas en el Apéndice I y su comercio deberá reglamentarse en consecuencia

•602 Los especímenes domesticados no están sujetos a las disposiciones de la Convención

${ }^{\circ} 603$ Los cupos de exportación anual para especímenes vivos y trofeos de caza se otorgan como sigue:

$\begin{array}{lr}\text { Botswana: } & 5 \\ \text { Namibia: } & 150 \\ \text { Zimbabwe: } & 50\end{array}$

El comercio de dichos especímenes está sujeto a las disposiciones del Artículo III de la Convención 
${ }^{\circ} 604$ Con el exclusivo propósito de permitir: 1) la exportación de trofeos de caza con fines no comerciales; 2) la exportación de animales vivos a destinatarios apropiados y aceptables (Namibia: únicamente con fines no comerciales); 3) la exportación de pieles (Zimbabwe únicamente); 4) la exportación de artículos de cuero y tallas de marfil con fines no comerciales (Zimbabwe únicamente). No se autorizará el comercio internacional de marfil hasta que hayan transcurrido 18 meses desde la fecha en que entre en vigor la transferencia al Apéndice II (es decir, el 18 de marzo de 1999). A partir de esa fecha, podrá exportarse a Japón marfil no trabajado, mediante cupos experimentales que no excedan 25,3 toneladas (Botswana), 13,8 toneladas (Namibia) y 20 toneladas (Zimbabwe), sujeto a las condiciones estipuladas en la Decisión No. 10.1 de la Conferencia de las Partes dirigidas a las Partes. Todos los demás especímenes deben considerarse como especímenes de especies incluidas en el Apéndice l y su comercio deberá reglamentarse en consecuencia

${ }^{\circ} 605$ Con el exclusivo propósito de permitir el comercio internacional de animales vivos a destinatarios apropiados y aceptables y trofeos de caza. Todos los demás especímenes deben considerarse como especímenes de especies incluidas en el Apéndice I y su comercio deberá reglamentarse en consecuencia

${ }^{\circ} 606$ Con el exclusivo propósito de permitir el comercio internacional de lana esquilada de vicuñas vivas, cuyas poblaciones están incluidas en el Apéndice II (véase +210 ) y de las existencias registradas en la novena reunión de la Conferencia de las Partes (noviembre de 1994) en Perú de $3.249 \mathrm{~kg}$ de lana, y de telas y artículos derivados, inclusive los artículos artesanales suntuarios y tejidos de punto fabricados. En el revés de las telas debe figurar el logotipo adoptado por los estados del área de distribución de la especie, signatarios del Convenio para la conservación y ordenación de la vicuña, y en los orillos la expresión "VICUÑA-PAIS DE ORIGEN", dependiendo del país de origen. Todos los demás especímenes deben considerarse como especímenes de especies incluidas en el Apéndice I y su comercio deberá reglamentarse en consecuencia

${ }^{\circ} 607$ Los fósiles no están sujetos a las disposiciones de la Convención

${ }^{\circ} 608$ Los especímenes reproducidos artificialmente de los siguientes híbridos y/o cultivares no están sujetos a las disposiciones de la Convención:

\author{
Hatiora $\times$ graeseri \\ Schlumbergera $\times$ buckleyi \\ Schlumbergera russelliana $\times$ Schlumbergera truncata \\ Schlumbergera orssichiana $\times$ Schlumbergera truncata \\ Schlumbergera opuntioides $\mathrm{x}$ Schlumbergera truncata \\ Schlumbergera truncata (cultivares)
}

Gymnocalycium mihanovichii (cultivares) formas que carecen de clorofila, injertadas en los siguientes patrones: Harrisia 'Jusbertii', Hylocereus trigonus o Hylocereus undatus Opuntia microdasys (cultivares)

○609 Los especimenes reproducidos artificialmente de cultivares de Euphorbia trigona no están sujetos a las disposiciones de la Convención

${ }^{\circ} 610$ Los cultivos de plántulas o de tejidos obtenidos in vitro, en medios sólidos o líquidos, que se transportan en envases estériles no están sujetos a las disposiciones de la Convención

${ }^{\circ} 611$ Los especímenes reproducidos artificialmente de cultivares de Cyclamen persicum no están sujetos a las disposiciones de la Convención. No obstante, esta exoneración no se aplica a los especímenes comercializados como tubérculos latentes. 
12. De conformidad con las disposiciones del subpártafo del párrafo b del Artículo I de la Convención, el signo (\#) seguido de un número colocado junto al nombre de una especie o de un taxón superior incluido en el Apéndice II designa las partes o derivados provenientes de esa especie o de ese taxón y se indican como sigue para los fines de la Convención:

\#1 Designa todas las partes y derivados, excepto:

a) las semillas, las esporas y el polen (inclusive las polinias); y

b) los cultivos de plántulas o de tejidos obtenidos in vitro, en medios sólidos o líquidos, que se transportan en envases estériles; y

c) las flores cortadas de plantas reproducidas artificialmente

\#2 Designa todas las partes y derivados, excepto:

a) las semillas y el polen;

b) los cultivos de plántulas o de tejidos obtenidos in vitro, en medios sólidos o líquidos, que se transportan en envases estériles;

c) las flores cortadas de plantas reproducidas artificialmente; y

d) los derivados químicos

\#3 Designa raíces enteras o en rodajas o partes de raíces, excluidas las partes o derivados manufacturados, tales como polvos, pastillas, extractos, tónicos, ies y otros preparados

\#4 Designa todas las partes y derivados, excepto:

a) las semillas, excepto las de las cactáceas mexicanas originarias de México, y el polen;

b) los cultivos de plántulas o de tejidos obtenidos in vitro, en medios sólidos o líquidos, que se transportan en envases estériles;

c) las flores cortadas de plantas reproducidas artificialmente;

d) los frutos, y sus partes y derivados, de plantas aclimatadas o reproducidas artificialmente; y

e) los elementos del tallo (ramificaciones), y sus partes y derivados, de plantas del género Opuntia subgénero Opuntia aclimatadas o reproducidas artificialmente

\#5 Designa trozas, madera aserrada y láminas de chapa de madera

Designa trozas, troceados de madera y material no elaborado

\#7 Designa todas las partes y derivados, excepto:

a) las semillas y el polen (inclusive las polinias);

b) los cultivos de plántulas o de tejidos obtenidos in vitro, en medios sólidos o líquidos, que se transportan en envases estériles;

c) las flores cortadas de ejemplares reproducidos artificialmente; y

d) los frutos, y sus partes y derivados, de plantas del género Vanilla reproducidas artificialmente

Designa todas las partes y derivados, excepto:

a) las semillas y el polen;

b) los cultivos de plántulas o de tejidos obtenidos in vitro, en medios sólidos o líquidos, que se transportan en envases estériles;

c) las flores cortadas de ejemplares reproducidos artificialmente; y

d) los productos farmacéuticos elaborados.

13. Habida cuenta de que ninguna de las especies o taxa superiores de FLORA incluidas en el Apéndice I están anotadas, en el sentido de que sus híbridos sean tratados de conformidad con las disposiciones del Artículo III de la Convención, los híbridos reproducidos artificialmente de una o más de estas especies o taxa pueden ser comercializados con un certificado de reproducción artificial. Además, las semillas, el polen (inclusive las polinias), las flores cortadas, los cultivos de plántulas o de tejidos obtenidos in vitro, en medios sólidos o líquidos, que se transportan en envases estériles de estos híbridos no están sujetos a las disposiciones de la Convención. 



\begin{abstract}
ANNEXES I ET II
telles qu'adoptées par la Conférence des Parties et valables à compter du 18 septembre 1997
\end{abstract}

\title{
INTERPRETATION
}

1. Les espèces figurant aux présentes annexes sont indiquées:

a) par le nom de l'espèce; ou

b) par l'ensemble des espèces appartenant à un taxon supérieur ou à une partie désignée dudit taxon.

2. L'abréviation "spp." sert à désigner toutes les espèces d'un taxon supérieur.

3. Les autres références à des taxons supérieurs à l'espèce sont données uniquement à titre d'information ou à des fins de classification.

4. Les abréviations suivantes sont utilisées pour des taxons végétaux inférieurs à l'espèce:
a) "ssp." sert à désigner une sous-espèce;
b) "var(s)." sert à désigner une variété ou des (variétés); et
c) "fa." sert à désigner une forma.

5. L'abréviation "p.e." sert à désigner des espèces peut-être éteintes.

6. Un astérisque $\left({ }^{*}\right)$ placé après le nom d'une espèce ou d'un taxon supérieur indique qu'une ou plusieurs populations géographiquement isolées, sous-espèces ou espèces, de ladite espèce ou dudit taxon, figurent à l'Annexe I et sont exclues de l'Annexe II.

7. Deux astérisques (**) placés après le nom d'une espèce ou d'un taxon supérieur indiquent qu'une ou plusieurs populations géographiquement isolées, sous-espèces ou espèces, de ladite espèce ou dudit taxon, figurent à l'Annexe II et sont exclues de l'Annexe I.

8. Le signe (-) suivi d'un nombre placé après le nom d'une espèce ou d'un taxon supérieur signifie que des populations géographiquement isolées, espèces, groupes d'espèces ou familles, de ladite espèce ou dudit taxon, sont exclus de l'annexe en question, comme suit:

-101 Population du Groenland occidental

-102 Populations du Bhoutan, de l'Inde, du Népal et du Pakistan

-103 Populations du Botswana, de la Namibie et du Zimbabwe

-104 Population de l'Australie

-105 Populations de Pecari tajacu des Etats-Unis d'Amérique et du Mexique

-106 - Argentine: la population de la province de Jujuy et les populations semi-captives des provinces de Jujuy, Salta, Catamarca, La Rioja et San Juan

- Bolivie: les populations des aires de conservation de Mauri-Desaguadero, Ulla Ulla et LípezChichas, avec un quota annuel d'exportation zéro

- Chili: une partie de la population de la province de Parinacota, région la. de Tarapacá

- Pérou: l'ensemble de la population Populations de l'Afghanistan, du Bhoutan, de l'Inde, du Myanmar, du Népal et du Pakistan

-108 Cathartidae

-109 Melopsittacus undulatus, Nymphicus hollandicus et Psittacula krameri

-110 Population de l'Argentine

-111 Population de l'Equateur, sous réserve de quotas annuels d'exportation zéro jusqu'à ce qu'un quota annuel d'exportation ait été approuvé par le Secrétariat CITES et le Groupe SSC/UICN de spécialistes des crocodiles 
Populations de l'Afrique du Sud, du Botswana, de l'Ehiopie, du Kenya, de Madagascar, du Malawi, du Mozambique, de l'Ouganda, de la République-Unie de Tanzanie, de la Zambie et du Zimbabwe

Outre les spécimens élevés en ranch, la République-Unie de Tanzanie autorisera l'exportation d'un maximum de 1100 spécimens sauvages (dont 100 trophées de chasse) en 1998, 1999 et 2000

-113 Populations de l'Australie, de l'Indonésie et de la Papouasie-Nouvelle-Guinée

-114 Population du Chili

-115 Toutes les espèces non succulentes

-116 Aloe vera; aussi appelé Aloe barbadensis.

9. Le signe (+) suivi d'un nombre placé après le nom d'une espèce, d'une sous-espèce ou d'un taxon supérieur signifie que seules des populations géographiquement isolées desdites espèce ou sous-espèce ou dudit taxon, sont inscrites à l'annexe en question, comme suit:

+201 Populations du Bhoutan, de l'Inde, du Népal et du Pakistan

+202 Populations du Bhoutan, de la Chine, du Mexique et de la Mongolie

+203 Populations du Cameroun et du Nigéria

+204 Population de l'Asie

+205 Populations de l'Amérique centrale et de l'Amérique du Nord

+206 Populations du Bangladesh, de l'Inde et de la Thaillande

+207 Population de l'Inde

+208 Populations du Botswana, de la Namibie et du Zimbabwe

+209 Population de l'Australie

+210 Population de l'Afrique du Sud

+211 - Argentine: la population de la province de Jujuy et les populations semi-captives des provinces de Jujuy, Salta, Catamarca, La Rioja et San Juan

- Bolivie: les populations des aires de conservation de Mauri-Desaguadero, Ulla Ulla et LípezChichas, avec un quota annuel d'exportation zéro

- Chili: une partie de la population de la province de Parinacota, région la. de Tarapacá

- Pérou: l'ensemble de la population

+212 Populations de l'Afghanistan, du Bhoutan, de l'Inde, du Myanmar, du Népal et du Pakistan

+213 Population du Mexique

+214 Populations de l'Algérie, du Burkina Faso, du Cameroun, du Mali, du Maroc, de la Mauritanie, du Niger, du Nigéria, de la République centrafricaine, du Sénégal, du Soudan et du Tchad

+215 Population des Seychelles

+216 Population de l'Europe, à l'exception des territoires qui constituaient antérieurement l'Union des Républiques socialistes soviétiques

+217 Population du Chili.

10. Le signe (=) suivi d'un nombre placé après le nom d'une espèce ou d'un taxon supérieur signifie que la dénomination de ladite espèce ou dudit taxon doit être interprétée comme suit:

\begin{tabular}{|c|c|}
\hline$=301$ & Aussi appelé Phalanger maculatus \\
\hline$=302$ & Comprend la famille Tupaiidae \\
\hline$=303$ & Précédemment inclus dans la famille Lemuridae \\
\hline$=304$ & Précédemment inclus en tant que sous-espèce de Callithrix jacchus \\
\hline$=305$ & Comprend le synonyme générique Leontideus \\
\hline$=306$ & Précédemment inclus dans l'espèce Saguinus oedipus \\
\hline$=307$ & Précédemment inclus dans l'espèce Alouatta palliata \\
\hline$=308$ & Précédemment inclus en tant que Alouatta palliata (villosa) \\
\hline$=309$ & Comprend le synonyme Cercopithecus roloway \\
\hline$=310$ & Précédemment inclus dans le genre Papio \\
\hline$=311$ & Comprend le synonyme générique Simias \\
\hline$=312$ & Comprend le synonyme Colobus badius kirkii \\
\hline$=313$ & Comprend le synonyme Colobus badius rufomitratus \\
\hline$=314$ & Comprend le synonyme générique Rhinopithecus \\
\hline$=315$ & Aussi appelé Presbytis entellus \\
\hline$=316$ & Aussi appelé Presbytis geei et Semnopithecus geei \\
\hline$=317$ & Aussi appelé Presbytis pileata et Semnopithecus pileatus \\
\hline
\end{tabular}


Comprend les synonymes Bradypus boliviensis et Bradypus griseus

Comprend le synonyme Priodontes giganteus

Comprend le synonyme Physeter macrocephalus

$=322$

Comprend le synonyme Eschrichtius glaucus

Précédemment inclus dans le genre Balaena

Précédemment inclus dans le genre Dusicyon

Comprend le synonyme Dusicyon fulvipes

Comprend le synonyme générique Fennecus

Aussi appelé Selenarctos thibetanus

Aussi appelé Aonyx microdon et Paraonyx microdon

Précédemment inclus dans le genre Lutra

Précédemment inclus dans le genre Lutra; comprend les synonymes Lutra annectens,

Lutra enudris, Lutra incarum et Lutra platensis

Comprend le synonyme Eupleres major

Aussi appelé Hyaena brunnea

Aussi appelé Felis caracal et Lynx caracal

Précédemment inclus dans le genre Felis

Aussi appelé Felis pardina et Felis lynx pardina

Précédemment inclus dans le genre Panthera

Aussi appelé Equus asinus

Précédemment inclus dans l'espèce Equus hemionus

Aussi appelé Equus caballus przewalskii

Aussi appelé Choeropsis liberiensis

Aussi appelé Cervus porcinus annamiticus

Aussi appelé Cervus porcinus calamianensis

Aussi appelé Cervus porcinus kuhlii

Aussi appelé Cervus dama mesopotamicus

Comprend le synonyme Bos frontalis

Comprend le synonyme Bos grunniens

Comprend le synonyme générique Novibos

Comprend le synonyme générique Anoa

Aussi appelé Damaliscus dorcas dorcas et Damaliscus pygargus dorcas

Précédemment inclus dans l'espèce Naemorhedus goral

Aussi appelé Capricornis sumatraensis

Comprend le synonyme Oryx tao

Comprend le synonyme Ovis aries ophion

Précédemment inclus en tant que Ovis vignei (voir aussi les décisions de la Conférence des

Parties à l'adresse des Parties en ce qui concerne l'inscription de Ovis vignei vignei à l'Annexe I)

Aussi appelé Rupicapra rupicapra ornata

Aussi appelé Pterocnemia pennata

Aussi appelé Sula abbotti

Aussi appelé Ciconia ciconia boyciana

Comprend les synonymes Anas chlorotis et Anas nesiotis

Aussi appelé Anas platyrhynchos laysanensis

Probablement un hybride entre Anas platyrhynchos et Anas superciliosa

Aussi appelé Aquila heliaca adalberti

Aussi appelé Chondrohierax wilsonii

Aussi appelé Falco peregrinus babylonicus et Falco peregrinus pelegrinoides

Aussi appelé Crax mitu mitu

Précédemment inclus dans le genre Aburria

$=366$

$=367$

$=368$

$=369$

$=370$

$=371$

$=372$

$=373$

$=374$

Précédemment inclus dans le genre Aburria; aussi appelé Pipile pipile pipile

Précédemment inclus dans l'espèce Crossoptilon crossoptilon

Précédemment inclus dans l'espèce Polyplectron malacense

Comprend le synonyme Rheinardia nigrescens

Aussi appelé Tricholimnas sylvestris

Aussi appelé Choriotis nigriceps

Aussi appelé Houbaropsis bengalensis

Aussi appelé Amazona dufresniana rhodocorytha

Souvent commercialisé sous le nom incorrect de Ara caninde

Aussi appelé Cyanoramphus novaezelandiae cookii 
Aussi appelé Opopsitta diophthalma coxeni

Aussi appelé Pezoporus occidentalis

Précédemment inclus dans l'espèce Psephorus chrysopterygius

Aussi appelé Psittacula krameri echo

Précédemment inclus dans le genre Gallirex; aussi appelé Tauraco porphyreolophus

Aussi appelé Otus gurneyi

Aussi appelé Ninox novaeseelandiae royana

Précédemment inclus dans le genre Glaucis

Comprend le synonyme générique Ptilolaemus

Précédemment inclus dans le genre Rhinoplax

Aussi appelé Pitta brachyura nympha

Aussi appelé Muscicapa ruecki et Niltava ruecki

Aussi appelé Dasyornis brachypterus longirostris

Aussi appelé Meliphaga cassidix

Comprend le synonyme générique Xanthopsar

Précédemment inclus dans le genre Spinus

Aussi mentionné dans le genre Damonia

Précédemment inclus en tant que Kachuga tecta tecta

Comprend les synonymes génériques Nicoria et Geoemyda (en partie)

Aussi appelé Geochelone elephantopus; aussi mentionné dans le genre Testudo

Aussi mentionné dans le genre Testudo

Aussi mentionné dans le genre Aspideretes

Précédemment inclus dans Podocnemis spp.

Comprend Alligatoridae, Crocodylidae et Gavialidae

Aussi appelé Crocodylus mindorensis

Aussi mentionné dans le genre Nactus

Comprend le synonyme générique Rhoptropella

Précédemment inclus dans Chamaeleo spp.

Comprend les synonymes génériques Calumma et Furcifer

Comprend les familles Bolyeriidae et Tropidophiidae en tant que sous-familles

Aussi appelé Constrictor constrictor occidentalis

Comprend le synonyme Python molurus pimbura

Comprend le synonyme Sanzinia manditra

Comprend le synonyme Pseudoboa cloelia

Aussi appelé Hydrodynastes gigas

Comprend les synonymes Naja atra, Naja kaouthia, Naja oxiana, Naja philippinensis,

Naja samarensis, Naja sputatrix et Naja sumatrana

Comprend le synonyme générique Megalobatrachus

Précédemment inclus dans Nectophrynoides spp.

Précédemment inclus dans Dendrobates spp.

Aussi mentionné dans le genre Rana

Sensu D'Abrera

Comprend les synonymes Pandinus africanus et Heterometrus roeseli

Comprend le synonyme générique Aphonopelma

Aussi appelé Conchodromus dromas

Aussi mentionné dans les genres Dysnomia et Plagiola

Comprend le synonyme générique Proptera

Aussi mentionné dans le genre Carunculina

Aussi appelé Megalonaias nickliniana

Aussi appelé Cyrtonaias tampicoensis tecomatensis et Lampsilis tampicoensis tecomatensis

Comprend le synonyme générique Micromya

Comprend le synonyme générique Papuina

Ne comprend que la famille Helioporidae avec une seule espèce: Heliopora coerulea

Aussi appelé Podophyllum emodi et Sinopodophyllum hexandrum

Comprend les synonymes génériques Neogomesia et Roseocactus

Aussi mentionné dans le genre Echinocactus

Aussi mentionné dans le genre Mammillaria; comprend le synonyme Coryphantha densispina

Aussi appelé Lobeira macdougallii et Nopalxochia macdougallii

Aussi appelé Echinocereus lindsayi

Aussi mentionné dans les genres Cereus et Wilcoxia 
Aussi mentionné dans le genre Coryphantha; comprend le synonyme Escobaria nellieae

Aussi mentionné dans le genre Coryphantha; comprend Escobaria leei en tant que sous-espèce

Comprend le synonyme Solisia pectinata

$=437$

Aussi appelé Backebergia militaris, Cephalocereus militaris et Mitrocereus militaris; comprend le synonyme Pachycereus chrysomallus

$=438$ Comprend Pediocactus bradyi ssp. despainii et Pediocactus bradyi ssp. winkleri et les synonymes Pediocactus despainii et Pediocactus winkleri

$=439$ Aussi mentionné dans le genre Toumeya

$=440$ Aussi mentionné dans les genres Navajoa, Toumeya et Utahia;

comprend Pediocactus peeblesianus var, fickeisenii

$=441$

$=442$

Aussi mentionné dans les genres Echinocactus et Utahia

$=443$

Comprend le synonyme générique Encephalocarpus

$=444$ Aussi mentionné dans les genres Echinomastus et Neolloydia;

Comprend les synonymes Ancistrocactus tobuschii et Ferocactus tobuschii

comprend les synonymes Echinomastus acunensis et Echinomastus krausei

$=445$

Comprend les synonymes Ferocactus glaucus, Sclerocactus brevispinus,

Sclerocactus wetlandicus et Sclerocactus wetlandicus ssp. ilseae

$=446$

$=447$

$=448$

$=449$

$=450$

$=451$

$=452$

$=453$

$=454$

$=455$

$=456$

$=457$

$=458$

$=459$

$=460$

$=461$

$=462$

$=463$

Aussi mentionné dans les genres Echinocactus, Echinomastus et Neolloydia

Aussi mentionné dans les genres Coloradoa, Echinocactus, Ferocactus et Pediocactus

Aussi mentionné dans les genres Echinocactus, Mammillaria, Pediocactus et Toumeya

Aussi mentionné dans les genres Echinocactus et Ferocactus

Aussi mentionné dans le genre Pediocactus

Comprend les synonymes Gymnocactus, Normanbokea et Rapicactus

Aussi appelé Saussurea lappa

Aussi appelé Euphorbia decaryi var. capsaintemariensis

Comprend Euphorbia cremersii fa. viridifolia et Euphorbia cremersii var. rakotozafyi

Comprend Euphorbia cylindrifolia ssp. tuberifera

Comprend Euphorbia decaryi var, ampanihyensis, robinsonii et spirosticha

Comprend Euphorbia moratii var. antsingiensis, bemarahensis et multiflora

Aussi appelé Euphorbia capsaintemariensis var. tulearensis

Aussi appelé Engelhardia pterocarpa

Comprend Aloe compressa var. rugosquamosa et Aloe compressa var. schistophila

Comprend Aloe haworthioides var. aurantiaca

Comprend Aloe laeta var. maniaensis

Comprend les familles Apostasiaceae et Cypripediaceae en tant que sous-famillies

Apostasioideae et Cypripedioideae

=464 Anacampseros australiana et $A$. kurtzii sont aussi mentionnés dans le genre Grahamia

=465 Précédemment inclus dans Anacampseros spp.

=466 Aussi appelé Sarracenia rubra ssp. alabamensis

$=467$ Aussi appelé Sarracenia rubra ssp. jonesii

$=468$ Précédemment inclus dans ZAMIACEAE spp.

$=469$ Comprend le synonyme Stangeria paradoxa

$=470$ Aussi appelé Taxus baccata ssp. wallichiana

$=471$ Comprend le synonyme Welwitschia bainesii.

11. Le signe $\left({ }^{\circ}\right)$ suivi d'un nombre placé après le nom d'une espèce ou d'un taxon supérieur doit être interprété comme suit:

${ }^{\circ} 601$ Un quota annuel d'exportation zéro a été établi. Tous les spécimens sont considérés comme des spécimens d'espèces inscrites à l'Annexe I et leur commerce est réglementé en conséquence

${ }^{\circ} 602$ Les spécimens de la forme domestiquée ne sont pas soumis aux dispositions de la Convention

${ }^{\circ} 603$ Des quotas annuels d'exportation d'animaux vivants et de trophées de chasse sont alloués comme suit:

Botswana:

Namibie:

Zimbabwe: 
○604 A seul fin de permettre l'exportation: 1) de trophées de chasse à des fins non commerciales;

2) d'animaux vivants vers des destinataires appropriés et acceptables (Namibie: à des fins non commerciales seulement); 3) de peaux (Zimbabwe seulement); 4) d'articles en cuir et de sculptures en ivoire à des fins non commerciales (Zimbabwe seulement). Aucun commerce international d'ivoire n'est autorisé pendant les 18 mois qui suivent l'entrée en vigueur du transfert à l'Annexe II (soit avant le 18 mars 1999). Par la suite, de l'ivoire brut pourra être exporté vers le Japon, selon des quotas expérimentaux de 25,3 tonnes (Botswana), 13,8 tonnes (Namibie) et 20 tonnes (Zimbabwe), dans les conditions établies par la décision de la Conférence des Parties relative à l'ivoire $n^{\circ} 10.1$. Tous les autres spécimens sont considérés comme des spécimens d'espèces inscrites à l'Annexe I et leur commerce est réglementé en conséquence

-605 A seule fin de permettre le commerce international d'animaux vivants vers des destinataires appropriés et acceptables et de trophées de chasse. Tous les autres spécimens sont considérés comme des spécimens d'espèces inscrites à l'Annexe I et leur commerce est réglementé en conséquence

${ }^{\circ} 606$ A seule fin de permettre le commerce international de la laine obtenue par la tonte de vigognes vivantes des populations inscrites à l'Annexe II (voir +211 ) et du stock de $3249 \mathrm{~kg}$ de laine qui existait au Pérou au moment de la neuvième session de la Conférence des Parties (novembre 1994), ainsi que de tissus et d'articles qui en dérivent, notamment d'articles artisanaux de luxe et d'articles tricotés. L'envers des tissus doit porter le logo adopté par les Etats de l'aire de répartition de l'espèce, signataires de la Convenio para la Consen ación y Manejo de la Vicuña, et les lisières les expressions "VICUÑA-PAYS D'ORIGINE", en fonction du pays d'origine. Tous les autres spécimens sont considérés comme des spécimens d'espèces inscrites à l'Annexe I et leur commerce est réglementé en conséquence

${ }^{\circ} 607$ Les fossiles ne sont pas soumis aux dispositions de la Convention

${ }^{\circ} 608$ Les spécimens reproduits artificiellement des hybrides evou cultivars suivants ne sont pas soumis aux dispositions de la Convention:

\section{Hatiora $\times$ graeseri \\ Schlumbergera $\mathrm{x}$ buckleyi \\ Schlumbergera russelliana $\times$ Schlumbergera truncata \\ Schlumbergera orssichiana $\times$ Schlumbergera truncata \\ Schlumbergera opuntioides $\mathrm{x}$ Schlumbergera truncata \\ Schlumbergera truncata (cultivars)}

Gymnocalycium mihanovichii (cultivars) formes sans chlorophylle, greffées sur les portegreffes suivants: Harrisia 'Jusbertii', Hylocereus trigonus ou Hylocereus undatus Opuntia microdasys (cultivars)

`609 Les spécimens reproduits artificiellement des cultivars d'Euphorbia trigona ne sont pas soumis aux dispositions de la Convention

॰610 Les cultures de plantules ou de tissus obtenues in vitro, en milieu solide ou liquide, et transportées en conteneurs stériles ne sont pas soumises aux dispositions de la Convention

¿611 Les spécimens reproduits artificiellement des cultivars de Cyclamen persicum ne sont pas soumis aux dispositions de la Convention. La dérogation ne s'applique cependant pas aux spécimens commercialisés sous forme de tubercules dormants.

12. Conformément aux dispositions de l'Article I, paragraphe b, alinéa iii), de la Convention, le signe (\#) suivi d'un nombre placé après le nom d'une espèce ou d'un taxon supérieur inscrit à l'Annexe II sert à désigner des parties ou produits obtenus à partir de ladite espèce ou dudit taxon et qui sont mentionnés comme suit aux fins de la Convention:

\#1 Sert à désigner toutes les parties et tous les produits, sauf:

a) les graines, les spores et le pollen ( $\mathrm{y}$ compris les pollinies); 
b) les cultures de plantules ou de tissus obtenues in vitro, en milieu solide ou liquide, et transportées en conteneurs stériles; et

c) les fleurs coupées des plantes reproduites artificiellement

\#2 Sert à désigner toutes les parties et tous les produits, sauf:

a) les graines et le pollen;

b) les cultures de plantules ou de tissus obtenues in vitro, en milieu solide ou liquide, et transportées en conteneurs stériles;

c) les fleurs coupées des plantes reproduites artificiellement; et

d) les produits chimiques

\#3 Sert à désigner racines entières et tranchées et les parties de racines, à l'exception des parties et produits transformés, tels que poudres, pilules, extraits, toniques, tisanes et autres préparations

\#4 Sert à désigner toutes les parties et tous les produits, sauf:

a) les graines, sauf celles des cactus mexicains provenant du Mexique, et le pollen;

b) les cultures de plantules ou de tissus obtenues in vitro, en milieu solice ou liquide, et transportées en conteneurs stériles;

c) les fleurs coupées des plantes reproduites artificiellement;

d) les fruits, et leurs parties et produits, des plantes acclimatées ou reproduites artificiellement; et

e) les éléments de troncs (raquettes), et leurs parties et produits, de plantes du genre Opuntia sous-genre Opuntia acclimatées ou reproduites artificiellement

Sert à désigner les grumes, les bois sciés et les placages

Sert à désigner les grumes, les copeaux et les matériaux déchiquetés non transformés

\#7 Sert à désigner toutes les parties et tous les produits, sauf:

a) les graines et le pollen ( $y$ compris les pollinies);

b) les cultures de plantules ou de tissus obtenues in vitro, en milieu solide ou liquide, et transportées en conteneurs stériles;

c) les fleurs coupées des plantes reproduites artificiellement; et

d) les fruits, et leurs parties et produits, de plantes du genre Vanilla reproduites artificiellement

Sert à désigner toutes les parties et tous les produits, sauf:

a) les graines et le pollen;

b) les cultures de plantules ou de tissus obtenues in vitro, en milieu solide ou liquide, et transportées en conteneurs stériles;

c) les fleurs coupées des plantes reproduites artificiellement; et

d) les produits pharmaceutiques finis.

13. Aucune des espèces ou aucun des taxons supérieurs de FLORA inscrits à l'Annexe I n'est annoté de manière à ce que ses hybrides soient traités conformément aux dispositions de l'Article III de la

Convention. Par conséquent, les hybrides reproduits artificiellement issus d'une ou plusieurs de ces espèces ou d'un ou plusieurs de ces taxons peuvent être commercialisés sous couvert d'un certificat de reproduction artificielle. En outre, les graines et le pollen (y compris les pollinies), les fleurs coupées, les cultures de plantules ou de tissus obtenues in vitro, cn milieu solide ou liquide, et transportées en conteneurs stériles de ces hybrides ne sont pas soumis aux dispositions de la Convention. 



\section{F A U N A}

\section{CHORDATA}

\section{MAMMALIA}

\section{MONOTREMATA}

Tachyglossidae

\section{DASYUROMORPHIA}

Dasyuridae

Thylacinidae

PERAMELEMORPHIA

Peramelidae

Chaeropus ecaudatus p.e.

Macrotis lagotis

Macrotis leucura

Perameles bougainville

\section{DIPROTODONTIA}

Phalangeridae

Sminthopsis longicaudata

Sminthopsis psammophila

Thylacinus cynocephalus p.e.
Zaglossus spp.
Vombatidae

Macropodidae

Potoroidae

\section{CHIROPTERA}

Pteropodidae

\section{PRIMATES}

Acerodon jubatus

Acerodon lucifer p.e.

Lagorchestes hirsutus

Lagostrophus fasciatus

Onychogalea fraenata

Onychogalea lunata

Bettongia spp.

Caloprymnus campestris p.e.

Phalanger orientalis

Spilocuscus maculatus $=301$

Dendrolagus inustus

Dendrolagus ursinus
Acerodon spp. *

Pteropus spp. *

Pteropus insularis

Pteropus mariannus

Pteropus molossinus

Pteropus phaeocephalus

Pteropus pilosus

Pteropus samoensis

Pteropus tonganus

PRIMATES spp. ${ }^{*}=302$

Lemuridae

Megaladapidae
Lemuridae spp.

Megaladapidae spp. $=303$ 
Cheirogaleidae

Indridae

Daubentoniidae

Callithricidae

Cebidae

Cercopithecidae

Hylobatidae

Hominidae

\section{XENARTHRA}

Myrmecophagidae

Bradypodidae

Dasypodidae
Cheirogaleidae spp.

Indridae spp.

Daubentonia madagascariensis

Callimico goeldii

Callithrix aurita $=304$

Callithrix flaviceps $=304$

Leontopithecus spp. $=305$

Saguinus bicolor

Saguinus geoffroyi $=306$

Saguinus leucopus

Saguinus oedipus

Alouatta coibensis $=307$

Alouatta palliata

Alouatta pigra $=308$

Ateles geoffroyi frontatus

Ateles geoffroyi panamensis

Brachyteles arachnoides

Cacajao spp.

Chiropotes albinasus

Lagothrix flavicauda

Saimiri oerstedii

Cercocebus galeritus galeritus

Cercopithecus dian $a=309$

Macaca silenus

Mandrillus leucophaeus $=310$

Mandrillus sphinx $=310$

Nasalis concolor $=311$

Nasalis larvatus

Presbytis potenziani

Procolobus pennantii kirkii $=312$

Procolobus rufomitratus $=313$

Pygathrix spp. $=314$

Semnopithecus entellus $=315$

Trachypithecus geei $=316$

Trachypithecus pileatus $=317$

Hylobatidae spp.

Gorilla gorilla

Pan spp.

Pongo pygmaeus

\section{PHOLIDOTA}

Manidae

Priodontes maximus $=319$

Myrmecophaga tridactyla

Bradypus variegatus $=318$

Chaetophractus nationi ${ }^{\circ} 601$ 


\section{LAGOMORPHA}

Leporidae

\section{RODENTIA}

Sciuridae

Muridae

Chinchillidae

CETACEA

Platanistidae

Ziphiidae

Physeteridae

Delphinidae

Phocoenidae

Eschrichtiidae

Balaenopteridae

Balaenidae

Neobalaenidae

\section{CARNIVORA}

Canidae
Caprolagus hispidus

Romerolagus diazi

Cynomys mexicanus

Leporillus conditor

Pseudomys praeconis

Xeromys myoides

Zyzomys pedunculatus

Chinchilla spp. ${ }^{\circ} 602$

CETACEA spp. *

Lipotes vexillifer

Platanista spp.

Berardius spp.

Hyperoodon spp.

Physeter catodon $=320$

Sotalia spp.

Sousa spp.

Neophocaena phocaenoides

Phocoena sinus

Eschrichtius robustus $=321$

Balaenoptera acutorostrata ** -101

Balaenoptera borealis

Balaenoptera edeni

Balaenoptera musculus

Balaenoptera physalus

Megaptera novaeangliae

Balaena mysticetus

Eubalaena spp. $=322$

Caperea marginata

Canis lupus $* *+201$

Speothos venaticus
Ratufa spp. 
Ursidae

Ailuropoda melanoleuca

Ailurus fulgens

Helarctos malayanus

Melursus ursinus

Tremarctos ornatus

Ursus arctos ** +202

Ursus arctos isabellinus

Ursus thibetanus $=326$

Mustelidae

Lutrinae

Mephitinae

Mustelinae

Viverridae

Prionodon pardicolor

Aonyx congicus $* *+203=327$

Enhydra lutris nereis

Lontra felina $=328$

Lontra longicaudis $=329$

Lontra provocax $=328$

Lutra lutra

Pteronura brasiliensis

Mustela nigripes

Hyaenidae

Felidae

Acinonyx jubatus ${ }^{\circ} 603$

Caracal caracal $* *+204=332$

Catopuma temminckii $=333$

Felis nigripes

Herpailurus yaguarondi $* *+205=333$

Leopardus pardalis $=333$

Leopardus tigrinus $=333$

Leopardus wiedii $=333$

Lynx pardinus $=334$

Neofelis nebulosa

Oncifelis geoffroyi $=333$

Oreailurus jacobita $=333$

Panthera leo persica

Panthera onca

Panthera pardus

Panthera tigris

Pardofelis marmorata $=333$

Prionailurus bengalensis bengalensis $+206=333$

Prionailurus planiceps $=333$

Prionailurus rubiginosus $* *+207=333$

Puma concolor coryi $=333$

Puma concolor costaricensis $=333$

Puma concolor couguar $=333$

Uncia uncia $=335$
Ursidae spp. *

Lutrinae spp. *

Conepatus humboldtii

Crypioprocta ferox

Cynogale bennettii

Eupleres goudotii $=\mathbf{3 3 0}$

Fossa fossana

Hemigalus derbyanus

Prionodon linsang

Parahyaena brunnea $=331$

Felidae spp. * ${ }^{\circ} 602$ 
Otariidae

Phocidae

\section{PROBOSCIDEA}

Elephantidae

Elephas maximus
Loxodonta africana $* *-103$

SIRENIA

Dugongidae

Trichechidae

\section{PERISSODACTYLA}

Equidae

Tapiridae

Rhinocerotidae

\section{ARTIODACTYLA}

Suidae

Tayassuidae

Hippopotamidae

\section{Camelidae}

Moschidae

Cervidae

\section{Arctocephalus townsendi}

Monachus spp.

Trichechus inunguis

Trichechus manatus

Equus hemionus hemionus

Equus onager khur $=337$

Equus przewalskii $=338$

Equus zebra zebra

Tapiridae spp. **

Rhinocerotidae spp. **

Catagonus wagneri

Axis calamianensis $=341$
Arctocephalus spp. *

Mirounga leonina

Loxodonta africana $*+208$ ${ }^{\circ} 604$

Dugong dugon * +209

Trichechus senegalensis

Equus hemionus *

Tapirus terrestris

Ceratotherium simum simum * $+210^{\circ} 605$

Tayassuidae spp. * -105

Hexaprotodon liberiensis $=$ 339

Hippopotamus amphibius

Lama guanicoe

Vicugna vicugna $*+211^{\circ} 606$

Moschus spp. * -107

Axis kuhlii $=342$

Axis porcinus annamiticus $=340$

Blastocerus dichotomus 
Cervidae (cont.)

Antilocapridae

Bovidae

Gazella dama

Hippotragus niger variani

Naemorhedus bailey $i=349$

Naemorhedus caudatus $=349$

Naemorhedus goral

Naemorhedus sumatraensis $=350$

Oryx dammah $=351$

Oryx leucoryx

Ovis ammon hodgsonii

Ovis ammon nigrimontana

Ovis orientalis ophion $=352$

Ovis vignei vignei $=353$

Pantholops hodgsonii

Pseudoryx nghetinhensis

Rupicapra pyrenaica ornata $=354$
Cervus elaphus bactrianus

Pudu mephistophiles

Ammotragus lervia

Bison bison athabascae

Budorcas taxicolor

Cephalophus dorsalis

Cephalophus monticola

Cephalophus ogilbyi

Cephalophus silvicultor

Cephalophus zebra

Damaliscus pygargus pygargus $=348$

Kobus leche

Ovis ammon *

Ovis canadensis +213

Saiga tatarica 
AVES

STRUTHIONIFORMES

Struthionidae

Struthio camelus +214

RHEIFORMES

Rheidae

Rhea pennata $=355$

Rhea americana

\section{TINAMIFORMES}

Tinamidae

Tinamus solitarius

\section{SPHENISCIFORMES}

Spheniscidae

Spheniscus humboldti

Spheniscus demersus

\section{PODICIPEDIFORMES}

Podicipedidae

Podilymbus gigas

PROCELLARIIFORMES

Diomedeidae

Diomedea albatrus

PELECANIFORMES

Pelecanidae

Pelecanus crispus

Sulidae

Papasula abbotti $=356$

Fregatidae

Fregata andrewsi

\section{CICONIIFORMES}

Balaenicipitidae

Balaeniceps rex

Ciconiidae

Ciconia boyciana $=357$

Jabiru mycteria

Mycteria cinerea

Ciconia nigra

Eudocimus ruber

Geronticus calvus

Geronticus eremita

Nipponia nippon

Platalea leucorodia

Phoenicopteridae spp.

Phoenicopteridae

\section{ANSERIFORMES}

Anatidae

Anas aucklandica $=358$

Anas bernieri

Anas formosa

Anas laysanensis $=359$

Anas oustaleti $=360$ 
Branta sandvicensis

Cairina scutulata

Rhodonessa caryophyllacea p.e.

\section{FALCONIFORMES}

Cathartidae

Accipitridae

Falconidae

\section{GALLIFORMES}

Megapodiidae

Cracidae

Phasianidae

Gymnogyps californianus

Vultur gryphus

Aquila adalberti $=361$

Aquila heliaca

Haliaeetus albicilla

Haliaeetus leucocephalus

Harpia harpyja

Pithecophaga jefferyi

Falco araea

Falco jugger

Falco newtoni ${ }^{* *}+215$

Falco pelegrinoides $=363$

Falco peregrinus

Falco punctatus

Falco rusticolus

Macrocephalon maleo

Crax blumenbachii

Miru mitu =364

Oreophasis derbianus

Penelope albipennis

Pipile jacuting $a=365 \mathrm{a}$

Pipile pipile $=365 \mathrm{~b}$

\section{Branta ruficollis}

Coscoroba coscoroba Cygnus melanocorypha Dendrocygna arborea Oxyura leucocephala

Sarkidiornis melanotos

FALCONIFORMES spp. * $-108$

Chondrohierax uncinatus wilsonii $=362$

Catreus wallichii

Argusianus argus

Colinus virginianus ridgwayi

Crossoptilon crossoptilon

Crossoptilon harmani $=366$

Crossoptilon mantchuricum

Lophophorus impejanus

Lophophorus Ihuysii

Lophophorus sclateri

Lophura edwardsi

Lophura imperialis

Lophura swinhaii
Gallus sonneratii

Ithaginis cruentus

Pavo muticus

Polyplectron bicalcaratum 
Rheinardia ocellata $=368$

Syrmaticus ellioti

Syrmaticus humiae

Syrmaticus mikado

Tetraogallus caspius

Tetraogallus tibetanus

Tragopan blythii

Tragopan caboti

Tragopan melanocephalus

Tympanuchus cupido attwateri
Polyplectron germaini Polyplectron malacense Polyplectron schleiermacheri $=367$

Gruidae spp. *

Grus americana

Grus canadensis nesiotes

Grus canadensis pulla

Grus japonensis

Grus leucogeranus

Grus monacha

Grus nigricollis

Grus vipio

Gallirallus sylvestris $=369$

Rhynochetos jubatus

Ardeotis nigriceps $=370$

Chlamydotis undulata

Eupodotis bengalensis $=371$

Otididae spp. *
Numenius borealis

Numenius tenuirostris

Tringa guttifer

Larus relictus

Laridae

COLUMBIFORMES

Columbidae

Caloenas nicobarica

Ducula mindorensis

\section{PSITTACIFORMES}

Psittacidae

\author{
Amazona arausiaca \\ Amazona barbadensis \\ Amazona brasiliensis \\ Amazona guildingii \\ Amazona imperialis
}

Gallicolumba luzonica Goura spp.

PSITTACIFORMES spp. * $-109$ 
Psittacidae (cont.)

\section{CUCULIFORMES}

Musophagidae

\section{STRIGIFORMES}

Tytonidae

Strigidae

\section{APODIFORMES}

Trochilidae

\author{
Amazona leucocephala \\ Amazona pretrei \\ Amazona rhodocorytha $=372$ \\ Amazona tucumana \\ Amazona versicolor \\ Amazona vinacea \\ Amazona viridigenalis \\ Amazona viltata \\ Anodorhynchus spp. \\ Ara ambigua \\ Ara glaucogularis $=373$ \\ Ara macao \\ Ara maracana \\ Ara militaris \\ Ara rubrogenys \\ Aratinga guarouba \\ Cacatua goffini \\ Cacatua haematuropygia \\ Cacatua moluccensis \\ Cyanopsitta spixii \\ Cyanoramphus auriceps forbesi \\ Cyanoramphus cookii $=374$ \\ Cyanoramphus novaezelandiae \\ Cyclopsitta diophthalma coxen $i=375$ \\ Eos histrio \\ Geopsittacus occidentalis p.e. $=376$ \\ Neophema chrysogaster \\ Ognorhynchus icterotis \\ Pezoporus wallicus \\ Pionopsitta pileata \\ Probosciger aterrimus \\ Psephotus chrysopterygius \\ Psephotus dissimilis $=377$ \\ Psephotus pulcherrimus p.e. \\ Psittacula echo $=378$ \\ Pyrrhura cruentata \\ Rhynchopsitta spp. \\ Strigops habroptilus \\ Vini ultramarina
}

Musophaga porphyreolopha $=379$

Tauraco spp.

STRIGIFORMES spp. *
Tyto soumagnei

Athene blewitti

Mimizuku gurneyi $=380$

Ninox novaeseelandiae undulata $=381$

Ninox squamipila natalis

Ramphodon dohrnii $=382$
Trochilidae spp. * 


\section{TROGONIFORMES}

Trogonidae

\section{CORACIIFORMES}

Bucerotidae

Aceros nipalensis

Aceros subruficollis

Buceros bicornis

Buceros vigil $=384$

\section{PICIFORMES}

Ramphastidae

Picidae

\section{PASSERIFORMES}

Cotingidae

Pittidae

Atrichornithidae

Hirundinidae

Pycnonotidae

Muscicapidae

Zosteropidae

Meliphagidae

Cotinga maculata

Pitta gurneyi

Pitta kochi

Picathartes oreas

Zosterops albogularis
Pharomachrus mocinno

Aceros spp. *

Anorrhinus spp. $=383$

Anthracoceros spp.

Buceros spp. *

Penelopides spp.

Pteroglossus aracari

Pteroglossus viridis

Ramphastos sulfuratus

Ramphastos toco

Ramphastos tucanus

Ramphastos vitellinus

Campephilus imperialis

Dryocopus javensis richardsi

Xipholena atropurpurea

Rupicola spp.

Pitta guajana

Pitta nympha $=385$

Atrichornis clamosus

Pseudochelidon sirintarae

Pycnonotus zeylanicus

Cyornis ruckii $=386$

Dasyornis broadbenti litoralis p.e.

Dasyornis longirostris $=387$

Leiothrix argentauris

Leiothrix lutea

Liocichla omeiensis

Picathartes gymnocephalus

Lichenostomus melanops cassidix

$=388$ 
Emberizidae

Icteridae

Fringillidae

Estrildidae

Sturnidae

Leucopsar rothschildi

Paradisaeidae

Agelaius flavus $=389$
Gubernatrix cristata

Paroaria capitata

Paroaria coronata

Tangara fastuosa

Carduelis cucullata $=390$

Carduelis yarrellii $=390$

Amandava formosa

Padda oryzivora

Poephila cincta cincta

Gracula religiosa

Paradisaeidae spp. 


\section{REPTILIA}

\section{TESTUDINATA}

Dermatemydidae

Dermatemys mawii

Emydidae

Batagur baska

Clemmys muhlenbergi

Geoclemys hamiltonii $=391$

Kachuga tect $a=392$

Melanochelys tricarinata $=393$

Morenia ocellata

Terrapene coahuila

Callagur borneoensis

Clemmys insculpta

\section{Terrapene spp. *}

Testudinidae

Geochelone nigra $=394$

Geochelone radiata $=395$

Geochelone yniphora $=395$

Gopherus flavomarginatus

Psammobates geometricus $=395$

Testudo kleinmanni

Cheloniidae

Cheloniidae spp.

Dermochelyidae

Dermochelys coriacea

Trionychidae

Trionyx ater $=396$

Triony'x gangeticus $=396$

Trionyx hurum $=396$

Trionyx nigricans $=396$

Pelomedusidae

Erymnochelys madagascariensis $=397$

Peliocephalus dumeriliana $=397$

Podocnemis spp.

\section{Chelidae}

Pseudemydura umbrina

CROCODYLIA

CROCODYLIA spp. $*=398$

Alligatoridae

Alligator sinensis

Caiman crocodilus apaporiensis

Caiman latirostris ** -110

Melanosuchus niger ** -111

Crocodylidae

Crocodylus acutus

Crocodylus cataphractus

Crocodylus intermedius

Crocodylus moreletii

Crocodylus niloticus **-112

Crocodylus novaeguineae mindorensis

=399

Crocodylus palustris

Crocodylus porosus ** -113

Crocodylus rhombifer 
Crocodylidae (cont.)

Gavialidae

\section{RHYNCHOCEPHALIA}

Sphenodontidae

\section{SAURIA}

Gekkonidae

Agamidae

Chamaeleonidae

Iguanidae

Brachylophus spp.

Cyclura spp.

Sauromalus varius

Gallotia simonyi

Lacertidae

Cordylidae

Teiidae

Scincidae

Xenosauridae

Helodermatidae

Varanidae
Cyrtodactylus serpensinsula $=400$

Phelsuma spp. $=401$

Uromastyx spp.

Bradypodion spp. $=402$

Chamaeleo spp. $=403$

Amblyrhynchus cristatus

Conolophus spp.

Iguana spp.

Phrynosoma coronatum

Podarcis lilfordi

Podarcis pityusensis

Cordylus spp.

Pseudocordylus spp.

Cnemidophorus hyperythrus

Crocodilurus lacertinus

Dracaena spp.

Tupinambis spp.

Corucia zebrata

Shinisaurus crocodilurus

Heloderma spp.

Varanus spp. *

Boidae spp. ${ }^{*}=404$

Acrantophis spp.

Boa constrictor occidentalis $=405$

Bolyeria multocarinata

Casarea dussumieri 
Boidae (cont.)

Colubridae

Elapidae

Viperidae
Epicrates inornatus

Epicrates monensis

Epicrates subflavus

Python molurus molurus $=406$

Sanzinia madagascariensis $=407$

Clelia clelia $=408$

Cyclagras gigas $=409$

Elachistodon westermanni

Ptyas mucosus

Hoplocephalus bungaroides

Naja naja $=410$

Ophiophagus hannah

Vipera ursinii +216

Vipera wagneri 


\section{AMPHIBIA}

\section{CAUDATA}

Ambystomidae

Ambystoma dumerilii

Ambystoma mexicanum

Cryptobranchidae

Andrias spp. $=411$

ANURA

Bufonidae

Altiphrynoides spp. $=412$

Atelopus varius zeteki

Bufo periglenes

Bufo superciliaris

Nectophrynoides spp.

Nimbaphrynoides spp. $=412$

Spinophrynoides spp. $=412$

Myobatrachidae

Dendrobatidae

Ranidae

Microhylidae

Dyscophus antongilii
Rheobairachus spp.

Allobates spp. $=413$

Dendrobates spp.

Epipedobates spp. $=413$

Minyobates spp. $=413$

Phobobates spp. $=413$

Phyllobates spp.

Mantella aurantiaca

Euphlyctis hexadactylus $=414$

Hoplobatrachus tigerinus $=414$ 


\section{PISCES}

\section{CERATODONTIFORMES}

Ceratodontidae

Neoceratodus forsteri

\section{COELACANTHIFORMES}

Latimeriidae

Latimeria chalumnae

PERCIFORMES

Sciaenidae

Cynoscion macdonaldi

\section{ACIPENSERIFORMES}

Acipenseridae

Acipenser brevirostrum

Acipenser sturio

Acipenser oxyrhynchus

Polyodor spathula

Polyodontidae

OSTEOGLOSSIFORMES

Osteoglossidae

Scleropages formosus

Arapaima gigas

\section{CYPRINIFORMES}

Cyprinidae

Probarbus jullieni

Caecobarbus geertsi

Chasmistes cujus

\section{SILURIFORMES}

Pangasiidae

Pangasianodon gigas

\section{'ACIPENSERIFORMES spp. *}

\section{Catostomidae}

1 This inclusion will enter into force on 1 April 1998. Esta inclusión entrará en vigor el 1 de abril de 1998. Cette inscription entrera en vigueur le $1^{\text {er }}$ avril 1998. 
ARTHROPODA

INSECTA

\section{LEPIDOPTERA}

Papilionidae

Ornithoptera alexandrae

Papilio chikae

Papilio homerus

Papilio hospiton
Bhutanitis spp.

Ornithoptera spp. ${ }^{*}=415$

Parnassius apollo

Teinopalpus spp.

Trogonoptera spp. $=415$

Troides spp. $=415$

\section{ARACHNIDA}

\section{SCORPIONES}

Scorpionidae

Pandinus dictator

Pandinus gambiensis

Pandinus imperator $=416$

\section{ARANEAE}

Theraphosidae

Brachypelma spp. $=417$

\section{ANNELIDA}

HIRUDINOIDEA

ARHYNCHOBDELLAE

Hirudinidae

Hirudo medicinalis 


\section{MOLLUSCA}

\section{BIVALVIA}

\section{VENEROIDA}

Tridacnidae

\section{UNIONOIDA}

\section{Unionidae}

Dromus dromas $=418$

Epioblasma curtisi $=419$

Epioblasma florentina $=419$

Epioblasma sampsoni $=419$

Epioblasma sulcata perobliqua $=419$

Epioblasma torulosa gubernaculum $=419$

Epioblasma torulosa torulosa $=419$

Epioblasma turgidula $=419$

Epioblasma walkeri $=419$

Fusconaia cuneolus

Fusconaia edgariana

Lampsilis higginsii

Lampsilis orbiculata orbiculata

Lampsilis satur

Lampsilis virescens

Plethobasus cicatricosus

Plethobasus cooperianus

Pleurobema plenum

Potamilus capax $=420$

Quadrula intermedia

Quadrula sparsa

Toxolasma cylindrella $=421$

Unio nickliniana $=422$

Unio tampicoensis tecomatensis $=423$

Villosa trabalis $=424$
Tridacnidae spp.

Cyprogenia aberti

Epioblasma torulosa rangiana $=419$

\section{Pleurobema clava}

\section{GASTROPODA}

\section{STYLOMMATOPHORA}

Achatinellidae

Achatinella spp.

\section{Camaenidae}

Papustyla pulcherrima $=425$

\section{MESOGASTROPODA}

Strombidae

Strombus gigas 
CNIDARIA

ANTHOZOA

COENOTHECALIA

COENOTHECALIA spp. $=426$

${ }^{\circ} 607$

STOLONIFERA

Tubiporidae

Tubiporidae spp. ${ }^{\circ} 607$

ANTIPATHARIA

ANTIPATHARIA spp.

SCLERACTINIA

SCLERACTINIA spp. ${ }^{\circ} 607$

HYDROZOA

MILLEPORINA

Milleporidae

Milleporidae spp. ${ }^{\circ} 607$

STYLASTERINA

Stylasteridae

Stylasteridae spp. ${ }^{\circ} 607$ 
F L O R A

AGAVACEAE

AMARYLLIDACEAE

APOCYNACEAE

Pachypodium ambongense

Pachypodium baronii

Pachypodium decaryi

ARALIACEAE

ARAUCARIACEAE

ASCLEPIADACEAE

BERBERIDACEAE

BROMELIACEAE

BYBLIDACEAE

\section{CACTACEAE}

Agave arizonica

Agave parviflora

Nolina interrata
Agave victoriae-reginae \#1

Galanthus spp. \#1

Sternbergia spp. \#1

Pachypodium spp. *\#1

Rauvolfia serpentina \#2

Panax q:ainquefolius \#3

Araucaria araucana $* *+217$

Araucaria araucana $*-114 \# 1$

Ceropegia spp.\#1

Frerea indica \#1

Podophyllum hexandrum

$=427$ \#2

Tillandsia harrisii \#1

Tillandsia kammii \#1

Tillandsia kautskyi \#1

Tillandsia mauryana \#1

Tillandsia sprengeliana \#1

Tillandsia sucrei \#1

Tillandsia xerographica \#1

Byblis spp. \#1

CACTACEAE spp. ${ }^{*}{ }^{\circ} 608$ \#4

Ariocarpus spp. $=428$

Astrophytum asterias $=429$

Aztekium ritteri

Coryphantha werdermannii $=430$

Discocactus spp.

Disocactus macdougallii $=431$

Echinocereus ferreirianus

ssp. lindsayi $=432$

Echinocereus schmollii $=433$

Escobaria minima $=434$

Escobaria sneedii $=435$

Mammillaria pectinifer $a=436$

Mammillaria solisioides

Melocactus conoideus

Melocactus deinacanthus

Melocactus glaucescens

Melocactus paucispinus

Obregonia denegrii

Pachycereus militaris $=437$

Pediocactus bradyi $=438$

Pediocactus knowltonii $=439$

Pediocactus paradinei

Pediocactus peeblesianus $=440$ 
Pediocactus siler $=441$

Pelecyphora spp. $=442$

Sclerocactus brevihamatus

ssp. tobuschii $=443$

Sclerocactus erectocentrus $=444$

Sclerocactus glaucus $=445$

Sclerocactus mariposensis $=446$

Sclerocactus mesae-verdae $=447$

Sclerocactus papyracanthus $=448$

Sclerocactus pubispinus $=449$

Sclerocactus wrightiae $=450$

Strombocactus spp.

Turbinicarpus spp. $=451$

Uebelmannia spp.

CARYOCARACEAE

CEPHALOTACEAE

COMPOSITAE

(ASTERACEAE)

CRASSULACEAE

CUPRESSACEAE

CYATHEACEAE

CYCADACEAE

DIAPENSIACEAE

DICKSONIACEAE

DIDIEREACEAE

DIOSCOREACEAE

DROSERACEAE

ERICACEAE

EUPHORBIACEAE
Cycas beddomei

Dudleya stolonifera

Dudleya traskiae

Fitzroya cupressoides

Pilgerodendron uviferum

\section{Caryocar costaricense \#1 \\ Cephalotus follicularis \#1}

CYATHEACEAE spp. \#1

CYCADACEAE spp. *\#1

Shortia galacifolia \#1

DICKSONIACEAE spp. \#1

DIDIEREACEAE spp. \#1

Dioscorea deltoidea \#1

Dionaea muscipula \#1

Kalmia cuneata \#1

Euphorbia spp. * - $115^{\circ} 609$ \#1

Euphorbia ambovombensis

Euphorbia capsaintemariensis $=453$

Euphorbia cremersii $=454$

Euphorbia cylindrifolia $=455$

Euphorbia decaryi $=456$

Euphorbia francoisii

Euphorbia moratii $=457$

Euphorbia parvicyathophora

Euphorbia quartziticola

Euphorbia tulearensis $=458$

Fouquieria fasciculata

Fouquieria columnaris \#1 
LEGUMINOSAE

(FABACEAE)

Dalbergia nigra

Aloe albida

Aloe albiflora

Aloe alfredii

Aloe bakeri

Aloe bellatula

Aloe calcairophila

Aloe compress $a=460$

Aloe delphinensis

Aloe descoingsii

Aloe fragilis

Aloe haworthioides $=461$

Aloe helenae

Aloe laeta $=462$

Aloe parallelifolia

Aloe parvula

Aloe pillansii

Aloe polyphylla

Aloe rauhii

Aloe suzannae

Aloe thorncroftii

Aloe versicolor

Aloe vossii

MELIACEAE

NEPENTHACEAE

Nepenthes khasiana

Nepenthes rajah

ORCHIDACEAE

Cattleya trianaei ${ }^{\circ} 610$

Dendrobium cruentum ${ }^{\circ} 610$

Laelia jongheana ${ }^{\circ} 610$

Laelia lobata ${ }^{\circ} 610$

Paphiopedilum spp. ${ }^{\circ} 610$

Peristeria elata ${ }^{\circ} 610$

Phragmipedium spp. ${ }^{\circ} 610$

Renanthera imschootiana ${ }^{\circ} 610$

Vanda coerulea ${ }^{\circ} 610$

\section{PALMAE}

(ARECACEAE)

PINACEAE

PODOCARPACEAE

Abies guatemalensis

Podocarpus parlatorei
Pericopsis elata \#5

Platymiscium pleiostachyum \#1

Pterocarpus santalinus \#6

Aloe spp. * -116 \#1
Swietenia humilis \#1

Swietenia mahagoni \#5

Nepenthes spp. *\#1

ORCHIDACEAE spp. $*=463$

\#7

Chrysalidocarpus

decipiens \#1

Neodypsis decaryi \#1 
PORTULACACEAE

PRIMULACEAE

PROTEACEAE

RANUNCULACEAE

ROSACEAE

RUBIACEAE

SARRACENIACEAE

Sarracenia alabamensis ssp. alabamensis $=466$

Sarracenia jonesii $=467$

Sarracenia oreophila

SCROPHULARIACEAE

STANGERIACEAE

TAXACEAE

THYMELEACEAE

(AQUILARIACEAE)

VALERIANACEAE

WELWITSCHIACEAE

ZAMIACEAE

Ceratozamia spp.

Chigua spp.

Encephalartos spp.

Microcycas calocoma
Anacampseros spp. $=464 \# 1$ Avonia spp. $=465$ \#1

Lewisia cotyledon \#1

Lewisia maguirei \#1

Lewisia serrata \#1

Cyclamen spp. ${ }^{\circ} 611$ \#1

Orothamnus zeyheri \#1

Protea odorata \#1

Hydrastis canadensis \#3

Prunus africana \#1

Darlingtonia californica \#1 Sarracenia spp. *\#1

Picrorhiza kurrooa \#3

Bowenia spp. $=468 \# 1$

Taxus wallichiana $=470 \# 8$

Aquilaria malaccensis \#1

Nardostachys grandiflora \#3

Welwitschia mirabilis $=471 \# 1$

ZAMIACEAE spp. *\#1

Hedychium philippinense \#1

Guaiacum officinale \#1

Guaiacum sanctum \#1 


\section{APPENDIX III}

\section{APENDICE III}

ANNEXE III 



\section{APPENDIX III}

valid from 19 March 1998

\section{INTERPRETATION}

1. References to taxa higher than species are for the purpose of information or classification only.

2. The symbol $(+)$ followed by a number placed against the name of a species denotes that only designated geographically separate populations of that species are included in Appendix III as follows:

+218 Population of Bolivia

+219 All populations of the species in the Americas.

3. The symbol $\Leftrightarrow$ followed by a number placed against the name of a species denotes that the name of that species shall be interpreted as follows:

$=472 \quad$ Also referenced as Vampyrops lineatus

$=473$ Formerly included as Tamandua tetradactyla (in part)

$=474$ Includes synonym Cabassous gymnurus

$=475$ Includes generic synonym Coendou

$=476$ Includes generic synonym Cuniculus

$=477$ Includes synonym Vulpes vulpes leucopus

$=478$ Formerly included as Nasua nasua

$=479$ Includes synonym Galictis allamandi

$=480$ Formerly included in Martes flavigula

$=481$ Includes generic synonym Viverra

$=482$ Formerly included as Viverra megaspila

$=483$ Formerly included as Herpestes auropunctatus

$=484$ Formerly included as Herpestes fuscus

$=485$ Formerly included as Bubalus bubalis (domesticated form)

$=486$ Also referenced as Boocercus eurycerus; includes generic synonym Taurotragus

$=487$ Also referenced as Ardeola ibis

$=488$ Also referenced as Egretta alba and Ardea alba

$=489$ Also referenced as Hagedashia hagedash

$=490$ Also referenced as Lampribis rara

$=491$ Also referenced as Spatula clypeata

$=492$ Also referenced as Nyroca nyroca

$=493$ Includes synonym Dendrocygna fulva

$=494$ Also referenced as Cairina hartlaubii

$=495$ Also referenced as Crax pauxi

$=496$ Formerly included as Arborophila brunneopectus (in part)

$=497$ Also referenced as Turturoena iriditorques; formerly included as Columba malherbii (in part)

$=498$ Also referenced as Nesoenas mayeri

$=499$ Formerly included as Treron australis (in part)

$=500$ Also referenced as Calopelia brehmeri; includes synonym Calopelia puella

$=501$ Also referenced as Tympanistria tympanistria

$=502$ Also referenced as Tchitrea bourbonnensis

$=503$ Formerly included as Serinus gularis (in part)

$=504$ Also referenced as Estrilda subflava or Sporaeginthus subflavus

$=505$ Formerly included as Lagonosticta larvata (in part)

$=506$ Includes generic synonym Spermestes

$=507$ Also referenced as Euodice cantans; formerly included as Lonchura malabarica (in part)

$=508$ Also referenced as Hypargos nitidulus

$=509$ Formerly included as Parmoptila woodhousei (in part)

$=510$ Includes synonyms Pyrenestes frommi and Pyrenestes rothschildi

$=511$ Also referenced as Estrilda bengala

$=512$ Also referenced as Malimbus rubriceps or Anaplectes melanotis 
$=513$ Also referenced as Coliuspasser ardens

$=514$ Formerly included as Euplectes orix (in part)

$=515$ Also referenced as Coliuspasser macrourus

$=516$ Also referenced as Ploceus superciliosus

$=517$ Includes synonym Ploceus nigriceps

$=518$ Also referenced as Sitagra luteola

$=519$ Also referenced as Sitagra melanocephala

$=520$ Formerly included as Ploceus velatus

$=521$ Also referenced as Hypochera chalybeata; includes synonyms Vidua amauropteryx, Vidua centralis, Vidua neumanni, Vidua okavangoensis and Vidua ultramarina

$=522$ Formerly included as Vidua paradisaea (in part)

$=523$ Also referenced as Pelusios subniger

$=524$ Formerly included in genus Natrix

$=525$ Formerly included as Talauma hodgsonii.

4. The names of the countries placed against the names of species are those of the Parties submitting these species for inclusion in this appendix.

5. Any animal, whether live or dead, of a species listed in this appendix, is covered by the provisions of the Convention, as is any readily recognizable part or derivative thereof.

6. In accordance with Article I, paragraph (b), sub-paragraph (iii), of the Convention, the symbol (\#) followed by a number placed against the name of a plant species included in Appendix III designates parts or derivatives which are specified in relation thereto for the purposes of the Convention as follows:

\#1 Designates all readily recognizable parts and derivatives, except:

a) seeds, spores and pollen (including pollinia);

b) seedling or tissue cultures obtained in vitro, in solid or liquid media, transported in sterile containers; and

c) cut flowers of artificially propagated plants

Designates logs, sawn wood and veneer sheets. 


\section{APENDICE III}

válido a partir del 19 de marzo de 1998

\section{INTERPRETACION}

1. Las referencias a los taxa superiores de la especie se indican únicamente para fines de información o de clasificación.

2. El signo (+) seguido de un número colocado junto al nombre de una especie significa que únicamente las poblaciones geográficamente aisladas de dicha especie están incluidas en el Apéndice III como sigue:

+218 Población de Bolivia

+219 Todas las poblaciones de la especie en el continente americano.

3. El signo (=) seguido de un número colocado junto al nombre de una especie significa que la denominación de dicha especie debe ser interpretada como sigue:

$=472$ También denominada Vampyrops lineatus

=473 Anteriormente incluida como Tamandua tetradactyla (en parte)

$=474$ Incluye el sinónimo Cabassous gymnurus

$=475$ Incluye el sinónimo genérico Coendou

$=476$ Incluye el sinónimo genérico Cuniculus

$=477$ Incluye el sinónimo Vulpes vulpes leucopus

$=478$ Anteriormente incluida como Nasua nasua

$=479$ Incluye el sinónimo Galictis allamandi

$=480$ Anteriormente incluida en Martes flavigula

$=481$ Incluye el sinónimo genérico Viverra

$=482$ Anteriormente incluida como Viverra megaspila

$=483$ Anteriormente incluida como Herpestes auropunctatus

$=484$ Anteriormente incluida como Herpestes fuscus

=485 Anteriormente incluida como Bubalus bubalis (forma doméstica)

=486 También denominada Boocercus eurycerus; incluye el sinónimo genérico Taurotragus

$=487$ También denominada Ardeola ibis

$=488$ También denominada Egretta alba y Ardea alba

$=489$ También denominada Hagedashia hagedash

$=490$ También denominada Lampribis rara

$=491$ También denominada Spatula clypeata

$=492$ También denominada Nyroca nyroca

=493 Incluye el sinónimo Dendrocy gna fulva

$=494$ También denominada Cairina hartlaubii

$=495$ También denominada Crax pauxi

$=496$ Anteriormente incluida como Arborophila brunneopectus (en parte)

=497 También denominada Turturoena iriditorques; anteriormente incluida como Columba malherbii (en parte)

$=498$ También denominada Nesoenas mayeri

$=499$ Anteriormente incluida como Treron australis (en parte)

$=500$ También denominada Calopelia brehmeri; incluye el sinónimo Calopelia puella

$=501$ También denominada Tympanistria tympanistria

$=502$ También denominada Tchitrea bourbonnensis

$=503$ Anteriormente incluida como Serinus gularis (en parte)

$=504$ También denominada Estrilda subflava o Sporaeginthus subflavus

$=505$ Anteriormente incluida como Lagonosticta larvata (en parte)

$=506$ Incluye el sinónimo genérico Spermestes

$=507$ También denominada Euodice cantans; anteriormente incluida como Lonchura malabarica (en parte)

$=508$ También denominada Hypargos nitidulus

=509 Anteriormente incluida como Parmoptila woodhousei (en parte) 
$=510$ Incluye los sinónimos Pyrenestes frommi y Pyrenestes rothschildi

=511 También denominada Estrilda bengala

=512 También denominada Malimbus rubriceps o Anaplectes melanotis

$=513$ También denominada Coliuspasser ardens

$=514$ Anteriormente incluida como Euplectes orix (en parte)

$=515$ También denominada Coliuspasser macrourus

$=516$ También denominada Ploceus superciliosus

$=517$ Incluye el sinónimo Ploceus nigriceps

$=518$ También denominada Sitagra luteola

$=519$ También denominada Sitagra melanocephala

$=520$ Anteriormente incluida como Ploceus velatus

=521 También denominada Hypochera chalybeata; incluye los sinónimos Vidua amauropteryx, Vidua centralis, Vidua neumanni, Vidua okavangoensis y Vidua ultramarina

$=522$ Anteriormente incluida como Vidua paradisaea (en parte)

=523 También denominada Pelusios subniger

$=524$ Anteriormente incluida en el género Natrix

$=525$ Anteriormente incluida como Talauma hodgsonii.

4. Los nombres de los países colocados junto a los nombres de las especies son los de las Partes que solicitaron la inclusión de esas especies en el presente Apéndice.

5. Todo animal, vivo o muerto, perteneciente a una especie incluida en el presente Apéndice, está al amparo de las disposiciones de la Convención, así como cualquier parte o derivado fácilmente identificable del mismo.

6. De conformidad con las disposiciones el subpárrafo iii) del párrafo b) del Artículo I de la Convención, el signo (\#) seguido de un número colocado junto al nombre de una especie vegetal incluida en el Apéndice III designa las partes o derivados provenientes de esa especie y se indican como sigue para los fines de la Convención:

\#1 Designa todas las partes y derivados fácilmente identificables, excepto:

a) las semillas, las esporas y el polen (inclusive las polinias);

b) los cultivos de plántulas o de tejidos obtenidos in vitro, en medios sólidos o líquidos, que se transportan en envases estériles; y

c) flores cortadas de plantas reproducidas artificialmente

\#5 Designa trozas, madera aserrada y láminas de chapa de madera. 


\section{ANNEXE III}

valable à compter du 19 mars 1998

\section{INTERPRETATION}

1. Les références à des taxons supérieurs à l'espèce sont données uniquement à titre d'information ou à des fins de classification.

2. Le signe (+) suivi d'un nombre placé après le nom d'une espèce signifie que seules des populations géographiquement isolées de ladite espèce sont inscrites à l'Annexe III, comme suit:

+218 Population de Bolivie

+219 Toutes les populations de l'espèce dans les Amériques.

3. Le signe ( $=$ ) suivi d'un nombre placé après le nom d'une espèce signifie que la dénomination de ladite espèce doit être interprétée comme suit:

$=472$ Aussi appelé Vampyrops lineatus

=473 Précédemment inclus en tant que Tamandua tetradactyla (en partie)

$=474$ Comprend le synonyme Cabassous gymnurus

=475 Comprend le synonyme générique Coendou

$=476 \quad$ Comprend le synonyme générique Cuniculus

$=477$ Comprend le synonyme Vulpes vulpes leucopus

$=478$ Précédemment inclus en tant que Nasua nasua

$=479$ Comprend le synonyme Galictis allamandi

=480 Précédemment inclus dans Martes flavigula

$=481$ Comprend le synonyme générique Viverra

$=482$ Précédemment inclus en tant que Viverra megaspila

$=483$ Précédemment inclus en tant que Herpestes auropunctatus

=484 Précédemment inclus en tant que Herpestes fuscus

=485 Précédemment inclus en tant que Bubalus bubalis (forme domestiquée)

$=486$ Aussi appelé Boocercus eurycerus; comprend le synonyme générique Taurotragus

$=487$ Aussi appelé Ardeola ibis

$=488$ Aussi appelé Egretta alba et Ardea alba

$=489$ Aussi appelé Hagedashia hagedash

$=490$ Aussi appelé Lampribis rara

$=491$ Aussi appelé Spatula clypeata

$=492$ Aussi appelé Nyroca nyroca

$=493$ Comprend le synonyme Dendrocygna fulva

$=494$ Aussi appelé Cairina hartlaubii

$=495$ Aussi appelé Crax pauxi

=496 Précédemment inclus en tant que Arborophila brunneopectus (en partie)

$=497$ Aussi appelé Turturoena iriditorques; précédemment inclus en tant que Columba malherbii (en partie)

$=498$ Aussi appelé Nesoenas mayeri

=499 Précédemment inclus en tant que Treron australis (en partie)

$=500$ Aussi appelé Calopelia brehmeri; comprend le synonyme Calopelia puella

$=501$ Aussi appelé Tympanistria tympanistria

$=502$ Aussi appelé Tchitrea bourbonnensis

=503 Précédemment inclus en tant que Serinus gularis (en partie)

$=504$ Aussi appelé Estrilda subflava et Sporaeginthus subflavus

$=505$ Précédemment inclus en tant que Lagonosticta larvata (en partie)

=506 Comprend le synonyme générique Spermestes

=507 Aussi appelé Euodice cantans; précédemment inclus en tant que Lonchura malabarica (en partie)

$=508$ Aussi appelé Hypargos nitidulus

=509 Précédemment inclus en tant que Parmoptila woodhousei (en partie) 
$=510$ Comprend les synonymes Pyrenestes frommi et Pyrenestes rothschild $i$

$=511$ Aussi appelé Estrilda bengala

$=512$ Aussi appelé Malimbus rubriceps et Anaplectes melanotis

$=513$ Aussi appelé Coliuspasser ardens

$=514$ Précédemment inclus en tant que Euplectes orix (en partie)

$=515$ Aussi appelé Coliuspasser macrourus

$=516$ Aussi appelé Ploceus superciliosus

$=517$ Comprend le synonyme Ploceus nigriceps

$=518$ Aussi appelé Sitagra luteola

$=519$ Aussi appelé Sitagra melanocephala

$=520$ Précédemment inclus en tant que Ploceus velatus

$=521$ Aussi appelé Hypochera chalybeata; comprend les synonymes Vidua amauropteryx,

Vidua centralis, Vidua neumanni, Vidua okavangoensis et Vidua ultramarina

$=522$ Précédemment inclus en tant que Vidua paradisaea (en partie)

$=523$ Aussi appelé Pelusios subniger

$=524$ Précédemment inclus dans le genre Natrix

$=525$ Précédemment inclus en tani que Talauma hodgsonii.

4. Les noms des pays placés après les noms des espèces sont ceux des Parties qui ont fait inscrire lesdites espèces à la présente annexe.

5. Tout animal, vivant ou mort, appartenant à une espèce mentionnée à la présente annexe, est couvert par les dispositions de la Convention, ainsi que toute partie ou tout produit facilement identifiable qui en dérive.

6. Conformément aux dispositions de l'Article I, paragraphe b), alinéa iii), de la Convention, le signe (\#) suivi d'un chiffre placé après le nom d'une espèce végétale inscrite à l'Annexe III sert à désigner des parties ou produits obtenus à partir de ladite espèce et qui sont mentionnés comme suit aux fins de la Convention:

\#1 Sert à désigner toutes les parties et tous les produits facilement identifiables, sauf:

a) les graines, les spores et le pollen (y compris les pollinies);

b) les cultures de plantules ou de tissus obtenues in vitro, en milieu solide ou liquide, et transportées en conteneurs stériles; et

c) les fleurs coupées des plantes reproduites artificiellement

sert à désigner les grumes, les bois sciés et les placages. 


\section{FAUNA}

\section{CHORDATA}

\section{MAMMALIA}

CHIROPTERA

Phyllostomidae

Platyrrhinus lineatus $=472$

Uruguay

XENARTHRA

Myrmecophagidae

Tamandua mexican $a=473$

Guatemala

Megalonychidae

Choloepus hoffmanni

Costa Rica

Dasypodidae

Cabassous centralis

Cabassous tatouay $=474$

Costa Rica

Uruguay

\section{RODENTIA}

Sciuridae

Epixerus ebii

Marmota caudata

Marmota himalayana

Sciurus deppei

Ghana

India

India

Costa Rica

Anomaluridae

Anomalurus beecrofii

Ghana

Anomalurus derbianus

Ghana

Anomalurus pelii

Idiurus macrotis

Ghana

Ghana

Hystricidae

Hystrix cristata

Ghana

Erethizontidae

Sphiggurus mexicanus $=475$

Honduras

Sphiggurus spinosus $=475$

Uruguay

Agoutidae

Agouti paca $=476$

Honduras

Dasyproctidae

Dasyprocta punctata

Honduras

CARNIVORA

Canidae

Canis aureus

Vulpes bengalensis

Vulpes vulpes griffithi

Vulpes vulpes montana

Vulpes vulpes pusilla $=477$
India
India
India
India
India
Costa Rica
Costa Rica
Honduras
Uruguay
Honduras

Bassaricyon gabbii

Bassariscus sumichrasti

Nasua nasua solitaria 
Mustelidae

Viverridae

Herpestidae

Hyaenidae

Odobenidae

\section{ARTIODACTYLA}

Tragulidae

Cervidae

Bovidae

\section{AVES}

\section{CICONIIFORMES}

Ardeidae

Ciconiidae
Eira barbara

Galictis vittata $=479$

Martes flavigula

Martes foina intermedia

Martes gwatkinsii $=480$

Mellivora capensis

Mustela altaica

Mustela erminea ferghanae

Mustela kathiah

Mustela sibirica

Arctictis binturong

Civettictis civetta $=481$

Paguma larvata

Paradoxurus hermaphroditus

Paradoxurus jerdoni

Viverra civettina $=482$

Viverra zibetha

Viverricula indica

Herpestes brachyurus fuscus $=484$

Herpestes edwardsii

Herpestes javanicus auropunctatus $=483$

Herpestes smithii

Herpestes urva

Herpestes vitticollis

Proteles cristatus

Odobenus rosmarus

Hyemoschus aquaticus

Cenvus elaphus barbarus

Mazama americana cerasina

Odocoileus virginianus mayensis

Antilope cervicapra

Bubalus arnee $=485$

Damaliscus lunatus

Gazella cuvieri

Gazella dorcas

Gazella leptoceros

Tetracerus quadricornis

Tragelaphus eurycerus $=486$

Tragelaphus spekii
Honduras

Costa Rica

India

India

India

Botswana, Ghana

India

India

India

India

India

Botswana

India

India

India

India

India

India

India

India

India

India

India

India

Botswana

Canada

Ghana

Tunisia

Guatemala

Guatemala

Nepal

Nepal

Ghana

Tunisia

Tunisia

Tunisia

Nepal

Ghana

Ghana

Ardea goliath

Bubulcus ibis $=487$

Ghana

Casmerodius albus $=488$

Ghana

Egretta garzetta

Ghana

Ghana

Ephippiorhynchus senegalensis

Ghana

Leptoptilos crumeniferus

Ghana 
Bostrychia hagedash $=489$

Bostrychia rara $=490$

Threskiornis aethiopicus
Ghana

Ghana

Ghana

Ghana

Ghana

Ghana

Ghana

Ghana

Ghana

Ghana

Ghana

Honduras

Honduras

Ghana, Honduras

Ghana

Ghana

Ghana

Ghana

Honduras

Sarcoramphus papa

Colombia

Colombia

Colombia

Colombia, Costa Rica,

Guatemala, Honduras

Guatemala, Honduras

Colombia

Honduras

Guatemala

Ghana

Guatemala

Malaysia

Malaysia

Malaysia

Malaysia

Malaysia

Malaysia

Malaysia

Malaysia

Malaysia

Nepal

Guatemala 


\section{COLUMBIFORMES}

Columbidae

Columba guinea

Ghana

Columba iriditorques $=497$

Ghana

Columba livia

Columba mayeri $=498$

Ghana

Columba unicincta

Mauritius

Oena capensis

Ghana

Streptopelia decipiens

Ghana

Streptopelia roseogrisea

Ghana

Streptopelia semitorquata

Ghana

Streptopelia senegalensis

Ghana

Streptopelia turtur

Ghana

Streptopelia vinacea

Ghana

Treron calva $=499$

Ghana

Treron waalia

Turtur abyssinicus

Turtur afer

Turtur brehmeri $=500$

Turtur tympanistria $=501$

Ghana

Ghana

Ghana

Ghana

Ghana

Ghana

\section{PSITTACIFORMES}

Psittacidae

Psittacula krameri

Ghana

\section{CUCULIFORMES}

Musophagidae

Conythaeola cristata

Crinifer piscator

Ghana

Musophaga violacea

Ghana

Ghana

\section{PICIFORMES}

Capitonidae

Semnornis ramphastinus

Colombia

Ramphastidae

Baillonius bailloni

Pteroglossus castanotis

Ramphastos dicolorus

Selenidera maculirostris

Argentina

Argentina

Argentina

Argentina

\section{PASSERIFORMES}

Cotingidae

Muscicapidae

Fringillidae

Estrildidae
Cephalopterus ornatus

Cephalopterus penduliger

Bebrornis rodericanus

Terpsiphone bourbonnensis $=502$

Serinus canicapillus $=503$

Serinus leucopygius

Serinus mozambicus

Amadina fasciata

Amandava subflava $=504$

Estrilda astrild

Estrilda caerulescens

Estrilda melpoda

Estrilda troglodytes

Lagonosticta rara

Lagonosticta rubricata
Colombia

Colombia

Mauritius

Mauritius

Ghana

Ghana

Ghana

Ghana

Ghana

Ghana

Ghana

Ghana

Ghana

Ghana

Ghana 
Lonchura bicolor $=506$

Ghana

Lonchura cantans $=507$

Ghana

Lonchura cucullata $=506$

Ghana

Lonchura fringilloides $=506$

Ghana

Mandingoa nitidula $=508$

Ghana

Nesocharis capistrata

Ghana

Nigrita bicolor

Nigrita canicapilla

Ghana

Nigrita fusconota

Ghana

Nigrita luteifrons

Ghana

Ortygospiza atricollis

Ghana

Parmoptila rubrifrons $=509$

Ghana

Pholidornis rushiae

Ghana

Pyrenestes ostrinus $=510$

Ghana

Pytilia hypogrammica

Ghana

Pytilia phoenicoptera

Ghana

Spermophaga haematina

Ghana

Uraeginthus bengalus =511

Ghana

Ghana

Amblyospiza albifrons

Anaplectes rubriceps $=512$

Ghana

Anomalospiza imberbis

Ghana

Bubalornis albirostris

Ghana

Euplectes afer

Euplectes ardens $=513$

Euplectes franciscanus $=514$

Ghana

Ghana

Ghana

Euplectes hordeaceus

Ghana

Euplectes macrourus $=515$

Ghana

Malimbus cassini

Ghana

Malimbus malimbicus

Ghana

Malimbus nitens

Ghana

Malimbus rubricollis

Ghana

Malimbus scutatus

Ghana

Pachyphantes superciliosus $=516$

Ghana

Passer griseus

Petronia dentata

Plocepasser superciliosus

Ghana

Ghana

Ghana

Ploceus albinucha

Ghana

Ploceus aurantius

Ghana

Ploceus cucullatus $=517$

Ghana

Ploceus heuglini

Ploceus luteolus $=518$

Ghana

Ghana

Ploceus melanocephalus $=519$

Ghana

Ploceus nigerrimus

Ghana

Ploceus nigricollis

Ghana

Ploceus pelzelni

Ghana

Ghana

Ploceus preussi

Ploceus tricolor

Ploceus vitellinus $=520$

Ghana

Ghana

Quelea erythrops

Ghana

Sporopipes frontalis

Ghana

Vidua chalybeat $a=521$

Ghana

Vidua interjecta

Ghana

Vidua larvaticola

Ghana

Vidua macrourn

Ghana

Vidua orientalis $=522$

Ghana

Vidua raricola

Ghana

Ghana 
REPTILIA

\section{TESTUDINATA}

Trionychidae

Pelomedusidae

\section{SERPENTES}

Colubridae

Elapidae

Viperidae

F L O R A

GNETACEAE

MAGNOLIACEAE

MELIACEAE

PAPAVERACEAE

PODOCARPACEAE

TETRACENTRACEAE
Trionyx triunguis

Ghana

Pelomedusa subrufa

Ghana

Pelusios adansonii

Pelusios castaneus

Pelusios gabonensis $=523$

Pelusios niger

Ghana

Ghana

Ghana

Ghana

India

India

India

Cerberus rhynchops

Xenochrophis piscator $=524$

Honduras

Honduras

Micrurus nigrocinctus

Honduras

Honduras

Honduras

Honduras

Honduras

Honduras

Honduras

India

Nepal

Nepal

Swietenia macrophylla $+218+219 \# 5$

Bolivia, Costa Rica

Meconopsis regia \#1

Nepal

Podocarpus neriifolius \#1

Nepal

Tetracentron sinense \#1

Nepal 


\section{CHECKLIST OF FAUNA}

\section{LISTA DE LAS ESPECIES DE FAUNA}

\section{LISTE DES ESPECES ANIMALES}





\section{CHECKLIST OF FAUNA}

\section{EXPLANATORY NOTE}

The checklist of Fauna contains the scientific names of all animal species listed in the CITES Appendices I and II and III (as of 18 September 1997) and, where available, English, French and Spanish common names. Some synonyms of scientific and common names are also listed but no attempt has been made to make these comprehensive. Two species of Psittaciformes not listed in the CITES appendices are included for information: Melopsittacus undulatus and Nymphicus hollandicus; these are the only species in this large order not covered by CITES provisions.

There are three types of entry in the list:

The first type commences with a scientific name, and is followed by English (E), Spanish (S) and French (F) common names (where available); after these the appendix (or appendices) in which the species is listed is given (or NC for the two non-CITES species), and finally a taxonomic letter code indicating the order or higher taxon in which the species is included. These letters are: $\mathrm{A}=$ amphibians, $\mathrm{B}=$ birds, $\mathrm{F}=$ fish, $\mathrm{In}=$ invertebrates, $\mathrm{M}=$ mammals and $\mathrm{R}$ $=$ reptiles.

The second type commences with a common name, either English (E), Spanish (S) or French (F), followed by the corresponding scientific name, the appendix number and the taxonomic letter code. The common names are listed only once, thus Golden Eagle but not also as Eagle, Golden. Some group names are included for information, indicating whether the species bearing that group name are in one (or more) CITES appendix and whether some species in the group are not listed in the appendices e.g. Parrots (E): PSITTACIFORMES spp. I/IIII/NC B.

The third type consists of a scientific synonym followed by = and then the accepted scientific name. The latter name should be looked up alphabetically to find the other relevant information about the species.

Nomenclature and taxonomy follow the CITES appendices but, for taxa listed above the species level in the appendices, the following references have been used:

Mammals:

Wilson, D. E. and Reeder, D. M. 1993. Mammal Species of the World: a Taxonomic and Geographic Reference. Second edition. Smithsonian Institution Press, Washington.

\section{Birds:}

Families

Species
Morony, J. J., Bock, W. J. and Farrand, J., Jr. 1975. A Reference List of the Birds of the World. American Museum of Natural History.

Sibley, C. G. and Monroe, B. L., Jr. 1990. Distribution and Taxonomy of Birds of the World. Yale University Press, New Haven; and

Sibley, C. G. and Monroe, B. L., Jr. 1993. Supplement to the Distribution and Taxonomy of Birds of the World. Yale University Press, New Haven. 
Frost, D. R., ed. 1985. Amphibian Species of the World. Allen Press, Inc. and The Association of Systematics Collections, Lawrence, Kansas; and

Duellman, W. E. 1993. Amphibian Species of the World: additions and corrections. University of Kansas Museum of Natural History, Special Publication No. 21.

Reptiles:

World Conservation Monitoring Centre. 1995. Checklist of Reptiles and Amphibians listed in the CITES Appendices. Sixth edition. Joint Nature Conservation Committee, Peterborough.

Invertebrates: World Conservation Monitoring Centre. 1995. Checklist of Fish and Invertebrates listed in the CITES Appendices. Third edition. Joint Nature Conservation Committee, Peterborough.

Note that the list does not contain the names of all known species of coral. 


\section{LISTA DE LAS ESPECIES DE FAUNA}

\section{NOTA EXPLICATIVA}

La Lista de las especies de Fauna contiene los nombres científicos de todos los animales incluidos en los Apéndices I, II y III de la CITES (al 18 de septiembre de 1997) y, en la medida de lo posible, sus nombres comunes en español, francés e inglés. Se indican también algunos sinónimos de los nombres científicos y comunes, aunque no de forma generalizada. Para su información, se incluyen dos especies de Psittaciformes que no figuran en los Apéndices de la CITES, a saber, Melopsittacus undulatus y Nymphicus hollandicus; se trata de las únicas especies de este amplio orden que no están protegidas por las disposiciones de la Convención.

La lista consta de tres tipos de entradas:

La primera comienza con un nombre científico, seguido de los nombres comunes en inglés (E), español $(S)$ y francés $(F)$ (cuando ha sido posible); a continuación figura el Apéndice (o Apéndices) en el que está incluida la especie (o NC para las dos especies no incluidas en los Apéndices) y, por último, una letra correspondiente al código taxonómico que indica el orden o taxón superior en que está incluida la especie: $\mathrm{A}=$ anfibios, $\mathrm{B}=$ aves, $\mathrm{F}=$ peces, $\mathrm{In}=$ invertebrados, $\mathrm{M}=$ mamíferos y $\mathrm{R}=$ reptiles.

La segunda comienza con un nombre común, ya sea inglés (E), español (S) o francés $(F)$, seguido del correspondiente nombre científico, el número del Apéndice y la letra correspondiente al código taxonómico. Los nombres comunes se indican una sola vez, por ejemplo, Aguila real, pero no como real, Aguila. Para su información se incluyen algunos nombres de grupos, indicando si la especie perteneciente a ese grupo está en uno (o más) Apéndices CITES y si algunas especies del grupo no están incluidas en los Apéndices, por ejemplo: Loros (S): PSITTACIFORMES spp. I/IIIII/NC B.

La tercera consiste en un sinónimo científico seguido de $=$ y luego el nombre científico aceptado. Este último deberá buscarse por orden alfabético para acceder a la información pertinente sobre la especie.

La nomenclatura y la taxonomía corresponden a las utilizadas en los Apéndices CITES, pero, en el caso de los taxa incluidos por encima del nivel de la especie en los Apéndices, se han utilizado las siguientes referencias:

Mamíferos:

Wilson, D. E. and Reeder, D. M. 1993. Mammal Species of the World: a Taxonomic and Geographic Reference. Second edition. Smithsonian Institution Press, Washington.

Aves:

Familias

Morony, J. J., Bock, W. J. and Farrand, J., Jr. 1975. A Reference List of the Birds of the World. American Museum of Natural History.

Especies

Sibley, C. G. and Monroe, B. L., Jr. 1990. Distribution and Taxonomy of Birds of the World. Yale University Press, New Haven; y 
Sibley, C. G. and Monroe, B. L., Jr. 1993. Supplement to the Distribution and Taxonomy of Birds of the World. Yale University Press, New Haven.

Anfibios:

Reptiles:

Invertebrados:
Frost, D. R., ed. 1985. Amphibian Species of the World. Allen Press, Inc. and The Association of Systematics Collections, Lawrence, Kansas; y

Duellman, W. E. 1993. Amphibian Species of the World: additions and corrections. University of Kansas Museum of Natural History, Special Publication No. 21.

World Conservation Monitoring Centre. 1995. Checklist of Reptiles and Amphibians listed in the CITES Appendices. Sixth edition. Joint Nature Conservation Committee, Peterborough.

World Conservation Monitoring Centre. 1995. Checklist of Fish and Invertebrates listed in the CITES Appendices. Third edition. Joint Nature Conservation Committee, Peterborough.

Sírvase tomar nota de que en esta lista no se incluyen los nombres de todas las especies conocidas de coral. 


\section{LISTE DES ESPECES ANIMALES}

\section{NOTE EXPLICATIVE}

La liste des espèces animales contient les noms scientifiques de toutes les espèces de faune sauvage inscrites aux annexes CITES I, II et III ( au 18 septembre 1997), avec leurs noms communs en anglais, en français et en espagnol lorsqu'ils sont connus. Certains synonymes de noms scientifiques et de noms communs sont indiqués mais la liste n'en est pas complète. Deux espèces de l'ordre des Psittaciformes (Melopsittacus undulatus et Nymphicus hollandicus) sont signalées pour information - bien que n'étant pas inscrites aux annexes - car ce sont les deux seules espèces de cet ordre très vaste à ne pas être couvertes par la Convention.

La liste des espèces animales comporte trois types d'entrées:

Le premier comprend le nom scientifique suivi, s'ils sont connus, des noms communs en anglais (E), en espagnol (S). et en français (F) Vient ensuite l'annexe (ou les annexes) à laquelle l'espèce est inscrite (ou la mention NC pour les deux espèces non inscrites aux annexes CITES). Enfin, un code taxonomique à une ou à deux lettres indique l'ordre ou le taxon supérieur auquel appartient l'espèce. Ces codes sont les suivants: $\mathrm{A}=$ amphibiens, $\mathrm{B}=$ oiseaux, $\mathrm{F}=$ poissons, In $=$ invertébrés, $M=$ mammifères et $R=$ reptiles.

Le deuxième comprend le nom commun, soit en anglais $(E)$, en espagnol $(S)$ ou en français $(F)$, suivi du nom scientifique, de l'annexe et du code taxonomique. Les noms communs apparaissent une seule fois. Par exemple: Aigle royal n'est pas complété par Royal, aigle. Certains noms de groupe sont donnés à titre d'information, en indiquant si les espèces du groupe sont inscrites à une ou plusieurs annexes et si certaines espèces n'y sont pas inscrites. Exemple: Perroquets (F): PSITTACIFORMES spp. I/II/II/NC B.

Le troisième est un synonyme scientifique suivi de $=$ puis du nom scientifique accepté. Ce dernier doit être recherché selon l'ordre alphabétique pour trouver les autres renseignements concernant l'espèce.

La nomenclature et la taxonomie utilisées sont celles des annexes CITES mais, en ce qui concerne les taxons supérieurs à l'espèce, les ouvrages de référence suivants ont été utilisés:

Mammifères:

Wilson, D. E. and Reeder, D. M. 1993. Mammal Species of the World: a Taxonomic and Geographic Reference. Second edition. Smithsonian Institution Press, Washington.

\section{Oiseaux:}

Familles

Morony, J. J., Bock, W. J. and Farrand, J., Jr. 1975. A Reference List of the Birds of the World. American Museum of Natural History.

Espèces

Sibley, C. G. and Monroe, B. L., Jr. 1990. Distribution and Taxonomy of Birds of the World. Yale University Press, New Haven; et

Sibley, C. G. and Monroe, B. L., Jr. 1993. Supplement to the Distribution and Taxonomy of Birds of the World. Yale University Press, New Haven. 
Amphibiens:

Reptiles:

Invertébrés:
Frost, D. R., ed. 1985. Amphibian Species of the World. Allen Press, Inc. and The Association of Systematics Collections, Lawrence, Kansas; et

Duellman, W. E. 1993. Amphibian Species of the World: additions and corrections. University of Kansas Museum of Natural History, Special Publication No. 21.

World Conservation Monitoring Centre. 1995. Checklist of Reptiles and Amphibians listed in the CITES Appendices. Sixth edition. Joint Nature Conservation Committee, Peterborough.

World Conservation Monitoring Centre. 1995. Checklist of Fish and Invertebrates listed in the CITES Appendices. Third edition. Joint Nature Conservation Committee, Peterborough.

Remarque: La liste des espèces animales ne donne pas tous les noms des espèces connues de coraux. 


\section{Aardwolf (E): Proteles cristatus III M}

Abaco Island Boa (E): Epicrates exsul II R

Abbé Allotte's Birdwing (E): Omithoptera allottei II In

Abbott's Booby (E): Papasula abbotti I B

Abbott's Day Gecko (E): Phelsuma abbotti II R

Abeillia abeillei: Emerald-chinned Hummingbird (E), Colibri barbiesmeralda (S), Colibri d'Abeillé (F) II B

Abejero colilargo (S): Henicopemis longicauda II B

Abejero de Célebes (S): Pemis celebensis II B

Abejero europeo (S): Pernis apivorus II B

Abejero negro (S): Henicopemis infuscatus II B

Abejero oriental (S): Pernis ptilorhyncus II B

Abolí (S): Euoricus elegantulus II M

Aboma (F): Epicrates cenchria II R

Abruzzo Chamois (E): Rupicapra pyrenaica omata I M

Aburria jacuring $a=$ Pipile jacutinga

Aburria pipile pipile $=$ Pipile pipile

Abyssinian Lovebird (E): Agapomis tarania II B

Abyssinian Owl (E): Asio abyssinicus II B

Acanthastrea amakusensis: II In

Acanthastrea bowerbanki: II In

Acanthastrea echinata: II in

Acanthastrea hemprichii: II In

Acanthastrea hillae: II In

Acanthastrea ishigakiensis: II ln

Acanthastrea lordhowensis: II in

Acanthastrea maxima: II in

Acanthastrea minuta: II in

Acanthastrea rotundoflora: II in

Acanthastrea simplex: II In

Acanthelia $=$ Echinopora

Acanthocyathus $=$ Caryophyllia

Acanthophyllia = Cynarina

Acanthopora = Echinopora

Acapulco Lesser Orange Tarantula (E): Brechypelma klaasi II In

Accipiter albogularis: Pied Goshawk (E), Pied Sparrowhawk (E), Gavilán pio (S), Autour pie (F) II B

Accipiter badius: Little Banded Sparrowhawk (E), Shikra (E), Gavilán chikra (S), Épervier shikra (F) II B

Accipiter bicolor: Bicolored Hawk (E), Bicolored Sparrowhawk (E), Azor bicolor (S), Esparvero bicolor (S), Gavilán bicolor (S), Gavilán pantalón (S), Épervier bicolore (F) II B

Accipiter brachyurus: New Britain Sparrowhawk (E), Gavilancito de Nueva Bretaña (S). Épervier de Nouvelle-Bretagne (F) II B

Accipiter brevipes: Levant Sparowhawk (E), Gavilån griego (S), Epervier à pieds courts (F) II B

Accipiter buergersi $=$ Erythrotriorchis buergersi

Accipiter butleri: Nicobar Shikra (E), Nicobar Sparrowhawk (E), Gavilán de Nicobar (S), Épervier des Nicobar (F) II B

Accipiter castanilius: Chestnut-bellied Sparrowhawk (E), Chestnutflanked Sparrowhawk (E), Gavilán flanquirrojo (S), Gavilán pechirrojo (S), Autour à flancs roux (F) II B

Accipiter chilensis $=$ Accipiter bicolor

Accipiter chionogaster: White-breasted Hawk (E), Gavilán pechiblanco (S), Gavilån ventriblanco (S), Épervier à poitrine blanche (F) II B

Accipiter cirrocephalus: Collared Sparrowhawk (E), Gavilán acollarado (S), Épervier à collier roux (F) II B

Accipiter collaris: Semi-collared Hawk (E), Semi-collared Sparrowhawk (E), Azer semicollarejo (S), Gavilancito torcaz (S), Gavilán acollarado (S), Épervier à collier interrompu (F) II B

Accipiter cooperii: Cooper's Hawk (E), Gavilán de Cooper (S), Épervier de Cooper (F) II B

Accipiter doriae $=$ Megatriorchis doriae

Accipiter erythrauchen: Moluccan Sparrowhawk (E), Rufous-necked Sparrowhawk (E), Gavilancito moluqueño (S), Épervier à gorge grise (F) II B

Accipiter erythronemius: Rufous-thighed Hawk (E), Épervier à cuisses rouges (F) II B

Accipiter erythropus: Red-thighed Sparowhawk (E), Gavilancito de Hartlaub (S), Gavilancito muslirrojo (S), Autour minulle (F), Épervier de Hartlaub (F) II B

Accipiter fasciatus: Australasian Goshawk (E), Brown Goshawk (E), Azor australiano (S), Autour australien (F) II B
Accipiter francesii: Frances's Goshawk (E), Gavilán de Frances (S), Épervier de Frances (F) II B

Accipiter genrilis: Northern Goshawk (E), Azor (S), Azor común (S), Gavilán azor (S), Autour des palombes (F) II B

Accipiter griseiceps: Sulawesi Goshawk (E), Gavilán de Célebes (S), Autour des Célèbes (F) II B

Accipiter griseogularis $=$ Accipiter novaehollandiae

Accipiter gularis: Japanese Sparrowhawk (E), Gavilancito japonés (S), Epervier du Japon (F) II B

Accipiter gundlachi: Gundlach's Hawk (E), Gavilán cubano (S), Epervier de Cuba (F) II B

Accipiter gundlachii $=$ Accipiter gundlach $i$

Accipiser haplochrous: New Caledonia Goshawk (E), White-bellied Goshawk (E), Gavilán de Nueva Caledonia (S), Autour à ventre blane (F) II B

Accipiter henicogrammus: Gray's Goshawk (E), Moluccan Barred Sparrowhawk (E), Moluccan Goshawk (E), Azor moluqueño (S), Autour des Moluques (F) II B

Accipiler henstii: Henst's Goshawk (E), Azor malgache (S), Autour de Henst (F) II B

Accipiter imitator: Imitator Sparrowhawk (E), Gavilán imitador (S), Autour imitateur (F) II B

Accipiter lureoschistaceus: Slaty-backed Goshawk (E), Slatymantled Sparrowhawk (E), Gavilán de Nueva Bretaña (S) Autour blue et gris (F) II B

Accipiter madagascariensis: Madagescar Sparrowhawk (E), Gavilán malgache (S), Épervier de Madagascar (F) II B

Accipiter melanochlamys: Black-mantled Goshawk (E), Gavilán rufinegro (S), Autour à manteau noir (F) II B

Accipiter melanoleucus: Black Goshawk (E), Black Sparrowhawk

(E), Great Sparrowhawk (E), Azor blanquinegro (S), Autour noir (F), Épervier pie (F) II B

Accipiter meyerianus: Meyer's Goshawk (E), Papuan Goshawk (E), Azor de Meyer (S), Autour de Meyer (F) II B

Accipiter minullus: African Little Sparrowhawk (E), Little Sparrowhawk (E), Gavilancito chico (S), Épervier minule (F) II B

Accipiter nanus: Small Sparrowhawk (E), Gavilancito de Célebes (S), Épervier des Célèbes (F) II B

Accipiter nisus: Eurasian Sparrowhawk (E), Gavilán común (S), Épervier d'Europe (F) II B

Accipiter novaehollandiae: Grey Goshawk (E), Grey-throated Goshawk (E), Variable Goshawk (E), White Goshawk (E), Azor variable (S), Autour blanc (F) II B

Accipiler ovampensis: Ovampo Sparrowhawk (E), Gavilán de Ovampo (S), Épervier de l'Ovampo (F) II B

Accipiler poliocephalus: Grey-headed Goshawk (E), New Guinea Grey-headed Goshawk (E), Gavilán cabecigris (S), Autour à tête grise (F) II B

Accipiter poliogaster: Grey-bellied Goshawk (E). Grey-bellied Hawk (E), Azor pechigrís (S), Azor ventigris (S), Esparvero pechigris (S), Gavilán vientrigris (S), Autour à ventre gris (F) II B

Accipiter princeps: New Britain Goshawk (E), Azor de Nueva Bretaña (S), Autour de Mayr (F) II B

Accipiter radiatus $=$ Erythrotriorchis radiatus

Accipiter rhodogaster. Vinous-breasted Sparrowhawk (E), Gavilán pechirrojo (S), Épervier à poitrine rousse (F) II $\mathrm{B}$

Accipiter rufitorques: Fiji Goshawk (E), Gavilán de las Fiji (S), Autour des Fidji (F) II B

Accipiter nufiventris: Rufous-chested Sparrowhawk (E), Gavilán papirufo (S), Épervier menu (F) II B

Accipiter soloensis: Chinese Goshawk (E), Chinese Sparrowhawk (E), Grey Frog Hawk (E), Gavilán ranero (S), Épervier de Horsfield (F) II B

Accipiter striatus: Sharp-shinned Hawk (E), Azor chico (S),

Esparvero chico (S), Gavilán americano (S), Gavilán arrastrador (S), Gavilán pajarero (S), Épervier brun (F) II B

Accipiter striatus chionogaster $=$ Accipiter chionogaster Accipiter striatus erythronemius $=$ Accipite erythronemius

Accipiter striatus ventralis $=$ Accipiter ventralis

Accipiter superciliosus: Tiny Hawk (E), Tiny Sparrowhawk (E),

Azor gris (S), Esparvero gris (S), Gavilancito americano (S) Gavilán enano (S), Epervier nain (F) II B

Accipiter tachiro: African Goshawk (E), Azor tachiro (S), Autour tachiro (F) II B

Accipiter tachiro toussenelii $=$ Accipiter sousseneli 
Accipiter toussenelii: Red-chested Goshawk (E), Azor de Toussenel (S), Gavilán pechirroseo (S), Autour de Toussenel (F) II B

Accipiter trinotarus: Spot-tailed Goshawk (E), Spot-tailed Sparrowhawk (E), Gavilán colipinto (S), Epervier à queue tachetée (F) II B

Accipiter trivirgatus: Asian Crested Goshawk (E), Crested Goshawk (E), Azor moñudo (S), Autour huppé (F) II B

Accipiser ventralis: Plain-breasted Hawk (E), Azor pechillano (S), Gavilán andino (S), Epervier à gorge rayée (F) II B

Accipiter virgatus: Besra (E), Besra Sparrowhawk (E), Gavilán besra (S), Épervier besta (F) II B

Accipiter virgatus gularis $=$ Accipiter gularis

Acerodon spp.: Fiying-foxes (E), Zorros voladores (S), Renardsvolants (F). Roussettes (F) II M

Acerodon celebensis: Sulawesi Flying-fox (E), Sulawesi Fruit Bat (E), Zorro volador de las Célebes (S) II M

Acerodon humilis: Talaud Flying-fox (E), Talaud Fruit Bat (E), Zorro volador de Talaud (S) II M

Acerodon jubatus: Golden-capped Fruit Bat (E), Zorro volador filipino (S) I M

Acerodon leucotis: Zorro volador de Calamian (S) II M

Acerodon lucifer. Panay Giant Fruit Bat (E), Zorro volador de la isla Panay (S) I M

Acerodon mackloti: Sunda Flying-fox (E), Zorro volador de Macklo (S) II M

Aceros spp.: Hombills (E), Buceros (S), Calaos (F) I/II B

Aceros cassidix: Knobbed Hombill (E), Calao à cimier (F) II B

Aceros comalus: White-crowned Hornbill (E), Calao coiffé (F) II B

Aceros corrugarus: Wrinkled Hornbill (E), Calao à casque rouge (F) II B

Aceros everetri: Sumba Hombill (E), Calao de Sumba (F) II B

Aceros leucocephalus: Writhed Hombill (E), Calao de Vieillot (F) II B

Aceros leucocephalus waldeni $=$ Aceros waldeni

Aceros narcondami: Narcondam Hornbill (E), Cálao de Narcondam (S), Calao de Narcondam (F) II B

Aceros nipalensis: Rufous-cheeked Hombill (E), Rufous-necked Hornbill (E), Calao de montagne (F), Calao à cou roux (F) I B

Aceros plicatus: Blyth's Hombill (E), Papuan Hombill (E), Calao papou (F) II B

Aceros plicatus subruficollis $=$ Aceros subruficollis

Aceros subruficollis: Plain-pouched Hombill (E), Calao à gorge claire (F), Calao à poche unie (F) I B

Aceros undulatus: Wreathed Hornbill (E), Calao festonné (F) II B

Aceros waldeni: Writhed-billed Hornbill (E), Calao de Walden (F) II B

Acestrura astreans: Santa Marta Woodstar (E), Colibri des Santa Marta (F) II B

Acestrura berlepschi: Esmeraldas Woodstar (E), Estrellita esmeraldeña (S), Colibri de Berlepsch (F) II B

Acestrura bombus: Little Woodstar (E). Estrellita chica (S), Colibri bourdon (F) Il B

Acestrura heliodor. Gorgeted Woodstar (E), Estrella cuellirrojo (S), Estrellita de gorguera (S), Colibri héliodore (F) II B

Acestrura heliodor astreans $=$ Acestrura astreans

Acestrura mulsant: White-bellied Woodstar (E), Estrellita ventriblanca (S), Colibri de Mulsant (F) II B

Achatinella spp:: Little agate shells (E), Oahu tree snails (E) I in

Achatinella abbreviata: I In

Achatinella apexfulva: I in

Achatinella bellula: I In

Achatinella buddii: I In

Achatinella bulimoides: I In

Achatinella byronii: I In

Achatinella caesia: I In

Achatinella casta: I in

Achatinella cestus: I in

Achatinella concavospira: I In

Achatinella curta: I In

Achatinella decipiens: I In

Achatinella decora: I In

Achatinella dimorpha: I in

Achatinella elegans: I in

Achatinella fulgens: I In

Acharinella fuscobasis: $I$ in

Achatinella juddii: I In
Achatinella juncea: I In

Achatinella lehuiensis: I in

Acharinella leucorrhaphe: I In

Achatinella lila: I in

Achatinella livida: I In

Achatinella lorata: I In

Achatinella musselina: I In

Achatinella papyracea: I In

Achatinella phaeozona: I In

Achatinella pulcherrima: I In

Achatinella pupukanioe: $\mathbf{I}$ in

Achatinella rose $=$ = Achatinella bulimoides rosea

Achatinella sowerbyana: I In

Achatinella spaldingi: I ln

Achatinella stewartii: I In

Achatinella swiftii: I In

Achatinella taeniolata: I in

Acharinella thaanumi: I in

Achatinella turgida: I in

Achasinella valida: I In

Acharinella viridans: I in

Achatinella vittata = Achatinella apexfulva vittata

Achatinella vulpina: I In

Achoque (E): Ambystoma dumerilii II A

Acinixys planicauda $=$ Pyxis planicauda

Acinonyx jubatus: Cheetah (E), Hunting Leopard (E), Chita (S), Guepardo (S), Guépard (F) I M

Acipenser baerii: Siberian Sturgeon (E), Esturgeon sibérien (F) II F Acipenser brevirostrum: Shortnose Sturgeon (E), Esturión

hociquicorto (S), Esturgeon à nez court $(\mathrm{F})$ I F

Acipenser dabryanus: Dabry's Sturgeon (E), Yangtze Sturgeon (E) II F

Acipenser fulvescens: Lake Sturgeon (E), Esturión lacustre (S), Esturgeon jaune (F) II F

Acipenser gueldenstaedtii: Russian Sturgeon (E) II F

Acipenser medirostris: Green Sturgeon (E), Esturgeon vert (F) II F Acipenser mikadoi: Sakhalin Sturgeon (E) II F

Acipenser naccarii: Adriatic Sturgeon (E), Storione cobice (Ital) (E), Esturión del Adriático (S), Esturgeon de l'Adriatique (F) II F

Acipenser nudiventris: Bastard Sturgeon (E), Fringebarbel Sturgeon (E), Ship Sturgeon (E), Spiny Sturgeon (E), Thom Sturgeon (E),

Esturión barba de flecos (S), Esturgeon à barbillons frangés (F) II $\mathbf{F}$

Acipenser oxyrhynchus: Atlantic Sturgeon (E), Esturión del Atlántico

(S), Esturgeon de l'Atlantique (F) II F

Acipenser persicus: Persian Sturgeon (E) II F

Acipenser ruthenus: Sterlet (E) II F

Acipenser schrencki: Amur Sturgeon (E) II F

Acipenser sinensis: Chinese Sturgeon (E) II F

Acipenser stellatus: Star Sturgeon (E), Stellate Sturgeon (E),

Esturión estrellado (S), Esturgeon étoilé (F) II F

Acipenser sturio: Baltic Sturgeon (E), Common Sturgeon (E),

Esturión común (S), Esturgeon commun (F) I F

Acipenser transmontanus: White Sturgeon $(E)$, Esturgeon blanc $(F)$ II F

ACIPENSERIFORMES spp.: Sturgeons (E), Esturións (S),

Esturgeons (F) I/II F

Acklin's Ground lguana (E): Cyclura rileyi I R

Acoupa de MacDonald (F): Cynoscion macdonaldi I F

Acrantophis spp.: Madagascar ground boas (E), Boas des savanes de Madagascar (F) I R

Acrantophis dumerili: Dumeril's Boa (E), Madagascar Ground Boa (E), Boa de Dumeril (S), Boa de Dumeril (F), Boa des savanes de Dumeril (F) I R

Acrantophis madagascariensis: Madagascar Boa (E), Malagasy

Ground Boa (E), Boa de Madagascar meridional (S), Boa de

Madagascar (F), Boa des savanes de Madagascar (F) I R

Acrhelia horrescens: II in

Acrocephalus rodericanus $=$ Bebromis rodericanus

Acropora abrolhosensis: II In

Acropora acenvata: II In

Acropora aculeus: II In

Acropora acuminasa: II In

Acropora akajimensis: II In

Acropora alces: II In

Acropora angulata: II In 
Acropora anthocercis: II In Acropora appressa: II in Acropora arabensis: II In Acropora aspera: II in Acropora attenuata: II In Acropora austera: II in Acropora azurea: II In Acropora batunai: II In Acropora borealis: II in Acropora branchi: 11 In Acropora brueggemanni: II In Acropora bushyensis: II In Acropora canalis: II in Acropora capillaris: II In Acropora cardenae: II In Acropora carduus: II In Acropora caroliniana: II In Acropora cerealis: II In

Acropora cervicomis: Staghom Coral (E), Corail comes de cerf (F) II in

Acropora ceylonica: II In

Acropora chesterfieldensis: II in

Acropora claihrata: II In

Acropora copiosa: II In

Acropora corymbosa: II In

Acropora corymbosa $=$ Acropora cytherea

Acropora crassa: II In

Acropora crateriformis: II In

Acropora cuneata: II in

Acropora cuspidata: II In

Acropora cyclopea: II in

Acropora cytherea: II In

Acropora danai: II In

Acropora demani: $\mathbf{I I}$ In

Acropora dendrum: 11 in

Acropora derawanensis: II in

Acropora desalwii: II In

Acropora diffusa: II In

Acropora digitifera: II In

Acropora diomedeae: II In

Acropora divaricata: II ln

Acropora donei: II In

Acropora dumosa: II in

Acropora echinata: II In

Acropora effluens: II In

Acropora elegans: II In

Acropora elliptica: II in

Acropora elseyi: II In

Acropora exquisita: II In

Acropora florida: II In

Acropora formosa: II In

Acropora forskalii: II In

Acropora gemmifera: II In

Acropora glauca: 11 In

Acropora glochiclados: II In

Acropora gonagra: II In

Acropora grandis: II In

Acropora granulosa: II In

Acropora hemprichii: II In

Acropora heteroclados: II In

Acropora hoeksemai: II In

Acropora horrida: II In

Acropora humilis: II In

Acropora hyacinthus: II In

Acropora implicata: II In

Acropora indiana: II In

Acropora indica: II In

Acropora indonesia: II In

Acropora insignis: II in

Acropora jacquelineae: II In

Acropora kirstyae: II In

Acropora kosurini: II In

Acropora latistella: II in

Acropora lianae: II In

Acropora listeri: II In

Acropora loisetteae: II In
Acropora lokani: II In

Acropora longicyathus: II Ir,

Acropora loripes: II in

Acropora lovelli: II In

Acropora lutkeni: II In

Acropora luzonica: $\mathbf{I}$ In

Acropora magnifica: II In

Acropora microclados: II In

Acropora microphthalma: II in

Acropora millepora: II In

Acropora mirabilis: II In

Acropora monticulosa: II In

Acropora mossambica: II In

Acropora multiacuta: II In

Acropora nana: II in

Acropora nasula: II in

Acropora natalensis: II In

Acropora nobilis: II In

Acropora ocellata: II In

Acropara oligocyathus: II in

Acropora pagoensis: II In

Acropora palifera: II In

Acropora palmata: Elkhorn Coral (E), Corail cornes d'élan (F) II In Acropora palmerae: II In

Acropora paniculata: II In

Acropora parilis: II In

Acropora parvistella: II In

Acropora pharaonis: II In

Acropora philippinensis: II In

Acropora platycyathus: II In

Acropora pocilloporina: II In

Acropora polymorpha: II In

Acropora polystoma: II In

Acropora profusa: II in

Acropora prolifera: Fused Staghom Coral (E) II In

Acropora prosirata: II In

Acropora pruinosa: II In

Acropora pulchra: II in

Acropora pumila: II In

Acropora rambleri: II In

Acropora ramiculosa: II In

Acropora rayneri: II In

Acropora retusa: II In

Acropora robusta: II in

Acropora rosaria: II In

Acropora russelli: II In

Acropora samoensis: II In

Acropora sammentosa: II In

Acropora scandens: II L

Acropora schmitti: II In

Acropora secale: II in

Acropora sekiseiensis: II In

Acropora selago: II In

Acropora solitaryensis: II In

Acropora sordiensis: II In

Acropora spicifera: II in

Acropora splendida: II In

Acropora squamosa: II in

Acropora squarrosa: II In

Acropora stigmaiaria: II in

Acropora stoddarti: II In

Acropora striata: II In

Acropora studeri: II In

Acropora subglabra: II In

Acropora subulata: II In

Acropora suharsonoi: II In

Acropora sukamoi: II In

Acropora syringodes: II In

Acropora tanegashimensis: II in

Acropora tenella: II In

Acropora tenuis: II in

Acropora teres: II in

Acropora teres $=$ Acropora microphthalma

Acropora thurstoni: II In

Acropora rogianensis: II In

Acropora torihalimeda: II In 
Acropora tortuosa: II In Acropora tuberculosa: II In Acropora tubicinaria: II In Acropora tubigera: II In Acropora tubulosa: II In Acropora tumida: II In Acropora turaki: II In

Acropora turbida: II In Acropora turbinata: II In Acropora valenciennesit: II In Acropora valida: II In Acropora vaughani: II In Acropora venweyi: II In Acropora wallaceae: II In Acropora willisae: II In Acropora yongei: II In Actinastrea pectinata: II In

Acuá (S): Herpetotheres cachinnans II B

Adalbert's Eagle (E): Aquila adalberi I B

Adanson's Mud Turtle (E): Pelusios adansonii III R

Addax (E/S/F): Addax nasomaculatus I M

Addax à nez tacheté $(\mathrm{F})$ : Addax nasomaculatus I M

Addax nasomaculatus: Addax (E), Addax (S), Addax (F), Addax à nez tacheté (F), Antilope blanche (F) I M

Addra Gazelle (E): Gazella dama I M

Adelomyia melanogenys: Speckled Hummingbird (E), Colibrí serrano gargantiazul (S), Picaflor gargantiazul (S), Colibri moucheté (F) II B

Adelopora crassilabrum: II In

Adelopora fragilis: II In

Adelopora moseleyi: II in

Adelopora pseudothyron: II In

Admiralty Flying-fox (E): Pteropus admiralitatum II M

Admiralty Hawk-Owl (E): Ninox meeki II B

Adorable Coquette (E): Lophomis adorabilis II B

Adriatic Sturgeon (E): Acipenser naccarii II F

Aegolius acadicus: Northern Saw-whet Owl (E), Saw-whet Owl (E), Tecolote-abetero norteño (S), Petite nyctale (F) II B

Aegolius funereus: Boreal Owl (E), Tengmalm's Owl (E), Lechuza de Tengmalm (S), Nyctale de Tengmalm (F) II B

Aegolius harrisii: Buff-fronted Owl (E), Carburé acanelado (S), Curucucú barriga amarilla (S), Lechucita acanelada (S), Chouette de Harris (F), Nyctale de Harris (F) II B

Aegolius ridgwayi $=$ Aegolius acadicus

Aegypius calvus = Sarcogyps calvus

Aegypius monachus: Black Vulture (E), Cinereous Vulture (E), Buitre negro (S), Vautour moine (F) II B

Aegypius occipitalis = Trigonoceps occipitalis

Aegypius trachelioms $=$ Torgos trachelioms

Afep Pigeon (E): Columba unicincta III B

Afghan Fox (E): Vulpes cana II M

Afghan Tortoise (E): Testudo horsfieldii II R

African Ass (E): Equus africanus I M

African Bared Owlet (E): Glaucidium capense II B

African Bay Owl (E); Phodilus prigoginet II B

African Baza (E): Aviceda cuculoides II B

African Black Eagle (E): Aquila verreauxii II B

African Black Terrapin (E): Pelusios niger III R

African Blind Barb Fish (E): Caecobarbus geertsi II F

African Burrowing Python (E): Calabaria reinhardtii II R

African Caracal (E): Caracal caracal I/II M

African Chameleon (E): Chamaeleo africanus II R

African Civet (E): Civertictis civetta $11 \mathrm{II} \mathrm{M}$

African Clawless Otter (E): Aonyx capensis II M

African Collared-Dove (E): Sireptopelia roseogrisea III B

African Crocodile (E): Crocodylus niloticus I/I R

African Crowned Eagle (E): Siephanocetus coronatus II B

African Cuckoo-Falcon (E): Aviceda cuculoides II B

African Cuckoo-Hawk (E): Aviceda cuculoides II B

African Dwarf Crocodile (E): Osteolaemus tetraspis I R

African Eagle-Owl (E): Bubo africanus II B

African Elephant (E): Loxodonta africana I/I M

African Firefinch (E): Lagonosticta rubricata III B

African Fish-Eagle (E): Haliaeetus vocifer II B

African Forest Turtle (E): Pelusios gabonensis III R

African Golden Cat (E): Profelis aurata II M
African Goshawk (E): Accipiser tachira II B

African Grass-Owl (E): Tylo capensis II B

African Green-Pigeon (E): Treron calva III B

African Gymnogene (E): Polyboroides typus II B

African Harrier-Hawk (E): Polyboroides typus II B

African Hawk-Eagle (E): Hieraaetus spilogaster II B

African Helmeted Turtle (E): Pelomedusa subrufa III R

African Hobby (E): Falco cuvieri II B

African Large-grain Lizard (E): Varanus exanthematicus II R

African Little Sparrowhawk (E): Accipiter minullus II B

African Long-tailed Hawk (E): Urotriorchis macrourus II B

African Manatec (E): Trichechus senegalensis II M

African Marsh Owl (E): Asio capensis II B

African Marsh-Harrier (E): Circus ranivorus 11 B

African Mouming Dove (E): Streptopelia decipiens III B

African Orange-bellied Parrot (E): Poicephalus rufiventris II B

African Pygmy Falcon (E): Polihierax semitorquatus II B

African Pygmy-goose (E): Netiapus auritus III B

African Python (E): Python sebae II R

African Quailfinch (E): Ortygospiza alricollis III B

African Red-tailed Buzzard (E): Buteo auguralis II B

African Rock Python (E): Python sebae II R

African Savanna Monitor (E): Varanus exanthematicus II R

African Serpent-Eagle (E): Dryotriorchis spectabilis II B

African Sharp-nosed Crocodile (E): Crocodylus caraphractus I R

African Silverbill (E): Lonchura cantans III B

African Slender-snouted Crocodile (E): Crocodylus cataphractus I R

African Small-grain Lizard (E): Varanus niloricus II R

African Soft-shell Turtle (E): Trionyx triunguis III R

African Spiny-tailed Lizard (E): Cordylus polyzonus II R

African Spurred Tortoise (E): Geochelone sulcala II R

African Swallow-tailed Kite (E): Chelictinia riocourii II B

African Tent Tortoise (E): Psammobates tentorius II R

African viviparous toads (E): Neclophrynoides spp. I A

African White-backed Vulture (E): Gyps africanus II B

African Wild Ass (E): Equus africanus I M

African Wood-Owl (E): Sirix woodfordii II B

African Wood-Figeon (E): Columba unicincta III B

Afro-Australian Fur Seal (E): Arctocephalus pusillus II M

Afrotis atra = Eupodotis afra

Agapomis canus: Grey-headed Lovebird (E), Madagascar Lovebird (E), Inseparable malgache (S), Inséparable à tête grise (F) II B

Agapomis fischeri: Fischer's Lovebird (E), Inseparable de Fischer

(S), Inséparable de Fischer (F), Perruche de Fischer (F) II B

Agapormis lilianae: Lilian's Lovebird (E), Nyasa Lovebird (E),

Inseparable del Nyasa (S), Inséparable de Lilian (F), Perruche de Lilian (F) II B

Agapornis lilianae nigrigenis $=$ Agapornis nigrigenis

Agapornis nigrigenis: Black-cheeked Lovebird (E), Inseparable cachetón (S), Inséparable à joues noires (F), Peruche à joue noire (F) II B

Agapomis personatus: Black-masked Lovebird (E), Masked Lovebird (E), Yellow-collared Lovebird (E), Inseparable cabecinegro (S), Inséparable masqué (F), Perruche masqué (F) II B

Agapomis pullarius: Red-faced Lovebird (E), Red-headed Lovebird (E), Inseparable carimojo (S), Inséparable à tête rouge (F), Perruche à tête rouge (F) II B

Agapomis roseicollis: Peach-faced Lovebird (E), Rosy-faced Lovebird (E), Inseparable de Namibia (S), Inséparable rosegorge (F), Inséparable à face rose (F), Peruche à face rose (F) II B

Agapomis swindemianus: Black-collared Lovebird $(E)$, Inseparable acollarado (S), Inseparable de collera (S), Inséparable de Swindem (F), Inséparable à collier noir (F), Perruche de Swindern (F) II B

Agapomis taranta: Abyssinian Lovebird (E), Black-winged Lovebird

(E), Inseparable Abisinio (S), Inséparable d'Abyssinie (F) II B

Agaricia agaricites: Leaf Coral (E) II In

Agaricia fragilis: Fragile Saucer Coral (E) II In

Agaricia grahamae: II In

Agaricia humilis: II In

Agaricia lamarcki: Sheet Coral (E) II In

Agaricia tenuifolia: Ribbon Coral (E) II In

Agaricia undala: Scroll Coral (E) II ln

Agariciella $=$ Gardineroseris

Agate shells (E): Achatinella spp. I In 
Agelaius flavus: Saffron-cowled Blackbird (E), Dragón (S), Mirlo americano pechiamarillo (S), Tordo amarillo (S), Tordo de cabeza amarilla (S), Carouge safran (F), Ictéride à tête jaune (F) I B

Agelastes meleagrides: White-breasted Guineafowl (E), Pintado de pecho blanco (S), Pintado pechiblanca (S), Pintade à poitrine blanche (F) III B

Agelecyathus = Polycyathus

Agile Gibbon (E): Hylobares agilis I M

Agile Mangabey (E): Cercocebus agilis II M

Agkistrodon bilineasus: Cantil (E), Ancistrodon à deux lignes (F), Mocassin à deux lignes (F) III $R$

Aglaeactis aliciae: Purple-backed Sunbearn (E), Colibri d'Alice (F) II B

Aglaeactis castelnaudii: White-tufted Sunbeam (E), Colibri de Castelneau (F) II B

Aglaeactis cupripennis: Shining Sunbeam (E), Rayito brillante (S), Colibri étincelant (F) II B

Aglaeactis pamela: Black-hooded Sunbeam (E), Colibri paméla ( $\mathrm{F}$ ) II B

Aglaiocercus coelestis: Violet-tailed Sylph (E), Silfo colivioleta (S). Sylphe à queue violette (F) II B

Aglaiocercus kingi: Long-tailed Sylph (E), Colibrí coludo azul (S), Silfo colilargo (S), Sylphe à queue d'azur (F) II B

Agouti (F): Agouti paca III M

Agouti paca: Spotted Paca (E), Paca (S), Agouti (F) III M

Agouti ponctué (F): Dasyprocia punctata III M

Agoutí rojizo (S): Dasyprocta punctata III M

Agoutis (E): Dasyprocia spp. IIINC M

Agra Lizard (E): Varanus griseus I R

Agra Monitor (E): Varanus griseus I R

Agriocharis ocellata: Ocellated Turkey (E), Guajolote ocelado (S), Dindon ocellé (F) III B

Agrionemys horsfieldii $=$ Tesiudo horsfieldii

Aguara guazu (S): Chrysocyon brachyurus II M

Aguila arpía (S): Harpia harpyja I B

Aguila audaz (S): Aquila audax II B

Aguila blanca (S): Leucoptemis polionota II B

Aguila blanquinegra (S): Spizastur melanoleucus II B

Aguila cabeciblanca (S): Haliaeetus leucocephalus I B

Aguila cabeza blanca (S): Haliaeetus leucocephalus I B

Aguila cafre (S): Aquila verreauxii II B

Aguila calzada (S): Hieraaetus pennatus II B

Aguila castaña (S): Oroaetus isidori II B

Aguila colorada (S): Busarellus nigricollis II B

Aguila comemonos (S): Pithecophaga jefferyi I B

Aguila copetuda africana (S): Lophaetus occipitalis II B

Aguila coronada (S): Harpyhaliaetus coronatus II B/ Stephanoaetus coronatus II B

Aguila coronada rabuda (S): Stephanoaetus coronatus II B

Aguila crestada (S): Morphnus guianensis II B

Aguila crestilarga (S): Lophaerus occipiralis II B

Aguila culebrera (S): Circaetus gallicus II B

Aguila culebrera colilarga (S): Dryotriorchis spectabilis II B

Aguila culebrera culiblanca (S): Circaeius cinerascens II B

Aguila culebrera de Brown (S): Circaetus cinereus II B

Aguila de Azara (S): Harpyhaliaetus coronatus II B

Aguila de Bonelli (S): Hieraaetus fasciatus II B

Aguila de Copete (S): Oroaerus isidori II B

Aguila de Penacho (S): Spizaetus omatus II B

Aguila de Walhberg (S): Aquila wahlbergi II B

Aguila elegante (S): Spizaetus omatus II B

Aguila escudada (S): Geranoaetus melanoleucus II B

Aguila estoparia (S): Aquila nipalensis II B

Aguila harpia (S): Harpia harpyja I B

Aguila imperial (S): Aquila heliaca I B

Aguila imperial ibérica (S): Aquila adalberti I B

Aguila imperial oriental (S): Aquila heliaca I B

Aguila marcial (S): Polemaerus bellicosus II B

Aguila milana (S): Ictinaetus malayensis II B

Aguila moluqueña (S): Aquila gumeyi II B

Aguila monera (S): Morphnus guianensis II B/ Pithecophaga jefferyi I B

Aguila mora (S): Geranoaetus melanoleucus II B

Aguila moteada (S): Aquila clanga II B

Aguila negra (S): Buteogallus urubitunga II B
Aguila perdicera (S): Hieraaelus fascialus II B

Aguila perdicera afnicana (S): Hieraaetus spilogaster II B

Aguila pescadora (S): Pandion haliaerus II B

Aguila poma (S): Oroaetus isidori II B

Aguila pomerana (S): Aquila pomarina II B

Aguila rapaz (S): Aquila rapax II B

Aguila real (S): Aquila chrysaetos II B/ Geranoaetus melanoleucus II $\mathrm{B}$

Aguila sangual (S): Pandion haliaetus II B

Aguila solitaria (S): Harpyhaliaetus solitarius II B

Aguila tirana (S): Spizaelus tyrannus II B

Aguila volatinera (S): Terathopius ecaudarus II B

Aguila-azor africana (S): Hieraaetus spilogaster II B

Aguila-azor blanca (S): Spizuetus omatus II B

Aguila-azor blanquinegra (S): Spizastur melanoleucus II B

Aguila-azor chica (S): Spizastur melanoleucus II B

Aquila-azor congoleña (S): Spizaetus africanus II B

Aguila-azor de Ayres (S): Hieraaetus ayresii II B

Aguila-azor de Célebes (S): Spizaetus lanceolatus II B

Aguila-azor de Java (S): Spizaerus bartelsi II B

Aguila-azor de Wallace (S): Spizaetus nanus II B

Aguila-azor filipina (S): Spizaetus philippensis II B

Aguila-azor galana (S): Spizaetus omarus II B

Aguila-azor Indonesia (S): Spizaetus alboniger II B

Aguila-azor montañesa (S): Spizaetus nipalensis II B

Aguila-azor negra (S): Spizaetus tyrannus II B

Aguila-azor perdicera (S): Hieraaetus fasciatus II B

Aguila-azor variable (S): Spizaetus cirrhatus II B

Aguila-azor ventrirroja (S): Hieraaetus kienerii II B

Aguilila blanca (S): Leucoptemis albicollis II B

Aguililla aluda (S): Buteo platypterus II B

Aguililla artica (S): Buteo lagopus II B

Aguililla aura (S): Buteo albonotatus II B

Aguililla australiana (S): Hieraaetus morphnoides II B

Aguililla calzada (S): Hieraaetus pennatus II B

Aguililla caminera (S): Buteo magnirostris II B

Aguililla coliblanca (S): Buteo albicaudatus II B

Aguililla colicorta (S): Buteo brachyurus II B

Aguililla colirroja (S): Buteo jamaicensis II B

Aguililla de Haris (S): Parabureo unicinctus II B

Aguililla de Swainson (S): Buseo swainsoni II B

Aguililla gris (S): Asturina plagiata II B

Aguililla langostera (S): Butastur rufipennis II B

Aguililla negra mayor (S): Buteogallus urubitunga II B

Aguililla negra menor (S): Buteogallus anthracinus II B

Aguililla pechirroja (S): Buteo lineatus II B

Aguililla real (S): Buteo regalis II B

Aguilucho cabecinegro (S): Bureo albicaudatus II B

Aguilucho cenizo (S): Circus pygargus II B

Aguilucho chico (S): Buteo albigula II B

Aguilucho cola colorada (S): Buteo ventralis II B

Aguilucho cola corta (S): Buteo brachyurus II B

Aguilucho colorada (S): Buteogallus meridionalis II B

Aguilucho común (S): Buteo polyosoma II B

Aguilucho de azara (S): Circus buffoni II B

Aguilucho de ciénaga (S): Circus buffoni II B

Aguilucho gris (S): Asturina nirida II B

Aguilucho lagunero (S): Circus aeruginosus II B

Aguilucho lagunero del pacifico (S): Circus approximans II B

Aguilucho lagunero etiópico (S): Circus ranivorus II B

Aguilucho lagunero malgache (S): Circus maillardi II B

Aguilucho lagunero occidental (S): Circus aeruginosus II B

Aguilucho lagunero oriental (S): Circus spilonotus II B

Aguilucho langostero (S): Buteo swainsoni II B

Aguilucho moteado (E): Circus assimilis II B

Aguilucho negro (S): Circus maurus Il B

Aguilucho pálido (S): Circus cyaneus II B

Aguilucho papialbo (S): Circus macrourus II B

Aguilucho pio (S): Circus melanoleucos II B

Aguilucho puna (S): Buteo poecilochrous II B

Aguilucho sabanero (S): Buteogallus meridionalis II B

Aguilucho vari (S): Circus cinereus II B

Aguilucho variado (S): Buteo polyosoma II B

Aguilucho-caricalvo común (S): Polyboroides typus II B

Aguilucho-caricalvo malgache (S): Polyboroides radiatus II B

Aï de Bolivie (F): Bradypus variegatus II M 
Aigle à épaules blanches (F): Aquila adalberti I B

Aigle à tête blanche (F): Haliceetus leucocephalus I B

Aigle à ventre roux (F): Hieraaetus kienerii II B

Aigle blanchard (F): Stephanoaetus coronatus II B

Aigle botté (F): Hieraaetus pennatus II B

Aigle couronné (F): Stephanoaetus coronatus II B

Aigle criard (F): Aquila clanga II B

Aigle d'Australie (F): Aquila audax II B

Aigle d'Ayres (F): Hieraaetus ayresii II B

Aigle de Blyth (F): Spizaetus alboniger II B

Aigle de Bonelli (F): Hieraaerus fasciatus II B

Aigle de Cassin (F): Spizaerus africanus II B

Aigle de Gurney (F): Aquila gumeyi II B

Aigle de Java (F): Spizaetus bartelsi II B

Aigle de Nouvelle-Guinée (F): Harpyopsis novaeguineae II B

Aigle de Verreaux (F): Aquila verreauxii II B

Aigle de Wahlberg (F): Aquila wahlbergi II B

Aigle de Wallace (F): Spizaetus nanus II B

Aigle des Célèbes (F): Spizaetus lanceolatus II B

Aigle des Philippines (F): Pithecophaga jefferyi I B/ Spizaetus philippensis II B

Aigle des singes (F): Pithecophaga jefferyi I B

Aigle des steppes (F): Aquila nipalensis II B

Aigle d'Isidore (F): Oroaetus isidori II B

Aigle doré (F): Aquila chrysaetos II B

Aigle fascié (F): Hieraaeius spilogaster II B

Aigle harpie (F): Harpia harpyja I B

Aigle huppard (F): Lophaetus occipitalis II B

Aigle huppé (F): Spizaetus cirrhatus II B

Aigle impérial (F): Aquila heliaca I B

Aigle impérial espagnol (F): Aquila adalberii I B

Aigle mangeur de singes (F): Pithecophaga jefferyi I B

Aigle martial (F): Polemaetus bellicosus $11 \mathrm{~B}$

Aigle montagnard (F): Spizaetus nipalensis II B

Aigle nain (F): Hieracietus morphnoides II B

Aigle noir $(\mathrm{F})$ : Icrinaetus malayensis II B

Ajgle noir et blanc ( $F$ ): Spizastur melanoleucus II B

Aigle orné (F): Spizaetus ornatus II B

Aigle pêcheur (F): Pandion haliaetus II B

Aigle pêcheur africain (F): Haliaeerus vocifer II B

Aigle pomarin (F): Aquila pomarina II B

Aigle ravisseur (F): Aauila rapar II B

Aigle royal (F): Aquila chrysaeros II B

Aigle serpentaire (F): Dryotriorchis spectabilis II B

Aigle tyran (F): Spizaetus tyrannus II B

Aigles (F): Accipitridae spp. I/II B

Aigle-autour fascié (F): Hieraaeius spilogaster II B

Aigrette garzette (F): Egretta garzesta III B

Aigrettes (F): Casmerodius albus/Egretı spp. III/NC B

Ailuropoda melanoleuca: Giant Panda (E), Panda gigante (S), Panda (F), Panda géant (F) I M

Ailurus fulgens: Lesser Panda (E), Red Cat-bear (E), Red Panda (E), Panda chico (S), Panda rojo (S), Panda éclatant (F), Petit panda (F) I M

Ajolote de lago Pátzquaro (S): Ambystoma dumerilii II A

Ajolote mexicano (S): Ambysfoma mexicanum II A

Akun Eagle-Owl (E): Bubo leucosticius II B

Ala de sable anteado (S): Campylopterus duidae II B

Ala de sable gris (S): Campylopterus largipennis II B

Ala de sable pechivioleta (S): Campyloprerus falcarus II B

Ala de sable rufo (S): Campylopterus hyperythrus II B

Ala de sable verde (S): Campylopierus ensipennis II B

Alabama Lamp Naiad (E): Lampsilis virescens I in

Alabama Lamp Pearly Mussel (E): Lampsilis virescens I in

Alabama Lampmussel (E): Lampsilis virescens I In

Alabama Sturgeon (E): Scaphirhynchus sultkusi II F

Alagoas Curassow (E): Miru mim I B

Alajuela Toad (E): Bufo periglenes I A

Alasable azulino (S): Campylopterus falcatus 11 B

Alasable del Napo (S): Campylopterus largipennis II B

Alatotrochus rubescens: II In

Alazafiro grande (S): Plerophanes cyanopterus II B

Albatros (F): Diomedeidae spp. INC B

Albatros à queue courte (F): Diomedea albalrus I B

Albatros colicorto (S): Diomedea albatrus I B

Albatros de Steller (F): Diomedea albatrus I B
Albatros rabón (S): Diomedea albatrus I B

Albatrosses (E): Diomedeidae spp. I/NC B

Albertine Owlet (E): Glaucidium albertinum II B

Alcaraván americana (S): Burhinus bistriarus III B

Alcaraván venozolano (S): Burhinus bistriatus III B

Alcatraz de Abbott (S): Papasula abbotti I B

Alcotán (S): Falco subbuteo II B

Alcotán africano (S): Falco cuvieri II B

Alcotán australiano (S): Falco longipennis II B

Alcotán cuellirrojo (S): Falco chicquera II B

Alcotán europeo (S): Falco subbuteo II B

Alcotán filipino (S): Falco severus II B

Alcotán turumti (S): Falco chicquera II B

Aldabra Day Gecko (E): Phelsuma abbotti II R

Aldabra Flying-fox (E): Pteropus aldabrensis II M

Aldabra Giant Tortoise (E): Geochelone giganiea II R

Aldabrachelys elephantina $=$ Geochelone gigantea

Alder Parrot (E): Amazona tucumana I B

Alecto à bec blanc (F): Bubalomis albirostris III B

Alectos (F): Bubalomis spp/Dinemellia dinemelli III/NC B

Aleutian Canada Goose (E): Brania canadensis leucopareia I B

Aleutian Goose (E): Branta canadensis leucopareia I B

Alexandra's Parrot (E): Polytelis alexandrae II B

Alexandrine Parakeet (E): Psittacula eupatria II B

Aligator de China (S): Alligaror sinensis I R

Aligator del Mississippi (S): Alligator mississippiensis II R

Aligators (S): Alligatoridae spp. V/II R

Alimoche común (S): Neophron percnopterus II B

Alimoche sombrío (S): Necrosyries monachus II B

Alisterus amboinensis: Ambon King-Parrot (E), Moluccan King-

Parrot (E), Papagayo Moluqueño (S). Perruche tricolore (F) II B

Alisterus chloropterus: Green-winged King-Parrot (E), Papuan King-

Parrot (E), Papagayo papú (S), Perruche à ailes vertes (F) II B

Alisterus scapularis: Australian King-Parrot (E), Papagayo australiano (S), Perruche royale (F) II B

Allenopithecus nigroviridis: Allen's Swamp Monkey (E),

Cercopithèque de Allen (F) II M

Allen's Bushbaby (E): Galago alleni II M

Allen's Galago (E): Galago alleni II M

Allen's Hummingbird (E): Selasphorus sasin II B

Allen's Swamp Monkey (E): Allenopithecus nigroviridis II M

Alligator américain (F): Alligator mississippiensis II R

Alligator de Chine (F): Alligator sinensis I R

Alligator du Mississippi (F): Alligator mississippiensis II R

Alligator mississippiensis: American Alligator (E), Aligator del

Mississippi (S), Alligator américain (F). Alligator du Mississippi

(F) II R

Alligator sinensis: China Alligator (E), Chinese Alligator (E), Aligator de China (S), Alligator de Chine (F) I R

Alligators (E/S): Alligatoridae spp. I/I R

Allobates spp.: Poison frogs (E), Ranas de puntas de flechas (S). Dendrobates (F) II A

Allobates femoralis: Brilliant-thighed Poison Frog (E), Epipedobate fémorale (F), Epipedobate simisimi (F), Simisimi (F) II A

Allocèbe (F): Allocebus rrichotis I M

Allocebus trichotis: Hairy-eared Dwarf Lemur (E), Lemur orejipeludo (S), Allocèbe (F), Chirogale aux oreilles poilues (F) I M

Allopathes desbonni: II In

Alopochen aegyptiacus: Egyptian Goose (E), Ganso del Nilo (S),

Oca del Nilo (S), Oie d'Egypte (F), Ouette d'Egypte (F) III B

Alouatta belzebul: Black-and-red Howler (E), Red-handed Howler

(E), Hurleur à mains rousses (F) II M

Alouatra beniensis = Alouana fusca

Alouatta caraya: Black Howler (E), Hurleur noir (F) I M

Alouatta coibensis: I M

Aloualla fusca: Brown Howler (E), Hurleur brun (F) II M

Alouatta guariba = Alouatta fusca

Alouatta palliata: Mantled Howler (E), Aullado (S), Guariba Peludo

(S), Mono congo (S), Mono negro (S), Mono saraguato (S),

Zaraguate (S) I M

Alouatta palliata coibensis = Alouatta coibensis

Aloualta pigra: Guatemalan Howler (E), Araguato de Guatemala (S),

Hurleur du Guatemala (F) 1 M

Alouatra sara: II M

Alouana seniculus: Red Howler (E), Hurleur roux (F) II M 
Alouatta seniculus sara = Alouatra sara

Alouatta villosa $=$ Alouatta pigra

Altiphrynoides spp.: Ethiopian toads (E), Sapos viviparos (S), Crapauds vivipares (F) I A

Altiphnnoides malcolmi: Malcolm's Ethiopian Toad (E), Sapo viviparo de Malcolm (S), Nectophrynoide de Malcolm (F) I A

Alto de Buey Poison Frog (E): Minyobates altobueyensis II A

Alveopora allingi: II In

Alveopora catalai: II In

Alveopora daedalea: 11 in

Alveopora excelsa: II In

Alveopora explanata: II In

Alveopora fenestrata: II in

Alveopora gigas: II in

Alveopora japonica: II In

Alveopora marionensis: II in

Alveopora ocellata: II In

Alveopora retusa: II in

Alveopora spongiosa: II In

Alveopora superficialis: II ln

Alveopora sizardi: II In

Alveopora verrilliana: II In

Alveopora viridis: II In

Amadina fasciala: Cut-throat (E), Ribbon Finch (E), Amadina gorgirroja (S), Amadine cou-coupé (F), Cou-coupé (F) III B

Amadina gorgiroja (S): Amadina fasciata III B

Amadine cou-coupé (F): Amadina fasciata III B

Amadines (F): Amadina spp. III/NC B

Amandava formosa: Green Avadavat (E), Bengali vert (F) II B

Amandava subflava: Golden-breasted Waxbill (E), Orange-breasted Waxbill (E), Zebra Waxbill (E), Astrilda pechigualda (S), Bengali zébré (F), Ventre orange (F) III B

Amapola (S): Amazona viridigenalis I B

Amarante à ventre noir (F): Lagonosticta rara III B

Amarante commun (F): Lagonosticia senegala III B

Amarante du Sénégal (F): Lagonosticia senegala III B

Amarante flambé (F): Lagonosticta rubricata III B

Amarante foncé (F): Lagonosticta rubricata III B

Amarante masqué (F): Lagonosticta vinacea III B

Amarante pointé (F): Lagonosticta rufopicta III B

Amarante rare (F): Lagonosticta rara III B

Amarante vineux (F): Lagonosticta vinacea III B

Amarantes (F): Lagonosticta spp. III/NC B

Amazilia amabilis: Blue-chested Hummingbird (E), Amazilia pechiazul (S), Ariane aimable (F) II B

Amazilia amabilis decora = Amazilia decora

Amazilia amazilia: Amazilia Hummingbird (E), Amazilia ventrirrufa (S), Ariane de Lesson (F) II B

Amazilia andina (S): Amazilia franciae II B

Amazilia beryllina: Berylline Hummingbird (E), Colibri de Berilo (S), Ariane béryl (F) II B

Amazilia boucardi: Mangrove Hummingbird (E), Ariane de Boucard (F) II B

Amazilia bronceada coliazul (S): Amazilia tobaci II B

Amazilia candida: White-bellied Emerald (E), Esmeralda vienteblanco (S), Ariane candide (F) II B

Amazilia castaneiventris: Chestnut-bellied Hummingbird $(E)$, Ariane à ventre roux (F) II B

Amazilia chionogaster: White-bellied Hummingbird (E), Picaflor ventriblanco (S), Ariane à ventre blanc $(\mathrm{F})$ II B

Amazilia chionopectus: White-chested Emerald (E), Diamante colidorado (S), Ariane à poitrine blanche $(\mathrm{F})$ II B

Amazilia colimorada (S): Amazilia viridigaster II B

Amazilia colirrufa (S): Amazilia tzacall II B

Amazilia cyanifrons: Indigo-capped Hummingbird $(E)$, Ariane à front bleu (F) II B

Amazilia cyanocephala: Azure-crowned Hummingbird (E), Colibri coroniazul (S), Ariane à couronne azur (F) II B

Amazilia cyanura: Blue-tailed Hummingbird (E), Colibrí coliazul (S), Ariane à queue bleue (F) II B

Amazilia decora: Charming Hummingbird (E), Ariane charmante (F) II B

Amazilia distans: Táchira Emerald (E), Diamante de Wetmore (S), Ariane du Tachira (F) II B

Amazilia edward: Snowy-breasted Hummingbird (E), Ariane d'Edward (F) II B
Amazilia fimbriata: Glittering-throated Emerald (E), Amazilia gorjibrillante (S), Diamante gargantiverde (S), Ariane de Linné (F) II B

Amazilia franciae: Andean Emerald (E), Amazilia andina (S), Ariane de Francia (F) II B

Amazilia gorjibrillante (S): Amazilia fimbriata II B

Amazilia handleyi $=$ Amazilia tzacall

Amazilia Hummingbird (E): Amazilia amazilia II B

Amazilia lactea: Sapphire-spangled Emerald (E), Diamante pechizafiro (S). Ariane saphirine (F) II B

Amazilia leucogaster. Plain-bellied Emerald (E), Diamante ventriblanco (S), Ariane vert-doré (F) II B

Amazilia luciae: Honduran Emerald (E), Esmeralda Hondurena (S), Ariane de Lucy (F) II B

Amazilia microrhyncha = Amazilia cyanocephala

Amazilia pechiazul (S): Amazilia amabilis II B

Amazilia pechimorada (S): Amazilia rosenbergi II B

Amazilia rosenbergi: Purple-chested Hummingbird (E), Amazilia pechimorada (S), Ariane de Rosenberg (F) II B

Amazilia rutila: Cinnamon Hummingbird (E), Colibrí canelo (S), Ariane cannelle (F) II B

Amazilia saucerrottei: Blue-vented Hummingbird (E), Steely-vented Hummingbird (E), Amazilia verde-azul (S), Ariane de Sophie (F) II B

Amazilia robaci: Copper-rumped Hummingbird (E), Amazilia bronceada coliazul (S), Ariane de Félicie (F) II B

Amazilia tzacatl: Rufous-tailed Hummingbird (E), Amazilia colimufa (S), Colibrí colirrufo (S), Ariane à ventre gris $(\mathrm{F})$ II B

Amazilia ventrirrufa (S): Amazilia amazilia II B

Amazilia verde-azul (S): Amazilia saucerrottei II B

Amazilia versicolor: Versicolored Emerald (E), Diamante multicolor (S), Picaflor pequeño verde (S), Ariane versicolore (F) II B

Amazilia violiceps: Violet-crowned Hummingbird (E), Colibrí corona-violeta (S), Ariane à couronne violette (F) II B

Amazilia viridicauda: Green-and-white Hummingbird (E), Ariane du Pérou (F) II B

Amazilia viridifrons: Green-fronted Hummingbird (E), Colibrí corona-verde $(\mathrm{S})$, Ariane à front vert $(\mathrm{F})$ II B

Amazilia viridigaster: Green-bellied Hummingbird (E), Amazilia colimorada (S), Ariane à ventre vert (F) II B

Amazilia wagneri $=$ Amazilia viridifrons

Amazilia yucatanensis: Buff-bellied Hummingbird (E), Fawnbreasted Hummingbird (E), Colibri vientre-canelo (S), Ariane du Yucatan (F) II B

Amazon River Dolphin (E): Inia geoffrensis II M

Amazon Tree Boa (E): Corallus enydris II R

Amazona aestiva: Blue-fronted Amazon (E), Blue-fronted Parrot (E) Turquoise-fronted Parrot (E), Amazona frentiazul (S), Loro hablador (S), Amazone à front bleu (F) II B

Amazona agilis: Black-billed Amazon (E), Black-billed Parrot (E), Amazona Jamaicana piquioscura (S), Amazonico activo (S), Amazonico jamaica (S), Amazonico todo verde (S), Amazone agile (F), Amazone verte (F) II B

Amazona albifrons: White-fronted Amazon (E), White-fronted Parrot (E), Amazona frentialba (S), Loro frente blanca (S), Perico manglero (S), Amazone à front blanc $(F)$ II B

Amazona alinaranja (S): Amazona amazonica II B

Amazona amazonica: Orange-winged Amazon (E), Orange-winged Parrot (E), Amazona alinaranja (S), Loro guaro (S), Amazone aourou $(\mathrm{F})$, Amazone à ailes oranges $(\mathrm{F})$, Amazone à ailes vertes (F) II B

Amazona arausiaca: Jacquot (E), Red-necked Amazon (E), Rednecked Parrot (E), Amazona cabenazul (S), Amazona cabeza azul (S), Amazona gorgirroja (S), Amazone de Bouquet (F), Amazone à cou rouge (F) I B

Amazona auropalliata: Yellow-naped Amazon (E), Yellow-naped Parrot (E), Loro nuquiamarillo (S), Amazone à nuque d'or (F) II B

Amazona autumnalis: Red-lored Amazon (E), Red-lored Parrot (E), Amazona frentirroja (S), Cotorra cucha (S), Loro frente roja (S), Loro frentirojo (S), Amazone diadème ( $F$ ), Amazone à joues jaunes (F) II B 
Amazona barbadensis: Yellow-shouldered Amazon (E), Yellowshouldered Parrot (E). Amazona de espalda amarilla (S). Amazona hombrogualda (S), Cotorra cabeciamarilla (S), Amazone de la Barbade (F), Amazone à épaulettes jaunes (F) I B

Amazona brasiliensis: Red-tailed Amazon (E), Red-tailed Parrot (E) Amazona colirroja (S), Loro cariazul (S), Papagayo de cara roja (S), Amazone du Brésil (F), Amazone à joues bleues (F). Amazone à queue rouge (F) I B

Amazona cabecirroja (S): Amazona pretrei I B

Amazona cabenazul (S): Amazona arausiaca I B

Amazona cabeza azul (S): Amazona arausiaca I B

Amazona cariazul (S): Amazona dufresniana II B

Amazona charao (S): Amazona pretrei I B

Amazona colirroja (S): Amazona brasiliensis I B

Amazona collaria: Yellow-billed Amazon (E), Yellow-billed Parrot

(E), Amazona Jamaicana piquiclara (S), Loro de Jamaica (S),

Amazone de la Jamaïque (F), Amazone sasabé (F) II B

Amazona coronirroja (S): Amazona rhodocorytha I B

Amazona crestirroja (S): Amazona rhodocorytha I B

Amazona Cubana (S): Amazona leucocephala I B

Amazona de cara amarilla (S): Amazona xanthops II B

Amazona de Cuba (S): Amazona leucocephala I B

Amazona de espalda amarilla (S): Amazona barbadensis I B

Amazona de Kawall (S): Amazona kawalli II B

Amazona de la Española (S): Amazona ventralis II B

Amazona de Puerto Rico (S): Amazona vitraia I B

Amazona de San Vincente (S): Amazona guildingii I B

Amazona de Santa Lucia (S): Amazona versicolor I B

Amazona del cerrado (S): Amazona xanthops 11 B

Amazona dufresniana: Blue-cheeked Amazon (E), Blue-cheeked

Parrot (E), Amazona cariazul (S), Loro cariazul (S), Amazone de

Dufresne (F), Amazone à joues bleues (F) $11 \mathrm{~B}$

Amazona dufresniana rhodocorytha = Amazona rhodocorythe

Amazona farinosa: Mealy Amazon (E), Mealy Parrot (E), Amazona

harinosa (S), Loro burrón (S), Loro cabeza azul (S), Loro verde (S), Amazone farineuse (F), Amazone poudrée (F) II B

Amazona festiva: Festive Amazon (E), Festive Parrot (E), Amazona festiva (S), Loro lomirrojo (S), Amazone festive (F). Amazone tavoua (F) $11 \mathrm{~B}$

Amazona festiva (S): Amazona festiva II B

Amazona finschi: Lilac-crowned Amazon (E). Lilac-crowned Parrot (E), Amazona guayabera (S), Cotorra frente roja (S), Loro corona-violeta (S), Amazone de Finsch (F), Amazone couronne lilas (F) II B

Amazona frentialba (S): Amazona albifrons II B

Amazona frentiazul (S): Amazona aestiva II B

Amazona frentirroja (S): Amazona aurumnalis II B

Amazona gorgirroja (S): Amazona arausiaca I B

Amazona guayabera (S): Amazona finschi 11 B

Amazona guildingii: Saint Vincent Amazon (E), Saint Vincent Parrot (E), St Vincent Amazon (E), Amazona de San Vincente (S), Amazone de Guilding (F), Amazone de Saint-Vincent (F) I B

Amazona harinosa (S): Amazona farinosa II B

Amazona hombrogualda ( $\mathrm{S}$ ): Amazona barbadensis I B

Amazona imperial (S): Amazona imperialis I B

Amazona imperialis: Imperial Amazon (E), Imperial Parrot (E) Amazona imperial (S), Amazone impériale (F) I B

Amazona Jamaicana piquiclara ( $\mathrm{S}$ ): Amazona collaria II B

Amazona Jamaicana piquioscura (S): Amazona agilis II B

Amazona kawall: Kawall's Amazon (E), Kawall's Parrot (E), Whitefaced Amazon (E), Amazona de Kawall (S), Amazone de Kawall (F) II B

Amazona leucocephala: Bahamas Parrot (E), Caribbean Amazon (E), Cuban Amazon (E), Cuban Parrot (E), Amazona Cubana (S), Amazona de Cuba (S), Amazone de Cuba (F), Amazone à tête blanche (F) I B

Amazona mercenaria: Scaly-naped Amazon (E), Scaly-naped Parrot (E), Amazona mercenaria (S), Loro verde (S), Amazone mercenaire (F) II B

Amazona mercenaria (S): Amazona mercenaria II B

Amazona ochrocephala: Yellow-crowned Amazon (E), Yellowcrowned Parrot (E), Yellow-fronted Amazon (E), Amazona real (S), Loro cabeza amarilla (S), Loro mona amarilla (S), Loro nuca amarilla (S), Loro real (S), Amazone à front jaune (F) II B

Amazona ochrocephala auropalliata = Amazona auropalliata
Amazona ochrocephala oratrix = Amazona oratrix

Amazona orarrix: Yellow-headed Amazon (E), Yellow-headed Parrot

(E), Loro cabeciamarillo (S), Amazone à tête jaune (F) II B

Amazona pechivinosa (S): Amazona vinacea I B

Amazona Portoriqueña (S): Amazona vintala I B

Amazona pretrei: Red-spectacled Amazon (E), Red-spectacled Parrot

(E), Amazona cabecirroja (S), Amazona charao (S), Loro

cabecirrojo (S), Loro de cabeza roja (S), Amazone de Prêtre (F) I B

Amazona real (S): Amazona ochrocephala II B

Amazona rhodocorytha: Red-browed Amazon (E), Red-browed

Parrot (E), Red-topped Amazon (E), Red-topped Parrot (E),

Amazona coronirroja (S), Amazona crestirroja (S), Amazone à

cronne rouge (F), Amazone à sourcils rouges (F) I B

Amazona Tamaulipeca (S): Amazona viridigenalis I B

Amazona lucumana: Alder Parrot (E), Tucuman Amazon (E),

Tucuman Parrot (E), Amazona tucumana (S), Loro alisero (S),

Amazone de Tucuman (F) I B

Amazona tucumana (S): Amazona tucumana I B

Amazona ventralis: Hispaniolan Amazon (E), Hispaniolan Parrot (E),

Amazona de la Española (S), Cotorra (S), Loro de Hispaniola

(S), Amazone d'Hispaniola (F), Amazone de San Domingo (F) II B

Amazona versicolor: Saint Lucia Amazon (E), Saint Lucia Partot (E),

St Lucia Amazon (E), Amazona de Santa Lucia (S), Amazone de Sainte-Lucie (F), Amazone versicolore (F) I B

Amazona vinacea: Vinaceous Amazon (E), Vinaceous Parrot (E), Amazona pechivinosa (S), Amazona vinosa (S), Loro

pechivinoso (S), Loro pecho vinoso (S), Amazone bourgogne (F), Amazone vineuse (F) I B

Amazona vinosa (S): Amazona vinacea I B

Amazona viridigenalis: Green-cheeked Amazon (E), Red-crowned Amazon (E), Red-crowned Parrot (E), Amapola (S), Amazona Tamaulipeca (S), Loro cabeza roja (S), Loro famaulipeco (S), Amazone à joues vertes $(F) I B$

Amazona vittaia: Puerto Rican Amazon (E), Puerto Rican Parrot (E), Red-fronted Amazon (E), Amazona Portorriqueña (S), Amazona de Puerto Rico (S), Amazone de Porto Rico (F), Amazone à bandeau rouge (F) I B

Amazona xantholora: Yellow-lored Amazon (E), Yellow-lored Parrot (E), Amazona Yucateca (S), Loro yucateci (S), Loro yucateco (S), Amazone du Yucatan (F), Amazone à lores jaunes (F) II B

Amazona xanthops: Yellow-faced Amazon (E), Yellow-faced Parrot (E), Amazona de cara amarilla (S), Amazona del cerrado (S), Amazone à face jaune (F) II B

Amazona Yucateca (S): Amazona xantholora II B Amazonas (S): Amazona spp. VII B

Amazone à ailes oranges (F): Amazona amazonica II B Amazone à ailes vertes (F): Amazona amazonica II B Amazone à bandeau rouge $(\mathrm{F})$ : Amazona vittata I B Amazone à cou rouge (F): Amazona arausiaca I B Amazone à couronne lilas (F): Amazona finschi II B Amazone à cronne rouge (F): Amazona rhodocorytha I B Amazone à épaulettes jaunes (F): Amazona barbadensis I B Amazone à face jaune (F): Amazona xanthops II B Amazone à front blanc (F): Amazona albifrons II B Amazone à front bleu (F): Amazona aestiva II B Amazone à front jaune (F): Amazona ochrocephala II B Amazone à joues bleues (F): Amazona brasiliensis I B/Amazono dufresniona II B

Amazone à joues jaunes (F): Amazona autumnalis II B Amazone à joues vertes $(\mathrm{F})$ : Amazona viridigenalis I B Amazone a lores jaunes (F): Amazona xantholora II B Amazone à nuque d'or (F): Amazona auropalliata II B Amazone à queue rouge (F): Amazona brasiliensis I B Amazone à sourcils rouges (F): Amazona rhodocorytha I B Amazone à tête blanche (F): Amazona leucocephala I B Amazone à tête jaune (F): Amazona orairix II B Amazone agile (F): Amazona agilis II B Amazone aourou (F): Amazona amazonica II B Amazone bourgogne (F): Amazona vinacea $1 \mathrm{~B}$ Amazone de Bouquet (F): Amazona arausiaca I B Amazone de Cuba (F): Amazona leucocephala I B Amazone de Dufresne (F): Amazona dufresniana II B Amazone de Finsch (F): Amazona finschi II B 
Amazone de Guilding (F): Amazona guildingii I B Amazone de Kawall (F): Amazona kawalli II B Amazone de la Barbade (F): Amazona barbadensis I B Amazone de la Jamaique (F): Amazona collaria II B Amazone de Porto Rico (F): Amazona vitnata I B Amazone de Prêtre (F): Amazona pretrei I B Amazone de Sainte-Lucie ( $F$ ): Amazona versicolor I B Amazone de Saint-Vincent (F): Amazona guildingii 1 B Amazone de San Domingo (F): Amazona ventralis II B Amazone de Tucuman (F): Amazona rucumana I B Amazone d'Hispaniola ( $\mathrm{F})$ : Amazona ventralis II $\mathrm{B}$ Amazone diadème ( $\mathrm{F}$ ): Amazona autumnalis II B Amazone du Brésil (F): Amazona brasitiensis I B Amazone du Yucatan (F): Amazona xantholora II B Amazone farineuse (F): Amazona forinosa II B Amazone festive (F): Amazona festiva II B Amazone impériale $(\mathrm{F})$ : Amazona imperialis I $\mathrm{B}$ Amazone mercenaire (F): Amazona mercenaria II B Amazone poudrée ( $F$ ): Amazona farinosa II B Amazone sasabé ( $F$ ): Amazona collaria II B Amazone tavoua (F): Amazona festiva II B Amazone versicolore (F): Amazona versicolor I B Amazone verte (F): Amazona agilis II B

Amazone vineuse ( $\mathrm{F}$ ): Amazona vinacea I $\mathrm{B}$ Amazones (F): Amazona spp. I/II B

Amazonian Manatee (E): Trichechus inunguis I M Amazonian Parakeet (E): Nannopsittaca dachilleae II B Amazonian Parrotlet (E): Nannopsitraca dachilleae II B Amazonian Poison Frog (E): Dendrobates ventrimacularus II A Amazonian Umbrellabird (E); Cephalopterus omatus III B Amazonico activo (S): Amazona agilis II $\mathrm{B}$

Amazonico jamaica (S): Amazona agilis II B

Amazonico todo verde (S): Amazona agilis II B

Amazons (E): Amazona spp. I/II B

Ambergris Cay Dwarf Boa (E): Tropidophis greenwayi II R Ambiky Chameleon (E): Chamaeleo suzetae II R

Amblonyx cinereus: Oriental Small-clawed Otter (E), Nutria cenicienta (S), Nutria inerme asiatica (S), Loutre cendrée (F) II $\mathrm{M}$

Amblyospiza albifrons: Grosbeak Weaver (E), Thick-billed Weaver (E), White-fronted Grosbeak (E), Tejedor picogordo (S), Amblyospize à front blanc (F), Gros-bec à front blanc (F) III B Amblyospize à front blanc (F): Amblyospiza albifrons III B Amblyrhynche à crête (F): Amblyrhynchus cristaus II R Amblyrhynchus cristatus: Galapagos Marine Iguana (E), Sea Iguana (E), Iguana marina (S), Amblyshynche à crête (F), lguane marin (F) II R

Ambon Flying-fox (E): Pteropus chrysoproctus II M Ambon King-Parrot (E): Alisterus amboinensis II B

Ambon Lizard (E): Varanus indicus II R

Ambystoma dumerilii: Achoque (E), Lake Patzcuaro Salamander (E), Ajolote de lago Pátzquaro (S), Salamandre du lac Pàtzcuaro (F) II A

Ambystoma mexicanum: Axolotl (E), Ajolote mexicano (S), Ambystome du Mexique (F), Axolotl (F), Salamandre du Mexique (F) II A

Ambystome du Mexique (F): Ambystoma mexicanum II A American Alligator (E): Alligator mississippiensis II R American Black Bear (E): Ursus americanus II M American Crocodile (E): Crocodylus acutus I R American Flamingo (E): Phoenicopterus ruber II B American Harpy Eagle (E): Harpia harpyja I B American Kestrel (E): Falco sparverius II B American Lynx (E): Lynx canadensis II M

American Manatee (E): Trichechus manatus I M American Swallow-tailed Kite (E): Elanoides forficatus II B Amethyst Woodstar (E): Calliphlox amethystina II B Amethystine (Rock) Python (E): Morelia amethistina II R Amethyst-throated Hummingbird (E): Lampornis amethystinus II B Amethyst-throated Sunangel (E): Heliangelus amethysticollis II B Ammotragus lervia: Aoudad (E), Barbary Sheep (E), Uaddan (E), Aoudad (F), Mouflon à manchettes (F) II M

Ampalagua (E): Boa constricior /II R

Amphelia atlantica: II In

Amphelia oculaia: II In

Amphelia ornata: II In
Amphihelia $=$ Amphelia

Amsterdam Island Fur Seal (E): Arctocephalus tropicalis II M Amur Falcon (E): Falco amurensis II B

Amur Sturgeon (E): Acipenser schrencki II F

Anaconda (E/S/F): Eunectes murinus II R

Anaconda à taches sombres (F): Eunectes deschauenseei II R

Anaconda amarilla (S): Eunectes notaeus II R

Anaconda commun ( $F$ ): Eunecles murinus II $R$

Anaconda de Barbour (F): Eunecles barbouri II R

Anaconda de Deschauensee (F): Eunectes deschauenseei II $R$

Anaconda du Paraguay (F): Eunectes notaeus II R

Anaconda jaune (F): Eunectes notoeus II $R$

Anaconda vert (F): Eunectes murinus II R

Anacondas (E): Eunectes spp. II R

Anacropora forbesi: II In

Anacropora gracilis: II In

Anacropora manthai: II th

Anacropora puertogalerae: II In

Anacropora reticulata: II In

Anacropora spinosa: II in

Anade rabudo (S): Anas acuta III B

Anade silbón (S): Anas penelope III B

Anamolure de Beecroft (F): Anomalurus beecrofit III M

Anamolure de Derby ( $F$ ): Anomalurus derbianus $111 \mathrm{M}$

Anamolure de Pel (F): Anomalurus pelii III M

Anamolure nain ( $\mathrm{F}$ ): Idiunis macrotis III M

Anaplectes melanotis $=$ Anaplectes rubricep

Anaplectes rubriceps: Red-headed Weaver (E), Mailmbo capirotado (S), Tisserin à ailes rouges (F), Tisserin écarlate (F) III B

Anas (S): Conepalus humboldtii II M

Anas acuta: Common Pintail (E), Northerm Pintail (E), Anade rabudo (S), Pato golondrino norteño (S), Pato rabudo (S), Canard pilet (F), Pilet (F) III B

Anas aucklandica: Brown Teal (E), Cerceta alicorta de Auckland (S), Sarcelle aptère (F), Sarcelle brune (F), Sarcelle de NouvelleZélande (F), Sarcelle terrestre des îles Auckland (F) I B

Anas bernieri: Madagascar Teal (E), Cerceta de Madagascar (S), Cerceta malgache (S), Pato de Bernier (S), Canard de Bernier (F), Sarcelle de Bernier (F), Sarcelle de Madagascar (F), Sarcelle malgache de Bernier (F) II B

Anas capensis: Cape Teal (E), Cape Wigeon (E), Cerceta del cabo (S), Canard du Cap (F) III B

Anas chlorotis = Anas aucklandica

Anas clypeata: Northem Shoveler (E), Pato cuchara (S), Pato cucharón norteño (S), Canard souchet (F), Souchet (F) III B

Anas crecca: Common Teal (E), Green-winged Teal (E), Cerceta aliverde (S), Cerceta común (S), Sarcelle d'hiver (F) III B

Anas formosa: Baikal Teal (E), Cerceta del Baikal (S), Sarcelle formose (F), Sarcelle élégante (F) II B

Anas laysanensis: Laysan Duck (E), Laysan Teal (E), Pato real de Laysan (S), Canard de Laysan (F), Sarcelle de Laysan (F) I B Anas nesiotis = Anas aucklandica

Anas oustaleti: Marianas Island Duck (E), Marianas Mallard (E), Oustalet's Grey Duck (E), Pato real marismeno (S), Canard aberrant (F), Canard d'Oustalet (F), Carnard des Mariannes (F) I B

Anas penelope: Eurasian Wigeon (E), Anade silbón (S), Pato silbón (S), Canard siffleur (F) III B

Anas platyrhynchos laysanensis $=$ Anas laysanensis

Anas platyrhynchos oustaleti $=$ Anas oustaleti

Anas querquedula: Garganey (E), Garganey Teal (E), Cerceta carretona (S), Cerceta cejiblanca (S), Sarcelle d'été (F) III B

Anathana ellioti: Madras Treeshrew (E), Musaraña arborícola de la India (S), Toupaie d'Elliot (F) II M

Anchieta's Chameleon (E): Chamaeleo anchierae II R

Anchor Coral (E): Euphyllia ancora II in

Ancistrodon à deux lignes $(\mathrm{F})$ : Agkistrodon bilineatus III R

Andaman Day Gecko (E): Phelsuma andamanensis II R

Andaman Hawk-Owl (E): Ninox affinis II B

Andaman Scops-Owl (E): Otus balli II B

Andaman Serpent-Eagle (E): Spilomis elgini II B

Andean Bear (E): Tremarctos omatus I M

Andean Cat (E): Oreailurus jacobita I M

Andean Cock-of-the-rock (E): Rupicola peruviana II B

Andean Condor (E): Vultur gryphus I B

Andean Emerald (E): Amazilia franciae II B 
Andean Flamingo (E): Phoenicopterss andinus II B Andean Hairy Armadillo (E): Chaetophractus nationi $11 \mathrm{M}$ Andean Hillstar (E): Oreotrochilus estella II B Andean Night Monkey (E): Aotus miconax II M Andean Parakeet (E): Bolborhynchus orbygnesius II B Andean Poison Frog (E): Minyobates opisthomelas II A Andean Pygmy-Owl (E): Glaucidium jardinii II B Andean Tapir (E): Tapirus pinchaque I M Andean Titi Monkey (E): Callicebus oenanthe II M Andean Wolf (E): Pseudalopex culpaeus II M Andrews' Beaked Whale (E): Mesoplodon bowdoini II M Andrews' Frigatebird (E): Fregata andrewsi I B Andrias spp.: Giant salamanders (E), Salamandras gigantes (S), Salamandre géante (F) I A

Andrias davidianus: Chinese Giant Salamander (E), Salamandra gigante de China (S), Salamandre du Père David (F), Salamandre géante de Chine (F) I A

Andrias japonicus: Japanese Giant Salamander (E), Salamandra gigante de Japón (S), Salamandre géante du Japon (F) I A

Androdon aequatorialis: Tooth-billed Hummingbird (E), Colibrí piquidentado (S), Colibri d'Équateur (F) II B

Andros Ground Iguana (E): Cyclura cyclura I R

Ane sauvage d'Afrique (F): Equus africanus I M

Ane sauvage d'Asie (F): Equus hemionus III M

Ane sauvage de l'Inde (F): Equus onager khur I M

Ane sauvage de Mongolie (F): Equus hemionus hemionus I M

Ane sauvage du Tibet $(\mathrm{F})$ : Equus kiang II M

Anegada Ground Iguana (E): Cyclura pinguis I R

Anegada Rock Iguana (E): Cyclura pinguis I R

Angel de Sol amatista (S): Heliangelus amerhysticollis II B

Angel de Sol cuelliocre (S): Heliangelus mavors II B

Angel de Sol de Mérida (S): Heliangelus spencei II B

Angel's Chameleon (E): Chamaeleo angeli II R

Angia $=$ Culicia

Angola Chameleon (E): Chamaeleo anchietae II R

Angolan Black-and-white Colobus (E): Colobus angolensis II M

Angolan Colobus (E): Colobus angolensis II M

Angolan Python (E): Python anchierae II R

Angonoka (E): Geochelone yniphora I R

Angulated Tortoise (E): Chersina angulata II R

Angwantibo (E/F): Arcrocebus calabarensis II M

Anisopsammia $=$ Enallopsammia

Anna's Hummingbird (E): Calypte anna II B

Annulated Tree Boa (E): Corallus annulatus II R

Anoa de Ilanura (S): Bubalus depressicornis I M

Anoa de montaña (S): Bubalus quarlesi I M

Anoa de Quarle (F): Bubalus quarlesi I M

Anoa depressicomis $=$ Bubalus depressicomis

Anoa des montagnes (F): Bubalus quarlesi I M

Anoa des plaines (F): Bubalus depressicomis I M

Anoa mindorensis $=$ Bubalus mindorensis

Anoa quarlesi $=$ Bubalus quarlesi

Anodorhynchus spp.: Blue macaws (E), Aras bleus (F) I B

Anodorhynchus glaucus: Glaucous Macaw (E), Ara azul (S), Guacamayo glauco (S), Guacamayo violáceo (S), Ara bleu (F), Ara glauque (F) I B

Anodorhynchus hyacinthinus: Hyacinth Macaw (E), Guacamayo azul (S), Guacamayo jacinto (S), Ara bleu (F), Ara hyacinthe (F), Ara jacinthe (F) I B

Anodorhynchus leari: Indigo Macaw (E), Lear's Macaw (E), Guacamayo cobalto (S), Guacamayo de Lear (S), Ara cobalt (F), Ara de Lear (F) I B

Anomalospiza imberbis: Cuckoo Weaver (E), Parasitic Weaver (E), Anomalospize parasite (F), Tisserin covcau (F) III B

Anomalospize parasite (F): Anomalospiza imberbis III B

Anomalurus beecrofti: Beecroft's Flying Squirrel (E), Anamolure de Beecroft (F), Écureuil volant de Beecroft (F) III M

Anomalurus derbianus: Lord Derby's Flying Squirrel (E), Anamolure de Derby (F), Écureuil volant de Derby (F) III M

Anomalurus pelii: Pel's Flying Squírel (E), Aramolure de Pel (F). Écureuil volant de Pel (F) III M

Anomalurus schoutedeni $=$ Anomalurus beecrofti

Anomastraea irregularis: II In

Anomocora carinata: II In

Anomocora fecunda: II In

Anorrhinus spp.: Hornbills (E), Buceros (S), Calaos (F) II B
Anorrhinus galerifus: Bushy-crested Hombill (E), Calao largup (F) II B

Anorrhinus tickelli: Brown Hombill (E), Calao brun (F) II B

Anserelle naine (F): Netrapus auritus III B

Anta (S): Tapirus terrestris II M

Anta brasileña (S): Tapirus terrestris П M

Antarctic Fur Seal (E): Arcrocephalus gazella II M

Antaresia childreni $=$ Morelia childreni

Antaresia maculosa = Morelia maculosa

Antaresia perthensis $=$ Morelia perthensis

Antaresia stimsoni = Morelia stimsoni

Anteaters (E): Myrmecophagidae spp. VII/NC M

Anteburro (S): Tapins bairdii I M

Antelopes (E): Bovidae spp. ИI/II/NC M

Anthemiphyllia dentata: II In

Anthemiphyllia frustum: II In

Anthemiphyllia pacifica: II In

Anthemiphyllia patera: II In

Anthocephala floriceps: Blossomcrown (E), Colibri à tête rose (F)

II B

Anthracoceros spp.: Hornbills (E), Buceros (S), Calaos (F) II B Anthracoceros albirosiris: Malaysian Pied-Hombill (E), Oriental

Pied-Hombill (E), Calao pie (F) II B

Anthracoceros convexus = Anthracoceros albirostris

Anthracuceros coronatus: Malabar Pied-Hornbill (E), Malabar Piedhombill (E), Calao de Malabar (F) II B

Anthracoceros malabaricus $=$ Anthracoceros albirostris

Anshracoceros malayanus: Black Hornbill (E), Calao charbonnier (F)

II B

Anthracoceros marchei: Palawan Hornbill (E), Calao de Palawan (F)

II B

Anthracoceros montani: Sulu Hombill (E), Calao des Sulu (F) II B

Anthracothorax dominicus: Antillean Mango (E), Dominican Mango

(E), Zumbador grande (S), Mango doré (F) II B

Anthracothorax mango: Jamaican Mango (E), Mango de la Jamaïque (F) II B

Anthracothorax nigricollis: Black-throated Mango (E), Mango (S),

Mango pechinegro (S), Picaflor ventrinegro (S), Mango à

cravate noire (F) II B

Anthracothorax prevostii: Green-breasted Mango (E), Veraguas

Mango (E), Mango pechiverde (S), Mango de Prévost (F) II B

Anthracothorax veraguensis $=$ Anthracothorax prevostii Anthracothorax viridigula: Green-throated Mango (E), Mango

gargantiverde (S), Mango à cravate verte (F) II B

Anthracothorax viridis: Green Mango (E), Mango vert (F) II B

Anthropoides paradisea $=$ Grus paradisea

Anthropoides virgo $=$ Grus virgo

Antillean Crested Hummingbird (E): Orthorhynchus cristatus II B

Antillean Mango (E): Anthracothorax dominicus II B

Antillia $=$ Trachyphyllia

Antilocapra americana: Mexican Pronghom (E), Pronghorn (E),

Berrendo (S), Antilocapre (F), Antilope américaine (F),

Pronghom (F) I/NC M

Antilocapre (F): Antilocapra americana $\mathbf{V} / \mathrm{NC} \mathrm{M}$

Antilope américaine (F): Antilocapra americana $\mathbf{I} \mathbf{N C} \mathrm{M}$ Antilope amizclero enano de agua ( $\$$ ): Hyemoschus aquaticus III M Antilope blanche (F): Addar nasomaculatus I M

Antilope cenvicapra: Blackbuck (E), Sasin (E), Cervicapra (S),

Antilope cervicapre (F) $111 \mathrm{M}$

Antilope cervicapre (F): Antilope cervicapra III M

Antilope de cuatrocuemos (S): Tetracerus quadricomis III M

Antilope del Tibet (S): Pantholops hodgsonii I M

Antilope du Tibet (F): Pantholops hodgsonii I M

Antilope sable negro (S): Hippotragus niger variani I M

Antílope saiga (S): Saiga fatarica II M

ANTIPATHARIA spp.: Black corals (E), Corales negros (S), Coraux noirs (F) II in

Antipathella $=$ Antipashes

Antipathes abies: II In

Antipathes aculeata: II in

Antipathes alata: II In

Antipathes americana: II In

Antipathes aperia: II In

Antipathes arborea: II In

Antipathes assimilis: II In

Antipathes allantica: II In 
Antipathes barbadensis: II In Antipathes bifaria: II In Antipathes boscii: II In Antipathes caribbeana: II In Antipathes catharinae: II In Antipathes ceylonensis: II In Antipathes chamaemorus: II In Antiparhes chota: II In Antipathes columnaris: II In Antipathes contorta: II in Antipathes crispa: II In Antipathes cupressus: II In Antipathes curvala: II In Antipathes cylindrica: II In Antipathes delicatula: II In Antipathes densa: 11 in Antipathes dichotoma: II in Antipathes dubia: II In Antipathes elegans: II In Antipathes ericoides: II In Antipathes erinaceus: II In Antipathes eupteridea: II In Antipathes fermandezii: II In Antipathes fiordensis: II In Antipathes flabellum: II In Antipathes fragilis: II In Antipathes fruticosa: II In Antipathes furcata: II In Antipathes galapagensis: II In Antipathes gallensis: 11 In Antipathes glutinata: II In Antipathes grandiflora: II In Antipathes grandis: II In Antipathes grayi: II In Antipathes hirta: II In Antipathes hypnoides: II In Antipathes indistincta: II In Antipathes intermedia: II In Antipathes lata: II In Antipathes lenta: II In Antipathes lentipinna: II In Antipathes longibrachiata: II In Antipathes mediterranea: II In Antipathes minor: II In Antipathes myriophylla: II In Antipathes nilanduensis: II In Antipathes panamensis: II In Antipathes paniculata: II In Antipathes pauroclema: II In Antipathes pectinata: II In Antipathes pedata: II In Antipathes pennacea: II In Antipathes plana: II In Antipathes plantagenista: II In Antipathes pluma: II In Antipathes pseudodichotoma: II In Antipathes punctata: II In Antipathes reticulata: II In Antipathes rhipidion: II In Antipathes rigida: II In Antipathes robillardi: II In Antipathes rugosa: II In Antipathes salicoides: II In Antipathes salix: II In Antipathes sarothamnoides: II In Antipathes sarothrum: II In Antipathes sealarki: II In Antipathes sibogae; II In Antipathes simplex: II in Antipathes speciosa: II in Antipathes spinescens: II In Antipathes spinosa: II In Antipathes squamosa: II in Antipathes stechowi: II In Antipathes strigosa: 11 In Antipathes subpinnaia: II In
Antipathes ranacetum: II In

Antipathes renuispina: II In

Antipathes tematensis: II in

Antipathes thamnea: II In

Antipathes thamnoides: II In

Antipathes tristis: II In

Antipathes ulex: II In

Antipathes umbratica: II In

Antipathes valdiviae: II in

Antipathes vericillata: II In

Antipathes viminalis: II in

Antipathes virgata: II In

Antipathes wollastoni: II In

Antipathes zoothallus: II In

Antipodes Green Parakeet (E): Cyanoramphus unicolor II B

Antipodes Parakeet (E): Cyanoramphus unicolor II B

Antpeckers (E): Parmoptila spp. IIU/NC B

Aonyx capensis: African Clawless Otter (E), Cape Clawless Otter

(E), Nutria africana (S), Nutria de cuello blanco (S), Loutre à joues blanches (F) $11 \mathrm{M}$

Aonyx cinerea $=$ Amblonyx cinereus

Aonyx conģicus: Cameroon Clawless Otter (E), Small-clawed Otter

(E), Small-toothed Clawless Otter (E), Zaire Clawless Otter (E),

Nutria inerme de Camerún (S), Loutre à joues blanches du

Cameroun (F), Paraonyx tacheté (F) III M

Aony $x$ microdon $=$ Aony $x$ congicus

Aonyx phillippsi $=$ Aonyx congicus

Aotus azarai: II M

Aorus brumbacki: Brumback's Night Monkey (E) II M

Aotus hershkoviuzi: II M

Aotus infulalus: $11 \mathrm{M}$

Aolus lemurinus: II M

Aotus miconax: Andean Night Monkey (E) II M

Aotus nancymaae: II M

Aorus nigriceps: II M

Aotus trivirgatus: Douroucouli (E), Night Monkey (E), Owl Monkey

(E), Douroucouli (F), Singe de nuit (F) II M

Aotus trivirgatus azarai $=$ Aolus azarai

Aotus rivirgafus brumbacki $=$ Aotus brumbacki

Aotus irivirgatus hershkovitzi = Aotus hershkovitzi

Aotus trivirgatus infularus $=$ Aorus infulatus

Aotus trivirgatus lemurinus = Aotus lemurinus

Aolus trivirgatus miconax $=$ Aolus miconax

Aotus trivirgatus nancymaae = Aotus nancymaae

Aotus trivirgatus nigriceps = Aotus nigriceps

Aotus irivirgaius vociferans $=$ Aolus vociferans

Aotus vociferans: II M

Aoudad (E/F): Ammotragus lenvia II M

Apalone spinifera ater $=$ Trionyx ater

Apaporis River Caiman (E): Caiman crocodilus apaporiensis I R

Apennine Chamois (E): Rupicapra pyrenaica omata I M

Apes (E): PRIMATES spp. I/I M

Aphanipathes abietina: II In

Aphanipathes cancellata: II in

Aphanipathes filix: $\mathbf{I}$ In

Aphanipathes hancocki: II In

Aphanipathes humilis: II In

Aphanipalhes reviculata: II In

Aphanipathes somervillei: II In

Aphanipathes thyoides: II In

Aphanipathes undulata: II In

Aphantochroa cirrochloris: Sombre Hummingbird (E), Colibri vert et gris (F) II B

Aphonopelma = Brachypelma

Aphonopelma pallida $=$ Brachypelma pallidum

Aphrasirea $=$ Favites

Aplocyathus $=$ Trochocyathus

Aplomado Falcon (E): Falco femoralis II B

Apodora papuanus = Morelia papuana

Apollo (E): Pamassius apollo II In

Apollo Butterfly (E): Pamassius apollo II in

Apollos (E): Pamassius apollo/Pamassius spp. IINC In

Apolo (S): Parnassius apollo II In

Appalachian Monkeyface (E): Quadrula sparsa I In

Appalachian Monkey-face Pearly Mussel (E): Quadrula sparsa I In 
Aprosmictus erythropterus: Red-winged Parrot (E), Papagayo alirrojo (S), Perruche érythroptère (F) II B

Aprosmictus jonquillaceus: Olive-shouldered Parrot (E), Timor Redwinged Parrot (E), Papagayo de Timor (S), Perruche jonquille (F) II B

Aquatic Box Turtle (E): Terrapene coahuila I R

Aquila adalberi: Adalbert's Eagle (E), Spanish Imperial Eagle (E), Aguila imperial ibérica (S), Aigle impérial espagnol (F), Aigle à épaules blanches (F) I B

Aquila audax: Wedge-tailed Eagle (E), Aguila audaz (S), Aigle d'Australie (F) II B

Aquila chrysaetos: Golden Eagle (E), Aguila real (S), Aigle doré (F), Aigle royal (F) II B

Aquila clanga: Greater Spotted Eagle (E), Spotted Eagle (E), Aguila moteada (S), Aigle criard (F) II B

Aquila gumeyi: Gurney's Eagle (E), Aguila moluqueña (S), Aigle de Gumey (F) II B

Aquila heliaca: Imperial Eagle (E), Aguila imperial (S), Aguila imperial oriental (S), Aigle impérial (F) I B

Aquila heliaca adalberti = Aquila adalberti

Aquila nipalensis: Steppe Eagle (E), Aguila estoparia (S), Aigle des steppes (F) II B

Aquila orientalis $=$ Aquila nipalensis

Aquila pomarina: Lesser Spotted Eagle (E), Aguila pomerana (S) Aigle pomarin (F) 11 B

Aquila rapax: Tawny Eagle (E), Aguila rapaz (S), Aigle ravisseur (F) II B

Aquila verreauxii: African Black Eagle (E), Black Eagle (E), Verreaux's Eagle (E), Aguila cafre (S), Aigle de Verreaux (F) II B

Aquila wahlbergi: Wahlberg's Eagle (E), Aguila de Walhberg (S), Aigle de Wahlberg (F) II B

Ara à collier jaune (F): Ara auricollis II B

Ara à face grise (F): Cyanopsitta spixii I $\mathbf{B}$

Ara à gorge bleue (F): Ara glaucogularis $1 \mathrm{~B}$

Ara aे nuque d'or (F): Ara auricollis II B

Ara ambigua: Buffon's Macaw (E), Great Green Macaw (E), Guacamayo ambiguo (S), Guacamayo verde mayor (S), Ara de Buffon (F) I B

Ara ararauna: Blue-and-gold Macaw (E), Blue-and-yellow Macaw (E), Guacamayo azul y amarillo (S), Guacamayo azulamarillo (S), Ara bleu (F) II B

Ara auricollis: Golden-collared Macaw (E), Yellow-collared Macaw (E), Guacamayo acollarado (S), Maracana cuellidorada (S), Ara à collier jaune (F). Ara à nuque d'or (F) II B

Ara azul (S): Anodorhynchus glaucus I B

Ara bleu (F): Anodorhynchus glaucus I B/Anodorhynchus hyacinthinus I B/Ara ararauna II B

Ara caninde $=$ Ara ararauna

Ara canindé (F): Ara glaucogularis I B

Ara chloroptère (F): Ara chloropterus II B

Ara chloropterus: Green-winged Macaw (E), Red-and-green Macaw (E), Guacamayo aliverde (S), Guacamayo rojo (S), Ara chloroptère (F) II B

Ara cobalt (F): Anodorhynchus leari I B

Ara couloni: Blue-headed Macaw (E), Guacamayo cabeciazul (S), Ara de Coulon (F) II B

Ara de Buffon (F): Ara ambigua I B

Ara de Coulon (F): Ara couloni II B

Ara de Lafresnaye (F): Ara rubrogenys I B

Ara de Lear (F): Anodorhynchus leari I B

Ara de Spix (F): Cyanopsitla spixii I B

Ara d'Illiger (F): Ara maracana I B

Ara glaucogularis: Blue-throated Macaw (E), Guacamayo amarillo (S), Guacamayo barbazul (S), Guacamayo caninde (S), Ara canindé (F), Ara à gorge bleue (F) I B

Ara glauque (F): Anodorhynchus glaucus I B

Ara hyacinthe (F): Anodorhynchus hyacinthinus I B

Ara jacinthe (F): Anodorhynchus hyacinthinus I B

Ara macao: Scarlet Macaw (E), Guacamayo bandera (S), Guacamayo macao (S), Lapa azul (S), Ara macao (F), Ara rouge (F) I B

Ara macao (F): Ara macao I B

Ara macavouanne (F): Ara manilata II B

Ara manilata: Red-bellied Macaw (E), Guacamayo barriga roja (S) Guacamayo ventrirrojo (S), Ara macavouanne (F) II B
Ara maracana: Blue-winged Macaw (E), llliger's Macaw (E), Guacarnayo maracaná (S), Maracana dorsirroja (S), Ara d'Illiger (F) I B

Ara militaire (F): Ara militaris I B

Ara militaris: Military Macaw (E), Guacamayo militar (S), Guacamayo verde (S), Ara militaire (F) I B

Ara nobilis: Noble Macaw (E), Red-shouldered Macaw (E),

Guacamayo enano (S), Guacamayo noble (S), Ara noble (F),

Petit perruche-ara (F) II B

Ara noble (F): Ara nobilis II B

Ara rouge (F): Ara macao I B

Ara rubrogenys: Red-fronted Macaw (E), Guacamayo de Cochabamba (S), Guacamayo frentirroja (S), Ara de Lafresnaye (F) I B

Ara severa: Chestnut-fronted Macaw (E), Severe Macaw (E), Guacamayo severo $(S)$, Ara sévère $(F)$, Ara vert $(F)$ II B Ara sévère (F): Ara severa II B

Ara vert (F): Ara severa II B

Arabian Bustard (E): Ardeotis arabs II B

Arabian Chameleon (E): Chamaeleo arabicus II R

Arabian Oryx (E): Oryx leucoryx I M

Arabian Sand Boa (E): Eryx jayakari II R

Araçari à cou noir (F): Pteroglossus aracari II B

Araçari à oreillons roux (F): Pteroglossus castanotis III B

Araçari grigri (F): Pteroglossus aracari II B

Araçari vert (F): Pteroglossus viridis II B

Aracaris (E): Preroglossus spp. IIIIINC B

Arachnopathes $=$ Antipathes

Araeacis $=$ Astreopora

Araguato de Guatemala (S): Alouatta pigra I M

Arapaima (E/S/F): Arapaima gigas II F

Arapaima gigas: Arapaima (E), Pirarucu (E), Arapaima (S), Paiche

(S), Arapaima (F), Paiche (F), Pirarucu (F) II F

Aras (F): Anodorhynchus spp/Ara spp/Cyanopsitta spp. ИII B

Aras bleus (F): Anodorhynchus spp. I B

Arasari orejicastano (S): Pteroglossus castanotis III B

Aratinga acuticaudata: Blue-crowned Conure (E), Blue-crowned

Parakeet (E), Aratinga cabeciazul (S), Carapaico (S), Cotorra de

los Palos (S), Conure à tête bleue (F) 11 B

Aratinga astec $=$ Aratinga nana

Aratinga aurea: Golden-crowned Conure (E), Peach-fronted Conure

(E), Peach-fronted Parakeet (E), Aratinga frentidorada (S),

Cotorra frentidorada (S), Conure couronné ( $F$ ), Perruche à front doré (F) II B

Aratinga auricapilla: Golden-capped Conure (E), Golden-capped

Parakeet (E), Aratinga testadorada (S), Conure à tête d'or (F) II B

Aratinga brevipes $=$ Aratinga holochlora

Aratinga cabeciazul (S): Aratinga acuticaudata II B

Aratinga cabecifusca (S): Aratinga weddellii II B

Aratinga cactácea (S): Araringa cacrorum II B

Aratinga cactorum: Caatinga Parakeet (E), Cactus Conure (E),

Cactus Parakeet (E), Aratinga cactácea (S), Conure des cactus

(F) II B

Aratinga canicularis: Orange-fronted Conure (E), Orange-fronted Parakeet (E), Aratinga frentinaranja (S), Perico frentinaranja (S), Conure à front rouge $(\mathrm{F})$ II B

Aratinga chloroptera: Hispaniolan Conure (E), Hispaniolan Parakeet (E), Aratinga de la Española (S), Perico (S), Conure maîtresse (F) II B

Aratinga Cubana (S): Aratinga euops II B

Aratinga de Finsch (S): Aratinga finschi II B

Aratinga de Guayaquil (S): Aratinga erythrogenys II B

Aratinga de la Española (S): Aratinga chloroptera II B

Aratinga de pinceles (S): Leptosittaca branickii II B

Aratinga de Wagler (S): Aratinga wagleri 11 B

Aratinga erythrogenys: Red-masked Conure (E), Red-masked Parakeet (E), Aratinga de Guayaquil (S), Perico caretirrojo (S), Conure à tête rouge (F) II B

Aratinga euops: Cuban Conure (E), Cuban Parakeet (E), Aratinga Cubana (S), Conure de Cuba (F) II B

Aratinga finschi: Crimson-fronted Conure (E), Crimson-fronted Parakeet (E), Finsch's Conure (E), Aratinga de Finsch (S), Conure de Finsch (F) II B

Aratinga frentidorada (S): Aratinga aurea II B

Aratinga frentinaranja (S): Aratinga canicularis II B 
Aratinga guarouba: Golden Conure (E), Golden Parakeet (E), Queen of Bavaria's Conure (E), Aratinga guaruba (S), Cotorra amarilla $(\mathrm{S})$, Conure de la Reine de Bavière $(\mathrm{F})$, Conure doré $(\mathrm{F})$, Guarouba (F), Pernuche dorée (F) I B

Aratinga guanuba (S): Aratinga guarouba I B

Aratinga holochlora: Green Conure (E), Green Parakeet (E), Aratinga verde (S), Perico verde (S), Conure vert (F) II B

Aratinga jandaya: Jandaya Conure (E), Jandaya Parakeet (E), Yellow-headed Parakeet (E), Aratinga jandaya (S), Conure jandaya (F), Perruche de Jenday (F) II B

Aratinga jandaya (S): Aratinga jandaya II B

Aratinga leucophthalmus: White-eyed Conure (E), White-eyed Parakeet (E), Aratinga ojiblanca (S), Cotorra verde (S), Perico ojo blanco (S), Conure pavouane (F) II B

Aratinga mitrada (S): Aratinga mitrata II B

Aratinga mitrala: Mitred Conure (E), Mitred Parakeet (E), Aratinga mitrada (S), Cotorra cariroja (S), Conure mitré (F) II B

Aratinga nana: Aztec Parakeet (E), Olive-throated Conure (E) Olive-throated Parakeet (E), Aratinga pechisucia (S), Perico pechisucio (S), Conure aztèque (F) II B

Aratinga ñanday (S): Nandayus nenday II B

Aratinga ojiblanca (S): Aratinga leucophihalmus II B

Aratinga orejigualda (S): Ognorhynchus icterotis I B

Aratinga pechisucia (S): Aratinga nana II B

Aratinga pertinax: Brown-throated Conure (E), Brown-throated Parakeet (E), Aratinga pertinaz (S), Perico cara sucia (S), Conure cuivré (F) II B

Aratinga pertinaz (S): Aratinga pertinax II B

Aratinga rubritorquis $=$ Aratinga holochlora

Aratinga sol (S): Aratinga solstitialis II B

Aratinga solstitialis: Sun Conure (E), Sun Parakeet (E), Aratinga sol (S), Perico dorado (S), Conure soleil (F), Perruche du soleil (F) II B

Aratinga strenua: Pacific Parakeet (E), Perico verde centroamericano (S), Conure de Ridgway (F) II B

Aratinga testadorada (S): Aratinga auricapilla II B

Aratinga verde (S): Aratinga holochlora II B

Aratinga wagleri: Red-fronted Conure (E), Scarlet-fronted Parakeet (E), Aratinga de Wagler (S), Chacaraco (S), Conure de Wagler (F) 11 B

Aratinga weddellii: Dusky-headed Conure (E), Dusky-headed Parakeet (E), Aratinga cabecifusca (S), Perico cabecioscuro (S), Conure de Weddell (F) II B

Aratingas (S): Aratinga spp/Leptosittaca branickii/Nandayus nenday/Ognorhynchus icterotis $\mathrm{I} / \mathrm{II} \mathrm{B}$

Arborófila de Sumatra (S): Arborophila orientalis III B

Arborófila pechicastaña (S): Arborophila charltonii III B

Arborophila charltonii: Chestnut-breasted Tree-Partridge (E),

Chestnut-necklaced Partridge (E), Arborófila pechicastaña (S), Perdiz de bosque de Charlton (S), Torquéole de Merlin (F). Torquéole des bois $(\mathrm{F})$, Torquéole à poitrine châtaine $(\mathrm{F})$ III $\mathrm{B}$

Arborophila orientalis: Bar-backed Partridge (E), Grey-breasted Partridge (E), Arborófila de Sumatra (S), Torquéole de Sumatra (F), Torquéole à poitrine brune (F) III B

Arch-beaked Whale (E): Mesoplodon carlhubbsi II M

Archer's Buzzard (E): Buteo archeri II B

Archibebe moteado (S): Tringa guttifer I B

Archilochus alexandri: Black-chinned Hummingbird (E), Colibrí barbinegro (S), Colibri à gorge noire (F) II B

Archilochus anna $=$ Calypte anna

Archilochus calliope $=$ Siellula calliope

Archilochus colubris: Ruby-throated Hummingbird (E), Colibri gorjirrubi (S), Colibri à gorge rubis (F) II B

Archohelia rediviva: II In

Arctictis binturong: Binturong (E), Binturong (S), Binturong (F) III $M$

Arctocèbe (F): Arctocebus calabarensis II M

Arctocebus aureus: Golden Potto (E) II M

Arctocebus calabarensis: Angwantibo (E), Poto dorado (S), Angwantibo (F), Arctocèbe (F), Potto de Calabar (F) II M Arctocebus calabarensis aureus = Arctocebus aureus Arctocéphale d'Afrique du Sud (F): Arctocephalus pusillus II M Arctocéphale d'Australie (F): Arctocephalus tropicalis II M Arctocéphale de Guadalupe (F): Arctocephalus townsendi I M Arctocéphale de Juan Femandez (F): Arctocephalus philippii II M Arctocéphale de Kerguelen (F): Arctocephalus gazella II M
Arctocéphale des Galapagos (F): Arctocephalus galapagoensis II M Arctocéphales du Sud (F): Arctocephalus spp. II M

Arctocephalus spp.: Southem fur seals (E), Osos marinos (S), Arctocéphales du Sud (F), Otaries à fourrure du Sud (F) II M

Arclocephalus australis: South American Fur Seal (E), Southem Fur Seal (E), Oso marino austral (S), Otarie à fourrure australe (F) II M

Arctocephalus doriferus $=$ Arctocephalus pusillus

Arcrocephalus forsteri: New Zealand Fur Seal (E), Oso marino de Neuva Zelanda (S) II M

Arctocephalus galapagoensis: Galapagos Islands Fur Seal (E), Galápagos Fur Seal (E), Oso marino de las Galápagos (S), Arctocéphale des Galapagos (F) II M

Arctocephalus gazella: Antarctic Fur Seal (E), Kerguelen Fur Seal (E), Arctocéphale de Kerguelen (F) II M

Arcrocephalus philippii: Juan Fernández Fur Seal (E), Oso marino de Chile (S). Arctocéphale de Juan Fernandez (F) II M

Arclocephalus pusillus: Afro-Australian Fur Seal (E), Cape Fur Seal (E), South African Fur Seal (E), Arctocéphale d'Afrique du Sud (F) II M

Arctocephalus tasmanicus $=$ Arctocephalus pusillus Arcrocephalus fownsendi: Guadalupe Fur Seal (E), Lower Califomian Fur Seal (E), Oso marino de Guadalupe (S), Arctocéphale de Guadalupe (F), Otarie à fourrure d'Amerique (F) I M

Arctocephalus tropicalis: Amsterdam Island Fur Seal (E). Subantarctic Fur Seal (E), Arctocéphale d'Australie (F) II M

Ardea goliath: Goliath Heron (E), Garza goliat (S), Héron goliath (F) III B

Ardeola ibis = Bubulcus ibis

Ardeotis arabs: Arabian Bustard (E), Avutarda árabe (S), Grande outarde arabe (F), Outarde arabe (F) II B

Ardeotis australis: Australian Bustard (E), Avutarda australiana (S), Outarde australienne (F), Outarde d'Australie (F) II B

Ardeotis kori: Kori Bustard (E), Avutarda kori (S), Outarde kori (F) II B

Ardeotis nigriceps: Great Indian Bustard (E), Indian Bustard (E),

Avutarda india (S), Avutarda índica (S), Outarde de l'Inde (F),

Outarde à tête noire (F) I B

Ardilla de Deppe (S): Sciurus deppei III M

Ardilla de los pinos (S): Sciurus deppei III M

Ardilla de palmera (S): Epixerus ebii III M

Ardilla voladera de orejas largas (S): Idiurus macrotis III M

Ardillas gigantes (S): Ratufa spp. II M

Areolated Tortoise (E): Homopus areolatus II R

Arfak Astrapia (E): Astrapia nigra II B

Arfak Parotia (E): Parofia sefilata II B

Argali (E/F): Ovis ammon I/II M

Argentine Boa Constrictor (E): Boa constrictor occidentalis I R

Argentine Grey Fox (E): Pseudalopex griseus II M

Argentine Teju (E): Tupinambis nufescens II R

Argentine Tortoise (E): Geochelone chilensis II R

Argos real (S): Argusianus argus II B

Argus (F): Argusianus argus/Rheinardia ocellata I/I B

Argus géant (F): Argusianus argus II B

Argus ocellé (F): Rheinardia ocellata I B

Argus Pheasant (E): Argusianus argus II B

Arguses (E): Argusianus argus/Rheinardia ocellata $\mathbf{V I I} \mathbf{B}$

Argusianus argus: Argus Pheasant (E), Great Argus (E), Argos real

(S), Faisán argos (S), Argus géant (F) II B

Ariane à couronne azur (F): Amazilia cyanocephala II B

Ariane à couronne violette (F): Amazilia violiceps II B

Ariane à front bleu (F): Amazilia cyanifrons II B

Ariane à front vert (F): Amazilia viridifrons II B

Ariane à poitrine blanche (F): Amazilia chionopectus II B

Ariane à queue bleue (F): Amazilia cyanura II B

Ariane à ventre blanc (F): Amazilia chionogaster $\mathbf{I}$ B

Ariane à ventre gris (F): Amazilia tzacasl II B

Ariane à ventre roux (F): Amazilia castaneiventris II B

Ariane à ventre vert $(\mathrm{F})$ : Amazilia viridigaster II B

Ariane aimable (F): Amazilia amabilis II B

Ariane béryl (F): Amazilia beryllina II B

Ariane candide (F): Amazilia candida II B

Ariane cannelle (F): Amazilia rutila II B

Ariane charmante (F): Amazilia decora II B

Ariane de Boucard (F): Amazilia boucardi II B 
Ariane de Félicie (F): Amazilia tobaci II B Ariane de Francia (F): Amazilia franciae II B Ariane de Lesson (F): Amazilia amazilia II B Ariane de Linné (F): Amazilia fimbriala II B Ariane de Lucy (F): Amazilia luciae II B Ariane de Rosenberg (F): Amazilia rosenbergi 11 B Ariane de Sophie (F): Amazilia saucerrottei II B Ariane d'Edward (F): Amazilia edward II B Ariane du Pérou (F): Amazilia viridicauda II B Ariane du Tachira (F): Amazilia distans II B Ariane du Yucatan (F): Amazilia yucatanensis II B Ariane saphirine (F): Amazilia lactea II B Ariane versicolore (F): Amazilia versicolor II B Ariane vert-doré (F): Amazilia leucogaster II B Arianes (F): Amazilia spp. II B

Arid Monitor (E): Varanus tristis II R Arirai (S): Pteronura brasiliensis I M Armadillo gigante (S): Priodonies maximus I M Armadillo Girdled Lizard (E): Cordylus cataphractus II R Armadillo Spiny-tailed Lizard (E): Cordylus calaphracius II R Armadillos (E): Dasypodidae spp. IIIINC M

Armado de zapilots (S): Cabassous centralis III M Armoured Teyou (E): Dracaena guianensis II R Amoux's Beaked Whale (E): Berardius amuxii I M Arowanas (E): Scleropages spp. UNC F

Arpia (S): Harpia harpyja I B

Arpia mayor (S): Harpia harpyja I B

Arpia menor (S): Morphnus guianensis II B

Arpía papúa (S): Harpyopsis novaeguineae II B Arrau (E): Podocnemis expansa II R

Ascagne (F): Cercopithecus ascanius II M Ashy-faced Owl (E): Tyto glaucops II B

Ashy-headed Flying-fox (E): Preropus caniceps II M Asian Arowana (E): Scleropages formosus I F

Asian Barred Owlet (E): Glaucidium cuculoides II B

Asian Baza (E): Aviceda jerdoni II B

Asian Black Eagle (E): Ictinaetus malayensis II B Asian Bonytongue (E): Scleropages formosus I F Asian Caracal (E): Caracal caracal I/II M

Asian Cobra (E): Naja naja II R

Asian Crested Goshawk (E): Accipiser Irivirgalus II B

Asian Elephant (E): Elephas maximus I M

Asian Giant Tortoise (E): Manouria emys II R

Asian Tapir (E): Tapirus indicus I M

Asian Tortoise (E): Manouria emys II R

Asian White-backed Vulture (E): Gyps bengalensis II B

Asian Wild Ass (E): Equus hemionus III M

Asian Wild Sheep (E): Ovis ammon III M

Asiatic Black Bear (E): Ursus thibetanus $1 \mathrm{M}$

Asiatic Golden Cat (E): Catopuma temminckii I M

Asiatic Lion (E): Panthera leo persica I M

Asiatic Rock Python (E): Python molurus $\mathbf{I} / \mathbf{I}$ R

Asiatic Wild Ass (E): Equus hemionus I/II M

Asiatic Wild Dog (E): Cuon alpinus II M

Asio abyssinicus: Abyssinian Owl (E), Hibou d'Abyssinie (F) II B

Asio capensis: African Marsh Owl (E), Marsh Owl (E), Lechuza mora (S), Hibou du Cap (F) II B

Asio clamator: Striped Owl (E), Búho-cornudo cariblanco (S), Lechuza listada (S), Lechuzón (S), Lechuzón orejas largas (S), Lechuzón orejudo (S), Hibou criard (F), Hibou strié (F) II B

Asio flammeus: Short-eared Owl (E), Búho orejicorto (S), Lechuza campestre (S), Lechuza orejicorta (S), Lechuzón campestre (S), Hibou brachyote (F), Hibou des marais (F) II B

Asio madagascariensis: Madagascar Owl (E), Lechuza orejita de Madagascar (S), Hibou de Madgascar (F), Hibou malgache (F) II B

Asio otus: Long-eared Owl (E), Búho chico (S), Búho-cornudo caricafe (S), Hibou moyen-duc (F) II B

Asio orus abyssinicus = Asio abyssinicus

Asio stygius: Stygian Owl (E), Búho-comudo oscuro (S), Lechuza estigia (S), Lechuzón negruzco (S), Hibou maître-bois (F) II B

Asno salvaje asiático (S): Equus hemionus I/II M

Asno salvaje de Africa (S): Equus africanus I M

Aspideretes gangeticus $=$ Trionyx gangeticus

Aspideretes hurum $=$ Trionyx hurum

Aspideretes nigricans $=$ Trionyx nigricans
Aspidites melanocephalus: Black-headed Python (E), Pitón de cabeza negra (S), Python à tête noire d'Australie (F), Woma (F) II R

Aspidites ramsayi: Ramsay's Python (E), Woma (E), Pitón de

Ramsay (S), Python de Ramsay (F), Woma (F) II R

Assam Macaque (E): Macaca assamensis II M

Assam Rabbit (E): Caprolagus hispidus I M

Assamese Macaque (E): Macaca assamensis II M

Asses (E): Equus spp. $\mathbf{I} \mathbf{I} / \mathrm{NC} \mathrm{M}$

Asterochelys radiata $=$ Geochelone radiata

Asterochelys yniphora $=$ Geochelone yniphora

Asteroseris = Pavona/Gardineroseris

Asterosmilia marchadi: II In

Asterosmilia prolifera: II In

Astraeosmilia connata: II in

Astrangia atrata: II In

Astrangia browni: II In

Astrangia caboensis: II In

Astrangia californica: II in

Astrangia concepcionensis: II In

Astrangia concinna: II In

Astrangia cortezi: II In

Astrangia costata: II in

Astrangia dentata: II In

Astrangia epithecata: II In

Astrangia equatorialis: 11 In

Astrangia haimei: II In

Astrangia hancocki: II In

Astrangia howardi: II In

Astrangia macrodentatu: II In

Astrangia minuta: II In

Astrangia vaxacensis: II In

Astrangia pederseni: II in

Astrangia poculata: II In

Astrangia pulchella: II In

Astrangia raihbuni: II In

Astrangia sanfelipensis: II In

Astrangia solitaria: II In

Astrangia tangolaensis: II In

Astrangia woodsi: II In

Astrapia mayeri: Ribbon-tailed Astrapia (E), Ribbon-tailed Bird-ofparadise (E), Paradisier à rubans (F) II B

Astrapia nigra: Arfak Astrapia (E), Hausse-col doré (F), Paradisier à gorge noire (F) II B

Astrapia rorhschildi: Huon Astrapia (E), Hausse-col de Rothschild (F), Paradisier de Rothschild (F) II B

Astrapia splendidissima: Splendid Astrapia (E), Hausse-col splendide (F), Paradisier splendide (F) II B

Astrapia stephaniae: Princess Stephanie's Bird-of-paradise (E), Stephanie's Astrapia (E), Hausse-col à queue rubanée (F), Paradisier de Stéphanie (F), Paradisier de la princesse Stéphanie (F) II B

Astrapias (E): Astrapia spp. II B

Astreoides $=$ Astroides

Astreopora cucullata: II In

Astreopora elliptica: II In

Astreopora expansa: II in

Astreopora explanata: II In

Astreopora gracilis: II In

Astreopora lambertsi: II In

Astreopora listeri: II in

Astreopora macrostoma: II In

Astreopora moretonensis: II In

Astreopora myriophthalma: II In

Astreopora ocellata: II In

Astreopora randalli: II In

Astreopora scabra: II In

Astreopora suggesta: II In

Astrild à joues oranges (F): Estrilda melpoda III B

Astrild cendré (F): Estrilda troglodytes III B

Astrild fourmilier (F): Parmoptila rubrifrons III B

Astrild mésange (F): Pholidomis rushiae III B

Astrild ondulé (F): Estrilda astrild III B

Astrild queue-de-vinaigre (F): Estrilda caerulescens III B

Astrild vert pointillé (F): Mandingoa nitidula III B

Astrilda aperdizada (S): Ortygospiza atricollis III B

Astrilda carirroja (S): Estrilda melpoda III B 
Astrilda ceniza del Senegal (S): Estrilda caerulescens III B Astrilda común (S): Estrilda astrild III B

Astrilda culinegra (S): Estrilda troglodytes 111 B

Astrilda pechigualda (S): Amandava subflava III B

Astrild-caille à lunettes (F): Ortygospiza arricollis III B

Astrild-cailles (F): Ortygospiza spp. II/NC B

Astrilds (F): Estrilda spp. III/NC B

Astroides calycularis: II In

Astrophyllia $=$ Favites

Astropsammia pedersenit: II in

Astroria $=$ Platygyra

Asturina nitida: Grey-lined Hawk (E), Aguilucho gris (S), Busardo gris (S), Gavilán gris (S), Buse cendrée (F) II B

Asturina plagiata: Grey Hawk (E), Aguililla gris (S), Buse grise (F) II B

Astya aspidopora: II In

Astya subviridis: II In

Atele à tête brune (F): Areles fusciceps II M

Atèle arachnoïde $(\mathrm{F})$ : Brachyteles arachnoides I M

Atele belzébuth (F): Aieles belzebuth II M

Atèle de Geoffroy (F): Areles geoffroyi $\mathbf{l} / \mathrm{II}$

Atèle de Geoffroy du Panama ( $F$ : Ateles geoffroyi panamensis I M

Atele du Costa Rica (F): Ateles geoffroyi frontatus I M

Atèle du Panama (F): Ateles geoffroyi panamensis I M

Atèle noir $(\mathrm{F})$ : Ateles paniscus II M

Ateles belzebuth: Long-haired Spider Monkey (E), White-bellied Spider Monkey (E), Atèle belzébuth (F) II M

Ateles belzebuth marginatus $=$ Ateles marginatus

Ateles chamek: II M

Areles fusciceps: Brown-headed Spider Monkey (E). Atèle à tête brune (F) II M

Ateles geoffroyi: Black-handed Spider Monkey (E), Geoffroy's Spider Monkey (E), Mono colorado (S), Atèle de Geoffroy (F) I/II M

Ateles geoffroyi frontatus: Black-browed Spider Monkey (E), Redbellied Spider Monkey (E), Mono araña maninegro (S), Atèle du Costa Rica (F), Singe araignée du Panama (F), Singe-araignée aux mains noires $(\mathrm{F})$ I $M$

Ateles geoffroyi panamensis: Panama Spider Monkey (E), Red Spider Monkey (E), Mono araña de panamá (S). Atèle de Geoffroy du Panama (F), Atèle du Panama (F) I M Ateles marginatus: II M

Ateles paniscus: Black Spider Monkey (E), Atèle noir (F) II M Ateles paniscus chamek = Ateles chamek

Atelope variable du Panama (F): Arelopus varius zereki I A

Atelopus varius zeteki: Cerro Campana Stubfoot Toad (E), Golden Arrow Poison Frog (E), Golden Frog (E), Zetek's Golden Frog (E), Rana dorada (S), Rana venenosa de Zetek (S), Atelope variable du Panama (F), Grenouille de Zetek (F) I A

Atelopus zeteki $=$ Atelopus varius zeteki

Athene blewitti: Forest Little Owl (E), Forest Owlet (E), Forest Spotted Owlet (E), Mochuelo forestal (S), Chevêche forestière (F), Chouette des forêts (F) I B

Athene brama: Spotted Little Owl (E), Spotted Owlet (E), Chevêche brame (F) II B

Athene cunicularia = Speatyto cunicularia

Athene noctua: Little Owl (E), Northem Little Owl (E), Tibet Owlet (E), Mochuelo común (S), Chouette chevêche (F) II B

Atitlan Grebe (E): Podilymbus gigas I B

Atlantic Coral Snake (E): Micrurus diastema III R

Atlantic Dolphin (E): Delphinus delphis II M

Atlantic Humpback Dolphin (E): Sousa reuszii I M

Atlantic Hump-backed Dolphin (E): Sousa teuszii I M

Atlantic Ridley (E): Lepidochelys kempi I R

Atlantic Spinner Dolphin (E): Stenella clymene II M

Atlantic Spotted Dolphin (E): Stenella frontalis II M

Atlantic Sturgeon (E): Acipenser oxyrhynchus II F

Atlantic White-sided Dolphin (E): Lagenorhynchus acutus II M

Atlas Deer (E): Cervus elaphus barbarus III M

Atretium schistosum: Olive Keel-back (E), Serpent ardoisé (F) III R

Atrichorne bruyant (F): Atrichomis clamosus $1 \mathrm{~B}$

Atrichomes (F): Atrichomis spp. INC B

Atrichomis clamosus: Noisy Scrub-bird (E), Western Scrubird (E), Corredor chillón (S), Atrichorne bruyant (F), Oiseau bruyant des buissons (F) I B

Atropoides nummifer $=$ Bothrops nummifer
Athis ellioti: Wine-throated Hummingbird (E) Zimbador centroamericano (S), Colibri d'Elliot (F) II B

Atthis heloisa: Bumblebee Hummingbird (E), Zimbador mexicano (S), Colibri héloïse (F) II B

Attwater's Prairie-Chicken (E): Tympanuchus cupido attwateri I B Augastes geoffroyi: Wedge-billed Hummingbird (E), Colibrí pico de cuña (S), Colibrí piquicunna (S), Colibri de Geoffroy (F) II B

Augastes lumachellus: Hooded Visorbearer (E), Colibri lumachelle (F) II B

Augastes scutatus: Hyacinth Visorbearer (E), Colibri superbe (F) II B

Augur Buzzard (E): Buteo augur II B

Aullado (S): Alouatta palliata I M

Aulocyathus atlanticus: II in

Aulocyathus juvenescens: II In

Aulocyathus matricidus: II in

Aulocyathus recidivus: II In

Aurora Finch (E): Pytilia phoenicoptera III B

Austral Conure (E): Enicognathus ferrugineus II B

Austral Parakeet (E): Enicognathus ferrugineus II B

Austral Pygmy-Owl (E): Glaucidium nanum II B

Australasian Goshawk (E): Accipiter fasciatus II B

Australian Black-shouldered Kite (E): Elanus axillaris II B

Australian Bustard (E): Ardeotis australis II B

Australian Fresh-water Crocodile (E): Crocodylus johnsoni II R

Australian Hobby (E): Falco longipennis II B

Australian Kestrel (E): Falco cenchroides II B

Australian King-Parrot (E): Alisterus scapularis II B

Australian Lungfish (E): Neoceratodus forsteri II F

Australian Masked-Owl (E): Tyto novaehollandiae II B

Australocyathus vincentinus: II In

Australogyra zelli: II In

Australomussa rowleyensis: II In

Australophocaena dioptrica: Spectacled Porpoise (E), Marsopa de anteojo (S), Marsouin de Lahille (F), Marsouin à lunettes (F) II $\mathrm{M}$

Autillo (S): Ous scops II B

Autillo de Guemey (\$): Mimizuku gurneyi I B

Autillo europeo (S): Otus scops II B

Autillo roborado (S): Otus roboratus II B

Autour à ailes grises (F): Melierax poliopterus II B

Autour à flancs roux (F): Accipiter castanilius II B

Autour à longue queue $(F)$ : Urotriorchis macrourus II B

Autour à manteau noir (F): Accipiter melanochlamys II B

Autour à tête grise (F): Accipiter poliocephalus II B

Autour à ventre blanc (F): Accipiter haplochrous II B

Autour à ventre gris (F): Accipiter poliogaster II B

Autour australien (F): Accipiter fasciatus II B

Autour blanc (F): Accipiter novaehollandiae II B

Autour blue et gris (F): Accipiter luteoschistaceus II B

Autour chanteur (F): Melierax canorus II B

Autour de Bürger (F): Erythrotriorchis buergersi II B

Autour de Doria (F): Megatriorchis doriae II B

Autour de Henst (F): Accipiter henstii II B

Autour de Mayr (F): Accipiter princeps II B

Autour de Meyer (F): Accipiter meyerianus II B

Autour de Toussenel (F): Accipiter loussenelii II B

Autour des Célèbes (F): Accipiter griseiceps II B

Autour des Fidji (F): Accipiter rufitorques II B

Autour des Moluques (F): Accipiter henicogrammus II B

Autour des palombes (F): Accipiter gentilis II B

Autour gabar (F): Melierax gabar II B

Autour huppé (F): Accipiter trivirgatus II B

Autour imitateur ( $F$ ): Accipiter imitator II B

Autour minulle (F): Accipiter erythropus II B

Autour noir (F): Accipiter melanoleucus II B

Autour pie (F): Accipiter albogularis II B

Autour rouge (F): Erythrotriorchis radiatus II B

Autour sombre (F): Melierax metabates II B

Autour tachiro (F): Accipiter tachiro II B

Autour unibande (F): Kaupifalco monogrammicus II B

Autours (F): Accipiter spp./Erythroiriorchis spp./Kaupifalco monogrammicus/Megatriorchis doriae/Melierax spp $/$ Micronisus gabar II B

Autruche d'Afrique (F): Struthio camelus I/NC B

Avahi $(\mathrm{E} / \mathrm{F})$ : Avahi laniger I $\mathrm{M}$ 
Avahi laineux (F): Avahi laniger I M

Avahi laniger. Avahi (E), Woolly Indris (E), Woolly Lemur (E), Indri lanudo (S), Avahi (F), Avahi laineux (F), Maki à bourre (F) I $\mathrm{M}$

Avahi occidentalis $=$ Avahi laniger

Aves del paraiso (S): Paradisaeidae spp. II B

Aves de rapiña (S): FALCONIFORMES spp. I/IIIINC B

Avestruz (S): Siruthio camelus INC B/ Rhea americana II B

Avestruz de Magallanes (S): Rhea pennata I B

Aviceda cuculoides: African Baza (E), African Cuckoo-Falcon (E), African Cuckoo-Hawk (E), Baza africano (S), Baza coucou (F), Faucon-coucou (F) II B

Aviceda jerdoni: Asian Baza (E), Jerdon's Baza (E), Baza oriental (S), Baza de Jerdon (F) II B

Aviceda leuphores: Black Baza (E), Baza negro (S), Baza huppard (F) II B

Aviceda madagascariensis: Madagascar Baza (E), Madagascar Cuckoo-Falcon (E), Madagascar Cuckoo-Hawk (E), Baza malgache (S), Baza malgache (F) II B

Aviceda subcristata: Crested Baza (E), Pacific Baza (E), Baza australiano (S), Baza huppé (F) II B

Avocettula recurvirostris: Fiery-tailed Awlbill (E), Colibrí pico lezna (S), Colibri avocette (F) II B

Avutarda (S): Otis tarda II B

Avutarda árabe (S): Ardeotis arabs II B

Avutarda australiana (S): Ardeotis australis II B

Avutarda bengalí (S): Eupodotis bengalensis I B

Avutarda cafre (S): Neotis denhami II B

Avutarda de Burchell (S): Neotis denhami II B

Avutarda de Denham (S): Neotis denhami II B

Avutarda de Heuglin (S): Neotis heuglinii II B

Avutarda de Ludwig (S): Neotis ludwigii II B

Avutarda de Namibia (S): Neotis ludwigii II B

Avutarda de Savile (S): Eupodotis savilei II B

Avutarda euroasiática (S): Otis tarda II B

Avutarda hubara (S): Chlamydoris undulata I B

Avutarda india (S): Ardeotis nigriceps I B

Avutarda indica (S): Ardeotis nigriceps I B

Avutarda kori (S): Ardeotis kori II B

Avutarda núbica (S): Neotis nuba II B

Avutarda somali (S): Neotis heuglinii II B

Avutardas (S): Otididae spp. UII B

Axhelia $=$ Madracis

Axis calamianensis: Calamanian Deer (E), Calamian Hog Deer (E), Philippine Deer (E), Ciervo de los Calamianes (S), Ciervo porquerizo de los Calamianes (S), Cerf-cochon calamien (F) I M

Axis kuhlii: Bawean Hog Deer (E), Kuhl's Hog Deer (E), Ciervo de Kuhl (S), Ciervo porquerizo de Kuhl (S), Cerf-cochon de Bawean (F) I M

Axis porcinus annamiticus: Indochinese Hog Deer (E), Thai Hog Deer (E), Ciervo porquerizo de Indochina (S), Cerf-cochon d'Indochine (F) I M

Axohelia $=$ Madracis

Axolotl (E/F): Ambystoma mexicanum II A

Aye-aye (E/S/F): Daubentonia madagascariensis I M

Ayres's Eagle (E): Hieraaerus ayresii II B

Ayres's Hawk-Eagle (E): Hieraaetus ayresii II B

Aythya nyroca: Ferruginous Duck (E), Ferruginous Pochard (E), White-eyed Pochard (E), Porrón pardo (S), Fuligule nyroca (F) III $\mathrm{B}$

Azara's Zorro (E): Pseudalopex gymnocercus II M

Azer semicollarejo (S): Accipirer collaris II B

Azor (S): Accipiter gentilis II B

Azor australiano (S): Accipiter fasciatus II B

Azor bicolor (S): Accipiter bicolor II B

Azor blanquinegro (S): Accipiter melanoleucus II B

Azor chico (S): Accipiter striatus II B

Azor común (S): Accipiter gentilis II B

Azor culebrero (S): Polyboroides radiatus II B

Azor de Bürgers (S): Erythrotriorchis buergersi II B

Azor de Doria (S): Megatriorchis doriae II B

Azor de Meyer (S): Accipiter meyerianus II B

Azor de Nueva Bretaña (S): Accipiter princeps II B

Azor de Toussenel (S): Accipiter roussenelii II B

Azor gris (S): Accipiter superciliosus II B

Azor lagartijero oscuro (S): Melierax metabates II B
Azor malgache (S): Accipirer hensrii II B

Azor moluqueño (S): Accipiter henicogrammus II B

Azor moñudo (S): Accipiter irivirgatus II B

Azor pechigrís (S): Accipiter poliogaster II B

Azor pechillano (S): Accipiter ventralis II B

Azor rabilargo (S): Urotriorchis macrourus II B

Azor rojo (S): Erythrotriorchis radiatus II B

Azor tachiro (S): Accipiter tachiro II B

Azor variable (S): Accipiter novachollandiae II B

Azor ventigris (S): Accipiter poliogaster II B

Azor zancón (S): Geranospiza caerulescens II B

Azor-lagartijero claro (S): Melierax canorus II B

Azor-lagartijero somali (S): Melierax poliopterus II B

Aztec Parakeet (E): Aratinga nana II B

Azure-crowned Hummingbird (E): Amazilia cyanocephala II B

Babiroussa (E/F): Babyrousa babyrussa I M

Babirusa (E/S): Babyrousa babyrussa I M

Babisuri (S): Bassariscus sumichrasti III M

Baboons (E): Papio spp./Theropithecus gelada II M

Babouin anubis (F): Papio hamadryas II M

Babouin chacma (F): Papio hamadryas II M

Babouin de Guinée (F): Papio hamadryas II M

Babouin jaune (F): Papio hamadryas II M

Baby Python (E): Eryx conicus II R

Babyrousa babyrussa: Babiroussa (E), Babirusa (E), Deer Hog (E),

Babirusa (S), Babiroussa (F) I M

Bactrian Deer (E): Cervus elaphus bactrianus II M

Bactrian Red Deer (E): Cervus elaphus bactrianus II M

Bactrian Wapiti (E): Cervus elaphus bactrianus II M

Badgers (E): Mustelidae spp. III/NC M

Baghdad Small-grain Lizard (E): Varanus griseus 1 R

Bahama Woodstar (E): Calliphlox evelynae II B

Bahamas Islands Boa (E): Epicrates chrysogaster II R

Bahamas Parrot (E): Amazona leucocephala I B

Bahamas Rock Iguana (E): Cyclura carinata I R

Baiji (E): Lipotes vexillifer I M

Baiji (F): Lipotes vexillifer I $M$

Baiji (S): Lipotes vexillifer I M

Baikal Teal (E): Anas formosa II B

Baillonius bailloni: Saffron Toucanet (E), Toucan de Baillon (F) III $\mathrm{B}$

Baird's Beaked Whale (E): Berardius bairdii I M

Baird's Tapir (E): Tapirus bairdii I M

Baka Indigobird (E): Vidua larvaricola III B

Balaena glacialis = Eubalaena glacialis

Balaena glacialis australis = Eubalaena australis

Balaena mysticerus: Bowhead Whale (E), Greenland Right Whale

(E), Ballena boreal (S), Baleine de grande baie (F), Baleine du Groenland (F) I M

Balaeniceps rex: Shoebill (E), Whale-headed Stork (E), Picozapato (S), Baléniceps rois (F), Bec-en-sabot (F) II B

Balaenoptera acutorostrata: Lesser Rorqual (E), Little Piked Whale (E), Minke Whale (E), Ballena minke (S), Rorcual menor (S), Baleinoptère à museau pointu (F), Petit rorqual (F) I/II M

Balaenoptera bonaerensis = Balaenoptera acutorostrata

Balaenoptera borealis: Coalfish Whale (E), Pollack Whale (E), Rudophi's Rorqual (E), Sei Whale (E), Ballena boba (S), Ballena sei (S), Rorcual boreal (S), Rorcual de Rudolphi (S), Rorcual norteno (S), Baleinoptère de Rudolphi (F), Rorqual boréal (F), Rorqual de Rudolphi (F), Rorqual sei (F) I M

Balaenoptera edeni: Bryde's Whale (E), Tropical Whale (E), Ballena de Bryde (S), Baleinoptère de Bryde (F), Rorqual d'Eden (F), Rorqual de Bryde (F), Rorqual tropical (F) I M

Baloenoptera musculus: Blue Whale (E), Sibbald's Rorqual (E), Sulphur-bottom Whale (E), Ballena azul (S), Rorcual azul (S), Baleine bleue $(F)$, Baleine d'Ostende $(F)$, Baleinoptère bleue $(F)$, Rorqual bleu (F), Rorqual de Sibbold (F), Rorqual à ventre cannelé (F) I M

Balaenopiera physalus: Common Rorqual (E), Fin Whale (E), Finbacked Whale (E), Finback (E), Finner (E), Herring Whale (E), Razorback (E), Ballena aleta (S), Ballena boba (S), Rorcual común $(S)$, Baleine fin $(F)$, Baleine à nageoires $(F)$, Baleinoptère commune (F), Rorqual commun (F) I M

Balanophyllia bairdiana: II In

Balanophyllia bayeri: II In

Balanophyllia bonaspei: II in 
Balanophyllia buccina: II In

Balanophyllia caribbeana: II in

Balanophyllia cedrosensis: II In

Balanophyllia cellulosa: II In

Balanophyllia chnous: II In

Balanophyllia cornu: II In

Balanophyllia crassitheca: II In

Balanophyllia cumingii: II In

Balanophyllia cyathoides: II In

Balanophyllia dentata: II In

Balanophyllia desmophyllioides: II in

Balanophyllia diffusa: II In

Balanophyllia dilatata: II in

Balanophyllia dinera: II In

Balanophyllia diomedeae: II In

Balanophyllia elegans: II In

Balanophyllia europaea: II In

Balanophyllia floridana: II In

Balanophyllia galapagensis: II In

Balanophyllia gemmifera: II In

Balanophyllia gigas: II in

Balanophyllia grandis: II In

Balanophyllia hadros: II In

Balanophyllia imperialis: II In

Balanophyllia incisa: II In

Balanophyllia italica: II In

Balanophyllia laysanensis: II In

Balanophyllia malouinensis: II In

Balanophyllia merguiensis: II In

Balanophyllia palifera: II In

Balanophyllia ponderosa: II In

Balanophyllia rediviva: II in

Balanophyllia regia: II In

Balanophyllia scabra: II In

Balanophyllia scabrosa: II In

Balanophyllia stimpsonii: II In

Balanophyllia teres: II In

Balanophyllia thalassae: II In

Balanophyllia verrucaria: II In

Balanophyllia wellsi: II In

Balanophyllia yongei: II In

Balbuzard pêcheur ( $F$ ): Pandion halicatus II B

Bald Eagle (E): Haliaeerus leucacephalus I B

Bald Ibis (E): Geronticus calvus II B

Bald Uakari (E): Cacajao calvus I M

Balearica pavonina: Black Crowned Crane (E), West African Crowned Crane (E), Grulla coronada (S), Grulla coronada cuellinegra (S), Grulla coronada del Africa Occidental (S), Grue couronnée de l'Afrique del'Ouest et du Soudan (F) II B

Balearica regulonum: Grey Crowned Crane (E), South African Crowned Crane (E), Grulla coronada cuelligris (S), Grulla coronada sudafricana (S), Grue couronnée de Cap (F), Grue couronnée de l'Afrique du Sud et de l'Est (F), Grue royale (F) II $\mathrm{B}$

Baleine à bosse (F): Megaptera novaeangliae I M

Baleine à nageoires $(\mathrm{F})$ : Balaenoptera physalus I M

Baleine à six bosses $(\mathrm{F})$ : Eschrichtius robustus I M

Baleine à taquet (F): Megaptera novaeangliae I M

Baleine australe (F): Eubalaena australis I M

Baleine bleue (F): Balaenoptera musculus I M

Baleine de Biscaye (F): Eubalaena glacialis I M

Baleine de grande baie ( $F$ ): Balaena mysticetus I M

Baleine des Basques (F): Eubalaena glacialis I M

Baleine d'Ostende (F): Balaenoptera musculus I M

Baleine du Groenland (F): Balaena mysticesus I M

Baleine fin (F): Balaenoptera physalus I M

Baleine grise (F): Eschrichtius robustus I M

Baleine pygmée ( $F$ ): Caperea marginata I M

Baleines (F): CETACEA spp. I/II M

Baleines à bec $(\mathrm{F})$ : Berardius spp. I M

Baleines franches (F): Eubalaena spp. I M

Baleines noires (F): Eubalaena spp. I M

Baleinoptère à museau pointu (F): Balaenoptera acutorostrata VII M

Baleinoptère bleue (F): Balaenoptera musculus I M

Baleinoptère commune (F): Balaenoptera physalus I M
Baleinoptère de Bryde (F): Balaenoptera edeni I M

Baleinoptère de Rudolphi $(\mathbf{F})$ : Balaenoptera borealis I M

Baléniceps rois (F): Balaeniceps rex II B

Bali Myna (E): Leucopsar rothschildi I B

Bali Starling (E): Leucopsar rolhschildi I B

Ball Python (E): Python regius II $R$

Ballena (S): Eubalaena glacialis I M

Ballena aleta (S): Balaenoptera physalus I M

Ballena azul (S): Balaenoptera musculus I M

Ballena blanca (S): Delphinapterus leucas II M

Ballena boba (S): Balaenoptera borealis I M/Baloenoptera physalus I $\mathrm{M}$

Ballena boreal (S): Balaena mysticetus I M

Ballena de Bryde (S): Baloenoptera edeni I M

Ballena de Cuvier (S): Ziphius cavirostris II M

Ballena de pico de Andrew (S): Mesoplodon bowdoini II M

Ballena de pico de Amoux (S): Berardius arnuxii I M

Ballena de pico de Baird (S): Berardius bairdii I M

Ballena de pico de Blainville (S): Mesoplodon densirostris II M

Ballena de pico de Gervais (S): Mesoplodon europaeus II M

Ballena de pico de Gray (S): Mesoplodon grayi II M

Ballena de pico de Héctor (S): Mesoplodon hectori II M

Ballena de pico de Hubbs (S): Mesoplodon carlhubbsi II M

Ballena de pico de Layard (S): Mesoplodon layardii II M

Ballena de pico de Longman (S): Indopacetus pacificus II M

Ballena de pico de Sowerby (\$): Mesoplodon bidens II M

Ballena de pico de Stejneger (S): Mesoplodon stejnegeri II M

Ballena de pico de True (S): Mesoplodon mirus II M

Ballena de Shepherd (S): Tasmacelus shepherdi II M

Ballena esperma (S): Physeter catodon I M

Ballena franca (S): Eubalaena australis I M

Ballena franca del Norte (S): Eubalaena glacialis I M

Ballena franca pigmea (S): Caperea marginata I M

Ballena gris (S): Eschrichtius robusius I M

Ballena hocico de botella del norte (S): Hyperoodon ampullatus I M

Ballena hocico de botella del sur (S): Hyperoodon planifrons I M

Ballena jorobada (S): Megaptera novaeangliae I M

Ballena minke (S): Balaenoptera acutorostrata VI M

Ballena sei (S): Balaenoptera borealis I M

Ballenas (S): CETACEA spp. I/II M

Ballenas de pico (S): Berardius spp. I M

Ballenas hocico de botella (S): Hyperoodon spp. I M

Ballenga (S): Eubalaena glacialis I M

Baltic Sturgeon (E): Acipenser sturio I F

Bamboo Lemurs (E): Hapalemur spp. I M

Banana Boa (E): Ungaliophis continentalis II R

Band-bellied Owl (E): Pulsatrix melanota II B

Banded Cotinga (E): Cotinga maculata I B

Banded Day Gecko (E). Phelsuma standingi II R

Banded Duiker (E): Cephalophus zebra II M

Banded Dwarf Boa (E): Tropidophis semicinctus II R

Banded Eagle-Owl (E): Bubo shelleyi II B

Banded Hare-wallaby (E): Lagostrophus fasciatus I M

Banded Kestrel (E): Falco zoniventris II B

Banded Langur (E): Presbytis femoralis II M

Banded Leaf Monkey (E): Presbytis femoralis II M

Banded Linsang (E): Prionodon linsang II M

Banded Musang (E): Hemigalus derbyanus II M

Banded Palm Civet (E): Hemigalus derbyanus II M

Banded Pitta (E): Pinta guajana II B

Banded Snake-Eagle (E): Circaetus cinerascens II B

Banded Tegu (E): Tupinambis teguixin II R

Banderón caballo (S): Myrmecophaga iridacryla II M

Bandicoot à pied de porc (F): Chaeropus ecaudatus I M

Bandicoot de Bougainville (F): Perameles bougainville I M

Bandicoot pieds de cochon (F): Chaeropus ecaudarus I M

Bandicoot-lapin à queue blanche (F): Macrotis leucura I M

Bandicoot-lapin mineur (F): Macrotis leucura I M

Bandicoots (E): Peramelidae spp./Peroryctidae spp. INC M

Band-tailed Bartchroat (E); Threnetes ruckeri II B

Band-tailed Fish-Eagle (E): Haliaeetus leucoryphus II B

Band-tailed Owl (E): Pulsatrix melanota II B

Bannerman's Turaco (E): Tauraco bannermani II B

Bantamia = Blastomussa/Galaxea

Barabartoia amicorum: II In

Barabattoia laddi: II In 


\section{Barabanoia mirabilis: II In}

Barasinga (S/F): Cervus duvauceli I M

Barasingha (E/F): Cervus duvauceli I M

Bar-backed Partridge (E): Arborophila orientalis III B

Barbary Ape (E): Macaca sylvanus II M

Barbary Deer (E): Cervus elaphus barbarus III M

Barbary Falcon (E): Falco pelegrinoides I B

Barbary Macaque (E): Macaca sylvanus II M

Barbary Red Deer (E): Cenus elaphus barbarus III M

Barbary Sheep (E): Ammotragus lervia II M

Barbary Stag (E): Cervus elaphus barbarus III M

Barbeau de Jullien (F): Probarbus jullieni I F

Barbita colibandeada (S): Threneses ruckeri II B

Barbita colipalida (S): Threnetes leucurus II B

Barbour's Anaconda (E): Eunectes barbouri II R

Barbour's Day Gecko (E): Phelsuma barbouri II R

Bar-breasted Firefinch (E): Lagonosticta rufopicta III B

Bar-breasted Waxbill (E): Lagonosticta rufopicta III B

Barbthroats (E): Threnetes spp. II B

Barbu aveugle (F): Caecobarbus geerisi II F

Bare-eyed Cockatoo (E): Cacatua sanguinea II B

Bare-faced Tamarin (E): Saguinus bicolor I M

Bare-headed Rockfowl (E): Picathartes gymnocephalus I B

Bare-legged Owl (E): Otus lawrencii II B

Bare-shanked Screech-Owl (E): Otus clarkii II B

Baribal (F): Ursus americanus II M

Barizo dorsirrojo (S): Saimiri oerstedii I M

Barking Owl (E): Ninox connivens II B

Bam Owl (E): Tyto alba II B

Bamacla canadiense aleutiana (S): Branta canadensis leucopareia I B

Bamacla cuellirroja (S): Branta ruficollis II B

Bamacla hawaiana (S): Branta sandvicensis I B

Bamacla nené (S): Branta sandvicensis I B

Bamardius bamardi $=$ Platycercus barnardi

Bamardius zonarius = Platycercus zonarius

Barnard's Parakeet (E): Platycercus bamardi II B

Barn-Owls (E): Tyto spp. VII B

Barraband Parakeet (E): Polytelis swainsonii II B

Barraband's Parrot (E): Pionopsitta barrabandi II B

Barred Bandicoot (E): Perameles bougainville I M

Barred Eagle-Owl (E): Bubo sumatranus II B

Barred Forest-Falcon (E): Micrastur ruficollis II B

Barred Hawk (E): Leucoptemis princeps II B

Barred Honey-buzzard (E): Pernis celebensis II B

Barred Jungle Owlet (E): Glaucidium radiatum 11 B

Barred Kestrel (E): Falco zoniventris II B

Barred Owl (E): Strix varia II B

Barred Owlet (E): Glaucidium capense II B

Barred Parakeet (E): Bolborhynchus lineola II B

Barred Python (E): Morelia boa II R

Barrington Land Iguana (E): Conolophus pallidus II R

Bartsch's Iguana (E): Cyclura carinata I R

Baryastrea $=$ Leptastrea

Basáride (S): Bassariscus sumichrasti III M

Basilinna leucotis $=$ Hylocharis leucoris

Basilinna xantusii = Hylocharis xantusii

Basket Coral (E): Sandalolitha robusta II in

Bassarai rusé (F): Bassariscus sumichrasti III M

Bassaricyon gabbii: Bushy-tailed Olingo (E), Chosna pericote (S),

Cuataquil (S), Cuchumbi (S), Lingo (S), Olingo (S), Cataquil (F), Olingo (F) III M

Bassariscus sumichrasti: Central American Cacomistle (E), Babisuri (S), Basáride (S), Cacomistle (S), Mico de noche (S), Mico rayado (S), Bassarai rusé (F) III M

Bastard Sturgeon ( $\mathrm{E})$ : Acipenser nudiventris II F

Bat Falcon (E): Falco rufigularis II B

Bat Hawk (E): Macheiramphus alcinus II B

Bat Kite (E): Macheiramphus alcinus II B

Batagur (E): Batagur baska I R

Batagur baska: Batagur (E), Common Batagur (E), Four-toed

Terrapin (E), River Terrapin (E), Galápago Batagur (S), Batagur malais ( $F$ ) I $R$

Batagur malais (F): Batagur baska I R

Bateleur (E/F): Terathopius ecaudatus II B

Bateleur des savanes (F): Terathopius ecaudatus II B
Bateleur Eagle (E): Terathopius ecaudatus II B

Bathelia candida: II In

Bathyactis = Fungiacyathus

Bathycyathus $=$ Phyllangia

Bathypathes alternata: II In

Bathypathes bifida: II in

Bathypathes erotema: II In

Bathypathes euantha: II in

Bathypathes galatheae: II In

Bathypathes heterorhodzos: II In

Bathypathes lyra: II In

Bathypathes patula: II in

Bathypathes platycaulus: II In

Bathypathes quadribrachiata: II In

Bathypathes scoparia: II In

Bathypathes tenuis: II In

Bathypsammia fallosocialis: II In

Bathypsammia tintinnabulum: II In

Bathytrochus $=$ Fungiacyathus

Batotrochus = Trematotrochus

Battersby's Dwarf Boa (E): Tropidophis battersbyi II R

Bawean Hog Deer (E): Axis kuhlii I M

Bay Cat (E): Catopuma badia II M

Bay Duiker (E): Cephalophus dorsalis II M

Bay Lynx (E): Lynx rufus II M

Bay Owl (E): Phodilus badius II B

Bay-Owls (E): Phodilus spp. II B

Bay-winged Hawk (E): Parabuteo unicinctus II B

Baza africano (S): Aviceda cuculoides II B

Baza australiano (S): Aviceda subcristata II B

Baza coucou (F): Aviceda cuculoides II B

Baza de Jerdon (F): Aviceda jerdoni II B

Baza huppard (F): Aviceda leuphotes II B

Baza huppé (F): Aviceda subcristata II B

Baza malgache (S/F): Aviceda madagascariensis II B

Baza negro (S): Aviceda leuphotes II B

Baza oriental (S): Aviceda jerdoni II B

Bazas (E/F): Aviceda spp. II B

Beach Cobra (E): Cyclagras gigas II R

Beaded Lizard (E): Heloderma horridum II R

Beaked Cape Tortoise (E): Homopus areolatus II R

Beaked whales (E): Ziphiidae spp. I/II M

Beak-head (E): Sphenodon puncrasus I R

Bear Macaque (E): Macaca arcioides II M

Bear Paw Clam (E): Hippopus hippopus II In

Bearded Helmetcrest (E): Oxypogon guerinii II B

Bearded Mountaineer (E): Oreonympha nobilis II B

Bearded Saki (E): Chiropotes satanas II M

Bearded Vulture (E): Gypaetus barbatus II B

Bears (E): Ursidae spp. VII M

Beaudouin's Snake-Eagle (E): Circaetus gallicus 11 B

Beaumarquet (F): Pytilia phoenicoptera III B

Beaumarquet à ailes jaunes (F): Pytilia hypogrammica III B

Beaumarquets (F): Pytilia spp. III/NC B

Beautiful Hummingbird (E): Calothorax pulcher II B

Beautiful Parakeet (E): Psephotus pulcherrimus I B

Beautiful Parrot (E): Pionopsitta pulchra II B

Beautiful-breasted Poison Frog (E): Epipedobates pulchripectus II A

Bebromis rodericanus: Rodrigues Brush-Warbler (E), Rodrigues

Warbler (E), Rousserolle de Rodriguez (F) III B

Bec de corail cendre (F): Estrilda troglodytes III B

Bec-d'argent (F): Lonchura cantans III B

Bec-en-faucille aigle (F): Eutoxeres aquila II B

Bec-en-faucille de la Condamine (F): Eutoxeres condamini II B

Bec-en-faucilles (F): Eutoxeres spp. II B

Bec-en-sabot (F): Balaeniceps rex II B

Bee Hummingbird (E): Mellisuga helenae II B

Beecroft's Flying Squirrel (E): Anomalurus beecrofi III M

Belalanda Chameleon (E): Chamaeleo belalandaensis II R

Belette à ventre jaune (F): Mustela kathiah $111 \mathrm{M}$

Belette alpine (F): Mustela alkaica III M

Belette de Sibérie (F): Mustela sibirica III M

Belette des Alpes (F): Mustela altaica III M

Belize Crocodile (E): Crocodylus moreletii I R

Bell's Dabb Lizard (E): Uromastyx acanthinurus II R

Bell's Hinged Tortoise (E): Kinixys belliana II R 
Bell's Hinged-backed Tortoise (E): Kinirys belliana II R Belouga (F): Delphinapterus leucas II M

Beluga (E): Delphinapterus leucas II M

Beluga (E/S/F): Huso huso II F

Bengal Black Lizard (E): Varanus bengalensis I R

Bengal Bustard (E): Eupodotis bengalensis I B

Bengal Eyed Terrapin (E): Morenia ocellata I R

Bengal Florican (E): Eupodotis bengalensis I B

Bengal Fox (E): Vulpes bengalensis III M

Bengal Leopard Cat (E): Prionailurus bengalensis bengalensis VII M

Bengal Lizard (E): Varanus bengalensis I R

Bengal Monitor (E): Varanus bengalensis I R

Bengali carinegro (S): Lagonosticta vinacea III B

Bengalí de Fraser (S): Lagonosticta rufopicta III B

Bengalí de Lichtenstein (S): Lagonosticta rubricala III B

Bengalí senegalés (S): Lagonosticta senegala III B

Bengali vert (F): Amandava formosa II B

Bengali zébré (F): Amandava subflava III B

Bengalis (F): Amandava spp. IU/II/NC B

Benguela Dolphin (E): Cephalorhynchus heavisidii II $M$

Beni Titi Monkey (E): Callicebus olallae II M

Bénitier de Rosewater (F): Tridacna rosewareri 11 in

Bénitier de Tevoro (F): Tridacna tevoroa II In

Bénitier géant (F): Tridacna gigas II In

Bénitiers ( $F$ ): Tridacnidae spp. II In

Bent's Mastigure (E): Uromastyx benti II R

Bérardien d'Amoux (F): Berardius amuxii I M

Bérardien de Baird (F): Berardius bairdii I M

Berardius spp.: Beaked whales (E), Ballenas de pico (S), Baleines à bec (F), Bérards (F) I M

Berardius arnuxii: Amoux's Beaked Whale (E), Southern Fourtoothed Whale (E), Ballena de pico de Amoux (S), Bérardien d'Amoux (F) I M

Berardius bairdii: Baird's Beaked Whale (E), Giant Bottle-nosed Whale (E), Northern Four-toothed Whale (E), Ballena de pico de Baird (S), Bérardien de Baird (F) I M

Berards (F): Berardius spp. I M

Berenicomis comatus = Aceros comalus

Berger's Cape Tortoise (E): Homopus bergeri II R

Berlandier's Tortoise (E): Gopherus berlandieri II R

Bernache à cou roux $(\mathrm{F})$ : Branta ruficollis II $\mathrm{B}$

Bernache des Aléoutiennes $(F)$ : Branta canadensis leucopareia I B

Bernache des Iles Sandwich (F): Branla sandvicensis I B

Bernache d'Hawaï (F): Branta sandvicensis I B

Bernache du Canada aléoute (F): Branta canadensis leucopareia I B

Bernache né-né (F): Branta sandvicensis I B

Bemaches (F): Anatidae spp. I/INC B

Berrendo (S): Antilocapra americana INC M

Berylline Hummingbird (E): Amazilia beryllina II B

Besra (E): Accipiter virgalus II B

Besra Spartowhawk (E): Accipiler virgatus II B

Bettongia spp.: Rat kangaroos (E), Canguro-ratas (S), Kangourourats (F) I M

Bettongia gaimardi: Eastern Bettong (E), Tasmanian Bettong (E), Tasmanian Rat Kangaroo (E), Canguro-rata de Tasmania (S), Kangourou-rat de Tasmanic (F) I M

Bettongia lesueur: Boodie (E), Burrowing Bettong (E), Lesueur's Rat Kangaroo (E), Canguro-rata de Lesueur (S), Bettongie de Lesueur (F), Kangourou-rat de Lesueur (F) I M

Bettongia penicillata: Brush-tailed Bettong (E), Brush-tailed Rat Kangaroo (E), Woylie (E), Canguro-rata colipeludo (S), Bettongie à queuc touffue $(F)$, Kangourou-rat à queue touffue (F) $1 \mathrm{M}$

Bettongia tropica $=$ Bettongia penicillata

Bettongie à queue touffue (F): Bettongia penicillata I M

Bettongie de Lesueur (F): Bettongia lesueur I M

Bettongs (E): Bettongia spp./Aepyprymnus rufescens $\mathbf{I N C ~ M ~}$

Bhutan Glory (E): Bhutanitis Lidderdalii II in

Bhutanitis spp.: Swallowtails (E), Macaones (F) II In Bhutanitis lidderdalii: Bhutan Glory (E) II In

Bhutanitis ludlowi: Ludlow's Bhutan Swallowtail (E) II In

Bhutanitis mansfieldi: Mansfield's Three-tailed Swallowtail (E) II In

Bhutanitis thaidina: Chinese Three-tailed Swallowtail (E) II In

Biak Red Lory (E): Eos cyanogenia II B

Bichichi (S): Saguinus oedipus I M
Bicolored Hawk (E): Accipiter bicolor II B

Bicolored Sparrowhawk (E): Accipiter bicolor II B

Biflabellum $=$ Monomyces

Big-eared Flying-fox (E): Pteropus macrotis II M

Big-headed Amazon River Turtle (E): Peltocephalus dumeriliano II $\mathrm{R}$

Big-headed Sideneck (E): Peltocephalus dumeriliana II R

Bighom Sheep (E): Ovis canadensis I/I M

Big-nosed Chameleon (E): Chamaeleo nasutus II R

Bikiniastrea $=$ Barabattoia

Bilbies (E): Macrotis spp. I M

Bilby (E): Macrotis lagoris I M

Binturong (E/S/F): Arctictis binturong III M

Biolat Poison Frog (E): Dendrobates biolat II A

Birds of paradise (E): Paradisaeidae spp, II B

Birds of prey (E): FALCONIFORMES spp. UIIII/NC B

Birdwing butterflies (E): Omithoptera spp. III In/Trogonoptera spp.

II in/Troides spp. II In

Birdwing Pearly Mussel (E): Conradilla caelata I In

Birdwings (E): Omithoptera spp./Trogonoptera spp./Troides spp III In

Bishops (E): Euplectes spp. III/NC B

Bismarck Flying-fox (E): Pleropus neohibemicus II M

Bismarck Hawk-Owl (E): Ninox variegata II B

Bismarck Masked-Owl (E): Tyto aurantia II B

Bismarck Ringed Python (E): Morelia boa II R

Bison (E): Bison spp./Bos gaurus I/NC M

Bison bison athabascae: Wood Bison (E), Bisonte americano de bosque (S), Bison des forêts (F) II M

Bison des forêts (F): Bison bison athabascae II M

Bisonte americano de bosque (S): Bison bison athabascae II M

Black Baza (E): Aviceda leuphotes II B

Black Bustard (E): Eupodotis afra II B

Black Caiman (E): Melanosuchus niger I/II R

Black Caracara (E): Daptrius ater II B

Black Colobus (E): Colobus satanas II M

Black corals (E): ANTIPATHARIA spp. II In

Black Crowned Crane (E): Balearica pavonina II B

Black Dolphin (E): Cephalorhynchus eutropia II M

Black Eagle (E): Aquila verreauxii II B/Ictinaetus malayensis II B

Black Falcon (E): Falco subniger II B

Black Finless Porpoise (E): Neophocaena phocaenoides I M

Black Fronted Curassow (E): Pipile jacuinga I B

Black Giant Squirrel (E): Rutufa bicolor II M

Black Giant Tortoise (E): Manouria emys II R

Black Gibbon (E); Hylobates concolor I M

Black Goshawk (E): Accipifer melanoleucus II B

Black Harrier (E): Circus maunus II B

Black Hawk-Eagle (E): Spizaetus tyrannus II B

Black Honey-buzzard (E): Henicopernis infuscatus II B

Black Hombill (E): Anthracoceros malayanus II B

Black Howler (E): Alouatta caraya II M

Black Inca (E): Coeligena prunellei II B

Black Jacobin (E): Melanorrochilus fuscus II B

Black Kite (E): Milvus migrans II B

Black Korhaan (E): Eupodotis afra II B

Black Leaf Monkey (E): Trachypithecus johnii II M

Black Lemur (E): Eulemur macaco I M

Black Lion Tamarin (E): Leontopithecus chrysopygus I M

Black Lory (E): Chalcopsitta aira II B

Black Metaltail (E): Melallura phoebe II B

Black Mud Turtle (E): Trionyx ater I R

Black Muntjac (E): Muntiacus crinifrons I M

Black Musk Deer (E): Moschus fuscus II M

Black Parrot (E): Coracopsis nigra II B

Black Partridge (E): Melanoperdix nigra III B

Black Pond Turtle (E): Geoclemys hamiltonii I R

Black Porpoise (E): Phocoena spinipinnis II M

Black Python (E): Morelia boeleni II R

Black Rhinoceros (E): Diceros bicornis I M

Black Right Whale (E): Eubalaena glacialis I M

Black Saki (E): Chiropotes satanas II M

Black Sand Monitor (E): Varanus rosenbergi II R

Black Sicklebill (E); Epimachus fastuosus II B

Black Side-necked Turtle (E): Pelusios niger III R

Black Soft-shell Turtle (E): Trionyx ater I R 
Black Solitary Eagle (E): Harpyhaliaetus solitarius II B Black Sparrowhawk (E): Accipiter melanoleucus II B Black Spider Monkey (E): Ateles paniscus II M

Black Spiny Soft-shell Turtle (E): Trionyx ater I R

Black Spiny-tailed Lizard (E): Uromastyx acanthinunus II R

Black Spotted Turtle (E): Geoclemys hamiltonii I R

Black Stork (E): Ciconia nigra II B

Black Tegu (E): Tupinambis teguixin II R

Black Tree Monitor (E): Varanus beccarii II R

Black Tree-kangaroo (E): Dendrologus ursinus II M

Black Uakari (E): Cacajao melanocephalus I M

Black Vulture (E): Aegypius monachus II B

Black Wood-Partridge (E): Melanoperdix nigra III B

Black-and-chestnut Eagle (E): Oroaetus isidori II B

Black-and-gold Birdwing (E): Troides helena II In

Black-and-red Howler (E): Alouana belzebul II M

Black-and-red Tamarin (E): Saguinus nigricollis II M

Black-and-white Hawk-Eagle (E): Spizastur melanoleucus II B

Black-and-white Mannikin (E): Lonchura bicolor III B

Black-and-white Munia (E): Lonchura bicolor III B

Black-and-white Owl (E): Strix nigrolineata II B

Black-and-white Saki (E): Pithecia albicans II M

Black-backed Thombill (E): Ramphomicron dorsale II B

Black-banded Coral Snake (E): Micrurus nigrocincius III R

Black-banded Owl (E): Strix huhula II B

Black-bearded Flying-fox (E): Preropus melanopogon II M

Black-bellied Bustard (E): Eupodotis melanogaster II B

Black-bellied Dwarf Boa (E): Tropidophis nigriventris II R

Black-bellied Firefinch (E): Lagonosticta rara III B

Black-bellied Hummingbird (E): Eupherusa nigriventris II B

Black-bellied Korhaan (E): Eupodotis melanogaster II B

Black-bellied Pangolin (E): Manis terradacryla II M

Black-bellied Seedcracker (E): Pyrenestes ostrinus III B

Black-bellied Thomtail (E): Popelairia langsdorfi II B

Black-bellied Waxbill (E): Lagonosticta rara III B

Black-bellied Whistling-Duck (E): Dendrocygna autumnalis III B

Black-billed Amazon (E): Amazona agilis II B

Black-billed Hanging-Partot (E): Loriculus stigmatus II B

Black-billed Parrot (E): Amazona agilis II B

Black-billed Sicklebill (E): Epimachus albertisi II B

Black-billed Turaco (E): Tauraco schuetrii II B

Black-billed Wood-Dove (E): Turiur abyssinicus III B

Black-billed Wood-Duck (E): Dendrocygna arborea II B

Blackbirds (E): Icteridae spp./Turdus spp. INC B

Black-breasted Buzzard (E): Hamirostra melanosiernon II B

Black-breasted Hillstar (E): Oreotrochilus melanogaster II B

Black-breasted Kite (E): Hamirostra melanosternon II B

Black-breasted Puffleg (E): Eriocnemis nigrivestis II B

Black-browed Spider Monkey (E): Aleles geoffroyi frontatus $1 \mathrm{M}$

Blackbuck (E): Antilope cenicapra III M

Black-capped Capuchin (E): Cebus apella II M

Black-capped Conure (E): Pyrrhura rupicola II B

Black-capped Lory (E): Lorius Lory II B

Black-capped Parakeet (E): Pyrrhura rupicola II B

Black-capped Screech-Owl (E): Otus atricapillus II B

Black-cheeked Lovebird (E): Agapornis nigrigenis II B

Black-cheeked White-nosed Monkey (E): Cercopithecus ascanius II M

Black-chested Buzzard-Eagle (E): Geranoaetus melanoleucus II B

Black-chested Eagle (E): Geranoaetus melanoleucus II B

Black-chested Hawk (E): Leucoplemis princeps II B

Black-chested Snake-Eagle (E): Circaetus pecroralis II B

Blackchin Dolphin (E): Lagenorhynchus ausiralis II M

Black-chinned Hummingbird (E): Archilochus alexandri II B

Black-Cockatoos (E): Calyptorhynchus spp. II B

Black-collared Hawk (E): Busarellus nigricollis II B

Black-collared Lovebird (E): Agapomis swindemianus II B

Black-crested Coquette (E): Lophornis helenae II B

Black-eared Fairy (E): Heliothryx aurita 11 B

Black-eared Kite (E): Milvus linearus II B

Black-eared Parrot (E): Hapalopsitraca melanoris II B

Black-eared Parrotlet (E): Touit melanonotus II B

Black-faced Firefinch (E): Lagonosticta vinacea III B

Black-faced Hawk (E): Leucopternis melanops II B

Black-faced Lion Tamarin (E): Leoniopishecus caissara I M

Black-footed Cat (E): Felis nigripes I M
Black-footed Ferret (E): Mustela nigripes I M

Black-footed Penguin (E): Spheniscus demersus II B

Black-fronted Hummingbird (E): Hylocharis xantusii II B

Black-fronted Piping-Guan (E): Pipile jacutinga I B

Black-handed Spider Monkey (E): Areles geoffroyi $\mathbf{I} / \mathbf{M}$

Black-Hawks (E): Buteogallus spp. II B

Black-headed Caique (E): Pionites melanocephala II B

Black-headed Conure (E): Nandayus nenday II B

Black-headed Dwarf Chameleon (E): Bradypodion melanocephalum II R

Black-headed Monitor (E): Varanus iristis II R

Black-headed Parakeet (E): Nandayus nenday II B

Black-headed Parrot (E): Pionires melanocephala II B

Black-headed Python (E): Aspidies melanocephalus II R

Black-headed Tragopan (E): Tragopan melanocephalus I B

Black-headed Uakari (E): Cacajao melanocephalus I M

Black-headeu Weaver (E): Ploceus melanocephalus III B

Black-hooded Sunbeam (E): Aglaeactis pamela II B

Blackish Squirrel Monkey (E): Saimiri vanzolinii II M

Black-legged Falconet (E): Microhierax fringillarius II B

Black-legged Poison Frog (E): Phyllobates bicolor II A

Black-lored Parrot (E): Tanygnathus gramineus II B

Black-mantled Goshawk (E): Accipiter melanochlamys II B

Black-mantled Tamarin (E): Saguinus nigricollis II M

Black-masked Lovebird (E): Agapornis personatus II B

Black-necked Aracari (E): Preroglossus aracari II B

Black-necked Crane (E): Grus nigricollis I B

Black-necked Swan (E): Cygnus melanoconpha II B

Black-necked Weaver (E): Ploceus nigricollis III B

Black-pencilled Marmoset (E): Callithrix penicillata II M

Black-rumped Waxbill (E): Estrilda troglodytes III B

Black-shouldered Kite (E): Elanus axillaris II B/Elanus caeruleus II B

Black-sided Falconet (E): Microhierax fringillarius U B

Black-tailed Marmoset (E): Callithrix argentara II M

Black-tailed Trainbearer (E): Lesbia victoriae II B

Black-thighed Falconet (E); Microhierax fringillarius II B

Black-thighed Puffleg (E): Eriocnemis derbyi II B

Black-throated Brilliant (E): Heliodoxa schreibersii II B

Black-throated Malimbe (E): Malimbus cassini III B

Black-throated Mango (E): Anthracolhorax nigricollis II B

Black-tip Crested Turaco (E): Tauraco macrorhynchus II B

Black-winged Bishop (E): Euplectes hordeaceus III B

Black-winged Kite (E): Elanus caeruleus II B

Black-winged Lory (E): Eos cyanogenia II B

Black-winged Lovebird (E): Agapomis taranta II B

Black-winged Parrot (E): Hapalopsittaca melanotis II B

Blade Chameleon (E): Chamaeleo gallus II R

Blainville's Beaked Whale (E): Mesoplodon densirostris II M

Blakiston's Fish-Owl (E): Ketupa blakistoni II B

Blanford's Fox (E): Vulpes cana II M

Blastocerus dichotomus: Marsh Deer (E), Ciervo de los pantanos (S), Ciervo marismeño (S), Cerf des marais (F) I M

Blastomussa loyae: II In

Blastomussa merleti: II in

Blastomussa wellsi: II In

Blastosmilia $=$ Pourtalosmilia

Blastotrochus nutrix: II in

Blaze-winged Conure (E): Pyrrhura devillei II B

Blaze-winged Parakeet (E): Pyrrhura devillei II B

Bleeding Heart Dove (E): Gallicolumba luzonica II B

Bleeding Heart Pigeon (E): Gallicolumba luzonica II 8

Bleeding-hearts (E): Gallicolumba spp. IINC B

Blind barb fish (E): Caecobarbus geertsi/Barbopsis devecchii/Caecocypris basimi/Garra barreimiae//ranocypris typhlops/Phreatichthys andruzzilTyphlogarra widdowsoni IINC F

Blood Pheasant (E): Ithaginis cruentus II B

Blood Python (E): Python curius II R

Blood-eared Parakeet (E): Pyrrhura hoematotis II B

Blossomcrown (E): Anthocephala floriceps II B

Blossom-headed Parakeet (E); Psittacula roseata II B

Blue Bird-of-paradise (E): Paradisaea rudolphi II B

Blue Bustard (E): Eupodotis caerulescens II B

Blue Coral (E): Heliopora coerulea II In/COENOTHECALIA spp. II In 


\section{Blue Crane (E): Grus paradisea II B}

Blue Crowned-Pigeon (E): Goura cristala II B

Blue Duiker (E): Cephalophus monticola II M

Blue Korhaan (E): Eupodotis caenulescens II B

Blue Lorikeet (E): Vini peruviana II B

Blue macaws (E): Anodorhynchus spp. I B

Blue Monkey (E): Cercopithecus mitis II M

Blue Plantain-eater (E): Conthaeola cristata III B

Blue Poison Frog (E): Dendrobates azureus II A

Blue Whale (E): Balaenoptera musculus I M

Blue-and-gold Macaw (E): Ara ararauna II B

Blue-and-yellow Macaw (E): Ara ararauna II B

Blue-backed Parrot (E): Tanygnathus sumatranus II B

Blue-bellied Parrot (E): Triclaria malachitacea II B

Blue-bellied Poison Frog (E): Minyobates minutus II A

Blue-billed Curassow (E): Crax alberti III B

Blue-billed Firefinch (E): Lagonosticta rubricata III B

Blue-billed Malimbe (E): Malimbus nitens III B

Blue-billed Mannikin (E): Lonchura bicolor III B

Blue-billed Weaver (E): Spermophaga haematina III B

Bluebills (E): Spermophaga haematina III/NC B

Bluebonnet (E): Northiella haematogaster II B

Blue-breasted Poison Frog (E): Epipedobates pulchripectus II A

Blue-capped Hummingbird (E): Eupherusa cyanophrys II B

Blue-capped Puffleg (E): Eriocnemis glaucopoides II B

Blue-cheeked Amazon (E): Amazona dufresniana II B

Blue-cheeked Parrot (E): Amazona dufresniana II B

Blue-chested Hummingbird (E): Amazilia amabilis II B

Blue-chested Parakeet (E): Pyrrhura cruentata I B

Blue-chinned Sapphire (E): Chlorestes notaius II B

Blue-collared Parrot (E): Geoffroyus simplex II B

Blue-crowned Conure (E): Aratinga acuticaudata II B

Blue-crowned Hanging-Parot (E): Loriculus galgulus II B

Blue-crowned Lorikeet (E): Vini australis II B

Blue-crowned Parakeet (E): Aratinga acuticaudata II B

Blue-crowned Racquet-tail (E): Prioniturus discurus II B

Blue-crowned Woodnymph (E): Thalurania colombica II B

Blue-eared Lory (E): Eos semilarvata II B

Blue-eyed Cockatoo (E): Cacatua ophthalmica II B

Blue-Flycatchers (E): Cyomis spp. II/NC B

Blue-fronted Amazon (E): Amazona aestiva II B

Blue-fronted Lancebill (E): Doryfera johannae II B

Blue-fronted Lorikeet (E): Charmosyna toxopei II B

Blue-fronted Parrot (E): Amazona aestiva II B

Blue-fronted Parrotlet (E): Touit dilectissima II B

Blue-headed Dove (E): Turrur brehmeri III B

Blue-headed Hummingbird (E): Cyanophaia bicolor II B

Blue-headed Macaw (E): Ara couloni II B

Blue-headed Parrot (E): Pionus menstruus II B

Blue-headed Racquet-tail (E): Prioniturus platenae II B

Blue-headed Sapphire (E): Hylocharis grayi II B

Blue-headed Wood-Dove (E): Turtur brehmeri III B

Blue-knobbed Curassow (E): Crax alberi III B

Blue-mantled Thombill (E): Chalcostigma stanleyi II B

Blue-naped Parrot (E): Tanygnathus lucionensis II B

Blue-rumped Parrot (E): Psittinus cyanurus II B

Blue-rumped Parrotlet (E): Forpus cyanopygius II B

Blue-spotted Girdled Lizard (E): Cordylus coeruleopunctaris II R

Blue-spotted Spiny-tailed Lizard (E): Cordylus coeruleopunctatus II $\mathbf{R}$

Blue-spotted Wood-Dove (E): Turtur afer III B

Blue-streaked Lory (E): Eos reficulaia II B

Blue-tailed Day Gecko (E): Phelsuma cepediana II R

Blue-tailed Emerald (E): Chlorostilbon mellisugus II B

Blue-tailed Hummingbird (E): Amazilia cyanura II B

Blue-tailed Monitor (E); Varanus doreanus II R

Blue-tailed Pitta (E): Pitta guajana II B

Blue-throated Goldentail (E): Hylocharis eliciae II B

Blue-throated Hummingbird (E): Lampormis clemenciae II B

Blue-throated Macaw (E): Ara glaucogularis I B

Blue-throated Parakeet (E): Pyrrhura cruentata I B

Blue-throated Starfrontlet (E): Coeligena helianthea II B

Blue-topped Hanging-Parrot (E); Loriculus galgulus II B

Blue-tufted Starthroat (E): Heliomaster furcifer II B

Blue-vented Hummingbird (E): Amazilia saucerrollei II B

Blue-winged Grass-Parakeet (E): Neophema chrysostoma II B
Blue-winged Macaw (E): Ara maracana I B

Blue-winged Parrot (E): Neophema chrysostoma II B

Blue-winged Parrotlet (E): Forpus xanthopterygius II B

Blue-winged Racquet-tail (E): Prioniturus vernicalis II B

Blunt-tailed Sand Boa (E): Eryx johnii II R

Blushing Star Coral (E): Stephanocoenia michelinii II In

Blyth's Hawk-Eagle (E): Spizaefus alboniger II B

Blyth's Hombill (E): Aceros plicatus II B

Blyth's Parakeet (E): Psintacula caniceps II B

Blyth's Tragopan (E): Tragopan blythii I B

Boa à trois bandes $(\mathrm{F})$ : Lichanura trivirgata II $\mathrm{R}$

Boa annelé (F): Corallus annulaus II R

Boa annulata $=$ Corallus annulatus

Boa arborícola anillada (S): Corallus annulatus II R

Boa arboricola de jardin (S): Corallus enydris II $\mathbf{R}$

Boa arborícola de Madagascar (S): Sanzinia madagascariensis I R

Boa arboricole annelé (F): Corallus annulatus II $R$

Boa arboricole d'Amazonie (F): Corallus enydris II R

Boa arboricole gracile (F): Epicrates gracilis II R

Boa arc-en-criel (F): Epicrates cenchria II R

Boa canin (F): Corallus caninus II R

Boa canina = Corallus caninus

Boa caoutchouc ( $\mathrm{F}$ ): Charina bottae II R

Boa constrictor: Ampalagua (E), Boa Constrictor (E), Giboya (E),

Masacuate (E), Boa constrictor (S), Boa constrictor (F) I/II R

Boa Constrictor (E): Boa constrictor I/II R

Boa constrictor (S/F): Boa constrictor III R

Boa constrictor occidental (F): Boa constrictor occidentalis I R

Boa constrictor occidentalis: Argentine Boa Constrictor (E), Boa constrictor occidental (F) I R

Boa cookii $=$ Corallus enydris

Boa costera de Brasil (S): Xenoboa cropanii II R

Boa de arena de Egipto (S): Eryx colubrinus II R

Boa de arena de la India (S): Eryx johnii II R

Boa de arena de Müller (S): Eryx muelleri II R

Boa de arena de Sri Lanka (S): Eryx conicus II $\mathrm{R}$

Boa de arena de Whitaker (S): Eryx whitakeri II R

Boa de arena somalí (S): Eryx somalicus II R

Boa de bosque (S): Tropidophis melanurus II R

Boa de Cook (F): Corallus enydris II R

Boa de Cropan (F): Xenoboa cropanii II R

Boa de Cuba (S/F): Epicrates angulifer II R

Boa de Dumeril (S/F): Acrantophis dumerili I R

Boa de Fiji (S): Candoia bibroni II R

Boa de Ford (F): Epicrates fordii II R

Boa de Gomme (S): Charina bottae II R

Boa de Jamaica (S): Epicrates subflavus I R

Boa de la Jamaïque (F): Epicrates subflavus I R

Boa de l'ile Abaco (F): Epicrates exsul II R

Boa de l'île Mona (F): Epicrates monensis I R

Boa de l'lle Ronde de Dussumier (F): Casarea dussumieri I R

Boa de l'île Turk (F): Epicrates chrysogaster II R

Boa de Madagascar $(\mathrm{F})$ : Acrontophis madagascoriensis $\mathbf{I}$

Boa de Madagascar meridional (S): Acrantophis madagascariensis I R

Boa de Nueva Guinea (S): Candoia aspera II R

Boa de Pono Rico (F): Epicrates inomalus I R

Boa de Puerto Rico (S): Epicrates inomatus I R

Boa de Round Island (S): Casarea dussumieri I R

Boa del Pacífico (S): Candoia carinata II R

Boa des forĺts de Madagascar (F): Sanzinia madagascariensis I R

Boa des jardins (F): Corallus enydris II $R$

Boa des sables à écailles rugueuses ( $F$ ): Eryx conicus II $R$

Boa des sables brun (F): Enyx johnii II R

Boa des sables couleuvrin (F): Eryx colubrinus II R

Boa des sables d'Arabie (F): Eryx jayakari II R

Boa des sables d'Asie centrale (F): Eryx elegans II R

Boa des sables de Müller (F): Eryx muelleri II R

Boa des sables de Somalie (F): Eryx somalicus II R

Boa des sables de Tatarie (F): Eryx tataricus II R

Boa des sables de Whitaker (F): Eryx whitakeri II R

Boa des sables d'Egypte (F): Eryx colubrinus II $R$

Boa des sables des Indes (F): Eryx conicus II R

Boa des sables du Kenya (F): Eryx colubrinus II R

Boa des sables élégant (F): Eryx elegans II R

Boa des sables miliaire (F): Enyx miliaris II R 
Boa des sables nain (F): Eryx miliaris II R

Boa des sables occidental (F): Eryx jaculus II R

Boa des savanes de Dumeril (F): Acrantophis dumerili I R

Boa des savanes de Madagascar (F): Acrantophis madagascariensis I R

Boa d'Haiti (F): Epicrales striatus II R

Boa d'Hispaniola (F): Epicrates gracilis II R

Boa émeraude ( $F$ ): Corallus caninus II $R$

Boa enana de las Bahamas (S): Tropidophis canus II R

Boa enana de las islas Caimán (S): Tropidophis caymanensis II R

Boa enana del norte de centroamérica (S): Ungaliophis continentalis II R

Boa enana mexicana (S): Exiliboa placala II R

Boa esmerelda (S): Corallus caninus II R

Boa excavadora de Round Island (S): Bolyeria multocarinata I R

Boa forestier à queue noire de l'ille Navassa (F): Tropidophis melanurus II $\mathrm{R}$

Boa forestier à ventre noir (F): Tropidophis nigriventris II $\mathbf{R}$

Boa forestier annelé (F): Tropidophis semicinctus II $\mathbf{R}$

Boa forestier brun (F): Tropidophis fuscus II R

Boa forestier d'Ambergris Cay (F): Tropidophis greenwayi II R

Boa forestier de Battersby (F): Tropidophis battersbyi II R

Boa forestier de Feick (F): Tropidophis feicki II R

Boa forestier de lîle du Grand Inagua (F): Tropidophis canus II R

Boa forestier de Pilsbry (F): Tropidophis pilsbryi II R

Boa forestier de Taczanowsky $(F)$ : Tropidophis taczanowskyi II R

Boa forestier de Wright (F): Tropidophis wrighti II R

Boa forestier des îles Cayman (F): Tropidophis caymanensis II R

Boa forestier d'Haït (F): Tropidophis haetianus II R

Boa forestier du Brésil (F): Tropidophis paucisquamis II R

Boa forestier léopard (F): Tropidophis pardalis II R

Boa forestier tacheté (F): Tropidophis macularus II R

Boa fouisseur de l'ile Maurice (F): Bolyeria multocarinata I $\mathrm{R}$

Boa fouisseur de l'lle Ronde (F): Bolyeria multocarinata I R

Boa hortulana $=$ Corallus enydris

Boa inisada (S): Epicrates cenchria II R

Boa jabalina (S): Eryx jaculus II R

Boa manditra = Sanzinia madagascariensis

Boa mexicana rosada (S): Lichanura trivirgara II R

Boa nain à carènes ( $F$ ): Candoia carinala II $R$

Boa nain à queue noire de lîle Navassa (F): Tropidophis melanurus II $\mathbf{R}$

Boa nain à ventre noir $(F)$ : Tropidophis nigriventris II $R$

Boa nain annelé (F): Tropidophis semicinctus II R

Boa nain brun (F): Tropidophis fuscus II R

Boa nain d'Ambergris Cay (F): Tropidophis greenwayi II R

Boa nain d'Amérique centrale ( $F)$ : Ungaliophis continentalis II R

Boa nain de Battersby (F): Tropidophis battersbyi II R

Boa nain de Bibron (F): Candoia bibroni II R

Boa nain de Boulenger (F): Trachyboa boulengeri II R

Boa nain de Feick (F): Tropidophis feicki II $\mathbf{R}$

Boa nain de l'Equateur (F): Trachyboa gularis II R

Boa nain de lîle du Grand Inagua (F): Tropidophis canus II R

Boa nain de Pilsbry (F): Tropidophis pilsbny II R

Boa nain de Taczanowsky (F): Tropidophis taczanowskyi II R

Boa nain de Wright (F): Tropidophis wrighti II R

Boa nain des îles Cayman $(F)$ : Tropidophis caymanensis II R

Boa nain des îles du Pacifique (F): Candoia bibroni II R

Boa nain des Salomons (F): Candoia carinasa II R

Boa nain d'Haiti (F): Tropidophis haetianus II R

Boa nain d'Oaxaca (F): Exiliboa placala II R

Boa nain du Brésil (F): Tropidophis paucisquamis II R

Boa nain du Panama (F): Ungaliophis panamensis II R

Boa nain léopard (F): Tropidophis pardalis II R

Boa nain tacheté (F): Tropidophis macularus II R

Boa rose (F): Lichanura trivirgata II R

Boa rugosa (S): Trachyboa boulengeri II R

Boa rugosa de Ecuador (S): Trachyboa gularis II R

Boa rugueux de Boulenger ( $F)$ : Trachyboa boulengeri II $\mathrm{R}$

Boa rugueux de l'Equateur (F): Trachyboa gularis II R

Boa sobre (F): Epicrates inomarus I R

Boa vipère de Nouvelle Guinée (F): Candoia aspera II $R$

Boa-javelot (F): Enyx jaculus II R

Boas (E/S/F): Boidae spp. I/I R

Boas des savanes de Madagascar (F): Acrantophis spp. I R

Bobcat (E): Lynx nufus II M
Bobwhites (E): Colinus spp. INC B

Bocage's Chameleon (E): Chamaeleo quilensis II R

Bockadam (E): Cerberus rhynchops III $R$

Boelen's Python (E): Morelia boeleni II R

Boella renella: II R

Boettger's Chameleon (E): Chamaeleo boengeri II R

Bog Turtle (E): Clemmys muhlenbergi I R

Bogert's Boa (E): Exiliboa placata II R

Bogert's Monitor (E): Varanus bogenti II R

Boidae spp.: Boas (E), Pythons (E), Boas (S), Pitons (S), Boas (F), Pythons (F) I/I R

Boissonneaua flavescens: Buff-railed Coronet (E), Colibri cabecidorado (S), Coronita colihabana (S), Colibri flavescent (F) II B

Boissonneaua jardini: Velvet-purple Coronet (E), Coronita aterciopelada (S), Colibri de Jardine (F) II B

Boissonneaua matthewsii: Chestnut-breasted Coronet (E), Coronita pechicastaña (S), Colibri de Matthews (F) II B

Bokharan Deer (E): Cenus elaphus bactrianus II M

Bolbopsittacus lunulatus: Guaiabero (E), Lorito guayabero (S), Perruche lunulée (F) II B

Bolborhynchus aurifrons: Golden-fronted Parakeet (E), Mountain Parakeet (E), Catita frentidorada (S), Catita serrana verde (S), Toui à bandeau jaune (F) II B

Bolborhynchus aymara: Grey-hooded Parakeet (E), Sierra Parakeet (E), Catita aimará (S), Catita serrana común (S), Perruche d'Aymara (F), Toui aymara (F) II B

Bolborhynchus ferrugineifrons: Rufous-fronted Parakeet (E), Catita frentirrufa (S). Toui à front roux (F) II B

Bolborhynchus lineola: Barred Parakeet (E). Catita barrada (S). Perico barreteado (S), Periquito barrado (S), Toui catherine (F) II B

Bolborhynchus orbygnesius: Andean Parakeet (E), Catita andina (S). Toui d'Orbigny (F) II B

Bolivian Hairy Armadillo (E): Chaetophractus nationi II M

Bolivian Poison Frog (E): Epipedobates bolivianus II A

Bolivian Three-toed Sloth (E): Bradypus variegarus II M

Bolson Tortoise (E): Gopherus flavomarginatus I R

Bolyeria multocarinata: Round Island Burrowing Boa (E), Boa excavadora de Round Island (S), Boa fouisseur de l'Ile Ronde (F), Boa fouisseur de l'île Maurice (F), Bolyeride (F) I R Bolyeride (F): Bolyeria multocarinata I R

Bondrée à longue queue (F): Henicopemis longicauda II B

Bondrée apivore (F): Permis apivorus II B

Bondrée des Célèbes (F): Pernis celebensis II B

Bondrée noire (F): Henicopernis infuscatus II B

Bondrée orientale (F): Pemis prilorhyncus II B

Bondrées (E): Pernis spp $/$ Henicopemis spp. II B

Bonelli's Eagle (E): Hieraaerus fasciarus II B

Bongo (E/S/F): Tragelaphus eurycerus III M

Bonin Fruit Bat (E): Pieropus pselaphon II M

Boninastrea boninensis: II In

Bonnet Macaque (E): Macaca radiata II M

Bonneted Langur (E): Trachypithecus pileatus I M

Bonobo (E/F): Pan paniscus I M

Bontebok (E/S/F): Damaliscus pygargus pygargus II M

Bonytongues (E): Osteoglossidae spp. I/UNC F

Boobies (E): Sula spp. INC B

Boobook owls (E): Ninox spp. I/II B

Boobooks (E): Ninox spp. VII B

Boocercus eurycerus = Tragelaphus eurycerus

Boodie (E): Betrongia lesueur I M

Booted Eagle (E): Hieraaetus pennatus II B

Booted Macaque (E): Macaca ochreala II M

Booted Racket-tail (E): Ocrearus underwoodii II B

Boreal Owl (E): Aegolius funereus II B

Boring Clam (E): Tridacna crocea II In

Bomean Falconet (E): Microhierax latifrons II B

Bomean Gibbon (E): Hylobates muelleri I M

Bornean Marbled Cat (E): Catopuma badia II M

Bornean Peacock-Pheasant (E): Polyplectron schleiermacheri II B

Bornean Smooth-tailed Treeshrew (E): Dendrogale melanura II M

Bornean Treeshrew (E): Tupaia longipes II M

Bomer's Day Gecko (E): Phelsuma minuthi II R

Borrego cimarrón (S): Ovis canadensis $\mathrm{VII} \mathrm{M}$

Bos frontalis $=$ Bos gaurus 
Bos gaurus: Gaur (E), Indian Bison (E), Indian Wild Ox (E), Saladang (E), Gaur (S), Gaur (F) I M

Bos grunniens $=$ Bos mutus

Bos murus: Wild Yak (E), Yak salvaje (S), Yack sauvage (F) I M

Bos sauveli: Kouprey (E), Kouprey (S), Toro cuprey (S), Kouprey (F) I M

Bosc's Monitor (E): Varanus exanthemaicus $11 \mathrm{R}$

Bostami Turtle (E): Trionyx nigricans I R

Bostnchia hagedash: Hadada Ibis (E), Ibis hadada (S), Ibis hagedash (F) III B

Bostrychia rara: Spot-breasted Ibis (E), Ibis moteado (S), Ibis rara (S), Ibis vermiculé (F) III B

Bothriéchide de Schlegel (F): Boihrops schlegelii III R

Bothriechis schlegelii = Bothrops schlegelii

Bothrochile (F): Morelia boa II R

Bothrochilus boa $=$ Morelia boa

Bothrops asper. Terciopolo (E), Trigonocéphale rugueux (F) III R

Bothrops atrox = Bothrops asper

Bothrops nasula: Homed Hog-nosed Pit Viper (E), Rainforest Hog nosed Pit Viper (E), Trigonocéphale à grand nez (F) III R

Bothrops nasutus $=$ Bothrops nasuta

Bothrops nummifer: Jumping Pit Viper (E), Trigonocéphale sauteur (F) III R

Bothrops nummifera $=$ Bothrops nummifer

Bothrops ophryomegas: Comiz (E), Slender Hog-nosed Pit Viper (E), Western Hog-nosed Viper (E), Trigonocéphale à groin de Bocourt (F) III R

Bothrops schlegelii; Eyelash Palm Pit Viper (E), Eyelash Viper (E), Horned Palm Viper (E), Bothriéchide đe Schlegel (F) III R

Boto (E): Inia geoffrensis II M

Bottlehead (E): Hyperoodon ampullatus I M

Bottlenose Dolphin (E): Tursiops truncarus II M

Bottlenose whales (E): Hyperoodon spp. I M

Bottle-nosed Dolphin (E): Tursiops truncarus II M

Boulenger's Cape Tortoise (E): Homopus boulengeri II R

Boulenger's Padloper (E): Homopus boulengeri II R

Bouquetin du Népal (F): Naemorhedus goral I M

Bourke's Parrot (E): Neopsephorus bourkii II B

Boumeotrochus stellulatus: II In

Boutu (E): Inia geoffrensis II M

Bowhead Whale (E): Balaena mysticelus I M

Bowsprit Tortoise (E): Chersina angulata II R

Box turtles (E): Terrapene spp. III R

Brace's Emerald (E): Chlorostilbon bracei II B

Brachlyophes (F): Brachylophus spp. I R

Brachylophus spp: Fijian iguanas (E), Iguanas de Fiji (S), Brachlyophes (F), Iguanes des Fidji (F) I R

Brachylophus fasciatus: Fiji Banded Iguana (E), South Pacific Banded Iguana (E), Iguane à bandes de Fidji (F) I R

Brachylophus vitiensis: Fiji Crested Iguana (E), Iguana crestada de Fiji (S), Iguane à crête de Fidji (F) I R

Brachymaeandrina = Platygyra

Brachypelma spp.: Tarantulas (E), Tarăntulas (S), Mygales (F), Tarentules (F) II In Brachypelma albopilisum: Curly-hair Tarantula (E) II In

Brachypelma angustum: II In

Brachypelma auratum: Flame-knee Tarantula (E) II In

Brachypelma aureoceps: Florida Golden Chestnut (E) II in

Brachypelma baumgarteni: Michoacan Orange Tarantula (E) II In

Brachypelma boehmei: Guerrero Orange Legs (E) II In

Brachypelma emilia: Mexican Black-cap (E), Mexican Red Leg (E), Orange-knee Tarantula (E), True Red Leg (E) II In

Brachypelma epicureanum: Yucatan Rust Rump Tarantula (E) II In

Brachypelma fossorium: II in

Brachypelma klaasi: Acapulco Lesser Orange Tarantula (E) II In

Brachypelma pallidum: II In

Brachypelma sabulosum: II In

Brachypelma smithi: Mexican Red-kneed Tarantula (E) II in

Brachypelma vagans: Red-rumped Tarantula (E) II In

Brachyteles arachnoides: Muriqui (E), Woolly Spider Monkey (E), Mono grande (S), Muriki (S), Atèle arachnoïde (F), Eroide (F), Singe-araignée laineux (F) I M

Brachyteles hypoxanthus = Brachyteles arachnoides

Brachytrochus = Heterocyathus $/$ Paracyathus

Bradype (F): Bradypus variegatus $1 \mathrm{M}$
Bradypodion spp.: Chameleons (E), Dwarf chameleons (E), Camaleónes enanos (S), Caméléons nain (F) II R

Bradypodion adolfifriderici: Ituri Forest Chameleon (E), Caméléon nain d'Ituri (F) II R

Bradypodion caffer. Transkei Dwarf Chameleon (E), Caméléon nain du Transkei (F) II R

Bradypodion caffrum $=$ Bradypodion caffer

Bradypodion carpenteri: Ruwenzori Mountain Chameleon (E), Caméléon nain de Carpenter (F) II R

Bradypodion damaranum: Knysna Dwarf Chameleon (E), Caméléon nain du Kenya (F) II R

Bradypodion dracomonianum: Drakensberg Dwarf Chameleon (E), Caméléon nain du Drakensberg (F) II R

Bradypodion fischeri: Fischer's Chameleon (E), Uluguru Twohomed Chameleon (E), Usambara Two-horned Chameleon (E), Caméléon nain de Fischer (F) II R

Bradypodion gutturale: Robertson's Dwarf Chameleon (E), Caméléon nain de Karro (F) II R

Bradypodion karroicum: Karoo Dwarf Chameleon (E), Caméléon nain à tête noire (F) II R

Bradypodion melanocephalum: Black-headed Dwarf Chameleon (E). Caméléon nain du Mont Ulanje (F) II R

Bradypodion mlanjensis: Mlanje Mountain Chameleon (E), Caméléon nain du Zululand (F) II R

Bradypodion nemorale: Zululand Dwarf Chamaeleon (E), Caméléon nain à nez rouge (F) II $R$

Bradypodion occidentale $=$ Bradypodion ventrale

Bradypodion oxyrhinum: Red-nosed Dwarf Chameleon (E), Uluguru One-homed Chameleon (E), Caméléon nain du Cap (F) II R

Bradypodion pumilum: Cape Dwarf Chameleon (E), Variegated Dwarf Chameleon (E), Caméléon nain de Setaro (F) II R

Bradypodion setaroi: Setaro's Dwarf Chameleon (E), Caméléon nain épineux (F) II R

Bradypodion spinosum: Prickly Chameleon (E), Rosette-nosed Chameleon (E), Spiny Chameleon (E), Caméléon nain de Smith (F) II R

Bradypodion taeniabronchum = Bradypodion taeniobronchum Bradypodion taeniobronchum: Smith's Dwarf Chameleon (E) II R

Bradypodion tavetanum: Kilimanjaro Two-homed Chameleon (E), Caméléon nain de Matschie (F) II R

Bradypodion renue: Matschie's Dwarf Chameleon (E), Single Softnosed Chameleon (E), Slender Chameleon (E), Usambara Softhorned Chameleon ( $E$ ), Caméléon nain du Natal (F) II R

Bradypodion thamnobates: Natal Midland Dwarf Chamaeleon (E), Caméléon nain du Transvaal (F) II $\mathrm{R}$

Bradypodion transwaalense: Transvaal Dwarf Chameleon (E), Caméléon nain de Muller (F) II R

Bradypodion uthmoelleri: Hanang Hornless Chameleon (E), Müller's Leaf Chameleon (E), Caméléon nain du sud et du Namaqua (F) II $\mathbf{R}$

Bradypodion ventrale: Namaqua Dwarf Chameleon ( $\mathrm{E})$, Southern Dwarf Chameleon (E), Caméléon nain des Monts Ruwenzori (F) II R

Bradypodion ventrale karroicum $=$ Bradypodion karroicum

Bradypodion xenorhinus: Single Welded-hom Chameleon (E) Strange-nosed Chameleon (E), Caméléon de Rüppell (F) II R Bradypus boliviensis = Bradypus variegatus

Bradypus griseus = Bradypus variegaius

Bradypus infuscatus = Bradypus variegatus

Bradypus variegarus: Bolivian Three-toed Sloth (E), Brown-throated Sloth (E), Perezoso grisaceo (S), Perezoso tridáctilo (S), Perico ligero (S), Aï de Bolivie (F), Bradype (F), Paresseux tridactyle (F), Paresseux tridactyle de Bolivie (F) II M

Brahminy Kite (E): Haliastur indus II B

Brain Coral (E): Diploria labyrinthiformis II In

Branta canadensis leucopareia: Aleutian Canada Goose (E), Aleutian Goose (E), Bamacla canadiense aleutiana (S), Ganso canadiense aleutiana (S), Bernache des Aléoutiennes (F), Bernache du Canada aléoure (F) I B

Branta ruficollis: Red-breasted Goose (E), Barnacla cuellirroja (S), Bemache à cou roux (F) II $\mathrm{B}$

Branta sandvicensis: Hawaiian Goose (E), Nene (E), Bamacla hawaiana (S), Bamacla nené (S), Bernache d'Hawaï (F), Bernache des lles Sandwich $(F)$, Bernache né-né (F), Oie néné (F) I B

Brasseyia $=$ Dendrophyllia 
Brazil Teju (E): Tupinambis nigropunctatus II R

Brazilian Dwarf Boa (E): Tropidophis paucisquamis II $\mathbf{R}$

Brazilian Giant Tortoise (E): Geochelone denticulata II R

Brazilian Poison Frog (E): Dendrobares vanzolinii II A

Brazilian Ruby (E): Clytolaema rubricauda II B

Brazilian Tapir (E): Tapirus terrestris II M

Brazil-nut Poison Frog (E): Dendrobates castaneoticus II A

Brehm's Tiger-Parrot (E): Psittacella brehmii II B

Brelich's Snub-nosed Monkey (E): Pygathrix brelichi I M

Brève à queue bleve (F): Pitia guajana II B

Breve azurine (F): Pirta guajana II B

Brève de Gumey (F): Pinta gumeyi I B

Brève de Koch (F): Pirla kochi I B

Brève du Japon (F): Pirla nympha II B

Brève migratrice (F): Pitra nympha II B

Breves (F): Pilla spp. I/INC B

Bridled Dolphin (E): Stenella atrenuata II M

Bridled Nailtail Wallaby (E): Onychogalea fraenaia I M

Bridled Wallaby (E): Onychogalea fraenaia I M

Bright-eyed Day Gecko (E): Phelsuma dubia II R

Brillant à bandeau bleu (F): Heliodoxa aurescens II B

Brillant à couronne verte (F): Heliodoxa xanthogonys II B

Brillant à front violet (F): Heliodoxa leadbeateri II B

Brillant à gorge noire (F): Heliodoxa schreibersii II B

Brillant à gorge rose (F): Heliodoxa gularis II B

Brillant de Branicki (F): Heliodoxa branickii II B

Brillant fer-de-lance (F): Heliodoxa jacula II B

Brillant impératrice (F): Heliodoxa imperatrix II B

Brillant rubinoïde (F): Heliodoxa rubinoides II B

Brillante coroniverde (S): Heliodoxa jacula II B

Brillante emperatriz (S): Heliodoxa imperatrix II B

Brillante frentivioleta (S): Heliodoxa leadbeaseri II B

Brillante gorjinegro (S): Heliodoxa schreibersii II B

Brillante gorjirrosado (S): Heliodoxa gularis II B

Brillante pechianteado (S): Heliodoxa rubinoides II B

Brillants (F): Heliodoxa spp. II B

Brilliants (E): Heliodoxa spp. II B

Brilliant-thighed Poison Frog (E): Allobates femoralis II A

Bristlebirds (E): Dasyomis spp. UNC B

Broad-billed Hummingbird (E): Cynanthus latirostris II B

Broad-headed Snake (E): Hoplocephalus bungaroides II R

Broad-nosed Caiman (E): Caiman latirostris II R

Broad-nosed Gentle Lemur (E): Hapalemur simus I M

Broad-snouted Caiman (E): Caiman latirostris II R

Broad-snouted Crocodile (E): Crocodylus palustris I R

Broad-tailed Hummingbird (E): Selasphorus plarycercus II B

Broad-tipped Hermit (E): Phaethornis gounellei II B

Broad-winged Hawk (E): Buteo plarypterus II B

Brockets (E): Mazama spp. II/NC M

Brolga (E): Grus rubicunda II B

Bronze Mannikin (E): Lonchura cucullata III B

Bronze Munia (E): Lonchura cucullara III B

Bronze-tailed Barbthroat (E): Threnetes leucurus II B

Bronze-tailed Comet (E): Polyonymus caroli II B

Bronze-tailed Plumeleteer (E): Chalybura urochrysia II B

Bronze-tailed Thombill (E): Chalcostigma heteropogon II B

Bronze-winged Mannikin (E): Lonchura cucullata III B

Bronze-winged Parrot (E): Pionus chalcopterus II B

Bronzy Hermit (E): Glaucis aenea II B

Bronzy Inca (E): Coeligena coeligena II B

Brother Islands Tuatara (E): Sphenodon guntheri I R

Brotogeris chirin: Yellow-chevroned Parakeet (E), Catita chiriri (S), Toui à ailes jaunes (F) II B

Brotogeris chrysopierus: Golden-winged Parakeet (E), Catita alidorada (S), Periquito ala dorada (S), Toui para (F) II B

Brotogeris cyanoptera: Cobalt-winged Parakeet (E), Catita aliazul (S), Periquito azul (S), Toui de Deville (F) II B

Brotogeris jugularis: Orange-chinned Parakeet (E), Catita churica (S), Churica (S), Periquito barbinaranja (S), Perruche tovi (F), Toui à menton d'or (F) II B

Brotogeris pyrrhopterus: Grey-cheeked Parakeet (E), Catita macareña (S), Perico cachetigris (S), Perruche orangé (F), Toui flamboyant (F) II B

Brotogeris sanctithomae: Tui Parakeet (E), Catita frentigualda (S), Perico tui (S), Toui à front d'or (F) II B
Brotogeris tirica: Plain Parakeet (E), Catita tirica (S), Toui tirica (F) II B

Brologeris versicolurus: Canary-winged Parakeet (E), Catita aliamarilla (S), Catita versicolor (S), Toui à ailes variées (F) II B Brotogeris versicolurus chiriri $=$ Brotogeris chiriri

Brow-antlered Deer (E): Cenus eldii I M

Brown Bear (E): Ursus arctos

Brown Caiman (E): Caiman crocodilus I/II R

Brown Eared-Pheasant (E): Crossoptilon mantchuricum I B

Brown Falcon (E): Falco berigora II B

Brown Fish-Owl (E): Ketupa zeylonensis II B

Brown Goshawk (E): Accipiter fasciarus II B

Brown Harrier-Eagle (E): Circaetus cinereus II B

Brown Hawk-Owl (E): Ninox scutulata 11 B

Brown Hombill (E): Anorrhinus tickelli II B

Brown Howler (E): Alouatta fusca II M

Brown Hyaena (E): Parahyaena brunnea II M

Brown Inca (E): Coeligena wilsoni II B

Brown Lemur (E): Eulemur fulvus I M

Brown Lory (E): Chalcopsitta duivenbodei II B

Brown Monitor (E): Varanus primordius II R

Brown Mouse-lemur (E): Microcebus rufus I M

Brown Pale-fronted Capuchin (E): Cebus albifrons II M

Brown Parrot (E): Poicephalus meyeri II B

Brown Sand Boa (E): Eryx johnii II R

Brown Sicklebill (E): Epimachus meyeri II B

Brown Snake-Eagle (E): Circaetus cinereus II B

Brown Soft-shelled Turtle (E): Trionyx hurum I R

Brown Teal (E): Anas aucklandica I B

Brown Violet-ear (E): Colibri delphinae II B

Brown Wood-Owl (E): Strix leptogrammica II B

Brown-backed Firefinch (E): Lagonosticta rubricata III B

Brown-backed Parrotlet (E): Touit melanonotus II B

Brown-blossom Naiad (E): Epioblasma walkeri I in

Brown-blossom Pearly Mussel (E): Epioblasma walkeri I In

Brown-breasted Parakeet (E): Pyrrhura calliptera II B

Brown-headed Parrot (E): Poicephalus cryptoxanthus II B

Brown-headed Spider Monkey (E): Aleles fusciceps II M

Brown-headed Tamarin (E): Saguinus fuscicollis II M

Brown-hooded Parrot (E): Pionopsitta haematotis II B

Brown-necked Parrot (E): Poicephalus robustus II B

Brown-throated Conure (E): Aratinga pertinax II B

Brown-throated Parakeet (E): Aratinga perinax II B

Brown-throated Sloth (E): Bradypus variegatus II M

Bruce's Green-Pigeon (E): Treron waalia III B

Brumback's Night Monkey (E): Aotus brumbacki II M

Brush-tailed Bettong (E): Bettongia penicillata I M

Brush-tailed Rat Kangaroo (E): Bettongia penicillata I M

Brush-turkeys (E): Megapodiidae spp. INC B

Brush-Warblers (E): Bebrornis spp. IIU/NC B

Bryde's Whale (E): Balaenopiera edeni I M

Brygoo's Chameleon (E): Chamaeleo peyrieresi II R

Bubalornis albirosiris: White-billed Buffalo Weaver (E), Alecto à bec blanc (F) III B

Bubalus amee: Wild Asiatic Buffalo (E), Wild Water Buffalo (E), Búfalo ami (S), Buffle de l'Inde (F), Buffle d'eau (F) III M

Bubalus depressicomis: Lowland Anoa (E), Anoa de llanura (S), Anoa des plaines (F) I M

Bubalus mindorensis: Tamaraw (E), Tamarou (E), Búfalo de Mindoro (S), Tamarau (S), Tamarau (F) I M

Bubalus quarlesi: Mountain Anoa (E), Anoa de montaña (S), Anoa de Quarle (F), Anoa des montagnes (F) I M

Bubble Coral (E): Physogyra lichtensteini II in

Bubo africanus: African Eagle-Owl (E), Spotted Eagle-Owl (E), Búho africano chico (S), Grand-duc africain (F) II B

Bubo ascalaphus: Pharaoh Eagle-Owl (E), Grand-duc ascalaphe (F) II B

Bubo bengalensis: Rock Eagle-Owl (E), Grand-duc indien (F) II B Bubo blakistoni $=$ Ketupa blakistoni

Bubo bubo: Eurasian Eagle-Owl (E), Búho real (S), Grand-duc d'Europe (F), Hibou grand duc (F) II B

Bubo bubo ascalaphus $=$ Bubo ascalaphus

Bubo bubo bengalensis $=$ Bubo bengalensis

Bubo capensis: Cape Eagle-Owl (E), Mackinder's Eagle-Owl (E), Mountain Eagle-Owl (E), Grand-duc du Cap (F) II B 
Bubo coromandus: Dusky Eagle-Owl (E), Grand-duc de Coromandel (F) II B

Bubo flavipes $=$ Ketupa flavipes

Bubo kesupu = Ketupa ketupu

Bubo lacteus: Giant Eagle-Owl (E), Milky Eagle-Owl (E),

Verreaux's Eagle-Owl (E), Gran búło etiópico (S), Grand-duc de Verreaux (F) II B

Bubo leucostictus: Akun Eagle-Owl (E), Sooty Eagle-Owl (E), Búho de Akun (S), Grand-duc tacheté (F) II B

Bubo nipalensis: Forest Eagle-Owl (E), Spot-bellied Eagle-Owl (E), Grand-duc du Népal (F) II B

Bubo philippensis: Philippine Eagle-Owl (E), Grand-duc des Philippines (F) II B

Bubo poensis: Fraser's Eagle-Owl (E), Grand-duc à aigrettes (F) II B

Bubo poensis vosseler $=$ Bubo vosseleri

Bubo shelleyi: Banded Eagle-Owl (E), Shelley's Eagle-Owl (E). Grand-duc de Shelley (F) II B

Bubo sumatranus: Barred Eagle-Owl (E), Grand-duc bruyant ( $F$ ) II B

Bubo virginianus: Great Horned Owl (E), Búho (S), Búho cornudo (S), Lechuzón orejudo '`, Nacurutú (S), Grand-duc d'Amérique (F) II B

Bubo vosseleri: Usambara Eagle-Owl (E), Grand-duc des Usambara (F) II B

Bubo zeylonensis = Kerupa zeylonensis

Bubulcus ibis: Cattle Egret (E), Espulgabueyes (S), Garcilla bueyera (S), Garcita resnera (S), Garza ganadera (S), Héron garde-boeuf (F) III B

Buceros (S): Bucerotidae spp. I/IV/Nc B

Buceros spp.: Hornbills (E), Buceros (S), Calaos (F) I/II B

Buceros bicomis: Great Hombill (E), Great Indian Hombill (E). Great Pied Hornbill (E), Cálao grande (S), Calao bicorne (F) I B

Buceros hydrocorax: Rufous Hombill (E), Cálao rojipardo (S), Calao des Philippines ( $F$ ), Calao d casque plat (F) II B

Buceros rhinoceros: Rhinoceros Hornbill (E), Cálao rinoceronte (S), Calao rhinocéros (F) II B

Buceros vigil: Helmeted Hombill (E), Cálao de casco (S), Calao à casque $(F)$, Calao à casque rond $(\mathrm{F}) \mathrm{I} \mathrm{B}$

Buckley's Forest-Falcon (E): Micrastur buckleyi II B

Budeng (F): Trachypiihecus crisıalus II M

Budgerigar (E): Melopsitracus undulatus NC B

Budgerigar ondulado (S): Melopsittacus undulatus NC B

Budorcas taxicolor: Takin (E), Takin (F) II M

Búfalo ami (S): Bubalus amee III M

Búfalo de Mindoro (S): Bubalus mindorensis I M

Bufeo (S): Inia geoffrensis II M

Bufeo africano (S): Sousa reuszii I M

Bufeo asiático (S): Sousa chinensis I M

Bufeo negro (S): Sotalia fluviatilis I M

Bufeos (S): Sotalia spp. I M

Buffalo (E): Bubalus arnee/Bubalus bubalis/Syncerus caffer III/NC M

Buffalo weavers (E): Bubalornis spp $/$ Dinemellia spp. III/NC B

Buff-bellied Hermit (E): Phaethornis subochraceus II B

Buff-bellied Hummingbird (E): Amazilia yucatanensis II B

Buff-breasted Sabrewing (E): Campylopterus duidae II B

Buff-cheeked Gibbon (E): Hylobates gabriellae I M

Buff-crested Bustard (E): Eupodotis gindiana II B

Buff-faced Pygmy-Parrot (E): Micropsinta pusio II B

Buff-fronted Owl (E): Aegolius harrisii II B

Buffle d'eau (F): Bubalus arnee III M

Buffle de l'Inde (F): Bubalus arnee III M

Buff-nosed Rat-kangaroo (E): Caloprymnus campestris I M

Buffon's Macaw (E): Ara ambigua I B

Buff-tailed Coronet (E): Boissonneaua flavescens II B

Buff-tailed Sicklebill (E); Eutoxeres condamini II B/Epimachus albertisi II B

Buff-winged Starfrontlet (E): Coeligena lutetiae II B

Buffy Fish-Owl (E): Ketupa ketupu II B

Buffy Hummingbird (E): Leucippus fallar II B

Buffy Saki (E): Pithecia albicans II M

Buffy-headed Marmoset (E): Callithrix flaviceps I M

Buffy-tufted-ear Marmoset (E): Callithrix aurita I M

Bufo osgoodi = Spinophrynoides osgoodi
Bufo periglenes: Alajuela Toad (E), Golden Toad (E), Monte Verde Toad (E), Orange Toad (E), Sapo dorado de Monteverde (S), Crapaud doré (F) I A

Bufo retiformis: Sonoran Green Toad (E), Sapo verde de Sonora (S), Crapaud vert du Sonora (F) II A

Bufo superciliaris: Cameroon Toad (E), Zaire Toad (E), Sapo del Camenin (S), Crapaud du Cameroun (F) I A

Bugeranus carunculatus $=$ Grus caruncularus

Buhito en Pernetas (S): Orus clarkii II B

Búho (S): Bubo virginianus II B

Búho africano chico (S): Bubo africanus II B

Búho aguilucho de Neuva Guinea (S): Uroglaux dimorpha II B

Búho barrado (S): Strix varia II B

Búho blanquinegro (S): Sirix nigrolineasa II B

Búho café (S): Strix virgata II B

Búho chico (S): Asio otus II B

Búho corniblanco (S): Lophosirix cristata II B

Búho cornudo (S): Bubo virginianus II B/Lophosirix cristąa II B

Búho de Akun (S): Bubo leucosticius II B

Búho de anteojos (S): Pulsatrix perspicillata II B

Búho de Guinea (S): Jubula lettii II B

Búho de Jamaica (S): Pseudoscops grammicus II B

Búho de las Islas Salomón (S): Nesasio solomonensis II B

Búho fulvo (S): Strix fulvescens II B

Búho llanero (S): Speotyto cunicularia II B

Búho manchado (S): Strix occidentalis II B

Búho nival (S): Nyclea scandiaca II B

Búho orejicorto (S): Asio flammeus II B

Búho pequeño de bigotes alargados (S): Xenoglaux loweryi II B

Búho pescador (S): Kelupa flavipes II B

Búho pescador rojizo (S): Scotopelia ussheri II B

Búho real (S): Bubo bubo II B

Búho-comudo cariblanco (S): Asio clamator II B

Búho-cornudo caricafe (S): Asio otus II B

Búho-comudo oscuro (S): Asio stygius II B

Buitre cabeciblanco (S): Trigonoceps occipitalis II B

Buitre cabecirrojo (S): Sarcogyps calvus II B

Buitre del Himalaya (S): Gyps himalayensis II B

Buitre dorsiblanco africano (S): Gyps africanus II B

Buitre dorsiblanco bengali (S): Gyps bengalensis II B

Buitre el cabo (S): Gyps coprotheres II B

Buitre leonado (S): Gyps fulvus II B

Buitre leonado bengalés ( $\mathrm{S}$ ): Gyps bengalensis II B

Buitre leonado común (S): Gyps fulvus II B

Buitre moteado (S): Gyps rueppellii II B

Buitre negro (S): Aegypius monachus II B

Buitre orejudo (S): Torgos tracheliotus II B

Buitre palmero (S): Gypohierax angolensis II B

Buitre picofino (S): Gyps indicus II B

Buitre piquirrojo (S): Trigonoceps occipitalis II B

Bulbul à tête jaune (F): Pycnonotus zeylanicus II B

Bulbuls (E/F): Pycnonotidae spp. ILNC B

Bullfrogs (E): Dicroglossus occipinalis/Hoplobatrachus spp/Pyxicephalus adspersus/Rana spp. II/NC A

Bumblebee Hummingbird (E): Althis heloisa II B

Bunch (E): Megaptera novaeangliae I M

Bungarra (E): Varanus gouldii II R

Bürger's Sparrowhawk (E): Erythrorriorchis buergersi II B

Burhinus bistriatus: Double-siriped Thick-knee (E), Alcaraván americana (S), Alcaraván venozolano (S), Dara (S), Oedicnème bistrié (F) III B

Burmeister's Porpoise (E): Phocoena spinipinnis II M

Burmese Brown Tortoise (E): Manouria emys II R

Burmese Eyed Turtle (E): Morenia ocellata I R

Burmese Mountain Tortoise (E): Manouria emys II R

Burmese Python (E): Python molurus I/II R

Burmese Starred Tortoise (E): Geochelone plarynota II R

Burrowing Bettong (E): Betrongia lesueur I M

Burrowing Owl (E): Speotyto cunicularia II B

Burrowing Parakeet (E): Cyanoliseus pasagonus II B

Burrowing Python (E): Loxocemus bicolor II R

Buru Opalescent Birdwing (E): Troides prattonum II in

Buru Racquet-tail (E): Prioniturus mada II B

Busard bariolé (F): Circus cinereus II B

Busard cendré ( $F$ ): Circus pygargus $\mathbf{U} \mathrm{B}$

Busard de Buffon (F): Circus buffoni II B 
Busard de Gould (F): Circus approximans II B Busard de Maillard (F): Circus maillardi II B

Busard de montagne (F): Circus pygargus II B

Busard de Wilson (F): Chondrohierax uncinatus wilsonii I B

Busard des roseaux (F): Circus aenaginosus II B

Busard d'Orient (F): Circus spilonotus II B

Busard grenouillard (F): Circus ranivorus II B

Busard maure (F): Circus maurus II B

Busard pâle (F): Circus macrourus II B

Busard Saint-Martin (F): Circus cyaneus II B

Busard tacheté (F): Circus assimilis II B

Busard tchoug (F): Circus melanoleucos II B

Busardo aliancho (S): Buteo platypterus II B

Busardo alimufo (S): Butastur liventer II B

Busardo augur meridional (S): Buteo nufofuscus II B

Busardo augur oriental (S): Buteo augur II B

Busardo augur somali (S): Buteo archeri II B

Busardo aura (S): Buteo albonotatus II B

Busardo azoreño (S): Leucopternis princeps II B

Busardo blanco (S): Leucopternis albicollis II B

Busardo blanquinegro (S): Leucoptemis polionota II B

Busardo calzado (S): Buteo lagopus II B

Busardo caminero (S): Buteo magnirostris II B

Busardo carigrís (S): Bulastur indicus II B

Busardo carinegro (S): Leucoptemis melanops II B

Busardo cejiblanco (S): Leucoptemis kuhli II B

Busardo chapulinero (S): Buteo swainsoni II B

Busardo coliblanco (S): Buteo albicaudatus 11 B

Busardo colicorto (S): Buteo brachyurus II B

Busardo colirrojo (S): Buteo jamaicensis II B

Busardo colorado (S): Busarellus nigricollis II B

Busardo cuelliblanco (S): Leucoptemis lacemulata II B

Busardo cuellirrojo (S): Buteo auguralis II B

Busardo culiblanco (S): Buteo leucorrhous II B

Busardo de Galápagos (S): Buteo galapagoensis II B

Busardo de la española (S): Buseo ridgwayi II B

Busardo dorsigris (S): Leucopternis occidentalis II B

Busardo dorsirnojo (S): Buteo polyosoma II B

Busardo gavilán (S): Kaupifalco monogrammicus II B

Busardo gorgiblanco (S): Buteo albigula II B

Busardo gris (S): Asturina nitida II B

Busardo hawaiano (S): Buteo solitarius II B

Busardo herrumbroso (S): Buteo regalis II B

Busardo hombrorrojo (S): Bufeo linealus II B

Busardo langostero (S): Butastur rufipennis II B

Busardo malgache (S): Buteo brachypterus II B

Busardo mixto (S): Parabuteo unicinctus II B

Busardo mongol (S): Buteo hemilasius II B

Busardo montañés (S): Bureo oreophilus II B

Busardo moro (S): Buteo rufinus II B

Busardo patagón (S): Buteo ventralis II B

Busardo pizarroso (S): Leucopternis schistacea II B

Busardo plomizo (S): Leucoptemis plumbea II B

Busardo puneño (S): Buteo poecilochrous II B

Busardo ratonero (S): Buteo buteo II B

Busardo sabanero (S): Buteogallus meridionalis II B

Busardo semiplomizo (S): Leucoptemis semiplumbea II B

Busardo tisa (S): Butastur teesa II B

Busardo-negro del atlántico (S): Buseogallus aequinoctialis II B

Busardo-negro del pacifico (S): Buseogallus subtilis II B

Busardo-negro norteño (S): Buteogallus anthracinus II B

Busardo-negro urubitinga (S): Buteogallus urubitunga II B

Busards (F): Circus spp. II B

Busarellus nigricollis: Black-collared Hawk (E), Collared Fishing

Hawk (E), Aguila colorada (S), Busardo colorado (S), Gavilán colorado (S). Buse à tête blanche (F) II B

Busautour à joues grises (F): Butastur indicus II B

Busautour aux yeux blancs (F): Butastur teesa II B

Busautour des sauterelles (F): Butastur rufipennis II B

Busautour pâle (F): Butastur liventer II B

Busautours (F): Butastur spp. II B

Buse à dos gris (F): Leucoptemis occidentalis II B

Buse à épaulettes (F): Buteo lineatus II B

Buse à face noire $(\mathbf{F})$ : Leucoptemis melanops II B

Buse à gorge blanche (F): Buteo albigula II B

Buse a gros bec (F): Buteo magnirostris II B
Buse à queue barté (F): Buteo albonotatus II B

Buse à queue blanche (F): Buteo albicaudatus II B

Buse à queue courte (F): Buteo brachyurus II B

Buse à queue rousse (F): Buteo auguralis II B/Buteo jamaicensis II B

Buse à sourcils blancs (F): Leucoptemis kuhli II B

Buse à tête blanche (F): Busarellus nigricollis II B

Buse aguia (F): Geranoaetus melanoleucus II B

Buse ardoisée (F): Leucoptemis schistacea II B

Buse augure (F): Buteo augur II B

Buse barrée (F): Leucopternis princeps II B

Buse blanche (F): Leucoptemis albicollis II B

Buse buson (F): Buteogallus aequinoctialis II B

Buse cendrée (F): Asturina nitida II B

Buse couronnée ( $\mathrm{F}$ ): Harpyhaliaetus coronatus II B

Buse cul-blanc (F): Bufeo leucorrhous II B

Buse d'Afrique ( $F)$ : Buteo auguralis II B

Buse d'Archer (F): Bureo archeri II B

Buse de Chine (F): Buteo hemilasius II B

Buse de Harris (F): Parabureo unicinclus II B

Buse de Madagascar (F): Buteo brachypterus II B

Buse de Patagonie (F): Buteo ventralis II B

Buse de Ridgway (F): Buteo ridgwayi II B

Buse de Swainson (F): Bureo swainsoni II B

Buse des Galapagos (F): Buteo galapagoensis II B

Buse des mangroves (F): Buteogallus subtilis II B

Buse d'Hawaï (F): Buteo solitarius II B

Buse du puna (F): Buteo poecilochrous II B

Buse échasse (F): Geranospiza caerulescens II B

Buse féroce (F): Buteo nufinus II B

Buse grise (F): Asturina plagiata II B

Buse lacernulée (F): Leucoptemis lacemulata II B

Buse mantelée (F): Leucoptemis polionota II B

Buse montagnarde (F): Buteo oreophilus II B

Buse noire (F): Buteogallus anthracinus II B

Buse pattue (F): Buteo lagopus II B

Buse plombée (F): Leucopternis plumbea II B

Buse rouilleuse (F): Buteo regalis II B

Buse rounoir (F): Butea nufofuscus II B

Buse roussâtre (F): Buteogallus meridionalis II B

Buse semiplombée (F): Leucopternis semiplumbea II B

Buse solitaire (F): Harpyhaliaerus solitarius II B

Buse tricolore (F): Buseo polyosoma II B

Buse urubu (F): Buteogallus urubitunga II B

Buse variable (F): Bureo buteo II B

Buses (F): Asturina spp./Busarellus nigricollis/Buteo

spp./Buteogallus spp./Geranoaetus melanoleucus/Geranospiza

caerulescens/Harpyhaliaetus spp./Leucopternis spp. II B

Bush Dog (E): Speothos venaticus I $M$

Bush Petronia (E): Petronia dentata III B

Bush Sparrow (E): Petronia dentata III B

Bushbabies (E): Galagonidae spp. II M

Bushy-crested Hombill (E): Anorrhinus galeritus II B

Bushy-tailed Olingo (E): Bassaricyon gabbii III M

Bustards (E): Otididae spp. I/II B

Butastur indicus: Grey-faced Buzzard (E), Grey-faced Buzzard-

Eagle (E), Busardo carigrís (S), Busautour à joues grises (F) II B

Butastur liventer: Rufous-winged Buzzard (E), Rufous-winged

Buzzard-Eagle (E), Busardo alirrufo (S), Busautour pâle (F) II B

Butastur rufipennis: Grasshopper Buzzard (E), Grasshopper

Buzzard-Eagle (E), Aguililla langostera (S), Busardo langostero

(S), Busautour des sauterelles (F) II B

Butastur teesa: White-eyed Buzzard (E), Busardo tisa (S), Busautour aux yeux blancs (F) II B

Buteo albicaudatus: White-tailed Hawk (E), Aguililla coliblanca (S), Aguilucho cabecinegro (S), Busardo coliblanco (S), Gavilán tejé (S), Buse à queue blanche (F) II B

Buteo albigula: White-throated Hawk (E), Aguilucho chico (S), Busardo gorgiblanco (S), Gavilán gargantiblanco (S), Buse à gorge blanche (F) II B

Buteo albonotatus: Zone-tailed Hawk (E), Aguililla aura (S),

Busardo aura (S), Gavilán albonotatus (S), Gavilán colifajeado (S), Buse à queue barrée (F) II B

Buteo archeri: Archer's Buzzard (E), Busardo augur somali (S), Buse d'Archer (F) II B 
Buteo augur. Augur Buzzard (E), Busardo augur oriental (S), Buse augure (F) II B

Buteo augur archeri $=$ Buteo archeri

Buteo auguralis: African Red-tailed Buzzard (E), Red-necked Buzzard (E), Red-tailed Buzzard (E), Busardo cuellirrojo (S), Ratonero cuellirrojo (S), Buse d'Afrique (F), Buse à queue rousse (F) II B

Buteo bannermani $=$ Buteo buteo

Buteo brachypterus: Madagascar Buzzard (E), Busardo malgache (S), Buse de Madagascar (F) II B

Buteo brachyurus: Short-tailed Hawk (E), Aguililla colicorta (S), Aguilucho cola corta (S), Busardo colicorto (S), Gavilán cola corta (S), Buse à queue courte (F) II B

Buteo brachyurus albigula $=$ Buteo albigula

Buteo buteo: Common Buzzard (E), Eurasian Buzzard (E), Busardo ratonero (S), Ratonero común (S), Buse variable (F) II B

Buteo galapagoensis: Galapagos Hawk (E), Busardo de Galápagos (S), Gavilán de Galapagos (S), Buse des Galapagos (F) II B

Buteo hemilasius: Upland Buzzard (E), Busardo mongol (S), Buse de Chine (F) II B

Buteo jamaicensis: Red-tailed Hawk (E), Aguililla colinoja (S), Busardo colirrojo (S), Guaraguao (\$), Buse à queue rousse (F) II B

Buteo lagopus: Rough-legged Buzzard (E), Rough-legged Hawk (E), Aguililla artica (S), Busardo calzado (S), Ratonero calzado (S), Buse pattue (F) II B

Buteo leucorrhous: Rufous-thighed Hawk (E), White-rumped Hawk (E), Busardo culiblanco (S), Gavilán negro (S), Gavilán rabadilla blanca (S), Buse cul-blanc (F) II B

Buteo lineatus: Red-shouldered Hawk (E), Aguililla pechirroja (S), Busardo hombrorrojo (S), Buse à épaulettes (F) II B

Buteo magnirostris: Roadside Hawk (E), Aguililla caminera (S), Busardo caminero (S), Gavilán alirrojizo (S), Gavilán común (S), Gavilán habado (S), Buse à gros bec (F) II B

Buteo nitidus = Asturina nitida

Buteo nitidus plagiatus = Asturina plagiata

Buteo oreophilus: Forest Buzzard (E), Mountain Buzzard (E), Busardo montañés (S), Buse montagnarde (F) II B

Buteo platypterus: Broad-winged Hawk (E), Aguililla aluda (S), Busardo aliancho (S), Gavilán bebehumo (S), Petite buse (F) II B

Buteo poecilochrous: Gurney's Hawk (E), Puna Hawk (E), Variable Hawk (E), Aguilucho puna (S), Busardo puneño (S), Gavilán punenno (S), Buse du puna (F) II B

Buteo polyosoma: Red-backed Hawk (E), Aguilucho común (S), Aguilucho variado (S), Busardo dorsirrojo (S), Buse tricolore (F) II B

Bureo regalis: Ferruginous Hawk (E), Aguililla real (S), Busardo herrumbroso (S), Buse rovilleuse (F) II B

Buteo ridgwayi: Hispaniolan Hawk (E), Ridgway's Hawk (E), Busardo de la española (S), Buse de Ridgway (F) II B

Bureo rufinus: Long-legged Buzzard (E), Busardo moro (S), Ratonero moro (S), Buse féroce (F) II B

Buteo rufofuscus: Jackal Buzzard (E), Busardo augur meridional (S), Buse rounoir (F) II B

Buteo solitarius: Hawaitan Hawk (E), Busardo hawaiano (S), Buse d'Hawaï (F) II B

Buteo swainsoni: Swainson's Hawk (E), Aguililla de Swainson (S) Aguilucho langostero (S), Busardo chapulinero (S), Gavilán langostero (S), Buse de Swainson (F) II B

Buteo tachardus $=$ Buteo oreophilus

Buteo ventralis: Red-tailed Hawk (E), Rufous-tailed Hawk (E), Aguilucho cola colorada (S), Busardo patagón (S), Buse de Patagonie (F) II B

Buteogallus aequinoctialis: Rufous Crab-Hawk (E), Busardo-negro del atlántico (S), Gavilán de Manglares (S), Buse buson (F) II B

Buteogallus anthracinus: Common Black-Hawk (E), Lesser BlackHawk (E), Aguililla negra menor (S), Busardo-negro norteño (S), Gavilán cangrejero (S), Buse noire (F) II B

Buteogallus meridionalis: Savanna Hawk (E), Aguilucho colorada (S), Aguilucho sabanero (S), Busardo sabanero (S), Gavilán pita venado (S), Gavilán sabanero (S), Buse roussâtre (F) II B

Buteogallus subrilis: Mangrove Black-Hawk (E), Busardo-negro del pacifico (S), Gavilán negro común (S), Buse des mangroves (F) II B
Buteogallus urubitunga: Great Black Hawk (E), Aguila negra (S), Aguililla negra mayor (S), Busardo-negro urubitinga (S). Gavilán negro grande (S), Buse urubu (F) II B

Butterflies (E): LEPIDOPTERA spp. I/I/NC in

Butterfly of Paradise (E): Omithoptera paradisea II in

Buzzards (E): Butastur spp/Buteo spp/Hamirostra melanosternon II B

Caatinga Parakeet (E): Aratinga cactorum II B

Caballo de Przewalski (S): Equus przewalskii I M

Caballo salvaje (S): Equus prewaiskii I M

Cabassous centralis: Northern Naked-tailed Armadillo (E), Armado de zapilots (S), Tatou (F) III M

Cabassous gymnurus = Cabassous tatouay

Cabassous tatouay: Greater Naked-tailed Armadillo (E), Cabasu de orejas largas (S), Tatú de rabo mol (S), Tatú de rabo molle (S), Tatou à queue nue (F) III M

Cabasu de orejas largas (S): Cabassous fatouay III M

Cabeza de viejo (S): Eira barbara III M

Cabezón (S): Peltocephalus dumeriliana II R

Cabot's Tragopan (E): Tragopan caboti I B

Caburé (S): Micrastur ruficoilis II B/Glaucidium brasilianum II B

Caburé patagónico (S): Glaucidium nanum II B

Cacajao (S): Cacajao calvus I M

Cacajao spp:: Uakaris (E), Ouakaris (F) I M

Cacajao calvus: Bald Uakari (E), Red-and-white Uakari (E), Cacajao

(S), Cacayao (S), Huapo color:do (S), Huapo rojo (S), Uacaries (S), Ouakari chauve (F) I M

Cacajao melanocephalus: Black Uakari (E), Black-headed Uakari (E), Ouakari à tête noire (F) I M

Cacajao rubicundus $=$ Cacajao calvus

Cacapo (S): Strigops habroptilus I B

Cacatoès (F): Cacatua spp/Callocephalon spp./Calyptorhynchus spp./Eolophus spp./Probusciger alerrimus I/II B

Cacatoès à bec gracile (F): Cacatua tenuirostris II B

Cacatoes à huppe blanche (F): Cacatua alba II B

Cacatoès à huppe jaune (F): Cacarua galerita II B

Cacatoès à huppe rouge $(\mathrm{F})$ : Cacatua moluccensis I B

Cacatoès à lores rouges (F): Cacatua pastinator II B

Cacatoès à oeil bleu (F): Cacarua ophthalmica II B

Cacatoès à oreilles blanches $(\mathrm{F})$ : Calyptorhynchus funereus II B

Cacatoès à oreilles jaunes (F): Calyptorhynchus furereus II B

Cacatoès à rectrices blanches $(\mathbf{F})$ : Calyptorhynchus latirostris II B

Cacatoès à tête brune (F): Calyptorhynchus lathami II B

Cacatoès à tête rouge (F): Callocephalon fimbriatum II B

Cacatoès à ventre rouge (F): Cacatua haematuropygia I B

Cacatoès aux yeux bleus (F): Cacatua ophthalmica II B

Cacatoès aux yeux nus (F): Cacatua passinator II B

Cacatoès banksien (F): Calyptorhynchus banksii II B

Cacatoès blanc (F): Cacatua alba II B

Cacatoès casqué (F): Callocephalon fimbriatum II B

Cacatoès corella (F): Cacatua sanguinea II B

Cacatoès de Banks (F): Calyptorhynchus banksii 11 B

Cacatoès de Baudin (F): Calypiorhynchus baudinii II B

Cacatoès de Ducorps (F): Cacatua ducorpsii II B

Cacatoès de Goffin (F): Cacatua goffini I B

Cacatoès de Latham (F): Calyptorhynchus lathami II B

Cacatoès de Leadbeater (F): Cacatua leadbeateri II B

Cacatoès des Moluques (F): Cacarua moluccensis I B

Cacatoès des Philippines (F): Cacatua haematuropygia I B

Cacatoès funèbre (F): Calyptorhynchus funereus II B

Cacatoès laboureur (F): Cacatua pastinator II B

Cacatoès nasique (F): Cacatua renuirostris II B

Cacatoès noir (F): Probosciger aterrimus I B

Cacatoès rosalbin $(\mathbf{F})$ : Eolophus roseicapillus II B

Cacatoès soufré (F): Cacalua sulphurea II B

Cacatua alba: Umbrella Cockatoo (E), White Cockatoo (E), Cacatúa alba $(S)$, Cacatúa blanca $(S)$, Cacatoès blanc $(F)$, Cacatoès à huppe blanche (F) II B

Cacarua alba (S): Cacarua alba II B

Cacatúa blanca (S): Cacatua alba II B

Cacatúa de cabeza roja (S): Callocephalon fimbriatum II B

Cacatúa de cola roja (S): Calyptorhynchus banksii II B

Cacatúa de cola sangrante (S): Cacatua haematuropygia I B

Cacatúa de cresta amarilla grande (S): Cacarua galerita II B

Cacatúa de Ducorps (S): Cacatua ducorpsii II B

Cacatúa de Goffin (S): Cacalua goffini I B 
Cacatía de las Molucas (S): Cacatua moluccensis I 8

Cacatúa de Leadbeater (S): Cacatua leadbeateri II B

Cacania de pico afilado (S): Cacarua pastinator II B

Cacatúa de pico afilado anaranjada (S): Cacatua tenuirostris II B

Cacatua ducorpsii: Ducorps's Cockatoo (E), Cacatúa de Ducorps (S), Cacatoès de Ducorps (F) II B

Cacatúa enlutada (S): Probosciger aterrimus I B

Cacatua galerita: Greater Sulphur-crested Cockatoo (E), Sulphurcrested Cocatoo (E), Cacatúa de cresta amarilla grande (S), Cacatoès à huppe jaune (F), Grand cacatoès à huppe jaune (F) II $\mathrm{B}$

Cacatua goffini: Goffin's Cockatoo (E). Tanimbar Cockatoo (E). Tanimbar Corella (E), Cacatúa de Goffin (S), Cacatoès de Goffin (F) I B

Cacatua haematuropygia: Philippine Cockatoo (E), Red-vented Cockatoo (E), Cacatúa de cola sangrante (S), Cacatoès des Philippines $(\boldsymbol{F})$, Cacatoès à ventre rouge $(\mathrm{F})$ I $\mathrm{B}$

Cacatúa Inca (S): Cacatua leadbeateri II B

Cacatua leadbeateri: Leadbeater's Cockatoo (E), Major Mitchell's Cockatoo (E), Pink Cockatoo (E), Cacanía Inca (S), Cacarúa de Leadbeater (S), Cacatoès de Leadbeater (F) II B

Cacalua moluccensis: Moluccan Cockatoo (E), Salmon-crested Cockatoo (E), Cacatúa de las Molucas (S), Cacatoès des Moluques (F), Cacatoès à huppe rouge (F) I B

Cacatúa negra de cola amarilla (S): Calyptorhynchus funereus II B

Cacatúa negra de cola roja (S): Calyptorhynchus banksii II B

Cacatúa negra de Latham (S): Calyprorhynchus larhami II B

Cacanía oftálmica (S): Cacatua ophthalmica II B

Cacarua ophthalmica: Blue-eyed Cockatoo (E), Cacatúa oftálmica (S), Cacatoès aux yeux bleus (F), Cacatoès à oeil bleu (F) II B

Cacarua pastinator. Westem Corella (E), Cacatúa de pico afilado (S), Cacatoès aux yeux nus ( $F$ ), Cacatoès laboureur $(F)$. Cacatoès à lores rouges (F) II B

Cacania rosada (S): Eolophus roseicapillus II B

Cacatua roseicapilla $=$ Eolophus roseicapillus

Cacatua sanguinea: Bare-eyed Cockatoo (E), Little Corella (E). Cacatoès corella (F) II B

Cacatua sulphurea: Lesser Sulphur-crested Cockatoo (E), Yellowcrested Cockatoo (E), Cacatoès soufré $(F)$, Petit cacatoès à huppe jaune (F) II B

Cacatua tenuirostris: Long-billed Corella (E), Cacatúa de pico afilado anaranjada (S), Cacatoès nasique $(F)$, Cacatoès à bec gracile (F) 11 B

Cacalua tenuirostris pastinator = Cacalua pastinator

Cacayao (S): Cacajao calvus I M

Cachalot (F): Physeter catodon I M

Cachalot nain (F): Kogia simus II M

Cachalot pygmée (F): Kogia breviceps II M

Cachalote (S): Physeter catodon I M

Cachalote cabeza chica (S): Kogia breviceps II M

Cachalote enano (S): Kogia simus II M

Cachalote pigmeo (S): Kogia breviceps II M

Cachelot (E): Physeter catodon I M

Cachorro de mato vinagre (S): Speothos venoticus I M

Cachorro vinagre (S): Speothos venaticus I M

Cacomistle (S): Bassariscus sumichrasti III M

Cacomistles (E): Bassariscus spp. III/NC M

Cactus Conure (E): Aratinga caciorum II B

Cactus Parakeet (E): Aratinga cactorum II B

Caebézon toucan (F): Semnomis ramphastinus III B

Caecobarbus geertsi: African Blind Barb Fish (E), Congo Blind Barb (E), Barbu aveugle (F), Poisson cavemicole d'Afrique (F) II $\mathrm{F}$

Caeloria $=$ Platygyra

Caica Parrot (E): Pionopsitta caica II B

Caiman (E): Alligatoridae spp. I/II R

Caimán (S): Caiman crocodilus I/II R

Caïman à lunettes (F): Caiman crocodilus III R

Caìman à museau large (F): Caiman latirosiris II R

Caïman à paupières osseuses (F): Palaeosuchus palpebrosus II R

Caiman crocodilus: Brown Caiman (E), Common Caiman (E). Spectacled Caiman (E), Caimán (S), Caïman à lunettes (F) III R

Caiman crocodilus apaporiensis: Apaporis River Caiman (E), Rio Apaporis (Spectacled) Caiman (E), Caimán del Río Apaporis

(S), Caïman du Rio Apaporis (F) I R

Caiman crocodilus matogrossiensis = Caiman yacare
Caiman crocodilus paraguavensis = Caiman yacare

Caiman crocodilus yacare = Caiman yacare

Caimán del Río Apaporis (S): Caiman crocodilus apaporiensis I R

Caïman du Rio Apaporis (F): Caiman crocodilus apaporiensis I R

Caimán enano (S): Palaeosuchus palpebrosus II R

Caimán frenuiplano (S): Palaeosuchus rrigonatus II R

Cä̈man hérissé (F): Palceosuchus irigonatus II R

Caimán hociquiancho (S): Caiman Latirostris II R

Caiman latirosiris: Broad-nosed Caiman (E), Broad-snouted Caiman

(E), Caimán hociquiancho (S), Yacaré de hocico ancho (S),

Yacaré overo (S), Caïman à museau large (F) II R

Caiman lizards (E): Dracaena spp. II $\mathrm{R}$

Caimán negro (S): Melanosuchus niger $\mathrm{III} \mathrm{R}$

Caiman noir (F): Melanosuchus niger I/I R

Caiman sclerops = Caiman crocodilus

Caiman sclerops apaporiensis = Caiman crocodilus apaporiensis

Caiman yacare: Yacare Caiman (E), Yacaré (S), Caïman yacare (F) II $\mathrm{R}$

Caïman yacare $(F)$ : Caiman yacare II $R$

Caïque à ailes noires (F): Hapalopsittaca melanotis II B

Caique à capuchon (F): Pionopsina hoematotis II B

Caique à face rouge (F): Hapalopsiuraca amazonina II B

Caique à joues roses (F): Pionopsinta pulchra II B

Caique à queue courte (F): Graydidascalus brachyurus II B

Caïque à tête noire (F): Pionopsitra caica II B

Caique à ventre blanc (F): Pionites leucogaster II B

Caïque de Barraband (F): Pionopsitra barrabandi II B

Caique de Bonapante (F): Pionopsina pyrilia II B

Caique de Fuertes (F): Hapalopsintaca fuertesi II B

Caique de Salvin (F): Hapalopsinaca pyrrhops II B

Caique maipourri (F): Pionires melanocephala II B

Caique mitré (F): Pionopsitia pileata I B

Caique vautourin (F): Gypopsitta vullurina II B

Caiques (E): Pionires spp. II B

Caiques (F): Graydidascalus brachyurus/Gypopsitta vulrurina/Hapalopsillaca spp./Pionires spp./Pionopsilta spp. III B

Cairina harlaubii = Pleronetta harlaubii

Cairina moschara: Muscovy Duck (E), Pato criollo (S), Pato real (S), Canard de Barbarie (F), Canard musqué (F) III B

Cairina scululata: White-winged Duck (E). White-winged Wood Duck (E), Pato almizclero aliblanco (S), Pato de jungla (S), Canard musqué à ailes blanches $(F)$, Canard à ailes blanches $(F)$ I B

Calabar Ground Python (E): Calabaria reinhardrii II $R$

Calabare de Reinhardt ( $F$ ): Calabaria reinhardiii II $R$

Calabaria de Reinhardt (F): Calabaria reinhardiii II R

Calabaria reinhardrii: African Burrowing Python (E), Calabar Ground Python (E), Pitón de Calabar (S), Calabare de Reinhard (F), Calabaria de Reinhardt (F) II R

Calamanian Deer (E): Axis calamianensis I M

Calamian Hog Deer (E): Aris calamianensis I M

Calao à casque (F): Buceros vigil I $\mathrm{B}$

Calao à casque plat (F): Buceros hydrocorax II B

$\mathrm{Calao}$ à casque rond (F): Buceros vigil I B

Calao à casque rouge $(F)$ : Aceros corrugatus II B

Calao à cimier (F): Aceros cassidix II B

Calao à cou roux (F): Aceros nipalensis I B

Calao à gorge claire (F): Aceros subruficollis I B

Calao à poche unie (F): Aceros subruficollis I B

Calao bicome (F): Buceros bicomis I B

Calao brun (F): Anorrhinus fickelli II B

Calao charbonnier (F): Anthracoceros malayanus II B

Calao coiffé ( $\mathbf{F})$ : Aceros comatus II B

Cálao de casco (S): Buceros vigil I B

Calao de Malabar (F): Anthracoceros coronatus II B

Calao de Manille (F): Penelopides manillae II B

Calao de Mindanao (F): Penelopides affinis II B

Calao de Mindoro (F): Penelopides mindorensis II B

Calao de montagne (F): Aceros nipalensis I B

Calao de Narcondam (F): Aceros narcondami II B

Cálao de Narcondam (S): Aceros narcondami II B

Calao de Palawan (F): Anthracoceros marchei II B

Calao de Samar (F): Penelopides samarensis II 8

Calao de Sumba (F): Aceros evereni II B

Calao de Vieillot (F): Aceros leucocephalus II B 


\section{Calao de Walden (F): Aceros waldeni II B} Calao des Célebes (F): Penelopides exarhatus II B Calao des Philippines (F): Buceros hydrocorax II B Calao des Sulu (F): Anthracoceros montani II B Calao festonné (F): Aceros undularus II B Cálao grande (S): Buceros bicomis I B

Calao largup (F): Anorrhinus galeritus II B

Calao papou (F): Aceros plicatus II B

Calao pie (F): Anthracoceros albirostris II B

Calao rhinocéros (F): Buceros rhinoceros II B

Cálao rinoceronte (S): Buceros rhinoceros II B

Cálao rojipardo (S): Buceros hydrocorax II B

Calao tarictic (F): Penelopides panini II B

Calaos (F): Bucerotidae spp. III/Nc B

Calcutta Oval-grain Lizard (E): Varanus flavescens I R

Caldrón negro (S): Globicephala macrorhynchus II M

Caldrón negro (\$): Globicephala melaena II M

California Condor (E): Gymnogyps califormianus I B

California Sea Otter (E): Enhydra lutris nereis I M

Callagur bomeoensis: Painted Batagur (E), Painted Terapin (E) Saw-jawed Turtle (E), Three-striped Batagur (E) II R

Callicebus brunneus: II M

Callicebus caligatus: II M

Callicebus cinerascens: II M

Callicebus cupreus: II M

Callicebus donacophilus: II M

Callicebus dubius: II M

Callicebus hoffmannsi: II M

Callicebus modestus: II M

Callicebus moloch: Dusky Titi (E), Titi molock (F) II M

Callicebus moloch brunneus $=$ Callicebus brunneus

Callicebus moloch caligatus $=$ Callicebus caligatus

Callicebus moloch cinerascens $=$ Callicebus cinerascens

Callicebus moloch cupreus = Callicebus cupreus

Callicebus moloch donacophilus = Callicebus donacophilus

Callicebus moloch dubius $=$ Callicebus dubius

Callicebus moloch hoffmannsi $=$ Callicebus hoffmannsi

Callicebus moloch modestus $=$ Callicebus modestus

Callicebus moloch oenanthe $=$ Callicebus oenanthe

Callicebus moloch olalloe $=$ Callicebus olallae

Callicebus oenanthe: Andean Titi Monkey (E) II M

Callicebus olallae: Beni Titi Monkey (E) II M

Callicebus personatus: Masked Titi (E), Titi à masque (F) II M

Callicebus forqualus: Widow Titi (E), Titi à collier (F) II M

Callimico goeldii: Goeldi's Marmoset (E), Goeldi's Monkey (E), Goeldi's Tamarin (E), Pichico negro (S), Tití de Goeldi (S), Tamarin de Goeldi (F), Tamarin sauteur (F) I M

Calliope Hummingbird (E): Stellula calliope II B

Calliphlox amethystina: Amethyst Woodstar (E), Estrellita amatista (S), Picaflor amatista (S), Tucusito amatista (S), Colibri améthyste (F) II B

Calliphlox bryantae $=$ Philodice bryantae

Calliphlox evelynae: Bahama Woodstar (E), Colibri des Bahamas (F) II B

Calliphlox mitchellii $=$ Philodice mitchelli

Calliste superbe (F): Tangara fastuosa II B

Callistes (F): Chlorochrysa spp/Iridophanes pulcherrima/Tangara spp. II/NC B

Callithrix argentata: Black-tailed Marmoset (E), Silvery Marmoset (E), Ouistiti argenté (F) II M

Callithrix aurita: Buffy-tufted-ear Marmoset (E), White-eared Marmoset $(\mathrm{E})$, Marmouset à oreilles blanches $(\mathrm{F})$, Oustiti oreillard (F), Oustiti à oreilles blanches (F) I M

Callithrix emiliae $=$ Callithrix argentata

Callithrix flaviceps: Buffy-headed Marmoset (E), Ouistiti à tête jaune (F) I M

Callithrix geoffroyi: Geoffroy's Tufted-ear Marmoset (E), Whitefronted Marmoset (E), Tití de caba blanca (S) II M

Callithrix humeralifer: Tassel-eared Marmoset (E), Marmouset Santarem (F) II M

Callithrix intermedius $=$ Callithrix argentata

Callithrix jacchus: Common Marmoset (E), Ouistiti (F) II M

Callithrix jacchus aurita $=$ Callithrix aurita

Callithrix jacchus flaviceps $=$ Callithrix flaviceps

Callithrix jacchus geoffroyi $=$ Callithrix geoffroyi

Callithrix jacchus kuhlii $=$ Callithrix kuhlii
Callithrix jacchus penicillata $=$ Callithrix penicillata

Callithrix kuhlii: II M

Callithrix leucippe $=$ Callithrix argentata

Callithrix penicillata: Black-pencilled Marmoset (E) II M

Callithrix pygmaea: Pygmy Marmoset (E), Ouistiti mignon (F) II M

Callocephalon fimbriatum: Gang-gang Cockatoo (E), Cacatúa de cabeza roja (S), Gan-Gan (S), Cacatoès casqué (F), Cacatoès à tête rouge (F) II B

Callogyra $=$ Trachyphyllia

Caloenas nicobarica: Nicobar Dove (E), Nicobar Pigeon (E), Paloma nicobar (S), Nicobar à camail (F), Pigeon nicobar (F), Pigeon à camail $(\mathrm{F})$, Pigeon à collerette $(\mathrm{F})$ I B

Caloperdix oculea: Ferruginous Partridge (E), Ferruginous WoodPartridge (E), Perdicilla herrumbrosa (S), Perdiz de bosque ferruginosa (S), Rouloul ocellé (F) III B

Caloprymnus campestris: Buff-nosed Rat-kangaroo (E), Desert Ratkangaroo (E), Plains Rat-kangaroo (E), Canguro-rata desértico (S), Kangourou-rat du désert (F) I M

Calopsitte élégante (F): Nymphicus hollandicus NC B Perruche calopsitte (F), Lorito de copete (S)

Calothorax eliza $=$ Doricha elize

Calothorax enicura $=$ Doricha enicura

Calothorax lucifer: Lucifer Hummingbird (E), Tijereta nortena (S), Colibri lucifer (F) II B

Calothorax pulcher: Beautiful Hummingbird (E), Tijereta Oaxaquena (S), Colibri charmant (F) II B

Calumma boettgeri $=$ Chamaeleo boettger

Calumma brevicomis $=$ Chamaeleo brevicomis

Calumma capuroni $=$ Chamaeleo capuroni

Calumma cucullata $=$ Chamaeleo cucullatus

Calumma fallax = Chamaeleo fallar

Calumma furcifer $=$ Chamaeleo furcifer

Calumma gallus = Chamaeleo gallus

Calumma gastrotaenia $=$ Chamaeleo gastrotaenia

Calumma globifer $=$ Chamaeleo globifer

Calumma guibe $\mathrm{i}=$ Chamaeleo guibei

Calumma linota $=$ Chamaeleo linotus

Calumma malthe $=$ Chamaeleo malthe

Calumma nasura $=$ Chamaeleo nasutus

Calumma oshaughnessyi = Chamaeleo oshaughnessyi

Calumma parsonii $=$ Chamaeleo parsonii

Calumma peyrieresi $=$ Chamaeleo peyrieresi

Calumma rigris $=$ Chamaeleo tigris

Calumma isaratananensis = Chamaeleo tsaratananensis

Calypte anna: Anna's Hummingbird (E), Colibri de Anna (S), Colibri d'Anna (F) II B

Calypte costae: Costa's Hummingbird (E), Colibri de Costa (F) II B

Calypte helenae $=$ Mellisuga helenae

Calyptopora reticulata: II In

Calyptopora sinuosa: II In

Calyptorhynchus banksii: Red-tailed Black-Cockatoo (E), Cacatúa de cola roja (S), Cacatúa negra de cola roja (S), Cacatoès banksien (F), Cacatoès de Banks (F) II $B$

Calyptorhynchus baudinii: White-tailed Black-Cockatoo (E), Cacatoès de Baudin (F) II B

Calyptorhynchus funereus: Yellow-tailed Black-Cockatoo (E), Cacatúa negra de cola amarilia (S), Cacatoès funèbre (F), Cacatoès à oreilles blanches $(F)$, Cacatoès à oreilles jaunes $(F)$ II B

Calyptorhynchus funereus baudinii = Calyptorhynchus baudinii Calyptorhynchus funereus latirostris = Calyptorhynchus latirostris Calyptorhynchus lathami: Glossy Black-Cockatoo (E), Cacatúa negra de Latham $(S)$, Cacatoès de Latham $(F)$, Cacatoès à tête brune (F) II B

Calyptorhynchus latirostris: Slender-billed Black-Cockatoo (E), Cacatoès à rectrices blanches (F) II B

Calyptorhynchus magnificus = Calyptorhynchus banksii

Calzonario colibronceado (S): Chalybura buffonii II B

Camaleón africano (S): Chamaeleo africanus II R

Camaleón crestado (S): Chamaeleo cristatus II R

Camaleón cuadricome (S): Chamaeleo quadricomis II R

Camaleón de Boettger (S): Chamaeleo boettgeri II R

Camaleón de casco (S): Chamaeleo hoehnelii II R

Camaleón de dos bandas (S): Chamaeleo biraeniarus II R

Camaleón de Elliot (S): Chamaeleo ellioti II R

Camaleón de Fuelleborn (S): Chamaeleo fuellebomi II R 
Camaleón de Jackson (S): Chamaeleo jacksonii II R

Camaleón de Johnston (S): Chamaeleo johnsıoni II R

Camaleón de Marshall (S): Chamaeleo marshalli II R

Camaleón de Meller (S): Chamaeleo melleri II R

Camaleón de montan̄a (S): Chamaeleo montium II R

Camaleón de Oustalet (S): Chamaeleo oustaleti II R

Camaleón de Owen (S): Chamaeleo oweni II R

Camaleón de Parson (S): Chamaeleo parsonii II R

Camaleón de Petter (S): Chamaeleo petteri II R

Camaleón de Pfeffer (S): Chamaeleo pfefferi II R

Camaleón de Schouteden (S): Chamaeleo schoutedeni II R

Camaleón de Schubotz (S): Chamaeleo schubotzi II R

Camaleón de Sri Lanka (S): Chamaeleo zeylanicus II R

Camaleón de Wemer (S); Chamaeleo wemeri II R

Camaleón de Wiedersheim (S): Chamaeleo wiedersheimi II R

Camaleón del Senegal (S): Chamaeleo senegalensis II R

Camaleón grácil (S): Chamaeleo gracilis II R

Camaleón lobulado (S): Chamaeleo dilepis II R

Camaleón rinoceronte (S): Chamaeleo rhinocerarus II R

Camaleón verrugoso (S): Chamaeleo verrucosus II $R$

Camaleónes (S): Chamaeleo spp. II R

Camaleónes enanos (S): Bradypodion spp. II R

Caméléon (F): Chamaeleo laevigatus II $R$

Caméléon à bandes latérales $(F)$ : Chamaeleo lateralis II $R$

Caméléon à capuchon (F): Chamaeleo cucullarus II R

Caméléon à casque élevé (F): Chamaeleo hoehnelii II R

Caméleon à casque plat (F): Chamaeleo globifer II R

Caméléton à cornes courtes $(\mathrm{F})$ : Chamaeleo brevicomis II $\mathrm{R}$

Caméléon à deux bandes (F): Chamaeleo bitaeniatus $11 \mathrm{R}$

Caméléon à deux comes (F): Chamaeleo bifídus II R

Caméléon à écailles doubles (F): Chamaeleo anchierae II R

Caméléon à lignes blanches (F): Chamaeleo antimena II R

Caméléon à nez fourchu (F): Chamaeleo bifidus II R/Chamaeleo furcifer II R

Caméléon à quatre cornes (F): Chamaeleo quadricornis II $R$

Caméléon à une come (F): Chamaeleo monoceras II R

Caméléon à voile du Cameroun (F): Chamaeleo montium II R

Caméléon africain (F): Chamaeleo africanus II R

Caméléon américain (F): Chamaeleo affinis II $\mathrm{R}$

Caméléon bilobé (F): Chamaeleo dilepis II $\mathbf{R}$

Caméléon casqué (F): Chamaeleo calyptratus II R

Caméléon commun (F): Chamaeleo chamaeleon II R

Caméleon común (S): Chamaeleo chamaeleon II R

Caméléon crêté (F): Chamaeleo cristatus II R

Caméléon d'Andrenalamivola (F): Chamaeleo ruzetae II R

Caméléon d'Angel (F): Chamaeleo angeli II R

Caméléon d'Angola (F): Chamaeleo anchietae II R

Caméléon d'Arabie (F): Chamaeleo arabicus II R

Caméléon d'Eisentraut (F): Chamaeleo eisentrauti II R

Caméléon d'Elliot (F): Chamaeleo ellioti II R

Caméléon d'Đlolo (F): Chamaeleo goeszei II R

Caméléon d'O'Shaughnessy (F): Chamaeleo oshaughnessyi II R

Caméléon d'Oustalet (F): Chamaeleo ousialeti II R

Caméléon d'Owen (F): Chamaeleo oweni II R

Caméléon de Belalanda (F): Chamaeleo belalandaensis II R

Caméléon de Bocage (F): Chamaeleo quilensis II R

Caméléon de Boettger (F): Chamaeleo boestgeri II R

Caméléon de Ceylan (F): Chamaeleo zeylanicus II R

Caméléon de Chapin (F): Chamaeleo chapini II R

Caméléon de Fea (F): Chamaeleo feae II R

Caméléon de Goetze (F): Chamaeleo goetzei II R

Caméléon de Guibé (F): Chamaeleo guibei II R

Caméléon de Günther (F): Chamaeleo minor II R

Caméléon de Jackson (F): Chamaeleo jacksonii II R

Caméléon de Johnston (F): Chamaeleo johnsioni II R

Caméléon de la canopée (F): Chamaeleo willsii II R

Caméléon de Laborde (F): Chamaeleo labordi II R

Caméléon de Lanza (F): Chamaeleo ruspolii II $R$

Caméléon de Leach (F): Chamaeleo dilepis II $\mathrm{R}$

Caméléon de Madagascar (F): Chamaeleo capuroni II R

Caméléon de Maroantsetra (F): Chamaeleo linorus II R

Caméléon de Marshall (F): Chamaeleo marshalli II R

Caméléon de Mayotte (F): Chamaeleo polleni II R

Caméléon de Meller (F): Chamaeleo melleri II R

Caméléon de Mocquard (F): Chamaeleo fallax II R

Caméléon de Parson (F): Chamaeleo parsonii II $\mathbf{R}$
Caméléon de Perinet (F): Chamaeleo gastrotaenia II R

Caméléon de Petter (F): Chamaeleo petteri II R

Caméléon de Peyrieras (F): Chamaeleo peyrieresi II R

Caméléon de Pfeffer (F): Chamaeleo pfefferi II R

Caméléon de Rüppell (F): Bradypodion xenorhinus II R

Caméléon de Schoudeten (F): Chamaeleo schouredeni II R

Caméléon de Schubotz (F): Chamaeleo schubotzi II R

Caméleon de Tilbury (F): Chamaeleo marsabitensis II R

Caméléon de Tsaratana (F): Chamaeleo tsaratananensis II R

Caméléon de Wemer (F): Chamaeleo wemeri II R

Caméléon de Wills (F): Chamaeleo willsii II R

Caméléon des Comores (F): Chamaeleo cephalolepis II R

Caméléon des montagnes de Tanzanie (F): Chamaeleo tempeli II R

Caméléon des Monts Uzungwe (F): Chamaeleo laterispinis II R

Caméléon du Cameroun (F): Chamaeleo camerunensis II R

Caméléon du Mont Lefo (F): Chamaeleo wiedersheimi II R

Caméléon du Mont Poroto (F): Chamaeleo incomutus II R

Caméléon du Namaqua (F): Chamaeleo namaquensis II R

Caméléon du Sénégal (F): Chamaeleo senegalensis II R

Caméléon du volcan Ngosi (F): Chamaeleo fuellebomi II R

Caméléon đu Yémen (F): Chamaeleo calcaricarens II R

Caméléon épée (F): Chamaeleo gallus II $R$

Caméléon forestier ( $F$ ): Chamaeleo balteatus II

Caméléon forestier de Madagascar (F): Chamaeleo campani II R

Caméléon gracile (F): Chamaeleo gracilis II $\mathbf{R}$

Caméléon gris (F): Chamaeleo chapini II R

Caméléon nain à nez rouge $(\mathrm{F})$ : Bradypodion nemorale II $\mathbf{R}$

Caméléon nain à tête noire $(\mathrm{F})$ : Bradypodion karroicum II $\mathrm{R}$

Caméléon nain d'Ituri (F): Bradypodion adolfifriderici II R

Caméléon nain de Carpenter (F): Bradypodion carpenteri II R

Caméléon nain de Fischer (F): Bradypodion fischeri II R

Caméléon nain de Karro (F): Bradypodion gutturale II R

Caméléon nain de Matschie (F): Bradypodion tavetanum II $\mathbf{R}$

Caméléon nain de Muller (F): Bradypodion iransvaalense II R

Caméléon nain de Setaro (F): Bradypodion pumilum II R

Cameléon nain de Smith (F): Bradypodion spinosum II $\mathrm{R}$

Caméléon nain des Monts Ruwenzori (F): Bradypodion ventrale II R

Caméléon nain du Cap (F): Bradypodion oxyrhinum II R

Caméléon nain du Drakensberg (F): Bradypodion dracomontanum II $\mathrm{R}$

Caméléon nain du Kenya (F): Bradypodion damaranum II R

Caméléon nain du Mont Ulanje (F): Bradypodion melanocephalum II $R$

Caméléon nain du Natal (F): Bradypodion tenue II R

Caméléon nain du sud et du Namaqua (F): Bradypodion uthmoelleri II $\mathrm{R}$

Caméléon nain du Transkei (F): Bradypodion caffer II R

Caméléon nain du Transvaal (F): Bradypodion thamnobates II $\mathbf{R}$

Caméléon nain du Zululand (F): Bradypodion mlanjensis II R

Caméléon nain épineux (F): Bradypodion selaroi II R

Caméléon nasique (F): Chamaeleo nasutus II R

Caméléon ondulé ( $F$ ): Chamaeleo deremensis II R

Caméléon panthère (F): Chamaeleo pardalis II R

Caméléon rhinocéros ( $\mathrm{F}$ ): Chamaeleo rhinoceratus II $\mathrm{R}$

Caméléon rude (F): Chamaeleo rudis II R

Caméléon tigre (F): Chamaeleo tigris II R

Caméléon verruqueux (F): Chamaeleo verrucosus II $\mathbf{R}$

Caméléon vert-et-jaune (F): Chamaeleo malthe II R

Caméléon vulgaire (F): Chamaeleo chamaeleon II R

Camélếon-joyau (F): Chamaeleo lateralis II R

Caméléon-moine (F): Chamaeleo monachus II R

Caméléons (F): Chamaeleo spp. II R

Caméléons nain (F): Bradypodion spp. II R

Cameroon Clawless Otter (E): Aonyx congicus /II M

Cameroon Sailfin Chameleon (E): Chamaeleo montium II R

Cameroon Toad (E): Bufo superciliaris I A

Campbell's Girdled Lizard (E): Cordylus campbelli II R

Campbell's Guenon (E): Cercopithecus campbelli II M

Campbell's Monkey (E): Cercopithecus campbelli II M

Campbell's Spiny-tailed Lizard (E): Cordylus campbelli II R

Campephilus imperialis: Imperial Woodpecker (E), Carpintero gigante (S), Pito imperial (S), Pitorreal ocotero (S), Pic impérial (F) I B

Campyloptère à queue blanche (F): Campyloplerus ensipennis II B

Campyloptère à ventre gris (F): Campylopterus largipennis II B

Campyloptère de Wetmore (F): Campylopterus excellens II B 
Campyloptère des Santa Marta (F): Campylopterus phainopeplus II B

Campyloptère du Napo (F): Campylopterus villaviscensio II B

Campyloptère lazulite (F): Campyloptenus falcatus II B

Campyloptère montagnard (F): Campylopterus duidae II B

Campyloptère pampa (F): Campylopterus curvipennis II B

Campyloptère rougeâtre (F): Campylopterus hyperythrus II B

Campyloptère roux (F): Campylopterus rufus II B

Campyloptère violet (F): Campylopterus hemileucurus II B

Campyloptères (F): Campylopterus spp. II B

Campyloptenus curvipennis: Wedge-tailed Sabrewing (E), Fandangero colicuna (S), Campyloptère pampa (F) II B

Campylopterus curvipennis excellens = Campylopterus excellens

Campylopterus duidae: Buff-breasted Sabrewing (E), Ala de sable anteado (S), Campyloptère montagnard (F) II B

Campylopterus ensipennis: White-tailed Sabrewing (E), Ala de sable verde (S), Campyloptère à queue blanche (F) II B

Campylopterus excellens: Long-tailed Sabrewing (E), Fandangero colilargo (S), Campyloptère de Wetmore (F) II B

Campylopterus falcatus: Lazuline Sabrewing (E), Ala de sable pechivioleta (S), Alasable azulino (S), Campyloptère lazulite (F) II B

Campyloptenus hemileucurus: Violet Sabrewing (E), Fandangero morado (S), Campyloptère violet (F) II B

Campylopterus hyperythrus: Rufous-breasted Sabrewing (E), Ala de sable rufo (S), Campyloptère rougeâtre (F) II B

Campyloptenus largipennis: Grey-breasted Sabrewing (E), Ala de sable gris $(\mathrm{S})$, Alasable del Napo (S), Campyloptère à ventre gris (F) II B

Campylopterus phainopeplus: Santa Marta Sabrewing (E) Campyloptère des Santa Marta (F) II B

Campylopterus rufus: Rufous Sabrewing (E), Fandangero rufo (S), Campyloptère roux (F) II B

Campylopterus villaviscensio: Napo Sabrewing (E), Campyloptère du Napo (F) II B

Canada Lynx (E): Lynx canadensis II M

Canal (S): Dermochelys coriacea I R

Canard à ailes blanches (F): Cairina scutulara I B

Canard à bosse bronzé (F): Sarkidiomis melanotos II B

Canard à tête rose (F): Rhodonessa caryophyllacea I B

Canard aberrant (F): Anas oustaleri I B

Canard armé (F): Plectropterus gambensis III B

Canard coronculé (F): Sarkidiornis melanotos II B

Canard de Barbarie (F): Cairina moschasa III B

Canard de Bemier (F): Anas bemieri II B

Canard de Hartlaub (F): Pteronetta hartlaubii III B

Canard de Laysan (F): Anas laysanensis I B

Canard d'Oustalet (F): Anas ousialeri I B

Canard du Cap (F): Anas capensis III B

Canard musqué (F): Cairina moschata III B

Canard musqué à ailes blanches (F): Cairina scumulata I B

Canard pilet (F): Anas acuta $111 \mathrm{~B}$

Canard siffleur (F): Anas penelope III B

Canard souchet (F): Anas clypeara III B

Canards (F): Anatidae spp. I/LIII/NC B

Canaries (E): Serinus spp. III/NC B

Canario de Mozambique (S): Serinus mozambicus III B

Canary-winged Parakeet (E): Brotogeris versicolurus II B

Candoia aspera: New Guinea Viper Boa (E), Papuan Ground Boa

(E), Boa de Nueva Guinea (S), Boa vipère de Nouvelle Guinée (F) II R

Candoia bibroni: Pacific Boa (E), Boa de Fiji (S), Boa nain de Bibron (F), Boa nain des îles du Pacifique (F) II R

Candoia carinata: Solomon Ground Boa (E), Tree Boa (E), Boa del Pacífico $(\mathrm{S})$, Boa nain des Salomons $(\mathrm{F})$, Boa nain à carènes $(\mathrm{F})$ II R

Cangurito narigudo coliblanco (S): Macrotis leucura I M

Cangurito narigudo grande (S): Macrotis lagotis I M Cangurito piedecerdo (S): Chaeropus ecaudatus I M

Canguro arborícola gris (S): Dendrolagus inustus II M Canguro arborícola negro (S): Dendrolagus ursinus II M Canguro rabipelado occidental (S): Onychogalea lunaia I M Canguro rabipelado oriental (S): Onychogalea fraenata I M

Canguro-liebre peludo (S): Lagorchestes hirsutus I M

Canguro-liebre rayado (S): Lagostrophus fasciatus I M

Canguro-rata colipeludo (S): Bettongia penicillaza I M
Canguro-rata de Lesueur (S): Betrongia lesueur I M

Canguro-rata de Tasmania (S): Bettongia gaimardi I M

Canguro-rata desértico (S): Caloprymnus campestris I M

Canguro-ratas (S): Betrongia spp. I M

Canis aureus: Golden Jackal (E), Chacal commun (F), Chacal doré (F) III M

Canis lupus: Common Wolf (E), Grey Wolf (E), Timber Wolf (E),

Wolf (E), Lobo común (S), Loup (F), Loup gris (F), Loup vulgaire (F) I/I M

Canopy Chameleon (E): Chamaeleo willsii II R

Cantharellus doederleini: II In

Cantharellus jebbi: II in

Cantharellus noumeae: II In

Cantil (E): Agkistrodon bilineatus III R

Caouanne (F): Caretta caretta I R

Cape Clawless Otter (E): Aonyx capensis II M

Cape Crag Lizard (E): Pseudocordylus microlepidotus II R

Cape Dwarf Chameleon (E): Bradypodion pumilum II R

Cape Eagle-Owl (E): Bubo capensis II B

Cape Fur Seal (E): Arctocephalus pusillus II M

Cape Girdled Lizard (E): Cordylus cordylus II R

Cape Griffon (E): Gyps coprotheres II B

Cape Mountain Zebra (E): Equus zebra zebra I M

Cape Pangolin (E): Manis remminckii II M

Cape Parrot (E): Poicephalus robustus II B

Cape Teal (E): Anas capensis III B

Cape Vulture (E): Gyps coprotheres II B

Cape Wigeon (E): Anas capensis III B

Caperea marginata: Pygmy Right Whale (E), Ballena franca pigmea

(S), Baleine pygmée (F) I M

Capped Gibbon (E): Hylobates pileatus I M

Capped Langur (E): Trachypithecus pileatus I M

Capped Leaf Monkey (E): Trachypithecus pileatus I M

Capped Monkey (E): Trachypithecus pileatus I M

Capra falconeri: Markhor (E), Markhor (S), Markhor (F) I M

Capricorne de Sumatra (F): Naemorhedus sumatraensis I M

Capricomis sumatraensis $=$ Naemorhedus sumatraensis

Caprolagus hispidus: Assam Rabbit (E), Hispid Hare (E), Conejo de Assam (S), Lapin de l'Assam (F) I M

Capuchins (E): Cebus spp. II M

Capucin bec-d'argent (F): Lonchura cantans III B

Capucin bicolore (F): Lonchura bicolor III B

Capucin nonnette (F): Lonchura cucullata III B

Capucin pie (F): Lonchura fringilloides III B

Capucins (F): Lonchura spp. III/NC B

Cárabo común (S): Strix aluco II B

Cárabo de Hume (S): Strix butleri II B

Cárabo de Sudamérica (S): Strix nigrolineata II B

Caracal (E/S/F): Caracal caracal $\mathbf{I} / \mathbf{I}$ M

Caracal caracal: African Caracal (E), Asian Caracal (E), Caracal

(E), Desert Lynx (E), Caracal (S), Lince africano (S), Caracal

(F), Lynx du désert (F) I/II M

Caracara à gorge blanche (F): Phalcoboenus albogularis II B

Caracara à gorge rouge $(\mathrm{F})$ : Daptrius americanus II B

Caracara à tête jaune $(\mathrm{F})$ : Milvago chimachima II B

Caracara à ventre rouge (F): Daptrius americanus II B

Caracara andino (S): Phalcoboenus megalopterus II B

Caracara araucano (S): Phalcoboenus albogularis II B

Caracara austral (S/F): Phalcoboenus australis II B

Caracara carancho (S): Polyborus plancus II B

Caracara caronculé (F): Phalcoboenus carunculatus II B

Caracara carunculado (S): Phalcoboenus carunculatus II B

Caracara chimachima (S/F): Milvago chimachima II B

Caracara chimango (S/F): Milvago chimango II B

Caracara comecacao (S): Daptrius americanus II B

Caracara commun (F): Polyborus plancus II B

Caracara común (S): Polyborus plancus II B

Caracara de Guadalupe (F): Polyborus lutosus II B

Caracara funèbre $(\mathbf{F})$ : Phalcoboenus australis II B

Caracara gorgirojo (S): Daptrius americanus II B

Caracara huppé (F): Polyborus plancus II B

Caracara montagnard (F): Phalcoboenus megalopterus II B

Caracara negro (\$): Daptrius ater II B

Caracara noir (F): Daptrius ater II B

Caracaras (E/F): Falconidae spp. II B

Carachupa Manan (S): Priodontes maximus I M 
Caracolero plomizo (S): Rostrhamus hamatus II B

Carancho (S): Polyborus plancus II B

Carancho andino (S): Phalcoboenus megalopterus II B

Carancho de Guadalupe (S): Polyborus lutosus II B

Carancho negro (S): Daptrius ater II B

Carancho ventri-blanco (S): Daptrius americanus II B

Carapaico (S): Aratinga acuricaudata II B

Carburé acanelado (S): Aegolius harrisii II B

Cardenal amarillo (S): Gubematrix cristara II B

Cardenal cabecirrojo (S): Paroaria capisata II B

Cardenal copetón (S): Paroaria coronata II B

Cardenalito (S): Carduelis cucullata I B

Cardinal Lory (E): Chalcopsitta cardinalis II B

Cardinal vert (F): Gubematrix cristala II B

Cardinals (E): Gubemarrix $\operatorname{spp} /$ Paroaria spp/Cardinalis spp. II/NC B

Carduelis cucullara: Red Siskin (E), Cardenalito (S), Lúgano cardenalito (S), Lugano encapuchado (S), Chardonneret rouge (F), Tatin rouge du Venezuela (F) I B

Carduelis yarrelli: Yellow-faced Siskin (E), Jilguero cara amarilla (S), Jilguero de Yarrell (S), Luigano de Yarrell (S), Chardonneret de Yarrell (F), Tatin de Yarrell (F) II B

Caret (F): Eretmochelys imbricata I R

Caretta caretla: Loggerhead (E), Cayuma (S), Tortuga boba (S), Caouanne (F), Tortue caouanne (F) I R

Caribbean Amazon (E): Amazona leucocephala I B

Caribbean Flamingo (E): Phoenicopterus ruber II B

Caribbean Manatee (E): Trichechus manatus I M

Caribbean Monk Seal (E): Monachus tropicalis I M

Caribs (E): Eulampis spp. II B

Caricare encrestado (S): Polyborus plancus II B

Caricare sabanero (S): Milvago chimachima II B

Carnard des Mariannes (F): Anas oustaleti I B

Carnation Coral (E): Pectinia lactuca II In

Camero del Canadá (S): Ovis canadensis I/I M

Camero salvaje (S): Ovis canadensis I/I M

Camifex (F): Micrastur spp/Spiziapteryx circumcinctus II B

Camifex à ailes tachetées (F): Spiziapteryx circumcincius II B

Camifex à collier (F): Micrastur semirorquatus II B

Camifex à cou roux (F): Micrastur ruficollis II B

Camifex à gorge cendrée (F): Micrastur gilvicollis II B

Carnifex ardoisé (F): Micrastur mirandollei II B

Camifex barré (F): Micrastur ruficollis II B

Carnifex de Buckley (F): Micrastur buckleyi II B

Camifex de Traylor (F): Micrasrur buckleyi II B

Camifex plombé (F): Micrastur plumbeus II B

Carocolero común (S): Rosirhamus sociabilis II B

Carola's Parotia (E): Parotia carolae II B

Carouge safran (F): Agelaius flavus I B

Carouges (F): Agelaius spp/Gymnomystax mexicanus/Nesopsar nigerrimus/Xanthocephalus xanthocephalus I/NC B

Carpet Chameleon (E): Chamaeleo lateralis II R

Carpet Python (E): Morelia spilota II R

Carpintero gigante (S): Campephilus imperialis I B

Carpophage de Mindoro (F): Ducula mindorensis I B

Carpophages (F): Ducula spp. UNC B

Carunculated Caracara (E): Phalcoboenus carunculatus II B

Carunculina glans = Toxolasma cylindrellus

Caryophyllia abyssonum: II In

Caryophyllia alaskensis: II In

Caryophyllia alberti: II in

Caryophyllia ambrosia: II in

Caryophyllia antarctica: II In

Caryophyllia antillarum: II In

Caryophyllia amoldi: II In

Caryophyllia aflantica: II In

Caryophyllia balaenacea: II In

Caryophyllia barbadensis: II In

Caryophyllia berteriana: II In

Caryophyllia burchae: II In

Caryophyllia calver: II in

Caryophyllia capensis: II In

Caryophyllia clavus = Caryophyllia smithii

Caryophyllia compressa: II In

Caryophyllia cornuformis: II In

Caryophyllia corrugata: II In
Caryophyllia cultrifera = Caryophyllia scobinosa

Caryophyllia cyashus: II In

Caryophyllia dentiformis: II In

Canyophyllia diomedeae: II In

Caryophyllia elongata: II In

Caryophyllia elianinae: II In

Canophyllia ephyala: II in

Caryophyllia epithecata: II In

Canyophyllia foresti: 11 In

Caryophyllia grandis: II In

Caryophyllia grayi: II In

Caryophyllia hawaiiensis: II In

Caryophyllia horologium: II In

Caryophyllia inomata: II In

Canyophyllia japonica: II In

Caryophyllia jogashimaensis: II In

Caryophyllia lamellifera: II in

Caryophyllia mabahithi: II In

Caryophyllia marmorea: II In

Caryophyllia ocsopali: II In

Caryophyllia panda = Caryophyllia atlantica

Caryophyllia paradoxus: II In

Caryophyllia parvula: II In

Caryophyllia paucipalata: II In

Caryophyllia pauciseptata: II in

Caryophyllia perculta: II In

Caryophyllia planilamellata: II In

Caryophyllia polygona: II In

Caryophyllia profunda: II In

Canyophyllia quadragenaria: II In

Caryophyllia ralphae: II in

Canyophyllia rugosa: II In

Caryophyllia sarsiae $=$ Caryophyllia diomedeae

Canyophyllia scillaemorpha: II In

Caryophyllia scobinosa: II In

Canyophyllia seguenzae: II In

Caryophyllia sewelli: II In

Caryophyllia smithii: Devonshire Cup Coral (E) II In

Canyophyllia solida: II In

Caryophyllia spinicarens: II In

Caryophyllia spiniger. II In

Caryophyllia squiresi: II In

Canophyllia transversalis: II In

Caryophyllia valdiviae: II In

Caryophyllia zopyros: II In

Casarea dussumieri: Round Island Keel-scaled Boa (E), Boa de Round Island (S), Boa de l'lle Ronde de Dussumier (F) I R

Cascabel (E): Croialus durissus III R

Cascavelle (F): Crotalus durissus III $\mathrm{R}$

Casmerodius albus: Great Egret (E), Great White Egret (E), Garceta grande (S), Garza blanca real (S), Garzetta grande (S), Grande aigrette (F) III B

Caspian Snowcock (E): Tetraogallus caspius I B

Cassin's Hawk-Eagle (E): Spizaerus africanus II B

Cassin's Malimbe (E): Malimbus cassini III B

Catagonus wagneri: Chacoan Peccary (E), Tagua (E), Chaco argentino (S), Quimilero (S), Pécari du Chaco (F) I M

Caralaphyllia jardinei: II In

Catalaphyllia okinawensis: II In

Catalaphyllia plicata: II In

Caralaphyllia sabiuraensis: II in

Cataquil (F): Bassaricyon gabbii III M

Catfish (E): SILURIFORMES spp. UNC F

Catita aimará (S): Bolborhynchus aymara II B

Catita aliamarilla (S): Brotogeris versicolurus II B

Catita aliazul (S): Brotogeris cyanoptera II B

Catita alidorada (S): Brotogeris chrysopierus II B

Catita andina (S): Bolborhynchus orbygnesius II B

Catita barrada (S): Bolborhynchus lineola II B

Catita chiriri (S): Brotogeris chiriri II B

Catita churica (S): Brotogeris jugularis II B

Catita común (S): Myiopsitta monachus II B

Catita enana (S): Forpus xanthopteryius II B

Catita frentidorada (S): Bolborhynchus aurifrons II B

Catita frentigualda (S): Brologeris sanctithomae II B

Catita frentimufa (S): Bolborhynchus ferrugineifrons II B 
Catita macareña (S): Brotogeris pyrrhopterus II B Catita serrana común (S): Bolborhynchus aymara II B Catita serrana verde (S): Bolborhynchus aurifrons II B Catita tirica (S): Brotogeris tirica II B

Catita versicolor (S): Brotogeris versicolurus II B

Catitas (S): Bolborhynchus spp/Brologeris spp. II B

Catopuma badia: Bay Cat (E), Bornean Marbled Cat (E), Chat bai (F) II M

Catopuma remminckii: Asiatic Golden Cat (E), Golden Cat (E), Temminck's Cat (E), Gato dorado asiático (S), Chat de Temminck (F), Chat doré d'Asie (F) I M

Carreus wallichii: Cheer Pheasant (E), Chir Pheasant (E), Wallich's Pheasant (E), Faisán chir (S), Faisán de Wallich (S), Faisan de Wallich (F), Faisan de l'Himalaya (F) I B

Cats (E): Felidae spp. I/I M

Catspaw mussels (E): Epioblasma spp. I/II/NC In

Catspaws (E): Epioblasma spp. I/U/NC In

Cattle Egret (E): Bubulcus ibis III B

Cauca Poison Frog (E): Minyobates bomberes II A

Caucasian Sand Boa (E): Eryx jaculus II R

Caucel (S): Leopardus tigrinus I M /Leopardus wiedii I M

Caulastraea curvata: II In

Caulastraea echinulaia: II In

Caulasiraea furcata: II In

Caulastraea plana: II in

Caulastraea tumida: II in

Cavernous Star Coral (E): Montastrea cavemosa II In

Cayenne Kite (E): Leptodon cayanensis II B

Cayman Islands Dwarf Boa (E): Tropidophis caymanensis II R

Cayman Islands Ground Iguana (E); Cyclura nubila I R

Caymans Ground Boa (E): Tropidophis caymanensis II R

Cayuma (S): Caretsa caretta I R

Cebra de Grévy (S): Equus grevyi I M

Cebra de Hartmann (S): Equus zebra harmannae II M

Cebra montanesa del Cabo (S): Equus zebra zebra I M

Cebuella pygmaea $=$ Callithrix pygmaea

Cebus albifrons: Brown Pale-fronted Capuchin (E), White-fronted Capuchin (E), Sajou à front blane (F) II M

Cebus apella: Black-capped Capuchin (E), Tufted Capuchin (E), Sajou apelle (F) II M

Cebus capucinus: White-faced Capuchin (E), White-throated Capuchin (E), Mono capuchino (S), Sajou à gorge blanche (F) II M

Cebus nigrivittatus $=$ Cebus olivaceus

Cebus olivaceus: Weeper Capuchin (E) II M

Cebus xanthosternos = Cebus apella

Celebes Black Macaque (E): Macaca nigra II M

Celebes Crested Macaque (E): Macaca nigra II M

Celebes Macaque (E): Macaca maura II M

Celebes Scops-Owl (E): Orus manadensis II B

Celebes Tortoise (E): Indotestudo forstenii II R

Celecanto (S): Latimeria chaluminae I F

Celestial Parrotlet (E): Forpus coelestis II B

Central American Agouti (E): Dasyprocia punctala III M

Central American Cacomistle (E): Bassariscus sumichrasti III M

Central American Coral Snake (E): Micrunus nigrocinctus III R

Central American Dwarf Boa (E): Ungaliophis panamensis II R

Central American Puma (E): Puma concolor costaricensis I M

Central American River Turtle (E): Dermatemys mawii II R

Central American Squirrel Monkey (E): Saimiri oerstedii 1 M

Central American Tapir (E): Topinus bairdii $1 \mathrm{M}$

Central Asian Sand Boa (E): Eryx elegans II R

Central Asian Stone Marten (E): Manes foina intermedia III M

Central Asian Tortoise (E): Testudo horsfieldii II R

Central Flying-fox (E): Pleropus alecto II M

Central Rock-rat (E): Zyzomys pedunculaus I M

Central Thick-tailed Rock-rat (E): Zyzomys pedunculatus I M

Céphalophe à bande dorsale noire (F): Cephalophus dorsalis II M.

Céphalophe à dos jaune (F): Cephalophus silvicultor II M

Céphalophe bai (F): Cephalophus dorsalis II M

Céphalophe bleu (F): Cephalophus monticola II M

Céphalophe de Jentink (F): Cephalophus jentinki I M

Céphalophe d'Ogilby (F): Cephalophus ogilbyi II M

Céphalophe géant (F): Cephalophus silvicultor II M

Céphalophe zèbré (F): Cephalophus zebra II M

Cephalophus dorsalis: Bay Duiker (E), Duiquero bayo (S),
Céphalophe bai (F), Céphalophe à bande dorsale noire (F) II M Cephalophus jentinki: Jentink's Duiker (E), Duiquero de Jentink (S), Céphalophe de Jentink (F) I M

Cephalophus monticola: Blue Duiker (E), Duiquero azul (S), Céphalophe bleu (F) II M

Cephalophus ogilbyi: Ogilby's Duiker (E), Duiquero de Ogilby (S), Céphalophe d'Ogilby (F) II M

Cephalophus silviculior: Yellow-backed Duiker (E), Duiquero de lomo amarillo (S), Céphalophe géant (F), Céphalophe à dos jaune (F) II M

Cephalophus zebra: Banded Duiker (E), Zebra Antelope (E), Zebra Duiker (E), Duiquero cebrado (S), Céphalophe zèbré (F) II M

Cephalopterus omatus: Amazonian Umbrellabird (E), Omate Umbrellabird (E), Pájaro-paraguas amazónico (S), Coracine omée (F) III B

Cephalopterus penduliger: Long-wattled Umbrellabird (E), Păjaroparaguas longuipendulo (S), Coracine casquée (F) III B

Cephalorhynchus commersonii: Commerson's Dolphin (E), Piebald Dolphin (E), Jacobita (S), Tunina overa (S), Dauphin de Cornmerson (F) II M

Cephalorhynchus eutropia: Black Dolfhin (E), Chilean Dolphin (E), White-bellied Dolphin (E), Delfin negro (S), Tunina de vientre blanco (S), Dauphin noir du Chili (F) II M

Cephalorhynchus heavisidit: Benguela Dolphin (E), Heaviside's Dolphin (E), Tunina de Heaviside (S), Dauphin de Heaviside (F) II M

Cephalorhynchus heciori: Hector's Dolphin (E), New Zealand Dolphin (E), White-headed Dolphin (E), Delfin de Héctor (S), Tunina de Héctor (S), Dauphin d'Hector (F) II M

Ceratocyathus $=$ Caryophyllia

Cératode (F): Neoceratodus forsteri II F

Ceratodus (E): Neoceratodus forsteri II F

Ceratotherium simum cottoni: Northem Square-lipped Rhinoceros (E), Northem White Rhinoceros (E), Rinoceronte blanco del norte (S), Rhinocéros blanc du Nord (F) I M

Ceratotherium simum simum: Southern Square-lipped Rhinoceros (E), Southem White Rhinoceros (E), Rinoceronte blanco (S), Rhinocéros blanc du Sud (F) I/II M

Ceratotrochus franciscana: II In

Ceratotrochus laxus: II in

Ceratotrochus magnaghii: II In

Cerberus rhynchops: Bockadam (E), Dog-faced Water Snake (E), Serpent d'eau à títe de chien (F), Serpent d'eau à ventre blanc (F) III $R$

Cerceta alicorta de Auckland (S): Anas aucklandica I B

Cerceta aliverde (S): Anas crecca III B

Cerceta carretona ( $S$ ): Anas querquedula III B

Cerceta cejiblanca (S): Anas querquedula III B

Cerceta común (\$): Aras crecca III B

Cerceta de Madagascar (S): Anas bernieri II B

Cerceta del Baikal (S): Anas formosa II B

Cerceta del cabo (S): Anas capensis III B

Cerceta malgache (S): Anas bemieri II B

Cercocèbe agile (F): Cercocebus agilis II M

Cercocebus agilis: Agile Mangabey (E), Cercocèbe agile (F), Mangabey agile (F) II M

Cercocebus albigena $=$ Lophocebus albigena

Cercocebus aternimus = Lophocebus albigena

Cercocebus atys = Cercocebus torqualus

Cercocebus galeritus: Crested Mangabey (E) I/I M

Cercocebus galeritus agilis = Cercocebus agilis

Cercocebus galeritus galeritus: Tana River Mangabey (E).

Mangabey crestado ventriblanco (S), Mangabey de la Tana (F), Mangabey à crête $(F)$ I M

Cercocebus torqualus: Collared Mangabey (E), Red-capped Mangabey (E), Sooty Mangabey (E), White-collared Mangabey (E), Mangabey de collar blanco (S), Mangabey couronné (F). Mangabey enfumé (F) II M

Cercopithecus aethiops $=$ Chlorocebus aethiops

Cercopithecus albogularis $=$ Cercopithecus mitis

Cercopithecus ascanius: Black-cheeked White-nosed Monkey (E), Red-tailed Monkey (E), Redtail Monkey (E), Schmidt's Guenon (E), Ascagne (F) II M 
Cercopithecus campbelli: Campbell's Guenon (E), Campbell's Monkey (E), Cercopithèque de Campbell (F) II M

Cercopithecus cephus: Moustached Monkey (E), Moustac bleu (F) II M

Cercopithecus denti $=$ Cercopishecus wolfi

Cercopithecus diana: Diana Guenon (E), Diana Monkey (E), Diana (S), Cercopithèque diane (F) I M

Cercopithecus dryas: Dryas Guenon (E), Dryas Monkey (E), Mono dryas (S) II M

Cercopithecus erythrogaster, Red-bellied Guenon (E), Red-bellied Monkey (E), White-throated Guenon (E), White-throated Monkey (E), Cercopithèque à ventre roux (F) II M

Cercopithecus erythrotis: Red-eared Guenon (E), Red-eared Monkey (E), Russet-eared Guenon (E), Mono de orejas rojas (S), Moustac à oreilles rouges (F) II M

Cercopithecus erythrotis sclateri = Cercopithecus sclaten

Cercopithecus hamlyni: Owl-faced Guenon (E), Owl-faced Monkey (E), Cercopithèque d'Hamlyn (F) II M

Cercopithecus thoesti: L'Hoest's Monkey (E), Mountain Monkey (E), Cercopithèque de I'Hoest (F) II M

Cercopithecus lhoesti preussi = Cercopithecus preussi

Cercopithecus mitis: Blue Monkey (E), Diademed Monkey (E), Sykes' Monkey (E), Cercopithèque à diadème (F) II M

Cercopithecus mona: Mona Monkey (E), Cercopitheque mone (F) II $\mathbf{M}$

Cercopithecus neglectus: De Brazza's Monkey (E), Cercopithèque de Brazza (F) II M

Cercopithecus nictitans: Greater White-nosed Monkey (E), Puttynosed Monkey (E), Spot-nosed Guenon (E), White-nosed Guenon (E), Hocheur (F) II M

Cercopithecus nigroviridis = Allenopithecus nigroviridis

Cercopithecus patas = Erythrocebus patas

Cercopithecus petaurista: Lesser Spot-nosed Guenon (E), Lesser White-nosed Guenon (E), Lesser White-nosed Monkey (E), Spot-nosed Monkey (E), Hocheur du Ghana (F) II M

Cercopithecus pogonias: Crowned Guenon (E), Crowned Monkey (E), Golden-bellied Guenon (E), Golden-bellied Monkey (E), Mono coronado (S), Guenon couronné (F), Mone (F) II M

Cercopithecus pogonias wolfi $=$ Cercopithecus wolfi

Cercopithecus preussi: Preuss's Guenon (E), Preuss's Monkey (E), Mono de Preuss (S) II M

Cercopithecus roloway $=$ Cercopithecus diana

Cercopithecus salongo $=$ Cercopithecus dryas

Cercopithecus sclateri: Sclater's Guenon (E), White-throated Guenon (E), Mono de Sclater (S) II M

Cercopithecus solatus: Sun-tailed Guenon (E), Sun-tailed Monkey (E), Mono del Gabón (S), Cercopithèque à queue de soleil (F) II $\mathrm{M}$

Cercopithecus talapoin = Miopithecus talapoin

Cercopithecus thomasi $=$ Cercopithecus thoesti

Cercopithecus wolfi: Wolf's Monkey (E), Mono de Wolf (S), Mone de Wolf (F) II M

Cercopithèque à diadème (F): Cercopithecus mitis II M

Cercopithèque à queue de soleil (F): Cercopithecus solatus II M

Cercopithèque à ventre roux (F): Cercopithecus erythrogaster II M

Cercopithèque de Allen (F): Allenopithecus nigroviridis II M

Cercopithèque de Brazza (F): Cercopithecus neglecius II M

Cercopithèque de Campbell (F): Cercopithecus campbelli II M

Cercopithèue de 1'Hoest (F): Cercopithecus thoesh II M

Cercopithèque d'Hamlyn (F): Cercopithecus hamlyni II M

Cercopithèque diane (F): Cercopithecus diana I M

Cercopithèque mone (F): Cercopithecus mona II M

Cerdocyon thous: Common Zorro (E), Crab-eating Zorro (E), Forest Fox (E), Zorro cangrejero (S) II M

Cerf de Bactriane (F): Cervus elaphus bactrianus II M

Cerf de Barbarie (F): Cenus elaphus barbarus III M

Cerf de Duvaucel (F): Cervus duvauceli I M

Cerf de Virginie SE (F): Odocoileus virginianus mayensis III M

Cerf d'Eld (F): Cervus eldii I M

Cerf des Andes méridionales (F): Hippocamelus bisulcus I M

Cerf des Andes septentrionales (F): Hippocamelus antisensis I M

Cerf des marais (F): Blastocerus dichotomus I M

Cerf des pampas (F): Ozotoceros bezoarticus I M

Cerf du Turkestan (F): Cervus elaphus bactrianus 11 M

Cerf élaphe du Cachemire (F): Cervus elaphus hanglu I M

Cerf élaphe du Turkestan (F): Cenus elaphus bactrianus II M
Cerf porte-musc (F): Moschus moschiferus I M

Cerf rouge du Turkestan (F): Cervus elaphus bactrianus II M

Cerf-cochon calamien ( $F$ ): Axis calamianensis $1 \mathrm{M}$

Cerf-cochon de Bawean (F): Axis kuhlii I M

Cerf-cochon d'Indochine (F): Axis porcinus annamiticus I M

Cerfs des Andes (F): Hippocamelus spp. I M

Ceriomorpha $=$ Blasıomussa

Cerise-throated Hummingbird (E): Selasphorus flammula II B

Cemicalo (S): Gampsonyx swainsonii II B

Cemícalo americano (S): Falco sparverius II B

Cemícalo australiano (S): Falco cenchroides II B

Cemicalo común (S): Falco tinnunculus II B

Cernícalo de Aldabra (S): Falco newroni I/II B

Cernícalo de Dickinson (S): Falco dickinsoni II B

Cernícalo de la Mauricio (S): Falco punctatus I B

Cernicalo de las Molucas (S): Falco moluccensis II B

Cernícalo de las Seychelles (\$): Falco araea I B

Cernicalo de Madagascar (S): Falco newtoni I/II B

Cemicalo del Amur (S): Falco amurensis II B

Cemícalo dorsinegro (S): Falco dickinsoni II B

Cemícalo gris (S): Falco ardosiaceus II B

Cernicalo malgache (S): Falco zoniventris II B

Cernicalo moluqueño (S): Falco moluccensis II B

Cernicalo ojiblanco (S): Falco rupicoloides II B

Cernícalo ojiblanco de Africa (S): Falco rupicoloides II B

Cernícalo patirrojo (S): Falco vespertinus II B

Cernícalo pizarroso (S): Falco ardosiaceus II B

Cernícalo primilla (S): Falco naumanni II B

Cernícalo vulgar (S): Falco tinnunculus II B

Cernícalo zorruno (S): Falco alopex II B

Cerro Campana Stubfoot Toad (E): Avelopus varius zeteki I A

Cervicapra (S): Antilope cervicapra III M

Cervus dama mesopotamicus = Dama mesopotamica

Cervus duvauceli: Barasingha (E), Swamp Deer (E), Barasinga (S), Ciervo de Duvaucel (S), Barasinga (F), Barasingha (F), Cerf de Duvaucel (F) I M

Cervus elaphus bactrianus: Bactrian Deer (E), Bactrian Red Deer (E), Bactrian Wapiti (E), Bokharan Deer (E), Ciervo bactriano (S), Cerf de Bactriane (F), Cerf du Turkestan (F), Cerf rouge du Turkestan (F), Cerf élaphe du Turkestan (F) II M

Cervus elaphus barbarus: Atlas Deer (E), Barbary Deer (E), Barbary Red Deer (E), Barbary Stag (E), Ciervo de la Berberia (S), Cerf de Barbarie (F) III M

Cervus elaphus hanglu: Hangul (E), Kashmir Deer (E), Kashmir Red Deer (E), Kashmir Stag (E), Ciervo de Cachemira (S), Cerf élaphe du Cachemire (F), Hangul (F) I M

Cervus eldii: Brow-antlered Deer (E), Eld's Deer (E), Thamin (E), Ciervo de Elde (S), Cerf d'Eld (F) I M

Cervus porcinus annamiticus = Axis porcinus annamilicus

Cervus porcinus calamianensis = Axis calamianensis

Cervus porcinus kuhlii $=$ Axis kuhlii

CETACEA spp.: Cetaceans (E), Dolphins (E), Porpoises (E), Whales (E), Ballenas (S), Cetáceos (S), Baleines (F) I/I M

Cetaceans (E): CETACEA spp. I/II M

Cetáceos (S): CETACEA spp. I/II M

Ceylon Hanging-Parrot (E): Loriculus beryllinus II B

Chacal commun (F): Canis aureus III M

Chacal doré (F): Canis aureus III M

Chacaraco (S): Aratinga wagleri II B

Chachalaca norteña (S): Ortalis vetula III B

Chacma Baboon (E): Papio hamadryas II M

Chaco argentino (S): Catagonus wagneri I M

Chaco Tortoise (E): Geochelone chilensis II R

Chacoan Peccary (E): Catagonus wagneri I M

Chaeropus ecaudarus: Pig-footed Bandicoot (E), Cangurito piedecerdo (S), Bandicoot pieds de cochon (F), Bandicoot à pied de $\operatorname{porc}(\mathbf{F})$ I M

Chaetocercus jourdani: Rufous-shafted Woodstar (E), Tucusito garganta rosa (S), Colibri de Jourdan (F) II B

Chaetophractus nationi: Andean Hairy Armadillo (E), Bolivian Hairy Armadillo (E) II M

Chalcopsitta atra: Black Lory (E), Lori negro (S), Lori noir (F) 11 B

Chalcopsitta cardinalis: Cardinal Lory (E), Lori cardenal (S), Lori cardinal (F) II B

Chalcopsitta duivenbodei: Brown Lory (E), Duyvenbode's Lory (E), Lori pardo (S), Lori de Duyvenbode (F) II B 
Chalcopsina sintillata: Greater Streaked Lory (E), Yellow-streaked Lory (E), Lori chispeado (S), Lori flamméché (F) II B

Chalcostigma herrani: Rainbow-bearded Thombill (E), Picoespina arcoiris (S), Métallure arc-en-ciel (F) II B

Chalcostigma heferopogon: Bronze-tailed Thombill (E), Pico espina bronceado (S), Métallure à queue bronzée (F) II B

Chalcostigma olivaceum: Olivaceous Thornbill (E), Métallure olivâtre (F) II B

Chalcostigma nuficeps: Rufous-capped Thornbill (E), Picoespina gorrirufa (S), Métallure à tête rousse (F) II B

Chalcostigma stanleyi: Blue-mantled Thombill (E), Picoespina dorsiazul (S), Métallure de Stanley (F) II B

Chalybura buffonii: White-vented Plumeleteer $(\mathrm{E})$, Calzonario colibronceado (S), Colibrí grande colinegro (S), Colibri de Buffon (F) II B

Chalybura urochrysia: Bronze-tailed Plumeleteer (E), Colibri à queue bronzée (F) II B

Chamaeleo spp.: Chameleons (E), Camaleónes (S), Caméléons (F) II $\mathbf{R}$

Chamaeleo adolfifriderici $=$ Bradypodion adolfifriderici

Chamaeleo affinis: Rüppell's Desert Chameleon (E), Caméléon américain (F) II R

Chamaeleo africanus: African Chameleon (E), Camaleón africano (S), Caméléon africain (F) II $R$

Chamaeleo anchietae: Anchieta's Charneleon (E), Angola Chameleon (E), Double-scaled Chameleon (E), Caméléon d'Angola (F), Caméléon à écailles doubles (F) II R

Chamaeleo angeli: Angel's Chameleon (E), Caméléon d'Angel (F) II $\mathbf{R}$

Chamaeleo antimena: White-lined Chameleon (E), Caméléon à lignes blanches (F) II R

Chamaeleo arabicus: Arabian Chameleon (E), Caméléon d'Arabie (F) II R

Chamaeleo baltearus: Rainforest Chameleon (E), Two-banded Chameleon (E), Caméléon forestier (F) II R

Chamaeleo belalandaensis: Belalanda Chameleon (E), Caméléon de Belalanda (F) II R

Chamaeleo bifidus: Two-homed Chameleon (E), Caméléon à deux cornes (F), Caméléon à nez fourchu (F) II R

Chamaeleo bifidus balteatus = Chamaeleo balteatus

Chamaeleo bitaeniatus: Montane Chamaeleon (E), Side-striped Chameleon (E), Two-lined Chameleon (E), Camaleón de dos bandas (S), Caméléon à deux bandes (F) II $\mathrm{R}$

Chamaeleo bitaeniatus ellioti = Chamaeleo elliot

Chamaeleo bitaeniatus kinetensis = Chamaeleo kinetensis

Chamaeleo boetsgeri: Boettger's Chameleon (E). Camaleón de Boettger (S), Caméléon de Boettger (F) II R

Chamaeleo brevicomis: Short-homed Chameleon (E), Caméléon à comes coures (F) II R

Chamaeleo calcaricarens: Caméléon du Yémen (F) II R

Chamaeleo calyptratus: Veiled Chameleon (E), Caméléon casqué ( $F$ ) II $\mathrm{R}$

Chamaeleo camerunensis: Caméléon du Cameroun (F) II R

Chamaeleo campani: Madagascar Forest Chameleon (E), Caméléon forestier de Madagascar (F) II R

Chamaeleo capuroni: Madagascar Chameleon (E), Caméléon de Madagascar (F) II R

Chamaeleo carpenteri $=$ Bradypodion carpenter

Chamaeleo cephalolepis: Comoro Islands Chameleon (E), Caméléon des Comores (F) II R

Chamaeleo chamaeleon: Common Chameleon (E), European Chameleon (E), Mediterranean Chameleon (E), Caméleon común (S), Caméléon commun (F), Caméléon vulgaire (F) II $R$

Chamaeleo chamaeleon zeylanicus = Chamaeleo zeylanicus

Chamaeleo chapini: Grey Chameleon (E), Caméléon de Chapin (F), Caméléon gris (F) II R

Chamaeleo cristatus: Crested Chameleon (E), Camaleón crestado (S). Caméléon crêté (F) II R

Chamaeleo cucullatus: Hooded Chameleon (E), Caméléon à capuchon (F) II R

Chamaeleo deremensis: Usambara Three-homed Chameleon (E) Wavy Chameleon (E), Caméléon ondulé (F) II R

Chamaeleo dilepis: Flap-necked Chameleon (E), Camaleón lobulado (S), Caméléon bilobé (F), Caméléon de Leach (F) II R

Chamaeleo dilepis ruspolii = Chamaeleo ruspoli

Chamaeleo eisentrauti: Caméléon d'Eisentraut (F) II R
Chamaeleo ellioti: Montane Side-striped Chameleon (E), Mountain Dwarf Chameleon (E), Camaleón de Elliot (S), Caméléon d'Elliot (F) II R

Chamaeleo fallax: Deceptive Chameleon (E), Caméléon de Mocquard (F) II R

Chamaeleo feae: Caméléon de Fea (F) II R

Chamaeleo fischeri $=$ Bradypodion fischer

Chamaeleo fischeri uthmoelleri = Bradypodion uthmoelleri

Chamaeleo fuellebomi: Ngosi Volcano Chameleon (E), Poroto Three-homed Chameleon (E), Camaleón de Fuellebom (S). Caméléon du volcan Ngosi (F) II R

Chamaeleo furcifer: Fork-nosed Chameleon (E), Forked Chameleon (E), Caméléon à nez fourchu (F) II R

Chamaeleo gallus: Blade Chameleon (E), Caméléon épée (F) II R

Chamaeleo gastrotaenia: Perinet Chameleon (E), Caméléon de Perinet (F) II R

Chamaeleo globifer. Flat-casqued Chameleon (E), Caméléon à casque plat (F) II R

Chamaeleo goefzei: Goetze's Chameleon (E), Dolo Chameleon (E), Tanganyika Chameleon ( $E$ ), Caméléon de Goetze (F), Caméléon d'Dolo (F) II R

Chamaeleo gracilis: Graceful Chameleon (E), Gracile Chameleon (E), Spur-heeled Chameleon (E), Camaleón grácil (S), Caméléon gracile (F) II R

Chamaeleo guentheri $=$ Chamaeleo pardalis

Chamaeleo guibei: Guibe's Chameleon (E), North-western Chameleon (E), Caméléon de Guibé (F) II R

Chamaeleo hoehnelii: Helmeted Chameleon (E), High-casqued Chameleon (E), Camaleón de casco (S), Caméléon à casque élevé (F) II R

Chamaeleo incomurus: Poroto Mountain Chameleon (E), Ukinga Homless Chameleon (E), Caméléon du Mont Poroto (F) II R

Chamaeleo jacksonit: Jackson's Three-homed Chameleon (E), Camaleón de Jackson (S), Caméléon de Jackson (F) II R

Chamaeleo johnstoni: Johnston's Chameleon (E), Ruwenzori Threehorned Chameleon (E), Camaleón de Johnston (S), Caméléon de Johnston (F) II R

Chamaeleo kinerensis: II R

Chamaeleo labordi: Caméléon de Laborde (F) II R

Chamaeleo laevigatus: Smooth Chameleon (E), Caméléon (F) II R

Chamaeleo lateralis: Carpet Chameleon (E), Jewelled Chameleon (E), Caméléon à bandes latérales (F), Caméléon-joyau (F) II R

Chamaeleo laterispinis: Spine-sided Chameleon (E). Uzungwe Mountain Chameleon (E), Caméléon des Monts Uzungwe (F) II $\mathrm{R}$

Chamaeleo linotus: Maroantsetra Chameleon (E), Caméléon de Maroantsetra (F) II R

Chamaeleo malthe: Yellow-green Chameleon (E), Caméléon vert-etjaune (F) II R

Chamaeleo marsabitensis: Tilbury's Chameleon (E), Caméléon de Tilbury (F) II R

Chamaeleo marshalli: Short-tailed Dwarf Chameleon (E), Camaleón de Marshall (S), Caméléon de Marshall (F) II R

Chamaeleo melleri: Giant One-homed Chameleon (E), Meller's Chameleon (E), Camaleón de Meller (S), Caméléon de Meller (F) II R

Chamaeleo minor. South-central Chameleon (E), Caméléon de Günther (F) II R

Chamaeleo mlanjensis $=$ Bradypodion mlanjensis

Chamaeleo monachus: Monarch Chameleon (E), Socotra Chameleon (E), Caméléon-moine (F) II R

Chamaeleo monoceras: One-homed Chameleon (E), Caméléon à une come (F) II R

Chamaeleo montium: Cameroon Sailfin Chameleon (E), Mountain Chameleon (E), Camaleón de montaña (S), Caméléon à voile du Cameroun (F) II R

Chamaeleo montium feae $=$ Chamaeleo feae

Chamaeleo namaquensis: Desert Chameleon (E), Namaqua Chameleon (E), Caméléon du Namaqua (F) II R

Chamacleo nasurus: Big-nosed Chameleon (E), Caméléon nasique (F) II R

Chamaeleo oshaughnessyi: O'Shaughnessy's Chameleon (E), Caméléon d'O'Shaughnessy (F) II $R$

Chamaeleo oustaleti: Oustalet's Giant Chameleon (E), Camaleón de Oustalet (S), Caméléon d'Oustalet (F) II R 
Chamaeleo oweni: Owen's Three-homed Chameleon (E), Camaleón de Owen (S), Caméléon d'Owen (F) II R

Chamaeleo pardalis: Panther Chameleon (E), Caméléon panthère (F) II $\mathbf{R}$

Chamaeleo parsonii: Parson's Giant Chameleon (E), Camaleón de Parson (S), Caméléon de Parson (F) II R

Chamaeleo petreri: Petter's Chameleon (E), Camaleón de Petter (S), Caméléon de Petter (F) II R

Chamaeleo peyrieresi: Brygoo's Chameleon (E), Caméléon de Peyrieras ( $F$ ) II $R$

Chamaeleo pfefferi: Pfeffer's Chameleon (E), Camaleón de Pfeffer (S), Caméléon de Pfeffer (F) II R

Chamaeleo polleni: Mayotte Chameleon (E), Caméléon de Mayotte (F) II R

Chamaeleo pumilis = Bradypodion pumilum

Chamaeleo quadricomis: Four-homed Chameleon (E), Camaleón cuadricorne (S), Caméléon à quatre cornes (F) II R

Chamaeleo quilensis: Bocage's Chameleon (E), Caméléon de Bocage (F) II R

Chamaeleo rhinoceratus: Rhinoceros Chameleon (E), Camaleón rinoceronte (S), Caméléon rhinocéros (F) II R

Chamaeleo rhinocerarus labordi $=$ Chamaeleo labordi

Chamaeleo rudis: Coarse Chameleon (E), Ruwenzori Side-striped Chameleon (E), Caméléon rude (F) II R

Chamaeleo rudis schouredeni $=$ Chamaeleo schouredeni

Chamaeleo rudis schubotzi $=$ Chamaeleo schubotz $i$

Chamaeleo ruspolii: Ruspoli's Chameleon (E), Caméléon de Lanza (F) II R

Chamaeleo schoutedeni: Schouteden's Chameleon (E), Camaleón de Schouteden (S), Caméléon de Schoudeten (F) II R

Chamaeleo schubotzi: Schubotz's Chameleon (E). Camaleón de Schubotz (S), Caméléon de Schubotz (F) II R

Chamaeleo senegalensis: Senegal Chameleon (E), Camaleón del Senegal (S), Caméléon du Sénégal (F) II R

Chamaeleo senegalensis laevigatus = Chamaeleo laevigatus

Chamaeleo spinosus $=$ Bradypodion spinosum

Chamaeleo sempeli: Tanzania Mountain Chameleon (E). Tuberclenosed Chameleon $(E)$, Caméléon des montagnes de Tanzanie ( $F$ ) II $\mathrm{R}$

Chamaeleo tenuis = Bradypodion senue

Chamaeleo tigris: Tiger Chameleon (E), Caméléon tigre (F) II R

Chamaeleo isaratananensis: Tsaratanan Chameleon (E), Caméléon de Tsaratana (F) II R

Chamaeleo tuzetae: Ambiky Chameleon (E), Caméléon d'Andrenalamivola (F) II R

Chamaeleo verrucosus: Madagascar Giant Chameleon (E), Warty Chameleon (E), Camaleón verrugoso (S), Caméléon verruqueux (F) II R

Chamaeleo wemeri: Uzungwe Three-homed Chameleon (E), Werner's Chameleon (E), Camaleón de Wemer (S), Caméléon de Werner (F) II R

Chamaeleo wiedersheimi: Mount Lefo Chameleon (E), Camaleón de Wiedersheim (S), Caméléon du Mont Lefo (F) II R

Chamaeleo willsii: Canopy Chameleon (E), Caméléon de Wills ( $\mathrm{F}$ ), Caméléon de la canopée (F) II R

Chamaeleo xenorhinum $=$ Bradypodion xenorhinus

Chamaeleo zeylanicus: Indian Chameleon (E), Camaleón de Sri Lanka (S), Caméléon de Ceylan (F) II R

Chameleons (E): Bradypodion spp./Brookesia sppJChamaeleo spp. II/NC R

Chamois (E): Rupicapra spp. INC M

Chamois des Abruzzes (F): Rupicapra pyrenaica omaia I M

Changeable Hawk-Eagle (E): Spizaetus cirrhatus II B

Changjiang Dolphin (E): Lipotes vexillifer I M

Channel-billed Toucan ( $\mathrm{E}$ ): Ramphassos vitellinus II B

Chanteur d'Afrique (F): Serinus leucopygius III B

Chanting-Goshawks (E): Melierax spp. II B

Chardonneret de Yarrell (F): Carduelis yarrellii II B

Chardonneret rouge (F): Carduelis cucullata I B

Chardonnerets (F): Carduelis spp. I/I/NC B

Charina bottae: Rubber Boa (E), Boa de Gomme (S), Boa caoutchouc (F), Serpent gomme (F) II R

Charming Hummingbird (E): Amazilia decora II B

Charmosyna amabilis: Red-throated Lorikeet (E), Lori gorgirrojo (S), Lori à gorge rouge (F) II B
Charmosyna diadema: New Caledonian Lorikeet (E), Lori diadema (S), Lori de Nouvelle-Calédonie (F), Lori à diadème (F) II B

Chamosyna josefinae: Josephine's Lorikeet (E), Josephine's Lory (E), Lori de Josefina (S), Lori de Joséphine (F) II B

Charmosyna margarethae: Duchess Lorikeet (E), Lori de Margarita (S), Lori de Margaret (F), Lori de Marguerite (F) II B

Charmosyna meeki: Meek's Lorikeet (E), Lori de Meek (S), Lori de Meek (F) II B

Charmosyna multistriata: Streaked Lorikeet (E), Striated Lorikeet (E), Lori estriado (S), Lori strié (F) II B

Chamosyna palmarum: Palm Lorikeet (E), Lori palmero (S), Lori des palmiers (F) II B

Charmosyna papou: Papuan Lorikeet (E), Lori rabilargo (S), Lori de Papouasie (F), Lori papou (F) II B

Charmosyna placentis: Red-flanked Lorikeet (E), Lori flanquirrojo (S), Lori croquet (F), Lori splendide (F) II B

Charmosyna pulchella: Fairy Lorikeet (E), Little Red Lorikeet (E), Lori lindo (S), Lori féérique (F) II B

Charmosyna rubrigularis: Red-chinned Lorikeet (E), Lori barbirrojo (S), Lori à menton rouge (F) II B

Charmosyna rubronotata: Red-fronted Lorikeet (E), Red-spotted Lorikeet (E), Lori frentirrojo (S), Lori à front rouge (F) II B

Charmosyna roxopei: Blue-fronted Lorikeet (E), Lori de Buru (S), Lori de Buru (F), Lori de l'ille Buru (F) II B

Charmosyna wilhelminae: Pygmy Lorikeet $(\mathrm{E})$, Wilhelmina's Lorikeet (E), Lori pigmeo (S), Lori de Wilhelmina (F) II B

Chasmistes cujus: Cui-ui (E) I F

Chat à pieds noirs (F): Felis nigripes I M

Chat à tête plate $(\mathrm{F})$ : Prionailurus planiceps I M

Chat bai (F): Catopuma badia II M

Chat de Biet (F): Felis bieti II M

Chat de Chine (F): Prionailurus bengalensis I/II M

Chat de Geoffroy (F): Oncifelis geoffroyi I M

Chat de jungle (F): Felis chaus II M

Chat de Temminck (F): Catopuma temminckii I M

Chat des Andes (F): Oreailurus jacobita I M

Chat des marais ( $\mathrm{F}$ ): Felis chaus II M

Chat des pampas (F): Oncifelis colocolo II M

Chat des sables (F): Felis margarita II M

Chat doré $(\mathrm{F})$ : Profelis aurata II M

Chat doré d'Asie (F): Catopuma temminckii I M

Chat manul (F): Orocolobus manul II M

Chat marbré (F): Pardofelis marmorata $1 \mathrm{M}$

Chat omé (F): Felis silvestris II M

Chat pêcheur (F): Prionailurus viverrinus II $\mathrm{M}$

Chat rougeâtre (F): Prionailurus rubiginosus VII M

Chat rubigineux (F): Prionailurus rubiginosus $\mathbf{1} / \mathrm{I}$ M/Felis silvestris II M

Chat sauvage (F): Lynx rufus II M

Chatham Island Yellow-fronted Parakeet (E): Cyanoramphus auriceps forbesi I B

Chat-léopard de l'Inde (F): Prionailurus rubiginosus VII M

Chat-léopard du Bengale (F): Prionailurus bengalensis I/II M

Chat-tacheté (F): Leopardus tigrinus I M

Chattering Lory (E): Lorius garnulus II B

Chat-tigre (F): Leopardus tigrinus I M Reptailurus serval II M

Chat-tigre du Bengale (F): Prionailurus bengalensis bengalensis I/II M

Cheer Pheasant (E): Catreus wallichii I B

Cheetah (E): Acinonyx jubarus I M

Cheiloporidion pulvinatum: II in

Cheirogale moyen (F): Cheirogaleus medius I M

Cheirogaleidae spp.: Dwarf lemurs (E), Chirogales (F) I M

Cheirogaleus major: Greater Dwarf Lemur (E), Gran lemur ardilla

(S), Lemur enano mayor (S), Makis coligordos (S), Grand cheirogale (F) I M

Cheirogaleus medius: Fat-tailed Dwarf Lemur (E), Lemur ardilla coligrueso (S), Lemur enano coligrueso (S), Makis coligordos (S), Cheirogale moyen (F), Petit cheirogale (F) I M

Cheirogaleus trichotis $=$ Allocebus trichotis

Chelictinia riocourii: African Swallow-tailed Kite (E), Scissor-tailed Kite (E), Elanio golondrina (S), Élanion naucler (F) II B

Chelonée à dos plat (F): Nasator depressus I R

Chelonia depress $a=$ Natator depressus 
Chelonia mydas: Green Turtle (E), Tortuga blanca (S), Tortuga verde (S), Tortue comestible $(\mathrm{F})$, Tortue franche $(\mathrm{F})$, Tortue verte $(\mathrm{F})$ I R

Cheloniidae spp.: Marine turtles (E), Tortugas marinas (S), Tortues de mer (F) I R

Chelonoidis carbonaria $=$ Geochelone carbonaria

Chelonoidis chilensis = Geochelone chilensis

Chelonoidis denticulata $=$ Geochelone denticulata

Chelonoidis elegans $=$ Geochelone elegans

Chelonoidis gigantea $=$ Geochelone gigantea

Chelonoidis nigra $=$ Geochelone nigra

Chequered Keelback (E): Xenochrophis piscator III R

Chersina angulata: Angulated Tortoise (E), Bowsprit Tortoise (E), South African Bowsprit Tortoise (E), Tortuga ariete (S), Tortuga de pechera (S), Tortue à soc d'Afrique du Sud (F) II R

Chestnut Hawk-Owl (E): Ninox theomacha II B

Chestnut Owlet (E): Glaucidium castaneum II B

Chestnut Terrapin (E): Pelusios castaneus III R

Chestnut-backed Owlet (E): Glaucidium castanonorum II B

Chestnut-bellied Hawk-Eagle (E): Hiereaetus kienerii II B

Chestnut-bellied Hummingbird (E): Amazilia castaneiventris II B

Chestnut-bellied Sparrowhawk (E): Accipiter castanilius II B

Chestnut-breasted Coronet (E): Boissonneaua manhewsii II B

Chestnut-breasted Negrofinch (E): Nigriza bicolor III B

Chestnut-breasted Tree-Partridge (E): Arborophila charltonii III B

Chestnut-crowned Sparrow-Weaver (E): Plocepasser superciliosus III B

Chestnut-eared Aracari (E): Pteroglossus castanotis III B

Chestnut-flanked Sparrowhawk (E): Accipiter castanilius 11 B

Chestnut-fronted Macaw (E): Ara severa II B

Chestnut-mantled Goshawk (E): Erythrotriorchis buergersi II B

Chestnut-necklaced Partridge (E): Arborophila charltonii III B

Chestnut-shouldered Goshawk (E): Erythrotriorchis buergersi II B

Chestnut-shouldered Hawk (E): Erythrotriorchis buergersi II B

Cheval de Przewalski (F): Equus przewalskii I M

Chevalier à gouttelette (F): Tringa gutrifer I $B$

Chevalier tacheté (F): Tringa guttifer I B

Chevaliers (F): Tringa spp. I/NC B

Chevềche brame (F): Athene brama II B

Chevêche des terriers (F): Speotyto cunicularia II B

Chevêche forestière (F): Athene blewitti I B

Chevêches (F): Athene spp/Speotyı cunicularia I/II B

Chevêchelte à collier $(F)$ : Glaucidium brodiei II B

Chevêchette à pieds jaunes $(\boldsymbol{F})$ : Glaucidium sephronotum II B

Chevêchette à queue barrée (F): Glaucidium sjostedri II B

Chevêchette australe (F): Glaucidium nanum II B

Chevêchette brune (F): Glaucidium brasilianum II B

Chevêchette cabouré (F): Glaucidium minutissimum II B

Chevêchette châtaine (F): Glaucidium castaneum II B

Chevêchette cuculoïde (F): Glaucidium cuculoides II B

Chevêchette d'Amazonie (F): Glaucidium hardyi II B

Chevêchette de Cuba (F): Glaucidium siju II B

Chevêchette de jungle (F): Glaucidium radiatum II B

Chevêchette de Ngami (F): Glaucidium ngamiense II B

Chevêchette de Scheffler (F): Glaucidium scheffleri II B

Chevêchette des Andes (F): Glaucidium jardinii II B

Chevêchette des Rocheuses $(\mathrm{F})$ : Glaucidium californicum II B

Chevêchette des saguaros (F): Micrathene whirneyi II B

Chevêchette d'Europe (F): Glaucidium passerinum II B

Chevêchette du Cap (F): Glaucidium capense II B

Chevêchette du Graben (F): Glaucidium alberinum II B

Chevêchette du Pérou (F): Glaucidium peruanum II B

Chevêchette naine (F): Glaucidium gnoma II B

Chevêchette nimbée (F): Xenoglaux loweryi II B

Chevêchette perlée (F): Glaucidium perlatum II B

Chevêchette spadicée (F): Glaucidium castanopterum II B

Chevêchette-elfe (F): Micrathene whitneyi II B

Chevêchettes (F): Glaucidium spp/Micrathene whimeyiXenoglaux loweryi II B

Chevrotain aquatique (F): Hyemoschus aquaticus III M

Chevrotain porte-musc (F): Moschus moschiferus I M

Chevrotains (E): Tragulidae spp. II/NC M

Chevrotains porte-musc (F): Moschus spp. I/II M

Chiapan Boa (E): Ungaliophis continentalis II R

Chichimen (S): Lontra felina I M

Chico Grey Fox (E): Pseudalopex griseus II M
Chien de prairie du Mexique (F): Cynomys mexicanus I M

Chien des buissons (F): Speothos venaticus I M

Chien sauvage d'Asie (F): Cuon alpinus II M

Children's (Rock) Python (E): Morelia childreni II R

Chilean Cat (E): Oncifelis guigna II M

Chilean Dolphin (E): Cephalorhynchus eutropia II M

Chilean Flamingo (E): Phoenicopterus chilensis II B

Chilean Guemal (E): Hippocamelus bisulcus I M

Chilean Huemul (E): Hippocamelus bisulcus I M

Chilean Pampa Cat (E): Oncifelis colocolo II M

Chilean Pudu (E): Pudu puda I M

Chilean Woodstar (E): Eulidia yarrellii II B

Chilla (S): Pseudalopex griseus II M

Chillón de Sundevall (S): Petronia dentata III B

Chiloe Fox (E): Pseudalopex griseus II M

Chimachima (\$): Milvago chimachima II B

Chimaera Birdwing (E): Omithoptera chimaera II In

Chimango (S): Milvago chimango II B

Chimango Caracara (E): Milvago chimango II B

Chimpancé (S): Pan troglodytes I M

Chimpancé pigmeo (\$): Pan paniscus I M

Chimpancés (S): Pan spp. $1 \mathrm{M}$

Chimpanzé (F): Pan troglodytes I M

Chimpanzé nain (F): Pan paniscus I M

Chimpanzé pygmée (F): Pan paniscus I M

Chimpanzee (E): Pan troglodytes I M

Chimpanzees (E): Pan spp. I M

Chimpanzés (F): Pan spp. $1 \mathrm{M}$

Chimpire (S): Podocnemis erythrocephala II R

China Alligator (E): Alligator sinensis I R

China Clam (E): Hippopus porcellanus II in

Chinalinda (S): Phalcoboenus albogularis II B

Chinchay (S): Oreailurus jacobila I M

Chinchilla à longue queue (F): Chinchilla lanigera I M

Chinchilla à queue courte $(F)$ : Chinchilla brevicaudata I M

Chinchilla spp.: Chinchillas (E), Chinchillas (S), Chinchillas (F) I M

Chinchilla brevicaudata: Short-tailed Chinchilla (E), Chinchilla de cola corta (S), Chinchilla à queue courte (F) I M

Chinchilla costina (S): Chinchilla lanigera I M

Chinchilla de cola corta (S): Chinchilla brevicaudata I M

Chinchilla de cola larga (S): Chinchilla lanigera I M

Chinchilla lanigera: Long-tailed Chinchilla (E), Chinchilla costina

(S), Chinchilla de cola larga (S), Chinchilla à longue queue (F) I M

Chinchillas (E/S/F): Chinchilla spp. I M

Chinchimen ( $\mathrm{S}$ ): Lonira felina I M

Chinese Alligator (E): Alligator sinensis I R

Chinese Barred-backed Pheasant (E): Syrmaticus ellioti I b

Chinese Crocodile Lizard (E): Shinisaurus crocodilurus II R

Chinese Desert Cat (E): Felis bieti II M

Chinese Giant Salamander (E): Andrias davidianus I A

Chinese Goral (E): Naemorhedus caudatus I M

Chinese Goshawk (E): Accipizer soloensis II B

Chinese Impeyan (E): Lophophorus thuysii I B

Chinese Lake Dolphin (E): Lipotes vexillifer I M

Chinese Monal (E): Lophophorus Ihuysii I B

Chinese Paddlefish (E): Psephunus gladius II F

Chinese Pangolin (E): Manis pentadactyla II M

Chinese Sparrowhawk (E): Accipiter soloensis II B

Chinese Sturgeon (E): Acipenser sinensis II F

Chinese Three-tailed Swallowtail (E): Bhutanitis thaidina II In

Chinese Xenosaur (E): Shinisaurus crocodilurus II R

Chingue de la Patagonia (S): Conepatus humboldtii II M

Chingungo (E): Lontra felina I M

Chir Pheasant (E): Catreus wallichii i B

Chirica (S): Nannopsittaca panychlora II B

Chiripepé común (S): Pyrrhura frontalis II B

Chiripepé salteño (S): Pyrrhura molinae II B

Chirogale aux oreilles poilues (F): Allocebus trichotis I M

Chirogales (F): Cheirogaleidae spp. I M

Chiropotes albinasus: Red-nosed Saki (E), White-nosed Bearded Saki (E), White-nosed Saki (E), Saki nariblanco (S), Saki à nez blanc (F) I M

Chiropotes salanas: Bearded Saki (E), Black Saki (E), Saki noir (F) II $M$

Chiru (E): Pantholops hodgsonii I M 
Chita (S): Acinonyx jubatus I M

Chittagong Soft-shell Turtle (E): Trionyx nigricans I R

Chivito de los Páramos (S): Oxypogon guerinii II B

Chlamydotis macqueen $i=$ Chlamydotis undulata

Chlamydotis undulata: Houbara Bustard (E), Avutarda hubara (S), Hubara (S), Houbara ondulé (F), Outarde houbara (F) I B

Chlorestes notatus: Blue-chinned Sapphire (E), Colibri verdecito (S), Zafiro barbiazul (S), Colibri à menton bleu (F) II B

Chlorocebus aethiops: Green Monkey (E), Grivet Monkey (E), Savanna Monkey (E), Tantalus Monkey (E), Vervet Monkey (E), Mono verde (S), Singe vert (F) II M

Chlorocebus pygerythrus $=$ Chlorocebus aethiops

Chlorocebus sabaeus $=$ Chlorocebus aethiops

Chlorocebus tantalus $=$ Chlorocebus aethiops

Chlorostilbon alice: Green-tailed Emerald (E), Esmeralda coliverde (S), Émeraude alice (F) II B

Chlorostilbon assimilis: Garden Emerald (E), Émeraude du Panama (F) II B

Chlorostilbon auratus $=$ Chlorostilbon poortman

Chlorostilbon aureoventris: Glittering-bellied Emerald (E), Picaflor común (S), Émeraude splendide (F) II B

Chlorostilbon auriceps $=$ Chlorostilbon canivetii

Chlorostilbon bracei: Brace's Emerald (E), Grace's Emerald (E), Émeraude de New Providence (F) II B

Chlorostilbon canivetii: Fork-tailed Emerald (E), Esmeralda de Canivet (S), Émeraude de Cavinet (F) II B

Chlorostilbon canivetii assimilis $=$ Chlorostilbon assimilis

Chlorostilbon forficalus $=$ Chlorostilbon canivetii

Chlorostilbon gibsoni: Red-billed Emerald (E), Esmeralda pico rojo (S), Émeraude de Gibson (F) II B

Chlorostilbon inexpectalus $=$ Chlorostilbon poortmani

Chlorostilbon maugaeus: Puerto Rican Emerald (E), Émeraude de Porto Rico (F) II B

Chlorostilbon maugeus bracei $=$ Chlorostilbon bracei

Chlorostilbon mellisugus: Blue-tailed Emerald (E), Esmeralda coliazul (S), Émeraude orvert (F) II B

Chlorostilbon poortmani: Short-tailed Emerald (E), Esmeralda cola corta (S), Émeraude à queue courte (F) II B

Chlorostilbon ricordii: Cuban Emerald (E), Émeraude de Ricord (F) II B

Chlorostilbon russatus: Coppery Emerald (E), Esmeralda bronceada (S), Émeraude cuivrée (F) II B

Chlorostilbon salvini $=$ Chlorostilbon caniverii

Chlorostilbon stenura: Narrow-tailed Emerald (E), Esmeralda cola de alambre (S), Emeraude à queue étroite (F) 1 I B

Chlorostilbon swainsonii: Hispaniolan Emerald (E), Zumbador (S), Émeraude d'Hispaniola (F) II B

Choeropsis liberiensis $=$ Hexaprotodon liberiensis

Choloepus hoffmanni: Hoffmann's Two-toed Sloth (E), Perezoso (S). Unau (S), Unau d'Hoffmann (F) III M

Chondrohierax uncinatus: Hook-billed Kite (E), Gavilán pico ganchudo (S), Milano picogarfio (S), Milan bec-en-croc (F) I/II B

Chondrohierax uncinatus wilsonii: Cuban Hook-billed Kite (E), Busard de Wilson (F), Milan de Cuba (F), Milan de Wilson (F) I B

Chondrohierax wilsonii $=$ Chondrohierax uncinatus wilsonii

Chondropython viridis = Morelia viridis

Choriotis arabs = Ardeotis arabs

Choriotis australis $=$ Ardeotis australis

Choriotis kori $=$ Ardeotis kori

Choriotis nigriceps $=$ Ardeotis nigriceps

Chorlito esquimal (S): Numenius borealis I B

Chorlo polar (S): Numenius borealis I B

Chosna (S): Potos flavus III M

Chosna pericote (S): Bassaricyon gabbii III M

Chouette à collier (F): Pulsairix melanota II B

Chouette a joues blanches (F): Sceloglaux albifacies II B

Chouette à lignes noires (F): Strix nigrolineata II B

Chouette a lunettes (F): Pulsarrix perspicillata 11 B

Chouette à sourcils jaunes (F): Pulsatrix koeniswaldiana II B

Chouette africaine (F): Strix woodfordii II B

Chouette baie (F): Phodilus badius II B

Chouette boobok (F): Ninox novaese landiae III B

Chouette boobok de l'ile Norfolk (F): Ninox novaeseelandiae undulata I B
Chouette chevêche (F): Athene noctua II B

Chouette chevêchette (F): Glaucidium passerinum II B

Chouette coucou (F): Ninox novaeseelandiae I/II B

Chouette de Butler (F): Strix bulleri II B

Chouette de Cuba (F): Orus lawrencii II B

Chouette de Harris (F): Aegolius harrisii II B

Chouette de Koeniswald (F): Pulsatrix koeniswaldiana II B

Chouette de l'Oural (F): Sirix uralensis II B

Chouette de Nouvelle-Guinée (F): Uroglaux dimorpha II B

Chouette de Sitchouan (F): Strix davidi II B

Chouette de terrier (F): Speotyto cunicularia II B

Chouette des forêts ( $\mathrm{F}$ ): Athene blewitti I B

Chouette des îles Salomons (F): Nesasio solomonensis II B

Chouette des Moluques (F): Ninox squamipila III B

Chouette des pagodes (F): Strix seloputo II B

Chouette dryade (F): Strix hylophila II B

Chouette d'Ussher (F): Scotopelia ussheri II B

Chouette effraie (F): Tyto alba II B

Chouette épervière (F): Ninox squamipila III B/Sumia ulula II B

Chouette épervière de l'ile Christmas (F): Ninox squamipila natalis I B

Chouette fasciée (F): Strix albitarsus II B

Chouette fauve (F): Strix fulvescens II B

Chouette harfang (F): Nyctea scandiaca II B

Chouette huhul (F): Strix huhula II B

Chouette hulotte (F): Strix aluco II B

Chouette hulotte de Hume (F): Strix butleri II B

Chouette lapone (F): Strix nebulosa II B

Chouette leptogramme (F): Strix leptogrammica II B

Chouette masquée (F): Strix rufipes II B

Chouette mouchetée (F): Sirix virgata II B

Chouette noire et blanche (F): Strix nigrolineasa II B

Chouette ocellée (F): Strix ocellata II B

Chouette pêcheuse (F): Scotopelia peli II B

Chouette rayée (F): Sirix varia II B

Chouette tachetée (F): Strix occidentalis II B

Chouette-pêcheuse de Bouvier (F): Scotopelia bouvieri II B

Chouette-pêcheuse de Pel (F): Scoropelia peli II B

Chouette-peccheuse rousse (F): Scotopelia ussheri II B

Chouette-pêcheuses (F): Scotopelia spp. II B

Chouettes (F): Pulsarrix spp/Strix spp/Sumia ulula II B

Chousingha (E): Tetracerus quadricomis III M

Christmas Hawk-Owl (E): Ninox squamipila natalis I B

Christmas Island Frigatebird (E): Fregara andrewsi I B

Christmas Island Hawk-Owl (E): Ninox squamipila natalis I B

Chrysocyon brachyurus: Maned Wolf (E), Aguara guazu (S), Lobo de crin (S), Loup à crinière (F) II M

Chrysolampis mosquitus: Ruby-topaz Hummingbird (E), Tucusito rubi (S), Colibri rubis-topaze (F) II B

Chrysuronia oenone: Golden-tailed Sapphire (E), Colibri cola de oro (S), Zafiro colidorado (S), Saphir oenome (F) II B

Chuckwalla de San Esteban (F): Sauromalus varius I R

Chuckwallas (E): Sauromalus spp. INC R

Chungungo (E/S/F): Lontra felina I M

Chupacacao negro (S): Daptrius ater II B

Chupacacao ventriblanco (S): Dapirius americanus II B

Churica (S): Brotogeris jugularis II B

Churiquita (S): Touit dilectissima II B

Chuuk Flying-fox (E): Pteropus insularis I M

Cibeta africana (S): Civettictis civelta III M

Cibeta atigrada (S): Prionodon linsang II M

Cibeta de Madagascar (S): Fossa fossana II M

Cibeta moteada (S): Prionodon pardicolor I M

Cibeta nutria (S): Cynogale bennettii II M

Cibeta palmirayada (S): Hemigalus derbyanus II M

Ciccaba albilarsus = Strix albitarsus

Ciccaba huhula = Strix huhula

Ciccaba nigrolineata $=$ Strix nigrolineata

Ciccaba virgata $=$ Strix virgata

Ciccaba woodfordii = Strix woodfordi

Cicinnurus magnificus: Magnificent Bird-of-paradise (E), Paradisier magnifique (F) II B

Cicinnurus regius: King Bird-of-paradise (E), Paradisier royal (F) II B

Cicinnurus respublica: Wilson's Bird-of-paradise (E), Paradisier républicain (F) II B 
Ciconia boyciana: Japanese White Stork (E), Oriental Stork (E), Oriental White Stork (E), Cigieña blanca coreana (S), Ciguieña oriental (S), Cigogne blanche de Corée (F), Cigogne blanche du Japon (F), Cigogne orientale (F), Cigogne à bec noir (F) I B Ciconia ciconia boyciana = Ciconia boyciana

Ciconia nigra: Black Stork (E), Cigüeña negra (S), Cigogne noire (F) II B

Cicupa (S): Pulsatrix melanota II B

Ciervo almizclero (S): Moschus moschiferus I M

Ciervo andino meridional (S): Hippocamelus bisulcus $1 \mathrm{M}$

Ciervo andino septentrional (S): Hippocamelus antisensis I M

Ciervo bactriano (S): Cervus elaphus bactrianus II M

Ciervo de Cachemira (S): Cervus elaphus hanglu I M

Ciervo de Duvaucel (S): Cervus duvauceli I M

Ciervo de Elde (S): Cervus eldii I M

Ciervo de Kuhl (S): Axis kuhlii I M

Ciervo de la Berberia (S): Cervus elaphus barbarus III M

Ciervo de las Pampas (S): Ozotoceros bezoarticus I M

Ciervo de los Calamianes (S): Axis calamianensis I M

Ciervo de los pantanos (S): Blastocerus dichotomus I M

Ciervo enano (S): Pudu puda I M

Ciervo marismeño (S): Blastocerus dichotomus I M

Ciervo parnpero (S): Ozotoceros bezoanicus I M

Ciervo porquerizo de Indochina (S): Axis porcinus annamiticus I M

Ciervo porquerizo de Kuhl (S): Axis kuhlii I M

Ciervo porquerizo de los Calamianes (S): Axis calamianensis I M

Ciervos almizcleros (S): Moschus spp. I/II M

Ciervos andinos (S): Hippocamelus spp. I M

Cigogne à bec noir (F): Ciconia boyciana I $\mathrm{B}$

Cigogne blanche de Corée (F): Ciconia boyciana I B

Cigogne blanche du Japon (F): Ciconia boyciana I B

Cigogne noire (F): Ciconia nigra II B

Cigogne orientale (F): Ciconia boyciana I B

Cigognes (F): Ciconiidae spp. I/IUII/NC B

Cigüeña blanca coreana (S): Ciconia boyciana I B

Cigüeña ensillada (S): Ephippiorhynchus senegalensis III B

Cigüeña negra (S): Ciconia nigra II B

Cigüeña oriental (S): Ciconia boyciana I B

Cinereous Harrier (E): Circus cinereus II B

Cinereous Vulture (E): Aegypius monachus II B

Cinnamon Hummingbird (E): Amazilia rutila II B

Cinnamon-throated Hermit (E): Phaethomis nattereri II B

Circaète à poitrine noire (F): Circaelus pectoralis II B

Circaète barté (F): Circaerus fasciolatus II B

Circaète brun (F): Circaetus cinereus II B

Circaète cendré (F): Circaetus cinerascens II B

Circaète Jean-le-Blanc (F): Circaetus gallicus II B

Circaètes (F): Circaetus spp. II B

Circaetus beaudouini $=$ Circaetus gallicus

Circaetus cinerascens: Banded Snake-Eagle (E), Smaller Banded Snake-Eagle (E), Aguila culebrera culiblanca (S), Culebrera coliblanca (S), Circaète cendré (F) II B

Circaetus cinereus: Brown Harrier-Eagle (E), Brown Snake-Eagle (E), Aguila culebrera de Brown (S), Culebrera sombría (S), Circaète brun (F) II B

Circaetus fasciolatus: Fasciated Snake-Eagle (E), Southern Banded Snake-Eagle (E), Circaète barté (F) II B

Circaetus gallicus: Beaudouin's Snake-Eagle (E), Short-toed Eagle (E), Short-toed Snake-Eagle (E), Aguila culebrera (S), Culebrera barreada (S), Culebrera europea (S), Circaète Jean-le-Blanc ( $F$ ) II B

Circaetus pectoralis: Black-chested Snake-Eagle (E), Culebrera pechinegra (S), Circaète à poitrine noire $(\mathrm{F})$ II B

Circus aeruginosus: Westem Marsh-Harrier (E), Aguilucho lagunero (S), Aguilucho lagunero occidental (S), Busard des roseaux (F) II B

Circus aeruginosus approximans $=$ Circus approximans

Circus aeruginosus maillard $=$ Circus maillardi

Circus aeruginosus spilonotus = Circus spilonotus

Circus approximans: Swamp Harrier (E), Aguilucho lagunero del pacifico (S), Busard de Gould (F) II B

Circus assimilis: Aguilucho moteado (E), Spotted Harrier (E), Busard tacheté (F) II B

Circus buffoni: Long-winged Harrier (E), Aguilucho de azara (S), Aguilucho de ciénaga (S), Gavilán grande (S), Busard de Buffon (F) II B
Circus cinereus: Cinereous Harrier (E), Aguilucho vari (S), Gavilán ceniciento (S), Busard bariolé (F) II B

Circus cyaneus: Hen Harrier (E), Marsh Hawk (E), Northem Harrier (E), Aguilucho pálido (S), Gavilán rastrero (S), Busard SaintMartin (F) II B

Circus macrouns: Pale Harrier (E), Pallid Harrier (E), Aguilucho papialbo (S), Busard pâle (F) II B

Circus maillardi: Madagascar Harrier (E), Madagascar MarshHarrier (E), Aguilucho lagunero malgache (S), Busard de Maillard (F) II B

Circus maurus: Black Harrier (E), Aguilucho negro (S), Busard maure (F) II B

Circus melanoleucos: Pied Harrier (E), Aguilucho pío (S), Busard tchoug (F) II B

Circus pygargus: Montagu's Harrier (E), Aguilucho cenizo (S) Busard cendré (F), Busard de montagne (F) II B

Circus ranivorus: African Marsh-Harier (E), Aguilucho lagunero etiópico (S), Busard grenouillard (F) II B

Circus spilonotus: Eastem Marsh-Harrier (E), Aguilucho lagunero oriental (S). Busard d'Orient (F) II B

Cirrhipathes aggregata: II In

Cirrhipathes anguina: II In

Cirrhipathes contorta: II In

Cirrhipathes musculosa: II In

Cirrhipathes nana: II In

Cirrhipathes rumphi: II In

Cirrhipathes saccula: II In

Cirrhipathes semiglabra: II In

Cirrhipathes sinensis: II In

Cirrhipathes solorensis: II In

Cirrhipathes spiralis: II In

Cirrhipathes translucens: 11 In

Cirrhipathes variabilis: II In

Cisne coscoroba (S): Coscoroba coscoroba II B

Cisne cuellinegro (S): Cygnus melanocorypha II B

Cisne cuello negro (S): Cygnus melanocorypha II B

Citharocyathus $=$ Notocyathus

Civeta de palmera común (S): Paradoxurus hermaphroditus III M

Civeta de palmera de Jerdon (S): Paradoxurus jerdoni III M

Civeta de palmera enmascarada (S): Paguma larvata III M

Civets (E): Vivernidae spp. II/IIINC M

Civette à bandes $(\mathrm{F})$ : Prionodon linsang $1 \mathrm{M}$

Civette africaine (F): Civertictis civenta III M

Civette d'Afrique (F): Civentictis civerta III M

Civette fossane (F): Fossa fossana II M

Civette malgache (F): Fossa fossana II M

Civette palmiste à bandes de Derby $(\mathrm{F})$ : Hemigalus derbyanus II $\mathrm{M}$

Civette palmiste rayée ( $F$ ): Hemigalus derbyanus II M

Civette-loutre de Sumatra (F); Cynogale bennettii II M

Civentictis civenta: African Civet $(E)$, Cibeta africana (S), Civetre africaine $(\mathrm{F})$, Civette d'Afrique (F) III M

Cladangia exusta: II In

Cladangia gemmans: II In

Cladangia gemmans = Schizoculina africana

Cladocora arbuscula: Ivory Tube Coral (E) II in

Cladocora cespitosa: II In

Cladocora debilis: II In

Cladocora pacifica: II In

Cladopathes plumosa: II In

Cladopsammia echinata: II In

Cladopsammia eguchii: II in

Cladopsammia gracilis: II In

Cladopsammia rolandi: II In

Clavarina $=$ Paraclavarina

Clawless otters (E): Aonyx congicus/Aonyx capensis $\mathbf{I I I} M$

Clelia clelia: Mussurana (E), Masurana (S), Mussurana (F) II R

Clemmyde de Muhlenberg (F): Clemmys muhlenbergi I R

Clemmyde sculptée (F): Clemmys insculpta II R

Clemmys insculpta: Wood Turtle (E), Clemmyde sculptée (F) II R

Clemmys muhlenbergi: Bog Turtle (E), Muhlenberg's Turtle (E), Galápago de Muhlenberg (S), Clemmyde de Muhlenberg (F), Tortue de Muhlenberg $(F)$, Tortue des marécages (F) I R

Clemmys muhlenbergii $=$ Clemmys muhlenbergi

Clouded Leopard (E): Neofelis nebulosa I M

Cloud-forest Screech-Owl (E): Otus marshalli II B

Club Finger Coral (E): Porises porites II In 
Club Naiad (E): Pleurobema clava II In

Clubshell (E): Pleurobema clava II In

Clubshell Pearly Mussel (E): Pleurobema clava II In

Clymene Dolphin (E): Sienella clymene II M

Clytolaema rubricauda: Brazilian Ruby (E), Colibri rubis-émeraude (F) II B

Cnemidophorus hyperythrus: Orange-throated Race-runner (E). Orange-throated Whiptail (E), Race-runner (E), Corredor gorguinaranja (S), Coureur à gorge orange (F) II R

Cnemophilus loriae: Loria's Bird-of-paradise (E), Paradisier de Loria (F), Paradisier loria (F) II B

Cnemophilus macgregorii: Crested Bird-of-paradise (E), Paradisier huppé (F) II B

Coahuila Box Turtle (E): Terrapene coahuila I R

Coahuila Turtle (E): Terrapene coahuila I R

Coalfish Whale (E): Balaenoptera borealis I M

Coarse Chameleon (E): Chamaeleo rudis II R

Coast Horned Lizard (E): Phrynosoma coronatum II R

Coastal Spiny-tailed Lizard (E): Cordylus macropholis II R

Coati (E): Nasua narica $\mathbf{I I} \mathrm{M}$

Coat́ (S): Nasua nasua solitaria III M

Coati roux (F): Nasua nasua solitaria III M

Coatis (E): Nasua spp. II/II/NC M

Cobalt-winged Parakeet (E): Brotogeris cyanoptera II B

Cobe lechwe (F): Kobus leche II M

Cobo de Lechwe (S): Kobus leche II M

Cobra à lunettes (F): Naja naja II R

Cobra escupidora asiática (S): Naja naja II R

Cobra royal (F): Ophiophagus hannah II $\mathbf{R}$

Cobras (E): Naja spp/Ophiophagus hannah/Paranaja spp/Pseudohaje spp. IU/NC R

Cochito (E/S/F): Phocoena sinus I M

Cockatiel (E/S): Nymphicus hollandicus NC B

Cockatoos (E): Cacatua spp/Callocephalon spp/Calyptorhynchus spp/Eolophus spp/Probosciger aterrimus III B

Cocks-of-the-rock (E): Rupicola spp. II B

Cocodrilo americano (S): Crocodylus acumus I R

Cocodrilo australiano (S): Crocodylus johnsoni II R

Cocodrilo chico africano (S): Osteolaemus tetraspis I R

Cocodrilo de Cuba (S): Crocodylus rhombifer I R

Cocodrilo de Mindoro (S): Crocodylus novaeguineae mindorensis I $\mathrm{R}$

Cocodrilo de Morelet (S): Crocodylus moreletii I R

Cocodrilo de Nueva Guinea (S): Crocodylus novaeguineae I/I R

Cocodrilo de Siam (S): Crocodylus siamensis I R

Cocodrilo del Nilo (S): Crocodylus niloticus VI R

Cocodrilo del Orinoco (S): Crocodylus intermedius I R

Cocodrilo hociquifino africano (S): Crocodylus catophractus I R

Cocodrilo marismeño (S): Crocodylus palustris I R

Cocodrilo poroso (S): Crocodylus porosus I/II $R$

Cocodrilos (S): CROCODYLIA spp, I/I R

Coelacanth (E): Latimeria chalumnae I F

Coelacanthe $(\mathrm{F})$ : Lasimeria chalumnae I $\mathrm{F}$

Coelastrea tenuis: II In

Coeligena bonapartei: Golden-bellied Starfrontlet (E), Colibrí inca dorado (S), Inca de Bonaparte (F) II B

Coeligena coeligena: Bronzy Inca (E), Colibri inca bronceado (S), Inca céleste (F) 11 B

Coeligena helianthea: Blue-throated Starfrontlet (E), Colibri inca ventrivioleta (S), inca porphyre (F) II B

Coeligena inca = Coeligena torquata

Coeligena iris: Rainbow Starfrontlet (E), Frentiestrella arcoiris (S), Inca iris (F) II B

Coeligena lutetiae: Buff-winged Starfrontlet (E), Frentiestrella alihabano (S), Inca à gemme bleue (F) II B

Coeligena orina: Dusky Starfrontlet (E), Inca de Wetmore (F) II B

Coeligena phalerata: White-tailed Starfrontlet (E), Inca à queue blanche (F) II B

Coeligena prunellei: Black Inca (E), Inca noir (F) II B

Coeligena forquata: Collared Inca (E), Colibri inca acollarado (S), Inca à collier (F) II B

Coeligena violifer: Violet-throated Starfrontlet (E), Inca violifere (F) II $\mathrm{B}$

Coeligena wilsoni: Brown inca (E), Inca cafe (S), Inca brun (F) II B

Coelocyathus $=$ Monomyces

Coeloseris mayeri: II In
Coelosmilia $=$ Desmophyllum

Coenangia conferia: II in

Coendou mexicanus $=$ Sphiggurus mexicanus

Coendou spinosus = Sphiggurus spinosus

Coenocyathus anthophyllites: II In

Coenocyathus bowersi: II In

Coenocyathus brooki: II In

Coenocyashus cylindricus; II in

Coenocyathus dohmi: II In

Coenocyathus dohmi = Caryophyllia inomata

Coenocyathus giesbrechti: II In

Coenocyathus giesbrechti = Caryophyllia inornata

Coenocyathus goreaui: II In

Coenocyarhus sagamiensis: II In

Coenocyathus zelandiae: II In

Coenopsammia aequiserialis: II In

Coenopsammia affinis: II In

Coenopsammia manni: II In

Coenopsammia radiata: II In

Coenosmilia inordinata: II in

COENOTHECALIA spp:: Blue Coral (E), Dont corail bleu (F) II In

Colacerda crestuda (S): Popelairia popelairii II B

Colacerda ventrinegra (S): Popelairia langsdorffi II B

Colacerda verde (S): Popelairia conversii II B

Colacinta colinegra (S): Lesbia victorice II B

Colacinta coliverde (S): Lesbia nuna II B

Colaespatula zamarito (S): Ocreatus underwoodii II B

Colangia immersa: II In

Colapinto ecuatoriano (\$): Phlogophilus hemileucurus II B

Colasisi (E): Loriculus philippensis II B

Coliazul bengali (S): Uraeginthus bengalus III B

Colibri à ailes saphir (F): Pierophanes cyanopterus II B

Colibrí à bec incurvé (F): Ramphodon dohmii I B

Colibri à calotte violette (F): Goldmania violiceps II B

Colibri à coiffe blanche (F): Microchera albocoronata $I I$ B

Colibri à dos noir (F): Ramphomicron dorsale II B

Colibri à épaulettes (F): Eupherusa eximia II B

Colibri à flancs blancs (F): Oreotrochilus leucopleurus II B

Colibri à gorge améthyste (F): Lampornis amethystinus II B

Colibri à gorge blanche (F): Leucochloris albicollis II B

Colibri à gorge bleue (F): Lampomis clemenciae II B

Colibri à gorge grenat (F): Lamprolaima rhami II B

Colibri à gorge lilas (F): Lampornis hemileucus II B

Colibri à gorge noire (F): Archilochus alexandri II B

Colibri à gorge rubis (F): Archilochus colubris II B

Colibri à menton bleu (F): Chlorestes notatus II B

Colibri à petit bec (F): Ramphomicron microrhynchum II B

Colibri à plastron noir (F): Oreotrochilus melanogaster II B

Colibri à poitrine violette (F): Stemoclyta cyanopectus II B

Colibri à queue bronzée (F): Chalybura urochrysia II B

Colibri à queue courte (F): Myrmia micrura II B

Colibri à queue en ciseaux (F): Hylonympha macrocerca II B

Colibri à queue large (F): Selasphorus platycercus II B

Colibri à queue mi-blanche (F): Phlogophilus hemileucurus II B

Colibri à queue singulière (F): Doricha enicura II B

Colibri à queue verte (F): Leucippus chlorocercus II B

Colibri à sous-caudales rousses (F): Urosticte ruficrissa II B

Colibri à tête bleue (F): Cyanophaia bicolor II B

Colibri à tête cuivrée (F): Elvira cupreiceps II B

Colibri à tête noire (F): Trochilus polyrmus II B

Colibri à tête rose (F): Anthocephala floriceps II B

Colibri à tête violette (F): Klais guimeti II B

Colibri à ventre blanc (F): Colibri serrirostris II B

Colibri à ventre châtain (F): Lampomis castaneoventris II B

Colibri à ventre noir (F): Eupherusa nigriventris $11 \mathrm{~B}$

Colibri adèle (F): Oreorrochilus adela II B

Colibri alicastano (S): Lamprolaima rhami II B

Colibri amethyste (F): Calliphlox amethystina II B

Colibri anaïs (F): Colibri coruscans II B

Colibri anteado (S): Leucippus fallax II B

Colibri ardent (F): Selasphorus ardens II B

Colibri aux huppes d'or (F): Heliactin cornuta II B

Colibri avocette (F): Avocettula recurvirostris II B

Colibri avocettin (F): Opisthoprora euryptera II B

Colibri barbiesmeralda (S): Abeillia abeillei II B

Colibri barbinegro (S): Archilochus alexandri II B 
Colibri bourdon (F): Acesirura bombus II B Colibri cabecidorado (S): Boissonneaua flavescens II B Colibrí cabecivioleta (S): Klais guimeti II B Colibri calliope (F): Stellula calliope II B Colibri canelo (S): Amazilia rutila II B Colibri casqué (F): Oxypogon guerinii II B Colibri charmant (F): Calothorax pulcher II B Colibri circe (F): Cynanthus latirostris II B Colibri cola de hoja (S): Ocreatus underwoodii II B Colibri cola de oro (S): Chrysuronia oenone II B Colibrí coliazul (S): Amazilia cyanura II B Colibri colirrayado (S): Eupherusa eximia II B Colibrí colirrufo (S): Amazilia tzacatl II B Colibrí colpinto (S): Tilmatura dupontii II B Colibri coludo azul (S): Aglaiocercus kingi II B Colibrí coludo verde (S): Lesbia nuna II B Colibri comète (F): Taphrolesbia griseiventris II B Colibri cora (F): Thaumastura cora II B

Colibri corinne (F): Heliomaster longirostris II B

Colibrí corona-verde (S): Amazilia viridifrons II B

Colibrí corona-violeta (S): Amazilia violiceps II B

Colibrí coroniazul (S): Amazilia cyanocephala II B

Colibri coruscans: Sparkling Violet-ear (E), Colibri orejivioleta grande (S). Picaflor ventriazul (S), Colibri anaïs (F) II B

Colibrí cuellicastaño (S): Heliodoxa aurescens II B

Colibri d'Abeillé (F): Abeillia abeillei II B

Colibri d'Alice (F): Aglaeactis aliciae II B

Colibri d'Allen (F): Selasphorus sasin II B

Colibri d'Angèle (F): Heliomaster furcifer II B

Colibri d'Anna (F): Calypte anna II B

Colibri d'Arica (F): Eulidia yarrellii II B

Colibri de Anna (S): Calypte anna II B

Colibri de Benjamin (F): Urosticte benjamini II B

Colibri de Berilo (\$): Amazilia beryllina II B

Colibri de Berlepsch (F): Acestrura berlepschi II B

Colibri de Bouguer (F): Urochroa bougueri II B

Colibri de Bourcier (F): Polyonymus caroli II B

Colibri de Buffon (F): Chalybura buffonii II B

Colibri de Burmeister (F): Microstilbon burmeisteri II B

Colibri de Castelneau (F): Aglaeacris castelnaudii II B

Colibri de Colliope (S): Stellula calliope II B

Colibri de Constant (F): Heliomaster constantii II B

Colibri de Costa (F): Calypte costae II B

Colibri de Cuvier (F): Phaeochroa cuvierii II B

Colibri de Delalande (F): Stephanoxis lalandi II B

Colibri de Delphine (F): Colibri delphinae II B

Colibri de Dohm (F): Ramphodon dohmii I B

Colibri de Geoffroy (F): Augastes geoffroyi II B

Colibri de Goudot (F): Lepidopyga goudoti II B

Colibri de Hartert (F): Phlogophilus harteri II B

Colibri de Jardine (F): Boissonneaua jardini II B

Colibri de Jourdan (F): Chaetocercus jourdanii II B

Colibri de Lafresnaye (F): Lafresnaya lafresnayi II B

Colibri de Lillian (F): Lepidopyga lilliae II B

Colibri de Matthews (F): Boissonneaua matthewsii II B

Colibri de Mitchell (F): Philodice mitchellii II B

Colibri de Mulsant (F): Acestrura mulsant II B

Colibri de Perijá (S): Metallura iracunda II B

Colibri de Rivoli (F): Eugenes fulgens II B

Colibri de Sybil (F): Lampomis sybillae II B

Colibri de Taczanowski (F): Leucippus taczanowskii II B

Colibri de Tumbes $(\mathrm{F})$ : Leucippus baeri II B

Colibri de Xantus (S): Hylocharis xantusii II B

Colibri d'Elliot (F): Atthis ellioti II B

Colibri delphinae: Brown Violet-ear (E), Orejavioleta café (S), Orejivioleta marón (S), Colibri de Delphine (F) II B

Colibri demi-deuil (F): Melanotrochilus fuscus II B

Colibri d'Équateur (F): Androdon aequatorialis II B

Colibri des Bahamas (F): Calliphlox evelynae II B

Colibri des Santa Marta (F): Acestrura astreans II B

Colibri des tépuis (F): Polysmus milleri II B

Colibri d'Helen (F): Mellisuga helenae II B

Colibri d'Oaxaca (F): Eupherusa cyanophrys II B

Colibri du Chili (F): Sephanoides sephaniodes II B

Colibri du Chimborazo (F): Oreotrochilus chimborazo II B

Colibri du Guerrero (F): Eupherusa poliocerca II B
Colibri du Pirré (F): Goethalsia bella II B

Colibri élise (F): Doricha eliza II B

Colibri elvire (F): Elvira chionura II B

Colibri estelle (F): Oreotrochilus estella II B

Colibri estrella picolargo (S): Heliomaster longirostris II B

Colibri étincelant (F): Aglaeactis cupripennis II B

Colibri falle-vert (F): Eulampis holosericeus II B

Colibri fanny (F): Myrtis fanny II B

Colibri faux-saphir (F): Lepidopyga coeruleogularis $11 \mathrm{~B}$

Colibri féérique (F): Heliothryx barroti II B

Colibri flamboyant (F): Topaza pyra II B

Colibri flammule (F): Selasphorus flammula II B

Colibri flavescent (F): Boissonneaua flavescens II B

Colibrí frentiverde (S): Heliodoxa xanthogonys II B

Colibrí gargantidorado (S): Polyımus guainumbi II B

Colibri géant (F): Patagona gigas II B

Colibrí gigante (S): Palagona gigas II B

Colibrí gorjimubi (S): Archilochus colubris II B

Colibrí grande colinegro (S): Chalybura buffonii II B

Colibri grivelé (F): Taphrospilus hypostičus II B

Colibri guaïnumbi (F): Polytmus guainumbi II B

Colibrí guerrerense (S): Eupherusa poliocerca II B

Colibrí hada orejazul (S): Heliothryx aurita II B

Colibri héliodore $(\mathrm{F})$ : Acestrura heliodor II $\mathrm{B}$

Colibri héloöse (F): Atthis heloisa II B

Colibri hirondelle (F): Eupetomena macroura II B

Colibri huppé (F): Ortharhynchus cristatus II B

Colibri inca acollarado (S): Coeligena torquata II B

Colibri inca bronceado (S): Coeligena coeligena II B

Colibrí inca dorado (S): Coeligena bonapartei $\mathbf{I I} \mathrm{B}$

Colibri inca ventrivioleta (S): Coeligena helianthea II B

Colibri insigne (F): Panterpe insignis II B

Colibri jacobin (F): Florisuga mellivora II B

Colibri julie (F): Damophila julie II B

Colibri lucifer ( $\mathrm{F}$ ): Calothorax lucifer II B

Colibri lumachelle (F): Augastes lumachellus II B

Colibri madère (F): Eulampis jugularis II B

Colibri magenta (F): Philodice bryantae II B

Colibri magnífico (S): Eugenes fulgens II B

Colıbri médiastin (F): Heliomaster squamosus II B

Colibri moucheté (F): Adelomyia melanogenys II B

Colibrí multipunteado (S): Taphrospilus hypostictus II B

Colibri nain (F): Mellisuga minima II B

Colibri noble (F): Oreonympha nobilis II B

Colibri nuca blanca (S): Florisuga mellivora II B

Colibrí oaxaqueño (S): Eupherusa cyanophrys II B

Colibrí olivipunteado (S): Leucippus chlorocercus II B

Colibri oreillard (F): Heliothryx aurita II B

Colibrí orejiblanco (S): Hylocharis leucotis II B

Colibri orejivioleta grande (S): Colibri coruscans II B

Colibri orejivioleta verde (S): Colibri thalassinus II B

Colibri paméla (F): Aglaeactis pamela II B

Colibrí pantalón cobrizo (S): Eriocnemis cupreoventris II B

Colibri pantalón verde (S): Eriocnemis vestirus II B

Colibrí pechiazul (S): Stemoclyta cyanopectus II B

Colibri pecho canela (S): Glaucis hirsuta II B

Colibrí pico de cuña (S): Augastes geoffroyi II B

Colibrí pico espada (S): Ensifera ensifera II B

Colibri pico espina (S): Ramphomicron microrhynchum II B

Colibri pico lezna (S): Avocettula recurvirostris II B

Colibri picosierra chico (S): Ramphodon dohmii I B

Colibri piquiancho (S): Cynanthus latirostris II B

Colibri piquiavoceta (S): Opisthoprora euryptera II B

Colibri piquicunna (S): Augastes geoffroyi II B

Colibri piquidentado (S): Androdon aequatorialis II B

Colibri porte-épée (F): Ensifera ensifera II B

Colibri prieto (S): Cynanthus sordidus II B

Colibri robinson (F): Sephanoides fernandensis II B

Colibri roux (F): Selasphorus rufus II B

Colibri r.sbis-émeraude (F): Clytolaema rubricauda II B

Colibri rubis-topaze (F): Chrysolampis mosquitus II B

Colibri sapho (F): Sappho sparganura II B

Colibri scintillant (F): Selasphorus scintilla II B

Colibri serrano gargantiazul (S): Adelomyia melanogenys II B

Colibri serrirostris: White-vented Violet-ear (E), Picaflor ventriverde (S), Colibri à ventre blanc (F) II B 
Colibri sombre (F): Cynanthus sordidus II B

Colibri superbe (F): Augastes scutatus II B

Colibri tacheté (F): Ramphodon naevius II B

Colibrí tepui (S): Polyrmus milleri II B

Colibrí terciopelo (S): Lafresnaya lafresnayi II B

Colibri thalassin (F): Colibri thalassinus II B

Colibri thalassinus: Green Violet-ear (E), Colibri orejivioleta verde

(S), Picaflor orejiazul (S), Colibri thalassin (F) II B

Colibri tijereta (S): Hylonympha macrocerca II B

Colibri topaze (F): Topaza pella II B

Colibri tout-vert (F): Polytmus theresiae II B

Colibri trompeur (F): Leucippus fallax II B

Colibrí ventrivioleta (S): Damophila julie II B

Colibri verde colimojo (S): Metallura tyrianthina II B

Colibrí verdecito (S): Chlorestes notatus II B

Colibri vert et gris (F): Aphantochroa cirrochloris II B

Colibri vert-d'eau $(\mathrm{F})$ : Lampormis viridipallens II B

Colibri vesper $(\mathrm{F})$ : Rhodopis vesper II B

Colibrí vientre-canelo (S): Amazilia yucatanensis II B

Colibri zémès (F): Tilmatura dupontii II B

Colibries (S): Trochilidae spp. I/II B

Colibris (F): Trochilidae spp. I/I B

Colibri-serrano gorjiamatisto (S): Lampomis amethystinus II B

Colibri-serrano gorjiazul (S): Lampornis clemenciae II B

Colibrí-serrano gorjiverde (S): Lampormis viridipallens II B

Colibri-serrano pechiverde (S): Lampormis svbillae II B

Colin de Ridgwayi (F): Colinus virginianus ridgwayi I B

Colin de Virginie masqué (F): Colinus virginianus ridgwayi I B

Colín virginiano de Ridgway (S): Colinus virginianus ridgwayi I B

Colins (F): Phasianidae spp. I/NC B

Colinus virginianus ridgwayi: Masked Bobwhite (E), Colín virginiano de Ridgway (S), Colin de Ridgway (F), Colin de Virginie masqué (F) I B

Coliuspasser ardens = Euplectes ardens

Coliuspasser macrourus $=$ Euplectes macrourus

Collared Falconet (E): Microhierax caerulescens II B

Collared Fishing Hawk (E): Busarellus nigricollis II B

Collared Forest-Falcon (E): Micrastur semitorquatus II B

Collared Inca (E): Coeligena torquata II B

Collared Lory (E): Phigys solinarius II B

Collared Mangabey (E): Cercocebus torquarus II M

Collared Owlet (E): Glaucidium brodiei II B

Collared Pygmy-Owl (E): Glaucidium brodiei II B

Collared Scops-Owl (E): Otus bakkamoena II B

Collared Sparrowhawk (E): Accipiter cirrocephalus II B

Collared-Doves (E): Streptopelia spp. III/NC B

Collins's Poison Frog (E): Minyobates abditus II A

Colobe (F): Colobus angolensis II M

Colobe à camail (F): Colobus polykomos II M

Colobe à épaules blanches (F): Colobus guereza II M

Colobe a huppe (F): Procolobus verus II M

Colobe à longs poils (F): Colobus polykomos II M

Colobe bai (F): Procolobus badius II M/Procolobus pennantii I/II M

Colobe bai d'Afrique occidentale (F): Procolobus badius II M

Colobe bai de la Tana (F): Procolobus rufomitratus I M

Colobe bai de Zanzibar (F): Procolobus pennantii kirkii I M

Colobe bai du Cameroun (F): Procolobus preussi II M

Colobe blanc et noir d'Afrique occidentale (F): Colobus polykomos II M

Colobe de l'Abyssinie (F): Colobus guereza II M

Colobe de Van Beneden (F): Procolobus verus II M

Colobe ferrugineux (F): Procolobus badius II M

Colobe guéréza (F): Colobus guereza II M

Colobe magistrat (F): Colobus polykomos II M

Colobe roux de la Tana (F): Procolobus rufomitratus I M

Colobe roux de Zanzibar (F): Procolobus pennantii II M

Colobe vert (F): Procolobus verus II M

Colobe vrai (F): Procolobus verus II M

Colobo blanco y negro de Africa occidental (S): Colobus polykomos II M

Colobo herrumbroso occidental (S): Procolobus badius II M

Colobo negro (S): Colobus satanas II M

Colobo rojo (S): Procolobus pennantii III M

Colobo rojo de Camerún (S): Procolobus preussi II M

Colobo rojo de Zanzibar (S): Procolobus pennantii kirkii I M

Colobo rojo guereza (S): Colobus guereza II M
Colobo rojo mitrado (S): Procolobus rufomitratus I M

Colobo verde (S): Procolobus verus $11 \mathrm{M}$

Colobus (E): Colobus spp/Procolobus spp. III M

Colobus abyssinicus $=$ Colobus guereza

Colobus angolensis: Angolan Black-and-white Colobus (E), Angolan Colobus (E), Colobe (F) II M

Colobus badius $=$ Procolobus badius

Colobus badius kirkii = Procolobus pennanvii kirkii

Colobus guereza: Eastern Black-and-white Colobus (E), Guereza (E), Magistrate Colobus (E), Colobo rojo guereza (S), Colobe de l'Abyssinie (F), Colobe guéréza (F), Colobe à épaules blanches (F) II M

Colobus pennanti $=$ Procolobus pennantii

Colobus polykomos: King Colobus (E), Western Black-and-white Colobus (E), Colobo blanco y negro de Africa occidental (S),

Colobe blanc et noir d'Afrique occidentale (F), Colobe magistrat (F), Colobe à camail (F), Colobe à longs poils (F) II M

Colobus preussi $=$ Procolobus preussi

Colobus rufomitratus $=$ Procolobus rufomitratus

Colobus saranas: Black Colobus (E), Colobo negro (S) II M

Colobus vellerosus $=$ Colobus polykomos

Colobus verus $=$ Procolobus verus

Colombar à front nu (F): Treron calva III B

Colombar waalia (F): Treron waalia III B

Colombars (F): Treron spp. III/NC B

Colombe poignardée (F): Gallicolumba luzonica II B

Colombian Screech-Owl (E): Ous ingens II B

Colourful Puffleg (E): Eriocnemis mirabilis II B

Colpeo Fox (E): Pseudalopex culpaeus II M

Colpophyllia amaranthus: II in

Colpophyllia natans: II In

Columba guinea: Speckled Pigeon (E), Speckled Rock Pigeon (E) Triangular-spotted Pigeon (E), Paloma de Guinea (S), Pigeon de Guinée (F), Pigeon roussard (F) III B

Columba iriditorques: Western Bronze-naped Pigeon (E), Pigeon a nuque bronzée (F) III B

Columba livia: Rock Dove (E), Rock Pigeon (E), Paloma bravía (S), Pigeon biset (F) III B

Columba mayeri: Pink Pigeon (E), Paloma de Mauricio (S), Pigeon rose (F) III B

Columba unicincta: Afep Pigeon (E), African Wood-Pigeon (E), Grey Wood-Pigeon (E), Paloma afep (S), Pigeon gris (F), Pigeon gris écailleux (F) $111 \mathrm{~B}$

Comadreja de montaña (S): Mustela altaica III M

Comadreja de Siberia (S): Mustela sibirica III M

Comadreja de vientre amarillo (S): Mustela kathiah III M

Comb Duck (E): Sarkidiomis melanotos II B

Combassou de Baka (F): Vidua larvaticola III B

Combassou de Jamdandu (F): Vidua raricola III B

Combassou de Wilson (F): Vidua wilsoni III B

Combassou du Sénégal (F): Vidua chalybeaia III B

Combassous (E/F): Vidua spp. IIINC B

Comets (E): Polyonymus caroli/Sappho sparganura/Taphrolesbia griseiventris II B

Commandeur huppé (F): Gubernatrix cristata II B

Commerson's Dolphin (E): Cephalorhynchus commersonii II M

Common Bam-Owl (E): Tyto alba II B

Common Batagur (E): Batagur baska I R

Common Bay Owl (E): Phodilus badius II B

Common Birdwing (E): Omithoptera priamus II IN/Troides helena II In

Common Black-Hawk (E): Buteogallus anthracinus II B

Common Box Turtle (E): Terrapene carolina II R

Common Buzzard (E): Buteo buteo II B

Common Caiman (E): Caiman crocodilus V/II R

Common Caracara (E): Polyborus plancus II B

Common Chameleon (E): Chamaeleo chamaeleon II R

Common Cock-of-the-Rock (E): Rupicola rupicola II B

Common Crane (E): Grus grus II B

Common Crowned Pigeon (E): Goura crisraia II B

Common Dolphin (E): Delphinus delphis II M

Common Gibbon (E): Hylobates lar I M

Common Girdled Lizard (E): Cordylus cordylus II R

Common Green Birdwing (E): Omithoptera priamus II in

Common Iguana (E): Iguana iguana II R

Common Kestrel (E): Falco tinnunculus II B 
Common Langur (E): Semnopithecus entellus $1 \mathrm{M}$ Common Marmoset (E): Callithrix jacchus II M Common Monitor ( $\mathrm{E})$ : Varanus bengalensis I R Common Otter (E): Lutra lutra I M

Common Palm Civet (E): Paradoxurus hermaphroditus III M Common Peacock-Pheasant (E): Polyplectron bicalcaratum II B Common Phalanger (E): Phalanger orientalis II M Common Pintail (E): Anas acuta III B

Common Porpoise (E): Phocoena phocoena II M

Common Rat Snake (E): Ptyas mucosus II R

Common Rhea (E): Rhea americana II B

Common Rorqual (E): Balaenoprera physalus I M

Common Scaled Water Snake (E): Xenochrophis piscator III R

Common Scops-Owl (E): Otus scops II B

Common Spiny-tailed Lizard (E); Cordylus cordylus II R

Common Spotted Cuscus (E): Spilocuscus maculatus II M

Common Squirrel Monkey (E): Saimiri sciureus II M

Common Sturgeon (E): Acipenser sturio I F

Common Teal (E): Anas crecca III B

Common Tegu (E): Tupinambis teguixin II R

Common Tortoise (E): Kinixys erosa II R/Testudo graeca II R

Common Tree Monitor (E): Varanus varius II R

Common Treeshrew (E): Tupaia belangeri II M

Common Water Monitor (E): Varanus salvałor II R

Common Waxbill (E): Estrilda astrild III B

Common Wolf (E): Canis lupus I/II M

Common Woolly Monkey (E): Lagothrix lagotricha II M

Common Zorro (E): Cerdocyon thous II M

Comoro Black Flying-fox (E): Pteropus livingstonei II M

Comoro Day Gecko (E): Phelsuma comorensis II R

Comoro Islands Chameleon (E): Chamaeleo cephalolepis II R

Compact Weaver (E): Pachyphantes superciliosus III B

Concentrotheca laevigata: II In

Concentrotheca vaughani: II In

Conchodromus dromas = Dromus dromas

Conchs (E): Strombidae spp. IINC In

Cóndor (S): Vultur gryphus I B

Cóndor andino (S): Vultur gryphus I B

Cóndor californiano (S): Gymnogyps califomianus I B

Cóndor de California (S): Gymnogyps califomianus I B

Condor de Californie (F): Gymnogyps califomianus I B

Condor des Andes (F): Vultur gryphus I B

Condors (E/F): Gymnogyps californianus/Vultur gryphus I B

Conejo de Assam (S): Caprolagus hispidus I M

Conejo de Díaz (S): Romerolagus diazi I M

Conejo de los volcanes (S): Romerolagus diazi I M

Conepatus castaneus = Conepatus humboldti

Conepatus humboldtii: Humboldt's Hog-nosed Skunk (E), Patagonian Hog-nosed Skunk (E), Anas (S), Chingue de la Patagonia (S), Zorrillo (S), Zorrino de la Patagonia (S), Moufette de Patagonie (F), Moufette à nez de cochon (F) II M

Confusing Poison Frog (S): Epipedobates macularus II A

Congo Bay-Owl (E): Phodilus prigoginei II B

Congo Blind Barb (E): Caecobarbus geertsi II F

Congo Serpent-Eagle (E): Dryotriorchis spectabilis II B

Conocyathus compressus: II In

Conocyathus fenestratus: II In

Conocyathus zelandiae: II In

Conolophes (F): Conolophus spp. II R

Conolophus pallidus: Barrington Land Iguana (E), Iguana terrestre de Barrington (S), Iguane terrestre de l'île Santa Fe (F) II R

Conolophus spp.: Land iguanas (E), Iguanas terrestres (S), Conolophes (F), Iguanes terrestres des Galapagos (F) II R

Conolophus subcristatus: Galapagos Land Iguana (E), Iguana terrestre de las Galápagos (S), Iguane terrestre des Galapagos (F) II $\mathrm{R}$

Conondale Gastric-brooding Frog (E): Rheobatrachus silus II A

Conopora adeta: II In

Conopora anthohelia: II In

Conopora candelabrum: II In

Conopora dura: II In

Conopora gigantea: II In

Conopora laevis: II In

Conopora major. II In

Conopora retrastichopora: II in

Conopora unifacialis: II In
Conopora verrucosa: II In

Conorrochus brunneus: II In

Conotrochus funicolumna: II In

Conradilla caelata: Birdwing Pearly Mussel (E), Rimose Naiad (E) I In

Constrictor constrictor $=$ Boa constrictor

Constrictor constrictor occidentalis $=$ Boa constrictor occidentalis Constrictors (E): Boidae spp. III R

Conure à cape noire (F): Pyrrhura rupicola II B

Conure à col blanc (F): Pyrrhura albipectus II B

Conure à front brun (F): Rhynchopsitta terrisi I B

Conure à front rouge (F): Arating a canicularis II B

Conure à gorge bleue (F): Pyrrhura cruentata I B

Conure à gros bec (F): Rhynchopsirta pachyrhyncha I B

Conure à long bec (F): Enicognathus leptorhynchus II B

Conure à oreilles jaunes (F): Ognorhynchus icterotis I B

Conure à oreillons (F): Pyrrhura hoematotis II B

Conure à pinceaux d'or (F): Leptosistaca branickii II B

Conure à poitrine brune (F): Pyrrhura calliptera II B

Conure à tête bleue (F): Aratinga acuticaudata II B

Conure à tête d'or (F): Arasinga auricapilla II $\mathrm{B}$

Conure à tête rouge (F): Aratinga erythrogenys II B

Conure à ventre rouge (F): Pyrrhura rhodogaster II B

Conure aile-de-feu (F): Pyrrhura egregia II B

Conure aztèque (F): Aratinga nana II $B$

Conure couronné (F): Aratinga aurea II B

Conure cuivré (F): Aratinga pertinax II B

Conure de Cuba (F): Aratinga euops II B

Conure de Deville (F): Pyrrhura devillei II B

Conure de Finsch (F): Arafinga finschi II B

Conure de Hoffmann (F): Pyrrhura hoffmanni II B

Conure de la Reine de Bavière (F): Aratinga guarouba I B

Conure de Molina (F): Pyrrhura molinae II B

Conure de Patagonie (F): Cyanoliseus patagonus II B

Conure de Ridgway (F): Aratinga strenua II B

Conure de Souancé (F): Pyrrhura melanura II B

Conure de Vieillot (F): Pyrrhura frontalis II B

Conure de Wagler (F): Aratinga wagleri II B

Conure de Weddell (F): Aratinga weddellii II B

Conure des cactus (F): Aratinga cactorum II B

Conure des Santa Marta (F): Pyrrhura viridicata II B

Conure d'Orcès (F): Pyrrhura orcesi II B

Conure doré (F): Aratinga guarouba I B

Conure emma (F): Pyrrhura leucotis II B

Conure jandaya $(\mathrm{F})$ : Aratinga jandaya II B

Conure magellanique (F): Enicognathus ferrugineus II B

Conure maîtresse (F): Aratinga chloroptera II B

Conure mitré (F): Aratinga mitrata II B

Conure nanday (F): Nardayus nenday II B

Conure pavouane (F): Aratinga leucophthalmus II B

Conure perlé (F): Pyrrhura perlaia II B

Conure soleil (F): Aratinga solstitialis II B

Conure tête-de-feu (F): Pyrrhura rhodocephala II B

Conure tiriba (F): Pyrrhura cruentata I B

Conure versicolore (F): Pyrrhura picta II B

Conure vert (F): Aratinga holochlora II B

Conure veuve (F): Myiopsinta monachus II B

Conures (E): Aratinga spp./Cyanoliseus patagonus/Enicognathus spp./Leptosittaca branickil/Nandayus nenday/Ognorhynchus icierotis/Pyrrhura spp. I/II B

Conures (F): Aratinga spp//Cyanoliseus patagonus/Enicognathus spp./Reptosittaca branickii/Myiopsitta monachus/Nandayus nenday/Ognorhynchus icterotis/Pyrrhura spp/Rhynchopsitta spp. I/II B

Cook Strait Tuatara (E): Sphenodon punctatus I R

Cook's Tree Boa (E): Corallus enydris II R

Cooper's Hawk (E): Accipiter cooperii II B

Copper-rumped Hummingbird (E): Amazilia tobaci II B

Coppery Emerald (E): Chlorosilibon russatus II B

Coppery Metaltail (E): Metallura theresiae II B

Coppery Thomtail (E): Popelairia letitiae II B

Coppery-bellied Puffleg (E): Eriocnemis cupreoventris II B

Coppery-headed Emerald (E): Elvira cupreiceps II B

Coq de Bois (F): Terpsiphone bourbonnensis ill B

Coq de Sonnerat (F): Gallus sonneratii II B

Coq-de-roche de Guyane (F): Rupicola rupicola II B 
Coq-de-roche orange (F): Rupicola rupicola II B

Coq-de-roche péruvien (F): Rupicola peruviana II B

Cogs (F): Gallus spp. II/NC B

Coqs-de-roche (F): Rupicola spp. II B

Coquerel's Dwarf Lemur (E): Microcebus coquereli I M

Coquerel's Mouse-lemur (E): Microcebus coquereli I M

Coqueta abanico canela (S): Lophomis omatus II B

Coqueta abanico pavo real (S): Lophomis pavoninus II B

Coqueta abanico puntiblanco (S): Lophornis chalybeus II B

Coqueta cola de Lira (S): Popelairia langsdorffi II B

Coqueta cola raqueta (S): Discosura longicauda II B

Coqueta coronada (S): Lophomis stictolophus II B

Coqueta cresticorta (S): Lophornis brachylopha II B

Coqueta crestinegra (S): Lophornis helenae II B

Coqueta festiva (S): Lophornis chalybeus II B

Coqueta lentejuelada (S): Lophomis stictolophus II B

Coquette à queue fine (F): Popelairia conversii II B

Coquette à raquettes (F): Discosura longicauda II B

Coquette adorable (F): Lophomis adorabilis II B

Coquette chalybée (F): Lophornis chalybeus II B

Coquette de Delattre (F): Lophornis delattrei II B

Coquette de Gould (F): Lophornis gouldii II B

Coquette de Langsdorff (F): Popelairia langsdorffi II B

Coquette de Létitia (F): Popelairia letitiae II B

Coquette de Popelaire (F): Popelairia popelaini II B

Coquette d'Hélène (F): Lophomis helenae II B

Coquette du Guerrero (F): Lophornis brachylopha II B

Coquette huppe-col (F): Lophomis ornasus II B

Coquette magnifique (F): Lophomis magnificus II B

Coquette pailletée (F): Lophornis stictolophus II B

Coquette paon (F): Lophormis pavoninus II B

Coquettes (E): Lophomis spp. II B

Coquettes (F): Discosura longicauda/Lophormis spp./Popelairia spp. II B

Coracine casquée (F): Cephalopterus penduliger III B

Coracine ornée (F): Cephalopterus ornaius III B

Coracines (F): Cephalopterus spp. III/NC B

Coracopsis nigra: Black Parrot (E), Loro negro (S), Perroquet noir (F), Perroquet petit vasa (F) II B

Coracopsis vasa: Vasa Parrot (E), Loro vasa (S), Perroquet vaza (F), Vasa géant (F) II B

Corail comes de cerf (F): Acropora cenicomis II In

Corail comes d'élan (F): Acropora palmata II in

Corail de feu (F): Milleporidae spp. II In

Corail limace (F): Herpolitha limax II In

Corail orgue (F): Tubiporidae spp. II In

Coral snakes (E): Elapidae spp. IIU/NC R

Corales negros (S): ANTIPATHARIA spp. II In

Corallus annulatus: Annulated Tree Boa (E), Boa aboricola anillada (S), Boa annelé (F), Boa arboricole annelé (F) II $R$

Corallus caninus: Emerald Tree Boa (E), Boa esmerelda (S), Boa canin (F), Boa émeraude (F), Faux jacquot (F) II R

Corallus cookii $=$ Corallus enydris

Corallus enydris: Amazon Tree Boa (E), Cook's Tree Boa (E), Garden Tree Boa (E), Boa arboricola de jardin (S), Boa arboricole d'Amazonie (F), Boa de Cook (F), Boa des jardins (F) II $\mathbf{R}$

Corallus hortulanus $=$ Corallus enydris

Corals (E): ANTHOZOA spp./HYDROZOA spp. IU/NC In

Coraux champignon (F): Fungia spp. II In

Coraux de feu (F): Millepora spp. II In

Coraux noirs (F): ANTIPATHARIA spp. II in

Cordon bleu à joures rouges (F): Uraeginthus bengalus III B

Cordonbleu (E): Uraeginthus bengalus III B

Cordonbleus (E/F): Uraeginthus spp. IIU/NC B

Cordyle à larges écailles (F): Cordylus macropholis II R

Cordyle à pustules $(\mathrm{F})$ : Cordylus pustulatus II $\mathrm{R}$

Cordyle à taches bleues (F): Cordylus coeruleopuncrarus II R

Cordyle africain (F): Cordylus polyzonus II R

Cordyle commun (F): Cordylus cordylus II R

Cordyle d'Armadillo (F): Cardylus cataphractus II R

Cordyle de Campbell (F): Cordylus campbelli II R

Cordyle de Cope (F): Cordylus iropidostemum II R

Cordyle de Hewitt (F): Cordylus peersi II R

Cordyle de Jordan (F): Cordylus jordani II R

Cordyle de Lawrence (F): Cordylus lawrencei II R
Cordyle de McLachlan (F): Cordylus mclachlani II R

Cordyle de Reichenow (F): Cordylus vitrifer II R

Cordyle de Rhodésie (F): Cordylus rhodesianus II R

Cordyle de Tasman (F): Cordylus tasmani II R

Cordyle de Warren (F): Cordylus warreni II R

Cordyle d'Oelofsen (F): Cordylus oelofseni II R

Cordyle du Namaqua (F): Cordylus namaquensis II R

Cordyle d'Ukinga (F): Cordylus ukingensis II R

Cordyle géant (F): Cordylus giganteus II R

Cordyle noir (F): Cordylus niger II R

Cordyles (F): Cordylus spp. II R

Cordylus spp.: Spiny-tailed lizards (E), Lagartos amadillos (S), Cordyles (F), Lézards à queue épineuse d'Afrique Australe (F) II $\mathbf{R}$

Cordylus campbelli: Campbell's Girdled Lizard (E), Campbell's Spiny-tailed Lizard (E), Cordyle de Campbell (F), Lézard à queue épineuse de Campbell (F) II R

Cordylus cataphracius: Armadillo Girdled Lizard (E), Amadillo Spiny-tailed Lizard (E), Cordyle d'Armadillo (F), Lézard à queue épineuse d'Armadillo (F) II R

Cordylus coeruleopunctatus: Blue-spotted Girdled Lizard (E), Bluespotted Spiny-tailed Lizard (E), Cordyle à taches bleues (F), Lézard à queve épineuse à taches bleues (F) II R

Cordylus cordylus: Cape Girdled Lizard (E), Common Girdled Lizard (E), Common Spiny-tailed Lizard (E), Rough-scaled Girdled Lizard (E), Cordyle commun (F), Lezard ì queue épineuse commun (F) II $\mathrm{R}$

Cordylus ardylus lawrence $=$ Cordylus lawrencei

Cordylus cordylus minor $=$ Cordylus minor

Cordylus cordylus niger $=$ Cordylus niger

Cordylus cordylus pustulatus $=$ Cordylus pustulatus

Cordylus cordylus rhodesianus = Cordylus rhodesianus

Cordylus cordylus tasmani = Cordylus tasmani

Cordylus cordylus tropidostemum = Cordylus tropidostemum

Cordylus giganieus: Giant Girdled Lizard (E), Giant Spiny-tailed Lizard (E), Giant Zonure (E), Sungazer (E), Cordyle géant (F), Lézard à queue épineuse géant (F) II R

Cordylus jonesii = Cordylus tropidostemum

Cordylus jordani: Jordan's Girdled Lizard (E), Cordyle de Jordan (F), Lézard à queue épineuse de Jordan (F) II R

Cordylus jordani $=$ Cordylus polyzonus

Cordylus lawrencei: Lawrence's Girdled Lizard (E), Cordyle de Lawrence (F), Lézard à queue épineuse de Lawrence (F) II R

Cordylus macropholis: Coastal Spiny-tailed Lizard (E), Large-scaled Girdled Lizard (E), Cordyle à larges écailles (F), Lézard à queue épineuse à larges écailles (F) II R

Cordylus mclachlani: McLachlan's Girdled Lizard (E), McLachlan's Spiny-tailed Lizard (E), Cordyle de McLachlan (F), Lézard à queue épineuse de McLachlan (F) II R

Cordylus minor: Dwarf Girdled Lizard (E), Petit cordyle (F), Petit lézard à queue épineuse (F) II R

Cordylus namaquensis: Namaqua Girdled Lizard (E), Namaqua Spiny-tailed Lizard (E), Cordyle du Namaqua (F), Lézard à queue épineuse du Namaqua (F) II R

Cordylus niger: Cordyle noir (F), Lézard à queue épineuse noir (F) II $R$

Cordylus oelofseni: Cordyle d'Oelofsen (F), Lézard à queue épineuse d'Oelofsen (F) II R

Cordylus peersi: Hewitt's Spiny-lailed Lizard (E), Peer's Girdled Lizard (E), Cordyle de Hewitt (F), Lézard à queue épineuse de Hewitt (F) II R

Cordylus polyzonus: African Spiny-tailed Lizard (E), Karoo Girdled Lizard (E), Cordyle africain ( $F)$, Lézard à queue épineuse africain (F) II R

Cordylus polyzonus jordani $=$ Cordylus jordani

Cordylus pustulatus: Herero Girdled Lizard (E), Cordyle à pustules (F), Lézard à queue épineuse à pustules (F) II R

Cordylus rhodesianus: Rhodesian Girdled Lizard (E), Zimbabwe Girdled Lizard $(E)$, Cordyle de Rhodésie $(F)$, Lézard à queue épineuse de Rhodésie (F) II R

Cordylus tasmani: Tasman's Girdled Lizard (E), Cordyle de Tasman (F), Lézard à queue épineuse de Tasman (F) II R

Cordylus tropidosternum: East African Spiny-tailed Lizard (E), Tropical Girdled Lizard (E), Tropical Spiny-tailed Lizard (E), Cordyle de Cope (F), Lézard à queue épineuse de Cope (F) II R 
Cordylus ukingensis: Ukinga Spiny-tailed Lizard (E), Cordyle d'Ukinga (F), Lézard à queue épineuse d'Ukinga (F) II R

Cordylus vinifer: Reichenow's Spiny-tailed Lizard (E), Transvaal Girdled Lizard (E), Cordyle de Reichenow (F), Lézard à queue épineuse de Reichenow (F) II R

Cordylus warreni: Warten's Girdled Lizard (E), Warren's Spinytailed Lizard (E), Cordyle de Warren (F), Lézard à queue épineuse de Warren (F) II R

Corellas (E): Cacarua spp. III B

Comeille de paradis (F): Lycocorax pyrrhopterus II B

Comiz (E): Bothrops ophryomegas III R

Corocoro colorado (S): Eudocimus ruber II B

Corocoro rojo (S): Eudocimus ruber II B

Coronets (E): Boissonneaua spp. II B

Coronita aterciopelada (S): Boissonneaua jardini II B

Coronita colihabana (S): Boissonneaua flavescens II B

Coronita pechicastaña (S): Boissonneaua matthewsii II B

Corredor chillón (S): Atrichomis clamosus I B

Corredor gorguinaranja (S): Cnemidophorus hyperyshrus II R

Corsac (E): Vulpes cana II M

Corsican Swallowtail (E): Papilio hospiton I In

Conucia zebrata: Prehensile-tailed Skink (E), Solomon Islands Giant Skink (E), Eslizón de las Islas Salomón (S), Scinque arboricole des Iles Salomons (F), Scinque géant des Iles Salomons (F). Scinque à queue préhensile des lles Salomons (F) II R

Coryllis (F): Loriculus spp. II B

Coryllis a front orange (F): Loriculus aurantiifrons II B

Coryllis à gorge jaune (F): Loriculus pusillus II B

Coryllis à tête bleue (F): Loriculus galgulus II B

Coryllis de Ceylan (F): Loriculus beryllinus II B

Coryllis de Wallace (F): Loriculus flosculus II B

Coryllis des Bismark (F): Loriculus tener II B

Coryllis des Célèbes (F): Loriculus stigmatus II B

Coryllis des Moluques (F): Loriculus amabilis $11 \mathrm{~B}$

Coryllis des Philippines (F): Loriculus philippensis II B

Coryllis des Sangi (F): Loriculus catamene II B

Coryllis vemal $(\mathrm{F})$ : Loriculus vernalis II $\mathrm{B}$

Coryllis vert $(\mathrm{F})$ : Loriculus exilis II $\mathrm{B}$

Corythaeola cristata: Blue Plantain-eater (E), Great Blue Turaco (E), Turaco gigante (S), Touraco géant (F) III B

Coscinarea $=$ Coscinastrea

Coscinastrea bottae: II In

Coscinastrea columna: II In

Coscinastrea crassa: II In

Coscinastrea exesa: II In

Coscinastrea hahazimaensis: II In

Coscinastrea marshae: II In

Coscinastrea mcneilli: II In

Coscinastrea monile: II In

Coscinastrea wellsi: II In

Coscoroba coscoroba: Coscoroba Swan (E), Cisne coscoroba (S), Ganso blanco (S), Cygne coscoroba (F) II B

Coscoroba Swan (E): Coscoroba coscoroba II B

Cosmoporites $=$ Porites

Costa Rican Puma (E): Puma concolor costaricensis I M

Costa's Hummingbird (E): Calypte costae II B

Cotinga à ailes blanches $(\mathrm{F})$ : Xipholena atropurpurea I B

Cotinga aliblanca (S): Xipholena atropurpurea I B

Cotinga cordon-bleu (F): Cotinga maculata I B

Cotinga de corbata (S): Cotinga maculata I B

Cotinga maculata: Banded Cotinga (E), Spotted Cotinga (E), Cotinga de corbata (S), Cotinga cordon-bleu (F), Cotinga maculé (F) I B

Cotinga macule $(\mathrm{F})$ : Cotinga maculata I $\mathrm{B}$

Cotinga porphyrion (F): Xipholena atropurpurea I B

Cotingas (E/F): Cotingidae spp. I/NC B

Cotorra (S): Amazona ventralis II B

Cotorra (S): Lepidochelys kempi I R

Cotorra alejandrina (S): Psintacula eupatria II B

Cotorra amarilla (S): Aratinga guarouba I B

Cotorra Argentina (S): Myiopsitta monachus II B

Cotorra austral (S): Enicognathus ferrugineus II B

Cotorra cabeciamarilla (S): Amazona barbadensis I B

Cotorra cabeciazul (S): Pionus menstruus II B

Cotorra cabeciblanca (S): Pionus tumultuosus II B

Cotorra cabecinegra (S): Nandayus nenday II B
Cotorra cachaña (S): Enicognathus ferrugineus II B Cotorra capirotada (S): Pyrrhura rupicola 11 B

Cotorra cariparda (S): Pyrrhura leucotis II B

Cotorra carirroja (S): Aratingr mitrata II B

Cotorra carirrosa (S): Psittacula roseasa II B

Cotorra catana (S): Pyrrhura hoffmanni II B

Cotorra chiripepé (S): Pyrrhura frontalis II B

Cotorra choroy (S): Enicognathus leptorhynchus II B

Cotorra ciruela (S): Psittacula cyanocephala II B

Cotorra colilarga (S): Psittacula longicauda II B

Cotorra colinegra (S): Pyrrhura melanura II B

Cotorra colirroja (S): Pyrrhura hoematotis II B

Cotorra coronirroja (S): Pyrrhura rhodocephala II B

Cotorra cucha (S): Amazona autumnalis II B

Cotorra cuelliblanca (S): Pyrrhura albipectus II B

Cotorra de ceilán (S): Psirzacula calthropae II B

Cotorra de Derby (S): Psittacula derbiana II B

Cotorra de Deville (S): Pyrrhura devillei II B

Cotorra de El Oro (S): Pyrrhura orcesi II B

Cotorra de Finsch (S): Psittacula finschii II B

Cotorra de Kramer (S): Psittacula krameri III B

Cotorra de los Palos (S): Aratinga acuticaudata II B

Cotorra de Malabar (S): Psittacula columboides II B

Cotorra de Mauricio (S): Psittacula echo I B

Cotorra de Molina (S): Pyrrhura molinae II B

Cotorra de Nicobar (S): Psittacula caniceps II B

Cotorra de Santa Marta (S): Pyrrhura viridicata II B

Cotorra del Himalaya (S): Psittacula himalayana II B

Cotorra egregia (S): Pyrrhura egregia II B

Cotorra frente roja (S): Amazona finschi II B

Cotorra frentidorada (S): Aratinga aurea II B

Cotorra intermedia (S): Psittacula intermedia II B

Cotorra morada (S): Pionus fuscus II B

Cotorra negra (S): Pionus chalcopterus II B

Cotorra pechiparda (S): Pyrrhura callipsera II B

Cotorra pechirroja (S): Psinacula alexandri II B

Cotorra perlada (S): Pyrrhura perlata II B

Cotorra pintada (S): Pyrrhura picta II B

Cotorra serranas (S): Rhynchopsitra spp. I B

Cotorra tiriba (S): Pyrrhura cruentata I B

Cotorra ventriroja (S): Pyrrhura rhodogaster II B

Cotorra verde (S): Aratinga leucophthalmus II B

Cotortas (S): Enicognathus spp/Myiopsitta monachus/Psittacula spp/Pyrrhura spp. IIIII B

Cotorra-serrana occidental (S): Rhynchopsitta pachyrhyncha I B

Cotorra-serrana oriental (S): Rhynchopsitta terrisi I B

Cotorrita aliazul (S): Forpus xanthopterygius II B

Cotorita alipinta (S): Touit stictoptera II B

Cotorrita alirroja (S); Touit huetii II B

Cotorrita amazónica (S): Nannopsittaca dachilleae II B

Cotorrita cariazul (S): Touit dilectissima II B

Cotorrita carigualda (S): Forpus xanthops II B

Cotorrita Costarricense (S): Touit costaricensis II B

Cotorrita culiverde (S): Forpus passerinus II B

Cotorita de Anteojos (S): Forpus conspicillatus II B

Cotorrita de Piura (S): Forpus coelestis II B

Cotorita de Sclater (S): Forpus sclateri II B

Cotorrita dorsinegra (S): Touit melanonotus II B

Cotorrita Mexicana (S): Forpus cyanopygius II B

Cotorrita purpurada (S): Touit purpurafa II B

Cotorrita sietecolores (S): Touit batavica II B

Cotorrita sorda ( ): Touit surda II B

Cotorita tepuí (S): Nannopsittaca panychlora II B

Cotorritas (S): Forpus spp/Nannopsittaca spp/Touit spp. II B

Cotton-headed Tamarin (E): Saguinus oedipus 1 M

Cotton-top Marmoset (E): Saguinus geoffroyi I M

Cotton-top Tamarin (E): Saguinus oedipus I M

Cotunto (S): Otus lawrencii II B

Cou-coupé (F): Amadina fasciala III B

Cougar (E): Puma concolor I/II M

Cougar de Floride (F): Puma concolor coryi I M

Cougouar (F): Puma concolor couguar I M

Couleuvre à damier (F): Xenochrophis piscator III R

Couleuvre de Westermann (F): Elachistodon westermanni II R

Couleuvre lisse du Brésil (F): Cyclagras gigas II R

Count Raggi's Bird-of-paradise (E): Paradisaea raggiana II B 
Coureur à gorge orange (F): Cnemidophorus hyperythrus II R

Courlis (F): Numenius spp. INC B

Courlis à bec grêle (F): Numenius tenuirostris I B

Courlis esquimau (F): Numenius borealis I B

Couscous de la Sonde (F): Phalanger orientalis II M

Couscous gris (F): Phalanger orientalis II M

Couscous tacheté (F): Spilocuscus maculatus II M

Coxen's Blue-browed Fig Parrot (E): Cyclopsitta diophthalma coxeni I B

Coxen's Double-eyed Fig-Parrot (E): Cyclopsinta diophthalma coxeni I B

Coxen's Two-eyed Fig Parrot (E): Cyclopsinta diophthalma coxeni I B

Crab-eating Macaque (E): Macaca fascicularis II M

Crab-eating Mongoose (E): Herpestes urva III M

Crab-eating Zorro (E): Cerdocyon thous II M

Crag lizards (E): Pseudocordylus spp. II R

Crane Hawk (E): Geranospiza caerulescens II B

Cranes (E): Gruidae spp. III B

Crapaud doré (F): Bufo periglenes I A

Crapaud du Cameroun (F): Bufo superciliaris I A

Crapaud rouge de Madagascar (F): Dyscophus antongilii I A

Crapaud vert du Sonora (F): Bufo retiformis II A

Crapauds vivipares (F): Nectophrynoides spp. I A/Altiphrynoides spp. I ANNimbaphrynoides spp. I AVSpinophrynoides spp. I A

Craterastrea $=$ Leptoseris

Crax alberti: Blue-billed Curassow (E), Blue-knobbed Curassow (E), Pavón piquiazul (S), Hocco d'Albert (F), Hocco à bec bleu (F) III B

Crax blumenbachii: Mutum (E), Red-billed Curassow (E), Muitú mamaco (S), Pavón piquirrojo (S), Hocco de Blumenbach (F), Hocco à bec rouge $(\mathrm{F}) \mathrm{I} \mathrm{B}$

Crax daubentoni: Yellow-knobbed Curassow (E), Pauji de Copete (S), Pavón poria (S), Hocco de Daubenton (F) III B

Crax globulosa: Wattled Curassow (E), Pavón carunculado (S), Hocco globuleux (F) III B

Crax mitu mitu $=$ Mitu mitu

Crax pauxi $=$ Pauxi pauxi

Crax rubra: Great Curassow (E), Hocofaisan (S), Pavón grande (S), Pavón norteña (S), Grand hocco (F) III B

Cream-coloured Giant Squirrel (E): Ratufa affinis II M

Crécerelle américaine (F): Falco sparverius II B

Crécerelle australienne (F): Falco cenchroides II B

Crécerelle aux yeux blancs (F): Falco rupicoloides II B

Crécerelle d'Aldabra (F): Falco newtoni I/II B

Crécerelle d'Amérique (F): Falco sparverius II B

Crécerelle d'Australie (F): Falco cenchroides II B

Crécerelle de Maurice (F): Falco punctatus I B

Crécerelle des Moluques (F): Falco moluccensis II B

Crécerelle des Seychelles (F): Falco araea I B

Crécerelle katitie (F): Falco araea I B

Crécerelle malgache (F): Falco newioni III B

Crécerelle renard (F): Falco alopex II B

Crécerelles (F): Falco spp. I/II B

Crescent Nailtail Wallaby (E): Onychogalea lunata I M

Crested Argus (E): Rheinardia ocellata I B

Crested Baza (E): Aviceda subcristata II B

Crested Bird-of-paradise (E): Cnemophilus macgregorii II B

Crested Caracara (E): Polyborus plancus II B

Crested Chameleon (E): Chamaeleo cristatus II R

Crested Eagle (E): Morphnus guianensis II B

Crested Fireback (E): Lophura ignita III B

Crested Gibbon (E): Hylobates concolor I M

Crested Goshawk (E): Accipiter trivirgatus II B

Crested Guan (E): Penelope purpurascens III B

Crested Hawk-Eagle (E): Spizaetus cirrhatus II B

Crested Ibis (E): Nipponia nippon I B

Crested Korhaan (E): Eupodotis ruficrista II B

Crested Malimbe (E): Malimbus malimbicus III B

Crested Mangabey (E): Cercocebus galeritus I/II M

Crested Owl (E): Lophostrix cristata II B

Crested Partridge (E): Rollulus rouloul III B

Crested Peacock-Pheasant (E): Polyplectron malacense II B

Crested Porcupine (E): Hystrix cristata III M

Crested porcupines (E): Hystrix cristata/Hystrix africaeaustralis III/NC M
Crested Serpent-Eagle (E): Spilomis cheela II B

Crested Turaco (E): Tauraco macrorhynchus II B

Crestless Fireback (E): Lophura erythrophthalma III B

Crestless Monal (E): Lophophorus sclateri I B

Crevice Monitor (E): Varanus kingorum II R

Crevice Tortoise (E): Malacochersus tomieri II R

Crick à ventre bleu (F): Triclaria malachiracea II B

Crimson Homed-Pheasant (E): Tragopan satyra III B

Crimson Rosella (E): Platycercus elegans II B

Crimson Shining-Parrot (E): Prosopeia splendens II B

Crimson Topaz (E): Topaza pella II B

Crimson-bellied Conure (E): Pyrrhura rhodogaster II B

Crimson-bellied Parakeet (E): Pyrrhura rhodogaster II B

Crimson-fronted Conure (E): Aratinga finschi II B

Crimson-fronted Parakeet (E): Aratinga finschi II B

Crimson-winged Pytilia (E): Pytilia phoenicoptera III B

Crinifer piscator: Grey Plantain-eater (E), Westem Grey Plantaineater (E), Turaco de Crin occidentale (S), Touraco gris (F) III B

Crinkle-collared Manucode (E): Manucodia chalybata II B

Crispatorrochus comu: II In

Crispatotrochus curvatus: II In

Crispatotrochus foxi: II In

Crispatotrochus galapagensis: II in

Crispatotrochus inomatus: II In

Crispatotrochus irregularis: II In

Crispatotrochus niinoi: II In

Crispatotrochus rubescens: II In

Crispatotrochus rugosus: II In

Crispatotrochus squiresi: II In

Crispatotrochus tydemani: II In

Crispatotrochus woodsi: II In

Crocodile à front large (F): Osteolaemus terraspis I R

Crocodile à museau allongé d'Afrique $(\mathrm{F})$ : Crocodylus cataphractus I R

Crocodile à museau étroit (F): Crocodylus cataphractus I R

Crocodile à nuque cuirassée (F): Osteolaemus tetraspis I R

Crocodile d'Amérique (F): Crocodylus acutus I R

Crocodile de Cuba (F): Crocodylus rhombifer I R

Crocodile de Johnstone ( $F$ ): Crocodylus johnsoni II R

Crocodile de l'Orénoque (F): Crocodylus intermedius I R

Crocodile de Morelet (F): Crocodylus moreletii I R

Crocodile de Nouvelle Guinée (F): Crocodylus novaeguineae I/II R

Crocodile des marais (F): Crocodylus palustris I R

Crocodile des Philippines (F): Crocodylus novaeguineae mindorensis I $R$

Crocodile d'estuaire (F): Crocodylus porosus I/II R

Crocodile du Nil (F): Crocodylus niloticus III R

Crocodile du Siam (F): Crocodylus siamensis I R

Crocodile marin ( $\mathrm{F}$ ): Crocodylus porosus I/II $\mathrm{R}$

Crocodile Monitor (E): Varanus salvadorii II R

Crocodile nain africain (F): Osteolaemus tetraspis I R

Crocodile paludéen (F): Crocodylus palustris I R

Crocodile palustre (F): Crocodylus palustris I R

Crocodiles (E/F): Crocodylidae spp. VII R

Crocodilians (E): CROCODYLIA spp. I/II R

Crocodilurus lacertinus: Dragon Lizard (E), Dragon Lizardet (E), Téju-Crocodile (F) II R

CROCODYLIA spp.: Alligators (E), Crocodiles (E), Crocodilians (E), Aligators (S), Cocodrilos (S), Alligators (F), Crocodiles (F) I/II R

Crocodylus acutus: American Crocodile (E), Cocodrilo americano (S), Crocodile d'Amérique (F) I R

Crocodylus cataphractus: African Sharp-nosed Crocodile (E), African Slender-snouted Crocodile (E), Long-snouted Crocodile (E), Cocodrilo hociquifino africano (S), Crocodile à museau allongé d'Afrique (F), Crocodile à museau étroit (F), Faux-gavial d'Afrique (F) I R

Crocodylus intermedius: Orinoco Crocodile (E), Cocodrilo del Orinoco (S), Crocodile de l'Orénoque (F) I R

Crocodylus johnsoni: Australian Fresh-water Crocodile (E), Johnstone's Crocodile (E), Cocodrilo australiano (S), Crocodile de Johnsione (F) II R

Crocodylus johnstoni $=$ Crocodylus johnsoni

Crocodylus mindorensis = Crocodylus novaeguineae mindorensis

Crocodylus morelevii: Belize Crocodile (E), Morelet's Crocodile (E), Cocodrilo de Morelet (S), Crocodile de Morelet (F) I R 
Crocodylus niloticus: African Crocodile (E), Nile Crocodile (E), Cocodrilo del Nilo (S), Crocodile du Nil (F) I/II R

Crocodylus novaeguineae: New Guinea Crocodile (E), Cocodrilo de Nueva Guinea (S), Crocodile de Nouvelle Guinée (F) I/II R

Crocodylus novaeguineae mindorensis: Mindoro Crocodile $(\mathrm{E})$, Philippine Crocodile (E), Cocodrilo de Mindoro (S), Crocodile des Philippines (F) I R

Crocodylus palustris: Broad-snouted Crocodile (E), Marsh Crocodile (E), Mugger (E), Cocodrilo marismeño (S), Crocodile des marais (F), Crocodile paludéen (F), Crocodile palustre (F) I R

Crocodylus porosus: Estuarine Crocodile (E), Salt-water Crocodile (E), Cocodrilo poroso (S), Crocodile d'estuaire (F), Crocodile marin (F) I/II R

Crocodylus raninus: $\mathbf{L I I} \mathbf{R}$

Crocodylus rhombifer. Cuban Crocodile (E), Cocodrilo de Cuba (S), Crocodile de Cuba (F) I R

Crocodylus siamensis: Siamese Crocodile (E), Cocodrilo de Siam (S), Crocodile du Siam (F) I R

Croco-Teju (E): Dracaena guianensis II R

Crocus Clam (E): Tridacna crocea II In

Cropan's Boa (E): Xenoboa cropanii 11 R

Crossoptilon crossoptilon: White Eared-Pheasant (E), Faisán orejudo blanco (S), Faisán orejudo tibetano (S), Faisan oreillard blanc (F), Hokki blanc (F) I B

Crossoptilon crossoptilon harmani $=$ Crossoptilon harmani

Crossoptilon harmani: Tibetan Eared-Pheasant (E), Tibetan Earedpheasant (E), Hokki de Harman (F), Hokki du Tibet (F) I B

Crossoptilon mantchuricum: Brown Eared-Pheasant (E), Brown Eared-pheasant (E), Manchurian Eared-Pheasant (E), Faisán orejudo de Manchuria (S), Faisán orejudo pardo (S), Faisan oreillard brun (F), Hokki brun (F) I B

Crotale durisse ( $\mathbf{F}$ : Crotalus durissus III $\mathbf{R}$

Crotalus durissus: Cascabel (E), Neotropical Rattlesnake (E), Cascavelle (F), Crotale durisse (F) III R

Crowned cranes (E): Balearica spp. II B

Crowned Eagle (E): Harpyhaliaetus coronatus II B/Stephanoaetus coronatus II B

Crowned Gibbon (E): Hylobates pilearus I M

Crowned Guenon (E): Cercopithecus pogonias II M

Crowned Hawk-Eagle (E): Stephanoaetus coronatus II B

Crowned Lemur (E): Eulemur coronatus I M

Crowned Monkey (E): Cercopishecus pogonias II M

Crowned Solitary Eagle (E): Harpyhaliaetus coronatus II B

Crowned-pigeons (E): Goura spp. II B

Cryptabacia $=$ Polyphyllia

Crypthelia affinis: II In

Crypthelia balio: II In

Crypthelia clausa: II In

Crypthelia cryptotrema: 11 in

Crypthelia curvata: II In

Crypthelia cymas: II In

Crypthelia dactylopoma: II In

Crypthelia eueides: II In

Crypthelia floridana: II In

Crypthelia formosa: II in

Crypthelia fragilis: II In

Crypthelia gigantea: II in

Crypthelia glebulenta: II in

Crypthelia glossopoma: II in

Crypthelia insolita: II In

Crypthelia japonica: II In

Crypthelia lacunosa: $\mathbf{U}$ in

Crypthelia medioatlantica: II In

Crypthelia micropoma: II In

Crypthelia papillosa: II In

Crypthelia peircei: II In

Crypthelia platypoma: II In

Crypthelia polypoma: II In

Crypthelia pudica: II In

Crypthelia ramosa: II In

Crypthelia robusta: II In

Crypthelia stenopoma: II in

Crypthelia studeri: II In

Crypthelia tenuiseptata: II in

Crypthelia trophostega: 11 in

Crypthelia vascomarquesi: II In
Cryptoprocta ferox: Fossa (E), Gato fossa de Madagascar (S) Cryptoprocte féroce (F), Fossa (F), Foussa (F) II M

Cryptoprocte féroce (F): Cryptoprocła ferox II M

Cryptotrochus carolinensis: II in

Cryptotrochus javanus: II In

Cryptotrochus venustus: II in

Crenacis albitentaculara: II In

Cienactis crassa: II In

Ctenactis echinata: 11 In

Crenella chagius: II In

Crenella laxa: II In

Cienophyllia $=$ Meandrina

Cuataquil (S): Bassaricyon gabbii III M

Cuatro Cienegas Soft-shell Turtle (E): Trionyx ater I R

Cuban Amazon (E): Amazona leucocephala I B

Cuban Black-tailed Dwarf Boa (E): Tropidophis melanurus II R

Cuban Boa (E): Epicrates angulifer II R

Cuban Conure (E): Aratinga euops II B

Cuban Crocodile (E): Crocodylus rhombifer I R

Cuban Emerald (E): Chlorostilbon ricordii II B

Cuban Ground Iguana (E): Cyclura nubila I R

Cuban Hook-billed Kite (E): Chondrohierax uncinatus wilsonii I B

Cuban Parakeet (E): Aratinga euops II B

Cuban Partot (E): Amazona leucocephala I B

Cuban Pygmy-Owl (E): Glaucidium siju II B

Cuban Sandhill Crane (E): Grus canadensis nesiotes I B

Cuban Tree Boa (E): Epicrates angulifer II R

Cuban Tree-Duck (E): Dendrocygna arborea II B

Cuchumbi (S): Bassaricyon gabbii III M/Potos flavus III M

Cuckoo Owlet (E): Glaucidium cuculoides II B

Cuckoo Weaver (E): Anomalospiza imberbis III B

Cuckoo-falcons (E): Aviceda spp. II B

Cuckoo-hawks (E): Aviceda spp. II B

Cuco (S): Otus lawrencii II B

Cucú (S): Speotyto cunicularia II B

Cuervo calvo de cuello blanco (S): Picathartes gymnocephalus I B

Cuervo calvo de cuello gris (S): Picathartes oreas I B

Cúiu cúiu (S): Pionopsitta pileåra I B

Cui-ui (E): Chasmistes cujus I F

Culebra boba (S): Tropidophis melanurus II R

Culebra tragahuevos india (S): Elachistodon westermanni II R

Culebrera azor (S): Eutriorchis astur II B

Culebrera barreada (S): Circaetus gallicus II B

Culebrera chilla (S): Spilomis cheela II B

Culebrera coliblanca (S): Circaetus cinerascens II B

Culebrera congoleña (S): Dryotriorchis spectabilis II B

Culebrera de Andamán (S): Spilomis elgini II B

Culebrera de Célebes (S): Spilomis rufipectus II B

Culebrera de Nicobar ( 3 ): Spilornis minimus II B

Culebrera del Kinabalu (S): Spilornis kinabaluensis II B

Culebrera europea (S): Circaesus gallicus II B

Culebrera filipina (S): Spilomis holospilus II B

Culebrera pechinegra (S): Circaetus pectoralis II B

Culebrera sombría (S): Circaetus cinereus II B

Culicia australiensis: II In

Culicia cuticulata: II In

Culicia excavata: II In

Culicia hoffmeisteri: II in

Culicia japonica: II in

Culicia rubeola: II In

Culicia smithii: II ln

Culicia stellata: II In

Culicia tenella: II in

Culicia truncata: II in

Culicia verreauxii: II In

Culpeo (E/S): Pseudalopex culpaeus 11 M

Cumberland Bean (E): Villosa trabalis I in

Cumberland Bean Pearly Mussel (E): Villosa trabalis I in

Cumberland Monkeyface (E): Quadrula intermedia I In

Cumberiand Monkey-face Pearly Mussel (E): Quadrula intermedia I in

Cuniculus paca $=$ Agouti paca

Cuon alpinus: Asiatic Wild Dog (E), Dhole (E). Indian Wild Dog

(E), Red Dog (E), Perro salvaje asiático (S), Chien sauvage

d'Asie (F), Cuon d'Asie (F), Dhole (F) II M

Cuon d'Asie (F): Cuon alpinus II M 
Cupiso (S): Podocnemis sexfuberculata II R

Curassows (E): Crax spp $/$ Miru spp $/$ Nothocrax spp $/$ Pawi spp. IIIVNC B

Curl-crested Manucode (E): Manucodia comrii II B

Curlews (E): Numenius spp. INC B

Curly-hair Tarantula (E): Brachypelma albopilisum II In

Curruca bigotuda piquilarga (S): Dasyomis longirostris I B

Curnca bigotuda rojiza (S): Dasyomis broadbenti litoralis I B

Curtis' Pearly Mussel (E): Epioblasma curtisii I In

Curtis' Riffleshell (E): Epioblasma curtisii I In

Curucucú barriga amarilla (S): Aegolius harrisii II B

Curucucú común (S): Otus choliba II B

Curucucú gargantiblanco (S): Otus albogularis II B

Curucucú orejudo (S): Otus warsonii II B

Cunucucú pálido (S): Otus ingens II B

Cunucucú vermiculado (S): Otus atricapillus II B

Cuscús gris (S): Phalanger orientalis II M

Cuscús manchado (S): Spilocuscus macularus II M

Cuscús oriental (S): Phalanger orientalis II M

Cuscuses (E): Ailurops spp/Phalanger spp/Spilocuscus spp/Sirigocuscus spp. IU/NC M

Cuspon (S): Priodontes maximus I M

Cusumbi (S): Potos flavus III M

Cut-throat (E): Amadina fasciata III B

Cuvier's Beaked Whale (E): Ziphius cavirostris II M

Cuvier's Gazelle (E): Gazella cuvieri III M

Cuvier's Smooth-fronted Caiman (E): Palaeosuchus palpebrosus II R

Cyanoliseus patagonus: Burrowing Parakeet (E), Patagonian Conure (E), Loro barranquero (S), Conure de Patagonie (F) II B

Cyanophaia bicolor: Blue-headed Hummingbird (E), Colibri à tête bleue (F) II B

Cyanopsitta spixii: Little Blue Macaw (E), Spix's Macaw (E), Guacamayito azul (S), Guacamayo de Spix (S), Ara de Spix (F), Ara à face grise (F) I B

Cyanoramphus auriceps: Yellow-crowned Parakeet (E), Yellowfronted Kakariki (E), Yellow-fronted Parakeet (E), Perico maor cabecigualdo (S), Perruche à front jaune (F), Perruche à tête d'or (F) I/II B

Cyanoramphus auriceps forbesi: Chatham Island Yellow-fronted Parakeet (E), Forbes's Kakariki (E), Forbes's Parakeet (E), Perico de las Chatham (S), Periquito frentiamarillo de Forbes (S), Perruche des îles Chatham (F). Perruche à front jaune de Forbes (F), Perruche à tête d'or de Forbes (F) I B

Cyanoramphus cookii: Norfolk Island Parakeet (E), Norfolk Parakeet (E), Perruche de Norfolk (F) I B

Cyanoramphus forbesi $=$ Cyanoramphus auriceps forbes

Cyanoramphus malherbi = Cyanoramphus auriceps

Cyanoramphus novaezelandiae: New Zealand Parakeet (E), Red Crowned Parakeet (E), Red-fronted Kakariki (E), Red-fronted Parakeet (E), Perico maori cabecirrojo (S), Periquito cabecirrojo (S), Perruche de Nouvelle-ZÉlande (F), Perruche de Sparrman (F), Perruche à bandeau rouge $(F)$, Perruche à bandeau rouge $(F)$ I B

Cyanoramphus novaezelandiae cookii = Cyanoramphus cookii

Cyanoramphus unicolor: Antipodes Green Parakeet (E), Antipodes Parakeet (E), Perico de las Antípodas (S), Perruche des Antipodes (F), Perruche unicolore (F) II B

Cyathelia axillaris: II In

Cyathina = Caryophyllia/Paracyathus

Cyathoceras $=$ Crispatotrochus

Cyathohelia $=$ Cyathelia

Cyathoirochus = Tropidocyathus

Cyclagras gigas: Beach Cobra (E), False Cobra (E), South American Water Cobra (E), Surucucu (E), Falsa cobra (S), Nakamina (S), Couleuvre lisse du Brésil (F). Faux cobra (F) II R

Cyclicia $=$ Culicia

Cyclohelia lamellara: II In

Cyclopsitta diophthalma: Double-eyed Fig-Parrot (E), Dwarf FigParrot (E), Lorito dobleojo (S), Perroquet masqué (F), Psirtacule double-oeil (F) I/II B

Cyclopsitta diophthalma coxeni: Coxen's Blue-browed Fig Parrot (E), Coxen's Double-eyed Fig-Parrot (E), Coxen's Two-eyed Fig Parrot (E), Lorito de Coxen (S), Lorito emmascarado de Coxen (S), Perroquet masqué de Coxen (F), Psittacule à double-ceil de Coxen (F) I B
Cyclopsitla gulielmitertii: Orange-breasted Fig-Partot (E), Lorito pechinaranja (S), Psittacule à poitrine orange (F) II B

Cycloseris = Fungia

Cyclosmilia $=$ Parasmilia

Cyclura spp.: Ground iguanas (E), Iguanas terrestres (S), Cyclures (F), Iguanes comus (F), Iguanes rhinocéros (F) I R

Cyclura baelopha = Cyclura cyclura

Cyclura carinata: Bahamas Rock Iguana (E), Bartsch's Iguana (E), Turks and Caicos Ground Iguana (E), Cyclure des iles Turks et Caiques (F), Iguane terrestre des îles Turks et Caïques (F) I R

Cyclura caymanensis $=$ Cyclura nubila

Cyclura collei: Jamaica Ground Iguana (E), Jamaican Iguana (E), Cyclure terrestre de la Jamaïque (F), Iguane terrestre de la Jamaique (F) I R

Cyclura comuta: Rhinoceros Iguana (E), Cyclure cornu (F), Iguane comu (F), Iguane terrestre cornu (F) I R

Cyclura cyclura: Andros Ground Iguana (E), Rock Iguana (E),

Cyclure de l'île Exuma $(F)$, Iguane terrestre de l'île Exuma (F) I $\mathrm{R}$

Cyclura figginsi $=$ Cyclura cyclura

Cyclura inomata $=$ Cyclura cyclura

Cyclura lophoma $=$ Cyclura collei

Cyclura macleayi = Cyclura nubila

Cyclura nubila: Cayman Islands Ground Iguana (E), Cuban Ground Iguana (E), Cyclure des lles Cayman (F), Iguane terrestre des lles Cayman (F) I $\mathbf{R}$

Cyclura pinguis: Anegada Ground Iguana (E), Anegada Rock Iguana (E), Cyclure de l'lle Anegada (F), Iguane terrestre de l'lle Anegada (F) I R

Cyclura ricordi: Hispaniolan Ground Iguana (E), Ricord's Ground Iguana (E), Ricord's Iguana (E), Cyclure d'Hispaniola (F), Cyclure de Ricord (F), Iguane terrestre d'Hispaniola (F) I R

Cyclura rileyi: Acklin's Ground Iguanz (E), San Salvador Ground Iguana (E), Watling Island Iguana (E), White Cay Ground Iguana (E), Cyclure des Bahamas (F), Iguane terrestre des Bahamas (F) I R

Cyclura stejnegeri $=$ Cyclura comuta

Cyclure comu (F): Cyclura cormuta I R

Cyclure de l'île Exuma (F): Cyclura cyclura I R

Cyclure de l'lle Anegada (F): Cyclura pinguis I R

Cyclure de Ricord (F): Cyclura ricordi $\mathbf{I} \mathbf{R}$

Cyclure des Bahamas (F): Cyclura rileyi I R

Cyclure des lles Cayman (F): Cyclura nubila I R

Cyclure des îles Turks et Caiques (F): Cyclura carinata I R

Cyclure d'Hispaniola (F): Cyclura ricordi I R

Cyclure terrestre de la Jamaique (F): Cyclura collei I $R$

Cyclures (F): Cyclura spp. I R

Cygne à cou noir (F): Cygnus melanocorypha II B

Cygne chanteur (F): Cygnus melanocorypha II B

Cygne coscoroba (F): Coscoroba coscoroba II B

Cygnus melanoconypha: Black-necked Swan (E), Cisne cuellinegro

(S), Cisne cuello negro (S), Cygne chanteur (F), Cygne à cou noir (F) II B

Cylicosmilia $=$ Parasmilia

Cylindrophyllia $=$ Peponocyarhus

Cylloseris = Leptoseris

Cynanthus doubledayi = Cynanthus latirostris

Cynanthus latirostris: Broad-billed Hummingbird (E), Colibri piquiancho (S), Colibri circé (F) II B

Cynanthus sordidus: Dusky Hummingbird (E), Colibrí prieto (S), Colibri sombre (F) 11 B

Cynarina lacrymalis: II In

Cynogale bennetti: Otter-civet (E), Cibeta nutria (S), Civette-loutre de Sumatra (F) II M

Cynogale lowei = Cynogale bennetrii

Cynomolgus Monkey (E): Macaca fascicularis II M

Cynomys mexicanus: Mexican Prairie Dog (E), Mexican Prairie Marmot (E), Perrito de las praderas (S), Perrito llanero Mejicano (S), Chien de prairie du Mexique (F) I M

Cynopithèque nègre $(\mathrm{F})$ : Macaca nigra II M

Cynoscion macdonaldi: MacDonald's Weakfish (E), Totoaba (E), Totoba (S), Acoupa de MacDonald (F) I F

Cyormis ruckii: Rueck's Blue-Flycatcher (E), Rueck's Niltava (E), Gobemouche de Rueck (F), Gobemouches bleu de Rueck (F) II B

Cyphastrea agassizi: II In 
Cyphastrea chalcidicum: II In

Cyphastrea decadia: II In

Cyphastrea japonica: II In

Cyphastrea microphihalma: II in

Cyphastrea nodulosa: II in

Cyphastrea ocellina: II In

Cyphastrea serailia: II In

Cyphastrea tanabensis: II In

Cyphastrea zhongjianensis: II In

Cyprian Wild Sheep (E): Ovis orientalis ophion I M

Cyprogenia abenti: Edible Naiad (E), Edible Pearly Mussel (E), Western Fan-shell (E), Western Fanshell Mussel (E) II In

Cyprus Mouflon (E): Ovis orientalis ophion I M

Cyrtodactyle de líle de Serpent (F): Cyrtodactylus serpensinsula II $\mathrm{R}$

Cyrodacrylus serpensinsula: Serpent Island Gecko (E), Cyrtodactyle de lîle de Serpent (F), Gecko de l'île Serpent (F) II R

Cyrtonaias tampicoensis tecomatensis = Unio tampicoensis tecomatensis

Dabb Lizard (E): Uromastyx aegyptius II R

Dabb's Mastigure (E): Uromastyx acanthinurus II R

Daboia russellii $=$ Vipera nussellii

Dabois (F): Vipera russellii III R

Dabry's Sturgeon (E): Acipenser dabryanus II F

Dactylotrochus cervicomis: II In

Daim de Mésopotamie (F): Dama mesopotamica I M

D'Albert's Python (E): Morelia albertisii II R

Dalgyte (E): Macroris lagotis I M

Dall's Porpoise (E): Phocoenoides dalli II M

Dalmatian Pelican (E): Pelecanus crispus I B

Dama Gazelle (E): Gazella dama I M

Dama mesopotamica: Mesopotamian Fallow Deer (E), Persian Fallow Deer (E), Dama pérsico (S), Gacela de Irán (S), Daim de Mésopotamie (F) I M

Dama pérsico (S): Dama mesopotamica I M

Damaliscus $d$. dorcas = Damaliscus pygargus pygargus

Damaliscus lunatus: Tsessebe (E), Damalisque (F), Hirola (F), Korrigum (F), Sassaby (F), Topi (F) III M

Damaliscus pygargus pygargus: Bontebok (E), Bontebok (S), Topi gacela (S), Bontebok (F) II M

Damalisque (F): Damaliscus lunarus III M

Damara Korhaan (E): Eupodotis rueppellii II B

Damonia hamiltonii $=$ Geoclemys hamilionii

Damophila julie: Violet-bellied Hummingbird (E), Colibrí ventrivioleta (S), Colibri julie (F) II B

Danafungia $=$ Fungia

Danta (S): Tapirus terrestris II M

Danta cordillerana (S): Tapirus pinchaque IM

Danta lanuda (S): Tapirus pinchaque I M

Danta negra (S): Tapirus pinchaque I M

Dante (S): Tapirus bairdii I M

Daptrius americanus: Red-throated Caracara (E), Caracara comecacao (S), Caracara gorgirrojo (S), Carancho ventri-blanco (S), Chupacacao ventriblanco (S), Caracara à gorge rouge $(\mathrm{F})$, Caracara à ventre rouge $(\mathrm{F})$ II $\mathrm{B}$

Daptrius ater: Black Caracara (E), Yellow-throated Caracara (E), Caracara negro (S), Carancho negro (S), Chupacacao negro (S), Caracara noir (F) II B

Dara (S): Burhinus bistriatus III B

Dark Chanting-Goshawk (E): Melierax metabates II B

Dark Serpent-Eagle (E): Spilomis elgini II B

Dark Soft-shell Turtle (E): Trionyx nigricans I R

Dark-coloured Soft-shell Turtle (E): Trionyx nigricans I R

Dark-handed Gibbon (E): Hylobares agilis I M

Dark-spotted Anacaonda (E): Eunectes deschauenseei II R

Darwin's Rhea (E): Rhea pennata I B

Dasmosmilia lymani: II In

Dasmasmilia pacifica: II in

Dasmosmilia valida: II In

Dasmosmilia variegata: II in

Dasyorne a long bec (F): Dasyomis longirostris I B

Dasyomerousse de l'Ouest (F): Dasyomis broadbenti litoralis I B

Dasyornes (F): Dasyormis spp. INC B

Dasyomis brachypterus longirostris $=$ Dasyomis longirostris
Dasyomis broadbenti litoralis: Lesser Rufous Bristlebird (E).

Rufous-headed Bristlebird (E), Western Rufous Bristlebird (E), Curruca bigotuda rojiza (S), Dasyomerousse de l'Ouest (F),

Fauvette rousse de l'Ouest (F) I B

Dasyomis longirostris: Long-billed Bristlebird (E), Western Bristlebird (E), Curruca bigotuda piquilarga (S), Dasyorne à

long bec (F), Fauvette à long bec (F), Malure occidental (F) I B

Dasyphyllia $=$ Caulastrea

Dasyprocta punctata: Central American Agouti (E), Agoutí rojizo

(S), Agouti ponctué (F) III M

Dasyprocla variegata $=$ Dasyprocta punctata

Daubentonia madagascariensis: Aye-aye (E), Aye-aye (S), Aye-aye (F) $1 \mathrm{M}$

Dauphin à bosse de l'Atlantique (F): Sousa teuszii I M

Dauphin à bosse de l' Indo-Pacifique (F): Sousa chinensis I M

Dauphin aptère austral (F): Lissodelphis peronii II M

Dauphin aptère boréal (F): Lissodelphis borealis II M

Dauphin blanc (F): Delphinapserus leucas II M

Dauphin bleu et blanc (F): Stenella coeruleoalba II M

Dauphin commun (F): Delphinus delphis II M

Dauphin crucigère (F): Lagenorhynchus cruciger II M

Dauphin de Clymène (F): Stenella clymene II M

Dauphin de Commerson (F): Cephalorhynchus commersonii II M

Dauphin de Fraser (F): Lagenodelphis hosei II M

Dauphin de Heaviside (F): Cephalorhynchus heavisidii II M

Dauphin de l'Amazone (F): Inia gecffrensis II M

Dauphin de la Plata (F): Pontoporia blainvillei II M

Dauphin de Risso (F): Grampus griseus II M

Dauphin d'Hector (F): Cephalorhynchus hectori II M

Dauphin fluviatile de Chine (F): Lipotes vexillifer I M

Dauphin longirostre (F): Stenella longirostris II M

Dauphin noir du Chili (F): Cephalorhynchus eutropia II M

Dauphin rayé (F): Stenella coeruleoalba II M

Dauphin tacheté de l'Atlantique (F): Stenella frontalis II M

Dauphin tacheté pantropical (F): Stenella antenuata II M

Dauphins blancs (F): Sousa spp. I M

Dauphins de rivière (F): Sotalia spp. I M

David's Owl (E): Strix davidi II B

Day geckos (E): Phelsuma spp. II R

De Brazza's Monkey (E): Cercopithecus neglectus II M

Deceptive Chameleon (E): Chamaeleo fallax II R

Deer (E): Cervidae spp./Moschidae spp. I/IIII/NC M

Deer Hog (E): Babyrousa babyrussa I M

Deer Tiger (E): Puma concolor VII M

Delfín austral (S): Lagenorhynchus australis II M

Delfín blanco africano (S): Sousa teuszii I M

Delfín blanco de China (S): Sousa chinensis I M

Delfín blanco y azul (S): Stenella coeruleoalba II M

Delfín común (S): Delphinus delphis II M

Delfin cruzado (S): Lagenorhynchus cruciger II M

Delfin de Borneo (S): Lagenodelphis hosei II M

Delfín de China (S): Lipotes vexillifer I M

Delfín de flancos blancos (S): Lagenorhynchus acutus II M

Delfín de Héctor (S): Cephalorhynchus heciori II M

Delfín de la Plata (S): Pontoporia blainvillei II M

Delfin de pico blanco (S): Lagenorhynchus albirosiris II M

Delfín de pico largo (S): Steno bredanensis II M

Delfín de Risso (S): Grampus griseus II M

Delfin del Ganges (S): Platanista gangetica I M

Delfín del Indo (S): Platanista minor I M

Delfin del Irrawaddy (S): Orcaella brevirostris II M

Delfín lagenor ringo ( $\mathbf{S}$ : Lagenorhynchus obliquidens II M

Delfin liso (S): Lissodelphis borealis II M

Delfin liso (S): Lissodelphis peronii II M

Delfín listado (S): Lagenorhynchus obscurus II M

Delfín manchado (S): Stenella attenuara II M

Delfín manchado del Atlántico (S): Stenella frontalis II M

Delfín negro (S): Cephalorhynchus eutropia II M

Delfín pintado (S): Sienella artenuata II M

Delfín tornillón (S): Stenella longirostris II M

Delfines blancos (S): Sousa spp. I M

Delphinaptère blanc (F): Delphinapterus leucas II M

Delphinapterus leucas: Beluga (E), White Whale (E), Balıena blanca

(S), Bélouga $(F)$, Dauphin blanc $(F)$, Delphinaptère blanc $(F)$

II $\mathrm{M}$

Delphinus capensis $=$ Delphinus delphis 
Delphinus delphis: Allantic Dolphin (E), Common Dolphin (E),

Pacific Dolphin (E), Saddle-backed Dolphin (E), Delfín común (S), Dauphin commun (F) 11 M

Delphinus tropicalis = Delphinus delphis

Delphinus tropicalis $=$ Delphinus delphis

Deltocyathoides $=$ Deltocyathus

Deltocyathus agassizi: II In

Deltocyathus andamanicus: 11 in

Deltocyathus calcar: II In

Deliocyarhus conicus: II In

Deltocyathus eccentricus: II In

Deltocyathus formosus: II In

Deliocyathus inalicus: II In

Deltocyathus magnificus: II In

Deltocyathus moseleyi: II in

Deltocyathus murrayi: II In

Deltocyathus nascomatus: II In

Deltocyathus omatus: II In

Deltocyathus pourtalesi: II in

Deltocyathus rotulus: II In

Deltocyathus sarsi: II in

Deltocyathus varians: II In

Deltocyathus vaughani: II In

Deltocyathus vincentinus: II In

Demidoff's Dwarf Galago (E): Galagoides demidoff II M

Demidoff's Galago (E): Galagoides demidoff II M

Demoiselle Crane (E): Grus virgo II B

Demoiselle de Numidie (F): Grus virgo II B

Demonic Poison Frog (E): Minyobates steyermarki II A

Dendrobate à cinq bandes (F): Dendrobates quinquevittatus II A

Dendrobate a tapirer (F): Dendrobates tinctorius II A

Dendrobate à trois bandes (F): Phobobates irivittatus II A

Dendrobate arboricole (F): Dendrobates arboreus 11 A

Dendrobate au ventre tacheté (F): Dendrobates ventrimaculatus II A

Dendrobate biolat (F): Dendrobates biolat II A

Dendrobate bleu (F): Dendrobates azureus II A

Dendrobate de Bassler (F): Phobobates bassleri II A

Dendrobate de Lamas (F): Dendrobates lamasi II A

Dendrobate de Lehman (F): Dendrobates lehmanni II A

Dendrobate de Silverstone (F): Phobobates silverstonei II A

Dendrobate de Sira (F): Dendrobates sirensis II A

Dendrobate de Vanzolini (F): Dendrobates vanzolinii II A

Dendrobate doré $(F)$ : Dendrobates auralus II A

Dendrobate du Rio Magdalena (F): Dendrobates truncatus II A

Dendrobate du Rio Santiago (F): Dendrobates captivus II A

Dendrobate du Tapajos (F): Dendrabates galactonotus II A

Dendrobate du Xingu (F): Dendrobates castaneoticus II A

Dendrobate fantastique (F): Dendrobates fantasticus II A

Dendrobate granuleux (F): Dendrobates granulifer II A

Dendrobate imitateur (F): Dendrobates imitator II A

Dendrobate jaune et noir (F): Dendrobates leucomelas II A

Dendrobate labié (F): Dendrobates labialis II A

Dendrobate mystérieux (F): Dendrobates mysteriosus II A

Dendrobate occulte (F): Dendrobates occulator II A

Dendrobate pumilio (F): Dendrobates pumilio II A

Dendrobate réticule (F): Dendrobates reticulatus II A

Dendrobate rougeâtre (F): Dendrobates rufulus II A

Dendrobate variable (F): Dendrobates variabilis II A

Dendrobates (F): Dendrobates spp. II A Allobates spp.

II AVpipedobates spp. II AMinyobates spp. II A Phobobates spp. II A

Dendrobates spp.: Poison frogs (E), Ranas de puntas de flechas (S), Dendrobates (F) II A

Dendrobates abditus $=$ Minyobates abditus

Dendrobates altobueyensis = Minyobates altobueyensis

Dendrobates andinus = Epipedobates andinus

Dendrobates anthonyi $=$ Epipedobates tricolor

Dendrobates arboreus: Polkadot Poison Frog (E), Rana de punta de flecha arbórea (S), Dendrobate arboricole (F) II A

Dendrobates auratus: Green Poison Frog (E), Green-and-black Poison Frog (E), Dendrobate doré (F) II A

Dendrobates azureiventris = Epipedobates azureiventris

Dendrobates azureus: Blue Poison Frog (E), Giant Poison Frog (E), Rana de punta de flecha azul (S), Dendrobate bleu (F) II A

Dendrobates bassleri $=$ Phobobates bassleri
Dendrobates biolat: Biolat Poison Frog (E), Dendrobate biolat (F) II A

Dendrobates bolivianus = Epipedobates bolivianus

Dendrobates bombetes $=$ Minyobates bombetes

Dendrobates boulengeri $=$ Epipedobates boulengeri

Dendrobates caprivus: Rio Santiago Poison Frog (E), Dendrobate du Rio Santiago (F) II A

Dendrobates castaneoticus: Brazil-nut Poison Frog (E), Dendrobate du Xingu (F) II A

Dendrobates erythromos $=$ Epipedobates erythromos

Dendrobates espinosai $=$ Epipedobates espinosai

Dendrobates fantasticus: Red-headed Poison Frog (E), Rana de punta de flecha fantástica (S), Dendrobate fantastique (F) II A

Dendrobates femoralis $=$ Allobates femoralis

Dendrobates fulguritus = Minyobates fulguritus

Dendrobates galactonotus: Splash-backed Poison Frog (E), Dendrobate du Tapajos (F) II A

Dendrobates granulifer: Rana de punta de flecha granulosa (S), Dendrobate granuleux (F), Granular Poison Frog (F) II A

Dendrobates histrionicus: Harlequin Poison Frog (E), Red-and-black Poison Frog (E), Rana de punta de flecha histriónica (S) II A

Dendrobates imitator: Mimic Poison Frog (E), Dendrobate imitateur (F) II A

Dendrobates ingeri $=$ Epipedobates ingeri

Dendrobates labialis: Dendrobate labié (F) II A

Dendrobates lamasi: Pasco Poison Frog (E), Dendrobate de Lamas (F) II A

Dendrobates lehmanni: Lehmann's Poison Frog (E), Red-banded Poison Frog (E), Rana de punta de flecha de Lehmann (S), Dendrobate de Lehman $(\boldsymbol{F})$ II A

Dendrobates leucomelas: Yellow-banded Poison Frog (E), Rana de punta de flecha de bandas amarillas (S), Dendrobate jaune et noir (F) II A

Dendrobates maculatus $=$ Epipedobates maculatus

Dendrobates minutus $=$ Minyobates minutus

Dendrobates myersi $=$ Epipedobates myersi

Dendrobates mysteriosus: Maranon Poison Frog (E), Rana de punta de flecha misteriosa (S), Dendrobate mystérieux (F) II A

Dendrobates occulator: La Brea Poison Frog (E), Dendrobate occulté (F) II A

Dendrobates opisthomelas = Minyobates opisthomelas

Dendrobates parvulus = Epipedobates parvulus

Dendrobates petersi $=$ Epipedobates petersi

Dendrobates pictus $=$ Epipedobates pictus

Dendrobates pulchripectus = Epipedobates pulchripectus

Dendrobates pumilio: Flaming Poison Frog (E), Red-and-blue Poison Frog (E), Rana de punta de flecha roja (S), Dendrobate pumilio (F), Strawberry Poison Frog (F) II A

Dendrobates quinquevittatus: Rio Madeira Poison Frog (E), Rana de punta de flecha pentaestriada (S), Dendrobate à cinq bandes (F) II A

Dendrobates reticulatus: Red-backed Poison Frog (E), Reticulated Poison Frog (E), Rana de punta de flecha reticulada (S), Dendrobate réticulé (F) II A

Dendrobates rufulus: Gorzula's Poison Frog (E), Dendrobate rougeâtre (F) II A

Dendrobates silverstone $i=$ Phobobates silverstonei

Dendrobates sirensis: Sira Poison Frog (E), Dendrobate de Sira (F) II A

Dendrobates smaragdinus = Epipedobates smaragdinus

Dendrobates speciosus: Splendid Poison Frog (E), Epipedobate brillant (F) II A

Dendrobates steyermarki = Minyobates steyermarki

Dendrobates tinctorius: Dyeing Poison Frog (E), Dendrobate à tapirer (F) II A

Dendrobates iricolor $=$ Epipedobates tricolor

Dendrobates trivittatus $=$ Phobobates irivintatus

Dendrobates truncalus: Yellow-striped Poison Frog (E), Rana de punta de flecha truncada (S), Dendrobate du Rio Magdalena ( $F$ ) II A

Dendrobates vanzolinii: Brazilian Poison Frog (E), Rana de punta de flecha de Vanzolini (S), Dendrobate de Vanzolini (F) II A

Dendrobates variabilis: Rana de punta de flecha variable (S), Dendrobate variable (F) II A

Dendrobaies ventrimacularus: Amazonian Poison Frog (E), Dendrobate au ventre tacheté (F) II A 
Dendrobates viridis $=$ Minyobates viridis

Dendrobates zaparo $=$ Epipedobates zaparo

Dendrocora fissipara: $\mathbf{I}$ in

Dendrocygna arborea: Black-billed Wood-Duck (E), Cuban TreeDuck (E), West Indian Tree-Duck (E), West Indian WhistlingDuck (E), Pato silbón de Cuba (S), Suiriri yaguaza (S). Dendrocygne des Antilles (F), Dendrocygne à bec noir (F) 11 B

Dendrocygna ausumnalis: Black-bellied Whistling-Duck (E), Redbilled Whistling-Duck (E), Guirirí (S), Pato silbón ventrinegro (S), Pijiji aliblanco (S), Suiriri piquirrojo (S), Dendrocygne à bec rouge (F) III B

Dendrocygna bicolor. Fulvous Tree-Duck (E), Fulvous WhistingDuck (E), Pato silbón común (S), Pijiji canelo (S), Suirirí bicolor (S), Suirirí leonado (S), Yaguaso colorado (S), Dendrocygne fauve (F) III B

Dendracygna fulva = Dendrocygna bicolor

Dendrocygna viduata: White-faced Tree-Duck (E), White-faced Whistling-Duck (E), Pato silbón cariblanco (S), Suirirí cariblanco (S), Yagouaso cariblanco (S), Dendrocygne veuf (F) III B

Dendrocygne à bec noir (F): Dendrocygna arborea II B

Dendrocygne à bec rouge (F): Dendrocygna autumnalis III B

Dendrocygne des Antilles (F): Dendrocygna arborea II B

Dendrocygne fauve (F): Dendrocygna bicolor III B

Dendrocygne veuf (F): Dendrocygna viduata III B

Dendrocygnes (F): Dendrocygna spp. II//J//NC B

Dendrogale melanura: Bomean Smooth-tailed Treeshrew (E), Tupaya de Borneo (S) II M

Dendrogale murina: Northern Smooth-tailed Treeshrew (E), Toupaie murin (F) $\mathbf{U} \mathrm{M}$

Dendrogyra cylindrus: Pillar Coral (E) II In

Dendrolague gris (F): Dendrolagus inusius II M

Dendrolague noir (F): Dendrolagus ursinus II M

Dendrolagus inustus: Grizzled Tree-kangaroo (E), Canguro arborícola gris (S), Dendrolague gris (F) II M

Dendrolagus ursinus: Black Tree-kangaroo (E), Vogelkop Treekangaroo (E), Canguro arborícola negro (S), Dendrolague noir (F) II M

Dendrophyllia alcocki: II In

Dendrophyllia alternata: II in

Dendrophyllia anastomozans: II In

Dendrophyllia arbuscula: II in

Dendrophyllia atrata $=$ Astrangia atrata

Dendrophyllia boschmai: II ln

Dendrophyllia californica: II in

Dendrophyllia carleenae: II in

Dendrophyllia cladonia: II In

Dendrophyllia coarctata: II in

Dendrophyllia comigera: II In

Dendrophyllia comucopia: II In

Dendrophyllia cribrosa: II In

Dendrophyllia danae: II In

Dendrophyllia dilatata: II In

Dendrophyllia fistula $=$ Eguchipsammia fistula

Dendrophyllia florulenta: II In

Dendrophyllia horst: II In

Dendrophyllia ijimai: II In

Dendrophyllia indica: II In

Dendrophyllia japonica = Eguchipsammia japonica

Dendrophyllia johnsoni: II In

Dendrophyllia Laboreli: 11 In

Dendrophyllia minuscula: II In

Dendrophyllia oahensis $=$ Eguchipsammia fistula

Dendrophyllia oldroydi: $\mathbf{I I}$ in

Dendrophyllia praecipua: II in

Dendrophyllia ramea: 11 in

Dendrophyllia robusta: II In

Dendrophyllia serpentina: II In

Dendrophyllia velata: II In

Dendrosmilia $=$ Lophelia

Denham's Bustard (E): Neotis denhami II B

Deppe's Squirrel (E): Sciurus deppei III M

Derbyan Parakeet (E): Psittacula derbiana II B

Dermatemyde de Mawe (F): Dermatemys mawii II R
Dermatemys mawii: Central American River Turte (E), Plana (S). Tortuga blanca (S), Dermatemyde de Mawe (F), Tortue de Tabasco (F) II R

Dermochelys coriacea: Leatherback (E), Leathery Turte (E), Luth (E), Trunkback turtle (E), Canal (S), Tinglada (S), Tortuga laud (S), Tortue luth (F) I R

Deroptyus accipitrinus: Hawk-headed Parrot (E), Red-fan Parrot (E), Loro cacique (S), Papegeai maillé (F) II B

Deschauensee's Anaconda (E): Eunectes deschauenseei II R

Desert Chameleon (E): Chamaeleo namaquensis II R

Desert Lynx (E): Caracal caracal UI M

Desert Monitor (E): Varanus griseus I R

Desert Owl (E): Strix butleri II B

Desert Pygmy Monitor (E): Varanus eremius II R

Desert Rat-kangaroo (E): Caloprymnus campestris I M

Desert Tortoise (E): Gopherus agassizii II R

Desmarest's Fig-Parrot (E): Psittaculirostris desmarestii II B

Desmophyllum dianthus: II In

Desmophyllum reesei: II in

Desmophyllum striatum: II In

Desmophyllum tenuescens $=$ Thalamophyllia tenuescens

Devil Fish (E): Eschrichtius robustus I M

Devonshire Cup Coral (E): Caryophyllia smithii II In

Dhaman (E): Pryas mucosus II R

Dhole (E/F): Cuon alpinus II M

Diademed Monkey (E): Cercopithecus mitis II M

Diademed Sifaka (E): Propithecus aiadema I M

Diafungia $=$ Micrabacia

Diamant aurore (F): Pytilia phoenicoptera III B

Diamant à bavette (F): Poephila cincta cincta II B

Diamant à face rouge (F): Pyrilia hypogrammica III B

Diamante colidorado (S): Amazilia chionopecfus II B

Diamante de pecho regro (S): Poephila cincta cincta II B

Diamante de Wetmore (S): Amazilia distans II B

Diamante gargantiverde (S): Amazilia fimbriata II B

Diamante multicolor (S): Amazilia versicolor II B

Diamante pechizafiro (S): Amazilia lactea II B

Diamante ventriblanco (S): Amazilia leucogaster II B

Diamants (F): Estrildidae spp. IL/NC B

Diamond Python (E): Morelia spilota II R

Diana (S): Cercopithecus diana I M

Diana Guenon (E): Cercopithecus diana I M

Diana Monkey (E): Cercopithecus diana I M

Diaseris $=$ Fungia

Dicerorhinus sumatrensis: Sumatran Rhinoceros (E), Rinoceronte de Sumatra (S), Rhinocéros de Sumatra (F) I M

Diceros bicomis: Black Rhinoceros (E), Hook-lipped Rhinoceros

(E), Rinoceronte negro (S), Rhinocéros noir (F) I M

Dichocoenia stellaris: Elliptical Star Coral (E) II In

Dichocoenia stokesii: II In

Dichopsammia granulosa: II In

Dickinson's Kestrel (E): Falco dickinsoni II B

Didermocerus sumatrensis $=$ Dicerorhinus sumatrensis

Diechoraea $=$ Alveopora

Dindon ocellé (F): Agriocharis ocellata III B

Dindons (F): Agriocharis ocellataMeleagris gallopavo IIUNC B

Diochs (E): Quelea spp. IIINC B

Diomedea albatrus: Short-tailed Albatross (E), Steller's Albatross

(E), Albatros colicorto (S), Albatros rabón (S), Albatros de Steller $(F)$, Albatros à queue courte $(F)$ I B

Diostedé pico acanelado (S): Ramphasıos virellinus II B

Diphyllodes magnificus = Cicinnurus magnificus

Diphyllodes respublica $=$ Cicinnurus respublica

Diploastrea heliopora: II In

Diploria clivosa: II Is

Diploria labyrinthiformis: Brain Coral (E) II In

Diploria strigosa: II Iת

Dipneuste (F): Neoceratodus forsteri II F

Dipsastrea $=$ Favia

Dipsochelys elephantina $=$ Geochelone gigantea

Discophus antongili $=$ Dyscophus antongilii

Discosura conversii $=$ Popelairia conversii

Discosura langsdorfi = Popelairia langsdorffi

Discosura letiriae $=$ Popelairia letitia

Discosura longicauda: Racket-tailed Coquette (E), Coqueta cola raqueta (S), Coquette à raquettes (F) II B 
Discosura popelairii $=$ Popelairia popelairi

Distichocyathus = Acropora

Distichopora anceps: II In

Distichopora anomala: II In

Distichopora barbadensis: II In

Distichopora borealis: II In

Distichopora cervina: II In

Distichopora coccinea: II In

Distichopora contoria: II In

Distichopora dispar. II In

Distichopora foliacea: II In

Distichopora gracilis: II In

Distichopora irregularis: II In

Distichopora laevigranulosa: II In

Distichopora livida: II In

Distichopora nitida: II In

Distichopora profunda: II In

Distichopora providentiae: II In

Distichopora rosalindae: II In

Distichopora serpens: II In

Distichopora sulcata: II In

Distichopora uniserialis: II In

Distichopora violacea: II In

Distichopora yucatanensis: II In

Dob (F): Uromastyx acanthinurus II R

Doederleinia $=$ Sandalolitha

Dog Fox (E): Vulpes cana II M

Dog-faced Water Snake (E): Cerberus rhynchops III $\mathrm{R}$

Dogs (E): Canidae spp. J/U/NC M

Dolphins (E): CETACEA spp. I/I M

Dominican Mango (E): Anthracothorax dominicus II B

Domoseris $=$ Leptoseris

Dont corail bleu (F): COENOTHECALIA spp. II In

Dont coraux récifaux (F): SCLERACTINIA spp. II In

Dorcas Gazelle (E): Gazella dorcas III M

Doria's Goshawk (E): Megatriorchis doriae II B

Doricha eliza: Mexican Sheartail (E), Tijereta yucateca (S), Colibri élise (F) 11 B

Doricha enicura: Slender Sheartail (E), Tijereta centroamericana (S). Colibri à queue singulière (F) II B

Doryfera johannae: Blue-fronted Lancebill (E), Pico lanza frentiazul (S), Porte-lance de Jeanne (F) II B

Doryfera ludovicae: Green-fronted Lancebill (E), Pico lanza frentiverde (S), Porte-lance de Louise (F) II B

Dos-vert à joues blanches (F): Nesocharis capisirata III B

Dos-verts (F): Nesocharis spp. IIINC B

Dot-eared Coquette (E): Lophomis gouldii II B

Double-eyed Fig-Parrot (E): Cyclopsitta diophthalma VI B

Double-scaled Chameleon (E): Chamaeleo anchietae II R

Double-striped Thick-knee (E): Burhinus bistriatus III B

Double-toothed Kite (E): Harpayus bidentatus II B

Douc (E/F): Pygathrix nemaeus I M

Douc Langur (E): Pygathrix nemaeus I M

Douc Monkey (E): Pygathrix nemaeus I M

Doucs (F): Pygathrix spp. I M

Douroucouli (E/F): Aons trivirgarus II M

Doves (E): Columbidae spp. I//IINC B

Dracaena spp.: Caiman lizards (E), Jacanuxi (S), Lagartos dragóns (S), Dracènes (F), Lézards caiman (F) II R

Dracaena guianensis: Armoured Teyou (E), Croco-Teju (E), Fourfoot Caiman (E), Guyana Caiman Lizard (E), Jacuruxi (E) Dracène de Guyane (F), Lézard-caïman de Guyane (F) II $R$

Dracaena paraguayensis: Paraguay Caiman Lizard (E), Dracène du Paraguay (F), Lézard-caìman du Paraguay (F) II $R$

Dracène de Guyane (F): Dracaena guianensis II $\mathrm{R}$

Dracène du Paraguay (F): Dracaena paraguayensis II R

Dracènes (F): Dracaena spp. II R

Dragón (S): Agelaius flavus I B

Dragon des Komodos (F): Varanus komodoensis I $R$

Dragon fish (E): Scleropages spp. I/NC F

Dragon Lizard (E): Crocodilunus lacertinus II $R$

Dragon Lizardet (E): Crocodilunus lacertinus II R

Drakensberg Crag Lizard (E): Pseudocordylus melanotus II R

Drakensberg Dwarf Chameleon (E): Bradypodion dracomontanum II $R$

Drepanomis albertisi $=$ Epimachus albertisi
Drepanornis bruijnii = Epimachus bruijnii

Drépanomis d'Albertis (F): Epimachus albertisi II B

Drépanomis de Bruijn (F): Epimachus bruijnii II B

Dril (S): Mandrillus leucophaeus $1 \mathrm{M}$

Drill (E/F): Mandrillus leucophaeus I M

Droguera (F): Papio hamadryas II M

Dromedary Naiad (E): Dromus dromas I In

Dromedary Pearly Mussel (E): Dromus dromas I In

Dromus dromas: Dromedary Naiad (E), Dromedary Pearly Mussel (E) I In

Dryade à queue fourchue (F): Thalurania furcala II B

Dryade couronnée (F): Thalurania colombica II B

Dryade de Fanny (F): Thalurania fannyi II B

Dryade de Waterton (F): Thalurania waterionii II B

Dryade du Mexique (F): Thalurania ridgwayi II B

Dryade glaucope (F): Thalurania glaucopis II B

Dryades (F): Thalurania spp. II B

Dryas Guenon (E): Cercopithecus dryas II M

Dryas Monkey (E): Cercopithecus dryas II M

Dry-bush Weasel Lemur (E): Lepilemur leucopus I M

Dryocopus javensis richardsi: Tristram's Woodpecker (E), Pito ventriblanco de Corea (S), Pic de Tristram (F), Pic à ventre blanc de Corée (F) I B

Dryotriorchis spectabilis: African Serpent-Eagle (E), Congo Serpent-

Eagle (E), Aguila culebrera colilarga (S), Culebrera congolen̄a

(S), Aigle serpentaire (F), Serpentaire du Congo (F) II B

Duc à aigrettes (F): Lophostrix cristala II B

Duc à crinière (F): Jubula lettii II B

Duchess Lorikeet (E): Chamosyna margarethae II B

Duck Hawk (E): Falco peregrinus I B

Duckbill Cat (E): Polyodon spathula $11 \mathrm{~F}$

Ducks (E): Anatidae spp. I/IIIU/NC B

Ducorps's Cockatoo (E): Cacatua ducorpsii II B

Ducs (F): Jubula letrivLophostrix cristata II B

Ducula mindorensis: Mindoro Imperial-Pigeon (E), Mindoro

Imperial-pigeon (E), Mindoro Zone-tailed Pigeon (E), Paloma de

Mindoro (S), Carpophage de Mindoro (F), Pigeon de Mindoro (F) \B

Dugon (S): Dugong dugon VI M

Dugong (E/S/F): Dugong dugon I/II M

Dugong dugon: Dugong (E), Sea Cow (E), Dugon (S), Dugong (S), Dugong (F) III M

Duikers (E): Cephalophus spp. I/I/NC M

Duiquero azul (S): Cephalophus monicola II M

Duiquero bayo (S): Cephalophus dorsalis II M

Duiquero cebrado (S): Cephalophus zebra II M

Duiquero de Jentink (S): Cephalophus jentinki I M

Duiquero de lomo amarillo (S): Cephalophus silvicultor II M

Duiquero de Ogilby (S): Cephalophus ogilbyi II M

Dumerilia madagascariensis = Erymnochelys madagascariensis

Dumeril's Boa (E): Acrantophis dumerili I R

Dumeril's Monitor (E): Varanus dumerilii II R

Duncania $=$ Gardineria

Duncanopsammia axifuga: II in

Dunnarts (E): Sminthopsis spp. INC M

Dunocyathus parasiticus: II In

Dura Turtle (E): Kachuga tecta I R

D'Urville's Birdwing (E): Ornithoptera unillianus 11 in

Dusicyon culpaeus = Pseudalopex culpaeus

Dusicyon griseus $=$ Pseudalopex griseus

Dusicyon gymnocercus $=$ Pseudalopex gymnocercus

Dusicyon thous $=$ Cerdocyon thous

Dusky Dolphin (E): Lagenorhynchus obscurus II M

Dusky Eagle-Owl (E): Bubo coromandus II B

Dusky Hummingbird (E): Cynanthus sordidus II B

Dusky Leaf Monkey (E): Trachypithecus obscurus II M

Dusky Lory (E): Pseudeos fuscata II B

Dusky Parrot (E): Pionus fuscus II B

Dusky Starfrontlet (E): Coeligena orina II B

Dusky Tamarin (E): Saguinus inusfus II M

Dusky Titi (E): Callicebus moloch II M

Dusky-billed Parrotlet (E): Forpus sclateri II B

Dusky-headed Conure (E): Aratinga weddellii II B

Dusky-headed Parakeet (E): Aratinga weddellii II B

Dusky-throated Hermit (E): Phaethornis squalidus II B

Duyvenbode's Lory (E): Chalcopsina duivenbodei II B 
Dwarf Bushbaby (E): Galogoides demidoff $11 \mathrm{M}$ Dwarf Caiman (E): Palaeosuchus palpebrosus II R Dwarf chameleons (E): Bradypodion spp. II R Dwarf Chimpanzee (E): Pan paniscus I M Dwarf Fig-Parrot (E): Cyclopsinta diophthalma VII B Dwarf Galago (E): Galagoides demidoff II M Dwarf Gibbon (E): Hylobates klossii I M

Dwarf Girdled Lizard (E): Cordylus minor II R

Dwarf lemurs (E): Cheirogaleidae spp. I $M$

Dwarf Monitor (E): Varanus storri II R

Dwarf Musk Deer (E): Moschus berezovskii $11 \mathrm{M}$

Dwarf Sand Boa (E): Eryx miliaris II R

Dwarf Sperm Whale (E): Kogia simus II M

Dyeing Poison Frog (E): Dendrobates tinctorius II A

Dyscophus antongilii: Tomato Frog (E), Crapaud rouge de Madagascar (F) I A

Dysnomia sulcata $=$ Epioblasma sulcata perobliqua

Dysnomia torulosa rangiana = Epioblasma torulosa gubemaculum

Dziggetai (E): Equus hemionus hemionus I M

Eagle-Owis (E): Bubo spp. II B

Eagles (E): Accipitridae spp. I/II B

Eared-Pheasants (E): Crossoptilon spp. INC B

East African Spiny-tailed Lizard (E): Cordylus tropidostemum II R

Eastern Bettong (E): Bettongia gaimardi I M

Eastern Black-and-white Colobus (E): Colobus guereza II M

Eastern Black-capped Lory (E): Lorius hypoinochrous II B

Eastem Box Turtle (E): Terrapene carolina II R

Eastern Chanting-Goshawk (E): Melierax poliopterus II B

Eastem Cougar (E): Puma concolor couguar I M

Eastem Grass-Owl (E): Tyro longimembris II B

Eastern Marsh-Harrier (E): Circus spilonotus II B

Eastern Needle-clawed Bushbaby (E): Galago matschiei II M

Eastem Needle-clawed Galago (E): Galago marschiei II M

Eastern Panther (E): Puma concolor couguar I M

Eastem Parotia (E): Parotia helenae II B

Eastem Puma (E): Puma concolor couguar I M

Eastem Red Colobus (E): Procolobus pennantii I/II M

Eastern Red-footed Falcon (E): Falco amurensis II B

Eastern Riflebird (E): Priloris intercedens II B

Eastern Rosella (E): Platycercus eximius II B

Eastern Screech-Owl (E): Oius asio II B

Eastem Tarsier (E): Tarsius specirum $11 \mathrm{M}$

Ebian's Palm Squirrel (E): Epixerus ebii III M

Ebony Leaf Monkey (E): Trachypithecus auratus II M

Echidnas (E): Zaglossus bruijni/Tachyglossus aculearus II/NC M

Echidné à bec courbe (F): Zaglossus bruijni II M

Echidné de Nouvelle-Guinée (F): Zaglossus bruijni II M

Echidnos narilargos (\$): Zaglossus bruijni II M

Echinastrea $=$ Echinopora

Echinophyllia aspera: II In

Echinophyllia echinata: II in

Echinophyllia echinoporoides: II In

Echinophyllia hirsuta: II In

Echinophyllia maxima: II in

Echinophyllia nishihirai: II In

Echinophyllia orpheensis: II In

Echinophyllia patula: II In

Echinophyllia subglabra: II in

Echinopora ashmorensis: II In

Echinopora forskaliana: II in

Echinopora gemmacea: $\mathbf{U}$ In

Echinopora hirsurissima: II In

Echinopora horrida: II In

Echinopora lamellosa: II in

Echinopora mammiformis: II in

Echinopora pacificus: II In

Eclectus Parrot (E): Eclectus roratus II B

Eclectus roratus: Eclectus Parrot (E), Loro ecléctico (S), Grand eclectus (F) II B

Ecuadorian Dwarf Boa (E): Tropidophis battersbyi II R

Ecuadorian Eyelash Boa (E): Trachyboa gularis II R

Ecuadorian Hillstar (E): Oreotrochilus chimborazo II B

Ecuadorian Piedtail (E): Phlogophilus hemileucurus II B

Écureuil de Deppe (F): Sciurus deppei III M

Écureuil de Raffles (F): Rarufa affinis II M

Écureuil d'Ebi (F): Epixerus ebii III M
Écurevil des palmiers (F): Epixerus ebii III M

Écureuil des pins (F): Sciurus deppei $\mathbf{\text { II M M }}$

Écureuil géant commun (F): Rarufa affinis II M

Écureuil géant de Ceylan (F): Ralufa macroura II M

Écureuil géant de l'Inde (F): Ratufa indica II M

Écureuil géant de Malaisie (F): Ratufa bicolor II M

Écureuil géant gris (F): Ratufa macroura II M

Écureuil palmiste (F): Epixerus ebii III M

Écureuil volant de Beecroft (F): Anomalunus beecrofii $111 \mathrm{M}$

Écureuil volant de Derby (F): Anomalurus derbianus III M

Écureuil volant de Pel (F): Anomalurus pelii III M

Écureuils géants (F): Ratufa spp. II M

Écureuils volants (F): Anomalurus spp. III M

Edıble Naiad (E): Cyprogenia aberi II In

Edible Pearly Mussel (E): Cyprogenia aberti II In

Edmi Gazelle (E): Gazella cuvieri III M

Edwardsotrochus spp.: II In

Edwards's Fig-Parrot (E): Psittaculirostris edwardsii II B

Edwards's Pheasant (E): Lophura edwardsi I B

Effraie africaine (F): Tyto alba II B

Effraie de Manus (F): Tyro manusi II B

Effraie de Minahassa (F): Tyto inexspectata II B

Effraie de prairie (F): Tyto longimembris II B

Effraje de Soumagne (F): Tyto soumagnei I B

Effraje de Taliabu (F): Tyto nigrobrunnea II B

Effraie de Tasmanie (F): Tyto castanops II B

Effraie des Célèbes (F): Tyto rosenbergii II B

Effraie des clochers (F): Tyıo alba II B

Effraie des Tanimbar (F): Tyto sororcula II B

Effraie d'Hispaniola (F): Tyto glaucops II B

Effraie dorée (F): Tyto aurantia II B

Effraie du Cap (F): Tyto capensis II B

Effraie masquée (F): Tyto novaehollandiae II B

Effraie ombrée (F): Tyro tenebricosa II B

Effraie piquetée (F): Tyto multipunctata II B

Effraie rousse de Madagascar (F): Tyto soumagnei I B

Effraies (F): Tyro spp. I/II B

Egg-eaters (E): Elachistodon westermanni/Dasypeltis spp. IU/NC R

Egg-eating snakes (E): Elachistodon westermanni/Dasypeltis spp. IUNC R

Egrets (E): Bubulcus ibis/Casmerodius albus/Egretta $\mathrm{spp} /$ Mesophoyx intermedia III/NC B

Egretla alba $=$ Casmerodius albus

Egrella garzetia: Little Egret (E), Garceta común (S), Aigrette garzette (F) III B

Eguchipsammia fistula: II in

Eguchipsammia gaditana: II In

Eguchipsammia japonica: II In

Eguchipsammia wellsi: II In

Egyptian Goose (E): Alopochen aegyptiacus III B

Egyptian Mastigure (E): Uromastyx aegyptius II R

Egyptian Spiny-tailed Lizard (E): Uromastyx aegyptius II R

Egyptian Tortoise (E): Testudo kleinmanni I R

Egyptian Vulture (E): Neophron percnopterus II B

Eira barbara: Tayra (E), Cabeza de viejo (S), Gato cutarra (S), Gato negro (S), Hurón mayor (S), Manco (S), Tejón (S), Tolumuco (S), Ucati (S), Ulamá (S), Tayra (F) III M

El Oro Conure (E): Pyrrhura orcesi II B

El Oro Parakeet (E): Pyrrhura orcesi II B

Elachistodon (F): Elachistodon wesiermanni II R

Elachistodon westermanni: Indian Egg-eater (E), Indian Egg-eating Snake (E), Westermann's Snake (E), Culebra tragahuevos india $(S)$, Couleuvre de Westermann (F), Elachistodon (F), Mangeur d'ouefs indien (F), Mangeur d'úufs asiatique (F) II R

Elanio australiano (S): Elanus axillaris II B

Elanio azul (S): Elanus caeruleus II B

Elanio bidentado (\$): Harpagus bidentatus II B

Elanio blanco (S): Elanus leucurus II B

Elanio chico (S): Gampsonyx swainsonii II B

Elanio ccmún (S): Elanus caeruleus II B

Elanio del Mississipi (S): Ictinia mississippiensis II B

Elanio enano (S): Gampsonyx swainsonii II B

Elanio escrito (S): Elanus scriptus II B

Elanio golondrina (S): Chelictinia riocourii II B

Elanio maromero (S): Elanus leucurus II B

Elanio muslirnfo (S): Harpagus diodon II B 
Elanio plomizo (S): Ictinia plumbea II B

Elanio tijereta (S): Elanoides forficatus II B

Élanion à queve blanche (F): Elanus leucurus II B

Élanion blanc (F): Elanus caeruleus II B

Élanion d'Australie (F): Elanus axillaris II B

Élanion lettré (F): Elanus scripsus $11 \mathrm{~B}$

Élanion naucler (F): Chelictinia riocourii II B

Élanion perle (F): Gampsonyx swainsonii II B

Elanions (F): Accipitridae spp. I/II B

Elanoides forficatus: American Swallow-tailed Kite (E), Swallowtailed Kite (E), Elanio tijereta (S), Gavilán tijereta (S), Milano tijereta (S), Milan à queue fourchue (F) II B

Elanus axillaris: Australian Black-shouldered Kite (E), Blackshouldered Kite (E), Elanio australiano (S), Élanion d'Australie (F) II B

Elanus caeruleus: Black-shouldered Kite (E), Black-winged Kite (E), Elanio azul (S), Elanio común (S), Élanion blanc (F) II B

Elanus caeruleus leucurus $=$ Elanus leucurus

Elanus leucurus: White-tailed Kite (E), Elanio blanco (S), Elanio maromero (S), Gavilán maromero (S), Milano coliblanco (S), Élanion à queue blanche (F) II B

Elanus notatus $=$ Elanus axillaris

Elanus rocourii $=$ Chelictinia riocourii

Elanus scriptus: Letter-winged Kite (E), Elanio escrito (S), Élanion lettré $(F)$ II B

Eld's Deer (E): Cervus eldii I M

Electra (S): Peponocephala electra II M

Elefante africano (S): Loxodonsa africana $\mathbf{V I} \mathbf{M}$

Elefante asiático (S): Elephas maximus I M

Elefante marino del sur (S): Mirounga leonina II M

Elegance Coral (E): Euphyllia picteti II In

Elegant Grass-Parakeet (E): Neophema elegans II B

Elegant Parrot (E): Neophema elegans II B

Elegant Sand Boa (E): Eryx elegans II R

Elegant Scops-Owl (E): Otus elegans II B

Eleonora's Falcon (E): Falco eleonorae II B

Eléphant africain (F): Loxodonta africana $\mathbf{H I I} \mathrm{M}$

Eléphant d'Afrique (F): Loxodonta africana VI M

Eléphant d'Asie (F): Elephas maximus I M

Eléphant de mer du sud (F): Mirounga leonina II M

Eléphant de mer méridional (F): Mirounga leonina II M

Eléphant d'Inde (F): Elephas maximus I M

Elephants (E): Elephantidae spp. III M

Elephant-seals (E): Mirounga spp. II/NC M

Elephas maximus: Asian Elephant (E), Indian Elephant (E), Elefante asiático (S), Eléphant d'Asie (F), Eléphant d'Inde (F) I M

Elf Owl (E): Micrathene whimeyi II B

Elkhom Coral (E): Acropora palmaia II in

Elliot's Pheasant (E): Syrmaticus ellioti I B

Elliptical Star Coral (E): Dichocoenia stellaris II In

Elongated Tortoise (E): Indotestudo elongata II R

Elvira chionura: White-tailed Emerald (E), Colibri elvire (F) II B

Elvira cupreiceps: Coppery-headed Emerald $(\mathrm{E})$, Colibri à tête cuivré (F) II B

Emerald Green Snail (E): Papustyla pulcherrima II In

Emerald Lorikeet (E): Neopsittacus pullicauda II B

Emerald Monitor (E): Varanus prasinus II R

Emerald Poison Frog (E): Epipedobates smaragdinus II A

Emerald Tree Boa (E): Corallus caninus II R

Emerald-bellied Puffleg (E): Eriocnemis alinae II B

Emerald-chinned Hummingbird (E): Abeillia abeillei II B

Emerald-collared Parakeet (E): Psittacula calthropae II B

Emeralds (E): Amazilia spp/Chlorostilbon spp. II B

Émeraude à queue courte (F): Chlorostilbon poortmani II B

Émeraude à queue étroite (F): Chlorostilbon stenura II B

Émeraude alice (F): Chlorostilbon alice II B

Émeraude cuivrée (F): Chlorostilbon russarus II B

Émeraude de Cavinet (F): Chlorostilbon canivesii II B

Émeraude de Gibson (F): Chlorostilbon gibsoni II B

Émeraude de New Providence (F): Chlorostilbon bracei II B

Émeraude de Porto Rico (F): Chlorosilbon maugaeus II B

Émeraude de Ricord (F): Chlorostilbon ricordii II B

Émeraude d'Hispaniola (F): Chlorostilbon swainsonii II B

Émeraude du Panama (F): Chlorostilbon assimilis II B

Émeraude orvert (F): Chlorostilbon mellisugus II B

Émeraude splendide (F): Chlorostilbon aureoventris II B
Émeraudes (F): Chlorostilbon spp. II B

Emerauldine à bec noir (F): Turur abyssinicus III B

Emerauldine à bec rouge (F): Turtur afer III B

Emouchel des Seychelles (F): Falco araea I B

Emperor Bird-of-paradise (E): Paradisaea guilielmi II B

Emperor of Germany's Bird-of-paradise (E): Paradisaea guilielmi II B

Emperor Scorpion (E): Pandinus imperator II In

Emperor Tamarin (E): Saguinus imperator II M

Empress Brilliant (E): Heliodoxa imperatrix II B

Emyde à ocelles de Birmanie (F): Morenia ocellata I R

Emyde indienne à trois carènes $(\mathrm{F})$ : Melanochelys tricarinata $\mathbf{I} \mathrm{R}$

Enallopsammia marenzelleri: II in

Enallopsammia profunda: II in

Enallopsammia pusilla: II In

Enallopsammia rostrata: II in

Endhelia $=$ Crypthelia

Endocyathopora laticostata: II In

Endopachys australiae: II In

Endopachys grayi: II In

Endopsammia philippensis: II In

Endopsammia pounalesi: II in

Enhydra lutris: Sea Onter (E), Nutria del Kamtchatka (S), Nutria marina (S), Loutre de mer (F) I/II M

Enhydra lutris nereis: California Sea Otter (E), Southem Sea Otter (E), Loutre de mer de Californie (F), Loutre de mer méridionale (F) I M

Enicognathus ferrugineus: Austral Conure (E), Austral Parakeet (E), Cotorra austral (S), Cotorra cachaña (S), Conure magellanique (F), Perruche émeraude (F) II B

Enicognathus leptorhynchus: Slender-billed Conure (E), Slenderbilled Parakeet (E), Cotorra choroy (S), Conure à long bec $(\mathbf{F})$, Perruche à bec gracille (F) II B

Ensifera ensifera: Sword-billed Hummingbird (E), Colibri pico espada (S), Colibri porte-épée (F) II B

Entelle (F): Semnopithecus entellus I M

Entelle de Pagi (F): Nasalis concolor I M

Entelle dorée (F): Trachypithecus geei I M

Entelle pileuse (F): Trachypithecus pileatus I M

Entellus Langur (E): Semnopithecus entellus I M

Enygnus asper $=$ Candoia aspere

Enygrus bibroni $=$ Candoia bibroni

Enygrus carinata $=$ Candoia carinata

Eolophus roseicapillus: Galah (E), Roseate Cockatoo (E), Cacatúa rosada ( $S$ ), Cacatoès rosalbin $(F)$ II $B$

Eos bornea: Moluccan Lory (E), Red Lory (E), Lori rojo (S), Lor écarlate (F) II $B$

Eos cyanogenia: Biak Red Lory (E), Black-winged Lory (E), Lori alinegro (S), Lori à joues bleues (F) II B

Eos histrio: Red-and-blue Lory (E), Lori de Sangir (S), Lori arlequin (F) I B

Eos reticulata: Blue-streaked Lory (E), Lori de las Tanimbar (S), Lori réticulé (F) II B

Eos semilarvata: Blue-eared Lory (E), Lori de Seram (S), Lori masqué (F) 11 B

Eos squamata: Moluccan Red Lory (E), Violet-necked Lory (E), Lori escamoso (S), Lori écaillé (F) II B

Epaulard (F): Orcinus orca II M

Epaulard pygmée (F): Feresa atsenuata $11 \mathrm{M}$

Eperonnier chinquis (F): Polyplectron bicalcaratum II B

Éperonnier de Bornéo (F): Polyplectron schleiermacheri II B

Éperonnier de Germain (F): Polyplectron germaini II B

Éperonnier de Hardwick (F): Polyplectron malacense II B

Éperonnier de Malaisie (F): Polypleciron malacense II B

Éperonnier de Palawan (F): Polyplectron emphanum I B

Eperonnier de Rothschild (F): Polypleciron inopinatum III B

Éperonnier gris (F): Polyplectron bicalcaratum II B

Éperonnier malais (F): Polyplectron malacense II B

Éperonnier Napoléon (F): Polyplectron emphanum I B

Éperonniers (F): Polyplectron spp. 1/IIIIINC B

Épervier à collier interrompu (F): Accipiter collaris II B

Épervier à collier roux (F): Accipiter cirrocephalus II B

Épervier à cuisses rouges (F): Accipiser erythronemius II B

Épervier à gorge grise (F): Accipiter enthrauchen II B

Épervier à gorge rayée (F): Accipizer ventralis II B

Épervier à pieds courts (F): Accipiter brevipes II B 
Épervier à poitrine blanche (F): Accipiter chionogaster II B Epervier à poitrine rousse (F): Accipiter rhodogaster II B Êpervier à queue tachetée (F): Accipiter trinotalus II B Epervier besra (F): Accipiter virgatus II B Epervier bicolore (F): Accipiser bicolor II B Épervier brun (F): Accipiter striatus II B Epervier de Cooper (F): Accipiter cooperii II B Épervier de Cuba (F): Accipiter gundlachi II B Epervier de Frances (F): Accipiter francesii II B Epervier de Hartlaub (F): Accipiter enthropus II B Épervier de Horsfield (F): Accipiter soloensis II B Épervier de l'Ovampo (F): Accipiter ovampensis II B

Epervier de Madagascar (F): Accipiter madagascariensis II B Épervier de Nouvelle-Bretagne (F): Accipizer brachyurus II B Épervier des Célèbes (F): Accipiter nanus II B Epervier des Nicobar (F): Accipiter butleri II B Epervier d'Europe (F): Accipiter nisus II B Epervier du Japon (F): Accipiter gularis II B Epervier menu (F): Accipiter rufiventris II B Épervier minule (F): Accipiter minullus II B Épervier nain (F): Accipiter superciliosus II B Épervier pie (F): Accipiter melanoleucus II B Epervier shikra (F): Accipiter badius II B

Eperviers (E): Accipiter spp. II B

Ephippiorhynchus senegalensis: Saddle-billed Stork (E), Saddlebill Stork (E), Cigüeña ensillada (S), Jabirí africano (S), Jabiru africain (F), Jabiru du Sénégal (F) III B

Epicrates angulifer: Cuban Boa (E), Cuban Tree Boa (E), Boa de Cuba (S), Maja de Sta. María (S), Boa de Cuba (F) II R

Epicrates cenchria: Rainbow boa (E), Boa irisada (S), Aboma (F), Boa arc-en-ciel (F) II R

Epicrates chrysogaster: Bahamas Islands Boa (E), Turks Islands Boa (E), Boa de líle Turk (F) II R

Epicrates exsul: Abaco Island Boa (E), Boa de l'île Abaco (F) II R

Epicrates fordii: Ford's Boa (E), Boa de Ford (F) II R

Epicrates gracilis: Haitian Vine Boa (E), Hispaniolan Boa (E), Boa arboricole gracile (F), Boa d'Hispaniola (F) II R

Epicrates inomatus: Puerto Rican Boa (E), Yellow Tree Boa (E) Boa de Puerto Rico (S), Boa de Porto Rico (F), Boa sobre (F) I R

Epicrates inomatus fordii $=$ Epicrates fordii

Epicrates monensis: Mona Island Boa (E), Virgin Islands Tree Boa (E), Boa de líle Mona (F) I R

Epicrates striatus: Fischer's Tree Boa (E), Haitian Boa (E), Boa d'Haìti (F) II R

Epicrates striatus chrysogaster $=$ Epicrates chrysogaster

Epicrates subflavus: Jamaican Boa (E), Yellow Snake (E), Boa de Jamaica (S), Boa de la Jamaique (F) I R

Epimachus albertisi: Black-billed Sicklebill (E), Buff-tailed Sicklebill (E), Drépanornis d'Albertis (F), Paradisier d'Albertis (F) II B

Epimachus bruijnii: Pale-billed Sicklebill (E), White-billed Sicklebill (E), Drépanornis de Bruijn (F), Paradisier à bec blanc (F) 11 B

Epimachus fastuosus: Black Sicklebill (E), Epimaque fastueux (F), Paradisier fastueux (F) II B

Epimachus meyeri: Brown Sicklebill (E), Epimaque de Mayer (F), Paradisier de Meyer (F) II B

Epimaque de Mayer (F): Epimachus meyeri II B

Epimaque fastueux (F): Epimachus fastuosus II B

Epioblasma curtisit: Curtis' Pearly Mussel (E), Curtis' Riffleshell (E) I In

Epioblasma florentina: Yellow Riffleshell (E), Yellow-blossom Pearly Mussel (E) I In

Epioblasma sampsonii: Sampson's Naiad (E), Sampson's Pearly Mussel (E), Sampson's Riffleshell (E), Wabash Riffleshell (E) I In

Epioblasma sulcata perobliqua: White Catspaw (E), White Catspaw Mussel (E) I in

Epioblasma torulosa gubemaculum: Green Riffle Shell (E), Greenblossorn Naiad (E), Green-blossom Pearly Mussel (E) I In

Epioblasma torulosa rangiana: Northern Riffleshell (E), Tanblossom Naiad (E), Tan-blossom Pearly Mussel (E) II In

Epioblasma torulosa torulosa: Tubercled-blossom Pearly Mussel (E), Turberculed Riffle Shell (E) I In

Epioblasma turgidula: Turgid Riffle Shell (E), Turgid-blossom Naiad (E), Turgid-blossom Pearly Mussel (E) I In
Epioblasma walkeri: Brown-blossom Naiad (E), Brown-blossom Pearly Mussel (E), Tan Riffleshell (E) I In

Epipedobate andin (F): Epipedobates andinus II A

Epipedobate au ventre bleu (F): Epipedobates azureiventris II A

Epipedobate bolivien (F): Epipedobates bolivianus II A

Epipedobate brillant (F): Dendrobares speciosus II A

Epipedobate calisterne (F): Epipedobates pulchripectus II A

Epipedobate de Bolivie (F): Epipedobates bolivianus II A

Epipedobate de Boulenger (F): Epipedobates boulengeri II A

Epipedobate de Myers (F): Epipedobates myersi II A

Epipedobate de Peters (F): Epipedobates perersi II A

Epipedobate d'Espinosa (F): Epipedobates espinosai II A

Epipedobate d'Inger (F): Epipedobates ingeri II A

Epipedobate du Rio Palenque (F): Epipedobates erythromos II A

Epipedobate émeraude (F): Epipedobates smaragdinus II A

Epipedobate fémorale (F): Allobates femoralis II A

Epipedobate minime (F): Epipedobates parvulus II A

Epipedobate peint ( $F$ ): Epipedobates pictus II A

Epipedobate simisimi (F): Allobates femoralis II A

Epipedobate tacheté (F): Epipedobates maculatus II A

Epipedobate tricolore (F): Epipedobates iricolor II A

Epipedobate zaparo (F): Epipedobates zaparo 11 A

Epipedobates spp.: Poison frogs (E), Ranas de puntas de flechas (S), Dendrobates $(F)$ II A

Epipedobates andinus: La Planada Poison Frog (E), Rana de punta de flecha andino (S), Epipedobate andin (F) II A

Epipedobates anthonyi $=$ Epipedobates tricolor

Epipedobates azureiventris: Sky-blue Poison Frog (E), Rana de punta de flecha de vientre azul (S), Epipedobate au ventre bleu (F) $11 \mathrm{~A}$

Epipedobates bassleri $=$ Phobobates bassleri

Epipedobates bolivianus: Bolivian Poison Frog (E), Rana de punta de flecha boliviana (S), Epipedobate bolivien (F), Epipedobate de Bolivie (F) II A

Epipedobates boulengeri: Marbled Poison Frog (E), Rana de punta de flecha de Boulenger (S), Epipedobate de Boulenger (F) II A

Epipedobates erythromos: Epipedobate du Rio Palenque (F) II A

Epipedobates espinosai: Espinosa Poison Frog (E), Turquoise-bellied Poison Frog (E), Epipedobate d'Espinosa (F) II A

Epipedobates ingeri: Niceforo's Poison Frog (E), Rana de punta de flecha de Inger (S), Epipedobate d'Inger (F) II A

Epipedobates maculasus: Confusing Poison Frog (S), Rana de punta de flecha maculada (S), Epipedobate tacheté (F) II A

Epipedobates myersi: Myers's Poison Frog (E), Rana de punta de flecha de Myers (S), Epipedobate de Myers (F) II A

Epipedobates parvulus: Ruby Poison Frog (S), Epipedobate minime (F) II A

Epipedobates petersi: Peruvian Poison Frog (E), Rana de punta de flecha de Peters (S), Epipedobate de Peters (F) II A

Epipedobates pictus: Spot-legged Poison Frog (E), Epipedobate peint (F), Rana de punta de flecha picta (F) II A

Epipedobates pulchripectus: Beautiful-breasted Poison Frog (E), Blue-breasted Poison Frog (E), Epipedobate calisterne (F) II A

Epipedobates silverstonei $=$ Phobobates silverstone $i$

Epipedobates smaragdinus: Emerald Poison Frog (E), Rana de punta de flecha esmeralda (S), Epipedobate émeraude (F) II A

Epipedobates tricolor. Phantasmal Poison Frog (E), Rana de punta de flecha tricolor (S), Epipedobate tricolore (F) II A

Epipedobates zaparo: Sanguine Poison Frog (E), Zaparo's Poison Frog (E), Epipedobate zaparo (F) II A

Epixerus ebii: Ebian's Palm Squirrel (E), Temminck's Giant Squirrel (E), Ardilla de palmera (S), Écureuil d'Ebi (F), Écureuil des palmiers (F), Écureuil palmiste (F) III M

Equatorial Saki (E): Pithecia aequatorialis II M

Equidna de Nueva Guinea (S): Zaglossus bruijni II M

Equus africanus: African Ass (E), African Wild Ass (E), Asno salvaje de Africa (S), Ane sauvage d'Afrique (F) IM

Equus asinus = Equus africanus

Equus caballus przewalskii $=$ Equus przewalskii

Equus ferus przewalskii = Equus przewalski

Equus grevyi: Grevy's Zebra (E), Cebra de Grévy (S), Zèbre de Grévy (F) I M

Equus hemionus: Asian Wild Ass (E), Asiatic Wild Ass (E), Asno salvaje asiático (S), Hemiono (S), Ane sauvage d'Asie (F), Hémione (F), Hémippe (F) I/II M 
Equus hemionus hemionus: Driggetai (E), Mongolian Wild Ass (E), North Mongolian Kulan (E), Hemiono mongol (S), Ane sauvage de Mongolie (F), Hémione de IInde (F) I M

Equus hemionus khur $=$ Equus onager khur

Equus hemionus kiang = Equus kiang

Equus hemionus onager $=$ Equus onager

Equus kiang: Kiang (E), Ane sauvage du Tibet (F), Kiang (F) II M

Equus luteus = Equus hemionus hemionus

Equus onager. Kulan (E), Onager Ass (E), Kulang (S), Onagro (S), Onagre (F) III M

Equus onager khur. Ghor-khar (E), Indian Wild Ass (E), Khur (E), Hemiono indostánico (S), Khur (S), Ane sauvage de l'Inde (F) $1 \mathrm{M}$

Equus przewalskii: Mongolian Wild Horse (E), Przewalski's Horse (E), Przewalski's Wild Horse (E), Takh (E), Caballo de Przewalski (S), Caballo salvaje (S), Cheval de Przewalski (F) I $\mathrm{M}$

Equus zebra hartmannae: Hartmann's Mountain Zebra (E), Cebra de Hartmann (S). Zèbre de Hartmann (F), Zèbre de montagne de Hartmann (F) II M

Equus zebra zebra: Cape Mountain Zebra (E), Cebra montanesa del Cabo (S), Zèbre de montagne du Cap (F) I M

Eretmochelys imbricata: Hawksbill Turle (E), Tortuga carey (S), Caret (F), Tortue caret (F), Tortue imbriquée (F), Tortue à bec faucon (F), Tortue à écailles (F) I R

Eriocnemis alinae: Emerald-bellied Puffleg (E), Zamarito pechiblanco (S), Érione d'Aline (F) II B

Eriocnemis cupreoventris: Coppery-bellied Puffleg (E), Colibri pantalón cobrizo (S), Érione à ventre cuivré (F) II B

Eriocnemis derbyi: Black-thighed Puffleg (E), Zamarrito muslinegro (S). Érione de Derby (F) II B

Eriocnemis glaucopoides: Blue-capped Puffleg (E), Picaflor frentivioleta (S), Érione à front bleu (F) II B

Eriocnemis godini: Turquoise-throated Puffleg (E), Zamarrito gorjiturquesa (S), Érione turquoise (F) II B

Eriocnemis luciani: Sapphire-vented Puffleg (E), Zamarnito colilargo (S), Érione Catherine (F) II B

Eriocnemis mirabilis: Colourful Puffleg (E), Érione multicolore (F) II B

Eriocnemis mosquera: Golden-breasted Puffleg (E), Zamarito pechidorado (S), Érione à poitrine d'or (F) II B

Eriocnemis nigrivestis: Black-breasted Puffleg (E), Zamarrito pechinegro (S), Érione à robe noire (F) II B

Eriocnemis vestitus: Glowing Puffleg (E), Colibri pantalón verde (S), Zamarrito luciente (S), Érione pattue (F) II B

Érione à front bleu (F): Eriocnemis glaucopoides II B

Érione à poitrine d'or (F): Eriocnemis mosquera II B

Érione à robe noire (F): Eriocnemis nigrivestis II B

Érione à ventre cuivré (F): Eriocnemis cupreoventris II B

Érione Catherine (F): Eriocnemis luciani II B

Érione d'Aline (F): Eriocnemis alinae II B

Érione d'Aurélie (F): Haplophaedia aureliae II B

Erione de Derby (F): Eriocnemis derbyi II B

Érione givrée (F): Haplophaedia lugens II B

Erione multicolore (F): Eriocnemis mirabilis II B

Erione pattue (F): Eriocnemis vestirus II B

Érione rurquoise (F): Eriocnemis godini II $\mathbf{B}$

Ériones (F): Eriocnemis spp/Haplophaedia spp. II B

Erismature à tête blanche (F): Oxyura leucocephala II B

Ermine (E): Mustela erminea ferghanae III M

Ermitañito barbignís (S): Phaethomis griseogularis II B

Ermitañito gargantifusco (S): Phaethomis squalidus II B

Ermitañito pequeño (S): Phaethomis longuemareus II B

Emitañito rufo (S): Phaethornis ruber II B

Ermitaño barbiblanco (S): Phaethornis hispidus II B

Ermitaño barbudo (S): Threneles ruckeri II B

Ermitano bigotiblanco (S): Phaethornis yaruqui II B

Emitaño bronceado (S): Glaucis aenea II B

Ermitaño carinegro (S): Phaethornis anthophilus II B

Ermitano chico (S): Phaethomis longuemareus II B

Ermitaño colilargo (S): Phaethornis superciliosus II B

Ermitaño de picorrecto (S): Phaethomis bourcieri II B

Ermitaño frentrinegro (S): Phaethornis pretrei II B

Ermitaño guayanés (S): Phaethornis superciliosus II B

Ermitaño manchado (S): Phaethomis eurynome II B

Ermitaño pechicanelo (S): Glaucis hirsuía II B
Ermitaño rojizo (S): Phaethomis nuber II B Ermitaño ventrihabano (S): Phaethomis symatophorus II B Ermitan̄o verde (S): Phaethornis guy II B

Ermite a brins blancs (F): Phaethornis superciliosus II B

Ermite a gorge grise (F): Phaethomis griseogularis II B

Ermite à long bec (F): Phaethomis malaris II B

Ermite à queue blanche (F): Threnetes leucurus II B

Ermite à ventre fauve (F): Phaethomis syrmatophorus II B

Emite anthophile (F): Phaethomis anthophilus II B

Ermite bronzé (F): Glaucis aenea II B

Ermite d'Antonie (F): Threnetes niger $\mathbf{U}$ B

Ermite d'Auguste (F): Phaethomis augusti II B

Emite de Bourcier (F): Phaethornis bourcieri II B

Ermite de Dohm (F): Ramphodon dohmii I B

Ermite de Filippi (F): Phaethomis philippii II B

Ermite de Gounelle (F): Phaethornis gounellei II B

Ermite de Koepcke (F): Phaethornis koepckeae II B

Ermite de Natterer (F): Phaethomis nattereri II B

Ermite de Prêtre (F): Phaethomis pretrei II B

Ermite de Rucker (F): Threnetes ruckeri II B

Ermite de Stuart (F): Phaethornis stuarti II B

Ermite d'Idalie (F): Phaethomis idaliae II B

Ermite d'Osery (F): Phaethornis hispidus II B

Emite eurynome (F): Phaethomis eurynome II B

Ermite hirsute (F): Glaucis hirsuta II B

Ermite nain (F): Phaethornis longuemareus II B

Ermite ocré (F): Phaethornis subochraceus II B

Ermite roussâtre (F): Phaethornis ruber 11 B

Ermite teme (F): Phaethomis squalidus II B

Ermite vert (F): Phacthornis guy II B

Ermite yaruqui (F): Phaethornis yaruqui II B

Ermites (F): Glaucis spp/Phaethomis spp $/$ Threnetes spp. II B

Eroïde (F): Brachyleles arachnoides I M

Errina altispina: II In

Errina antarctica: II In

Errina aspera: II In

Errina atlantica: II In

Errina bicolor: II In

Errina boschmai: II In

Errina capensis: II In

Errina chathamensis: II In

Errina cheilopora: II In

Errina cochleata: II in

Errina cruenta: II in

Errina dabneyi: II In

Errina dendyi: II In

Errina fissurata: II In

Errina gracilis: II In

Errina hicksoni: II In

Errina kerguelensis: II In

Errina Laevigata: II In

Errina laterorifa: II In

Errina macrogastra: II In

Errina novaezelandiae: II In

Errina reticulata: II In

Errina rubra: II in

Errina sinuosa: II In

Errinopora cestoporina: II In

Errinopora cyclopora: II In

Errinopora latifundara: II In

Errinopora nanneca: II In

Errinopora porifera: II In

Errinopora pounalesii: II In

Errinopora stylifera: II In

Errinopora zarhyncha: II In

Errinopsis fenestrata: II in

Errinopsis reticulum: II In

Erymnochelys madagascariensis: Madagascar Big-headed Turtle (E), Madagascar Sideneck Turtle (E), Podocnémide de Madagascar (F) II R

Enthrastrea flabellata: II In

Enthrastrea wellsi: II in

Erythrocebus patas: Patas Monkey (E), Patas (F) II M 
Erythrotriorchis buergersi: Bürger's Sparrowhawk (E), Chestnutmantled Goshawk (E), Chestnut-shouldered Goshawk (E), Chestnut-shouldered Hawk (E), Azor de Bürgers (S), Autour de Bürger (F) II B

Erythrotriorchis radiatus: Red Goshawk (E), Azor rojo (S), Autour rouge (F) $\mathbf{I} \mathrm{B}$

Eryx colubrinus: Kenyan Sand Boa (E), Theban Sand Boa (E), Boa de arena de Egipto (S), Boa des sables couleuvrin (F), Boa des sables d'Egypte (F), Boa des sables du Kenya (F) II R

Eryx conicus: Baby Python (E), Indian Sand Boa (E), Rough-scaled Sand Boa (E), Russell's Sand Boa (E), Boa de arena de Sri Lanka (S), Boa des sables des Indes (F), Boa des sables à écailles rugueuses (F) II $R$

Eryx elegans: Central Asian Sand Boa (E), Elegant Sand Boa (E), Boa des sables d'Asie centrale (F), Boa des sables élégant (F) II $\mathbf{R}$

Eryx jaculus: Caucasian Sand Boa (E), Javelin (E), Spotted Sand Boa (E), Boa jabalina (S), Boa des sables occidental (F), Boa-javelot (F) II R

Eryx jayakari: Arabian Sand Boa (E), Jayakar's Sand Boa (E), Boa des sables d'Arabie (F) II R

Eryx johnii: Blunt-tailed Sand Boa (E), Brown Sand Boa (E), Boa de arena de la India (S), Boa des sables brun (F) II R

Enx loveridgii $=$ Eryx colubrinus

Eryx miliaris: Dwarf Sand Boa (E), Boa des sables miliaire (F), Boa des sables nain (F) II $R$

Eryx muelleri: Müller's Sand Boa (E), Boa de arena de Müller (S), Boa des sables de Müller (F) II R

Eryx somalicus: Somali Sand Boa (E), Boa de arena somalí (S), Boa des sables de Somalie (F) II R

Eryx tataricus: Tartary Sand Boa (E), Boa des sables de Tatarie (F) II R

Eryx whitakeri: Whitaker's Boa (E), Boa de arena de Whitaker (S), Boa des sables de Whitaker (F) II R

Eschrichtius gibbosus $=$ Eschrichtius robustus

Eschrichtius glaucus $=$ Eschrichtius robustus

Eschrichtius robustus: Devil Fish (E), Gray Back (E), Grey Whale (E), Hard Head (E), Mussel Digger (E), Rip Sack (E), Ballena gris $(\mathrm{S})$, Baleine grise $(\mathrm{F})$, Baleine à six bosses $(\mathrm{F})$ I $\mathrm{M}$

Escorpión (S): Heloderma horridum II R

Escorpión de Gambia (S): Pandinus gambiensis II In

Escorpión emperador (S): Pandinus imperator II In

Escorpión gigante (S): Pandinus imperator II In

Escorpión magnifico (S): Pandinus dictator II In

Eskimo Curlew (E): Numenius borealis I B

Eslizón de las Islas Salomón (S): Corucia zebrata II R

Esmeralda bronceada (S): Chlorostilbon russatus II B

Esmeralda cola corta (S): Chlorostilbon poortmani II B

Esmeralda cola de alambre (S): Chlorosiilbon sienura II B

Esmeralda coliazul (S): Chlorostilbon mellisugus II B

Esmeralda coliverde (S): Chlorosilibon alice II B

Esmeralda de Canivet (S): Chlorostilbon caniverii II B

Esmeralda Hondurena (S): Amazilia luciee II B

Esmeralda pico rojo (S): Chlorostilbon gibsoni II B

Esmeralda viente-blanco (S): Amazilia candida II B

Esmeraldas Woodstar (E): Acestrura berlepschi II B

Esmerejón (S): Falco columbarius II B

Espadarte (S): Orcinus orca II M

Esparvero bicolor (S): Accipiter bicolor II B

Esparvero chico (S): Accipiter striatus II B

Esparvero gris (S): Accipiter superciliosus II B

Esparvero pechigrís (S): Accipiter poliogaster II B

Espátula blanca (S): Platalea leucorodia II B

Espátula común (S): Platalea leucorodia $I I$ B

Espermófaga hematina (S): Spermophaga haemarina III B

Espinosa Poison Frog (E): Epipedobates espinosai II A

Espolonero chinquis (S): Polyplectron bicalcaratum II B

Espolonero de Borneo (S): Polyplectron schleiermacheri II B

Espolonero de Germain (S): Polyplectron germaini II B

Espolonero de Palawan (S): Polyplectron emphanum I B

Espolonero de Rothschild (S): Polyplectron inopinatum III B

Espolonero malayo (S): Polypleciron malacense II B

Espulgabueyes (S): Bubulcus ibis III B

Estornino de Rothschild (S): Leucopsar rothschildi I B

Estrella coliblanca (S): Urochroa bougueri II B

Estrella cuellirrojo (S): Acesinura heliodor II B
Estrella ecuatoriana (S): Oreotrochilus chimborazo II B

Estrellita amatista (S): Calliphlox amethystina II B

Estrellita chica (S): Acestrura bombus II B

Estrellita colicorta (S): Myrmia micrura II B

Estrellita de gorguera (S): Acestrura heliodor II B

Estrellita esmeraldeña (S): Acestrura berlepschi II B

Estrellita gargantillada (S): Myrtis fanny II B

Estrellita gorjipurpura (S): Philodice mitchellit II B

Estrellita ventriblanca (S): Acestrura mulsant II B

Estrilda astrild: Common Waxbill (E), Saint Helena Waxbill (E),

Astrilda común (S), Astrild ondulé (F), Senegal ondulé (F) III B

Estrilda bengala $=$ Uraeginshus bengalus

Esirilda caerulescens: Lavender Firefinch (E), Lavender Waxbill

(E), Astrilda ceniza del Senegal (S), Astrild queue-de-vinaigre

(F), Queve de vinaigre (F) III B

Estrilda formosa = Amandava formosa

Estrilda melpoda: Orange-cheeked Waxbill (E), Astrilda carirroja

(S), Astrild à joues oranges (F), Joues-oranges (F) III B

Estrilda subflava $=$ Amandava subflava

Esirilda troglodytes: Black-rumped Waxbill (E), Pink-cheeked

Waxbill (E), Red-eared Waxbill (E), Astrilda culinegra (S),

Astrild cendré (F), Bec de corail cendré (F) III B

Estuarine Crocodile (E): Crocodylus porosus $\mathbf{I I I} \mathbf{R}$

Estuarine Dolphin (E): Sotalia fluviarilis I M

Esturgeon à barbillons frangés (F): Acipenser nudiventris II F

Esturgeon à nez court (F): Acipenser brevirostrum I F

Esturgeon blanc (F): Acipenser transmontanus II F

Esturgeon commun (F): Acipenser sturio I F

Esturgeon de l'Adriatique (F): Acipenser naccarii II F

Esturgeon de l'Atlantique (F): Acipenser oxyrhynchus II F

Esturgeon étoilé (F): Acipenser stellatus II F

Esturgeon jaune (F): Acipenser fulvescens II F

Esturgeon sibérien (F): Acipenser baerii II F

Esturgeon ver (F): Acipenser medirostris II F

Esturgeons (F): ACIPENSERIFORMES spp. I/II F

Esturión barba de flecos (S): Acipenser nudiventris II F

Esturión común (S): Acipenser sturio I F

Esturión del Adriático (S): Acipenser naccarii II F

Esturión del Atlántico (S): Acipenser oxyrhynchus II F

Esturión estrellado (S): Acipenser siellatus II F

Esturión hociquicorto (S): Acipenser brevirostrum I F

Esturión lacustre (S): Acipenser fulvescens II F

Esturións (S): ACIPENSERIFORMES spp. I/II F

Ethiopian toads (E): Altiphrynoides spp. I A/Spinophrynoides spp. I A

Étoumeau de Rothschild (F): Leucopsar rothschildi I B

Étoumeaux (F): Stumidae spp. I/NC B

Eubalaena spp.: Right whales (E), Baleines franches (F), Baleines noires (F) I M

Eubalaena australis: Southem Right Whale (E), Ballena franca (S), Baleine australe (F) I M

Eubalaena glacialis: Black Right Whale (E), Northern Right Whale (E), Right Whale (E), Ballena (S), Ballena franca del Norte (S), Ballenga (S), Baleine de Biscaye (F), Baleine des Basques (F) I M

Eudocimus ruber: Scarlet Ibis (E), Corocoro colorado (S), Corocoro rojo (S), Ibis escarlata (S), Ibis rouge (F) II B

Eugenes fulgens: Magnificent Hummingbird (E), Rivoli's Hummingbird (E), Colibri magnifico (S), Colibri de Rivoli (F) II B

Eulampis holosericeus: Green-throated Carib (E), Colibri falle-vert (F) II B

Eulampis jugularis: Purple-throated Carib (E), Colibri madère (F) II B

Eulemur coronatus: Crowned Lemur (E), Lemur coronado (S), Lémur à couronne (F) I M

Eulemur fulvus: Brown Lemur (E), Lemur leonado (S), Lémur brun (F), Maki brun (F) I M

Eulemur macaco: Black Lemur (E), Lemur negro (S), Lémur macaco (F), Lémur noir (F), Maki noir (F) I M

Eulemur mongoz: Mongoose Lemur (E), Lemur mangosta (S), Lémur mongoz (F), Maki mongoz (F) I M

Eulemur mongoz coronatus = Eulemur coronatus

Eulemur rubriventer: Red-bellied Lemur (E), Lemur de vientre rojo

(S), Lémur à ventre rouge (F) I M

Eulidia yarrelli: Chilean Woodstar (E), Colibri d'Arica (F) II B 
Eumadrepora = Acropora

Eunectes barbouri: Barbour's Anaconda (E), Anaconda de Barbour (F) II R

Eunectes deschauenseei: Dark-spotted Anacaonda (E), Deschauensee's Anaconda (E), Anaconda de Deschauensee (F). Anaconda à taches sombres (F) II R

Eunectes murinus: Anaconda (E), Green Anaconda (E), Water Boa (E), Anaconda (S), Sucury (S), Anaconda (F), Anaconda commun ( $\mathrm{F}$ ), Anaconda vert (F) II $\mathrm{R}$

Eunectes notoeus: Yellow Anaconda (E), Anaconda amarilla (S), Anaconda du Paraguay (F), Anaconda jaune (F) II R

Eungella Gastric-brooding Frog (E): Rheobatrachus vitellinus II A

Eunymphicus comutus: Homed Parakeet (E), Perico comudo (S), Perruche comue (F), Perruche huppé (F) II B

Euodice cantans = Lonchura cantans

Euoticus elegantulus: Westem Needle-clawed Bushbaby (E), Westem Needle-clawed Galago (E). Abolí (S), Gálago elegante (S), Galago élégant (F) II M

Euoticus inustus = Galago matschiei

Euoricus pallidus: Northern Needle-clawed Bushbaby (E), Northern Needle-clawed Galago (E) II M

Eupetomena macroura: Swallow-tailed Hummingbird (E), Colibri hirondelle (F) II $\mathrm{B}$

Eupherusa cyanophnys: Blue-capped Hummingbird (E), Oaxaca Hummingbird (E), Colibrí oaxaqueño (S), Colibri d'Oaxaca (F) II B

Eupherusa eximia: Stripe-tailed Hummingbird (E), Colibrí colirrayado (S), Colibri à épaulettes (F) II B

Eupherusa nigriventris: Black-bellied Hummingbird (E), Colibri à ventre noir (F) II B

Eupherusa poliocerca: White-tailed Hummingbird (E), Colibri guerterense (S), Colibri du Guerrero (F) II B

Euphlyctis hexadactylus: Six-fingered Frog (E), Grenouille du Bengale (F), Grenouille hexadactyle (F) II A

Euphrosyne Dolphin (E): Stenella coeruleoalba II M

Euphyllia ancora: Anchor Coral (E) II in

Euphyllia cristata: II In

Euphyllia divisa: II in

Euphyllia glabrescens: II In

Euphyllia paraancora: II In

Euphyllia paradivisa: II In

Euphyllia paraglabrescens: II in

Euphyllia picteti: Elegance Coral (E), Tooth Coral (E) II In

Euphyllia yoeyamaensis: II In

Euplecte à dos d'or (F): Euplectes macrourus III B

Euplecte franciscain (F): Euplectes franciscanus III B

Euplecte monseigneur (F): Euplectes hordeaceus III B

Euplecte veuve-noire (F): Euplectes ardens III B

Euplecte vorabé (F): Euplectes afer III B

Euplectes (F): Euplectes spp. III/NC B

Euplectes afer: Golden Bishop (E), Napoleon Weaver (E), Yellowcrowned Bishop (E), Euplectes amarillo (S), Euplecte vorabé (F), Vorabé (F) III B

Euplectes alinegro (S): Euplectes hordeaceus III B

Euplectes amarillo (S): Euplectes afer III B

Euplectes ardens: Red-collared Whydah (E), Red-collared Widowbird (E), Falsa viuda pechirroja (S), Euplecte veuve-noire (F), Veuve-noire (F) III B

Euplectes franciscanus: Orange Bishop (E), Orange Weaver (E), Euplectes rojo (S), Euplecte franciscain (F), lgnicolore (F) III B

Euplecies hordeaceus: Black-winged Bishop (E), Fire-crowned Bishop (E), Euplectes alinegro (S), Euplecte monseigneur (F), Monseigneur (F) III B

Euplectes macrourus: Yellow-backed Whydah (E), Yellow-mantled Whydah (E), Yellow-mantled Widowbird (E), Falsa viuda dorsigualda (S), Euplecte à dos d'or (F), Veuve à dos d'or (F) III B

Euplectes orix franciscanus = Euplectes franciscanus

Euplectes rojo (S): Euplectes franciscanus III B

Euplère de Goudot (F): Eupleres goudorii II M

Eupleres goudori: Falanouc (E), Malagasy Mongoose (E), Slender Fanalouc (E), Small-toothed Mongoose (E), Fanaloca (S), Mangosta dentipequeno (S), Euplère de Goudot (F) II M

Eupleres major $=$ Eupleres goudotii
Eupodotis afra: Black Bustard (E), Black Korhaan (E), Sisón negro (S), Sisón negro alioscuro (S), Outarde korhaan (F), Outarde noire (F) II B

Eupodotis afra afraoides $=$ Eupodotis afraoides

Eupodoris afraoides: White-quilled Bustard (E), Sisón negro aliclaro (S), Outarde à miroir blanc (F) II B

Eupodoris bengalensis: Bengal Bustard (E), Bengal Florican (E), Avutarda bengalí (S), Sisón bengali (S), Outarde de l'Inde (F), Outarde du Bengale (F) I B

Eupodotis caerulescens: Blue Bustard (E), Blue Korhaan (E), Sisón azul (S), Sisón azulado (S), Outarde plombée (F) II B

Eupodotis gindiana: Buff-crested Bustard (E), Sisón moñudo etiope (S), Outarde d'Oustalet (F) II B

Eupodoris hartlaubii: Hartlaub's Bustard (E), Sisón de Hartlaub (S), Sisón ventrinegro de Hartaub (S), Outarde de Hartlaub (F) II B

Eupodotis humilis: Little Brown Bustard (E), Sisón somalí (S), Outarde somalienne (F) II B

Eupodoris indica: Lesser Florican (E), Likh (E), Sisón de penacho (S), Sisón indio (S), Outarde naine de l'Inde (F), Outarde passarage (F) II B

Eupodotis melanogaster: Black-bellied Bustard (E), Black-bellied Korhaan (E), Sisón ventrinegro (S), Sisón ventrinegro común (S), Outarde à ventre noir (F) II B

Eupodotis rueppellii: Damara Korhaan (E), Rueppell's Bustard (E), Sisón de Damaraland (S), Sisón de Rüppell (S), Outarde de Rüppell (F) II B

Eupodotis ruficrista: Crested Korhaan (E), Red-crested Bustard (E). Sisón moñirrojo (S), Sisón moñudo austral (S), Outarde houppette (F), Outarde naine (F) II B

Eupodotis ruficrista gindiana = Eupodotis gindiana

Eupodoris savilei: Savile's Bustard (E), Avutarda de Savile (S), Sisón moñudo de saheliano (S), Outarde de Savile (F) II B

Eupodotis senegalensis: Senegal Bustard (E), White-bellied Bustard (E), White-bellied Korhaan (E), Sisón senegalés (S), Outarde du Sénégal (F) II B

Eupodotis vigorsii: Karoo Bustard (E), Karoo Korhaan (E), Vigors's Bustard (E), Sisón de Vigors (S), Sisón del karroo (S), Outarde de Vigors (F) II B

Eupsammia spp.: II In

Eurasian Buzzard (E): Buteo buteo II B

Eurasian Eagle-Ow] (E): Bubo bubo II B

Eurasian Griffon (E): Gyps fulvus II B

Eurasian Griffon Vulture (E): Gyps fulvus II B

Eurasian Hobby (E): Falco subbureo II B

Eurasian Kestrel (E): Falco finnunculus II B

Eurasian Lynx (E): Lynx lynx II M

Eurasian Otter (E): Lutra lutra I M

Eurasian Pygmy-Owl (E); Glaucidium passerinum II B

Eurasian Scops-Owl (E): Orus scops II B

Eurasian Sparrowhawk (E): Accipiter nisus II B

Eurasian Spoonbill (E): Platalea leucorodia II B

Eurasian Wigeon (E): Anas penelope III B

European Chameleon (E): Chamaeleo chamaeleon II R

European Honey-buzzard (E): Pernis apivonus II B

European River Otter (E): Lutra lutra I M

European Sturgeon (E): Huso huso II F

European Turtle-Dove (E): Sirepiopelia turtur III B

Eusmilia aspera: II In

Eusmilia fastigiata: Flower Coral (E) II In

Eusthenotrochus $=$ Sphenotrochus

Euroxeres aquila: White-tipped Sicklebill (E), Pico-de-Hoz puntiblanco (S), Bec-en-faucille aigle (F) II B

Eutoxeres condamini: Buff-tailed Sicklebill (E), Pico-de-Hoz colihabano (S), Bec-en-faucille de la Condamine (F) II B

Eutriorchis astur: Madagascar Serpent-Eagle (E), Culebrera azor (S), Serpentaire de Madagascar (F) II B

Everglade Kite (E): Rostrhamus sociabilis II B

Exiliboa placata: Bogert's Boa (E), Oaxacan Boa (E), Oaxacan Dwarf Boa (E), Boa enana mexicana (S), Boa nain d'Oaxaca (F) II $\mathbf{R}$

Explanaria $=$ Turbinaria

Eyed Dabb Lizard (E): Uromastyx ocellatus II R

Eyelash Palm Pit Viper (E): Bothrops schlegelii III R

Eyelash Viper (E): Bothrops schlegelii III R

Eyra Cat (E): Herpailurus yaguarondi $\mathbf{M I} \mathrm{M}$

Fabo calderón (S): Grampus griseus II M 
Fairies (E): Heliothryx spp. II B

Fairy Lorikeet (E): Charmosyna pulchella II B

Fairy Pitta (E): Pitta nympha II B

Faisan à queue bartée (F): Symaticus humiae I B

Faisan à queue rousse (F): Lophura erythrophthalma III B

Faisán argos (S): Argusianus argus II B

Faisán chir (S): Catreus wallichii I B

Faisán colicanelo (S): Lophura erythrophthalma III B

Faisan de Birmanie (F): Syrmaticus humiae I B

Faisán de caníncula azul (S): Lophura erythrophthalma III B

Faisán de caníncula azul crestado (S): Lophura ignita III B

Faisán de cola ocelada de Germain (S): Polyplectron germaini II B

Faisán de cola ocelada gris (S): Polyplectron bicalcaratum II B

Faisán de cola ocelada indómito (S): Polyplectron inopinatum III B

Faisán de cola ocelada malayo (S): Polyplectron malacense II B

Faisán de Edwards (S): Lophura edwardsi I B

Faisán de Elliot (S): Symaticus ellioti I B

Faisán de Formosa (S): Lophura swinhoii I B

Faisan de Formose (F): Lophura swinhoii I B

Faisan de Hume (F): Symaticus humiae 1 B

Faisán de Hume (S): Syrmaticus humiae I B

Faisan de l'Himalaya (F): Calreus wallichii I B

Faisán de Rheinard (S): Rheinardia ocellata I B

Faisan de Swinhoe (F): Lophura swinhoii I B

Faisán de Swinhoe (S): Lophura swinhoii I B

Faisan de Wallich (F): Catreus wallichii I B

Faisán de Wallich (S): Catreus wallichii I B

Faisan d'Edwards (F): Lophura edwardsi I B

Faisan d'Elliot (F): Syrmaticus ellioti I B

Faisán ensangrentado (S): Tthaginis cruentus II B

Faisan imperial (F): Lophura imperialis I B

Faisán imperial (S): Lophura imperialis I B

Faisan mikado (F): Symaticus mikado I B

Faisán mikado (S): Syrmaticus mikado I B

Faisán monal chino (S): Lophophorus thuysii I B

Faisán monal de Sclater (S): Lophophorus sclaseri I B

Faisán monal del Himalaya (S): Lophophorus impejanus I B

Faisan noble (F): Lophura ignita III B

Faisán noble (S): Lophura ignita III B

Faisan oreillard blanc (F): Crossoptilon crossoptilon I B

Faisan oreillard brun (F): Crossoptilon mantchuricum I B

Faisán orejudo blanco (S): Crossoptilon crossoptilon I B

Faisán orejudo de Manchuria (S): Crossoptilon mantchuricum I B

Faisán orejudo pardo (S): Crossoptilon mantchuricum I B

Faisán orejudo tibetano (S): Crossoptilon crossoptilon I B

Faisán real de Germain (S): Polyplectron gemaini II B

Faisán real de Palaguán (S): Polyplectron emphanum I B

Faisán real gris (S): Polyplectron bicalcarałum II B

Faisán real malayo (S): Polyplectron malacense II B

Faisan sanguin (F): lthaginis cruentus II B

Faisán sanguineo (S): Ithaginis cruentus II B

Faisans (F): Phasianidae spp. I/IIIIINC B

Falanouc (E): Eupleres goudotii II M

Falcatoflabellum raoulensis: II In

Falco alexanderi $=$ Falco innunculus

Falco alopex: Fox Kestrel (E), Cernícalo zorruno (S), Crécerelle renard $(F)$, Faucon-renard (F) II B

Falco altaicus $=$ Falco cherrug

Falco amurensis: Amur Falcon (E), Eastem Red-footed Falcon (E), Manchurian Red-footed Falcon (E), Cernícalo del Amur (S), Faucon de l'Amour (F) II B

Falco araea: Seychelles Kestrel (E), Cemícalo de las Seychelles (S), Crécerelle des Seychelles (F), Crécerelle katitie (F), Emouchel des Seychelles (F), Faucon crécerelle des Seychelles (F) I B

Falco ardosiaceus: Grey Kestrel (E), Cemícalo gris (S), Cemícalo pizarroso (S), Faucon ardoisé (F) II B

Falco berigora: Brown Falcon (E), Halcón berigora (S), Faucon berigora (F) II B

Falco biarmicus: Lanner (E), Lanner Falcon (E), Halcón borní (S), Faucon lanier (F) II B

Falco cenchroides: Australian Kestrel (E), Nankeen Kestrel (E), Cemícalo australiano (S), Crécerelle australienne (F), Crécerelle d'Australie (F) II B

Falco cherrug: Saker (E), Saker Falcon (E), Halcón sacre (S), Faucon sacre (F) II B
Falco chicquera: Red-headed Falcon (E), Red-headed Merlin (E), Red-necked Falcon (E), Alcotán cuellirrojo (S), Alcotán turumt (S), Halcón de cabeza roja (S), Faucon chicquera (F), Faucon à cou roux (F) II B

Falco columbarius: Merlin (E), Pigeon Hawk (E), Esmerejon (S), Halcón migratorio (S), Halcón palomero (S), Faucon émerillon (F) II B

Falco concolor. Sooty Falcon (E), Halcón pardo (S), Halcón pizarroso (S), Faucon concolore (F) II B

Falco cuvieri: African Hobby (E), Alcotán africano (S), Halcón de Cuvier (S), Faucon de Cuvier (F), Hobereau africain (F) II B

Falco deiroleucus: Orange-breasted Falcon (E), Halcón pechianaranjado (S), Halcón pechirrojo (S), Halcón pechirrufo (S), Haicón plomizo (S), Faucon orangé (F) U B

Falco dickinsoni: Dickinson's Kestrel (E), White-rumped Kestrel (E), Cernícalo de Dickinson (S), Cernícalo dorsinegro (S), Faucon de Dickinson (F) II B

Falco eleonorae: Eleonora's Falcon (E), Halcón de Eleonora (S), Faucon d'Eléonore (F) II B

Falco fasciinucha: Taita Falcon (E), Teita Falcon (E), Halcón taita (S), Faucon taita (F) II B

Falco femoralis: Aplomado Falcon (E), Halcón aleto (S), Halcón aplomado (S), Halcón azulado (S), Halcón perdiguero (S), Faucon aplomado (F) II B

Falco hypoleucos: Grey Falcon (E), Halcón gris (S), Halcón gris de Australia (S), Faucon gris (F) II B

Falco jugger. Laggar Falcon (E), Halcón yággar (S), Faucon laggar (F) I B

Falco kreyenborgi $=$ Falco peregrinus

Falco longipennis: Australian Hobby (E), Little Falcon (E), Alcotán australiano (S), Halconcito australiano (S), Petit faucon (F) II B

Falco madens = Falco peregrinus

Falco mexicanus: Prairie Falcon (E), Halcón de las praderas (S), Halcón mejicano (S), Halcón pradeño (S), Faucon des prairies (F) II B

Falco moluccensis: Moluccan Kestrel (E), Spotted Kestrel (E), Cemícalo de las Molucas (S), Cemícalo moluqueño (S), Crécerelle des Moluques (F) II B

Falco naumanni: Lesser Kestrel (E), Cemícalo primilia (S), Faucon crécerellette (F) II B

Falco neglectus $=$ Falco tinnunculus

Folco newtoni: Madagascar Kestrel (E), Newton's Kestrel (E), Cernícalo de Aldabra (S), Cemícalo de Madagascar (S), Crécerelle d'Aldabra (F), Crécerelle malgache (F) I/I B

Falco novaezeelandiae: New Zealand Falcon (E), Halcón de Nueva Zelandia (S), Halcón maori (S), Faucon de Nouvelle-Zélande (F) II $\mathrm{B}$

Falco pelegrinoides: Barbary Falcon (E), Halcón peregrino de Babilonia (S), Faucon de Barbarie (F) I B

Falco peregrinus: Duck Hawk (E), Peregrine (E), Peregrine Falcon (E), Halcón blancuzco (S), Halcón común (S), Halcón peregrino (S), Halcón real (S), Halcón viajero (S), Faucon pèlerin (F) I B

Falco peregrinus babylonicus $=$ Falco pelegrinoides babylonicus

Falco peregrinus pelegrinoides $=$ Falco pelegrinoides

Falco puncratus: Mauritius Kestrel (E), Cemícalo de la Mauricio (S), Crécerelle de Maurice (F), Faucon de l'île Maurice (F) I B

Falco rufigularis: Bat Falcon (E), Halcón golondrina (S), Halcón murcielaguero (S), Halcón plomizo menor (S), Faucon des chauves-souris (F) II B

Falco rupicoloides: Greater Kestrel (E), White-eyed Kestrel (E), Cemícalo ojiblanco (S), Cemícalo ojiblanco de Africa (S), Crécerelle aux yeux blancs (F) II B

Falco rusticolus: Gyr Falcon (E), Gyrfalcon (E), Halcón gerifalte (S), Faucon gerfaut (F), Gerfaut (F) I B

Falco severus: Oriental Hobby (E), Aicotán filipino(S), Faucon aldrovandin (F), Hobereau à poitrine rousse (F) II B

Falco sparverius: American Kestrel (E), Cernicalo americano (S), Halconcito (S), Halconcito común (S), Halcón primito (S), Crécerelle américaine (F), Crécerelle d'Amérique (F) II B

Falco subbuteo: Eurasian Hobby (E), Northem Hobby (E), Alcotán (S), Alcotán europeo (S), Faucon hobereau (F) II B

Falco subniger. Black Falcon (E), Halcón negro (S), Halcón negro de Australia (S), Faucon noir (F) II B

Falco sinnunculus: Common Kestrel (E), Eurasian Kestrel (E), Cernícalo común (S), Cernícalo vulgar (S), Faucon crécerelle (F) II B 
Falco vespertinus: Red-footed Falcon (E), Westem Red-footed Falcon (E), Cemicalo patirrojo (S), Faucon kobez (F) II B

Falco zoniveniris: Banded Kestrel (E), Barred Kestrel (E), Cernicalo malgache (S), Faucon à ventre rayé (F) II B

Falconete acollarado (S): Microhierax caerulescens II B

Falconete de Bomeo (S): Microhierax larifrons II B

Falconete filipino (S): Microhierax erythrogenys II B

Falconete indonesio (S): Microhierax fringillarius II B

Falconete pio (S): Microhierax melanoleucos II B

Falconets (E): Microhierax spp/Spiziapteryx spp. II B

FALCONIFORMES spp.: Birds of prey (E), Raptors (E), Aves de rapiña (S), Rapaces diumes (F) IIIII/NC B

Falcons (E): Falconidae spp. III B

Falsa cobra (S): Cyclagras gigas $11 \mathrm{R}$

Falsa viuda dorsigualda (S): Euplectes macrourus III B

Falsa viuda pechirroja (S): Euplectes ardens III B

False Cobra (E): Cyclagras gigas II R

False Gavial (E): Tomistoma schlegelii I R

False Gharial (E): Tomistoma schlegelii I R

False Girdled Lizard (E): Pseudocordylus capensis II R

False Killer Whale (E): Pseudorca crassidens II M

False Shovelnose Sturgeon (E): Pseudoscaphirhynchus kaufmanni II $\mathbf{F}$

False Swamp Rat (E): Xeromys myoides I M

False Water-rat (E): Xeromys myoides I M

Falso gavial malayo (S): Tomistoma schlegelii I R

Falso lagarto armadillo de Lang (S): Pseudocordylus langi II R

Falso lagarto armadillo del cabo (S): Pseudocordylus capensis II R

Falso lagarto armadillo espinoso (S): Pseudocordylus spinosus II R

Falsos lagartos amadillos (S): Pseudocordylus spp. II R

Fanaloca (S): Eupleres goudotii II M

Fanaloka (E/F): Fossa fossana II M

Fandangero colicuna (S): Campylopterus curvipennis II B

Fandangero colilargo (S): Campylopterus excellens II B

Fandangero morado (S): Campylopterus hemileucurus II B

Fandangero pechiescamosa (S): Phaeochroa cuvierii II B

Fandangero rufo (S): Campylopterus rufus II B

Fan-tailed Day Gecko (E): Phelsuma serraticauda II R

Fasciated Snake-Eagle (E): Circaetus fasciolatus II B

Fat Pocketbook (E): Potamilus capax I In

Fat Pocketbook Pearly Mussel (E): Potamilus capax I In

Fat-tailed Dwarf Lemur (E): Cheirogaleus medius I M

Faucon à cou roux (F): Falco chicquera II B

Faucon à ventre rayé (F): Falco zoniventris II B

Faucon aldrovandin (F): Falco sevenus II B

Faucon aplomado (F): Falco femoralis II B

Faucon ardoisé (F): Falco ardosiaceus II B

Faucon berigora (F): Falco berigora II B

Faucon chicquera (F): Falco chicquera II B

Faucon concolore (F): Falco concolor II B

Faucon crécerelle (F): Falco tinnunculus II B

Faucon crécerelle des Seychelles (F): Falco araea I B

Faucon crécerellette (F): Falco naumanni II B

Faucon de Barbarie (F): Falco pelegrinoides I B

Faucon de Cuvier (F): Falco cuvieri II B

Faucon de Dickinson (F): Falco dickinsoni II B

Faucon de l'ille Maurice (F): Falco punctatus I B

Faucon de l'Amour (F): Falco amurensis II B

Faucon de Nouvelle-Zélande (F): Falco novaezeelandiae II B

Faucon d'Eléonore (F): Falco eleonorae II B

Faucon des chauves-souris (F): Falco rufigularis II B

Faucon des prairies (F): Falco mexicanus II B

Faucon émerillon (F): Falco columbarius II B

Faucon gerfaut $(F)$ : Falco rusticolus I B

Faucon gris (F): Falco hypoleucos II B

Faucon hobereau (F): Falco subbuteo II B

Faucon kobez (F): Falco vespertinus II B

Faucon laggar (F): Falco jugger I B

Faucon lanier (F): Falco biarmicus II B

Faucon noir (F): Falco subniger II B

Faucon orangé (F): Falco deiroleucus II B

Faucon pelerin (F): Falco peregrinus I B

Faucon sacre (F): Falco cherrug II B

Faucon taita (F): Falco fasciinucha II B

Faucon-coucou (F): Aviceda cuculoides II B

Fauconnet à collier (F): Microhierax caerulescens II B
Fauconnet à pattes jaunes (F): Polihierax insignis II B Fauconnet d'Afrique (F): Polihierax semilorquatus II B Fauconnet d'Amérique (F): Spiziapteryx circumcincrus II B Fauconnet de Boméo (F): Microhierax latifrons II B Fauconnet des Philippines (F): Microhierax eryihrogenys II B Fauconnet moineau (F): Microhierax fringillarius II B Fauconnet noir-et-blanc (F): Microhierax melanoleucos II B Fauconnets (F): Microhierax spp/Polihierax spp. II B Faucon-renard (F): Falco alopex II B Faucons (F): Falco spp. III B

Fausse souris de la baie de Shark (F): Pseudomys praeconis I M Fauvette a long bec $(\mathrm{F})$ : Dasyomis longirosiris I B

Fauvette rousse de l'Ouest (F): Dasyornis broadbenti litoralis I B Faux cobra (F): Cyclagras gigas II $\mathbf{R}$

Faux gavial malais (F): Tomistoma schlegelii I R

Faux jacquot (F): Corallus caninus II $R$

Faux rat d'eau (F): Xeromys myoides I $M$

Faux-gavial d'Afrique (F): Crocodylus cataphracrus I R

Faux-orque (F): Pseudorca crassidens II M

Favia affinis: II In

Favia danae: II In

Favia danai: II In

Favia favus: II in

Favia fragum: Golfball Coral (E) II In

Favia gravida: II In

Favia helianthoides: 11 In

Favia laxa: II In

Favia leptophylla: II In

Favia lizardensis: II In

Favia lylei: II In

Favia maritima: II In

Favia manhaii: II In

Favia maxima: II In

Favia pallida: II In

Favia rotulosa: II In

Favia rotumana: II In

Favia rolundata: II In

Favia speciosa: II In

Favia stelligera: II In

Favia valenciennesii: II In

Favia veroni: II In

Favia whitfieldi: II In

Favia wisseli: II in

Favites abdita: Honeycomb Coral (E) II In

Favites chinensis: II in

Favires complanata: II In

Favites flexuosa: II In

Favites halicora: II In

Favires melicerum: II In

Favites pentagona: II In

Favites peresi: II In

Favites polarensis: II In

Favites rufa: II In

Favites russelli: II in

Favites snlifera: II In

Favositopora $=$ Alveopora

Fawn-breasted Brilliant (E): Heliodoxa rubinoides II B

Fawn-breasted Hummingbird (E): Amazilia yucalanensis II B

Fearful OwI (E): Nesasio solomonensis II B

Feather-toed Hawk-Eagle (E): Spizaetus nipalensis II B

Feick's Dwarf Boa (E): Tropidophis feicki II R

Felidae spp.: Cats (E), Felinos (S), Félidés (F), Félins (F) I/I M

Félidés (F): Felidae spp. VII M

Felinos (S): Felidae spp. I/II M

Félins (F): Felidae spp. III M

Felis aurata $=$ Profelis aurata

Felis badia $=$ Catopuma badia

Felis bengalensis = Prionailurus bengalensis

Felis bengalensis bengalensis = Prionailurus bengalensis bengalensis

Felis bieti: Chinese Desert Cat (E), Gato de Biet (S), Gato del desierto de China (S), Chat de Biet (F) II M

Felis canadensis $=$ Lynx canadensis

Felis caracal = Caracal caracal 
Felis chaus: Jungle Cat (E), Reed Cat (E), Swamp Cat (E), Gato de la jungla (S), Gato de los pantanos (S), Chat de jungle (F), Chat des marais (F) $11 \mathrm{M}$

Felis colocolo $=$ Oncifelis colocolo

Felis colocolo budinii = Oncifelis colocolo budinii

Felis colocolo crespoi $=$ Oncifelis colocolo crespoi

Felis colocolo pajeros = Oncifelis colocolo pajeros

Felis concolor $=$ Puma concolor

Felis concolor coryi $=$ Puma concolor coryi

Felis concolor costaricensis $=$ Puma concolor costaricensis

Felis concolor couguar $=$ Puma concolor couguar

Felis geoffroyi $=$ Oncifelis geoffroyi

Felis guigna = Oncifelis guigna

Felis iriomotensis = Prionailurus bengalensis

Felis jacobita $=$ Oreailurus jacobira

Felis lybica $=$ Felis silvestris

Felis lynx $=$ Lynx lynx

Felis lynx isabellina $=$ Lynx lynx isabellinus

Felis lynx pardinus = Lynx pardinus

Felis manul = Otocolobus manul

Felis margarita: Sand Cat (E), Sand Dune Cat (E), Gato de las arenas (S), Gato del Sahara (S). Chat des sables (F) I M

Felis marmorata $=$ Pardofelis marmorata

Felis nigripes: Black-footed Cat (E), Small-spotted Cat (E), Gato de pies negros (S), Gato patinegro (S), Chat à pieds noirs (F) I M

Felis ormata $=$ Felis silvestris

Felis pardalis = Leopardus pardalis

Felis pardina $=$ Lynx pardinus

Felis planiceps $=$ Prionailurus planiceps

Felis rubiginosa $=$ Prionailurus rubiginosus

Felis rufa = Lynx rufus

Felis serval = Leptailurus serval

Felis silvestris: Wild Cat $(\mathrm{E})$, Wildcat $(\mathrm{E})$, Gato montés ( $\mathrm{S})$, Gato silvestre (S), Chat orné (F), Chat sauvage (F) II M

Felis temminckii $=$ Catopuma temmincki

Felis tigrina $=$ Leopardus tigrinus

Felis viverrina $=$ Prionailurus viverrinus

Felis wiedii = Leopardus wiedi

Felis yaguarondi $=$ Herpailunus yaguarond $i$

Fenicoptéridos (S): Phoenicopteridae spp. II B

Fennec (S/F): Vulpes zerda II M

Fennec Fox (E): Vulpes zerda II M

Fennecus zerda $=$ Vulpes zerda

Feresa attenuata: Pygmy Killer Whale (E), Slender Blackfish (E),

Orca pigmeo (S), Epaulard pygmée (F), Orque pygmée (F) II M

Feresa occulta $=$ Feresa attenuata

Ferrets (E): Mustela spp. I/NC M

Ferruginous Duck (E): Aythya nyroca III B

Ferruginous Hawk (E): Buteo regalis II B

Ferruginous Partridge (E): Caloperdix oculea III B

Ferruginous Pochard (E): Aythya nyroca III B

Ferruginous Pygmy-Owl (E): Glaucidium brasilianum II B

Ferruginous Wood-Partridge (E): Caloperdix oculea III B

Festive Amazon (E): Amazona festiva II B

Festive Coquette (E): Lophomis chalybeus II B

Festive Parrot (E): Amazona festiva II B

Fielden's Falconet (E): Polihierax insignis II B

Fiery Topaz (E): Topaza pyra II B

Fiery-shouldered Conure (E): Pyrrhura egregia II B

Fiery-shouldered Parakeet (E): Pyrrhura egregia II B

Fiery-tailed Awlbill (E): Avocettula recunvirostris II B

Fiery-throated Hummingbird (E): Panterpe insignis $11 \mathrm{~B}$

Fig-Parrots (E): Cyclopsitta spp/Psilfaculirostris spp. I/I B

Fiji Banded Iguana (E): Brachylophus fasciatus I R

Fiji Crested Iguana (E): Brachylophus vitiensis I R

Fiji Goshawk (E): Accipiter rufitorques II B

Fijian iguanas (E): Brachylophus spp. I R

Fin Whale (E): Balaenoptera physalus I M

Finback (E): Balaenopiera physalus I M

Fin-backed Whale (E): Balaenoptera physalus I M

Finches (E): Emberizidae spp./Estrildidae spp/Fringillidae spp. IIIIUNC B

Fine-rayed Pigtoe (E): Fusconaia cuneolus I In

Fine-rayed Pigtoe Pearly Mussel (E): Fusconaia cuneolus I in

Finless Black Porpoise (E): Neophocaena phocaenoides I M

Finless Porpoise (E): Neophocaena phocaenoides I M
Finner (E): Balaenoptera physalus I M

Finsch's Conure (E): Aratinga finschi II B

Finsch's Pygmy-Parrot (E): Micropsitta finschii II B

Fire corals (E): Milleporidae spp. II In

Firebacks (E): Lophura spp. III/NC B

Fire-crowned Bishop (E): Euplectes hordeaceus III B

Firecrowns (E): Sephanoides spp. II B

Firefinches (E): Lagonosticta spp. III/NC B

Fire-throated Metaltail (E): Metallura eupogon II B

Fischer's Chameleon (E): Bradypodion fischeri II R

Fischer's Lovebird (E): Agapomis fischeri II B

Fischer's Tree Boa (E): Epicrates striatus II R

Fischer's Turaco (E): Tauraco fischeri II B

Fish Lizard (E): Varanus dumerilii II R

Fish-Eagles (E): Haliaeetus spp//chthyophaga spp. III B

Fish-eating Crocodile (E): Gavialis gangeticus I R

Fishing Cat (E): Prionailurus viverrinus II M

Fishing Snake (E): Xenochrophis piscator III R

Fishing-Owls (E): Scotopelia spp. II B

Fish-Owls (E): Ketupa spp. II B

Fissicella = Favio

Flabellum alabastrum: II In

Flabellum angiostomum: II in

Flabellum angulare: II in

Flabellum aolearoa: II in

Flabellum apertum: II in

Flabellum areum: II in

Flabellum atlanticum: II in

Flabellum australe: II in

Flabellum campanulatum: II in

Flabellum chunii: II In

Flabellum conuis: II In

Flabellum cunatum: II In

Flabellum daphnense: II In

Flabellum deludens: II In

Flabellum dens = Truncatoflabellum dens

Flabellum flexuosum: II In

Flabellum fragile: II In

Flabellum gardineri: II In

Flabellum hoffmeisieri: II in

Flabellum impensum: II In

Flabellum japonicum: II in

Flabellum knoxi: II In

Flabellum lamellulosum: II In

Flabellum lowekeyesei: II In

Flabellum macandrewi: II in

Flabellum magnificum: II In

Flabellum marcus: II In

Flabellum marenzelleri. II In

Flabellum messum: II in

Flabellum moseleyi: II in

Flabellum ongulense: II In

Flabellum patens: II In

Flabellum pavoninum: II In

Flabellum planus: II In

Flabellum politum: II In

Flabellum raukawaensis: II In

Flabellum sexcostatum: II In

Flabellum sibogae: II in

Flabellum thouarsii: II In

Flabellum transversale: II In

Flabellum futhilli: II In

Flabellum vaughani: II In

Flamant de Cuba (F): Phoenicopterus ruber II B

Flamant de James (F): Phoenicopterus jamesi II B

Flamant des Andes (F): Phoenicopterus andinus II B

Flamant du Chili (F): Phoenicopterus chilensis II B

Flamant nain (F): Phoenicopterus minor II B

Flamant rose (F): Phoenicoptenus nuber II B

Flamant rouge (F): Phoenicopterus ruber II B

Flamants (F): Phoenicopteridae spp. II B

Flame-knee Tarantula (E): Brachypelma auratum II In

Flamenco (S): Phoenicopterus ruber II B

Flamenco andino (S): Phoenicopterus andinus II B

Flamenco andino chico (S): Phoenicopterus jamesi II B

Flamenco chileno (S): Phoenicopterus chilensis II B 
Flamenco común (S): Phoenicopterus ruber II B Flamenco común de Chile (S): Phoenicopterus chilensis II B Flamenco de Cuba (S): Phoenicopterus ruber II B Flamenco de James (S): Phoenicopterus jamesi II B Flamenco enano (S): Phoenicopterus minor II B Flamenco rojo (S): Phoenicopterus ruber II B Flamencos (S): Phoenicopteridae spp. II B Flame-rumped Sapphire (E): Hylocharis pyropygia II B Flame-winged Conure (E): Pyrrhura calliptera II B Flame-winged Parakeet (E): Pyrrhura calliptera II B Flaming Poison Frog (E): Dendrobates pumilio II A Flamingos (E): Phoenicopteridae spp. II B Flammulated Owl (E): Otus flammeolus II B Flap-necked Chameleon (E): Chamaeleo dilepis II R Flatback (E): Natator depressus I R

Flatback Tortoise (E): Geochelone platynora II R

Flat-backed Spider Tontoise (E): Pyxis planicauda II R

Flat-casqued Chameleon (E): Chamaeleo globifer II R

Flat-headed Cat (E): Prionailurus planiceps I M

Flat-shelled Spider Tortoise (E): Pyxis planicauda II R

Flat-tailed Day Gecko (E): Phelsuma laticauda II R

Flores Hanging-Parrot (E): Loriculus flosculus II B

Floricans (E): Eupodotis spp. I/II B

Florida Cougar (E): Puma concolor coryi I M

Florida Golden Chestnut (E): Brachypelma aureoceps II In

Florida Panther (E): Puma concolor coryi I M

Florida Puma (E): Puma concolor coryi I M

Florisuga mellivora: White-necked Jacobin (E), Colibrí nuca blanca (S), Jacobino collarejo (S), Jacobino nuquiblanco (S), Colibri jacobin (F) II B

Flower Coral (E): Eusmilia fastigiata II In

Flower Lizard (E): Varanus indicus II R

Fluted Clam (E): Tridacna squamosa II In

Fluted Giant Clam (E): Tridacna squamosa II in

Flying squirrels (E): Sciuridae: Petauristinae spp. III/NC M

Flying-foxes (E): Acerodon spp./Pteropus spp./Pteralopex spp. ИINC M

Foca fraile de Hawaii (S): Monachus schauinslandi I M

Foca fraile del Caribe (S): Monachus tropicalis I M

Focas fraile (S): Monachus spp. I M

Focas monje (S): Monachus spp. I M

Folioseris $=$ Leptoseris

Forbes's Kakariki (E): Cyanoramphus auriceps forbesi I B

Forbes's Parakeet (E): Cyanoramphus auriceps forbesi I B

Ford's Boa (E): Epicrates fordii II R

Forest Buzzard (E): Buteo oreophilus II B

Forest Eagle-Owl (E): Bubo nipalensis II B

Forest Fox (E): Cerdocyon thous II M

Forest Hinged Tortoise (E): Kinixys erosa II R

Forest Little Owl (E): Athene blewitti I B

Forest Musk Deer (E): Moschus berezovskii II M

Forest Owlet (E): Athene blewirti I B

Forest Spotted Owlet (E): Athene blewitti I B

Forest Tortoise (E): Geochelone denticulata II R

Forest-Falcons (E): Micrastur spp. II B

Forked Chameleon (E): Chamaeleo furcifer II R

Fork-marked Lemur (E): Phaner furcifer I M

Fork-marked Mouse-lemur (E): Phaner furcifer I M

Fork-nosed Chameleon (E): Chamaeleo furcifer II R

Fork-tailed Emerald (E): Chlorostilbon canivetii II B

Fork-tailed Woodnymph (E): Thalurania furcata II B

Formosan Rock Macaque (E): Macaca cyclopis II M

Forpus coelestis: Celestial Parrotlet (E), Pacific Parrotlet (E), Cotorrita de Piura (S), Periquito del Pacífico (S), Toui céleste (F) II B

Forpus conspicillatus: Spectacled Parrotet (E), Cotorrita de Anteojos (S), Periquito ojiazul (S), Toui à lunettes (F) II B

Forpus cyanopygius: Blue-rumped Parrotlet (E), Mexican Parrotlet (E), Cotorrita Mexicana (S), Periquito mexicano (S), Toui du Mexique (F) II B

Forpus passerinus: Green-rumped Parrotlet (E), Cotorrita culiverde (S), Periquito (S), Perruche aux ailes bleues (F), Toui été (F) II B

Forpus sclateri: Dusky-billed Parrotlet (E), Sclater's Parrotlet (E), Cotorrita de Sclater (S), Periquito obscuro (S), Toui de Sclater (F) II B
Forpus xanthops: Yellow-faced Partotlet (E), Cotorrita carigualda

(S), Toui à tête jaune (F) II B

Forpus xanthopterygius: Blue-winged Parrotlet (E), Catita enana (S),

Cotorrita aliazul (S), Periquito aliazul (S), Toui de Spix (F) II B

Forsten's Tortoise (E): Indotestudo forstenii II R

Forster's Caracara (E): Phalcoboenus australis II B

Fossa (E/F): Cryptoprocta ferox II M

Fossa fossa $=$ Fossa fossana

Fossa fossana: Fanaloka (E), Malagasy Civet (E), Striped Civet (E),

Cibeta de Madagascar (S), Civette fossane (F), Civette malgache (F), Fanaloka (F), Fossana (F) II M

Fossana (F): Fossa fossana II M

Fou d'Abbott (F): Papasula abbotti I B

Fouette-queue d'Egypte (F): Uromastyx aegyptius II R

Fouette-queue d'Iran (F): Uromastyx asmussi II R

Fouette-queue d'O'Shaughnessy (F): Uromastyx princeps II R

Fouette-queue de Bent (F): Uromastyx benti II R

Fouette-queue de Hardwick (F): Uromastyx hardwickii II R

Fouette-queue de Mésopotamie (F): Uromastyx loricatus II R

Fouette-queue de Somalie (F): Uromastyx macfadyeni II R

Fouette-queue de Thomas (F): Uromastyx thomasi II R

Fouette-queue du Sahara (F): Uromastyx geyri II R

Fouette-queue épineux (F): Uromastyx acanthinurus II R

Fouette-queue indien (F): Uromastyx hardwickii II R

Fouette-queue ocellé (F): Uromastyx ocellatus II R

Fouette-queue princier (F): Uromastyx princeps II R

Fouettes-queue (F): Uromasnyx spp. II R

Fouine (F): Martes foina intermedia III M

Four-fool Caiman (E): Dracaena guianensis II R

Four-homed Antelope (E): Tetracerus quadricomis $111 \mathrm{M}$

Four-homed Chameleon (E): Chamaeleo quadricomis II R

Four-spot Day Gecko (E): Phelsuma quadriocellata II R

Four-toed Terrapin (E): Baragur baska I R

Four-toed Tortoise (E): Testudo horsfieldii II R

Fous (F): Sula spp. 1/NC B

Foussa (F): Cryptoprocta ferax II M

Fox Kestrel (E): Falco alopex II B

Foxes (E): Canidae spp. II/IILNC M

Fragile Saucer Coral (E): Agaricia fragilis II In

Fragilocyathus $=$ Aulocyathus

Frances's Goshawk (E): Accipiter francesii II B

Franciscana (E): Pontoporia blainvillei II M

François's Langur (E): Trachypithecus francoisi II M

François's Leaf Monkey (E): Trachypithecus francoisi II M

Fraser's Dolphin (E): Lagenodelphis hosei II M

Fraser's Eagle-Owl (E): Bubo poensis II B

Freckled Monitor (E): Varanus tristis II R

Fregata andrewsi: Andrews' Frigatebird (E), Christmas Island

Frigatebird (E), Rabihorcado de la Christmas (S), Rabihorcado

ventriblanco (S), Frégate d'Andrews (F), Frégate de l'ile

Christmas (F) I B

Frégate d'Andrews (F): Fregata andrewsi I B

Frégate de l'île Christmas (F): Fregata andrewsi I B

Frégates (F): Fregata spp. INC B

Frentiestrella alihabano (S): Coeligena lutetiae II B

Frentiestrella arcoiris (S): Coeligena iris II B

Frentijoya (S): Heliodoxa aurescens II B

Frigatebirds (E): Fregata spp. I/NC B

Frilled Coquette (E): Lophornis magnificus II B

Fringebarbel Sturgeon (E): Acipenser nudiventris II F

Frogs (E): ANURA spp. I/IUNC A

Fruit bats (E): Pteropodidae spp. I/II/NC M

Fuertes's Parrot (E): Hapalopsittaca fuertesi II B

Fuligule nyroca (F): Aythya nyroca III B

Fulvous Owl (E): Strix fulvescens II B

Fulvous Tree-Duck (E): Dendrocygna bicolor III B

Fulvous Whistling-Duck (E): Dendrocygna bicolor III B

Fungia concinna: II In

Fungia cossulata: II In

Fungia curvata: II in

Fungia cyclolites: II In

Fungia distorta: II In

Fungia fragilis: II In

Fungia fralinae: II In

Fungia fungites: Mushroom Coral (E) II in

Fungia granulosa: II In 
Fungia gravis: II In

Fungia hexagonalis: II in

Fungia horrida: II In

Fungia margaretae: II in

Fungia moluccensis: II In

Fungia paumotensis: II In

Fungia repanda: II In

Fungia scabra: II In

Fungia scruposa: II in

Fungia scutaria: II In

Fungia seychellensis: II ln

Fungia sinensis: II In

Fungia somervillei: II In

Fungia spinifer: II In

Fungia spp.: Coraux champignon (F) II In

Fungia taiwanensis: II In

Fungia tenuis: II In

Fungia vaughani: II in

Fungiacyathus aleuricus = Fungiacyathus symmetricus

Fungiacyathus crispus: II In

Fungiacyathus dennani: II In

Fungiacyathus durus $=$ Fungiacyathus symmetricus

Fungiacyathus fissilis: II In

Fungiacyathus fragilis = Fungiacyathus symmetricus

Fungiacyathus granulosus: II In

Fungiacyathus hydra: II in

Fungiacyathus marenzelleri: II In

Fungiacyathus paliferus: II In

Fungiacyathus pliciseptus: II In

Fungiacyathus pseudostephanus: II In

Fungiacyathus pusillus: 11 In

Fungiacyathus sibogae: II In

Fungiacyathus stephanus: II In

Fungiacyathus symmetricus: II In

Fungiacyathus turbinolioides: II In

Fungiacyathus variegalus: II In

Fur seals (E): Arcrocephalus spp/Callorhinus ursinus $\mathrm{MLNC}$ M

Furcifer angeli $=$ Chamaeleo angeli

Furcifer antimena $=$ Chamaeleo antimena

Furcifer balieatus $=$ Chamaeleo balteatus

Furcifer belalandaensis = Chamaeleo belalandaensis

Furcifer bifidus $=$ Chamaeleo bifidus

Furcifer campani = Chamaeleo campani

Furcifer cephalolepis = Chamaeleo cephalolepis

Furcifer labordi = Chamaeleo labordi

Furcifer lateralis $=$ Chamaeleo lateralis

Furcifer minor $=$ Chamaeleo minor

Furcifer monoceras = Chamaeleo monoceras

Furcifer oustalet $=$ Chamaeleo oustaleti

Furcifer pardalis = Chamaeleo pardalis

Furcifer petteri $=$ Chamaeleo petteri

Furcifer polleni $=$ Chamaeleo polleni

Furcifer rhinoceratus = Chamaeleo rhinoceratus

Furcifer tuzetae $=$ Chamaeleo luzetae

Furcifer verrucosus = Chamaeleo verrucosus

Furcifer willsii = Chamaeleo willsii

Fusconaia cuneolus: Fine-rayed Pigtoe (E), Fine-rayed Pigtoe Pearly Mussel (E) I In

Fusconaia edgariana: Shiny Pigtoe (E), Shiny Pigtoe Pearly Mussel (E) I ln

Fused Staghorn Coral (E): Acropora prolifera II in

Gabar Goshawk (E): Melierax gabar II B

Gabon Terrapin (E): Pelusios gabonensis III R

Gabon Turtle (E): Pelusios gabonensis III R

Gacela de Irán (S): Dama mesopotamica I M

Gafudo gorgiblanco (S): Zosierops albogularis I B

Galago à queue touffue (F): Otolemur crassicaudatus II M

Galago alleni: Allen's Bushbaby (E), Allen's Galago (E), Gálago de Allen (S), Galago d'Allen (F) II M

Galago d'Allen (F): Galago alleni II M

Gálago de Allen (S): Galago alleni II M

Gálago de cola gruesa (S): Otolemur crassicaudatus II M

Galago de Demidoff (F): Galagoides demidoff II M

Gálago de Senegal (S): Galago senegalensis II M

Gálago de Zanzibar (S): Galagoides zanzibaricus II M

Galago demidovii $=$ Galagoides demidoff
Galago du Congo (F): Galago matschiei II M

Galago du Sénégal (F): Galago senegalensis II M

Galago élégant (F): Euoticus ele gantulus II M

Gálago elegante (S): Euoticus eleganiulus II M

Galago ele gantulus = Euoricus elegantulus

Gálago enano (S): Galagoides demidoff II M

Gálago etíope (S): Galago gallarum II M

Galago gallarum: Somali Galago (E), Gálago etíope (S) II M

Galago inustus = Galago matschie

Galago marschiei: Eastem Needle-clawed Bushbaby (E), Eastem Needle-clawed Galago (E), Lesser Needle-clawed Galago (E), Galago du Congo (F) II M

Galago moholi: Mohol's Galago (E) II M

Galago senegalensis: Lesser Bushbaby (E), Lesser Galago (E), Senegal Galago (E), Gálago de Senegal (S), Galago du Sénégal (F) II M

Galago senegalensis gallarum = Galago gallarum

Galago senegalensis moholi $=$ Galaga moholi

Galago senegalensis zanzibaricus = Galagoides zanzibaricus

Galago zanzibaricus $=$ Galagoides zanzibaricus

Galagoides demidoff: Demidoff's Dwarf Galago (E), Demidoff's Galago (E), Dwarf Bushbaby (E), Dwarf Galago (E), Gálago enano (S), Galago de Demidoff (F) II M

Galagoides granti $=$ Galagoides zanzibaricus

Galagoides orinus $=$ Galagoides demidoff

Galagoides thomasi $=$ Galagoides demidoff

Galagoides zanzibaricus: Zanzibar Bushbaby (E), Zanzibar Galago (E), Gálago de Zanzíbar (S) II M

Galagos (E): Galagonidae spp. II M

Galah (E): Eolophus roseicapillus II B

Galápago Batagur (S): Batagur baska I R

Galápago caja Mexicano (S): Terrapene coahuila I R

Galápago conchiblando del Ganges (S): Trionyx gangeticus I R

Galápago conchiblando negro (S): Trionyx ater I R

Galápago conchiblando ocelado (S): Trionyx hurum I R

Galápago conchiblando oscuro (S): Trionyx nigricans I R

Galápago de Muhlenberg (S): Clemmys muhlenbergi I R

Galápago ocelado de Birmania (S): Morenia ocellata I R

Galápago rayado (S): Geoclemys hamiltonii I R

Galápago terrestre (S): Melanochelys tricarinata I R

Galápagos cajas (S): Terrapene spp. I/I R

Galápagos Fur Seal (E): Arctocephalus galapagoensis II M

Galapagos Giant Tortoise (E): Geochelone nigra I R

Galapagos Hawk (E): Buteo galapagoensis II B

Galapagos Islands Fur Seal (E): Arctocephalus galapagoensis II M

Galapagos Land Iguana (E): Conolophus subcristatus II R

Galapagos Marine Iguana (E): Amblyrhynchus cristatus II R

Galarea alia: II In

Galarea astreata: II in

Galaxea fascicularis: II in

Galarea lauensis: II In

Galarea paucisepta: II In

Galictis allamandi $=$ Galictis vitrata

Galictis vittata: Greater Grison (E), Grisón (S), Hurón (S), Hurón

grange (S), Tejón (S), Grison d'Allemand (F) III M

Gallicolombe poignardée (F): Gallicolumba luzonica II B

Gallicolombes (F): Gallicolumba spp. II/NC B

Gallicolumba luzonica: Bleeding Heart Dove (E), Bleeding Heart

Pigeon (E), Luzon Bleeding-hear (E), Paloma apuñalada de

Luzón (S), Colombe poignardée (F), Gallicolombe poignardée

(F) II B

Gallina de palo (S): Iguana iguana II R

Gallinazo rey (S): Sarcoramphus papa III B

Gallirallus sylvestris: Lord Howe Island Woodhen (E), Lord Howe

Rail (E), Rascón de Isla Lord Howe (S), Râle de l'île Lord Howe

(F), Râle sylvestre (F) I B

Gallirex porphyreolophus = Musophaga porphyreolopha

Gallito de las Rocas (S): Rupicola rupicola II B

Gallito de las Sierras (S): Rupicola peruviana II B

Galkito de pradera (S): Tympanuchus cupido attwateri 1 B

Gallito de roca anaranjado (S): Rupicola rupicola II B

Gallito de roca peruano (S): Rupicola peruviana II B

Gallitos de las rocas (S): Rupicola spp. II B

Gallo de Sonnerat (S): Gallus sonneratii II B

Gallo gris (S): Gallus sonneratii II B

Gallo salvaje del Caspio (S): Tetraogallus caspius I B 
Gallo salvaje del Tibet (S): Tetraogallus tibetanus I B

Galloria simonyi: Hierro Giant Lizard (E), Lézard de Simony (F) I R Gallus sonnerati: Grey Junglefowl (E), Sonneraf's Junglefowl (E), Gallo de Sonnerat (S), Gallo gris (S), Coq de Sonnerat (F) II B Gampsonyx swainsonii: Pearl Kite (E), Cemícalo (S), Elanio chico (S), Elanio enano (S), Elanion perle (F) II B

Gamuza de los Abruzzos (S): Rupicapra pyrenaica ormasa I M

Gan-Gan (S): Callocephalon fimbriatum II B

Ganges River Dolphin (E): Platanista gangetica I M

Ganges Soft-shell Turtle (E): Trionyx gangeticus I R

Ganges Susu (E): Platanista gangetica I M

Gang-gang Cockatoo (E): Callocephalon fimbriatum II B

Ganso blanco (S): Coscoroba coscoroba II B

Ganso canadiense aleutiana (S): Branta canadensis leucopareia I B

Ganso de Gambia (S): Plectropterus gambensis III B

Ganso del Nilo (S): Alopochen aegyptiacus III B

Ganso espolonado (S): Plectropterus gambensis III B

Garceta común (S): Egretta garzetta III B

Garceta grande (S): Casmerodius albus III B

Garcilla bueyera (S): Bubulcus ibis III B

Garcita resnera (S): Bubulcus ibis III B

Garden Emerald (E): Chlorostilbon assimilis II B

Garden Tree Boa (E): Corallus enydris II R

Gardineria aniarcica: II In

Gardineria capensis: II In

Gardineria hawaiiensis: II In

Gardineria minor: 11 In

Gardineria musorstomica: II In

Gardineria paradoxa: II In

Gardineria philippinensis: II In

Gardineria simplex: II In

Gardineroseris planulata: II In

Garganey (E): Anas querquedula III B

Garganey Teal (E): Anas querquedula III B

Garganta de oro coliverde (S): Polytmus theresiae II B

Garganta lanza coliblanca (S): Threneres leucurus II B

Gamet-throated Hummingbird (E): Lamprolaima rhami II B

Garrulaxe de l'Omei (F): Liocichla omeiensis II B

Garrulaxes (F): Garrulax spp $/$ iocichla spp. IUNC B

Garza blanca real (S): Casmerodius albus III B

Garza ganadera (S): Bubulcus ibis III B

Garza goliat (S): Ardea goliath III B

Garzetta grande (S): Casmerodius albus III B

Garzón soldado (S): Jabiru mycteria I B

Gastric-brooding frogs (E): Rheobatrachus spp. II A

Gato andino (S): Oreailurus jacobita I M

Gato atigrado (S): Leopardus tigrinus I M

Gato bengalí (S): Prionailurus bengalensis II M/Prionailurus bengalensis bengalensis $\mathbf{I}$ I M

Gato cabeciancho (S): Prionailurus planiceps I M

Gato colorado (S): Herpailurus yaguarondi III M

Gato cutarra (S): Eira barbara III M

Gato de agua (S): Lontra longicaudis I M

Gato de Bengala (S): Prionailurus bengalensis $\mathbf{L I} \mathrm{M}$

Gato de Biet (S): Felis bieti II M

Gato de la jungla (S): Felis chaus II M

Gato de las arenas (S): Felis margarita II M

Gato de los pajonales (S): Oncifelis colocolo II M

Gato de los pantanos (S): Felis chaus II M

Gato de mar (S): Lontra felina I M

Gato de mato (S): Oncifelis geoffroyi I M

Gato de Pallas (S): Otocolobus manul II M

Gato de pies negros (S): Felis nigripes I M

Gato del desierto de China (S): Felis bieti II M

Gato del Sahara (S): Felis margarita II M

Gato dorado (S): Profelis aurata II M

Gato dorado asiático (S): Catopuma temminckii I M

Gato fossa de Madagascar (S): Cryptoprocia ferox II M

Gato jaspeado (S): Pardofelis marmorata I M

Gato lince (S); Oreailurus jacobita I M

Gato manul (S): Otocolobus manul II M

Gato marino (S): Lontra felina I M

Gato montes (S): Felis silvesiris II M/Leopardus wiedii I M/Lynx rufus II $\mathrm{M}$

Gato montés común (S): Oncifelis geoffroyi I M

Gato moro (S): Herpailurus yaguarondi $\mathbf{M I} \mathbf{M}$
Gato negro (S): Eira barbara III M

Gato onza (S): Leopardus pardalis I M

Gato pajero (S): Oncifelis colocolo II M

Gato patinegro (S): Felis nigripes I M

Gato pescador (S): Prionailurus viverrinus II M

Gato rojizo (S): Prionailurus rubiginosus $\mathbf{I I I} \mathbf{M}$

Gato rubiginosa (S): Prionailurus rubiginosus $\mathbf{I I} \mathbf{M}$

Gato silvestre (S): Felis silvestris II M

Gato tigre (S): Leopardus tigrinus I M

Gaur (E/S/F): Bos gaurus I M

Gavial (E): Gavialis gangeticus I R

Gavial del Ganges (S): Gavialis gangeticus I R

Gavial du Gange (F): Gavialis gangeticus I R

Gavialis gangeticus: Fish-eating Crocodile (E), Gavial (E), Gharial

(E), Long-nosed Crocodile (E), Gavial del Ganges (S), Gavial du Gange (F) I R

Gavials (E): Gavialis gangeticus/Tomistoma schlegelii I R

Gavilán acollarado (S): Accipiter cirrocephalus II B/Accipiter collaris II B

Gavilán albonotatus (S): Buteo albonotatus II B

Gavilán alirrojizo (S): Buteo magnirostris II B

Gavilán americano (S): Accipiter striatus II B

Gavilán andapié (S): Parabuteo unicinctus II B

Gavilán andino (S): Accipiter ventralis II B

Gavilán amastrador (S): Accipiter striatus II B

Gavilán azor (S): Accipiter gentilis II B

Gavilán azul (S): Leucoptemis schistacea II B

Gavilán bebehumo (S): Buteo platypterus II B

Gavilán besra (S): Accipiter virgarus II B

Gavilán bicolor (S): Accipiter bicolor II B

Gavilán bidente (S): Harpagus bidentatus II B

Gavilán blanco (S): Leucoptemis albicollis II B

Gavilán cabecigrís (S): Accipiter poliocephalus II B

Gavilán cangrejero (S): Buteogallus anthracinus II B

Gavilán caracolero (S): Rostrhamus sociabilis II B

Gavilán carinegro (S): Leucopternis melanops II B

Gavilán ceniciento (S): Circus cinereus II B

Gavilán chikra (S): Accipiter badius II B

Gavilán cola corta (S): Buteo brachyurus II B

Gavilán colifajeado (S): Buteo albonotatus II B

Gavilán colipinto (S): Accipiter trinotatus II B

Gavilán colorado (S): Busarellus nigricollis II B

Gavilán común (S): Accipiter nisus II B/Buteo magnirostris II B

Gavilán cubano (S): Accipiter gundlachi II B

Gavilán cueillirojo (S): Micrastur ruficollis II B

Gavilán de Célebes (S): Accipiter griseiceps II B

Gavilán de collar (S): Micrastur gilvicollis II B

Gavilán de Cooper (S): Accipiter cooperiï II B

Gavilán de Frances (S): Accipiter francesii II B

Gavilán de Galapagos (S): Buteo galapagoensis II B

Gavilán de las Fiji (S): Accipiter rufitorques II B

Gavilän de Manglares (S): Buteogallus aequinoctialis II B

Gavilán de Nicobar (S): Accipiter butleri II B

Gavilán de Nueva Bretaña (S): Accipiter luteoschistaceus II B

Gavilán de Nueva Caledonia (S): Accipiter haplochrous II B

Gavilán de Ovampo (S): Accipiter ovampensis II B

Gavilán de Traylor (S): Micrastur buckleyi II B

Gavilán dorsigris (S): Leucoptemis occidentalis II B

Gavilán enano (S): Accipiler superciliosus II B

Gavilán flanquirrojo (S): Accipiter castanilius II B

Gavilán gabar (S): Melierax gabar II B

Gavilán gargantiblanco (S): Buteo albigula II B

Gavilán grande (S): Circus buffoni II B

Gavilán griego (S): Accipiter brevipes II B

Gavilán gris (S): Asturina nitida II B

Gavilán habado (S): Buteo magnirostris II B

Gavilán imitador (S): Accipiter imitator II B

Gavilán langostero (S): Buteo swainsoni II B

Gavilán malgache (S): Accipiter madagascariensis II B

Gavilán maromero (S): Elanus leucurus II B

Gavilán mix to (S): Parabuteo unicincrus II B

Gavilán negro (S): Buteo leucorrhous II B

Gavilán negro común (S): Buteogallus subtilis II B

Gavilán negro grande (S): Buteogallus urubitunga II B

Gavilán pajarero (S): Accipiter striatus II B

Gavilán palomero (S): Leptodon cayanensis II B 
Gavilán pantalón (S): Accipiter bicolor II B

Gavilán papirrufo (S): Accipiter rufiventris II B

Gavilán patilargo (S): Geranospiza caerulescens II B

Gavilán pechiblanco (S): Accipiter chionogaster II B

Gavilán pechirrojo (S): Accipiter castanilius II B/Accipiter rhodogaster II B

Gavilán pechirroseo (S): Accipiter toussenelii II B

Gavilán pescador (S): Pandion haliaetus II B

Gavilán pico de $\mathrm{Hoz}$ (S): Rostrhamus hamatus II B

Gavilán pico ganchudo (S): Chondrohierax uncinarus $\mathbf{I}$ I B

Gavilán pio (S): Accipiter albogularis II B

Gavilán pita venado (S): Buteogallus meridionalis II B

Gavilán pizarroso (S): Leucopternis schistacea II B

Gavilán plomizo (S): Icrinia plumbea II B/Leucopternis plumbea II $\mathrm{B}$

Gavilán principe (S): Leucoptemis princeps II B

Gavilán punenno (S): Buteo poecilochrous II B

Gavilán rabadilla blanca (S): Buteo leucorrhous II B

Gavilán ranero (S): Accipiter soloensis II B

Gavilán rastrero (S): Circus cyaneus II B

Gavilán rufinegro (S): Accipirer melanochlamys II B

Gavilán sabanero (S): Buteogallus meridionalis II B

Gavilán semiplomizo (S): Leucopternis semiplumbea II B

Gavilán tejé (S): Buteo albicaudarus II B

Gavilán tijereta (S): Elanoides forficatus II B

Gavilán ventriblanco (S): Accipiler chionogaster II B

Gavilán vientrigris (S): Accipiter poliogaster II B

Gavilán zancón (S): Geranospiza caenulescens II B

Gavilán zancudo (S): Geranospiza caerulescens II B

Gavilancito americano (S): Accipiter superciliosus II B

Gavilancito chico (S): Accipiter minullus II B

Gavilancito de Célebes (S): Accipiter nanus II B

Gavilancito de Hartlaub (S): Accipiter erythropus II B

Gavilancito de Nueva Bretaña (S): Accipiter brachyurus II B

Gavilancito japonés (S): Accipiter gularis II B

Gavilancito moluqueño (S): Accipiter erythrauchen II B

Gavilancito muslirrojo (S): Accipiter erythropus II B

Gavilancito torcaz (S): Accipiter collaris II B

Gavilán-reloj (S): Micrastur semiłorquatus II B

Gaviota de Mongolia (S): Larus relictus I B

Gaviota relicta (S): Larus relictus I B

Gazella cuvieri: Cuvier's Gazelle (E), Edmi Gazelle (E), Idmi (E), Gazelle de Cuvier (F) III M

Gazella dama: Addra Gazelle (E), Dama Gazelle (E), Gazelle dama (F) $1 \mathrm{M}$

Gazella dorcas: Dorcas Gazelle (E), Gazelle dorcas (F) III M

Gazella leptoceros: Rhim (E), Sand Gazelle (E), Slender-homed Gazelle (E), Gazelle leptocère (F), Gazelle à comes fines $(F)$, $\mathrm{Rhim}$ (F) III M

Gazelie à comes fines (F): Gazella leptoceros III M

Gazelle dama (F): Gazella dama I M

Gazelle de Cuvier (F): Gazella cuvieri III M

Gazelle dorcas (F): Gazella dorcas III M

Gazelle leptocère (F): Gazella leptoceros III M

Gazelles (E): Gazella spp/Procapra spp. IIIV/NC M

Gecko de l'tle Serpent (F): Cyrtodactylus serpensinsula II R

Gecko diume à bandes noires $(\mathrm{F})$ : Phelsuma nigristriata II $\mathrm{R}$

Gecko diurne à courte tête (F): Phelsuma breviceps II R

Gecko diume à gorge jaune (F): Phelsuma flavigularis II R

Gecko diume à large queue (F): Phelsuma laticauda II R

Gecko diume à quatre ocelles (F): Phelsuma quadriocellata II R

Gecko diume à queue bleue (F): Phelsuma cepediana II R

Gecko diume à queue chamue (F): Phelsuma mutabilis II R

Gecko diume à queue plate (F): Phelsuma serraticauda II R

Gecko diume à tête jaune (F): Phelsuma klemmeri II R

Gecko diume à trois lignes (F): Phelsuma irilineaia II R

Gecko diume d'Aldabra (F): Phelsuma abbotti II R

Gecko diume de Barbour (F): Phelsuma barbouri II R

Gecko diume de Befotaka (F): Phelsuma befolakensis II R

Gecko diume de Boettger (F): Phelsuma v-nigra II R

Gecko diume de Bomer (F): Phelsuma minuthi II R

Gecko diume de Bourbon (F): Phelsuma borbonica II R

Gecko diume de Cheke (F): Phelsuma chekei II R

Gecko diume de Grandidier (F): Phelsuma mutabilis II R

Gecko diume de Klemmer (F): Phelsuma klemmeri II R

Gecko diume de la région Antanosy (F): Phelsuma antanosy II $R$
Gecko diume de la Réunion (F): Phelsuma borbonica II R Gecko diume de I'lle Maurice (F): Phelsuma guimbeaui II R Gecko diume de l'lle Pemba (F): Phelsuma parkeri II R Gecko diume de l'Jle Ronde (F): Phelsuma guentheri II R Gecko diume de Madagascar (F): Phelsuma madagascariensis II $\mathbf{R}$ Gecko diume de Meier (F): Phelsuma nigristriata II R Gecko diume de Minuth (F): Phelsuma minuthi II R Gecko diume de Parker (F): Phelsuma parkeri II R Gecko diume de Peacock (F): Phelsuma quadriocellata II R Gecko diume de Robert Mertens (F): Phelsuma robertmertensi II R Gecko diume de Rodrigues (F): Phelsuma edwardnewtonii II R Gecko diume de Seipp (F): Phelsuma seippi II R Gecko diume de Standing ( $F$ ): Phelsuma standingi II R Gecko diume de Zanzibar (F): Phelsuma dubia II R Gecko diume des Comores (F): Phelsuma comorensis II R Gecko diurne des Iles Andaman (F): Phelsuma andamanensis II R Gecko diume des Seychelles (F): Phelsuma longinsulae II R Gecko diume du Nord de Madagascar (F): Phelsuma chekei II R Gecko diume du nord-ouest de Madagascar (F): Phelsuma befotakensis II R

Gecko diume du sud-ouest de Madagascar (F): Phelsuma leiogaster II $\mathrm{R}$

Gecko diume géant de Rodrigues (F): Phelsuma gigas II R Gecko diume géant des Seychelles (F): Phelsuma sundbergi II R Gecko diume ligné (F): Phelsuma lineara II $\mathrm{R}$ Gecko diume modeste (F): Phelsuma modesta II R Gecko diume nain (F): Phelsuma pusilla II R Gecko diume ocellé (F): Phelsuma ocellata II R Gecko diume orné de l'ile Maurice (F): Phelsuma ornata II R Gecko diume poussière-d'or (F): Phelsuma laticauda II R Gecko diume rayé (F): Phelsuma lineata II R

Gecko diume sombre (F): Phelsuma dubia II R Gecko diume tacheté (F): Phelsuma gutrata II R Geckos (E): Gekkonidae spp. IUNC R

Geckos diumes (F): Phelsuma spp. II R

Geckos diumes de lOcéan Indien (F): Phelsuma spp. II R Geco diume de Abbott (S): Phelsuma abboti II R

Geco diumo de Andaman (S): Phelsuma andamanensis II R Geco diumo de bandas (S): Phelsuma lineata II R

Geco diumo de Barbour (S): Phelsuma barbouri II R

Geco diumo de cola azul (S): Phelsuma cepediana II R

Geco diurno de cola dentada (S): Phelsuma serraticauda II R Geco diumo de cola gruesa (S): Phelsuma laticauda II R Geco diumo de estrias negras (S): Phelsuma nigristriata II R Geco diumo de garganta amarilla (S): Phelsuma flavigularis II R Geco diumo de Guenther (S): Phelsuma guentheri II R Geco diumo de Guimbeau (S): Phelsuma guimbeaui II R Geco diumo de Klemmer (S): Phelsuma klemmeri II R Geco diumo de las Comores (S): Phelsuma comorensis II R Geco diumo de Madagascar (S): Phelsuma madagascariensis II R Geco diumo de Robert Mertens (S): Phelsuma robertmertensi II R Geco diumo de Standing (S): Phelsuma standingi II R

Geco diumo de Sundberg (S): Phelsuma sundbergi II R Geco diumo de tres bandas (S): Phelsuma trilineata II R Geco diumo de Zanzibar (S): Phelsuma dubia II R

Geco diumo ocelado (S): Phelsuma ocellata II R

Geco diumo omado (S): Phelsuma ornata II $\mathbf{R}$

Gecos diumos (S): Phelsuma spp. II R

Geelvink Bay Fruit Bat (E): Preropus pohlei II M

Geelvink Pygmy-Parrot (E): Micropsitta geelvinkiana II B

Geese (E): Anatidae spp, I/I/II/NC B

Gelada (F): Theropithecus gelada II M

Gelada Baboon (E): Theropithecus gelada II M

Gemmipora $=$ Turbinaria

Gemmulatrochus $=$ Hoplangia

Gentle lemurs (E): Hapalemur spp. I M

Geochelone carbonaria: Red-footed Tortoise (E), Wood Tortoise (E), Jabuti (S), Karumbé (S), Tortuga de patas rojas (S), Tortue charbonnière (F) II R

Geochelcne chilensis: Argentine Tortoise (E), Chaco Tortoise (E), Southern Wood Tortoise (E), Tortuga terrestre argentina (S), Tortue de la Pampa (F) II R 
Geochelone denticulaia: Brazilian Giant Tortoise (E), Forest Tortoise (E), South American Tortoise (E), South American Yellow-footed Tortoise (E). Yellow-footed Tortoise (E) Morrocoy (S), Motelo (S), Tortue de l'Amérique du Sud (F), Tortue dentelée (F). Tortue denticuléc (F) II R

Geochelone donosobarrosi = Geochelone chilensis

Geochelone elegans: Indian Star Tortoise (E), Star Tortoise (E), Tortuga estrellada de la India (S), Tortue étoilé de l'Inde (F) II R

Geochelone elephantopus = Geochelone nigra

Geochelone elongata = Indotestudo elongata

Geochelone emys = Manouria emys

Geochelone forstenii $=$ Indotestudo forstenii

Geochelone gigantea: Aldabra Giant Tortoise (E), Tortuga gigante de Aldabra (S), Tortue géante (F), Tortue géante d'Aldabra (F) II R

Geochelone impressa $=$ Manouria impressa

Geochelone nigra: Galapagos Giant Tortoise (E), Tortuga gigante de las Galápagos (S), Tortue géante des Galapagos (F), Tortue éléphantine (F) I R

Geochelone pardalis: Leopard Tortoise (E), Mountain Tortoise (E). Tortuga leopardo (S), Tortue-léopard du Cap (F) II R

Geochelone petersi $=$ Geochelone chilensis

Geochelone platynota: Burmese Starred Tortoise (E), Flatback Tortoise (E), Tortuga estrellada de Burma (S), Tortue étoilée de Birmanie (F) II R

Geochelone radiata: Radiated Tortoise (E), Tortuga estrellada de Madagascar (S), Tortuga rayada (S), Tortue radiée de Madagascar (F), Tortue rayonnée (F) I R

Geochelone sulcata: African Spurred Tortoise (E), Grooved Tortoise (E), Tortuga con púas (S), Tortue sillonnée (F) II R

Geochelone travancorica =Indotestudo forstenii

Geochelone yniphora: Angonoka (E), Madagascar Angulated Tortoise (E), Madagascar Tortoise (E), Tortuga de Madagascar (S), Tortuga globulosa malagache (S), Tortue de Madagascar (F), Tortue à plastron éperonné $(F)$, Tortue à soc de Madagascar (F), Tortue à éperon (F) I R

Géoclemmyde d' Hamilton (F): Geoclemys hamiltonii I R

Geoclemys hamiltonii: Black Pond Turtle (E), Black Spotted Turtle (E), Hamilton's Terrapin (E), Spotted Pond Turtle (E), Galápago rayado (S), Géoclemmyde d' Hamilton (F), Tortue de Hamilton (F) I R

Geoemyda tricarinata $=$ Melanochelys tricarinata

Géoémyde tricarénée (F): Melanochelys iricarinata I R

Geoffroy's Cat (E): Oncifelis geoffroyi I M

Geoffroy's Spider Monkey (E): Ateles geoffroyi UII M

Geoffroy's Tamarin (E): Saguinus oedipus I M

Geoffroy's Tufted-ear Marmoset (E): Callithrix geoffroyi II M

Geoffroyus geoffroyi: Red-cheeked Parrot (E), Lorito carirnojo (S), Perruche de Geoffroy (F) II B

Geoffroyus heteroclitus: Singing Parrot (E), Lorito heteróclito (S), Perruche hétéroclite (F) II B

Geoffroyus simplex: Blue-collared Parrot (E), Lorito acollarado (S), Perruche à col bleu (F) II B

Geometric Tortoise (E): Psammobates geometricus I R

Geopsillacus occidentalis: Night Parrot (E), Loro noctumo (S), Perico noctumo (S), Perruche noctume (F) I B

Geranoaetus melanoleucus: Black-chested Buzzard-Eagle (E), Black-chested Eagle (E), Grey Buzzard-Eagle (E), Aguila escudada (S), Aguila mora (S), Aguila real (S), Buse aguia (F) II B

Geranospiza caerulescens: Crane Hawk (E), Azor zancón (S), Gavilán patilargo (S), Gavilán zancudo (S), Gavilán zancón (S), Buse échasse (F) II B

Gerfaut (F): Falco rusticolus I B

Germain's Peacock-Pheasant (E): Polyplectron germaini II B

Geronticus calvus: Bald Ibis (E), Southem Bald Ibis (E), Ibis calvo (S), Ibis chauve de l'Afrique du Sud (F), Ibis noir (F) I B

Geronticus eremita: Hermit Ibis (E). Northern Bald Ibis (E), Waldrapp (E), Ibis eremita (S), Ibis chauve (F) I B

Gervais' Beaked Whale (E): Mesoplodon europaeus II M

Gharial (E): Gavialis gangeticus I R

Gharials (E): Gavialis gangericus/Tomistoma schlegelii I R

Ghor-khar (E): Equus onager khur I M

Giant Anteater (E): Myrmecophaga tridactyla II M

Giant Armadillo (E): Priodontes maximus I M
Giant Bottle-nosed Whale (E): Berardius bairdii I M Giant Brazilian Otter (E): Pteronura brasiliensis I M Giant Catfish (E): Pangasianodon gigas I F

Giant Clam (E): Tridacna gigas II In

Giant clams (E): Tridacnidae spp. II In

Giant Eagle-Owl (E): Bubo lacteus II B

Giant Girdled Lizard (E): Cordylus giganteus II R

Giant Grebe (E): Podilymbus gigas I B

Giant Ground Pangolin (E): Manis gigantea II M

Giant Hummingbird (E): Patagona gigas II B

Giant Muntjac (E): Megamuntiacus vuquanghensis I M

Giant One-homed Chameleon (E): Chamaeleo melleri II R

Giant Otter (E): Pteronura brasiliensis I M

Giant Panda (E): Ailuropoda melanoleuca I M

Giant Pangolin (E): Manis gigantea II M

Giant Pied-billed Grebe (E): Podilymbus gigas I B

Giant Poison Frog (E): Dendrobates azureus II A

Giant Sable Antelope (E): Hippotragus niger variani I M

Giant salamanders (E): Andrias spp. I A

Giant Scops-Owl (E): Mimizuku gumeyi I B

Giant skinks (E): Corucia zebrata/Mabuya brevicollis IINC R

Giant South American Turtle (E): Podocnemis expansa II $\mathbf{R}$

Giant Spiny-tailed Lizard (E): Cordylus giganteus II R

Giant squirrels (E): Ratufa spp. I M

Giant Sturgeon (E): Huso huso II F

Giant Zonure (E): Cordylus giganteus II R

Gibbon à favoris blancs (F): Hylobates concolor I M

Gibbon à mains blanches (F): Hylobates lar I M

Gibbon agile (F): Hylobates agilis I M

Gibbon cendré (F): Hylobates moloch $1 \mathrm{M}$

Gibbon de Muller (F): Hylobates muelleri I M

Gibbon lar (F): Hylobates lar I M

Gibbon noir (F): Hylobates concolor I M

Gibbons (E/F): Hylobatidae spp. I M

Gibón ceniciento (S): Hylobates moloch I M

Gibón de cresta negra (S): Hylobates pileatus I M

Gibón de manos blancas (S): Hylobates lar I M

Gibón de Mueller (S): Hylobates muelleri I M

Gibón hulock (S): Hylobates hoolock I M

Gibones (S): Hylobatidae spp. I M

Giboya (E): Boa constrictor I/II R

Gila Monster (E): Heloderma suspectum II R

Gilded Hummingbird (E): Hylocharis chrysura II B

Gilliard's Flying-fox (E): Pteropus gilliardi II M

Ginkgo-toothed Beaked Whale (E): Mesoplodon ginkgodens II M

Girdled lizards (E): Pseudocordylus spp. II R

Glaucidium alberinum: Albertine Owlet (E), Prigogine's Owlet (E), Chevêchette du Graben (F) II B

Glaucidium bolivianum: Yungas Pygmy-Owl (E) II B

Glaucidium brasilianum: Ferruginous Pygmy-Owl (E), Caburé (S), Pavita ferruginea (S), Tecolotito común (S), Chevechette brune (F) II $B$

Glaucidium brodiei: Collared Owlet (E), Collared Pygmy-Owl (E), Chevêchette à collier (F) II B

Glaucidium californicum: Northern Pygmy-Owl (E), Tecolotito del cabo (S), Chevêchette des Rocheuses (F) II B

Glaucidium capense: African Barred Owlet (E), Barred Owlet (E), Chevêchette du Cap (F) II B

Glaucidium capense castaneum = Glaucidium castaneum

Glaucidium capense ngamiense = Glaucidium ngamiense

Glaucidium capense scheffleri = Glaucidium scheffleri

Glaucidium castaneum: Chestnut Owlet (E), Chevêchette châtaine (F) II B

Glaucidium castanonotum: Chestnut-backed Owlet (E) II B

Glaucidium castanopterum: Javan Owlet (E), Chevêchette spadicée (F) II B

Glaucidium cuculoides: Asian Barred Owlet (E), Cuckoo Owlet (E), Chevêchette cuculoìde (F) II B

Glaucidium gnoma: Mountain Pygrny-Owl (E), Tecolotito serano (S), Chevêchette naine (F) II B

Glaucidium gnoma califormicum = Glaucidium califormicum

Glaucidium griseiceps = Glaucidium minutissimum

Glaucidium hardyi: Hardy's Pygmy-Owl (E), Chevêchette d'Amazonie (F) II B

Glaucidium hoskinsii $=$ Glaucidium califomicum 
Glaucidium jardinii: Andean Pygmy-Owl (E), Pavita andina (S) Chevêchette des Andes (F) II B

Glaucidium minutissimum: Least Pygmy-Owl (E), Chevêchette cabouré (F) II B

Glaucidium nanum: Austral Pygmy-Owl (E), Caburé patagónico (S) Chevêchette australe (F) II B

Glaucidium ngamiense: Ngami Owlet (E), Chevêchette de Ngami (F) II B

Glaucidium palmarum $=$ Glaucidium minutissimum

Glaucidium passerinum: Eurasian Pygmy-Owl (E), Mochuelo chico (S). Chevêchette d'Europe (F), Chouette chevêchette (F) II B

Glaucidium perlarum: Pearl-sported Owlet (E), Mochuelín perlado (S), Chevêchette perlée (F) II B

Glaucidium peruanum: Peruvian Pygmy-Owl (E), Chevêchette du Pérou (F) II B

Glaucidium radiatum: Barred Jungle Owlet (E), Jungle Owlet (E), Chevêchette de jungle (F) II B

Glaucidium radiatum castanono $\mathrm{lum}=$ Glaucidium castanonotum

Glaucidium sanchezi $=$ Glaucidium minutissimum

Glaucidium scheffleri: Scheffler's Owlet (E), Chevêchette de Scheffler (F) II B

Glaucidium siju: Cuban Pygmy-Owl (E), Siju (S), Sijucito (S), Chevêchette de Cuba (F) II B

Glaucidium sjostedri: Sjostedt's Owlet (E), Mochelo de Sjöstedt (S), Mochuelo chico (S), Chevêchette à queue barrée (F) II B

Glaucidium tephronotum: Red-chested Owlet (E), Yellow-legged Owlet (E), Chevêchette à pieds jaunes (F) II B

Glaucis aenea: Bronzy Hermit (E), Ermitaño bronceado (S), Ermite bronzé (F) II B

Glaucis dohmii = Ramphodon dohmii

Glaucis hirsuta: Rufous-breasted Hermit (E), Colibri pecho canela (S), Emnitaño pechicanelo (S), Ermite hirsute (F) II B

Glaucous Macaw (E): Anodorhynchus glaucus I B

Glauert's Monitor (E): Varanus glauerti II R

Glittering-bellied Emerald (E): Chlorostilbon aureoventris II B

Glittering-throated Emerald (E): Amazilia fimbriata II B

Globicephala edwardii = Globicephala melaena

Globicephala macrorhynchus: Pacific Pilot Whale (E), Short-finned Pilot Whale (E), Caldrón negro (S), Globicéphale tropical (F) II $\mathrm{M}$

Globicephala melaena: Long-finned Pilot Whale (E), Caldrón negro (S), Globicéphale commun (F) II M

Globicephala sieboldii = Globicephala macrorhynchus

Globicéphale commun (F): Globicephala melaena II M

Globicéphale tropical (F): Globicephala macrorhynchus II M

Glossopsina concinna: Musk Lorikeet (E), Lori almizclero (S), Lori musc (F), Lori à bandeau rouge (F) II B

Glossopsitta porphyrocephala: Purple-crowned Lorikeet (E), Lori coronipúrpura (S), Lori à couronne pourpre (F) II B

Glossopsinta pusilla: Little Lorikeet (E), Lori carirojo (S), Lori à masque rouge (F), Petit lori musc (F) II B

Glossy Black-Cockatoo (E): Calyptorhynchus lathami II B

Glossy-mantled Manucode (E); Manucodia atra II B

Glowing Puffleg (E): Eriocnemis vestitus II B

Glow-throated Hummingbird (E): Selasphorus ardens II B

Goanna Monitor (E): Varanus acanthurus II R

Goannas (E): Varanus spp. II R

Goazu (F): Ozotoceros bezoarticus I M

Gobemouche de Rueck (F): Cyomis ruckii II B

Gobernouches (F): Muscicapidae: Muscicapinae spp. II/NC B

Gobemouches bleu de Rueck (F): Cyomis nuckii II B

Godoy (S): Potos flavus $111 \mathrm{M}$

Goéland de Mongolie (F): Larus relictus I B

Goéland relique (F): Larus relictus I B

Goeldi's Marmoset (E): Callimico goeldii I M

Goeldi's Monkey (E): Callimico goeldii I M

Goeldi's Tamarin (E): Callimico goeldii I M

Goethalsia bella: Rufous-cheeked Hummingbird (E), Colibri du Pirré (F) II B

Goetze's Chameleon (E): Chamaeleo goetzei II R

Goffin's Cockatoo (E): Cacatua goffini I B

Gold-dust Day Gecko (E): Phelsuma laticauda II R

Golden Arowana (E): Scleropages formosus I F

Golden Arrow Poison Frog (E): Atelopus varius zeteki I A

Golden Bamboo Lemur (E): Hapalemur aureus I M
Golden Birdwing (E): Troides aeacus II II/Troides amphrysus $\mathbf{I I}$ In/Troides cuneifer II in

Golden Bishop (E): Euplectes afer III B

Golden Cat (E): Catopuma semminckii I M/ Profelis aurata II M

Golden Conure (E): Aratinga guarouba I B

Golden Dragon Fish (E): Scleropages formosus I F

Golden Eagle (E): Aquila chrysaetos II B

Golden Frog (E): Atelopus varius zeteki I A

Golden Jackal (E): Conis aureus III M

Golden Kaiserihind (E): Teinopalpus aureus II In

Golden Langur (E): Trachypithecus geei I M

Golden Leaf Monkey (E): Trachypithecus geei I M

Golden Lemur (E): Hapalemur aureus I M

Golden Lion Tamarin (E): Leontopithecus rosalia $1 \mathrm{M}$

Golden Mantella (E): Mantella aurantiaca II A

Golden Owl (E): Tyto aurantia II B

Golden Parakeet (E): Aratinga guarouba I B

Golden Poison Frog (E): Minyobates altabueyensis II A/ Phyllobates terribilis II A

Golden Potto (E): Arctocebus aureus II M

Golden Snub-nosed Monkey (E): Pyga'hrix roxellana I M

Golden Toad (E): Bufo periglenes I A

Golden-backed Weaver (E): Ploceus preussi III B

Golden-bellied Guenon (E): Cercopithecus pogonias II M

Golden-bellied Monkey (E): Cercopithecus pogonias II M

Golden-bellied Starfrontlet (E): Coeligena bonapanei II B

Golden-bellied Treeshrew (E): Tupaia chrysogaster II M

Golden-breasted Puffleg (E): Eriocnemis mosquera II B

Golden-breasted Waxbill (E): Amandava subflava III B

Golden-capped Conure (E): Aratinga auricapilla II B

Golden-capped Fruit Bat (E): Acerodon jubatus I M

Golden-capped Parakeet (E): Aratinga auricapilla II B

Golden-collared Macaw (E): Ara auricollis II B

Golden-crowned Conure (E): Arating a aurea II B

Golden-crowned Sifaka (E): Propithecus tattersalli I M

Golden-fronted Parakeet (E): Bolborhynchus aurifrons II B

Golden-headed Fig-Parrot (E): Psittaculirostris desmarestii II B

Golden-headed Lion Tamarin (E): Leontopithecus chrysomela I M

Golden-mantled Racquet-tail (E): Prionirurus plasurus II B

Golden-mantled Rosella (E): Platycercus eximius II B

Golden-mantled Saddle-back Tamarin (E): Saguinus Iripartitus II M

Golden-plumed Conure (E): Leptosintaca branickii II B

Golden-plumed Parakeet (E): Leptosittaca branickii II B

Golden-rumped Lion Tamarin (E): Leontopithecus chrysopygus I M

Golden-shouldered Parrot (E): Psephotus chrysopterygius I B

Golden-tailed Parrotlet (E): Touit surda II B

Golden-tailed Sapphire (E); Chrysuronia oenone II B

Goldenthroats (E); Polytmus spp. II B

Golden-winged Parakeet (E): Brotogeris chrysopterus II B

Goldie's Bird-of-paradise (E): Paradisaea decora II B

Goldie's Lorikeet (E): Psitteuteles goldiei II B

Goldmania violiceps: Violet-capped Hummingbird (E), Colibri à calotte violette (F) II B

Golfball Coral (E): Favia fragum II in

Golfoduicean Poison Frog (E): Phyllobates vittatus II A

Goliath Birdwing (E): Omithoptera goliath II in

Goliath Heron (E): Ardea goliath III B

Gombessa (E): Latimeria chalumnae I F

Gongylophis conicus $=$ Eryx conicus

Goniastrea aspera: II In

Goniastrea australensis: II It

Goniastrea columella: II in

Goniastrea deformis: II In

Goniastrea edwardsi: II In

Goniastrea favulus: III In

Goniastrea palauensis: II In

Goniastrea pectinata: II In

Goniastrea retiformis: II In

Goniastrea spectabilis: II In

Goniastrea varia: II In

Goniocorella dumosa: II in

Goniocyathus = Caryophyllia

Goniopora arbuscula: II In

Goniopora bemardi: II in

Goniopora burgosi: II In

Goniopora cellulosa: II In 
Goniopora columna: II In

Goniopora crassa: II In

Goniopora djiboutiensis: II In

Goniopora eclipsensis: II in

Goniopora fruticosa: II

Goniopora gracilis: II In

Goniopora granulosa: II In

Goniopora klunzingeri: II In

Goniopora lobata: II In

Goniopora minor: II In

Goniopora muscosa: II in

Goniopora norfolkensis: II In

Goniopora palmensis: II In

Goniopora pandoraensis: II In

Goniopora parvistella: II In

Goniopora pedunculata: II in

Goniopora pendulus: II In

Goniopora planulata: II In

Goniopora polyformis: II In

Goniopora pulvinula: II In

Goniopora sagamiensis: II in

Goniopora samoa: II In

Goniopora savignyi: II In

Goniopora somaliensis: II In

Goniopora stokesi: II In

Goniopora stutchburyi: II In

Goniopora tenella: II In

Goniopora senuidens: II In

Goniopora traceyi: II in

Goniopora viridis: II In

Goniopora wotouensis: II In

Goose-beaked Whale (E): Ziphius cavirostris II M

Gopher Tortoise (E): Gopherus polyphemus II R

Gophère à bords jaunes (F): Gopherus flavomarginatus I R

Gophère d'Agassiz (F): Gopherus agassizii II R

Gophère du Texas (F): Gopherus berlandieri II R

Gophère polyphème (F): Gopherus polyphemus II R

Gopherus agassizii: Desert Tortoise (E), Tortuga del desierto (S), Gophère d'Agassiz (F), Tortue d'Agassiz (F) II R

Gopherus berlandieri: Berlandier's Tortoise (E), Texas Tortoise (E), Tortuga de Texas (S), Gophère du Texas (F), Tortue du Texas (F) II R

Gopherus flavomarginatus: Bolson Tortoise (E), Mexican Giant Tortoise (E), Yellow-bordered Tortoise (E). Tortuga grande (S), Tortuga llanero (S), Tortuga topo (S), Gophère à bords jaunes (F), Tortue du Mexique (F), Tortue à bords jaunes (F) I R

Gophenus polyphemus: Gopher Tortoise (E), Tortuga terrestre de Florida (S), Gophère polyphème (F), Tortue de la Floride (F), Tortue gaufrée (F) II R

Gopherus polyphemus agassizii = Gopherus agassizii

Gopherus polyphemus berlandieri = Gopherus berlandieri

Goral (E/S/F): Naemorhedus goral I M

Goral à queue longue (F): Naemorhedus caudaius I M

Gorals (E): Naemorhedus spp. I M

Goreaugyra $=$ Meandrina

Gorgeted Sunangel (E): Heliangelus strophianus II B

Gorgeted Woodstar (E): Acestrura heliodor II B

Gorila (S): Gorilla gorilla I M

Gorilla (E): Gorilla gorilla I M

Gorilla gorilla: Gorilla (E), Gorila (S), Gorille (F) I M

Gorille (F): Gorilla gorilla I M

Gorontalo Macaque (E): Macaca nigra II M

Gorrión pardillo (S): Passer griseus III B

Gorzula's Poison Frog (E): Dendrobates rufulus II A

Goshawks (E): Accipiter spp $/$ Erythrotriorchis spp/Megatriorchis doriae/Micronisus gabar II B

Gould's Jewelfront (E): Heliodoxa aurescens II B

Gould's Monitor (E): Varanus gouldii II R

Goura couronné (F): Goura cristata II B

Goura spp.: Crowned-pigeons (E), Gouras (E), Palomas crestadas (S), Gouras (F) II B

Goura cristata: Blue Crowned-Pigeon (E). Common Crowned

Pigeon (E), Great Goura (E), Western Crowned-Pigeon (E),

Paloma crestada azul (S), Goura couronné (F) II B

Goura de Scheepmaker (F): Goura scheepmakeri II B

Goura de Sclater (F): Goura scheepmakeri II B
Goura de Victoria (F): Goura victoria II B

Goura scheepmakeri: Maroon-breasted Crowned-Pigeon (E), Masked Goura (E), Scheepmaker's Crowned-Pigeon (E), Southem Crowned-Pigeon (E), Paloma crestada de

Scheepmaker (S), Goura de Scheepmaker (F), Goura de Sclater (F) II B

Goura victoria: Victoria Crowned-Pigeon (E), Victoria Crownedpigeon (E), Victoria Goura (E), Paloma crestada victoria (S), Goura de Victoria (F) II B

Gouras (E/S): Goura spp. II B

Graceful Chameleon (E): Chamaeleo gracilis II R

Graceful Crag Lizard (E): Pseudocordylus capensis II R

Grace's Emerald (E): Chlorostilbon bracei II B

Gracile Chameleon (E): Chamaeleo gracilis II R

Gracula religiosa: Hill Myna (E), Miná de la India (S), Mainate religieux (F), Merles des Indes (F) II B

Grampus (F): Grampus griseus II M

Grampus griseus: Grey Dolphin (E), Risso's Dolphin (E), Delfín de Risso (S), Fabo calderón (S), Dauphin de Risso (F), Grampus (F) $11 \mathrm{M}$

Gran autillo veliblanco (S): Otus leucotis II B

Gran búho etiópico (S): Bubo lacieus II B

Gran civeta de la India (S): Viverra zibetha III M

Gran lemur ardilla (S): Cheirogaleus major I M

Gran sifaka blanco (S): Propithecus verreauxi I M

Grand bandicoot-lapin (F): Macrotis lagotis I M

Grand cacatoès à huppe jaune (F): Cacatua galerita II B

Grand cheirogale (F): Cheirogaleus major I M

Grand Comoro Scops-Owl (E): Otus pauliani II B

Grand dauphin (F): Tursiops truncatus II M

Grand eclectus (F): Eclectus roratus II B

Grand fourmilier (F): Myrmecophaga iridacnla II M

Grand galago (F): Otolemur crassicaudarus II M

Grand hapalémur (F): Hapalemur simus $\mathbf{I} \mathbf{M}$

Grand hocco (F): Crax rubra III B

Grand hocco à bec rasoir (F): Mitu mitu I B

Grand lépilémur (F): Lepilemur mustelinus I $\mathrm{M}$

Grand pangolin (F): Manis gigantea II M

Grand pangolin de l'Inde (F): Manis crassicaudata II M

Grand paradisier (F): Paradisaea apoda II B

Grand scorpian du Sénégal (F): Pandinus gambiensis II In

Grand téju (F): Tupinambis teguixin II R

Grand téjus (F): Tupinambis spp. II R

Grand-duc à aigrettes (F): Bubo poensis II B

Grand-duc africain (F): Bubo africanus II B

Grand-duc ascalaphe (F): Bubo ascalaphus II B

Grand-duc bruyant (F): Bubo sumairanus II B

Grand-duc d'Amérique (F): Bubo virginianus II B

Grand-duc de Coromandel (F): Bubo coromandus II B

Grand-duc de Shelley (F): Bubo shelleyi II B

Grand-duc de Verreaux (F): Bubo lacteus II B

Grand-duc des Philippines (F): Bubo philippensis II B

Grand-duc des Usambara (F): Bubo vosseleri II B

Grand-duc d'Europe (F): Bubo bubo II B

Grand-duc du Cap (F): Bubo capensis II B

Grand-duc du Népal (F): Bubo nipalensis II B

Grand-duc indien (F): Bubo bengalensis II B

Grand-duc tacheté (F): Bubo leucostictus II B

Grand-ducs (F): Bubo spp. II B

Grande aigrette (F): Casmerodius albus III 8

Grande mélampitte (F): Melampitta gigantea II B

Grande outarde (F): Otis tarda II B

Grande outarde arabe (F): Ardeotis arabs II B

Grande toupaie (F): Tupaia tana II M

Granular Poison Frog (F): Dendrobates granulifer 11 A

Grass Owl (E): Tyto capensis II B

Grasshopper Buzzard (E): Butastur rufipennis II B

Grasshopper Buzzard-Eagle (E): Butastur rufipennis II B

Grass-Owls (E): Tyto spp. II B

Grass-Parakeets (E): Neophema spp. III B

Gray Back (E): Eschrichrius robustus I M

Graydidascalus brachyurus: Short-tailed Parrot (E), Lorito colicorto

(S), Loro colicorto (S), Caique à queue courte (F) II B

Gray's Beaked Whale (E): Mesoplodon grayi II M

Gray's Brush-turkey (E): Macrocephalon maleo I B

Gray's Goshawk (E): Accipiter henicogrammus II B 
Gray's Malimbe (E): Malimbus nirens III B Gray's Monitor (E): Varanus olivaceus II R Great Argus (E): Argusianus argus II B Great Black Hawk (E): Buteogallus urubitunga II B Great Blue Turaco (E): Corythaeola cristata III B Great Bustard (E): Oris tarda II B Great Curassow (E): Crax rubra III B Great Egret (E): Casmerodius albus III B Great Goura (E): Goura cristata II B Great Green Macaw (E): Ara ambigua I B Great Grey Owl (E): Strix nebulosa II B Great Hombill (E): Buceros bicomis I B Great Horned Owl (E): Bubo virginianus II B Great Inagua Island Dwarf Boa (E): Tropidophis canus II R Great Indian Bustard (E): Ardeotis nigriceps I B Great Indian Hornbill (E): Buceros bicomis I B Great Indian Rhinoceros (E): Rhinoceros unicomis I M Great Philippine Eagle (E): Pithecophaga jefferyi I B Great Pied Hombill (E): Buceros bicomis I B Great Sapphirewing (E): Pterophanes cyanopterus II B Great Sparrowhawk (E): Accipiter melanoleucus II B Great Sturgeon (E): Huso huso II F

Great Tibetan sheep (E): Ovis ammon hodgsonii I M Great White Egret (E): Casmerodius albus III B Great-billed Hermit (E): Phaethomis malaris II B Great-billed Parrot (E): Tanygnathus megalorynchos II B Greater Bamboo Lemur (E): Hapalemur simus I M Greater Bilby (E): Macrotis lagotis I M Greater Bird-of-paradise (E): Paradisaea apoda II B Greater Bushbaby (E): Otolemur crassicaudatus II M Greater Dwarf Lemur (E): Cheirogaleus major I M Greater Flamingo (E): Phoenicopterus ruber II B Greater Grison (E): Galictis virtala III M Greater Kestrel (E): Falco rupicoloides II B

Greater Mascarene Flying-fox (E): Pteropus niger II M Greater Melampitta (E): Melampitta gigantea II B

Greater Naked-tailed Amadillo (E): Cabassous tatouay III M Greater Padloper (E): Homopus femoralis II R

Greater Rabbit-eared Bandicoot (E): Macrotis lagotis I M Greater Rhea (E): Rhea americana II B

Greater Sooty-Owl (E): Tyto tenebricosa II B

Greater Sportive Lemur (E): Lepilemur mustelinus I M

Greater Spotted Eagle (E): Aquila clanga II B

Greater Stick-nest Rat (E): Leporillus conditor I M

Greater Streaked Lory (E): Chalcopsitta sintillata II B

Greater Sulphur-crested Cockatoo (E): Cacatua galerita II B

Greater Weasel Lemur (E): Lepilemur mustelinus I M

Greater White-nosed Monkey (E): Cercopithecus nictitans II M

Grèbe du lac Atilan (F): Podilymbus gigas I B

Grèbe géant (F): Podilymbus gigas I B

Grebes (E): Podicipedidae spp. I/NC B

Grèbes (F): Podicipedidae spp. I/NC B

Greek Tortoise (E): Testudo graeca II R

Green Anaconda (E): Eunectes murinus II R

Green Aracari (E): Pteroglossus viridis II B

Green Avadavat (E): Amandava formosa II B

Green Cactus Coral (E): Madracis decactis II in

Green Conure (E): Aratinga holochlora II B

Green Hanging-Parrot (E): Loriculus exilis II B

Green Hermit (E): Phaethornis guy II B

Green Iguana (E): Iguana iguana II R

Green Indigobird (E): Vidua chalybeata 111 B

Green Leek Partot (E): Polytelis swainsonii II B

Green Mango (E): Anthracothorax viridis II B

Green Monkey (E): Chlorocebus aethiops II M

Green Parakeet (E): Aratinga holochlora II B

Green Peafowl (E): Pavo muricus II B

Green Poison Frog (E): Dendrobates auratus II AMMinyobates viridis II A

Green Racquet-tail (E): Prioniturus luconensis II B

Green Riffle Shell (E): Epioblasma torulosa gubemaculum I In Green Rosella (E): Platycercus caledonicus II B

Green Singing Finch (E): Serinus mozambicus III B

Green Sturgeon (E): Acipenser medirostris II F

Green Thomtail (E): Popelairia conversii II B

Green Tree Pychon (E): Morelia viridis II R
Green Tree Snail (E): Papustyla pulcherrima II In Green Turtle (E): Chelonia mydas I R

Green Twinspot (E): Mandingoa nitidula III B

Green Violet-ear (E): Colibri shalassinus II B

Green-and-black Poison Frog (E): Dendrobates auratus II A Green-and-white Hummingbird (E): Amazilia viridicauda II B

Green-backed Firecrown (E): Sephanoides sephaniodes II B

Green-backed Twinspot (E): Mandingoa nitidula III B

Green-bellied Hummingbird (E): Amazilia viridigaster II B

Green-blossom Naiad (E): Epioblasma torulosa gubemaculum I In

Green-blossom Pearly Mussel (E): Epioblasma torulosa gubernaculum I In

Green-breasted Mango (E): Anthracoshorax prevostii II B

Green-breasted Mountain-gem (E): Lampornis sybillae II B

Green-cheeked Amazon (E): Amazona viridigenalis I B

Green-cheeked Conure (E): Pyrrhura molinae II B

Green-cheeked Parakeet (E): Pyrrhura molinae II B

Green-crowned Brilliant (E): Heliodoxa jacula II B

Green-crowned Racket-tailed Parrot (E): Prioniturus luconensis II B

Green-crowned Woodnymph (E): Thalurania fannyi II B

Green-fronted Hanging-Parrot (E): Loriculus tener II B

Green-fronted Hummingbird (E): Amazilia viridifrons II B

Green-fronted Lancebill (E): Doryfera ludovicae II B

Green-headed Racket-tailed Parrot (E): Prioniturus luconensis II B Greenish Puffleg (E): Haplophaedia aureliae II B

Greenland Right Whale (E): Balaen r mysticetus I M

Green-naped Lorikeet (E): Trichoglossus haematodus II B

Green-necked Peafowl (E): Pavo muticus II B

Green-Pigeons (E): Treron spp. III/NC B

Green-rumped Parrotlet (E): Forpus passerinus II B

Greenshanks (E): Tringa spp. VNC B

Green-tailed Emerald (E): Chlorostilbon alice II B

Green-tailed Goldenthroat (E): Polytmus theresiae II B

Green-tailed Trainbearer (E): Lesbia nuna II B

Green-throated Carib (E): Eulampis holosericeus II B

Green-throated Mango (E): Anthracothorax viridigula II B

Green-throated Mountain-gem (E): Lampomis viridipallens II B

Green-winged King-Parrot (E): Alisterus chloropterus II B

Green-winged Macaw (E): Ara chloroprerus II B

Green-winged Teal (E): Anas crecca III B

Grenouille à incubation gastrique (F): Rheobatrachus vitellinus II A

Grenouille de Zetek (F): Atelopus varius zeteki 1 A

Grenouille du Bengale (F): Euphlyctis hexadactylus II A

Grenouille hexadactyle (F): Euphlyctis hexadactylus II A

Grenouille plate à incubation gastrique (F): Rheobatrachus silus II A

Grenouille tigre (F): Hoplobatrachus tigerinus II A

Grenouille tigrée (F): Hoplobatrachus rigerinus II A

Grenouilles à incubation gastrique (F): Rheobatrachus spp. II A

Grevy's Zebra (E): Equus grevyi I M

Grey Buzzard-Eagle (E): Geranoaetus melanoleucus II B

Grey Chameleon (E): Chamaeleo chapini II R

Grey Crowned Crane (E): Balearica regulorum II B

Grey Cuscus (E): Phalanger orientalis II M

Grey Dolphin (E): Grampus griseus II M

Grey Falcon (E): Falco hypoleucos II B

Grey Flying-fox (E): Pteropus griseus II M

Grey Frog Hawk (E): Accipiter soloensis II B

Grey Gentle Lemur (E): Hapalemur griseus I M

Grey Gibbon (E): Hylobates muelleri I M

Grey Goshawk (E): Accipiter novaehollandiae II B

Grey Hawk (E): Asturina plagiala II B

Grey Junglefowl (E): Gallus sonneratii II B

Grey Kestrel (E): Falco ardosiaceus II B

Grey Langur (E): Semnopithecus entellus I M

Grey Leaf Monkey (E): Presbytis hosei II M

Grey Monitor (E): Varanus griseus I R

Grey Mouse-lemur (E): Microcebus murinus I M

Grey Owl (E): Strix nebulosa II B

Grey Parrot (E): Psittacus erithacus II B

Grey Peacock-Pheasant (E): Polyplectron bicalcaratum II B

Grey Plantain-eater (E): Crinifer piscator III B

Grey Sea Eagle (E): Haliaeetus albicilla I B

Grey Singing Finch (E): Serinus leucopygius III B

Grey Snub-nosed Monkey (E): Pygathrix brelichi I M

Grey Whale (E): Eschrichtius robustus I M

Grey Wolf (E): Canis lupus VI M 
Grey Wood-Pigeon (E): Columba unicincta III B Grey Zorro (E): Pseudalopex griseus II M

Grey-backed Hawk (E): Leucoptemis occidentalis II B Grey-backed Sportive Lemur (E): Lepilemur dorsalis I M Grey-bellied Comet (E): Taphrolesbia griseiventris II B Grey-bellied Goshawk (E): Accipiter poliogaster II B Grey-bellied Hawk (E): Accipiter poliogaster II B Grey-bellied Tragopan (E): Tragopan blythii I B Grey-breasted Parakeet (E): Myiopsilla monachus II B Grey-breasted Partridge (E): Arborophila orientalis III B Grey-breasted Sabrewing (E): Campyloptenus largipennis II B Grey-cheeked Mangabey (E): Lophocebus albigena II M Grey-cheeked Parakeet (E): Brotogeris pyrrhopterus II B Grey-chinned Hermit (E): Phaethomis griseogularis II B Grey-crowned Negrofinch (E): Nigrita canicapilla III B Grey-faced Buzzard (E): Butastur indicus II B Grey-faced Buzzard-Eagle (E): Butastur indicus II B Grey-headed Fish-eagle (E): Ichthyophaga ichthyaetus II B Grey-headed Fishing Eagle (E): Ichthyophaga ichthyaetus II B Grey-headed Flying-fox (E): Pteropus poliocephalus II M Grey-headed Goshawk (E): Accipiter poliocephalus II B Grey-headed Kite (E): Leptodon cayanensis II B Grey-headed Lovebird (E): Agapomis canus II B Grey-headed Negrofinch (E): Nigrisa canicapilla III B Grey-headed Olive-back (E): Nesocharis capistrata III B Grey-headed Parakeet (E): Psintacula finschii II B Grey-headed Sparrow (E): Passer griseus III B Grey-hooded Parakeet (E); Bolborhynchus aymara II B Grey-lined Hawk (E): Asturina nitida II B Grey-necked Picathartes (E): Picathartes oreas I B Grey-necked Rockfowl (E): Picathartes oreas I B Grey-throated Goshawk (E): Accipiter novaehollandiae II B Griffons (E): Gyps spp. II B

Grisón (S): Galictis vittata III M

Grison d'Allemand (F): Galictis vittata III M

Grisons (E); Galictis spp. III/NC B

Grivet Monkey (E): Chlorocebus aethiops II M

Grizzled Giant Squirrel (E): Ratufa macroura II M

Grizzled Leaf Monkey (E): Presbytis comata II M

Grizzled Tree-kangaroo (E): Dendrolagus inusius II M

Grizzly (F): Ursus arctos I/I M

Grizzly Bear (E): Ursus arctos I/II M

Grooved Mountain Tortoise (E): Homopus areolarus II R

Grooved Tortoise (E): Geochelone sulcata II R

Grosbeak Weaver (E): Amblyospiza albifrons III B

Grosbeaks (E): Amblyospiza albifrons/Emberizidae spp./Fringillidae $\operatorname{spp}$. III/NC B

Gros-bec à front blanc (F): Amblyospiza albifrons III B

Gros-bec ponceau à venure noir (F): Pyrenestes ostrinus III B

Gros-bec sanguin (F): Spermophaga haematina III B

Grosse cateau verte de Maurice (F): Psittacula echo I B

Ground boas (E): Acrantophis spp/Candoia spp/Tropidophis spp. I/II R

Ground iguanas (E): Cyclura spp. I R

Ground iguanas (E): Cyclura spp/Cienosaura spp/Leiocephalus spp. I/NC R

Ground Parakeet (E): Pezoporus wallicus I B

Ground Parrot (E): Pezoporus wallicus I B

Ground Python (E): Loxocemus bicolor II R

Grue à cou blanc (F): Grus vipio I B

Grue à cou noir (F): Grus nigricollis I B

Grue antigone (F): Grus antigone II B

Grue antigone tropicale (F): Grus antigone II B

Grue blanche (F): Grus americana $1 \mathrm{~B}$

Grue blanche d'Amérique (F): Grus americana I B

Grue blanche d'Asie (F): Grus leucogeranus I B

Grue blanche du Japon (F): Grus japonensis I B

Grue bleue (F): Grus paradisea II B

Grue brolga (F): Grus rubicunda II B

Grue canadienne (F): Grus canadensis III B

Grue canadienne de Cuba (F): Grus canadensis nesiores I B

Grue canadienne du Mississippi (F): Grus canadensis pulla I B

Grue caronculée (F): Grus caruncularus II B

Grue cendrée (F): Grus grus II B

Grue couronnée de Cap (F): Balearica regulorum II B
Grue couronnée de l'Afrique del'Ouest et du Soudan: Balearica pavonina II B

Grue couronnée de l'Afrique du Sud et de l'Est (F): Balearica regulorum II B

Grue d'Australie (F): Grus rubicunda II B

Grue de Mandchourie (F): Grus japonensis I B

Grue de paradis (F): Grus paradisea II B

Grue de Sibérie (F): Grus leucogeranus I B

Grue demoiselle (F): Grus virgo II B

Grue du Canada (F): Grus canadensis $\mathbf{I} / \mathrm{I}$ B

Grue moine (F): Grus monacha I B

Grue royale (F): Balearica regulorum II B

Grues (F): Gruidae spp. I/II B

Gruidae spp.: Cranes (E), Grullas (S), Grúidos (S), Grues (F) I/I B

Grúidos (S): Gruidae spp. VII B

Grulla americana (S): Grus americana I B

Grulla australiana (S): Grus rubicunda II B

Grulla azul (S): Grus paradisea II B

Grulla blanca asiática (S): Grus leucogeranus I B

Grulla blanco cuello (S): Grus antigone II B

Grulla brolga (S): Grus rubicunda II B

Grulla canadiense ( S): Grus canadensis I/II B

Grulla carunculada (S): Grus carunculatus II B

Grulla cenicienta (S): Grus canadensis I/II B

Grulla cenicienta de Cuba (S): Grus canadensis nesiotes I B

Grulla cenicienta del Misisipi (S): Grus canadensis pulla I B

Grulla común (\$): Grus grus II B

Grulla coronada (S): Balearica pavonina II B

Grulla coronada cuelligris (S): Balearica regulorum II B

Grulla coronada cuellinegra (S): Balearica pavonina II B

Grulla coronada del Africa Occidental (S): Balearica pavonina II B

Grulla coronada sudafricana (S): Balearica regulorum II B

Grulla cuelliblanca (S): Grus vipio I B

Grulla cuellinegra (S): Grus nigricollis I B

Grulla darnisela (S): Grus virgo II B

Grulla de Manchuria (S): Grus japonensis I B

Grulla de paraiso (S): Grus paradisea II B

Grulla gritona (S): Grus americana I B

Grulla manchú (S): Grus juponensis I B

Grulla monje (S): Grus monacha I B

Grulla monjita (S): Grus monacha I B

Grulla monuna (S): Grus virgo II B

Grulla sarus (\$): Grus antigone II B

Grulla siberiana (S): Grus leucogeranus I B

Grulla trompetera (S): Grus americana I B

Grulla zarzo (S): Grus carunculatus II B

Grullas (S): Gruidae spp. VII B

Grus americana: Whooping Crane (E), Grulla americana (S), Grulla gritona (S), Grulla trompetera (S), Grue blanche (F), Grue blanche d'Amérique (F) I B

Grus antigone: Sarus Crane (E), Grulla blanco cuello (S), Grulla sarus (S), Grue antigone (F), Grue antigone tropicale (F) II B

Grus canadensis: Sandhill Crane (E), Grulla canadiense (S), Grulla cenicienta (S), Grue canadienne (F), Grue du Canada (F) I/II B

Grus canadensis nesiotes: Cuban Sandhill Crane (E), Grulla cenicienta de Cuba (S), Grue canadienne de Cuba (F) I B

Grus canadensis pulla: Mississippi Sandhill Crane (E), Grulla cenicienta del Misisipi (S), Grue canadienne du Mississippi (F) I B

Grus carunculatus: Wattled Crane (E), Grulla carunculada (S), Grulla zarzo (S), Grue caronculée (F) II B

Grus grus: Common Crane (E), Grulla común (S), Grue cendrée (F) II B

Grus japonensis: Japanese Crane (E), Manchurian Crane (E), Redcrowned Crane (E), Grulla de Manchuria (S), Grulla manchú (S), Grue blanche du Japon (F), Grue de Mandchourie (F) I B

Grus leucogeranus: Siberian Crane (E), Siberian White Crane (E), Snow Crane (E), Grulla blanca asiática (S), Grulla siberiana (S), Grue blanche d'Asie (F), Grue de Sibérie (F), Leucogéranne (F) I B

Grus monacha: Hooded Crane (E), Grulla monje (S), Grulla monjita (S), Grue moine (F) I B

Grus nigricollis: Black-necked Crane (E), Tibetan Crane (E), Grulla cuellinegra (S), Grue à cou noir (F) I B

Grus paradisea: Blue Crane (E), Stanley Crane (E), Grulla azul (S), Grulla de paraiso (S), Grue bleve (F), Grue de paradis (F) II B 
Grus rubicunda: Brolga (E), Grulla australiana (S), Grulla brolga (S), Grue brolga (F), Grue d'Australie (F) II B

Grus vipio: White-naped Crane (E), White-necked Crane (E), Grulla cuelliblanca (S), Grue à cou blanc (F) I B

Grus virgo: Demoiselle Crane (E), Grulla damisela (S), Grulla moruna (S), Demoiselle de Numidie (F), Grue demoiselle (F) II B

Guacamayito azul (S): Cyanopsitta spixii I B

Guacamayo acollarado (S): Ara auricollis II B

Guacamayo aliverde (S): Ara chloropterus II B

Guacamayo amarillo (S): Ara glaucogularis I B

Guacamayo ambiguo (S): Ara ambigua I B

Guacamayo azul (S): Anodorhynchus hyacinthinus I B

Guacamayo azul y amarillo (S): Ara ararauna II B

Guacamayo azulamarillo (S): Ara ararauna II B

Guacamayo bandera (S): Ara macao I B

Guacamayo barbazul (S): Ara glaucogularis I B

Guacamayo barriga roja (S): Ara manilata II B

Guacamayo cabeciazul (S): Ara couloni II B

Guacamayo caninde (S): Ara glaucogularis I B

Guacamayo cobal to (S): Anodorhynchus leari I B

Guacamayo de Cochabamba (S): Ara rubrogenys I B

Guacamayo de Lear (S): Anodorhynchus leari I B

Guacamayo de Spix (S): Cyanopsinta spixii I B

Guacamayo enano (S): Ara nobilis II B

Guacamayo frentirroja (S): Ara rubrogenys I B

Guacamayo glauco (S): Anodorhynchus glaucus I B

Guacamayo jacinto (S): Anodorhynchus hyacinthinus I B

Guacamayo macao (S): Ara macao I B

Guacamayo maracaní (S): Ara maracana I B

Guacamayo militar (S): Ara militaris I B

Guacamayo noble (S): Ara nobilis II B

Guacamayo rojo (S): Ara chloropterus II B

Guacamayo severo (S): Ara severa II B

Guacamayo ventrirrojo (S): Ara manilata II B

Guacamayo verde (S): Ara militaris I B

Guacamayo verde mayor (S): Ara ambigua I B

Guacamayo violáceo (S): Anodorhynchus glaucus I B

Guacamayos (S): Anodorhynchus spp/Ara spp/Cyanopsitta spixii III B

Guadalupe Caracara (E): Polyborus lutosus II B

Guadalupe Fur Seal (E): Arcrocephalus townsendi I M

Guaiabero (E): Bolbopsitracus lunulatus II B

Guajolote ocelado (S): Agriocharis ocellata III B

Guam Flying-fox (E): Pteropus iokudae II M

Guam Fruit Bat (E): Pteropus tokudae II M

Guan comudo (S): Oreophasis derbianus I B

Guanaco (E/S/F): Lama guanicoe II M

Guans (E): Cracidae spp. 1/III/NC B

Guaraguao (S): Buteo jamaicensis 11 B

Guarahuau cordillerano (S): Phalcoboenus albogularis II B

Guariba Peludo (S): Alouatta palliata I M

Guarouba (F): Aratinga guarouba I B

Guaruba guarouba $=$ Aratinga guarouba

Guatemalan Howler (E): Alouatta pigra I M

Guatemalan Red Brocket (E): Mazama americana cerasina III M

Guatemalan White-tailed Deer (E): Odocoileus virginianus mayensis III $M$

Gubarte (S): Megaptera novaeangliae I M

Gubematrix cristaia: Yellow Cardinal (E), Cardenal amarillo (S) Cardinal vert (F), Commandeur huppé (F) II B

Guemal (S): Hippocamelus antisensis I M

Guémal péruvien (F): Hippocamelus antisensis I M

Guemals (E): Hippocamelus spp. I M

Guémaux (F): Hippocamelus spp. I M

Guenon couronné (F): Cercopithecus pogonias II M

Guenons (E): Cercopithecus spp. I//I M

Guépard (F): Acinonyx jubarus I M

Guepardo (\$): Acinonyx jubatus I M

Guereza (E): Colobus guereza II M

Guerrero Orange Legs (E): Brachypelma boehmei II in

Guianan Cock-of-the-rock (E): Rupicola rupicola II B

Guianan Crested Eagle (E): Morphnus guianensis II B

Guianian River Dolphin (E): Sotalia fluviatilis I M

Guib d'eau (F): Tragelaphus spekii III M

Guibe's Chameleon (E): Chamaeleo guibei II R
Guigna (F): Oncifelis guigna II M

Guina (S): Oncifelis guigna II M

Guincho (S): Pandion haliaetus II B

Guinea Baboon (E): Papio hamadryas II M

Guinea Turaco (E): Tauraco persa II B

Guineafowl (E): Acryllium vulturinum/Agelastes spp/Guttera $\operatorname{spp}$ /Numida meleagris III/NC B

Guirin (S): Dendrocygna autumnalis 111 B

Guizhou Snub-nosed Monkey (E): Pygathrix brelichi I M

Gulf of Califomia Harbour Porpoise (E): Phocoena sinus I M

Gulf Porpoise (E): Phocoena sinus I M

Gulf Ridley (E): Lepidochelys kempi I R

Gulf Stream Beaked Whale (E): Mesoplodon europaeus II M

Gulls (E): Laridae spp. INC B

Gundlach's Hawk (E): Accipiter gundlachi II B

Gumey's Eagle (E): Aquila gumeyi II B

Gumey's Hawk (E): Buteo poecilochrous II B

Gumey's Pitta (E): Pitta gumeyi I B

Guyana Caiman Lizard (E): Dracaena guianensis II R

Guynia annulara: II In

Gymnogène d'Afrique (F): Polyboroides typus II B

Gymnogène de Madagascar ( $\mathrm{F}$ : : Polyboroides radiatus II B

Gymnogenes (E): Polyboraides spp. II B

Gymnogènes (F): Polyboroides spp. II B

Gymnoglaux lawrencii = Otus lawrencii

Gymnogyps californianus: California Condor (E), Cóndor californiano (S), Cóndor de California (S), Condor de Californie (F) I B

Gypaète barbu (F): Gypaetus barbatus II B

Gypaetus barbatus: Bearded Vulture (E), Lammergeier (E), Quebrantahuesos (S), Gypaète barbu (F) II B

Gypohierax angolensis: Palm-nut Vulture (E), Vulturine Fish-Eagle (E), Buitre palmero (S), Palmiste africain (F) II B

Gypopsitta vulturina: Vulturine Parrot (E), Lorito vulturino (S), Cäque vautourin (F) II B

Gyps africain (F): Gyps bengalensis II B

Gyps africanus: African White-backed Vulture (E), White-backed Vulture (E), Buitre dorsiblanco africano (S), Vautour africain (F) II B

Gyps bengalensis: Asian White-backed Vulture (E), Oriental Whitebacked Vulture $(E)$, White-rumped Vulture $(E)$, Buitre dorsiblanco bengalí (S), Buitre leonado bengalés (S), Gyps africain (F), Vautour chaugoun (F) II B

Gyps coprotheres: Cape Griffon (E), Cape Vulture (E), Buitre el cabo (S), Vautour chassefiente (F) II B

Gyps fulvus: Eurasian Griffon (E), Eurasian Griffon Vulture (E), Buitre leonado (S), Buitre leonado común (S), Vantour fauve (F) II B

Gyps himalayensis: Himalayan Griffon (E), Himalayan Griffon Vulture (E), Buitre del Himalaya (S), Vautour de l'Himalaya (F) II B

Gyps indicus: Long-billed Griffon (E), Long-billed Vulture (E), Buitre picofino (S), Vautour indien (F) II B

Gyps rueppellit: Rueppell's Griffon (E), Rueppell's Griffon Vulture (E), Rueppell's Vulture (E), Buitre moteado (S), Vautour de Rüppell (F) II B

Gyr Falcon (E): Falco rusticolus I B

Gyrfalcon (E): Falco rusticolus I B

Gyropora africana: II In

Gyrosmilia interrupta: II In

Hada coronimorada (S): Heliothryx barroti II B

Hada gorrimorada (S): Heliothryx barroti II B

Hada orejinegro (S): Heliothryx aurita II B

Hadada Ibis (E): Bostrychia hagedash III B

Hagedashia hagedash = Bostrychia hagedash

Hairy-eared Dwarf Lemur (E): Allocebus trichotis I M

Hairy-nosed Otter (E): Lutra sumatrana II M

Haitian Boa (E): Epicrates striatus II R

Haitian Dwarf Boa (E): Tropidophis haetianus II R

Haitian Ground Boa (E): Tropidophis haetianus II R

Haitian Vine Boa (E): Epicrates gracilis II R

Halcón abejero (S): Pernis apivorus II B

Halcón aleto (S): Falco femoralis II B

Halcón aplomado (S): Falco femoralis II B

Halcón azulado (S): Falco femoralis II B

Halcón berigora (S): Falco berigora II B 
Halcón blancuzco (S): Falco peregrinus $1 \mathrm{~B}$

Halcón borni (S): Falco biarmicus II B

Halcón común (S): Falco peregrinus I B

Halcón cuellimufo (S): Micrastur ruficollis II B

Halcón de cabeza roja (S): Falco chicquera II B

Halcón de Cuvier (S): Falco cuvieri II B

Halcón de Eleonora (S): Falco eleonorae II B

Halcón de las praderas (S): Falco mexicanus II B

Halcón de lomo pizarreño (S): Micrastur mirandollei II B

Halcón de Nueva Zelandia (S): Falco novaezeelandiae II B

Halcón enano de la India (S): Microhierax caerulescens II B

Halcón gerifalte (S): Falco nusticolus I B

Halcón golondrina (S): Falco nufigularis II B

Halcón gris (S): Falco hypoleucos II B

Halcón gris de Australia (S): Falco hypoleucos II B

Halcón guaco (S): Herpeiotheres cachinnans II B

Halcón guaicurú (S): Herpetotheres cachinnans II B

Halcón macagua (S): Herpetotheres cachinnans II B

Halcón maori (S): Falco novaezeelandiae II B

Halcón mejicano (S): Falco mexicanus II B

Halcón migratorio (S): Falco columbarius II B

Halcón murcielaguero (S): Falco rufigularis II B

Halcón negro (S): Falco subniger II B

Halcón negro de Australia (S): Falco subniger II B

Halcón palomero (S): Falco columbarius II B

Halcón palomero (S): Micrastur ruficollis II B

Halcón palomero del sur (S): Micrastur gilvicollis II B

Halcón pardo (S): Falco concolor II B

Halcón pechianaranjado (S): Falco deiroleucus II B

Halcón pechirrojo (S): Falco deiroleucus II B

Halcón pechimufo (S): Falco deiroleucus II B

Halcón perdiguero (S): Falco femoralis II B

Halcón peregrino (S): Falco peregrinus I B

Halcón peregrino de Babilonia (S): Falco pelegrinoides I B

Halcón pizarroso (S): Falco concolor II B

Halcón plomizo (S): Falco deiroleucus II B

Halcón plomizo menor (S): Falca rufigularis II B

Halcón pradeño (S): Falco mexicanus II B

Halcón primito (S): Falco sparverius II B

Halcón real (S): Falco peregrinus I B

Halcón reidor (S): Herpetotheres cachinnans II B

Halcón sacre (S): Falco cherrug II B

Halcón semiacollarado (S): Micrastur semitorqualus II B

Halcón taita (S): Falco fasciinucha II B

Halcón viajero (S): Falco peregrinus I B

Halcón yággar (S): Falco jugger I B

Halconcito (S): Falco spanerius II B

Halconcito africano (S): Polihierax semitorquasus II B

Halconcito argentino (S): Spiziapteryx circumcinctus II B

Halconcito asiático (S): Polihierax insignis II B

Halconcito australiano (S): Falco longipennis II B

Halconcito colilargo de Asia (S): Polihierar insignis II B

Halconcito común (S): Falco sparverius II B

Halconcito de Bomeo (S): Microhierax latifrons II B

Halconcito de las Filipinas (S): Microhierax erythrogenys II B

Halconcito gris (S): Spiziapteryx circumcinctus II B

Halconcito gris americano (S): Spiziapteryx circumcinctus II B

Halconcito malayo (S): Microhierax fringillarius II B

Halcón-montés agavilanado (S): Micrastur ruficollis II B

Halcón-montés cabecigrís (S): Micrastur gilvicollis II B

Halcón-montés collarejo (S): Micrastur semitorquatus II B

Halcón-montés de Buckley (S): Micrastur buckleyi II B

Halcón-montés dorsigrís (S): Micrastur mirandollei II B

Halcón-montés plomizo (S): Micrastur plumbeus II B

Halcón-selvático collarejo (S): Micrastur semitorquatus II B

Haliaeetus albicilla: Grey Sea Eagle (E), White-tailed Eagle (E),

Pigargo coliblanco (S), Pigargo coliblanco de Groenlandia (S),

Pigargo europeo (S), Pygargue commun (F), Pygargue à queue blanche (F) I B

Haliaeetus leucocephalus: Bald Eagle (E), White-headed Eagle (E), Aguila cabeciblanca (S), Aguila cabeza blanca (S), Pigargo americano (S), Pigargo cabeciblanco (S), Pigargo cabeciblanco meridional (S), Aigle à tête blanche (F), Pygargue à tête blanche (F) I B
Halineetus leucogaster. White-bellied Fish-Eagle (E), White-bellied Sea-Eagle (E), Pigargo de vientre blanco (S), Pigargo oriental (S), Pygargue blagre (F), Pygargue à ventre blanc (F) II B

Haliaeetus leucoryphus: Band-tailed Fish-Eagle (E), Pallas's FishEagle (E), Pallas's Sea-Eagle (E), Pigargo de Pallas (S), Pygargue de Pallas (F) II B

Haliaeelus pelagicus: Steller's Sea-Eagle (E), Pigargo de Steller (S), Pigargo gigante (S), Pygargue de Steller (F), Pygargue empereur (F) II B

Haliaeetus sanfordi: Sanford's Fish-eagle (E), Sanford's Sea-Eagle (E), Solomon Fish-Eagle (E), Solomon Sea-Eagle (E), Pigargo de Sanford (S), Pigargo de las Salomón (S), Pygargue de Sanford (F) II B

Haliaeetus vocifer, African Fish-Eagle (E), River Eagle (E), Pigargo vocinglero $(S)$, Aigle pêcheur africain $(F)$. Pygargue vocifere $(F)$ II B

Haliaeetus vociferoides: Madagascar Fish-Eagle (E), Pigargo de Madagascar (S), Pigargo malgache (S), Pygargue de Madagascar (F) II B

Haliastur indus: Brahminy Kite (E), Milano brahmán (S), Milan sacré (F) II B

Haliastur sphenurus: Whistling Kite (E), Milano silbador (S), Milan siffleur (F) II B

Haliglossa $=$ Herpolitha

Halomitra clavator: II In

Halomitra pileus: II In

Haloseris $=$ Leptoseris

Hamadryad (E): Ophiophagus hannah II R

Hamadryade (F): Ophiophagus hannah II R

Hamadryas (F): Papio hamadryas II M

Hamadryas Baboon (E): Papio hamadryas II M

Hamilton's Terrapin (E): Geoclemys hamiltonii I R

Hamirostra melanostemon: Black-breasted Buzzard (E), Black breasted Kite (E), Milano pechinegro (S), Milan à plastron (F) II B

Hanang Homless Chameleon (E): Bradypodion uthmoelleri II R

Hanging-Parrots (E): Loriculus spp. II B

Hangul (E/F): Cervus elaphus hanglu I M

Hanuman Langur (E): Semnopithecus entellus I M

Hapalemur aureus: Golden Bamboo Lemur (E), Golden Lemur (E) Lemur cariancho (S), Hapalémur doré (F) I M

Hapalémur doré (F): Hapalemur aureus I M

Hapalemur gallieni = Hapalemur simus

Hapalémur gris (F): Hapalemur griseus I M

Hapalemur griseus: Grey Gentle Lemur (E), Lemur cariancho (S), Hapalérnur gris (F), Petit hapalémur (F) I M

Hapalémur simien (F): Hapalemur simus I M

Hapalemur simus: Broad-nosed Gentle Lemur (E), Greater Bamboo Lemur (E), Lemur cariancho (S), Grand hapalémur (F). Hapalémur simien (F) I M

Hapalemur spp.: I M

Hapalopsittaca amazonina: Rusty-faced Parrot (E). Lorito amazonino (S), Perico multicolor (S), Caüque à face rouge $(F)$ II $\mathbf{B}$

Hapalopsittaca amazonina fuertesi = Hapalopsittaca fuertesi

Hapalopsittaca amazonina pyrrhops = Hapalopsittaca pyrrhops

Hapalopsittaca fuertesi: Fuertes's Partot (E), Indigo-winged Parrot (E), Lorito de Fuertes (S), Caique de Fuertes (F) II B

Hapalopsittaca melanotis: Black-eared Parrot (E), Black-winged Parrot (E), Lorito alinegro ( $S$ ), Caïque à ailes noires (F) II B

Hapalopsituaca pyrrhops: Red-faced Parrot (E), Lorito ecuatoriano (S), Caïque de Salvin (F) II B

Haplophaedia aureliae: Greenish Puffleg (E), Zamarrito verdoso (S), Érione d'Aurélie (F) II B

Haplophaedia lugens: Hoary Puffleg (E), Zamarrito canoso (S), Érione givrée (F) II B

Haplophyllia $=$ Gardineria

Harbour Porpoise (E): Phocoena phocoena II M

Hard Head (E): Eschrichiius robustus I M

Hardwick's Civet (E): Hemigalus derbyanus II M

Hardwick's Spiny-tailed Lizard (E): Uromastyx hardwickii II R

Hardy's Pygmy-Owl (E): Glaucidium hardyi II B

Hares (E): Caprolagus hispidus/Lepus spp. I/NC M

Hare-wallabies (E): Lagorchestes spp/Lagostrophus fasciatus I/NC M

Harfang des neiges (F): Nycrea scandiaca II B 
Harlequin Monitor (E): Varanus rudicollis II $R$

Harlequin Poison Frog (E): Dendrobates histrionicus II A

Harpagus bidentatus: Double-toothed Kite (E), Elanio bidentado (S), Gavilán bidente (S), Milano bidentado (S), Milan bidenté (F) II B

Harpagus diodon: Rufous-thighed Kite (E), Elanio muslirrufo (S), Milano dentado (S), Milano muslimufo (S), Milan diodon (F) II B

Harpía (S): Harpia harpyja I B

Harpia harpyja: American Harpy Eagle (E), Harpy Eagle (E), Aguila arpía (S), Aguila harpía (S). Arpía (S), Arpía mayor (S), Harpía (S), Aigle harpie (F), Harpie féroce (F), Harpye (F) I B

Harpie féroce (F): Harpia harpyja I B

Harpie huppé (F): Morphnus guianensis II B

Harpies (F): Harpia harpyja/Morphnus guianensis I/I B

Harpy Eagle (E): Harpia harpyja I B

Harpye (F): Harpia harpyja I B

Harpyhaliaetus coronatus: Crowned Eagle (E), Crowned Solitary Eagle (E), Aguila coronada (S), Aguila de Azara (S), Buse couronné (F) II B

Harpyhaliaetus solitarius: Black Solitary Eagle (E), Solitary Eagle (E), Aguila solitaria (S), Buse solitaire (F) II B

Harpyopsis novaeguineae: New Guinea Eagle (E), New Guinea Harpy Eagle (E), Arpia papúa (S), Aigle de Nouvelle-Guinée (F) II $\mathrm{B}$

Harrier-Eagles (E): Circaetus spp. II B

Harrier-Hawks (E): Polyboroides spp. II B

Harriers (E): Circus spp. II B

Harris's Hawk (E): Parabuteo unicinctus II B

Hartlaub's Bustard (E): Eupodotis hartlaubii II B

Hartlaub's Duck (E): Pteronena hartaubii III B

Hartlaub's Turaco (E): Tauraco harllaubi II B

Hartmann's Mountain Zebra (E): Equus zebra hartmannae II M

Hatteria de Günther (F): Sphenodon guniheri I R

Hatteria ponctué (F): Sphenodon punctatus I R

Hausse-col à queue rubanée (F): Astrapia stephaniae II B

Hausse-col de Rothschild (F): Astrapia rothschildi 11 B

Hausse-col doré (F): Astrapia nigra II B

Hausse-col splendide (F): Astrapia splendidissima II B

Haut-de-chausses à palettes (F): Ocreaus undenwoodii II B

Hawaiian Goose (E): Branta sandvicensis I B

Hawailan Hawk (E): Buteo solitarius II B

Hawaiian Monk Seal (E): Monachus schauinslandi I M

Hawk Owl (E): Surnia ulula II B

Hawk-Eagles (E): Spizaetus spp/Spizastur melanoleucus/Stephanoaetus coronarus II B

Hawk-headed Parrot (E): Deroptyus accipitrinus II B

Hawk-Owls (E): Ninox spp/Sumia ulula I/I B

Hawks (E): Accipitridae spp. VII B

Hawksbill Turtle (E): Eretmochelys imbricata I R

Heaviside's Dolphin (E): Cephalorhynchus heavisidii II M

Hector's Beaked Whale (E): Mesoplodon heciori II M

Hector's Dolphin (E): Cephalorhynchus hectori II M

Helarctos malayanus: Malayan Sun Bear (E), Sun Bear (E), Oso de sol (S), Oso malayo (S), Ours des cocotiers (F), Ours malais (F) I $\mathrm{M}$

Heliactin bilophum $=$ Heliactin cornuta

Heliactin comuta: Homed Sungem (E), Colibri aux huppes d'or (F) II B

Héliange à queue bleue (F): Heliangelus strophianus II B

Héliange clarisse (F): Heliangelus amethysticollis II B

Héliange de Mérida (F): Heliangelus spencei II B

Héliange mars (F): Heliangelus mavors II B

Héliange royale (F): Heliangelus regalis II B

Heliange tourmaline (F): Heliangelus exonis II B

Héliange violette (F): Heliangelus viola II B

Heliangelus amethysticollis: Amethyst-throated Sunangel (E), Angel de Sol amatista (S), Solangel gorjiamatista (S), Héliange clarisse (F) II B

Heliangelus exorris: Little Sunangel (E), Tourmaline Sunangel (E), Solangel turmalino (S), Héliange tourmaline (F) II B

Heliangelus mavors: Orange-throated Sunangel (E), Angel de Sol cuelliocre (S), Héliange mars (F) II B

Heliangelus micraster $=$ Heliangelus exortis

Heliangelus regalis: Royal Sunangel (E), Héliange royale (F) II B
Heliangelus spencei: Merida Sunangel (E), Angel de Sol de Mérida (S), Héliange de Mérida (F) II B

Heliangelus strophianus: Gorgeted Sunangel (E), Solangel de gorguera (S), Héliange à queue bleue (F) II B

Heliangelus viola: Purple-throated Sunangel (E), Solangel gorjipurpura (S), Héliange violette (F) II B

Hélianges (F): Heliangelus spp. II B

Heliastrea $=$ Montastrea

Helicolestes hamatus = Rostrhamus hamatus

Heliodoxa aurescens: Gould's Jewelfront (E), Colibrí cuellicastaño (S), Frentijoya (S), Brillant à bandeau bleu (F) II B

Heliodoxa branickii: Rufous-webbed Brilliant (E), Brillant de Branicki (F) II B

Heliodoxa frentiazul (S): Heliodoxa leadbeateri II B

Heliodoxa gularis: Pink-throated Brilliant (E), Brillante gorjirrosado (S), Brillant à gorge rose (F) II B

Heliodoxa imperatrix: Empress Brilliant (E), Brillante emperatriz (S), Brillant impératrice (F) II B

Heliodoxa jacula: Green-crowned Brilliant (E), Brillante coroniverde (S), Brillant fer-de-lance (F) II B

Heliodoxa leadbeateri: Violet-fronted Brilliant (E), Brillante frentivioleta (S), Heliodoxa frentiazul (S), Brillant à front violet (F) II B

Heliodoxa nubinoides: Fawn-breasted Brilliant (E), Brillante pechianteado (S), Brillant rubinoïde (F) II B

Heliodoxa schreibersii: Black-throated Brilliant (E), Brillante gorjinegro (S), Brillant à gorge noire (F) II B

Heliodoxa xanthogonys: Velvet-browed Brilliant (E), Velvetcrowned Brilliant (E), Colibri frentiverde (S), Brillant à couronne verte (F) II B

Heliofungia actiniformis: II In

Heliomaster constanii: Plain-capped Starthroat (E), Picolargo coronioscuro (S), Colibri de Constant (F) II B

Heliomaster furcifer: Blue-tufted Starthroat (E), Picaflor de Barbijo (S), Colibri d'Angèle (F) II B

Heliomaster longirostris: Long-billed Starthroat (E), Colibrí estrella picolargo (S), Heliomaster piquilargo (S), Picolargo coroniazu] (S), Colibri corinne (F) II B

Heliomaster piquilargo (S): Heliomaster longirostris II B

Heliomaster squamosus: Stripe-breasted Starthroat (E), Picaflor escamado (S), Colibri médiastin (F) II B

Heliopora coerulea: Blue Coral (E) II In

Helioseris = Leptoseris

Heliothryx aurita: Black-eared Fairy (E), Colibri hada orejazul (S), Hada orejinegro (S), Colibri oreillard (F) II B

Heliothryx barroti: Purple-crowned Fairy (E), Hada coronimorada (S), Hada gorrimorada (S), Colibri féérique (F) II B

Heliotrope-throated Hummingbird (E): Selasphorus flam,zula II B

Helmet Dolphin (E): Stenella clymene II M

Helmeted Chameleon (E): Chamaeleo hoehnelii II R

Helmeted Curassow (E): Pauxi pauxi III B

Helmeted Honeyeater (E): Lichenostomus melanops cassidix I B

Helmeted Hornbill (E): Buceros vigil I B

Helmeted Turaco (E): Tauraco corythaix II B

Helmeted Turtle (E): Pelomedusa subrifa III R

Heloderma spp.: Poisonous lizards (E), Hélodermes (F), Lézards perlés $(F)$, Lézards venimeux (F) II R

Heloderma horridum: Beaded Lizard (E), Escorpión (S), Lagarto de Cuentas (S), Héloderme granuleux (F), Héloderme horrible (F) II $\mathbf{R}$

Heloderma suspectum: Gila Monster (E), Monstruo de Gila (S), Lézard perlé (F), Monstre de Gila (F) II R

Héloderme granuleux (F): Heloderma horridum II R

Héloderme horrible (F): Heloderma horridum II R

Hélodermes (F): Heloderma spp. II R

Hémigale de Derby (F): Hemigalus derbyanus II M

Hemigalus derbyanus: Banded Musang (E), Banded Palm Civet (E) Hardwick's Civet (E), Cibeta palmirayada (S), Civette palmiste rayée (F), Civette palmiste à bandes de Derby (F), Hémigale de Derby (F) II M

Hémione (F): Equus hemionus I/II M

Hémione de I'Inde (F): Equus hemionus hemionus I M

Hemiono (S): Equus hemionus III M

Hemiono indostánico (S): Equus onager khur I M

Hemiono mongol (S): Equus hemionus hemionus I M

Hémippe (F): Equus hemionus I/II M 
Hen Harrier (E): Circus cyaneus II B

Henderson Lorikeet (E): Vini stepheni II B

Henicopemis infuscatus: Black Honey-buzzard (E), New Britain Honey-buzzard (E), Abejero negro (S), Bondrée noire (F) II B

Henicopemis longicauda: Long-tailed Honey-buzzard (E), Papuan Honey-buzzard (E), Abejero colilargo (S), Bondrée à longue queue $(F)$ II B

Henst's Goshawk (E): Accipiter henstii II B

Herero Girdled Lizard (E): Cordylus pustulatus II R

Hermann's Tortoise (E): Testudo hemanni II R

Hermine (F): Mustela erminea ferghanae III M

Hermit lbis (E): Geronticus eremita I B

Hermits (E): Glaucis spp /Phaeihomis spp/Ramphodon spp. VII B

Héron garde-boeuf (F): Bubulcus ibis III B

Héron goliath (F): Ardea goliath III B

Herons (E): Ardeidae spp. III/NC B

Hérons (F): Ardeidae spp. IIINC B

Herpailurus yaguarondi: Eyra Cat (E), Jaguanundi (E), Gato colorado (S), Gato moro (S), Leoncillo (S), León brenero (S), Onza (S), Tigrillo (S), Yaguarundi (S), Jaguarundi (F) I/II M Herpestes auropunctatus $=$ Herpestes javanicus auropunctatus Herpestes brachyurus fuscus: Indian Brown Mongoose (E). Mangosta colicorta oscura (S), Mangouste à queue courte de l'Inde (F) III M

Herpestes edwardsii: Indian Grey Mongoose (E), Mangosta gris de la India (S), Mangouste grise de l'Inde (F) III M

Herpestes fuscus = Herpestes brachyurus fuscus

Herpestes javanicus auropunctatus: Small Indian Mongoose (E), Mangosta de manchas doradas (S), Petite mangouste indienne (F) III M

Herpestes smithii: Ruddy Mongoose (E), Mangosta roja de la India (S), Mangousie de Smith (F) III M

Herpestes urva: Crab-eating Mongoose (E), Mangosta cangrejera (S) III $M$

Herpestes vitticollis: Stripe-necked Mongoose (E), Mangosta de nuca rayada (S) $I 11 \mathrm{M}$

Herpetoglossa $=$ Crenactis

Herpetolitha $=$ Herpolitha

Herpetotheres cachinnans: Laughing Falcon (E), Acuá (S), Halcón guaco (S), Halcón guaicurí (S), Halcón macagua (S), Halcón reidor (S), Macagua rieur (F) II B

Herpolitha limax: Slipper Coral (E), Corail limace (F) II In

Herring Whale (E): Balaenoptera physalus I M

Heterocyathus aequicostatus: II In

Heterocyathus altermatus: II In

Heterocyathus sulcatus: II In

Helerometrus roeseli $=$ Pandinus imperator

Heteropora $=$ Acropora

Heleropsammia cochlea: II in

Heseropsammia eupsammides: II In

Heterospizias meridionalis = Buteogallus meridionalis

Heuglin's Bustard (E): Neoris heuglinii II B

Heuglin's Masked-Weaver (E): Ploceus heuglini III B

Hewitt's Spiny-tailed Lizard (E): Cordylus peersi II R

Hexapathes heterosticha: II In

Hexaprotodon liberiensis: Pygmy Hippopotamus (E), Hipopótamo enano (S), Hipopótamo pigmeo (S), Hippopotame nain (F), Hippopotame pygmée (F) II M

Hibou a bec jaune ( $\mathrm{F})$ : Jubula letrii II B

Hibou à casque $(\mathrm{F})$ : Lophostrix cristata II B

Hibou brachyote (F): Asio flammeus II B

Hibou criard (F): Asio clamator II B

Hibou d'Abyssinie (F): Asio abyssinicus II B

Hibou de la Jamaique (F): Pseudoscops grammicus II B

Hibou de Madgascar (F): Asio madagascariensis II B

Hibou de Tweeddale (F): Mimizuku gumeyi I B

Hibou des marais (F): Asio flammeus II B

Hibou du Cap (F): Asio capensis II B

Hibou grand duc (F): Bubo bubo II B

Hibou maître-bois (F): Asio stygius II B

Hibou malgache (F): Asio madagascariensis II B

Hibou moyen-duc (F): Asio otus II B

Hibou pêcheur roux (F): Ketupa flavipes II B

Hibou petit-duc (F): Ous scops II B

Hibou redoutable (F): Nesasio solomonensis II B

Hibou strié (F): Asio clamator II B
Hibous (F): Asio spp $/$ Nesasio solomonensis/Pseudoscops grammicus II $\mathrm{B}$

Hiena dorada (S): Parahyaena brunnea II M

Hiena parda (S): Parahyaena brunnea II M

Hieraaetus ayresii: Ayres's Eagle (E), Ayres's Hawk-Eagle (E), Aguila-azor de Ayres (S), Aigle d'Ayres (F) II B

Hieraaefus bellicosus = Polemaetus bellicosus

Hieraaetus dubius = Hieraaetus ayresii

Hieraaetus fasciarus: Bonelli's Eagle (E), Aguila de Bonelli (S), Aguila perdicera (S), Aguila-azor perdicera (S), Aigle de Bonelli (F) II B

Hieraaetus fascialus spilogaster = Hieraaetus spilogaster

Hieraaetus kienerii: Chestnut-bellied Hawk-Eagle (E), Rufousbellied Eagle (E), Aguila-azor ventrimoja (S), Aigle à ventre roux (F) $11 \mathrm{~B}$

Hieraaetus morphnoides: Little Eagle (E), Aguililla australiana (S), Aigle nain (F) II B

Hieraaetus pennatus: Booted Eagle (E), Aguila calzada (S), Aguililla calzada (S), Aigle botté (F) II B

Hieraaetus spilogaster. African Hawk-Eagle (E), Aguila perdicera africana (S), Aguila-azor africana (S), Aigle fascié (F), Aigleautour fascié (F) II B

Hierro Giant Lizard (E): Gallotia simonyi I R

Higgins' Eye Pearly Mussel (E): Lampsilis higginsii I In

High-casqued Chameleon (E): Chamaeleo hoehnelii II R

Highland Guan (E): Penelopina nigra III B

Hill Myna (E): Gracula religiosa II B

Hillopathes ramosa: II In

Hillstars (E): Oreotrochilus $\operatorname{spp} /$ Urochroa bougueri II B

Himalayan Black Bear (E): Ursus thibetanus I M

Himalayan Brown Bear (E): Ursus arctos isabellinus I M

Himalayan Goral (E): Naemorhedus goral I M

Himalayan Griffon (E): Gyps himalayensis II B

Himalayan Griffon Vulture (E): Gyps himalayensis II B

Himalayan Marmot (E): Marmota himalayana III M

Himalayan Monal (E): Lophophorus impejanus I B

Himalayan Musk Deer (E): Moschus chrysogaster III M

Hipopótamo anfibio (S): Hippopotamus amphibius II M

Hipopótamo enano (S): Hexaprotodon liberiensis II M

Hipopótamo pigmeo (S): Hexaprotodon liberiensis II M

Hippocamelus spp.: Huemuls (E), Ciervos andinos (S), Huemules (S), Cerfs des Andes (F), Guémaux (F) I M

Hippocamelus antisensis: North Andean Deer (E), North Andean Huemul (E), North Andean Huemul (E), Peruvian Guemal (E). Peruvian Huemul (E), Tanca (E), Ciervo andino septentrional (S), Guemal (S), Taruca (S), Cerf des Andes septentrionales (F), Guémal péruvien (F), Huémul des Andes septentrionales (F) I M

Hippocamelus bisulcus: Chilean Guemal (E), Chilean Huemul (E), South Andean Deer (E), South Andean Huemul (E), Ciervo andino meridional (S), Huemul (S), Cerf des Andes méridionales (F), Huémul des Andes méridionales (F) I M

Hippopotame (F): Hippopotanus amphibius II M

Hippopotame nain (F): Hexaprotodon liberiensis II M

Hippopotame pygmée (F): Hexaprotodon liberiensis II M

Hippopotamus (E): Hippopotamus amphibius II M

Hippopotamus amphibius: Hippopotamus (E), Large Hippo (E), Hipopótamo anfibio (S), Hippopotame (F) II M

Hippopotamuses (E): Hippopotamidae spp. 11 M

Hippopus hippopus: Bear Paw Clam (E), Horse's Hoof Clam (E), Strawberry Clam (E) II In

Hippopus porcellanus: China Clam (E) II In

Hippotrague géant de l'Angola (F): Hippotragus niger variani I M

Hippotrague noir géant (F): Hippotragus niger variani I M

Hippotragus niger variani: Giant Sable Antelope (E), Antilope sable negro (S), Hippotrague géant de l'Angola (F), Hippotrague noir géant (F) I M

Hirola (F): Damaliscus lunatus III $\mathrm{M}$

Hirondelle à lunettes (F): Pseudochelidon sirintarae I B

Hirudo medicinalis: Medicinal Leech (E), Sangsue médicinale (F), Sangsue officinale (F) II In

Hispaniolan Amazon (E): Amazona ventralis II B

Hispaniolan Boa (E): Epicrates gracilis II R

Hispaniolan Conure (E): Aratinga chloroptera II B

Hispaniolan Emerald (E): Chlorostilbon swainsonii II B

Hispaniolan Ground Iguana (E): Cyclura ricordi I R

Hispaniolan Hawk (E): Buteo ridgwayi II B 
Hispaniolan Parakeet (E): Aratinga chloroptera II B Hispaniolan Parrot (E): Amazona ventralis II B Hispid Hare (E): Caprolagus hispidus I M Hoary Fox (E): Vulpes cana II M

Hoary Puffleg (E): Haplophaedia lugens II B Hobbies (E): Falco spp. II B

Hobereau à poitrine rousse (F): Falco severus II B

Hobereau africain (F): Falco cuvieri II B

Hocco à bec bleu (F): Crax alberti III B

Hocco à bec rouge (F): Crax blumenbachii I B

Hocco à pierre (F): Pauxi pauxi III B

Hocco d'Albert (F): Crax alberti 111 B

Hocco de Blumenbach (F): Crax blumenbachii I B

Hocco de Daubenton (F): Crax daubentoni III B

Hocco globuleux (F): Crax globulosa III B

Hocco huppé (F): Oreophasis derbianus I B

Hocco mitou (F): Mitu milu I B

Hoccos (F): Crax spp/Mitu spp/Nothocrar spp/Pauxi spp. I/IIINC B

Hocheur (F): Cercopithecus nictitans II M

Hocheur du Ghana ( $\mathrm{F}$ ): Cercopithecus petaurisıa II M

Hocofaisan (S): Crax rubra III B

Hodgson's Hawk-Eagle (E): Spizaetus nipalensis II B

Hoffmann's Conure (E): Pyrrhura hoffmanni II B

Hoffmann's Two-toed Sloth (E): Choloepus hoffmanni III M

Hog-nosed skunks (E): Conepatus spp. II/NC M

Hogs (E): Suidae spp. INC M

Hokki blanc (F): Crossoptilon crossoptilon I B

Hokki brun (F): Crossoptilon manichuricum I B

Hokki de Harman (F): Crossoptilon harmani I B

Hokki du Tibet (F): Crossoptilon harmani I B

Hokkis (F): Crossoprilon spp. I/NC B

Holcotrochus crenulatus: II in

Holcotrochus scriptus: II In

Homerus Swallowtail (E): Papilio homerus I In

Home's Hinge-back Tortoise (E); Kinixys homeana II R

Home's Hinged Tortoise (E): Kinirys homeana II R

Home's Hinged-backed Tortoise (E): Kinixys homeana II R

Homophyllia $=$ Scolymia

Homopode à éperon (F): Homopus femoralis II R

Hornopode aréolé (F): Homopus areolatus II $\mathrm{R}$

Homopode de Boulenger (F): Homopus boulengeri II R

Homopode de Namibie (F): Homopus bergeri II R

Homopode éperonné (F): Homopus femoralis II R

Homopode marqué ( $\mathrm{F})$ : Homopus signatus II R

Homopus areolatus: Areolated Tortoise (E), Beaked Cape Tortoise (E), Grooved Mountain Tortoise (E), Parrot-beaked Tortoise (E), Tortuga pico de loro (S), Homopode aréolé (F) II R

Homopus bergeri: Berger's Cape Tortoise (E), Homopode de Namibie (F) II R

Homopus boulengeri: Boulenger's Cape Tortoise (E), Boulenger's Padloper (E), Tortuga terrestre (S), Homopode de Boulenger (F) II $R$

Homopus femoralis: Greater Padloper (E), Karoo Cape Tortoise (E), Homopode à éperon (F), Homopode éperonné (F) II R

Homopus signatus: Speckled Cape Tortoise (E), Speckled Tortoise (E), Tortuga manchada (S), Homopode marqué (F) II R

Honduran Emerald (E): Amazilia luciae II B

Honey Badger (E): Mellivora capensis III M

Honey-buzzards (E): Pernis spp /Henicopemis spp. II B

Honeycornb Coral (E): Fovites abdita II In

Honeyeaters (E): Meliphagidae spp. I/NC B

Hooded Chameleon (E): Chamaeleo cucullatus II R

Hooded Crane (E): Grus monacha I B

Hooded Parrot (E): Psephorus dissimilis I B

Hooded Visorbearer (E): Augastes lumachellus II B

Hooded Vulture (E): Necrosyrtes monachus II B

Hook-billed Hermit (E): Ramphodon dohmii I B

Hook-billed Kite (E): Chondrohierax uncinatus III B

Hooked-beaked Terrapin (E): Pelusios niger III R

Hook-lipped Rhinoceros (E): Diceros bicomis I M

Hoolock (F): Hylobates hoolock I M

Hoolock Gibbon (E): Hylobates hoolock I M

Hoplangia durotrix: II in

Hoplobatrachus tigerinus: Indian Bullfrog (E), Tiger Frog (E), Grenouille tigre (F), Grenouille tigree (F) II A
Hoplocéphale de Schlegel (F): Hoplocephalus bungaroides II R

Hoplocephalus bungaroides: Broad-headed Snake (E), Hoplocéphale de Schlegel (F) II R

Horastrea indica: II In

Hormiguero gigante (S): Myrmecophaga iridacryla II M

Hombills (E): Bucerotidae spp. IIINC B

Horn Lizard (E): Uromastyx asmussi II R

Homed Guan (E): Oreophasis derbianus I B

Homed Hog-nosed Pit Viper (E): Bothrops nasuta III R

Homed lizards (E): Phrynosoma spp. IU/NC R

Homed Palm Viper (E): Bothrops schlegelii III R

Homed Parakeet (E): Eunymphicus comutus II B

Homed Sungem (E): Heliactin comura II B

Horned-Pheasants (E): Tragopan spp. I/III/NC B

Horses (E): Equus przewalskii/Equus caballus $\mathbf{V} / \mathrm{NC} M$

Horse's Hoof Clam (E): Hippopus hippopus II In

Horsfield's Tarsier (E): Tarsius bancanus II M

Horsfield's Tortoise (E): Testudo horsfieldii II R

Hose's Leaf Monkey (E): Presbytis hosei II M

Houbara Bustard (E): Chlamydotis undulata I B

Houbara ondulé (F): Chlamydotis undulata I B

Houbaropsis bengalensis $=$ Eupodotis bengalensis

Houleman (F): Semnopithecus entellus I M

Hourglass Dolphin (E): Lagenorhynchus cruciger II M

House-building Rat (E): Leporillus conditor I M

Howlers (E): Alouatia spp. I/II M

Huallaca ( $S$ ): Lontra felina I M

Huapo colorado (S): Cacajao calvus I M

Huapo rojo (S): Cacajao calvus I M

Hubara (S): Chlamydotis undulata I B

Hubbs's Beaked Whale (E): Mesoplodon carlhubbsi II M

Huemul (S): Hippocamelus bisulcus I M

Huérnul des Andes méridionales (F): Hippocamelus bisulcus I M

Huémul des Andes septentrionales (F): Hippocamelus antisensis I M

Huemules (S): Hippocamelus spp. I M

Huemuls (E): Hippocamelus spp. I M

Huillin (E/F): Lontra provocax I M

Huillin (S): Lonsra provocax I M

Hulotte africaine (F): Strix woodfordii II B

Humboldt Penguin (E): Spheniscus humboldti I B

Humboldt's Hog-nosed Skunk (E): Conepatus humboldtii II M

Humboldt's Woolly Monkey (E): Lagothrix lagotricha II M

Hume's Bar-tailed Pheasant (E): Syrmaticus humiae I B

Hume's Owl (E): Strix butleri II B

Hume's Pheasant (E): Syrmaticus humiae I B

Hume's Tawny Owl (E): Strix butleri II B

Hume's Wood-Owl (E): Strix butleri II B

Hummingbirds (E): Trochilidae spp. I/II B

Hump Whale (E): Megaptera novaeangliae I M

Humpback dolphins (E): Sousa spp. I M

Humpback Whale (E): Megaptera novaeangliae I M

Hunchbacked Whale (E): Megaptera novaeangliae I M

Hunting Leopard (E): Acinonyx jubatus I M

Huon Astrapia (E): Astrapia rothschildi II B

Hurleur à mains rousses (F): Alouatta belzebul II M

Hurleur brun (F): Alouatta fusca II M

Hurleur du Guatemala (F): Alouatta pigra I M

Hurleur noir (F): Alouatta caraya II M

Hurleur roux (F): Alouatta seniculus II M

Hurón (S): Galictis vittata III M

Hurón grange (S): Galictis vittata $\mathbf{\text { II M }}$

Hurón mayor (S): Eira barbara III M

Huso dauricus: Kaluga (E) II F

Huso huso: Beluga (E), European Sturgeon (E), Giant Sturgeon (E), Great Sturgeon (E), Beluga (S), Beluga (F) II F

Hyacinth Macaw (E): Anodorhynchus hyacinthinus I B

Hyacinth Visorbearer (E): Augastes scutatus II B

Hyaena brunnea $=$ Parahyaena brunnea

Hyaenas (E): Crocuta crocuta/Hyaena hyaena/Parahyaena brunnea II/NC M

Hyalopathes = Antipathes

Hydnophora bonsai: II In

Hydnophora exesa: II In

Hydnophora grandis: II In

Hydnophora microconos: II In

Hydnophora pilosa $=$ Hydnophora exesa 


\section{Hydnophora rigida: II In}

Hydnophorella $=$ Hydnophora

Hydrictis maculicollis = Lutra maculicollis

Hydrodynastes gigas = Cyclagras gigas

Hyemoschus aquaticus: Water Chevrotain (E), Antilope amizclero enano de agua (S), Chevrotain aquatique (F) III M

Hyène brune (F): Parahyaena brunnea II M

Hylobates agilis: Agile Gibbon (E), Dark-handed Gibbon (E), Gibbon agile (F) I M

Hylobates concolor: Black Gibbon (E), Crested Gibbon (E) Indochinese Gibbon (E), Gibbon noir (F), Gibbon à favoris blancs (F) I M

Hylobates concolor gabriellae $=$ Hylobates gabriellae

Hylobates concolor leucogenys $=$ Hylobales leucogenys

Hylobates gabriellae: Buff-cheeked Gibbon (E) I M

Hylobates hoolock: Hoolock Gibbon (E), Gibón hulock (S), Hoolock (F) $1 \mathrm{M}$

Hylobates klossii: Dwarf Gibbon (E), Kloss's Gibbon (E), Mentawai Gibbon (E), Siamang de Kloss (S), Siamang enano (S), Siamang de Kloss (F) I M

Hylobates lar: Common Gibbon (E), Lar Gibbon (E), White-handed Gibbon (E), Gibón de manos blancas (S), Gibbon lar (F), Gibbon à mains blanches (F) I M

Hylobates lar agilis = Hylobates agilis

Hylobates lar moloch = Hylobates moloch

Hylobates lar mueller $=$ Hylobates muelleri

Hylobates lar pileatus $=$ Hylobates pilearus

Hylobates leucogenys: White-cheeked Gibbon (E) I M

Hylobates leucogenys gabriellae $=$ Hylobates gabriellae

Hylobates moloch: Javan Gibbon (E), Silvery Gibbon (E), Gibón ceniciento (S), Gibbon cendré (F) I M

Hylobates muelleri: Bornean Gibbon (E), Grey Gibbon (E), Müller's Gibbon (E), Gibón đe Mueller (S), Gibbon de Muller (F) I M

Hylobates pileatus: Capped Gibbon (E), Crowned Gibbon (E), Pileated Gibbon (E), Gibón de cresta negra (S) I M

Hylobates syndactylus: Siamang (E), Siamang (S), Siamang (F) I M Hylobatidae spp.: Gibbons (E), Gibones (S), Gibbons (F) I M

Hylocharis chrysura: Gilded Hummingbird (E), Picaflor bronceado (S), Picaflor dorado (S), Saphir à queue d'or (F) II B

Hylocharis cyanus: White-chinned Sapphire (E), Picaflor cabeciazul (S), Zafiro cabecimorado (S), Saphir azuré (F) II B

Hylocharis eliciae: Blue-throated Goldentail (E), Zafiro gorjiazul (S), Saphir d'Elicia (F) II B

Hylocharis grayi: Blue-headed Sapphire (E), Zafiro cabeciazul (S), Saphir ulysse (F) II B

Hylocharis leucotis: White-eared Hummingbird (E), Colibr orejiblanco (S), Saphir à oreilles blanches (F) II B

Hylocharis pyropygia: Flame-rumped Sapphire (E), Saphir embrasé (F) II B

Hylocharis sapphirina: Rufous-throated Sapphire (E), Picaflor pechiazul (S), Zafiro gargantiru fo (S), Saphir à gorge rousse (F) II $\mathrm{B}$

Hylocharis xantusii: Black-fronted Hummingbird (E), Xantus's Hummingbird (E), Colibri de Xantus (S), Saphir de Xantus (F) II B

Hylonympha macrocerca: Scissor-tailed Hummingbird (E), Colibrí tijereta (S), Colibri à queue en ciseaux (F) II B

Hypargos nitidula $=$ Mandingoa nitidula

Hyperoodon spp.: Bottlenose whales (E), Ballenas hocico de botella (S) I M

Hyperoodon ampullatus: Bottlehead (E), Northern Bottlenose Whale (E), Ballena hocico de botella del norte (S), Hyperoodon boréal (F) I M

Hyperoodon austral (F): Hyperoodon planifrons I M

Hyperoodon boréal (F): Hyperoodon ampullatus I M

Hyperoodon planifrons: Southern Bottlenose Whale (E), Ballena hocico de botella del sur (S), Hyperoodon austral (F) I M

Hyperoodon rostratus $=$ Hyperoodon ampullatus

Hypochera chalybeata = Vidua chalybeata

Hypochera wilsoni $=$ Vidua wilsoni

Hystrix cristata: Crested Porcupine (E), North African Crested Porcupine (E), Porc-épic du nord de l'Afrique (F) III M

Hystrix spp.: III M

laça (S): Podocnemis sextuberculata II R

Iberian Lynx (E): Lynx pardinus I M

Ibis (F): Threskiomithidae spp. I/IIIUNC B
Iois à crête du Japon (F): Nipponia nippon I B

Ibis blanc (F): Nipponia nippon I B

Ibis blanc du Japon (F): Nipponia nippon $1 \mathrm{~B}$

Ibis calvo (S): Geronticus calvus II B

Ibis chauve (F): Geronticus eremita I B

Ibis chauve de l'Afrique du Sud (F): Geronticus calvus II B

Ibis eremita (S): Geronticus eremita I B

This escarlata (S): Eudocimus ruber II B

Ibis hadada (S): Bostrychia hagedash III B

Ibis hagedash (F): Bosirychia hagedash III B

Ibis moñudo japonés (S): Nipponia nippon $1 \mathrm{~B}$

Ibis moteado (S): Bostrychia rara III B

Ibis nipón (S): Nipponia nippon I B

Ibis nippon (F): Nipponia nippon I B

Ibis noir (F): Geronticus calvus II B

lbis rara (S): Bostrychia rara III B

Ibis rouge (F): Eudocimus nuber II B

lbis sacré (F): Threskiomis aethiopicus III B

Ibis sagrado (S): Threskiomis aethiopicus III B

Ibis vermicule (F): Bosirychia rara III B

Ibises (E): Threskiomithidae spp. VIUIINC B

Ibiza Wall Lizard (E): Podarcis pityusensis II $\mathrm{R}$

Ichthyophaga humilis: Lesser Fish-eagle (E), Lesser Fishing Eagle

(E), Pigarguillo menor (S), Pygargue nain (F) II B

Ichthyophaga ichthyaetus: Grey-headed Fish-eagle (E), Grey-headed Fishing Eagle (E), Pigarguillo común (S), Pygargue à tête grise (F) $11 \mathrm{~B}$

Ich hyophaga nana = Ichthyophaga humilis

Ictéride à tête jaune (F): Agelaius flavus I B

Icrinaetus malayensis: Asian Black Eagle (E), Black Eagle (E), Indian Black Eagle (E), Aguila milana (S), Aigle noir (F) II B

Ictinia mississippiensis: Mississippi Kite (E), Elanio del Mississipí (S), Milano del Misisipi (S), Milano misisipero (S), Milan du Mississippi (F) II B

Ictinia plumbea: Plumbeous Kite (E), Elanio plomizo (S), Gavilán plomizo (S), Milano plomizo (S), Milan bleuâtre (F) II B

Idiorrochus kikurii: II In

Idiotrochus perexigua: II In

ldiurus kivuensis = Idiurus macrotis

Idiurus macrotis: Long-eared Flying Squirrel (E), Ardilla voladera de orejas largas (S), Anamolure nain (F) III M

Idiurus spp.: III M

Idmi (E): Gazella cuvieri III M

Ignicolore (F): Euplectes franciscanus III B

Iguana crestada de Fiji (S): Brachylophus vitiensis I R

Iguana spp.; Iguanas (E), Iguanas (S), Iguanes communs (F), Iguanes vrais (F) II $R$

Iguana delicatissima: Lesser Antillean Iguana (E), West Indian Iguana (E), Iguane des Antilles (F) II R

Iguana iguana: Common Iguana (E), Green Iguana (E), Gallina de palo (S), Iguane commun (F), Iguane vert (F) II $R$

Iguana marina (S): Amblyrhynchus cristatus II $\mathbf{R}$

Iguana rhinolopha = Iguana iguana

Iguana terrestre de Barrington (S): Conolophus pallidus II R

Iguana terrestre de las Galápagos ( $\mathbf{S}$ ): Conolophus subcristatus II R Iguanas (E): Iguana spp./Iguanidae spp. I/IINC R Iguanas de Fiji (S): Brachylophus spp. I R

Iguanas terrestres (S): Conolophus spp. II R/ Cyclura spp. I R

Iguane à bandes de Fidji (F): Brachylophus fasciatus I R

Iguane à crête de Fidji (F): Brachylophus viriensis I R

Iguane commun (F): Iguana iguana $11 \mathrm{R}$

Iguane comu (F): Cyclura comula I R

Iguane des Antilles (F): Iguana delicatissima II R

Iguane marin (F): Amblyrhynchus cristatus II R

Iguane terrestre comu (F): Cyclura comuta I R

Iguane terrestre de l'île Exuma (F): Cyclura cyclura I R

Iguane terrestre de l'île Santa Fe (F): Conolophus pallidus II R

Iguane terrestre de la Jamaique $(\mathrm{F})$ : Cyclura collei I R

Iguane terrestre de l'lle An'egada (F): Cyclura pinguis I R

Iguane terrestre des Bahamas (F): Cyclura rileyi I $R$

Iguane terrestre des Galapagos (F): Conolophus subcristatus II R Iguane terrestre des lles Cayman (F): Cyclura nubila I R

Iguane terrestre des îles Turks et Caiques (F): Cyclura carinaia I R Iguane terrestre d'Hispaniola (F): Cyclura ricordi I $\mathrm{R}$

Iguane vert (F): Iguana iguana II R

Iguanes communs (F): Iguana spp. II R 


\section{Iguanes cornus (F): Cyclura spp. I R} Iguanes des Fidji (F): Brachylophus spp. I R Iguanes rhinocéros (F): Cyclura spp. I R Iguanes terrestres des Galapagos (F): Conolophus spp. II R Iguanes vrais (F): Iguana spp. II R lliger's Macaw (E): Ara maracana I B Dlolo Chameleon (E): Chamaeleo goetzei II R Imitator Sparrowhawk (E): Accipiter imitator II B Imperial Amazon (E): Amazona imperialis I B Imperial Eagle (E): Aquila heliaca I B Imperial Parrot (E): Amazona imperialis I B Imperial Pheasant (E): Lophura imperialis I B Imperial Woodpecker (E): Campephilus imperialis I B Imperial-Pigeons (E): Ducula spp. I/NC B Impeyan Pheasant (E): Lophophorus impejanus I B Impressed Tortoise (E): Manouria impressa II R Inca à collier (F): Coeligena torquata II B Inca à gemme bleue $(\mathrm{F})$ : Coeligena luteriae II B Inca à queue blanche (F): Coeligena phalerata II B Inca brun (F): Coeligena wilsoni II B Inca café (S): Coeligena wilsoni II B Inca céleste (F): Coeligena coeligena II B Inca de Bonaparte $(\mathrm{F})$ : Coeligena bonapartei II $\mathrm{B}$ Inca de Wetmore (F): Coeligena orina II B Inca iris (F): Coeligena iris II B Inca noir (F): Coeligena prunellei II B Inca porphyre (F): Coeligena helianshea II B Inca violifere (F): Coeligena violifer II B Incas (E/F): Coeligena spp. II B Indian Bison (E): Bos gaurus I M Indian Black Eagle (E): Ictinaetus malayensis II B Indian Black Vulture (E): Sarcogyps calvus II B Indian Brown Mongoose (E): Herpestes brachyurus fuscus III M Indian Bullfrog (E): Hoplobatrachus tigerinus II A Indian Bustard (E): Ardeotis nigriceps I B Indian Chameleon (E): Chamaeleo zeylanicus II R Indian Egg-eater (E): Elachistodon westermanni II R Indian Egg-eating Snake (E): Elachistodon westermanni II R Indian Elephant (E): Elephas maximus I M Indian Flap-shell Turtle (E): Lissemys punctata II R Indian Flying-fox (E): Pteropus giganteus II M Indian Giant Squirrel (E): Ratufa indica II M Indian Grey Mongoose (E): Herpestes edwardsii $11 \mathrm{M}$ Indian Lion (E): Panthera leo persica I M Indian Mastigure (E): Uromastyx hardwickii II R Indian Monitor (E): Varanus bengalensis I R Indian Oval-grain Lizard (E): Varanus flavescens I R Indian Pangolin (E): Manis crassicaudata II M Indian Pond Turtle (E): Kachuga tecra I R Indian Python (E): Python molurus molurus I R Indian Sand Boa (E): Eryx conicus II R Indian Sawback Turtle (E): Kachuga lecta I R Indian Small-grain Lizard (E): Varanus bengalensis I R Indian Smooth-coated Otter (E): Lutrogale perspicillata II M Indian Soft-shell Turtle (E): Trionyx gangericus I R Indian Spectacled Cobra (E): Naja naja II R Indian Star Tortoise (E): Geochelone elegans II R Indian Tapir (E): Tapirus indicus I M Indian Tragopan (E): Tragopan satyra III B Indian Wild Ass (E): Equus onager khur I M Indian Wild Dog (E): Cuon alpinus II M Indian Wild Ox (E): Bos gaurus I M Indigo Macaw (E): Anodorhynchus leari I B Indigobirds (E): Vidua spp. III/NC B Indigo-capped Hummingbird (E): Amazilia cyanifrons II B Indigo-winged Parrot (E): Hapalopsintaca fuertesi II B Indochinese Gibbon (E): Hylobates concolor I M Indochinese Hog Deer (E): Axis porcinus annamiticus I M Indo-Gangetic Flap-shell (E): Lissemys punctata II R

Indopacetus pacificus: Indo-pacific Beaked Whale (E), Longman's

Beaked Whale (E), Ballena de pico de Longman (S), Mesoplodon de Longman (F) II M Indo-pacific Beaked Whale (E): Indopacetus pacificus II M Indo-pacific Humpback Dolphin (E): Sousa chinensis I M Indophyllia macassarensis: II in
Indorestudo elongata: Elongated Tortoise (E). Pineapple Tortoise (E), Red-nosed Tortoise (E), Yellow Tortoise (E), Yellowheaded Tortoise (E), Tortue à tête jaune (F) II R

Indotestudo forstenii: Celebes Tortoise (E), Forsten's Tortoise (E), Travancore Tortoise (E), Tortuga marrón de la India (S), Tortue de Tranvancore (F), Tortue des Celèbes (F) II R Indri (F): Indri indri I M

Indri à queue courte $(\mathrm{F})$ : Indri indri I $\mathrm{M}$

Indri colicorto ( $($ ): Indri indri I M

Indri indri: Indris (E), Indri coliconto (S), Indri (F), Indri à queue courte (F) I M

Indri lanudo (S): Avahi laniger I M

Indridae spp.: Indris (E), Indris (S), Indris (F) I M

Indris (E): Indri indri $1 \mathrm{M}$

Indris (E/S/F): Indridae spp. I M

Indris sifaca (S): Propithecus spp. I M

Indus Dolphin (E): Platanista minor I M

Indus River Dolphin (E): Platanista minor I M

Inferiolabiata labiata: II In

Inferiolabiata lowei: II In

Inferiolabiala spinosa: II In

Inia (F): Inia geoffrensis II M

Inia boliviensis = Inia geoffrensis

Inia geoffrensis: Amazon River Dolphin (E), Boto (E), Boutu (E), Bufeo (S), Dauphin de l'Amazone (F), Inia (F) II M

Inséparable à collier noir (F): Agap.rmis swindernianus II B Inséparable à face rose (F): Agapomis roseicollis II B Inséparable à joues noires (F): Agapornis nigrigenis II B Inséparable à tête grise (F): Agapornis canus II B Inséparable à tête rouge (F): Agapornis pullarius II $\mathrm{B}$ Inseparable Abisinio (S): Agapomis taranta II B Inseparable acollarado ( $\$$ ): Agapomis swindernianus II B Inseparable cabecinegro (S): Agapornis personatus II B Inseparable cachetón (S): Agapornis nigrigenis II B Inseparable carirojo (S): Agapornis pullarius II B Inséparable d'Abyssinie (F): Agapomis taranta II B Inseparable de collera (S): Agapomis swindemianus II B inséparable de Fischer (F): Agapomis fischeri II B Inseparable de Fischer (S): Agapornis fischeri II B Inséparable de Lilian (F): Agapornis lilianae II B Inseparable de Namibia (S): Agapornis roseicollis II B Inséparable de Swindern (F): Agapornis swindemianus II B Inseparable del Nyasa (S): Agapomis lilianae II B Inseparable malgache (S): Agapomis canus II B Inséparable masqué (F): Agapomis personatus II B Inséparable rosegorge $(\mathrm{F})$ : Agapomis roseicollis II B Inséparables (F): Agapornis spp. II B Inseparables (S): Agapornis spp. II B Insular Flying-fox (E): Pteropus tonganus I M Intermediate Parakeet (E): Psittacula intermedia II B Iranian Mastigure (E): Uromastyx asmussi II R Irbis (F): Uncia uncia I M Iris Lorikeet (E): Psitteuteles iris II B Iris Lory (E): Psitteureles iris II B Iris Peacock-Pheasant (E): Polyplectron bicalcaratum II B Inawaddy Dolphin (E): Orcaella brevirostris II M Isidor's Eagle (E): Oroaetus isidori II B

Island Day Gecko (E): Phelsuma nigristriata II R

Isophyllastrea rigida: 11 in

Isophyllia multiflora: II in

Isophyllia sinuosa: II In

Isopora $=$ Acropore

Isthmian Dwarf Boa (E): Ungaliophis continentalis II R Ithagine ensanglantée (F): lthaginis cruentus II B

lthaginis cruentus: Blood Pheasant (E), Faisán ensangrentado (S),

Faisån sanguineo (S), Faisan sanguin (F), Ithagine ensanglantée (F) II B

Itombwe Owl (E): Phodilus prigoginei II B

Ituri Forest Chameleon (E): Bradypodion adolfifriderici II R

Ivory Bush Coral (E): Oculina diffusa II In

Ivory Tree Coral (E): Oculina valenciennesi II in Ivory Tube Coral (E): Cladocora arbuscula II in Jabalí enano (S): Sus salvanius I M

Jabalí pigmeo (S): Sus salvanius I M

Jabiru (E): Jabiru mycreria I B

Jabinú (S): Jabiru mycteria I B 
Jabiru africain (F): Ephippiorhynchus senegalensis III B

Jabirú africano (S): Ephippiorhynchus senegalensis III B

Jabiru américain (F): Jabiru mycteria I B

Jabirú americano (S): Jabiru mycieria I B

Jabiru d'Amérique (F): Jabiru mycteria I B

Jabiru du Sénégal (F): Ephippiorhynchus senegalensis III B

Jabiru mycteria: Jabiru (E), Garzón soldado (S), Jabirú (S), Jabirú americano (S), Jabiru américain (F), Jabiru d'Amérique (F) I B Jabuti (S): Geochelone carbonaria II R

Jacaruxi (S): Dracaena spp. II R

Jackal Buzzard (E): Buteo rufofuscus II B

Jackals (E): Canis spp. IIU/NC M

Jackass Penguin (E): Spheniscus demersus II B

Jacko (F): Psinacus erithacus II B

Jackson's Three-homed Chameleon (E): Chamaeleo jacksonii II R

Jacobino collarejo (S): Florisuga mellivora II B

Jacobino nuquiblanco (S): Florisuga mellivora II B

Jacobita (S): Cephalorhynchus commersonii II M

Jacquot (E): Amazona arausiaca I B

Jacquot (F): Psittacus erithacus II B

Jacuruxi (E): Dracaena guianensis II R

Jaguar (E/S/F): Panthera onca I M

Jaguarundi (E/F): Herpailurus yaguarondi $\mathbf{V I} \mathbf{M}$

Jamaica Ground Iguana (E): Cyclura collei I R

Jamaican Boa (E): Epicrates subflavus I R

Jamaican Iguana (E): Cyclura collei I R

Jamaican Mango (E): Anthracothorax mango II B

Jamaican Owl (E): Pseudoscops grammicus II B

Jambandu Indigobird (E): Vidua raricola III B

Jameson's Antpecker (E): Parmoptila rubrifrons III B

James's Flamingo (E): Phoenicopterus jamesi II B

Jandaya Conure (E): Aratinga jandaya II B

Jandaya Parakeet (E): Aratinga jandaya II B

Japanese Crane (E): Grus japonensis I B

Japanese Crested Ibis (E): Nipponia nippon I B

Japanese Giant Salamander (E): Andrias japonicus I A

Japanese Macaque (E): Macaca fuscaia II M

Japanese Sparrowhawk (E): Accipiter gularis II B

Japanese White Stork (E): Ciconia boyciana I B

Jardine's Parrot (E): Poicephalus gulielmi II B

Java Leaf Monkey (E): Presbytis comata II M

Java Rock Python (E): Python reticulatus II R

Java Sparrow (E): Padda oryzivora II B

Javan Gibbon (E): Hylobases moloch I M

Javan Hawk-Eagle (E): Spizaetus bartelsi II B

Javan Owlet (E): Glaucidium castanopierum II B

Javan Rhinoceros (E): Rhinoceros sondaicus I M

Javan Scops-Owl (E): Otus angelinae II B

Javan Treeshrew (E): Tupaia javanica II M

Javania antarctica: II In

Javania borealis: II In

Javania cailleti: II In

Javania californica: II in

Javania insignis: II In

Javania lamprotichum: II In

Javania pachytheca: II in

Javania pseudoalabastra: II In

Javelin (E): Eryx jaculus II R

Jayakar's Sand Boa (E): Ervx jayakari II R

Jentink's Duiker (E): Cephalophus jentinki I M

Jerdon's Baza (E): Aviceda jerdoni II B

Jerdon's Palm Civet (E): Paradoxurus jerdoni III M

Jewelled Chameleon (E): Chamaeleo lateralis II R

Jilguero cara amarilla (S): Carduelis yarrellii II B

Jilguero de Yarrell (S): Carduelis yarrellii II B

Jobi Manucode (E): Manucodia jobiensis II B

John's Langur (E): Trachypithecus johnii II M

Johnstone's Crocodile (E): Crocodylus johnsoni II R

Johnstone's Lorikeet (E): Trichoglossus johnstoniae II B

Johnston's Chameleon (E): Chamaeleo johnstoni II R

Jordan's Girdled Lizard (E): Cordylus jordani II R

Jorobada (S): Megaptera novaeangliae I M

Josephine's Lorikeet (E): Charmosyna josefinae II B

Josephine's Lory (E): Charmosyna josefinae II B

Jote real (S): Sarcoramphus papa III B

Joues-oranges (F): Estrilda melpoda III B
Juan Fernández Firecrown (E): Sephanoides femandensis II B

Juan Fernández Fur Seal (E): Arctocephalus philippii II M

Jubarte (F): Megaptera novaeangliae I M

Jubula lettii: Maned Owl (E), Búho de Guinea (S), Duc à crinière

(F), Hibou a bec jaune (F) II B

Jumping Pit Viper (E): Bothrops nummifer III R

Jungle Cat (E): Felis chaus II M

Jungle Hawk-Owl (E): Ninox theomacha II B

Jungle Owlet (E): Glaucidium radiatum II B

Junglefowl (E): Gallus spp. II/NC B

Kachuga à dos en toit (F): Kachuga tecia I R

Kachuga tecta: Dura Turtle (E), Indian Pond Turtle (E), Indian

Sawback Turtle (E), Roofed Turtle (E), Tent Turtle (E), Tortuga

india de visera (S), Kachuga à dos en toit (F) I R

Kagou (F): Rhynochetos jubatus I B

Kagou huppé (F): Rhynochetos jubatus I B

Kagu (E): Rhynocheros jubatus I B

Kagú (S): Rhynochetos jubatus I B

Kaiserihind (E): Teinopalpus imperialis II In

Kaiserihinds (E): Teinopalpus spp. II In

Kaka (E): Nestor meridionalis II B

Kaka (S): Nestor meridionalis II B

Kakapo (E/S/F): Strigops habroptilus I B

Kakarikis (E): Cyanoramphus spp. I/II B

Kaluga (E): Huso dauricus II F

Kangourou-rat à queue touffue (F): Beltongia penicillata I M

Kangourou-rat de Lesueur (F): Bettongia lesueur I M

Kangourou-rat de Tasmanie (F): Bettongia gaimardi I M

Kangourou-rat du désert (F): Caloprymnus campestris I M

Kangourou-rats (F): Bentongia spp. I M

Kara Tau Argali (E): Ovis ammon nigrimoniana I M

Karoo Bustard (E): Eupodonis vigorsii II B

Karoo Cape Tortoise (E): Homopus femoralis II R

Karoo Dwarf Chameleon (E): Bradypodion karroicum II R

Karoo Girdled Lizard (E): Cordylus polyzonus II R

Karoo Korhaan (E): Eupodotis vigorsii II B

Karumbé (S): Geochelone carbonaria II R

Kashmir Deer (E): Cervus elaphus hanglu I M

Kashmir Red Deer (E): Cenus elaphus hanglu I M

Kashmir Stag (E): Cenus elaphus hanglu I M

Kaupifalco monogrammicus: Lizard Buzzard (E), Busardo gavilán (S), Autour unibande (F) II B

Kawall's Amazon (E): Amazona kawalli 11 B

Kawall's Parrot (E): Amazona kawalli II B

Kea (E/S/F): Nestor notabilis II B

Keelbacks (E): Colubridae spp. IIV/NC R

Keel-billed Toucan (E): Ramphastos sulfuratus II B

Keeled-scaled Python (E): Morelia carinata II R

Kelesa (E): Scleropages formosus I F

Kemp's Ridley (E): Lepidochelys kempi I R

Kenyan Sand Boa (E): Eryx colubrinus II R

Kerguelen Fur Seal (E): Arctocephalus gazella II M

Kestrels (E): Falco spp. VII B

Kétoupa brun (F): Ketupa zeylonensis II B

Kétoupa de Blakiston (F): Ketupa blakistoni II B

Kétoupa malais (F): Ketupa ketupu II B

Kétoupa roux (F): Kelupa flavipes II B

Kétoupas (F): Kerupa spp. II B

Ketupa blakistoni: Blakiston's Fish-Owl (E), Kétoupa de Blakiston (F) II B

Ketupa flavipes: Tawny Fish-Owl (E), Búho pescador (S), Hibou pêcheur roux (F), Kétoupa roux (F) II B

Ketupa ketupu: Buffy Fish-Owl (E), Malay Fish-Owl (E), Kétoupa malais (F) II B

Ketupa zeylonensis: Brown Fish-Owl (E), Kétoupa brun (F) II B

Khur (E/S): Equus onager khur I M

Kiang (E/F): Equus kiang II M

Kilimanjaro Two-homed Chameleon (E): Bradypodion tavetanum II $\mathbf{R}$

Killer Whale (E): Orcinus orca II M

Kinabalu Serpent-Eagle (E): Spilomis kinabaluensis II B

King Bird-of-paradise (E): Cicinnurus regius II B

King Cobra (E): Ophiophagus hannah II R

King Colobus (E): Colobus polykomos II M

King Vulture (E): Sarcoramphus papa III B

King-of-Saxony Bird-of-paradise (E): Pteridophora alberti II B 
King-Parrots (E): Alisterus spp. II B

King's Monitor (E): Varanus kingorum II R

Kinixys belliana: Bell's Hinged Tortoise (E), Bell's Hinged-backed Tortoise (E), Kinixys de Bell (F) II R

Kinixys belliana natalensis $=$ Kinixys natalensis

Kinixys de Bell (F): Kinixys belliana II R

Kinixys de Home (F): Kinixys homeana II R

Kinixys du Natal (F): Kinirys natalensis II R

Kinirys erosa: Common Tortoise (E), Forest Hinged Tortoise (E), Schweigger's Tortoise (E), Serrated Hinge-back Tortoise (E), Serrated Hinge-backed Tortoise (E), Serrated Tortoise (E), Kinixys rongée (F), Tortue articulée d'Afrique (F) II R

Kinixys homeana: Home's Hinge-back Tortoise (E), Home's Hinged Tortoise (E), Home's Hinged-backed Tortoise (E), Kinixys de Home (F) II R

Kinixys natalensis: Natal Hinge-back Tortoise (E), Natal Hingebacked Tortoise (E), Natal Hinged Tortoise (E), Kinixys du Natal (F) II R

Kinixys rongée (F): Kinixys erosa II R

Kinkajou (E): Potos flavus III M

Kionotrochus suteri: II ln

Kirk's Colobus (E): Procolobus pennantii kirkii I M

Kirk's Red Colobus (E): Procolobus pennantii kirkii I M

Kites (E): Accipitridae spp. VII B

Klais guimeti: Violet-headed Hummingbird (E), Colibrí cabecivioleta (S), Tucusito cabeza azul (S), Colibri à tête violette (F) II B

Kleinmann's Tortoise (E): Testudo kleinmanni I R

Kloss's Gibbon (E): Hylobates klossii I M

Knobbed Hombill (E): Aceros cassidix II B

Knob-billed Goose (E): Sarkidiomis melanotos II B

Knysna Dwarf Chameleon (E): Bradypodion damaranum II R

Knysna Turaco (E): Tauraco corythaix II B

Kobus leche: Lechwe (E), Cobo de Lechwe (S), Cobe lechwe (F), Lechwe (F) II M

Koch's Pitta (E): Pitta kochi I B

Kodkod (E): Oncifelis guigna II M

Koepcke's Hermit (E): Phaethomis koepckeae II B

Koepcke's Screech-Owl (E): Otus koepckeae II B

Kogia breviceps: Pygmy Sperm Whale (E), Cachalote cabeza chica (S), Cachalote pigmeo (S), Cachalot pygmée (F) II M

Kogia simus: Dwarf Sperm Whale (E), Cachalote enano (S), Cachalot nain (F) II M

Kokoe Poison Frog (E): Phyllobates aurotaenia II A

Komodo Dragon (E): Varanus komodoensis I R

Komodo Monitor (E): Varanus komodoensis I R

Korhaans (E): Eupodotis spp. II B

Kori Bustard (E): Ardeotis kori II B

Korrigum (F): Damaliscus lunatus III M

Kouprey (E/S/F): Bos sauveli I M

Kuhl's Hog Deer (E): Axis kuhlii I M

Kuhl's Lorikeet (E): Vini kuhlii II B

Kuhl's Tortoise (E): Psammobates oculiferus II R

Kulan (E): Equus onager I/II M

Kulang (S): Equus onager I/II M

La Brea Poison Frog (E): Dendrobates occulator II A

La chimère (F): Omithoptera chimaera II In

La Planada Poison Frog (E): Epipedobates andinus II A

La Plata River Dolphin (E): Pontoporia blainvillei II M

Labiopora $=$ Errina

Laborioso cabecirrojo (S): Quelea erythrops III B

Labyrinthocyathus delicatus: II In

Labyrinthocyathus facesus: II In

Labyrinthocyathus kondoi: II In

Labyrinthocyothus langi: II In

Labyrinthocyathus limatulus: II In

Labyrinthocyathus quaylei: II In

Lace corals (E): Stylasteridae spp. II In

Lace Monitor (E): Varanus varius II $R$

La-Digue Day Gecko (E): Phelsuma sundbergi II R

Lafresnaya lafresnayi: Mountain Velvetbreast (E), Colibrí terciopelo

(S), Colibri de Lafresnaye (F) II B

Lagartija balear (S): Podarcis lilfordi II R

Lagartija de las Pitiusas (S): Podarcis piryusensis II R

Lagarto cocodrilo chino (S): Shinisaurus crocodilurus II R

Lagarto de cola espinosa común (S): Uromastyx acanthinurus II R

Lagarto de cola espinosa de Geyr (S): Uromastyx geyri II R
Lagarto de cola espinosa de Macfadyen (S): Uromastyx macfadyeni II $\mathbf{R}$

Lagarto de cola espinosa de Thomas (S): Uromastyx thomasi II R

Lagarto de cola espinosa egipcio (S): Uromastyx aegyptius II R

Lagarto de cola espinosa indo (S): Uromastyx hardwickii II R

Lagarto de cola espinosa ocelado (S): Uromastyx acellatus II R

Lagarto de Cuentas (S): Heloderma horridum II R

Lagarto negro (S): Melanosuchus niger I/II R

Lagartos (\$): Tupinambis spp. II R

Lagartos armadillos (S): Cordylus spp. II R

Lagartos dragóns (S): Dracaena spp. II R

Lagenodelphis hosei: Fraser's Dolphin (E), Sarawak Dolphin (E), Delfín de Bomeo (S), Dauphin de Fraser (F) II M

Lagenorhynchus acutus: Atlantic White-sided Dolphin (E), Delfin de flancos blancos (S), Lagénorhynque à flanc blanc de l'Atlantique (F) II M

Lagenorhynchus albirostris: White-beaked Dolphin (E), Delfín de pico blanco (S), Lagénorhynque à bec blanc de l'Atlantique (F) II $\mathrm{M}$

Lagenorhynchus australis: Blackchin Dolphin (E), Peale's Dolphin (E), Delfín austral (S), Lagénorhynque de Peale (F) II M

Lagenorhynchus cruciger: Hourglass Dolphin (E), Delfin cruzado (S), Dauphin crucigère $(F)$, Lagénorhynque crucigère (F) II M

Lagenorhynchus obliquidens: Pacific White-sided Dolphin (E), Delfín lagenor ringo (S), Lagénorhynque à flanc blanc du Pacifique (F) II M

Lagenorhynchus obscurus: Dusky Dolphin (E), Delfín listado (S), Lagénorhynque sombre (F) II M

Lagenorhynchus superciliosus = Lagenorhynchus obscurus

Lagénorhynque à bec blanc de l'Atlantique (F): Lagenorhynchus albirostris II M

Lagénorhynque à flanc blanc de l'Atlantique (F): Lagenorhynchus acutus II M

Lagénorhynque à flanc blanc du Pacifique (F): Lagenorhynchus obliquidens II M

Lagénorhynque crucigère (F): Lagenorhynchus cruciger II M

Lagénorhynque de Peale (F): Lagenorhynchus australis II M

Lagénorhynque sombre (F): Lagenorhynchus obscurus II M

Laggar Falcon (E): Falco jugger I B

Lagonosticla larvata vinacea $=$ Lagonosticta vinacea

Lagonosticia rara: Black-bellied Firefinch (E), Black-bellied Waxbill $(E)$, Amarante rare $(F)$, Amarante à ventre noir $(F)$ III B

Lagonosticia rubricata: African Firefinch (E), Blue-billed Firefinch (E), Brown-backed Firefinch (E), Bengalí de Lichtenstein (S), Amarante flambé (F), Amarante foncé (F) III B

Lagonosticia nufopicta: Bar-breasted Firefinch (E), Bar-breasted Waxbill (E), Bengali de Fraser (S), Amarante pointé (F) III B

Lagonosticta senegala: Red-billed Firefinch $(E)$, Senegal Firefinch (E), Bengali senegalés ( $S$ ), Amarante commun (F), Amarante du Sénégal (F) III B

Lagonosticta vinacea: Black-faced Firefinch (E), Vinaceous Firefinch (E), Vinaceous Waxbill (E), Bengalí carinegro (S), Amarante masqué (F), Amarante vineux (F) III B

Lagorchestes hirsutus: Ormala (E), Rufous Hare-wallaby (E), Western Hare-wallaby (E), Wurnup (E), Canguro-liebre peludo (S), Wallaby-lièvre de l'ouest (F), Wallaby-lièvre roux (F) I M

Lagostrophus fasciatus: Banded Hare-wallaby (E), Munning (E), Canguro-liebre rayado (S), Wallaby-lièvre rayé (F), Wallabylièvre à bandes $(\mathrm{F})$ I M

Lagothriche de Humboldt (F): Lagothrix lagotricha II M

Lagothrix flavicauda: Yellow-tailed Woolly Monkey (E), Singe laineux à queue jaune (F) I M

Lagothrix hendeei = Lagothrix flavicauda

Lagothrix lagotricha: Common Woolly Monkey (E), Humboldt's Woolly Monkey (E), Lagothriche de Humboldt (F), Singe laineux (F) II M

Lake Patzcuaro Salamander (E): Ambystoma dumerilii II A

Lake Sturgeon (E): Acipenser fulvescens II F

Lama guanicoe: Guanaco (E), Guanaco (S), Guanaco (F) II M

Lamantin d'Afrique (F): Trichechus senegalensis II M

Lamantin d'Amérique du Nord (F): Trichechus manarus I M

Lamantin d'Amérique du Sud (F): Trichechus inunguis I M

Lamantin de l'Amazone (F): Trichechus inunguis I M

Lamantin des Antilles (F): Trichechus manatus I M

Lamantin des Caraibes (F): Trichechus manatus I M

Lamantin du Sénégal (F): Trichechus senegalensis II M 
Lamantino amazónico (S): Trichechus inunguis I M

Lamantino norteamericana (S): Trichechus manatus I M

Lambis (F): Strombus gigas II in

Lammergeier (E): Gypaenus barbatus II B

Lampornis amethystinus: Amethyst-throated Hummingbird (E). Colibri-serrano gorjiamatisto (S), Colibri à gorge améthyste (F) II B

Lampornis calolaema = Lampomis castaneoventris

Lampornis castaneoventris: Purple-throated Mountain-gem (E), Variable Mountain-gem (E), White-throated Mountain-gem (E), Colibri à ventre châtain (F) II B

Lampornis cinereicauda = Lampornis castaneoventris

Lampornis clemenciae: Blue-throated Hummingbird (E), Colibriserrano goriazul (S), Colibri à gorge bleue (F) II B

Lampormis hemileucus: White-bellied Mountain-gem (E), Colibri à gorge lilas (F) II B

Lampornis sybillae: Green-breasted Mountain-gem (E), Colibriserrano pechiverde (S), Colibri de Sybil (F) II B

Lampornis viridipallens: Green-throated Mountain-gem (E). Colibriserrano gorjiverde (S), Colibri vert-d'eau (F) II B

Lampornis viripallens sybillae = Lampomis sybillae

Lampribis rara $=$ Bostrychia rara

Lamprolaima rhami: Gamet-throated Hummingbird (E), Colibrí alicastano (S), Colibri à gorge grenat (F) II B

Lampsilis abrupta = Lampsilis orbiculata orbiculata

Lampsilis higginsii: Higgins' Eye Pearly Mussel (E) I In

Lampsilis orbiculata orbiculata: Pink Mucket Pearly Mussel (E) I In

Lampsilis salur. Plain Pocketbook Pearly Mussel (E), Sandback Pocketbook Mussel (E) I In

Lampsilis tampicoensis tecomatensis = Unio tampicoensis tecomatensis

Lampsilis virescens: Alabama Lamp Naiad (E). Alabama Lamp Pearly Mussel (E), Alabama Lampmussel (E) I In

Lancebills (E): Doryfera spp. II B

Land iguanas (E): Conolophus spp. II R

Lang's Crag Lizard (E): Pseudocordylus langi II R

Lang's Girdled Lizard (E): Pseudocordylus langi II R

Langur à capuchon (F): Trachypithecus pilearus I M

Langur à queue de cochon (F): Nasalis concolor I M

Langur capuchino (S): Trachypithecus pileasus I M

Langur chato (S): Pygaihrix roxellana I M

Langur colilargo (S): Presbytis potenziani I M

Langur común (S): Semnopithecus entellus I M

Langur crestado (\$): Trachypithecus cristatus II M

Langur de capa (S): Trachypirhecus pileatus I M

Langur de cara roja (S): Trachypithecus verulus II M

Langur de cresta (S): Presbyris melalophos II M

Langur de François (F): Trachypithecus francoisi II M

Langur de Francois (S): Trachypithecus francoisi II M

Langur de frente blanca (S): Presbytis frontata II M

Langur de Hose (S): Presbytis hosei II M

Langur de Mentawi (S): Presbytis potenziani I M

Langur de Nilgiri (S): Trachypithecus johnii II M

Langur de Thomas (S): Presbyris thomasi II M

Langur des îles Mentawi (F): Nasalis concolor I M

Langur dorado (S): Trachypithecus geei I M

Langur doré (F): Trachypithecus geei I M

Langur douc (S): Pygathrix nemaeus $1 \mathrm{M}$

Langur du Nilgiri (F): Trachypithecus johnii II M

Langur gris (S): Presbyris comala II M

Langur hanuman (S): Semnopithecus entellus I M

Langur mitrado (S): Presbytis femoralis II M

Langur obscuro (S): Trachypirhecus obscurus II M

Langur rabicerdo (S): Nasalis concolor I M

Langur rubicundo (S): Presbytis rubicunda II M

Langurs (E): Presbytis spp/Semnopithecus entellus/Trachypithecus spp. VII M

Lanner (E): Falco biarmicus II B

Lanner Falcon (E): Falco biarmicus II B

Lapa azul (S): Ara macao I B

Lapin de Diaz (F): Romerolagus diazi I M

Lapin de l'Assam (F): Caprolagus hispidus I M

Lapin des volcans (F): Romerolagus diazi I M

Lapland Owl (E): Sirix nebulosa II B

Lapp Owl (E): Sirix nebulosa II B

Lappet-faced Vulture (E): Torgos tracheliofus II B
Lar Gibbon (E): Hylobates lar I M

Large Amu-Dar Shovelnose Sturgeon (E): Pseudoscaphirhynchus kaufmanni II F

Large Desert Marsupial-Mouse (E): Sminthopsis psammophila I M

Large Fig-Parrot (E): Psitraculirostris desmarestii II B

Large Flower Coral (E): Mussa angulosa II ln

Large Flying-fox (E): Preropus vampyrus II M

Large Hippo (E): Hippopotamus amphibius II M

Large Indian Civet (E): Viverra zibetha III M

Large Palau Flying-fox (E): Pteropus pilosus I M

Large Treeshrew (E): Tupaia tana $11 \mathrm{M}$

Large-blotched Python (E): Morelia stimsoni II R

Large-Eared Greater Galago (E): Otolemur crassicaudarus II M

Large-scaled Girdled Lizard (E): Cordylus macropholis II R

Larus relictus: Relicı Gull (E), Gaviota de Mongolia (S), Gaviota relicta (S), Goéland de Mongolie (F), Goéland relique (F), Mouette relique (F) I B

Lasiorhinus bamardi = Lasiorhinus $k$ refftii

Lasiorhinus kreffii: Northem Hairy-nosed Wombat (E), Queensland Hairy-nosed Wombat (E), Oso marsupial del Rio Moonie (S). Wombat à nez poilu de Queensland (F) I M

Lashamus discolor. Swift Parrot (E), Periquito migrador (S), Perruche de Latham (F) II B

Latimeria chalumnae: Coelacanth (E), Gombessa (E), Celecanto (S), Coelacanthe (F) I F

Laughing Dove (E): Streptopelia senegalensis III B

Laughing Falcon (E): Herpetotheres cachinnans II B

Laughing Owl (E): Sceloglaux albifacies II B

Lavender Firefinch (E): Estrilda caerulescens III B

Lavender Waxbill (E): Estrilda caerulescens III B

Lawes's Parotia (E): Parotia lawesii II B

Lawrence's Girdled Lizard (E): Cordylus lawrencei II R

Layard's Beaked Whale (E): Mesoplodon layardii II M

Layard's Black-headed Weaver (E): Ploceus cucullatus III B

Layard's Parakeet (E): Psintacula calihropae II B

Laysan Duck (E): Anas laysanensis I B

Laysan Teal (E): Anas laysanensis I B

Lazuline Sabrewing (E): Campylopterus falcatus II B

Leadbeater's Cockatoo (E): Cacatua leadbeateri II B

Leaf Coral (E): Montipora foliosa II In/Agaricia agaricites II In

Leaf monkeys (E): Presbytis spp $/$ Trachypirhecus spp. VI M

Lear's Macaw (E): Anodorhynchus leari I B

Least Flying-fox (E): Pleropus woodfordi II M

Least Fruit Bat (E): Pieropus woodfordi II M

Least Pygmy-Owl (E): Glaucidium minutissimum II B

Leatherback (E): Dermochelys coriacea I R

Leathery Turtle (E): Dermochelys coriacea I R

Lechucita acanelada (S): Aegolius harrisii II B

Lechucita común (S): Otus choliba II B/ Speotyto cunicularia II B

Lechucita de las viscacheras (S): Speoryto cunicularia II B

Lechucita rojiza (S): Otus atricapillus II B

Lechuza bataraz (S): Sirix rufipes II B

Lechuza baya de Madagascar (S): Phodilus badius II B

Lechuza blanquinegra (S): Sirix nigrolineata II B

Lechuza campestre (S): Asio flammeus II B

Lechuza colilarga (S): Sirix albitarsus II B/Strix virgata II B

Lechuza común (S): Tyto alba II B

Lechuza copetona (S): Lophostrix cristata II B

Lechuza de campanario (S): Tyto alba II B

Lechuza de Madagascar (S): Tyro soumagnei I B

Lechuza de Tengmalm (S): Aegolius funereus II B

Lechuza estigia (S): Asio stygius II B

Lechuza gavilana (S): Sumia ulula II B

Lechuza gavilana de las Molucas (S): Ninox squamipila I/I B

Lechuza gavilana de Norfolk (S): Ninox novaeseelandiae undulata I B

Lechuza listada (S): Asio clamator II B/Strix hylophila II B

Lechuza mora (S): Asio capensis II B

Lechuza negra (S): Strix huhula II B

Lechuza orejicorta (S): Asio flammeus II B

Lechuza orejita de Madagascar (S): Asio madagascariensis II B

Lechuza patiblanca (S): Sirix albitarsus II B

Lechuza pescadora (S): Scoropelia peli II B

Lechuzas (S): STRIGIFORMES spp. III B

Lechuzón (S): Asio clamator II B

Lechuzón anteojo (S): Pulsatrix perspicillata II B 
Lechuzón anteojo menor (S): Pulsatrix koeniswaldiana II B Lechuzón campestre ( $($ ): Asio flammeus II B

Lechuzón cariblanco (S): Sceloglaux albifacies II B

Lechuzón de Anteojos (S): Pulsatrix perspicillata II B

Lechuzón de Woodford (S): Strix woodfordii II B

Lechuzón negruzco (S): Asio stygius II B

Lechuzón orejas largas (S): Asio clamaior II B

Lechuzón orejudo (S): Asio clamator II B/Bubo virginianus II B

Lechuzón pardo menor (S): Pulsatrix koeniswaldiana II B

Lechwe (E/F): Kobus leche II M

Leeches (E): HIRUDINOIDEA spp. II/NC In

Lehmann's Poison Frog (E): Dendrobates lehmanni II A

Leiopathes expansa: II In

Leiopathes glaberrima: II In

Leiopathes grimaldit: 11 in

Leiopython albertisii = Morelia alberisii

Léiothrix à joues argent (F): Leiothrix argentauris II B

Leiothrix argentauris: Silver-eared Mesia (E), Léiothrix à joues argent (F) II B

Léiothrix jaune ( $\mathrm{F})$ : Leiothrix lutea II B

Leiothrix lusea: Red-billed Leiothrix (E), Léiothrix jaune (F) II B

Léiothrixs (F): Leiothrix spp. II B

Leith's Tortoise (E): Tesiudo kleinmanni I R

Lemiox rimosus = Conradilla caelata

Lémur à couronne (F): Eulemur coronatus I M

Lémur à ventre rouge $(F)$ : Eulemur rubriventer I M

Lemur ardilla coligrueso ( $\mathrm{S}$ ): Cheirogaleus medius I M

Lémur brun (F): Eulemur fulvus I M

Lemur cariancho (S): Hapalemur aureus I M

Lemur cariancho (S): Hapalemur griseus I M

Lemur cariancho (S): Hapalemur simus I M

Lemur catra: Ring-tailed Lemur (E), Lemur colianillado (S), Lémur catta (F), Maki catta (F) I M

Lémur catta (F): Lemur catta I M

Lemur colianillado (S): Lemur carta I M

Lemur comadreja de Ambanja (S): Lepilemur dorsalis I M

Lemur comadreja de dientes pequeños ( $S$ ): Lepilemur microdon I M

Lemur comadreja de Edwards (S): Lepilemur edwardsi I M

Lemur comadreja de pies blancos (S): Lepilemur leucopus I M

Lemur comadreja septentrional (S): Lepilemur septentrionalis I M

Lemur coronado (S): Eulemur coronatus I M

Lemur coronatus = Eulemur coronatus

Lemur de collar (\$): Varecia variegata I M

Lemur de Gola (\$): Varecia variegata $1 \mathrm{M}$

Lemur de Gorguera (S): Varecia variegaia I M

Lemur de vientre rojo (S): Eulemur rubriventer I M

Lemur enano coligrueso (S): Cheirogaleuś medius I M

Lemur enano mayor (S): Cheirogaleus major I M

Lemur fulvus = Eulemur fulvus

Lemur leonado (S): Eulemur fulvus I M

Lemur macaco $=$ Eulemur macaco

Lémur macaco (F): Eulemur macaco I M

Lemur mangosta (S): Eulemur mongoz I M

Lemur mongoz = Eulemur mongoz

Lémur mongoz (F): Eulemur mongoz I M

Lemur negro (S): Eulemur macaco I M

Lémur noir (F): Eulemur macaco I M

Lemur orejipeludo (S): Allocebus trichotis I M

Lemur rubriventer $=$ Eulemur rubriventer

Lemur spp.: I M

Lémur vari (F): Varecia variegata I M

Lemur variegatus = Varecia variegata

Lemures (S): Avahi laniger/Cheirogaleidae spp./Lemuridae spp/Megaladapidae spp. I M

Lemuridae spp.: Lemurs $(E)$, Lemures $(S)$, Lémurs $(F)$, Lémuriens (F) $I \mathrm{M}$

Lémuriens (F): Avahi laniger/Cheirogaleidae spp./emuridae spp/Megaladapidae spp. I M

Lemurs (E): Avahi laniger/Cheirogaleidae spp./Lemuridae spp./Megaladapidae spp. I M

Lémurs (F): Lemuridae spp. I M

León (S): Panthera leo I/II M

León americano (S): Puma concolor I/II M

León bayo (S): Puma concolor I/II M

León brenero (S): Herpailurus yaguarondi $\mathbf{I I I} \mathrm{M}$

León costarricense (S): Puma concolor costaricensis I M
León de Florida (S): Puma concolor coryi I M

León indostảnico (S): Panthera leo persica I M

Leoncillo (S): Herpailunus yaguarondi $\mathbf{~} \mathbf{I I} \mathrm{M}$

Leontideus caissara $=$ Leontopithecus caissara

Leontideus chrysomela = Leonsopithecus chrysomela

Leontideus chrysopygus = Leontopithecus chrysopygus

Leontideus rosalia = Leontopithecus rosalia

Leontideus spp. = Leontopithecus spp.

Leonsopithecus caissara: Black-faced Lion Tamarin (E) I M

Leontopithecus chrysomela: Golden-headed Lion Tamarin (E), Tamarino león de cabeza dorada (S) I M

Leontopithecus chrysopygus: Black Lion Tamarin (E), Goldenrumped Lion Tamarin (E) I M

Leontopithecus rosalia: Golden Lion Tamarin (E), Singe-lion (F), Tamarin soyeux (F) I M

Leontopithecus rosalia caissara $=$ Leontopithecus caissara

Leontopithecus rosalia chrysomela = Leontopithecus chrysomela Leontopithecus rosalia chrysopygus = Leontopithecus chrysopygus

Leontopithecus rosalia rosalia = Leontopithecus rosalia

Leontopithecus spp.: I M

Leopard (E): Panthera pardus IM

Léopard (F): Panthera pardus I M

Leopard Cat (E): Prionailurus bengalensis I/II M

Léopard des neiges (F): Uncia uncia I M

Leopard Dwarf Boa (E): Tropidophis pardalis II R

Leopard Tortoise (E): Geochelone fardalis II R

Leopardo (S): Panthera pardus I M

Leopardo nival (S): Uncia uncia I M

Leopards (E): Neofelis nebulosa/Panthera pardus/Uncia uncia I M

Leopardus pardalis: Ocelot (E), Gato onza (S), Manigordo (S), Ocelote (S), Ocelot (F) I M

Leopardus tigrinus: Little Spotted Cat (E), Oncilla (E), Tiger Cat (E), Caucel (S), Gato atigrado (S), Gato tigre (S), Tigrillo (S), Tirica (S), Chat-tacheté (F), Chat-tigre (F), Oncille (F) I M

Leopardus wiedii: Margay (E), Tree Ocelot (E), Caucel (S), Gato montés (S), Margay (S), Tigrillo (S), Margay (F) I M

Lépidochelyde de Kemp (F): Lepidochelys kempi I R

Lepidochelys kempi: Atlantic Ridley (E), Gulf Ridley (E), Kemp's Ridley (E), Mexican Ridley (E), Cotorra (S), Tortuga iora (S), Tortuga marina bastarda (S), Lépidochelyde de Kemp (F), Ridley de Kemp (F), Tortue de Kemp (F) I R

Lepidochelys olivacea: Olive Ridley (E), Pacific Ridley (E), Tortuga golfina (S), Tortuga olivacea (S), Ridley du Pacifique (F).

Tortue bâtarde (F), Tortue de Ridley (F), Tortue olivâtre (F) I R

Lepidocyathus = Acropora

Lepidopora acrolophos: II in

Lepidopora biserialis: II In

Lepidopora carinata: II In

Lepidopora clavigera: II In

Lepidopora cryptocymas: II In

Lepidopora decipiens: II in

Lepidopora dendrostyius: II In

Lepidopora diffusa: II In

Lepidopora glabra: II In

Lepidopora granulosa: II In

Lepidopora hicksoni: II In

Lepidopora microstylus: II In

Lepidopora polystichopora: II In

Lepidopora sarmentosa: II In

Lepidopora symmetrica: II in

Lepidopyga coeruleogularis: Sapphire-throated Hummingbird $(E)$, Colibri faux-saphir (F) II B

Lepidopyga goudori: Shining-green Hummingbird (E), Tucusito pico curvo (S), Colibri de Goudot (F) II B

Lepidopyga lilliae: Sapphire-bellied Hummingbind (E), Colibri de Lillian (F) II B

Lepidotheca altispina: II In

Lepidotheca brochi: II in

Lepidotheca cenicornis: II In

Lepidotheca chauliostylus: II In

Lepidotheca fascicularis: II In

Lepidotheca hachijoensis: II in

Lepidorheca harrida: II In

Lepidotheca inconsuta: II In

Lepidotheca japonica: II In

Lepidotheca macropora: II In 
Lepidotheca pourtalesi: II In

Lepidotheca ramosa: II In

Lepidotheca robusta: II In

Lepidotheca renuistylus: 11 In

Lepilemur dorsalis: Grey-backed Sportive Lemur (E), Nossi-bé Sportive Lemur (E), Lemur comadreja de Ambanja (S) I M

Lepilemur edwardsi: Milne-Edwards's Sportive Lemur (E), Lemur comadreja de Edwards (S) I M

Lepilemur leucopus: Dry-bush Weasel Lemur (E), White-footed Sportive Lemur (E), Lemur comadreja de pies blancos (S) I M

Lepilemur microdon: Light-necked Sportive Lemur (E), Lemur comadreja de dientes pequeños (S) I M

Lepilemur mustelinus: Greater Sportive Lemur (E), Greater Weasel Lemur (E), Grand lépilémur (F) I M

Lepilemur mustelinus dorsalis = Lepilemur dorsalis

Lepilemur mustelinus edwardsi = Lepilemur edwardsi

Lepilemur mustelinus leucopus = Lepilemur leucopus

Lepilemur mustelinus microdon = Lepilemur microdon

Lepilemur mustelinus ruficaudarus = Lepilemur ruficaudatus

Lepilemur mustelinus septentrionalis = Lepilemur septentrionalis

Lepilemur rufescens = Lepilemur edwardsi

Lepilemur ruficaudarus: Lesser Weasel Lemur (E), Red-tailed Sportive Lemur (E), Petit lépilémur (F) I M

Lepilemur septentrionalis: Northern Sportive Lemur (E), Lemur comadreja septentrional (S) I M

Lepilemur spp.: I M

Leporillus conditor. Greater Stick-nest Rat (E), House-building Rat (E), Rata arquitecto (S), Rat architecte (F) I M

Leptailurus serval: Serval (E), Serval (S), Chat-tigre (F), Serval (F) II $\mathbf{M}$

Leptastrea bewickensis: II in

Leptastrea botiae: II in

Leptastrea humilis: II In

Leplastrea inaequalis: II In

Leptastrea pruinosa: II In

Leptastrea purpurea: II In

Leptastrea solidocolumella: II In

Leptastrea transversa: II In

Leptodon cayanensis: Cayenne Kite (E), Grey-headed Kite (E),

Gavilán palomero (S), Milano blanquigrís (S), Milano cabecigris (S), Milan de Cayenne (F) II B

Leptodon forbesi: White-collared Kite (E), Milano acollarado (S), Milan de Forbes (F) II B

Leptopenus antarcticus: 11 In

Leptopenus discus: II In

Leptopenus hypocoelus: 11 in

Leptopenus solidus: II In

Leptopsammia britannica: II In

Lepropsammia chevalier: II In

Leptopsammia formosa: II In

Leptopsammia microcardia: II In

Lepropsammia pruvoti: II In

Leptopsammia queenslandiae: II In

Leptopsammia stokesiana: II In

Leptopsammia irinitaris: II In

Leptoptilos crumeniferus: Marabou Stork (E), Marabú africano (S), Marabout africain (F) III B

Leproria irregularis: II In

Leptoria phrygia: II In

Leptoseris amitoriensis: II In

Leproseris cailleti: II In

Leptoseris cucullata: II In

Leptoseris explanata: II In

Leptoseris foliosa: II In

Leptoseris fragilis: II In

Leptoseris gardineri: II In

Leptoseris glabra: II In

Leptoseris hawaiiensis: II In

Leptoseris incrustans: II In

Leptoseris mycetoseroides: II In

Leptoseris papyracea: II In

Leptoseris scabra: II in

Leptoseris solida: II In

Leptoseris tenuis: II In

Leptoseris yabei: II In
Leptosillaca branickii: Golden-plumed Conure (E), Golden-plumed Parakeet (E), Aratinga de pinceles (S), Conure a pinceaux d'or (F), Perruche de Branicki (F) II B

Leptosmilia = Euphyllia

Lesbia nuna: Green-tailed Trainbearer (E), Colacinta coliverde (S), Colibrí coludo verde (S), Porte-traîne nouna (F) II B

Lesbia victoriae: Black-tailed Trainbearer $(E)$, Colacinta colinegra (S), Porte-traîne lesbie (F) II B

Lesser Antillean Iguana (E): Iguana delicatissima II R

Lesser Beaked Whale (E): Mesoplodon peruvianus II M

Lesser Bilby (E): Macrotis leucura $1 \mathrm{M}$

Lesser Bird-of-paradise (E): Paradisaea minor II B

Lesser Black-Hawk (E): Buteogallus anthracinus II B

Lesser Bushbaby (E): Galago senegalensis II M

Lesser Collared Forest-Falcon (E): Micrastur buckleyi II B

Lesser Eagle-Owl (E): Mimizuku gumeyi I B

Lesser Fish-eagle (E): Ichthyophaga humilis II B

Lesser Fishing Eagle (E): Ichihyophaga humilis II B

Lesser Flamingo (E): Phoenicopterus minor II B

Lesser Florican (E): Eupodotis indica II B

Lesser Flying-fox (E): Pleropus mahaganus II M

Lesser Galago (E): Galago senegalensis II M

Lesser Kestrel (E): Falco naumanni II B

Lesser Mascarene Flying-fox (E): Pteropus subniger II M

Lesser Masked-Owl (E): Tyto sororcula II B

Lesser Melampitta (E): Melampitta lugubris II B

Lesser Mouse-lemur (E): Microcebus murinus I M

Lesser Needle-clawed Galago (E): Galago masschiei II M

Lesser Panda (E): Ailurus fulgens I M

Lesser Rabbit-cared Bandicoot (E): Macrotis leucura I M

Lesser Rhea (E): Rhea pennata I B

Lesser Rorqual (E): Balaenoptera acutorosirata I/I M

Lesser Rufous Bristlebird (E): Dasyornis broadbenti lioralis I B

Lesser Slow Loris (E): Nycticebus pygmaeus II M

Lesser Sooty-Owl (E): Tyto multipunctata II B

Lesser Spectral Tarsier (E): Tarsius pumilus II M

Lesser Spot-nosed Guenon (E): Cercopithecus petaurista II M

Lesser Spotted Eagle (E): A quila pomarina II B

Lesser Sulphur-crested Cockatoo (E): Cacanua sulphurea II B

Lesser Sunda Scops-Owl (E): Otus silvicola II B

Lesser Treeshrew (E): Tupaia minor II M

Lesser Weasel Lemur (E): Lepilemur ruficaudatus I M

Lesser White-nosed Guenon (E): Cercopithecus petaurista II M

Lesser White-nosed Monkey (E); Cercopithecus petaurista II M

Lesueur's Rat Kangaroo (E): Bettongia lesueur I M

Letepsammia fissilis: II In

Letepsammia formosissima: II In

Letepsammia franki: II in

Letter-winged Kite (E): Elanus scriprus II B

Leucippus baeri: Tumbes Hummingbird (E), Colibri de Tumbes (F) II B

Leucippus chlorocercus: Olive-spotted Hummingbird (E), Colibri olivipunteado (S), Colibri à queue verte (F) II B

Leucippus fallax: Buffy Hummingbird (E), Colibri anteado (S), Colibri trompeur (F) II B

Leucippus taczanowski: Spot-throated Hummingbird (E), Colibri de Taczanowski (F) II B

Leucochloris albicollis: White-throated Hummingbird (E), Picaflor cuelliblanco (S), Colibri à gorge blanche (F) II B

Leucogéranne (F): Grus leucogeranus I B

Leucopsar rothschildi: Bali Myna (E), Bali Starling (E), Rothschild's Mynah (E), Rothschild's Starling (E), White Starling (E), Estornino de Rothschild (S), Miná de Rothschild (S), Mainate de Rothschild (F), Martin de Rothschild (F), Sansonnet de Rothschild (F), Étoumeau de Rothschild (F) I B

Leucopternis albicollis: White Hawk (E), Aguilila blanca (S), Busardo blanco (S), Gavilán blanco (S), Buse blanche (F) II B

Leucoptemis kuhli: White-browed Hawk (E), Busardo cejiblanco (S), Buse à sourcils blancs (F) II B

Leucoptemis lacemulata: White-necked Hawk (E), Busardo cuelliblanco (S), Buse lacernulée (F) II B

Leucopternis melanops: Black-faced Hawk (E), Masked Hawk (E), Busardo carinegro (S), Gavilán carinegro (S), Buse à face noire (F) II B

Leucopternis occidentalis: Grey-backed Hawk (E), Busardo dorsigrís (S), Gavilán dorsigris (S), Buse à dos gris (F) II B 
Leucopternis plumbea: Plumbeous Hawk (E), Busardo plomizo (S), Gavilán plomizo (S), Buse plombée (F) II B

Leucopternis polionota: Mantled Hawk (E), Aguila blanca (S), Busardo blanquinegro (S), Buse mantelée (F) II B

Leucopternis princeps: Barred Hawk (E), Black-chested Hawk (E), Busardo azoreño (S), Gavilán principe (S), Buse barrée (F) II B

Leucopternis schislacea: Slate-colored Hawk (E), Busardo pizarroso (S), Gavilán azul (S), Gavilán pizarroso (S), Buse ardoisée (F) II B

Leucopternis semiplumbea: Semiplumbeous Hawk (E), Busardo semiplomizo (S), Gavilán semiplomizo (S), Buse semiplombée (F) II B

Levant Sparrowhawk (E); Accipiter brevipes II B

Lézard à ceinture épineuse (F): Pseudocordylus spinosus II R

Lézard à fausse ceinture de Lang (F): Pseudocordylus langi II $R$

Lézard à fausse ceinture des rochers (F): Pseudocordylus microlepidotus II R

Lézard à fausse ceinture du Cap (F): Pseudocordylus capensis II R

Lézard à queue épineuse à larges écailles (F): Cordylus macropholis II $\mathrm{R}$

Lézard à queue épineuse à pustules (F): Cordylus pustulatus II $\mathbf{R}$

Lézard à queue épineuse à laches bleues (F): Cordylus coeruleopunctatus II R

Lézard à queue épineuse africain (F): Cordylus polyzonus II R

Lézard à queue épineuse commun (F): Cordylus cordylus II R

Lézard à queue épineuse d'Armadillo (F): Cordylus cataphractus II $\mathbf{R}$

Lézard à queue épineuse de Campbell (F): Cordylus campbelli II R

Lézard à queue épineuse de Cope (F): Cordylus tropidostemum II R

Lézard à queue épineuse de Hewitt (F): Cordylus peersi II R

Lézard à queue épineuse de Jordan (F): Cordylus jordani II R

Lézard à queue épineuse de Lawrence (F): Cordylus lawrencei II R

Lézard à queue épineuse de McLachlan (F): Cordylus mclachlani II $R$

Lézard à queuse épineuse de Reichenow (F): Cordylus vitufer II R

Lézard à queue épineuse de Rhodésie (F): Cordylus rhodesianus II R

Lézard à queue épineuse de Tasman (F): Cordylus tasmani II $k$ K

Lézard à queue épineuse de Warren (F): Cordylus warreni II R

Lézard à queue épineuse d'Oelofsen (F): Cordylus oelofseni II R

Lézard à queue épineuse du Narnaqua (F): Cordylus namaquensis II $R$

Lézard à queue épineuse d'Ukinga (F): Cordylus ukingensis II R

Lézard à queue épineuse géant $(\mathrm{F})$ : Cordylus giganteus II R

Lézard à queue épineuse noir (F): Cordylus niger II R

Lézard cornu (F): Phrynosama coronatum II R

Lézard crocodile de Chine (F): Shinisaurus crocodilurus II R

Lézard de Lilford (F): Podarcis lilfordi II R

Lézard de Simony (F): Gallotia simonyi I R

Lézard des Baléares (F): Podarcis lilfordi II R

Lézard des Pityuses (F): Podarcis pityusensis II R

Lézard des rochers (F): Pseudocordylus microlepidotus II R

Lézard des rochers du Drakensberg (F): Pseudocordylus melanotus II $\mathbf{R}$

Lézard perlé (F): Heloderma suspectum II R

Lézard-caïman de Guyane (F): Dracaena guianensis II R

Lézard-caïman du Paraguay (F): Dracaena paraguayensis II R

Lézards à fausse ceinture (F): Pseudocordylus spp. II R

Lézards à queue épineuse d'Afrique Australe (F): Cordylus spp. II R

Lézards caiman (F): Dracaena spp. II R

Lézards fouette-queue (F): Uromastyx spp. II R

Lézards perlés (F): Heloderma spp. II R

Lézards venimeux (F): Heloderma spp. II R

L'Hoest's Monkey (E): Cercopithecus lhoesti II M

Liasis alberisii = Morelia alberisii

Liasis amethistinus = Morelia amerhistina

Liasis boa $=$ Morelia boa

Liasis boeleni $=$ Morelia boeleni

Liasis carinata $=$ Morelia carinata

Liasis childreni $=$ Morelia childreni

Liasis fuscus albertisii = Morelia albertisii

Liasis mackloti Liasis fuscus = Morelia macklon

Liasis maculosus = Morelia maculosa

Liasis maximus = Morelia papuana

Liasis olivaceus $=$ Morelia olivacea

Liasis papuanus $=$ Morelia papuana

Liasis perthensis $=$ Morelia perthensis
Liasis sauvuensis = Morelia mackloti

Liasis taronga $=$ Morelia bueleni

Liberia Nimba Toad (E): Nimbaphrynoides liberiensis I A

Lichunotus laniger $=$ Avahi laniger

Lichanura roseofusca $=$ Lichanura rivirgaia

Lichanura irivirgata: Rosy Boa (E), Boa mexicana rosada (S), Boa rose (F), Boa à trois bandes (F) II $R$

Lichenostomus cassidix = Lichenostomus melanops cassidix

Lichenostomus melanops cassidix: Helmeted Honeyeater (E), Melero de casco (S), Méliphage casqué (F), Méliphage cornu (F) I B

Lichenostomus melanops cassidix = Lichenostomus melanops cassidix

Light-necked Sportive Lemur (E): Lepilemur microdon I M

Likh (E): Eupodotis indica II B

Lilac-crowned Amazon (E): Amazona finschi II B

Lilac-crowned Parrot (E): Amazona finschi II B

Lilac-tailed Parrotlet (E): Touir batavica II B

Lilford's Wall Lizard (E); Podarcis lilfordi II R

Lilian's Lovebird (E): Agapomis lilianae II B

Limpiacasa (S): Phaethomis augusti II B

Lince (S): Lynx tynx II M

Lince africano (S): Caracal caracal I/II M

Lince del Canadá (S): Lynx canadensis II M

Lince ibérico (S): Lynx pardinus I M

Lince rojo (S): Lynx rufus II M

Lined Day Gecko (E): Phelsuma lineata II R

Lined Forest-Falcon (E): Micrastur gilvicollis II B

Lingo (S): Bassaricyon gabbii III M

Linsang à taches (F): Prionodon pardicolor I M

Linsang rayé (F): Prionodon linsang II M

Linsang tacheté (F): Prionodon pardicolor I M

Linsangs (E): Prionodon spp/Poiana richardsonii //U/NC M

Liocichla omeiensis: Mount Omei Liocichla (E), Omei Shan Liocichla (E), Garrulaxe de l'Omei (F) II B

Lion (E/F): Panthera leo $\mathbf{V} / \mathbf{I} \mathrm{M}$

Lion d'Afrique (F): Panthera leo II M

Lion d'Asie (F): Panthera leo persica I M

Lion tamarins (E): Leontopithecus spp. I M

Lion-tailed Macaque (E): Macaca silenus I M

Lipotes vexillifer: Baiji (E), Changjiang Dolphin (E), Chinese Lake Dolphin (E), White Flag Dolphin (E), Whitefin Dolphin (E),

Baiji (S), Delfín de China (S), Baiji (F), Dauphin fluviatile de Chine (F) I M

Lissemys puncrata: Indian Flap-shell Turtle (E), Indo-Gangetic Flapshell (E), Spotted Turtle (E), Tortuga de concha blanda moteada (S), Tortuga plana indiana (S), Lyssémyde ponctuée (F), Tortue de l'Inde (F), Trionyx de l'Inde (F) II R

Lissodelphis borealis: Northem Right Whale Dolphin (E), Delfín liso (S), Dauphin aptère boréal (F) II M

Lissodelphis peronit: Southern Right Whale Dolphin (E), Delfin liso (S), Tunina sinaleta (S), Dauphin aptère austral (F) II M

Lissotis hartaubii $=$ Eupodotis hartlaubii

Lissotis melanogaster $=$ Eupodotis melanogaster

Liszt Monkey (E): Saguinus geoffroyi I M

Litharea $=$ Goniopora

Lithomyces $=$ Flabellum

Lithophyllia = Scolymia

Lithophyllon mokai: II In

Lithophyllon undularum: II In

Little agate shells (E): Achatinella spp. I In

Little Banded Sparrowhawk (E): Accipiter badius 11 B

Little Blue Macaw (E): Cyanopsitta spixil I B

Little Brown Bustard (E): Eupodotis humilis II B

Little Bustard (E): Tetrax tetrax II B

Little Corella (E): Cacarua sanguinea II B

Little Eagle (E): Hieraaetus morphnoides II B

Little Egret (E): Egretta garzetia III B

Little Falcon (E): Falco longipennis II B

Little Fox (E): Pseudalopex griseus II M

Little Golden-mantled Flying-fox (E): Pteropus pumilus II M

Little Hermit (E): Phaethomis longuemareus II B

Little Lorikeet (E): Glossopsirta pusilla II B

Little Owl (E): Athene noctua II B

Little Piked Whale (E): Balaenopiera acusorostraia VII M

Little Red Flying-fox (E): Pteropus scapulatus II M

Little Red Lorikeet (E): Charmosyna pulchella II B 
Litule Spotted Cat (E): Leopardus tigrinus I M Litte Sunangel (E): Heliangelus exoris II B Little Weaver (E): Ploceus luteolus III B Little Woodstar (E): Acestrura bombus II B Livingstone's Turaco (E): Tauraco livingstonii II B Lizard Buzzard (E): Kaupifalco monogrammicus II B Lizards (E): SAURIA spp. IIINC R

Llanos Sideneck Turtle (E): Podocnemis vogli II R

Lobactis $=$ Fungia

Lobed Star Coral (E): Solenastrea hyades II In

Lobito de cola ancha (S): Pteronura brasiliensis I M

Lobito de Río (S): Lontra longicaudis I M

Lobito del Plata (S): Lontra longicaudis I M

Lobito patagonica (S): Lontra provocax I M

Lobo común (S): Canis lupus I/II M

Lobo de crin (S): Chrysocyon brachyurus II M

Lobo de Río Grande (S): Pteronura brasiliensis I M

Lobo de Tasamania (S): Thylacinus cynocephalus I M

Lobo de tierra (S): Proteles cristatus III M

Lobo del río (S): Preronura brasiliensis I M

Lobo gargantilla (S): Pteronura brasiliensis I M

Lobo marsupial (S): Thylacinus cynocephalus I M

Loboparadisea sericea: Wattle-billed Bird-of-paradise (E), Yellowbreasted Bird-of-paradise (E), Paradisier soyeux (F) II B

Lobophyllia corymbosa: II In

Lobophyllia diminuta: II In

Lobophyllia hataii: II In

Lobophyllia hemprichii: II In

Lobophyllia pachysepta: II In

Labophyllia robusta: II In

Lochmaeotrochus oculeus: II In

Loddigesia mirabilis: Marvellous Spatuletail (E), Loddigésie admirable (F) II B

Loddigésie admirable (F): Loddigesia mirabilis II B

Lofóforo chino (S): Lophophorus Ihuysii I B

Lofóforo de Sclater (S): Lophophorus sclateri I B

Lofóforo del Himalaya (S): Lophophorus impejanus I B

Loggerhead (E): Carelra carenta I R

Lombok Flying-fox (E): Pteropus lombocensis II M

Lonchura bicolor: Black-and-white Mannikin (E), Black-and-white Munia (E), Blue-billed Mannikin (E), Capucin bicolore (F), Spermète à bec bleu $(\mathrm{F})$ III B

Lonchura cantans: African Silverbill (E), Monjita pico-de-plata (S), Bec-d'argent (F), Capucin bec-d'argent (F) III B

Lonchura cucullata: Bronze Mannikin (E), Bronze Munia (E). Bronze-winged Mannikin (E), Negrita bronceada (S), Capucin nonnette $(F)$. Spermète nonnerte $(F)$ III B

Lonchura fringilloides: Magpie Mannikin (E), Magpie Munia (E). Pied Mannikin (E), Negrita picanza (S), Capucin pie (F), Spermète pie (F) III B

Lonchura malabarica cantans = Lonchura cantans Long-beaked Dolphin (E): Stenella longirostris II M Long-beaked Echidna (E): Zaglossus bruijni II M

Long-billed Bristlebird (E): Dasyomis longirostris I B

Long-billed Corelia (E): Cacatua tenuirostris II B

Long-billed Curlew (E): Numenius renuirostris I B

Long-billed Griffon (E): Gyps indicus II B

Long-billed Partridge (E): Rhizothera longirostris III B

Long-billed Starthroat (E): Heliomaster longirostris II B

Long-billed Vulture (E): Gyps indicus II B

Long-crested Eagle (E): Lophaerus occipitalis II B

Long-eared Flying Squirrel (E): Idiurus macrotis III M

Long-eared Owl (E): Asio otus II B

Long-finned Pilot Whale (E): Globicephala melaena II M

Long-haired Spider Monkey (E): Ateles belzebuth II M

Long-legged Buzzard (E): Buteo rufinus II B

Longman's Beaked Whale (E): Indopacerus pacificus II M

Long-nosed Bandicoot (E): Perameles bougainville I M

Long-nosed Crocodile (E): Gavialis gangericus I R

Long-nosed Echidna (E): Zaglossus bruijni II M

Long-nosed Monkey (E): Nasalis larvatus I M

Long-nosed Spiny Anteater (E): Zaglossus bruijni II M

Long-snouted Crocodile (E): Crocodylus cataphracrus I R

Long-snouted Dolphin (E): Stenella longirostris II M

Long-tailed Chinchilla (E): Chinchilla lanigera I M

Long-tailed Dove (E): Oena capensis III B
Long-tailed Dunnart (E): Sminthopsis longicaudata I M Long-tailed Hawk (E): Urotriorchis macrourus II B

Long-tailed Hermit (E): Phaethomis superciliosus II B

Long-tailed Honey-buzzard (E): Henicopemis longicauda II B

Long-tailed Langur (E): Presbytis potenziani I M

Long-tailed Macaque (E): Macaca fascicularis II M

Long-tailed Marmot (E): Marmota caudata III M

Long-tailed Marsupial-Mouse (E): Sminthopsis longicaudata I M

Long-tailed Otter (E): Lontra longicaudis I M

Long-tailed Pangolin (E): Manis tetradactyla II M

Long-tailed Paradigalla (E): Paradigalla carunculata II B

Long-tailed Paradise-Whydah (E): Vidua interjecta III B

Long-tailed Parakeet (E): Psittacula longicauda II B

Long-tailed Rock Monitor (E): Varanus glebopalma II R

Long-tailed Sabrewing (E): Campylopterus excellens II B

Long-tailed Sminthopsis (E): Sminthopsis longicaudata I M

Long-tailed Sylph (E): Aglaiocercus kingi II B

Long-tailed Woodnymph (E): Thalurania watertonii II B

Long-tufted Screech-Owl (E): Otus sanctaecatarinae II B

Long-wattled Umbrellabird (E): Cephalopterus penduliger III B

Long-whiskered Owlet (E): Xenoglawx loweryi II B

Long-winged Harrier (E): Circus buffoni II B

Lontra annectens $=$ Lontra longicaudis

Lontra canadensis: North American Otter (E), North American River Otter (E), Nutria de Canadá (S), Nutria norteamericana (S), Loutre du Canada (F) II M

Lontra enudris = Lontra longicaudis

Lontra felina: Chingungo (E), Chungungo (E), Marine Otter (E), Sea Cat (E), Chichimen (S), Chinchimen (S), Chungungo (S), Gato de mar (S), Gato marino (S), Huallaca (S), Nutria de mar (S), Chungungo $(F)$, Loutre de mer $(F)$ I M

Lontra incarum $=$ Lontra longicaudis

Lontra longicaudis: Long-tailed Otter (E), Neotropical River Otter (E), South American River Otter (E), Gato de agua (S), Lobito de Río (S), Lobito del Plata (S), Nutria de agua (S), Perro de agua (S), Taira (S), Loutre d'Amérique du Sud (F), Loutre à longue queue (F) I M

Lontra mesopetes $=$ Lontra longicaudis

Lontra platensis $=$ Lontra longicaudis

Lontra provocax: Huillin (E), Southem River Otter (E), Huillín (S), Lobito patagonica (S), Nutria de Chile (S), Huillin (F), Loutre du Chili (F) I M

Lophaetus occipitalis: Long-crested Eagle (E), Aguila copetuda africana (S). Aguila crestilarga (S), Aigle huppard (F) II B

Lophelia periusa: II in

Lophocebus albigena: Grey-cheeked Mangabey (E), White-cheeked Mangabey (E), Mangabey de mejillas grises (S) II M

Lophocebus aterrimus = Lophocebus albigena

Lophohelia $=$ Lophelia

Lophoictinia isura: Square-tailed Kite (E), Milano colicuadrado (S), Milan à queue carrée (F) II B

Lophophore de Lhuys (F): Lophophorus thuysii I B

Lophophore de Sclater (F): Lophophorus sclateri I B

Lophophore resplendissant (F): Lophophorus impejanus I B

Lophophores (F): Lophophorus spp. I B

Lophophorus impejanus: Himalayan Monal (E). Impeyan Pheasant (E), Monal Pheasant (E), Faisán monal del Himalaya (S). Lofóforo del Himalaya (S), Monal colirrojo (S), Lophophore resplendissant (F), Monal de l'Himalaya (F) I B

Lophophorus thuysii: Chinese Impeyan (E), Chinese Monal (E). Faisán monal chino (S), Lofoforo chino (S), Monal coliverde (S), Lophophore de Lhuys (F), Monal chinois (F) I B

Lophophorus sclateri: Crestless Monal (E), Sclater's Monal (E), Faisán monal de Sclater (S), Lofóforo de Sclater (S), Monal coliblanco (S), Lophophore de Sclater (F), Monal birman (F) I B

Lophorina superba: Superb Bird-of-paradise (E), Paradisier superbe (F) II B

Lophomis adorabilis: Adorable Coquette (E), White-crested Coquette (E), Coquette adorable (F) II B

Lophomis brachylopha: Short-crested Coquette (E), Coqueta cresticorta (S), Coquette du Guerrero (F) II B

Lophornis chalybeus: Festive Coquette (E), Coqueta abanico puntiblanco (S), Coqueta festiva (S), Picaflor abanico verde (S), Coquette chalybée (F) II B 
Lophomis delartrei: Rufous-crested Coquette (E), Coquette de Delattre (F) II B

Lophomis delattrei brachylopha $=$ Lophomis brachylopha

Lophomis gouldii: Dot-eared Coquette (E), Coquette de Gould (F) II B

Lophomis helenae: Black-crested Coquette (E), Coqueta crestinegra (S), Coquette d'Hélène (F) II B

Lophornis magnificus: Frilled Coquette $(\mathrm{E})$, Coquette magnifique $(\mathrm{F})$ II B

Lophornis omatus: Tufted Coquette (E), Coqueta abanico canela (S), Coquette huppe-col (F) II B

Lophomis pavoninus: Peacock Coquette (E), Coqueta abanico pavo real (S), Coquette paon (F) II B

Lophornis stictolophus: Spangled Coquette (E), Coqueta coronada (S), Coqueta lentejuelada (S), Coquette pailleté (F) II B

Lophoseris = Pavona

Lophosmilia = Oxysmilia

Lophostrix cristata: Crested Owl (E), Búho comiblanco (S), Búho comudo (S), Lechuza copetona (S), Duc à aigrettes (F), Hibou à casque (F) II B

Lophostrix lettii $=$ Jubula lettii

Lophotis ruficrista = Eupodotis ruficrista

Lophotis savilei $=$ Eupodotis savilei

Lophura edwardsi: Edwards's Pheasant (E), Faisán de Edwards (S), Faisan d'Edwards (F) I B

Lophura erythrophthalma: Crestless Fireback (E), Rufous-tailed Pheasant (E), Faisán colicanelo (S), Faisán de carúncula azul (S), Faisan à queue rousse (F) III B

Lophura ignita: Crested Fireback (E), Vieillot's Fireback (E), Faisán de caríncula azul crestado (S), Faisán noble (S), Faisan noble (F) III B

Lophura imperialis: Imperial Pheasant (E), Faisán imperial (S), Faisan impérial (F) I B

Lophura swinhoii: Swinhoe's Pheasant (E), Faisán de Formosa (S), Faisán de Swinhoe (S), Faisan de Formose (F), Faisan de Swinhoe (F) I B

Lord Derby's Flying Squirrel (E): Anomalurus derbianus III M

Lord Howe Island Woodhen (E): Gallirallus sylvestris I B

Lord Howe Rail (E): Gallirallus sylvestris I B

Lori à bandeau rouge $(F)$ : Glossopsitta concinna II B

Lori à collier jaune (F): Lorius chlorocercus II B

Lori à couronne pourpre (F): Glossopsitta porphyrocephala II B

Lori à diadème (F): Charmosyna diadema II B

Lori à front rouge (F): Charmosyna rubronotata II B

Lori à gorge rouge (F): Charmosyna amabilis II B

Lori a joues bleues (F): Eos cyanogenia II B

Lori à masque rouge (F): Glossopsina pusilla II B

Lori à menton rouge (F): Chamosyna rubrigularis II B

Lori à nuque blanche (F): Lorius albidinuchus II B

Lori à ventre violet $(\mathrm{F})$ : Lorius hypoinochrous II B

Lori acollarado (S): Lorius chlorocercus II B

Lori adomado (S): Trichoglossus omatus II B

Lori alinegro (S): Eos cyanogenia II B

Lori almizclero (S): Glossopsitta concinna II B

Lori arcoiris (S): Trichoglossus haematodus II B

Lori arlequin (F): Eos histrio I B

Lori barbirrojo (S): Charmosyna rubrigularis II B

Lori bigotudo (S): Oreopsittacus arjaki II B

Lori bridé (F): Oreopsittacus arfaki II B

Lori cardenal (S): Chalcopsitta cardinalis II B

Lori cardinal (F): Chalcopsitta cardinalis II B

Lori carirrojo (\$): Glossopsitta pusilla II B

Lori chispeado (S): Chalcopsitta sintillata II B

Lori coronipúrpura (S): Glossopsina porphyrocephala II B

Lori croquet (F): Charmosyna placentis II B

Lori damisela (S): Lorius domicella II B

Lori de Buru (F): Charmosyna toxopei II B

Lori de Buru (S): Chamosyna toxopei II B

Lori de Duyvenbode (F): Chalcopsitta duivenbodei II B

Lori de Goldie (S): Psitteuteles goldiei II B

Lori de Josefina (S): Charmosyna josefinae II B

Lori de Joséphine (F): Charmosyna josefinae II B

Lori de Kuhl (F): Vini kuhlii II B

Lori de las Tanimbar (S): Eos reticulata II B

Lori de l'île Buru (F): Charmosyna toxopei II B

Lori de Margaret (F): Charmosyna margarethae II B
Lori de Margarita (S): Charmosyna margarethae II B

Lori de Marguerite (F): Charmosyna margarethae II B

Lori de Meek (F): Charmosyna meeki II B

Lori de Meek (S): Charmosyna meeki II B

Lori de Mindanao (S): Trichoglossus johnstoniae II B

Lori de Musschenbroek (F): Neopsittacus musschenbroekii II B

Lori de Nouvelle-Calédonie (F): Charmosyna diadema II B

Lori de Papouasie (F): Charmosyna papou II B

Lori de Ponapé (S): Trichoglossus rubiginosus II B

Lori de Rimitara (S): Vini kuhlii II B

Lori de Samoa (S): Vini australis II B

Lori de Sangir (S): Eos histrio I B

Lori de Seram (S): Eos semilarvata II B

Lori de Stephen (F): Vini stepheni II B

Lori de Stephen (S): Vini stepheni II B

Lori de Wilhelmina (F): Charmosyna wilhelminae II B

Lori des dames (F): Lorius domicella II B

Lori des Fidji (F): Phigys solitarius II B

Lori des Moluques (F): Lorius lory II B

Lori des montagnes (F): Oreopsitracus arfaki II B

Lori des palmiers (F): Charmosynu palmanum II B

Lori diadema (S): Charmosyna diadema II B

Lori écaillé (F): Eos squamata II B

Lori écarlate (F): Eos bomea II B

Lori émeraude (F): Neopsintacus pullicauda II B

Lori escamoso (S): Eos squamria II B

Lori escuamiverde (S): Trichoglossus chlorolepidotus II B

Lori estriado (S): Charmosyna multistriata II B

Lori féérique (F): Charmosyna pulchella II B

Lori flamméché (F): Chalcopsinta sintillata II B

Lori flanquirrojo (S): Charmosyna placentis II B

Lori frentirrojo (S): Chanmosyna rubronotata II B

Lori fringillaire (F): Vini australis II B

Lori gárrulo (S): Lorius garrulus II B

Lori gorgirrojo (S): Chamosyna amabilis II B

Lori humilde (S): Trichoglossus euteles II B

Lori iris (S): Psitteuteles iris II B

Lori lindo (S): Charmosyna pulchella II B

Lori masqué $(\mathrm{F})$ : Eos semilarvata II B

Lori monjita (S): Vini peruviana II B

Lori montano chico (S): Neopsitracus pullicauda II B

Lori montano grande (S): Neopsittacus musschenbroekii II B

Lori musc (F): Glossopsitta concinna II B

Lori negro (S): Chalcopsitta atra II B

Lori noir (F): Chalcopsitta atra II B

Lori noira (F): Lorius garrulus II B

Lori nonnette (F): Vini peruviana II B

Lori nuquiblanco (S): Lorius albidinuchus II B

Lori orange sombre (F): Pseudeos fuscata II B

Lori palmero (S): Charmosyna palmarum II B

Lori papou (F): Charmosyna papou II B

Lori pardo (S): Chalcopsinta duivenbodei II B

Lori pigmeo (S): Charmosyna wilhelminae II B

Iori rabilargo (S): Charmosyna papou II B

Lori réticulé (F): Eos reticulata II B

Lori rojo (S): Eos bomea II B

Lori solitaire des îles Fidji (F): Phigys solitarius II B

Lori solitario (S): Phigys solitarius II B

Lori sombre (F): Pseudeos fuscata II B

Lori sombrio (S): Pseudeos fuscata II B

Lori splendide (F): Charmosyna placentis II B

Lori strié (F): Charmosyna multistriata II B

Lori tricolor (S): Lorius lory II B

Lori tricolore (F): Lorius lory II B

Lori ultramar (S): Vini ultramarina I B

Lori ultramarin (F): Vini ultramarina I B

Lori ventrivinoso (S): Lorius hypoinochrous II B

Lori verdigualdo (S): Trichoglossus flavoviridis II B

Lori versicolor (S): Psitteuteles versicolor II B

Loria loriae $=$ Cnemophilus loriae

Loria's Bird-of-paradise (E): Cnemophilus loriae II B

Lorículo amable (S): Loriculus amabilis II B

Lorículo coroniazul (S): Loriculus galgulus II B

Lorículo de Ceilán (S): Loriculus beryllinus II B

Lorículo de Célebes (S): Loriculus srigmarus II B

Lorículo de Flores (S): Loriculus flosculus II B 
Lorículo de Java (S): Loriculus pusillus II B

Lorículo de la Sangihe (S): Loriculus catamene II B

Lorículo de las Bismarck (S): Loriculus tener II B

Lorículo exiguo (S): Loriculus exilis II B

Lorículo Filipino (S): Loriculus philippensis II B

Lorículo papú (S): Loriculus aurantiifrons II B

Lorículo vemal (S): Loriculus vemalis II B

Loriculos (S): Loriculus spp. II B

Loriculus amabilis: Moluccan Hanging-Parrot (E), Lorículo amable (S), Coryllis des Moluques (F) II B

Loriculus amabilis catamene $=$ Loriculus catamene

Loriculus aurantiifrons: Orange-fronted Hanging-Parrot (E), Papuan Hanging-Parrot (E), Lorículo papú (S), Coryllis à front orange (F) II B

Loriculus beryllinus: Ceylon Hanging-Parrot (E), Sri Lanka Hanging-Parrot (E), Lorículo de Ceilán (S), Coryllis de Ceylan (F) II B

Loriculus catamene: Sangihe Hanging-Parrot (E), Lorículo de la Sangihe ( $\mathrm{S}$ ), Coryllis des Sangi (F) II B

Loriculus exilis: Green Hanging-Parrot (E), Red-billed Hanging. Parrot (E), Loriculo exiguo (S), Coryllis vert (F) II B

Loriculus flosculus: Flores Hanging-Parrot (E), Wallace's HangingParrot (E), Lorículo de Flores (S), Coryllis de Wallace (F) II B

Loriculus galgulus: Blue-crowned Hanging-Parrot (E), Blue-topped Hanging-Parrot (E), Lorículo coroniazul (S), Coryllis à tête bleue ( $F$ ), Loriculus malais (F) II B

Loriculus malais (F): Loriculus galgulus II B

Loriculus philippensis: Colasisi (E), Philippine Hanging-Parrot (E), Lorículo Filipino (S), Coryllis des Philippines (F) II B

Loriculus pusillus: Yellow-throated Hanging-Parrot (E), Lorículo de Java (S), Coryllis à gorge jaune (F) II B

Loriculus stigmatus: Black-billed Hanging-Parrot (E), Maroonrumped Hanging-Parrot (E), Sulawesi Hanging-Parrot (E), Lorículo de Célebes (S), Coryllis des Célèbes (F) II B

Loriculus stigmatus tener $=$ Loriculus tener

Loriculus tener. Green-fronted Hanging-Parrot (E), Lorículo de las Bismarck (S), Coryllis des Bismark (F) II B

Loriculus vernalis: Vernal Hanging-Parrot (E), Lorículo vernal (S), Coryllis vemal (F) II B

Lories (E): Chalcopsitta spp./Eos spp./Lorius spp./Phigys solitarius/Pseudeos fuscata $\mathbf{1} / \mathbf{I I}$ B

Lorikeets (E): Charmosyna spp./Glossopsirta spp./Neopsintacus $\mathrm{spp}$ /Oreopsittacus arfaki/Psitteuteles spp./Trichoglossus spp. Nini spp. I/II B

Loriquet à col rouge (F): Trichoglossus rubritorquis II B

Loriquet à tête bleue (F): Trichoglossus haematudus II B

Loriquet de Goldie (F): Psitteuteles goldiei II B

Loriquet de Johnstone (F): Trichoglossus johnstoniae II B

Loriquet de Ponapé (F): Trichoglossus rubiginosus II B

Loriquet eutèle (F): Trichoglossus euseles II B

Loriquet inis (F): Psinteuteles iris II B

Loriquet jaune et vert (F): Trichoglossus flavoviridis II B

Loriquet omé (F): Trichoglossus ornalus II B

Loriquet versicolore (F): Psirteuteles versicolor II B

Loriquet vert (F): Trichoglossus chlorolepidotus II B

Loriquets (F): Psitteuteles spp $/$ Trichoglossus spp. II B

Loris (F): Chalcopsitta spp./Charmosyna spp./Eos spp./Glossopsitta spp./Lorius spp./Neopsinacus spp./Oreopsittacus arfaki/Phigys solitarius/Pseudeos fuscata/Vini spp. I/II B

Loris (S): Chalcopsina spp./Charmosyna spp./Eos spp./Glossopsitta $\mathrm{spp}$ /Lorius spp./Neopsittacus spp./Oreopsittacus arfaki/ Phigys solitarius/Pseudeos fuscata/Psitteuteles spp./Trichoglossus spp. Vini spp. I/II B

Loris fino (S): Loris tardigradus II M

Loris grèle (F): Loris tardigradus II M

Loris lent (F): Nycticebus coucang II M

Loris lento (S): Nycticebus coucang II M

Loris tardigradus: Slender Loris (E), Loris fino (S), Loris grèle (F) II $\mathrm{M}$

Lorises (E): Loris tardigradus/Nycticebus spp. II M

Lorito acollarado (S): Geoffroyus simplex II B

Lorito alinegro (S): Hapalopsittaca melanotis II B

Lorito amazonino (S): Hapalopsintaca amazonina II B

Lorito cabecigualdo (S): Pionopsitta pyrilia II B

Lorito cabecipardo (S): Poicephalus cryproxanthus II B

Lorito caica (S): Pionopsitta caica II B
Lorito carigualdo (S): Poicephalus flavifrons II B

Lorito carinaranja (S): Pionopsitta barrabandi II B

Lorito carirrojo (S): Geoffroyus geoffroyi 11 B

Lorito carirrojo (S): Pionopsinta pileata I B

Lorito carirrosado (S): Pionopsitta pulchra II B

Lorito chirlecrés (S): Pionites melanocephala II B

Lorito colicorto (S): Graydidascalus brachyurus II B

Lorito de copete (S): Nymphicus hollandicus NC B

Lorito de Coxen (S): Cyclopsitta diophthalma coxeni I B

Lorito de Desmarest (S): Psitraculirostris desmarestii II B

Lorito de Edwards (S): Psittaculirostris edwardsii II B

Lorito de Fuertes (S): Hapalopsittaca fuertesi II B

Lorito de Meyer (S): Poicephalus meyeri II B

Lorito de Rüppell (S): Poicephalus rueppellii II B

Lorito de Salvadori (S): Psittaculirostris salvadoni II B

Lorito dobleojo (S): Cyclopsitta diophthalma I/II B

Lorito dorsiazul (S): Psirtinus cyanurus II B

Lorito ecuatoriano (S): Hapalopsittaca pyrrhops II B

Lorito emmascarado de Coxen (S): Cyclopsitta diophthalma coxeni $1 \mathrm{~B}$

Lorito encapuchado (S): Pionopsitta haematotis II B

Lorito frentimojo (S): Poicephalus gulielmi II B

Lorito guayabero (S): Bolbopsittacus lunulatus II B

Lorito heteróclito (S): Geoffroyus heteroclitus II B

Lorito niam-niam (S): Poicephalus crassus II B

Lorito pechinaranja (S): Cyclopsina gulielmitertii II B

Lorito pileado (S): Pionopsitta pileata I B

Lorito robusto (S): Poicephalus robustus II B

Lorito rubio (S): Pionites leucogaster II B

Lorito Senegalés (S): Poicephalus senegalus II B

Lorito ventrirrojo (S): Poicephalus rufiventris II B

Lorito vulturino (S): Gypopsitta vulturina II B

Lorito-momoto amarillento (S): Prioniturus flavicans II B

Lorito-momoto coroniazul (S): Prioniturus discurus II B

Lorito-momoto de Buru (S): Prioniturus mada II B

Lorito-momoto de las Sulu (S): Prionilurus verticalis II B

Lorito-momoto de Luzón (S): Prioniturus luconensis II B

Lorito-momoto de Mindanao (S): Prioniturus waterstradit II B

Lorito-momoto de Palawan (S): Prioniturus platenae II B

Lorito-momoto dorsidorado (\$): Prioniturus platurus II B

Lorito-momoto montano (S): Prioniturus montanus II B

Lorito-momotos (S): Prioniturus spp. II B

Loritos (S): Bolbopsittacus lunulatus/Cyclopsitta spp/Geoffroyus $\mathrm{spp} /$ Graydidascalus brachyurus/Gypopsitta

vulturina/Hapalopsinaca spp./Pionites spp./Pionopsitta

spp./Poicephalus spp./Psittaculirostris spp./Psittinus cyanurus III B

Lorito-tigre de Brehm (S): Psittacella brehmii II B

Lorito-tigre de Madarasz (S): Psittacella madaraszi II B

Lorito-tigre modesto (S): Psirtacella modesta II B

Lorito-tigre pintado (S): Psittacella picta II B

Lorito-tigres (S): Psitacella spp. II B

Lorius albidinuchus: White-naped Lory (E), Lori nuquiblanco (S), Lori à nuque blanche (F) II B

Lurius chlorocercus: Yellow-bibbed Lory (E), Lori acollarado (S), Lori à collier jaune (F) II B

Lorius domicella: Purple-capped Lory (E), Purple-naped Lory (E), Lor damisela (S), Lori des dames (F) II B

Lorius garrulus: Chattering Lory (E), Yellow-backed Lory (E), Lori gárrulo (S), Lori noira (F) II B

Lorius hypoinochrous: Eastem Black-capped Lory (E), Purplebellied Lory (E), Lori ventrivinoso (S), Lori à ventre violet $(F)$ II B

Lorius lory: Black-capped Lory (E), Western Black-capped Lory (E), Lori tricolor (S), Lori des Moluques (F), Lori tricolore (F) I B

Lorius vibialis $=$ Lorius domicella

Loro aguilen̄o (S): Psittrichas fulgidus II B

Loro alibronceado (S): Pionus chalcopterus II B

Loro alisero (S): Amazona Pucumana I B

Loro barranquero (S): Cyanoliseus patagonus II B

Loro burrón (S): Amazona farinosa II B

Loro cabeciamarillo (S): Amazona oratrix II B

Loro cabeciazul (S): Pionus menstruus II B

Loro cabecirrojo (S): Amazona pretrei I B

Loro cabeza amarilla (S): Amazona ochrocephala II B

Loro cabeza azul (S): Amazona farinosa II B 
Loro cabeza roja (S): Amazona viridigenalis I B

Loro cachtirtosa (S): Pionopsitta pulchra II B

Loro cacique (S): Deropryus accipirninus II B

Loro cariazul (S): Amazona brasiliensis I B/Amazona dufresniana II $\mathrm{B}$

Loro caroniblanco (S): Pionus senilis II B

Loro choclero (S): Pionus maximiliani II B

Loro colicorto (S): Graydidascalus brachyurus II B

Loro corona-violeta (S): Amazona finschi II B

Loro de Buru (S): Tanygnathus gramineus II B

Loro de cabeza roja (S): Amazona pretrei I B

Loro de Hispaniola (S): Amazona ventralis II $\mathrm{B}$

Loro de Jamaica (S): Amazona collaria II B

Loro de Müller (S): Tanygnathus sumairanus II B

Loro del paraíso (S): Psephofus pulcherrimus I B

Loro ecléctico (S): Eclectus roratus II B

Loro famaulipeco (S): Amazona viridigenalis I B

Loro frente blanca (S): Amazona albifrons II B

Loro frente roja (S): Amazona autumnalis II B

Loro frentirrojo (S): Amazona autumnalis II B

Loro guaro (S): Amazona amazonica II B

Loro hablador (S): Amazona aestiva II B

Loro hombroamarillo (S): Psephotus chrysopterygius I B

Loro lomirrojo (S): Amazona fesriva II B

Loro mona amarilla (S): Amazona ochrocephala II B

Loro morado (S): Pionus fuscus II B

Loro negro (S): Coracopsis nigra II B

Loro noctumo (S): Geopsittacus occidentalis I B

Loro nuca amarilla (S): Amazona ochrocephala II B

Loro nuquiamarillo (S): Amazona auropalliara II B

Loro nuquiazul (S): Tanygnathus lucionensis II B

Loro orejirrojo (S): Pionopsina haematotis II B

Loro pechivinoso (S): Amazona vinacea I $\mathrm{B}$

Loro pecho vinoso (S): Amazona vinacea I B

Loro picogrodo (S): Tanygnathus megalorynchos II B

Loro piquigordo (S): Rhynchopsitra pachyrhyncha I B

Loro piquirrojo (S): Pionus sordidus $11 \mathrm{~B}$

Loro rabudo collar (S): Psittacula krameri III B

Loro real (S): Amazona ochrocephala II B

Loro senil (S): Pionus senitis II B

Loro tumultuoso (S): Pionus tumultuosus II B

Loro vasa (S): Coracopsis vasa II B

Loro ventriazul (S): Triclaria malachitacea II B

Loro ventrinaranja (S): Neophema chrysogaster I B

Loro verde (S): Amazona farinosa II B/Amazona mercenaria II B

Loro yaco (S): Psitiacus erithacus II B

Loro yucateci (S): Amazona xantholora II B

Loro yucateco (S): Amazona xantholora II B

Loros (S): Coracopsis spp./Cyanoliseus patagonus/Deroptyus accipitrinus/Eclectus roratus/Pionus spp./Psittacus erithacus/Psitrichas fulgidus/Tanygnathus spp./Triclaria malachitacea II B

Loup (F): Canis lupus I/II M

Loup à crinière (F): Chrysocyon brachyurus II M

Loup gris (F): Canis lupus VII M

Loup marsupial (F): Thylacinus cynocephalus I M

Loup vulgaire (F): Canis lupus $\mathbf{I I} \mathrm{M}$

Loutre à cou tacheté (F): Lutra maculicollis $11 \mathrm{M}$

Loutre à joues blanches $(\mathrm{F})$ : Aonyx capensis II M

Loutre à joues blanches du Cameroun (F): Aonyx congicus III M

Loutre à longue queue (F): Lontra longicaudis I M

Loutre cendrée (F): Amblonyx cinereus II M

Loutre commune (F): Lutra lutra I M

Loutre d'Amérique du Sud (F): Lontra longicaudis I M

Loutre d'Asie (F): Lutrogale perspicillata II M

Loutre de mer (F): Enhydra lutris II M/Lonira felina I M

Loutre de mer de Califomie (F): Enhydra luris nereis I M

Loutre de mer méridionale (F): Enhydra lutris nereis I M

Loutre de rivière (F): Lutra lutra I M

Loutre de Sumatra (F): Lutra sumatrana II M

Loutre d'Europe (F): Lutra lutra I M

Loutre du Canada (F): Lontra canadensis II M

Loutre du Chili (F): Lontra provocax I M

Loutre géante du Brésil (F): Pieronura brasiliensis I M

Loutres (F): Lutrinae spp. I/I M

Lovebirds (E): Agapormis spp. II B
Lovely Poison Frog (E): Phyllobares lugubris II A

Lower Californian Fur Seal (E): Arctocephalus townsendi I M

Lowland Anoa (E): Bubalus depressicomis I M

Lowland Tapir (E): Tapirus terrestris II M

Loxocemus bicolor: Burrowing Python (E), Ground Python (E), New World Python (E), Pitón excavadora (S), Python d'Amérique centrale (F), Python fouisseur du Mexique (F) II R

Loxodonia africana: African Elephant (E), Elefante africano (S), Eléphant africain (F), Eléphant d'Afrique (F) I/II M

Lucifer Hummingbird (E): Calothorax lucifer II B

Ludlow's Bhutan Swallowtail (E): Bhutanitis ludlowi II In

Ludwig's Bustard (E): Neotis ludwigii II B

Lúgano cardenalito (S): Carduelis cucullara I B

Lúgano de Yarrell (S): Carduelis yarrellii II B

Lúgano encapuchado (S): Carduelis cucullata I B

Luth (E): Dermochelys coriacea I R

Lutra canadensis = Lonira canadensis

Lutra felina $=$ Lontra felina

Lutra longicaudis $=$ Lontra longicaudis

Lutra lutra: Common Otter (E), Eurasian Otter (E), European River Otter (E), Old World Otter (E), Nutria común (S), Loutre commune $(\mathbf{F})$, Loutre d'Europe $(\mathbf{F})$, Loutre de rivière $(F)$ I $M$

Lutra maculicollis: Speckle-throated Otter (E), Spot-necked Otter (E), Nutria de cuello manchado (S), Loutre à cou tacheté (F) II $\mathrm{M}$

Lutra nippon = Lutra lutra

Lutra perspicillata $=$ Lutrogale perspicillato

Lutra provocax = Lontra provocax

Lutra sumatrana: Hairy-nosed Otter (E), Nutria de Sumatra (S), Loutre de Sumatra (F) II M

Lutrinae spp: Otters (E), Nutrias (S), Loutres (F) I/II M

Lutrogale perspicillata: Indian Smooth-coated Otter (E), Smoothcoated Otter (E), Nutria lisa (S), Nutria simung (S), Loutre d'Asie (F) II M

Luzon Bleeding-heart (E): Gallicolumba luzonica II B

Luzon Hombill (E): Penelopides manillae II B

Luzon Peacock Swallowtail (E): Papilio chikae I In

Luzon Racquet-tail (E): Prioniturus montanus II B

Luzon Scops-Owl (E): Otus longicomis II B

Lycocorax pyrrhopterus: Paradise-Crow (E), Silky-Crow (E), Corneille de paradis (F), Paradisier corvin (F) II B

Lyle's Flying-fox (E): Pleropus lylei II M

Lynx (F): Lynx lynx II M

Lynx canadensis: American Lynx (E), Canada Lynx (E), Lince del Canadá (S), Lynx du Canada (F) II M

Lynx caracal $=$ Caracal caracal

Lynx d'Espagne (F): Lynx pardinus I M

Lynx du Canada (F): Lynx canadensis II M

Lynx du désert (F): Caracal caracal I/II M

Lynx lynx: Eurasian Lynx (E), Lince (S), Lynx (F) II M

Lynx lynx canadensis = Lynx canadensis

Lynx lynx pardinus = Lynx pardinus

Lynx pardelle ( $\mathrm{F})$ : Lynx pardinus I M

Lynx pardinus: Iberian Lynx (E), Pardel Lynx (E), Spanish Lynx (E), Lince ibérico (S), Lynx d'Espagne (F), Lynx pardelle (F) I M

Lynx, roux (F): Lynx rufus II M

Lynx rufus: Bay Lynx (E), Bobcat (E), Gato montés (S), Lince rojo (S), Chat sauvage (F), Lynx roux (F) II M

Lynxes (E): Lynx spp. VII M

Lyssémyde ponctuée (F): Lissemys punctata II R

Macaca arctoides: Bear Macaque (E), Stump-tailed Macaque (E), Stumptail Macaque (E), Macaca ursin (S), Macaque brun (F) II $\mathrm{M}$

Macaca assamensis: Assam Macaque (E), Assamese Macaque (E), Macaca del Himalaya (S), Macaque d' Assam (F) II M

Macaca cangrejera (S): Macaca fascicularis II M

Macaca cola de cerdo (S): Macaca nemestrina II M

Macaca crestada de Sulawesi (S): Macaca ochreata II M

Macaca cyclopis: Formosan Rock Macaque (E), Taiwan Macaque

(E), Macaca de Formosa (S), Macaque de Formose (F) II M

Macaca cynomolgus = Macaca fascicularis

Macaca de Formosa (S): Macaca cyclopis II M

Macaca de Madras (S): Macaca radiata II M

Macaca de Sri Lanka (S): Macaca sinica II M

Macaca de Tonkean (S): Macaca ionkeana II M

Macaca del Himalaya (S): Macaca assamensis II M 
Macaca del Tibet (S): Macaca thiberana II M

Macaca fascicularis: Crab-eating Macaque (E), Cynomolgus

Monkey (E), Long-tailed Macaque (E), Macaca cangrejera (S), Macaque crabier (F), Macaque de Buffon (F) II M

Macaca fuscala: Japanese Macaque (E), Macaca japonesa (S), Macaque japonais (F), Macaque à face rouge (F) II M

Macaca hecki = Macaca lonkeana

Macaca irus = Macaca fascicularis

Macaca japonesa (S): Macaca fuscata II M

Macaca leonina (S): Macaca silenus I M

Macaca maura: Celebes Macaque (E), Moor Macaque (E), Macaca mora (S), Macaque maure (F) II M

Macaca mora (S): Macaca maura II M

Macaca mulatta: Rhesus Macaque (E), Rhesus Monkey (E), Mono resus (S), Macaque rhésus (F) II M

Macaca negra (S): Macaca nigra II M

Macaca nemestrina: Pig-tailed Macaque (E), Pigtail Macaque (E), Macaca cola de cerdo (S), Macaque à queue de cochon (F) II M

Macaca nigra: Celebes Black Macaque (E), Celebes Crested Macaque (E), Gorontalo Macaque (E), Sulawesi Macaque (E). Macaca negra (S), Cynopithèque nègre (F), Macaque des Célèbes (F) II M

Macaca nigrescens $=$ Macaca nigra

Macaca ochreata: Booted Macaque (E), Macaca crestada de Sulawesi (S) II M

Macaca pagensis $=$ Macaca nemestrina

Macaca radiata: Bonnet Macaque (E), Macaca de Madras (S) Macaque bonnet chinois (F), Macaque commun (F) II M

Macaca silenus: Lion-tailed Macaque (E), Wanderoo (E), Macaca leonina ( $\mathrm{S}$ ), Macaco barbudo (S), Macaque ouandérou $(\mathrm{F})$. Macaque à queue de lion (F), Ouandérou (F) I M

Macaca sinica: Toque Macaque (E), Macaca de Sri Lanka (S), Macaque couronné (F), Macaque toque (F) II M

Macaca speciosa $=$ Macaca arcioides

Macaca speciosa = Macaca fuscala

Macaca sylvanus: Barbary Ape (E), Barbary Macaque (E), Mono de Berberea (S), Mono de Gibraltar (S), Macaque de Gibraltar (F). Magot (F), Magot commun (F) II M

Macaca thibetana: Père David's Macaque (E), Short-tailed Tibetan Macaque (E), Tibetan Macaque (E), Macaca del Tibet (S), Macaque du Thibet (F) II M

Macaca tonkeana: Tonkean Black Macaque (E), Tonkean Macaque (E), Macaca de Tonkean (S), Macaque de Tonkea (F) II M

Macaca ursin (S): Macaca arctoides II M

Macaco barbudo (S): Macaca silenus I M

Macagua rieur (F): Herpetotheres cachinnans II B

Macaones (F): Bhutanitis spp. II In

Macaque à face rouge (F): Macaca fuscata II M

Macaque à queue de cochon (F): Macaca nemestrina II M

Macaque à queue de lion (F): Macaca silenus I M

Macaque bonnet chinois (F): Macaca radiata II M

Macaque brun (F): Macaca arctoides II M

Macaque commun (F): Macaca radiata II M

Macaque couronné (F): Macaca sinica II M

Macaque crabier (F): Macaca fascicularis II M

Macaque d' Assam (F): Macaca assamensis II M

Macaque de Buffon (F): Macaca fascicularis II M

Macaque de Formose (F): Macaca cyclopis II M

Macaque de Gibraltar (F): Macaca sylvanus II M

Macaque de Tonkea (F): Macaca tonkeana II M

Macaque des Célèbes (F): Macaca nigra II M

Macaque du Thibet (F): Macaca thibetana II M

Macaque japonais (F): Macaca fuscata II M

Macaque maure (F): Macaca maura II M

Macaque ouandérou ( $F$ ): Macaca silenus I M

Macaque thésus (F): Macaca mulatı II M

Macaque toque (F): Macaca sinica II M

Macaques (E): Macaca spp. VII M

Macaws (E): Anodorhynchus spp/Ara spp/Cyanopsita spp. VII B

MacDonald's Weakfish (E): Cynoscion macdonaldi I F

Macdonnell Range Rock-rat (E): Zyzomys pedunculatus I M

Macgregoria pulchra: MacGregor's Bird-of-paradise (E), Paradisier de Macgregor (F) II B

MacGregor's Bird-of-paradise (E): Macgregoria pulchra 11 B

Machaerhamphus alcinus = Macheiramphus alcinus

Macheiramphus alcinus: Bat Hawk (E), Bat Kite (E), Milano murcielaguero (S), Milan des chauves-souris (F) II B

Mackinder's Eagle-Owl (E): Bubo capensis II B

Macklot's Python (E): Morelia mackloti II R

Macrocephalon maleo: Gray's Brush-turkey (E), Maleo (E), Maleo Megapode (E), Megapodio maleo (S), Talégalo maleo (S), Mégapode maléo (F) I B

Macrotis lagotis: Bilby (E), Dalgyte (E), Greater Bilby (E), Greater Rabbit-eared Bandicoot (E), Cangurito narigudo grande (S), Grand bandicoot-lapin (F) I M

Macrotis leucura: Lesser Bilby (E), Lesser Rabbit-eared Bandicoot (E), White-tailed Rabbit-eared Bandicoot (E), Yallara (E),

Cangurito narigudo coliblanco (S), Bandicoot-lapin mineur (F), Bandicoot-lapin à queue blanche $(F)$, Petit bandicoot-lapin $(F)$ I M

Macuco (S): Tinamus solitarius I B

Madagascar Angulated Tortoise (E): Geochelone yniphora I R

Madagascar Baza (E): Aviceda madagascariensis II B

Madagascar Big-headed Turtle (E): Erymnochelys madagascariensis II $\mathbf{R}$

Madagascar Boa (E): Acrantophis madagascariensis I R

Madagascar Buzzard (E): Buteo brachypterus II B

Madagascar Chameleon (E): Chamaeleo capuroni II R

Madagascar Cuckoo-Falcon (E): Aviceda madagascariensis II B

Madagascar Cuckoo-Hawk (E): Aviceda madagascariensis II B

Madagascar Day Gecko (E): Phelsuma madagascariensis II R

Madagascar Fish-Eagle (E): Haliaeetus vociferoides II B

Madagascar Flat-shelled Tortoise (E): Pyxis planicauda II R

Madagascar Flying-fox (E): Pteropus rufus II M

Madagascar Forest Chameleon (E): Chamaeleo campani II R

Madagascar Fruit Bat (E): Pleropus rufus II M

Madagascar Giant Chameleon (E): Chamaeleo verrucosus II R

Madagascar Grass Owl (E): Tyro soumagnei I B

Madagascar Ground Boa (E): Acrantophis dumerili I R

Madagascar ground boas (E): Acrantophis spp. I R

Madagascar Gymnogene (E): Polyboroides radiatus II B

Madagascar Harrier (E): Circus maillardi II B

Madagascar Harier-Hawk (E): Polyboroides radialus II B

Madagascar Hawk-Owl (E): Ninox superciliaris II B

Madagascar Kestrel (E): Falco newtoni III B

Madagascar Lovebird (E): Agapomis canus II B

Madagascar Marsh-Harrier (E): Circus maillardi II B

Madagascar Owl (E): Tyto soumagnei I B/Asio madagascariensis II B

Madagascar Red Owl (E): Tyto soumagnei I B

Madagascar Scops-Owl (E): Otus rutilus II B

Madagascar Serpent-Eagle (E): Eutriorchis astur II B

Madagascar Sideneck Turtle (E): Enymnochelys madagascariensis II $\mathbf{R}$

Madagascar Sparrowhawk (E): Accipiser madagascariensis II B

Madagascar Teal (E): Anas bemieri II B

Madagascar Tortoise (E): Geochelone yniphora I R

Madagascar Tree Boa (E): Sanzinia madagascariensis I R

Madarasz's Tiger-Parrot (E): Psittacella madaraszi II B

Madracis asanoi: II In

Madracis asperula: II In

Madracis brueggemanni: II In

Madracis decacris: Green Cactus Coral (E) II in

Madracis formosa: II In

Madracis interjecta: II in

Madracis kauaiensis: II In

Madracis kirbyi: II In

Madracis mirabilis: Yellow Pencil Coral (E) II In

Madracis myriaster: II In

Madracis profunda: II In

Madracis senaria: II In

Madras Treeshrew (E): Anathana ellioti II M

Madrepora arbuscula: II In

Madrepora candida: II In

Madrepora carolina: II In

Madrepora exigua: II In

Madrepora formosa: II In

Madrepora kauaiensis: II In

Madrepora oculata: II in

Maeandra $=$ Meandrina

Magdalena River Turtle (E): Podocnemis lewyana II R

Magenta-throated Woodstar (E): Philodice bryantae $\mathbf{I}$ B 
Magistrate Colobus (E): Colobus guereza II M

Magnificent Bird-of-paradise (E): Cicinnurus magnificus II B

Magnificent Hummingbird (E): Eugenes fulgens II B

Magnificent Quetzal (E): Pharomachrus mocinno I B

Magnificent Riflebird (E): Priloris magnificus II B

Magot (F): Macaca sylvanus II M

Magot commun (F): Macaca sylvanus II M

Magpie Mannikin (E): Lonchura fringilloides III B

Magpie Munia (E): Lonchura fringilloides III B

Mahali à calotie marron (F): Plocepasser superciliosus III B

Mahalis (F): Plocepasser spp. IIINC B

Mailmbo capirotado (S): Anaplectes rubriceps III B

Mainate de Rothschild (F): Leucopsar rothschildi I B

Mainate religieux (F): Gracula religiosa II B

Mainates (F): Gracula spp. II/NC B

Mainland Serow (E): Naemorhedus sumatraensis I M

Maja chico (S): Tropidophis melanurus II R

Maja de Sta. María (S): Epicrates angulifer II R

Major Mitchell's Cockatoo (E): Cacatua leadbeateri II B

Maki à bourre (F): Avahi laniger I M

Maki ardilla (S): Phaner furcifer I M

Maki brun (F): Eulemur fulvus I M

Maki catta (F): Lemur cana I M

Maki horquilla (S): Phaner furcifer I M

Maki mongoz (F): Eulemur mongoz I M

Maki noir (F): Eulemur macaco I M

Maki ratón (S): Microcebus murinus I M/ Microcebus rufus I M

Maki ratón de Coquerel (S): Microcebus coquereli I M

Maki vari (F): Varecia variegata I M

Makis coligordos (S): Cheirogaleus spp. I M

Makisratone (S): Microcebus coquereli I M

Makisratone (S): Microcebus murinus I M

Malabar Large-spotted Civet (E): Viverra civettina III M

Malabar Parakeet (E): Psiltacula columboides II B

Malabar Pied-Hombill (E): Anthracoceros coronatus II B

Malacochersus tomieri: Crevice Tortoise (E), Pancake Tortoise (E), Softshell Tortoise (E), Tomier's Tortoise (E), Tortuga de cuña (S), Tortue de Tomier (F), Tortue à carapace souple (F) II R

Malagasy Civet (E): Fossa fossana II M

Malagasy Ground Boa (E): Acrantophis madagascariensis I R

Malagasy Mongoose (E): Eupleres goudotii II M

Malay Birdwing (E): Troides amphrysus II in

Malay Fish-Owl (E): Ketupa ketupu II B

Malayan Gharial (E): Tomistoma schlegelii I R

Malayan Monitor (E): Varanus salvator II $\mathbf{R}$

Malayan Pangolin (E): Manis javanica II M

Malayan Peacock-Pheasant (E): Polyplectron malacense II B

Malayan Sun Bear (E): Helarcios malayanus I M

Malayan Tapir (E): Tapirus indicus I M

Malaysian Pied-Hombill (E): Anthracoceros albirostris II B

Malaysian Treeshrew (E): Tupaia glis II M

Malcolm's Ethiopian Toad (E): Altiphrynoides malcolmi I A

Maleo (E): Macrocephalon maleo I B

Maleo Megapode (E): Macrocephalon maleo I B

Malimbe à bec bleu (F): Malimbus nitens III B

Malimbe à gorge noire (F): Malimbus cassini III B

Malimbe à queue rouge (F): Malimbus scutatus III B

Malimbe à tête rouge (F): Malimbus rubricollis $111 \mathrm{~B}$

Malimbe de Cassin (F): Malimbus cassini III B

Malimbe huppé (F): Malimbus malimbicus III B

Malimbes (E/F): Malimbus spp. II/NC B

Malimbo cabecinegro (S): Malimbus nitens III B

Malimbo cuellirrojo (S): Malimbus rubricollis III B

Malimbo culirrojo (S): Malimbus scutatus III B

Malimbo moñudo (S): Malimbus malimbicus III B

Malimbus cassini: Black-throated Malimbe (E), Cassin's Malimbe (E), Malimbe de Cassin (F), Malimbe à gorge noire (F) III B

Malimbus malimbicus: Crested Malimbe (E), Malimbo moñudo (S). Malimbe huppé (F) III B

Malimbus nitens: Blue-billed Malimbe (E), Gray's Malimbe (E), Malimbo cabecinegro (S), Malimbe à bec bleu (F) 11 B B

Malimbus rubriceps $=$ Anaplectes rubriceps

Malimbus rubricollis: Red-headed Malimbe (E), Red-headed Weaver (E), Malimbo cuellirrojo (S), Malimbe à tête rouge (F) III B
Malimbus scutatus: Red-vented Malimbe (E), Malimbo culirrojo (S), Malimbe à queue rouge (F) III B

Mallards (E): Anas oustaletiAnas platyrhynchos INC B

Mallee Ringneck (E): Platycercus bamardi II B

Malure occidental (F): Dasyomis longirostris I B

Malvasía (S): Oxyura leucocephala II B

Manatees (E): Trichechus spp. VII M

Manatí amazónico (S): Trichechus inunguis I M

Manati de Senegal (S): Trichechus senegalensis II M

Manati norteamericano (S): Trichechus manatus I M

Manchot de Humboldt (F): Spheniscus humboldti I B

Manchot du Cap (F): Spheniscus demersus II B

Manchots (F): Spheniscidae spp. I/IINC B

Manchurian Crane (E): Grus japonensis I B

Manchurian Eared-Pheasant (E): Crossoptilon manichuricum I B

Manchurian Red-footed Falcon (E): Falco amurensis II B

Manco (S): Eira barbara III M

Mandingoa nitidula: Green Twinspot (E), Green-backed Twinspot

(E), Astrild vert pointillé ( $F$ ), Sénégali vert (F) III B

Mandril (S): Mandrillus sphinx I M

Mandrill (E/F): Mandrillus sphinx I M

Mandrillus leucophaeus: Drill (E), Dril (S), Drill (F) I M

Mandrillus sphinx: Mandrill (E), Mandril (S), Mandrill (F) I M

Maned Owl (E): Jubula letiii II B

Maned Wolf (E): Chrysocyon brachyurus II M

Mangabey à crête $(\mathbf{F})$ : Cercocebus galeritus galeritus I M

Mangabey agile (F): Cercocebus agilis II $\mathbf{M}$

Mangabey couronné (F): Cercocebus torquaius II M

Mangabey crestado ventriblanco (S): Cercocebus galeritus galeritus I M

Mangabey de collar blanco (S): Cercocebus torquatus II M

Mangabey de la Tana (F): Cercocebus galeritus galeritus I M

Mangabey de mejillas grises (S): Lophocebus albigena II M

Mangabey enfumé (F): Cercocebus torquatus II M

Mangabeys (E): Cercocebus spp $\Omega$ Lophocebus albigena I/I M

Mangeur d'ouefs indien (F): Elachistodon westermanni II R

Mangeur d'úufs asiatique (F): Elachistodon westermanni II R

Mango (S): Anthracothorax nigricollis II B

Mango à cravate noire (F): Anthracothorax nigricollis II B

Mango a cravate verte (F): Anthracothorar viridigula II B

Mango de la Jamaique (F): Anthracothorax mango II B

Mango de Prévost (F): Anthracothorax prevostii II B

Mango doré (F): Anthracothorax dominicus II B

Mango gargantiverde (S): Anthracoshorax viridigula II B

Mango pechinegro (S): Anthracothorax nigricollis II B

Mango pechiverde (S): Anthracothorax prevostii II B

Mango vert (F): Anthracothorax viridis II B

Mangos (E/F): Anthracothorax spp. II B

Mangosta cangrejera (S): Herpestes urva III M

Mangosta coliconta oscura (S): Herpestes brachyurus fuscus III M

Mangosta de manchas doradas (S): Herpestes javanicus auropunctaius III M

Mangosta de nuca rayada (S): Herpestes vitticollis III M

Mangosta dentipequeno (S): Eupleres goudotii II M

Mangosta gris de la India (S): Herpestes edwardsii III M

Mangosta roja de la India (S): Herpestes smithii III M

Mangouste à queue courte de l'Inde (F): Herpestes brachyurus fuscus III $\mathrm{M}$

Mangouste de Smith (F): Herpestes smithii III M

Mangouste grise de l'Inde (F): Herpestes edwardsii III M

Mangrove Black-Hawk (E): Buteogallus subrilis II B

Mangrove Hummingbird (E): Amazilia boucardi II B

Mangrove Monitor (E): Varanus indicus II R/Varanus semiremex II $\mathbf{R}$

Manicina areolata: Rose Coral (E) II In

Manigordo (S): Leopardus pardalis I M

Manis spp.: Pangolins (E), Pangolínos (S), Pangolins (F) II M

Manis crassicaudara: Indian Pangolin (E), Thick-tailed Pangolin (E), Pangolín indio (S), Grand pangolin de I'Inde (F), Pangolin à grosse queue (F) II M

Manis gigantea: Giant Ground Pangolin (E), Giant Pangolin (E) Pangolin gigante (S), Grand pangolin (F), Pangolin géant (F) II $\mathrm{M}$

Manis javanica: Malayan Pangolin (E), Pangolín malayo (S), Pangolin javanais (F), Pangolin malais (F) II M

Manis longicaudara $=$ Manis tetradactyla 
Manis pentadactyla: Chinese Pangolin (E), Pangolín chino (S), Pangolin de Chine (F), Pangolin à queue courte (F) II M

Manis temminckii: Cape Pangolin (E), Scaly Anteater (E), South African Pangolin (E), Temminck's Ground Pangolin (E), Pangolín del Cabo (S), Pangolin de Temminck (F), Pangolin terrestre du Cap (F) II M

Manis tetradacryla: Black-bellied Pangolin (E), Long-tailed Pangolin (E), Pangolín de cola larga (S), Pangolin tétradactyle (F), Pangolin à longue queue (F) II M

Manis iricuspis: Three-cusped Pangolin (E), Tree Pangolin (E), White-bellied Pangolin (E), Pangolin commun (F), Pangolin a écailles tricuspides (F). Tricuspide (F) II M

Mannikins (E): Lonchura spp. IIL/NC B

Manopora = Montipora

Manouria emys: Asian Giant Tortoise (E), Asian Tortoise (E), Black Giant Tortoise (E), Burmese Brown Tortoise (E), Burmese Mountain Tortoise (E), Six-legged Tortoise (E), Tortue brune (F) II R

Manouria impressa: Impressed Tortoise ( $\mathrm{E}$ ), Tortuga marrón de Burma (S), Tortue imprimé (F) II R

Mansfield's Three-tailed Swallowtail (E): Bhutanitis mansfieldi II In

Mantanani Scops-Owl (E): Otus mantananensis II B

Mantella aurantiaca: Golden Mantella (E), Rana dorada (S), Ranita dorada de Madagascar (S), Mantelle dorée (F) II A

Mantellas (E): Mantella spp. IUNC A

Mantelle dorée (F): Mantella aurantiaca II A

Mantled Hawk (E): Leucopternis polionota II B

Mantled Howler (E): Alouatta palliata I M

Manucaude de Comrie (F): Manucodia comrii II B

Manucaude de Jobi (F): Manucodia jobiensis II B

Manucaude de Keraudren (F): Manucodia keraudrenii II B

Manucaude noir (F): Manucodia atra II B

Manucaude vert (F): Manucodia chalybata II B

Manucodes (E): Manucodia spp. II B

Manucodia atra: Glossy-mantled Manucode (E), Manucaude noir (F), Paradisier noir (F) II B

Manucodia chalybasa: Crinkle-collared Manucode (E), Manucaude vert (F), Paradisier vert (F) II B

Manucodia comrii: Curl-crested Manucode (E), Manucaude de Comrie (F), Paradisier d'Entrecasteaux (F) II B

Manucodia jobiensis: Jobi Manucode (E), Manucaude de Jobi (F), Paradisier de Jobi (F) II B

Manucodia keraudrenii: Trumpet Manucode (E), Trumpetbird (E), Manucaude de Keraudren (F), Paradisier de Keraudren (F) II B

Manus Green Tree Snail (E): Papustyla pulcherrima II In

Manus Hawk-Owl (E): Ninox meeki II B

Manus Masked-Owl (E): Tyto manusi II B

Manus Masked-owl (E): Tyto manusi II B

Many-coloured Parakeet (E): Psephotus varius II B

Many-spotted Hummingbird (E): Taphrospilus hypostictus II B

Marabou Stork (E): Leptoptilos crumeniferus III B

Marabout africain (F): Leptoptilos crumeniferus III B

Marabú africano (S): Leptoptilos crumeniferus III B

Maracana cuellidorada (S): Ara auricollis II B

Maracana dorsirroja (S): Ara maracana I B

Maranon Poison Frog (E): Dendrobates mysteriosus II A

Marbled Cat (E): Pardofelis marmorata I M

Marbled Poison Frog (E): Epipedobales boulengeri II A

Marco Polo Sheep (E): Ovis ammon I/II M

Margaretta's Hermit (E): Phaethornis malaris II B

Margay (E/S/F): Leopardus wiedii I M

Marginated Tortoise (E): Testudo marginata II R

Margined Tortoise (E): Testudo marginata II R

Marianas Flying-fox (E): Preropus mariannus I M

Marianas Island Duck (E): Anas oustaleti I B

Marianas Mallard (E): Anas oustaleti I B

Marine Otter (E): Lontra felina I M

Marine turtles (E): Cheloniidae spp. I R

Mariposa apollo (S): Parnassius apollo II in

Markhor (E/S/F): Capra falconeri I M

Marl (E): Perameles bougainville I M

Marmosets (E): Callimico goeldiv/Callithrix spp. I/II M

Marmota caudata: Long-tailed Marmot (E), Marmota de cola larga (S) III M
Marmota de cola larga (S): Marmota caudata III M Marmota del Himalaya (S): Marmota himalayana III M

Marmota himalayana: Himalayan Marmot (E), Marmota del Himalaya (S) III M

Marmots (E): Marmota spp. IIL/NC M

Marmouset à oreilles blanches (F): Callithrix aurita I M

Marmouset Santarem (F): Callithrix humeralifer II M

Maroantsetra Chameleon (E): Chamaeleo linorus II R

Maroon Langur (E): Presbytis rubicunda II M

Maroon Leaf Monkey (E): Presbytis rubicunda II M

Maroon-bellied Parakeet (E): Pyrrhura frontalis II B

Maroon-breasted Crowned-Pigeon (E): Goura scheepmakeri II B

Maroon-faced Parakeet (E): Pyrrhura leucotis II B

Maroon-fronted Parrot (E): Rhynchopsitta terrisi I B

Maroon-rumped Hanging-Parrot (E): Loriculus stigmatus II B

Maroon-tailed Conure (E): Pyrrhura melanura II B

Maroon-tailed Parakeet (E): Pyrrhura melanura II B

Marsh Crocodile (E): Crocodylus palustris I R

Marsh Deer (E): Blastocerus dichotomus I M

Marsh Hawk (E): Circus cyaneus II B

Marsh Owl (E): Asio capensis II B

Marsh Terrapin (E): Pelomedusa subrufa III R

Marshbuck (E): Tragelaphus spekii III M

Marsh-Hariers (E): Circus spp. II B

Marsopa de anteojo (S): Australophocaena dioptrica II M

Marsopa de Dall (S): Phocoenoides dalli II M

Marsopa espinosa (S): Phocoena spinipinnis II M

Marsopa negra (S): Neophocaena phocaenoides I M

Marsouin à lunettes (F): Australophocaena dioptrica II M

Marsouin aptère (F): Neophocaena phocaenoides I M

Marsouin commun (F): Phocoena phocoena II M

Marsouin de Burmeister (F): Phocoena spinipinnis II M

Marsouin de Dall (F): Phocoenoides dalli II M

Marsouin de Lahille (F): Australophocaena dioptrica II M

Marsouin du Golfe de Californie (F): Phocoena sinus I M

Marsouin sans nageoires (F): Neophocaena phocaenoides I M

Marta (S): Potos flavus III M

Marta de cuello amarillo (S): Martes flavigula III M

Martens (E): Martes spp. IIL/NC M

Martes flavigula: Yellow-throated Marten (E), Marta de cuello amarillo (S), Martre à gorge jaune (F) III M

Martes flavigula gwatkinsii $=$ Martes gwatkinsii

Martes foina intermedia: Central Asian Stone Marten (E), Fouine (F) III M

Martes gwaskinsii: Nilgiri Marten (E), Martre de l'Inde du Sud (F) III $M$

Martial Eagle (E): Polemaetus bellicosus II B

Martin de Rothschild (F): Leucopsar rothschildi I B

Martre à gorge jaune (F): Mantes flavigula III M

Martre de l' Inde du Sud (F): Martes gwatkinsii III M

Martucha (S): Potos flavus III M

Marvellous Spatuletail (E): Loddigesia mirabilis II B

Masacuate (E): Boa constrictor $1 / 11 \mathrm{R}$

Mascarene Paradise-Flycatcher (E): Terpsiphone bourbonnensis III B

Masked Bobwhite (E): Colinus virginianus ridgwayi I B

Masked Dove (E): Oena capensis III B

Masked Flying-fox (E): Pteropus personatus II M

Masked Fruit Bat (E): Preropus personatus II M

Masked Goura (E): Goura scheepmakeri II B

Masked Hawk (E): Leucopternis melanops II B

Masked Lovebird (E): A gapomis personatus II B

Masked Palm Civet (E): Paguma larvata III M

Masked Shining-Parrot (E): Prosopeia personata II B

Masked Titi (E): Callicebus personatus II M

Masked-Owls (E): Tyto spp. II B

Masked-Weavers (E): Ploceus spp. III/NC B

Mastigures (E): Uromastyx spp. II R

Masurana (S): Clelia clelia II R

Matamico blanco (S): Phalcoboenus albogularis II B

Matamico cordillerano (S): Phalcoboenus megalopterus II B

Matamico estriado (S): Phalcoboenus australis II B

Matamico grande (S): Phalcoboenus australis II B

Matschie's Dwarf Chameleon (E): Bradypodion tenue II R

Mauritius Day Gecko (E): Phelsuma guimbeaui II R

Mauritius Greater Day Gecko (E): Phelsuma cepediana II R 
Mauritius Kestrel (E): Falco punctatus I B

Mauritius Lowland Forest Day Gecko (E): Phelsuma guimbeaui II R

Mauritius Parakeet (E): Psittacula echo I B

Mauritius Ring-necked Parakeet (E): Psitracula echo I B

Maxwell's Black Weaver (E): Ploceus albinucha III B

Mayotte Chameleon (E): Chamaeleo polleni II R

Mazama americana cerasina: Guatemalan Red Brocket (E), Middle American Red Brocket (E) III M

McLachlan's Girdled Lizard (E): Cordylus mclachlani II R

McLachlan's Spiny-tailed Lizard (E): Cordylus mclachlani II R

Meadow Viper (E): Vipera ursinii INC R

Mealy Amazon (E): Amazona farinosa II B

Mealy Parrot (E): Amazona farinosa II B

Mealy Rosella (E): Platycercus adscifus II B

Meandrina alveolus: II In

Meandrina meandrites: II In

Meandrina memorialis: II In

Meandrina spinulosa: II In

Medicinal Leech (E): Hirudo medicinalis II In

Mediterranean Chameleon (E): Chamaeleo chamaeleon II R

Mediterranean Monk Seal (E): Monachus monachus I M

Meek's Lorikeet (E): Charmosyna meeki II B

Meek's Pygmy-Parrot (E): Micropsitta meeki II B

Megalobatrachus davidianus = Andrias davidianus

Megalobairachus japonicus = Andrias japonicus

Megalonaias nickliniana = Unio nickliniana

Megamuntiacus vuquanghensis: Giant Muntjac (E), Muntjac géant (F) $1 \mathrm{M}$

Mégapode maléo (F): Macrocephalon maleo I $\mathrm{B}$

Megapodio maleo (S): Macrocephalon maleo I B

Megaprera novaeangliae: Bunch (E), Hump Whale (E), Humpback Whale (E), Hunchbacked Whale (E), Ballena jorobada (S), Gubarte (S), Jorobada (S), Rorcual jorobado (S), Baleine à bosse (F), Baleine à taquet (F), Jubarte (F), Mégaptère (F), Rorqual du Cap (F), Rorqual à bosse (F) I M

Mégaptère (F): Megaptera novaeangliae I M

Megatriorchis doriae: Doria's Goshawk (E), Azor de Doria (S), Autour de Doria (F) II B

Melampitta gigantea: Greater Melampitta (E), Grande mélampitte (F) II B

Melampitta lugubris: Lesser Melampitta (E), Petite mélampirte (F) II $\mathrm{B}$

Melampittas (E): Melampirza spp. II B

Melanochelys iricarinata: Three-keeled Land Tortoise (E), Threekeeled Land Turtle (E), Tricarinate Hill Turtle (E), Galápago terrestre $(\mathrm{S})$, Emyde indienne à trois carènes $(\boldsymbol{F})$, Géoémyde tricarénée (F), Tortue tricarénée (F) I R

Melanoperdix nigra: Black Partridge (E), Black Wood-Partridge (E), Perdiz de bosque negra (S), Perdiz negra (S), Perdrix noire (F), Rouloul noir (F) III B

Melanosuchus niger: Black Caiman (E), Caimán negro (S), Lagarto negro (S), Caiman noir (F) I/I R

Melanotrochilus fuscus: Black Jacobin (E), Picaflor negro (S), Colibri demi-deuil (F) II B

Meleagris ocellata $=$ Agriocharis ocellata

Melero de casco (S): Lichenostomus melanops cassidix I B

Melierax canorus: Pale Chanting-Goshawk (E), Azor-lagartijero claro (S), Autour chanteur (F) II B

Melierax canorus poliopterus $=$ Melierax poliopterus

Melierax gabar: Gabar Goshawk (E), Gavilán gabar (S), Autour gabar (F) II B

Melierax metabates: Dark Chanting-Goshawk (E), Azor lagartijero oscuro (S), Autour sombre (F) II B

Melierax poliopterus: Eastem Chanting-Goshawk (E), Azorlagartijero somali (S), Autour à ailes grises (F) II B

Meliphaga cassidix = Lichenostomus melanops cassidix

Méliphage casqué (F): Lichenostomus melanops cassidix I B

Méliphage cornu (F): Lichenosiomus melanops cassidix I B

Méliphages (F): Meliphagidae spp. INC B

Meller's Chameleon (E): Chamaeleo melleri II $\mathbf{R}$

Mellisuga helenae: Bee Hummingbird (E), Colibri d'Helen (F) II B

Mellisuga minima: Vervain Hummingbird (E), Zumbadorcito (S), Colibri nain (F) II B

Mellivora capensis: Honey Badger (E), Ratel (E), Ratel (S), Tejón melivoro (S), Ratel (F) III M
Melon-headed Whale (E): Peponocephala electra II M

Melopsitiacus undulatus: Budgerigar (E), Budgerigar ondulado (S) Perruche inséparable (F), Perruche ondulée (F), NC B

Melursus ursinus: Sloth Bear (E), Oso perezoso (S), Ours lippu de l'Inde (F), Ours prochile lippu (F) I M

Mentaur Scops-Owl (E): Otus umbra II B

Mentawai Gibbon (E): Hylobates klossii I M

Mentawai Langur (E): Presbyris potenziani I M

Mentawai Leaf Monkey (E): Presbytis potenziani I M

Mentawai Scops-Owl (E): Otus mentawi II B

Mentawai Treeshrew (E): Tupaia chrysogaster II M

Mentawi Islands Snub-nosed Langur (E): Nasalis concolor I M

Merida Sunangel (E): Heliangelus spencei II B

Merles des Indes (F): Gracula religiosa II B

Merlin (E): Falco columbarius II B

Merlins (E): Falco columbarius/Falco chicquera II B

Merrin (E): Onychogalea fraenata I M

Mertens's Day Gecko (E): Phelsuma robertmentensi II R

Mertens's Water Monitor (E): Varanus mertensi II R

Merulina ampliata: II In

Merulina scabricula: II In

Merulina scheeri: II In

Merulina togianensis: II In

Mésangette rayée (F): Pholidornis rushiae III B

Mesoplodon de Nishiwaki (F): Mesoplodon ginkgodens II M

Mesoplodon de True (F): Mescolodon mirus II M

Mesoplodon bidens: Sowerby's Beaked Whale (E), Ballena de pico de Sowerby (S), Mesoplodon de Sowerby (F) II M

Mesoplodon bowdoini: Andrews' Beaked Whale (E). Splaytooth Beaked Whale (E), Ballena de pico de Andrew (S), Mesoplodon de Bowdoin (F) II M

Mesoplodon carlhubbsi: Arch-beaked Whale (E), Hubbs's Beaked Whale (E), Ballena de pico de Hubbs (S), Mesoplodon de Hubbs (F) II M

Mesoplodon de Blainville (F): Mesoplodon densirostris II M

Mesoplodon de Bowdoin (F): Mesoplodon bowdoini II M

Mesoplodon de Gervais (F): Mesoplodon europaeus II M

Mesoplodon de Gray (F): Mesoplodon grayi II M

Mesoplodon de Hubbs (F): Mesoplodon carlhubbsi II M

Mesoplodon de Layard (F): Mesoplodon Layardii II M

Mesoplodon de Longman (F): Indopacerus pacificus II M

Mesoplodon de Sowerby (F): Mesoplodon bidens II M

Mesoplodon de Stejneger (F): Mesoplodon siejnegeri II M

Mesoplodon densirostris: Blainville's Beaked Whale (E), Ballena de pico de Blainville (S), Mesoplodon de Blainville (F) II M

Mesoplodon d'Hector (F): Mesoplodon hectori II M

Mesoplodon europaeus: Gervais' Beaked Whale (E), Gulf Stream Beaked Whale (E), Ballena de pico de Gervais (S), Mesoplodon de Gervais (F, II M

Mesoplodon ginkgodens: Ginkgo-toothed Beaked Whale (E), Mesoplodon de Nishiwaki (F) II M

Mesoplodon grayi: Gray's Beaked Whale (E), Southem Beaked Whale (E), Ballena de pico de Gray (S), Mesoplodon de Gray (F) $\Pi \mathrm{M}$

Mesoplodon heciori: Hector's Beaked Whale (E), Skew-beaked Whale (E), Ballena de pico de Héctor (S), Mesoplodon d'Hector (F) $11 \mathrm{M}$

Mesoplodon Layardii: Layard's Beaked Whale (E), Strap-toothed Whale (E), Ballena de pico de Layard (S), Mesoplodon de Layard (F) II M

Mesoplodon mirus: True's Beaked Whale (E), Ballena de pico de True (S), Mesoplodon de True (F) II M

Mesoplodon pacificus = Indopacetus pacificus

Mesoplodon peruvianus: Lesser Beaked Whale (E), Mesoplodon pygmée (F) II M

Mesoplodon pygmée (F): Mesoplodon peruvianus II M

Mesoplodon stejnegeri: Stejneger's Beaked Whale (E), Ballena de pico de Stejneger (S), Mesoplodon de Stejneger (F) II M

Mesopotamian Fallow Deer (E): Dama mesopotamica I M

Mesopotamian Mastigure (E): Uromastyx loricalus II R

Mesopotamian Spiny-tailed Lizard (E): Uromastyx loricatus II R

Messager sagittaire (F): Sagittarius sementarius II B

Metallura aeneocauda: Scaled Metaltail (E), Métallure à queue d'airain (F) II B

Metallura baroni: Violet-throated Metaltail (E), Metalura gorivivioleta (S), Métallure de Baron (F) II B 
Metallura eupogon: Fire-throated Metaltail (E), Métallure à gorge feu (F) II B

Metallura iracunda: Perija Metaltail (E), Perijá Metaltail (E), Colibrí de Perijá (S), Métallure dorée (F) II B

Metallura malagae $=$ Metallura aeneocauda

Metallura odomae: Neblina Metaltail (E), Métallure du Chinguela (F) II B

Metallura phoebe: Black Metaltail (E), Métallure phébé (F) II B

Metallura theresiae: Coppery Metaltail (E), Métallure de Thérèse (F) $11 \mathrm{~B}$

Metallura tyrianthina: Tyrian Metaltail (E), Colibri verde colirrojo (S), Metalura tiria (S), Métallure émeraude (F) II B

Metallura williami: Viridian Metaltail (E), Metalura verde (S), Métallure verte (F) II B

Métallure à gorge feu (F): Melallura eupogon II B

Métallure à queue bronzée (F): Chalcostigma heteropogon II B

Métallure à queue d'airain (F): Metallura aeneocauda II B

Métallure à tête rousse (F): Chalcostigma ruficeps II B

Métallure arc-en-ciel (F): Chalcostigma herrani II B

Métallure de Baron (F): Metallura baroni II B

Métallure de Stanley (F): Chalcostigma stanleyi II B

Métallure de Thérèse (F): Metallura theresiae II B

Métallure dorée (F): Metallura iracunda II B

Métallure du Chinguela (F): Mesallura odomae II B

Métallure émeraude (F): Metallura tyrianthina II B

Métallure olivâtre (F): Chalcostigma olivaceum II B

Métallure phébé (F): Metallura phoebe II B

Métallure verte (F): Metallura williami II B

Metallures (F): Chalcostigma spp/Metallura spp. II B

Metaltails (E): Metallura spp. II B

Metalura gorjivioleta (S): Metallura baroni II B

Metalura tiria (S): Metallura tyrianthina II B

Metalura verde (S): Metallura williami II B

Metastrea $=$ Favite

Mexican Black-cap (E): Brachypelma emilia II in

Mexican Giant Tortoise (E): Gopherus flavomarginatus I R

Mexican Grizzly Bear (E): Ursus arctos nelsoni I M

Mexican Parrotlet (E): Forpus cyanopygius II B

Mexican Prairie Dog (E): Cynomys mexicanus I M

Mexican Prairie Marmot (E): Cynomys mexicanus I M

Mexican Pronghom (E): Antilocapra americana $\mathbf{L}$ NC M

Mexican Red Leg (E): Brachypelma emilia II In

Mexican Red-kneed Tarantula (E): Brachypelma smithi II In

Mexican Ridley (E): Lepidochelys kempi I R

Mexican Sheartail (E): Doricha eliza II B

Mexican Tree Porcupine (E): Sphiggurus mexicanus III M

Mexican Woodnymph (E): Thalurania ridgwayi II B

Meyer's Goshawk (E): Accipiter meyerianus II B

Meyer's Lorikeet (E): Trichoglossus flavoviridis II B

Meyer's Parrot (E): Poicephalus meyeri II B

Michoacan Orange Tarantula (E): Brachypelma baumgarteni II In

Mico de noche (S): Bassariscus sumichrasti III M/Potos flavus III M

Mico rayado (S): Bassariscus sumichrasti III M

Micoleón (S): Potos flavus III M

Micrabacia spp.: II In

Micrastur buckleyi: Buckley's Forest-Falcon (E), Lesser Collared Forest-Falcon (E), Traylor's Forest-Falcon (E), Gavilán de Traylor (S), Halcón-montés de Buckley (S), Camifex de Buckley (F), Camifex de Traylor (F) II B

Micrasiur gilvicollis: Lined Forest-Falcon (E), Gavilán de collar (S), Halcón palomero del sur (S), Halcón-montés cabecigrís (S), Camifex à gorge cendrée (F) II B

Micrastur mirandollei: Slaty-backed Forest-Falcon (E), Halcón de lomo pizarreño (S), Halcón-montés dorsigris (S), Camifex ardoisé (F) II B

Micrastur plumbeus: Plumbeous Forest-Falcon (E), Halcón-montés plomizo (S), Carnifex plombé (F) II B

Micrastur ruficollis: Barred Forest-Falcon (E), Caburé (S), Gavilán cueillirojo (S), Halcón cuellirrufo (S), Halcón palomero (S), Halcón-montés agavilanado (S), Camifex barté (F), Camifex à cou roux (F) II B

Micrastur ruficollis gilvicollis = Micrastur gilvicollis
Micrastur semitorquatus: Collared Forest-Falcon (E), Gavilán-reloj (S), Halcón semiacollarado (S), Halcón-montés collarejo (S). Halcón-selvático collarejo (S), Camifex à collier (F) II B

Micrathene whimeyi: Elf Owl (E), Mochuelo duende (S), Tecolotito enano (S), Chevêchette des saguaros (F), Chevêchette-elfe (F) II $\mathrm{B}$

Microcèbe de Coquerel (F): Microcebus coquereli I M

Microcèbe murin (F): Microcebus murinus I M

Microcebus coquereli: Coquerel's Dwarf Lemur (E), Coquerel's Mouse-lemur (E), Maki ratón de Coquerel (S), Makisratone (S), Microcèbe de Coquerel (F) I M

Microcebus murinus: Grey Mouse-lemur (E), Lesser Mouse-lemur (E), Maki ratón (S), Makisratone (S), Microcèbe murin (F), Petit microcèbe (F) $1 \mathrm{M}$

Microcebus murinus rufus = Microcebus rufus

Microcebus myoxinus = Microcebus murinus

Microcebus rufus: Brown Mouse-lemur (E), Rufous Mouse-lemur (E), Russet Mouse-lemur (E), Maki ratón (S) I M

Microchera albocoronata: Snowcap (E), Colibri à coiffe blanche (F) II B

Microcyathus = Hoplangia

Microglosse noir (F): Probosciger aterrimus I B

Microhierax caerulescens: Collared Falconet (E), Red-legged Falconet (E), Red-thighed Falconet (E), Falconete acollarado (S). Halcón enano de la India (S), Fauconnet à collier (F) II B

Microhierax erythrogenys: Philippine Falconet (E), Falconete filipino (S), Halconcito de las Filipinas (S), Fauconnet des Philippines (F) II B

Microhierax fringillarius: Black-legged Falconet (E), Black-sided Falconet (E), Black-thighed Falconet (E), Falconete indonesio (S), Halconcito malayo (S), Fauconnet moineau (F) II B

Microhierax latifrons: Bomean Falconet (E), White-fronted Falconet (E), Falconete de Borneo (S), Halconcito de Borneo (S), Fauconnet de Boméo (F) II B

Microhierax melanoleucos: Pied Falconet (E), Falconete pio (S), Fauconnet noir-et-blanc (F) II B

Microloro de Finsch (S): Micropsinta finschii II B

Microloro de Geelvink (S): Micropsitta geelvinkiana II B

Microloro de las Kai (S): Micropsitla keiensis II B

Microloro de Meek (S): Micropsinta meeki II B

Microloro pechirrojo (S): Micropsitta bruijnii II B

Microloro pusio (S): Micropsitra pusio II B

Microloros (S): Micropsitta spp. II B

Micromya irabalis = Villosa trabalis

Micronesian Flying-fox (E): Pteropus mariannus I M

Micronisus gabar = Melierax gabar

Micropsitta bruijnii: Red-breasted Pygmy-Parrot (E), Microloro pechirrojo (S), Micropsitte de Bruijn (F), Perruche pygmée de Brujin (F) II B

Micropsitta finschii: Finsch's Pygmy-Parrot (E), Microloro de Finsch (S), Micropsitte de Finsch (F) II B

Micropsinta geelvinkiana: Geelvink Pygmy-Parrot (E), Geelvink Pygmy-parrot (E), Microloro de Geelvink (S), Micropsitte de Geelvink (F), Perruche pygmée de Schlegel (F) II B

Micropsitna keiensis: Yellow-capped Pygmy-Parrot (E), Microloro de las Kai (S), Micropsitte pygmée (F), Pernche pygmée de Salvadori (F) II B

Micropsitta meeki: Meek's Pygmy-Parrot (E), Microloro de Meek (S), Micropsitte de Meek (F), Perruche pygmée de Meek (F) II B

Micropsina pusio: Buff-faced Pygmy-Parrot (E), Microloro pusio (S), Micropsitte à tête fauve (F), Perruche pygmée de Sclater (F) II B

Micropsitte à tête fauve (F): Micropsinta pusio II B

Micropsitte de Bruijn (F): Micropsirta bruijnii II B

Micropsitte de Finsch (F): Micropsitta finschii II B

Micropsitte de Geelvink (F): Micropsitta geelvinkiana II B

Micropsitte de Meek (F): Micropsitta meeki II B

Micropsitte pygmée (F): Micropsitta keiensis II B

Micropsittes (F): Micropsitla spp. II B

Microstilbon burmeisteri: Slender-tailed Woodstar (E), Picaflor enano (S), Colibri de Burmeister (F) II B

Microtrochus = Placotrochus

Micrure à bandes noires (F): Micrumes nigrocincrus III $\mathbf{R}$

Micrure distancé (F): Micrurus diastema III R 
Micrurus diastema: Allantic Coral Snake (E), Variable Coral Snake (E), Micrure distancé (F) III R

Micrurus nigrocincrus: Black-banded Coral Snake (E), Central American Coral Snake (E), Micrure à bandes noires (F) III R

Middle American Red Brocket (E): Mazama americana cerasina III M

Mikado Pheasant (E): Symaticus mikado I B

Milan a long bec (F): Rostrhamus hamatus II B

Milan à plastron (F): Hamirostra melanostemon II B

Milan a queue carrée (F): Lophoictinia isura II B

Milan à queue fourchue (F): Elanoides forficatus II B

Milan bec-en-croc (F): Chondrohierax uncinatus I/I B

Milan bidenté (F): Harpagus bidentatus II B

Milan bleuâtre (F): Ictinia plumbea II B

Milan brun (F): Milvus lineatus II B

Milan de Cayenne (F): Leptodon cayanensis II B

Milan de Cuba (F): Chondrohierax uncinarus wilsonii I B

Milan de Forbes (F): Leptodon forbesi II B

Milan de Wilson (F): Chondrohierax uncinarus wilsonii I B

Milan des chauves-souris (F): Macheiramphus alcinus II B

Milan des marais (F): Rostrhamus sociabilis II B

Milan diodon (F): Harpagus diodon II B

Milan du Mississippi (F): Ictinia mississippiensis II B

Milan noir (F): Milvus migrans II B

Milan royal (F): Milvus milvus II B

Milan sacré (F): Haliastur indus II B

Milan siffleur (F): Haliastur sphenurus II B

Milano acollarado (S): Leptodon forbesi II B

Milano bidentado (S): Harpagus bidentarus II B

Milano blanquigrís (S): Leptodon cayanensis II B

Milano brahmán (S): Haliastur indus II B

Milano cabecigris (S): Leprodon cayanensis II B

Milano caracolero (S): Rostrhamus sociabilis II B

Milano coliblanco (S): Elanus leucurus II B

Milano colicuadrado (S): Lophoictinia isura II B

Milano del Misisipi (S): Icrinia mississippiensis II B

Milano dentado (S): Harpagus diodon II B

Milano misisipero (S): Ictinia mississippiensis II B

Milano murcielaguero (S): Macheiramphus alcinus II B

Milano muslimufo (S): Harpagus diodon II B

Milano negro (S): Milvus migrans II B

Milano pechinegro (S): Hamirostra melanosternon II B

Milano picogarfio (S): Chondrohierax uncinatus IJH B

Milano plomizo (S): Ictinia plumbea II B

Milano real (S): Milvus milvus II B

Milano silbador (S): Haliastur sphenurus II B

Milano tijereta (S): Elanoides forficalus II B

Milans (F): Accipitridae spp. VII B

Milanus nigro (S): Milvus migrans II B

Military Macaw (E): Ara militaris I B

Milky Eagle-Owl (E): Bubo lacteus II B

Milky Stork (E): Mycleria cinerea I B

Millepora alcicornis: II In

Millepora boschmai: II In

Millepora braziliensis: II In

Millepora complanaia: 11 in

Millepora cruzi: II In

Millepora dichotoma: II In

Millepora exaesa: II In

Millepora fasciculata: II In

Millepora intricata: II In

Millepora Latifolia: II In

Millepora murrayi: II In

Millepora nitida: II In

Millepora platyphylla: II In

Millepora plicata: II In

Millepora pumila: II In

Millepora ramosa: II In

Millepora spp.: Coraux de feu (F) II In

Millepora squarrosa: II In

Millepora siriata: II In

Millepora tenera: II In

Millepora tuberosa: II In

Millepora xishaensis: II in

Milleporidae spp.: Fire corals (E), Corail de feu (F) II In

Milne-Edwards's Sportive Lemur (E): Lepilemur edwardsi I M
Milvago chimachima: Yellow-headed Caracara (E), Caracara chimachima (S), Caricare sabanero (S), Chimachima (S). Caracara chimachima (F), Caracara à tête jaune (F) II B

Milvago chimango: Chimango Caracara (E), Caracara chimango (S), Chimango (S), Caracara chimango (F) II B

Milvus fasciicaudala $=$ Milvus milvus

Milvus linearus: Black-eared Kite (E), Milan brun (F) II B

Milvus migrans: Black Kite (E), Pariah Kite (E), Yellow-billed Kite

(E), Milano negro (S), Milanus nigro (S), Milan noir (F) II B

Milvus migrans linearus $=$ Milvus linearus

Milvus milvus: Red Kite (E), Milano real (S), Milan royal (F) II B

Mimic Poison Frog (E): Dendrobates imitator II A

Mimizuku gumeyi: Giant Scops-Owl (E), Lesser Eagle-Owl (E), Autillo de Guerney (S), Hibou de Tweeddale (F), Petit-duc de

Gumey (F), Petit-duc géant (F), Scops géant de Guerney (F) I B

Miná de la India (S): Gracula religiosa II B

Miná de Rothschild (S): Leucopsar rothschildi I B

Minahassa Bam-Owl (E): Tyto inexspectala II B

Minahassa Masked-Owl (E): Tyto inexspectata II B

Minahassa Owl (E): Tyto inexspectata II B

Mindanao Hombill (E): Penelopides affinis II B

Mindanao Lorikeet (E): Trichoglossus johnstoniae II B

Mindanao Racquet-tail (E): Prioniturus waterstradit II B

Mindanao Scops-Owl (E): Orus mirus II B

Mindoro Crocodile (E): Crocodylus novaeguineae mindorensis I R

Mindoro Hombill (E): Penelopides mindorensis II B

Mindoro Imperial-Pigeon (E): Ducula mindorensis I B

Mindoro Scops-Owl (E): Otus mindorensis II B

Mindoro Zone-tailed Pigeon (E): Ducula mindorensis I B

Minke Whale (E): Balaenopiera acutorosirata III M

Minute Hermit (E): Phaethornis idaliae II B

Minute Tree Toad (E): Nectophrynoides minutus I A

Minyobate bourdonnant (F): Minyobates bombetes II A

Minyobate d'Alto del Buey (F): Minyobates altobueyensis II A

Minyobate de Steyermark (F): Minyobates steyermarki II A

Minyobate étincelant (F): Minyobates fulgurius II A

Minyobate menu (F): Minyobates minutus II A

Minyobate opisthomèle (F): Minyobates opisthomelas II A

Minyobate secret $(\mathrm{F})$ : Minyobates abditus II A

Minyobate vert (F): Minyobates viridis II A

Minyobates spp.: Poison frogs (E), Ranas de puntas de flechas ( $\mathrm{S}$ ), Dendrobates (F) II A

Minyobates abditus: Collins's Poison Frog (E), Minyobate secret (F) II A

Minyobates altobueyensis: Alto de Buey Poison Frog (E), Golden Poison Frog (E), Minyobate d'Alto del Buey (F) II A

Minyobates bombetes: Cauca Poison Frog (E), Minyobate bourdonnant (F) II A

Minyobates fulgurius: Yellow-bellied Poison Frog (E), Minyobate étincelant (F) II A

Minyobates minutus: Blue-bellied Poison Frog (E), Minyobate menu (F) II A

Minyobates opisthomelas: Andean Poison Frog (E), Minyobate opisthomèle (F) II A

Minyobates steyermarki: Demonic Poison Frog (E), Rana de punta de flecha de Steyermark (S), Minyobate de Steyermark (F) II A

Minyobates viridis: Green Poison Frog (E), Rana de punta de flecha verde (S), Minyobate vert (F) II A

Miopithecus talapoin: Southem Talapoin (E), Talapoin (E), Talapoin (S), Talapoin (F) II M

Mirlo americano pechiamarillo (S): Agelaius flavus I B

Mirounga leonina: South Atlantic Elephant-seal (E), Southern

Elephant-seal (E), Elefante marino del sur (S), Eléphant de mer du sud (F), Eléphant de mer méridional (F) II M

Mirza coquereli $=$ Microcebus coquereli

Mississippi Kite (E): Ictinia mississippiensis II B

Mississippi Sandhill Crane (E): Grus canadensis pulla I B

Mitchell's Water Monitor (E): Varanus mitchelli II R

Mitred Conure (E): Aratinga mitrata II B

Mitred Leaf Monkey (E): Presbytis melalophos II M

Mitred Parakeet (E): Aratinga mirrata II B

Mitu mitu: Alagoas Curassow (E), Razor-billed Curassow (E). Pauji de Alagoas (S), Paují menor (S), Grand hocco à bec rasoir (F),

Hocco mitou (F) I B

Mitu miru mitu $=$ Miru miru

Mitzli (S): Puma concolor I/II M 
Mlanje Mountain Chameleon (E): Bradypodion mlanjensis II R Mocassin à deux lignes (F): Agkistrodon bilinearus III R Mochelo de Sjöstedt (S): Glaucidium sjostedii II B Mochuelín perlado (S): Glaucidium perlatum II B Mochuelo chico (S): Glaucidium passerinum II B Mochuelo chico (S): Glaucidium sjostedti II B Mochuelo común (S): Athene noctua II B Mochuelo de Hoyo (S): Speoryro cunicularia II B Mochuelo duende (S): Micrathene whitneyi II B Mochuelo forestal (S): Athene blewiti I B

Modest Day Gecko (E): Phelsuma modesta II R

Modest Tiger-Parrot (E): Psittacella modesta II B

Mohol's Galago (E): Galago moholi II M

Moineau gris (F): Passer griseus III B

Moineau quadrillé (F): Sporopipes frontalis III B

Moineaus (F): Passer spp/Petronia spp. IIINC B

Moineau-tisserin (F): Plocepasser superciliosus III B

Moluccan Barred Sparrowhawk (E): Accipiter henicogrammus II B

Moluccan Cockatoo (E): Cacatua moluccensis I B

Moluccan Goshawk (E): Accipiter henicogrammus II B

Moluccan Hanging-Parrot (E): Loriculus amabilis II B

Moluccan Hawk-Owl (E): Ninox squamipila I/I B

Moluccan Kestrel (E): Falco moluccensis II B

Moluccan King-Parrot (E): Alisterus amboinensis II B

Moluccan Lory (E): Eos bornea II B

Moluccan Red Lory (E): Eos squamata II B

Moluccan Scops-Owl (E): Otus magicus II B

Moluccan Sparrowhawk (E): Accipiser enthrauchen II B

Mona Island Boa (E): Epicrates monensis I R

Mona Monkey (E): Cercopithecus mona II M

Monachus spp:: Monk seals (E), Focas fraile (S), Focas monje (S), Phoque-moines (F) I M

Monachus monachus: Mediterranean Monk Seal (E), Phoque-moine méditerranéen ( $\mathrm{F}$ ) I M

Monachus schauinslandi: Hawaiian Monk Seal (E), Foca fraile de Hawaii (S) I M

Monachus tropicalis: Caribbean Monk Seal (E), West Indian Monk Seal (E), West Indian Seal (E), Foca fraile del Caribe (S) I M

Monal birman (F): Lophophorus sclateri I B

Monal chinois (F): Lophophorus Ihuysii I B

Monal coliblanco (S): Lophophorus sclateri I B

Monal colirrojo (S): Lophophorus impejanus I B

Monal coliverde (S): Lophophorus lhuysii I B

Monal de l'Himalaya (F): Lophophorus impejanus I B

Monal Pheasant (E): Lophophorus impejanus I B

Monals (E): Lophophorus spp. I B

Monarch Chameleon (E): Chamaeleo monachus II R

Mone (F): Cercopithecus pogonias II M

Mone de Wolf (F): Cercopithecus wolfi II M

Mongolian Wild Ass (E): Equus hemionus hemionus I M

Mongolian Wild Horse (E): Equus przewalskii I M

Mongoose Lemur (E): Eulemur mongoz I M

Mongooses (E): Herpestidae spp. IIUNC M

Monitor lizards (E): Varanus spp/Lanthonotus borneensis VII NCR

Monitors (E): Varanus spp.

Monjita pico-de-plata (S): Lonchura cantans III B

Monk Parakeet (E): Myiopsitta monachus II B

Monk Saki (E): Pithecia monachus II M

Monk seals (E): Monachus spp. I M

Monkey faces (E): Quadrula INC In

Monkey-eating Eagle (E): Pithecophaga jefferyi I B

Monkeys (E): PRIMATES spp. III M

Mono araña de panamá (S): Ateles geoffroyi panamensis I M

Mono araña maninegro (S): Ateles geoffroyi frontatus I M

Mono capuchino (S): Cebus capucinus II M

Mono colorado (E): Areles geoffroyi III M

Mono congo (S): Alouatia palliata I M

Mono coronado (S): Cercopithecus pogonias II M

Mono de Berberea (S): Macaca sylvanus II M

Mono de Gibraltar (S): Macaca sylvanus II M

Mono de orejas rojas (S): Cercopithecus erythrotis II M

Mono de Preuss (S): Cercopithecus preussi II M

Mono de Sclater (S): Cercopithecus sclateri II M

Mono de Wolf (S); Cercopithecus wolf II M

Mono del Gabón (S): Cercopithecus solarus II M

Mono dryas (S): Cercopithecus dryas II M
Mono grande (S): Brachyteles arachnoides I M

Mono narigudo (S): Nasalis larvatus I M

Mono negro (S): Alouatta palliata I M

Mono pigatrix (S): Pygathrix nemaeus I M

Mono resus (S): Macaca mulatta II M

Mono saraguato (S): Alouatta palliata I M

Mono titi (S): Saimiri oerstedii I M

Mono verde (S): Chlorocebus aethiops II M

Monocarya $=$ Parasmilia

Monodon monoceros: Narwhal (E), Unicom Whale (E), Narval (S), Narval (F) II M

Monomyces pygmaea: II In

Monomyces rubrum: II In

Monopora $=$ Montipora

Monos (S): PRIMATES spp. I/II M

Monseigneur (F): Euplectes hordeaceus III B

Monstre de Gila (F): Heloderma suspectum II R

Monstruo de Gila (S): Heloderma suspectum II R

Montagu's Harrier (E): Circus pygargus II B

Montane Chamaeleon (E): Chamaeleo bitaeniatus II R

Montane Racquet-tail (E): Prioniturus montanus II B

Montane Side-striped Chameleon (E): Chamaeleo elliori II R

Montastrea annularis: Mountainous Star Coral (E) II in

Montastrea annuligera: II In

Montastrea cavemosa: Cavemous Star Coral (E) II In

Montastrea curta: II In

Montastrea forskaelana: II In

Montastrea magnistellata: II in

Montastrea multipunctata: II In

Montastrea valenciennesii: II In

Monte Verde Toad (E): Bufo periglenes I A

Monticularia $=$ Hydnophora

Montigyra kenti: II In

Montipora acutala: II In

Montipora aequiruberculata: II In

Montipora altasepta: II In

Montipora alveopora: II In

Montipora angulata: II in

Monipora aspera: 11 In

Montipora ausiraliensis: II In

Montipora bemardi: II in

Montipora berryi: II In

Montipora bilaminata: II In

Montipora cactus: II In

Montipora calcarea: II In

Montipora caliculata: II In

Montipora capilata: II In

Montipora capricornis: II In

Montipora cebuensis: II In

Monsipora circumvallara: II In

Montipora cocosensis: II In

Montipora columnaris: II In

Montipora complanata: II In

Montipora confusa: II In

Montipora corbettensis: II In

Montipora crassituberculata: II In

Montipora cristagalli: II In

Montipora danae: II In

Monipora digitata: II In

Montipora dilatata: II In

Montipora edwardsi: II In

Montipora efflorescens: II In

Montipora effusa: II In

Montipora ehrenbergii: II In

Montipora elschneri: II In

Montipora explanafa: II In

Montipora exserta: II In

Montipora flabellata: II In

Montipora florida: II In

Montipora floweri: II In

Montipora foliosa: Leaf Coral (E) II In

Montipora foveolata: II In

Montipora fragosa: II In

Montipora friabilis: II In

Montipora fruricosa: II In

Montipora gaimardi: II In 
Montipora gracilis: II in Montipora grandifolia: II In Montipora granifera: II In Montipora granulosa: II In Montipora grisea: II In Montipora hirsula: II In Montipora hispida: II In Montipora hoffmeisteri: II in Montipora incognita: II in Montipora incrassata: II In Montipora informis: II In Montipora lichen: II in Montipora lobulata: II In

Montipora mactanensis: II In Montipora malampaya: II In Montipora maldivensis: II in Montipora manauliensis: II in Montipora marshallensis: II In Montipora millepora: II In

Montipora mollis: II In

Montipora monasteriata: II In

Montipora multipapillosa: II In

Montipora nodosa: II In

Montipara orientalis: II in

Montipora palmata: II In

Montipora papillosa: II In

Montipora patula: II In

Montipora peliformis: II in

Montipora perforata: II in

Montipora poritiformis: II In

Montipora profunda: II In

Montipora rigida: II In

Montipora samarensis: II In

Montipora savea: II In

Montipora scabricula: II in

Montipora setosa: II In

Montipora sinuosa: II In

Montipora solanderi: II In

Montipora spongiosa: II In

Montipora spongodes: II In

Montipora spumosa: II In

Montipora stellata: II In

Montipora stilosa: II In

Montipora studeri: II in

Montipora sulcata: II In

Montipora sumilonensis: II In

Montipora suvadivae: II In

Montipora tenuicaulis: II In

Montipora tenuissima: II In

Montipora trabeculata: II In

Montipora tuberculosa: II In

Montipora turgescens: II In

Montipora turtensis: II In

Montipora undata: II In

Montipora venosa: II In

Montipora verrucosa: II in

Moor Macaque (E): Macaca maura II M

Moorish Tortoise (E): Testudo graeca II R

Morden's Scops-Owl (E): Otus ireneae II B

Morelet's Crocodile (E): Crocodylus moreletii I R

Morelia alberisii: D'Albert's Python (E), White-lipped Python (E), Pitón de Albert (S), Python de Nouvelle-Guinée (F), Python à lèvres blanches (F) II R

Morelia amethistina: Amethystine (Rock) Python (E), Scrub Python (E), Pitón ametista (S), Python améthiste (F), Python de la brousse (F), Python des rochers (F) II R

Morelia boa: Barred Python (E), Bismarck Ringed Python (E), Ringed Python (E), Pitón anillada de Bismarck (S), Bothrochile (F) II R

Morelia boeleni: Black Python (E), Boelen's Python (E), Pitón de Boelen (S), Python de Boelen (F) II R

Morelia carinata: Keeled-scaled Python (E), Rough-scaled Python (E), Python caréné (F), Python à écailles rugueuses (F) II R

Morelia childreni: Children's (Rock) Python (E), Pitón enana (S), Python de Children (F) II R
Morelia mackloti: Macklot's Python (E), Water Python (E), Pitón de Macklot (S), Python de Macklot (F) II R

Morelia maculosa: Spotted Python (E), Python tacheté (F) II R

Morelia oenpelliensis: Oenpelli Python (E), South Australian Water Python (E), Python d'Oenpelli (F) II R

Morelia olivacea: Olive (Rock) Python (E), Pitón oliva (S), Python olive (F) II R

Morelia papuana: Papuan Python (E), Pitón acuática de Papua (S), Python de Papouasie (F) II R

Morelia perthensis: Perth Pygmy Python (E), Pygmy Python (E), Python pygmée de Perth (F) II R

Morelia spilota: Carpet Python (E), Diamond Python (E), Pitón diamantina (S), Python tapis (F) II R

Morelia spilota spilotus $=$ Morelia spilota

Morelia spilota variegata $=$ Morelia spilota

Morelia stimsoni: Large-blotched Python (E), Stimson's Python (E) Python de Stimson (F), Python à large tache (F) II R

Morelia viridis: Green Tree Python (E), Python arboricole vert australien (F) II R

Morenia ocellata: Bengal Eyed Terrapin (E), Burmese Eyed Turtle (E), Peacock Turte (E), Swamp Turte (E), Galápago ocelado de Birmania (S), Emyde à ocelles de Birmanie (F), Tortue de Birmanie (F) I R

Morepork (E): Ninox novaeseelandiae III B

Morogoro Tree Toad (E): Nectophrynoides viviparus I A

Morphnus guianensis: Crested Eagle (E), Guianan Crested Eagle (E), Aguila crestada (S), Aguila monera (S), Arpía menor (S), Harpie huppée (F) II B

Morrocoy (S): Geochelone denticulata II R

Morse (F): Odobenus rosmarus III M

Mortlock Flying-fox (E): Pteropus phaeocephalus I M

Mortlock Islands Flying-fox (E): Pleropus phaeocephalus I M

Moschus spp.: Musk deer (E), Ciervos almizcleros (S), Chevrotains porte-musc (F) I/II M

Moschus anhuiensis = Moschus berezovskii

Moschus berezovskii: Dwarf Musk Deer (E), Forest Musk Deer (E), South China Forest Musk Deer (E) II M

Moschus chnysogaster: Himalayan Musk Deer (E) $\mathbf{l} I \mathbf{M}$

Moschus chrysogaster berezovskii $=$ Moschus berezovskii

Moschus chrysogaster fuscus = Moschus fuscus

Moschus fuscus: Black Musk Deer (E) II M

Moschus leucogaster $=$ Moschus chrysogaster

Moschus moschiferus: Siberian Musk Deer (E). Ciervo almizclero

(S), Cerf porte-musc (F), Chevrotain porte-musc (F), Ponte-musc (F) I M

Moschus sifanicus = Moschus chrysogaster

Moseleya latistellata: II in

Motelo (S): Geochelone denticulata II R

Mottled Owl (E): Strix virgata II B

Mottled Wood-Owl (E): Strix acellata II B

Mottle-faced Tamarin (E): Saguinus inustus II M

Mouette relique ( $\mathrm{F}$ ): Larus relictus I B

Mouettes (F): Larus spp. $\mathbf{I} / \mathrm{NC}$ B

Moufette à nez de cochon (F): Conepatus humboldtii II M

Moufette de Patagonie (F): Conepatus humboldrii II M

Mouflon à manchettes (F): Ammotragus lervia II M

Mouflon d'Amérique ( $F$ ): Ovis canadensis $\mathbf{1}$ II M

Mouflon d'Asie (F): Ovis ammon VII M

Mouflon de Chypre (F): Ovis orientalis ophion I M

Mouflon de l'Himalaya (F): Ovis ammon hodgsonii $1 \mathrm{M}$

Mouflon des montagnes (F): Ovis ammon hodgsonii $1 \mathrm{M}$

Mouflon d'Eurasie (F): Ovis ammon I/II M

Mouflon du Canada ( $\mathrm{F}$ ): Ovis canadensis VII M

Mouflon du Ladak (F): Ovis vignei vignei I M

Mouflon pachycère $(\mathrm{F})$ : Ovis canadensis $\mathbf{\text { VII }} \mathrm{M}$

Mouflon vrai (F): Ovis ammon III M

Mount Lefo Chameleon (E): Chamaeleo wiedersheimi II R

Mount Omei Liocichla (E): Liocichla omeiensis II B

Mountain Anoa (E): Bubalus quarlesi I M

Mountain Apollo (E): Parmassius apollo II In

Mountain Avocetbill (E): Opisthoprora euryptera II B

Mountain Buzzard (E): Buteo oreophilus II B

Mountain Caracara (E): Phalcoboenus megalopterus II B

Mountain Cat (E): Oreailurus jacobita I M

Mountain Chameleon (E): Chamaeleo montium II R

Mountain Dwarf Chameleon (E): Chamaeleo ellioti II R 
Mountain Eagle-Owl (E): Bubo capensis II B

Mountain Hawk-Eagle (E): Spizaetus nipalensis II B

Mountain Lion (E): Puma concolor I/II M

Mountain Monkey (E): Cercopithecus lhoesti II M

Mountain Parakeet (E): Bolborhynchus aurifrons II B

Mountain Peacock-Pheasant (E): Polyplectron inopinatum III B

Mountain Pygmy-Owl (E): Gloucidium gnoma II B

Mountain Scops-Owl (E): Orus spilocephalus II B

Mountain Serpent-Eagle (E): Spilomis kinabaluensis II B

Mountain Sheep (E): Ovis canadensis I/II M

Mountain Tapir (E): Tapirus pinchaque I M

Mountain Tortoise (E): Geochelone pardalis II R

Mountain Treeshrew (E): Tupaia montana II M

Mountain Velvetbreast (E): Lafresnaya lafresnayi II B

Mountain Weasel (E): Mustela altaica III M

Mountain-gems (E): Lampornis spp. II B

Mountainous Star Coral (E): Moniastrea annularis II in

Moumful Tree Monitor (E): Varanus tristis II R

Mouming Collared-Dove (E): Sireptopelia decipiens III B

Mouse-lemurs (E): Microcebus spp/Phaner furcifer I M

Moustac à oreilles rouges (F): Cercopithecus enthroris II M

Moustac bleu (F): Cercopithecus cephus II M

Moustached Monkey (E): Cercopithecus cephus II M

Moustached Parakeet (E): Psittacula alexandri II B

Moustached Tamarin (E): Saguinus mystax II M

Mt Nimba Viviparous Toad (E): Nimbaphrynoides occidentalis I A

Mud rurtles (E): Pelusios spp/Kinostemon spp. IIINC R

Muflón Argal (S): Ovis ammon V/I M

Muflón Argal Tibetano (S): Ovis ammon hodgsonii I M

Mufón de Chipre (S): Ovis orienlalis ophion I M

Muflón de Marco Polo (S): Ovis ammon I/II M

Mugger (E): Crocodylus palustris I $\mathbf{R}$

Muhlenberg's Turtle (E); Clemmys muhlenbergi I R

Muitú mamaco (S): Crax blumenbachii I B

Mulga Parrot (E): Psephotus varius II B

Müller's Gibbon (E): Hylobates muelleri I M

Müller's Leaf Chameleon (E): Bradypodion uthmoelleri II R

Müller's Parrot (E): Tanygnathus sumatranus II B

Müller's Sand Boa (E): Enyx muelleri II R

Munias (E): Lonchura spp. III/NC B

Munning (E): Lagostrophus fasciatus I M

Muntiacus crinifrons: Black Muntjac (E), Muntjac negro (S), Muntjac noir (F) I M

Muntiacus vuquanghensis = Megamuntiacus vuquanghensis

Muntjac géant (F): Megamuntiacus vuquanghensis I M

Muntjac negro (S): Muntiacus crinifrons $1 \mathrm{M}$

Muntjac noir (F): Muntiacus crinifrons I M

Muntjacs (E): Muntiacus spp/Megamuntiacus vuquanghensis INC M

Murciélago de estrias blancas (S): Platyrrhinus lineatus III M

Muriki (S): Brachyleles arachnoides I M

Muriqui (E): Brachyteles arachnoides I M

Musaraña arborícola coliplumada (S): Prilocercus lowiî II M

Musaraña arboricola de Filipinas (S): Urogale evereti II M

Musaraña arborícola de la india (S): Anathana elliori II M

Musaraña común (S): Tupaia glis II M

Musaraña grande (S): Tupaia tana II M

Musaraña pigmea (S): Tupaia minor II M

Muscicapa ruecki $=$ Cyomis rucki

Muscory Duck (E): Cairina moschata III B

Mushroom Coral (E): Fungia fungires II in

Musk deer (E): Moschus spp. I/II M

Musk Lorikeet (E): Glossopsitta concinna II B

Musophaga porphyreolopha: Purple-crested Turaco (E), Violetcrested Turaco (E), Turaco crestivioleta (S), Touraco à huppe pourprée (F), Touraco à huppe splendide (F), Touraco à huppe violette (F) II B

Musophaga violacea: Violet Plantain-eater (E), Violet Turaco (E), Turaco azul (S), Touraco violet (F) III B

Mussa angulosa: Large Flower Coral (E) II In

Mussa cactus: II In

Mussa cerebriformis: II In

Musschenbroek's Lorikeet (E): Neopsittacus musschenbroekii II B

Mussel Digger (E): Eschrichrius robustus I M

Mussels (E): BIVALVIA spp. IIUNC in

Mussismilia braziliensis: II In
Mussismilia hartii: 11 In

Mussismilia hispida: II In

Mussurana (E/F): Clelia clelia II R

Mustard Hill Coral (E): Porites astreoides II in

Mustela altaica: Mountain Weasel (E), Comadreja de montan̄a (S), Belette alpine (F), Belette des Alpes (F) III M

Mustela eminea ferghanae: Ermine (E), Stoat (E), Hermine (F) III M

Mustela kathiah: Yellow-bellied Weasel (E), Comadreja de vientre amarillo (S), Belette à ventre jaune (F) III M

Mustela nigripes: Black-footed Ferret (E), Turón patinegro americano (S), Putois a pieds noirs (F) I M

Mustela sibirica: Siberian Weasel (E), Comadreja de Siberia (S), Belette de Sibérie (F), Vison sibérien (F) III M

Mutum (E): Crax blumenbachii I B

Mycedia $=$ Agaricia

Mycedium elephantotus: II In

Mycedium mancaoi: II In

Mycedium robokaki: II In

Mycelophyllia aliciae: II In

Mycetophyllia daniana: II In

Mycetophyllia ferox: II In

Mycetophyllia lamarckiana: II In

Mycetophyllia reesi: II In

Mycteria cinerea: Milky Stork (E), Tantale blanc (F) I B

Myers's Poison Frog (E): Epipedobates myersi II A

Mygales (F): Brachypelma spp. II In

Myiopsinta monachus: Grey-breasted Parakeet (E), Monk Parakeet

(E), Quaker Parakeet (E), Catita común (S), Cotorra Argentina

(S), Conure veuve (F), Perruche-souris (F) II B

Mynas (E): Stumidae spp. I/II/NC B

Myrmecophaga jubata $=$ Myrmecophaga tridactyla

Myrmecophaga tridactyla: Giant Anteater (E), Banderón caballo (S), Hormiguero gigante (S), Oso caballo (S), Oso hormiguero (S), Grand fourmilier (F), Tamanoir (F) II M

Myrmia micrura: Short-tailed Woodstar (E), Estrellita colicorta (S) Colibri a queue courte (F) II B

Myrtis fanny: Purple-collared Woodstar (E), Estrellita gargantillada (S), Colibri fanny (F) II B

Nactus serpensinsula $=$ Cyrtodactylus serpensinsula

Nacurutú (S): Bubo virginianus II B

Naemorhedus baileyi: Red Goral (E) I M

Naemorhedus caudatus: Chinese Goral (E), Goral à queue longue (F) I M

Naemorhedus cranbrooki $=$ Naemorhedus baileyi

Naemorhedus goral: Goral (E), Himalayan Goral (E), Goral (S), Bouquetin du Népal (F), Goral (F) I M

Naemorhedus goral baileyi = Naemorhedus baileyi

Naemorhedus goral caudatus = Naemorhedus caudatus

Naemorhedus sumatraensis: Mainland Serow (E), Serau (S). Capricome de Sumatra (F) I M

Naiads (E): Unionidae spp. I/II In

Nailtail wallabies (E): Onychogalea spp. V/NC M

Naja à lunettes (F): Naja naja II R

Naja atra = Naja naja

Naja indien (F): Naja naja II R

Naja kaouthia $=$ Naja najo

Naja naja: Asian Cobra (E), Indian Spectacled Cobra (E), Cobra escupidora asiática (S), Cobra à lunettes (F), Naja indien (F), Naja à lunettes (F) II R

Naja oxiana $=$ Naja naja

Naja philippensis = Naja naja

Naja samurensis = Naja naja

Naja sputairix = Naja naja

Naja sumatrana = Naja naja

Nakamina (S): Cyclagras gigas II R

Naked-tailed armadillos (E): Cabassous spp. IIINC M

Namaqua Chameleon (E): Chamaeleo namaquensis II R

Namaqua Day Gecko (E): Phelsuma ocellata II R

Namaqua Dove (E): Oena capensis III B

Namaqua Dwarf Chameleon (E): Bradypodion ventrale II R

Narnaqua Girdled Lizard (E): Cordylus namaquensis II R

Namaqua Spiny-tailed Lizard (E): Cordylus namaquensis II R

Nanday Conure (E): Nandayus nenday II B

Nanday Parakeet (E): Nandayus nenday II B 
Nandayus nenday: Black-headed Conure (E), Black-headed Parakeet (E), Nanday Conure (E), Nanday Parakeet (E), Aratinga ñanday (S), Cotorra cabecinegra (S), Conure nanday (F), Pernuche Nanday (F) II B

Nandou commun (F): Rhea americana II B

Nandou d'Amérique (F): Rhea americana II B

Nandou de Darwin (F): Rhea pennata I B

Nandou gris (F): Rhea americana II B

Nandous (F): Rhea spp. III B

N̄andú (S): Rhea americana II B

Ñandú común (S): Rhea americana II B

Ñandú cordillerano (S): Rhea pennata I B

Ñandú petizo (S): Rhea pennata I B

Nankeen Kestrel (E): Falco cenchroides II B

Nannopsittaca dachilleae: Amazonian Parakeet (E), Amazonian Parrotlet (E), Cotorrita amazónica (S), Toui de Achille (F) II B

Nannopsitraca panychlora: Tepui Parakeet (E), Chirica (S), Cotorrit tepuí (S), Toui des tépuis (F) II B

Napo Sabrewing (E): Campylopterus villaviscensio II B

Napoleon Weaver (E): Euplecies afer III B

Napoleon's Peacock-Pheasant (E): Polyplectron emphanum I B

Napopora $=$ Porites

Narcondam Hornbill (E): Aceros narcondami II B

Narrow-snouted Dolphin (E): Stenella antenuata II M

Narrow-tailed Emerald (E): Chlorostilbon stenura II B

Narval (S/F): Monodon monoceras II M

Narwhal (E): Monodon monoceros II M

Nasalis spp.: I M

Nasalis concolor. Mentawi Islands Snub-nosed Langur (E), Pagai Island Langur (E), Pig-tailed Langur (E), Langur rabicerdo (S), Entelle de Pagi $(F)$, Langur des îles Mentawi $(F)$, Langur à queue de cochon (F) I M

Nasalis lanarus: Long-nosed Monkey (E), Proboscis Monkey (E), Mono narigudo (S), Nasique (F) I M

Nasique (F): Nasalis lanvalus I M

Nasua narica: Coati (E) III M

Nasua nasua solitaria: South Brazilian Coati (E), Coati (S), Coati roux (F) III M

Natal Hinge-back Tortoise (E): Kinixy's notalensis II R

Natal Hinge-backed Tortoise (E): Kinixys natalensis II R

Natal Hinged Tortoise (E): Kinixys natalensis II R

Natal Midland Dwarf Chamaeleon (E): Bradypodion thamnobates II $\mathrm{R}$

Natator depressus: Flatback (E), Tortuga franca oriental (S), Chelonée à dos plat $(F)$, Tortue marine à dos plat $(F)$ I R

Natrix piscator $=$ Xenochrophis piscator

Navassa Island Black-tailed Dwarf Boa (E): Tropidophis melanurus II R

Neblina Metaltail (E): Melallura odomae II B

Necrosyrtes monachus: Hooded Vulture (E), Alimoche sombrío (S), Percnoptère brun $(F)$, Vautour charognard (F) II B

Nectophrynoide cryptique (F): Neciophrynoides cryprus I A

Nectophrynoide de Malcolm (F): Aliphrynoides malcolmi I A

Nectophrynoide de Tomier (F): Nectophrynoides tomieri I A

Nectophrynoide des Monts Uluguru (F): Nectophrynoides viviparus I A

Nectophrynoide des Monts Usambara (F): Nectophrynoides tomieri I A

Nectophrynoide des Monts Uzungwe (F): Nectophrynoides wendyae I A

Nectophrynoide d'Ethiopie (F): Spinophrynoides osgoodi I A

Nectophrynoide d'Osgood (F): Spinophrynoides osgoodi I A

Nectophrynoide du Liberia (F): Nimbaphrynoides liberiensis I A

Nectophrynoide du Mont Nimba (F): Nimbaphrynoides occidentalis I A

Nectophrynoide menu (F): Necrophrynoides minutus I A

Nectophrynoides (F): Nectophrynoides spp. I A

Nectophrynoides spp.: African viviparous toads (E), Tree toads (E), Sapos vivíparos africanos (S), Crapauds vivipares (F),

Nectophrynoides cryptus: Secret Tree Toad (E), Nectophrynoide cryptique (F) I A

Nectophrynoides liberiensis $=$ Nimbaphrynoides liberiensis

Nectophrynoides malcolmi $=$ Alriphrynoides malcolmi

Nectophrynoides minutus: Minute Tree Toad (E), Nectophrynoide menu (F) I A

Nectophrynoides occidentalis $=$ Nimbaphrynoides occidentalis
Nectophrynoides osgoodi $=$ Spinophrynoides osgood $i$

Nectophrynoides (F) I A

Nectophnyoides tomieri: Tomier's Tree Toad (E), Usambara Viviparous Toad (E), Nectophrynoide de Tomier (F), Nectophrynoide des Monts Usambara (F) I A

Nectophrynoides viviparus: Morogoro Tree Toad (E), Rungwe Viviparous Toad (E), Nectophrynoide des Monts Uluguru (F) I A

Nectophrynoides wendyae: Uzungwe Scarp Tree Toad (E), Nectophrynoide des Monts Uzungwe (F) I A

Needle Coral (E): Seriaropora hystrix II In

Needle-billed Hernit (E): Phaethornis philippii II B

Negrita bronceada (S): Lonchura cucullata III B

Negrita cana (S): Nigrina canicapilla III B

Negrita frentigualda (S): Nigrita luteifrons III B

Negrita pechiblanca (S): Nigrita fusconota III B

Negrita pechirroja (S): Nigrita bicolor 111 B

Negrita picanza (S): Lonchura fringilloides III B

Negro Tamarin (E): Saguinus midas II M

Negrofinches (E): Nigrita spp. III B

Nemenzophyllia $=$ Plerogyra

Nene (E): Branta sandvicensis I B

Neoceratodus forsteri: Australian Lungfish (E), Ceratodus (E) Queensland Lungfish (E), Pez pulmonado australiano (S), Cératode (F), Dipneuste (F) II F

Neofelis nebulosa: Clouded Leopard (E), Pantera del Himalaya (S), Pantera longibanda (S), Pantera nebulosa (S), Panthère longibande (F), Panthère nébuleuse (F) I M

Neohelia porcellana: II In

Neophema bourkii $=$ Neopsephotus bourkii

Neophema chrysogaster. Orange-bellied Parakeet (E), Orangebellied Parrot (E), Loro ventrinaranja (S), Periquito ventrinaranja $(S)$, Perruche à lunettes vertes $(F)$, Perruche à ventre orange $(F)$ I B

Neophema chrysostoma: Blue-winged Grass-Parakeet (E), Bluewinged Parrot (E), Periquito crisóstomo (S), Pernuche à bouche d'or (F) II B

Neophema elegans: Elegant Grass-Parakeet (E), Elegant Parrot (E), Periquito elegante (S), Perruche élégante (F) II B

Neophema petrophila: Rock Parrot (E), Periquito roquero (S), Perruche des rochers (F), Perruche des écueils (F) II B

Neophema pulchella: Turquoise Grass-Parakeet (E), Turquoise Parrot (E), Periquito turquesa (S), Pemuche turquoisine (F) II B Neophema splendida: Scarlet-chested Parrot (E), Splendid GrassParakeet (E), Periquito espléndido (S), Perruche splendide (F), Perruche à poitrine écarlate (F) II B

Neophocaena phocaenoides: Black Finless Porpoise (E), Finless Black Porpoise (E), Finless Porpoise (E), Marsopa negra (S), Marsouin aptère (F), Marsouin sans nageoires (F) I M

Neophron percnopterus: Egyptian Vulture (E), Scavenger Vulture (E). Alimoche común (S), Percnoptère d'Egypte $(F)$. Vautour percnoptère (F) II B

Neoporites $=$ Porites

Neopsephotus bourkii: Bourke's Parrot (E), Periquito rosado (S), Perruche de Bourke (F) II B

Neopsittacus musschenbroekii: Musschenbroek's Lorikeet (E), Yellow-billed Lorikeet (E), Lori montano grande (S), Lori de Musschenbroek (F) II B

Neopsittacus pulticauda: Emerald Lorikeet (E), Orange-billed Lorikeet (E), Lori montano chico (S), Lori émeraude (F) II B Neotis burchellii $=$ Neotis denhami

Neotis cafra $=$ Neotis denhami

Neotis denhami: Denham's Bustard (E), Stanley Bustard (E), Avutarda cafre (S), Avutarda de Burchell (S), Avutarda de Denham (S), Outarde de Burchell (F), Outarde de Denham (F) II B

Neoris heuglinii: Heuglin's Bustard (E), Avutarda de Heuglin (S), Avutarda somali (S), Outarde de Heuglin (F) II B

Neotis ludwigii: Ludwig's Bustard (E), Avutarda de Ludwig (S), Avutarda de Namibia (S), Outarde de Ludwig (F) II B

Neotis nuba: Nubian Bustard (E), Avutarda núbica (S), Outarde de Nubie (F), Outarde nubienne (F) II B

Neotropical Ratlesnake (E): Crotalus durissus III R

Neotropical River Otter (E): Lontra longicaudis I M

Nesasio solomonensis: Fearful Owl (E), Búho de las Islas Salomón (S), Chouette des îles Salomons (F), Hibou redoutable (F) II B 
Nesocharis capistrata: Grey-headed Olive-back (E), White-cheeked Olive Weaver (E), Olivino cariblanco (S), Dos-vert à joues blanches (F), Sénégali vert à joues blanches (F) III B

Nesochen sandvicensis = Branta sandvicensis

Nesoenas mayeri $=$ Columba mayer

Nestor de Norfolk (F): Nestor productus II B

Nestor kéa (F): Nestor notabilis II B

Nestor meridionalis: Kaka (E), New Zealand Kaka (E), Kaka (S), Nestor superbe (F) II B

Nestor notabilis: Kea (E), Kea (S), Kea (F), Nestor kéa (F) II B

Nestor productus: Norfolk Island Kaka (E), Norfolk Kaka (E), Nestor de Norfolk (F) II B

Nestor superbe (F): Nestor meridionalis II B

Nestors (F): Nestor spp. II B

Nenapus aurizus: African Pygmy-goose (E), Patito africano (S), Anserelle naine (F), Sarcelle à oreillons (F) III B

New Britain Goshawk (E): Accipiter princeps II B

New Britain Hawk-Owl (E): Ninox odiosa II B

New Britain Honey-buzzard (E): Henicopemis infuscarus II B

New Britain Masked-Owl (E): Tyto aurantia 11 B

New Britain Sparrowhawk (E): Accipiter brachyurus II B

New Caledonia Goshawk (E): Accipiter haplochrous II B

New Caledonian Lorikeet (E): Charmosyna diadema II B

New Guinea Birdwing (E): Ornithoptera priamus II In

New Guinea Crocodile (E): Crocodylus novaeguineae I/II R

New Guinea Eagle (E): Harpyopsis novaeguineae II B

New Guinea Grey-headed Goshawk (E): Accipiter poliocephalus II B

New Guinea Harpy Eagle (E): Harpyopsis novaeguineae II B

New Guinea Long-nosed Echidna (E): Zaglossus bruijni II M

New Guinea Viper Boa (E): Candoia aspera II R

New Ireland Hawk-Owl (E): Ninox variegata II B

New World Python (E): Loxocemus bicolor II R

New Zealand Dolphin (E): Cephalorhynchus hectori II M

New Zealand Falcon (E): Falco novaezeelandiae II B

New Zealand Fur Seal (E): Arctocephalus forsteri II M

New Zealand Kaka (E): Nestor meridionalis II B

New Zealand Parakeet (E): Cyanoramphus novaezelandiae I B

Newton's Kestrel (E): Falco newtoni I/II B

Ngami Owlet (E): Glaucidium ngamiense II B

Ngosi Volcano Chameleon (E): Chamaeleo fuellebomi II R

Niam-niam Parrot (E): Poicephalus crassus II B

Niceforo's Poison Frog (E): Epipedobates ingeri II A

Nicklin's Pearly Mussel (E): Unio nickliniana I In

Nicobar à camail (F): Caloenas nicobarica I B

Nicobar Dove (E): Caloenas nicobarica I B

Nicobar Parakeet (E): Psintacula caniceps II B

Nicobar Pigeon (E): Caloenas nicobarica I B

Nicobar Serpent-Eagle (E): Spilomis minimus II B

Nicobar Shikra (E): Accipiter bulleri II B

Nicobar Sparrowhawk (E): Accipiter butleri II B

Nicobar Treeshrew (E): Tupaia nicobarica II M

Nicoria tricarinata $=$ Melanochelys sricarinata

Nifa mexicana (S): Thalurania ridgwayi II B

Night Monkey (E): Aotus trivirgatus II M

Night Parrot (E): Geopsittacus occidentalis I B

Nigrette à calotte grise (F): Nigrita canicapilla III B

Nigrette à front jaune (F): Nigrita luteifrons III B

Nigrette à ventre blanc (F): Nigrita fusconota III B

Nigrette à ventre roux $(F)$ : Nigrita bicolor III $\mathrm{B}$

Nigrettes (F): Nigrita spp. III B

Nigrita bicolor: Chestnut-breasted Negrofinch (E), Negrita pechirroja $(S)$, Nigrette à ventre roux $(F)$, Sénégali brun à ventre roux (F) III B

Nigrifa canicapilla: Grey-crowned Negrofinch (E), Grey-headed Negrofinch (E), Negrita cana (S), Nigrette à calotte grise (F) Sénégali nègre (F) III B

Nigrita fusconota: White-breasted Negrofinch (E), Negrita pechiblanca $(\mathrm{S})$, Nigrette à ventre blanc $(\mathrm{F})$, Sénégali brun à ventre blanc (F) III B

Nigrita luteifrons: Pale-fronted Negrofinch (E), Negrita frentigualda (S), Nigrette à front jaune (F). Sénégali nègre à front jaune (F) III B

Nile Crocodile (E): Crocodylus niloticus I/II R

Nile Monitor (E): Varanus niloticus II R

Nile Soft-shell Turtle (E): Trionyx triunguis III R
Nilgiri Langur (E): Trachypithecus johnii II M Nilgiri Leaf Monkey (E): Trachypithecus johnii II M

Nilgiri Marten (E): Martes gwatkinsii III M

Nillava ruecki $=$ Cyomis ruckii

Niltavas (E): Cyomis spp/Niltava spp. IU/NC B

Nimba toads (E): Nimbaphrynoides spp. 1 A

Nimbaphrynoides spp.: Nimba toads (E), Sapos viviparos (S), Crapauds vivipares (F) I A

Nimbaphrynoides liberiensis: Liberia Nimba Toad (E), Sapo vivíparo liberiano (S), Nectophrynoide du Liberia (F) I A

Nimbaphrynoides occidentalis: Mt Nimba Viviparous Toad (E), Westem Nimba Toad (E), Sapo vivíparo de Africa occidental (S), Nectophrynoide du Mont Nimba (F) I A

Ninfa coronada (S): Thalurania colombica II B/Thalurania fannyi II B

Ninox affinis: Andaman Hawk-Owl (E), Ninoxe des Andaman (F) II B

Ninox boobook: Southern Boobook (E), Ninoxe d'Australie (F) II B Ninox connivens: Barking Owl (E), Winking Owl (E), Ninoxe aboyeuse (F) II B

Nino goldii $=$ Nino $x$ theomacha

Ninox jacquinoti: Solomon Hawk-Owl (E), Ninoxe de Jacquinot (F) II B

Ninox meeki: Admiralty Hawk-Owl (E), Manus Hawk-Owl (E), Ninoxe de l'Amirauté (F) II B

Ninox novaeseelandiae: Morepork (E), Chouette boobok (F), Chouette coucou (F), Ninoxe boubouk (F) III B

Ninox novaeseelandiae boobook $=$ Ninox boobook

Ninox novaeseelandiae royana = Ninox novaeseelandiae undulata

Ninox novaeseelandiae rudolf $\mathrm{i}=$ Ninox rudolf $i$

Ninox novaeseelandiae undulata: Norfolk Boobook (E), Norfolk Island Boobook Owl (E), Lechuza gavilana de Norfolk (S), Chouette boobok de lîle Norfolk ( $F$ ), Ninoxe boubouk de l'ìle Norfolk (F) I B

Ninox ochracea: Ochre-bellied Hawk-Owl (E), Ninoxe ocrée (F) II B

Ninox odiosa: New Britain Hawk-Owl (E), Russet Hawk-Owl (E), Ninoxe odieuse (F) II B

Ninox perversa $=$ Ninox ochracea

Ninox philippensis: Philippine Hawk-Owl (E), Ninoxe des Philippines (F) II B

Ninox punctulata: Speckled Hawk-Owl (E), Ninoxe pointillée (F) II B

Ninox rudolfi: Sumba Boobook (E), Ninoxe de Sumba (F) II B Ninox rufa: Rufous Owl (E), Ninoxe rousse (F) II B

Ninox scurulata: Brown Hawk-Owl (E), Ninoxe hirsute (F) II B Ninox solomonis $=$ Ninox variegata

Ninox squamipila: Moluccan Hawk-Owl (E), Lechuza gavilana de las Molucas ( $\mathrm{S}$ ), Chouette des Moluques ( $\mathrm{F}$ ), Chouette épervière (F), Ninoxe des Moluques (F) I/II B

Ninox squamipila natalis: Christmas Hawk-Owl (E), Christmas Island Hawk-Owl (E), Chouette épervière de l'ile Christmas (F) I B

Ninox strenua: Powerful Owl (E), Ninoxe puissante (F) II B

Ninox superciliaris: Madagascar Hawk-Owl (E), White-browed Ow (E), Ninoxe à sourcils blancs (F) II B

Ninox theomacha: Chestnut Hawk-Owl (E), Jungle Hawk-Owl (E), Sooty-backed Hawk-Owl (E), Ninoxe brune (F) II B

Ninox variegata: Bismarck Hawk-Owl (E), New Ireland Hawk-Owl (E), Ninoxe bariolée (F) II B

Ninoxe à sourcils blanes $(\mathbf{F})$ : Ninox superciliaris II B

Ninoxe aboyeuse (F): Ninox connivens II B

Ninoxe bariolé (F): Ninox variegata II B

Ninoxe boubouk (F): Ninox novaeseelandiae I/II B

Ninoxe boubouk de líle Norfolk (F): Ninox novaeseelandiae undulata I B

Ninoxe brune (F): Ninox theomacha II B

Ninoxe d'Australie (F): Ninox boobook II B

Ninoxe de Jacquinot (F): Ninox jacquinoti $11 \mathrm{~B}$

Ninoxe de l'Amirauté (F): Ninox meeki II B

Ninoxe de Sumba (F): Ninox rudolfi II B

Ninoxe des Andaman (F): Ninox affinis II B

Ninoxe des Moluques (F): Ninox squamipila II B

Ninoxe des Philippines (F): Ninox philippensis II B

Ninoxe hirsute (F): Ninox scutulata II B

Ninoxe ocree (F): Ninox ochracea II B 
Ninoxe odieuse (F): Ninox odiosa II B

Ninoxe papoue (F): Uroglawx dimorpha II B

Ninoxe pointillée (F): Ninox puncrulata II B

Ninoxe puissante (F): Ninox strenua II B

Ninoxe rieuse (F): Sceloglaux albifacies II B

Ninoxe rousse (F): Ninox rufa II B

Ninoxes (F): Ninox spp/Sceloglaux albifacies/Uroglaux dimorpha I/I B

Nipponia nippon: Crested Ibis (E), Japanese Crested Ibis (E), Ibis moñudo japonés (S), lbis nipón (S), lbis blanc (F), lbis blanc du Japon (F), Ibis nippon (F), Ibis à crête du Japon (F) I B

Noble Macaw (E): Ara nobilis II B

Noisy Scrub-bird (E): Atrichomis clamosus I B

No-mark Lizard (E): Varanus salvator II R

Nomlandia califomica: II In

Nordmann's Greenshank (E): Tringa gutrifer I B

Norfolk Boobook (E): Ninox novaeseelandiae undulata I B

Norfolk Island Boobook Owl (E): Ninox novaeseelandiae undulata I B

Norfolk Island Kaka (E): Nestor producius II B

Norfolk Island Parakeet (E): Cyanoramphus cookii I B

Norfolk Kaka (E): Nestor productus II B

Norfolk Parakeet (E): Cyanoramphus cookii I B

North African Crested Porcupine (E): Hystrix cristata III M

North American Manatee (E): Trichechus manarus I M

North American Otter (E): Lontra canadensis II M

North American River Otter (E): Lontra canadensis II M

North Andean Deer (E): Hippocamelus antisensis I M

North Andean Huemul (E): Hippocamelus antisensis I M

North Andean Huemul (E): Hippocamelus antisensis I M

North Mongolian Kulan (E): Equus hemionus hemionus I M

Northem Bald Ibis (E): Geronticus eremita I B

Northem Bottlenose Whale (E): Hyperoodon ampullatus I M

Northem Common Cuscus (E): Phalanger orientalis II M

Northem Day Gecko (E): Phelsuma chekei II R

Northern Eyelash Boa (E): Trachyboa boulengeri II $R$

Northern Four-toothed Whale (E): Berardius bairdii I M

Northern Goshawk (E): Accipiter gentilis II B

Northem Hairy-nosed Wombat (E): Lasiorhinus kreffii I M

Northem Harrier (E): Circus cyaneus II B

Northem Hawk Owl (E): Surnia ulula II B

Northern Helmeted Curassow (E): Pauxi pauxi III B

Northern Hobby (E): Falco subbuteo II B

Northem Little Owl (E): Athene noctua II B

Northem Naked-tailed Armadillo (E): Cabassous centralis III M

Northern Needle-clawed Bushbaby (E): Euoricus pallidus II M

Northern Needle-clawed Galago (E): Euoticus pallidus II M

Northem Paradise-Whydah (E): Vidua orientalis III B

Northern Pintail (E): Anas acuta III B

Northem Pudu (E): Pudu mephistophiles II M

Northern Pygmy-Owl (E): Glaucidium californicum II B

Northern Riffleshell (E): Epioblasma torulosa rangiana II In

Northern Right Whale (E): Eubalaena glacialis I M

Northern Right Whale Dolphin (E): Lissodelphis borealis II M

Northern Rosella (E): Platycercus venustus II B

Northem Savanna Monitor (E): Varanus exanthematicus II $R$

Northem Saw-whet Owl (E): Aegolius acadicus II B

Northern Shoveler (E): Anas clypeata III B

Northern Smooth-tailed Treeshrew (E): Dendrogale murina II M

Northern Sportive Lemur (E): Lepilemur septentrionalis I M

Northern Square-lipped Rhinoceros (E): Ceratotherium simum cottoni I M

Northerm Tamandua (E): Tamandua mexicana III M

Northern White Rhinoceros (E): Ceratotherium simum cottoni I M

Northiella haematogaster: Bluebonnet (E), Perico cariazul (S),

Perruche à bonnet bleu (F) II B

North-westem Chameleon (E): Chamaeleo guibei II R

North-western Day Gecko (E): Phelsuma befotakensis II R

Nossi-bé Sportive Lemur (E): Lepilemur dorsalis I M

Norocyathus conicus: II In

Notocyathus venustus: II In

Notophyllia etheridgei: II In

Notophyllia recta: II In

Notophyllia variolaris: II In

Nototrochus $=$ Notocyathus

Novibos sauveli $=$ Bos sauveli
Nubian Bustard (E): Neoris nuba II B

Nubian Vulture (E): Torgos tracheliorus II B

Numenius borealis: Eskimo Curlew (E), Chorlito esquimal (S),

Chorlo polar (S), Zarapito boreal (S), Zarapito esquimal (S),

Zarapito polar (S), Courlis esquimau (F) I B

Numenius tenuirostris: Long-billed Curlew (E), Slender-billed

Curlew (E), Zarapito fino (S), Courlis à bec grêle (F) I B

Nutria africana (S): Aonyx capensis II M

Nutria cenicienta (S): Amblonyx cinereus II M

Nutria común (S): Lutra lutra I M

Nutria de agua (S): Lontra longicaudis I M

Nutria de Canadá (S): Lontra canadensis $11 \mathrm{M}$

Nutria de Chile (S): Lontra provocax I M

Nutria de cuello blanco (S): Aonyx capensis II M

Nutria de cuello manchado (S): Lutra maculicollis II M

Nutria de mar (S): Lonira felina I M

Nutria de Sumatra (S): Lutra sumatrana I/II M

Nutria del Kamichatka (S): Enhydra lutris II M

Nutria inerme asiatica (S): Amblonyx cinereus II M

Nutria inerme de Camerún (S): Aonyx congicus III M

Nutria lisa (S): Lutrogale perspicillata II M

Nutria marina (S): Enhydra lutris I/II M

Nutria norteamericana (S): Lontra canadensis II M

Nutria simung (S): Lutrogale perspicillata II M

Nutrias (S): Lutrinae spp. I/I M

Nyan (E): Ovis ammon hodgsonii I M

Nyasa Lovebird (E): Agapornis lilianae II B

Nyctale de Harris (F): Aegolius harrisii II B

Nyctale de Tengmalm (F): Aegolius funereus II B

Nyctales (F): Aegolius spp. II B

Nyctea scandiaca: Snowy Owl (E), Búho nival (S), Chouette harfang (F), Harfang des neiges (F) II B

Nycricebus coucang: Slow Loris (E), Loris lento (S), Loris lent (F) II $\mathrm{M}$

Nycticebus intermedius $=$ Nycticebus pygmaeus

Nycticebus pygmaeus: Lesser Slow Loris (E), Pygmy Loris (E) II M

Nymphicus hollandicus: Cockatiel (E/S), Lorito de copete (S)

Calopsitte élégante (F), Perruche calopsitte (F) NC B

Nyroca nyroca $=$ Aythya nyroca

Oasis Hummingbird (E): Rhodopis vesper II B

Oaxaca Hummingbird (E): Eupherusa cyanophrys II B

Oaxacan Boa (E): Exiliboa placata II R

Oaxacan Dwarf Boa (E): Exiliboa placata II R

Oca del Nilo (S): Alopochen aegyptiacus III B

Ocellated Day Gecko (E): Phelsuma ocellata II R

Ocellated Mastigure (E): Uromastyx ocellatus II R

Ocellated Pheasant (E): Rheinardia ocellata I B

Ocellated Turkey (E): Agriocharis ocellata III B

Ocelot (E/F): Leopartus pardalis I M

Ocelote (S): Leopardus pardalis I M

Ochre-bellied Hawk-Owl (E): Ninox ochracea II B

Ochre-marked Parakeet (E): Pyrrhura cruentata I B

Ocreatus undenwoodii: Booted Racket-tail (E), Colaespatula zamarrito (S), Colibrí cola de hoja (S), Haut-de-chausses à palettes (F) II B

Oculina banksi: II In

Oculina diffusa: Ivory Bush Coral (E) II In

Oculina patagonica: II In

Oculina profunda: II In

Oculina robusta: II in

Oculina tenella: II In

Oculina valenciennesi: Ivory Tree Coral (E) II In

Oculina varicosa: II In

Oculina virgosa: 11 in

Odobenus rosmarus: Walrus (E), Morse (F) III M

Odocoileus bezoarticus = Ozotoceros bezoaricus

Odocoileus virginianus mayensis: Guatemalan White-tailed Deer (E), Cerf de Virginie SE (F) III M

Odontocyathus coronatus: II in

Odontocyathus sexradiis: II In

Odontocyathus stella: II in

Oedicnème bistrié (F): Burhinus bistriatus III B

Oedicnèmes (F): Burhinus spp. IIVNC B

Oena capensis: Long-tailed Dove (E), Masked Dove (E), Namaqua Dove (E), Palomita especulada capense (S), Tourtelette masquée (F), Tourterelle du Cap (F), Tourterelle à masque de fer (F) III B 
Oenpelli Python (E): Morelia oenpelliensis II R Ogilby's Duiker (E): Cephalophus ogilbyi II M

Ognorhynchus icterotis: Yellow-eared Conure (E), Yellow-eared

Parrot (E), Aratinga orejigualda (S), Periquito orejiamarillo (S),

Conure à oreilles jaunes (F), Perruche aux oreilles jaunes (F) I B

Oie d'Egypte (F): Alopochen aegyptiacus III B

Oie néné (F): Branta sandvicensis I B

Oiseau bruyant des buissons (F): Atrichomis clamosus I B

Oiseaux-mouches (F): Trochilidae spp. I/I B

Old World Otter (E): Lutra lutra I M

Olingo (S/F): Bassaricyon gabbii III M

Olingos (E): Bassaricyon spp. IIUNC M

Olivaceous Thombill (E): Chalcostigma olivaceum II B

Olive (Rock) Python (E): Morelia olivacea II R

Olive Baboon (E): Papio hamadryas II M

Olive Colobus (E): Procolobus verus II M

Olive Keel-back (E): Atretium schistosum III R

Olive Ridley (E): Lepidochelys olivacea I R

Olive-backs (E): Nesocharis spp. III/NC B

Olive-headed Lorikeet (E): Trichoglossus euteles II B

Olive-shouldered Parrot (E): Aprosmictus jonquillaceus II B

Olive-spotted Hummingbird (E): Leucippus chlorocercus II B

Olive-throated Conure (E): Aratinga nana II B

Olive-throated Parakeet (E): Aratinga nana II B

Olivino cariblanco (S): Nesocharis capistrata III B

Omei Shan Liocichla (E): Liocichla omeiensis II B

Onager Ass (E): Equus onager $\mathbf{I} / \mathbf{I I} \mathbf{M}$

Onagre (F): Equus onager I/II M

Onagro (S): Equus onager $\mathbf{I I I} \mathrm{M}$

Once (F): Uncia uncia I M

Oncifelis colocolo: Chilean Pampa Cat (E), Pampas Cat (E), Gato de los pajonales (S), Gato pajero (S), Osio (S), Chat des pampas (F) II M

Oncifelis geoffroyi: Geoffroy's Cat (E), Gato de mato (S), Gato montés común (S), Chat de Geoffroy (F) I M

Oncifelis guigna: Chilean Cat (E), Kodkod (E), Guina (S), Guigna (F) II M

Oncifelis pajeros $=$ Oncifelis colocolo

Oncilla (E): Leopardus tigrinus I M

Oncille (F): Leopardus rigrinus I M

One-homed Chameleon (E): Chamaeleo monoceras II R

Onychogale bridé (F): Onychogalea fraenata $1 \mathrm{M}$

Onychogale croissant ( $\mathrm{F}$ ): Onychogalea lunata I M

Onychogalea fraenata: Bridled Nailtail Wallaby (E), Bridled Wallaby (E), Merrin (E), Canguro rabipelado oriental (S) Onychogale bridé (F) I M

Onychogalea lunata: Crescent Nailtail Wallaby (E). Wurung (E) Canguro rabipelado occidental (S), Onychogale croissant (F), Wallaby à queue comée (F) I M

Onza (S): Herpailurus yaguarondi $\mathbf{L I} \mathrm{M}$

Onza bermeja (S): Puma concolor I/I M

Ophiophagus hannah: Hamadryad (E), King Cobra (E), Cobra royal (F), Hamadryade (F) II R

Opisthoprora euryptera: Mountain Avocetbill (E), Colibrí piquiavoceta (S), Colibri avocettin (F) II B

Opopsinta diophthalma $=$ Cyclopsitta diophthalma

Opopsitta diophthalma coxeni = Cyclopsitra diophthalma coxeni

Opopsitta gulielmitertii = Cyclopsitta gulielmitertii

Ora (E): Varanus komodoensis I R

Orange Bishop (E): Euplectes franciscanus III B

Orange Cock-of-the-rock (E): Rupicola rupicola II B

Orange Toad (E): Bufo periglenes I A

Orange Tube Coral (E): Tubastraea coccinea II In

Orange Weaver (E): Euplectes franciscanus 111 B/ Ploceus aurantius III B

Orange-and-black Poison Frog (E): Phyllobates vitiatus II A

Orange-bellied Parakeet (E): Neophema chrysogaster I B

Orange-bellied Parrot (E): Neophema chrysogaster I B

Orange-billed Lorikeet (E); Neopsinacus pullicauda II B

Orange-breasted Falcon (E): Falco deiroleucus II B

Orange-breasted Fig-Parrot (E): Cyclopsitta gulielmitertii II B

Orange-breasted Waxbill (E): Amandava subflava III B

Orange-cheeked Parrot (E): Pionopsirra barrabandi II B

Orange-cheeked Waxbill (E): Estrilda melpoda III B

Orange-chinned Parakeet (E): Brotogeris jugularis II B

Orangefoot Pimpleback (E): Plethobasus cooperianus I in
Orange-footed Pimpleback Mussel (E): Pleshobasus cooperianus I In Orange-fronted Conure (E): Aratinga canicularis II B

Orange-fronted Hanging-Parrot (E): Loriculus aurantiifrons II B

Orange-fronted Parakeet (E): Aratinga canicularis II B

Orange-knee Tarantula (E): Brachypelma emilia II in

Orange-throated Race-runner (E): Cnemidophorus hyperythrus II R

Orange-throated Sunangel (E): Heliangelus mavors II B

Orange-throated Whiptail (E): Cnemidophorus hyperythrus II R

Orange-winged Amazon (E): Amazona amazonica II B

Orange-winged Parrot (E): Amazona amazonica II B

Orang-outan (F): Pongo pygmaeus I M

Orang-utan (E): Pongo pygmaeus I M

Orangután (S): Ponga pygmaeus I M

Orbicella $=$ Montasirea

Orca (E/S): Orcinus orca II M

Orca falsa (S): Pseudorca crassidens II M

Orca pigmeo (S): Feresa attenuata II M

Orcaella brevirostris: Irawaddy Dolphin (E), Snubfin Dolphin (E), Delfin del Irrawaddy (S), Orcelle (F) II M

Orcelle (F): Orcaella brevirostris II M

Orcinus glacialis = Orcinus orca

Orcinus orca: Killer Whale (E), Orca (E), Espadarte (S), Orca (S), Epaulard (F), Orque (F) II M

Oreailurus jacobita: Andean Cat (E), Mountain Cat (E), Chinchay (S), Gato andino (S), Gato lince (S), Osjo (S), Chat des Andes (F) I M

Orejavioleta café (S): Colibri delphinae II B

Orejivioleta marrón (S): Colibri delphinae II B

Oreonympha nobilis: Bearded Mountaineer (E), Colibri noble (F) II B

Oréophase comu (F): Oreophasis derbianus I B

Oreophasis derbianus: Homed Guan (E), Guan comudo (S), Pauji cornudo (S), Pava pajuil (S), Pavón (S), Pavón comudo (S), Hocco huppé (F), Oréophase cornu (F), Pénélope comue (F), Pénélope de Derby $(F)$ I B

Oreopsillacus arfaki: Plum-faced Lorikeet (E), Whiskered Lorikeet (E), Lori bigotudo (S), Lori bridé (F), Lori des montagnes (F) II B

Oreotrochilus adela: Wedge-tailed Hillstar (E), Colibri adèle (F) II B

Oreotrochilus chimborazo: Ecuadorian Hillstar (E), Estrella ecuatoriana (S), Colibri du Chimborazo (F) II B

Oreotrochilus estella: Andean Hillstar (E), Picaflor serrano ventricanela (S), Colibri estelle (F) II B

Oreotrochilus estella chimborazo = Oreotrochilus chimborazo

Oreotrochilus leucopleurus: White-sided Hillstar (E), Picaflor serrano ventrinegro (S), Colibri à flancs blancs (F) II B

Oreotrochilus melanogaster: Black-breasted Hillstar (E), Colibri à plastron noir (F) II B

Organ-pipe Coral (E): Tubipora musica II In

Oricou (F): Torgos tracheliotus II B

Oriental Bay-Owl (E): Phodilus badius II B

Oriental Hobby (E): Falco severus II B

Oriental Honey-buzzard (E): Pernis ptilorhyncus II B

Oriental Pied-Hombill (E): Anthracoceros albirostris II B

Oriental Rat Snake (E): Pryas mucosus II R

Oriental Scops-Owl (E): Otus scops II B

Oriental Small-clawed Otter (E): Amblonyx cinereus II M

Oriental Stork (E): Ciconia boyciana I B

Oriental White Stork (E): Ciconia boyciana I B

Oriental White-backed Vulture (E): Gyps bengalensis II B

Orinoco Crocodile (E): Crocodylus intermedius I R

Orix de Arabia (S): Oryx leucoryx I M

Orix de Cimitara (S): Oryx dammah I M

Ormala (E): Lagorchestes hirsutus I M

Ornate Box Turle (E): Terrapene omara II R

Ornate Day Gecko (E): Phelsuma omata II R

Omate Hawk-Eagle (E): Spizaetus ornatus II B

Ornate Lorikeet (E): Trichoglossus ornatus II B

Ornate Lory (E): Trichoglossus omatus II B

Omate Umbrellabird (E): Cephalopterus ornatus III B

Omithoptera spp.: Birdwing butterflies (E), Omithoptères (F) III In

Ornithoptera aesacus: II In

Omithoptera akakeae: II In

Omithoptera alexandrae: Queen Alexandra's Birdwing (E), Omithoptère de la reine Alexandra (F) I In 
Omithoptera allonei: Abbé Allotte's Birdwing (E), Omithoptère de l'abbé Allotte (F) II In

Omithoptera caelestis: II In

Ornithoptera chimaera: Chimaera Birdwing $(\mathrm{E})$, La chimère (F), Omithoptère chimère (F) II In

Omithoptera croesus: II In

Ornithoptera goliath: Goliath Birdwing (E), Omithoptère goliath (F) II In

Ornithoptera meridionalis: Omithoptère méridional (F) II In Ornithoptera paradisea: Butterfly of Paradise (E), Paradise Birdwing (E), Tailed Birdwing (E), Omithoptere de paradis (F) II In Omithoptera priamus: Common Birdwing (E), Common Green Birdwing (E), New Guinea Birdwing (E), Priam's Birdwing (E) II In

Ornithoptera richmondia: Richmond Birdwing (E) II In

Ornithoptera rothschildi: Rothschild's Birdwing (E) II In Omithoptera tithonus: II In

Omithoptera urvillianus: D'Urville's Birdwing (E) II in

Omithoptera victoriae: Queen Victoria's Birdwing (E), Omithoptère de la reine Victoria (F) II in

Omithoptère chimère (F): Ornithoptera chimaera II In

Omithoptère d'obi $(\mathrm{F})$ : Troides aeacus $\mathbf{\amalg}$ in

Omithopterre de Brooke (F): Trogonoptera brookiana II In

Omithoptère de la reine Alexandra (F): Omithoptera alexandrae I In

Omithoptère de la reine Victoria (F): Omishoptera victoriae II In

Omithoptère de l'abbé Allotte (F): Ornithoptera allottei II ln

Omithoptère de paradis (F): Ornithoptera paradisea $\mathbf{I I}$ ln

Omithoptere goliath (F): Omithoptera goliath 11 in

Omithoptère meridional (F): Omithoptera meridionalis II In

Omithoptères (F): Omithoptera spp. I/II In/Trogonoptera spp. II In/Troides spp. II In

Oroaetus isidori: Black-and-chestnut Eagle (E), Isidor's Eagle (E), Aguila castaña (S), Aguila de Copete (S), Aguila poma (S), Aigle d'Isidore (F) II B

Orong (E): Pantholops hodgsonii $1 \mathrm{M}$

Orque (F): Orcinus orca II M

Orque pygmée (F): Feresa attenuata II M

Orsini's Viper (E): Vipera ursinii I/NC R

Ortalide chacamel $(\mathrm{F})$ : Ortalis vetula III B

Ortalide du Mexique (F): Ortalis vefula III B

Ortalides (F): Ortalis spp. IIL/NC B

Ortalis vetula: Plain Chachalaca (E), Chachalaca norteña (S).

Ortalide chacamel (F), Ortalide du Mexique (F) III B

Orthonymus metanevrus tuberosus = Quadrula sparsa

Orthorhynchus cristatus: Antillean Crested Hummingbird (E), Colibri huppé (F) II B

Ortygospiza atricollis: African Quailfinch (E), Astrilda aperdizada (S), Astrild-caille à lunettes (F) III B

Oryx algazelle (F): Oryx dammah I M

Oryx blanc (F): Oryx leuconyx I M

Oryx dammah: Sahara Oryx (E), Scimitar-homed Oryx (E), Orix de Cimitarra (S), Oryx algazelle (F), Oryx de Libye (F) I M

Oryx d'Arabie (F): Oryx leucoryx I M

Oryx de Libye (F): Oryx dammah I M

Oryx leucoryx: Arabian Oryx (E), White Oryx (E), Orix de Arabia (S), Oryx blanc (F), Oryx d'Arabie (F) I M

Oryx tao = Oryx dammah

Oryxes (E): Oryx spp. INC M

Oryzotrochus stephensoni: II In

Osgood's Ethiopian Toad (E): Spinophrynoides osgoodi I A

O'Shaughnessy's Chameleon (E): Chamaeleo oshaughnessyi II R

Osio (S): Oncifelis colocolo II M

Osjo (S): Oreailurus jacobita I M

Oso caballo (S): Myrmecophaga tridactyla II M

Oso de anteojos (S): Tremarcios omatus I M

Oso de collar (S): Ursus thibetanus I M

Oso de sol (S): Helarctos malayanus I M

Oso frontino (S): Tremarcios omatus I M

Oso hormiguero (S): Myrmecophaga tridactyla II M

Oso malayo (S): Helarctos malayanus I M

Oso marino austral (S): Arctocephalus australis II M

Oso marino de Chile (S): Arctocephalus philippii II M

Oso marino de Guadalupe (S): Arctocephalus townsendi I M

Oso marino de las Galápagos (S): Arctocephalus galapagoensis II M

Oso marino de Neuva Zelanda (S): Arctocephalus forsteri II M

Oso marsupial del Rio Moonie (S): Lasiorhinus krefftii I M
Oso negro americano (S): Ursus americanus II M

Oso negro de Asia (S): Ursus thibetanus I M

Oso pardo (S): Ursus arclos I/II M

Oso pardo del Mimalaya (S): Ursus arcios isabellinus I M

Oso perezoso (S): Melursus ursinus I M

Oso polar (S): Ursus maritimus $11 \mathrm{M}$

Oso real (S): Tremarctos omalus I M

Osos (S): Ursidae spp. MI M

Osos marinos (S): Arctocephalus spp. II M

Osprey (E): Pandion haliaetus II B

Osteolaemus tetraspis: African Dwarf Crocodile (E), West African Dwarf Crocodile (E), Cocodrilo chico africano (S), Crocodile nain africain $(\mathrm{F})$, Crocodile à front large $(\mathrm{F})$, Crocodile à nuque cuirassée (F) I R

Ostrich (E): Strushio camelus $\mathbf{I N C}$ B

Otarie à fourrure australe (F): Arctocephalus australis II M

Otarie à fourrure d'Amérique (F): Arciocephalus townsendi I M

Otaries à fourrure du Sud (F): Arctocephalus spp. II M

Otididae spp.: Bustards (E), Avutardas (S), Otídidos (S), Sisones (S), Outardes (F) I/I B

Otídidos (S): Otididae spp. I/II B

Oris tarda: Great Bustard (E), Avutarda (S), Avutarda euroasiática (S), Grande outarde (F), Outarde barbue (F) II B

Otis tetrax = Tetrax tetra

Otocolobus manul: Pallas's Cat (E), Gato de Pallas (S), Gato manul (S), Chat manul (F) II M

Otolemur crassicaudatus: Greater bushbaby (E), Large-Eared Greater Galago (E), Thick-tailed Bushbaby (E), Gálago de cola gruesa (S), Galago à queue touffue (F), Grand galago (F) II M

Otolemur crassicaudatus gamettii = Otolemur gamettii

Otolemur gamettii: Small-eared Greater Galago (E) II M

Otorongo (S): Panthera onca I M

Otrer-civet (E): Cynogale bennettii II M

Otters (E): Lutrinae spp. VII M

Otus albogularis: White-throated Screech-Owl (E), Curucucú gargantiblanco (S), Petit-duc à gorge blanche (F) II B

Otus alfredi $=$ Otus magicus

Otus angelinae: Javan Scops-Owl (E), Petit-duc de Java (F) II B

Otus asio: Eastem Screech-Owl (E), Tecolote oriental (S), Petit-duc maculé (F) II B

Otus asio kennicottii = Otus kennicottii

Orus atricapillus: Black-capped Screech-Owl (E), Variable ScreechOwl (E), Curucucú vermiculado (S), Lechucita rojiza (S), Petitduc à mèches noires $(\mathrm{F})$ II $\mathrm{B}$

Olus bakkamoena: Collared Scops-Owl (E), Petit-duc à collier (F) II B

Otus bakkamoena fuliginosus = Otus fuliginosu

Otus bakkamoena megalotis = Otus megalotis

Otus bakkamoena mentawi = Orus mentawi

Otus balli: Andaman Scops-Owl (E), Petit-duc des Andaman (F) II B

Otus barbarus: Santa Barbara Screech-Owl (E), Tecolote barbudc

(S), Petit-duc bridé (F) II B

Otus beccarii = Otus magicus

Otus brookii: Rajah Scops-Owl (E), Petit-duc radjah (F) II B

Otus brucei: Pallid Scops-Owl (E), Striated Scops-Owl (E), Petit-duc de Bruce (F) II B

Otus capnodes = Olus rutilus

Otus choliba: Tropical Screech-Owl (E), Curucucú común (S), Lechucita común (S), Petit-duc choliba (F) II B

Otus clarkii: Bare-shanked Screech-Owl (E), Buhito en Pernetas (S), Petit-duc de Clark (F) II B

Otus colombianus $=$ Otus ingens

Ous cooperi = Otus kennicottii

Otus elegans: Elegant Scops-Owl (E), Petit-duc élégant (F) II B

Otus enganensis $=$ Orus magicus

Otus flammeolus: Flammulated Owl (E), Tecolote flameado (S), Petit-duc nain (F) II B

Otus fuliginosus: Palawan Scops-Owl (E), Petit-duc de Palawan (F) II B

Otus guatemalae $=$ Otus atricapillus

Otus guatemalae vermiculatus $=$ Otus vermiculatus

Orus gurneyi $=$ Mimizuku gurneyi

Otus hartlaubi: Sao Tome Scops-Owl (E), Petit-duc de Sao Tomé (F) II B

Otus hoyi $=$ Otus atricapillus 
Otus icterorhynchus: Sandy Scops-Owl (E), Petit-duc à bec jaune (F) II B

Otus ingens: Colombian Screech-Owl (E), Rufescent Screech-Owl (E), Cuncucú pálido (S), Petit-duc de Salvin (F) II B

Orus insularis $=$ Orus magicus

Otus ireneae: Morden's Scops-Owl (E), Sokoke Scops-Owl (E), Petit-duc d'Irène (F) II B

Onus kenniconti: Western Screech-Owl (E), Tecolote occidental (S), Petit-duc des montagnes (F) II B

Otus koepckeae: Koepcke's Screech-Owl (E), Petit-duc de Koepcke (F) II B

Otus lawrencii: Bare-legged Owl (E), Cotunto (S), Cuco (S), Siju cotunto (S), Chovette de Cuba (F). Petit-duc de Cuba (F) II B

Otus lempiji = Otus bakkamoena

Otus leucotis: White-faced Scops-Owl (E), Gran autillo veliblanco (S), Petit-duc à face blanche (F) II B

Otus longicomis: Luzon Scops-Owl (E), Petit-duc longicome (F) II B

Otus magicus: Moluccan Scops-Owl (E), Papuan Scops-Owl (E), Petit-duc mystérieux (F) II B

Otus manadensis: Celebes Scops-Owl (E), Sulawesi Scops-Owl (E), Petit-duc Manado (F) II B

Orus manadensis magicus = Otus magicus

Otus mantananensis: Mantanani Scops-Owl (E), Petit-duc de Mantanani (F) II B

Otus marshalli: Cloud-forest Screech-Owl (E), Petil-duc de Marshall (F) II B

Otus megalotis: Philippine Scops-Owl (E), Petit-duc de Luçon (F) II B

Otus mentawi: Mentawai Scops-Owl (E), Petit-duc des Mentawi (F) II B

Otus mindorensis: Mindoro Scops-Owl (E), Petit-duc de Mindoro (F) II B

Otus mirus: Mindanao Scops-Owl (E), Petit-duc de Mindanao (F) II B

Otus nudipes: Puerto Rican Screech-Owl (E), Petit-duc de Porto Rico (F) II B

Otus pauliani: Grand Comoro Scops-Owl (E), II B

Otus pembaensis $=$ Otus rusilus

Otus petersoni $=$ Otus marshalli

Otus podarginus: Palau Owl (E), Petit-duc des Palau (F) II B

Otus roboratus: West Peruvian Screech-Owl (E), Autillo roborado (S), Petit-duc du Pérou (F) II B

Ous rufescens: Reddish Scops-Owl (E), Petit-duc roussâtre (F) II B

Otus rutilus: Madagascar Scops-Owl (E), Pemba Scops-Owl (E),

Petit-duc malgache (F) II B

Otus rutilus pauliani $=$ Orus pauliani

Otus sagittatus: White-fronted Scops-Owl (E), Petit-duc à front blanc (F) II B

Otus sanctaecatarinae: Long-tufted Screech-Owl (E), Petit-duc à aigrettes longues ( $\mathrm{F}$ ) II B

Otus scops: Common Scops-Owl (E), Eurasian Scops-Owl (E),

Oriental Scops-Owl (E), Autillo (S), Autillo europeo (S), Hibou petit-duc (F), Petit-duc africain (F), Petit-duc scops (F) II B

Orus scops elegans = Orus elegans

Orus scops longicomis = Orus longicomis

Otus scops mantananensis = Otus mantananensis

Otus scops mindorensis = Otus mindorensis

Otus scops minus $=$ Otus mirus

Otus seductus = Otus kennicotti

Orus senegalensis = Otus scops

Otus silvicola: Lesser Sunda Scops-Owl (E), Wallace's Scops-Owl (E), Petit-duc Wallace (F) II B

Otus spilocephalus: Mountain Scops-Owl (E), Spotted Scops-Owl (E), Petit-duc tacheté (F) II B

Otus spilocephalus angelinae $=$ Otus angelinae

Orus sunia $=$ Otus scops

Otus trichopsis: Whiskered Screech-Owl (E), Tecolote bigotudo (S), Petit-duc à moustaches (F) II B

Otus umbra: Mentaur Scops-Owl (E), Simeulue Scops-Owl (E), Petit-duc de Simalur (F) II B

Otus usta $=$ Otus watsonii

Orus vermicularus: Vermiculated Screech-Owl (E), Petit-duc vermiculé (F) II B

Otus watsonii: Tawny-bellied Screech-Owl (E), Curucucú orejudo (S), Petit-duc de Watson (F) II B
Ouakari à tête noire (F): Cacajao melanocephalus I M Ouakari chauve (F): Cacajao calvus I M

Ouakaris (F): Cacajao spp. I M

Ouandérou ( $\mathbf{F}$ ): Macaca silenus I M

Ouette d'Egypte (F): Alopochen aegyptiacus III B

Ouistiti (F): Callithrix jacchus II M

Ouistiti à tête jaune (F): Callithrix flaviceps I M

Ouistiti argenté (F): Callishrix argentata II M

Ouistiti mignon (F): Callithrix pygmaea II M

Oulangia bradleyi: II ln

Oulangia stokesiana: II In

Oulastrea crispata: 11 in

Oulophyllia bennertae: II in

Oulophyllia crispa: II In

Ounce (E): Uncia uncia I M

Ours (F): Ursidac spp. I/I M

Ours à collier (F): Ursus thibetanus I M

Ours à lunettes (F): Tremarctos omatus I M

Ours andin (F): Tremarctos omatus I M

Ours blanc (F): Ursus maritimus II M

Ours brun (F): Ursus arctos $\mathbf{V} / \mathrm{I} \mathrm{M}$

Ours brun de l'Himalaya (F): Ursus arctos isabellinus I M

Ours brun du Tibet $(\mathrm{F})$ : Ursus arctos pruinosus I M

Ours brun isabelle ( $F$ ): Ursus arctos isabellinus I M

Ours de l'Himalaya (F): Ursus thibetanus I M

Ours des cocotiers (F): Helarctos malayanus I M

Ours du Tibet (F): Ursus thibetanus I M

Ours grizzly (F): Ursus arctos I/II M

Ours isabelle (F): Ursus arctos isabellinus I M

Ours lippu de l'Inde (F): Melursus ursinus I M

Ours malais ( $\mathrm{F}$ ): Helarctos malayanus $1 \mathrm{M}$

Ours noir américain (F): Ursus americanus II M

Ours noir d'Asie (F): Ursus thibetanus I M

Ours polaire (F): Ursus maritimus II M

Ours prochile lippu (F): Melursus ursinus I M

Oustalet's Giant Chameleon (E): Chamaeleo oustaleti II R

Oustalet's Grey Duck (E): Anas oustaleti I B

Oustiti à oreilles blanches (F): Callithrix aurita I M

Oustiti oreillard (F): Callithrix aurita I M

Outarde à miroir blanc $(F)$ : Eupodotis afraoides II B

Outarde à tête noire (F): Ardeotis nigriceps I B

Outarde à ventre noir $(\mathrm{F})$ : Eupodotis melanogaster II B

Outarde arabe (F): Ardeotis arabs II B

Outarde australienne (F): Ardeotis australis II B

Outarde barbue (F): Oris tarda II B

Outarde canepetière (F): Tetrax tetrax II B

Outarde d'Australie (F): Ardeoris australis II B

Outarde de Burchell (F): Neotis denhami II B

Outarde de Denham (F): Neotis denhami II B

Outarde de Hartlaub (F): Eupodotis hartlaubii II B

Outarde de Heuglin (F): Neotis heuglinii II B

Outarde de l'Inde (F): Ardeotis nigriceps I B

Outarde de l'Inde (F): Eupodoris bengalensis I B

Outarde de Ludwig (F): Neotis ludwigii II B

Outarde de Nubie (F): Neoris nuba II B

Outarde de Rüppell (F): Eupodotis rueppellii II B

Outarde de Savile (F): Eupodoris savilei II B

Outarde de Vigors (F): Eupodotis vigorsii II B

Outarde d'Oustalet (F): Eupadotis gindiana II B

Outarde du Bengale (F): Eupodotis bengalensis I B

Outarde du Sénégal (F): Eupodotis senegalensis II B

Outarde houbara (F): Chlamydotis undulata I B

Outarde houppette (F): Eupodotis ruficrista U B

Outarde korhaan (F): Eupodotis afra II B

Outarde kori (F): Ardeotis kori II B

Outarde naine (F): Eupodotis nuficrista II B

Outarde naine de l'Inde (F): Eupodotis indica II B

Outarde noire (F): Eupodotis afra II B

Outarde nubienne (F): Neoris nuba II B

Outarde passarage (F): Eupodotis indica II B

Outarde plombée (F): Eupodotis caerulescens II B

Outarde somalienne (F): Eupodotis humilis II B

Outardes (F): Otididae spp. VI B

Ovampo Sparrowhawk (E): Accipirer ovampensis II B 
Ovis ammon: Argali (E), Asian Wild Sheep (E), Marco Polo Sheep (E), Muflón Argal (S), Muflón de Marco Polo (S), Argali (F), Mouflon d'Asie (F), Mouflon d'Eurasie (F), Mouflon vrai (F) I/I M

Ovis ammon hodgsonii: Great Tibetan sheep (E), Nyan (E), Tibetan Argali (E), Muflón Argal Tibetano (S), Mouflon de l'Himalaya (F), Mouflon des montagnes (F) I M

Ovis ammon musimon pro parte $=$ Ovis orientalis ophion

Ovis ammon nigrimontana: Kara Tau Argali (E) I M

Ovis ammon vignei $=O v i$ vignei vignei

Ovis aries ophion $=$ Ovis orientalis ophion

Ovis canadensis: Bighom Sheep (E), Mountain Sheep (E), Borrego cimarnón (S), Camero del Canadá (S), Camero salvaje (S) Mouflon d'Amérique (F), Mouflon du Canada (F), Mouflon pachycère (F) U/I M

Ovis orientalis ophion: Cyprian Wild Sheep (E). Cyprus Mouflon (E), Muflón de Chipre (S), Mouflon de Chypre (F) I M

Ovis orientalis vignei $=$ Ovis vignei vignei

Ovis vignei vignei: Red Sheep (E), Shapo (E), Shapu (E), Urial (E), Urial (S), Mouflon du Ladak (F), Urial (F) I M

Owen's Three-homed Chameleon (E): Chamaeleo oweni II R

Owl Monkey (E): Aotus irivirgatus II M

Owl Partot (E): Strigops habroptilus I B

Owlets (E): Strigidae spp. II B

Owl-faced Guenon (E): Cercopithecus hamlyni II M

Owl-faced Monkey (E): Cercopithecus hamlyni II M

Owls: STRIGIFORMES spp. I/II B

Oxyphyllia = Echinophyllia

Oxypogon guerinii: Bearded Helmetcrest (E), Chivito de los Páramos

(S), Colibri casqué (F) II B

Oxypora crassispinosa: II In

Oxypora glabra: II In

Oxypora lacera: II In

Oxypora titizimaensis: II In

Oxysmilia portoricensis: II In

Oxysmilia rotundifolia: II in

Oxyura leucocephala: White-headed Duck (E), Malvasía (S), Érismature à tête blanche (F) II B

Ozotoceros bezoarticus: Pampas Deer (E), Ciervo de las Pampas (S) Ciervo pampero (S), Venado campero (S), Venado de campo (S), Venado de las Pampas (S), Cerf des pampas (F), Goazu (F) I $\mathrm{M}$

Paca (S): Agouti paca III M

Pachyphantes superciliosus: Compact Weaver (E), Tejedor rollizo (S), Tisserin gros-bec (F) III B

Pachypsammia $=$ Coenopsammia

Pachyseris carinata: II In

Pachyseris foliosa: II In

Pachyseris gemmae: II In

Pachyseris rugosa: II In

Pachyseris speciosa: II In

Pacific Baza (E): Aviceda subcristata II B

Pacific Boa (E): Candoia bibroni II R

Pacific Dolphin (E): Delphinus delphis II M

Pacific Monitor (E): Varanus indicus II R

Pacific Parakeet (E): Aratinga strenua II B

Pacific Parrotlet (E): Forpus coelestis II B

Pacific Pilot Whale (E): Globicephala macrorhynchus II M

Pacific Ridley (E): Lepidochelys olivacea I R

Pacific White-sided Dolphin (E): Lagenorhynchus obliquidens II M

Padda de Java (F): Padda oryzivora II B

Padda oryzivora: Java Sparrow (E), Padda de Java (F) II B

Paddas (F): Padda spp. II/NC B

Paddlefish (E): Polyodon spathula II F

Pagai Island Langur (E): Nasalis concolor I M

Paguma larvata: Masked Palm Civet (E), Civeta de palmera enmascarada (S) III M

Paiche (S/F): Arapaima gigas II F

Painted Batagur (E): Callagur bomeoensis II R

Painted Conure (E): Pyrrhura picta II B

Painted Parakeet (E): Pyrrhura picta II B

Painted Terrapin (E): Callagur bomeoensis II R

Painted Tiger-Partot (E): Psittacella picta II B

Painted Treeshrew (E): Tupaia picta II M

Pájaro-paraguas amazónico (S): Cephalopterus omatus III B

Pájaro-paraguas longuipendulo (S): Cephalopterus penduliger III B
Pajuil (S): Penelopina nigra III B

Palceosuchus palpebrosus: Cuvier's Smooth-fronted Caiman (E), Dwarf Caiman (E), Caimán enano (S), Caìman à paupières osseuses (F) II R

Palaeosuchus trigonatus: Schneider's Smooth-fronted Caiman (E), Caimán frentiplano (S), Caïman hérissé (F) II R

Palau Fruit Bat (E): Pteropus pilosus I M

Palau Owl (E): Otus podarginus II B

Palauastrea ramosa: II in

Palauphyllia $=$ Lobophyllia

Palawan Hombill (E): Anthracoceros marchei II B

Palawan Peacock-Pheasant (E): Polyplectron emphanum I B

Palawan Racket-tailed Parrot (E): Prioniturus discurus II B

Palawan Scops-Owl (E): Otus fuliginosus II B

Palawan Treeshrew (E): Tupaia palawanensis II M

Pale Chanting-Goshawk (E): Melierax canorus II B

Pale Harrier (E): Circus macrourus II B

Pale Lilliput Naiad (E): Toxolasma cylindrellus I in

Pale Lilliput Pearly Mussel (E): Toxolasma cylindrellus I In

Pale-bellied Hermit (E): Phaethomis anthophilus II B

Pale-billed Sicklebill (E): Epimachus L ruijnii II B

Pale-fronted Negrofinch (E): Nigrila luteifrons III B

Pale-headed Rosella (E): Platycercus adscitus II B

Pale-headed Saki (E): Pithecia pithecia II M

Pale-tailed Barbthroat (E): Threnetes leucurus II B

Palette à couronne bleue (F): Prioniturus discunus II B

Palette à manteau d'or (F): Prioniturus platurus II B

Palette de Buru (F): Prioniturus mada II B

Palette de Cassin (F): Prioniturus flavicans II B

Palette de Mindanao (F): Prioniturus waterstradi II B

Palette de Palawan (F): Prioniturus platenae II B

Palette des Sulu (F): Prioniturus verticalis II B

Palette momot (F): Prioniturus montanus II B

Palette verte (F): Prioniturus luconensis II B

Palettes (F): Prioniturus spp. II B

Pale-winged Indigobird (E): Vidua wilsoni III B

Pallas's Cat (E): Osocolobus manul II M

Pallas's Fish-Eagle (E): Haliaeetus leuconyphus II B

Pallas's Sea-Eagle (E): Haliaeerus leucoryphus II B

Pallid Harrier (E): Circus macrourus II B

Pallid Scops-Owl (E): Otus brucei II B

Pallid Sturgeon (E): Scaphirhynchus albus II F

Palm civets (E): Viverridae spp. II/L/NC M

Palm Cockatoo (E): Probosciger aterrimus I B

Palm Dove (E): Streptopelia senegalensis III B

Palm Lorikeet (E): Charmosyna palmarum II B

Palm squirrels (E): Epixerus spp/Funambulus spp. III/NC M

Palmiste africain (F): Gypohierax angolensis II B

Palm-nut Vulture (E): Gypohierax angolensis II B

Paloma afep (S): Columba unicincia III B

Paloma apuñalada de Luzón (S): Gallicolumba luzonica II B

Paloma bravia (S): Columba livia III B

Paloma crestada azul (S): Goura cristata II B

Paloma crestada de Scheepmaker (S): Goura scheepmakeri II B

Paloma crestada victoria (S): Goura victoria II B

Paloma de Guinea (S): Columba guinea III B

Paloma de Mauricio (S): Columba mayeri III B

Paloma de Mindoro (S): Ducula mindorensis I B

Paloma nicobar (S): Caloenas nicobarica I B

Palomas crestadas (S): Goura spp. II B

Palomita especulada abisinica (S): Turtur abyssinicus III B

Palomita especulada africana (S): Turtur afer III B

Palomita especulada capense (S): Oena capensis III B

Palomita especulada cobriza (S): Turtur brehmeri III B

Pampa Fox (E): Pseudalopex griseus II M/ Pseudalopex gymnocercus II M

Pampas Cat (E): Oncifelis colocolo II M

Pampas Deer (E): Ozotoceros bezoarticus I M

Pan spp:: Chimpanzees (E), Chimpancés (S), Chimpanzés (F) I M

Pan paniscus: Bonobo (E), Dwarf Chimpanzee (E), Pygmy

Chimpanzee (E), Chimpancé pigmeo (S), Bonobo (F).

Chimpanzé nain (F), Chimpanzé pygmée (F) I M

Pan troglodyzes: Chimpanzee (E), Chimpancé (S), Chimpanzé (F) I M

Panama Spider Monkey (E): Aleles geoffroyi panamensis I M

Paramanian Dwarf Boa (E): Ungaliophis panamensis II R 
Panay Giant Fruit Bat (E): Acerodon lucifer I M

Pancake Tonoise (E): Malacochersus tornieri 11 R

Panda (F): Ailuropoda melanoleuca I M

Panda chico (S): Ailurus fulgens I M

Panda éclatant (F): Ailurus fulgens I M

Panda géant (F): Ailuropoda melanoleuca I M

Panda gigante (S): Ailuropoda melanoleuca I M

Panda rojo (S): Ailurus fulgens I M

Pandas (E): Ailuropoda spp/Ailurus spp. I M

Pandinus africanus $=$ Pandinus imperator

Pandinus dictator: Escorpión magnifico (S) II In

Pandinus gambiensis: Escorpión de Gambia (S), Grand scorpian du Sénégal (F) II In

Pandinus imperator: Emperor Scorpion (E), Escorpión emperador (S), Escorpión gigante (S) II In

Pandion haliaetus: Osprey (E), Aguila pescadora (S), Aguila sangual (S), Gavilán pescador (S), Guincho (S), Aigle pêcheur (F), Balbuzard pêcheur (F) II B

Pangasianodon gigas: Giant Catfish (E), Siluro gigante (S), Silure de verre géant (F) I $F$

Pangolin a écailles tricuspides (F): Manis tricuspis II M

Pangolin à grosse queue (F): Manis crassicaudata II M

Pangolin a longue queue (F): Manis terradactyla II M

Pangolin à queue courte (F): Manis pentadacryla II M

Pangolín chino ( $\mathrm{S}$ ): Manis pentadactyla II M

Pangolin commun (F): Manis ericuspis $11 \mathrm{M}$

Pangolin de Chine (F): Manis pentadactyla II M

Pangolín de cola larga (S): Manis tetradactyla II M

Pangolin de Temminck (F): Manis temminckii II M

Pangolín del Cabo (S): Manis temminckii II M

Pangolin géant (F): Manis gigantea II M

Pangolín gigante (S): Manis gigantea II M

Pangolin indio (S): Manis crassicaudata II M

Pangolin javanais $(F)$ : Manis javanica II M

Pangolin malais (F): Manis javanica II M

Pangolín malayo (S): Manis javanica II M

Pangolin terrestre du Cap (F): Manis temminckii II M

Pangolin tétradactyle (F): Manis tetradacnyla II M

Pangolínos (S): Manis spp. II M

Pangolins (E/F): Manis spp. II M

Pantera (S): Panthera pardus I M

Pantera de la nieves (S): Uncia uncia I M

Pantera del Himalaya (S): Neofelis nebulosa I M

Pantera longibanda (S): Neofelis nebulosa I M

Pantera nebulosa (S): Neofelis nebulosa I M

Panterpe insignis: Fiery-throated Hummingbird (E), Colibri insigne (F) II B

Panther Chameleon (E): Chamaeleo pardalis II R

Panthera leo: Lion (E), León (S), Lion d'Afrique (F) I/II M

Panthera leo persica: Asiatic Lion (E), Indian Lion (E), León indostánico (S), Lion d'Asie (F) I M

Panthera onca: Jaguar (E), Jaguar (S), Otorongo (S), Tigre americano (S), Tigre real (S), Yaguar (S), Yaguarete (S), Jaguar (F) I M

Panthera pardus: Leopard (E), Leopardo (S), Pantera (S), LEopard (F), Panthère (F) I M

Panthera rigris: Tiger (E), Tigre (S), Tigre (F) I M

Panthera uncia $=$ Uncia uncia

Panthère (F): Panthera pardus I M

Panthère des neiges (F): Uncia uncia I M

Panthère longibande ( $\mathrm{F}$ ): Neofelis nebulosa I M

Panthère nébuleuse (F): Neofelis nebulosa I M

Panthers (E): Panthera pardus/Puma concolor I/I M

Pantholops hodgsonit: Chiru (E), Orong (E), Tibetan Antelope (E), Antilope del Tibet (S), Antilope du Tibet (F). Tchirou (F) I M

Pantropical Spotted Dolphin (E): Sfenella attenuara II M

Paon spicifere (F): Pava muticus II B

Paon vert (F): Pavo muticus II B

Paons (F): Pavo spp. II/NC B

Papagayo alirrojo (S): Aprosmictus erythropterus II B

Papagayo australiano (S): Alisterus scapuloris II B

Papagayo de cara roja (S): Amazona brasiliensis I B

Papagayo de Gulielm (S): Poicephalus gulielmi II B

Papagayo de Timor (S): Aprosmictus jonquillaceus II B

Papagayo enmascarado (S): Prosopeia personata II B

Papagayo escarlata (S): Prosopeia splendens II B
Papagayo granate (S): Prosopeia tabuensis II B

Papagayo Moluqueño (S): Alisterus amboinensis II B

Papagayo papú (S): Alisterus chloropierus II B

Papagayo robusto (S): Poicephalus robustus II B

Papagayo senegalés (S): Poicephalus senegalus II B

Papagayos (S): Alisterus spp/Aprosmictus spp/Prosopeia spp. II B

Papasula abbotti: Abbott's Booby (E), Alcatraz de Abbott (S).

Piquero de Abbott (S), Fou d'Abbott (F) I B

Papegeai maille (F): Deroptyus accipitrinus II B

Paphosia adorabilis = Lophomis adorabilis

Paphosia helenae $=$ Lophorn is helenae

Papilio chikae: Luzon Peacock Swallowtail (E) I In

Papilio homerus: Homerus Swallowtail (E) I In

Papilio hospiton: Corsican Swallowtail (E) I In

Papio anubis = Papio hamadryas anubis

Papio cyanocephalus = Papio hamadryas cynocephalus

Papio hamadryas: Chacma Baboon (E), Guinea Baboon (E).

Hamadryas Baboon (E), Olive Baboon (E), Yellow Baboon (E),

Papión de Guinea (S), Papión negro (S), Papión oliva (S),

Babouin anubis (F), Babouin chacma (F), Babouin de Guinée

(F), Babouin jaune (F), Droguera (F), Hamadryas (F) II M

Papio hamadryas $=$ Papio hamadryas

Papio leucophaeus $=$ Mandrillus leucophaeus

Papio papio = Papio hamadryas

Papio sphinx $=$ Mandrillus sphinx

Papio ursinus = Papio hamadryas

Papión de Guinea (S): Papio hamadryas II M

Papión negro (S): Papio hamadryas II M

Papión oliva (S): Papio hamadryas II M

Papuan Goshawk (E): Accipifer meverianus II B

Papuan Ground Boa (E): Candoia aspera II R

Papuan Hanging-Parrot (E): Loriculus aurantiifrons II B

Papuan Hawk-Owl (E): Uroglaux dimorpha II B

Papuan Honey-buzzard (E): Henicopernis longicauda II B

Papuan Hombill (E): Aceros plicatus II B

Papuan King-Parrot (E): Alisterus chloropterus II B

Papuan Lorikeet (E): Charmosyna papou II B

Papuan Monitor (E): Varanus salvadorii II R

Papuan Python (E): Morelia papuana II $R$

Papuan Scops-Owl (E): Otus magicus II B

Papuina pulcherrima = Papustyla pulcherrima

Papusnila pulcherrima: Emerald Green Snail (E), Green Tree Snail (E), Manus Green Tree Snail (E) II In

Parabuteo unicinctus: Bay-winged Hawk (E), Haris's Hawk (E), Aguililla de Harris (S), Busardo mixto (S), Gavilán andapié (S), Gavilán mixto (S), Buse de Harris (F) II B

Paraclavarina triangularis: II In

Paraconotrochus zeidleri: II In

Paracyathus andersoni: II In

Paracyathus arcuatus: II In

Paracyashus caeruleus: II In

Paracyathus cavatus: II In

Paracyathus clathra: II In

Paracyathus conceptus: II In

Paracyathus coronatus: II In

Paracyathus ebonensis: II In

Paracyathus fulvus: II In

Paracyathus humilis: II In

Paracyathus indicus: II In

Paracyathus laxus: II in

Paracyathus merguiensis: II In

Paracyathus molokensis: II In

Paracyathus montereyensis: II In

Paracyathus persicus: II In

Paracyathus porphyreus: II In

Paracyathus profundus: II In

Paracyathus pruinosus: II In

Paracyathus pulchellus: II in

Paracyathus steamsii: II In

Paracyathus slokesii: II In

Paracyathus vinatus: II in

Paradellocyathus = Peponocyathus

Paradichocoenia $=$ Dichocoenia

Paradigalla brevicauda: Short-tailed Paradigalla (E), Paradigalle a queue courte (F), Paradisier à queue courte (F) II B 
Paradigalla carunculata: Long-tailed Paradigalla (E), Paradigalle caronculée (F), Paradisier caronculé (F) II B

Paradigallas (E): Paradigalla spp. II B

Paradigalle à queue coune (F): Paradigalla brevicauda II B

Paradigalle caronculée (F): Paradigalla carunculata II B

Paradisaea apoda: Greater Bird-of-paradise (E), Grand paradisier (F), Paradisier grand-émeraude (F) II B

Paradisaea decora: Goldie's Bird-of-paradise (E). Paradisier de Goldie (F) II B

Paradisaea guilielmi: Emperor Bird-of-paradise (E), Emperor of Germany's Bird-of-paradise (E), Paradisier de Guillaume (F) Paradisier de l'empereur Guillaume (F) II B

Paradisaea minor. Lesser Bird-of-paradise (E), Paradisier petitémeraude (F), Petit paradisier (F) II B

Paradisaea raggiana: Count Raggi's Bird-of-paradise (E), Raggiana Bird-of-paradise (E), Paradisier de Raggi (F), Paradisier du comte Raggi (F) II B

Paradisaea rubra: Red Bird-of-paradise (E), Paradisier rouge (F) II B

Paradisaea nudolphi: Blue Bird-of-paradise (E), Paradisier bleu (F), Paradisier de Rodolphe (F) II B

Paradisaeidae spp.: Birds-of-paradise (E), Aves del paraiso (S). Paradisiers (F) II B

Paradise Birdwing (E): Omithoptera paradisea II in

Paradise Parrot (E): Psephotus pulcherrimus I B

Paradise Riflebird (E): Priloris paradiseus II B

Paradise-Crow (E): Lycocorax pyrrhopterus II B

Paradise-Flycatchers (E): Terpsiphone spp. IIINC B

Paradise-Whydahs (E): Vidua spp. III/NC B

Paradisier à bec blanc (F): Epimachus bruijnii II B

Paradisier à gorge noire (F): Astrapia nigra II B

Paradisier à queue courte (F): Paradigalla brevicauda II B

Paradisier a rubans $(\mathrm{F})$ : Astrapia mayeri II B

Paradisier bleu (F): Paradisaea rudolphi II B

Paradisier caronculé (F): Paradigalla carunculata II B

Paradisier corvin (F): Lycocorax pyrrhopterus II B

Paradisier d'Albert (F): Pteridophora albeni II B

Paradisier d'Albertis (F): Epimachus albertisi II B

Paradisier de Carola (F): Parotia carolae II B

Paradisier de Goldie (F): Paradisaea decora II B

Paradisier de Guillaume (F): Paradisaea guilielmi II B

Paradisier de Jobi (F): Manucodia jobiensis II B

Paradisier de Keraudren (F): Manucodia keraudrenii II B

Paradisier de la princesse Stéphanie (F): Astrapia stephaniae II B

Paradisier de Lawes (F): Parotia lawesii II B

Paradisier de l'empereur Guillaume (F): Paradisaea guilielmi II B

Paradisier de Loria (F): Cnemophilus loriae II B

Paradisier de Macgregor (F): Macgregoria pulchra II B

Paradisier de Meyer (F): Epimachus meyeri II B

Paradisier de Raggi (F): Paradisaea raggiana II B

Paradisier de Rodolphe (F): Paradisaea rudolphi II B

Paradisier de Rothschild (F): Astrapia rothschildi II B

Paradisier de Stéphanie (F): Astrapia stephaniae II B

Paradisier de Victoria (F): Priloris victoriae II B

Paradisier de Wahnes (F): Parotia wahnesi II B

Paradisier de Wallace (F): Semioptera wallacii II B

Paradisier dEntrecasteaux (F): Manucodia comrii II B

Paradisier d'Helena (F): Parotia helenae II B

Paradisier du comte Raggi (F): Paradisaea raggiana II B

Paradisier du prince Albert (F): Pteridophora alberti II B

Paradisier fastueux (F): Epimachus fastuosus II B

Paradisier festonné (F): Ptiloris paradiseus II B

Paradisier gorge-d'acier (F): Priloris magnificus II B

Paradisier grand-émeraude (F): Paradisaea apoda II B

Paradisier huppé (F): Cnemophilus macgregorii II B

Paradisier loria (F): Cnemophilus loriae II B

Paradisier magnifique (F): Cicinnurus magnificus II B

Paradisier multifil (F): Seleucidis melanoleuca II B

Paradisier noir $(\mathrm{F})$ : Manucodia atra Il B

Paradisier petit-émeraude (F): Paradisaea minor II B

Paradisier républicain (F): Cicinnurus respublica II B

Paradisier rouge (F): Paradisaea rubra II B

Paradisier royal (F): Cicinnurus regius II B

Paradisier sifilet (F): Parotia sefilata II B

Paradisier soyeux (F): Loboparadisea sericea II B

Paradisier splendide (F): Astrapia splendidissima II B
Paradisier superbe (F): Lophorina superba II B

Paradisier vert (F): Manucodia chalybata II B

Paradisiers (F): Paradisaeidae spp. II B

Paradoxurus hermaphrodirus: Common Palm Civet (E), Civeta de palmera común (S) $111 \mathrm{M}$

Paradoxurus jerdoni: Jerdon's Palm Civet (E), Civeta de palmera de Jerdon (S) III M

Paraerrina decipiens: II ln

Paraguay Caiman Lizard (E): Dracaena paraguayensis II R

Parahalomitra $=$ Sandalolitha

Parahyaena brunnea: Brown Hyaena (E), Hiena dorada (S), Hiena parda (S), Hyène brune (F) II M

Parakeets (E): Psittacidae spp. I/II B

Parantipathes laricides: II In

Paranipathes larix: II In

Parantipathes tilliei: II In

Parantipathes strigosa: II In

Parantipathes terrasticha: II In

Parantipathes iristicha: II In

Parantipathes wolffi: II In

Paraonyx microdon $=$ Aony $x$ congicus

Paraonyx tacheté ( $\mathrm{F}$ ): Aonyx congicus I/I M

Parascolymia $=$ Scolymia

Parasimplastrea simplicitexta: II in

Parasitic Weaver (E): Anomalospiza imberbis III B

Parasmilia poculum: II In

Parastrea $=$ Favia

Paratrochocyathus = Trochocyathus

Pardel Lynx (E): Lynx pardinus I M

Pardofelis marmorata: Marbled Cat (E), Gato jaspeado (S), Chat marbré $(\mathrm{F})$ I $\mathrm{M}$

Paresseux tridactyle (F): Bradypus variegatus II M

Paresseux tridactyle de Bolivie (F): Bradypus variegalus II M

Pariah Kite (E): Milvus migrans II B

Parihuana (S): Phoenicopterus chilensis II B

Parina chica (S): Phoenicopterus jamesi II B

Parina grande (S): Phoenicopterus andinus II B

Pariona (S): Phoenicopterus chilensis II B

Parker's Day Gecko (E): Phelsuma parkeri II R

Parmoptila rubrifrons: Jameson's Antpecker (E), Red-fronted Antpecker (E). Astrild fourmilier (F), Parmoptile à front rouge (F) III B

Parmoptila woodhousei rubrifrons = Parmoptila nubrifrons

Parmoptile à front rouge (F): Parmoptila rubrifrons III B

Parmoptiles (F): Parmoptila spp. HINC B

Pamassius apollo: Apollo (E), Apollo Butterfly (E), Mountain Apollo (E), Apolo (S), Mariposa apollo (S) II In

Paroare à bec jaune (F): Paroaria capitata II B

Paroare huppé (F): Poroaria coronała II B

Paroares (F): Paroaria spp. IU/NC B

Paroaria capitata: Yellow-billed Cardinal (E), Cardenal cabecirrrjo (S), Paroare à bec jaune (F) II B

Paroaria coronata: Red-crested Cardinal (E), Cardenal copetón (S), Paroare huppé (F) II B

Parotia carolae: Carola's Parotia (E), Queen Carola's Parotia (E), Paradisier de Carola (F), Sifilet de Carola (F) II B

Parotia helenae: Eastern Parotia (E), Paradisier d'Helena (F), Sifilet d'Hélène (F) II B

Parotia Lawesii: Lawes's Parotia (E), Paradisier de Lawes (F), Sifilet de Lawe (F) II B

Parotia lawesii helenae $=$ Parotia helenae

Parotia sefilata: Arfak Parotia (E), Six-plumed Parotia (E), Westem Parotia (E), Paradisier sifilet (F), Sifilet d'Arfak (F) II B

Parotia wahnesi: Wahnes's Parotia (E), Paradisier de Wahnes (F), Sifilet de Wahnes (F) II B

Parotias (E): Parotia spp. II B

Parrot-beaked Tortoise (E): Homopus areolatus II R

Parrotlets (E): Forpus spp $/$ Touir spp. II B

Parrots (E): PSITTACIFORMES spp. I/III/NC B

Parson's Giant Chameleon (E): Chamaeleo parsonii II R

Partridges (E): Phasianidae spp. III/NC B

Pasco Poison Frog (E): Dendrobates lamasi II A

Passer griseus: Grey-headed Sparrow (E), Gorrión pardillo (S), Moineau gris (F) III B

Patagona gigas: Giant Hummingbird (E), Colibri gigante (S), Picaflor gigante (S), Colibri géant (F) II B 
Patagonian Conure (E): Cyanoliseus patagonus II B Patagonian Hog-nosed Skunk (E): Conepasus humboldtii II M Patas (F): Erythrocebus patas II M

Patas Monkey (E): Erythrocebus patas II M

Paterocyathus $=$ Caryophyllia

Patito africano (S): Nettapus auritus III B

Pato almizclero aliblanco (S): Cairina scutulata I B

Pato cabecirrosa (S): Rhodonessa caryophyllacea I B

Pato crestón (S): Sarkidiomis melanotos II B

Pato crestudo (S): Sarkidiomis melanotos II B

Pato criollo (S): Cainina moschata III B

Pato cuchara (S): Anas clypeata III B

Pato cucharón norteño (S): Anas clypeata III B

Pato de Bemier (S): Anas bernieri II B

Pato de Hartlaub (S): Pteronetta hartlaubii III B

Pato de jungla (S): Cairina scutulata I B

Pato de moco (S): Sarkidiornis melanotos II B

Pato de monte (S): Sarkidiomis melanotos II B

Pato golondrino norteño (S): Anas acuta III B

Pato rabudo (S): Anas acuta III B

Pato real (S): Cairina moschata III B

Pato real de Laysan (S): Anas laysanensis I B

Pato real marismeno (S): Anas oustaleti I B

Pato silbón (S): Anas penelope III B

Pato silbón cariblanco (S): Dendrocygna viduata 111 B

Pato silbón común (S): Dendrocygna bicolor III B

Pato silbón de Cuba (S): Dendrocygna arborea II B

Pato silbón ventrinegro (S): Dendrocygna aulumnalis III B

Pauji copete de Piedra (S): Pauxi pauxi III B

Paují comudo (S): Oreophasis derbianus I B

Pauji de Alagoas (S): Miru mitu I B

Paují de Copete (S): Crax daubentoni III B

Paují de yelmo (S): Pauxi pauxi III B

Paují menor (S): Mitu mitu I B

Pauxi pauxi: Helmeted Curassow (E), Northem Helmeted Curassow (E), Paují copete de Piedra (S), Pauji de yelmo (S), Hocco à pierne (F) III B

Pava aliblanca (S): Penelope albipennis I B

Pava cojolita (S): Penelope purpurascens III B

Pava culirroja (S): Penelope purpurascens III B

Pava de Trinidad (S): Pipile pipile I B

Pava pajuil (S): Oreophasis derbianus I B

Pava rajadora (S): Pipile pipile I B

Pava yacutinga (S): Pipile jacutinga I B

Pavita andina (S): Glaucidium jardinii II B

Pavita ferruginea (S): Glaucidium brasilianum II B

Pavo muticus: Green Peafowl (E), Green-necked Peafowl (E), Pavoreal cuelliverde (S), Pavo-real verde (S), Paon spicifere (F), Paon vert (F) II B

Pavón (S): Oreophasis derbianus I B

Pavón carunculado (S): Crar globulosa $\mathrm{HI}$ B

Pavón comudo (S): Oreophasis derbianus I B

Pavón grande (S): Crax rubra III B

Pavón norteña (S): Crax rubra III B

Pavón piquiazul (S): Crax alberti III B

Pavón piquirrojo (S): Crax blumenbachii I B

Pavón poría (S): Crax daubentoni III B

Pavona acuticarinata: II In

Pavona bipartita: II in

Pavona cactus: II In

Pavona calicifera: II In

Pavona clavus: II In

Pavona complanata: II in

Pavona danai: II In

Pavona decussata: II in

Pavona diffluens: II In

Pavona divaricata: II In

Pavona duerdeni: II In

Pavona explanulata: II in

Pavona foliosa: II In

Pavona formosa: II In

Pavona frondifera: II in

Pavona galapagensis: II In

Pavona gigantea: II In

Pavona intermedia: II In

Pavona maldivensis: II in
Pavona minor: II ln

Pavona minuta: II In

Pavona repens: II In

Pavona varians: II In

Pavona venosa: II In

Pavona xarifae: II In

Pavona yamanari: II In

Pavonia $=$ Pavona

Pavo-real cuelliverde (S): Pavo muricus II B

Pavo-real verde (S): Pavo muticus II B

Peach-faced Lovebird (E): Agapomis roseicollis II B

Peach-fronted Conure (E): Aratinga aurea II B

Peach-fronted Parakeet (E): Aratinga aurea II B

Peachthroat Monitor (E): Varanus jobiensis II R

Peacock Coquette (E): Lophomis pavoninus II B

Peacock Day Gecko (E): Phelsuma quadriocellata II R

Peacock Soft-shell Turtle (E): Trionyx hurum I R

Peacock Turtle (E): Morenia ocellata I R

Peacock-marked Soft-shelled Turtle (E): Trionyx hurum I R

Peacock-Pheasants (E): Polyplectron spp. I/IIIINC B

Peafowl (E): Pavo spp. IUNC B

Peale's Dolphin (E): Lagenorhynchus australis II M

Pearl Kite (E): Gampsonyx swainsonii II B

Pearl-spotted Owlet (E): Glaucidium perlatum II B

Pearly Conure (E): Pyrrhura perlata II B

Pearly mussels (E): Unionidae spp. I/INC In

Pearly Parakeet (E): Pyrrhura perlasa II B

Pécari à barbe blanche (F): Tayassu pecari II M

Pécari aux babines blanches (F): Tayassu pecari II M

Pecarí de labio blanco (S): Tayassu pecari II M

Pécari du Chaco (F): Calagonus wagneri I M

Pécaries (F): Tayassuidae spp. II M

Pecaries (S): Tayassuidae spp. VII M

Peccaries (E): Tayassuidae spp. I/II M

Pectinia alcicomis: II In

Pecrinia elongara: II In

Pectinia lactuca: Camation Coral (E) II In

Pectinia laxa: II In

Pectinia paeonia: II in

Pectinia symphylloides: II In

Pectinia teres: II In

Pedicellocyaihus keyesi: II In

Peer's Girdled Lizard (E): Cordylus peersi II R

Pelecanus crispus: Dalmatian Pelican (E), Pelícano ceñudo (S),

Pelícano rizado (S), Pélican dalmate (F). Pélican frisé ( $F)$ I B

Pélican dalmate (F): Pelecanus crispus I B

Pélican frisé (F): Pelecanus crispus I B

Pelícano ceñudo (S): Pelecanus crispus I B

Pelícano rizado (S): Pelecanus crispus I B

Pelicans (E): Pelecanus spp. INC B

Pélicans (E): Pelecanus spp. INC B

Pelomedusa subrufa: African Helmeted Turte (E), Helmeted Turle

(E), Marsh Terrapin (E), Tortuga plana (S), Peloméduse roussâtre (F) III $R$

Peloméduse roussâtre (F): Pelomedusa subrufa III R

Pel's Fishing-Owl (E): Scotopelia peli II B

Pel's Flying Squirrel (E): Anomalurus pelii III M

Peltocephale de Duméril (F): Peltocephalus dumeriliana II R

Peliocephalus dumeriliana: Big-headed Amazon River Turtle (E)

Big-headed Sideneck (E), Cabezón (S), Peltocephale de Duméril

(F), Podocnémide de Duméril (F) II R

Peltocephalus dumerilianus = Peltocephalus dumeriliano

Peltocephalus tracaxa $=$ Peltocephalus dumeriliana

Péluse à bec crochu (F): Pelusios niger III R

Péluse d'Adanson (F): Pelusios adansonii UI R

Péluse de Schweigger (F): Pelusios castaneus III R

Péluse du Gabon (F): Pelusios gabonensis III R

Péluse noire (F): Pelusios niger III R

Péluses (F): Pelusios spp. III R

Pelusios spp.: Péluses (F) III R

Pelusios adansonit: Adanson's Mud Turtle (E), Sahelian Terrapin (E), White-breasted Side-necked Turtle (E), Tortuga negra de Adanson (S), Péluse d'Adanson (F) III R

Pelusios castaneus: Chestnut Terrapin (E), Schweigger's Terrapin (E), West African Mud Turtle (E), Péluse de Schweigger (F) III $R$ 
Pelusios gabonensis: African Forest Turle (E), Gabon Terrapin (E), Gabon Turtle (E), Stripe-backed Side-necked Turtle (E), Tortuga negra del Gabón (S), Péluse du Gabon (F) III R

Pelusios niger. African Black Terrapin (E), Black Side-necked Turtle (E), Hooked-beaked Terrapin (E), West African Black Forest Turtle (E), Tortuga negra de Africa occidental (S), Péluse noire (F), Péluse à bec crochu (F) III R

Pemba Flying-fox (E): Pieropus voelizkowi II M

Pemba Scops-Owl (E): Otus nutilus II B

Penang Lizard (E): Varanus bengalensis I R

Pénélope à ailes blanches (F): Penelope albipennis I B

Pénélope à front noir (F): Pipile jacutinga I B

Pénélope à plastron (F): Pipile jacutinga I B

Pénélope à ventre blanc (F): Penelope purpurascens III B

Penelope albipennis: White-winged Guan (E), Pava aliblanca (S), Pénélope à ailes blanches (F) I B

Pénélope comue (F): Oreophasis derbianus I B

Pénélope de Derby (F): Oreophasis derbianus I B

Pénélope huppée (F): Penelope purpurascens III B

Pénélope noire (F): Penelopina nigra III B

Pénélope pajuil (F): Penelopina nigra III B

Pénélope panachée (F): Penelope purpurascens III B

Penelope purpurascens: Crested Guan (E), Pava cojolita (S), Pava culirroja (S), Pénélope huppée (F), Pénélope panachée (F) Pénélope à ventre blanc (F) III B

Pénélope siffleuse (F): Pipile jacutinga I B

Pénélope siffleuse de la trinité (F): Pipile pipile I B

Pénélopes (F): Cracidae spp. IUI/NC B

Penelopides spp.: Hornbills (E), Buceros (S), Calaos (F) II B

Penelopides affinis: Mindanao Hornbill (E), Calao de Mindanao (F) II B

Penelopides exarhatus: Sulawesi Hornbill (E), Calao des Célèbes (F) II B

Penelopides manillae: Luzon Hombill (E), Calao de Manille (F) II B

Penelopides mindorensis: Mindoro Hombill (E), Calao de Mindoro (F) II B

Penelopides panini: Tarictic Hombill (E), Visayan Hornbill (E), Calao tarictic (F) II B

Penelopides panini affinis $=$ Penelopides affinis

Penelopides panini manillae $=$ Penelopides manillae

Penelopides panini panini $=$ Penelopides panini

Penelopides panini samarensis = Penelopides samarensis

Penelopides samarensis: Samar Hombill (E), Calao de Samar (F) II B

Penelopina nigra: Highland Guan (E), Pajuil (S), Pénélope noire (F), Pénélope pajuil (F) III B

Penguins (E): Spheniscidae spp. I/IINC B

Pennant's Parakeet (E): Platycercus elegans II B

Pen-tailed Treeshrew (E): Prilocercus lowii II M

Peponocephala electra: Melon-headed Whale (E), Electra (S), Péponocéphale (F) II M

Péponocéphale (F): Peponocephala electra II M

Peponocyathus australiensis: II in

Peponocyathus dawsoni: II In

Peponocyathus folliculus: II in

Pequeña civeta de la India (S): Viverricula indica III M

Perameles bougainville: Barred Bandicoot (E), Long-nosed Bandicoot (E), Marl (E), Westem Barred Bandicoot (E), Tejon marsupial rayado (S), Bandicoot de Bougainville (F) I M

Percnoptère brun (F): Necrosynes monachus II B

Percnoptere d'Egypte (F): Neophron percnopterus II B

Perdicilla herrumbrosa (S): Caloperdix oculea III B

Perdigallo caspio (S): Tetraogallus caspius I B

Perdigallo tibetano (S): Tetraogallus tibetanus I B

Perdiz de bosque crestada (S): Rollulus rouloul III B

Perdiz de bosque de Charlton (S): Arborophila charltonii III B

Perdiz de bosque de pico largo (S): Rhizothera longirostris III B

Perdiz de bosque ferruginosa (S): Caloperdix oculea III B

Perdiz de bosque negra (S): Melanoperdix nigra III B

Perdiz negra (S): Melanoperdix nigra III B

Perdiz piquilarga (S): Rhizothera longirostris III B

Perdiz real del Caspio (S): Tetraogallus caspius I B

Perdiz real del Tibet (S): Tetraogallus tibetanus I B

Perdiz rulrul (S): Rollulus rouloul III B

Perdrix (F): Phasianidae spp. IIL/NC B

Perdrix à long bec (F): Rhizothera longirostris III B
Perdrix des neiges de la Caspienne (F): Tetraogallus caspius I B Perdrix des neiges du Tibet (F): Tetraogallus tiberanus I B Perdrix noire (F): Melanoperdix nigra III B

Père David's Macaque (E): Macaca thibetana II M

Peregrine (E): Falco peregrinus I B

Peregrine Falcon (E): Falco peregrinus I B

Perentie (E/F): Varanus giganteus II R

Perezoso (S): Choloepus hoffmanni III M

Perezoso grisaceo (S): Bradypus variegalus II M

Perezoso tridáctilo (S): Bradypus variegatus II M

Perfect Lorikeet (E): Trichoglossus euteles II B

Perico (S): Aratinga chloroptera II B

Perico aligualdo (S): Psephotus chrysopterygius I B

Perico barreteado (S): Bolborhynchus lineola 11 B

Perico cabecidorado (S): Pionopsinta pyritia II B

Perico cabecinegro (S): Pionopsinta caica II B

Perico cabecioscuro (S): Aratinga weddellii II B

Perico cabecirrojo (S): Pyrrhura rhodocephala II B

Perico cabecirrosado (S): Pyrrhura picta II B

Perico cachete amarillo (S): Pionopsitta barrabandi II B

Perico cachetigris (S): Brotogeris pyrrinopterus II B

Perico calzoncito (S): Pionites melanocephala II B

Perico capelo (S): Purpureiccphalus spurius II B

Perico capirotado (S): Psephotus dissimilis I B

Perico cara sucia (S): Aratinga pertinax II B

Perico caretirrojo (S): Aratinga erythrogenys II B

Perico cariazul (S): Northiella haematogaster II B

Perico carigualdo (S): Platycercus icterotis II B

Perico cola negra (S): Pyrrhura melanura II B

Perico cola roja (S): Pyrrhura hoematotis II B

Perico colimorado (S): Pyrrhura egregia II B

Perico cornudo (S): Eunymphicus cornutus II B

Perico de Bamard (S): Platycercus bamardi II B

Perico de El Oro (S): Pyrrhura orcesi II B

Perico de las Antípodas (S): Cyanoramphus unicolor II B

Perico de las Chatham (S): Cyanoramphus auriceps forbesi I B

Perico de Port Lincoln (S): Platycercus zonarius II B

Perico de Tasmania (S): Platycercus caledonicus II B

Perico del Paraíso (S): Psephotus pulcherrimus I B

Perico dorado (S): Aratinga solstitialis II B

Perico dorsirrojo (S): Psephotus haematonorus II B

Perico elegante (S): Platycercus elegans II B

Perico frentinaranja (S): Aratinga canicularis II B

Perico gracioso (S): Platycercus venustus II B

Perico grande (S): Pyrrhura cruentata I B

Perico gualda (S): Platycercus flaveolus II B

Perico ligero (S): Bradypus variegatus II M

Perico manglero (S): Amazona albifrons II B

Perico maori cabecigualdo (S): Cyanoramphus auriceps VII B

Perico maori cabecirrojo (S): Cyanoramphus novaezelandiae I B

Perico multicolor (S): Hapalopsittaca amazonina II B

Perico multicolor (S): Plarycercus eximius II B

Perico nocturno (S): Geopsittacus occidentalis I B

Perico ojo blanco (S): Aratinga leucophthalmus II B

Perico pálido (S): Planycercus adscirus II B

Perico pechiblanco (S): Pyrrhura albipectus II B

Perico pechisucio (S): Aratinga nana II B

Perico pico rojo (S): Pionus sordidus II B

Perico pintado (S): Pyrrhura leucotis II B

Perico princesa (S): Polytelis alexandrae II B

Perico regente (S): Polytelis anthopeplus II B

Perico siete colores (S): Touit batavica II B

Perico soberbio (S): Polytelis swainsonii II B

Perico terrestre (S): Pezoponus wallicus I B

Perico tui (S): Brotogeris sanctithomae II B

Perico variado (S): Psephotus varius II B

Perico verde (S): Aratinga holochlora II B

Perico verde centroamericano (S): Aratinga strenua II B

Pericos (\$): Cyanoramphus spp./Eunymphicus comutus/Geopsittacus occidentalis/Northiella haematogasier/Pezoporus wallicus/Plarycercus spp./Polytelis spp./Psephotus spp./Purpureicephalus spurius $\mathbf{1}$ II B

Perija Metaltail (E): Metallura iracunda II B

Perijá Metaltail (E): Metallura iracunda U B

Perinet Chameleon (E): Chamaeleo gastrotaenia II R

Periquito (S): Forpus passerinus II B 
Periquito ala dorada (S): Brotogeris chrysopterus II B Periquito aliazul (S): Forpus xanthopterygius II B Periquito alipunteado (S): Touit stictopsera II B Periquito azul (S): Brotogeris cyanoptera II B Periquito azul alirrojo (S): Touit huetii II B Periquito barbinaranja (S): Brotogeris jugularis II B Periquito barrado (S): Bolborhynchus lineola II B Periquito cabecirrojo (S): Cyanoramphus novaezelandiae I B

Periquito crisóstomo (S): Neophema chrysostoma II B Periquito de collar africano (S): Psittacula krameri III B

Periquito de espalda dorada (S): Psephotus chrysopterygius I B

Periquito de pico grueso (S): Rhynchopsitta pachyrhyncha I B

Periquito de tierra (S): Pezoporus wallicus I B

Periquito del Pacífico (S): Forpus coelestis II B

Periquito elegante (S): Neophema elegans II B

Periquito encapuchado (S): Psephotus dissimilis I B

Periquito espléndido (S): Neophema splendida II B

Periquito frentiamarillo de Forbes (S): Cyanoramphus auriceps forbesi I B

Periquito frentiazul (S): Touit dilectissima II B

Periquito mexicano (S): Forpus cyanopygius II B

Periquito migrador (S): Lathamus discolor II B

Periquito obscuro (S): Forpus sclateri II B

Periquito ojiazul (\$): Forpus conspicillatus II B

Periquito orejiamarillo (S): Ognorhynchus icterotis I B

Periquito rabadilla púrpura (S): Touit purpuraia II B

Periquito roquero (S): Neophema petrophila II B

Periquito rosado (S): Neopsepholus bourkii II B

Periquito turquesa (S): Neophema pulchella II B

Periquito ventrinaranja (S): Neophema chrysogaster I B

Periquitos (S): Lathamus discolor/Melopsitlacus undulatus/Neophema spp/Neopsephotus bourkii IIINC B

Pemis apivorus: European Honey-buzzard (E), Abejero europeo (S), Halcón abejero (S), Bondrée apivore (F) II B

Permis celebensis: Barred Honey-buzzard (E), Abejero de Célebes (S), Bondrée des Célèbes (F) II B

Pemis ptilorhyncus: Oriental Honey-buzzard (E), Abejero oriental (S), Bondrée orientale (F) II B

Perodicticus potto: Potto (E), Potto Gibbon (E), Poto de Bosman (S), Potto (F) II M

Perrito de las praderas (S): Cynomys mexicanus I M

Perrito llanero Mejicano (S): Cynomys mexicanus I M

Perro de agua (S): Speothos venaticus/Lontra longicaudis/Preronura brasiliensis I M

Perro de monte (S): Speothos venaticus I M

Perro salvaje asiático (S): Cuon alpinus II M

Perroquet à calotte rouge (F): Poicephalus gulielmi II B

Perroquet à couronne (F): Tanygnathus lucionensis II B

Perroquet à face jaune (F): Poicephalus flavifrons II B

Perroquet à oreilles (F): Pionopsitta pileata I B

Perroquet à tête brune (F): Poicephalus cryptoxanthus II B

Perroquet à tête grise (F): Poicephalus senegalus II B

Perroquet à ventre rouge (F): Poicephalus rufiventris II B

Perroquet de Meyer (F): Poicephalus meyeri II B

Perroquet de Rüppell (F): Poicephalus rueppellii II B

Perroquet des niam-niam (F): Poicephalus crassus II B

Perroquet du Cap (F): Poicephalus robustus II B

Perroquet gris du Gabon (F): Psittacus erithacus II B

Perroquet jaco (F): Psittacus erithacus II B

Perroquet masqué (F): Cyclopsitta diophthalma I/II B

Perroquet masqué de Coxen (F): Cyclopsitta diophthalma coxeni I B

Perroquet nocturne (F): Strigops habroptilus I B

Perroquet noir (F): Coracopsis nigra II B

Perroquet petit vasa (F): Coracopsis nigra II B

Perroquet robuste (F): Poicephalus robustus II B

Perroquet vaza (F): Coracopsis vasa II B

Perroquet vert à calotte rouge (F): Poicephalus gulielmi II B

Perroquet vert du Congo (F): Poicephalus gulielmi II B

Perroquet youyou (F): Poicephalus senegalus II B

Perroquet-hibou (F): Strigops habroptilus I B

Perroquets (F): Coracopsis spp./Poicephalus spp./Psirfacus erithacus II B

Perruche à ailes vertes (F): Alisterus chloropterus II B

Perruche à bandeau rouge (F): Cyanoramphus novaezelandiae I B

Perruche à bandeau rouge $(\mathrm{F})$ : Cyanoramphus novaezelandiae I B

Perruche à bec de sang (F): Tanygnathus megalorynchos II B
Perruche aे bec gracille (F): Enicognathus leptorhynchus II B Perruche à bonnet bleu (F): Nonhiella haematogaster II B Perruche à bouche d'or (F): Neophema chrysostoma II B Perruche à calotte bleue (F): Polytelis alexandrae II B/Tanygnathus lucionensis II B

Perruche à capuchon noir $(\mathrm{F})$ : Psephotus dissimilis I B

Perruche à col bleu (F): Geoffroyus simplex II B

Perruche à collier (F): Psintacula krameri III B

Perruche à collier de Maurice (F): Psittacula echo I B

Perruche à collier jaune (F): Platycercus zonarius II B

Perruche à croupion bleu (F): Psittinus cyanunus II B

Perruche à croupion rouge (F): Psephotus haematonotus II $\mathrm{B} / \mathrm{Psephotus} \mathrm{varius} \mathrm{II} \mathrm{B}$

Perruche à dos rouge (F): Psephorus haematonotus II B

Perruche à épaules dorées (F): Psephotus chrysopterygius I B

Perruche à face rose (F): Agapomis roseicollis II B

Perruche à front doré (F): Aratinga aurea II B

Perruche à front jaune (F): Cyanoramphus auriceps I/II B

Perruche à front jaune de Forbes $(\mathrm{F})$ : Cyanoramphus auriceps forbesi I B

Perruche à gros bec (F): Rhynchopsitfa pachyrhyncha I B

Perruche à joue noire (F): Agapomis nigrigenis II B

Perruche à longs brins (F): Psinacula longicauda II B

Perruche à lunettes vertes (F): Neophema chrysogaster I B

Perruche à moustaches $(\mathrm{F})$ : Psittacula alexandri II $\mathrm{B}$

Perruche à oreilles jaunes (F): Platycercus icterotis II B

Perruche à poitrine écarlate (F): Neophema splendida II B

Perruche à tête ardoisée (F): Psittacula himalayana II B

Perruche à tête d'or (F): Cyanoramphus auriceps $\mathrm{VII} \mathrm{B}$

Perruche à tête d'or de Forbes (F): Cyanoramphus auriceps forbesi I B

Perruche à tête pâle (F): Plancercus adscinus II B

Perruche à tête pourpre (F): Purpureicephalus spurius II B

Perruche à tête prune (F): Psittacula cyanocephala II B

Perruche à tête rose (F): Psittacula roseata II B

Perruche à tête rouge (F): Agapornis pullarius II B

Perruche à ventre bleu (F): Triclaria malachiracea II B

Perruche à ventre jaune (F): Platycercus caledonicus II B

Perruche à ventre orange (F): Neophema chrysogaster I B

Perruche alexandre (F): Psintacula eupatria II B

Perruche ara (F): Rhynchopsitra pachyrhyncha I B

Perruche aux ailes bleues (F): Forpus passerinus II B

Perruche aux ailes d'or (F): Psephotus chrysopterygius I B

Perruche aux oreilles jaunes (F): Ognorhynchus icterotis I B

Perruche calédonienne (F): Planycercus caledonicus II B

Perruche calopsitte (F): Nymphicus hollandicus NC B

Perruche cornue (F): Eunymphicus comutus II B

Perruche d'Alexandra (F): Polytelis alexandrae II B

Perruche d'Aymara (F): Bolborhynchus aymara II B

Perruche de Bamard (F): Plarycercus barnardi II B

Perruche de Barraband (F): Polytelis swainsonii II B

Perruche de Bourke (F): Neopsephotus bourkii II B

Perruche de Branicki (F): Leptosittaca branickii II B

Perruche de Brehm (F): Psittacella brehmii II B

Perruche de Buru (F): Tanygnathus gramineus II B

Perruche de Derby (F): Psittacula derbiana II B

Perruche de Finsch (F): Psittacula finschii II B

Perruche de Fischer (F): Agapomis fischeri II B

Perruche de Geoffroy (F): Geoffroyus geoffroyi II B

Perruche de Jenday (F): Aratinga jandaya II B

Perruche de Latham (F): Lathamus discolor II B

Perruche de Layard (F): Psittacula calthropae II B

Perruche de l'Himalaya (F): Psittacula himalayana II B

Perruche de Lilian (F): Agapomis lilianae II B

Perruche de Luçon (F): Tanygnathus lucionensis II B

Perruche de Madarasz (F): Psittacella madaraszi II B

Perruche de Malabar (F): Psittacula columboides II B

Perruche de Maurice (F): Psittacula echo I B

Perruche de Müller (F): Tanygnathus sumarranus II B

Perruche de Norfolk (F): Cyanoramphus cookii I B

Perruche de Nouvelle-Zélande ( $F$ ): Cyanoramphus novaezelandiae I B

Perruche de paradis (F): Psephotus pulcherrimus I B

Perruche de Pennant (F): Platycercus elegans II B

Perruche de Sparman (F): Cyanoramphus novaezelandiae I B

Perruche de Stanley (F): Platycercus icterotis II B 
Perruche de Swindern (F): Agapomis swindemianus II B Perruche de terre (F): Pezoporus wallicus I B Perruche des Antipodes (F): Cyanoramphus unicolor II B Perruche des écueils (F): Neophema petrophila II B Perruche des îles Chatham (F): Cyanoramphus auriceps forbesi I B Perruche des Nicobar (F): Psittacula caniceps II B Perruche des rochers (F): Neophema petrophila II B Perruche doré (F): Aratinga guarouba I B Perruche du soleil (F): Aratinga solsritialis II B Perruche écarlate (F): Prosopeia splendens II B Perruche élégante (F): Neophema elegans II B Perruche émeraude (F): Enicognathus ferrugineus II B Pernche érythroptère (F): Aprosmictus erythropterus II B Perruche flavéole (F): Plarycercus flaveolus II B Perruche gracieuse (F): Platycercus venusius II B Perruche hétéroclite (F): Geoffroyus heteroclitus II B Perruche huppée (F): Eunymphicus cornulus II B Perruche inséparable (F): Melopsitracus undulatus NC B Perruche intermédiaire (F): Psittacula intermedia II B Perruche jonquille (F): Aprosmicrus jonquillaceus II B Perruche lunulée (F): Bolbopsittacus lunulatus II B Perruche magnifique (F): Psephotus pulcherrimus I B Pertuche masqué (F): Agapomis personąus II B Perruche masquée (F): Prosopeia personata II B Perruche mélanure (F): Polytelis anthopeplus II B Perruche modeste (F): Psittacella modesta II B Perruche multicolore (F): Psephotus varius II B Perruche nanday (F): Nandayus nenday II B Perruche noctume (F): Geopsittacus occidentalis I B Perruche ondulée (F): Melopsirfacus undularus: NC B Perruche omnicolore (F): Platycercus eximius II B Perruche orangé (F): Brotogeris pyrrhopterus II B Perruche palliceps (F): Platycercus adscitus II B Perruche peinte (F): Psittacella picta II B Perruche pompadour (F): Prosopeia tabuensis II B Perruche princesse (F): Polytelis alexandrae II B Perruche pygmée de Brujin (F): Micropsitta bruijnii II B Perruche pygmée de Meek (F): Micropsitta meeki II B Perruche pygmée de Salvadori (F): Micropsinta keiensis II B Perruche pygmée de Schlegel (F): Micropsitta geelvinkiana II B Perruche pygmée de Sclater (F): Micropsitta pusio II B Perruche royale (F): Alisterus scapularis II B Perruche splendide (F): Neophema splendida 11 B Perruche terrestre (F): Pezoporus wallicus I B Perruche tiriba (F): Pyrrhura cruentala I B Perruche tovi (F): Brotogeris jugularis II B Perruche tricolore (F): Alisterus amboinensis II B Perruche turquoisine (F): Neophema pulchella II B Perruche unicolore (F): Cyanoramphus unicolor II B Perruches (F): PSITTACIFORMES spp. I/I/III B Perruche-souris (F): Myiopsitta monachus II B Persian Fallow Deer (E): Dama mesopotamica I M Persian Sturgeon (E): Acipenser persicus II F Perth Pygmy Python (E): Morelia penhensis II R Peruvian Cock-of-the-Rock (E): Rupicola peruviana II B Peruvian Guemal (E): Hippocamelus antisensis I M Peruvian Huemul (E): Hippocamelus antisensis I M Peruvian Penguin (E): Spheniscus humboldti I B Peruvian Piedtail (E): Phlogophilus harterti II B Peruvian Poison Frog (E): Epipedobates petersi II A Penuvian Pygmy-Owl (E): Glaucidium peruanum II B Peruvian Sheartail (E): Thaumastura cora II B Pesquet's Parrot (E): Psittrichas fulgidus II B Petit bandicoot-lapin (F): Macrotis leucura I M Petit cacatoès à huppe jaune (F): Cacarua sulphurea II B Petit cheirogale (F): Cheirogaleus medius I M Petit cordyle (F): Cordylus minor II R Petit faucon (F): Falco longipennis II B Petit flamant (F): Phoenicopterus minor II B Petit gecko diume des Seychelles (F): Phelsuma astriata II R Petit hapalémur (F): Hapalemur griseus I M Petit lépilémur (F): Lepilemur nuficaudaius I M Petit lézard à queue épineuse (F): Cordylus minor II $\mathrm{R}$ Petit lori musc (F): Glossopsitta pusilla II B Petit microcèbe (F): Microcebus murinus I M Petit moineau soulcie (F): Petronia dentata III B
Petit panda ( $F$ ): Ailurus fulgens I M

Petit paradisier (F): Paradisaea minor II B

Petit perruche-ara (F): Ara nobilis II B

Petit phelsume des Seychelles (F): Phelsuma astriata II R

Petit rorqual (F): Balaenopsera acutorostrata I/I M

Petit serpentaire (F): Polyboroides radiarus II B

Petit-duc à aigrettes longues (F): Otus sanciaecatarinae II B

Petit-duc à bec jaune ( $F$ : Otus icterorhynchus II B

Petit-duc à collier (F): Orus bakkamoena II B

Petit-duc à face blanche (F): Otus leucofis II B

Petit-duc à front blanc (F): Otus sagittatus II B

Petit-duc à gorge blanche (F): Otus albogularis II B

Petit-duc à mèches noires (F): Otus atricapillus II B

Petit-duc à moustaches (F): Otus trichopsis II B

Petit-duc africain (F): Otus scops II B

Petit-duc bridé (F): Otus barbarus II B

Petit-duc choliba (F): Onus choliba II B

Petit-duc de Bruce (F): Otus brucei II B

Petit-duc de Clark (F): Otus clarkii II B

Petit-duc de Cuba (F): Otus lawrencii II B

Petit-duc de Gumey (F): Mimizuku gurneyi I B

Petit-duc de Java (F): Orus angelinae II B

Petit-duc de Koepcke (F): Otus koepckeae II B

Petit-duc de Luçon (F): Otus megalotis II B

Petit-duc de Mantanani (F): Otus mantananensis II B

Petit-duc de Marshall (F): Otus marshalli II B

Petit-duc de Mindanao (F): Otus mirus II B

Petit-duc de Mindoro (F): Otus mindorensis II B

Petit-duc de Palawan (F): Otus fuliginosus II B

Petit-duc de Porto Rico (F): Otus nudipes II B

Petit-duc de Salvin (F): Otus ingens II B

Petit-duc de Sao Tomé (F): Otus hartlaubi II B

Petit-duc de Simalur (F): Otus umbra II B

Petit-duc de Watson (F): Otus watsonii II B

Petit-duc des Andaman (F): Otus balli II B

Petit-duc des Mentawi (F): Otus mentawi II B

Petit-duc des montagnes (F): Otus kennicottii II B

Petit-duc des Palau (F): Otus podarginus II B

Petit-duc d'Irène (F): Otus ireneae II B

Petit-duc du Pérou (F): Olus roboratus II B

Petit-dusc élégant (F): Otus elegans II B

Petit-duc géant (F): Mimizuku gumeyi 1 B

Petit-duc longicome (F): Otus longicomis II B

Petit-duc maculé (F): Orus asio II B

Petit-due malgache (F): Otus rutilus II B

Petit-duc Manado (F): Otus manadensis II B

Petit-duc mystérieux (F): Orus magicus II B

Petit-duc nain (F): Otus flammeolus II B

Petit-duc radjah (F): Gius brookii II B

Petit-duc roussâtre (F): Otus rufescens II B

Petit-duc scops (F): Otus scops II B

Petit-duc tacheté (F): Otus spilocephalus II B

Petit-duc vermiculé (F): Oıus vermiculatus II B

Petit-duc Wallace (F): Otus silvicola II B

Petit-ducs (F): Orus spp/Mimizuku gumeyi ИI В

Petite buse (F): Buteo platypterus II B

Petite mangouste indienne (F): Herpestes javanicus auropunctatus III $M$

Petite mélampitte (F): Melampitta lugubris II B

Petite nyctale (F): Aegolius acadicus II B

Petronia dentata: Bush Petronia (E), Bush Sparrow (E), Chillón de Sundevall (S), Petit moineau soulcie (F) III B

Petter's Chameleon (E): Chamaeleo petteri II R

Petterus coronatus = Eulemur coronatus

Pez lengüihueso malayo (S): Scleropages formosus I F

Pez mular (S): Tursiops iruncatus II M

Pez pulmonado australiano (\$): Neoceratodus forsseri II F

Pezoporus occidentalis $=$ Geopsittacus occidentalis

Pezoporus wallicus: Ground Parakeet (E), Ground Parrot (E),

Swamp Parakeet (E), Perico terrestre (S), Periquito de tierra (S),

Perruche de terre (F), Perruche terrestre (F) I B

Pfeffer's Chameleon (E): Chamaeleo pfefferi II R

Phacelocyathus flos: II in

Phaeochroa cuvierii: Scaly-breasted Hummingbird (E), Fandangero pechiescamosa (S), Colibri de Cuvier (F) II B 
Phaethomis anthophilus: Pale-bellied Hermit (E), Ermitaño carinegro (S), Emite anthophile (F) II B

Phaethornis augusti: Sooty-capped Hermit (E), Limpiacasa (S), Ermite d'Auguste (F) II B

Phaethornis bourcieri: Straight-billed Hermit (E), Ermitaño de picorrecto (S), Ermite de Bourcier (F) 11 B

Phaethomis eurynome: Scale-throated Hermit (E), Enmitaño manchado (S), Ermite eurynôme (F) II B

Phaethomis gounellei: Broad-tipped Hermit (E), Ermite de Gounelle (F) 11 B

Phaethomis griseogularis: Grey-chinned Hermit (E), Porculla Hermit (E), Ermitañito barbigris (S), Emite à gorge grise (F) II B

Phaethomis guy: Green Hermit (E), Ermitan̄o verde (S), Ermite vert (F) II B

Phaethornis hispidus: White-bearded Hermit (E), Ermitan̄o barbiblanco (S), Ermite d'Osery (F) II B

Phaethornis idaliae: Minute Hermit (E), Ermite d'Idalie (F) II B

Phoethomis koepckeae: Koepcke's Hermit (E), Ermite de Koepcke (F) II B

Phaethornis longuemareus: Little Hermit (E), Ermitañito pequeño (S), Ermitaño chico (S), Ermite nain (F) II B

Phaethomis malaris: Great-billed Hermit (E), Margaretta's Hermit (E), Ermite à long bec (F) II B

Phaethornis maranhaoensis = Phaethomis nattereri

Phaethornis margarenae $=$ Phaethornis malaris

Phaethomis mexicanus $=$ Phaethomis superciliosus

Phathomis nattereri: Cinnamon-throated Hermit (E), Ermite de Natterer (F) II B

Phaethomis nigrirostris = Phaethomis eunnome

Phaethomis philippii: Needle-billed Hermit (E), Ermite de Filippi (F) II B

Phaethomis porcullae $=$ Phaethornis griseogularis

Phaethornis pretrei: Planalto Hermit (E), Ermitan̄o frentrinegro (S), Errnite de Prêtre (F) II B

Phaethornis ruber: Reddish Hermit (E), Ermitañito rufo (S), Ermitaño rojizo (S), Ermite roussâtre (F) II B

Phaethomis squalidus: Dusky-throated Hermit (E), Ermitañito gargantifusco (S), Ermite terne (F) II B

Phaethomis strigularis = Phaethomis longuemareus

Phaethomis stuarti: White-browed Hermit (E), Ermite de Stuart (F) II B

Phaethomis subochraceus: Buff-bellied Hermit (E), Ermite ocré (F) II B

Phaethomis superciliosus: Long-tailed Hermit (E), Emitaño colilargo (S), Ermitaño guayanés (S), Ermite à brins blancs (F) II B

Phaethomis symatophorus: Tawny-bellied Hermit (E), Ermitaño ventrihabano (S), Ermite à ventre fauve (F) II B

Phaethornis yaruqui: White-whiskered Hermil (E), Emitano bigotiblanco (S), Emite yaruqui (F) II B

Phalanger maculatus $=$ Spilocuscus maculatus

Phalanger orientalis: Common Phalanger (E), Grey Cuscus (E), Northem Common Cuscus (E), Cuscús gris (S), Cuscús oriental (S), Couscous de la Sonde (F), Couscous gris (F) II M

Phalanger tacheté (F): Spilocuscus macularus II M

Phalangers (E): Phalanger spp/Spilocuscus spp. IU/NC M

Phalangopora regularis: II In

Phalcoboenus albogularis: White-throated Caracara (E), Caracara araucano (S), Chinalinda (S), Guarahuau cordillerano (S), Matamico blanco (S), Caracara à gorge blanche (F) II B

Phalcoboenus australis: Forster's Caracara (E), Striated Caracara (E), Caracara austral (S), Matamico estriado (S), Matamico grande (S), Tiuque cordillerano austral (S), Caracara austral (F), Caracara funèbre (F) II B

Phalcoboenus carunculatus: Carunculated Caracara (E), Caracara carunculado (S), Caracara caroncule (F) II B

Phalcoboenus megalopterus: Mountain Caracara (E), Caracara andino (S), Carancho andino (S), Matamico cordillerano (S), Caracara montagnard (F) II B

Phalcoboenus megalopterus albogularis = Phalcoboenus albogularis Phaner (F): Phaner furcifer I M

Phaner furcifer: Fork-marked Lemur (E), Fork-marked Mouse-lemur (E), Maki ardilla (S), Maki horquilla (S), Phaner (F) I M

Phantasmal Poison Frog (E): Epipedobates tricolor II A

Pharaoh Eagle-Owl (E): Bubo ascalaphus II B
Pharomachrus mocinno: Magnificent Quetzal (E), Resplendent Quetzal (E), Quetzal centroamericano (S), Quetzal resplendissant $(F)$. Trogon couroucou (F) I B

Phataginus gigantea $=$ Manis gigantea

Phataginus temmincki $=$ Manis remminckii

Phataginus tetradactyla $=$ Manis tetradactyla

Phataginus tricuspis = Manis tricuspis

Phayre's Langur (E): Trachypithecus phayrei II M

Phayre's Leaf Monkey (E): Trachypithecus phayrei II M

Pheasants (E): Phasianidae spp. I/IIII/NC B

Phelsuma spp.: Day geckos (E), Gecos diumos (S), Geckos diumes (F), Geckos diumes de l'Océan Indien (F), Phelsumes (F) II R

Phelsuma abboti: Abbott's Day Gecko (E), Aldabra Day Gecko (E), Geco diume de Abbott (S), Gecko diume d'Aldabra (F), Phelsume d'Aldabra (F) II R

Phelsuma abbotti parkeri $=$ Phelsuma parkeri

Phelsuma abbotti v-nigra = Phelsuma v-nigra

Phelsuma agalega $=$ Phelsuma borbonica

Phelsuma andamanensis: Andaman Day Gecko (E), Geco diumo de Andaman (S), Gecko diume des lles Andaman (F), Phelsume des Iles Andaman (F) II R

Phelsuma antanosy: Gecko diurne de la région Antanosy (F), Phelsume de la région Antanosy (F) II R

Phelsuma astriata: Seychelles Day Gecko (E), Petit gecko diume des Seychelles (F), Petit phelsume des Seychelles (F) II R

Phelsuma barbouri: Barbour's Day Gecko (E), Geco diumo de Barbour (S), Gecko diume de Barbour (F), Phelsume de Barbour (F) $I I \mathbf{R}$

Phelsuma befotakensis: North-western Day Gecko (E), Gecko diume de Befotaka (F), Gecko diume du nord-ouest de Madagascar (F), Phelsume de Befotaka (F) II R

Phelsuma bimaculata $=$ Phelsuma quadriocellata

Phelsuma borbonica: Réunion Day Gecko (E), Gecko diume de Bourbon (F), Gecko diume de la Réunion (F), Phelsume de Bourbon (F) II R

Phelsuma breviceps: Gecko diume à courte tête (F), Phelsume à courte tête (F) II R

Phelsuma cepediana: Blue-tailed Day Gecko (E), Mauritius Greater Day Gecko (E), Geco diumo de cola azul (S), Gecko diume à queue bleue (F), Phelsume à queue bleue (F) II R

Phelsuma cepediana borbonica = Phelsuma borbonica

Phelsuma chekei: Northem Day Gecko (E), Gecko diume de Cheke (F), Gecko diurne du Nord de Madagascar (F), Phelsume de Cheke (F) II R

Phelsuma comorensis: Comoro Day Gecko (E), Geco diurno de las Comores (S), Gecko diume des Comores (F), Phelsume des Comores (F) II R

Phelsuma dubia: Bright-eyed Day Gecko (E), Zanzibar Day Gecko (E), Geco diumo de Zanzibar (S), Gecko diume de Zanzibar (F), Gecko diume sombre (F), Phelsume de Zanzibar (F) II R

Phelsuma dubia comorensis = Phelsuma comorensis

Phelsuma edwardnewtonii: Rodrigues Day Gecko (E), Gecko diume de Rodrigues (F), Phelsume de Rodrigues (F) II R

Phelsuma edwardnewronii = Phelsuma gigas

Phelsuma flavigularis: Yellow-throated Day Gecko (E), Geco diumo de garganta amarilla (S), Gecko diume à gorge jaune (F), Phelsume à gorge jaune (F) II $R$

Phelsuma flavigularis serraticauda = Phelsuma serraticauda

Phelsuma gigas: Gecko diume géant de Rodrigues (F), Phelsume de géant Rodrigues (F) II R

Phelsuma guentheri: Round Island Day Gecko (E), Geco diumo de Guenther (S), Gecko diume de l'He Ronde (F), Phelsume de l' lle Ronde (F) II R

Phelsuma guimbeaui: Mauritius Day Gecko (E), Mauritius Lowland Forest Day Gecko (E), Geco diurno de Guimbeau (S), Gecko diume de l'lle Maurice (F), Phelsume de l'Ile Maurice (F) I R

Phelsuma gutrata: Speckled Day Gecko (E), Spotted Day Gecko (E), Gecko diume tacheté (F), Phelsume tacheté (F) II R

Phelsuma Klemmeri: Geco diumo de Klemmer (S), Gecko diume de Klemmer (F), Gecko diume à tête jaune (F), Phelsume de Klemmer (F) II R

Phelsuma laticauda: Flat-tailed Day Gecko (E), Gold-dust Day Gecko (E), Geco diumo de cola gruesa (S), Gecko diume poussière-d'or (F), Gecko diume à large queue (F), Phelsume poussière-d'or (F) II R 
Phelsuma leiogaster: Gecko diume du sud-ouest de Madagascar (F), Phelsume du sud-ouest de Madagascar (F) II R

Phelsuma lineasa: Lined Day Gecko (E), Side-striped Day Gecko (E), Striped Day Gecko (E), Geco diumo de bandas (S), Gecko diume ligné (F), Gecko diurne rayé (F), Phelsume rayé (F) II R

Phelsuma lineata pusilla = Phelsuma pusilla

Phelsuma longinsulae: Gecko diume des Seychelles (F), Phelsume des Seychelles (F) II R

Phelsuma longinsulae $=$ Phelsuma sundbergi

Phelsuma madagascariensis: Madagascar Day Gecko (E), Geco diumo de Madagascar (S), Gecko diume de Madagascar (F), Phelsume de Madagascar (F) II R

Phelsuma minuthi: Borner's Day Gecko (E), Gecko diume de Borne (F), Gecko diume de Minuth (F), Phelsume de Minuth (F) II R

Phelsuma modesta: Modest Day Gecko ( $\mathrm{E}$ ), Gecko diume modeste (F), Phelsume modeste (F) II R

Phelsuma mutabilis: Thick-tailed Day Gecko (E), Gecko diume de Grandidier $(F)$, Gecko diurne à queue chamue $(F)$, Phelsume variable (F) II $\mathrm{R}$

Phelsuma mutabilis breviceps $=$ Phelsuma breviceps

Phelsuma newtonii $=$ Phelsuma gigas

Phelsuma nigristriata: Island Day Gecko (E), Geco diumo de estrias negras (S), Gecko diurne de Meier (F), Gecko diume à bandes noires (F), Phelsume de Meier (F) II R

Phelsuma ocellata: Namaqua Day Gecko (E), Ocellated Day Gecko (E), Spotted Day Gecko (E), Geco diumo ocelado (S), Gecko diume ocellé (F), Phelsume ocellé (F) II R

Phelsuma ornata: Ornate Day Gecko (E), Geco diurno omado (S), Gecko diume omé de l'lle Maurice (F). Phelsume omé de l'lle Maurice (F) II R

Phelsuma parkeri: Parker's Day Gecko (E), Gecko diume de Parker (F), Gecko diurne de l'Lle Pemba (F), Phelsume de Parker (F) II $\mathrm{R}$

Phelsuma pronki: II R

Phelsuma pusilla: Gecko diume nain (F), Phelsume nain (F) II R

Phelsuma quadriocellata: Four-spot Day Gecko (E), Peacock Day Gecko (E), Gecko diume de Peacock (F), Gecko diume à quatre ocelles (F), Phelsume à quatre ocelles (F) II R

Phelsuma robertmertensi: Mertens's Day Gecko (E), Geco diumo de Robert Mertens (S), Gecko diurne de Robert Mertens (F). Phelsume de Robert Mertens (F) II R

Phelsuma seippi: Seipp's Day Gecko (E), Gecko diume de Seipp (F), Phelsume de Seipp (F) II R

Phelsuma serraticauda: Fan-tailed Day Gecko (E), Serrated Day Gecko (E), Geco diumo de cola dentada (S), Gecko diume à queue plate (F), Phelsume à queue plate (F) II R

Phelsuma standingi: Banded Day Gecko (E). Standing's Day Gecko (E), Geco diumo de Standing (S), Gecko diume de Standing (F), Phelsume de Standing (F) II R

Phelsuma sundbergi: La-Digue Day Gecko (E), Seychelles Giant Day Gecko (E), Geco diumo de Sundberg (S), Gecko diurne géant des Seychelles (F), Phelsume géant des Seychelles (F) II R

Phelsuma trilineata: Three-lined Day Gecko (E), Geco diumo de tres bandas (S), Gecko diume à trois lignes $(\mathrm{F})$, Phelsume à trois lignes (F) II R

Phelsuma vinsoni $=$ Phelsuma omata

Phelsuma v-nigra: Gecko diume de Boettger (F), Phelsume de Boettger (F) II R

Phelsume à courte tête (F): Phelsuma breviceps II R

Phelsume à gorge jaune (F): Phelsuma flavigularis II R

Phelsume à quatre ocelles (F): Phelsuma quadriocellata II R

Phelsume à queue bleue (F): Phelsuma cepediana II R

Phelsume à queue plate (F): Phelsuma serraticauda II R

Phelsume à trois lignes (F): Phelsuma trilineata II R

Phelsume d'Aldabra (F): Phelsuma abbotti II R

Phelsume de Barbour (F): Phelsuma barbouri II R

Phelsume de Befotaka (F): Phelsuma beforakensis II R

Phelsume de Boettger (F): Phelsuma v-nigra II R

Phelsume de Bourbon (F): Phelsuma borbonica II R

Phelsume de Cheke (F): Phelsuma chekei II R

Phelsume de géant Rodrigues (F): Phelsuma gigas II R

Phelsume de Klemmer (F): Phelsuma klemmeri II R

Phelsume de la région Antanosy (F): Phelsuma antanosy II R

Phelsume de l'Ile Maurice (F): Phelsuma guimbeaui II R

Phelsume de l'lle Ronde (F): Phelsuma guentheri II R

Phelsurne de Madagascar (F): Phelsuma madagascariensis II R
Phelsume de Meier (F): Phelsuma nigristriata II R Phelsume de Minuth (F): Phelsuma minuthi II R Phelsume de Parker (F): Phelsuma parkeri II R Phelsume de Robert Mertens (F): Phelsuma roberimertensi II R Phelsume de Rodrigues (F): Phelsuma edwardnewtonii II R Phelsume de Seipp (F): Phelsuma seippi II R

Phelsume de Standing (F): Phelsuma standingi II R Phelsume de Zanzibar (F): Phelsuma dubia II R

Phelsume des Comores (F): Phelsuma comorensis II R

Phelsume des Iles Andaman (F): Phelsuma andamanensis II R

Phelsume des Seychelles (F): Phelsuma longinsulae II R

Phelsume du sud-ouest de Madagascar (F): Phelsuma leiogaster II R

Phelsume géant des Seychelles (F): Phelsuma sundbergi II R

Phelsume modeste (F): Phelsuma modesia II R

Phelsume nain (F): Phelsuma pusilla II R

Phelsume ocelle (F): Phelsuma ocellata II R

Phelsume omé de l'ile Maurice (F): Phelsuma omata II $\mathrm{R}$

Phelsume poussière-d'or (F): Phelsuma laricauda II R

Phelsume rayé ( $F)$ : Phelsuma lineata II $R$

Phelsume tacheté (F): Phelsuma guttata II R

Phelsume variable (F): Phelsuma mutabilis II R

Phelsumes (F): Phelsuma spp. II R

Phigys solitarius: Collared Lory (E), Lori solitario (S), Lori des Fidji

(F), Lori solitaire des îles Fidji (F) II B

Philantomba manticola $=$ Cephalophus monticola

Philippine Cockatoo (E): Cacatua haematuropygia I B

Philippine Crocodile (E): Crocodylus novaeguineae mindorensis I R

Philippine Deer (E): Axis calamianensis I M

Philippine Eagle (E): Pithecophaga jefferyi I B

Philippine Eagle-Owl (E): Bubo philippensis II B

Philippine Falconet (E): Microhierax erythrogenys II B

Philippine Hanging-Parrot (E): Loriculus philippensis II B

Philippine Hawk-Eagle (E): Spizaetus philippensis II B

Philippine Hawk-Owl (E): Ninox philippensis II B

Philippine Scops-Owl (E): Otus megalotis II B

Philippine Serpent-Eagle (E): Spilormis holospilus II B

Philippine Tarsier (E): Tarsius syrichta II M

Philippine Treeshrew (E): Urogale everent II M

Phillipine Tarsier (E): Tarsius syrichta II M

Philodice bryantae: Magenta-throated Woodstar (E), Colibri magenta (F) II B

Philodice dupontii = Tilmatura dupontii

Philodice evelynae $=$ Calliphlox evelynae

Philodice mitchellii: Purple-throated Woodstar (E), Estrellita goripurpura (S), Colibri de Mitchell (F) II B

Phloeocyathus $=$ Conorrochus

Phlogophilus harterti: Peruvian Piedtail (E), Colibri de Hartert (F) II B

Phlogophilus hemileucunus: Ecuadorian Piedtail (E), Colapinto ecuatoriano (S), Colibri à queue mi-blanche (F) II B

Phobobate à trois bandes (F): Phobobates trivintunus II A

Phobobate de Bassler (F): Phobobates bassleri II A

Phobobate de Silverstone (F): Phobobates silverstonei II A

Phobobates spp.: Poison frogs (E), Ranas de puntas de flechas (S). Dendrobates (F) II A

Phobobates bassleri: Pleasing Poison Frog (E), Rana de punta de flecha de Bassler (S), Dendrobate de Bassler (F), Phobobate de Bassler (F) II A

Phobobates silversionei: Silverstone's Poison Frog (E), Rana de punta de flecha de Silversione (S), Dendrobate de Silverstone (F), Phobobate de Silverstone (F) II A

Phobobates trivittatus $=$ Three-striped Poison Frog $(\mathrm{E})$, Dendrobate a trois bandes (F), Phobobate a trois bandes (F) II A

Phocoena dioptrica $=$ Australophocaena dioptrica

Phocoena phocoena: Common Porpoise (E), Harbour Porpoise (E), Marsouin commun (F) II M

Phocoena sinus: Cochito (E), Gulf Porpoise (E), Gulf of California Harbour Porpoise (E), Vaquita (E), Cochito (S), Vaquita (S), Cochito (F), Marsouin du Golfe de Califomie (F), Vaquita (F) I M

Phocoena spinipinnis: Black Porpoise (E), Burmeister's Porpoise (E), Marsopa espinosa (S), Marsouin de Bumneister (F) II M

Phocoena vomerina $=$ Phocoena phocoeno

Phocoenoides dalli: Dall's Porpoise (E), White-flanked Porpoise (E), Marsopa de Dall (S), Marsouin de Dall (F) II M

Phodile calong (F): Phodilus badius II B 
Phodile de Prigogine (F): Phodilus prigoginei II B

Phodiles (F): Phodilus spp. II B

Phodilus badius: Bay Owl (E), Common Bay Owl (E), Oriental BayOwl (E), Lechuza baya de Madagascar (S), Chouette baie (F), Phodile calong (F) II B

Phodilus prigoginei: African Bay Owl (E), Congo Bay-Owl (E), Itombwe Owl (E), Phodile de Prigogine (F) 11 B

Phoeniconaias minor $=$ Phoenicopterus minor

Phoenicoparrus andinus = Phoenicoplerus andinus

Phoenicoparrus jamesi = Phoenicopterus jamesi

Phoenicopteridae spp.: Flamingos (E), Flamencos (S), Fenicoptéridos (S), Flamants (F) II B

Phoenicopterus andinus: Andean Flamingo (E), Flamenco andino (S), Parina grande (S), Flamant des Andes (F) II B

Phoenicopterus chilensis: Chilean Flamingo (E), Flamenco chileno (S), Flamenco común de Chile (S), Parihuana (S), Pariona (S), Flamant du Chili (F) II B

Phoenicopterus jamesi: James's Flamingo (E), Puna Flamingo (E), Flamenco andino chico (S), Flamenco de James (S), Parina chica (S), Flamant de James (F) II B

Phoenicopterus minor: Lesser Flamingo (E), Flamenco enano (S), Flamant nain (F), Petit flamant (F) II B

Phoenicopterus roseus $=$ Phoenicopterus ruber

Phoenicopterus ruber: American Flamingo (E), Caribbean Flamingo (E), Greater Flamingo (E), Flamenco (S), Flamenco común (S), Flamenco de Cuba (S), Flamenco rojo (S), Tococo (S), Flamant de Cuba (F), Flamant rose (F), Flamant rouge (F) II B

Pholidomis rushiae: Tit-hylia (E), Astrild mésange (F), Mésangette rayée (F) III B

Phonygammus keraudrenii = Manucodia keraudrenii

Phoque-moine méditerranéen (F): Monachus monachus I M

Phoque-moines (F): Monachus spp. I M

Phrynosoma coronatum: Coast Homed Lizard (E). Lézard comu (F) Phrynosome couronné (F) II R

Phrynosome couronné (F): Phrynosoma coronatum II R

Phyllangia americana: II In

Phyllangia consagensis: II In

Phyllangia dispersa: II ln

Phyllangia fuegoensis: II In

Phyllangia granulata: II In

Phyllangia hayamaensis: II In

Phyllangia mouchezii: II In

Phyllangia papuensis: II in

Phyllastraea $=$ Mycedium

Phyllobate (F): Phyllobates spp. II A

Phyllobate à bande (F): Phyllobares virtatus II A

Phyllobate à bande dorée (F): Phyllobates aurotaenia II A

Phyllobate bicolore (F): Phyllobates bicolor II A

Phyllobate lugubre (F): Phyllobates lugubris II A

Phyllobate temible (F): Phyllobates terribilis II A

Phyllobares spp.: Poison frogs (E), Ranas de puntas de flechas (S), Phyllobate (F) II A

Phyllobates anthonyi $=$ Epipedobates iricolor

Phyllobates aurotaenia: Kokoe Poison Frog (E), Phyllobate à bande dorée (F) II A

Phyllobates azureiventris = Epipedobates azureiventris

Phyllobates bicolor: Black-legged Poison Frog (E), Two-toned Poison Frog (E), Phyllobate bicolore (F) II A

Phyllobates espinosai $=$ Epipedobates espinosai

Phyllobates lugubris: Lovely Poison Frog (E), Rana de punta de flecha lúgubre (S), Phyllobate lugubre (F) II A

Phyllobates petersi $=$ Epipedobates petersi

Phyllobates pulchripectus = Epipedobates pulchripectus

Phyllobates smaragdinus = Epipedobates smaragdinus

Phyllobates terribilis: Golden Poison Frog (E), Rana de punta de flecha terrible (S), Phyllobate terrible (F) II A

Phyllobates viliatus: Golfoduicean Poison Frog (E), Orange-andblack Poison Frog (E), Rana de punta de flecha rayada (S), Phyllobate à bande (F) II A

Phyllobates zaparo $=$ Epipedobates zaparo

Phyllopora $=$ Astreopora/Stylophora

Phymastrea $=$ Favia

Physeter catodon: Cachelot (E), Pot whale (E), Sperm Whale (E), Spermacet whale (E), Ballena esperma (S), Cachalote (S), Cachalot (F) I M

Physeter macrocephalus $=$ Physeter catodon
Physogyra astraeiformis: II In

Physogyra exerta: 11 In

Physogyra gravieri: II In

Physogyra lichtensteini: Bubble Coral (E) II in

Physogyra somaliensis: II In

Physophyllia ayleni: II In

Piapoco pico rojo (S): Ramphastos tucanus II B

Piapoco pico verde (S): Ramphastos sulfuratus II B

Pic à ventre blanc de Corée (F): Dryocopus javensis richardsi I B

Pic de Tristram (F): Dryocopus javensis richardsi I B

Pic impérial (F): Campephilus imperialis I B

Picaflor abanico verde (S): Lophornis chalybeus II B

Picaflor amatista (S): Calliphlox amethystina II B

Picaflor bronceado (S): Hylocharis chrysura II B

Picaflor cabeciazul (S): Hylocharis cyanus II B

Picaflor cabeza granate (S): Sephanoides sephaniodes II B

Picaflor coliverde (S): Polytmus guainumbi II B

Picaflor coludo rojo (S): Sappho sparganura II B

Picaflor común (S): Chlorostilbon aureoventris II B

Picaflor copetón (S): Stephanoxis lalandi II B

Picaflor corona azul (S): Thalurania glaucopis II B

Picaflor cuelliblanco (S): Leucochloris albicollis II B

Picaflor de Barbijo (S): Heliomaster furcifer II B

Picaflor dorado (S): Hylocharis chrysura II B

Picaflor enano (S): Microstilbon burmeisteri II B

Picaflor escamado (S): Heliomaster squamosus II B

Picaflor frentivioleta (S): Eriocnemis glaucopoides II B

Picaflor gargantiazul (S): Adelomyia melanogenys II B

Picaflor gargantiverde (S): Thalurania furcata II B

Picaflor gigante (S): Patagona gigas II B

Picaflor negro (S): Melanotrochilus fuscus II B

Picaflor orejiazul (S): Colibri thalassinus II B

Picaflor pechiazul (S): Hylocharis sapphirina II B

Picaflor pequeño verde (S): Amazilia versicolor II B

Picaflor serrano ventricanela (S): Oreotrochilus estella II B

Picaflor serrano ventrinegro (S): Oreotrochilus leucopleurus II B

Picaflor ventriazul (S): Colibri coruscans II B

Picaflor ventriblanco (S): Amazilia chionogaster II B

Picaflor ventrinegro (S): Anthracothorax nigricollis II B

Picaflor ventriverde (S): Colibri serrirostris II B

Picaflores (S): Trochilidae spp. I/II B

Picatartes cuelliblanco (S): Picathartes gymnocephalus I B

Picatartes cuelligris (S): Picathartes oreas I B

Picatharte à cou blanc (F): Picathartes gymnocephalus I B

Picatharte à cou gris (F): Picathares oreas I B

Picatharte à tête rouge (F): Picathartes oreas I B

Picatharte chauve (F): Picathartes gymnocephalus I B

Picatharte de Guinée (F): Picathartes gymnocephalus I B

Picatharte du Cameroun (F): Picathartes oreas I B

Picathartes (E/F): Picatharies spp. I B

Picathartes gymnocephalus: Bare-headed Rockfowl (E). Whitenecked Picathartes (E), White-necked Rockfowl (E), Yellowheaded Rockfowl (E), Cuervo calvo de cuello blanco (S).

Picatartes cuelliblanco (S), Picatharte chauve (F), Picatharte de Guinée (F), Picatharte à cou blanc (F) I B

Picathartes oreas: Grey-necked Picathartes (E), Grey-necked Rockfowl (E), Red-headed Rockfowl (E), Cuervo calvo de cuello gris (S), Picatartes cuelligris (S), Picathante du Cameroun (F), Picatharte à cou gris (F), Picatharte à tête rouge $(F)$ I B

Pichico negro (S): Callimico goeldii I M

Pico espina bronceado (S): Chalcostigma heteropogon II B

Pico lanza frentiazul (S): Doryfera johannae II B

Pico lanza frentiverde (S): Doryfera ludovicae II B

Pico-de-Hoz colihabano (S): Eutoxeres condamini II B

Pico-de-Hoz puntiblanco (S): Eutoxeres aquila II B

Picoespina arcoiris (S): Chalcostigma herrani II B

Picoespina dorsiazul (S): Chalcostigma stanleyi II B

Picoespina dorsipurpura (S): Ramphomicron microrhynchum II B

Picoespina gorrirufa (S): Chalcostigma ruficeps II B

Picolargo coroniazul (S): Heliomaster longirostris II B

Picolargo coronioscuro (S): Heliomaster constantii II B

Picozapato (S): Balaeniceps rex II B

Pics (F): Picidae spp. INC B

Piebald Dolphin (E): Cephalorhynchus commersonii II M

Pied Falconet (E): Microhierax melanoleucos 11 B

Pied Goshawk (E): Accipiter albogularis II B 
Pied Harrier (E): Circus melanoleucos II B

Pied Mannikin (E): Lonchura fringilloides III B

Pied Sparrowhawk (E): Accipiser albogularis II B

Pied Tamarin (E): Saguinus bicolor I M

Pied-Hombills (E): Anthracoceros spp $/$ Tockus fasciatus IL/NC B Piedtails (E): Phlogophilus spp. II B

Pigargo americano (S): Haliaeetus leucocephalus I B

Pigargo cabeciblanco (S): Haliaeelus leucocephalus I B

Pigargo cabeciblanco meridional (S): Haliaeetus leucocephalus I B

Pigargo coliblanco (S): Haliaeetus albicilla I B

Pigargo coliblanco de Groenlandia (S): Haliaeetus albicilla I B

Pigargo de las Salomón (S): Haliaeetus sanfordi II B

Pigargo de Madagascar (S): Haliaeetus vociferoides II B

Pigargo de Pallas (S): Haliaeelus leucoryphus II B

Pigargo de Sanford (S): Haliaeetus sanfordi II B

Pigargo de Steller (S): Haliaeefus pelagicus II B

Pigargo de vientre blanco (S): Haliaeetus leucogaster II B

Pigargo europeo (S): Haliaeerus albicilla I B

Pigargo gigante (S): Haliaeetus pelagicus II B

Pigargo malgache (S): Haliaeetus vociferoides II B

Pigargo oriental (S): Haliaeefus leucogaster II B

Pigargo vocinglero (S): Haliaeetus vocifer II B

Pigarguillo común (S): Ichthyophaga ichthyaetus II B

Pigarguillo menor (S): Ichthyophaga humilis II B

Pigeon à camail (F): Caloenas nicabarica $1 \mathrm{~B}$

Pigeon à collerette (F): Caloenas nicabarica I B

Pigeon à nuque bronzée (F): Columba iriditorques III B

Pigeon biset (F): Columba livia III B

Pigeon de Guinée (F): Columba guinea III B

Pigeon de Mindoro (F): Ducula mindorensis I B

Pigeon gris (F): Columba unicincia III B

Pigeon gris écailleux (F): Columba unicincta III B

Pigeon Hawk (E): Falco columbarius II B

Pigeon nicobar (F): Caloenas nicobarica I B

Pigeon rose (F): Columba mayeri III B

Pigeon roussard (F): Columba guinea III B

Pigeon vert à épaulettes violettes (F): Treron waalia III B

Pigeon vert à front nu (F): Streptopelia vinacea III B

Pigeons (E): Columbidae spp. I/I/IIUNC B

Pigeons (F): Columba spp. III/NC B

Pig-footed Bandicoot (E): Chaeropus ecaudarus I M

Pigtail Macaque (E): Macaca nemestrina II M

Pig-tailed Langur (E): Nasalis concolor I M

Pig-tailed Macaque (E): Macaca nemestrina II M

Pigtoes (E): Fusconaia spp. III In

Pijiji aliblanco (S): Dendrocygna autumnalis III B

Pijiji canelo (S): Dendrocygna bicolor III B

Pilbara Monitor (E): Varanus pilbarensis II R

Pileated Gibbon (E): Hylobares pileatus I M

Pileated Parakeet (E): Purpureicephalus spurius II B

Pileated Parrot (E): Pionopsilla pileala I B

Pilet (F): Anas acuta III B

Pillar Coral (E): Dendrogyra cylindrus II in

Pilot whales (E): Globicephala spp. II M

Pilsbry's Dwarf Boa (E): Tropidophis pilsbryi II R

Pimpleback mussels (E): Plethobasus spp. I In

Pinchaque (S): Tapirus pinchaque I M

Pinche Marmoset (E): Saguinus geoffroyi I M

Pineapple Tortoise (E): Indotesiudo elongata II R

Pingüino de Humboldt (S): Spheniscus humboldti I B

Pingüino del Cabo (S): Spheniscus demersus II B

Pink Cockatoo (E): Cacatua leadbeateri II B

Pink Conch (E): Strombus gigas II In

Pink Mucket Pearly Mussel (E): Lampsilis orbiculata orbiculata I In

Pink Pigeon (E): Columba mayeri III B

Pink-cheeked Waxbill (E); Estrilda troglodytes III B

Pink-headed Dove (E): Streptopelia roseogrisea III B

Pink-headed Duck (E): Rhodonessa caryophyllacea I B

Pink-throated Brilliant (E): Heliodoxa gularis II B

Pintade à poitrine blanche (F): Agelastes meleagrides III B

Pintades (F): Acryllium vulturinum/Agelastes spp/Guttera spp Numida meleagris III/NC B

Pintado de pecho blanco (S): Agelastes meleagrides III B

Pintado pechiblanca (S): Agelastes meleagrides III B

Pin-tailed Whydah (E): Vidua macroura III B
Pintails (E): Anas acuta/Anas bahamensis/Anas eatoni/Anas georgica IIL/NC B

Pione à bec rouge (F): Pionus sordidus II B

Pione à couronne blanche (F): Pionus senilis II B

Pione à tête bleue (F): Pionus mensiruus II B

Pione de Maximilien (F): Pionus maximiliani II B

Pione noire (F): Pionus chalcopterus II B

Pione pailletée (F): Pionus rumultuosus II B

Pione violette (F): Pionus fuscus II B

Piones (F): Pionus spp. II B

Pionites leucogaster. White-bellied Caique (E), White-bellied Parrot (E), Lorito rubio (S), Caïque à ventre blanc (F) II B

Pionites melanocephala: Black-headed Caique (E), Black-headed Parrot (E), Lorito chirlecrés (S), Perico calzoncito (S), Cä̈que maïpouri (F) II B

Pionopsitra barrabandi: Barraband's Parrot (E), Orange-cheeked Parrot (E), Lorito carinaranja (S), Perico cachete amarillo (S), Caique de Barraband (F) II B

Pionopsitta caica: Caica Parrot (E), Lorito caica (S), Perico cabecinegro (S), Caïque à tête noire (F) II B

Pionopsitta haematotis: Brown-hoodec' Parrot (E), Lorito encapuchado (S), Loro orejirrojo (S), Caïque à capuchon $(\mathrm{F})$ II B

Pionopsitta pileata: Pileated Parrot (E), Red-capped Parrot (E), Cúiu cúiu (S), Lorito carirrojo (S), Lorito pileado (S), Caïque mitré (F), Perroquet à oreilles $(\mathrm{F}) \mathbf{I}$

Pionopsitta pulchra: Beautiful Parrot (E), Rose-faced Parrot (E), Lorito carirrosado (\$), Loro cachtirrosa (S), Caïque à joues roses (F) II B

Pionopsinta pyrilia: Saffron-headed Parrot (E), Lorito cabecigualdo (S), Perico cabecidorado (S), Caique de Bonaparte (F) II B

Pionus chalcopterus: Bronze-winged Parrot (E), Cotorra negra (S), Loro alibronceado (S), Pione noire (F) II B

Pionus fuscus: Dusky Parrot (E), Cotorra morada (S), Loro morado (S), Pione violette (F) II B

Pionus maximiliani: Scaly-headed Parrot (E), Loro choclero (S), Pione de Maximilien (F) II B

Pionus menstruus: Blue-headed Parrot (E), Red-vented Parrot (E), Cotorra cabeciazul (S), Loro cabeciazul (S), Pione à tête bleue (F) II B

Pionus senilis: White-capped Parrot (E), White-crowned Parrot (E), Loro caroniblanco (S), Loro senil (S), Pione à couronne blanche (F) II B

Pionus seniloides $=$ Pionus tumultuosus

Pionus sordidus: Red-billed Panrot (E), Loro piquirojo (S), Perico pico rojo (S), Pione à bec rouge (F) II B

Pionus rumultuosus: Plum-crowned Parrot (E), Cotorra cabeciblanca (S), Loro tumultuoso (S), Pione pailletée (F) II B

Pipile jacutinga: Black Fronted Curassow (E), Black-fronted PipingGuan (E), Pava yacutinga (S), Yacutingá frentinegra (S), Pénélope siffleuse $(F)$, Pénélope à front noir $(F)$, Pénélope à plastron $(\mathrm{F})$ I B

Pipile pipile: Trinidad Piping-Guan (E). Trinidad White-headed Curassow (E), Trinidad White-headed Piping Guan (E), Pava de Trinidad (S), Pava rajadora (S), Yacutinga cariazul (S), Pénélope siffleuse de la trinité (F) I B

Piping-Guans (E): Pipile spp. INC B

Piquero de Abbott (S): Papasula abbotti I B

Pirarucu (E/F): Arapaima gigas II F

Pirenestes ventrinegro (S): Pyrenestes ostrinus III B

Pit vipers (E): Bothrops spp/Calloselasma $\mathrm{spp} /$ Trimeresurus $\operatorname{spp} /$ Tropidolaemus wagleri III/NC R

Pita de Koch (S): Pitta kochi I B

Pita nympha (S): Pitta nympha II B

Pithecia aequatorialis: Equatorial Saki (E) II M

Pithecia albicans: Black-and-white Saki (E), Buffy Saki (E), Saki amazónico (S) II M

Pithecia hirsuta $=$ Pithecia monachus

Pithecia irrorata: Saki de cara pelada (S) II M

Pithecia monachus: Monk Saki (E), Red-bearded Saki (E), Saki chevelu (F) II M

Pithecia monachus irrorata = Pithecia irrorata

Pithecia pithecia: Pale-headed Saki (E), White-faced Saki (E), Saki à tête pâle (F) II M 
Pithecophaga jeffenyi: Great Philippine Eagle (E), Monkey-eating Eagle (E), Philippine Eagle (E), Aguila comemonos (S), Aguila monera (S), Aigle des Philippines (F), Aigle des singes (F), Aigle mangeur de singes $(F)$. Pithécophage des Philippines $(F)$ I B

Pithécophage des Philippines (F): Pithecophaga jefferyi I B

Pitilia alirroja (S): Pyilia phoenicoptera III B

Pitiu (S): Podocnemis sextuberculata II R

Pito imperial (S): Campephilus imperialis I B

Pito ventriblanco de Corea (S): Dryocopus javensis richardsi I B

Pitón acuática de Papua (S): Morelia papuana II R

Pitón ametista (S): Morelia amethistina II R

Pitón anillada de Bismarck (S): Morelia boa II R

Pitón de Albert (S): Morelia albertisii II R

Pitón de Angola (S): Python anchietae II R

Pitón de Boelen (S): Morelia boeleni II R

Pitón de cabeza negra (S): Aspidites melanocephalus II R

Pitón de Calabar (S): Calabaria reinhardtii II R

Pitón de la India (S): Python molurus molurus I R

Pitón de Macklot (S): Morelia mackloti II R

Pitón de Ramsay (S): Aspidites ramsayi II R

Pitón de Seba (S): Python sebae II R

Pitón de Timor (S): Python timoriensis II R

Pitón diamantina (S): Morelia spilota II R

Pitón enana (S): Morelia childreni II R

Pitón excavadora (S): Loxocemus bicolor II R

Pitón malaya (S): Python curtus II R

Pitón oliva (S): Morelia olivacea II R

Pitón real (S): Python regius II R

Pirón reticulada (S): Python reficulatus II R

Pitóns (S): Boidae spp. I/II R

Pitorreal ocotero (S): Campephilus imperialis I B

Pitta brachyura nympha = Pitta nympha

Pitta guajana: Banded Pitta (E), Blue-tailed Pitta (E), Brève azurine (F), Brève à queue bleue (F) II B

Pitta gurneyi: Gumey's Pitta (E), Brève de Gurney (F) I B

Pitta kochi: Koch's Pitta (E), Whiskered Pitta (E), Pita de Koch (S), Brève de Koch (F) I B

Pitta nympha: Fairy Pitta (E), Pita nympha (S), Brève du Japon (F), Brève migratrice (F) II B

Pittas (E): Pitta spp. I/IINC B

Placocyathus $=$ Meandrina

Placotrochides alabastrum: II In

Placotrochides frustra: II in

Placotrochides scaphula: II In

Placotrochus fuscus: II In

Placotrochus laevis: II In

Placotrochus pedicellatus: II In

Plagiola florentina $=$ Epioblasma curtisii

Plagiola florentina $=$ Epioblasma walker

Plagiola obliquata $=$ Epioblasma sulcata perobliqua

Plagiola torulosa $=$ Epioblasma torulosa gubernaculum

Plagiola torulosa = Epioblasma torulosa rangiana

Plain Chachalaca (E); Ortalis vetula III B

Plain Lizard (E): Varanus salvator II R

Plain Parakeet (E): Brotogeris tirica II B

Plain Pocketbook Pearly Mussel (E): Lampsilis satur I In

Plain-bellied Emerald (E): Amazilia leucogaster II B

Plain-breasted Hawk (E): Accipiter ventralis II B

Plain-capped Starthroat (E): Heliomaster constantii II B

Plain-pouched Hornbill (E): Aceros subruficollis I B

Plains Rat-kangaroo (E): Caloprymnus campestris I M

Plana (S): Dermatemys mawii II R

Planalto Hermit (E): Phaethornis pretrei II B

Plantain-eaters (E): Corythaeola cristata/Crinifer $\operatorname{spp} /$ Musophaga spp. II/II/NC B

Platanista spp.: Susus (E), Platanistes (F), Sousous (F) I M

Platalea leucorodia: Eurasian Spoonbill (E), White Spoonbill (E), Espátula blanca (S), Espátula común (S), Spatule blanche (F) II B

Platanista gangetica: Ganges River Dolphin (E), Ganges Susu (E), Delfin del Ganges (S), Plataniste du Gange (F), Sousou (F) I M

Platanista gangetica minor $=$ Platanista minor

Platanista indi $=$ Platanista minor

Platanista minor: Indus Dolphin (E), Indus River Dolphin (E), Susu (E), Delfin del Indo (S), Plataniste de l'Indus (F) I M
Plataniste de l'Indus (F): Platanista minor $1 \mathrm{M}$

Plataniste du Gange (F): Platanista gangetica I M

Platanistes (F): Platanista spp. I M

Platycercus adelaidae $=$ Platycercus elegans $x$ Platycercus flaveolus

Platycercus adscitus: Mealy Rosella (E), Pale-headed Rosella (E), Perico pálido (S), Perruche palliceps (F), Pernche à tête pâle (F) II B

Platycercus bamardi: Barnard's Parakeet (E), Mallee Ringneck (E), Perico de Barnard (S), Perruche de Bamard (F) II B

Platycercus caledonicus: Green Rosella (E), Tasmanian Rosella (E), Perico de Tasmania (S), Perruche calédonienne (F), Perruche à ventre jaune (F) II B

Platycercus elegans: Crimson Rosella (E), Pennant's Parakeet (E), Perico elegante (S), Perruche de Pennant (F) II B

Platycercus eximius: Eastern Rosella (E), Golden-mantled Rosella (E), Perico multicolor (S), Perruche omnicolore (F) II B

Platycercus flaveolus: Yellow Rosella (E), Perico gualda (S), Perruche flavéole (F) II B

Plarycercus icterotis: Stanley Parakeet (E), Westem Rosella (E), Perico carigualdo (S), Perruche de Stanley (F), Perruche à oreilles jaunes (F) II B

Platycercus venustus: Northem Rosella (E), Perico gracioso (S), Perruche gracieuse (F) II B

Platycercus zonarius: Port Lincoln Ringneck (E), Twenty-eight Parakeet (E), Perico de Port Lincoln (S), Perruche à collier jaune (F) $11 \mathrm{~B}$

Platycyathus spp.: II In

Platygyra contorta: II In

Platygyra daedalea: II In

Plarygyra lamellina: II In

Platygyra pini: II In

Platygyra nukyuensis: II In

Platygyra sinensis: II In

Platygyra verweyi: II In

Platygyra yaeyamaensis: II In

Platypus Frog (E): Rheobatrachus silus II A

Platyrrhinus lineatus: White-lined Bat (E), Murcielago de estrias

blancas (S), Sténoderme pseudo-vampire (F) $111 \mathrm{M}$

Platytrochus compressus: II In

Platytrochus hastatus: II In

Platytrochus laevigatus: II In

Platyirochus parseptatus: II in

Platyirochus stokesi: II In

Pleasing Poison Frog (E): Phobobates bassleri II A

Plectropterus gambensis: Spur-winged Goose (E), Ganso de Gambia (S), Ganso espolonado (S), Canard armé (F) III B

Plerogyra eurysepta: II In

Plerogyra simplex: II In

Plerogyra sinuosa: II In

Plerogyra turbida: II In

Plesiastrea lilli: II In

Plesiastrea versipora: II In

Plesioseris australiae: II In

Plethobasus cicatricosus: White Warty-back Pearly Mussel (E), White Wartyback (E) I In

Plethobasus cooperianus: Orange-footed Pimpleback Mussel (E), Orangefoot Pimpleback (E) I In

Pleuractis $=$ Fungia

Pleurobema clava: Club Naiad (E), Clubshell (E), Clubshell Pearly Mussel (E) II in

Pleurobema plenum: Rough Pigtoe (E), Rough Pigtoe Pearly Mussel (E) I In

Pleurocyathus $=$ Conotrochus

Pliobothrus echinatus: II In

Pliobothrus gracilis: II In

Pliobothrus spinosa: II In

Pliobothrus symmetricus: II In

Pliobothrus tubulatus: II In

Plocepasser superciliosus: Chestnut-crowned Sparrow-Weaver (E), Tejedor pardal capirotado (S), Mahali à calotte marron (F). Moineau-tisserin (F) III B

Ploceus albinucha: Maxwell's Black Weaver (E), White-naped Weaver (E), Tejedor negro albinuca (S), Tisserin de Maxwell (F) III B

Ploceus aurantius: Orange Weaver (E), Tejedor naranja (S), Tisserin orangé (F) III B 
Ploceus capitalis $=$ Ploceus melanocephalus

Ploceus cucullatus: Layard's Black-headed Weaver (E), Spot-backed Weaver (E), Village Weaver (E), Tejedor cogullado (S), Tisserin gendarme ( $F$ ) III B

Ploceus heuglini: Heuglin's Masked-Weaver (E), Tejedor de Heuglin (S), Tisserin masqué (F) III B

Ploceus luteolus: Little Weaver (E), Sitragra chico común (S), Tisserin minule (F) III B

Ploceus melanocephalus: Black-headed Weaver (E), Yellow-backed Weaver (E), Tejedor cabecinegro (S), Tisserin à tête noire (F) III B

Ploceus nigerrimus: Vieillot's Black Weaver (E), Tejedor negro de Vieillot (S), Tisserin noir (F), Tisserin noir de Vieillot (F) III B Ploceus nigriceps $=$ Ploceus cucullatus

Ploceus nigricollis: Black-necked Weaver (E), Tejedor cuellinegro (S), Tisserin à cou noir (F), Tisserin à lunettes (F) III B

Ploceus pelzelni: Slender-billed Weaver (E), Sitagra de Pelzeln (S), Tisserin de Pelzeln (F), Tisserin nain (F) III B

Ploceus preussi: Golden-backed Weaver (E), Preuss's Weaver (E), Yellow-capped Weaver (E), Tisserin de Preuss (F), Tisserin à dos doré (F) III B

Ploceus superciliosus = Pachyphantes superciliosus

Ploceus tricolor: Yellow-mantled Weaver (E), Tejedor tricolor (S) Tisserin tricolore (F) III B

Ploceus velatus vitellinus $=$ Ploceus vitellinus

Ploceus vitellinus: Vitelline Masked-Weaver (E), Tejedor velado (S), Tisserin vitellin (F), Tisserin à tête rousse (F) III B

Plovercrest (E): Stephanoxis lalandi II B

Plumbeous Forest-Falcon (E): Micrastur plumbeus II B

Plumbeous Hawk (E): Leucopternis plumbea II B

Plumbeous Kite (E): Ictinia plumbea II B

Plum-crowned Parrot (E): Pionus tumultuosus II B

Plumeleteers (E): Chalybura spp. II B

Plum-faced Lorikeet (E): Oreopsittacus arfaki II B

Plum-headed Parakeet (E): Psittacula cyanocephala II B

Poc de Atitán (S): Podilymbus gigas I B

Pochards (E): Aythya spp Nette spp. IIU/NC B

Pocillopora ankeli: II In

Pocillopora capitata: II In

Pocillopora clavaria: II In

Pocillopora damicomis: II In

Pocillopora diomedeae: II in

Pocillopora eydouxi: II in

Pocillopora frondosa: II in

Pocillopora informis: II In

Pocillopora lacera: II in

Pocillopora ligulata: 11 In

Pocillopora mauritiana: II In

Pocillopora meandrina: II In

Pocillopora molokensis: II in

Pocillopora plicara: II In

Pocillopora porosa: II In

Pocillopora robusta: II in

Pocillopora setchelli: II in

Pocillopora solida: II In

Pocillopora squarrosa: II in

Pocillopora symmetrica: II in

Pocillopora verrucosa: II in

Pocillopora woodjonesi: II In

Pocketbook mussels (E): Lampsilis spp. I/NC In

Podabacia crustacea: II In

Podabacia motuporensis: II In

Podarcis lilfordi: Lilford's Wall Lizard (E), Lagartija balear (S), Lézard de Lilford (F), Lézard des Baléares (F) II R

Podarcis pityusensis: Ibiza Wall Lizard (E), Lagartija de las Pitiusas (S), Lézard des Pityuses (F) II R

Podilymbus gigas: Atitlan Grebe (E), Giant Grebe (E), Giant Piedbilled Grebe (E), Poc de Atitlán (S), Somormujo de Atitlán (S), Zambullidor de Atitlán (S), Zampullín del Atitlán (S), Grèbe du lac Atitlan (F), Grèbe géant (F) I B

Podocnémide à tête rouge (F): Podocnemis erythrocephala II R

Podocnémide de Cayenne (F): Podocnemis unifilis II R

Podocnémide de Duméril (F): Pelocephalus dumeriliana II $\mathrm{K}$

Podocnémide de Léwy (F): Podocnemis lewyana II R

Podocnémide de l'Orénoque (F): Podocnemis vogli II R
Podocnémide de Madagascar (F): Erymnochelys madagascariensis II R

Podocnémide de Vogl (F): Podocnemis vogli II R

Podocnémide élargie (F): Podocnemis expansa II R

Podocnémide tuberculée (F): Podocnemis sextuberculata II R

Podocnémides (F): Podocnemis spp. II R

Podocnemis spp.: South American river turtles (E), Podocnémides (F), Tortues fluviatiles (F) II R

Podocnemis cayennensis $=$ Podocnemis erythrocephala

Podocnemis dumeriliana $=$ Pelrocephalus dumeriliana

Podocnemis erythrocephala: Red-headed Amazon River Turtle (E), Red-headed River Turtle (E), Red-headed Sideneck (E), Chimpire (S), Podocnémide à tête rouge (F) II R

Podocnemis expansa: Arau (E), Giant South American Turtle (E), South American River Turtle (E), Tartaruga (E), Podocnémide élargie (F) II R

Podocnemis lewyana: Magdalena River Turtle (E), Rio Magdalena River Turtle (E), Tortuga de agua (S), Podocnémide de Léwy (F) II R

Podocnemis madagascariensis $=$ Eymnochelys madagascariensis

Podocnemis sextuberculata: Six-tubercled Amazon River Turle (E), Six-tubercled River Turtle (E), Cupiso (S), Iaça (S), Pitiu (S), Podocnémide tuberculée (F) II R

Podocnemis unifilis: Yellow-headed Sideneck (E). Yellow-spotted River Turte (E), Yellow-spotted Sideneck Turtle (E), Terecay (S), Podocnémide de Cayenne (F) II R

Podocnemis vogli: Llanos Sideneck Turtle (E), Savanna Sideneck Turtle (E), Podocnémide de Vogl (F), Podocnémide de l'Orénoque (F) II $\mathrm{K}$

Poephila cincra cincia: Southem Black-throated Finch (E), Diamante de pecho negro (S), Diamant à bavette (F) II B

Pohnpei Flying-fox (E): Pteropus molossinus I M

Pohnpei Lorikeet (E): Trichoglossus rebiginosus II B

Pohnpei Lory (E): Trichoglossus rubiginosus II B

Poicephalus crassus: Niam-niam Partot (E), Lorito niam-niam (S), Perroquet des niam-niam (F) II B

Poicephalus cryptoxanthus: Brown-headed Parrot (E), Lorito cabecipardo (S), Perroquet à tête brune (F) II B

Poicephalus flavifrons: Yellow-fronted Parrot (E), Lorito carigualdo (S), Perroquet à face jaune (F) II B

Poicephalus gulielmi: Jardine's Parrot (E), Red-crowned Parrot (E), Red-fronted Parrot (E), Lorito frentirojo (S), Papagayo de Gulielm (S), Perroquet vert du Congo (F), Perroquet vert à calotie rouge (F), Perroquet à calotte rouge (F) II B

Poicephalus meyeri: Brown Parrot (E), Meyer's Parrot (E), Lorito de Meyer (S), Perroquet de Meyer (F) II B

Poicephalus robustus: Brown-necked Parrot (E), Cape Parrot (E), Lorito robusto (S), Papagayo robusto (S), Perroquet du Cap (F), Perroquet robuste (F) II B

Poicephalus rueppellit: Rueppell's Parrot (E), Lorito de Rüppell (S), Rüppell's Parrot (S), Perroquet de Rüppell (F) II B

Poicephalus rufiventris: African Orange-bellied Parrot (E), Redbellied Parrot $(E)$, Lorito ventrirrojo (S), Perroquet à ventre rouge (F) II B

Poicephalus senegalus: Senegal Parrot (E), Lorito Senegalés (S), Papagayo senegalés (S), Perroquet youyou (F), Perroquet à tête grise (F), Youyou (F) II B

Poison frogs (E): Dendrobates spp. II AVPhyllobates spp. II A/Allobaies spp. II A/Epipedobales spp. II $A$ Minyobates spp. II A/Phobobates spp. II A

Poisonous lizards (E): Heloderma spp. II R

Poisson cavemicole d'Afrique (F): Caecobarbus geertsi II F

Poisson spatule (F): Polyodon spothula II F

Polar Bear (E): Ursus maritimus II M

Polemaetus bellicosus: Martial Eagle (E), Aguila marcial (S), Aigle martial (F) II B

Polihierax insignis: Fielden's Falconet (E), White-rumped Falcon (E), White-rumped Falconet (E), Halconcito asiático (S), Halconcito colilargo de Asia (S), Fauconnet à pattes jaunes $(F)$ II I

Polihierax semitorquatus: African Pygmy Falcon (E), Pygmy Falcon (E), Halconcito africano (S), Fauconnet d'Afrique (F) II B

Polkadot Poison Frog (E): Dendrobates arboreus II A

Pollack Whale (E): Balaenoptera borealis I M

Polyastra $=$ Pavona 
Polyboroides radialus: Madagascar Gymnogene (E), Madagascar Harrier-Hawk (E), Aguilucho-caricalvo malgache (S), Azor culebrero (S), Gymnogène de Madagascar (F), Petit serpentaire (F) II B

Polyboroides typus: African Gymnogene (E), African Harrier-Hawk (E), Aguilucho-caricalvo común (S), Gymnogène d'Afrique (F) II B

Polyborus lutosus: Guadalupe Caracara (E), Carancho de Guadalupe (S), Caracara de Guadalupe (F) II B

Polyborus plancus: Common Caracara (E), Crested Caracara (E), Caracara carancho (S), Caracara común (S), Carancho (S), Caricare encrestado (S), Traro (S), Caracara commun (F), Caracara huppé (F) II B

Polycyathus andamanensis: II In

Polycyathus allanticus: II In

Polycyathus banyulensis: II In

Polycyathus conceptus: II In

Polycyathus difficilis: II In

Polycyarhus fulvus: II In

Polycyathus furanaensis: II In

Polycyathus fuscomarginatus: II In

Polycyathus hodgsoni: II In

Polycyathus hondaensis: II In

Polycyathus isabela: II In

Polycyathus marigondoni; II In

Polycyathus mediterraneus: II In

Polycyarhus muellerae: II In

Polycyathus norfolkensis: II In

Polycyathus palifera: II In

Polycyathus pallidus: II in

Polycyathus senegalensis: II in

Polycyathus verrilli: II In

Polymyces fragilis: II In

Polymyces montereyensis: II In

Polymyces wellsi: II In

Polyodon spathula: Duckbill Cat (E), Paddlefish (E), Spadefish (E) Spoonbill Cat (E), Poisson spatule (F) II F

Polyonymus caroli: Bronze-tailed Comet (E), Colibri de Bourcier (F) II B

Polyphyllia novaehibemiae: II In

Polyphyllia talpina: II In

Polyplancta aurescens $=$ Heliodoxa aurescens

Polyplectron bicalcaratum: Common Peacock-Pheasant (E), Grey Peacock-Pheasant (E), Iris Peacock-Pheasant (E), Espolonero chinguis ( $\mathrm{S}$ ), Faisán de cola ocelada gris (S), Faisán real gris (S), Éperonnier chinquis (F), Éperonnier gris (F) II B

Polyplectron emphanum: Napoleon's Peacock-Pheasant (E), Palawan Peacock-Pheasant (E), Espolonero de Palawan (S), Faisán real de Palaguăn (S), Éperonnier Napoléon (F), Éperonnier de Palawan (F) I B

Polyplectron germaini: Germain's Peacock-Pheasant (E), Espolonero de Germain (S), Faisán de cola ocelada de Germain (S). Faisán real de Germain (S), Éperonnier de Germain (F) II B

Polyplectron inopinatum: Mountain Peacock-Pheasant (E), Rothschild's Peacock-Pheasant (E), Espolonero de Rothschild (S), Faisán de cola ocelada indómito (S), Éperonnier de Rothschild (F) III B

Polyplectron malacense: Crested Peacock-Pheasant (E), Malayan Peacock-Pheasant (E), Espolonero malayo (S), Faisán de cola ocelada malayo (S), Faisán real malayo (S), Éperonnier de Hardwick (F), Éperonnier de Malaisie (F), Éperonnier malais (F) II B

Polyplectron malacense schleiermacheri = Polyplectron schleiermacheri

Polyplectron schleiermacheri: Bornean Peacock-Pheasant (E), Espolonero de Borneo (S), Éperonnier de Bornéo (F) II B

Polystachys $=$ Acropora

Polytelis alexandrae: Alexandra's Parrot (E), Princess Parrot (E), Princess of Wales Parakeet (E), Perico princesa (S), Perruche d'Alexandra $(\boldsymbol{F})$, Perruche princesse $(\mathbf{F})$, Perruche à calotte bleue (F) II B

Polytelis anthopeplus: Regent Parro (E), Rock Peppler (E). Perico regente (S), Perruche mélanure (F) II B

Polytelis swainsonii: Barraband Parakeet (E), Green Leek Parrot (E), Superb Parrot (E), Perico soberbio (S), Perruche de Barraband (F) II B
Polytmus guainumbi: White-tailed Goldenthroat (E), Colibri gargantidorado (S), Picaflor coliverde (S), Colibri guaïnumbi (F) II B

Polytmus milleri: Tepui Goldenthroat (E), Colibrí tepui (S), Colibri des tépuis $(F)$ II $B$

Polytmus theresiae: Green-tailed Goldenthroat (E), Garganta de oro coliverde (S), Colibri tout-vert (F) II B

Pondicherry Vulture (E): Sarcogyps calvus II B

Pongo pygmaeus: Orang-utan (E), Orangután (S), Orang-outan (F) I M

Pontoporia blainvillei: Franciscana (E), La Plata River Dolphin (E), Delfín de la Plata (S), Tonina (S), Dauphin de la Plata (F) II M

Popelairia conversii: Green Thomtail (E), Colacerda verde (S), Coquette a queue fine (F) $\mathbf{I} \mathbf{B}$

Popelairia langsdorffi: Black-bellied Thomtail (E), Colacerda ventrinegra (S), Coqueta cola de Lira (S), Coquette de Langsdorff (F) II B

Popelairia letitiae: Coppery Thomtail (E), Coquette de Létitia (F) II B

Popelairia popelairi: Wire-crested Thomtail (E), Colacerda crestuda (S), Coquette de Popelaire (F) II B

Porc-épic du nord de l'Afrique (F): Hystrix cristata III M

Porc-épic préhensile (F): Sphiggurus mexicanus III M

Porculla Hermit (E): Phaethomis griseogularis II B

Porcupines (E): Hystricidae spp/Erethizontidae spp. IIINC M

Porites annae: II In

Porites aranetai: II In

Porites astreoides: Mustard Hill Coral (E) II In

Porites attenuata: II In

Porites australiensis: II In

Porites baracoensis: II In

Porites bemardi: II In

Porifes branneri: II In

Porites brighami: II In

Porifes califomica: II In

Porites cocosensis: II In

Porites colonensis: II In

Porites columnaris: 11 In

Porites compressa: II In

Porites cribripora: 11 in

Porites cumulatus: II In

Porites cylindrica: II In

Porites danae: II In

Porites deformis: II In

Porites densa: II In

Porites duerdeni: II In

Porites echinulata: II In

Puriles eridani: II In

Porites erosa: II in

Porites evermanni: II In

Porites exilis: II In

Porites exsena: II In

Poriles faustinoi: II In

Poriles favosa: II In

Porites gaimardi: II In

Porites galeata: II in

Porites hawaiiensis: II In

Porites heronensis: II In

Porites horizontalata: II In

Porites irregularis: II In

Porites laristellata: II In

Parites lichen: II In

Porites limosa: II In

Porires lobata: II In

Porites lutea: II In

Porites mannarensis: II In

Porites matthait: II In

Porites mayeri: II In

Porites minicoiensis: II In

Porires mordax: II In

Porites mucronata: II In

Porites murrayensis: II In

Porites myrmidonensis: II In

Poriles negrosensis: II In

Porites nigrescens: II In

Porites nodifera: II In 
Porites nodulosa: II In Porites okinawensis: II In Porites palmata: II in Porites parvistellata: II In Porites porites: Club Finger Coral (E) II in Porites portoricensis: II In Porites profundus: II In Porites pukoensis: II In Porites rus: II In

Porites saccharata: II In Porites sillimaniani: II In Porites solida: II ln Porites somaliensis: II In Poriles stephensoni: II In Porites studeri: II In Porites superflua: II In Porites sverdrupi: II In Porites tenuis: II in Porites trimurata: II In Poriles umbellifera: II In Porites vaughani: II In Porites waylandi: II In

Poroto Mountain Chameleon (E): Chamaeleo incomusus II R Poroto Three-horned Chameleon (E): Chamaeleo fuellebomi II R Porpoises (E): CETACEA spp. I/II M

Porrón pardo (S): Aythya nyroca III B

Port Lincoln Ringneck (E): Platycercus zonarius II B

Porte-lance de Jeanne (F): Doryfera johannae II B

Porte-lance de Louise (F): Doryfera ludovicae II B

Porte-lances (F): Doryfera spp. II B

Porte-musc (F): Moschus moschiferus I M

Porte-traîne lesbie (F): Lesbia victoriae II B

Porte-traîne nouna (F): Lesbia nuna II B

Porte-traines (E): Lesbia spp. II B

Porthidium nasutum $=$ Bothrops nasuta

Porthidium nummifer $=$ Bothrops nummifer

Porthidium ophryomegas $=$ Bothrops ophryomegas

Pot whale (E): Physeter catodon I M

Potamilus capax: Fat Pocketbook (E), Fat Pockerbook Pearly Mussel (E) I In

Poto de Bosman (S): Perodicticus potto II M

Poto dorado (\$): Arctocebus calabarensis II M

Potos (F): Potos flavus III M

Potos flavus: Kinkajou (E), Chosna (S), Cuchumbi (S), Cusumbi (S), Godoy (S), Marta (S), Martucha (S), Mico de noche (S). Micoleón (S), Potos (F) $111 \mathrm{M}$

Potto (E/F): Perodicticus porto II M

Potto de Calabar (F): Arctocebus calabarensis II M

Potto Gibbon (E): Perodicticus potto II M

Poudou du nord (F): Pudu mephistophiles II M

Poudou du sud (F): Pudu puda I M

Poule de prairie d'Attwater (F): Tympanuchus cupido attwateri I B

Pounalocyathus hispidus: 11 in

Pourtalosmilia anthophyllites: II in

Pourtalosmilia conferta: II In

Powerful Owl (E): Ninox strenua II B

Prairie dogs (E): Cynomys spp. I/NC M

Prairie Falcon (E): Falco mexicanus II B

Prairie marmots (E): Cynomys spp. I/NC M

Prairie-Chickens (E): Tympanuchus spp. INC B

Prehensile-tailed Skink (E): Corucia zebrata II R

Premocyathus $=$ Caryophyllia

Presbytis auratus = Trachypithecus auratus

Presbytis aygula $=$ Presbytis comata

Presbytis comata: Grizzled Leaf Monkey (E), Java Leaf Monkey (E), Langur gris ( ) II M

Presbytis comata hose $i=$ Presbytis hose $i$

Presbytis comata thomasi $=$ Presbytis thomas $i$

Presbytis cristata $=$ Trachypithecus cristatus

Presbytis entellus = Semnopithecus entellus

Presbytis femoralis: Banded Langur (E), Banded Leaf Monkey (E), Langur mitrado (S) II M

Presbytis francoisi $=$ Trachypithecus francoisi

Presbyris fredericae $=$ Presbyris comata
Presbytis frontata: White-faced Langur (E), White-fronted Leaf Monkey (E). Langur de frente blanca (S), Semnopithèque à front blanc (F) II M

Presbyis geei $=$ Trachypithecus geei

Presbytis hosei: Grey Leaf Monkey (E), Hose's Leaf Monkey (E), Langur de Hose (S), Semnopithèque de Hose (F) II M

Presbytis johnii $=$ Trachypithecus johnii

Presbytis melalophos: Mitred Leaf Monkey (E), Langur de cresta (S), Semnopithèque mélalophe (F) II M

Presbytis melalophos femoralis $=$ Presbytis femoralis

Presbytis obscura $=$ Trachypithecus obscurus

Presbytis phayrei $=$ Trachypithecus phayrei

Presbytis pileata $=$ Trachypithecus pileatus

Presbytis potenziani: Long-tailed Langur (E). Mentawai Langur (E), Mentawai Leaf Monkey (E), Langur colilargo (S), Langur de Mentawi (S), Semnopithèque de Mentawi (F) I M

Presbytis rubicunda: Maroon Langur (E), Maroon Leaf Monkey (E), Langur rubicundo (S), Semnopithèque rubicond (F) II M

Presbytis senex $=$ Trachypithecus vetulus

Presbytis thomasi: Thomas's Langur (E), Thomas's Leaf Monkey (E), Langur de Thomas (S), Semnopittèque de Thomas (F) II M

Presbyris vetulus $=$ Trachypithecus vetulus

Preuss's Colobus (E): Procolobus preussi II M

Preuss's Guenon (E): Cercopithecus preussi II M

Preuss's Monkey (E): Cercopithecus preussi II M

Preuss's Red Colobus (E): Procolobus preussi II M

Preuss's Weaver (E): Ploceus preussi III B

Priam's Birdwing (E): Omithoptera priamus II In

Prickly Chameleon (E): Bradypodion spinosum II R

Prickly Girdled Lizard (E): Pseudocordylus spinosus II R

Prigogine's Owlet (E): Glaucidium albertinum II B

Primates (E/S/F): PRIMATES spp. III M

PRIMATES spp.: Apes (E), Monkeys (E), Primates (E), Monos (S), Primates (S), Primates (F) //II M

Prince Ruspoli's Turaco (E): Tauraco nuspolii II B

Princely Mastigure (E): Uromastyx princeps II R

Princess of Wales Parakeet (E): Polytelis alexandrae II B

Princess Parrot (E): Polytelis alexandrae II B

Princess Stephanie's Bird-of-paradise (E): Astrapia stephaniae II B Priodontes giganteus $=$ Priodonies maximus

Priodontes maximus: Giant Armadillo (E), Armadillo gigante (S), Carachupa Manan (S), Cuspon (S), Tatú carreta (S), Tatú guazú (S), Tatou géant (F) I M

Prionailurus bengalensis: Leopard Cat (E), Gato bengalí (S), Gato de Bengala (S), Chat de Chine (F), Chat-léopard du Bengale (F) I/II M

Prionailurus bengalensis bengalensis: Bengal Leopard $\mathrm{Cat}(\mathrm{E}), \mathrm{Gato}$ bengali (S), Chat-tigte du Bengale (F) III M

Prionailurus iriomotensis $=$ Prionailurus bengalensis

Prionailurus planiceps: Flat-headed Cat (E), Gato cabeciancho (S), Chat à tête plate (F) I M

Prionailurus rubiginosus: Rusty-spotted Cat (E), Gato rojizo (S), Gato rubiginosa (S), Chat rougeâtre (F), Chat rubigineux (F), Chat-léopard de l'Inde (F) III M

Prionailurus viverrinus: Fishing Cat (E), Gato pescador (S), Chat pêcheur (F) II M

Prionastrea $=$ Favites

Prioniturus discurus: Blue-crowned Racquet-tail (E), Palawan Racket-tailed Parrot (E), Lorito-momoto coroniazul (S), Palette couronne bleue (F) II B

Prioniturus discurus platenae $=$ Prioniturus platenae

Prioniturus discurus verticalis $=$ Prioniturus verticalis

Prioniturus flavicans: Yellow-breasted Racquet-tail (E), Yellowishbreasted Racquet-tai] (E), Lorito-momoto amarillento (S), Palette de Cassin (F) II B

Prioniturus luconensis: Green Racquet-tail (E), Green-crowned Racket-tailed Parrot (E), Green-headed Racket-tailed Parrot (E). Lorito-momoto de Luzón (S), Palette verte (F) II B

Prioniturus mada: Buru Racquet-tail (E), Lorito-momoto de Bur (S), Palette de Buru (F) II B

Prioniturus montanus: Luzon Racquet-tail (E), Montane Racquet-tail (E), Lorito-momoto montano (S), Palette momot (F) II B

Prioniturus montanus waterstradti $=$ Prioniturus waterstradti

Prioniturus platenae: Blue-headed Racquet-tail (E), Lorito-momoto de Palawan (S), Palette de Palawan (F) II B 
Prionifurus platurus: Golden-mantled Racquet-tail (E), Loritomomoto dorsidorado (S), Palette à manteau d'or (F) II B

Prioniturus verticalis: Blue-winged Racquet-tail (E), Lorito-momoto de las Sulu (S), Palette des Sulu (F) II B

Prioniturus waterstradti: Mindanao Racquet-tail (E), Lorito-momoto de Mindanao (S), Palette de Mindanao (F) II B

Prionodon linsang: Banded Linsang (E), Cibeta atigrada (S), Civette à bandes (F), Linsang rayé (F) II $M$

Prionodon pardicolor. Spotted Linsang (E), Tiger Civet (E), Cibeta moteada (S), Linsang tacheté (F), Linsang à taches (F) I M

Probarbus jullieni: Barbeau de Jullien (F) I F

Probosciger aterrimus: Palm Cockatoo (E), Cacatúa enlutada (S), Cacatoès noir (F), Microglosse noir (F) I B

Proboscis Monkey (E): Nasalis larvatus I M

Procolobus badius: Red Colobus (E), Western Red Colobus (E), Colobo herrumbroso occidental (S), Colobe bai (F), Colobe bai d'Afrique occidentale (F), Colobe ferrugineux (F) II M

Procolobus badius preussi $=$ Procolobus preussi

Procolobus badius rufomitratus = Procolobus rufomitrafus

Procolobus kirkii = Procolobus pennantii kirkii

Procolobus pennanti preussi $=$ Procolobus preussi

Procolobus pennantii: Eastem Red Colobus (E), Colobo rojo (S), Colobe bai (F) $\mathbf{I} / \mathbf{I} \mathbf{M}$

Procolobus pennantii kirkii: Kirk's Colobus (E), Kirk's Red Colobus (E), Zanzibar Red Colobus (E), Colobo rojo de Zanzibar (S) Colobe bai de Zanzibar (F) Colobe roux de Zanzibar (F) I M

Procolobus pennantii kirkii = Procolobus pennantii kirkii

Procolobus preussi: Preuss's Colobus (E), Preuss's Red Colobus (E). Colobo rojo de Camerún (S), Colobe bai du Cameroun (F) II M

Procolobus rufomitratus: Tana River Colobus (E), Colobo rojo mitrado (S), Colobe bai de la Tana (F), Colobe roux de la Tana (F) I M

Procolobus tholloni $=$ Procolobus pennantii

Procolobus verus: Olive Colobus (E), Van Beneden's Colobus (E), Colobo verde (S), Colobe de Van Beneden (F), Colobe vert (F), Colobe vrai (F), Colobe à huppe (F) II M

Profelis aurata: African Golden Cat (E), Golden Cat (E), Gato dorado (S), Chat doré (F) II M

Proméfil (F): Priloris magnificus II B

Pronghom (E/F): Anrilocapra americana V/NC M

Propithecus diadema: Diademed Sifaka (E), Indris sifaca (S), Sifaka diademado $(\mathrm{S})$, Propithèque à diadème $(\mathrm{F})$ I M

Propithecus tattersalli: Golden-crowned Sifaka (E), Tattersall's Sifaka (E), Indris sifaca (S) I M

Propithecus verreauxi: Verreaux's Sifaka (E), Gran sifaka blanco (S), Indris sifaca (S), Propithèque de Verreaux (F), Sifaka (F) I M

Propithèque à diadème (F): Propithecus diadema I M

Propitheque de Verreaux (F): Propithecus verreauxi I M

Prosopeia personata: Masked Shining-Parrot (E), Papagayo enmascarado (S), Perruche masquée (F) II B

Prosopeia splendens: Crimson Shining-Parrot (E), Papagayo escarlata (S), Perruche écarlate (F) II B

Prosopeia tabuensis: Red Shining-Parrot (E), Papagayo granate (S), Perruche pompadour (F) II B

Prosopeia rabuensis splendens = Prosopeia splendens

Protèle (F): Proteles cristatus $111 \mathrm{M}$

Proteles cristatus: Aardwolf (E), Lobo de tierra (S), Protèle (F) III M

Protolobophyllia $=$ Cynarino

Protomussa $=$ Mussismilia

Przewalski's Horse (E): Equus przewalskii I M

Przewalski's Wild Horse (E): Equus przewalskii I M

Psammobates geometricus: Geometric Tortoise (E), Sacafi (S), Tortuga geométrica (S), Sakafi (F), Tortue géométrique (F) I R

Psammobates oculiferus: Kuhl's Tortoise (E), Serrated Star Tortoise (E), Toothed Cape Tortoise (E), Tortuga estrellada sudafricana de caparazón (S), Tortue ocelée (F) II R

Psammobates tentorius: African Tent Tortoise (E), South African Tent Tortoise (E), Tortuga estrellada sudafricana (S), Tortue bosselée ( $\boldsymbol{F}$ ) II $\mathbf{R}$

Psammocora brighami: II In

Psammocora contigua: II In

Psammocora decussala: II In

Psammocora digitata: II In

Psammocora explanulata: II in

Psammocora folium: II In
Psammocora haimiana: II In

Psammocora nierstraszi: II In

Psammocora obtusangula: II In

Psammocora profundacella: II in

Psammocora stellata: II In

Psammocora superficialis: II In

Psammocora vaughani: II in

Psammocora verrilli: II In

Psammoseris $=$ Heterocyathus

Psephotus chrysopterygius: Golden-shouldered Parrot (E), Loro hombroamarillo (S), Perico aligualdo (S), Periquito de espalda dorada (S), Perruche aux ailes d'or (F), Perruche à épaules dorées (F) I B

Psephorus chrysopterygius dissimilis $=$ Psephotus dissimilis

Psephotus dissimilis: Hooded Parrot (E), Perico capirotado (S), Periquito encapuchado (S), Peruche à capuchon noir (F) I B

Psephotus haematogasier $=$ Northiella haematogaster

Psephotus haematogaster narethae $=$ Northiella haematogaster narethae

Psephorus haematonotus: Red-rumped Parrot (E), Perico dorsirrojo (S), Perruche à croupion rouge (F), Perruche à dos rouge (F) II B

Psephotus pulcherrimus: Beautiful Parakeet (E), Paradise Parrot (E), Loro del paraíso (S), Perico del Paraíso (S), Perruche de paradis (F), Perruche magnifique (F) I B

Psephorus varius: Many-coloured Parakeet (E), Mulga Parrot (E), Perico variado (S), Perruche multicolore (F), Pernuche a croupion rouge (F) II B

Psephurus gladius: Chinese Paddlelish (E) II F

Pseudalopex culpaeus: Andean Wolf (E), Colpeo Fox (E), Culpeo (E), Red Fox (E), Culpeo (S), Zorro andino (S), Zorro colorado (S), Renard colfeo (F) II M

Pseudalopex fulvipes $=$ Pseudalopex griseus

Pseudalopex griseus: Argentine Grey Fox (E), Chico Grey Fox (E), Chiloe Fox (E), Grey Zorro (E), Little Fox (E), Pampa Fox (E), Chilla (S), Zorro chico (S), Zorro de la Isla Chiloe (S), Zorro gris (S), Renard de Chiloé (F), Renard gris d'Argentine (F) II M

Pseudalopex gymnocercus: Azara's Zorro (E), Pampa Fox (E), Renard d'Azara (F) II M

Pseudemydura umbrina: Western Short-necked Turtle (E), Western Swamp Turtle (E), Tortuga serpentina occidental (S), Pseudémydure de Perth (F), Tortue à col court (F) I R

Pseudémydure de Perth (F): Pseudemydura umbrina I R

Pseudeos fuscala: Dusky Lory (E), Lori sombrio (S), Lori orange sombre (F), Lori sombre (F) II B

Pseudoboa cloelia $=$ Clelia clelia

Pseudochelidon sirintarae: White-eyed River-Martin (E), Hirondelle à lunettes (F), Pseudolangrayen d'Asie (F) I B

Pseudocolumnastrea $=$ Pavona

Pseudocordyle de Lang (F): Pseudocordylus langi II R

Pseudocordyle des rochers (F): Pseudocordylus microlepidolus II R

Pseudocordyle du Cap (F): Pseudocordylus capensis II R

Pseudocordyle du Drakensberg (F): Pseudocordylus melanotus II R

Pseudocordyle épineux (F): Pseudocordylus spinosus II R

Pseudocordyles (F): Pseudocordylus spp. II R

Pseudocordylus spp.: Crag lizards (E), Girdled lizards (E), Falsos lagartos armadillos (S), Lézards à fausse ceinture (F),

Pseudocordylus capensis: False Girdled Lizard (E), Graceful Crag Lizard (E), Falso lagarto ammadillo del cabo (S), Lézard à fausse ceinture du Cap (F), Pseudocordyle du Cap (F) II R

Pseudocordylus langi: Lang's Crag Lizard (E), Lang's Girdled Lizard (E), Falso lagarto armadillo de Lang (S), Lézard à fausse ceinture de Lang (F), Pseudocordyle de Lang (F) II R

Pseudocordylus melanotus: Drakensberg Crag Lizard (E), Lézard des rochers du Drakensberg (F), Pseudocordyle du Drakensberg (F) II $\mathbf{R}$

Pseudocordylus microlepidotus: Cape Crag Lizard (E), Lézard des rochers $(F)$, Lézard à fausse ceinture des rochers $(F)$, Pseudocordyle des rochers (F) II R

Pseudocordylus microlepidotus melanorus = Pseudocordylus melanotus

Pseudocordylus robertsi $=$ Pseudocordylus capensis

Pseudocordylus spinosus: Prickly Girdled Lizard (E). Spiny Crag

Lizard (E), Falso lagarto armadillo espinoso (S), Lézard à

ceinture épineuse (F), Pseudocordyle épineux (F) II R

Pseudocordyles (F) II R 
Pseudocrypthelia pachypoma: II In

Pseudocyathoceras avis: II In

Pseudolangrayen d'Asie (F): Pseudochelidon sirintarae I B

Pseudolangrayens (F): Pseudochelidon spp. UNC B

Pseudomys praeconis: Shark Bay Mouse (E), Ratón bastardo peludo

(S). Fausse souris de la baie de Shark (F) I M

Pseudoptynx philippensis $=$ Bubo philippensis

Pseudorca crassidens: False Killer Whale (E), Orca falsa (S), Fauxorque (F) II M

Pseudonyx nghetinhensis: Saola (E), Vu Quang Ox (E), Saola (S) Ver nota pie de página (S), Saola (F) I M

Pseudoscaphirhynchus fedischenkoi: Syr-Dar Shovelnose Sturgeon (E) II F

Pseudoscaphirhynchus hermanni: Small Amu-Dar Shovelnose Sturgeon (E) II F

Pseudoscaphirhynchus kaufmanni: False Shovelnose Sturgeon (E) Large Amu-Dar Shovelnose Sturgeon (E) II F

Pseudoscops grammicus: Jamaican Owl (E), Búho de Jamaica (S), Hibou de la Jamaique (F) II B

Pseudosiderastrea tayamai: II In

Psittacella brehmii: Brehm's Tiger-Parrot (E), Lorito-tigre de Brehm (S), Perruche de Brehm (F) II B

Psinacella madaraszi: Madarasz's Tiger-Parrot (E), Lorito-tigre de Madarasz (S), Perruche de Madarasz (F) II B

Psittacella modesta: Modest Tiger-Parrot (E), Lorito-tigre modesto (S), Perruche modeste (F) II B

Psittacella picta: Painted Tiger-Parrot (E), Lonto-tigre pintado (S), Pernuche peinte (F) II B

PSITT ACIFORMES spp.: Parrots (E), Psittacines (E), Loros (S), Papagayos (S), Perruches (F) I/IL/III/NC B

Psittacines (E): PSITTACIFORMES spp. I/UIUNC B

Psittacula alexandri: Moustached Parakeet (E), Red-breasted Parakeet $(E)$, Cotorra pechirroja $(S)$, Perruche à moustaches $(F)$ II B

Psitracula calthropae: Emerald-collared Parakeet (E), Layard's Parakeet (E), Cotorra de ceilán (S), Perruche de Layard (F) II B Psittacula caniceps: Blyth's Parakeet (E), Nicobar Parakeet (E), Cotorra de Nicobar (S), Perruche des Nicobar (F) II B

Psittacula columboides: Malabar Parakeet (E), Cotorra de Malabar (S), Perruche de Malabar (F) II B

Psittacula cyanocephala: Plum-headed Parakeet (E), Cotorra ciruela (S), Perruche à tête prune (F) II B

Psillacula derbiana: Derbyan Parakeet (E), Cotorra de Derby (S), Perruche de Derby (F) II B

Psittacula echo: Mauritius Parakeet (E), Mauritius Ring-necked Parakeet (E), Cotorra de Mauricio (S), Grosse cateau verte de Maurice (F), Perruche de Maurice (F), Perruche à collier de Maurice (F) I B

Psitracula eques echo $=$ Psittacula echo

Psittacula eupatria: Alexandrine Parakeet (E), Cotora alejandrina (S), Perruche alexandre (F) II B

Psittacula finschii: Grey-headed Parakeet (E), Cotora de Finsch (S), Perruche de Finsch (F) II B

Psittacula himalayana: Slaty-headed Parakeet (E), Cotorra del Himalaya (S), Perruche de l'Himalaya (F), Perruche à tête ardoisée (F) II B

Psittacula himalavana finschii = Psintacula finschii

Psittacula intermedia: Intermediate Parakeet (E), Rothschild's Parakeet (E), Cotorra intermedia (S), Perruche intermédiaire (F) II B

Psittacula krameri: Ring-necked Parakeet (E), Rose-ringed Parakeet (E), Cotorra de Kramer (S), Loro rabudo collar (S), Periquito de collar africano (S), Perruche à collier (F) III B

Psittacula krameri echo $=P$ sittacula echo

Psittacula longicauda: Long-tailed Parakeet (E), Cotorra colilarga (S), Perruche à longs brins (F) II B

Psittacula roseata: Blossom-headed Parakeet (E), Cotorra carirosa (S), Perruche à tête rose (F) II B

Psittacule à double-oeil de Coxen (F): Cyclopsitta diophihalma coxeni I B

Psittacule à poitrine orange (F): Cyclopsitra gulielmitertil II B

Psittacule de Desmarest (F): Psittaculirostris desmarestii II B

Psittacule de Salvadori (F): Psittaculirostris salvadorii II B

Psittacule d'Edwards (F): Psittaculirostris edwardsii II B

Psittacule double-oeil (F): Cyclopsitta diophthalma I/II B

Psittacules (F): Cyclopsinta spp/Psittaculirostris spp. V/II B
Psittaculirostris desmarestii: Desmarest's Fig-Parrot (E), Goldenheaded Fig-Parrot (E), Large Fig-Parrot (E), Lorito de Desmarest (S), Psittacule de Desmarest (F) II B

Psittaculirostris edwardsii: Edwards's Fig-Parrot (E), Lorito de Edwards (S), Psittacule d'Edwards (F) II B

Psittaculirostris salvadorii: Sal vadori's Fig-Parrot (E), Lorito de Salvadori (S), Psittacule de Salvadori (F) II B

Psittacus erithacus: Grey Parrot (E), Loro yaco (S), Yaco (S), Jacko (F), Jacquot (F), Perroquet gris du Gabon (F), Perroquet jaco (F) II $\mathrm{B}$

Psitteuteles goldiei: Goldie's Lorikeet (E), Lori de Goldie (S), Loriquet de Goldie (F) II B

Psitteuteles iris: Lris Lorikeet (E), Iris Lory (E), Lori ins (S), Loriquet iris (F) II B

Psitteuteles versicolor. Varied Lorikeet (E), Lori versicolor (S), Loriquet versicolore (F) II B

Psittinus cyanurus: Blue-rumped Parrot (E), Lorito dorsiazul (S), Perruche à croupion bleu (F) II B

Psittrichas de Pesquet (F): Psittrichas fulgidus II B

Psittrichas fulgidus: Pesquet's Parrot (E), Vulturine Parrot (E), Loro aguileño (S), Psittrichas de Pesquat (F) II B

Pieridophora alberi: King-of-Saxony Bird-of-paradise (E), Paradisier d'Albert (F), Paradisier du prince Albert (F) II B

Plerocnemia pennala $=$ Rhea pennata

Pteroglossus aracari: Black-necked Aracari (E), Tilingo cuellinegro (S), Araçari grigri (F), Araçari à cou noir (F) II B

Pteroglossus castanotis: Chesenut-eared Aracari (E), Arasari orejicastano (S), Tucán-Tilingo verde (S), Araçari à oreillons roux (F) III B

Pteroglossus viridis: Green Aracari (E), Tilingo limón (S), Araçari vert (F) II B

Pteronetta hartlaubii: Hartlaub's Duck (E), Pato de Hartlaub (S) Canard de Hartlaub (F) III B

Prerunura brasiliensis: Giant Brazilian Otter (E), Giant Otter (E). Arirai (S). Lobito de cola ancha (S), Lobo de Río Grande (S), Lobo del río (S), Lobo gargantilla (S), Perro de agua (S), Loutre géante du Brésil (F) I M

Pteropathes $=$ Antipathes

Pterophanes cyanopterus: Great Sapphirewing (E), Alazafiro grande (S), Colibri à ailes saphir (F) II B

Pteropus spp.: Flying-foxes (E), Roussettes (F) II M

Pteropus admiralitatum: Admiralty Flying-fox (E), Zorro volador de las islas de almirantazgo (S) II M

Pteropus aldabrensis: Aldabra Flying-fox (E), Zorro volador de Aldabra (S) II M

Pteropus aldabrensis $=$ Pteropus seychellensis

Pteropus alecto: Central Flying-fox (E), Zorro volador negro (S) II M

Pteropus anetianus: 'White Flying-fox (E) II M

Pteropus argentasus: Silvery Flying-fox (E), Silvery Fruit Bat (E), Zorro volador argénteo (S) II M

Pteropus arquatus $=$ Acerodon celebensis

Pteropus balurus $=$ Pteropus pumilus

Pteropus brunneus: Zorro volador de las islas Percy (\$) II M

Pteropus caniceps: Ashy-headed Flying-fox (E), Zorro volador de cabeza ceniciento (S) II M

Preropus chrysoproctus: Ambon Flying-fox (E), Zorro volador de Amboina (S) II M

Pteropus cognatus $=$ Pteropus rayneri

Preropus conspicillatus: Spectacled Flying-fox (E), Zorro volador de anteojos (S) II M

Pieropus dasymallus: Ryukyu Flying-fox (E), Zorro volador de RyuKyu (S) II M

Preropus faunulus: II M

Pteropus fundatus: Zorro volador de las islas Banks (S) II M

Pteropus giganteus: Indian Flying-fox (E), Zorro volador de la india (S) II M

Pteropus gilliardi: Gilliard's Flying-fox (E), Zorno volador de Gilliard (S) II M

Pteropus griseus: Grey Flying-fox (E), Zorro volador gris (S) II M

Pteropus howensis: Zorro volador de Howens (S) II M

Preropus hypomelanus: Small Flying-fox (E), Zorro volador pequeño (S) II M

Pteropus hypomelanus meamsi $=$ Pteropus mearns

Pteropus hypomelanus santacrucis = Preropus santacrucis 
Preropus insularis: Chuuk Flying-fox (E), Truk Flying-fox (E), Roussette des îles Truk (F) I M

Pleropus intermedius = Preropus vampyrus

Pteropus leucopterus: Zorro volador de Luzón (S) II M

Pleropus leucotis $=$ Acerodon leucotis

Preropus livingstonei: Comoro Black Flying-fox (E), Zorro volador de Livingston (S) II M

Pteropus lombocensis: Lombok Flying-fox (E), Zorro volador de Lombok (S) II M

Pteropus loochooensis $=$ Pteropus mariannus

Pteropus lylei: Lyle's Flying-fox (E), Zorro volador de Lyle (S) II M

Pteropus macrotis: Big-eared Flying-fox (E), Zorro volador de orejas grandes (S) II M

Pteropus macrotis pohlei $=$ Pteropus pohlei

Pteropus mahaganus: Lesser Fying-fox (E), Zorro volador de Bougainville (S) II M

Pteropus mariannus: Marianas Flying-fox (E), Micronesian Flyingfox (E), Roussette des îles Marianes (F) I M

Pteropus meamsi: Zorro volador de Mearns (S) II M

Pteropus melanopogon: Black-bearded Flying-fox (E), Zorro volador de barba negra (S) II M

Pteropus melanorus: Zorro volador de Andaman (S) II M

Pteropus molossinus: Pohnpei Flying-fox (E), Renard volant de Ponape (F) I M

Pleropus neohibemicus: Bismarck Flying-fox (E), Zorro volador de Bismarck (S) II M

Pteropus niger. Greater Mascarene Flying-fox (E), Zorro volador negro de Mauricio (S) II M

Pteropus nitendiensis: Zorro volador de la isla Ndeni (S) II M

Pteropus ocularis: Seram Flying-fox (E), Seram Fruit Bat (E), Zorro volador de las isla Ceram (S) II M

Pteropus ornatus: Zorro volador de las islas de la Leal (S) II M

Pteropus pelewensis $=$ Pteropus mariannus

Pteropus personatus: Masked Flying-fox (E), Masked Fruit Bat (E), Zorro volador enmascarado (S) II M

Pieropus phaeocephalus: Mortlock Flying-fox (E), Mortlock Islands Flying-fox (E), Roussette de l'ile Mortlock (F) I M

Pteropus pilosus: Large Palau Flying-fox (E), Palau Fruit Bat (E). Zorro volador de las islas Palau (S) I M

Pteropus pohlei: Geelvink Bay Fruit Bat (E), Zorro volador de las isla Japen (S) II M

Preropus poliocephalus: Grey-headed Flying-fox (E), Zorro volador de cabeza gris (S) II M

Pteropus pselaphon: Bonin Fruit Bat (E), Zorro volador de las isla Bonin (S) II M

Pteropus pumilus: Little Golden-mantled Flying-fox (E), Zorro volador de Taylor (S) II M

Pieropus rayneri: Solomon Flying-fox (E), Zorro volador de las Salomón (S) II M

Pteropus rodricensis: Rodrigues Flying-fox (E), Zorro volador de la isla Rodrigues (S) II M

Pteropus rufus: Madagascar Flying-fox (E), Madagascar Fruit Bat (E), Zorro volador de Madagascar (S) II M

Pteropus samoensis: Samoan Flying-fox (E), Roussette des îles Samoa (F) I M

Pteropus santacrucis: Zorro volador de la isla Santa Cruz (S) II M

Pteropus scapulatus: Little Red Flying-fox (E), Zorro volador de Queensland (S) II M

Pteropus seychellensis: Seychelles Flying-fox (E), Zorro volador de las Seychelles (S) II M

Preropus seychellensis aldabrensis = Preropus aldabrensis

Pieropus speciosus: II M

Preropus subniger. Lesser Mascarene Flying-fox (E), Zorro volador oscuro de Mauricio (S) II M

Pteropus tablasi $=$ Pteropus pumilus

Pieropus temmincki: Temminck's Flying-fox (E), Zorro volador de Temminck (S) II M

Pteropus tokudae: Guam Flying-fox (E), Guam Fruit Bat (E), Zorro volador de Tokuda (S) II M

Pteropus tonganus: Insular Flying-fox (E), Roussette des îles Tonga (F) I M

Pteropus tuberculatus: Zorro volador de la isla Jawi (S) II M

Pteropus ualanus $=$ Pteropus mariannus

Pteropus vampyrus: Large Flying-fox (E), Zorro volador de cuello rojo (S) II M

Pteropus veiulus: Zorro volador de Nueva Caledonia (S) $11 \mathrm{M}$
Pleropus voelizkowi: Pemba Flying-fox (E), Zorro volador de Voettzkow (S) II M

Preropus woodfordi: Least Flying-fox (E), Least Fruit Bat (E), Zorro volador de Woodford (S) II M

Pteropus yapensis = Pteropus mariannus

Prilocercus lowit: Pen-tailed Treeshrew (E), Musaraña arborícola coliplumada (S), Ptilocerque de Low (F) II M

Ptilocerque de Low (F): Prilocercus lowii II M

Pilolaemus tickelli $=$ Anorrhinus sickelli

Ptiloris de Victoria (F): Priloris victoriae II B

Pfiloris intercedens: Eastern Riflebird (E) II B

Priloris magnificus: Magnificent Riflebird (E), Paradisier gorged'acier (F), Proméfil (F) II B

Ptiloris paradis (F): Ptiloris paradiseus II B

Psiloris paradiseus: Paradise Riflebird (E), Paradisier festonné (F), Ptiloris paradis (F) II B

Ptiloris victoriae: Queen Victoria's Riflebird (E), Victoria's Riflebird (E), Paradisier de Victoria (F), Ptiloris de Victoria (F) II B

Pryas mucosus: Common Rat Snake (E), Dhaman (E), Oriental Rat Snake (E), Serpent ratier oriental (F) II R

Pudu du Nord (F): Pudu mephistophiles II M

Pudu du Sud (F): Pudu puda I M

Pudu mephistophiles: Northem Pudu (E), Pudu norteño (S),

Sachacabra (S), Venadito de los páramos (S), Venado conejo (S), Poudou du nord (F), Pudu du Nord (F) II M

Pudu meridional (S): Pudu puda I M

Pudu norteño (S): Pudu mephistophiles II M

Pudu puda: Chilean Pudu (E), Southem Pudu (E), Ciervo enano (S). Pudu meridional (S), Venadito (S), Poudou du sud (F), Pudu du Sud (F) I M

Pudus (E/F/S): Pudu spp. I/I M

Puerto Rican Amazon (E): Amazona vittata I B

Puerto Rican Boa (E): Epicrates inomatus I R

Pueno Rican Emerald (E): Chlorostilbon maugaeus $11 \mathrm{~B}$

Puerto Rican Parrot (E): Amazona viltata I B

Puerto Rican Screech-Owl (E): Otus nudipes II B

Pufflegs (E): Eriocnemis spp//Maplophaedia spp. II B

Pulsatrix koeniswaldiana: Tawny-browed Owl (E), White-chinned Owl (E), Lechuzón anteojo menor (S), Lechuzón pardo menor (S), Chouette de Koeniswald (F), Chouette à sourcils jaunes (F) II B

Pulsatrix melanota: Band-bellied Owl (E), Band-tailed Owl (E), Rusty-barred Owl (E), Cicupa (S), Chouette à collier (F) II B

Pulsatrix perspicillata: Spectacled Owl (E), Búho de anteojos (S), Lechuzón anteojo (S), Lechuzón de Anteojos (S), Chouette à lunettes (F) II $\mathbf{B}$

Puma (E/S/F): Puma concolor I/II M

Puma concolor: Cougar (E), Deer Tiger (E), Mountain Lion (E), Puma (E), Red Tiger (E), León americano (S), León bayo (S), Mitzli (S), Onza bermeja (S), Puma (S), Puma (F) I/II M

Puma concolor coni: Florida Cougar (E), Florida Panther (E), Florida Puma (E), León de Florida (S), Puma de Florida (S), Cougar de Floride (F), Puma de Floride (F) I M

Puma concolor cosiaricensis: Central American Puma (E), Costa Rican Puma (E), León costarricense (S), Puma costarricense (S). Puma d'Amérique centrale (F) I M

Puma concolor couguar: Eastern Cougar (E), Eastem Panther (E), Eastern Puma (E), Puma oriental (S), Cougouar (F), Puma de l'est de l'Amérique du Nord (F) I M

Puma costarricense (S): Puma concolor costaricensis I M

Puma d'Amérique centrale (F): Puma concolor costaricensis I M Puma de Florida (S): Puma concolor coryi I M

Puma de Floride (F): Puma concolor coryi I M

Puma de l'est de l'Amérique du Nord (F): Puma concolor couguar I M

Puma oriental (S): Puma concolor couguar I M

Puna Flamingo (E): Phoenicopterus jamesi II B

Puna Hawk (E): Bureo poecilochrous II B

Puna Rhea (E): Rhea pennata I B

Punta blanca (S): Urosticte benjamini II B

Purple-backed Sunbeam (E): Aglaeactis aliciae II B

Purple-backed Thombill (E): Ramphomicron microrhynchum II B

Purple-bellied Lory (E): Lorius hypoinochrous II B

Purple-bellied Parrot (E): Triclaria malachitacea I B

Purple-bibbed Whitetip (E): Urosticte benjamini II B

Purple-capped Lory (E): Lorius domicella II B 
Purple-chested Hummingbird (E): Amazilia rosenbergi II B Purple-collared Woodstar (E): Myris fanny II B

Purple-crested Turaco (E): Musophaga porphyreolopha II B Purple-crowned Fairy (E): Heliothryx barroti II B

Purple-crowned Lorikeet (E): Glossopsitta porphyrocephala II B

Purple-faced Leaf Monkey (E): Trachypithecus vefulus II M

Purple-naped Lory (E): Lorius domicella II B

Purple-throated Carib (E): Eulampis jugularis II B

Purple-throated Mountain-gem (E): Lampomis castaneoventris II B

Purple-throated Sunangel (E): Heliangelus viola II B

Purple-throated Woodstar (E): Philodice mitchellii II B

Purpureicephalus spurius: Pileated Parakeet (E), Red-capped Parrot

(E), Perico capelo (S), Perruche à tête pourpre (F) II B

Putois à pieds noirs (F): Mustela nigripes I M

Putty-nosed Monkey (E): Cercopithecus nictitans II M

Pycnonotus zeylanicus: Straw-crowned Bulbul (E), Straw-headed

Bulbul (E), Bulbul à tête jaune (F) II B

Pygargue à queue blanche (F): Haliaeetus albicilla I $\mathrm{B}$

Pygargue à tête blanche (F): Haliaee us leucocephalus I B

Pygargue à tête grise (F): Ichthyophaga ichthyaetus II B

Pygargue à ventre blanc (F): Haliaeetus leucogaster II B

Pygargue blagre (F): Haliaeetus leucogaster II B

Pygargue commun (F): Haliaeetus albicilla I B

Pygargue de Madagascar (F): Haliaeetus vociferoides II B

Pygargue de Pallas (F): Haliaeerus leucoryphus II B

Pygargue de Sanford $(F)$ : Haliaeetus sanfordi II B

Pygargue de Steller ( $\mathrm{F}$ : Haliaeetus pelagicus II B

Pygargue empereur (F): Haliaeetus pelagicus II B

Pygargue nain (F): Ichthyophaga humilis II B

Pygargue vocifere (F): Haliaeetus vocifer II B

Pygargues (F): Haliaeetus spp/Ichthyophaga spp. I/II B

Pygathrix spp.: Snub-nosed monkeys (E), Doucs (F), Rhinopithèques (F) I M

Pygathrix avunculus: Tonkin Snub-nosed Monkey (E) Rhinopithèque du Tonkin (F) I M

Pygathrix bieti: Yunnan Snub-nosed Monkey (E) I M

Pygathrix brelichi: Brelich's Snub-nosed Monkey (E), Grey Snubnosed Monkey (E), Guizhou Snub-nosed Monkey (E), Rhinopithèque jaune doré (F) I M

Pygathrix nemaeus: Douc (E), Douc Langur (E), Douc Monkey (E), Red-shanked Douc Langur (E), Langur douc (S), Mono pigatrix (S), Douc $(\mathrm{F})$, Rhinopithèque douc $(\mathrm{F})$ I M

Pygathrix roxellana: Golden Snub-nosed Monkey (E), Sichuan Snub-nosed Monkey (E), Langur chato (S), Rhinopithèque de Roxellane (F), Rhinopithèque doré (F) I M

Pygathrix roxellana brelichi $=$ Pygathrix brelichi

Pygathrix roxellanae bieti $=$ Pygathrix bieti

Pygmomis longuemareus = Phaethornis longuemareus

Pygmomis striigularis $=$ Phaethomis longuemareus

Pygmy Chimpanzee (E): Pan paniscus I M

Pygmy Falcon (E): Polihierax semitorquatus II B

Pygmy Hippopotamus (E): Hexaprotodon liberiensis II M

Pygmy Hog (E): Sus salvanius I M

Pygmy Killer Whale (E): Feresa attenuata II M

Pygmy Lorikeet (E): Charmosyna wilhelminae II B

Pygmy Loris (E): Nycticebus pygmaeus II M

Pygmy Marmoset (E): Callithrix pygmaea II M

Pygmy Mulga Monitor (E): Varanus gilleni II R

Pygmy Python (E): Morelia perthensis II R

Pygmy Right Whale (E): Caperea marginata I M

Pygmy Sperm Whale (E): Kogia breviceps $11 \mathrm{M}$

Pygmy Tarsier (E): Tarsius pumilus II M

Pygmy Treeshrew (E): Tupaia minor II M

Pygmy-Geese (E): Nettapus spp. III/NC B

Pygmy-Owls (E): Glaucidium spp. II B

Pygmy-Parrots (E): Micropsitta spp. II B

Pyrénestes (F): Pyrenestes spp. IIINC B

Pyrenestes frommi $=$ Pyrenestes osirinus

Pyrenestes maximus = Pyrenestes ostrinus

Pyrenestes ostrinus: Black-bellied Seedcracker (E), Pirenestes ventrinegro $(\mathrm{S})$, Gros-bec ponceau à ventre noir $(\mathrm{F})$, Pyrénestes ponceau (F) III B

Pyrénestes ponceau (F): Pyrenestes osirinus III B

Pyrenestes rothschildi $=$ Pyrenestes ostrinus

Pyrophyllia = Guynia

Pyrrhura albipectus: White-breasted Parakeet (E), White-necked
Conure (E), White-necked Parakeet (E), Cotorra cuelliblanca (S). Perico pechiblanco (S), Conure à col blanc (F) II B

Pyrrhura calliptera: Brown-breasted Parakeet (E), Flame-winged Conure (E), Flame-winged Parakeet (E), Cotorra pechiparda (S), Conure à poitrine brune (F) $11 \mathrm{~B}$

Pyrrhura cruentata: Blue-chested Parakeet (E), Blue-throated Parakeet (E), Ochre-marked Parakeet (E), Red-eared Conure (E), Cotorra tiriba (S), Perico grande (S), Conure tiriba (F), Conure à gorge bleue (F), Perruche tiriba (F) I B

Pyrrhura devillei: Blaze-winged Conure (E), Blaze-winged Parakeet (E), Cotorra de Deville (S), Conure de Deville (F) II B

Pyrrhura egregia: Fiery-shouldered Conure (E), Fiery-shouldered Parakeet (E), Cotorra egregia (S), Perico colimorado (S), Conure aile-de-feu (F) II B

Pyrrhura fronıalis: Maroon-bellied Parakeet (E), Red-bellied Conure (E), Reddish-bellied Parakeet (E), Chiripepé común (S), Cotorra chiripepé (S), Conure de Vieillot (F) II B

Pyrrhura hoematotis: Blood-eared Parakeet (E), Red-breasted Conure (E), Red-eared Parakeet (E), Cotorra colirroja (S), Perico cola roja (S), Conure à oreillons (F) II B

Pyrrhura hoffmanni: Hoffmann's Conure (E), Sulphur-winged Parakeet (E), Cotorra catana (S), Conure de Hoffmann (F) II B

Pyrrhura hypoxantha = Pyrrhura molinae

Pyrrhura leucotis: Maroon-faced Parakeet (E), White-eared Conure (E), White-eared Parakeet (E), Cotorra cariparda (S), Perico pintado (S), Conure emma (F) II B

Pyrrhura melanura: Maroon-tailed Conure (E), Maroon-tailed Parakeet (E), Cotorra colinegra (S), Perico cola negra (S), Conure de Souancé (F) II B

Pyrrhura molinae: Green-cheeked Conure (E), Green-cheeked Parakeet (E), Chiripepé salteño (S), Cotorra de Molina (S), Conure de Molina (F) II B

Pyrrhura orcesi: El Oro Conure (E), El Oro Parakeet (E), Cotorra de El Oro (S), Perico de El Oro (S), Conure d'Orcès (F) II B

Pyrrhura perlata: Pearly Conure (E), Pearly Parakeet (E), Cotorra perlada (S), Conure perlé (F) II B

Pyrrhura picta: Painted Conure (E), Painted Parakeet (E), Cotorra pineada (S), Perico cabecirrosado (S), Conure versicolore (F) II B

Pyrrhura rhodocephala: Rose-crowned Conure (E), Rose-headed Conure (E), Rose-headed Parakeet (E), Cotorra coronirroja (S), Perico cabecirrojo (S), Conure tête-de-feu (F) II B

Pyrrhura rhodogasier: Crimson-bellied Conure (E), Crimson-bellied Parakeet $(\mathrm{E})$, Cotorra ventrinoja (S), Conure à ventre rouge $(\mathrm{F})$ II B

Pyrrhura rupicola: Black-capped Conure (E), Black-capped Parakeet (E), Rock Parakeet (E), Cotorra capirotada (S), Conure à cape noire (F) II B

Pyrrhura vinidicaia: Santa Marta Conure (E), Santa Marta Parakeet (E), Cotorra de Santa Marta (S), Conure des Santa Marta (F) II B

Pyrroglaux podargina $=$ Otus podarginus

Python à écailles rugueuses (F): Morelia carinata II R

Python à large tache (F): Morelia stimsoni II R

Python à lèvres blanches (F): Morelia alberisii II R

Pyshon à tête noire d'Australie (F): Aspidites melanocephalus II R

Python améthiste (F): Morelia amethistina II R

Python amethistinus = Morelia amethistina

Python anchietae: Angolan Python (E), Pitón de Angola (S), Python d'Angola (F) II R

Python arboricole vert australien (F): Morelia viridis II $\mathbf{R}$

Python boeleni $=$ Morelia boeleni

Python bredli $=$ Morelia spilota

Python caréné (F): Morelia carinata II R

Python curtus: Blood Python (E), Short-tailed Python (E), Pitón malaya (S), Python malais (F), Python sanguin (F) II $R$

Python d'Amérique centrale (F): Loxocemus bicolor II R

Python d'Angola (F): Python anchietae II R

Python de Boelen (F): Morelia boeleni II R

Pythorı de Children (F): Morelia childreni II R

Python de la brousse (F): Morelia amethistina II R

Python de Macklot (F): Morelia macklori II R

Python de Nouvelle-Guinée (F): Morelia albertisii II R

Python de Papouasie (F): Morelia papuana II R

Python de Ramsay (F): Aspidites ramsayi II R

Python de Seba (F): Python sebae II R 
Python de Stimson (F): Morelia stimsoni II R Python de Timor (F): Python timoriensis II R Python des rochers (F): Morelia amethistina II $\mathbf{R}$ Python d'Oenpelli (F): Morelia oenpelliensis II R

Python fouisseur du Mexique (F): Loxocemus bicolor II R

Python malais (F): Python curtus II R

Python molure (F): Python molurus I/II R

Python molure indien (F): Python molurus molurus I R

Python molurus: Asiatic Rock Python (E), Burmese Python (E), Tiger Python (E), Python molure (F), Python tigre (F) I/I R

Python molurus molurus: Indian Python (E), Pitón de la India (S) Python molure indien (F) I R

Python molurus pimbura $=$ Python molurus molurus

Python molurus pimbura = Python molurus molurus

Python oenpelliensis = Morelia oenpelliensis

Python olive (F): Morelia olivacea II R

Python pygmée de Perth (F): Morelia perthensis II R

Python regius: Ball Python (E), Royal Python (E), Pitón real (S), Python royal (F) II R

Python reticulatus: Java Rock Python (E), Regal Python (E), Reticulated Python (E), Pitón reticulada (S), Python réticulé (F) II R

Python réticulé (F): Python reticularus II $R$

Python royal (F): Python regius II R

Python sanguin (F): Python curtus II R

Python saxuloides $=$ Python sebae

Python sebae: African Python (E), African Rock Python (E), Pitón de Seba (S), Python de Seba (F) II R

Python spilotus = Morelia spilota

Python tacheté (F): Morelia maculosa II R

Python tapis (F): Morelia spilota II R

Python tigre (F): Python molurus I/II R

Python timorensis $=$ Python timoriensis

Python timoriensis: Timor Python (E), Pitón de Timor (S), Python de Timor (F) II R

Pythons (E/F): Boidae spp. I/II R

Pytilia hypogrammica: Red-faced Pytilia (E), Yellow-winged Pytilia (E), Beaumarquet à ailes jaunes $(F)$, Diamant à face rouge (F) III B

Pytilia phoenicoptera: Aurora Finch (E), Crimson-winged Pytilia (E), Red-winged Pytilia (E), Pitilia alimoja (S), Beaumarquet (F), Diamant aurore (F) III B

Pytilias (E): Pytilia spp. III/NC B

Pyxide à queue platte (F): Pyxis planicauda II R

Pyxide arachnoide (F): Pyxis arachnoides II $\mathrm{R}$

Pyxis arachnoides: Spider Tortoise (E), Tortuga araña (S), Tortuga de plastrón articulado (S), Pyxide arachnoide (F) II R

Pyxis planicauda: Flat-backed Spider Tortoise (E), Flat-shelled Spider Tortoise (E), Madagascar Flat-shelled Tortoise (E), Tortue de cola plana (S), Pyxide à queue platte (F) II R

Quadrula cor = Fusconaia edgariana

Quadrula intermedia: Cumberland Monkey-face Pearly Mussel (E). Cumberland Monkeyface (E) I In

Quadrula sparsa: Appalachian Monkey-face Pearly Mussel (E) Appalachian Monkeyface (E) I In

Quadrula striata $=$ Plethobasus cooperianus

Quailfinches (E): Ortygospiza spp. III/NC B

Quaker Parakeet (E): Myiopsina monachus II B

Quebrantahuesos (S): Gypaetus barbarus II B

Queen Alexandra's Birdwing (E): Omithoptera alexandrae I In

Queen Carola's Parotia (E): Parotia carolae II B

Queen Conch (E): Strombus gigas II In

Queen of Bavaria's Conure (E): Aratinga guarouba I B

Queen Victoria's Birdwing (E): Omithoptera victoriae II In

Queen Victoria's Riflebird (E): Ptiloris victoriae II B

Queensland Hairy-nosed Wombat (E): Lasiorhinus kreffii I M

Queensland Lungfish (E): Neoceratodus forsteri II F

Queensland Tree Monitor (E): Varanus teriae II R

Quelea erythrops: Red-headed Dioch (E), Red-headed Quelea (E), Laborioso cabecirrojo (S), Travailleur à tête rouge (F) III B

Queleas (E): Quelea spp. IIU/NC B

Quetzal centroamericano (S): Pharomachnus mocinno I B

Quetzal resplendissant (F): Pharomachrus mocinna I B

Quetzals (E/F): Pharomachnis spp. INC B

Queue de vinaigre (F): Estrilda caerulescens III B

Quimilero (S): Catagonus wagneri I M
Rabbits (E): Leporidae spp. INC M

Rabihorcado de la Christmas (S): Fregaia andrewsi I B

Rabihorcado ventriblanco (S): Fregata andrewsi I B

Race-runner (E): Cnemidophorus hyperythrus II R

Race-runners (E): Cnemidophorus spp. II/NC R

Racket-tailed Coquette (E): Discosura longicauda II B

Racket-tailed parrots (E): Prionirurus spp. II B

Racquet-tails (E): Prioniturus spp. II B

Radiated Tortoise (E): Geochelone radiata $\mathbf{I} \mathbf{R}$

Raggiana Bird-of-paradise (E): Paradisaea raggiana II $B$

Rails (E): Rallidae spp. I/NC B

Rainbow Boa (E): Epicrates cenchria II R

Rainbow Lorikeet (E): Trichoglossus haematodus II B

Rainbow Lory (E): Trichoglossus haematodus 11 B

Rainbow Starfrontlet (E): Coeligena iris II B

Rainbow-bearded Thombill (E): Chalcostigma herrani II B

Rainforest Chameleon (E): Chamaeleo balteatus II R

Rainforest Hog-nosed Pit Viper (E): Bothrops nasula III R

Rajah Brooke's Birdwing (E): Trogonoptera brookiana II In

Rajah Scops-Owl (E): Otus brookii II B

Râle de l'île Lord Howe (F): Gallirallus sylvestris I B

Râle sylvestre $(F)$ : Gallirallus sylvestris I B

Râles (F): Rallidae spp. I/NC B

Ramphastos dicolorus: Red-breasted Toucan (E), Tucán rojo y amarillo (S), Toucan à ventre rouge (F) $111 \mathrm{~B}$

Ramphastos sulfurarus: Keel-billed Toucan (E), Piapoco pico verde (S), Tucán pico-multicolor (S), Toucan à carène (F) II B

Ramphastos toco: Toco Toucan (E), Tucán grande (S), Toucan toco (F) II B

Ramphastos tucanus: Red-billed Toucan (E), Piapoco pico rojo (S), Toucan a bec rouge (F) II B

Ramphastos virellinus: Channel-billed Toucan (E), Diostedé pico acanelado $(S)$, Toucan ariel $(F)$, Toucan à gorge jaune et blanche (F) II B

Ramphodon dohmii: Hook-billed Hermit (E), Colibrí picosierra chico (S), Colibri de Dohm (F), Colıbri à bec incurvé (F), Emite de $\operatorname{Dohm}(F)$ I B

Ramphodon naevius: Saw-billed Hermit (E), Colibri tacheté (F) II B

Ramphomicron dorsale: Black-backed Thornbill (E), Colibri à dos noir (F) II B

Ramphomicron microrhynchum: Purple-backed Thombill (E), Colibrí pico espina (S), Picoespina dorsipurpura (S), Colibri à petit bec (F) II B

Ramsay's Python (E): Aspidites ramsayi II $\mathrm{R}$

Rana de punta de flecha andino (S): Epipedobates andinus II A

Rana de punta de flecha arbórea (S): Dendrobates arboreus II A

Rana de punta de flecha azul (S): Dendrobates azureus II A

Rana de punta de flecha boliviana (S): Epipedobates bolivianus II A

Rana de punta de flecha de bandas amarillas (S): Dendrobates leucomelas II A

Rana de punta de flecha de Bassler (S): Phobobates bassleri II A

Rana de punta de flecha de Boulenger (S): Epipedobates boulenger II A

Rana de punta de flecha de Inger (S): Epipedobates ingeri II A

Rana de punta de flecha de Lehmanr: (S): Dendrobates lehmanni II A

Rana de punta de flecha de Myers (S): Epipedobates myersi II A

Rana de punta de flecha de Peters (S): Epipedobates petersi II A

Rana de punta de flecha de Silverstone (S): Phobobates silverstonei II A

Rana de punta de flecha de Steyermark (S): Minyobates steyermarki II A

Rana de punta de flecha de Vanzolini (S): Dendrobates vanzolini II A

Rana de punta de flecha de vientre azul (S): Epipedobates azureiventris II A

Rana de punta de flecha esmeralda (S): Epipedobates smaragdinus II A

Rana de punta de flecha fantástica (S): Dendrobates fantasticus II A Rana de punta de flecha granulosa (S): Dendrobates granulifer II A Rana de punta de flecha histriónica (S): Dendrobates histrionicus II A

Rana de punta de flecha lúgubre (S): Phyllobates lugubris II A

Rana de punta de flecha maculada (S): Epipedobates macularus II A 
Rana de punta de flecha misteriosa (S): Dendrobates mysteriosus II A

Rana de punta de flecha pentaestriada (S): Dendrobates quinquevintaius II A

Rana de punta de flecha picta (F): Epipedobates pictus II A Rana de punta de flecha rayada (S): Phyllobates vittatus II A Rana de punta de flecha reticulada (S): Dendrobates reticularus II A Rana de punta de flecha roja (S): Dendrobates pumilio II A Rana de punta de flecha terrible (S): Phyllobates terribilis II A Rana de punta de flecha tricolor (S): Epipedobates trirolor II A Rana de punta de flecha truncada (S): Dendrobates truncatus II A Rana de punta de flecha variable (S): Dendrobates variabilis II A Rana de punta de flecha verde (S): Minyobates viridis II A

Rana dorada (S): Atelopus varius zeteki I AMantella aurantiaca II A

Rana hexadactyla $=$ Euphlyctis hexadactylus

Rana tigerina $=$ Hoplobatrachus tigerinus

Rana venenosa de Zetek (S): Atelopus varius zeteki I A

Ranas de puntas de flechas (S): Dendrobates spp. II AVhyllobates spp. II A Allobates spp. II A/Epipedobates spp. II AVMinyobates spp. II APhobobates spp. II A

Ranita dorada de Madagascar (S): Mantella aurantiaca If A Rapaces diumes (F): FALCONIFORMES spp. I/UIU/NC B Rapaces noctumes (F): STRIGIFORMES spp. UII B Raptors (E): FALCONIFORMES spp. IIUII/NC B Rascón de Isla Lord Howe (S): Gallirallus sylvestris I B

Rat à grosse queue $(\mathrm{F})$ : Zyzomys pedunculatus I M

Rat architecte $(F)$ : Leporillus conditor $\mathbf{I} \mathrm{M}$

Rat kangaroos (E): Bettongia spp. I M

Rata arquitecto (S): Leporillus conditor I M

Rata bastarda de agua (S): Xeromys myoides I M

Rata coligorda (S): Zyzomys pedunculatus I M

Ratel (E/S/F): Mellivora capensis III M

Ratón bastardo peludo (S): Pseudomys praeconis I M

Ratón marsupial colilargo (S): Sminthopsis longicaudata I M

Ratón marsupial desértico (S): Sminthopsis psammophila I M

Ratonero calzado (S): Buteo lagopus II B

Ratonero común (S): Buteo buteo II B

Ratonero cuellirrojo (S): Buteo auguralis II B

Ratonero moro (S): Buteo rufinus II B

Rattlesnakes (E): Crotalus spp/Sistrurus spp. IIINC R

Ratufa spp:: Giant squirrels (E), Ardillas gigantes (S), Écureuils géants (F) II M

Ratufa affinis: Cream-coloured Giant Squirrel (E), Ratufe dorée (F) Écureuil de Raffles (F), Écureuil géant commun (F) II M

Ratufa bicolor: Black Giant Squirrel (E), Écureuil géant de Malaisie (F) II M

Ratufa indica: Indian Giant Squirrel (E), Écureuil géant de l'Inde (F) II $\mathbf{M}$

Ratufa macroura: Grizzled Giant Squirrel (E), Écureuil géant de Ceylan (F), Écureuil géant gris (F) II M

Ratufe doree (F): Ratufa affinis II M

Rayito brillante (S): Aglaeactis cupripennis II B

Razorback (E): Balaenoptera physalus I M

Razor-billed Curassow (E): Mitu mitu I B

Rebeco de los Abruzzos (S): Rupicapra pyrenaica omata I M

Red Bear (E): Ursus arcios isabellinus I M

Red Bird-of-paradise (E): Paradisaea rubra II B

Red Cat-bear (E): Ailurus fulgens I M

Red Cock-of-the-rock (E): Rupicola peniviana II B

Red Colobus (E): Procolobus badius II M

Red Crowned Parakeet (E): Cyanoramphus novaezelandiae I B

Red Dog (E): Cuon alpinus II M

Red Fox (E): Pseudalopex culpaeus II M

Red Goral (E): Naemorhedus baileyi I M

Red Goshawk (E): Erythrotriorchis radiatus II B

Red Howler (E): Alouatta seniculus II M

Red Kite (E): Milvus milvus II B

Red Lory (E): Eos bornea II B

Red Panda (E): Ailurus fulgens I M

Red Sheep (E): Ovis vignei vignei I M

Red Shining-Parrot (E): Prosopeia tabuensis II B

Red Siskin (E): Carduelis cucullata I B

Red Spider Monkey (E): Ateles geoffroyi panamensis I M

Red Teju (E): Tupinambis rufescens II R

Red Tiger (E): Puma concolor I/I M
Red-and-black Poison Frog (E): Dendrobares histrionicus II A

Red-and-blue Lory (E): Eos histrio I B

Red-and-blue Poison Frog (E): Dendrobates pumilio II A

Red-and-green Macaw (E): Ara chloropterus II B

Red-and-white Uakari (E): Cacajao calvus I M

Red-backed Hawk (E): Buteo polyosoma II B

Red-backed Poison Frog (E): Dendrobates reticulatus 11 A

Red-backed Squirrel Monkey (E): Saimiri oerstedii I M

Red-banded Poison Frog (E): Dendrobates lehmanni II A

Red-bearded Saki (E): Pithecia monachus II M

Red-bellied Conure (E): Pyrrhura frontalis II B

Red-bellied Guenon (E): Cercopithecus erythrogaster II M

Red-bellied Lemur (E): Eulemur rubrivenier I M

Red-bellied Macaw (E): Ara manilata II B

Red-bellied Monkey (E): Cercopithecus erythrogaster II M

Red-bellied Parrot (E): Poicephalus rufiventris II B

Red-bellied Spider Monkey (E): Areles geoffroyi frontatus I M

Red-bellied Tamarin (E): Saguinus labiarus II M

Red-billed Curassow (E): Crax blumenbachii I B

Red-billed Emerald (E): Chlorostilbon gibsoni II B

Red-billed Firefinch (E): Lagonostictu senegala III B

Red-billed Hanging-Parrot (E): Loriculus exilis II B

Red-billed Leiothrix (E): Leiothrix lutea II B

Red-billed Parrot (E): Pionus sordidus II B

Red-billed Toucan (E): Ramphastos iucanus II B

Red-billed Whistling-Duck (E): Dendrocygna autumnalis III B

Red-billed Wood-Dove (E): Turiur afer III B

Red-breasted Conure (E): Pyrrhura hoemarotis II B

Red-breasted Goose (E): Branta ruficollis II B

Red-breasted Parakeet (E): Psittacula alexandri II B

Red-breasted Pygmy-Parrot (E): Micropsinta bruijnii II B

Red-breasted Toucan (E): Ramphastos dicolorus III B

Red-browed Amazon (E): Amazona rhodocontha I B

Red-browed Parrot (E): Amazona rhodocorytha I B

Red-capped Mangabey (E): Cercocebus torquatus II M

Red-capped Parrot (E): Pionopsitta pileata I B/ Purpureicephalus spurius II B

Red-cheeked Cordonbleu (E): Uraeginthus bengalus III B

Red-cheeked Parrot (E); Geoffroyus geoffroyi II B

Red-chested Goshawk (E): Accipiter roussenelii II B

Red-chested Owlet (E): Glaucidium tephronotum II B

Red-chested Tamarin (E): Saguinus labiatus II M

Red-chinned Lorikeet (E): Charmosyna rubrigularis II B

Red-collared Lorikeet (E): Trichoglossus rubritorquis II B

Red-collared Whydah (E): Euplectes ardens III B

Red-collared Widowbird (E): Euplectes ardens III B

Red-crested Bustard (E): Eupodotis ruficrista II B

Red-crested Cardinal (E): Paroaria coronata II B

Red-crested Turaco (E): Tauraco erythrolophus II B

Red-crowned Amazon (E): Amazona viridigenalis I B

Red-crowned Crane (E): Grus japonensis I B

Red-crowned Parrot (E): Amazona viridigenalis I B/Poicephalus gulielmi II $\mathrm{B}$

Reddish Hermit (E): Phaethornis ruber II B

Reddish Scops-Owl (E): Otus rufescens II B

Reddish-bellied Parakeet (E): Pyrrhura frontalis II B

Red-eared Conure (E): Pyrrhura cruentata I B

Red-eared Guenon (E): Cercopithecus erythrotis II M

Red-eared Monkey (E): Cercopithecus erythrotis II M

Red-eared Parakeet (E): Pyrrhura hoematotis II B

Red-eared Waxbill (E): Estrilda troglodytes III B

Red-eyed Dove (E): Streptopelia semirorquata III B

Red-faced Lovebird (E): Agapomis pullarius II B

Red-faced Parrot (E): Hapalopsittaca pyrrhops II B

Red-faced Pytilia (E): Pytilia hypogrammica III B

Red-fan Parrot (E): Deroptyus accipitrinus II B

Red-flanked Lorikeet (E): Charmosyna placentis II B

Red-footed Falcon (E): Falco vespertinus II B

Red-footed Tortoise (E): Geochelone carbonaria II R

Red-fronted Amazon (E): Amazona vittata I B

Red-fronted Antpecker (E): Parmoptila rubrifrons III B

Red-fronted Conure (E): Aratinga wagleri II B

Red-fronted Kakariki (E): Cyanoramphus novaezelandiae I B

Red-fronted Lorikeet (E): Charmosyna rubronotaia II B

Red-fronted Macaw (E): Ara rubrogenys I B

Red-fronted Parakeet (E): Cyanoramphus novaezelandiae I B 
Red-fronted Parrot (E): Poicephalus gulielmi II B Red-fronted Parrotlet (E): Touil costaricensis II B Red-handed Howler (E): Alouanta belzebul II M Red-handed Tamarin (E): Saguinus midas II M Red-headed Amazon River Turtle (E): Podocnemis erythrocephala II $\mathrm{R}$

Red-headed Dioch (E): Quelea erythrops III B

Red-headed Falcon (E): Falco chicquera II B

Red-headed Lovebird (E): Agapormis pullarius II B

Red-headed Malimbe (E): Malimbus rubricollis III B

Red-headed Merlin (E): Falco chicquera II B

Red-headed Poison Frog (E): Dendrobates fantasticus II A

Red-headed Quelea (E): Quelea erythrops III B

Red-headed River Turtle (E): Podocnemis erythrocephala II R

Red-headed Rockfowl (E): Picathartes oreas I B

Red-headed Sideneck (E): Podocnemis enthrocephala II R

Red-headed Vulture (E): Sarcogyps calvus II B

Red-headed Weaver (E): Anaplectes rubriceps III B/Malimbus rubricollis III B

Red-legged Falconet (E): Microhierax caerulescens II B

Red-lored Amazon (E): Amazona autumnalis II B

Red-lored Parrot (E): Amazona autumnalis II B

Red-masked Conure (E): Aratinga erythrogenys II B

Red-masked Parakeet (E): Aratinga erythrogenys II B

Red-necked Amazon (E): Amazona arausiaca I B

Red-necked Buzzard (E): Buteo auguralis II B

Red-necked Falcon (E): Falco chicquera II B

Red-necked Partot (E): Amazona arausiaca I B

Red-nosed Dwarf Chameleon (E): Bradypodion oxyrhinum II R

Red-nosed Saki (E): Chiropotes albinasus I M

Red-nosed Tortoise (E): Indotestudo elongata II R

Red-rumped Parrot (E): Psephotus haemasonotus II B

Red-rumped Tarantula (E): Brachypelma vagans II in

Red-shanked Douc Langur (E): Pygathrix nemaeus I M

Red-shouldered Hawk (E): Buteo lineatus II B

Red-shouldered Macaw (E): Ara nobilis II B

Red-spectacled Amazon (E): Amazona pretrei I B

Red-spectacled Partot (E): Amazona pretrei I B

Red-spotted Lorikeet (E): Charmosyna rubronotala II B

Redtail Monkey (E): Cercopithecus ascanius II M

Red-tailed Amazon (E): Amazona brasiliensis I B

Red-tailed Black-Cockatoo (E): Calyptorhynchus banksii II B

Red-tailed Buzzard (E): Buteo auguralis II B

Red-tailed Comet (E): Sappho sparganura II B

Red-tailed Hawk (E): Buteo jamaicensis II B/Buteo ventralis II B

Red-tailed Monkey (E): Cercopithecus ascanius II M

Red-tailed Parrot (E): Amazona brasiliensis I B

Red-tailed Sportive Lemur (E): Lepilemur ruficaudatus I M

Red-thighed Falconet (E): Microhierax caerulescens II B

Red-thighed Sparrowhawk (E): Accipiler enthropus II B

Red-throated Caracara (E): Daperius americanus II B

Red-throated Lorikeet (E): Charmosyna amabilis II B

Red-topped Amazon (E): Amazona rhodocorytha I B

Red-topped Parrot (E): Amazona rhodocorytha I B

Red-vented Cockatoo (E): Cacalua haematuropygia I B

Red-vented Malimbe (E): Malimbus scutatus III B

Red-vented Parrot (E): Pionus menstruus II B

Red-winged Parrot (E): Aprosmictus erythropterus II B

Red-winged Pytilia (E): Pyrilia phoenicoptera III B

Reed Cat (E): Felis chaus II M

Regal Python (E): Python reticulatus II $\mathbf{R}$

Regent Parrot (E): Polytelis anthopeplus II B

Reichenow's Spiny-tailed Lizard (E): Cordylus vitnifer II R

Relict Gull (E): Larus relictus I B

Renard colfeo (F): Pseudalopex culpaeus II M

Renard d'Azara (F): Pseudalopex gymnocercus II M

Renard de Blanford (F): Vulpes cana II M

Renard de Chiloé (F): Pseudalopex griseus II M

Renard du Bengale (F): Vulpes bengalensis III M

Renard gris d'Argentine (F): Pseudalopex griseus II M

Renard volant de Ponape (F): Pteropus molossinus I M

Renards-volants (F): Acerodon spp. II M

Resplendent Quetzal (E): Pharomachrus mocinno I B

Reticulated Poison Frog (E): Dendrobates reticulatus II A

Reticulated Python (E): Python reticulatus II R

Réunion Day Gecko (E): Phelsuma borbonica II R
Reussastrea = Pavona

Rey zamuro (S): Sarcoramphus papa III B

Rhabdocyathus = Acropora

Rhampholeon marshalli $=$ Chamaeleo marshall $i$

Rhea americana: Common Rhea (E), Greater Rhea (E), Avestruz (S), N̄andú (S), Ñandú común (S), Nandou commun (F), Nandou d'Amérique (F). Nandou gris $(F)$ II B

Rhea pennata: Darwin's Rhea (E), Lesser Rhea (E), Puna Rhea (E), Avestruz de Magallanes (S), Nandú cordillerano (S), Ñandú petizo (S), Nandou de Darwin (F) I B

Rheas (E): Rhea spp. I/II B

Rheinardia nigrescens $=$ Rheinardia ocellata

Rheinardia ocellata: Crested Argus (E), Ocellated Pheasant (E), Faisán de Rheinard (S), Argus ocellé (F), Rheinarte ocellé (F) I B

Rheinarte ocelle (F): Rheinardia ocellata I B

Rheobatrachus spp.: Gastric-brooding frogs (E), Grenouilles a incubation gastrique (F) II A

Rheobatrachus silus: Conondale Gastric-brooding Frog (E), Platypus

Frog (E), Grenouille plate à incubation gastrique (F) II A

Rheobatrachus vitellinus: Eungella Gastric-brooding Frog (E),

Southem Gastric-brooding Frog (E), Grenouille à incubation gastrique (F) II A

Rhesus Macaque (E): Macaca mulatta II M

Rhesus Monkey (E): Macaca mulatta II M

Rhim (E/F): Gazella leproceros III M

Rhinocéros (F): Rhinocerotidae spp. I/I M

Rhinocéros blanc du Nord (F): Ceratotherium simum coltoni I M

Rhinocéros blanc du Sud (F): Ceratotherium simum simum III M

Rhinocelos Chameleon (E): Chamaeleo rhinoceratus II R

Rhinocéros de la Sonde (F); Rhinoceros sondaicus I M

Rhinocéros de Sumatra (F): Dicerorhinus sumatrensis I M

Rhinoceros Hombill (E): Buceros rhinoceros II B

Rhinoceros Iguana (E): Cyclura comuta I R

Rhinocéros noir (F): Diceros bicomis I M

Rhinoceros sondaicus: Javan Rhinoceros (E), Rinoceronte de Java

(S), Rhinocéros de la Sonde (F) I M

Rhinocéros unicome de l'Inde (F): Rhinoceros unicomis I M

Rhinoceros unicornis: Great Indian Rhinoceros (E), Rinoceronte unicomio índico (S), Rhinocéros unicorne de l'Inde (F) I M

Rhinoceroses (E): Rhinocerotidae spp. I/II M

Rhinocerotidae spp.: Rhinoceroses (E), Rinocerontes (S), Rhinocéros (F) $\mathbf{I I I ~ M}$

Rhinopithecus avunculus = Pygathrix avunculus

Rhinopithecus bieti $=$ Pygathrix bieti

Rhinopithecus brelichi = Pygathrix brelichi

Rhinopithecus nemaeus = Pygathrix nemaeus

Rhinopithecus roxellana $=$ Pygathrix roxellana

Rhinopithèque de Roxellane (F): Pygathrix roxellana I M

Rhinopithèque doré (F): Pygathrix roxellana I M

Rhinopithèque douc (F): Pygathrix nemaeus I M

Rhinopithèque du Tonkin (F): Pygathrix avunculus I M

Rhinopithèque jaune doré (F): Pygarhrix brelichi I M

Rhinopithèques (F): Pygathrix spp. I M

Rhinoplax vigil = Buceros vigil

Rhinoptynx clamator $=$ Asio clamator

Rhipidipathes = Antipathes

Rhizopsammia annae: II In

Rhizopsammia chamissoi: II In

Rhizopsammia compacta: II In

Rhizopsammia manuelensis: II In

Rhizopsammia minuta: II In

Rhizopsammia pulchra: II In

Rhizopsammia verrill: II In

Rhizopsammia wellingtoni: II In

Rhizopsammia wettsteini: II In

Rhizosmilia gerdae: II In

Rhizosmilia maculata: II In

Rhizosmilia robusta: II In

Rhizothera longirostris: Long-billed Partridge (E), Perdiz de bosque

de pico largo (S), Perdiz piquilarga (S), Perdrix à long bec (F),

Rouloul à long bec (F) III B

Rhizotrochus flabelliformis: II In

Rhizotrochus levidensis: II In

Rhizorrochus radiatus: II In

Rhizotrochus typus: II In 
Rhodaraea $=$ Goniopora

Rhodesian Girdled Lizard (E): Cordylus rhodesianus II R

Rhodocyathus $=$ Scolymia

Rhodonessa caryophyllacea: Pink-headed Duck (E), Pato cabecirrosa (S), Canard à tête rose (F) I B

Rhodopis vesper. Oasis Hummingbird (E), Colibri vesper (F) II B

Rhodopsammia = Balanophyllia

Rhombopsammia niphada: II in

Rhombopsammia squiresi: II In

Rhoptropella ocellata $=$ Phelsuma ocellata

Rhynchopsitta spp.: I B

Rhynchopsina pachyrhyncha: Thick-billed Parrot (E), Cotorraserrana occidental (S), Loro piquigordo (S), Periquito de pico grueso $(\mathrm{S})$, Conure à gros bec (F), Perruche ara (F), Perruche à gros bec (F) I B

Rhynchopsitta pachyrhyncha terrisi $=$ Rhynchopsitta terris

Rhynchopsitta terrisi: Maroon-fronted Parrot (E), Cotorra-serrana oriental (S), Conure à front brun (F) I B

Rhynochetos jubatus: Kagu (E), Kagú (S), Kagou (F), Kagou huppé (F) \B

Rhyticeros cassidix $=$ Aceros cassidix

Rhyticeros corrugatus $=$ Aceros corrugatus

Rhyticeros everetti = Aceros everetti

Rhyticeros leucocephalus = Aceros leucocephalus

Rhyriceros leucocephalus waldeni $=$ Aceros waldeni

Rhyticeros narcondami $=$ Aceros narcondami

Rhyticeros plicatus = Aceros plicatus

Rhyriceros subruficollis $=$ Aceros subruficollis

Rhyticeros undulatus = Aceros undulatus

Ribbon Coral (E): Agaricia tenuifolia II In

Ribbon Finch (E): Amadina fasciata III B

Ribbon-tailed Astrapia (E): Astrapia mayeri II B

Ribbon-tailed Bird-of-paradise (E): Astrapia mayeri II B

Rice Lizard (E): Varanus salvator II R

Richmond Birdwing (E): Ornithoptera richmondia II In

Ricord's Ground Iguana (E): Cyclura ricordi I R

Ricord's Iguana (E): Cyclura ricordi I R

Ridge-tailed Monitor (E): Varanus acanthurus/Varanus primordius II $\mathbf{R}$

Ridgway's Hawk (E): Buteo ridgwayi II B

Ridley de Kemp (F): Lepidochelys kempi I R

Ridley du Pacifique (F): Lepidochelys olivacea I R

Ridleys (E): Lepidochelys spp. I R

Riffleshells (E): Epioblasma spp. I/IINC In

Riflebirds (E): Priloris spp. II B

Right Whale (E): Eubalaena glacialis I M

Right whales (E): Eubalaena $\operatorname{spp}$ /Caperea marginata I M

Rimose Naiad (E): Conradilla caelata I in

Ring Lizard (E): Varanus salvator II R

Ringed Python (E): Morelia boa II R

Ring-necked Parakeet (E): Psinacula krameri III B

Ringnecks (E): Platycercus spp. II B

Ring-tailed Lemur (E): Lemur catta I M

Rinoceronte blanco (S): Ceratotherium simum simum III M

Rinoceronte blanco del norte (S): Ceratotherium simum cottoni I M

Rinoceronte de Java (S): Rhinoceros sondaicus I M

Rinoceronte de Sumatra (S): Dicerorhinus sumarrensis $1 \mathrm{M}$

Rinoceronte negro (S): Diceros bicomis I M

Rinoceronte unicomio índico (S): Rhinoceros unicomis I M

Rinocerontes (S): Rhinocerotidae spp. I/I M

Rio Apaporis (Spectacled) Caiman (E): Caiman crocodilus apaporiensis I R

Rio Madeira Poison Frog (E): Dendrobates quinquevittatus II A

Rio Magdalena River Turtle (E): Podocnemis lewyana II R

Rio Napo Tamarin (E): Saguinus fuscicollis II M

Rio Santiago Poison Frog (E): Dendrobates captivus II A

Rip Sack (E): Eschrichtius robusius I M

Risso's Dolphin (E): Grampus griseus II M

River dolphins (E): Platanistidae spp./Soralia spp. I /II M

River Eagle (E): Haliaeetus vocifer II B

River Terrapin (E): Batagur baska I R

River-Martins (E): Pseudochelidon spp. INC B

Rivoli's Hummingbird (E): Eugenes fulgens II B

Roadside Hawk (E): Buteo magnirostris II B

Robertson's Dwarf Chameleon (E): Bradypodion gutturale II R

Rock Dove (E): Columba livia III B
Rock Eagle-Owl (E): Bubo bengalensis II B

Rock Iguana (E): Cyclura cyclura I R

Rock Monitor (E): Varanus albigularis II $\mathrm{R}$

Rock Parakeet (E): Pyrrhura rupicola II B

Rock Parrot (E): Neophema petrophila II B

Rock Peppler (E): Polyrelis anthopeplus II B

Rock Pigeon (E): Columba livia III B

Rockfowl (E): Picathartes spp. I B

Rock-rats (E): Zyzomys spo. I/NC M

Rodrigues Brush-Warbler (E): Bebromis rodericanus III B

Rodrigues Day Gecko (E): Phelsuma edwardnewtonii II R

Rodrigues Flying-fox (E): Pteropus rodricensis II M

Rodrigues Warbler (E): Bebromis rodericanus III B

Roi des vautours (F): Sarcoramphus papa III $\mathbf{B}$

Rollulus rouloul: Crested Partridge (E), Roulroul (E), Perdiz de bosque crestada (S), Perdiz nulrul (S), Rouloul couronné (F), Rouloul à couronne (F) III B

Romerolagus diazi: Volcano Rabbit (E), Conejo de Díaz (S), Conejo de los volcanes (S), Teporingo (S), Zacatuche (S), Lapin de Diaz (F), Lapin des volcans (F) I M

Roofed Turtle (E): Kachuga iecta $1 \mathrm{R}$

Rorcual azul (S): Balaenoptera musculus I M

Rorcual boreal (S): Balaenoptera borealis I M

Rorcual común (S): Balaenoptera physalus I M

Rorcual de Rudolphi (S): Balaenoptera borealis I M

Rorcual jorobado (S): Megapiera rovaeangliae I M

Rorcual menor (S): Balaenoptera acutorostrata $\mathbf{I I I} \mathrm{M}$

Rorcual norteno (S): Balaenoptera borealis I M

Rorqual à bosse (F): Megaptera novaeangliae I M

Rorqual à ventre cannelé (F): Balaenoptera musculus I M

Rorqual bleu (F): Balaenoptera musculus I M

Rorqual boréal (F): Balaenoptera borealis I M

Rorqual commun (F): Balaenoptera physalus I M

Rorqual de Bryde (F): Balaenoptera edeni I M

Rorqual de Rudolphi (F): Balaenoptera borealis I M

Rorqual de Sibbold (F): Balaenoptera musculus I M

Rorqual d'Eden (F): Balaenoptera edeni I M

Rorqual du Cap (F): Megaptera novaeangliae I M

Rorqual sei (F): Balaenoptera borealis I M

Rorqual tropical (F): Balaenoptera edeni I M

Rorquals (E): Balaenoptera spp. I/II M

Rose Coral (E): Manicina areolata II In

Roseate Cockatoo (E): Eolophus roseicapillus II B

Rose-crowned Conure (E): Pyrrhura rhodocephala II B

Rose-faced Partot (E): Pionopsitta pulchra II B

Rose-grey Dove (E): Sireptopelia roseogrisea III B

Rose-headed Conure (E): Pyrrhura rhodocephala II B

Rose-headed Parakeet (E): Pyrrhura rhodocephala II B

Rosellas (E): Platycercus spp. II B

Rosenberg's Monitor (E): Varanus rosenbergi II $\mathrm{R}$

Rose-ringed Parakeet (E): Psittacula krameri III B

Rosette-nosed Chameleon (E): Bradypodion spinosum II R

Rossell Island Tree Monitor (E): Varanus telenestes II R

Rostrhamus hamatus: Slender-billed Kite (E), Caracolero plomizo

(S), Gavilán pico de $\mathrm{Hoz}$ (S), Milan à long bec (F) II B

Rostrhamus sociabilis: Everglade Kite (E), Snail Kite (E),

Carocolero común (S), Gavilán caracolero (S), Milano

caracolero (S), Milan des marais (F) II B

Rosy Boa (E): Lichanura trivirgata II R

Rosy-faced Lovebird (E): Agapomis roseicollis II B

Rothschild's Birdwing (E): Omithoptera rothschildi II In

Rothschild's Mynah (E): Leucopsar rothschildi I B

Rothschild's Parakeet (E): Psitiacula intermedia II B

Rothschild's Peacock-Pheasant (E): Polyplectron inopinatum III B

Rothschild's Starling (E): Leucopsar rothschildi I B

Rough Pigtoe (E): Pleurobema plenum I In

Rough Pigtoe Pearly Mussel (E): Pleurobema plenum I In

Rough Starlet Coral (E): Siderastrea radians II in

Rough-legged Buzzard (E): Buteo lagopus II B

Rough-legged Hawk (E): Buteo lagopus II B

Rough-necked Monitor (E): Varanus rudicollis II R

Rough-scaled Boa (E): Trachyboa boulengeri II R

Rough-scaled Girdled Lizard (E): Cordylus cordylus II R

Rough-scaled Python (E): Morelia carinata II R

Rough-scaled Sand Boa (E): Eny conicus II R

Rough-toothed Dolphin (E): Steno bredanensis II M 
Rouloul à couronne (F): Rollulus rouloul III B

Rouloul a long bec (F): Rhizothera longirostris III B

Rouloul couronné (F): Rollulus rouloul III B

Rouloul noir (F): Melanoperdix nigra III B

Rouloul ocelle (F): Caloperdix oculea III B

Roulouls (F): Caloperdix oculea/Haemarortyx sanguiniceps/Rollulus rouloul IIINC B

Roulroul (E): Rollulus rouloul III B

Round Island Burrowing Boa (E): Bolyeria multocarinata I R

Round Island Day Gecko (E): Phelsuma guentheri II R

Round Island Keel-scaled Boa (E): Casarea dussumieri I R

Rousserolle de Rodriguez (F): Bebromis rodericanus III B

Rousserolles (F): Acrocephalus spp/Bebromis spp. IIINC B

Roussette de lile Mortlock (F): Preropus phaeocephalus I M

Roussette des îles Marianes (F): Pteropus mariannus I M

Roussette des îles Samoa (F): Pleropus samoensis I M

Roussette des îles Tonga (F): Preropus tonganus I M

Roussette des îles Truk (F): Preropus insularis I M

Roussettes (F): Pteropodidae spp. I/IVNC M

Royal Python (E): Python regius II R

Royal Sunangel $(\mathrm{E})$ : Heliangelus regalis II $\mathrm{B}$

Rubber Boa (E): Charina bottae II R

Ruby Poison Frog (S): Epipedobates parvulus II A

Ruby-throated Hummingbird (E); Archilochus colubris II B

Ruby-topaz Hummingbird (E): Chrysolampis mosquitus II B

Ruddy Mongoose (E): Herpestes smithii III M

Ruddy Snub-nosed Monitor (E): Varanus flavescens I R

Ruddy Treeshrew (E): Tupaia splendidula II M

Rudophi's Rorqual (E): Balaenoptera borealis I M

Rueck's Blue-Flycatcher (E): Cyomis ruckii II B

Rueck's Niltava (E): Cyomis ruckii II B

Rueppell's Bustard (E): Eupodotis rueppellii II B

Rueppell's Griffon (E): Gyps rueppellii II B

Rueppell's Griffon Vulture (E): Gyps rueppellii II B

Rueppell's Parrot (E): Poicephalus rueppellii II B

Rueppell's Vulture (E): Gyps rueppellii II B

Rufescent Screech-Owl (E): Otus ingens II B

Ruffed Lemur (E): Varecia variegala I M

Rufous Crab-Hawk (E): Buteogallus aequinocrialis II B

Rufous Fishing-Owi (E): Scotopelia ussheri II B

Rufous Hare-wallaby (E): Lagorchestes hirsutus I M

Rufous Hombill (E): Buceros hydrocorax II B

Rufous Hummingbird (E): Selasphorus rufus II B

Rufous Mouse-lemur (E): Microcebus rufus I M

Rufous Owl (E): Ninox rufa II B

Rufous Sabrewing (E): Campylopterus rufus II B

Rufous-banded Owl (E): Strix albitarsus II B

Rufous-bellied Eagle (E): Hieranetus kienerii II B

Rufous-breasted Hermit (E): Glaucis hirsufa II B

Rufous-breasted Sabrewing (E): Campylopterus hyperythrus II B

Rufous-capped Thombill (E): Chalcostigma nificeps II B

Rufous-cheeked Hombill (E): Aceros nipalensis I B

Rufous-cheeked Hummingbird (E): Goethalsia bella II B

Rufous-chested Sparrowhawk (E): Accipizer nufiventris II B

Rufous-crested Coquette (E): Lophomis delattrei II B

Rufous-fronted Parakeet (E): Bolborhynchus ferrugineifrons II B

Rufous-headed Bristlebird (E): Dasyomis broadbenti litoralis I B

Rufous-legged Owl (E): Sirix rufipes II B

Rufous-naped Tamarin (E): Saguinus geoffroyi/Saguinus oedipus I M

Rufous-necked Hornbill (E): Aceros nipalensis I B

Rufous-necked Sparrowhawk (E): Accipiter erythrauchen II B

Rufous-shafted Woodstar (E); Chaetocercus jourdanii II B

Rufous-tailed Hawk (E): Buteo ventralis II B

Rufous-tailed Hummingbird (E): Amazilia tzacall II B

Rufous-tailed Pheasant (E): Lophura enthrophthalma III B

Rufous-tailed Treeshrew (E): Tupaia splendidula II M

Rufous-thighed Hawk (E): Accipiter erythronemius II B/Buteo leucorrhous II B

Rufous-thighed Kite (E): Harpagus diodon II B

Rufous-throated Sapphire (E): Hylocharis sapphirina II B

Rufous-vented Whitetip (E): Urosticte ruficrissa II B

Rufous-webbed Brilliant (E): Heliodoxa branickii II B

Rufous-winged Buzzard (E): Butastur liventer II B

Rufous-winged Buzzard-Eagle (E): Busastur liventer II B

Rungwe Viviparous Toad (E): Nectophrynoides viviparus I A
Rupicapra pyrenaica omata: Abruzzo Chamois (E), Apennine Chamois (E), Gamuza de los Abruzzos (S), Rebeco de los Abruzzos (S), Chamois des Abruzzes (F) I M

Rupicapra rupicapra omata = Rupicapra pyrenaica omata

Rupicola spp.: Cocks-of-the-rock (E), Gallitos de las rocas (S), Coqsde-roche (F) II B

Rupicola peruviana: Andean Cock-of-the-rock (E), Peruvian Cockof-the-Rock (E), Red Cock-of-the-rock (E), Scarlet Cock-of-therock (E), Gallito de las Sierras (S), Gallito de roca peruano (S), Tunqui (S), Coq-de-roche péruvien (F) 11 B

Rupicola rupicola: Common Cock-of-the-Rock (E), Guianan Cockof-the-rock (E), Orange Cock-of-the-rock (E), Smaller-Cock-ofthe-rock (E), Gallito de las Rocas (S), Gallito de roca anaranjado (S), Coq-de-roche de Guyane (F), Coq-de-roche orange (F) II B

Rüppell's Desert Chameleon (E): Chamaeleo affinis II R

Rüppell's Parrot (S): Poicephalus rueppellii II B

Ruspoli's Chameleon (E): Chamaeleo ruspolii II R

Ruspoli's Turaco (E): Tauraco ruspolii II B

Russell's Sand Boa (E): Eryx conicus II R

Russell's Viper (E): Vipera russellii III $R$

Russet Hawk-Owl (E): Ninox odiosa II B

Russet Mouse-lemur (E): Microcebus rufus I M

Russet-eared Guenon (E): Cercopithecus erythrotis II M

Russian Sturgeon (E): Acipenser gueldensiaedrii II F

Rusty Monitor (E): Varanus semiremex II R

Rusty-barred Owl (E): Pulsarrix melanota II B/Strix hylophila II B

Rusty-faced Parrot (E): Hapalopsiltaca amazonina II B

Rusty-spotted Cat (E): Prionailurus rubiginosus VI M

Ruwenzori Mountain Chameleon (E): Bradypodion carpenteri II R

Ruwenzori Side-striped Chameleon (E): Chamaeleo rudis II R

Ruwenzori Three-horned Chameleon (E): Chamaeleo johnsioni II R

Ryukyu Flying-fox (E): Pleropus dasymallus II M

Sabinotrochus = Stephanocyathus

Sable Antelope (E): Hippotragus niger $\mathbf{I} / \mathrm{NC}$ M

Sabrewings (E): Campylopterus spp. II B

Sacafi (S): Psammobates geometricus I R

Sachacabra (S): Pudu mephistophiles II M

Sachavaca (S): Tapirus terrestris II M

Sacred Black Mud Turtle (E): Trionyx nigricans I R

Sacred Ibis (E): Threskiomis aethiopicus III B

Saddle-back Tamarin (E): Saguinus fuscicollis II M

Saddle-backed Dolphin (E): Delphinus delphis II M

Saddlebill Stork (E): Ephippiorhynchus senegalensis III B

Saddle-billed Stork (E): Ephippiorhynchus senegalensis III B

Saffron Toucanet (E): Baillonius bailloni III B

Saffron-coloured Clam (E): Tridacna crocea II in

Saffron-cowled Blackbird (E): Agelaius flavus I B

Saffron-headed Parrot (E): Pionopsina pyrilia II B

Sagittarius sementarius: Secretary-bird (E), Secretario (S)

Serpentario (S), Messager sagittaire (F), Secrétaire des serpents (F), Serpentaire (F) II B

Saguinus bicolor: Bare-faced Tamarin (E), Pied Tamarin (E), Tamarín bicolor (S). Tamarin bicolore (F) I M

Saguinus fuscicollis: Brown-headed Tamarin (E), Rio Napo Tamarin (E), Saddle-back Tamarin (E), Tamarin à manteau rouge (F) II M

Saguinus fuscicollis triparitus = Saguinus tripartitus

Saguinus geoffroyi: Cotton-top Marmoset (E), Liszt Monkey (E),

Pinche Marmoset (E), Rufous-naped Tamarin (E), Tamarino de Geoffroy (S), Tamarin de Geoffroy (F) I M

Saguinus graellsi = Saguinus nigricollis

Saguinus imperator. Emperor Tamarin (E), Tamarino emperador (S) II M

Saguinus inustus: Dusky Tamarin (E), Mottle-faced Tamarin (E), Tamarino de caba manchada (S) II M

Saguinus labiatus: Red-bellied Tamarin (E), Red-chested Tamarin (E), White-lipped Tamarin (E), Tamarino de labios blancos (S) II M

Saguinus leucopus: White-footed Tamarin (E), Tamarin de manos blancas (S), Tamarin à pieds blancs (F) I M

Saguinus martinsi = Saguinus bicolor

Saguinus melanoleucus $=$ Saguinus fuscicollis

Saguinus midas: Negro Tamarin (E), Red-handed Tamarin (E), Tamarin aux mains rousses (F) II $\mathbf{M}$

Saguinus mystax: Moustached Tamarin (E), Tamarin à moustaches (F) II M 
Saguinus nigricollis: Black-and-red Tamarin (E), Black-mantled Tamarin (E), Tamarin rouge et noir (F) II M

Saguinus oedipus: Cotton-headed Tamarin (E), Cotton-top Tamarin (E), Geoffroy's Tamarin (E), Rufous-naped Tamarin (E),

Bichichi (S), Tamarin d'Oedipe (F), Tamarin pinché (F),

Tamarin à perruque (F) I M

Saguinus oedipus geoffroyi = Saguinus geoffroyi

Saguinus pileatus = Saguinus mystax

Saguinus tamarin = Saguinus midas

Saguinus tripartitus: Golden-mantled Saddle-back Tamarin (E),

Tamarino de espalda dorada (S) II M

Sahara Mastigure (E): Uromastyx geyri II R

Sahara Oryx (E): Oryx dammah I M

Sahelian Terrapin (E): Pelusios adansonii III R

Saiga (E/S): Saiga tatarica II M

Saiga (F): Saiga tatarica II M

Saiga Antelope (E): Saiga tatarica II M

Saiga tatarica: Saiga (E), Saiga Antelope (E), Antilope saiga (S), Saiga (S), Saiga (F) II M

Saïmiri à dos roux (F): Saimiri oerstedii I M

Saimiri boliviensis: II M

Saïmiri écureuil (F): Saimiri sciureus II M

Saimiri oerstedii: Central American Squirrel Monkey (E), Redbacked Squirrel Monkey (E), Barizo dorsirrojo (S), Mono tití (S), Saïmiri à dos roux $(F)$, Singe-écureuil à dos rouge $(F)$. Singe-écureuil à dos roux (F) I M

Saimiri saimiri ustus = Saimiri ustus

Saimiri sciureus: Common Squirrel Monkey (E), Saïmiri écureuil (F) II M

Saimiri sciureus boliviensis $=$ Saimiri boliviensis

Saimin ustus: II M

Saimiri vanzolinii: Blackish Squirrel Monkey (E) II M

Saint Helena Waxbill (E): Estrilda astrild III B

Saint Lucia Amazon (E): Amazona versicolor I B

Saint Lucia Partot (E): Amazona versicolor I B

Saint Vincent Amazon (E): Amazona guildingii I B

Saint Vincent Parrot (E): Amazona guildingii I B

Sajou à front blanc (F): Cebus albifrons II M

Sajou à gorge blanche (F): Cebus capucinus II M

Sajou apelle (F): Cebus apella II M

Sakafi (F): Psammubaies geometricus I R

Saker (E): Falco cherrug II B

Saker Falcon (E): Falco cherrug II B

Sakhalin Sturgeon (E): Acipenser mikadoi II F

Saki à nez blanc $(F)$ : Chiropotes albinasus I M

Saki à tête pâle (F): Pithecia pithecia II M

Saki amazónico (S): Pithecia albicans II M

Saki chevelu (F): Pithecia monachus II M

Saki de cara pelada (S): Pithecia irrorata II M

Saki nariblanco (S): Chiropotes albinasus I M

Saki noir (F): Chiropotes satanas II M

Sakis (E): Chiropotes spp/Pithecia spp. III M

Saladang (E): Bos gaurus I M

Salamanders (E): CAUDATA spp. I/II/NC A

Salamandra gigante de China (S): Andrias davidianus I A

Salamandra gigante de Japón (S): Andrias japonicus I A

Salamandras gigantes (S): Andrias spp. I A

Salamandre du lac Pàtzcuaro (F): Ambystoma dumerilii II A

Salamandre du Mexique (F): Ambystoma mexicanum II A

Salamandre du Père David (F): Andrias davidianus I A

Salamandre géante (F): Andrias spp. I A

Salamandre géante de Chine (F): Andrias davidianus I A

Salamandre géante du Japon (F): Andrias japonicus I A

Salmon-crested Cockatoo (E): Cacatua moluccensis I B

Salt-water Crocodile (E): Crocodylus porosus I/II R

Salvadori's Fig-Parrot (E): Psitnaculirostris salvadorii II B

Samar Hornbill (E): Penelopides samarensis II B

Samoan Flying-fox (E): Pteropus samoensis I M

Sampson's Naiad (E): Epioblasma sampsonii I In

Sampson's Pearly Mussel (E): Epioblasma sampsonii I In

Sampson's Riffleshell (E): Epioblasma sampsonii II In

San Esteban Island Chuckwalla (E): Sauromalus varius I R

San Salvador Ground Iguana (E): Cyclura rileyi I R

Sand boas (E): Eryx spp. II R

Sand Cat (E): Felis margarita II M

Sand Dune Cat (E): Felis margarita II M
Sand Gazelle (E): Gazella leptoceros III M

Sand Goanna (E): Varanus rosenbergi II $R$

Sand Monitor (E): Varanus gouldii II R

Sand Sturgeon (E): Scaphirhynchus platorynchus II F

Sandalolitha dentata: II In

Sandalolitha robusta: Basket Coral (E) II In

Sandback Pocketbook Mussel (E): Lampsilis sasur 1 In

Sandhill Crane (E): Grus canadensis III B

Sandhill Dunnart (E): Sminthopsis psammophila I M

Sandhill Sminthopsis (E): Sminthopsis psammophila I M

Sandy Scops-Owl (E): Otus icterorhynchus II B

Sanford's Fish-eagle (E): Haliaeetus sanfordi II B

Sanford's Sea-Eagle (E): Haliaeetus sanfordi II B

Sangihe Hanging-Parrot (E): Loriculus catamene II B

Sanglier nain (F): Sus salvanius I M

Sanglier pygmée ( $F$ ): Sus salvanius I M

Sangsue médicinale (F): Hirudo medicinalis II In

Sangsue officinale (F): Hirudo medicinalis II in

Sanguine Poison Frog (E): Epipedobates zaparo II A

Sansonnet de Rothschild (F): Leucopsar rothschildi I B

Santa Barbara Screech-Owl (E): Otus varbarus II B

Santa Marta Conure (E): Pyrrhura viridicata II B

Santa Marta Parakeet (E): Pyrrhura viridicata II B

Santa Marta Sabrewing (E): Campylopterus phainopeplus II B

Santa Marta Woodstar (E): Acestrura astreans II B

Sanzinia (E): Sanzinia madagascariensis I $R$

Sanzinia madagascariensis: Madagascar Tree Boa (E), Sanzinia (E),

Boa arborícola de Madagascar (S), Boa des foríts de Madagascar (F) I R

Sanzinia manditra $=$ Sanzinia madagascariensis

Sao Tome Scops-Owl (E): Otus hartlaubi II B

Saola (E/S/F): Pseudonyx nghetinhensis I M

Saphir à gorge rousse (F): Hylocharis sapphirina II B

Saphir à oreilles blanches (F): Hylocharis leucotis II B

Saphir à queue d'or (F): Hylocharis chrysura II B

Saphir azuré (F): Hylocharis cyanus II B

Saphir de Xantus (F): Hylocharis xantusii II B

Saphir d'Elicia (F): Hylocharis eliciae II B

Saphir embrasé (F): Hylocharis pyropygia II B

Saphir oenome (F): Chrysuronia oenone II B

Saphir ulysse (F): Hylocharis grayi II B

Saphirs (F): Chrysuronia oenone/Hylocharis spp. II B

Sapo del Camerún (S): Bufo superciliaris I A

Sapo dorado de Monteverde (S): Bufo periglenes I A

Sapo verde de Sonora (S): Bufo retiformis II A

Sapo viviparo de Africa occidental (S): Nimbaphrynoides occidentalis I A

Sapo viviparo de Malcolm (S): Altiphrynoides malcolmi I A

Sapo viviparo de Osgcod (S): Spinophrynoides osgoodi I A

Sapo viviparo liberiano (S): Nimbaphrynoides liberiensis I A

Sapos viviparos (S): Altiphrynoides Spp/Nimbaphrynoides spp/Spinophrynoides spp. I A

Sapos vivíparos africanos (S): Nectophrynoides spp. $1 \mathrm{~A}$

Sapphire-bellied Hummingbird (E): Lepidopyga lilliae II B

Sapphire-rumped Parrotlet (E): Touis purpurata II B

Sapphires (E): Chlorestes notatus/Chrysuronia oenone/Hylocharis spp. II B

Sapphire-spangled Emerald (E): Amazilia lactea II B

Sapphire-throated Hummingbird (E): Lepidopyga coeruleogularis II $B$

Sapphire-vented Puffleg (E): Eriocnemis luciani II B

Sappho sparganura: Red-tailed Comet (E), Picaflor coludo rojo (S), Colibri sapho (F) II B

Sarawak Dolphin (E): Lagenodelphis hosei II M

Sarcelle à oreilions $(F)$ : Nenapus auritus III B

Sarcelle aptère (F): Anas aucklandica I B

Sarcelle brune (F): Anas aucklandica I B

Sarcelle de Bernier (F): Anas bemieri II B

Sarcelle de Laysan (F): Anas laysanensis I B

Sarcelle de Madagascar (F): Anas bernieri II B

Sarcelle de Nouvelle-Zélande (F): Anas aucklandica I B

Sarcelle d'été (F): Anas querquedula III B

Sarcelle d'hiver (F): Anas crecca III B

Sarcelle élégante (F): Anas formosa II B

Sarcelle formose (F): Anas formosa II B

Sarcelle malgache de Bernier (F): Anas bemieri II B 
Sarcelle terrestre des îles Auckland (F): Anas aucklandica I B

Sarcidiome à crête (F): Sarkidiomis melanotos II B

Sarcinula $=$ Galarea

Sancogyps calvus: Indian Black Vulture (E), Pondicherry Vulture (E), Red-headed Vulture (E), Buitre cabecirrojo (S), Vautour royal (F) II B

\section{Sarcoramphe roi (F): Sarcoramphus papa III B}

Sarcoramphus papa: King Vulture (E), Gallinazo rey (S), Jote real (S), Rey zamuro (S), Zopilote rey (S), Roi des vautours (F), Sarcoramphe roi (F), Vautour page (F), Vautcur royal (F) III B

Sarkidiomis melanotos: Comb Duck (E), Knob-billed Goose (E). Pato crestudo (S), Pato crestón (S), Pato de moco (S), Pato de monte (S), Canard coronculé (F), Canard a bosse bronzé (F), Sarcidiome à crête (F) II B

Sarus Crane (E): Grus antigone II B

Sasin (E): Antilope cervicapra III M

Sassaby (F): Damaliscus lunatus III M

Satyr Tragopan (E): Tragopan satyra III B

Sauromalus varius: San Esteban Island Chuckwalla (E), Chuckwalla de San Esteban (F) I R

Sauvegarde (F): Tupinambis teguixin II R

Savagliopsis = Antipathes

Savanna Hawk (E): Bureogallus meridionalis II B

Savanna Monkey (E): Chlorocebus aethiops II M

Savanna Sideneck Turtle (E): Podocnemis vogli II R

Savannah Dog (E): Speothos venaticus I M

Savile's Bustard (E): Eupodotis savilei II B

Saw-billed Hermit (E): Ramphodon naevius II B

Saw-jawed Turtle (E): Callagur bomeoensis II R

Saw-whet Owl (E): Aegolius acadicus II B

Scaled Metaltail (E): Metallura aeneocauda II B

Scale-throated Hermit (E): Phaethornis eurynome II B

Scaly Anteater (E): Manis remminckii II M

Scaly Clam (E): Tridacna squamosa II In

Scaly-breasted Hummingbird (E): Phaeochroa cuvierii II B

Scaly-breasted Lorikeet (E): Trichoglossus chlorolepidotus II B

Scaly-fronted Weaver (E): Sporopipes frontalis III B

Scaly-headed Parrot (E): Pionus maximiliani II B

Scaly-naped Amazon (E): Amazona mercenaria II B

Scaly-naped Parrot (E): Amazona mercenaria II B

Scaphirhynchus albus: Pallid Sturgeon (E) II F

Scaphirhynchus platonnchus: Sand Sturgeon (E), Shovelnose

Sturgeon (E) II F

Scaphirhynchus sutikusi: Alabama Sturgeon (E) II F

Scapophyllia cylindrica: II In

Scapiochelys agassizii = Gopherus agassizii

Scaptochelys berlandieri $=$ Gopherus berlandie $i$

Scarlet Cock-of-the-rock (E): Rupicola peruviana II B

Scarlet Ibis (E): Eudocimus ruber II B

Scarlet Macaw (E): Ara macao I B

Scarlet-breasted Lorikeet (E): Vini kuhlii II B

Scarlet-chested Parrot (E): Neophema splendida II B

Scarlet-fronted Parakeet (E): Aratinga wagleri II B

Scarlet-shouldered Parrotlet (E): Touit huetii II B

Scavenger Vulture (E): Neophron percnopterus II B

Sceloglaux albifacies: Laughing Owl (E), White-faced Owl (E), Lechuzón cariblanco (S), Chouette à joues blanches (F), Ninoxe rieuse (F) II B

Schalow's Turaco (E): Tauraco schalowi II B

Scheepmaker's Crowned-Pigeon (E): Goura scheepmakeri II B

Scheffler's Owlet (E): Glaucidium scheffleri II B

Schistes geoffroyi $=$ Augastes geoffroyi

Schizoculina africana: II In

Schizoculina arbuscula: II In

Schizoculina fissipara: II In

Schizocyathus fissilis: II In

Schizopathes affinis: II In

Schizoparhes amplispina: II In

Schizopashes conferta: II In

Schizopathes crassa: II In

Schizopsammia = Dichopsammia

Schmidt's Guenon (E): Cercopithecus ascanius II M

Schmidt's Monitor (E): Varanus jobiensis II R

Schneider's Smooth-fronted Caiman (E): Palaeosuchus trigonatus II $\mathbf{R}$

Schouteden's Chameleon (E): Chamaeleo schoutedeni II R
Schubotz's Chameleon (E): Chamaeleo schuborzi II R

Schweigger's Terrapin (E): Pelusios castaneus III R

Schweigger's Tortoise (E): Kinixys erosa II $\mathbf{R}$

Scimitar-horned Oryx (E); Oryx dammah I M

Scinque à queue préhensile des lles Salomons (F): Corucia zebrata II $\mathrm{R}$

Scinque arboricole des Iles Salomons (F): Corucia zebrata II R

Scinque géant des lles Salomons (F): Corucia zebrata II R

Scintillant Hummingbird (E): Selasphorus scintilla II B

Scissor-tailed Hummingbird (E): Hylonympha macrocerca II B

Scissor-tailed Kite (E): Chelictinia riocourii II B

Sciuns deppei: Deppe's Squirrel (E), Ardilla de Deppe (S), Ardilla de los pinos (S), Écureuil de Deppe (F), Écureuil des pins (F) III M

Sclater's Guenon (E): Cercopithecus sclateri II M

Sclater's Monal (E): Lophophorus sclateri I B

Sclater's Parrotlet (E): Forpus sclateri II B

SCLERACTINIA spp.: Stony corals (E), Dont coraux récifaux (F) II In

Sclerhelia dubia: II In

Sclerhelia hirtella: II In

Sclerohelia $=$ Sclerhelia

Scléropage d'Asie (F): Scleropages formosus I F

Scléropage formosus (F): Scleropages formosus I F

Scleropages formosus: Asian Arowana (E), Asian Bonytongue (E), Golden Arowana (E), Golden Dragon Fish (E), Kelesa (E), Pez lenguiihueso malayo (S), Scléropage d'Asie (F), Scléropage formosus (F) I F

Sclerophyllia $=$ Cynarina

Scolymia australis: II In

Scolymia cubensis: Solitary Disk Coral (E) II In

Scolymia vitiensis: II In

Scops géant de Guemey (F): Mimizuku gurneyi I B

Scops-Owls (E): Otus spp/Mimizuku gumeyi I/I B

Scorpions (E): SCORPIONES spp. II/NC In

Scotopelia bouvieri: Vermiculated Fishing-Owl (E), Chouettepêcheuse de Bouvier (F) II B

Scotopelia peli: Pel's Fishing-Owl (E), Lechuza pescadora (S), Chouetie pêcheuse (F), Chouette-pêcheuse de Pel (F) II B

Scotopelia ussheri: Rufous Fishing-Owl (E), Búho pescador rojizo (S), Chouette d'Ussher (F), Chouette-pêcheuse rousse (F) II B

Screech-Owls (E): Oius spp. II B

Scroll Coral (E): Agaricia undata II In

Scrub Python (E): Morelia amethistina II R

Scrub-birds (E): Atrichornis spp. I/NC B

Sea Cat (E): Lonira felina I M

Sea Cow (E): Dugong dugon I/I M

Sea Iguana (E): Amblyrhynchus crisıatus II R

Sea Otter (E): Enhydra lutris VII M

Sea-Eagles (E): Haliaeetus spp. I/I B

Secret Tree Toad (E): Nectophrynoides cryptus I A

Secrétaire des serpents (F): Sagitiarius serpentarius II B

Secretario (S): Sagittarius serpentarius II B

Secretary-bird (E): Sagittarius serpentarius II B

Seedcrackers (E): Pyrenestes spp. III/NC B

Seedeaters (E): Amaurospiza spp./Catamenia spp./Dolospingus fringilloides/Serinus spp/Sporophila spp. IIINC B

Sei Whale (E): Balaenoptera borealis I M

Seipp's Day Gecko (E): Phelsuma seippi II R

Selasphorus ardens: Glow-throated Hummingbird (E), Colibri ardent (F) II B

Selasphons ellioti $=$ Atrhis ellioti

Selasphorus flammula: Cerise-throated Hummingbird (E), Heliotrope-throated Hummingbird (E), Volcano Hummingbird (E), Colibri flammule (F) II B

Selasphorus heloisa $=$ Auhis heloisa

Selasphorus platycercus: Broad-tailed Hummingbird (E), Zumbador coliancho (S), Colibri à queue large (F) II B

Selasphorus rufus: Rufous Hummingbird (E), Zumbador rufo (S), Colibri roux (F) II B

Selasphorus sasin: Allen's Hummingbird (E), Zumbador de Allen (S), Colibri d'Allen (F) II B

Selasphorus scintilla: Scintillant Hummingbird (E), Colibri scintillant (F) II B

Selasphorus simoni = Selasphorus flammula

Selasphorus torridus = Selasphorus flammula 
Selenarctos thibetanus = Ursus thibetanus

Selenidera maculirostris: Spot-billed Toucanet (E), Tucancito picomaculado (S), Toucanet à bec tacheté (F) III B

Seleucidis melanoleuca: Twelve-wired Bird-of-paradise (E), Paradisier multifil (F) II B

Semi-collared Hawk (E): Accipiter collaris II B

Semi-collared Sparrowhawk (E): Accipiter collaris II B

Semioptera wallacii: Standard-wing Bird-of-paradise (E) Standardwing (E), Wallace's Standardwing (E), Paradisier de Wallace (F) II B

Semiplumbeous Hawk (E): Leucoptemis semiplumbea II B

Semnopithecus entellus: Common Langur (E), Entellus Langur (E), Grey Langur (E), Hanuman Langur (E), True Langur (E) Langur común (S), Langur hanuman (S), Entelle (F), Houleman (F) I M

Semnopithecus geei = Trachypithecus geei

Semnopithecus hypoleucos = Semnopithecus entellus

Semnopithecus pileatus $=$ Trachypithecus pileatus

Semnopithèque à front blanc $(\mathrm{F})$ : Presbytis frontata II M

Sernnopithèque blanchâtre (F): Trachypithecus vetulus II M

Semnopitheque de François (F): Trachypithecus francoisi II M

Semnopithèque de Gee (F): Trachypithecus geei I M

Semnopithèque de Hose (F): Presbyris hosei II M

Semnopithèque de Mentawi ( $F$ ): Presbytis potenziani I M

Semnopitheque de Phayre (F): Trachypithecus phayrei II M

Semnopithèque de Thomas (F): Presbytis thomasi II M

Semnopithèque des Nilgiris (F): Trachypithecus johnii II M

Semnopithèque doré (F): Trachypithecus geei I M

Semnopithèque mélalophe (F): Presbytis melalophos II M

Semnopithèque obscur (F): Trachypithecus obscurus II M

Semnopithèque rubicond (F): Presbytis mbicunda II M

Semnomis ramphastinus: Toucan Barbet (E), Caebézon toucan (F) III B

Senegal Bustard (E): Eupodotis senegalensis II B

Senegal Chameleon (E): Chamaeleo senegalensis II R

Senegal Combassou (E): Vidua chalybeata III B

Senegal Dove (E): Streptopelia senegalensis III B

Senegal Firefinch (E): Lagonosticta senegala III B

Senegal Galago (E): Galago senegalensis II M

Senegal ondulé (F): Estrilda astrild III B

Senegal Parrot (E): Poicephalus senegalus II B

Sénégali brun à ventre blanc $(F)$ : Nigrita fusconota $111 \mathrm{~B}$

Sénégali brun à ventre roux (F): Nigrira bicolor III B

Sénégali nègre $(\mathbf{F})$ : Nigriła canicapilla III B

Sénégali nègre à front jaune $(\mathrm{F})$ : Nigrito luteifrons III $\mathrm{B}$

Sénégali sanguin (F): Spermophaga haematina III B

Sénégali vert (F): Mandingoa nitidula III B

Sénégali vert à joues blanches (F): Nesocharis capistrata III B

Sénégalis (F): Clytospiza monteiri/Cryptospiza spp./Euschistospiza

spp./Hypargos spp./Mandingoa nitidula/Spermophaga spp. III $N$ C B

Sephanoides fernandensis: Juan Fernández Firecrown (E), Colibri robinson (F) II B

Sephanoides sephaniodes: Green-backed Firecrown (E), Picaflor cabeza granate (S), Colibri du Chili (F) II B

Sepik Monitor (E): Varanus jobiensis II R

Seram Flying-fox (E): Pteropus ocularis II M

Seram Fruit Bat (E): Pteropus ocularis II M

Serau (S): Naemorhedus sumatraensis I M

Seriatopora caliendrum: II In

Seriatopora crassa: II In

Seriatopora hystrix: Needle Coral (E) II In

Seriatopora lineata: II in

Seriatopora spinosa: II In

Seriatopora stellata: II In

Seriatopora siricta: II in

Sericotes holosericeus $=$ Eulampis holosericeus

Serin a croupion blanc (F): Serinus leucopygius III B

Serin culiblanco (S): Serinus leucopygius III B

Serin du Mozambique (F): Serinus mazambicus III B

Serin gris à tête blanche (F): Serinus canicapillus III B

Serín mofletudo (S): Serinus canicapillus III B

Serin ouest-africain (F): Serinus canicapillus III B

Serins (F): Serinus spp. III/NC B

Serinus canicapillus: West African Seedeater (E), Serin mofletudo

(S), Serin gris à tête blanche (F), Serin ouest-africain (F) III B
Serinus gularis canicapillus = Serinus canicapillus

Serinus leucopygius: Grey Singing Finch (E), White-rumped Seedeater (E), Serin culiblanco (S), Chanteur d'Afrique (F). Serin à croupion blanc (F) III B

Serinus mozambicus: Green Singing Finch (E), Yellow-fronted Canary (E), Canario de Mozambique (S), Serin du Mozambique (F) III B

Serows (E): Naemorhedus crispus/Naemorhedus sumairaensis/Naemorhedus swinhoei I/NC M

Serpent aquatique caréné à damier (F): Xenochrophis piscator III R

Serpent ardoise (F): Atretium schistosum III R

Serpent d'eau à títe de chien (F): Cerberus rhynchops III R

Serpent d'eau à ventre blanc (F): Cerberus rhynchops III R

Serpent gomme (F): Charina boltae II R

Serpent Island Gecko (E): Cyrtodactylus serpensinsula II R

Serpent ratier oriental (F): Pryas mucosus II R

Serpentaire (F): Sagittarius serpentarius II B

Serpentaire bacha (F): Spilomis cheela II B

Serpentaire de Madagascar (F): Eutriorchis astur II B

Serpentaire des Andaman (F): Spilornis elgini II B

Serpentaire des Célèbes (F): Spilomis rufipectus II B

Serpentaire des Kinabalu (F): Spilomis kinabaluensis II B

Serpentaire des Nicobar (F): Spilomis minimus II B

Serpentaire des Philippines (F): Spilornis holospilus II B

Serpentaire du Congo (F): Dryotriorchis spectabilis II B

Serpentaires (F): Dryotriorchis spectabilis/Eutriorchis astur/Spilomis spp. II B

Serpentario (S): Sagittarius serpentarius II B

Serpent-Eagles (E): Dryotriorchis spectabilis/Eutriorchis astur/Spilomis spp. II B

Serrated Day Gecko (E): Phelsuma serraticauda II R

Serrated Hinge-back Tortoise (E): Kinixys erosa II R

Serrated Hinge-backed Tortoise (E): Kinixys erosa II R

Serrated Star Tortoise (E): Psammobates oculiferus II R

Serrated Tortoise (E): Kinixys erosa II R

Serval (E/S/F): Lepiailunus serval II M

Setaro's Dwarf Chameleon (E): Bradypodion setaroi II R

Seven-colored Parrotlet (E): Touil batavica II B

Seven-colored Tanager (E): Tangara fastuosa II B

Severe Macaw (E): Ara severa II B

Seychelles Day Gecko (E): Phelsuma astriata II R

Seychelles Flying-fox (E): Pteropus seychellensis II M

Seychelles Giant Day Gecko (E): Phelsuma sundbergi II R

Seychelles Kestrel (E): Falco araea I B

Shapo (E): Ovis vignei vignei I M

Shapu (E): Ovis vignei vignei I M

Shark Bay Mouse (E): Pseudomys praeconis I M

Sharp-shinned Hawk (E): Accipiter striatus II B

Sheartails (E): Doricha spp. II B

Sheep (E); Ovis spp. UI/NC M

Sheet Coral (E): Agaricia lamarcki II in

Shelley's Eagle-Owl (E): Bubo shelleyi II B

Shepherd's Beaked Whale (E): Tasmacelus shepherdi II M

Shikra (E): Accipiter badius II B

Shining Sunbeam (E): Aglaeactis cupripennis II B

Shining-green Hummingbird (E): Lepidopyga goudoti II B

Shining-Parrots (E): Prosopeia spp. II B

Shinisaurus crocodilurus: Chinese Crocodile Lizard (E), Chinese

Xenosaur (E), Lagarto cocodrilo chino (S), Lézard crocodile de

Chine (F) II R

Shiny Pigtoe (E): Fusconaia edgariana I In

Shiny Pigtoe Pearly Mussel (E): Fusconaia edgariana I In

Ship Sturgeon (E): Acipenser nudiventris II F

Shoebill (E): Balaeniceps rex II B

Short-crested Coquette (E): Lophomis brachylopha II B

Short-eared Owl (E): Asio flammeus II B

Short-finned Pilot Whale (E): Globicephala macrorhynchus II M

Short-horned Chameleon (E): Chamaeleo brevicomis II R

Shortnose Sturgeon (E): Acipenser brevirosinum I F

Short-tailed Albatross (E): Diomedea albatrus I B

Short-tailed Chinchilla (E): Chinchilla brevicaudata I M

Short-tailed Dwarf Chameleon (E): Chamaeleo marshalli II R

Short-tailed Emerald (E): Chlorostilbon poormani II B

Short-tailed Hawk (E): Buteo brachyurus II B

Short-tailed Paradigalla (E): Paradigalla brevicauda II B

Short-tailed Parrot (E): Graydidascalus brachyurus II B 
Short-tailed Pygmy Monitor (E): Varanus brevicauda II R Short-tailed Python (E): Python curlus II R

Short-tailed Tibetan Macaque (E): Macaca thibetana II M

Short-tailed Woodstar (E): Myrmia micrura II B

Short-toed Eagle (E): Circaetus gallicus II B

Short-toed Snake-Eagle (E): Circaetus gallicus II B

Shovelers (E): Anas clypeata/Anas platalea/Anas rhynchotis/Anas smithii III/NC B

Shovelnose Sturgeon (E): Scaphirhynchus platorynchus II F

Siamang (E/S/F): Hylobates syndactylus I M

Siamang de Kloss (F): Hylobates klossii I M

Siamang de Kloss (S): Hylobates klossii I M

Sianang enano (S): Hylobates klossii I M

Siamese Crocodile (E): Crocodylus siamensis I R

Sibbald's Rorqual (E): Balaenopiera musculus I M

Siberian Crane (E): Grus leucogeranus I B

Siberian Musk Deer (E): Moschus moschifenus I M

Siberian Sturgeon (E): Acipenser baerii II F

Siberian Weasel (E): Mustela sibirica III M

Siberian White Crane (E): Grus leucogeranus I B

Sibopathes gephura: II In

Sibopathes macrospina: II In

Sichuan Snub-nosed Monkey (E): Pygathrix roxellana I M

Sichuan Wood-Owl (E): Sirix davidi II B

Sicklebills (E): Epimachus spp/Eutoxeres spp/Falculea palliata II/NC B

Side-necked turtles (E): Pelomedusidae spp. IIIIU/NC R

Sidenecks (E): Peltocephalus dumeriliana/Podocnemis spp. II R

Siderastrea glynni: II In

Siderastrea radians: Rough Starlet Coral (E) II In

Siderastrea savignyana: II in

Siderastrea siderea: Smooth Starlet Coral (E) II In

Siderastrea stellata: II In

Siderina $=$ Siderastrea

Side-striped Chameleon (E): Chamaeleo biraeniatus II R

Side-striped Day Gecko (E): Phelsuma lineata II R

Sierra Parakeet (E): Bolborhynchus aymara II B

Sifaka (F): Propithecus verreauxi I M

Sifaka diademado (S): Propithecus diadema I M

Sifakas (E): Propithecus spp. I M

Sifilet d'Arfak (F): Parotia sefilala II B

Sifilet de Carola (F): Parotia carolae II B

Sifilet de Lawe (F): Parotia lawesii II B

Sifilet de Wahnes (F): Parotia wahnesi II B

Sifilet d'Hélène (F): Parotia helenae II B

Siju (S): Glaucidium siju II B

Siju cotunto (S): Otus lawrencii II B

Sijucito (S): Glaucidium siju II B

Silfo colilargo (S): Aglaiocercus kingi II B

Silfo colivioleta (S): Aglaiocercus coelestis II B

Silky-Crow (E): Lycocorax pyrrhopterus II B

Silure de verre géant $(F)$ : Pangasianodon gigas I F

Siluro gigante (S): Pangasianodon gigas IF

Silverbills (E): Lonchura cantans/Lonchura griseicapilla/Lonchura malabarica III/NC B

Silver-eared Mesia (E): Leiothrix argentauris II B

Silvered Langur (E): Trachypithecus cristatus II M

Silvered Leaf Monkey (E): Trachypithecus cristatus II M

Silvereyes (E): Zosterops spp. INC B

Silverstone's Poison Frog (E): Phobobates silverstonei II A

Silvery Flying-fox (E): Pteropus argentatus II M

Silvery Fruit Bat (E): Pteropus argentatus II M

Silvery Gibbon (E): Hylobares moloch I M

Silvery Marmoset (E): Callithrix argentata II M

Simeulue Scops-Owl (E): Otus umbra II B

Simias concolor $=$ Nasalis concolor

Simisimi (F): Allobates femoralis II A

Simplastrea vesicularis: II In

Singe araignée du Panama (F): Areles geoffroyi frontatus I M

Singe de nuit (F): Aotus trivirgasus II M

Singe laineux (F): Lagothrix lagotricha II M

Singe laineux à queue jaune (F): Lagothrix flavicauda I M

Singe vert (F): Chlorocebus aethiops II M

Singe-araignée aux mains noires $(\mathrm{F})$ : Areles geoffroyi frontafus I M

Singe-araignée laineux (F): Brachyieles arachnoides I M

Singe-écureuil à dos rouge (F): Saimiri oerstedii I M
Singe-écureuil à dos roux (F): Saimiri oerstedii 1 M

Singe-lion (F): Leontopithecus rosalia I M

Singing Parrot (E): Geoffroyus heteroclitus II B

Single Soft-nosed Chameleon (E): Bradypodion ienue II R

Single Welded-hom Chameleon (E): Bradypodion xenorhinus II R

Sira Poison Frog (E): Dendrobates sirensis II A

Siskins (E): Carduelis spp. I/LI/NC B

Sisón (S): Tetrax letrax II B

Sisón azul (S): Eupodotis caerulescens II B

Sisón azulado (S): Eupodotis caerulescens II B

Sisón bengali (S): Eupodotis bengalensis I B

Sisón de Damaraland (S): Eupodotis rueppellii II B

Sisón de Hartaub (S): Eupodotis harlaubii II B

Sisón de penacho (S): Eupodotis indica II B

Sisón de Rüppell (S): Eupodotis rueppellii II B

Sisón de Vigors (S): Eupodotis vigorsii II B

Sisón del karroo (S): Eupodotis vigorsii II B

Sisón indio (S): Eupodotis indica II B

Sisón mon̄irrojo (S): Eupodotis ruficrista II B

Sisón moñudo austral (S): Eupodoris ruficrisıa II B

Sisón moñudo de saheliano (S): Eupodotis savilei II B

Sisón moñudo etiope (S): Eupodotis gindiana II B

Sisón negro (S): Eupodotis afra II B

Sisón negro aliclaro (S): Eupodotis afraoides II B

Sisón negro alioscuro (S): Eupodotis afra II B

Sisón senegalés (S): Eupodotis senegalensis II B

Sisón somalí (S): Eupodotis humilis II B

Sisón ventrinegro (S): Eupodotis melanogaster II B

Sisón ventrinegro común (S): Eupodotis melanogaster II B

Sisón ventrinegto de Hartlaub (S): Eupodotis hartlaubii II B

Sisónes (S): Otididae spp. I/II B

Sitagra de Pelzeln (S): Ploceus pelzelni III B

Siragra melanocephala $=$ Ploceus melanocephalus

Sitatunga (E/S/F): Tragelaphus spekii III M

Sitragra chico común (S): Ploceus luteolus III B

Six-fingered Frog (E): Euphlyctis hexadacrylus II A

Six-legged Tortoise (E): Manouria emys II R

Six-plumed Parotia (E): Parotia sefilata II B

Six-tubercled Amazon River Turle (E): Podocnemis sextuberculata II $\mathbf{R}$

Six-tubercled River Turtle (E): Podocnemis sextuberculasa II R

Sjostedi's Owlet (E): Glaucidium sjostedi II B

Skew-beaked Whale (E): Mesoplodon hectori II M

Skinks (E): Scincidae spp. IINC R

Skunks (E): Coneparus spp/Mephitis spp/Spilogale spp. II/NC M

Sky-blue Poison Frog (E): Epipedabates azureiventris II A

Slate-colored Hawk (E): Leucoptemis schistacea II B

Slaty-backed Forest-Falcon (E): Micrastur mirandollei II B

Slaty-backed Goshawk (E): Accipiter luteoschistaceus II B

Slaty-headed Parakeet (E): Psittacula himalayana II B

Slaty-mantled Sparrowhawk (E): Accipiser luteoschistaceus II B

Slender Blackfish (E): Feresa atienuata II M

Slender Chameleon (E): Bradypodion tenue II R

Slender Fanalouc (E): Eupleres goudotii II M

Slender Hog-nosed Pit Viper (E): Bothrops ophryomegas III R

Slender Loris (E): Loris tardigradus II M

Slender Sheartail (E): Doricha enicura II B

Slender Treeshrew (E): Tupaia gracilis II M

Slender-billed Black-Cockatoo (E): Calyptorhynchus latirostris II B

Slender-billed Conure (E): Enicognathus leptorhynchus II B

Slender-billed Curlew (E): Numenius tenuirostris I B

Slender-billed Kite (E): Rosirhamus hamatus II B

Slender-billed Parakeet (E): Enicognathus leptorhynchus II B

Slender-billed Weaver (E): Ploceus pelzelni III B

Slender-horned Gazelle (E): Gazella leptoceros III M

Slender-tailed Woodstar (E): Microstilbon burmeisteri II B

Slipper Coral (E): Herpolitha limax II In

Sloth Bear (E): Melursus ursinus I M

Sloths (E): Bradypodidae spp./Megalonychidae spp. II/NC M

Slow Loris (E): Nycticebus coucang II M

Small Amu-Dar Shovelnose Sturgeon (E): Pseudoscaphirhynchus hermanni II $\mathrm{F}$

Small Birdwing (E): Troides aeacus II in

Small Flying-fox (E): Preropus hypomelanus II M

Smail Giant Clam (E): Tridacna maxima II In

Small Indian Civet (E): Viverricula indica III M 
Small Indian Mongoose (E): Herpestes javanicus auropunctatus III $\mathrm{M}$

Small Sparrowhawk (E): Accipiser nanus II B

Small-clawed Otter (E): Aonyx congicus III M

Small-eared Greater Galago (E): Otolemur gametrii II M

Smaller Banded Snake-Eagle (E): Circaetus cinerascens II B

Smaller-Cock-of-the-rock (E): Rupicola rupicola II B

Small-spotted Cat (E): Felis nigripes I M

Small-toothed Clawless Otter (E): Aonyx congicus III M

Small-toothed Mongoose (E): Eupleres goudotii II M

Sminthopsis (E): Sminthopsis spp. INC M

Sminthopsis longicaudata: Long-tailed Dunnart (E), Long-tailed Marsupial-Mouse (E), Long-tailed Sminthopsis (E), Ratón marsupial colilargo (S), Souris marsupiale à longue queue (F) I $M$

Sminthopsis psammophila: Large Desert Marsupial-Mouse (E), Sandhill Dunnart (E), Sandhill Sminthopsis (E), Ratón marsupial desértico $(S)$, Souris marsupiale du désert $(F)$ I M

Sinith's Dwarf Chameleon (E): Bradypodion taeniobronchum II R

Smooth Chameleon (E): Chamaeleo laevigatus II R

Smooth Starlet Coral (E): Siderastrea siderea II In

Smooth-coated Otter (E): Lutrogale perspicillata II M

Snail Kite (E): Rostrhamus sociabilis II B

Snails (E): GASTROPODA spp. VIVNC in

Snake-Eagles (E): Circaetus spp. II B

Snakes (E): SERPENTES spp. I/III/NC R

Snow Crane (E): Grus leucogeranus I B

Snow Leopard (E): Uncia uncia I M

Snowcap (E): Microchera albocoronata II B

Snowcocks (E): Tetraogallus spp. I/NC B

Snowy Owl (E): Nyclea scandiaca II B

Snowy-breasted Hummingbird (E): Amazilia edward II B

Snubfin Dolphin (E): Orcaella brevirosiris II M

Snub-nosed monkeys (E): Pygathrix spp. I M

Socotra Chameleon (E): Chamaeleo monachus II R

Softshell Tortoise (E): Malacochersus tomieri II R

Soft-shell turtles (E): Trionychidae spp. I/III/NC R

Sokoke Scops-Owl (E): Olus ireneae II B

Solangel de gorguera (S): Heliangelus strophianus II B

Solangel gorjiamatista (S): Heliangelus amethysticollis II B

Solangel gorjipurpura (S): Heliangelus viola II B

Solangel turmalino (S): Heliangelus exortis II B

Solenastrea hyades: Lobed Star Coral (E) II In

Solenosmilia variabilis: II in

Solitary Disk Coral (E): Scolymia cubensis II in

Solitary Eagle (E): Harpyhaliaetus solitarius II B

Solitary Tinamou (E): Tinamus solisarius I B

Soljania $=$ Hoplangia

Solomon Fish-Eagle (E): Haliaeetus sanfordi II B

Solomon Flying-fox (E): Pteropus rayneri $11 \mathrm{M}$

Solomon Ground Boa (E): Candoia carinata II R

Solomon Hawk-Owl (E): Ninox jacquinoti II B

Solomon Islands Giant Skink (E): Corucia zebrata II R

Solomon Islands Spiny Monitor (E): Varanus spinulosus II R

Solomon Sea-Eagle (E): Haliaeetus sanfordi II B

Somali Galago (E): Galago gallarum II M

Somali Mastigure (E): Uromastyx macfadyeni II R

Somali Sand Boa (E): Eryx somalicus II R

Sombre Hummingbird (E): Aphantochroa cirrochloris II B

Somormujo de Atitlán (S): Podilymbus gigas I B

Sonnerat's Junglefowl (E): Gallus sonneratii II B

Sonoran Green Toad (E): Bufo retiformis II A

Sooty Barbthroat (E): Threnetes niger II B

Sooty Eagle-OwI (E): Bubo leucostictus II B

Sooty Falcon (E): Falco concolor II B

Sooty Mangabey (E): Cercocebus torquatus II M

Sooty-backed Hawk-Owl (E): Ninox theomacha II B

Sooty-capped Hermit (E): Phaethomis augusti II B

Sooty-Owls (E): Tyto multipunctata/Tyto tenebricosa II B

Sotalia spp.: River dolphins (E), Bufeos (S), Toninas (S), Dauphins

de rivière (F), Sotalies de l'Amérique du Sud (F) I M

Sotalia brasiliensis = Sotalia fluviatilis

Sotalia fluviatilis: Estuarine Dolphin (E), Guianian River Dolphin

(E). Tucuxi (E), Bufeo negro (S), Sotalie (F) I M

Sotalia guianensis $=$ Soralia fluviatilis

Sotalie (F): Sotalia fluviatilis I M
Sotalies africaines et asiatiques (F): Sousa spp. I M

Sotalies de l'Amérique du Sud (F): Sotalia spp. I M

Souchet (F): Anas clypeata III B

Souffleur (F): Tursiops truncatus II M

Soumagne's Owl (E): Tyto soumagnei I B

Souris marsupiale à longue queue (F): Sminthopsis longicaudata I M

Souris marsupiale du désert (F): Sminthopsis psammophila I M

Sousa spp.: Humpback dolphins (E), Delfines blancos (S), Dauphins

blancs $(\mathrm{F})$, Sotalies africaines et asiatiques (F), Sousas (F) I M

Sousa bomeensis $=$ Sousa chinensis

Sousa chinensis: Indo-pacific Humpback Dolphin (E), Bufeo asiático

$(\mathrm{S})$, Delfín blanco de China (S), Dauphin à bosse de l'IndoPacifique (F) I M

Sousa lentiginosa $=$ Sousa chinensis

Sousa plumbea $=$ Sousa chinensis

Sousa reuszii: Atlantic Hump-backed Dolphin (E), Atlantic

Humpback Dolphin (E), Bufeo africano (S), Delfín blanco

africano (S), Dauphin à bosse de l'Atlantique (F) I M

Sousas (F): Sousa spp. I M

Sousou (F): Platanista gangetica I M

Sousous (F): Platanista spp. I M

South African Bowsprit Tortoise (E): Chersina angulata II R

South African Crowned Crane (E): Balearica regulorum II B

South African Fur Seal (E): Arctocephalus pusillus II M

South African Pangolin (E): Manis temminckii II M

South African Tent Tortoise (E): Psammobates tentorius II R

South American Fur Seal (E): Arctocephalus australis II M

South American Manatee (E): Trichechus inunguis I M

South American River Otter (E): Lontra longicaudis I M

South American River Turtle (E): Podocnemis expansa II R

South American river turtles (E): Podocnemis spp. II R

South American Tapir (E): Tapirus terrestris II M

South American Tortoise (E): Geochelone denticulata II R

South American Water Cobra (E): Cyclagras gigas II R

South American Yellow-footed Tortoise (E): Geochelone denticulata II $\mathbf{R}$

South Andean Deer (E): Hippacamelus bisulcus I M

South Andean Huemul (E): Hippocamelus bisulcus I M

South Atlantic Elephant-seal (E): Mirounga leonina II M

South Australian Water Python (E): Morelia oenpelliensis II R

South Brazilian Coati (E): Nasua nasua solitaria III M

South China Forest Musk Deer (E): Moschus berezovskii II M

South Pacific Banded Iguana (E): Brachylophus fasciatus I R

South-central Chameleon (E): Chamaeleo minor II R

Southern Bald Ibis (E): Geronticus calvus II B

Southern Banded Snake-Eagle (E): Circaelus fasciolatus II B

Southern Beaked Whale (E): Mesoplodon grayi II M

Southem Black-throated Finch (E): Poephila cincta cincta II B

Southern Boobook (E): Ninox boobook II B

Southern Botdenose Whale (E): Hyperoodon planifrons I M

Southern Crowned-Pigeon (E): Goura scheepmakeri II B

Southem Dwarf Chameleon (E): Bradypodion ventrale II R

Southern Elephant-seal (E): Mirounga leonina II M

Southem Eyelash Boa (E): Trachyboa gularis II R

Southern Four-toothed Whale (E): Berardius amuxii I M

Southern Fur Seal (E): Arctocephalus australis II M

Southern fur seals (E): Arcrocephalus spp. II M

Southern Gastric-brooding Frog (E): Rheobatrachus vitellinus II A

Southem Giant Clam (E): Tridacna derasa II In

Southern Pudu (E): Pudu puda I M

Southern Right Whale (E): Eubalaena australis I M

Southern Right Whale Dolphin (E): Lissodelphis peronii II M

Southern River Otter (E): Lontra provocax I M

Southem Savanna Monitor (E): Varanus albigularis II R

Southern Sea Otter (E): Enhydra lutris nereis I M

Southern Square-lipped Rhinoceros (E): Ceratotherium simum simum VII M

Southern Talapoin (E): Miopithecus talapoin II M

Southem White Rhinoceros (E): Ceratotherium simum simum III M

Southern Wood Tortoise (E): Geochelone chilensis II R

Sowerby's Beaked Whale (E): Mesoplodon bidens II M

Spadefish (E): Polyodon spathula II F

Spangled Coquette (E): Lophornis stictolophus II B

Spanish Imperial Eagle (E): Aquila adalberti I B

Spanish Lynx (E): Lynx pardinus I M

Sparkling Violet-ear (E): Colibri coruscans II B 
Sparkling-tailed Barbthroat (E): Tilmalura dupontii II B Sparkling-tailed Hummingbird (E): Tilmatura dupontii II B Sparrowhawks (E): Accipiter spp. II B

Sparrows (E): Ploceidae spp/Emberizidae spp. IIU/NC B Sparrow-Weavers (E): Plocepasser spp. IILNC B Spatula clypeata $=$ Anas clypeata

Spatule blanche (F): Platalea leucorodia II B

Spatules (F): Platalea spp. II/NC B

Speckled Cape Tortoise (E): Homopus signatus II R

Speckled Day Gecko (E): Phelsuma guttata II R

Speckled Hawk-Owl (E): Ninox puncrulata II B

Speckled Hummingbird (E): Adelomyia melanogenys II B

Speckled Pigeon (E): Columba guinea III B

Speckled Rock Pigeon (E): Columba guinea III B

Speckled Tortoise (E): Homopus signatus II R

Speckle-fronted Weaver (E): Sporopipes frontalis III B

Speckle-throated Otter (E): Lutra maculicollis II M

Spectacled Bear (E): Tremarctos omasus I M

Spectacled Caiman (E): Caiman crocodilus I/II R

Spectacled Flying-fox (E): Pteropus conspicillatus II M

Spectacled Langur (E): Trachypithecus obscurus II M

Spectacled Leaf Monkey (E): Trachypithecus obscurus II M

Spectacled Owl (E): Pulsatrix perspicillata II B

Spectacled Parrotlet (E): Forpus conspicillatus II B

Spectacled Porpoise (E): Australophocaena dioptrica II M

Spectral Tarsier (E): Tarsius spectrum II M

Spencer's Monitor (E): Varanus spenceri II R

Speoshos venaticus: Bush Dog (E), Savannah Dog (E), Cachorro de mato vinagre (S), Cachorro vinagre (S), Perro de agua (S), Репro de monte (S), Umba (S), Zorro vinagre (S), Chien des buissons (F) I M

Speoryto cunicularia: Burrowing Owl (E), Búho llanero (S), Cucú (S), Lechucita común (S), Lechucita de las viscacheras (S), Mochuelo de Hoyo (S), Chevêche des terriers (F), Chouette de terrier (F) II B

Sperm Whale (E): Physeter catodon I M

Spermacer whale (E): Physeter catodon I M

Spermestes bicolor $=$ Lonchura bicolor

Spermestes cucullatus $=$ Lonchura cucullata

Spermestes fringilloides $=$ Lonchura fringilloides

Spermète à bec bleu (F): Lonchura bicolor III B

Spermète nonnette $(F)$ : Lonchura cucullata III B

Spermète pie (F): Lonchura fringilloides III B

Spermophaga haematina: Blue-billed Weaver (E), Western Bluebill (E), Espermófaga hematina (S), Gros-bec sanguin (F), Sénégali sanguin (F) III B

Spheniscus demersus: Black-footed Penguin (E), Jackass Penguin (E), Pingüino del Cabo (S), Manchot du Cap (F) II B

Spheniscus humboldi: Humboldt Penguin (E), Peruvian Penguin (E), Pinguino de Humboldt (S), Manchot de Humboldt (F) I B

Sphenodon (E): Sphenodon punciatus I R

Sphénodon de Günther (F): Sphenodon guntheri I R

Sphenodon spp.: Sphénodons (F), Tuataras (F) I R

Sphenodon guntheri: Brother Islands Tuatara (E), Hatteria de Günther $(\mathrm{F})$, Sphénodon de Günther (F) I R

Sphénodon ponctué (F): Sphenodon punciatus I R

Sphenodon punctatus: Beak-head (E), Cook Strait Tuatara (E). Sphenodon (E), Tuatara (E), Tuátara (S), Hatteria ponctué (F), Sphénodon poncrué (F) I R

Sphénodons (F): Sphenodon spp. I R

Sphenophyllia = Meandrina

Sphenotrochus andrewianus: II In

Sphenotrochus aurantiacus: II In

Sphenorrochus auritus: II in

Sphenotrochus evexicostatus: II In

Sphenotrochus excavalus: II In

Sphenotrochus gardineri: II In

Sphenotrochus gilchristi: II In

Sphenotrochus hancocki: II In

Sphenotrochus imbricatocostatus: 11 In

Sphenotrochus ralphae: II In

Sphenotrochus squiresi: II in

Sphiggurus mexicanus: Mexican Tree Porcupine (E), Porc-épic préhensile (F) III M

Sphiggurus paragayensis $=$ Sphiggurus spinosus

Sphiggurus spinosus: Spiny Tree Porcupine (E) III M
Spider monkeys (E): Areles spp/Brachyreles arachnoides $\mathbf{I} \mathbf{I I} \mathbf{M}$ Spider Tortoise (E): Pyxis arachnoides II R

Spilocuscus macularus: Common Spotted Cuscus (E), Spotted Phalanger (E), Cuscús manchado (S), Couscous tacheté (F), Phalanger tachete (F) II M

Spilomis cheela: Crested Serpent-Eagle (E), Culebrera chilla (S), Serpentaire bacha (F) II B

Spilomis cheela holospilus $=$ Spilornis holospilus

Spilomis cheela kinabaluensis = Spilomis kinabaluensis

Spilomis elgini: Andaman Serpent-Eagle (E), Dark Serpent-Eagle

(E), Culebrera de Andamán (S), Serpentaire des Andaman (F) II B

Spilomis holospilus: Philippine Serpent-Eagle (E), Culebrera filipina (S), Serpentaire des Philippines (F) II B

Spilomis kinabaluensis: Kinabalu Serpent-Eagle (E), Mountain Serpent-Eagle (E), Culebrera del Kinabalu (S), Serpentaire des Kinabalu (F) II B

Spilomis minimus: Nicobar Serpent-Eagle (E), Culebrera de Nicobar (S), Serpentaire des Nicobar (F) II B

Spilomis rufipecıus: Sulawesi Serpent-Eagle (E), Culebrera de Célebes (S), Serpentaire des Célèbes (F) II B

Spine-sided Chameleon (E): Chamaeleo laterispinis II R

Spinifex Monitor (E): Varanus eremius II $R$

Spinner Dolphin (E): Stenella longirostris II M

Spinophrynoides spp.: Ethiopian toads (E), Sapos viviparos (S), Crapauds vivipares (F) I A

Spinophrynoides osgoodi: Osgood's Ethiopian Toad (E), Sapo vivíparo de Osgood (S), Nectophrynoide d'Ethiopie (F). Nectophrynoide d'Osgood (F) I A

Spinus cucullatus = Carduelis cucullato

Spinus yarrelli $=$ Carduelis yarrellii

Spiny Boa (E): Trachyboa gularis II R

Spiny Chameleon (E): Bradypodion spinosum II R

Spiny Crag Lizard (E): Pseudocordylus spinosus II R

Spiny Sturgeon (E): Acipenser nudiventris II F

Spiny Tree Porcupine (E): Sphiggurus spinosus III M

Spiny-tailed lizards (E): Uromastyx spp. II R/Cordylus spp. II R

Spiny-tailed Pygmy Monitor (E): Varanus acanthurus II R

Spix's Macaw (E): Cyanopsitta spixii I B

Spizaetus africanus: Cassin's Hawk-Eagle (E), Aguila-azor congoleña (S), Aigle de Cassin (F) II B

Spizaeius alboniger: Blyth's Hawk-Eagle (E), Aguila-azor Indonesia (S), Aigle de Blyth (F) II B

Spizaetus bartelsi: Javan Hawk-Eagle (E), Aguila-azor de Java (S), Aigle de Java (F) II B

Spizaeius cirrhatus: Changeable Hawk-Eagle (E), Crested HawkEagle (E), Aguila-azor variable (S), Aigle huppé (F) II B

Spizaetus coronatus $=$ Stephanoaetus coronatus

Spizaetus isidori $=$ Oroaetus isidor

Spizaetus lanceolatus: Sulawesi Hawk-Eagle (E), Aguila-azor de Célebes (S), Aigle des Célèbes (F) II B

Spizaetus nanus: Wallace's Hawk-Eagle (E), Aguila-azor de Wallace (S), Aigle de Wallace (F) II B

Spizaeius nipalensis: Feather-toed Hawk-Eagle (E), Hodgson's Hawk-Eagle (E), Mountain Hawk-Eagle (E), Aguila-azor montañesa (S), Aigle montagnard (F) II B

Spizaetus occipitalis $=$ Lophaelus occipitalis

Spizaetus omatus: Omate Hawk-Eagle (E), Aguila de Penacho (S), Aguila elegante (S), Aguila-azor blanca (S), Aguila-azor galana (S), Aigle orné (F) II B

Spizaetus philippensis: Philippine Hawk-Eagle (E), Aguila-azor filipina (S), Aigle des Philippines (F) II B

Spizaetus tyrannus: Black Hawk-Eagle (E), Tyrant Hawk-Eagle (E), Aguila tirana (S), Aguila-azor negra (S), Aigle tyran (F) II B

Spizastur melanoleucus: Black-and-white Hawk-Eagle (E), Aguila blanquinegra (S), Aguila-azor blanquinegra (S), Aguila-azor chica (S), Aigle noir et blanc (F) II B

Spiziapteryx circumcinctus: Spot-winged Falcon (E), Spot-winged Falconet (E), Halconcito argentino (S), Halconcito gris (S),

Halconcito gris americano (S), Camifex à ailes tachetées (F). Fauconnet d'Amérique (F) II B

Splash-backed Poison Frog (E): Dendrobates galactonotus II A

Splaytooth Beaked Whale (E): Mesoplodon bowdoini $11 \mathrm{M}$

Splendid Astrapia (E): Astrapia splendidissima II B

Splendid Grass-Parakeet (E): Neophema splendida II B

Splendid Poison Frog (E): Dendrobates speciosus II A 
Spongiocyathus $=$ Heterocyathus Spoonbill Cat (E): Polyodon spathula II F Spoonbills (E): Platalea spp. I/NC B Sporadopora dichotoma: II in Sporadopora micropoma: II In Sporadopora morienseni: II in Sporaeginthus subflavus = Amandava subflava Sporopipe quadrillé (F): Sporopipes fronialis III B Sporopipes (F): Sporopipes spp. IIUNC B

Sporopipes frontalis: Scaly-fronted Weaver (E), Speckle-fronted

Weaver (E), Tejedorcito punteado (S), Moineau quadrillé (F), Sporopipe quadrillé (F) III B

Sportive lemurs (E): Megaladapidae spp. I M Spot-backed Weaver (E): Ploceus cucullatus III B Spot-bellied Eagle-Owl (E): Bubo nipalensis II B Spot-billed Toucanet (E): Selenidera maculirostris III B Spot-breasted Ibis (E): Bostrychia rara III B

Spot-legged Poison Frog (E): Epipedobates picrus II A Spot-necked Otter (E): Lutra maculicollis II M Spot-nosed Guenon (E): Cercopithecus nictirans II M Spot-nosed Monkey (E): Cercopithecus petaurista II M Spot-tailed Goshawk (E): Accipiter trinotatus II B Spot-tailed Sparrowhawk (E): Accipiter Irinotatus II B Spotted Box Turte (E): Terrapene nelsoni II R Spotted Cotinga ( $E$ ): Cotinga maculata I B Spotted Day Gecko (E): Phelsuma gutiata II R/Phelsuma ocellata II $\mathbf{R}$

Spotted Dwarf Boa (E): Tropidophis maculatus II R Spotted Eagle (E): Aquila clanga II B Spotted Eagle-Owl (E): Bubo africanus II B Spotted Greenshank (E): Tringa gutifer I B Spotted Harrier (E): Circus assimilis II B Spotted Kestrel (E): Falco moluccensis II B Spotted Linsang (E): Prionodon pardicolor I M Spotted Little Owl (E): Athene brama II B Spotted Owl (E): Strix occidentalis II B Spotted Owlet (E): Athene brama II B Spotted Paca (E): Agouti paca III M Spotted Phalanger (E): Spilocuscus macularus II M Spotted Pond Turtle (E): Geoclemys hamiltonii I R Spotted Python (E): Morelia maculosa II R Spotted Sand Boa (E): Eryx jaculus II R Spotted Scops-Owl (E): Otus spilocephalus II B Spotted Tree Monitor (E): Varanus timorensis II R Spotted Turtle (E): Lissemys punctata II R Spotted Wood-Owl (E): Sirix selopuro II B Spot-throated Hummingbird (E): Leucippus taczanowskii II B Spot-winged Falcon (E): Spiziapteryx circumcinctus II B Spot-winged Falconet (E): Spiziapteryx circumcincius II B Spot-winged Parrotlet (E): Touit stictoptera II B Spur-heeled Chameleon (E): Chamaeleo gracilis II R Spur-thighed Tortoise (E): Testudo graeca II $\mathrm{R}$ Spur-winged Goose (E): Plectropterus gambensis III B Square-tailed Kite (E): Lophoictinia isura II B Squirrel monkeys (E): Saimiri spp. I/I M Squirrels (E): Sciuridae spp. IIIII/NC M Sri Lanka Hanging-Parrot (E): Loriculus beryllinus II B St Lucia Amazon (E): Amazona versicolor I B St Vincent Amazon (E); Amazona guildingii I B Staghom Coral (E): Acropora cenvicomis II In Stags (E): Cervus spp. I/III/NC M Standardwing (E): Semioptera wallacii II B

Standard-wing Bird-of-paradise (E): Semioptera wallacii II B Standing's Day Gecko (E): Phelsuma standingi II R Stanley Bustard (E): Neotis denhami II B Stanley Crane (E): Grus paradisea II B Stanley Parakeet (E): Platycercus icterotis II B Star Sturgeon (E): Acipenser stellatus II F Star Tortoise (E): Geochelone elegans II R Starfrontlets (E): Coeligena spp. II B Starlings (E): Stumidae spp. I/NC B Starhhroats (E): Heliomaster spp. II B Steely-vented Hummingbird (E): Amazilia saucerrottei II B Stejneger's Beaked Whale (E): Mesoplodon stejnegeri II M Stellangia $=$ Astrangia Stellapora echinata: II In
Stellate Sturgeon (E): Acipenser stellatus II F

Steller's Albatross (E): Diomedea albatrus I B

Steller's Sea-Eagle (E): Haliaeetus pelagicus $\mathbf{I}$ B

Stellula calliope: Calliope Hummingbird (E), Colibri de Colliope (S), Colibri calliope (F) II B

Stenella attenuata: Bridled Dolphin (E), Narrow-snouted Dolphin (E), Pantropical Spotted Dolphin (E), Delfin manchado (S), Delfín pintado (S), Dauphin tacheté pantropical (F) II M

Stenella attenuata frontalis $=$ Stenella fronialis

Stenella clymene: Atlantic Spinner Dolphin (E), Clymene Dolphin (E), Helmet Dolphin (E), Dauphin de Clymène (F) II M

Stenella coeruleoalba: Euphrosyne Dolphin (E), Striped Dolphin (E), Delfín blanco y azul (S), Dauphin bleu et blanc (F), Dauphin rayé (F) II M

Stenella dubia $=$ Stenella attenuata

Stenella dubia attenuata = Stenella attenuata

Stenella fronsalis: Atlantic Spotted Dolphin (E), Delfin manchado del Atlántico (S), Dauphin tacheté de l'Atlantique (F) II M

Stenella graffmani = Stenella attenuata

Stenella longirostris: Long-beaked Dolphin (E), Long-snouted Dolphin (E), Spinner Dolphin (E), Delfín tomillón (S), Dauphin longirostre (F) II M

Stenella malayana $=$ Stenella antenuata

Stenella microps = Stenella longirostris

Stenella plagiodon = Stenella frontalis

Stenella roseiventris $=$ Stenella longirostris

Stenella styx = Stenella coeruleoalba

Sténo (F): Steno bredanensis II M

Sieno bredanensis: Rough-toothed Dolphin (E), Delfín de pico largo (S), Sténo (F) $11 \mathrm{M}$

Stenocyathus vermiformis: II In

Sténoderme pseudo-vampire (F): Platyrrhinus lineatus III M

Sienohelia concinna: II In

Stenohelia conferia: II In

Stenohelia echinata: II In

Stenohelia maderensis: II In

Stenohelia minima: II in

Stenohelia pauciseptata: II In

Stenohelia profunda: II In

Sienohelia robusta: II In

Stenohelia siliata: II In

Stenohelia umbonata: II In

Stenohelia yabei: II In

Stephanaria $=$ Psammocora

Stephanie's Astrapia (E): Astrapia stephaniae II B

Stephanoaerus coronarus: African Crowned Eagle (E), Crowned Eagle (E), Crowned Hawk-Eagle (E), Aguila coronaca (S), Aguila coronada zabuda ( $\mathrm{S}$ ), Aigle blanchard (F), Aigle couronné (F) II B

Stephanocoenia michelinii: Blushing Star Coral (E) II in

Stephanocora $=$ Echinopora/Psammocora

Stephanocyathus campaniformis: II In

Stephanocyathus crassus: II in

Stephanocyathus diadema: II in

Stephanocyarhus discoides: II In

Stephanocyathus explanans: II In

Stephanocyathus laevifundus: II In

Stephanocyathus moseleyanus: II in

Stephanocyathus nobilis: II In

Stephanocyathus paliferus: II In

Stephanocyathus platypus: II In

Siephanocyashus spiniger. II in

Stephanocyathus weberianus: II In

Stephanophyllia complicata: II In

Stephanophyllia fungulus: II In

Stephanophyllia neglecta: II In

Stephanopsammia $=$ Stephanophyllia

Stephanoseris $=$ Heterocyathus

Stephanotrochus $=$ Stephanocyathus

Stephanoxis lalandi: Plovercrest (E), Picaflor copetón (S), Colibri de Delalande (F) II B

Stephen's Lorikeet (E): Vini stepheni II B

Steppe Eagle (E): Aquila nipalensis II B

Steppe Fox (E): Vulpes cana II M

Steppe Tortoise (E): Testudo horsfieldii II R

Sterlet (E): Acipenser ruthenus II F 
Stemoclyta cyanopectus: Violet-chested Hummingbind (E), Colibri pechiazul (S), Colibri à poitrine violette (F) II B

Stichopathes abyssicola: II In

Stichopathes alcocki: II In

Srichopathes boumei: II In

Stichopaihes ceylonensis: II in

Stichopathes contorta: II In

Stichoparhes echinulara: II In

Stichopathes filiformis: II In

Stichopathes flagellum: II In

Stichopathes gracilis: II In

Stichopathes longispina: II In

Stichopathes lurkeni: II In

Srichoparhes papillosa: 11 in

Srichopathes paucispina: II In

Stichopathes regularis: II In

Stichopathes semiglabra: II In

Stichopathes seychellensis: II In

Sichopathes spiessi: II In

Srichopathes variabilis: II In

Stick-nest rats (E): Leporillus spp. I/NC M

Stimson's Python (E): Morelia stimsoni II R

Stoat (E): Mustela erminea ferghanae III M

Stone Monitor (E): Varanus baritii II R

Stony corals (E): SCLERACTINIA spp. II in

Storione cobice (Ital) (E): Acipenser naccarii II F

Storks (E): Ciconiidae spp. VI/III/NC B

Straight-billed Hermit (E): Phaethornis bourcieri II B

Strange-nosed Chameleon (E): Bradypodion xenorhinus II R

Strap-toothed Whale (E): Mesoplodon layardii II M

Strawberry Clam (E): Hippopus hippopus II In

Strawberry Poison Frog (F): Dendrobates pumilio II A

Straw-crowned Bulbul (E): Pycnonotus zeylanicus II B

Straw-headed Bulbul (E): Pycnonotus zeylanicus II B

Streaked Lorikeet (E): Charmosyna multistriata II B

Streak-tailed Monitor (E): Varanus caudolineatus II R

Streamertail (E): Trochilus polytmus II B

Streptopelia decipiens: African Mouming Dove (E), Mouming

Collared-Dove (E), Tórtola engañosa (S), Tourterelle pleureuse (F) III B

Streptopelia roseogrisea: African Collared-Dove (E), Pink-headed Dove (E), Rose-grey Dove (E), Tóntola rosigris (S), Tourterelle rieuse (F) III B

Streptopelia semitorquata: Red-eyed Dove (E), Tórtola rizum (S). Tourterelle à collier (F) III B

Streptopelia senegalensis: Laughing Dove (E), Palm Dove (E), Senegal Dove (E), Tórtola senegalesa (S), Tourterelle maillée (F) III B

Sireptopelia turrur: European Turte-Dove (E), Turtle Dove (E), Western Turtle-Dove (E), Tóntola común (S), Tounterelle des bois (F) III B

Streptopelia vinacea: Vinaceous Dove (E), Tórtola vinosa (S), Pigeon vert à front nu (F), Tourterelle vineuse (F) III B

Striated Caracara (E): Phalcoboenus australis II B

Striated Lorikeet (E): Charmosyna multistriata II B

Striated Scops-Owl (E): Otus brucei II B

STRIGIFORMES spp.: Owls (E), Lechuzas (S), Rapaces noctumes (F) I/II B

Strigocuscus mimicus $=$ Phalanger orientalis

Sirigops habroptilus: Kakapo (E), Owl Parrot (E), Cacapo (S), Kakapo (S), Kakapo (F), Perroquet noctume (F), Perroquethibou (F), Strigops kakapo (F) I B

Strigops kakapo (F): Strigops habroptilus I B

Stripe-backed Side-necked Turtle (E): Pelusios gabonensis III R

Stripe-breasted Starthroat (E): Heliomaster squamosus II B

Striped Civet (E): Fossa fossana II M

Striped Day Gecko (E); Phelsuma lineata II R

Striped Dolphin (E): Stenella coeruleoalba II M

Striped Owl (E): Asio clamaior II B

Striped Treeshrew (E): Tupaia dorsalis II M

Stripe-necked Mongoose (E): Herpesies vitticollis III M

Stripe-tailed Hummingbird (E): Eupherusa eximia II B

Stripe-tailed Monitor (E): Varanus caudolineatus II R

Strix albilarsus: Rufous-banded Owl (E), Lechuza colilarga (S), Lechuza patiblanca (S), Chouette fasciée (F) II B
Strix aluco: Tawny Owl (E), Tawny Wood-Owl (E), Cárabo común (S), Chouette hulotte (F) II B

Sirix butleri: Desen Owl (E), Hume's Owl (E), Hume's Tawny Owl (E), Hume's Wood-Owl (E), Cárabo de Hume (S), Chouette de Butler (F), Chouette hulotte de Hume (F) II B

Strix davidi: David's Owl (E), Sichuan Wood-Owl (E), Chouette de Sitchouan (F) II B

Sirix fulvescens: Fulvous Owl (E), Búho fulvo (S), Chouette fauve (F) II B

Strix huhula: Black-banded Owl (E), Lechuza negra (S), Chouette huhul (F) II B

Strix hylophila: Rusty-barred Owl (E), Lechuza listada (S), Chouente dryade (F) II B

Strix leptogrammica: Brown Wood-Owl (E), Chouette leptogramme (F) II B

Sirix nebulosa: Great Grey Owl (E), Grey Owl (E), Lapland Owl (E), Lapp Owl (E), Chouette lapone (F) II B

Strix nigrolineata: Black-and-white Owl (E), Búho blanquinegro (S) Cárabo de Sudamérica (S), Lechuza blanquinegra (S), Chouette noire et blanche $(F)$, Chouette à lignes noires (F) II B

Strix occidentalis: Spotted Owl (E), Búho manchado (S), Chouette tachetée (F) II B

Strix ocellata: Mottled Wood-Owl (E), Chouette ocellée (F) II B

Sirix rufipes: Rufous-legged Owl (E), Lechuza bataraz (S), Chouette masquée (F) II B

Sirix seloputo: Spotted Wood-Owl (E), Chouette des pagodes (F) II $\mathrm{B}$

Strix uralensis: Ural Owl (E), Chouette de l'Oural (F) II B

Strix uralensis davidi $=$ Sirix davidi

Sirix varia: Barred Owl (E), Búho barrado (S), Chouette rayée (F) II B

Sirix varia fulvescens $=$ Strix fulvescens

Sirix virgasa: Mottled Owl (E), Búho café (S), Lechuza colilarga (S). Chouette mouchetée (F) II B

Strix woodfordii: African Wood-Owl (E), Woodford's Owl (E), Lechuzón de Woodford (S), Chouette africaine (F), Hulotte africaine (F) II B

Strombe géant (F): Strombus gigas II in

Strombus gigas: Pink Conch (E), Queen Conch (E), Lambis (F), Strombe géant (F) II In

Siruthio camelus: Ostrich (E), Avestruz (S), Autruche d'Afrique (F) INC B

Stumptail Macaque (E): Macaca arctoides II M

Stump-tailed Macaque (E): Macaca arctoides II M

Sturgeons (E): ACIPENSERIFORMES spp. II F

Stygian Owl (E): Asio stygius II B

Stylanheca papillosa: II In

Srylantheca petrograpta: 11 In

Srylantheca porphyra: II In

Stylaraea punctata: II In

Stylasier alaskanus: II In

Stylaster amphiheloides: II In

Silasier antillarum: II In

Srylaster asper: II in

Stylaster atlanticus: II In

Stylaster aurantiacus: II in

Srylaster bellus: II In

Srylaster bilobatus: II In

Stylaster bithalamus: II In

Srylasier blanteus: II In

Stylaster bocki: II In

Stylaster boreopacificus: II In

Stylaster boschmai: II In

Stylaster brochi: II In

Stylaster brunneus: II In

Stylaster califomicus: II In

Stylaster campylecus: II In

Stylaster cancellatus: II In

Stylaster carinatus: II In

Srylaster complanatus: II In

Stylaster corallium: II In

Stylaster crassior: II In

Srylaster densicaulis: II in

Stylaster dentatus: II in

Srylasier divergens: II In

Srylaster duchassaingii: II In 
Stylaster eguchii: II in Stylaster elassotomus: II In Stylaster erubescens: II In Stylaster filogranus: $\mathbf{I}$ in Stylaster flabelliformis: II in Stylaster galapagensis: II In Stylaster gemmascens: II In Stylaster gracilis: II In Stylaster granulosus: II In Stylaster hattorii: II In Srylaster horologium: II In Stylaster ibericus: II In Stylaster imbricarus: II In Stylaster incompletus: II in Stylaster inomatus: II in Stylaster laevigatus: II In Stylaster lonchitis: II in Stylaster marenzelleri: II in Srylaster maroccanus: II In Stylaster marshae: II In Stylaster microstriatus: II In Stylaster milleri: II In Stylaster miniatus: II In Stylaster moseleyanus: II in Stylaster multiplex: II In Stylaster nobilis: II In Stylaster norvegicus: II In Stylaster papillosa: 11 in Stylaster papuensis: II In Stylaster polymorphus: II In Stylaster polyorchis: II In Stylaster profundiporus: II In Stylaster profundus: II In Srylaster pulcher. II In Stylaster punctatus: II In Stylaster purpuratus: II In Stylaster ramosus: II In Stylaster robustus: II In Srylaster rosaceus: II In Stylaster roseus: II In Stylaster sanguineus: II In Stylaster scabiosus: II In Stylaster solidus: II In Stylaster spafula: II In Stylaster stejnegeri: II In Stylaster stellulatus: II In Stylaster subviolaceus: II In Stylaster ienisonwoodsi: II In Stylaster venustus: 11 in Stylaster verrillii: II In Stylasteridae spp.: Lace corals (E) II In Stylocoeniella ammata: II In Stylocoeniella cocosensis: II In Srylocoeniella guentheri: II In Sylophora compressa: II In Stylophora kuehlmanni: II In Stylophora lobata: II In Stylophora mamillata: II In Stylophora mordax: II ln Stylophora pistillata: II In Sylophora rugosa: II In Srylophora stellata: II In Stylophora wellsi: II in Subantarctic Fur Seal (E): Arctocephalus tropicalis II M Sucury (S): Eunectes murinus II R Suirirí bicolor (S): Dendrocygna bicolor III B Suiriri cariblanco (S): Dendracygna viduata III B Suirí leonado (S): Dendrocygna bicolor III B Suiriri piquirrojo (S): Dendrocygna autumnalis III B Suiriri yaguaza (S): Dendrocygna arborea II B Sula abbotti = Papasula abbotti

Sula Barn-Owl (E): Tyto nigrobrunnea II B Sulawesi Flying-fox (E): Acerodon celebensis II M Sulawesi Fruit Bat (E): Acerodon celebensis II M Sulawesi Goshawk (E): Accipiter griseiceps II B Sulawesi Hanging-Parrot (E): Loriculus stigmatus II B
Sulawesi Hawk-Eagle (E): Spizaetus lanceolatus II B Sulawesi Hornbill (E): Penelopides exarhatus II B

Sulawesi Macaque (E): Macaca nigra II M

Sulawesi Owl (E): Tyto rosenbergii II B

Sulawesi Scops-Owl (E): Otus manadensis II B

Sulawesi Serpent-Eagle (E): Spilomis rufipectus II B

Sulawesi Tarsier (E): Tarsius spectrum II M

Sulphur-bottom Whale (E): Balaenoptera musculus I M

Sulphur-crested Cocatoo (E): Cacatua galerita II B

Sulphur-winged Parakeet (E): Pyrrhura hoffmanni II B

Sulu Hombill (E): Anthracoceros montani II B

Sumatran Rhinoceros (E): Dicerorhinus sumatrensis I M

Sumba Boobook (E): Ninox rudolfi II B

Sumba Hornbill (E): Aceros everetti II B

Sun Bear (E): Helarctos malayanus I M

Sun Conure (E): Aratinga solstitialis II B

Sun Parakeet (E): Aratinga solstitialis II B

Sunangels (E): Heliangelus spp. II B

Sunbeams (E): Aglaeactis spp. II B

Sunda Flying-fox (E): Acerodon mactloni II M

Sungazer (E): Cordylus giganteus II R

Sun-tailed Guenon (E): Cercopithecus solatus II M

Sun-tailed Monkey (E): Cercopithecus solatus II M

Superb Bird-of-paradise (E): Lophorina superba II B

Superb Parrot (E): Polytelis swainsonii II B

Sumia ulula: Hawk Owl (E), Nortiem Hawk Owl (E), Lechuza gavilana (S), Chouette épervière (F) II B

Surucucu (E): Cyclagras gigas II R

Sus salvanius: Pygmy Hog (E), Jabalí enano (S), Jabalí pigmeo (S), Sanglier nain (F), Sanglier pygmée (F) I M

Susu (E): Platanista minor I M

Susus (E): Platanista spp. I M

Swainson's Hawk (E): Buteo swainsoni II B

Swallow-tailed Hummingbird (E): Eupetomena macroura II B

Swallow-tailed Kite (E): Elanoides forficatus II B

Swallowtails (E): Papilionidae spp. I/UNC \# In

Swamp Cat (E): Felis chaus II M

Swamp Deer (E): Cervus duvauceli I M

Swamp Harrier (E): Circus approximans II B

Swamp Parakeet (E): Pezoporus wallicus I B

Swamp Turtle (E): Morenia ocellata I R

Swans (E): Coscoroba coscoroba/Cygnus spp. IV/NC B

Swift Parrot (E): Lathamus discolor II B

Swinhoe's Pheasant (E): Lophura swinhoii I B

Sword-billed Hummingbird (E): Ensifera ensifera II B

Sykes' Monkey (E): Cercopithecus mitis II M

Sylphe à queue d'azur (F): Aglaiocercus kingi II B

Sylphe à queue violette (F): Aglaiocercus coelestis II B

Sylphes (F): Aglaiocercus spp. II B

Sylphs (E): Aglaiocercus spp. II B

Symphalangus syndactylus = Hylobates syndactylus

Symphyllia agaricia: II In

Symphyllia erythraea: II In

Symphyllia radians: II In

Symphyllia recta: II In

Symphyllia simplex: II In

Symphyllia valenciennesii: II In

Symphyllia wilsoni: II In

Synaraea $=$ Porites

Sypheotides indica $=$ Eupodotis indica

Syr-Dar Shovelnose Sturgeon (E): Pseudoscaphirhynchus fedischenkoi II F

Syrmaticus ellioti: Chinese Barred-backed Pheasant (E), Elliot's Pheasant (E), Faisán de Elliot (S), Faisan d'Elliot (F) 1 B

Syrmaticus humiae: Hume's Bar-tailed Pheasant (E), Hume's Pheasant (E), Faisán de Hume (S). Faisan de Birmanie (F), Faisan de Hume (F), Faisan à queue barrée (F) I B

Symaticus mikado: Mikado Pheasant (E), Faisán mikado (S), Faisan mikado (F) I B

Systemopora ornata: II In

Táchira Emerald (E): Amazilia disians II B

Taczanowsky's Dwarf Boa (E): Tropidophis laczanowskyi II R

Tagua (E): Catagonus wagneri I M

Tahitian Lorikeet (E): Vini peruviana II B

Tailed Birdwing (E): Omithoptera paradisea II In

Taira (S): Lonira longicaudis I M 
Taita Falcon (E): Falco fasciinucha II B Taiwan Macaque (E): Macaca cyclopis II M

Takh (E): Equus przewalskii I M

Takin (E/F): Budorcas taxicolor II M

Talagoya Lizard (E): Varanus bengalensis I R

Talapoin (E/S/F): Miopithecus talapoin II M

Talaud Black Birdwing (E): Troides dohertyi II in

Talaud Flying-fox (E): Acerodon humilis II M

Talaud Fruit Bat (E): Acerodon humilis II M

Talégalo maleo (S): Macrocephalon maleo I B

Taliabu Masked-Owl (E): Tyto nigrobrunnea II B

Taliabu Owl (E): Tyso nigrobrunnea II B

Tamandua mexicana: Northern Tamandua (E) III M

Tamanoir (F): Myrmecophaga tridactyla II M

Tamarau (S/F): Bubalus mindorensis I M

Tamaraw (E): Bubalus mindorensis $1 \mathrm{M}$

Tamarin à manteau rouge (F): Saguinus fuscicollis II M

Tamarin à moustaches (F): Saguinus mystax II M

Tamarin à perruque (F): Saguinus oedipus I M

Tamarin à pieds blancs (F): Saguinus leucopus I M

Tamarin aux mains rousses (F): Saguinus midas II M

Tamarín bicolor (S): Saguinus bicolor I M

Tamarin bicolore (F): Saguinus bicolor I M

Tamarin de Geoffroy (F): Saguinus geoffroyi I M

Tamarin de Goeldi (F): Callimico goeldii I M

Tamarín de manos blancas (S): Saguinus leucopus I M

Tamarin d'Oedipe (F): Saguinus oedipus I M

Tamarin pinché (F): Saguinus oedipus I M

Tamarin rouge et noir (F): Saguinus nigricollis II M

Tamarin sauteur (F): Callimico goeldii I M

Tamarin soyeux (F): Leontopithecus rosalia $1 \mathrm{M}$

Tamarino de caba manchada (S): Saguinus inustus II M

Tamarino de espalda dorada (S): Saguinus tripartitus II M

Tamarino de Geoffroy (S): Saguinus geoffroyi I M

Tamarino de labios blancos (S): Saguinus labiaius II M

Tamarino emperador (S): Saguinus imperator II M

Tamarino león de cabeza dorada (S): Leontopithecus chrysomela I M

Tamarins (E): Leontopithecus spp/Saguinus spp. I/II M

Tamarou (E): Bubalus mindorensis I M

Tambourine Dove (E): Turtur tympanistria III B

Tampico Pearly Mussel (E): Unio tampicoensis tecomatensis I In

Tan Riffleshell (E): Epioblasma walkeri I In

Tana (F): Tupaia tana II M

Tana River Colobus (E): Procolobus rufomitratus I M

Tana River Mangabey (E): Cercocebus galeritus galeritus I M

Tanagers (E): Emberizidae:Thraupinae spp. IUNC B

Tan-blossom Najad (E): Epioblasma ionulosa rangiana II In

Tan-blossom Pearly Mussel (E): Epioblasma torulosa rangiona II In

Tanganyika Chameleon (E): Chamaeleo goetzei II R

Tangara fastuosa: Seven-colored Tanager (E), Calliste superbe (F) II B

Tanimbar Cockatoo (E): Cacatua goffini I B

Tanimbar Corella (E): Cacatua goffini I B

Tanimbar Owl (E): Tyto sororcula II B

Tantale blanc (F): Mycteria cinerea I B

Tantalus Monkey (E): Chlorocebus aethiops II M

Tanygnathus gramineus: Black-lored Parrot (E), Loro de Buru (S), Perruche de Buru (F) II B

Tanygnathus heterurus = Tanygnathus sumatranus

Tanygnathus lucionensis: Blue-naped Parrot (E), Loro nuquiazul (S), Perroquet à couronne (F), Perruche de Luçon (F), Perruche à calotte bleue (F) II B

Tanygnathus megalorynchos: Great-billed Parrot (E), Loro picogrodo (S), Perruche à bec de sang (F) 11 B

Tanygnathus sumatranus: Blue-backed Parrot (E), Müller's Parrot (E), Loro de Müller (S), Perruche de Müller (F) II B

Tanzania Mountain Chameleon (E): Chamaeleo tempeli II R

Taphrolesbia griseiventris: Grey-bellied Comet (E), Colibri comète (F) II B

Taphrospilus hypostictus: Many-spotted Hummingbird (E), Colibrí multipunteado (S), Colibri grivelé (F) II B

Tapir à chabraque $(\mathrm{F})$ : Tapirus indicus $\mathbf{I} \mathrm{M}$

Tapir à dos blanc (F): Tapirus indicus I M

Tapir andino (S): Tapinus pinchaque I M

Tapir brasileño (S): Tapirus terrestris II M

Tapir centroamericano (S): Tapirus bairdii I M
Tapir d'Amérique (F): Tapinus serrestris II M

Tapir de Baird (F): Tapirus bairdii I M

Tapir de la India (S): Tapirus indicus I M

Tapir de l'Inde (F): Tapirus indicus I M

Tapir des Andes (F): Tapirus pinchaque I M

Tapir malais (F): Tapirus indicus I M

Tapir pinchaque (F): Tapirus pinchaque I M

Tapir terrestre (F): Tapirus terrestris II M

Tapires (S): Tapiridae spp. I M

Tapiridae spp.: Tapirs (E), Tapires (S), Tapirs (F) I M

Tapirs (E/F): Tapiridae spp. I M

Tapirus bairdii: Baird's Tapir (E), Central American Tapir (E). Anteburro (S), Dante (S), Tapir centroamericano (S), Tapir de Baird (F) I M

Tapirus indicus: Asian Tapir (E), Indian Tapir (E), Malayan Tapir (E), Tapir de la India (S), Tapir de l'Inde (F), Tapir malais (F), Tapir à chabraque (F), Tapir à dos blanc (F) I M

Tapirus pinchaque: Andean Tapir (E), Mountain Tapir (E), Woolly Tapir (E), Danta cordillerana (S), Danta lanuda (S), Danta negra (S), Pinchaque (S), Tapir andino (S), Tapir des Andes (F), Tapir pinchaque (F) I M

Tapirus roulinii $=$ Tapirus pinchaque

Tapirus terrestris: Brazilian Tapir (E), Lowland Tapir (E), South American Tapir (E), Anta (S), Anta hrasileña (S), Danta (S), Sachavaca (S), Tapir brasileño (S), I apir d'Amérique (F), Tapir terrestre (F) II M

Tarantulas (E): Brachypelma spp/Theraphosidae spp. II/NC in

Tarántulas (S): Brachypelma spp. II In

Tarentules (F): Brachypelma spp. II In

Tarictic Hombill (E): Penelopides panini II B

Tarsero piemeno (S): Tarsius pumilus II M

Tarsier de Boméo (F): Tarsius bancanus II M

Tarsier des Célèbes (F): Tarsius spectrum II M

Tarsier des Philippines (F): Tarsius syrichta II M

Tarsiers (E): Tarsius spp. I/II M

Tarsius bancanus: Horsfield's Tarsier (E), Westem Tarsier (E), Tarsier de Boméo (F) II M

Tarsius dianae: II M

Tarsius pelengensis $=$ Tarsius spectrum

Tarsius pumilus: Lesser Spectral Tarsier (E), Pygmy Tarsier (E), Tarsero piemeno (S) II M

Tarsius sangirensis $=$ Tarsius spectrum

Tarsius spectrum: Eastern Tarsier (E), Spectral Tarsier (E), Sulawesi Tarsier (E), Tarsier des Célèbes (F) II M

Tarsius spectrum pumilus $=$ Tarsius pumilus

Tarsius syrichta: Philippine Tarsier (E), Phillipine Tarsier (E), Tarsier des Philippines (F) II M

Tartaruga (E): Podocnemis expansa II R

Tartary Sand Boa (E): Eryx lataricus II R

Taruca (E/S): Hippocamelus antisensis I M

Tasmacète (F): Tasmacetus shepherdi II M

Tasmacerus shepherdi: Shepherd's Beaked Whale (E), Tasman Whale (E), Ballena de Shepherd (S), Tasmacète (F) II M

Tasman Whale (E): Tasmacelus shepherdi II M

Tasmanian Bettong (E): Betrongia gaimardi I M

Tasmanian Masked-Owl (E): Tyto castanops II B

Tasmanian Rat Kangaroo (E): Bettongia gaimardi I M

Tasmanian Rosella (E): Planycercus caledonicus II B

Tasmanian Tiger (E): Thylacinus cynocephalus I M

Tasmanian Wolf (E): Thylacinus cynocephalus I M

Tasman's Girdled Lizard (E): Cordylus tasmani II R

Tassel-eared Marmoset (E); Callithrix humeralifer II M

Tatin de Yarrell (F): Carduelis yarrellii II B

Tatin rouge du Venezuela (F): Carduelis cucullata I B

Tatou (F): Cabassous centralis III M

Tatou à queue nue (F): Cabassous tatouay III M

Tatou géant (F): Priodontes maximus I M

Tattersall's Sifaka (E): Propithecus tattersalli I M

Tatú carreta (S): Priodontes maximus I M

Tatú de rabo mol (S): Cabassous tatouay III M

Tatú de rabo molle (S): Cabassous iatouay III M

Tatú guazú (S): Priodontes maximus I M

Tauraco spp.: Touracos (E, F), Turacos (S) II B

Tauraco bannermani: Bannerman's Turaco (E), Turaco de Bannerman (S), Touraco de Bannerman (F), Touraco doré (F) II B 
Tauraco corythaix: Helmeted Turaco (E), Knysna Turaco (E) Turaco sudafricano $(S)$, Touraco lourie (F), Touraco vert d'Afrique du Sud (F) II B

Tauraco corythaix fischer $=$ Tauraco fischeri

Tauraco corythaix livingstonii $=$ Tauraco livingstonii

Tauraco corythair persa $=$ Tauraco persa

Tauraco corythaix schalowi $=$ Tauraco schalowi

Tauraco corythaix schuetii $=$ Tauraco schuettii

Tauraco erythrolophus: Red-crested Turaco (E), Touraco pauline (F) II B

Tauraco fischeri: Fischer's Turaco (E), Touraco de Fischer (F) II B

Touraco hartlaubi: Hartlaub's Turaco (E), Touraco de Hartlaub (F) II B

Tauraco leucolophus: White-crested Turaco (E), Turaco capuchablanca (S), Touraco à huppe blanche (F) II B

Tauraco leucotis: White-cheeked Turaco (E), Touraco à joues blanches (F) II B

Tauraco livingstonii: Livingstone's Turaco (E), Touraco de Livingstone (F) II B

Tauraco macrorhynchus: Black-tip Crested Turaco (E), Crested Turaco (E), Verreaux's Turaco (E), Yellow-billed Turaco (E), Turaco capucha-franjeada (S), Touraco à gros bec (F) II B

Tauraco persa: Guinea Turaco (E), Turaco capucha-verde de Guinea (S), Touraco vert (F) II B

Tauraco persa corythaix = Tauraco conythaix

Tauraco porphyreolophus = Musophaga porphyreolopha

Tauraco ruspolii: Prince Ruspoli's Turaco (E), Ruspoli's Turaco (E), Touraco de Ruspoli (F) II B

Tauraco schalowi: Schalow's Turaco (E), Touraco de Schalow (F) II B

Tauraco schuettii: Black-billed Turaco (E), Touraco à bec noir $(F)$ II B

Taurotragus eurycerus = Tragelaphus eurycerus

Tawny Eagle (E): Aquila rapax II B

Tawny Fish-Owl (E): Kelupa flavipes II B

Tawny Owl (E): Strix aluco II B

Tawny Wood-Owl (E): Strix aluco II B

Tawny-bellied Hermit (E): Phaethomis symatophorus II B

Tawny-bellied Screech-Owl (E): Otus watsonii II B

Tawny-browed Owl (E): Pulsatrix koeniswaldiana II B

Taxipashes recia: II In

Tayassu pecari: White-lipped Peccary (E), Pecari de labio blanco (S), Pécari aux babines blanches (F), Pécari à barbe blanche (F) II $\mathrm{M}$

Tayassuidae spp.: Peccaries (E), Pecaries (S), Pécaries (F) I/II M

Tayra (E/F): Eira barbara III M

Tchirou (F): Pantholops hodgsonit I M

Tchitrea bourbonnensis = Terpsiphone bourbonnensis

Tchitrec des Mascareignes (F): Terpsiphone bourbonnensis III B

Tchitrecs (F): Elminia spp/Eutrichomyias rowleyi/Hypothymis spp /Terpsiphone spp. III/NC B

Teal (E): Anatidae spp. $1 / 11 / 11 / \mathrm{NC} B$

Tecolote barbudo (S): Otus barbarus $\amalg$ B

Tecolote bigotudo (S): Otus trichopsis II B

Tecolote flameado (S): Otus flammeolus II B

Tecolote occidental (S): Otus kennicottii II B

Tecolote oriental (S): Otus asio Il B

Tecolote-abetero norteño (S): Aegolius acadicus II B

Tecolotito común (S): Glaucidium brasilianum II B

Tecolotito del cabo (S): Glaucidium californicum II $\mathrm{P}$

Tecolotito enano (S): Micrathene whitneyi II B

Tecolotito serano (S): Glaucidium gnoma II B

Tegu lizards (E): Tupinambis spp. II R

Tegus (E): Tupinambis spp. II R

Tégus (F): Tupinambis spp. II R

Teinopalpus spp.: Kaiserihinds (E) II In Teinopalpus aureus: Golden Kaiserihind (E) II in

Teinopalpus imperialis: Kaiserihind (E) II In

Teita Falcon (E): Falco fasciinucha II B

Tejedor bruñido de Wilson (S): Vidua wilsoni III B

Tejedor bruñido verdoso (S): Vidua chalybeata III B

Tejedor cabecinegro (S): Ploceus melanocephalus III B

Tejedor cogullado (S): Ploceus cucullatus III B

Tejedor cuellinegro (S): Ploceus nigricollis III B

Tejedor de Heuglin (S): Ploceus heuglini III B

Tejedor naranja (S): Ploceus aurantius III B
Tejedor negro albinuca (S): Ploceus albinucha III B

Tejedor negro de Vieillot (S): Ploceus nigerrimus III B

Tejedor pardal capirotado (S): Plocepasser superciliosus III B

Tejedor picogordo (S): Amblyospiza albifrons III B

Tejedor rollizo (S): Pachyphantes superciliosus III B

Tejedor tricolor (S): Ploceus tricolor III B

Tejedor velado (S): Ploceus vitellinus 111 B

Tejedorcito punteado (S): Sporopipes frontalis III B

Tejón (S): Eira barbara III M/Galictis vittata III M

Tejón marsupial rayado (S): Perameles bougainville I M

Tejón melívoro (S): Mellivora capensis III M

Téju à taches noires (F): Tupinambis nigropunctatus II $\mathbf{R}$

Téju noir (F): Tupinambis reguixin II $\mathbf{R}$

Téju rouge (F): Tupinambis rufescens II R

Téju-Crocodile (F): Crocodilurus lacertinus II R

Téjus (F): Tupinambis spp. II R

Temminck's Cat (E): Całopuma temminckii I M

Temminck's Flying-fox (E): Preropus temmincki II M

Temminck's Giant Squirrel (E): Epixerus ebii III M

Temminck's Ground Pangolin (E): Manis temminckii II M

Temnotrochus kermadecensis: II In

Tengma'm's Owl (E): Aegolius funereus II B

Tent Turtle (E): Kachuga tecta I R

Teporingo (S): Romerolagus diazi I M

Tepui Goldenthroat (E): Polytmus milleri II B

Tepui Parakeet (E): Nannopsittaci panychlora II B

Terathopius ecaudatus: Bateleur (E), Bateleur Eagle (E), Aguila volatinera (S), Bateleur (F), Bateleur des savanes (F) II B

Terciopolo (E); Bothrops asper III R

Terecay (S): Podocnemis unifilis II R

Terpsiphone bourbonnensis: Mascarene Paradise-Flycatcher (E), Coq de Bois (F), Tchitrec des Mascareignes (F) III B

Terropene spp:: Box turtles (E), Galápagos cajas (S), Tortugas caja (S), Tortue-boîtes (F) U/II R

Terrapene carolina: Common Box Turtle (E), Eastern Box Turtle (E), Tortue-boîte de Caroline (F) II R

Terrapene coahuila: Aquatic Box Turtle (E), Coahuila Box Turtle (E), Coahuila Turtle (E), Water Box Turtle (E), Galápago caja Mexicano (S), Tortuga Coahuila (S), Tortue-boîte de Coahuila (F), Tortue-boîte du Mexique (F) I R

Terrapene nelsoni: Spotted Box Turtle (E), Tortue-bôtte du Mexique (F) II R

Terrapene omata: Ornate Box Turtle (E), Western Box Turtle (E), Tortue-boîte omée commune (F) II R

Terrapins (E): Emydidae spp. I/NC R

Testudinidae spp:: Tortoises (E), Tortugas (S), Testudínidos (S), Tortues terrestres (F) $\mathbf{I} \mathbf{I} \mathbf{R}$

Testudo angulat $=$ Chersina angulata

Testudo elephantopus $=$ Geochelone nigra

Testudo geometrica $=P$ sammobates geometricus

Testudo graeca: Common Tortoise (E), Greek Tortoise (E), Moorish Toroise (E), Spur-thighed Tortoise (E), Tortuga mora (S), Tortue mauresque (F) II R

Testudo hermanni: Hermann's Tortoise (E), Tortuga mediterránea (S), Tortue d'Hermann (F) II R

Testudo horsfieldii: Afghan Tortoise (E), Central Asian Tortoise (E), Four-toed Tortoise (E), Horsfield's Tortoise (E), Steppe Tortoise (E), Tortuga terrestre afgana (S), Tortue d'Horsfield (F), Tortue des steppes (F) II R

Testudo kleinmanni: Egyptian Tortoise (E), Kleinmann's Tortoise (E), Leith's Tortoise (E), Tortuga de plastrón articulado (S), Tortue d'Egypte (F), Tortue de Kleinmann (F) I R

Testudo marginata: Marginated Tortoise (E), Margined Tortoise (E), Tortuga marginada (S), Tortue bordée (F) $11 \mathrm{R}$

Tesiudo nutapundi $=$ Manouria emys

Testudo planicauda $=$ Pyxis planicauda

Testudo radiata $=$ Geochelone radiata

Testudo yniphora = Geochelone yniphora

Tethocyathus cylindraceus: 11 in

Tethocyathus minor: II In

Tethocyathus recurvalus: $\mathbf{I I}$ in

Tethocyathus variabilis: II In

Tethocyathus virgatus: II In

Tétracère (F): Tetracenus quadricomis III M

Tetracenus quadricomis: Chousingha (E), Four-homed Antelope (E), Antílope de cuatrocuemos (S), Tétracère (F) III M 
Téraogalle de Perse (F): Tetraogallus caspius I B

Tétraogalle du Tibet (F): Tetraogallus tibetanus I B

Tétraogalles (F): Tetraogallus spp. INC B

Tetraogallus caspius: Caspian Snowcock (E), Gallo salvaje del Caspio (S), Perdigallo caspio (S), Perdiz real del Caspio (S) Perdrix des neiges de la Caspienne (F). Tétraogalle de Perse (F), Tétrogalle de la Caspienne (F) I B

Tetraogallus tibetanus: Tibetan Snowcock (E), Gallo salvaje del Tibet (S), Perdigallo tibetano (S), Perdiz real del Tibet (S), Perdrix des neiges du Tibet (F), Tétraogalle du Tibet (F) I B

Tétras (F): Phasianidae spp. INC B

Tétras cupidon d'Attwater (F): Tympanuchus cupido attwateri 1 B

Tetrax tetrax: Little Bustard (E), Sisón (S), Outarde canepetière (F) II B

Tétrogalle de la Caspienne (F): Tetraogallus caspius I B

Tevoro Clam (E): Tridacna tevoroa II In

Texas Tortoise (E): Gopherus berlandieri II R

Textu atrogularis $=$ Ploceus heuglini

Thai Hog Deer (E): Axis porcinus annamiticus I M

Thalamophyllia gasti: II In

Thalamophyllia gombergi: II in

Thalamophyllia rusei: II In

Thalamophyllia tenuescens: II in

Thalarctos maritimus = Ursus maritimus

Thalassiotrochus telegraphicus: II In

Thalurania colombica: Blue-crowned Woodnymph (E), Ninfa coronada (S), Dryade couronnée (F) II B

Thalu rania colombica fannyi $=$ Thalurania fannyi

Thalu rania colombica ridgwayi $=$ Thalurania ridgwayi

Thalurania fannyi: Green-crowned Woodnymph (E), Ninfa coronada (S), Dryade de Fanny (F) II B

Thalurania furcata: Fork-tailed Woodnymph (E), Picaflor gargantiverde (S), Tucusito moradito (S), Dryade à queue fourchue (F) II B

Thalurania furcata colombica $=$ Thalurania colombica

Thalurania glaucopis: Violet-capped Woodnymph (E), Picaflor corona azul (S), Dryade glaucope (F) II B

Thalurania ridgwayi: Mexican Woodnymph (E), Nifa mexicana (S), Dryade du Mexique (F) II B

Thalurania watertonii: Long-tailed Woodnymph (E), Dryade de Waterton (F) II B

Thamin (E): Cervus eldii I M

Thaumastura cora: Peruvian Sheartail (E), Colibri cora (F) II B

Theban Sand Boa (E): Eryx colubrinus II R

The cocyathus = Tethocyathus

Thecopsammia elongata: II in

The copsammia gemma: II In

Thecopsammia socialis: II In

Theropithecus gelada: Gelada Baboon (E), Gelada (F) II M

Thick-billed Parrot (E): Rhynchopsitia pachyrhyncha I B

Thick-billed Weaver (E): Amblyospiza albifrons III B

Thick-knees (E): Burhinus spp. III/NC B

Thick-tailed Bushbaby (E): Otolemur crassicaudatus II M

Thick-tailed Day Gecko (E): Phelsuma murabilis II R

Thick-tailed Pangolin (E): Manis crassicaudata II M

Thomas's Langur (E): Presbytis thomasi II M

Thomas's Leaf Monkey (E): Presbytis thomasi II M

Thomas's Mastigure (E): Uromastyx thomasi II R

Thom Sturgeon (E): Acipenser nudiventris II F

Thornbills (E): Chalcostigma spp/Ramphomicron spp/Acanthiza spp. II/NC B

Thomtails (E): Popelairia spp. II B

Three-cusped Pangolin (E): Manis tricuspis II M

Three-keeled Land Tortoise (E): Melanochelys iricarinata I R

Three-keeled Land Turtle (E): Melanochelys tricarinata I R

Three-lined Day Gecko (E): Phelsuma irilineata II R

Three-striped Batagur (E): Callagur bomeoensis II R

Three-striped Poison Frog (E): Phobobates trivittatus II A

Threneres cristinae $=$ Threnetes leucurus

Threnetes grimeki $=$ Glaucis hirsuta

Threnetes leucurus: Bronze-tailed Barbthroat (E), Pale-tailed Barbthroat (E), Bartita colipalida (S), Garganta lanza coliblanca (S), Ermite à queue blanche (F) II B

Threnetes loehkeni $=$ Threnetes leucurus

Threnetes niger. Sooty Barbthroat (E), Ermite d'Antonie (F) II B
Threnetes ruckeri: Band-tailed Barbthroat (E), Barbita colibandeada (S), Ermitaño barbudo (S), Tucuso de Barba (S), Ermite de Rucker (F) II B

Threskiomis aethiopicus: Sacred Ibis (E), Ibis sagrado (S), Ibis sacré (F) III B

Threskiornis bemieri $=$ Threskiomis aethiopicus

Thrypticotrochus multilobatus: II In

Thrypticotrochus petserdi: II In

Thunder Snake (E): Tropidophis pardalis II R

Thylacine (E/F): Thylacinus cynocephalus I M

Thylacinus cynocephalus: Tasmanian Tiger (E), Tasmanian Wolf

(E), Thylacine (E), Lobo de Tasamania (S), Lobo marsupial (S), Loup marsupial (F), Thylacine (F) I M

Tibet Owlet (E): Athene noctua II B

Tibetan Antelope (E): Pantholops hodgsonii I M

Tibetan Argali (E): Ovis ammon hodgsonii I M

Tibetan Crane (E): Grus nigricollis I B

Tibetan Eared-Pheasant (E): Crossoptilon harmani I B

Tibetan Macaque (E): Macaca thibetana II M

Tibetan Snowcock (E): Tetraogallus tibetanus I B

Tichopora $=$ Goniopora

Tichoseris $=$ Pavona

Tiger (E): Panthera tigris I M

Tiger Cat (E): Leopardus tigrinus I M

Tiger Chameleon (E): Chamaeleo tigris II R

Tiger Civet (E): Prionodon pardicolor I M

Tiger Frog (E): Hoplobatrachus tigerinus II A

Tiger Python (E): Pyrhon molurus I/II R

Tiger-Parrots (E): Psittacella spp. II B

Tigre (S/F): Panthera tigris I M

Tigre americano (S): Panthera onca I M

Tigre real (S): Panthera onca I M

Tigrillo (S): Herpailurus yaguarondi VI M/Leopardus tigrinus I $\mathrm{M} /$ Leopardus wiedii I M

Tijereta centroamericana (S): Doricha enicura II B

Tijereta nortena (S): Calothorax lucifer II B

Tijereta Oaxaquena (S): Calothorax pulcher II B

Tijereta yucateca (S): Doricha eliza II B

Tilbury's Chameleon (E): Chamaeleo marsabitensis II R

Tilingo cuellinegro (S): Preroglossus aracari II B

Tilingo limón (S): Pteroglossis viridis II B

Tilmatura dupontii: Sparkling-tailed Barbthroat (E), Sparkling-tailed

Hummingbird $(E)$, Colibrí colpinto $(S)$, Colibri zémès $(F)$ II B

Timber Wolf (E): Canis lupus I/II M

Timor Python (E): Python timoriensis II R

Timor Red-winged Parrot (E): Aprosmictus jonquillaceus II B

Timor Tree Monitor (E): Varanus timorensis II R

Tinamou solitaire ( $F$ ): Tinamus solitarius I B

Tinamous (E/F): Tinamidae spp. INC B

Tinamu grande (S): Tinamus solitarius I B

Tinamu macuco (S): Tinamus solitarius I B

Tinamus solitarius: Solitary Tinamou (E), Macuco (S), Tinamu grande (S), Tinamu macuco (S), Tinamou solitaire (F) I B

Tinglada (S): Dermochelys coriacea I R

Tiny Hawk (E): Accipiser superciliosus II B

Tiny Sparrowhawk (E): Accipiter superciliosus II B

Tirica (S): Leopardus tigrinus I M

Tisserin à ailes rouges (F): Anaplectes nubriceps III B

Tisserin à cou noir (F): Ploceus nigricollis III B

Tisserin à dos doré (F): Ploceus preussi III B

Tisserin à lunettes (F): Ploceus nigricollis III B

Tisserin à tête noire (F): Ploceus melanocephalus III B

Tisserin à tête rousse $(\mathrm{F})$ : Ploceus vitellinus III B

Tisserin covcau (F): Anomalospiza imberbis III B

Tisserin de Maxwell (F): Ploceus albinucha III B

Tisserin de Pelzeln (F): Ploceus pelzelni III B

Tisserin de Preuss (F): Ploceus preussi III B

Tisserin écarlate (F): Anaplecies rubriceps III B

Tisserin gendarme (F): Ploceus cucullatus III B

Tisserin gros-bec (F): Pachyphantes superciliosus III B

Tísserin masqué (F): Ploceus heuglini III B

Tisserin minule (F): Ploceus luteolus III B

Tisserin nain (F): Ploceus pelzelni III B

Tisserin noir (F): Ploceus nigerrimus III B

Tisserin noir de Vieillot (F): Ploceus nigerrimus III B

Tisserin orangé (F): Ploceus aurantius III B 
Tisserin tricolore (F): Ploceus tricolor III B

Tisserin vitellin (F): Ploceus visellinus III B

Tisserins (F): Anaplectes rubriceps/Pachyphanies superciliosus/Ploceus spp. IIINC B

Tit-hylia (E): Pholidomis rushiae III B

Titi à collier (F): Callicebus torquatus II M

Titi à masque (F): Callicebus personatus II M

Tití de caba blanca (S): Callithrix geoffroyi II M

Tití de Goeldi (S): Callimico goeldii I M

Titi molock (F): Callicebus moloch II M

Titis (E): Callicebus spp. II M

Tiuque cordillerano austral (S): Phalcoboenus australis II B

Toads (E): ANURA spp. IIINC A

Toco Toucan (E): Ramphastos toco II B

Tococo (S): Phoenicopterus nuber II B

Togo Paradise-Whydah (E): Vidua togoensis III B

Tolumuco (S): Eira barbara III M

Tomato Frog (E): Dyscophus antongilii I A

Tomistoma (E): Tomistoma schlegelii I R

Tomistoma schlegelii: False Gavial (E), False Gharial (E), Malayan Gharial (E), Tomistoma (E), Falso gavial malayo (S), Faux gavial malais (F) I R

Tonina (S): Pontoporia blainvillei II M

Toninas (S): Sotalia spp. I M

Tonkean Black Macaque (E): Macaca tonkeana II M

Tonkean Macaque (E): Macaca sonkeana II M

Tonkin Leaf Monkey (E): Trachypithecus francoisi II M

Tonkin Snub-nosed Monkey (E): Pygathrix avunculus I M

Tooth Coral (E): Euphyllia picteti II In

Tooth-billed Hummingbird (E): Androdon aequatorialis II B

Toothed Cape Tortoise (E): Psammobates oculiferus II R

Topacio candela colicanelo (S): Topaza pella II B

Topacio candela colimorado (S): Topaza pyra II B

Topacio carmesi (S): Topaza pella II B

Topacio fuego (S): Topaza pyra II B

Topaza pella: Crimson Topaz (E). Topacio candela colicantio (S), Topacio carmesi (S), Colibri topaze (F) II B

Topaza pyra: Fiery Topaz (E), Topacio candela colimorado (S), Topacio fuego (S), Colibri flamboyant (F) II B

Topazes (E): Topaza spp. II B

Topi (F): Damaliscus lunarus III M

Topi gacela (S): Damaliscus pygargus pygargus II M

Toque Macaque (E): Macaca sinica II M

Tordo amarillo (S): Agelaius flavus I B

Tordo de cabeza amarilla (S): Agelaius flavus I B

Torgos calvus = Sarcogyps calvus

Torgos eracheliotus: Lappet-faced Vulture (E), Nubian Vulture (E), Buitre orejudo (S), Oricou (F), Vautour oricou (F) II B

Tornier's Tortoise (E): Malacochersus tomieri II R

Tomier's Tree Toad (E): Nectophrynoides tomieri I A

Toro cuprey (S): Bos sauveli I M

Torquéole à poitrine brune $(\mathrm{F})$ : Arborophila orientalis III B

Torquéole à poitrine châtaine (F): Arborophila charltonii III B

Torquéole de Merlin (F): Arborophila charltonii III B

Torquéole de Sumatra (F): Arborophila orientalis III B

Torquéole des bois (F): Arborophila charlionii III B

Torquéoles (F): Arborophila spp. IIU/NC B

Tortoises (E): Testudinidae spp. I/I R

Tórtola común (S): Streptopelia rurtur III B

Tórtola engañosa (S): Streptopelia decipiens III B

Tórtola rizum (S): Sireptopelia semitorquata III B

Tórtola rosignis (S): Streptopelia roseogrisea III B

Tórtola senegalesa (S): Sireptopelia senegalensis III B

Tórtola tamborilera (S): Turtur tympanistria III B

Tórtola vinosa (S): Streptopelia vinacea III B

Tortue à bec faucon (F): Eretmochelys imbricata I R

Tortue à bords jaunes (F): Gophenus flavomarginatus I R

Tortue à carapace souple (F): Malacochersus tomieri II R

Tortue à col court (F): Pseudemydura umbrina I R

Tortue à écailles (F): Eretmochelys imbricata I R

Tortue à éperon (F): Geochelone yniphora I R

Tortue à plastron éperonné (F): Geochelone yniphora I $\mathrm{R}$

Tortue à soc d'Afrique du Sud (F): Chersina angulata II R

Tortue à soc de Madagascar (F): Geochelone yniphora I R

Tortue à tête jaune (F): Indotestudo elongata II $R$

Tortue articulee d'Afrique (F): Kinixys erosa II R
Tortue bâtarde (F): Lepidochelys olivacea I R

Tortue bordee (F): Testudo marginala II R

Tortue bosselee (F): Psammobates tentorius II R

Tortue brune (F): Manouria emys II R

Tortue caouanne (F): Carena carena I R

Tortue caret (F): Eretmochelys imbricata I R

Tortue charbonnière (F): Geochelone carbonaria II R

Tortue comestible (F): Chelonia mydas I R

Tortue d'Agassiz (F): Gopherus agassizii II R

Tortue de Birmanie (F): Morenia ocellata I R

Tortue de cola plana (S): Pyxis planicauda II R

Tortue de Hamilton (F): Geoclemys hamiltonii I R

Tortue de Kemp (F): Lepidochelys kempi I R

Tortue de Kleinmann (F): Testudo kleinmanni I R

Tortue de la Floride (F): Gopherus polyphemus II R

Tortue de la Pampa (F): Geochelone chilensis II R

Tortue de l'Amérique du Sud (F): Geochelone denticulata II R

Tortue de l'Inde (F): Lissemys punctata II R

Tortue de Madagascar (F): Geochelone yniphora I R

Tortue de Muhlenberg (F): Clemmys muhlenbergi I R

Tortue de Ridley (F): Lepidochelys olivacea I R

Tortue de Tabasco (F): Dematemys mawii II R

Tortue de Tomier (F): Malacochersus tomieri II R

Tortue de Tranvancore (F): Indotestudo forstenii II R

Tortue d'Egypte (F): Testudo kleinmanni I R

Tortue dentelée (F): Geochelone denticulata II R

Tortue denticulé (F): Geochelone denticulata II R

Tortue des Celèbes (F): Indotestudo forsienii II R

Tortue des marécages (F): Clemmys muhlenbergi I R

Tortue des steppes (F): Testudo horsfieldii II R

Tortue d'Hermann (F): Testudo hermanni II R

Tortue d'Horsfield (F): Tesiudo horsfieldii II R

Tortue du Gange (F): Trionyx gangeticus I R

Tortue du Mexique (F): Gopherus flavomarginatus I R

Tortue du Texas (F): Gopherus berlandieri II R

Tortue éléphantine (F): Geochelone rigra I R

Tortue étoilée de Birmanie (F): Geochelone plarynota II R

Tortue étoilée de l'Inde (F): Geochelone elegans II R

Tortue franche (F): Chelonia mydas I R

Tortue gaufrée (F): Gopherus polyphemus II R

Tortue géante (F): Geochelone gigantea II R

Tortue géante d'Aldabra (F): Geochelone gigantea II R

Tortue géante des Galapagos (F): Geochelone nigra I R

Tortue géométrique (F): Psammobates geometricus I R

Tortue imbriquéc (F): Eretmochelys imbricala I R

Tortue imprimée (F): Manouria impressa II R

Tortue luth (F): Dermochelys coriacea I R

Tortue marine à dos plat (F): Natator depressus I R

Tortue mauresque (F): Testudo graeca II R

Tortue noire (F): Trionyx ater I R

Tortue ocelée (F): Psammobales oculiferus II R

Tortue olivâtre (F): Lepidochelys olivacea I R

Tortue radiée de Madagascar (F): Geochelone radiata $\mathbf{I}$

Tortue rayonnée (F): Geochelone radiata I $\mathbf{R}$

Tortue sillonnée (F): Geochelone sulcata II R

Tortue tricarénée (F): Melanochelys tricarinata I R

Tortue verte (F): Chelonia mydas I R

Tortue-boitte de Caroline (F): Terrapene carolina II R

Tortue-boite de Coahuila (F): Terrapene coahuila I R

Tortue-boite du Mexique (F): Terrapene coahuila I R/Terrapene nelsoni II $\mathrm{R}$

Tortue-boîte ornée commune (F): Terrapene omata II R

Tortue-boites (F): Terrapene spp. III R

Tortue-léopard du Cap (F): Geochelone pardalis II R

Tortues de mer (F): Cheloniidae spp. I R

Tortues fluviatiles (F): Podocnemis spp. II R

Tortues terrestres (F): Testudinidae spp. III R

Tortuga araña (S): Pyxis arachnoides II $\mathbf{R}$

Tortuga ariete (S): Chersina angulata II $\mathbf{R}$

Tortuga blanca (S): Dermatemys mawil II R/Chelonia mydas I R

Tortuga boba (S): Caretta caretta I R

Tortuga carey (S): Eretmochelys imbricata I R

Tortuga Coahuila (S): Terrapene coahuila I R

Tortuga con púas (S): Geochelone sulcaia II R

Tortuga de agua (S): Podocnemis lewyana II R

Tortuga de concha blanda moteada (S): Lissemys punctata II R 
Tortuga de cuña (S): Malacochersus iornieri II R Tortuga de Madagascar (S): Geochelone yniphora I R Tortuga de patas rojas (S): Geochelone carbonaria II R Tortuga de pechera (S): Chersina angulata II R

Tortuga de plastrón articulado (S): Pyxis arachnoides II R Tortuga de plastrón articulado (S): Testudo kleinmanni I R Tortuga de Texas (S): Gopherus berlandieri II R Tortuga del desierto (S): Gopherus agassizii II R Tortuga del Nilo (S): Trionyx triunguis III $R$

Tortuga estrellada de Burma (S): Geochelone platynota II $R$ Tortuga estrellada de la India (S): Geochelone elegans II R Tortuga estrellada de Madagascar (S): Geochelone radiata I $\mathbf{R}$ Tortuga estrellada sudafricana (S): Psammobates tentorius II R Tortuga estrellada sudafricana de caparazón (S): Psammobates oculiferus II R

Tortuga franca oriental (S): Natator depressus I R

Tortuga geométrica (S): Psammabates geometricus I R

Tortuga gigante de Aldabra (S): Geochelone gigantea II R

Tortuga gigante de las Galápagos (S): Geochelone nigra I R

Tortuga globulosa malagache (S): Geochelone yniphora I R

Tortuga golfina (S): Lepidochelys olivacea I R

Tortuga grande (S): Gopherus flavomarginatus I R

Tortuga india de visera (S): Kachuga tecta I R

Tortuga iora (S): Lepidochelys kempi I R

Tortuga laud (S): Dermochelys coriacea I R

Tortuga leopardo (S): Geochelone pardalis II R

Tortuga llanero (S): Gopherus flavomarginatus I R

Tortuga manchada (S): Homopus signatus II R

Tortuga marginada (S): Testudo marginata II R

Tortuga marina bastarda (S): Lepidochelys kempi I R

Tortuga manrón de Burma (S): Manouria impressa II R

Tortuga marrón de la India (S): Indotesiudo forsienii II R

Tortuga mediterránea (S): Testudo hermanni II R

Tortuga mora (S): Testudo graeca II R

Tortuga negra de Adanson (S): Pelusios adansonii III R

Tortuga negra de Africa occidental (S): Pelusios niger III R

Tortuga negra del Gabón (S): Pelusios gabonensis III R

Tortuga olivacea (S): Lepidochelys olivacea I R

Tortuga pico de loro (S): Homopus areolatus II R

Tortuga plana (S): Pelomedusa subrufa III R

Tortuga plana indiana (S): Lissemys punctata II $\mathrm{R}$

Tortuga rayada (S): Geochelone radiaia I R

Tortuga serpentina occidental (S): Pseudemydura umbrina I R

Tortuga terrestre (S): Homopus boulengeri II R

Tortuga terrestre afgana (S): Testudo horsfieldii II R

Tortuga terrestre argentina (S): Geochelone chilensis II R

Tortuga terrestre de Florida (S): Gopherus polyphemus II R

Tortuga topo (S): Gopherus flavomarginatus I R

Tortuga verde (S): Chelonia mydas I R

Tortugas (S): Testudinidae spp. I/II R

Tortugas caja (S): Terrapene spp. III R

Tortugas de espaldars articulados (S): Kinixys spp. II R

Tortugas dentadas (S): Kinixys spp. II R

Tortugas marinas (S): Cheloniidae spp. I R

Totoaba (E): Cynoscion macdonaldi I F

Totoba (S): Cynoscion macdonaldi I F

Toucan à bec rouge (F): Ramphastos tucanus II B

Toucan à carène (F): Ramphastos sulfuratus II B

Toucan à gorge jaune et blanche (F): Ramphastos vitellinus II B

Toucan à ventre rouge (F): Ramphastos dicolorus III B

Toucan ariel (F): Ramphastos vitellinus II B

Toucan Barbet (E): Semnomis ramphastinus III B

Toucan de Baillon (F): Baillonius bailloni III B

Toucan toco (F): Ramphastos toco II B

Toucanet à bec tacheté (F): Selenidera maculirostris III B

Toucanets (E): Aulacorhynchus spp/Baillonius bailloni/Selenidera spp. IIU/NC B

Toucanets (F): Aulacorhynchus spp/Selenidera spp. III/NC B

Toucans (E): Ramphastos spp. IIIIINC B

Toucans (F): Andigena spp/Baillonius bailloni/Ramphastos spp. IIIINC B

Toui à ailes jaunes (F): Brotogeris chiriti II B

Toui à ailes variées (F): Brotogeris versicolurus II B

Toui à bandeau jaune (F): Bolborhynchus aurifrons II B

Toui à dos noir (F): Toui melanonorus $11 \mathrm{~B}$

Toui à front bleu (F): Touit dilectissima II B
Toui à front d'or (F): Brotogeris sanctishomae II B

Toui à front roux (F): Bolborhynchus ferrugineifrons II B

Toui à lunettes (F): Forpus conspicillatus II B

Toui à menton d'or (F): Brotogeris jugularis II B

Toui à queue d'or (F): Touit surda II B

Toui à queue pourpree (F): Touil purpurata 11 B

Toui à sept couleurs (F): Touir batavica II B

Toui à tête jaune (F): Forpus xanthops II B

Toui aymara (F): Bolborhynchus aymara II B

Toui catherine (F): Bolborhynchus lineola II B

Toui céleste (F): Forpus coelestis II B

Toui de Achille (F): Nannopsittaca dachilleae II B

Toui de Deville (F): Brotogeris cyanopsera II B

Toui de Huet (F): Touit huetii II B

Toui de Sclater (F): Forpus sclateri II B

Toui de Spix (F): Forpus xanthopterygius II B

Toui des tépuis (F): Nannopsitraca panychlora II B

Toui d'Orbigny (F): Bolborhynchus orbygnesius II B

Toui du Costa Rica (F): Touit costaricensis II B

Toui du Mexique (F): Forpus cyanopygius II B

Toui été (F): Forpus passerinus II B

Toui flamboyant ( $\mathrm{F})$ : Brologeris pyrrhopterus II B

Toui para (F): Brotogeris chrysopterus II B

Toui tacheté (F): Touit stictoptera II B

Toui tirica (F): Brotogeris tirica II B

Touis (F): Bolborhynchus spp./Brotogeris spp./Forpus spp./Nannopsittaca spp./Touit spp. II B

Touit batavica: Lilac-tailed Parrotlet (E), Seven-colored Parrotlet (E), Cotorrita sietecolores (S), Perico siete colores (S), Toui à sept couleurs (F) II B

Touit costaricensis: Red-fronted Parrotlet (E), Cotorrita Costarricense (S), Toui du Costa Rica (F) II B

Touit dilectissima: Blue-fronted Partotlet (E), Churiquita (\$), Cotomita cariazul (S), Periguito frentiazul (S), Toui à front bleu (F) II B

Touit dilectissima costaricensis $=$ Touit costaricensis

Touit huetii: Scarlet-shouldered Parrotlet (E), Cotorrita alirroja (S), Periquito azul alirojo (S), Toui de Huet (F) II B

Touit melanonotus: Black-eared Parrotiet (E), Brown-backed Parrotlet (E), Cotorrita dorsinegra (S), Toui à dos noir (F) II B

Touit purpurata: Sapphire-rumped Parrotlet (E), Cotorrita purpurada (S), Periquito rabadilla púrpura (S), Toui à queue pourprée (F) II B

Touit stictoptera: Spot-winged Parrotlet (E), Cotorrita alipinta (S). Periquito alipunteado (S), Toui tacheté (F) II B

Touit surda: Golden-tailed Parrotlet (E), Cotorrita sorda (S), Toui à queue d'or (F) II B

Toupaie commun (F): Tupaia glis II M

Toupaie de Java (F): Tupaia javanica II M

Toupaïe de l'île Palawan (F): Tupaia palawanensis II M

Toupaïe de Müller (F): Tupaia minor II M

Toupaie d'Elliot (F): Anathana elliosi II M

Toupaïe des îles Nicobar (F): Tupaia nicobarica II M

Toupaïe des montagnes (F): Tupaia montana II M

Toupaie murin (F): Dendrogale murina II M

Touraco à bec noir (F): Tauraco schuettii II B

Touraco à gros bec (F): Tauraco macrorhynchus II B

Touraco à huppe blanche (F): Tauraco leucolophus II B

Touraco à huppe pourprée (F): Musophaga porphyreolopha II B

Touraco a huppe splendide (F): Musophaga porphyreolopha II B

Touraco à huppe violette (F): Musophaga porphyreolopha II B

Touraco à joues blanches (F): Tauraco leucoris II B

Touraco de Bannerman (F): Tauraco bannermani II B

Touraco de Fischer (F): Tauraco fischeri II B

Touraco de Hartlaub (F): Tauraco hartlaubi II B

Touraco de Livingstone (F): Tauraco livingsionii II B

Touraco de Ruspoli (F): Tauraco ruspolii II B

Touraco de Schalow (F): Tauraco schalowi II B

Touraco doré (F): Tauraco banne mani II B

Touraco géant (F): Corythaeola cristata 111 B

Touraco gris (F): Crinifer piscalor III B

Touraco lourie (F): Tauraco corythair II B

Touraco pauline (F): Tauraco erythrolophus II B

Touraco vert (F): Tauraco persa II B

Touraco vert d'Afrique du Sud (F): Tauraco corythaix II B

Touraco violet (F): Musophaga violacea III B 
Touracos (F): Musophagidae spp. IIJINC B

Tourmaline Sunangel (E): Heliangelus exortis II B

Tourtelette améthystine (F): Turtur afer III B

Tourtelette d'Abyssinie (F): Turtur abyssinicus III B

Tourtelette demoiselle (F): Turtur brehmeri 111 B

Tourtelette masquée (F): Oena capensis III B

Tourtelette tambourette (F): Turtur tympanistria III B

Tourtelettes (F): Oena capensis/Turtur spp. IIINC B

Tourterelle à collier (F): Streptopelia semitorquata III B

Tourterelle à masque de fer (F): Oena capensis III B

Tourterelle à tête bleưe (F): Turtur brehmeri III B

Tourterelle des bois (F): Streptopelia iurtur III B

Tourterelle du Cap (F): Oena capensis III B

Tourterelle maillee (F): Streptopelia senegalensis III B

Tourterelle pleureuse (F): Streptopelia decipiens III B

Tourterelle rieuse (F): Streptopelia roseogrisea III B

Tourterelle tambourette (F): Turtur tympanistria III B

Tourterelle vineuse (F): Streptopelia vinacea III B

Tourterelles (F): Streptopelia spp/Zenaida spp. III/NC B

Toxolasma cylindrellus: Pale Lilliput Naiad (E), Pale Lilliput Pearly Mussel (E) I in

Trachyboa boulengeri: Northem Eyelash Boa (E), Rough-scaled Boa (E), Boa rugosa (S), Boa nain de Boulenger (F), Boa rugueux de Boulenger (F) II R

Trachyboa gularis: Ecuadorian Eyelash Boa (E), Southem Eyelash Boa (E), Spiny Boa (E), Boa rugosa de Ecuador (S), Boa nain de l'Equateur (F), Boa rugueux de l'Equateur (F) II R

Trachylopora = Acropora

Trachyphyllia geoffroyi: II In

Trachypithecus auratus: Ebony Leaf Monkey (E) II M

Trachypithecus cristata auratus = Trachypithecus auratus

Trachypithecus cristatus: Silvered Langur (E), Silvered Leaf Monkey (E), Langur crestado (S), Budeng (F) II M

Trachypithecus delacouri $=$ Trachypithecus francoisi

Trachypithecus francoisi: François's Langur (E), François's Leaf Monkey (E), Tonkin Leaf Monkey (E), Langur de Frantois (S), Langur de François (F), Semnopithèque de François (F) II M

Trachypithecus geei: Golden Langur (E), Golden Leaf Monkey (E), Langur dorado $(\mathrm{S})$, Entelle dorée (F), Langur doré (F). Semnopithèque de Gee (F), Semnopithèque doré (F) I M

Trachypithecus johnii: Black Leaf Monkey (E), John's Langur (E), Nilgiri Langur (E), Nilgiri Leaf Monkey (E), Langur de Nilgiri (S), Langur du Nilgiri (F), Semnopithèque des Nilgiris (F) II M

Trachypithecus laotum $=$ Trachypithecus francois $i$

Trachypithecus obscurus: Dusky Leaf Monkey (E), Spectacled Langur (E), Spectacled Leaf Monkey (E), Langur obscuro (S), Semnopithèque obscur (F) II M

Trachypithecus phayrei: Phayre's Langur (E), Phayre's Leaf Monkey (E), Semnopithèque de Phayre (F) II M

Trachypithecus pilearus: Bonneted Langur (E), Capped Langur (E), Capped Leaf Monkey (E), Capped Monkey (E), Langur capuchino (S), Langur de capa (S), Entelle pileuse (F), Langur à capuchon (F) I M

Trachypithecus poliacephalus $=$ Trachypithecus francoisi

Trachypithecus vetulus: Purple-faced Leaf Monkey (E), Langur de cara roja (S), Sernnopithèque blanchâtre (F) II M

Trachypora = Oxypora

Tragelaphus eurycerus: Bongo (E), Bongo (S), Bongo (F) III M

Tragelaphus spekii: Marshbuck (E), Sitarunga (E), Sitatunga (S), Guib d'eau (F), Sitatunga (F) III M

Tragopan à tête noire (F): Tragopan melanocephalus I B

Tragopan arlequin (F): Tragopan caboti I B

Tragopán arlequín (S): Tragopan caboti I B

Tragopan blythii: Blyth's Tragopan (E), Grey-bellied Tragopan (E), Tragopán de Blyth (S), Tragopán oriental (S), Tragopan de Blyth (F), Tragopan de Molesworth (F). Tragopan oriental (F) I B

Tragopan caboti: Cabot's Tragopan (E), Yellow-billed Tragopan (E), Tragopán arlequín (S), Tragopán chino (S), Tragopán de Cabot (S), Tragopan arlequin (F), Tragopan de Cabot (F) I B

Tragopán chino (S): Tragopan caboti I B

Tragopan de Blyth (F): Tragopan blythii I B

Tragopan de Blyth (S): Tragopan blythii I B

Tragopán de cabeza negra (S): Tragopan melanocephalus I B

Tragopan de Cabot (F): Tragopan caboti I B

Tragopán de Cabot (S): Tragopan caboti I B

Tragopan de Hastings (F): Tragopan melanocephalus I B
Tragopan de Molesworth (F): Tragopan blythii I B

Tragopán dorsigrís (S): Tragopan melanocephalus I B

Tragopan melanocephalus: Black-headed Tragopan (E), Western Homed-Pheasant (E), Westem Tragopan (E), Tragopán de cabeza negra (S), Tragopán dorsigrís (S), Tragopán occidental (S), Tragopan de Hastings (F), Tragopan occidental (F), Tragopan à tête noire (F) 1 B

Tragopan occidental (F): Tragopan melanocephalus I B

Tragopản occidental (S): Tragopan melanocephalus I B

Tragopan oriental (F): Tragopan blythii I B

Tragopán oriental (S): Tragopan blythii I B

Tragopán sátiro (S): Tragopan satyra III B

Tragopan satyra: Crimson Homed-Pheasant (E), Indian Tragopan (E), Satyr Tragopan (E), Tragopán sátiro (S), Tragopan satyre (F) III B

Tragopan satyre (F): Tragopan satyra III B

Tragopans (E/F): Tragopan spp. IIIU/NC B

Trainbearers (E): Lesbia spp. II B

Transkei Dwarf Chameleon (E): Bradypodion caffer II R

Transvaal Dwarf Chameleon (E): Bradypodion transvaalense II R

Transvaal Girdled Lizard (E): Cordylus vitnifer II R

Traro (S): Polyborus plancus II B

Travailleur à tête rouge (F): Quelea erythrops III B

Travailleurs (F): Brachycope anomala/Quelea spp. IIINC B

Travancore Tortoise (E): Indotestudo forstenii II R

Traylor's Forest-Falcon (E): Micrastur buckleyi II B

Tree Boa (E): Candoia carinata II R

Tree Coral (E): Tubastraea micrantha II In

Tree Crocodile (E): Varanus salvadorii II R

Tree Lizard (E): Varanus rudicollis II R

Tree Ocelot (E): Leopardus wiedii I M

Tree Pangolin (E): Manis tricuspis II M

Tree snails (E): GASTROPODA spp. VIINC In

Tree toads (E): Nectophnynoides spp. I A

Tree-Kangaroos (E): Dendrolagus spp. IUNC M

Tree-Partridges (E): Arborophila spp. IIINC B

Treeshrews (E): Tupaiidae spp. II M

Tremarctos omatus: Andean Bear (E), Spectacled Bear (E), Oso de anteojos (S), Oso frontino (S), Oso real (S), Ours andin (F), Ours à lunetres $(\mathrm{F})$ I M

Trematotrochus alternans: II In

Trematoirochus corbicula: II in

Trematotrochus hedleyi: II in

Trematotrochus verconis: II In

Treron australis calva $=$ Treron calva

Treron calva: African Green-Pigeon (E), Vinago obeng (S), Colombar à front nu (F) III B

Treron waalia: Bruce's Green-Pigeon (E), Yellow-bellied GreenPigeon (E), Vinago waalia (S), Colombar waalia (F), Pigeon ver à épaulettes violettes (F) III B

Triangular-spotted Pigeon (E): Columba guinea III B

Tricarinate Hill Turtle (E): Melanochelys tricarinata I R

Trichechus inunguis: Amazonian Manatee (E), South American Manatee (E), Lamantino amazónico (S), Manatí amazónico (S), Vaca marina amazónica (S), Lamantin d'Amérique du Sud (F), Lamantin de l'Amazone (F) I M

Trichechus manatus: American Manatee (E), Caribbean Manatee (E), North American Manatee (E), West Indian Manatee (E), Lamantino norteamericana (S), Manatí norteamericano (S), Lamantin d'Amérique du Nord (F), Lamantin des Antilles (F). Lamantin des Caraibes (F) I M

Trichechus senegalensis: African Manatee (E), West African Manatee (E), Manatí de Senegal (S), Lamantin d'Afrique (F), Lamantin du Sénégal (F) II M

Trichoglossus chlorolepidorus: Scaly-breasted Lorikeet (E), Lori escuamiverde (S), Loriquet vert (F) II B

Trichoglossus euteles: Olive-headed Lorikeet (E), Perfect Lorikeet (E), Lori humilde (S), Loriquet eutèle (F) II B

Trichoglossus flavoviridis: Meyer's Lorikeet (E), Yellow-and-green Lorikeet (E), Lori verdigualdo (S), Loriquet jaune et vert (F) Il B

Trichoglossus goldiei $=$ Psitteuteles goldiei

Trichoglossus haematodus: Green-naped Lorikeet (E), Rainbow Lorikeet (E), Rainbow Lory (E), Lori arcoiris (S), Loriquet à tête bleue (F) II B

Trichoglossus haematodus rubritorquis $=$ Trichoglossus rubritorquis 
Trichoglossus iris $=$ Psitteuteles iris

Trichoglossus johnstoniae: Johnstone's Lorikeet (E). Mindanao Lorikeet (E), Lori de Mindanao (S), Loriquet de Johnstone (F) II B

Trichoglossus omatus: Omate Lorikeet (E), Omate Lory (E), Lori adornado (S), Loriquet orné (F) II B

Trichoglossus rubiginosus: Pohnpei Lorikeet (E), Pohnpei Lory (E), Lori de Ponapé (S), Loriquet de Ponapé (F) II B

Trichoglossus rubritorquis: Red-collared Lorikeet (E), Loriquet à col rouge (F) II B

Trichoglossus versicolor $=$ Psinteuteles versicolor

Tricholimnas sylvestris = Gallirallus sylvestris

Triclaria malachitacea: Blue-bellied Parrot (E), Purple-bellied Parrot (E), Loro ventriazul (S), Crick à ventre bleu (F), Perruche à ventre bleu (F) II B

Tricuspide (F): Manis tricuspis II M

Tridacna crocea: Boring Clam (E), Crocus Clam (E), Saffroncoloured Clam (E) II In

Tridacna derasa: Southern Giant Clam (E) II In

Tridacna gigas: Giant Clam (E), Bénitier géant (F) II ln

Tridacna maxima: Small Giant Clam (E) II In

Tridacna mbulvuana = Tridacna tevoroa

Tridacna rosewateri: Bénitier de Rosewater (F) II In

Tridacna squamosa: Fluted Clam (E), Fluted Giant Clam (E), Scaly Clam (E) II In

Tridacna tevoroa: Tevoro Clam (E), Bénitier de Tevoro (F) II In

Tridacnidae spp.: Giant clams (E), Bénitiers (F) II ln

Tridacophyllia $=$ Pectinia

Trigonocéphale à grand nez (F): Borhrops nasuta III R

Trigonocéphale à groin de Bocourt (F): Bothrops ophryomegas III R

Trigonocéphale rugueux (F): Bothrops asper III R

Trigonocéphale sauteur (F): Bothrops nummifer III R

Trigonoceps occipitalis: White-headed Vulture (E), Buitre cabeciblanco (S), Buitre piquirrojo (S), Vautour huppé (F) Vautour à tête blanche (F) II B

Tringa gutnifer. Nordmann's Greenshank (E), Spotted Greenshank (E), Archibebe moteado (S), Chevalier tacheté (F), Chevalier à gouttelette (F) I B

Trinidad Piping-Guan (E): Pipile pipile I B

Trinidad White-headed Curassow (E): Pipile pipile I B

Trinidad White-headed Piping Guan (E): Pipile pipile I B

Trionyx à ocelles $(\mathrm{F})$ : Trionyx hurum I R

Trionyx arer, Black Mud Turte (E), Black Soft-shell Turtle (E), Black Spiny Soft-shell Turtle (E), Cuatro Cienegas Soft-shell Turtle (E), Galápago conchiblando negro (S), Tortue noire (F) Trionyx noir (F) I R

Trionyx de l'Inde (F): Lissemys punctaia II $\mathbf{R}$

Trionyx du Gange (F): Trionyx gangeticus I R

Trionyx du Nil (F): Trionyx iriunguis III $\mathrm{R}$

Trionyx gangeticus: Ganges Soft-shell Turtle (E), Indian Sofi-shell Turtle (E), Galápago conchiblando del Ganges (S), Tortue du Gange (F), Trionyx du Gange (F) I R

Trionyx hurum: Brown Soft-shelled Turtle (E), Peacock Soft-shell Turtle (E), Peacock-marked Soft-shelled Turtle (E), Galápago conchiblando ocelado (S). Trionyx à ocelles (F) I R

Trionyx nigricans: Bostami Turtle (E), Chittagong Soft-shell Turtle (E), Dark Soft-shell Turtle (E), Dark-coloured Soft-shell Turtle (E), Sacred Black Mud Turtle (E), Galápago conchiblando oscuro (S), Trionyx noirâtre (F) I R

Trionyx noir (F): Trionyx ater I R

Trionyx noirâtre $(\mathrm{F})$ : Trionyx nigricans I $\mathrm{R}$

Trionyx spiniferus ater $=$ Trionyx aier

Trionyx triunguis: African Soft-shell Turtle (E), Nile Soft-shell Turtle (E), Tortuga del Nilo (S), Trionyx du Nil (F) III R

Tristram's Woodpecker (E): Dryocopus javensis richardsi I B

Trochilidae spp.: Hummingbirds (E), Colibries (S), Picaflores (S), Colibris (F), Oiseaux-mouches (F) I/II B

Trochilus polytmus: Streamertail (E), Colibri à tête noire (F) II B

Trochilus scitulus = Trochilus polytmus

Trochocyathus aithoseptatus: II In

Trochocyathus caryophylloides: II in

Trochocyathus cepulla: II in

Trochocyathus cooperi: II in

Trochocyathus decamera: II In

Trochocyathus fasciatus: II in

Trochocyathus fossulus: II In
Trochocyathus gardineri: II In

Trochocyathus gordoni: II In

Trochocyathus japonicus: II in

Trochocyathus maculaiss: II In

Trochocyathus mauiensis: II In

Trochocyathus mediterraneus: II In

Trochocyathus meridionalis: II In

Trochocyathus oahensis: II In

Trochocyathus rawsonii: II In

Trochocyathus rhombocolumna: II In

Trochocyathus spinosocostatus: II In

Trochocyathus tenuicalyx = Trochocyathus rhombocolumna

Trochocyathus victoriae: II In

Trochocyathus virgatus = Tethocyathus virgatus

Trochopsammia infundibulum: II in

Trochopsammia togata: II In

Trogon couroucou (F): Pharomachrus mocinno I B

Trogonoptera spp.: Birdwing butterflies (E), Birdwing butterflies

(E), Ornithoptères (F), Ornithoptères (F) II In

Trogonoptera brookiana: Rajah Brooke's Birdwing (E), Omithoptère de Brooke (F) II ln

Trogonoptera trojana: II In

Troides spp.: Birdwing butterflies (E), Birdwing butterflies (E), Ornithoptères (F). Ornithoptères (F) II In

Troides aeacus: Golden Birdwing (E), Small Birdwing (E) Omithoptère d'obi (F) II In

Troides amphrysus: Golden Birdwing (E), Malay Birdwing (E) II In

Troides andromache: 11 in

Troides criton: II In

Troides cuneifer: Golden Birdwing (E) II in

Troides darsius: II In

Troides dohertyi: Talaud Black Birdwing (E) II In

Troides haliphron: II in

Troides helena: Black-and-gold Birdwing (E), Common Birdwing (E) II In

Troides hypolitus: II In

Troides magellanus: II in

Troides minos: II In

Troides miranda: II In

Troides oblongomaculatus: II In

Troides plateni: II In

Troides plato: II In

Troides prattorum: Buru Opalescent Birdwing (E) II in

Troides rhadamantus: II In

Troides riedeli: II In

Troides vandepolli: II In

Tropical Girdled Lizard (E): Cordylus tropidosternum II R

Tropical Screech-Owl (E): Otus choliba II B

Tropical Spiny-tailed Lizard (E): Cordylus tropidostemum II R

Tropical Whale (E): Balaenoptera edeni I M

Tropicoperdix charlionii $=$ Arborophila charlionil

Tropidocyathus lessonii: II In

Tropidocyathus pileus: II In

Tropidopathes saliciformis: II in

Tropidophis battersbyi: Battersby's Dwarf Boa (E), Ecuadorian Dwarf Boa (E), Boa forestier de Battersby (F), Boa nain de Battersby (F) II R

Tropidophis canus: Great Inagua Island Dwarf Boa (E), Wood Snake (E), Boa enana de las Bahamas (S), Boa fonestier de l'île du Grand Inagua (F), Boa nain de lîle du Grand Inagua (F) II R

Tropidophis caymanensis: Cayman Islands Dwarf Boa (E), Caymans Ground Boa (E), Bon enana de las islas Caimán (S), Boa forestier des îles Cayman (F), Boa nain des îles Cayman (F) II R

Tropidophis feicki: Feick's Dwarf Boa (E), Boa forestier de Feick (F), Boa nain de Feick (F) II R

Tropidophis fuscus: Boa forestier brun (F), Boa nain brun (F) II R

Tropidophis greenwayi: Ambergris Cay Dwarf Boa (E), Boa forestier d'Ambergris Cay (F), Boa nain d'Ambergris Cay (F) II R

Tropidophis haetianus: Haitian Dwarf Boa (E), Haitian Ground Boa (E), Boa forestier d'Haiti (F), Boa nain d'Haitti (F) II R

Tropidophis maculatus: Spotted Dwarf Boa (E), Boa forestier tacheté (F), Boa nain tacheté (F) II R 
Tropidophis melanurus: Cuban Black-tailed Dwarf Boa (E), Navassa Island Black-tailed Dwarf Boa (E), Wood Snake (E), Boa de bosque (S), Culebra boba (S), Maja chico (S), Boa forestier à queue noire de lìle Navassa $(F)$, Boa nain à queue noire de l'île Navassa (F) II R

Tropidophis nigriventris: Black-bellied Dwarf Boa (E), Boa forestier à ventre noir $(F)$, Boa nain à ventre noir $(F)$ II $R$

Tropidophis pardalis: Leopard Dwarf Boa (E), Thunder Snake (E), Boa forestier léopard ( $F$ ), Boa nain léopard (F) II R

Tropidophis paucisquamis: Brazilian Dwarf Boa (E), Boa forestier du Brésil (F), Boa nain du Brésil (F) II R

Tropidophis pilsbryi: Pilsbry's Dwarf Boa (E), Boa forestier de Pilsbry (F), Boa nain de Pilsbry (F) II R

Tropidophis semicinctus: Banded Dwarf Boa (E), Boa forestier annelé (F), Boa nain annelé (F) II $R$

Tropidophis taczanowskyi: Taczanowsky's Dwarf Boa (E), Boa forestier de Taczanowsky (F), Boa nain de Taczanowsky (F) II R

Tropidophis wrighti: Wright's Dwarf Boa (E), Boa forestier de Wright (F), Boa nain de Wright (F) II R

True Langur (E): Semnopithecus entellus I M

True Red Leg (E): Brachypelma emilia II in

True's Beaked Whale (E): Mesoplodon mirus II M

Truk Flying-fox (E): Pteropus insularis I M

Trumpet Manucode (E): Manucodia keraudrenii II B

Trumpetbird (E): Manucodia keraudrenii II B

Truncatoflabellum aculeatum: II In

Truncatoflabellum arcuatum: II In

Truncatoflabellum candeanum: II in

Truncatoflabellum carinatum: II in

Truncaloflabellum compressum: II In

Truncatoflabellum corbicula: II In

Truncatoflabellum crassum: II In

Truncatoflabellum cumingii: II in

Truncatoflabellum dens: II In

Truncatoflabellum formosum: II In

Truncatoflabellum gardineri: II In

Truncatoflabellum gippslandicus: II Is

Truncatoflabellum inconstans: II In

Truncatoflabellum incrustatum: II In

Truncaloflabellum irregulare: II in

Truncatoflabellum mariensii: II In

Truncatoflabellum multispinosum: II In

Truncaroflabellum paripavoninum: II in

Truncatoflabellum phoenix: II in

Truncatoflabellum pusillum: II In

Truncaroflabellum spheniscus: 11 in

Truncatoflabellum stabile: II in

Truncatoflabellum stokesii: II In

Truncatoflabellum trapezoideum: II In

Truncatoflabellum inuncum: II in

Truncatoflabellum variabile: II In

Truncatoflabellum zuluense: II In

Truncatoguynia irregularis: II In

Trunkback turtle (E): Dermochelys coriacea I R

Tsaratanan Chameleon (E): Chamaeleo tsaratananensis II R

Tsessebe (E): Damaliscus lunatus III M

Tuatara (E): Sphenodon punctatus I R

Tuátara (S): Sphenodon punctatus I R

Tuataras (E/F): Sphenodon spp. I R

Tubastraea coccinea: Orange Tube Coral (E) II In

Tubastraea diaphana: II In

Tubastraea faulkneri: II In

Tubastraea floreana: II In

Tubastraea micrantha: Tree Coral (E) II In

Tubastraea tagusensis: II In

Tubercled-blossom Pearly Mussel (E): Epioblasma tonulosa iorulosa I in

Tubercle-nosed Chameleon (E): Chamaeleo tempeli II R

Tubipora musica: Organ-pipe Coral (E) II In

Tubiporidae spp.: Organpipe corals (E), Corail orgue (F) II In

Tucán grande (S): Ramphası́os toco II B

Tucán pico-multicolor (S): Ramphastos sulfuratus II B

Tucán rojo y amarillo (S): Ramphastos dicolorus III B

Tucancito picomaculado (S): Selenidera maculirostris III B

Tucán-Tilingo verde (S): Pteroglossus castanotis III B
Tucuman Amazon (E): Amazona tucumana I B

Tucuman Parrot (E): Amazona fucumana I B

Tucusito amatista (S): Calliphlox amethystina II B

Tucusito cabeza azul (S): Klais guimeti II B

Tucusito garganta rosa (S): Chaetocercus jourdanii II B

Tucusito moradito (S): Thalurania furcata II B

Tucusito pico curvo (S): Lepidopyga goudoti II B

Tucusito rubí (S): Chrysolampis mosquitus II B

Tucuso de Barba (S): Threnetes ruckeri II B

Tucuxi (E): Solalia fluviarilis I M

Tufted Capuchin (E): Cebus apella II M

Tufted Coquette (E): Lophornis ornatus II B

Tui Parakeet (E): Brotogeris sanctithomae II B

Tumbes Hummingbird (E): Leucippus baeri II B

Tunina de Heaviside (S): Cephalorhynchus heavisidii II M

Tunina de Héctor (S): Cephalorhynchus hectori II M

Tunina de vientre blanco (S): Cephalorhynchus eutropia II M

Tunina overa (S): Cephalorhynchus commersonii II M

Tunina sinaleta (S): Lissodelphis peronii II M

Tunqui (S): Rupicola perwiviana II E

Tupaia belangeri: Common Treeshrew (E), Tupaya norteña (S) II M

Tupaia chrysogasier: Golden-bellied Treeshrew (E), Mentawai

Treeshrew (E), Tupaya de vientre dorado (S) II M

Tupaia de Boméo (F): Tupaia tana II M

Tupaia dorsalis: Suriped Treeshrew (E), Tupaya rayada (S) II M

Tupaia glis: Malaysian Treeshrew (E), Musaraña común (S),

Toupaie commun (F) II M

Tupaia glis belangeri $=$ Tupaia belanger

Tupaia glis chrysogaster $=$ Tupaia chrysogaster

Tupaia glis longipes = Tupaia longipes

Tupaia glis palawanensis = Tupaia palawanensis

Tupaia gracilis: Slender Treeshrew (E), Tupaya esbelta (S) II M

Tupaia javanica: Javan Treeshrew (E), Toupaïe de Java (F) II M

Tupaia longipes: Bomean Treeshrew (E), Tupaya de pies largos (S)

II M

Tupaia minor: Lesser Treeshrew (E), Pygmy Treeshrew (E),

Musaraña pigmea (S), Toupaïe de Müller (F) II M

Tupaia montana: Mountain Treeshrew (E), Toupaie des montagnes

(F) II M

Tupaia nicobarica: Nicobar Treeshrew (E), Toupäie des îles Nicobar (F) II M

Tupaia palawanensis: Palawan Treeshrew (E), Toupaïe de l'ile Palawan (F) II M

Tupaia picta: Painted Treeshrew (E), Tupaya pintada (S) II M

Tupaia splendidula: Ruddy Treeshrew (E), Rufous-tailed Treeshrew (E), Tupaya de cola marrón (S) II M

Tupaia tana: Large Treeshrew (E), Musaraña grande (S), Grande toupaïe (F), Tara (F), Tupaia de Boméo (F) II M

Tupaya de Borneo (S): Dendrogale melanura II M

Tupaya de cola marrón (S): Tupaia splendidula II M

Tupaya de pies largos (S): Tupaia longipes II M

Tupaya de vientre dorado (S): Tupaia chrysogaster II M

Tupaya esbelta (S): Tupaia gracilis II M

Tupaya norteña (S): Tupaia belangeri II M

Tupaya pintada (S): Tupaia picta II M

Tupaya rayada (S): Tupaia dorsalis II M

Tupinambis spp.: Tegu lizards (E), Lagartos (S), Grand téjus (F), Tégus (F), Téjus (F) II R

Tupinambis duseni $=$ Tupinambis rufescens

Tupinambis nigropunciarus: Brazil Teju (E), Téju à taches noires (F) II R

Tupinambis rufescens: Argentine Teju (E), Red Teju (E), Téju rouge (F) II R

Tupinambis reguixin: Banded Tegu (E), Black Tegu (E), Common Tegu (E), Grand téju (F), Sauvegarde (F), Téju noir (F) II R

Turaco azul (S): Musophaga violacea III B

Turaco capucha-blanca (S): Tauraco leucolophus II B

Turaco capucha-franjeada (S): Tauraco macrorhynchus II B

Turaco capucha-verde de Guinea (S): Tauraco persa II B

Turaco crestivioleta (S): Musophaga porphyreolopha II B

Turaco de Bannerman (S): Tauraco bannermani II B

Turaco de Crin occidentale (S): Crinifer piscator III B

Turaco gigante (S): Corythaeola cristata III B

Turaco sudafricano (S): Tauraco corythaix II B

Turacos (E): Corythaeola cristata/Musophaga spp /Tauraco spp. IV/III/NC B 
Turberculed Riffle Shell (E): Epioblasma sorulosa forulosa I In Turbinaria aspera: II In

Turbinaria auricularis: II In

Turbinaria bifrons: II In

Turbinaria brassica: II In

Turbinaria brueggemanni: II In

Turbinaria calicularis: II In

Turbinaria cinerascens: II In

Turbinaria conica: II In

Turbinaria conspicua: II In

Turbinaria contoria: II In

Turbinaria crater. II In

Turbinaria danae: II In

Turbinaria elegans: II In

Turbinaria foliosa: II In

Turbinaric indens: II In

Turbinaria heronensis: II In

Turbinaria irregularis: II In

Turbinaria laminata: II in

Turbinaria manronae: II In

Turbinaria mamorea: II In

Turbinaria mesenterina: II In

Turbinaria mollis: II In

Turbinaria orbicularis: II In

Turbinaria parvistella: II In

Turbinaria patula: II In

Turbinaria peltata: II In

Turbinaria porcellanea: II In

Turbinaria pulcherrima: II In

Turbinaria quincuncialis: II In

Turbinaria radicalis: II In

Turbinaria reniformis: II In

Turbinaria rugosa: II In

Turbinaria schistica: 11 In

Turbinaria sinensis: II In

Turbinaria speciosa: II In

Turbinaria stellulata: II In

Turbinaria tayamai: II In

Turbinaria tubifera: II In

Turbinaria undata: II In

Turgid Riffle Shell (E): Epioblasma turgidula I In

Turgid-blossom Naiad (E): Epioblasma iurgidula I In

Turgid-blossom Pearly Mussel (E): Epioblasma turgidula I In

Turkeys (E): Agriocharis ocellata/Meleagris gallopavo IIINC B

Turks and Caicos Ground Iguana (E): Cyclura carinara I R

Turks Islands Boa (E): Epicrates chrysogaster II R

Turón patinegro americano (S): Mustela nigripes I M

Turquoise Grass-Parakeet (E): Neophema puichella II B

Turquoise Parrot (E): Neophema pulchella II B

Turquoise-bellied Poison Frog (E): Epipedobares espinosai II A

Turquoise-fronted Parrot (E): Amazona aestiva II B

Turquoise-throated Puffleg (E): Eriocnemis godini II B

Tursión (S): Tursiops truncarus II M

Tursiops (F): Tursiops truncatus II M

Tursiops aduncus $=$ Tursiops iruncarus

Tursiops gillii = Tursiops iruncalus

Tursiops nuuanu = Tursiops iruncatus

Tursiops truncarus: Bottle-nosed Dolphin (E), Bottlenose Dolphin

(E), Pez mular (S), Tursión (S), Grand dauphin (F), Souffleur (F). Tursiops (F) II M

Turte Dove (E): Sireptopelia turtur III B

Turtle-Doves (E): Streptopelia spp. IIINC B

Turtles (E): TESTUDINATA spp. I/I/IIINC R

Turrur abyssinicus: Black-billed Wood-Dove (E), Palomita especulada abisinica (S), Emerauldine a bec noir (F), Tourtelette d'Abyssinie ( $F$ ) III B

Turur afer. Blue-spotted Wood-Dove (E), Red-billed Wood-Dove (E), Palomita especulada africana (S), Emerauldine à bec rouge (F). Tourtelette améthystine (F) III B

Turtur brehmeri: Blue-headed Dove (E), Blue-headed Wood-Dove (E), Palomita especulada cobriza (S), Tourtelette demoiselle (F). Tourterelle à tête bleue (F) III B

Turrur tympanistria: Tambourine Dove (E), Tórtola tamborilera (S) Tourtelette tambourette (F), Tourterelle tambourette (F) III B

Twelve-wired Bird-of-paradise (E): Seleucidis melanoleuca II B

Twenty-eight Parakeet (E): Platycercus zonarius II B
Twinspots (E): Clytospiza monteiri/Euschistospiza spp/Hypargos spp/Mandingoa nitidula IIUNC B

Two-banded Chameleon (E): Chamaeleo balieatus II R

Two-banded Monitor (E): Varanus salvator II R

Two-homed Chameleon (E): Chamaeleo bifidus II $R$

Two-lined Chameleon (E): Chamaeleo bitaeniatus II R

Two-toned Poison Frog (E): Phyllobates bicolor II A

Tylopathes $=$ Antipathes

Tylopora $=$ Acropora

Tympanuchus cupido attwateri: Attwater's Prairie-Chicken (E)

Gallito de pradera (S), Poule de prairie d'Attwater (F). Tétras cupidon d'Attwater (F) I B

Tyrant Hawk-Eagle (E): Spizaetus tyrannus II B

Tyrian Metaltail (E): Metallura hrianthina 11 B

Tyto alba: Bam Owl (E), Common Barn-Owl (E), Lechuza común (S). Lechuza de campanario (S), Chouette effraie (F), Effraie africaine (F). Effraie des clochers (F) II B

Tylo alba glaucops $=$ Tyro glaucops

Tyto aurantia: Bismarck Masked-Owl (E), Bismarck Masked-owl (E), Golden Owl (E). New Britain Masked-Owl (E), Effraie dorée (F) 11 B

Tyıo capensis: African Grass-Owl (E), Grass Owl (E), Effraie du Cap (F) II B

Tyto capensis longimembris = Tyro longimembris

Tyto castanops: Tasmanian Masked-Owi (E), Effraie de Tasmanie (F) II B

Tyto detoria $=$ Tyro $\mathrm{alba}$

Tyto glaucops: Ashy-faced Owl (E), Effraie d'Hispaniola (F) II B

Tyto inexspectata: Minahassa Bam-Owl (E), Minahassa Masked-Ow] (E), Minahassa Owl (E), Effraic de Minahassa (F) II B

Tyto longimembris: Eastem Grass-Owl (E), Effraie de prairie (F) II B

Tyto manusi: Manus Masked-Owl (E), Manus Masked-owl (E), Effraie de Manus (F) II B

Tyto mulfipunctata: Lesser Sooty-Owl (E), Effraie piquetee (F) II B Tyto nigrobrunnea: Sula Barn-Owl (E), Taliabu Masked-Owl (E). Taliabu Owl (E), Effraie de Taliabu (F) II B

Tyıo novachollandiae: Australian Masked-Owl (E), Effraie masquée (F) II B

Tyto novaehollandiae castanops $=$ Tyto castanops

Tyto novaehollandiae manusi = Tyto manusi

Tyto novachollandiae sororcula = Tyto sororcula

Tyto rosenbergii: Sulawesi Owl (E), Effraie des Célèbes (F) II B

Tyto sororcula: Lesser Masked-Owl (E), Tanimbar Owl (E), Effraie des Tanimbar (F) II B

Tyto soumagnei: Madagascar Grass Owl (E), Madagascar Owl (E). Madagascar Red Owl (E), Soumagne's Owl (E), Lechuza de Madagascar (S), Effraie de Soumagne (F), Effraie rousse de Madagascar (F) I B

Ty to tenebricosa: Greater Sooty-Owl (E), Effraie ombrée (F) II B

Tyto tenebricosa multipunciata $=$ Tyto multipunctata

Uacaries (S): Cacajao calvus I M

Uaddan (E): Ammotragus lenvia II M

Uakaris (E): Cacajao spp. I M

Ucati (S): Eira barbara III M

Uelle Paradise-Whydah (E): Vidua interjecta III B

Ukinga Homless Chameleon (E): Chamaeleo incomutus II R

Ukinga Spiny-tailed Lizard (E): Cordylus ukingensis II R

Ulamá (S): Eira barbara III M

Ulangia $=$ Oulangia

Ulocyathus = Flabellum

Ulophyllia = Oulophyllia

Ultramarine Lorikeet (E): Vini ultramarina I B

Ultramarine Lory (E): Vini ultramarna I B

Uluguru One-homed Chameleon (E): Bradypodion oxyrhinum II R

Uluguru Two-horned Chameleon (E): Bradypodion fischeri II R

Umba (S): Speothos venaticus I M

Umbrella Cockatoo (E): Cacatua alba II B

Umbrellabirds (E): Cephalopterus spp. IIU/NC B

Unau (S): Choloepus hoffmanni III M

Unau d'Hoffmann (F): Choloepus hoffmanni III M

Uncia uncia: Ounce (E), Snow Leopard (E), Leopardo nival (S),

Pantera de la nieves (S), Irbis (F), Léopard des neiges (F), Once

(F), Panthère des neiges (F) I M

Undaria $=$ Agaricia 
Ungaliophis continentalis: Banana Boa (E), Chiapan Boa (E), Isthmian Dwarf Boa (E), Boa enana del norte de centroamérica (S), Boa nain d'Amérique centrale (F) II R

Ungaliophis panamensis: Central American Dwarf Boa (E), Panamanian Dwarf Boa (E), Boa nain du Panama (F) II R Ungaliophis paucisquamis $=$ Tropidophis paucisquamis Unicom Whale (E): Monodon monoceros II M

Unio nickliniana: Nicklin's Pearly Mussel (E) I in

Unio tampicoensis tecomatensis: Tampico Pearly Mussel (E) I ln Upland Buzzard (E): Buteo hemilasius 11 B

Uraeginthus bengalus: Cordonbleu (E), Red-cheeked Cordonbleu (E), Coliazul bengali (S), Cordon bleu à joures rouges (F) III B

Ural Owl (E): Strix uralensis II B

Urial (E/S/F): Ovis vignei vignei $1 \mathrm{M}$

Urochroa bougueri: White-tailed Hillstar (E), Estrella coliblanca (S), Colibri de Bouguer (F) II B

Urogale de Mindanao (F): Urogale everesti II M

Urogale d'Everett (F): Urogale everetti II M

Urogale everetti: Philippine Treeshrew (E), Musaraña arboricola de Ftlipinas (S), Urogale d'Everett (F), Urogale de Mindanao (F) II M

Uroglaur dimorpha: Papuan Hawk-Owl (E), Búho aguilucho de Neuva Guinea (S), Chouette de Nouvelle-Guinée (F), Ninoxe papoue (F) II B

Uromastyx spp.: Mastigures (E), Spiny-tailed lizards (E), Fouettesqueue (F), Lézards fouette-queue (F) II R

Uromastyx acanthinurus: Bell's Dabb Lizard (E), Black Spiny-tailed Lizard (E), Dabb's Mastigure (E), Lagarto de cola espinosa común (S), Dob (F), Fouette-queue épineux (F) II R

Uromastyx aegyptius: Dabb Lizard (E), Egyptian Mastigure (E) Egyptian Spiny-tailed Lizard (E), Lagarto de cola espinosa egipcio (S), Fouette-queue d'Egypte (F) II R

Uromastyx asmussi: Horn Lizard (E), Iranian Mastigure (E), Fouettequeue d' $\operatorname{tran}(\mathrm{F})$ II R

Uromastyx benti: Bent's Mastigure $(\mathrm{E})$, Fouette-queue de Bent $(\mathrm{F})$ II $R$

Uromastyx dispar = Uromastyx acanthinures

Uromastyx geyri: Sahara Mastigure (E), Lagarto de cola espinosa de Geyr (S), Fouette-queue du Sahara (F) II R

Uromasryx hardwickii: Hardwick's Spiny-tailed Lizard (E), Indian Mastigure (E), Lagarto de cola espinosa indo (S), Fouette-queue de Hardwick (F), Fouette-queue indien (F) II $R$

Uromastyx loricatus: Mesopotamian Mastigure (E), Mesopolamian Spiny-tailed Lizard (E), Fouette-queue de Mésopotamie (F) II R

Uromastyx macfadyeni: Somali Mastigure (E), Lagarto de cola espinosa de Macfadyen (S). Fouette-queue de Somalie (F) II R

Uromastyx microlepis = Uromastyx aegyptius

Uromastyx ocellatus: Eyed Dabb Lizard (E), Ocellated Mastigure (E), Lagarto de cola espinosa ocelado (S), Fouette-queue ocellé (F) II R

Uromastyx ornatus = Uromasty $x$ ocellatus

Uromastyx philbyi = Uromastyx ocellatus

Uromastyx princeps: Princely Mastigure (E), Fouette-queue d'O'Shaughnessy (F), Fouette-queue princier (F) II R

Uromastyx thomasi: Thomas's Mastigure (E), Lagarto de cola espinosa de Thomas (S), Fouette-queue de Thomas (F) II R

Urosticte benjamini: Purple-bibbed Whitetip (E), Punta blanca (S), Colibri de Benjamin (F) II B

Urosticle benjamini ruficrissa $=$ Urosticle ruficrissa

Urosticte ruficrissa: Rufous-vented Whitetip (E), Colibri à souscaudales rousses (F) II B

Urotriorchis macrourus: African Long-tailed Hawk (E), Long-tailed Hawk (E), Azor rabilargo (S), Autour à longue queue (F) II B

Ursidae spp.: Bears (E), Osos (S), Ursidos (S), Ours (F) I/II M

Ursidos (S): Ursidae spp. III M

Ursus americanus: American Black Bear (E), Oso negro americano (S), Baribal (F), Ours noir américain (F) II M

Ursus arctos: Brown Bear (E), Grizzly Bear (E), Oso pardo (S), Grizzly (F), Ours brun (F), Ours grizzly (F) III M

Ursus arcios isabellinus: Himalayan Brown Bear (E), Red Bear (E), Oso pardo del Himalaya (S), Ours brun de l'Himalaya (F), Ours brun isabelle (F), Ours isabelle (F) I M

Ursus arctos nelsoni: Mexican Grizzly Bear (E) I M

Ursus arctos pruinosus: Ours brun du Tibet (F) I M

Ursus maritimus: Polar Bear (E), Oso polar (S), Ours blanc (F), Ours polaire (F) II M
Ursus thibetanus: Asiatic Black Bear (E), Himalayan Black Bear (E), Oso de collar (S), Oso negro de Asia (S), Ours de l'Himalaya (F), Ours du Tibet (F), Ours noir d'Asie (F), Ours à collier (F) I M

Usambara Eagle-Owl (E): Bubo vosseleri II B

Usambara Soft-homed Chameleon (E): Bradypodion tenue II R

Usambara Three-homed Chameleon (E): Chamaeleo deremensis II R

Usambara Two-horned Chameleon (E): Bradypodion fischeri II R

Usambara Viviparous Toad (E): Nectophrynoides tomieri I A

Uzungwe Mountain Chameleon (E): Chamaeleo laterispinis II R

Uzungwe Scarp Tree Toad (E): Nectophrynoides wendyae I A

Uzungwe Three-homed Chameleon (E): Chamaeleo werneri II R

Vaca marina amazónica (S): Trichechus inunguis I M

Van Beneden's Colobus (E): Procolobus verus II M

Vantour fauve (F): Gyps fulvus II B

Vaquita (E/S/F): Phocoena sinus I M

Varan à deux bandes ( $F$ ): Varanus salvator II $R$

Varan à gorge blanche (F): Varanus albigularis II R

Varan à queue bleue $(\mathbf{F})$ : Varanus doreanus II $\mathbf{R}$

Varan à queue courte (F): Varanus brevicauda II R

Varan à queue épineuse (F): Varanus acanthurus II R/Varanus primordius II $\mathrm{R}$

Varan à queue rayée (F): Varanus coudolineatus II R

Varan à tête noire (F): Varanus tristis II R

Varan aquatique commun (F): Varanus salvator II R

Varan aquatique de Mertens (F): Varanus mertensi II R

Varan aquatique de Mitchell (F): Varanus mitchelli II R

Varan arboricole de l'ile Rossell (F): Varanus telenestes II R

Varan arboricole du Queensland (F): Varanus terize II R

Varan arboricole noir (F): Varanus beccarii II R

Varan arboricole tacheté (F): Varanus timorensis II R

Varan bigarté ( $F$ ): Varanus varius II $R$

Varan brun (F): Varanus primordius II R

Varan cou rugueux (F): Varanus rudicollis II R

Varan crocodile $(\mathrm{F})$ : Varanus salvadorii II R

Varan de Bogert $(\mathrm{F})$ : Varanus bogerti II $\mathrm{R}$

Varan de Duméril (F): Varanus dumerilii II R

Varan de Gillen (F): Varanus gilleni II R

Varan de Glauert (F): Varanus glauerti II R

Varan de Gould (F): Varanus gouldii II R

Varan de Gray (F): Varanus olivaceus II R

Varan de Kimberley (F): Varanus panoples II $R$

Varan de King (F): Varanus kingorum II R

Varan de Komodo (F): Varanus komodoensis I R

Varan de Papouasie (F): Varanus salvadorii II R

Varan de Pilbara (F): Varanus pilbarensis II R

Varan de Rosenberg (F): Varanus rosenbergi II R

Varan de Schlegel (F): Varanus tristis II R

Varan de Schmidt (F): Varanus jobiensis II R

Varan de Spencer (F): Varanus spenceri II R

Varan de Storr (F): Varanus storri II R

Varan de Timor (F): Varanus timorensis II R

Varan dentelle $(F)$ : Varanus varius II $R$

Varan des cavemes (F): Varanus eremius II $\mathbf{R}$

Varan des crevaces (F): Varanus kingorum II R

Varan des Indes (F): Varanus indicus II $R$

Varan des mangroves (F): Varanus indicus II RNaranus semiremex II $\mathbf{R}$

Varan des rochers à longue queue $(\mathrm{F})$ : Varanus glebopalma II $\mathrm{R}$

Varan des sables noir (F): Varanus rosenbergi II $R$

Varan des savanes (F): Varanus exanthematicus II R

Varan des spinifex (F): Varanus eremius II R

Varan des steppes (F): Varanus exanshematicus II R

Varan du Bengale $(F)$ : Varanus bengalensis I R

Varan du désert (F): Varanus griseus I R

Varan du Nil (F): Varanus niloticus II R

Varan du nord de l'Australie (F): Varanus similis II R

Varan du Yemen (F): Varanus yemenensis II R

Varan cu Yunnan (F): Varanus irrawadicus II $R$

Varan émeraude (F): Varanus prasinus II $R$

Varan épineux des lles Salomon (F): Varanus spinulosus II $R$

Varan gigantesque (F): Varanus giganteus II R

Varan gris (F): Varanus griseus I R

Varan jaune $(F)$ : Varanus flavescens I $R$

Varan nain (F): Varanus storri II R

Varan pierre (F): Varanus baritji II R 
Varan pygmée de Mulga (F): Varanus gilleni II R

Varan rouille (F): Varanus semiremex II R

Varano amarillo (S): Varanus flavescens I R

Varano arboricola común (S): Varanus varius II $R$

Varano arborícola manchado (S): Varanus timorensis II R

Varano colicorto (S): Varanus brevicauda II R

Varano colilargo de las rocas (S): Varanus glauerti II $R$

Varano cuellingoso (S): Varanus rudicollis II R

Varano de agua de Mertens (S): Varanus mertensi 11 R

Varano de agua de Mitchell (S): Varanus mitchelli II R

Varano de arena (S): Varanus gouldii II R

Varano de Bengala (S): Varanus bengalensis I $R$

Varano de cabeza negra (S): Varanus tristis II $\mathbf{R}$

Varano de cola crestada (S): Varanus acanthurus II RWaranus primordius $\mathbf{I I} \mathbf{R}$

Varano de cola rayada (S): Varanus caudolineatus II R

Varano de dos bandas (S): Varanus salvator II R

Varano de Dumeril (S): Varanus dumerilii II $\mathrm{R}$

Varano de Gillen (S): Varanus gilleni II R

Varano de Gould (S): Varanus gouldii II R

Varano de King (S): Varanus kingonum II R

Varano de Komodo (S): Varanus komodoensis I R

Varano de manglar (S): Varanus indicus II R

Varano de Papua (S): Varanus salvadorii II R

Varano de Rosenberg (S): Varanus rosenbergi II R

Varano de Schmidt (S): Varanus jobiensis II R

Varano de Spencer (S): Varanus spenceri II R

Varano del Nilo (S): Varanus niloticus II R

Varano desértico (S): Varanus griseus I R

Varano enano (S): Varanus storri II R

Varano gigante (S): Varanus giganteus II R

Varano pardo (S): Varanus semiremex II R

Varano pigmeo del desierto (S): Varanus eremius II R

Varano verde (S): Varanus prasimus II R

Varanos (S): Varanus spp. I/I R

Varans (F): Varanus spp. I/II R

Varanus spp.: Monitor lizards (E), Monitors (E), Varanos (S), Varans (F) I/II R

Varanus acanthurus: Goanna Monitor (E), Ridge-tailed Monitor (E), Spiny-tailed Pygmy Monitor (E), Varano de cola crestada (S), Varan à queue épineuse (F) II R

Varanus acanthurus primordius = Varanus primordius

Varanus albigularis: Rock Monitor (E), Southern Savanna Monitor (E), White-throated Monitor (E), Varan à gorge blanche (F) II R Varanus baritii: Stone Monitor (E), Varan pierre (F) II R

Varanus beccarii: Black Tree Monitor $(E)$, Varan aboricole noir (F) II $\mathbf{R}$

Varanus bengalensis: Bengal Black Lizard (E), Bengal Lizard (E), Bengal Monitor (E), Common Monitor (E), Indian Monitor (E), Indian Small-grain Lizard (E), Penang Lizard (E), Talagoya Lizard (E), Varano de Bengala (S), Varan du Bengale (F) I R

Varanus bivittarus = Varanus salvator

Varanus bogerti: Bogert's Monitor (E), Varan de Bogen (F) II R

Varanus brevicauda: Short-tailed Pygmy Monitor (E), Varano colicorto (S), Varan à queue courte (F) II R

Varanus bulliwallah = Varanus mertensi

Varanus caudolineatus: Streak-tailed Monitor (E), Stripe-tailed Monitor $(E)$, Varano de cola rayada $(S)$, Varan à queue rayée $(F)$ II $\mathbf{R}$

Varanus cumingi $=$ Varanus salvator

Varanus doreanus: Blue-tailed Monitor (E), Varan à queue bleue (F) II $\mathbf{R}$

Varanus dumerilii: Dumeril's Monitor (E), Fish Lizard (E), Varano de Dumeril (S), Varan de Duméril (F) II R

Varanus eremius: Desert Pygmy Monitor (E), Spinifex Monitor (E), Varano pigmeo del desierto (S), Varan des cavernes (F), Varan des spinifex (F) II $\mathbf{R}$

Varanus exanthematicus: African Large-grain Lizard (E), African Savanna Monitor (E), Bosc's Monitor (E), Northern Savanna Monitor (E), Varan des savanes (F), Varan des steppes (F) II R

Varanus exanthematicus albigularis = Varanus albigularis

Varanus flavescens: Calcutta Oval-grain Lizard (E), Indian Ovalgrain Lizard (E), Ruddy Snub-nosed Monitor (E), Yellow Land Lizard (E), Yellow Monitor (E), Varano amarillo (S), Varan jaune (F) I R
Varanus giganteus: Perentie (E), Varano gigante (S), Perentie (F) Varan gigantesque (F) II $R$

Varanus gilleni: Pygmy Mulga Monitor (E), Varano de Gillen (S), Varan de Gillen (F), Varan pygmée de Mulga (F) II R

Varanus glaueri: Glauert's Monitor (E), Varano colilargo de las rocas (S), Varan de Glauert (F) II R

Varanus glebopalma: Long-tailed Rock Monitor (E), Varan des rochers à longue queue (F) II $R$

Varanus gouldii: Bungarra (E), Gould's Monitor (E), Sand Monitor (E), Varano de Gould (S), Varano de arena (S), Varan de Gould (F) II R

Varanus grayi $=$ Varanus olivaceus

Varanus griseus: Agra Lizard (E), Agra Monitor (E), Baghdad Small-grain Lizard (E), Desert Monitor (E), Grey Monitor (E), Varano desértico (S), Varan du désert (F), Varan gris (F) I R

Varanus indicus: Ambon Lizard (E), Flower Lizard (E), Mangrove Monitor (E), Pacific Monitor (E), Varano de manglar (S), Varan des Indes (F), Varan des mangroves (F) II R

Varanus indicus spinulosus = Varanus spinulosus

Varanus irrawadicus: Varan du Yunnan (F) II R

Varanus jobiensis: Peachthroat Monitor (E), Schmidt's Monitor (E), Sepik Monitor (E), Varano de Schmidt (S), Varan de Schmidt (F) II R

Varanus kalabeck = Varanus indicus

Varanus karlschmidti = Varanus jobiensis

Varanus kingorum: Crevice Monitor (E), King's Monitor (E), Varano de King (S), Varan de King (F), Varan des crevaces (F) II R

Varanus komodoensis: Komodo Dragon (E), Komodo Monitor (E), Ora (E), Varano de Komodo (S), Dragon des Komodos (F), Varan de Komodo (F) I R

Varanus kordensis $=$ Varanus bogerfi

Varanus meriensi: Mertens's Water Monitor (E), Varano de agua de Mertens (S), Varan aquatique de Mertens (F) II R

Varanus milchelli: Mitchell's Water Monitor (E), Varano de agua de Mitchell (S), Varan aquatique de Mitchell (F) II R

Varanus monitor = Varanus bengalensis

Varanus nebulosus = Varanus bengalensis

Varanus niloticus: African Small-grain Lizard (E), Nile Monitor (E). Varano del Nilo (S), Varan du Nil (F) II R

Varanus olivaceus: Gray's Monitor (E), Varan de Gray (F) II R

Varanus panopies: Varan de Kimberley (F) II R

Varanus pilbarensis: Pilbara Monitor (E), Varan de Pilbara (F) II R

Varanus prasinus: Emerald Monitor (E), Varano verde (S), Varan émeraude (F) II R

Varanus prasinus beccarii $=$ Varanus beccari

Varanus prasinus bogerti = Varanus bogerti

Varanus primordius: Brown Monitor (E), Ridge-tailed Monitor (E), Varano de cola crestada (S), Varan brun (F), Varan a queue épineuse (F) II R

Varanus rosenbergi: Black Sand Monitor (E), Rosenberg's Monitor (E), Sand Goanna (E), Varano de Rosenberg (S), Varan de Rosenberg (F), Varan des sables noir (F) II R

Varanus rudicollis: Harlequin Monitor (E), Rough-necked Monitor (E). Tree Lizard (E), Varano cuellirugoso (S), Varan cou rugueux (F) II $\mathbf{R}$

Varanus salvadorii: Crocodile Monitor (E), Papuan Monitor (E), Tree Crocodile (E), Varano de Papua (S), Varan crocodile (F), Varan de Papouasie (F) II R

Varanus salvator: Common Water Monitor (E), Malayan Monitor (E), No-mark Lizard (E), Plain Lizard (E), Rice Lizard (E), Ring Lizard (E), Two-banded Monitor (E), Water Monitor (E), Varano de dos bandas (S), Varan aquatique commun (F), Varan à deux bandes (F) II R

Varanus semiremex: Mangrove Monitor (E), Rusty Monitor (E), Varano pardo $(S)$, Varan des mangroves $(F)$, Varan rouille ( $F$ II R

Varanus similis: Varan du nord de l'Australie (F) II R

Varanus spenceri: Spencer's Monitor (E), Varano de Spencer (S), Varan de Spencer (F) II R

Varanus spinulosus: Solomon Islands Spiny Monitor (E), Varan épineux des lles Salomon (F) II R

Varanus storri: Dwarf Monitor (E), Varano enano (S), Varan de Stor (F), Varan nain (F) II R

Varanus telenestes: Rossell Island Tree Monitor (E), Varan arboricole de l'lle Rossell (F) II R 
Varanus teriae: Queensland Tree Monitor (E), Varan arboricole du Queensland (F) II R

Varanus timorensis: Spotted Tree Monitor (E), Timor Tree Monitor (E), Varano arborícola manchado (S), Varan arboricole tacheté (F), Varan de Timor (F) II R

Varanus timorensis scalaris $=$ Varanus similis

Varanus rimorensis similis = Varanus similis

Varanus togianus $=$ Varanus salvator

Varanus tristis: Arid Monitor (E), Black-headed Monitor (E), Freckled Monitor (E), Moumful Tree Monitor (E), Varano de cabeza negra (S), Varan de Schlegel (F), Varan à tête noire (F) II $\mathbf{R}$

Varanus varius: Common Tree Monitor (E), Lace Monitor (E), Varano arborícola común (S), Varan bigarré (F), Varan dentelle (F) II R

Varanus yemenensis: Yemen Monitor (E), Varan du Yemen (F) II R

Varecia variegata: Ruffed Lemur (E), Lemur de Gola (S), Lemur de Gorguera (S), Lemur de collar (S), Lémur vari (F), Maki vari (F) I M

Variable Coral Snake (E): Micrunus diastema III R

Variable Goshawk (E): Accipiter novaehollandiae II B

Variable Hawk (E): Buteo poecilochrous II B

Variable Mountain-gem (E): Lampomis castaneoventris II B

Variable Screech-Owl (E): Otus atricapillus II B

Varied Lorikeet (E): Psitteuteles versicolor II B

Variegated Dwarf Chameleon (E): Bradypodion pumilum II R

Vasa géant (F): Coracopsis vasa II B

Vasa Partot (E): Coracopsis vasa II B

Vasillum $=$ Rhizotrochus

Vaughanella concinna: II in

Vaughanella margaritata: II In

Vaughanella mulripalifera: II in

Vautour à tête blanche (F): Trigonoceps occipitalis II B

Vautour africain (F): Gyps africanus II B

Vautour charognard (F): Necrosyrtes monachus II B

Vautour chassefiente (F): Gyps coprotheres II B

Vautour chaugoun ( $\mathrm{F}$ ): Gyps bengalensis II $\mathrm{B}$

Vautour de l'Himalaya (F): Gyps himalayensis II B

Vautour de Rüppell (F): Gyps rueppellii II B

Vautour huppé (F): Trigonoceps occipitalis II B

Vautour indien (F): Gyps indicus II B

Vautour moine (F): Aegypius monachus II B

Vautour oricou (F): Torgos tracheliotus II B

Vautour page (F): Sarcoramphus papa III B

Vautour percnoptère (F): Neophron percnopierus II B

Vautour royal (F): Sarcoramphus papa III B/Sarcogyps calvus II B

Vautours (F): Aegypius monachus/Gyps spp./Necrosyrtes monachus/Neophron percnopterus/Sarcogyps calvus/Torgos tracheliotus/Trigonoceps occipitalis II B

Veiled Chameleon (E): Chamaeleo calyptratus II R

Velvet-browed Brilliant (E): Heliodoxa xanthogonys II B

Velvet-crowned Brilliant (E): Heliodoxa xamihogonys II B

Velvet-purple Coronet (E): Boissonneaua jardini II B

Venadito (S): Pudu puda I M

Venadito de los páramos (S): Pudu mephistophiles II M

Venado campero (S): Ozotoceros bezoarticus I M

Venado conejo (S): Pudu mephistophiles II M

Venado de campo (S): Ozotoceros bezoaricus I M

Venado de las Pampas (S): Ozotoceros bezoarticus I M

Ventre orange (F): Amandava subflava III B

Ver nota pie de página (S): Pseudoryx nghetinhensis I M

Veraguas Mango (E): Anthracothorax prevostii II B

Vermiculated Fishing-Owl (E): Scotopelia bouvieri II B

Vermiculated Screech-Owl (E): Otus vermiculatus II B

Vemal Hanging-Parrot (E): Loriculus vernalis II B

Verreaux's Eagle (E): Aquila verreauxii II B

Verreaux's Eagle-Owl (E): Bubo lacteus II B

Verreaux's Sifaka (E): Propithecus verreauxi I M

Verreaux's Turaco (E): Tauraco macrorhynchus II B

Verrillofungia $=$ Fungia

Versicolored Emerald (E): Amazilia versicolor II B

Vervain Hummingbird (E): Mellisuga minima II B

Vervet Monkey (E): Chlorocebus aethiops II M

Veuve à collier d'or ( $F$ ): Vidua orientalis III B

Veuve à dos d'or (F): Euplectes macrourus III $\mathrm{B}$

Veuve à queue large (F): Vidua inserjecta III B
Veuve dominicaine (F): Vidua macroura III B

Veuve du Togo (F): Vidua togoensis III B

Veuve nigérienne (F): Vidua interjecra III B

Veuve paradisier (F): Vidua orientalis III B

Veuve-noire (F): Euplectes ardens III B

Veuves (F): Vidua spp. III/NC B

Victoria Crowned-Pigeon (E): Goura victoria II B

Victoria Goura (E): Goura victoria II B

Victoria's Riflebird (E): Ptiloris victoriae II B

Vicugna (E): Vicugna vicugna $\mathbf{L I} \mathbf{M}$

Vicugna vicugna: Vicugna (E), Vicuña (E), Vicuña (S), Vigogne (F) III M

Vicuña (E/S): Vicugna vicugna VII M

Vidua amauropteryx $=$ Vidua chalybeata

Vidua camerunensis $=$ Vidua wilsoni

Vidua centralis $=$ Vidua chalybeata

Vidua chalybeata: Green Indigobird (E), Senegal Combassou (E). Village Indigobird (E), Tejedor bruñido verdoso (S), Combassou du Sénégal $(F)$ III B

Vidua incognita $=$ Vidua wilsoni

Vidua interjecta: Long-tailed Paradise-Whydah (E), Uelle ParadiseWhydah $(E)$, Veuve nigérienne $(F)$, Veuve à queue large $(F)$ III B

Vidua larvaticola: Baka Indigobird (E), Combassou de Baka (F) III B

Vidua lorenzi $=$ Vidua wilsoni

Vidua macroura: Pin-tailed Whydah (E), Viuda colicinta (S), Veuve dominicaine (F) III B

Vidua neumanni $=$ Vidua chalybeata

Vidua okavangoensis = Vidua chalybeato

Vidua orientalis: Northem Paradise-Whydah (E), Viuda del paraiso oriental (S), Veuve paradisier (F), Veuve à collier d'or (F) III B

Vidua orientalis interjecta $=$ Vidua interjecta

Vidua orientalis togoensis $=$ Vidua togoensis

Vidua paradisaea orientalis = Vidua orientalis

Vidua raricola: Jambandu Indigobird (E), Combassou de Jamdandu (F) III B

Vidua logoensis: Togo Paradise-Whydah (E), Veuve du Togo (F) III B

Vidua ultramarina $=$ Vidua chalybeata

Vidua wilsoni: Pale-winged Indigobird (E), Wilson's Indigobird (E). Tejedor bruñido de Wilson (S), Combassou de Wilson (F) III B

Vieillot's Black Weaver (E): Ploceus nigerrimus III B

Vieillot's Fireback (E): Lophura ignita III B

Vigogne (F): Vicugna vicugna III M

Vigors's Bustard (E): Eupodotis vigorsii II B

Village Indigobird (E): Vidua chalybeata III B

Village Weaver (E): Ploceus cucullarus III B

Villosa trabalis: Cumberland Bean (E), Cumberland Bean Pearly Mussel (E) I In

Vinaceous Amazon (E): Amazona vinacea I B

Vinaceous Dove (E): Streptopelia vinacea III B

Vinaceous Firefinch (E): Lagonosticta vinacea III B

Vinaceous Parrot (E): Amazona vinacea I B

Vinaceous Waxbill (E): Lagonosticta vinacea III B

Vinago obeng (S): Treron calva III B

Vinago waalia (S): Treron waalia III B

Vini australis: Blue-crowned Lorikeet (E), Lori de Samoa (S), Lori fringillaire (F) II B

Vini kuhlii: Kuhl's Lorikeet (E), Scarlet-breasted Lorikeet (E), Lori de Rimitara (S), Lori de Kuhl (F) II B

Vini peruviana: Blue Lorikeet (E), Tahitian Lorikeet (E), Lori monjita (S), Lori nonnette (F) II B

Vini stepheni: Henderson Lorikeet (E), Stephen's Lorikeet (E), Lori de Stephen (S), Lori de Stephen (F) II B

Vini ultramarina: Ultramarine Lorikeet (E), Ultramarine Lory (E). Lori ultramar (S), Lori ultramarin (F) I B

Vinous-breasted Sparrowhawk (E): Accipiter rhodogaster II B

Violet Plantain-eater (E): Musophaga violacea III B

Violet Sabrewing (E): Campylopterus hemileucurus II B

Violet Turaco (E): Musophaga violacea III B

Violet-bellied Hummingbird (E): Damophila julie II B

Violet-capped Hummingbird (E): Goldmania violiceps II B

Violet-capped Woodnymph (E): Thalurania glaucopis II B

Violet-chested Hummingbird (E): Stemoclyta cyanopectus II B

Violet-crested Turaco (E): Musophaga porphyreolopha II B 
Violet-crowned Hummingbird (E): Amazilia violiceps II B

Violet-ears (E): Colibri spp. II B

Violet-fronted Brilliant (E): Heliodoxa leadbeateri II B

Violet-headed Hummingbird (E): Klais guimeri II B

Violet-necked Lory (E): Eos squamata II B

Violet-tailed Sylph (E): Aglaiocercus coelestis II B

Violet-throated Metaltail (E): Metallura baroni II B

Violet-throated Starfrontlet (E): Coeligena violifer II B

Vipera russelii = Vipera russellii

Vipera russellii: Russell's Viper (E), Dabois (F), Vipere de Russell (F) III R

Vipera ursinii: Meadow Viper (E), Orsini's Viper (E), Vipère d'Orsini (F), Vipère des steppes $(F)$ INC R

Vipera wagneri: Wagner's Viper (E), Vipère de Wagner (F) II R

Vipère de Russell (F): Vipera russellii III R

Vipère de Wagner (F): Vipera wagneri II R

Vipère des steppes (F): Vipera ursinii INC R

Vipère d'Orsini (F): Vipera ursinii $\mathbf{L}$ NC R

Vipers (E): Viperidae spp. I/UIII R

Virgin Islands Tree Boa (E): Epicrates monensis I R

Viridian Metaltail (E): Metallura williami II B

Visayan Hornbill (E): Penelopides panini II B

Vison sibérien (F): Mustela sibirica III M

Visorbearers (E): Augasies spp. II B

Vitelline Masked-Weaver (E): Ploceus vitellinus III B

Viuda colicinta (S): Vidua macroura III B

Viuda del paraiso oriental (S): Vidua orientalis III B

Viverra civetta $=$ Civettictis civetta

Viverra civettina: Malabar Large-spotted Civet (E) III M

Viverra megaspila civenina $=$ Viverra civerina

Viverro zibetha: Large Indian Civet (E), Gran civeta de la India (S) III $\mathrm{M}$

Viverricula indica: Small Indian Civet (E), Pequeña civeta de la India (S) III M

Viviparous toads (E): Altiphrynoides $\operatorname{spp} /$ Nimbaphnnoides $\operatorname{spp} /$ Necrophrnoides spp/Spinophnnoides spp. I A

Vogelkop Tree-kangaroo (E): Dendrolagus ursinus II M

Volcano Hummingbird (E): Selasphorus flammula II B

Volcano Rabbit (E): Romerolagus diazi I M

Vorabé (F): Euplectes afer III B

Vu Quang Ox (E): Pseudoryx nghetinhensis I M

Vulpes bengalensis: Bengal Fox (E), Zorro de Bengala (S), Renard du Bengale (F) III M

Vulpes cana: Afghan Fox (E), Blanford's Fox (E), Corsac (E), Dog Fox (E), Hoary Fox (E), Steppe Fox (E), Zorro de Blanford (S), Renard de Blanford (F) II M

Vulpes vulpes griffithi: III M

Vulpes vulpes leucopus = Vulpes vulpes pusilla

Vulpes vulpes montana: III M

Vulpes vulpes pusilla: III M

Vulpes zerda: Fennec Fox (E), Fennec (S), Fennec (F) II M

Vultur gryphus: Andean Condor (E), Cóndor (S), Cóndor andino (S), Condor des Andes (F) I B

Vultures (E): Aegypius monachus/Cathartes spp/Coragyps atratus/Gypohierax angolensis/Gyps spp/Necrosyrtes monachus/Neophron percnopterus/Sarcogyps calvus/Sarcoramphus paparTorgos iracheliotus/Trigonoceps occipitalis IU/IJNC B

Vulturine Fish-Eagle (E): Gypohierax angolensis II B

Vulturine Parrot (E): Gypopsifta vulturina II B/Psinrichas fulgidus II B

Wabash Riffleshell (E): Epioblasma sampsonii I In

Wagner's Viper (E): Vipera wagneri II R

Wahlberg's Eagle (E): Aquila wahlbergi II B

Wahnes's Parotia (E): Parotia wahnesi II B

Waldrapp (E): Geronticus eremita I B

Wallabies (E): Macropodidae spp. I/NC M

Wallaby à queue comée $(\mathrm{F})$ : Onychogalea lunata I $\mathrm{M}$

Wallaby-lière à bandes (F): Lagostrophus fasciatus I $M$

Wallaby-lière de l'ouest (F): Lagorchestes hirsutus I M

Wallaby-lièvre rayé (F): Lagosirophus fasciarus I M

Wallaby-lièvre roux (F): Lagorchestes hirsutus I $M$

Wallace's Hanging-Parrot (E): Loriculus flosculus II B

Wallace's Hawk-Eagle (E): Spizaetus nanus II B

Wallace's Scops-Owl (E); Otus silvicola II B

Wallace's Standardwing (E): Semioptera wallacii II B
Wallich's Pheasant (E): Catreus wallichit I B

Walrus (E): Odobenus rosmarus III M

Wanderoo (E): Macaca silenus I M

Wapitis (E): Cervus elaphus IU/NC M

Warblers (E): Muscicapidae spp./Panlidae spp. IIUNC B

Warten's Girdled Lizard (E): Cordylus warreni II R

Warren's Spiny-tailed Lizard (E): Cordylus warreni II R

Warty Chameleon (E): Chamaeleo verrucosus II R

Water Boa (E): Eunecies murinus II R

Water Box Turtle (E): Terrapene coahuila I R

Water Chevrotain (E): Hyemoschus aquaticus III M

Water Monitor (E): Varanus salvator II $\mathbf{R}$

Water Python (E): Morelia mackloti II R

Water-rats (E): Muridae spp. INC M

Watling Island Iguana (E): Cyclura rileyi I $\mathbf{R}$

Wattle-billed Bird-of-paradise (E): Loboparadisea sericea II B

Wattled Crane (E): Grus caruncularus II B

Wattled Curassow (E): Crax globulosa III B

Wavy Chameleon (E): Chamaeleo deremensis II R

Waxbills (E): Estrildidae spp. IIINC B

Weasel lemurs (E): Lepilemur spp. I M

Weasels (E): Mustela spp $/$ Lyncodon patagonicus/Ictonyx libycalPoecilogale albinucha III/NC M

Weavers (E): Ploceidae spp. IIINC B

Wedge-billed Hummingbird (E): Augastes geoffroyi II B

Wedge-tailed Eagle (E): Aquila audax II B

Wedge-tailed Hillstar (E): Oreotrochilus adela II B

Wedge-tailed Sabrewing (E): Campylopterus curvipennis II B

Weeper Capuchin (E): Cebus olivaceus II M

Wellsophyllia = Trachyphyllia

Wemer's Chameleon (E): Chamaeleo wermeri II R

West African Black Forest Turtle (E): Pelusios niger III R

West African Crowned Crane (E): Balearica pavonina II B

West African Dwarf Crocodile (E): Osteolaemus tetraspis I R

West African Manatee (E): Trichechus senegalensis II M

West African Mud Turtle (E): Pelusios castaneus III R

West African Seedeater (E): Serinus canicapillus III B

West Indian Iguana (E): Iguana delicatissima II R

West Indian Manatee (E): Trichechus manatus I M

West Indian Monk Seal (E): Monachus tropicalis I M

West Indian Seal (E): Monachus tropicalis I M

West Indian Tree-Duck (E): Dendrocygna arborea II B

West Indian Whistling-Duck (E): Dendrocygna arborea II B

West Peruvian Screech-Owl (E): Otus roboratus II B

Westermann's Snake (E): Elachisrodon westermanni II R

Westem Barred Bandicoot (E): Perameles bougainville I M

Western Black-and-white Colobus (E): Colobus polykomos II M

Western Black-capped Lory ( $\overline{\mathbf{E}}$ ): Lorius lory II B

Western Bluebill (E): Spermophaga haematina III B

Western Box Turtle (E): Terrapene ornata II R

Western Bristlebird (E): Dasyornis longirostris I B

Western Bronze-naped Pigeon (E): Columba iriditorques III B

Western Corella (E): Cacatua pastinator II B

Westem Crowned-Pigeon (E): Goura cristata II B

Westem Fan-shell (E): Cyprogenia aberi II In

Western Fanshell Mussel (E): Cyprogenia aberti II In

Western Grey Plantain-eater (E): Crinifer piscator III B

Western Hare-wallaby (E): Lagorchestes hirsutus I M

Westem Hog-nosed Viper (E): Bothrops ophryomegas III R

Westem Homed-Pheasant (E): Tragopan melanocephalus I B

Westem Marsh-Harrier (E): Circus aeruginosus II B

Western Needle-clawed Bushbaby (E): Euoticus elegantulus II M

Western Needle-clawed Galago (E): Euoticus elegantulus II M

Western Nimba Toad (E): Nimbaphrynoides occidentalis I A

Westem Parotia (E): Parotia sefilata II B

Western Red Colobus (E): Procolobus badius II M

Westem Red-footed Falcon (E): Falco vespertinus II B

Westem Rosella (E): Platycercus icterotis II B

Westem Rufous Bristlebird (E): Dasyomis broadbenti litoralis I B

Western Screech-Owl (E): Otus kennicortii II B

Western Scrubird (E): Atrichomis clamosus I B

Western Short-necked Turtle (E): Pseudemydura umbrina I R

Westem Swamp Turtle (E): Pseudemydura umbrina I R

Western Tarsier (E): Tarsius bancanus II M

Western Tragopan (E): Tragopan melanocephalus I B

Western Turtle-Dove (E): Streptopelia turiur III B 
Whale-headed Stork (E): Balaeniceps rex II B Whales (E): CETACEA spp. VI M Whiptails (E): Cnemidophorus spp. II R Whiskered Lorikeet (E): Oreopsittacus arfaki II B Whiskered Pitta (E): Pitta kochi I B

Whiskered Screech-Owl (E): Otus trichopsis II B Whistling Kite (E): Haliastur sphenurus II B Whistling-Ducks (E): Dendrocygna spp. 11/11/NC B Whitaker's Boa (E): Eryx whitakeri II R

White Catspaw (E): Epioblasma sulcata perobliqua I In White Catspaw Mussel (E): Epioblasma sulcata perobliqua I In White Cay Ground Iguana (E): Cyclura rileyi I R White Cockatoo (E): Cacatua alba II B

White Eared-Pheasant (E): Crossoptilon crossoptilon I B

White Flag Dolphin (E): Lipotes vexillifer I $M$

White Flying-fox (E): Pteropus anetianus II M White Goshawk (E): Accipiler novaehollandiae II B White Hawk (E): Leucoptemis albicollis II B White Oryx (E): Oryx leucoryx I M

White Spoonbill (E): Platalea leucorodia II B White Starling (E): Leucopsar rothschildi I B White Sturgeon (E): Acipenser transmontanus II F White Wartyback (E): Plethobasus cicatricosus I In White Warty-back Pearly Mussel (E): Plethobasus cicairicosus I In White Whale (E): Delphinapterus leucas II M White-backed Vulture (E): Gyps africanus II B White-beaked Dolphin (E): Lagenorhynchus albirostris II M White-bearded Hermit (E): Phaethomis hispidus II B White-bellied Bustard (E): Eupodotis senegalensis II B White-bellied Caique (E): Pionites leucogaster II B White-bellied Dolphin (E): Cephalorhynchus eusropia II M White-bellied Emerald (E): Amazilia candida II B White-bellied Fish-Eagle (E): Haliaeetus leucogaster II B White-bellied Goshawk (E): Accipiter haplochrous II B White-bellied Hummingbird (E): Amazilia chionogaster II B White-bellied Korhaan (E): Eupodotis senegalensis II B White-bellied Mountain-gem (E): Lampornis hemileucus II B White-bellied Pangolin (E): Manis tricuspis II M White-bellied Parrot (E): Pionites leucogaster II B White-bellied Sea-Eagle (E): Haliaeetus leucogaster II B White-bellied Spider Monkey (E): Ateles belzebuth II M White-bellied Woodstar (E): Acestrura mulsant II B White-billed Buffalo Weaver (E): Bubalomis albirostris III B White-billed Sicklebill (E): Epimachus bruijnii II B White-breasted Guineafowl (E): Agelastes meleagrides III B White-breasted Hawk (E): Accipiter chionogaster II B White-breasted Negrofinch (E): Nigrita fusconota III B White-breasted Parakeet (E): Pyrrhura albipectus II B White-breasted Side-necked Turtle (E): Pelusios adansonii III R White-breasted Silvereye (E): Zosterops albogularis I B White-breasted White-eye (E): Zosterops albogularis I B White-browed Hawk (E): Leucoptemis kuhli II B White-browed Hermit (E); Phaethomis stuarti II B White-browed Owl (E): Ninox superciliaris II B White-capped Parrot (E): Pionus senilis II B White-cheeked Gibbon (E): Hylobates leucogenys I M White-cheeked Mangabey (E): Lophocebus albigena II M White-cheeked Olive Weaver (E): Nesocharis capistrata III B White-cheeked Turaco (E): Tauraco leucotis II B White-chested Emerald (E): Amazilia chionopectus II B White-chested White-eye (E): Zosterops albogularis I B White-chinned Owl (E): Pulsatrix koeniswaldiana II B White-chinned Sapphire (E): Hylocharis cyanus II B White-collared Kite (E): Leptodon forbesi II B White-collared Mangabey (E): Cercocebus torquatus II M White-crested Coquette (E): Lophomis adorabilis II B White-crested Turaco (E): Tauraco leucolophus II B White-crowned Hombill (E): Aceros comatus II B White-crowned Parrot (E): Pionus senilis II B White-eared Conure (E): Pyrrhura leucotis II B White-eared Hummingbird (E): Hylocharis leucotis II B White-eared Marmoset (E): Callithrix aurita I M White-eared Parakeet (E): Pyrrhura leucotis II B White-eyed Buzzard (E): Butastur ieesa II B White-eyed Conure (E): Aratinga leucophthalmus II B White-eyed Kestrel (E): Falco rupicoloides II B
White-eyed Parakeet (E): Aratinga leucophthalmus II B White-eyed Pochard (E): Aythya nyroca III B

White-eyed River-Martin (E): Pseudochelidon sirintarae I B White-eyes (E): Zosterops spp. I/NC B

White-faced Amazon (E): Amazona kawalli II B

White-faced Capuchin (E): Cebus capucinus II M

White-faced Langur (E): Presbytis frontata II M

White-faced Owl (E): Sceloglawx albifacies II B

White-faced Saki (E): Pithecia pithecia II M

White-faced Scops-Owl (E): Otus leucotis II B

White-faced Tree-Duck (E): Dendrocygna viduata III B

White-faced Whistling-Duck (E): Dendrocygna viduata 11 B

Whitefin Dolphin (E): Lipotes vexillifer I M

White-flanked Porpoise (E): Phocoenoides dalli II M

White-footed Sportive Lemur (E): Lepilemur leucopus I M

White-footed Tamarin (E): Saguinus leucopus I M

White-fronted Amazon (E): Amazona albifrons II B

White-fronted Capuchin (E): Cebus albifrons II M

White-fronted Falconet (E): Microhierax latifrons II B

White-fronted Grosbeak (E): Amblyaspiza albifrons III B

White-fronted Leaf Monkey (E): Presbytis frontaia II M

White-fronted Marmoset (E): Callithrix geoffroyi II M

White-fronted Parrot (E): Amazona albifrons II B

White-fronted Scops-Owl (E): Otus sagittalus II B

White-handed Gibbon (E): Hylobates lar I M

White-headed Dolphin (E): Cephalorhynchus hectori II M

White-headed Duck (E): Oxyura leucocephala II B

White-headed Eagle (E): Haliaeetus leucocephalus I B

White-headed Vulture (E): Trigonoceps occipitalis II B

White-lined Bat (E): Platyrrhinus lineatus III M

White-lined Chameleon (E): Chamaeleo antimena II R

White-lipped Peccary (E): Tayassu pecari II M

White-lipped Python (E): Morelia albertisii II R

White-lipped Tamarin (E): Saguinus labiatus II M

White-naped Crane (E): Grus vipio I B

White-naped Lory (E): Lorius albidinuchus II B

White-naped Weaver (E): Ploceus albinucha III B

White-necked Conure (E): Pyrrhura albipectus II B

White-necked Crane (E): Grus vipio I B

White-necked Hawk (E): Leucoptemis lacernulata II B

White-necked Jacobin (E): Florisuga mellivora II B

White-necked Parakeet (E): Pyrrhura albipectus II B

White-necked Picathartes (E): Picathartes gymnocephalus I B

White-necked Rockfowl (E): Picathartes gymnocephalus I B

White-nosed Bearded Saki (E): Chiropotes albinasus I M

White-nosed Guenon (E): Cercopithecus nictitans II M

White-nosed Saki (E): Chiropotes albinasus I M

White-quilled Bustard (E): Eupodotis afraoides II B

White-rumped Falcon (E): Polihierax insignis II B

White-rumped Falconet (E): Polihierax insignis II B

White-rumped Hawk (E): Buteo leucorrhous II B

White-rumped Kestrel (E): Falco dickinsoni II B

White-rumped Seedeater (E): Serinus leucopygius III B

White-rumped Vulture (E): Gyps bengalensis II B

White-sided Hillstar (E): Oreotrochilus leucopleurus II B

White-tailed Black-Cockatoo (E): Calyptorhynchus baudinii II B

White-tailed Eagle (E): Haligeetus albicilla I B

White-railed Emerald (E): Elvira chionura II B

White-tailed Goldenthroat (E): Polytmus guainumbi II B

White-tailed Hawk (E): Buteo albicaudatus II B

White-tailed Hillstar (E): Urochroa bougueri II B

White-tailed Hummingbird (E): Eupherusa poliocerca II B

White-tailed Kite (E): Elanus leucurus II B

White-tailed Rabbit-eared Bandicoot (E): Macrotis leucura I M

White-tailed Sabrewing (E): Campylopterus ensipennis II B

White-tailed Starfrontlet (E): Coeligena phalerata II B

White-throated Capuchin (E): Cebus capucinus II M

White-throated Caracara (E): Phalcoboenus albogularis II B

White-throated Guenon (E): Cercopithecus erythrogaster II M/Cercopithecus sclateri II M

White-throated Hawk (E): Buteo albigula II B

White-throated Hummingbird (E): Leucochloris albicollis II B White-throated Monitor (E): Varanus albigularis II R

White-throated Monkey (E): Cercopithecus erythrogaster II M

White-throated Mountain-gem (E): Lampornis castaneoventris II B

White-throated Screech-Owl (E): Olus albogularis II B 
White-tipped Sicklebill (E): Eutoxeres aquila II B Whitetips (E): Urosticie spp. II B

White-tufted Sunbeam (E): Aglaeactis castelnaudii II B White-vented Plumeleteer (E); Chalybura buffonii II B White-vented Violet-ear (E): Colibri serrirostris II B

White-whiskered Hermit (E): Phaethomis yaruqui II B

White-winged Cotinga (E): Xipholena atropurpurea I B

White-winged Duck (E): Cairina scutulata I B

White-winged Guan (E): Penelope albipennis I B

White-winged Wood Duck (E): Cairina scutulata I B

Whooping Crane (E): Grus americana I B

Whydahs (E): Vidua spp/Euplectes spp. III/NC B

Widow Titi (E): Callicebus torquatus II M

Widowbirds (E): Euplectes spp. III/NC B

Wigeons (E): Anas penelope/Anas americana/Anas sibilatrix IIINC B

Wild Asiatic Buffalo (E): Bubalus amee III M

Wild Cat (E): Felis silvestris II M

Wild Water Buffalo (E): Bubalus arnee III M

Wild Yak (E): Bos mutus I M

Wildcat (E): Felis silvestris II M

Wilhelmina's Lorikeet (E): Charmosyna wilhelminae II B

Wilson's Bird-of-paradise (E): Cicinnurus respublica II B

Wilson's Indigobird (E): Vidua wilsoni III B

Wine-throated Hummingbird (E): Arthis ellioti II B

Winking Owl (E): Ninox connivens II B

Wire-crested Thomtail (E): Popelairia popelaini II B

Wolf (E): Canis lupus III M

Wolfs Monkey (E): Cercopithecus wolfi II M

Wolves (E): Canis lupus/Canis rufus/Chrysocyon brachyurus/Pseudalopex culpaeus I/LNC M

Woma (E): Aspidites ramsayi II R

Woma (F): Aspidites melonocephalus II R/Aspidites ramsayi II $\mathrm{R}$

Wombat à nez poilu de Queensland (F): Lasiorhinus kreffiii I M

Wombats (E): Lasiorhinus $\operatorname{spp}$ Nombatus ursinus $\mathrm{V} / \mathrm{NC} \mathrm{M}$

Wood Bison (E): Bison bison athabascae II M

Wood ducks (E): Cairina scutulata/Dendrocygna arborea/Aix sponsa/Chenonetsa jubata V/I/NC B

Wood rails (E): Gallirallus australis/Aramides spp. IU/NC B

Wood Snake (E): Tropidophis canus II RTropidophis melanurus II $\mathrm{R}$

Wood Tortoise (E): Geochelone carbonaria II R

Wood Turtle (E): Clemmys insculpta II R

Wood-Doves (E): Turtur spp. III/NC B

Woodford's Owl (E): Strix woodfordii II B

Woodnymphs (E): Thalurania spp. II B

Wood-Owls (E): Strix spp. II B

Wood-Partidges (E): Caloperdix oculea/Melanoperdix nigra/Rhizothera longirostris/Rollulus rouloulDendrorbyx spp III/NC B

Woodpeckers (E): Picidae spp. I/NC B

Wood-Pigeons (E): Columba spp. IIU/NC B

Woodstars (E): Acestrura spp/Calliphlox spp/Chaetocercus jourdaniiVEulidia yarrelliiMicrostilbon burmeisteriMyrmia micrura/Myrtis fanny/Philodice spp. II B

Woolly Indris (E): Avahi laniger I M

Woolly Lernur (E): Avahi laniger I M

Woolly monkeys (E): Lagothrix spp. III M

Woolly Spider Monkey (E): Brachyteles arachnoides I M

Woolly Tapir (E): Tapirus pinchaque I M

Woylie (E): Bettongia penicillata I M

Wreathed Hombill (E): Aceros undulatus II B

Wright's Dwarf Boa (E): Tropidophis wrighti II R

Wrinkled Hombill (E): Aceros corrugarus II B

Writhed Hombill (E): Aceros leucocephalus II B

Writhed-billed Hombill (E): Aceros waldeni II B

Wurrung (E): Onychogalea lunara I M

Wurrup (E): Lagorchestes hirsutus I M

Xanthopsar flavus = Agelaius flavus

Xantus's Hummingbird (E): Hylocharis xantusii II B

Xenoboa cropanii: Cropan's Bca (E), Boa costera de Brasil (S), Boa de Cropan (F) II R

Xenochrophis piscator. Chequered Keelback (E), Common Scaled Water Snake (E), Fishing Snake (E), Couleuvre à damier (F). Serpent aquatique caréné à damier (F) III R
Xenoglaux loweryi: Long-whiskered Owlet (E), Búho pequeño de bigotes alargados (S), Chevêchette nimbée (F) II B

Xenosaurs (E): Xenosauridae spp. II/NC R

Xerobates agassizii $=$ Gopherus agassizii

Xerobates berlandier $=$ Gopherus berlandier

Xeromys myoides: False Swamp Rat (E), False Water-rat (E), Rata bastarda de agua (S), Faux rat d'eau (F) I M

Xipholena atropurpurea: White-winged Cotinga (E), Cotinga aliblanca $(S)$, Cotinga porphyrion $(F)$, Cotinga à ailes blanches (F) I B

Xishasiderastrea $=$ Coeloseris

Yacaré (S): Caiman yacare II R

Yacare Caiman (E): Caiman yacare II R

Yacaré de hocico ancho (S): Caiman latirostris II R

Yacaré overo (S): Caiman latirostris II R

Yack sauvage (F): Bos murus I M

Yaco (S): Psitracus erithacus II B

Yacutinga cariazul (S): Pipile pipile I B

Yacutingá frentinegra (S): Pipile jacutinga I B

Yagouaso cariblanco (S): Dendrocygna viduata III B

Yaguar (S): Panthera onca I M

Yaguarete (S): Panthera onca I M

Yaguarundí (S): Herpailurus yaguarondi VII M

Yaguaso colorado (S): Dendrocygna bicolor III B

Yak salvaje (S): Bos mutus I M

Yallara (E): Macrotis leucura I M

Yangtze Sturgeon (E): Acipenser dabryanus II F

Yellow Anaconda (E): Eunectes notaeus II R

Yellow Baboon (E): Papio hamadryas II M

Yellow Cardinal (E): Gubematrix cristata II B

Yellow Land Lizard (E): Varanus flavescens I R

Yellow Monitor (E): Varcnus flavescens I R

Yellow Pencil Coral (E): Madracis mirabilis II In

Yellow Riffleshell (E): Epioblasma florentina I in

Yellow Rosella (E): Platycercus flaveolus II B

Yellow Snake (E): Epicrates subflavus I R

Yellow Tortoise (E): Indotestudo elongata II R

Yellow Tree Boa (E): Epicrales inornarus I R

Yellow-and-green Lorikeet (E): Trichoglossus flavoviridis II B

Yellow-backed Duiker (E): Cephalophus silviculior II M

Yellow-backed Lory (E): Lorius garrulus II B

Yellow-backed Weaver (E): Ploceus melanocephalus III B

Yellow-backed Whydah (E): Euplecies macrourus III B

Yellow-banded Poison Frog (E): Dendrobates leucomelas II A

Yellow-bellied Green-Pigeon (E): Treron waalia III B

Yellow-bellied Poison Frog (E): Minyobates fulguritus II A

Yellow-bellied Weasel (E): Musteia kathiah III M

Yellow-bibbed Lory (E): Lorius chlorocercus II B

Yellow-billed Amazon (E): Amazona collaria II B

Yellow-billed Cardinal (E): Paroaria capiraia II B

Yellow-billed Kite (E): Milvus migrans II B

Yellow-billed Lorikeet (E): Neopsittacus musschenbroekii II B

Yellow-billed Parrot (E): Amazona collaria II B

Yellow-billed Tragopan (E): Tragopan caboti I B

Yellow-billed Turaco (E): Tauraco macrorhynchus II B

Yellow-blossom Pearly Mussel (E): Epioblasma florentina I In

Yellow-bordered Tortoise (E): Gopherus flavomarginatus I R

Yellow-breasted Bird-of-paradise (E): Loboparadisea sericea II B

Yellow-breasted Racquet-tail (E): Prioniturus favicans II B

Yellow-capped Pygmy-Parrot (E): Micropsitla keiensis II B

Yellow-capped Weaver (E): Ploceus preussi III B

Yellow-chevroned Parakeet (E): Brologeris chiriri II B

Yellow-collared Lovebird (E): Agapomis personatus II B

Yellow-collared Macaw (E): Ara auricollis II B

Yellow-crested Cockatoo (E): Cacatua sulphurea II B

Yellow-crowned Amazon (E): Amazona ochrocephala II B

Yellow-crowned Bishop (E): Euplectes afer III B

Yellow-crowned Parakeet (E): Cyanoramphus auriceps I/I B

Yellow-crowned Parrot (E): Amazona ochrocephala II B

Yellow-eared Conure (E): Ognorhynchus icterotis I B

Yellow-eared Parrot (E): Ognorhynchus icterotis I B

Yellow-faced Amazon (E): Amazona xanthops II B

Yellow-faced Parrot (E): Amazona xanthops II B

Yellow-faced Parrotlet (E): Forpus xanthops II B

Yellow-faced Siskin (E); Carduelis yarrellii II B

Yellow-footed Tortoise (E): Geochelone denticulata II R 
Yellow-fronted Amazon (E): Amazona ochrocephala II B Yellow-fronted Canary (E): Serinus mozambicus III B Yellow-fronted Kakariki (E): Cyanoramphus auriceps III B Yellow-fronted Parakeet (E): Cyanoramphus auriceps $\mathbf{1}$ II B Yellow-fronted Parrot (E): Poicephalus flavifrons II B Yellow-green Chameleon (E): Chamaeleo malthe II R Yellow-headed Amazon (E): Amazona oratrix II B Yellow-headed Caracara (E): Milvago chimachima II B Yellow-headed Parakeet (E): Aratinga jandaya II B Yellow-headed Parrot (E): Amazona oratrix II B Yellow-headed Rockfowl (E): Picarhartes gymnocephalus I B Yellow-headed Sideneck (E): Podocnemis unifilis II R Yellow-headed Tortoise (E): Indotestudo elongata II R Yellowish-breasted Racquet-tail (E): Prionimusus flavicans II B Yellow-knobbed Curassow (E): Crax daubentoni III B Yellow-legged Owlet (E): Glaucidium tephronotum II B Yellow-lored Amazon (E): Amazona xantholora II B Yellow-lored Parrot (E): Amazona xantholora II B Yellow-mantled Weaver (E): Ploceus tricolor III B Yellow-mantled Whydah (E): Euplectes macrourus III B Yellow-mantled Widowbird (E): Euplectes macrourus III B Yellow-naped Amazon (E): Amazona auropalliata II B Yellow-naped Parrot (E): Amazona auropalliata II B Yellow-shouldered Amazon (E): Amazona barbadensis I B Yellow-shouldered Parrot (E): Amazona barbadensis I B Yellow-spotted River Turtle (E): Podocnemis unifilis II R Yellow-spotted Sideneck Turtle (E): Podocnemis unifilis II R Yellow-streaked Lory (E): Chalcopsitta sintillata II B Yellow-striped Poison Frog (E): Dendrobates truncatus II A Yellow-tailed Black-Cockatoo (E): Calyprorhynchus funereus II B Yellow-tailed Woolly Monkey (E): Lagothrix flavicauda I M Yellow-throated Caracara (E): Daptrius ater II B Yellow-throated Day Gecko (E): Phelsuma flavigularis II R Yellow-throated Hanging-Parrot (E): Loriculus pusillus II B Yellow-throated Marten (E): Martes flavigula III M

Yellow-winged Pytilia (E): Pyrilia hypogrammica III B Yemen Monitor (E): Varanus yemenensis II R

Youyou (F): Poicephalus senegalus II B

Yucatan Rust Rump Tarantula (E): Brachypelma epicureanum II In Yungas Pygmy-Owl (E): Glaucidium bolivianum II B Yunnan Snub-nosed Monkey (E): Pygathrix bieti I M Zacatuche (S): Romerolagus diazi I M

Zafiro barbiazul (S): Chloresies notatus II B

Zafiro cabeciazul (S): Hylocharis grayi II B

Zafiro cabecimorado ( $($ ): Hylocharis cyanus 11 B

Zafiro colidorado (S): Chrysuronia oenone II B

Zafiro gargantirnufo (S): Hylocharis sapphirina II B

Zafiro gorjiazul (S): Hylocharis eliciae II B

Zaglossus spp.: II M

Zaglossus bruijni: Long-beaked Echidna (E), Long-nosed Echidna

(E), Long-תosed Spiny Anteater (E), New Guinea Long-nosed Echidna (E), Echidnos narilargos (S), Equidna de Nueva Guinea (S), Echidné de Nouvelle-Guinée (F), Echidné à bec coure (F) II M

Zaire Clawless Otter (E): Aonyx congicus $\mathbf{\text { /II M }}$

Zaire Toad (E): Bufo superciliaris I A

Zamarrito canoso (S): Haplophaedia lugens II B

Zamarrito colilargo (S): Eriocnemis luciani II B

Zamarrito gorjiturquesa (S): Eriocnemis godini II B

Zamarrito luciente (S): Eriocnemis vestitus II B

Zamarrito muslinegro (S): Eriocnemis derbyi II B

Zamarrito pechiblanco (S): Eriocnemis alinae II B

Zamarito pechidorado (S): Eriocnemis mosquera II B

Zamarrito pechinegro (S): Eriocnemis nigrivestis II B

Zamarito verdoso (S): Haplophaedia aureliae II B

Zambullidor de Atitlán (S): Podilymbus gigas I B

Zampullin del Atitlán (S): Podilymbus gigas I B

Zanzibar Bushbaby (E): Galagoides zanzibaricus II M

Zanzibar Day Gecko (E): Phelsuma dubia II R

Zanzibar Galago (E): Galagoides zanzibaricus II M

Zanzibar Red Colobus (E): Procolobus pennantii kirkii $1 \mathrm{M}$

Zaparo's Poison Frog (E): Epipedobates zaparo II A

Zaraguate (S): Alouana palliata $1 \mathrm{M}$

Zarapito boreal (S): Numenius borealis I B

Zarapito esquimal (S): Numenius borealis I B

Zarapito fino (S): Numenius tenuirostris I B
Zarapito polar (S): Numenius borealis I B Zebra Antelope (E): Cephalophus zebra II M Zebra Duiker (E): Cephalophus zebra II M Zebra Waxbill (E): Amandava subflava III B Zebras (E): Equus grevyi/Equus zebra/Equus burchellii II/NC M Zèbre de Grévy (F): Equus grevyi I M

Zèbre de Hartmans (F): Equus zebra hartmannae II M

Zebre de montagne de Hartmann (F): Equus zebra hartmannae II M

Zèbre de montagne du Cap (F): Equus zebra zebra I M

Zetek's Golden Frog (E): Atelopus varius zeteki I A

Zimbabwe Girdled Lizard (E): Cordylus rhodesianus II R

Zimbador centroamericano (S): Atthis ellioti II B

Zimbador mexicano (S): Atthis heloisa II B

Ziphio de Cuvier (S): Ziphius cavirostris II M

Ziphius (F): Ziphius cavirostris II M

Ziphius cavirostris: Cuvier's Beaked Whale (E), Goose-beaked

Whale (E), Ballena de Cuvier (S), Ziphio de Cuvier (S), Ziphius (F) II M

Zone-tailed Hawk (E): Bureo albonotalus II B

Zonures (E): Cordylus spp. II R

Zoopilus echinatus: II in

Zopilote rey (S): Sarcoramphus papa III B

Zomillo (S): Conepatus humboldtii II M

Zorrino de la Patagonia (S): Conepatus humboldtii II M

Zorro andino (S): Pseudalopex culpaeus II M

Zorro cangrejero (S): Cerdocyon : hous II M

Zorto chico (S): Pseudalopex griseus II M

Zorro colorado (S): Pseudalopex culpaeus II M

Zorro de Bengala (S): Vulpes bengalensis III M

Zorro de Blanford (S): Vulpes cana II M

Zorro de la Isla Chiloe (S): Pseudalopex griseus II M

Zorro gris (S): Pseudalopex griseus II M

Zorro vinagre (S): Speothos venaticus I M

Zorro volador argénteo (S): Pteropus argentatus II M Zorro volador de Aldabra (S): Pteropus aldabrensis II M Zorro volador de Amboina (S): Pteropus chrysoproctus II M Zorro volador de Andaman (S): Pteropus melanotus II M Zorro volador de anteojos (S): Pteropus conspicillatus II M Zoro volador de barba negra (S): Pieropus melanopogon II M Zorro volador de Bismarck (S): Pteropus neohibemicus II M Zorro volador de Bougainville (S): Pteropus mahaganus II M Zorro volador de cabeza ceniciento (S): Pteropus caniceps II M Zoro volador de cabeza gris (S): Pteropus poliocephalus II M Zorro volador de Calamian (S): Acerodon leucotis II M Zorro volador de cuello rojo (S): Pteropus vampyrus $11 \mathrm{M}$ Zorro volador de Gilliard (S): Preropus gilliardi II M Zorro volador de Howens (S): Pteropus howensis II M Zorro volador de la India (S): Pleropus giganieus II M Zorro volador de la isla Jawi (S): Pteropus tuberculatus II M Zoro volador de la isla Ndeni (S): Pteropus nitendiensis II M Zorro volador de la isla Panay (S): Acerodon lucifer I M Zorro volador de la isla Rodrígues (S): Pteropus rodricensis II M Zorro volador de la isla Santa Cruz (S): Pteropus santacrucis II M Zorro volador de las Célebes (S): Acerodon celebensis II M Zorro volador de las isla Bonin (S): Pteropus pselaphon II M Zorro volador de las isla Ceram (S): Pteropus ocularis II M Zorro volador de las isla Japen (S): Pteropus pohlei II M Zorro volador de las islas Banks (S): Pteropus fundatus II M Zorro volador de las islas de almirantazgo (S): Pteropus admiralitatum II M

Zorro volador de las islas de la Leal (S): Pteropus ornatus II M Zorro volador de las islas Palau (S): Pieropus pilosus I M Zorro volador de las islas Percy (S): Preropus brunneus II M Zorro volador de las Salomón (S): Pteropus rayneri II M Zorro volador de las Seychelles (S): Preropus seychellensis II M Zorro volador de Livingston (S): Pteropus livingstonei II M Zorro volador de Lombok (S): Pteropus lombocensis II M Zoro volador de Luzón (S): Pteropus leucopterus II M Zorro volador de Lyle (S): Pteropus lylei II M Zorro volador de Macklot (S): Acerodon mackloti II M Zorro volador de Madagascar (S): Pteropus rufus II M Zorro volador de Meams (S): Pteropus meamsi II M Zorro volador de Nueva Caledonia (S): Pteropus vetulus II M Zorro volador de orejas grandes (S): Pteropus macrotis II M Zorro volador de Queensland (S): Pteropus scapulatus II M Zorro volador de Ryu-Kyu (S): Pieropus dasymallus II M 
Zorro volador de Talaud (S): Acerodon humilis II M Zorro volador de Taylor (S): Pteropus pumilus II M Zorro volador de Temminck (S): Preropus temmincki II M Zorro volador de Tokuda (S): Pteropus tokudae II M Zorro volador de Voeltzkow (S): Pieropus voelizkowi II M Zorro volador de Woodford (S): Pleropus woodfordi II M Zorro volador enmascarado (S): Pteropus personatus II M Zorro volador filipino (S): Acerodon jubatus I M

Zorro volador gris (S): Pieropus griseus II M

Zorro volador negro (S): Pieropus alecto II M

Zorro volador negro de Mauricio (S): Preropus niger II M

Zorro volador oscuro de Mauricio (S): Pteropus subniger II M

Zorro volador pequeño (S): Pteropus hypomelanus II M

Zorros (E): Cerdocyon thous/Pseudalopex spp/Atelocynus microtis II/NC M

Zorros voladores (S): Acerodon spp. II M

Zostérops (F): Zosterops spp. UNC B
Zostérops à gorge blanche de l'île de Norfolk (F): Zosterops albogularis I B

Zostérops à poitrine blanche (F): Zosterops albogularis I $B$

Zosterops albogularis: White-breasted Silvereye (E), White-breasted White-eye (E), White-chested White-eye (E), Gafudo gorgiblanco (S), Zostérops à gorge blanche de l'île de Norfolk (F), Zostérops à poitrine blanche (F) I B

Zululand Dwarf Chamaeleon (E): Bradypodion nemorale II R

Zumbador (S): Chlorostilbon swainsonii II B

Zumbador coliancho (S): Selasphorus plarycercus II B

Zumbador de Allen (S): Selasphorus sasin II B

Zumbador grande (S): Anthracothorax dominicus II B

Zumbador rufo (S): Selasphorus rufus II B

Zumbadorcito (S): Mellisuga minima II B

Zyzomys pedunculatus: Central Rock-rat (E), Central Thick-tailed Rock-rat (E), Macdonnell Range Rock-rat (E), Rata coligorda (S), Rat à grosse queue (F) I M 


\section{CHECKLIST OF FLORA}

\section{LISTA DE LAS ESPECIES DE FLORA}

\section{LISTE DES ESPECES VEGETALES}





\section{CHECKLIST OF FLORA}

\section{EXPLANATORY NOTE}

The checklist of Flora contains the scientific names of all species (except Appendix-II Cactaceae and Orchidaceae which are included at the generic level only) listed in the CITES Appendices I, II and III (as of 18 September 1997) and, where available, English, French and Spanish common names. Some scientific and common name synonyms are also listed but these are mainly scientific synonyms of Appendix-I species and generic synonyms of Cactaceae and Orchidaceae. Hybrid generic names of Cactaceae and Orchidaceae are not included. One species that is not listed in the appendices (Aloe vera) is included for information because it is the only species in the large genus Aloe which is not listed.

There are three types of entry in the list.

The first type commences with a scientific name together with the name of the author(s) (in the case of species or infraspecific taxa), and is followed by English (E), Spanish (S) and French (F) common names (where available). In the case of taxa listed in Appendices II and III, these names are then followed by the symbol "\#" and a number, which denotes the parts and derivatives that are covered. Then the appendix (or appendices) in which the species is listed (or NC for unlisted species) is given, and finally the family in which the species is included.

The second type commences with a common name, either English (E), Spanish (S) or French (F), followed by the corresponding scientific name and the other information as described above. Some group names are included for information, indicating whether the species bearing that group name are in one (or more) appendix, and whether some species bearing that group name are not listed in the appendices e.g. Aloes (E): Aloe spp. I/II/NC.

The third type consists of a scientific synonym, followed by $=$ and then the accepted scientific name. The latter name should be looked up alphabetically to find the other relevant information about the species/genus.

Nomenclature and taxonomy follow the CITES appendices but, for taxa listed above the species level in the appendices, the following references have been used:

\section{Genera}

Mabberley, D. J. 1990. The Plant-Book. Cambridge University Press, Cambridge.

Brummitt, R. K. 1992. Vascular Plant Families and Genera. Royal Botanic Gardens, Kew.

Hunt, D. In press. CITES Cactaceae Checklist. Second edition.

Willis, J. C. 1973. A Dictionary of Flowering Plants and Ferns. Eighth edition, revised by H. K. Airy Shaw. Cambridge University Press, Cambridge 


\section{Species}

Davis, A. P., Grey-Wilson, C., Mathew, B. and McGough, H. N. In press. CITES Bulb Checklist, for the genera: Cyclamen, Galanthus, Sternbergia. The Royal Botanic Gardens, Kew.

Eggli, U. and Carter, S. 1997. The CITES Checklist of Succulent Euphorbia Taxa (Euphorbiaceae). German Federal Agency for Nature Conservation, Bonn.

Eggli, U. and Taylor, N. P., compilers. 1993. Repertorium Plantarum Succulentarum 44. Royal Botanic Gardens, Kew. [Pachypodium, Ceropegia, Euphorbia, Aloe]

Eggli, U. and Taylor, N. P., eds. 1994. List of Names of Succulent Plants other than Cacti published 1950-1992. Royal Botanic Gardens, Kew. [Pachypodium, Ceropegia, Euphorbia, Aloe]

Grey-Wilson, C. 1988. The Genus Cyclamen. Kew Monograph. [Cyclamen]

Hunt, D. In press. CITES Cactaceae Checklist. Second edition. [Cactaceae]

Jacobsen, H. 1977. Lexicon of Succulent Plants. Blandford, Poole, U.K. [Pachypodium, Ceropegia, Euphorbia, Aloe]

Roberts, J. A., Beale, C. R., Benseler, J. C., McGough, H. N. and Zappi, D. C. 1995. CITES Orchid Checklist. Volume 1. Royal Botanic Gardens, Kew. [Paphiopedilum, Phragmipedium]

Rowley, G. D. 1994. Anacampseros and allied genera - a reassessment. Bradleya 12: 105112. [Anacampseros]

Simpson, R. B. 1994. Pitchers in Trade, a Conservation Review of the Carnivorous Plant Genera Sarracenia, Darlingtonia and Heliamphora. Kew Conservation Review, Royal Botanic Gardens, Kew. [Sarracenia]

Stevenson, D. W., Osborne, R. and Hill, K. D. 1995. A world list of cycads. In: P. Vorster (Ed.) Proceedings of the Third International Conference on Cycad Biology: 56-64. Cycad Society of South Africa, Stellenbosch. [Cycadaceae, Zamiaceae] 


\section{LISTA DE LAS ESPECIES DE FLORA}

\section{NOTA EXPLICATIVA}

La Lista de las especies de Flora contiene los nombres científicos de todas las especies incluidas en los Apéndices I, II y III de la CITES (al 18 de septiembre de 1997), excepto las especies de Cactaceae y Orchidaceae del Apéndice II, que están incluidas a nivel genérico. En la medida de lo posible, se indican sus nombres comunes en español, francés e inglés. Se indican también algunos sinónimos de los nombres científicos y comunes, aunque se trata principalmente de sinónimos científicos de especies incluidas en el Apéndice I y sinónimos genéricos de Cactaceae y Orchidaceae. No se incluyen nombres genéricos de híbridos de Cactaceae y Orchidaceae. Para su información, se incluye una especie que no figuran en los Apéndices de la CITES, a saber, (Aloe vera) ya que se trata de la única especie de este amplio género Aloe que no está incluida en los Apéndices.

La lista consta de tres tipos de entradas:

La primera comienza con un nombre científico junto con el nombre del autor (en el caso de especies o taxa infraespecíficos), seguido de los nombres comunes en inglés (E), español (S) y francés (F) (cuando ha sido posible). Cuando se trata de un taxa incluido en los Apéndices II y III, estos nombres van seguidos del símbolo "\#" y un número, que indica las partes y derivados que están cubiertos. A continuación figura el Apéndice (o Apéndices) en que está incluida la especie (o NC para las especies no incluidas en los Apéndices) y, por último, la familia a la que pertenece la especie.

La segunda comienza con un nombre común, ya sea inglés (E), español (S), o francés (F) seguido del correspondiente nombre científico y el resto de la información mencionada en el párrafo anterior. Para su información, se incluyen algunos nombres de grupos, indicando si la especie perteneciente a ese grupo está en uno (o más) Apéndices CITES y si algunas especies del grupo no están incluidas en los Apéndices, por ejemplo: Aloes (S): Aloe spp. I/I/NC.

La tercera consiste en un sinónimo científico seguido de $=$ y luego el nombre científico aceptado. Este último deberá buscarse por orden alfabético para acceder a la información pertinente sobre la especie.

La nomenclatura y la taxonomía corresponden a las utilizadas en los Apéndices CITES, pero, en el caso de los taxa incluidos por encima del nivel de la especie en los Apéndices, se han utilizado las siguientes referencias:

\section{Género}

Mabberley, D. J. 1990. The Plant-Book. Cambridge University Press, Cambridge.

Brummitt, R. K. 1992. Vascular Plant Families and Genera. Royal Botanic Gardens, Kew.

Hunt, D. In press. CITES Cactaceae Checklist. Second edition.

Willis, J. C. 1973. A Dictionary of Flowering Plants and Ferns. Eighth edition, revised by H. K. Airy Shaw. Cambridge University Press, Cambridge 


\section{Especies}

Davis, A. P., Grey-Wilson, C., Mathew, B. and McGough, H. N. In press. CITES Bulb Checklist, for the genera: Cyclamen, Galanthus, Sternbergia. The Royal Botanic Gardens, Kew.

Eggli, U. and Carter, S. 1997. The CITES Checklist of Succulent Euphorbia Taxa (Euphorbiaceae). German Federal Agency for Nature Conservation, Bonn.

Eggli, U. and Taylor, N. P., compilers. 1993. Repertorium Plantarum Succulentarum 44. Royal Botanic Gardens, Kew. [Pachypodium, Ceropegia, Euphorbia, Aloe]

Eggli, U. and Taylor, N. P., eds. 1994. List of Names of Succulent Plants other than Cacti published 1950-1992. Royal Botanic Gardens, Kew. [Pachypodium, Ceropegia, Euphorbia, Aloe]

Grey-Wilson, C. 1988. The Genus Cyclamen. Kew Monograph. [Cyclamen]

Hunt, D. In press. CITES Cactaceae Checklist. Second edition. [Cactaceae]

Jacobsen, H. 1977. Lexicon of Succulent Plants. Blandford, Poole, U.K. [Pachypodium, Ceropegia, Euphorbia, Aloe]

Roberts, J. A., Beale, C. R., Benseler, J. C., McGough, H. N. and Zappi, D. C. 1995. CITES Orchid Checklist. Volume 1. Royal Botanic Gardens, Kew. [Paphiopedilum, Phragmipedium]

Rowley, G. D. 1994. Anacampseros and allied genera - a reassessment. Bradleya 12: 105112. [Anacampseros]

Simpson, R. B. 1994. Pitchers in Trade, a Conservation Review of the Camivorous Plant Genera Sarracenia, Darlingtonia and Heliamphora. Kew Conservation Review, Royal Botanic Gardens, Kew. [Sarracenia]

Stevenson, D. W., Osborne, R. and Hill, K. D. 1995. A world list of cycads. In: P. Vorster (Ed.) Proceedings of the Third International Conference on Cycad Biology: 56-64. Cycad Society of South Africa, Stellenbosch. [Cycadaceae, Zamiaceae] 


\section{LISTE DES ESPECES VEGETALES}

\section{NOTE EXPLICATIVE}

La liste des espèces végétales contient les noms scientifiques de toutes les espèces inscrites aux Annexes CITES I , II et III (au 18 septembre 1997) à l'exception des espèces de Cactaceae et d'Orchidaceae de l'Annexe II, qui sont inscrites au niveau du genre. Les noms communs en anglais, en français et en espagnol sont indiqués lorsqu'ils sont connus. Certains synonymes de noms scientifiques et de noms communs sont indiqués; il s'agit principalement de synonymes scientifiques d'espèces de l'Annexe I et de synonymes génériques de Cactaceae et d'Orchidaceae. Les noms génériques des hybrides des Cactaceae des Orchidaceae ne figurent pas sur la liste. Une espèce (Aloe vera) est signalée pour information - bien que n'étant pas inscrite aux annexes CITES - car c'est la seule de ce genre très vaste à ne pas être couverte par la Convention.

La liste des espèces végétales comporte trois types d'entrées:

Le premier comprend le nom scientifique et le nom du ou des auteurs (dans le cas des espèces ou des taxons infraspécifiques) suivis, s'ils sont connus, des noms communs en anglais (E), en espagnol (S) et en français (F). Les noms communs des taxons des Annexes II et III sont suivis du symbole \# et d'un nombre indiquant les parties et produits couverts. Vient ensuite l'annexe (ou les annexes) à laquelle l'espèce est inscrite (ou la mention NC pour les espèces non inscrites) et enfin, le nom de la famille à laquelle appartient l'espèce.

Le deuxième commence par le nom commun, soit en anglais ( $E)$, en espagnol $(S)$, ou en français (F), suivi du nom scientifique et des autres renseignements indiqués ci-dessus. Certains noms de groupe sont donnés à titre d'information, en indiquant si les espèces portant ce nom sont inscrites à une ou à plusieurs annexes et si certaines ne sont pas inscrites aux annexes. Exemple: Aloès (F): Aloe spp. I/II/NC.

Le troisième est un synonyme scientifique suivi de = puis du nom scientifique accepté. Ce dernier doit être recherché selon l'ordre alphabétique pour trouver les autres renseignements concernant l'espèce ou le genre.

La nomenclature et la taxonomie sont celles utilisées dans les annexes CITES mais, en ce qui concerne les taxons inscrits aux annexes à un niveau supérieur à l'espèce, les ouvrages de référence suivants ont été utilisés:

\section{Genres}

Mabberley, D. J. 1990. The Plant-Book. Cambridge University Press, Cambridge.

Brummitt, R. K. 1992. Vascular Plant Families and Genera. Royal Botanic Gardens, Kew.

Hunt, D. In press. CITES Cactaceae Checklist. Second edition.

Willis, J. C. 1973. A Dictionary of Flowering Plants and Ferns. Eighth edition, revised by H. K. Airy Shaw. Cambridge University Press, Cambridge 


\section{Espèces}

Davis, A. P., Grey-Wilson, C., Mathew, B. and McGough, H. N. In press. CITES Bulb Checklist, for the genera: Cyclamen, Galanthus, Sternbergia. The Royal Botanic Gardens, Kew.

Eggli, U. and Carter, S. 1997. The CITES Checklist of Succulent Euphorbia Taxa (Euphorbiaceae). German Federal Agency for Nature Conservation, Bonn.

Eggli, U. and Taylor, N. P., compilers. 1993. Repertorium Plantarum Succulentarum 44. Royal Botanic Gardens, Kew. [Pachypodium, Ceropegia, Euphorbia, Aloe]

Eggli, U. and Taylor, N. P., eds. 1994. List of Names of Succulent Plants other than Cacti published 1950-1992. Royal Botanic Gardens, Kew. [Pachypodium, Ceropegia, Euphorbia, Aloe]

Grey-Wilson, C. 1988. The Genus Cyclamen. Kew Monograph. [Cyclamen]

Hunt, D. In press. CITES Cactaceae Checklist. Second edition. [Cactaceae]

Jacobsen, H. 1977. Lexicon of Succulent Plants. Blandford, Poole, U.K. [Pachypodium, Ceropegia, Euphorbia, Aloe]

Roberts, J. A., Beale, C. R., Benseler, J. C., McGough, H. N. and Zappi, D. C. 1995. CITES Orchid Checklist. Volume 1. Royal Botanic Gardens, Kew. [Paphiopedilum, Phragmipedium]

Rowley, G. D. 1994. Anacampseros and allied genera - a reassessment. Bradleya 12: 105112. [Anacampseros]

Simpson, R. B. 1994. Pitchers in Trade, a Conservation Review of the Carnivorous Plant Genera Sarracenia, Darlingtonia and Heliamphora. Kew Conservation Review, Royal Botanic Gardens, Kew. [Sarracenia]

Stevenson, D. W., Osborne, R. and Hill, K. D. 1995. A world list of cycads. In: P. Vorster (Ed.) Proceedings of the Third International Conference on Cycad Biology: 56-64. Cycad Society of South Africa, Stellenbosch. [Cycadaceae, Zamiaceae] 
A-ga-ru (E): Aquilaria malaccensis Lamk.: \#1 II Thymelaeaceae Aa spp. \#7 II Orchidaceae

Abdominea spp. \#7 II Orchidaceae

Abies guatemalensis Rehder var. guatemalensis: Guatemalan Fir (E),

Pinabere (E), Sapin du Guatemala (F) I Pinaceae

Abies guatemalensis Rehder var. jaliscana Martinez: I Pinaceae Abies guatemalensis Rehder var. tacanensis (Lundell) Martinez: I Pinaceae

Abola $=$ Caucaea

Aborchis $=$ Disa

Acacallis = Aganisia

Acajou (F): Swietenia macrophylla King: \#1 III Meliaceae

Acampe spp. \#7 II Orchidaceae

Acanthephippium spp. \#7 II Orchidaceae

Acanthocalycium spp. \#4 II Cactaceae

Acanthocephala $=$ Parodia

Acanthocereus spp. \#4 II Cactaceae

Acanthoglossum $=$ Pholidota

Acantholobivia $=$ Echinopsis

Acanthopetalus $=$ Echinopsis

Acanthophippium $=$ Acanthephippium

Acanthorhipsalis $=$ Lepismium

Aceras spp. \#7 II Orchidaceae

Aceraforchis spp. \#7 II Orchidaceae

Achroanthes = Malaxis

Acianthera $=$ Pleurorhallis

Acianthus spp. \#7 II Orchidaceae

Acineta spp. \#7 II Orchidaceae

Ackermania spp. \#7 II Orchidaceae

Aclinia $=$ Dendrobium

Acoidium $=$ Trichocentrum

Acoridium spp. \#7 II Orchidaceae

Acostaea spp. \#7 II Orchidaceae

Acraea $=$ Pterichis

Acriopsis spp. \#7 II Orchidaceae

Acroanthes $=$ Malaxis

Acrochaene $=$ Monomerio

Acrolophia spp. \#7 II Orchidaceae

Acronia $=$ Pleurothallis

Acropera $=$ Gongora

Acrorchis spp. \#7 II Orchidaceae

Acrostylia = Cynorkis

Actinophlebia $=$ Cyathea

Acuña Cactus (E): Sclerocactus erectocentrus var. acunensis (W. T.

Marsh) H. Bravo: I Cactaceae

Ada spp. \#7 II Orchidaceae

Adacrylus = Apostasia

Adelopetalum $=$ Bulbophyllum

Adeneleuterophora $=$ Elleanthus

Adeneleuthera $=$ Elleanshus

Adenochilus spp. \#7 II Orchidaceae

Adenoncos spp. \#7 II Orchidaceae

Adenostylis = Zeuxine

Adipe $=$ Bifrenaria

Adnula $=$ Pelexia

Adrorhizon spp. \#7 II Orchidaceae

Aeeridium $=$ Aerides

Aenhenrya spp. \#7 II Orchidaceae

Aeonia $=$ Oeonia

Aerangis spp. \#7 II Orchidaceae

Aeranthes spp. \#7 II Orchidaceae

Aerides spp. \#7 II Orchidaceae

Aeridium $=$ Aerides

Aeridostachya $=$ Eria

Aerobion $=$ Angraecum

Aetheria $=$ Stenorrhynchos

African Cherry (E): Prunus africana (Hook. f.) Kalkm.: \#1 II Rosaceae

African Teak (E): Pericopsis elata (Harms) van Meeuwen: \#1 II Leguminosae

Afrormosia (E): Pericopsis elata (Harms) van Meeuwen: \#1 II Leguminosae

Aganisia spp. \#7 II Orchidaceae

Agarwood (E): Aquilaria malaccensis Lamk.; \#1 II Thymelaeaceae

Agave arizonica Gentry \& J. H. Weber: Arizona Agave (E) I

Agavaceae
Agave parviflora Tor. ssp. flexiflora H. S. Gentry: I Agavaceae Agave parviflora Torr. ssp. parviflora: Little Princess Agave (E), Small Flower Agave (E) I Agavaceae

Agave victoriae-reginae T. Moore: Queen Agave (E) \#1 I Agavaceae

Aggeianthus = Porpax

Aglossorhyncha spp. \#7 II Orchidaceae

Agrostophyllum spp. \#7 II Orchidaceae

Airampoa $=$ Opuntio

Ajillo (S): Caryocar costaricense J. D. Sm.: \#1 II Caryocaraceae

Ají (S): Caryocar costaricense J. D. Sm. \#I II Caryocaraceae

Ajo (S): Caryocar costaricense J. D. Sm.: \#1 II Caryocaraceae

Akersia $=$ Cleistocactu

Alabama Canebrake Pitcherplant (E): Sarracenia rubra Walter ssp. alabamensis (F. W. \& R. B. Case) Schnell: I Sarraceniaceae

Alamania spp. \#7 II Orchidaceac

Albany Pitcherplant (E): Cephalotus follicularis Labill. \#I II Cephalotaceae

Alerce (E): Fitzroya cupressoides (Molina) I. M. Johnston: I Cupressaceae

Algum (E): Pterocarpus santalinus Linn.f.: \#I II Leguminosae

Algum (S): Pterocarpus santalinus Linn.f.: \#1 II Leguminosae

Alipsea $=$ Liparis

Alismorkis $=$ Calanthe

Allochilus $=$ Goodyera

Allucudia ascendens (Drake) Drake \#I II Didiereaceae

Alluaudia comosa Drake \#1 II Didiereaceae

Alluaudia dumosa Drake \#I II Didiereaceae

Alluaudia humbertii Choux \#1 II Didiereaceae

Alluaudia montagnacii Rauh \#l II Didiereaceae

Alluandia procera Drake \#1 II Didereaceae

Alluaudiopsis fiherenensis Humbert and Choux \#1 II Didiereaceae

Alluaudiopsis mamieriana Rauh \#1 II Didiereaceae

Almendrillo (S): Caryocar costaricense J. D. Sm.: \#1 II

Caryocaraceae

Almendrón (S): Caryocar costaricense J. D. Sm.: \#1 II

Caryocaraceae

Aloe spp. Aloes (E), Aloes (S), Aloès (F) \#1 I/II Liliaceae

Aloe barbadensis Mill.: \#1 II Liliaceae

Aloe ferox Mill.: \#1 II Liliaceae

Aloe aageodonta L. E. Newton: \#I II Liliaceae

Aloe abyssicola Lavranos \& Bilaidi: \#1 II Liliaceae

Aloe abyssinica Lam.: \#l II Liliaceae

Aloe aculeata Pole Evans: \#1 II Liliaceae

Aloe acutissima H. Perrier var. acutissima: \#l II Liliaceae

Aloe acutissima H. Perrier var. antanimorensis G. Reyn.: \#1 II Liliaceae

Aloe adigratana G. Reyn.: \#1 II Liliaceae

Aloe affinis A. Berger: \#I II Liliaceae

Aloe africana Mill.: \#1 II Liliaceae

Aloe albida (Stapf) Reynolds: Aloès blanchâtre (F) I Liliaceae

Aloe albiflora Guillaumin: I Liliaceae

Aloe albovestita S. Carter \& Brandham: \#1 II Liliaceae

Aloe alfredii Rauh: I Liliaceae

Aloe alooides (Bolus) van Druten: \#I II Liliaceae

Aloe ambigens Chiov.: \#1 II Liliaceae

Aloe amiconum Newton: \#l II Liliaceae

Aloe ammophila G. Reyn.: \#1 II Liliaceae

Aloe amudatensis G. Reyn.: \#1 II Liliaceae

Aloe andongensis Baker var. andongensis: \#1 II Liliaceae

Aloe andongensis Baker var. repens Leach: \#1 II Liliaceae

Aloe andringitrensis $\mathrm{H}$. Perrier; \#1 II Liliaceae

Aloe angelica Pole Evans: \#1 II Liliaceae

Aloe angolensis Baker: \#1 II Liliaceae

Aloe antondroi (Decary) H. Perrier: \#1 II Liliaceae

Aloe arborescens Mill.: \#1 II Litiaceae

Aloe archeri Lavranos: \#1 II Liliaceae

Aloe arenicola Reynolds: \#1 II Liliaceae

Aloe argenticauda Merxmuller \& Giess: \#1 II Liliaceae

Aloe aristata Haw.: \#1 II Liliaceae

Aloe asperifolia A. Berger: \#1 II Liliaceae

Aloe audhalica Lavr. \& Hardy: \#1 II Liliaceae

Aloe ausana $\mathrm{Dtr} .=$ Aloe variegata $\mathrm{L}$

Aloe babatiensis Christian \& Verdoom: \#1 II Liliaceae

Aloe bainesii Dyer: \#1 II Liliaceae

Aloe bakeri Scott Elliot: I Liliaceae 


\section{Aloe ballii: \#1 II Liliaceac}

Aloe ballyi G. Reyn.: Rat Aloe (E) \#1 II Liliaceae

Aloe barbadensis Mill. = Aloe vera $(\mathrm{L}$.) N. L. Burman var. vera Aloe barbertoniae Pole Evans: \#1 II Liliaceae

Aloe bargalensis Lavranos: \#1 II Liliaceae

Aloe bella G. Rowley: \#1 II Liliaceae

Aloe bellatula G. Reynolds: I Liliaceae

Aloe berhana G. Reyn.: \#I II Liliaceae

Aloe betsiliensis H. Perrier: \#1 II Liliaceae

Aloe bicomitum Leach: \#1 II Liliaceae

Aloe boehmii Engl. = Aloe lateritia Engler var. lateritia

Aloe boiteaui Guillaumin: \#1 II Liliaceae

Aloe boscawenii Christian: \#1 II Liliaceae

Aloe bowiea Schult. \& Schult.f.: \# I II Liliaceae

Aloe boylei Baker: \#1 II Liliaceae

Aloe brachystachys Baker: \#1 II Liliaceae

Aloe branddraaiensis Groenew.: \#1 II Liliaceae

Aloe brandhamii S. Carter: \#1 II Liliaceae

Aloe brevifolia (L.) Mill.: \# I II Liliaceae

Aloe brevifolia Mill. var. brevifolia: \#1 II Liliaceae

Aloe brevifolia Mill. var. depressa (Haw.) Baker = Aloe brevifolia

(L.) Mill.

Aloe brevifolia Mill. var, postgenita (Roem. \& Schult.) Baker = Aloe brevifolia (L.) Mill.

Aloe breviscapa G. Reyn. \& Bally: \#1 II Liliaceae

Aloe broomii Schoenl. var. broomii: \#1 II Liliaceae

Aloe broomii Schoenl. var. tarkaensis G. Reyn.: \#1 II Liliaceae

Aloe brunneostriata Lavranos \& S. Carter: \#1 II Liliaceae

Aloe buchananii Baker: \#I II Liliaceae

Aloe buchlohii Rauh: \#1 II Liliaceae

Aloe buetneri A. Berger: \#I II Liliaceae

Aloe buhrii Lavranos: \#1 II Liliaceae

Aloe bukobana G. Reyn.; \#1 II Liliaceae

Aloe bulbicaulis Christian: \#1 II Liliaceae

Aloe bulbillifera $\mathrm{H}$. Perrier var. bulbillifera: \#1 II Liliaceae

Aloe bulbillifera H. Perrier var. pauliana G. Reyn.: \#I II Liliaceae

Aloe bullockii G. Reyn.: \#1 II Liliaceae

Aloe burgersfortensis G. Reyn.: \#1 II Liliaceae

Aloe bussei A. Berger: \#1 II Liliaceae

Aloe calcairophila G. Reyn.: I Liliaceae

Aloe calidophila G. Reyn: \#1 II Liliaceae

Aloe cameronii Hemsl. var. bondana G. Reyn.: \#I II Liliaceae

Aloe cameronii Hemsl. var. cameronii: \#I II Liliaceae

Aloe cameronii Hemsl, var. christiani Reynolds: \#1 II Liliaceae

Aloe cameronii Hemsl. var. dedzana G. Reyn.: \#1 II Liliaceae

Aloe camperi Schweinf.: \#I II Liliaceae

Aloe canarina S. Carter: \#I II Liliaceae

Aloe candelabrum A. Berger: \# I II Liliaceae

Aloe cannellii Leach: \#1 II Liliaceae

Aloe capitata Baker var. capitata: \#1 II Liliaceae

Aloe capitala Baker var. cipolinicola H. Perrier: \#1 II Liliaceae

Aloe capitata Baker var. gneissicola $\mathrm{H}$. Perrier: \#1 II Liliaceae

Aloe capitata Baker var. quartziticola $\mathrm{H}$. Perrier: \# 1 II Liliaceae

Aloe capitala Baker var. silvicola $\mathrm{H}$. Perrier: \# I II Liliaceae

Aloe caricina $\mathrm{Bgr} .=$ Aloe myriacantha (Haw.) Schult.

Aloe castanea Schoenl.: \#1 II Liliaceae

Aloe castellorum J. R. I. Wood: \#1 II Liliaceae

Aloe catengiana $\mathrm{G}$. Reyn.: \#1 II Liliaceae

Aloe chabaudii Schoenl. var, chabaudii: \#1 II Liliaceae

Aloe chabaudii Schoenl. var, mlanjeana Christian: \#1 II Liliaceae

Aloe chabaudii Schoenl, var, verekeri Christian: \#1 II Liliaceae

Aloe cheranganiensis S. Carter \& Brandham: \#1 II Liliaceae

Aloe chlorantha Lavranos: \#1 11 Liliaceae

Aloe chortolinioides A. Berger var. boastii (Letty) G. Reyn.: \# I II

Liliaceae

Aloe choriolirioides A. Berger var, chortolinioides: \#I II Liliaceae

Aloe choriolirioides Berger var, woolliana (Pole Evans) Glen \& Hardy: \#1 II Liliaceae

Aloe christianii G. Reyn.: \#1 II Liliaceae

Aloe chrysostachys Lavranos \& Newton: \#1 II Liliaceae

Aloe ciliaris Haw.: \#1 II Liliaceae

Aloe cirrina S. Carter \& Brandham: \#1 II Liliaceae

Aloe classenii G. Reyn.; \#1 II Liliaceae

Aloe claviflora Burchell: \#I II Liliaceae

Aloe commixra A. Berger: \#1 II Liliaceae
Aloe comosa Marloth \& A.Berger: Clanwilliam Alce (E) \#1 II

Liliaceae

Aloe compacta Reynolds = Aloe macrosiphon Baker

Aloe compressa $\mathrm{H}$. Perrier var, compressa: I Liliaceae

Aloe compressa H. Perrier var. rugosquamosa H. Perrier: I Liliaceae

Aloe compressa H. Perrier var. schistophila H. Perrier: I Liliaceae

Aloe compronii G. Reyn.: \#1 II Liliaceae

Aloe confusa Engl.: \#1 II Liliaceae

Aloe congdonii S. Carter: \#1 II Liliaceae

Aloe congolensis De Wild. \& Dur: \#1 II Liliaceae

Aloe conifera H. Perrier \#1 II Liliaceae

Aloe constricta Baker: \#1 II Liliaceae

Aloe contigua (Perr.) Reyn. = Aloe imalotensis G. Reyn.

Aloe cooperi Baker ssp. cooperi: \#1 II Liliaceae

Aloe cooperi Baker ssp. pulchra Glen \& Hardy: \# I II Liliaceae

Aloe corallina I.Verd.: \#1 II Liliaceae

Aloe crassipes Baker: \# I II Liliaceae

Aloe cremersii Lavranos: \#1 II Liliaceae

Aloe cremnophila G. Reyn. \& Bally: \#1 II Liliaceae

Aloe cryptoflora G. Reyn.: \#1 II Liliaceae

Aloe cryptopoda Baker: \#I II Liliaceae

Aloe dabenorisana Van Jaarsv.: \#1 II Liliaceae

Aloe davyana Schoenl. var. davyana: \#1 II Liliaceae

Aloe davyana Schoenl. var, subolifera Groenew.: \#1 II Liliaceae

Aloe dawei A. Berger: \#1 II Liliaceae

Aloe decaryi Guillaumin: \#I II Liliaceae

Aloe decorsei H. Perrier: \#1 II Liliaceae

Aloe decurva G. Reyn: \#1 II Liliaceae

Aloe decunidens Groenew. = Aloe komatiensis G. Reyn. II Liliaceae

Aloe defalcata Chiov.: \#1 II Liliaceav

Aloe delphinensis Rauh: I Liliaceae

Aloe delioideodonta Baker var. brevifolia H. Perrier: \#I II Liliaceae

Aloe deltoideodonta Baker var. candicans H. Perrier: \#1 II Liliaceae

Aloe deltoideodonta Baker var. deltoideodonta: \# I II Liliaceae

Aloe descoingsii G. Reyn.: I Liliaceae

Aloe deserti Engl.: \#1 II Liliaceae

Aloe dewetii G. Reyn.: \#1 II Liliaceae

Aloe dewinteri Giess: \#I II Liliaceae

Aloe dhalensis Lavr.: \#1 II Liliaceae

Aloe dhufarensis Lavranos: \#1 II Liliaceae

Aloe dichotoma Masson: \#1 II Liliaceae

Aloe dinteri A.Berger: \#1 II Liliaceae

Aloe dispar Bgr.: \#1 II Liliaceae

Aloe distans Haw.: \#1 II Liliaceae

Aloe divaricata A. Berger var. divaricata: \#1 II Liliaceae

Aloe divaricata A. Berger var. rosea (Decary) G. Reyn.: \#1 II Liliaceac

Aloe doei Lavranos: \# 1 II Liliaceae

Aloe dolomitica Groenew.: \#1 II Liliaceae

Aloe dominella Reynolds: \#1 II Liliaceae

Aloe dorothea A. Berger: \#I II Liliaceae

Aloe duckeri Christian: \#1 II Liliaceae

Aloe dumeiorum B. Mathew \& Brandham = Aloe ellenbeckii A. Berger

Aloe dyeri Schoenl.: \#I II Liliaceae

Aloe ecklonis Salm-Dyck: \#1 II Liliaceae

Aloe elata S. Carter \& L. E. Newton: \#1 II Liliaceae

Aloe elegans Tod.; \#1 II Liliaceae

Aloe elgonica Bullock: \#1 II Liliaceae

Aloe ellenbeckii A. Berger: \#1 II Liliaceae

Aloe eminens G. Reyn. \& Bally: \#1 II Liliaceae

Aloe enotata Leach: \#1 II Liliaceae

Aloe eremophila Lavranos: \#I II Liliaceae

Aloe erensii Christian: \# I II Liliaceae

Aloe erice torum Bosser: \#1 II Liliaceae

Aloe erinacea D. S. Hardy: \#1 II Liliaceae

Aloe eru A. Berger: \#1 II Liliaceae

Aloe erythrophylla Bosser: \#1 II Liliaceae

Aloe esculenia Leach: \#I II Liliaceae

Aloe excelsa A. Berger var, breviflora Leach; \#I II Liliaceae

Aloe excelsa A. Berger var. excelsa: \#1 II Liliaceae

Aloe falcaia Baker: \#1 II Liliaceae

Aloe ferox Mill.: Cape Alce (E) \#1 II Liliaceae

Aloe fibrosa Lavranos \& Newton: \#1 II Liliaceae

Aloe fieverii G. Reyn.: \#1 II Liliaceae

Aloe fleurentinorum Lavranos \& Newton: \#1 II Liliaceae 
Aloe flexilifolia Christian: \#1 II Liliaceae Aloe forbesii Balf. f.: \#1 II Liliaceae Aloe fosteri Pill: \#I II Liliaceae

Aloe fouriei D. S. Hardy \& Glen: \#1 11 Liliaceae Aloe fragilis Lavranos \& Roosli: 1 Liliaceae Aloe framesii L. Bolus: \#1 II Liliaceae Aloe francombei L. E. Newton: \#1 II Liliaceae Aloe fulleri Lavranos: \#I II Liliaceae Aloe gariepensis Pill: \#1 II Liliaceae Aloe gersineri Reynolds: \#1 II Liliaceae Aloe gigas Res.: \#1 II Liliaceae

Aloe gilbertii T. Reynolds ex Sebsebs Demissew \& Brandham: \#! II Liliaceae

Aloe gillilandi G. Reyn.: \#1 II Liliaceae

Aloe glabrescens (G. Reyn. \& Bally) S. Carter \& Brandham = Aloe sabaea Schweinf.

Aloe glauca Mill. var. glauca: \#1 II Liliaceae

Aloe glauca Mill. var. muricata (Schult.) Baker: \#1 II Liliaceae

Aloe globuligemma Pole Evans: \#I II Liliaceae

Aloe gloveri Reyn. \& Bally = Aloe hildebrandrii Baker

Aloe gossweileri G. Reyn.: \#1 II Liliaceae

Aloe gracilicaulis G. Reyn. \& Bally: \#1 II Liliaceae

Aloe graciliflora Groenew. = Aloe greatheadii Schonl, var, davyana (Schonl.) Glen \& D. S. Hardy

Aloe gracilis Haw. var. decumbens G. Reyn.: \#1 II Liliaceae

Aloe gracilis Haw. var. gracilis: \#1 II Liliaceae

Aloe graminicola G. Reyn. = Aloe lateritia Engler var. graminicola (Reynolds) S. Carter

Aloe graminifolia $\mathrm{Bgr}$. = Aloe myriacantha (Haw.) Schult.

Aloe grandidentata Salm-Dyck: \#I II Liliaceae

Aloe grata G. Reyn.: \#1 II Liliaceae

Aloe greatheadii Schoenl:; \#1 II Liliaceae

Aloe greatheadii Schonl. var. davyana (Schonl.) Glen \& D. S. Hardy = Aloe davyana Schoenl. var. davyana

Aloe greenii Baker: \#I II Liliaceae

Aloe greenwayi Reynolds = Aloe leptosiphon A. Berger

Aloe grisea S. Carter \& Brandham: \#I II Liliaceac

Aloe guerrai G. Reyn.: \#! II Liliaceae

Aloe guillaumetii Cremers: \#1 II Liliaceae

Aloe haemanthifolia A.Berger \& Marloth: \#1 II Liliaceae

Aloe hardyi Glen: \#I II Liliaceae

Aloe harlana G. Reyn.: \#1 II Liliaceae

Aloe harmsii Berger $=$ Aloe dorothea A. Berger

Aloe haworthioides Baker var. aurantiaca $\mathrm{H}$. Perrier: I Liliaceae

Aloe haworthioides Baker var. haworthioides: 1 Liliaceae

Aloe hazeliana G. Reyn.: \#I II Liliaceae

Aloe helenae Danguy: I Liliaceae

Aloe heliderana Lavranos: \#! II Liliaceae

Aloe hemmingii Reyn. \& Bally: \#1 II Liliaceae

Aloe hendrickxii G. Reyn.: \#1 II Liliaceae

Aloe hereroensis Engl. var. hereroensis: \#1 II Liliaceae

Aloe hereroensis Engl. var. lutea A. Berger: \#l II Liliaceae

Aloe hildebrandrii Baker: \#1 II Liliaceae

Aloe hlangapies Groenew: \#1 II Liliaceae

Aloe howmanii G. Reynolds: \#1 II Liliaceae

Aloe humberii H. Perrier: \#1 II Liliaceae

Aloe humilis (L.) Mill.: \#1 II Liliaceae

Aloe humilis (L.) Mill. var. acuminata (Haw.) Baker: \#1 II Liliaceae Aloe humilis (L.) Mill. var. echinara (Willd.) Baker: \#I II Liliaceae

Aloe humilis (L.) Mill, var. humilis: \#1 II Liliaceae

Aloe humilis (L.) Mill. var. incurva Haw.: \#I II Liliaceae

Aloe humilis (L.) Mill. var, suberecta (Haw.) Baker: \#1 II Liliaceae

Aloe humilis (L.) Mill. var, subtuberculata (Haw.) Baker: \#1 II Liliaceae

Aloe ibitiensis H. Perrier: \#I II Liliaceae

Aloe imalotensis G. Reyn.: \#1 II Liliaceae

Aloe immaculata Pill.: \#1 II Liliaceae

Aloe inamara Leach: \#1 II Liliaceae

Aloe inconspicua Plowes: \#1 II Liliaceae

Aloe inermis Forsk.: \#1 II Liliaceae

Aloe integra G. Reyn.: \#1 II Liliaceae

Aloe inyangensis Christian: \#1 II Liliaceae

Aloe isaloensis H. Perrier: \#I II Liliaceae

Aloe itremensis G. Reyn.: \#I II Liliaceae

Aloe jacksonii G. Reyn.: \#I II Liliaceae

Aloe jucunda G. Reyn.: \#1 II Liliaceae
Aloe juvenna Brandham \& S. Carter: \#1 II Liliaceae

Aloe karasbergensis Pill. = Aloe striata Haw. ssp, karasbergensis

(Pill.) Glen \& Hardy

Aloe keayi Reyn.: \#1 II Liliaceae

Aloe kedongensis G. Reyn.: \#I II Liliaceae

Aloe keithii Reynolds: \#I II Liliaceae

Aloe kelabrowniorum L. E. Newton: \#1 II Liliaceae

Aloe khamiesensis Pillans: Namaqua Aloe (E) \#1 II Liliaceae

Aloe kilifiensis Christian: \#1 II Liliaceae

Aloe kirkii Baker: \#1 II Liliaceae

Aloe kniphofioides Baker: \#1 II Liliaceae

Aloe komaggasensis Kritzinger \& van Jaarsveld: \#I II Liliaceae

Aloe komatiensis G. Reyn.; \#1 II Liliaceae

Aloe krapohliana Marloth: \#1 II Liliaceae

Aloe krapohliana Marloth var. dumoulinii Lavranos: \#1 II Liliaceae

Aloe krapohliana Marloth var. krapohliana: \#1 II Liliaceae

Aloe krausii Bak: \#I II Liliaceae

Aloe kulalensis Newton \& Beentje: \#1 II Liliaceae

Aloe labworana (Reynolds) S. Carter: \#1 II Liliaceae

Aloe laeta A. Berger var. laeta: I Liliaceae

Aloe laeta A. Berger var. maniaensis H. Perrier: I Liliaceae

Aloe lastii Baker = Aloe brachystachys Baker

Aloe lateritia Engler var. graminicola (Reynolds) S. Carter: \#I II Liliaceae

Aloe lateritia Engler var. kitaliensis (G. Reyn.) G. Reyn. = Aloe wollastonii Rendle

Aloe lateritia Engler var. lateritia: \#1 II Liliaceae

Aloe latifolia Haw. = Aloe saponaria (Ait.) Haw.

Aloe lavranosii G. Reyn.: \#1 II Liliaceae

Aloe leachii Reynolds: \#1 II Liliaceae

Aloe Léandrii Bosser: \#1 II Liliaceae

Aloe leedalii S. Carter: \#1 II Liliaceae

Aloe lensayuensis Lavranos \& Newton: \#1 II Liliaceae

Aloe lepida Leach: \#1 II Liliaceae

Aloe leptophylla N. E. Br. ex Baker = Aloe saponaria (Ait.) Haw.

Aloe leptosiphon A. Berger: \#1 II Liliaceae

Aloe lettyae G. Reyn.: \#l II Liliaceae

Aloe leucantha A. Berger: \#1 II Liliaceae

Aloe linearifolia A. Berger: \#1 II Liliaceae

Aloe lineata (Ait.) Haw. var. lineata: \#I II Liliaceae

Aloe lineata (Ait.) Haw. var, muirii (Marloth) G. Reyn.: \#! II Liliaceae

Aloe littoralis Baker: \#1 II Liliaceae

Aloe longibracteata Pole Evans: \#1 II Liliaceae

Aloe longistyla Baker: \#I II Liliaceae

Aloe luapulana Leach: \#1 II Liliaceae

Aloe lutescens Groenew.: \#1 II Liliaceae

Aloe macleayi G. Reyn.: \#I II Liliaceae

Aloe macracantha Baker: \#1 II Liliaceae

Aloe macrocarpa Tod.: \#1 II Liliaceae

Aloe macrocarpa Tod. var. macrocarpa: \#I II Liliaceae

Aloe macrocarpa Tod. var. major A. Berger: \#1 II Liliaceae

Aloe macroclada Baker: \#1 II Liliaceae

Aloe macrosiphon Baker: \#1 II Liliaceae

Aloe maculata All: \#1 II Liliaceae

Aloe madecassa H. Perrier var, lutea Guillaumin: \# I II Liliaceae

Aloe madecassa $\mathrm{H}$. Perrier var. madecassa: \#1 II Liliaceae

Aloe marlothii A. Berger var. bicolor G. Reyn.: \#1 II Liliaceae

Aloe marlothii A. Berger var. marlothii: \#1 II Liliaceae

Aloe marsabitensis Verdoorn \& Christian = Aloe secundiflora Engler var, secundiflora

Aloe massawana G. Reyn.: \#I II Liliaceae

Aloe mawii Christian: \#1 II Liliaceae

Aloe mayottensis A. Berger: \#! II Liliaceae

Aloe mcloughlinii Christian: \#1 II Liliaceae

Aloe medishiana G. Reyn, \& Bally: \#1 II Liliaceae

Aloe megalacantha Baker: \#I II Liliaceae

Aloe melanacantha A. Berger: \#1 II Liliaceae

Aloe menachensis (Schweinf.) Blatter: \# I II Liliaceae

Aloe mendesii G. Reyn.: \#1 II Liliaceae

Aloe menyharthii Baker: \#1 II Liliaceae

Aloe me ruana Lavranos: \#I II Liliaceae

Aloe mesallica Engl. \& Gilg.: \#1 II Liliaceae

Aloe meyeri Van Jaarsv.: \#1 II Liliaceae

Aloe microcantho Haw.: \#1 II Liliaceae

Aloe microdonia Chiov: \#I II Liliaceae 
Aloe microstigma Salm-Dyck: \#1 II Liliaceae

Aloe millorii G. Reynolds: \#1 II Liliaceac

Aloe milne-redheadii Christian: \#| II Liliaceae

Aloe minima Baker. \#I II Liliaceae

Aloe minima Baker var, blyderivierensis (Groenew.) G. Reyn.: \# II Liliaceae

Aloe minima Baker var minima: \# II Liliaceae

Aloe mitriformis Mill: \#1 II Liliaceae

Aloe modesta Reynolds: \#1 II Liliaceae

Aloe molederana Lavranos \& Glen: \#1 II Liliaceae

Aloe monotropa I. Verd.: \# I II Liliaceae

Aloe monteiroi Baker: \#1 II Liliaceae

Aloe monticola G. Reyn.: \#1 II Liliaceae

Aloe morijensis S. Carter \& Brandham: \#1 II Liliaceae

Aloe morogoroensis Christian = Aloe bussei A. Berger

Aloe mubendiensis Christian: \#1 II Liliaceae

Aloe mudenensis G. Reyn.: \#I II Liliaceae

Aloe mulricolor L. E. Newton: \#1 II Liliaceae

Aloe munchii Christian: \#1 II Liliaceae

Aloe murina L. E. Newton: \#1 II Liliaceae

Aloe musapana G. Reyn.: \#I II Liliaceae

Aloe mutabilis Pill.: \#1 II Liliaceae

Aloe mutans G. Reyn.: \#1 II Liliaceae

Aloe myriacantha (Haw.) Schult.: \#1 II Liliaceae

Aloe mzimbana Christian: \# II Liliaceae

Aloe namibensis Giess: \#1 II Liliaceac

Aloe ngobitensis Reyn. $=$ Aloe nyeriensis Christian

Aloe ngongensis Christian: \#1 II Liliaceae

Aloe niebuhriana Lavranos: \#I II Liliaceae

Aloe nubigena Groenew.: \#1 II Liliaceae

Aloe nuttii Baker: \#I II Liliaceae

Aloe nyeriensis Christian: \#I II Liliaceae

Aloe nyeriensis Christian ssp. kedongensis (G. Reyn.) S. Carter = Aloe kedongensis G. Reyn.

Aloe obscura Mill.: \#1 11 Liliaceae

Aloe officinalis Forsk. = Aloe vera (L.) N. L. Burman var. officinalis (Forssk.) Baker

Aloe ortholopha Christian \& Milne-Redh.: \#I II Liliaceac

Aloe otallensis Baker var. elongata A. Berger = Aloe rugosifolia

M. G. Gilbert \& Sebsebe Demissew

Aloe otallensis Baker var. orallensis: \#1 II Liliaceae

Aloe pachygaster Dinter: \#1 II Liliaceae

Aloe palmiformis Baker: \#1 II Liliaceae

Aloe parallelifolia $\mathrm{H}$. Perrier: I Liliaceae

Aloe parvibracteata Schoenl. var. parvibracteata: \#I II Liliaceae

Aloe parvibracteata Schoenl. var. zuluensis (G. Reyn.) G. Reyn.: \#]

II Liliaceae

Aloe parvidens M. G. Gilbert \& Sebsebe Demissew: \#1 II Liliaceae

Aloe parviflora $\mathrm{Baker}=$ Aloe minima $\mathrm{Baker}$

Aloe parvula A. Berger: I Liliaceae

Aloe patersonii B. Mathew: \#1 II Liliaceae

Aloe pearsonii Schönland: \# I II Liliaceae

Aloe peckii Bally \& Verdoom: \#1 II Liliaceae

Aloe peglerae Schönland: \#1 II Liliaceae

Aloe pendens Forssk.: \#1 II Liliaceae

Aloe penduliflora Baker: \#1 II Liliaceae

Aloe percrassa Tod.: \# II Liliaceae

Aloe perfoliata L.: \#1 II Liliaceae

Aloe perrieri G. Reynolds: \#I II Liliaceae

Aloe permi Baker: \#1 II Liliaceae

Aloe perricola Pole Evans: \#1 II Liliaceae

Aloe petrophila Pillans: \#I II Liliaceae

Aloe peyrierasii Cremers: \#I II Liliaceae

Aloe pictifolia D. S.Hardy: \#I II Liliaceae

Aloe pillansii L.Guthrie: Bastard Quiver Tree (E) I Liliaceae

Aloe pirotrae G. Reynolds = Aloe parvidens M. G. Gilbert \& Sebsebe Demissew

Aloe plicatilis (L) Mill.: \#1 II Liliaceac

Aloe plowesii G. Reyn.: \#1 II Liliaceae

Aloe pluridens Haw.: \#1 II Liliaceae

Aloe pole-evansii Christian = Aloe dawei A. Berger

Aloe polyphylla Schönland ex Pillans: Spiral Aloe (E) Aloès spiralé (F) I Liliaceae

Aloe powysiorum Newton \& Beentje: \# III Liliaceae

Aloe pratensis Baker: \#1 II Liliaceae

Aloe pretoriensis Pole Evans: \#I II Liliaceae
Aloe principis (Haw.) Stearn; \#1 II Liliaceae

Aloe prinslooi I. Verd. \& D. S.Hardy: \#1 II Liliaceae

Aloe procera Leach: \#I II Liliaceae

Aloe pruinosa Reynolds: \# II Liliaceae

Aloe pubescens G. Reyn.: \#1 II Liliaceae

Aloe purpurascens (Aiton) Haw. = Aloe succotrina All.

Aloe pustuligemma L. E. Newton: \#1 II Liliaceae

Aloe rabaiensis Rendle; \#1 II Liliaceae

Aloe ramosissima Pillans: Maiden's Quiver Tree (E) \#1 II Liliaceae

Aloe rauhii G. Reynolds: I Liliaceae

Aloe reitzii G. Reyn. var, reitzi: \#1 II Liliaceae

Aloe reilzii G. Reyn. var. vemalis Hardy: \# I II Liliaceae

Aloe retrospiciens G. Reyn. \& Bally: \#1 II Liliaceae

Aloe reynoldsii Letty: \#1 II Liliaceae

Aloe rhodesiana Rendle: \#I II Liliaceae

Aloe richardsiae Reynolds: \#1 II Liliaceae

Aloe richiersveldensis H. J. T. Venter \& Beukes: \#1 II Liliaceae

Aloe rigens G. Reyn. \& Bally var, morimeri Lavranos: \#1 II

Liliaceae

Aloe rigens G, Reyn. \& Bally var, rigens: \#1 II Liliaceae

Aloe rivae Baker: \#1 II Liliaceae

Aloe rivieri Lavranos \& Newton: \#1 II Liliaceae

Aloe rubroviolacea Schweinf.: \#1 II Liliaceae

Aloe nugosifolia M. G. Gilbert \& Sebsebe Demissew: \#1 II Liliaceac

Aloe runcinata Bgr.: \#1 11 Liliaceae

Aloe rupestris Baker: \#I II Liliaceae

Aloe rupicola G. Reyn.: \#1 II Liliaceae

Aloe ruspoliana Baker var. dracaeniformis A. Berger: \# I II Liliaceae

Aloe ruspoliana Baker var, ruspoliana: \#1 II Liliaceae

Aloe sabaea Schweinf.: \#1 II Liliaceae

Aloe salm-dyckiana Schult. \& Schult.f. = Aloe principis (Haw.) Stearn

Aloe saponaria (Ait.) Haw.: \#1 II Liliaceae

Aloe saponaria (Ait.) Haw. var. ficksburgensis G. Reyn.: \#1 II Liliaceae

Aloe saundersiae (Reynolds) Reynolds: \#1 II Liliaceae

Aloe scabrifolia Newton \& Lavranos: \#1 II Liliaceae

Aloe schelpei G. Reyn: \#1 II Liliaceae

Aloe schliebenii Lavranos = Aloe brachystachys Baker

Aloe schoelleri Schweinf: \# I II Liliaceae

Aloe schomeri Rauh: \# II Liliaceae

Aloe schweinfurthii Baker: \#1 II Liliaceae

Aloe schweinfurthii Baker var. labworana Reynolds = Aloe labworana (Reynolds) S. Carter

Aloe scobinifolia G. Reyn. \& Bally: \#1 II Liliaceae

Aloe scorpioides Leach: \#1 II Liliaceae

Aloe secundiflora Engler var. secundiflora: \#I II Liliaceae

Alve secundiflora Engl. var. sobolifera S. Carter, 1 II Liliaceae

Aloe sereli De Wild.: \#1 II Liliaceae

Aloe serriyensis Lavranos: \# II Liliaceae

Aloe sessiliflora Pole Evans: \#I II Liliaceae

Aloe sheilae Lavranos: \#1 II Liliaceae

Alce silicicola H. Perrier: \#1 II Liliaceae

Aloe simi Pole Evans: \#1 II Liliaceae

Aloe sinana G. Reyn.: \# I II Liliaceae

Aloe sinkalana G. Reyn.: \#1 II Liliaceae

Aloe sladeniana Pole Evans: \#1 II Liliacese

Aloe somaliensis W. Watson: \#1 II Liliaceae

Aloe soutpansbergensis I.Verd.: \#1 II Liliaceae

Aloe speciosa Baker: \#1 II Liliaceae

Aloe speciabilis Reynolds: \#1 II Liliaceae

Aloe splendens Lavranos: \#1 II Liliaceae

Aloe squarrosa Baker: \#1 II Liliaceae

Aloe steudneri Schweinf.: \#1 II Liliaceae

Aloe striala Haw.: \#1 II Liliaceae

Aloe striata Haw. ssp. karasbergensis (Pill.) Glen \& D. S. Hardy: \#1 II Liliaceae

Aloe striata Haw. ssp. komaggasensis (Kritzinger \& Van Jaarsv.)

Glen \& D. S.Hardy: \#1 II Liliaceae

Aloe striatula Haw. var. caesia G. Reyn.: \#1 II Liliaceae

Aloe striatula Haw, var. striatula: \#1 II Liliaceae

Aloe siuhlmannii Bak. = Aloe volkensii Engl. ssp. volkensii

Alce suarezensis H. Perrier: \#I II Liliaceae

Aloe subacurissima G. Rowley: \#I II Liliaceae

Aloe succorrina All.: \# I II Liliaceae

Aloe suffulta G. Reyn.: \#I II Liliaceae 
Aloe suprafoliaı Pole Evans: \#1 II Liliaceae Aloe suzannae Decary: I Liliaceae Aloe swynnerionii Rendle: \#1 II Liliaceae Aloe tauri Leach: \#1 II Liliaceae Aloe tenuior Haw. var. decidua G. Reyn.: \#1 II Liliaceae Aloe senuior Haw. var. densiflora G. Reyn.: \#I II Liliaceae Aloe tenuior Haw. var. rubriflora G. Reyn.: \#1 II Liliaceae Aloe tenuior Haw. var. tenuior. \#I II Liliaceae Aloe thompsoniae Groenew.: \#1 II Liliaceae Aloe thomcrofiii Pole Evans: Aloès de Thorncroft (F) I Liliaceae Aloe thraskii Baker: \#1 II Liliaceae

Aloe tidmarshii (Schoenl.) Muller: \#1 II Liliaceae

Aloe tomentosa Defl.: \#I II Liliaceae

Aloe tororoana G. Reyn.: \#1 II Liliaceae

Aloe torrei Verdoom \& Christian: \#1 II Liliaceae

Aloe trachyticola (H. Perrier) G. Reyn.: \#1 II Liliaceae

Aloe transvaalensis Kuntze var. stenacaniha Groenw.; \#1 II Liliaceae

Aloe transvaalensis Kuntze var, transvaalensis: \#1 II Liliaceae Aloe trichosantha A. Berger: \#I II Liliaceae

Aloe trigonantha Leach: \#I II Liliaceae

Aloe trothae A. Berger: \#I II Liliaceae

Aloe tugenensis Newton \& Lavranos: \#1 II Liliaceae

Aloe turkanensis Christian: \#I II Liliaceae

Aloe rweediae Christian: \#I II Liliaceae

Aloe ukambensis G. Reyn: \#1 II Liliaceae

Aloe umfoloziensis G. Reyn.: \#1 II Liliaceae

Aloe vacillans Forssk.: \#1 II Liliaceae

Aloe vallaris Leach: \#1 II Liliaceae

Aloe vanbalenii Pill.: \#1 II Liliaceae

Aloe vandermerwei Reynolds: \#1 11 Liliaceae

Aloe vaombe Decorse \& L. Poisson var. poissonii Decary: \#1 II Liliaceae

Aloe voombe Decorse \& L. Poisson var. vaombe: \#1 II Liliaceae Aloe vaotsanda Decary: \#1 II Liliaceae

Aloe variegata L.: Partridge-breasted Aloe (E) \#1 II Liliacese

Aloe venenosa Engl.: \#1 II Liliaceae

Aloe venusia Reynolds = Aloe bicomitum Leach

Aloe vera (L.) Burm.f.: NC Liliaceae

Aloe vera (L.) N. L. Burman var. officinalis (Forssk.) Baker: NC Liliaceae

Aloe vera (L.) N. L. Burman var, vera: Barbados Aloe (E), Common Aloe (E), Yellow Aloe (E), Curaçao Aloe (S) NC Liliaceae Aloe verdoomiae G. Reyn.: \#1 II Liliaceac Aloe verecunda Pole Evans: \#I II Liliaceae Aloe versicolor Guillaumin: I Liliaceae Aloe veseyi G. Reyn.: \#1 II Liliaceae Aloe viguieri $\mathrm{H}$. Perrier: \#I II Liliaceae Aloe viridiflora Reynolds: \#1 II Liliaceae Aloe viruensis Baker: \#1 II Liliaceae

Aloe vogtsii Reynolds: \#1 II Liliaceae

Aloe volkensii Engl. ssp. multicaulis S. Carter \& L. E. Newton: \#I II Liliaceae

Aloe volkensii Engl. ssp. volkensii: \#1 II Liliaceae

Aloe vossii Reynolds: Aloés de Voss (F) I Liliaceae

Aloe vryheidensis Groenew.: \#1 II Liliaceae

Aloe whitcombii Lavaranos: \#1 11 Liliaceae

Aloe wickensii Pole Evans var. lurea G. Reyn.: \#! II Liliaceae

Aloe wickensii Pole Evans var. wickensii: \#1 II Liliaceae

Aloe wildii (G. Reyn.) G. Reyn.: \#1 II Liliaceae

Aloe wilsonii G. Reyn.: \#1 II Liliaceae

Aloe wollastonii Rendle: \#1 II Liliaceae

Aloe woolliana Pole Evans = Aloe chortolirioides Berger var. woolliana (Pole Evans) Glen \& D. S. Hardy

Aloe wrefordii G. Reyn.: \#1 II Liliaceae

Aloe yavellana G. Reyn.: \#1 II Liliaceae

Aloe yemenica J. R. I. Wood: \#1 II Liliaceae

Aloe zanzibarica Milne-Redh. = Aloe squarrosa Baker

Aloe zebrina Baker: \#1 II Liliaceae

Aloes (E/S): Aloe spp. \#1 I/I Liliaceae

Aloès (F): Aloe spp. \#1 I/I Liliaceae

Aloès blanchâtre (F): Aloe albida (Stapf) Reynolds I Liliacene Aloès de Thomcroft (F): Aloe thomcroftii Pole Evans: I Liliaceae Aloès de Voss (F): Aloe vossii Reynolds: I Liliaceae Aloès spiralé $(F)$ : Aloe polyphylla Schönland ex Pillans I Liliaceae Alsophila abbotii (Maxon) Tryon = Cyathea abbottii Maxon
Alsophila amintae Conant: \#1 11 Cyatheaceae

Alsophila brooksii $($ Maxon) R. Tryon = Cyathea brooksii Maxon

Alsophila cuspidata (Kunze) Conant: \#1 II Cyatheaceae

Alsophila denticulata Bak.: \#1 II Cyatheaceae

Alsophila engelii $\mathbf{R}$. Tryon = Cyathea elongata Karsten

Alsophila erinacea (Karsten) Conant: \#I II Cyatheaceae

Alsophila firma (Baker) Conant: \#1 II Cyatheaceae

Alsophila manniana (Hook.) R. Tryon: \#1 11 Cyatheaceae

Alsophila minor $($ D. Eaton) Tryon = Cyathea minor D. Eaton

Alsophila polystichoides Christ: \#1 11 Cyatheaceae

Alsophila salvinii Hooker: Chimón (S) \#I II Cyatheaceae

Alsophila spinulosa (Wallich ex Hook.) Tryon = Cyathea spinulosa Wallich Ex. Hook.

Alsophila tryoniana (Gastony) Conant: \#l II Cyatheaceae

Alsophila urbanii (Brause) Tryon $=$ Cyathea urbanii Brause

Alsensteinia spp. \#7 II Orchidaceae

Alvisia $=$ Eria

Alwisia $=$ Taeniophyllum

Amalia $=$ Laelia

Amaryllis aetnensis Raf. $=$ Stembergia cochiciflora Waldst. \& Kit.

Amanyllis cirrina Sibth. \& Sm. = Stembergia cochiciflora Waldst. \& Kit

Amaryllis clusiana Ker Gawl. = Stembergia clusiana (Ker Gawl.) Spreng.

Amaryllis lutea M. Bieb. = Siembergia fischeriana (Herb.) M. Roem.

Amaryllis lutea L. = Stembergia lutea (L.) Ker Gawl. ex Spreng. Amaryllis vemalis Mill. = Sternbergia fischeriana (Herb.) M. Roem. Amblostoma spp. \#7 II Orchidaceae

Amblyanthe $=$ Dendrobium

Amblyanthus $=$ Dendrobium

Amblyglottis $=$ Calanthe

Ambrella spp. \#7 II Orchidaceae

Americar Ginseng (E): Panax quinquefolius L.: \#1 II Araliaceae

American Mahogany (E): Swietenia mahagoni (L.) Jacq.: \#1 II Meliaceae

American Pitcherplant (E): Cephalotus follicularis Labill.: \#1 II Cephalotaceae

Amerorchis spp. \#7 II Orchidaceae

Amesia $=$ Epipactis

Amesiella spp. \#7 11 Orchidaceae

Amitostigma spp. \#7 II Orchidaceae

Amparoa spp. \#7 II Orchidaceae

Amphicosmia $=$ Cyathea

Amphigena spp. \#7 11 Orchidaceae

Amphiglottis $=$ Epidendrum

Amphorchis = Cynorkis

Anacampseros spp. Pourpiers (F) \#1 II Portulacaceae

Anacampseros affinis Pears \& Steph. = Anacampseros lanceolata (Haw.) Sweet ssp. lanceolata

Anacampseros albidiftora Poelln.: \#1 II Portulacaceae

Anacampseros albissima Marl. = Avonia albissima (Marloth) Rowl.

Anacampseros alstonii Schoenl. = Avonia quinaria (E. Meyer ex Fenzl) ssp. alstonii (Schönl) Rowl.

Anacampseros alta Poelln. = Anacampseros filamentosa (Haw.)

Sims ssp. namaquensis (Pearson \& Stephens) Rowl.

Anacampseros angustifolia (Haw.) $S$ weet $=$ Anacampseros lanceolata (Haw.) Sweet ssp. lanceolata

Anacampseros arachnoides (Haw.) Sims: \#1 II Portulacaceae

Anacampseros australiana J. M. Black: \#I II

Anacampseros baeseckei Dinter: \#I II Portulacaceae

Anacampseros bayeriana S. A. Hammer. \#1 II Portulacaceae

Anacampseros bremekampii Poelln $=$ Avonia rhodesica

(N. E. Br.) Rowl

Anacampseros buderiana v. Poelln. = Avonia recurvala $($ Schönl)

Rowl. ssp. recurvate

Anacampseros comptonii Pillans: \#1 II Portulacaceae

Anacampseros crinita Dir. = Anacampseros baeseckei Dinter Anacanpseros decipiens v. Poelln = Avonia rhodesica (N. E. Br.) Rowl

Anacampseros densifolia Dtr. = Anacampseros filamentosa $($ Haw . Sims ssp. tomentosa (A. Berger) Gerbaulet

Anacampseros depauperata (Bgr.) Poelln. = Anacampseros arachnoides (Haw.) Sims

Anacampseros dielsiana Dr.: \#1 II Portulacaceae

Anacampseros dinteri Schinz = Avonia dinteri $($ Schinz) Rowl 
Anacampseros filamentosa (Haw.) Sims ssp. filamentosa: \#I II Portulacaceae

Anacampseros filamentosa (Haw.) Sims ssp. namaquensis (Pearson \& Stephens) Rowl.: \#1 II Portulacaceae

Anacampseros filamentosa (Haw.) Sims ssp. tomentosa (A. Berger) Gerbaulet: \#1 II Portulacaceae

Anacampseros fissa Poelin. = Avonia rhodesica (N. E. Br.) Rowl.

Anacampseros gracilis v. Poelln. = Anacampseros arachnoides (Haw.) Sims

Anacampseros herreana Poelln. = Avonia herreana (v. Poelln.) Rowl.

Anacampseros karasmontana Dinter = Anacampseros dielsiana Dtr.

Anacampseros kunzii Bacigalupo: \#1 II Portulacaceae

Anacampseros lanceolata (Haw.) Sweet ssp. lanceolata: \#1 II Portulacaceae

Anacampseros lanceolata (Haw.) Sweet ssp. nebrownii (Poelln.) Gerbaulet: \# I II Portulacaceae

Anacampseros lanigera Burch. = Anacampseros filamentosa (Haw.) Sims ssp. filamentosa

Anacampseros lubbersii M. B. Bleck = Anacampseros subnuda Poelln. ssp. lubbersii (M. B. Bleck) Gerbaulet

Anacampseros marlorhii v. Poelln.: \#1 II Portulacaceae

Anacampseros meyeri v. Poelln. = Avonia papyracea $($ E. Meyer ex Fenzl) ssp. namaensis (Gerbaulet) Rowl.

Anacampseros namaquensis Pears \& Stephens = Anacampseros filamentosa (Haw.) Sims ssp, namaquensis (Pearson \& Stephens) Rowl.

Anacampseros nebrownit v. Poelln. = Anacampseros lanceolata (Haw.) Sweet ssp. nebrownii (v. Poelln.) Gerbaulet

Anacampseros nitida v. Poelln. = Anacampseros retusa v. Poelln

Anacampseros papyracea E. Meyer ex Fenzl ssp. namaensis Gerbaulet = Avonia papyracea $(\mathrm{E}$. Meyer ex Fenzl) ssp. namaensis (Gerbaulet) Rowl.

Anacampseros papyracea E. Meyer ex Fenzl ssp. papyracea = Avonia papyracea (E. Meyer ex Fenzl) Rowl. ssp. papyracea

Anacampseros paradoxa $v$. Poelln. = Anacampseros filamentosa (Haw.) Sims ssp. tomentosa (A. Berger) Gerbaulet

Anacampseros parviflora v. Poelln. = Anacampseros baeseckei Dinter

Anacampseros quinaria E.Mey. ex Fenzl = Avonia quinaria (E. Meyer ex Fenzl) Rowl.

Anacampseros recurvata Schoenl. ssp. buderiana (v. Poelln.) Gerbaulet = Avonia recurvata (Schönl) Rowl. ssp. recunata

Anacampseros recurvata Schoenl. ssp. minuta Gerbaulet = Avonia recurvata (Schönl) Rowl. ssp. minuta (Gerbaulet) Rowl.

Anacampseros recurvata Schoenl. ssp. recurvata $=$ Avonia recurvata (Schönl) Rowl. ssp. recurvata

Anacampseros retusa v. Poelln.: \#I II Portulacaceae

Anacampseros rhodesica N. E. Br. = Avonia rhodesica (N. E. Br.) Rowl.

Anacampseros rubroviridis v. Poelln. = Anacampseros arachnoides (Haw.) Sims

Anacampseros rufescens (Haw.) Sweet: \#1 II Portulacaceae

Anacampseros ruschii Dinter ex v. Poelln. = Avonia ruschii (Dinter ex y. Poelln.) Rowl.

Anacampseros schoenlandii v. Poelln. = Anacampseros arachnoides (Haw.) Sims

Anacampseros scopata G.Will: \#I II Portulacaceae

Anacampseros starkiana v. Poelln. = Anacampseros subnuda v Poelin. ssp. subnuda

Anacampseros subnuda v. Poelln. ssp. lubbersii (Bleck) Gerbaulet: \#1 II Portulacaceae

Anacampseros subnuda v. Poelln. ssp. subnuda: \#1 II Portulacaceae

Anacampseros telephiastrum DC.: \#I II Portulacaceae

Anacampseros tomentosa A. Berger.. = Anacampseros filamentosa (Haw.) Sims ssp. tomentosa (A. Berger) Gerbaulet

Anacampseros trigona DC. = Anacampseros lanceolata (Haw.) Sweet ssp. Lanceolata

Anacampseros truncata $v$. Poelin. = Anacampseros retusa $\mathrm{v}$. Poelln

Anacampseros ustulata E. Mey. = Avonia ustulata (E. Meyer ex Fenzl) Rowl

Anacampseros variabilis v. Poelln. = Avonia papyracea (E. Meyer ex Fenzl) Rowl. ssp, namaensis (Gerbaulet) Rowl.

Anacampseros wischkonii Dtr. \& v. Poelln. = Avonia dinteri (Schinz) Rowl.

Anacamptis spp. \#7 II Orchidaceae
Anacheilium = Epidendrum

Anaphora $=$ Malaxis

Anathallis $=$ Pleurothallis

Ancisirocacrus = Sclerocactus

Ancistrocactus tobuschii (W. T. Marsh.) W. T. Marsh. ex Backeberg = Sclerocactus brevihamatus (Engelmann) D. Hunt ssp. lobuschii (W, T. Mrsh.) Tayl.

Ancistrochilus spp. \#7 II Orchidaceae

Ancistrorhynchus spp. \#7 11 Orchidaceae

Andenea $=$ Echinopsis

Andreetaea $=$ Pleurothallis

Androchilus spp. \#7 II Orchidaceae

Androconys spp. \#7 II Orchidaceae

Androgyne $=$ Panisea

Anectochilus $=$ Anoectochilus

Angianthus $=$ Porpax

Angorchis = Angraecum

Angraecopsis spp. \#7 II Orchidaceae

Angraecum spp. \#7 II Orchidaceae

Anguloa spp. \#7 II Orchidaceae

Anhalonium $=$ Ariocarpus

Anhalonium trigonum Weber = Ariocarpus trigonus

(F. A. C. Weber) K. Schum

Anhalonium turbiniforme (Pfeiffer) Weber = Strombocactus disciformis (DC.) Britton \& Rose

Ania spp. \#7 II Orchidaceae

Anisocereus $=$ Pachycereus

Anisoperalum $=$ Bulbophyllum

Anisophyllum atoto (G. Forster) Klotzsch \& Garcke = Euphorbia atoto $\mathrm{G}$. Forster

Anistylis = Liparis

Ankylocheilos $=$ Taeniophyllum

Anneliesia spp. \#7 II Orchidaceae

Anocheile = Epidendrum

Anochilus spp. \#7 II Orchidaceae

Anoectochilus spp. \#7 II Orchidaceae

Anota $=$ Rhynchostylis

Ansellia spp. \#7 II Orchidaceae

Anteriorchis $=$ Orchis

Anshacantha desmetiana Lemaire = Euphorbia heptagona L. var heptagona

Anthacantha mamillosa Lemaire = Euphorbia squarrosa Haworth

Anshericlis = Tipularia

Anthogonium spp. \#7 II Orchidaceae

Anthogyas = Bletia

Anthosiphon spp. \#7 II Orchidaceae

Anticheirostylis = Prasophyllum

Antillanorchis spp. \#7 II Orchidaceae

Aopla $=$ Herminium

Aorchis $=$ Chusua

Apateles $=$ Liparis

Apation = Liparis

Apatostelis $=$ Stelis

Apaturia $=$ Pachystoma

Apetalon $=$ Didymoplexis

Aphyllorchis spp. \#7 II Orchidaceae

Apista $=$ Podochilus

Aplectrum spp, \#7 II Orchidaceae

Aporocactus $=$ Disocactus

Aporocereus $=$ Aporocactus

Aporosnylis spp. \#7 II Orchidaceae

Aporum $=$ Dendrobium

Apostasia spp. \#7 II Orchidaceae

Appendicula spp. \$7 11 Orchidaceae

Aquilaria malaccensis Lamk.: A-ga-ru (E), Agarwood (E),

Eaglewood (E) \#1 II Thymelaeaceae

Aracamunia spp. \#7 II Orchidaceae

Arachnanthe $=$ Arachnis

Arachnis spp. \#7 II Orchidaceae

Arachniles $=$ Ophrys

Araucaria araucana (Mol.) K. Koch: Monkey Puzzle (E), Pehuén (S), Pino Araucana (S), Pino Chileno (S), Pin du Chili (F) U/I Araucariaceae

Archineoria spp. $\$ 7$ II Orchidaceae

Arequipa spp. Cactaceae

Arequipiopsis $=$ Oreocereus 
Arethusa spp. \#7 II Orchidaceae

Arethusantha $=$ Cymbidium

Argyrorchis $=$ Macodes

Arhynchium $=$ Armodorum

Arietinum $=$ Cypripedium

Ariocarpus spp. I Cactaceae

Ariocarpus agavoides (Castaneda) E. F. Anderson: Tamaulipas Living-rock Cactus (E) I Cactaceae

Ariocarpus aselliformis (Ehrenb.) A. Weber = Pelecyphora aselliformis Ehrenb.

Ariocarpus bravoanus H. M. Hem \& E. F. Anderson: I Cactaceae Ariocarpus denegrii (Fric) W. Marshall = Obregonia denegrii Fric Ariocarpus elongatus (Salm Dyck) M. H. Lee: I Cactaceae

Ariocarpus fissurasus (Engelm.) K. Schum var. fissuratus: I Cactaceae

Ariocarpus fissuratus (Engelm.) K. Schum var, hintonit Stuppy \& N. P. Taylor: I Cactaceae

Ariocarpus fissuratus (Engelm.) K. Schum var. lloydii (Rose) W. T. Marsh.: I Cactaceae

Ariocarpus furfuraceus (Wats.) C. Thompson = Ariocarpus relusus Scheidw.

Ariocarpus kotschoubeyanus (Lemaire) Schumann: Pata de venado (S), Pezuña de venado (S) I Cactaceae

Ariocarpus retusus Scheidw.: Chaute (F), Chautle (F) I Cactaceae

A riocarpus retusus Scheidw. var. furfuraceus (Wats.) Frank = A riocarpus retusus Scheidw.

Ariocarpus scaphirostris Bödeker: Nuevo Leon Living-rock Cactus (E), Orejas de conejo (S), Orejitas (S) I Cactaceae

Ariocarpus strabiliformis Werdermann = Pelecyphora strobiliformis (Werdermann) Fric \& Schelle

A riocarpus sulcatus K. Schumann = Ariocarpus kotschoubeyanus (Lemaire) Schumann

Ariocarpus trigonus (F. A. C. Weber) K. Schum.: Living Rock

Cactus (E), Chaute (F), Chautle (F) I Cactaceae

Arisanorchis $=$ Cheirostylis

Aristolelea $=$ Spiranthes

Arizona Agave (E): Agave arizonica Gentry \& J. H. Weber: I Agavaceae

Armatocereus spp. \#4 II Cactaceae

Armodorum spp. \#7 II Orchidaceae

Arnedina $=$ Arundina

Amottia spp. \#7 II Orchidaceae

Arpophyllum spp. \#7 11 Orchidaceae

Arhychium $=$ Arachnis

Arrojadoa spp. \#4 II Cactaceae

Arthrocereus spp. \#4 II Cactaceae

Arhrochilium $=$ Epipactis

Arthrochilus spp. \#7 II Orchidaceae

Arthrothamnus = Euphorbia

Arthrothamnus brachiatus Klotzsch = Euphorbia brachiata (Klotzsch) Boissier

Arthrothamnus burmannii Klotzsch $=$ Euphorbia burmannit (Klotzsch) E. Meyer ex Boissier

Arthrothamnus cassythoides (Boissier) Millspaugh $=$ Euphorbia cassythoides Boissier

Arthrothamnus densiflorus Klotzsch \& Garcke = Euphorbia mundtii N. E. Brown in Thiselton-Dyer

Arthrothamnus schimperi (Presl) Balf.f. $=$ Euphorbia schimperi Presl

Arthrothamnus scopiformis Klotzsch = Euphorbia arceuthobioides Boissier

Arhrothamnus tirucalli (L. ) Klotzsch \& Garcke = Euphorbia tirucalli L.

Artichoke Cactus (E): Obregonia denegrii Fric: I Cactaceae

Artomeria $=$ Erio

Artorima spp. \#7 II Orchidaceae

Arundina spp. \#7 II Orchidaceae

Asarca $=$ Chloraea

Ascidieria spp. \#7 II Orchidaceae

Ascocentrum spp. \#7 II Orchidaceae

Ascochilopsis spp. \#7 II Orchidaceae

Ascochilus spp. \#7 II Orchidaceae

Ascoglossum spp. \#7 II Orchidaceae

Ascolabium $=$ Ascocentrum

Ascotainia $=$ Tainia

Aspasia spp. \#7 II Orchidaceae

Aspegrenia $=$ Octomerio
Aspidogyne spp. \#7 II Orchidaceae

Aspla $=$ Herminium

Assamela (E): Pericopsis elata (Harms) van Meeuwen: \#1 II Leguminosae

Astroglossus $=$ Stellilabium

Astrophyton = Astrophytum

Astrophytum spp. \#4 I/I Cactaceae

Astrophylum asterias (Zucc)) Lem.: Sea Urchin Cactus (E), Star Cactus (E) I Cactaceae

Ate $=$ Habenaria

Aucklande (F): Saussurea costus (Falc.) Lipsch I Compositae

Aulacophyllum $=$ Zamia

Aulacothele $=$ Coryphantha

Auliza $=$ Epidendrum

Aulosepalum spp. \#7 II Orchidaceae

Aulostylis $=$ Calanthe

Aureilobivia $=$ Echinopsis

Australian Tree Fem (E): Dicksonia antarctica Labill. \#I I Dicksoniaceae

Australorchis $=$ Dendrobium

Austrocactus spp. \#4 II Cactaceae

Austrocephalocereus $=$ Micranthocereus

Austrocylindropuntia = Opuntia

Auxopus spp. \#7 II Orchidaceae

Aviceps $=$ Satyrium

Avonia spp. \#1 II Portulacaceae

Avonia albissima (Marloth) Rowl. \#1 II Portulacaceae

Avonia dinteri (Schinz) Rowl. \# II Portulacaceae

Avonia herreana (v. Poelln.) Rowl. \# II Portulacaceae

Avonia papyracea (E. Meyer ex Fenzl) Rowl. ssp. namaensis (Gerbaulet) Rowl. \# II Portulacaceae

Avonia papyracea (E. Meyer ex Fenzl) Rowl, ssp. papyracea (Gerbaulet) Rowl. \# II Portulacaceae

Avonia quinaria (E. Meyer ex Fenzl) Rowl. ssp. alstonii (Schönl) Rowl. \# II Portulacaceae

Avonia quinaria (E. Meyer ex Fenzl) Rowl. ssp. quinaria (Schönl) Rowl. \# II Portulacaceae

Avonia recurvata (Schönl.) Rowl. ssp. minuta (Gerbaulet) Rowl. \# II Portulacaceae

Avonia recurvata (Schönl.) Rowl. ssp. recurvata (Gerbaulet) Rowl. \# II Portulacaceae

Avonia rhodesica (N. E. Br.) Rowl.

Avonia ruschii (Dinter ex y. Poelln.) Rowl.

Avonia ustulata (E. Meyer ex Fenzl.) Rowl.

Aylostera $=$ Rebutia

Azadehdelia $=$ Cribbia

Aztec Cactus (E): Aztekium ritteri (Bödeker) Bödeker: I Cactaceae

Azsekium spp. \#4 I/L Cactaceae

Aztekium ritteri (Bödeker) Bödeker: Aztec Cactus (E) I Cactaceae

Azureocereus $=$ Browningia

Backebergia $=$ Pachycereus

Backebergia chrysomalla (Lemaire) Bravo = Pachycereus militaris (Audot) D. Hunt

Backebergia militaris (Audot) H. Bravo-Holl. \& Sanchez-Mej. = Pachycereus militaris (Audot) D. Hunt

Bahia Rosewood (E): Dalbergia nigra Allem. ex Benth.: I Leguminosae

Balantium = Dicksonia

Balmea stormiae Martinez: I Rubiaceae

Baptistonia spp. \#7 II Orchidaceae

Barbados Aloe (E): Aloe vera (L.) N. L. Burman var. vera: NC Liliaceae

Barbosella spp. \#7 II Orchidaceae

Barbrodria spp. \#7 II Orchidaceae

Barkeria spp. \#7 II Orchidaceae

Barlaea $=$ Cynorkis

Barlia spp. \#7 II Orchidaceae

Barombia spp. \#7 II Orchidaceae

Bartholina spp. \#7 II Orchidaceae

Bartschella = Mammillario

Basigyne = Dendrochilum

Basiphyllaea spp. \#7 II Orchidaceae

Baskenvillea spp. \#7 II Orchidaceae.

Bastard uiver Tree (E): Aloe pillansii L. Guthrie: I Liliaceae

Batemannia spp. \#7 II Orchidaceae

Bathiea $=$ Neobathiea 
Beadlea $=$ Cyclopogon

Beclardia spp. \#7 II Orchidaceae

Beloglonis = Spiranthes

Benthamia spp. \#7 II Orchidaceae

Benzingia spp. \#7 II Orchidaceae

Bergerocactus spp. \#4 II Cactaceae

Bergerocereus $=$ Bergerocactus

Bergkambro (E): Pachypodium succulentum (L.f.) A. DC: \#1 II Apocynaceae

Bicchia $=$ Habenaria

Bicomella $=$ Cynorkis

Bieneria $=$ Chloraea

Biermannia spp. \#7 II Orchidaceae

Bifolium $=$ Listera

Bifrenaria spp. \#7 II Orchidaceae

Bigleaf Mahogany (E): Swietenia macrophylla King: \# III Meliaceae

Bilabrella $=$ Habenaria

Binghamia $=$ Espostoa

Binotia spp. \#7 II Orchidaceae

Bipinnula spp. \#7 II Orchidaceae

Birchea $=$ Luisia

Bisnaga $=$ Ferocactus

Black Tree Fern (E): Cyarhea medullaris (G. Forster) Swartz: \#! II Cyatheaceae

Blephariglottis $=$ Platanthera

Bletia spp. \#7 II Orchidaceae

Bletia boothiana (Reichb.f.) Reichb.f. = Laelia lobata (Lindl.) Veitch

Bletia jongheana Reichb.f. = Laelia jongheana Reichb.f.

Bletia lobata (Lindl.) Reichb.f. = Laelia lobata (Lindl.) Veitch

Bletiana $=$ Bletia

Bletilla spp. \#7 II Orchidaceae

Blossfeldia spp. \#4 II Cactaceae

Blue Vanda (E): Vanda coerulea Griffith ex Lindley: I Orchidaceae

Bogoria spp. \#7 II Orchidaceae

Bolbidium $=$ Dendrobium

Bolbophyllaria $=$ Bulbophyllum

Bolbophyllopsis $=$ Bulbophyllum

Bolborchis = Coelogyne/Nervilia

Bolivicereus = Cleistocactus

Bollea spp. \#7 II Orchidaceae

Bolusiella spp. \#7 II Orchidaceae

Bonatea spp. \#7 II Orchidaceae

Bonifazia = Disocactus

Bonniera spp. \#7 II Orchidaceae

Boojum Tree (E): Fouquieria columnaris Kellogg ex Curran: \#1 II Fouquieriaceae

Borzicactella $=$ Cleistocactus

Borzicactus $=$ Cleistocactus

Borzicereus $=$ Cleistocactus

Bothriochilus spp. \#7 II Orchidaceae

Bowenia sernulata (W. Bull) Chamberlain: \#1 II Stangeriaceae

Bowenia spectabilis Hook. ex Hook.f.: \#I II Stangeriaceae

Braasiella = Oncidium

Brachionidium spp. \#7 II Orchidaceae

Brachtia spp. \#7 II Orchidaceae

Brachycalycium $=$ Gymnocalycium

Brachycereus spp. \#4 II Cactaceae

Brachycorythis spp. \#7 II Orchidaceae

Brachypeza spp, \#7 II Orchidaceae

Brachystele spp. \#7 II Orchidaceae

Brachystepis = Deonia

Bracisepalum spp. \#7 II Orchidaceae

Brady's Pincushion Cactus (E): Pediocactus bradyi L. Benson: I Cactaceae

Braemia $=$ Houlletia

Brasilicacrus $=$ Parodia

Brasilicereus spp. \#4 II Cactaceae

Brasiliopuntia $=$ Opuntia

Brasiliparodia $=$ Parodia

Brasilocactus $=$ Parodia

Brassavola spp. \#7 II Orchidaceae

Brassia spp. \#7 II Orchidaceae

Brazilian Mahogany (E): Swietenia macrophylla King: \#I III Meliaceae
Brazilian Rosewood (E): Dalbergia nigra Allem. ex Benth.: I Leguminosae

Brenesia $=$ Pleurothallis

Bridgesia $=$ Rebutia

Briegeria spp. \#7 II Orchidaceae

Bristly Tree Fern (E): Dicksonia antarctica Labill.: \#I II Dicksoniaceae

Britronia $=$ Ferocactus

Britronrosea $=$ Stenocactus

Bromheadia spp. \#7 II Orchidaceae

Brooks's Alsophila (E): Alsophila brooksii (Maxon) R. Tryon: \#I II Cyatheaceae

Broughtonia spp. \#7 II Orchidaceae

Browningia spp. \#4 II Cactaceae

Brownleea spp. \#7 II Orchidaceae

Bryobium $=$ Eria

Bucculina $=$ Holothrix

Buchtienia spp. \#7 II Orchidaceae

Buesiella $=$ Rusbyella

Buiningia $=$ Coleocephalocereus

Bulbophyllaria $=$ Bulbophyllum

Bulbophyllum spp. \#7 II Orchidaceae

Bulleyia spp. \#7 II Orchidaceae

Burlingtonia $=$ Rodriguezia

Burnertia spp. \#7 II Orchidaceae

Bumsbaloghia spp. \#7 II Orchidaceae

Byblis spp. \#1 II Byblidaceae

Byblis gigantea J. Lindley: Rainbow Plant (E), Southem Byblis (E) \#1 II Byblidaceac

Byblis liniflora Salisb.: \#1 II Byblidaceae

Cachimbo (S): Platymiscium pleiostachyum Donn. Sm.: \#I II Leguminosae

Cactodendron $=$ Opuntia

Cactus = Mammillaria/Melocactus/Opuntia

Caclus cloporté $(\mathrm{F})=$ Pelecyphora aselliformis Ehrenb.: I Cactaceae

Cacrus disciformis $($ DC. $)$ Kuntze $=$ Strombocactus disciformis $(D C$.) Britton \& Rose

Cactus hache (F): Pelecyphora aselliformis Ehrenb.: I Cactaceae

Cactus Iurbinatus Kuntze = Strombocactus disciformis (DC.) Britton \& Rose

Cadetia spp. \#7 II Orchidaceae

Caelia $=$ Coelia

Caeloglossum $=$ Coeloglossum

Caguí (S): Caryocar costaricense J. D. Sm.: \#1 II Caryocaraceae Caladenia spp. \#7 II Orchidaceae

Calanthe spp. \#7 II Orchidaceae

Calanthidum $=$ Calanthe

Calcearia $=$ Corybas

Calceolaria $=$ Cypripedium

Calceolus $=$ Cypripedium

Caleana spp. \#7 II Orchidaceae

Caleya $=$ Caleana

Califomia Pitcherplant (E): Darlingsonia californica Torrey: \#1 II Sarraceniaceae

Calipogon $=$ Calopogon

Calliphyllon = Epipactis

Callista $=$ Dendrobium

Callithronum $=$ Cephalanthera

Callostylis $=$ Eria

Calochilus spp. \#7 II Orchidaceae

Calochlaena blepharodes (Maxon) G. B. Nair: \#1 II Dicksoniaceae

Calochlaena dubia (R. Br.) M. D. Turner \& R. A. White: Common Ground Fern (E), False Bracken (E), Rainbow Fern (E) \#1 II Dicksoniaceae

Calochlaena javanica (Blume) M. D. Turner \& R. A. White: \#1 II Dicksoniaceae

Calochlaena novae-guineae (Rosenstock) M. D. Turner \& R. A. White: \#1 II Dicksoniaceae

Calochlaena straminea (Labill.) M. D. Tumer \& R. A. White: \#1 II Dicksoniaceae

Calochlaena villosa (C. Chr.) M. D. Tumer \& R. A. White; \#1 II Dicksoniaceae

Caloglossum $=$ Cymbidiella

Calophyllum $=$ Epipactis

Calopogon spp. \#7 II Orchidaceae

Calorchis $=$ Ponthieva 
Calostylis $=$ Eria

Calota $=$ Ceratandra

Caluera spp. \#7 II Orchidaceae

Calymmanthera spp. \#7 II Orchidaceae

Calymmanthium spp. \#4 II Cactaceae

Calypso spp. \#7 II Orchidaceae

Calypsodium $=$ Calypso

Calyptrochilum spp. \#7 II Orchidaceae

Camaridium = Maxillario

Camarotis $=$ Micropera

Camelosialix $=$ Pholidoto

Camilleugenia $=$ Cynorkis

Campanulorchis $=$ Eria

Campylocentrum spp. \#7 II Orchidaceae

Canacorchis $=$ Bulbophyllum

Candelilla (S): Euphorbia antisyphilitica Zucc.: \#1 II Euphorbiaceae

Canebrake Pitcherplant (E): Sarracenia rubra Walter ssp. alabamensis (F. W. \& R. B. Case) Schnell: I Sarraceniaceae

Cántaros (S): Sarracenia spp.\#I I/II Sarraceniaceae

Caoba (S): Sivietenia macrophylla King: \#1 III Meliaceae

Capanemia spp. \#7 II Orchidaceae

Cape Aloe (E): Aloe ferox Mill.: \#1 II Liliaceae

Cardiochilos spp. \#7 II Orchidaceae

Cardiophyllum $=$ Listera

Cardoncillo de Tenerife (S): Ceropegia chrysantha Svent.: \#I II Asclepiadaceae

Cardoncillos (S): Ceropegia spp, \#1 II Asclepiadaceae

Cardones (S): Euphorbia spp. III Euphorbiaceae

Cariocar du Costa Rica (F): Caryocar costaricense J. D. Sm. \#1 II Caryocaraceae

Camegiea spp. \#4 II Cactaceae

Carteretia $=$ Cleisostoma

Carteria = Basiphyllaea

Caryocar costaricense J. D. Sm.: Ajillo (S), Ajo (S), Aji (S),

Almendrillo (S), Almendrón (S), Cagüí (S), Manú (S), Plomillo (S), Cariocar du Costa Rica (F) \#I II Caryocaraceae

Cassyra $=$ Rhipsalis

Castellanosia $=$ Browningia

Catachaetum $=$ Catasefum

Calasetum spp. \#7 II Orchidaceae

Cathea $=$ Calopogon

Cattleya spp. \#7 II Orchidaceae

Cattley a bogotensis Linden = Canleya trianae Linden \& Reichb.f.

Cattleya kimballiana L. Linden \& Rodigas = Cattleya trianae Linden \& Reichb.f.

Cantleya lobata Lindl. = Laelia lobara $($ Lindl.) Veitch

Cattleya trianaei Linden \& Reichb.f.: Christmas Orchid (E) I Orchidaceae

Cantleyopsis spp. \$7 II Orchidaceae

Caucaea spp. \#7 II Orchidaceae

Caularthron spp. \#7 11 Orchidaceae

Celery-leaved Panax (E): Panax quinquefolius L. \#3 II Araliaceae

Centranthera $=$ Pleurothallis

Centrochilus = Habenaria

Centrogenium $=$ Stenorrhynchos

Centroglossa spp. \#7 II Orchidaceae

Centropetalum $=$ Fernandezia

Centrosia $=$ Calanthe

Centrosis $=$ Calanthe

Centrostigma spp. \#7 II Orchidaceae

Cephalangraecum $=$ Ancistrorhynchus

Cephalanthera spp. \#7 II Orchidaceac

Cephalantheropsis spp. \#7 II Orchidaceae

Cephalocereus spp. \#4 II Cactaceae

Cephalocereus chrysomallus (Lemaire) K. Schumann = Pachycereus militaris (Audot) D. Hunt

Cephalocereus militaris (Audot) H. E. Moore $=$ Pachycereus militaris (Audot) D. Hunt

Cephalocleistocactus spp. \#4 II Cactaceae

Cephalomammillaria = Mammillaria

Cephalotus follicularis Labill.: Albany Pitcherplant (E), American Pitcherplant (E) \#1 II Cephalotaceae

Ceraia $=$ Dendrobium

Ceratandra spp. \#7 II Orchidaceae

Ceratandropsis $=$ Ceratandra

Ceratistes $=$ Eriosyce
Ceratium $=$ Eria

Ceratocentron spp. \#7 II Orchidaceae

Ceralochilus spp. \#7 II Orchidaceae

Ceratopsis = Epipogium

Ceratostylis spp. \#7 II Orchidaceae

Ceratozamia spp. \#I II Zamiaceae

Ceratozamia euryphyllidia Vazquez Torres, Sabato \& D. Stevenson: I Zamiaceae

Ceratozamia hildae Landry \& M. Wilson: I Zamiaceae

Ceratozamia kuesteriana Regel: I Zamiaceae

Ceratozamia latifolia Miq.: I Zamiaceae

Ceratozamia matudae Lundell: I Zamiaceae

Ceratozamia mexicana Brongn. I Zamiaceae

Ceratozamia microstrobila Vovides \& Rees: I Zamiaceae

Ceratozamia miqueliana H. Wendl : I Zamiaceae

Ceratozamia norstogii D. Stevenson: I Zamiacene

Cerasozamia robusta Miq.: I Zamiaceae

Cerarozamia sabatoi Vovides, Vazques Tores, Schutzman \& Inglesias: I Zamiaceae

Ceralozamia zaragozae Medellin-Leal: I Zamiaceae

Cereus spp. \#4 II Cactaceae

Cereus chrysomallus (Lemaire) Hemsley = Pachycereus milizaris (Audot) D. Hunt

Cereus militaris Audot $=$ Pachycereus miliraris (Audot) D. Hunt

Cereus schmollii Weingart = Echinocereus schmollii (Weingart) N.P. Taylor

Cerochilus $=$ Hetaeria

Ceropegia spp. Cardoncillos (S) \#I II Asclepiadaceae

Ceropegia abyssinica (A. Rich) Decne.: \#I II Asclepiadaceae

Ceropegia abyssinica var. abyssinica: \#1 II Asclepiadaceae

Ceropegia abyssinica Decne. var. songeensis Huber: \#1 II Asclepiadaceae

Ceropegia achtenii De Wild.: \#1 II Asclepiadaceae

Ceropegia achtenii De Wild, ssp, achtenii: \#1 II Asclepiadaceae

Ceropegia achenii De Wild. ssp. adolfii (Werdermann) H. Huber: \#1 II Asclepiadaceae

Ceropegia achtenii De Wild. ssp. togoensis H. Huber: \#1 II Asclepiadaceae

Ceropegia affinis Vatke: \#I II Asclepiadaceae

Ceropegia africana R. Br, ssp. africana: \#I II Asclepiadaceae

Ceropegia africana $\mathrm{R}$. Br, ssp. barklyi Bruyns: \#I II Asclepiadaceae

Ceropegia africana $\mathrm{R}$. Br. ssp. fortuita $(\mathrm{R}$. A. Dyer) H. Huber = Ceropegia fonuita R. A. Dyer

Ceropegia albisepta Jum, \& H. Perrier var. albisepta: \#1 II Asclepiadaceae

Ceropegia albisepra Jum. \& H. Perrier var, bruceana H. Huber: \#1 II Asclepiadaceae

Ceropegia albisepta Jum. \& H. Perrier var. robynsiana (Werdermann) H. Huber: \#1 II Asclepiadaceae

Ceropegia albisepta Jum. \& H. Perrier var. Iruncata $\mathrm{H}$. Huber = Ceropegia viridis Choux var. Iruncata $(H$. Huber) $H$. Huber

Ceropegia albisepta Jum. \& H. Perrier var. viridis (Choux) H. Huber = Ceropegia viridis Choux var. viridis

Ceropegia ampliata E. Meyer ssp, ampliata: \#l II Asclepiadaceae

Ceropegia ampliata E. Meyer ssp. madagascariensis Lavranos: \#1 II Asclepiadaceae

Ceropegia ampliata E. Meyer ssp. oxyloba H. Huber: \#1 II Asclepiadaceae

Ceropegia anceps S. Moore: \#1 II Asclepiadaceae

Ceropegia angusta N. E. Brown: \#1 II Asclepiadaceae

Ceropegia angustifolia Wight: \#1 II Asclepiadaceae

Ceropegia angustilimba Merrill: \#1 II Asclepiadaceae

Ceropegia antennifera Schltr.: \#l II Asclepiadaceae

Ceropegia arabica H. Huber var. abbreviala Bruyns: \#I II Asclepiadaceae

Ceropegia arabica H. Huber var, arabica: \#1 II Asclepiadaceae

Ceropegia arabica H. Huber var. superba (D. V. Field \& Collenette) Bruyns: \#1 II Asclepiadaceae

Ceropcgia arenaria R. A. Dyer: \#1 II Asclepiadaceae

Ceropegia aridicola W. W. Smith: \#1 II Asclepiadaceae

Ceropegia aristolochioides Decne. ssp. albertina (Moore) H. Huber: \#1 II Asclepiadaceae

Ceropegia aristolochioides Decne. ssp. aristolochioides: \#1 II Asclepiadaceae

Ceropegia aristolochioides Decne. ssp. deflersiana Bruyns: \#1 II Asclepiadaceae 
Ceropegia armandii Rauh: \#I II Asclepiadaceae Ceropegia amottiana Wight: \#I II Asclepiadaceae Ceropegia atacorensis A. Chevalier: \#1 II Asclepiadaceae Ceropegia attenuata Hook.: \#1 II Asclepiadaceae Ceropegia balfouriana Schlechter: \# I I Asclepiadaceae Ceropegia ballyana Bullock: \#1 II Asclepiadaceae Ceropegia barbata R. A. Dyer: \#1 II Asclepiadaceae

Ceropegia barbertonensis N. E. Brown = Ceropegia linearis E. Mey. ssp. woodii (Schlechter) H. Huber

Ceropegia barbigera Bruyns: \#1 11 Asclepiadaceae

Ceropegia barkleyi Hook.f. = Ceropegia africana $\mathrm{R} . \mathrm{Br}$ Bruyns ssp. barklyi Bruyns

Ceropegia bamesii Bruce \& Chatterjee: \#1 II Asclepiadaceae

Ceropegia batesii S. Moore: \#! II Asclepiadaceae

Ceropegia beccariana Martelli: \# 1 II Asclepiadaceae

Ceropegia beddomei Hook.f.: \#1 II Asclepiadaceae

Ceropegia bequaeni De Wild.: \#1 II Asclepiadaceae

Ceropegia bhutanica Hara: \#1 II Asclepiadaceae

Ceropegia blanteri McCann: \#1 II Asclepiadaceae

Ceropegia boerhaaviifolia Defl.: \#1 II Asclepiadaceae

Ceropegia bonafouxii Schumann: \#1 II Asclepiadaceae

Ceropegia bonafouxii Schumann var. linearifolia Stopp: \#111 Asclepiadaceae

Ceropegia bomeensis Merrill: \#1 II Asclepiadaceae

Ceropegia bosseri Rauh \& Buchloh: \#I II Asclepiadaceae

Ceropegia bosseri Rauh \& Buchloh var. razafindratsirana Rauh \& Buchloh = Ceropegia razafindratsirana (Rauh \& Buchloh) Rauh

Ceropegia botnys K. Schum.: \#1 II Asclepiadaceae

Ceropegia bowkeri Harv. ssp. bowkeri: \#1 II Asclepiadaceae

Ceropegia bowkeri Harv. ssp. sororia (Harvey ex Hook.f.) R. A. Dyer: \#1 II Asclepiadaceae

Ceropegia brevicollis Hook.f. = Ceropegia decaisneana Wight var. brevicollis (Hook.f.) H. Huber

Ceropegia brevirosiris Bally \& Field: \#1 II Asclepiadaceae

Ceropegia bulbosa Roxb.: \#1 II Asclepiadaceae

Ceropegia campanulata G. Don var, abinsica (N. E. Brown) H. Huber: \#1 II Asclepiadaceae

Ceropegia campanulata G. Don var. campanulata: \#1 II Asclepiadaceae

Ceropegia campanulata G. Don var. porphyrotricha (W. W. Smith) H. Huber = Ceropegia porphyrotricha W. W. Smith

Ceropegia campanulata $\mathrm{G}$. Don var. pulchella $\mathrm{H}$. Huber $=$ Ceropegia insignis R. A. Dyer

Ceropegia cancellata Rchb.: \#1 II Asclepiadaceae

Ceropegia candelabrum L. var, biflora (L.) M. Ansari: \#I II Asclepiadaceae

Ceropegia candelabrum L. var. candelabrum: \#1 II Asclepiadaceae

Ceropegia candelabrum L. var. tuberosa (Roxburgh) N. P. Singh: \#1 II Asclepiadaceae

Ceropegia camosa E. Mey: \#1 II Asclepiadaceae

Ceropegia cataphyllaris Bullock: \#1 II Asclepiadaceae

Ceropegia ceratophora Svent.: \#1 II Asclepiadaceae

Ceropegia chipiaensis Stopp: \#1 II Asclepiadaceae

Ceropegia chortophylla Werderm.: \#I II Asclepiadaceae

Ceropegia chrisienseniana Hand.-Mazz: \#1 II Asclepiadaceae

Ceropegia chrysantha Svent.: Cardoncillo de Tenerife (S) \#1 II Asclepiadaceae

Ceropegia chrysochroma Huber: \#1 II Asclepiadaceae

Ceropegia ciliata Wight ssp. ciliata: \#1 II Asclepiadaceae

Ceropegia ciliata Wight ssp. ensifolia (Beddome) H. Huber: \#1 II Asclepiadaceae

Ceropegia cimiciodora Oberm.: \#1 II Asclepiadaceae

Ceropegia claviloba Werderm.: \#1 II Asclepiadaceae

Ceropegia connivens $\mathbf{R}$. A. Dyer = Ceropegia fimbriata $\mathbf{E}$. Mey. ssp. connivens (R. A. Dyer) Bruyns

Ceropegia conrathii Schlechter: \#1 II Asclepiadaceae

Ceropegia convolvuloides A. Rich.: \#1 II Asclepiadaceae

Ceropegia copleyae E. A. Bruce \& P. R. O. Bally = Ceropegia crassifolia Schltr. var. copleyae (E. A. Bruce \& P. R. O. Bally) H. Huber

Ceropegia crassifolia Schler, var. copleyae (E. A. Brice \& P. R. O. Bally) H. Huber; \#1 II Asclepiadaceae

Ceropegia crassifolia Schltr. var. crassifolia: \#1 II Asclepiadaceae

Ceropegia cufodonti Chiovenda: \#1 II Asclepiadaceae

Ceropegia cumingiana Decne. ssp. cumingiana: \#1 II Asclepiadaceac
Ceropegia cumingiana Decne. ssp. horsfieldiana (Miquel) H. Huber: \#1 II Asclepiadaceae

Ceropegia cumingiana Decne. ssp. merrillii (Schlechter) $\mathbf{H}$. Huber: \# I II Asclepiadaceae

Ceropegia cycniflora R. A. Dyer: \#1 II Asclepiadaceae

Ceropegia damannii Stopp: \#1 II Asclepiadaceae

Ceropegia decaisneana Wight var, brevicollis (Hook.f.) H. Huber \#1 II Asclepiadaceae

Ceropegia decaisneana Wight var, decaisneana: \#1 II Asclepiadaceae

Ceropegia decidua E. A. Bruce ssp. decidua: \#1 II Asclepiadaceae

Ceropegia decidua E. A. Bruce ssp.pretoriensis R. A. Dyer: \#1 II Asclepiadaceae

Ceropegia decumbens Bally: \#1 II Asclepiadaceae

Ceropegia deightonii Hutch. \& Dalz. ssp. conjuncia H. Huber: \#I II Asclepiadaceae

Ceropegia deightonit Hutch. \& Dalz. ssp. deightonii: \#1 II Asclepiadaceae

Ceropegia deightonii Hutch. \& Dalz. ssp. tisserantii Huber: \#1 II Asclepiadaceac

Ceropegia denticulata K. Sch. ex. Engl. var. brownii (Ledger) P. R. O. Bally: \#I II Asclepiadaceae

Ceropegia denticulata K. Sch. ex. Engl. var. denticulata: \#1 II Asclepiadaceae

Ceropegia devecchii Chiov, var. adelaidae P. R. O. Bally = Ceropegia variegata (Forssk.) Decne.

Ceropegia devecchii Chiov, var. devecchii = Ceropegia variegata (Forssk.) Decne.

Ceropegia dichotoma Haw. ssp. dichotoma: \#1 II Asclepiadaceae Ceropegia dichotoma Haw, ssp. krainzii (Sventenius) Bruyns = Ceropegia krainzii Svent.

Ceropegia dimorpha Humbert: \#1 II Asclepiadaceae

Cerupegia dinteri Schlechter: \#I II Asclepiadaceae

Ceropegia distincta N. E. Br. forma pubescens $\mathbf{H}$. Huber: \#1 $\mathbf{U}$ Asclepiadaceae

Ceropegia distincta N. E. Br. ssp. distincta: \#1 II Asclepiadaceae

Ceropegia distincta N. E. Br. ssp. haygarthii (Schlecter) H. Huber = Ceropegia haygarthii Schlechter

Ceropegia distincta N. E. Br. ssp. lugardiae (N. E. Brown) H. Huber = Ceropegia lugardiae N. E. Brown

Ceropegia distincta $\mathrm{N}$. E. Br. ssp. verruculosa $\mathrm{R}$. A. Dyer = Ceropegia verruculosa (R. A. Dyer) D. V. Field

Ceropegia dorjei C. E. C. Fischer: \#1 II Asclepiadaceae

Ceropegia dubia R. A.Dyer = Ceropegia meyeri-johannis Engl. var. angiensis (De Wild.) H. Huber

Ceropegia effusa $\mathbf{H}$. Huber: \#1 II Asclepiadaceae

Ceropegia elegans Wallich var. elegans: \#1 II Asclepiadaceae

Ceropegia elegans Wallich var. gardneri (Thwaites) H. Huber: \#1 II Asclepiadaceae

Ceropegia euryacme Schltr.: \#1 II Asclepiadaceae

Ceropegia evansii McCann: \#1 II Asclepiadaceae

Ceropegia fantastica Sedgw.: \#1 II Asclepiadaceae

Ceropegia filiformis (Burch.) Schltr: \#1 II Asclepiadaceae

Ceropegia filipendula $\mathrm{K}$. Schum.: \#I II Asclepiadaceae

Ceropegia fimbriata E. Mey. ssp. connivens (R. A. Dyer) Bruyns: \# II Asclepiadaceae

Ceropegia fimbriata E. Mey. ssp. fimbriata: \#1 II Asclepiadaceae

Ceropegia fimbriata E. Mey. ssp. geniculata (R. A. Dyer) Bruyns: \#1 II Asclepiadaceae

Ceropegia fimbriifera Beddome: \#1 II Asclepiadaceae

Ceropegia floribunda N. E. Br.: \#1 II Asclepiadaceae Ceropegia foliosa Bnyns: \#1 II Asclepiadaceae

Ceropegia fortuita R. A. Dyer: \#1 II Asclepiadaceae Ceropegia furcata Werdermann: \#1 11 Asclepiadaceae Ceropegia fusca Bolle: \#1 II Asclepiadaceae Ceropegia fusiformis N. E. Brown: \#1 II Asclepiadaceae Ceropegia galeata H. Huber: \#1 II Asclepiadaceae Ceropegia gemmifera K. Sch.: \#1 II Asclepiadaceae

Ceropegia geniculata R. A. Dyer = Ceropegia fimbriata E. Mey. ssp. geniculata (R. A. Dyer) Bruyns

Ceropegia gilgiana Werdermann: \#1 II Asclepiadaceae

Ceropegia haygarthii Schlechter: \#1 11 Asclepiadaceae Ceropegia hians Svent. var, hians: \#1 II Asclepiadaceae Ceropegia hians Svent. var. striata Sventenius: \#1 II Asclepiadaceae Ceropegia hirsuta Wight \& Am.: \#1 II Asclepiadaceae Ceropegia hofstaetteri Rauh: \#1 II Asclepiadaceae 
Ceropegia hookeri Clarke ex Hook. f. var. hookeri: \#1 II Asclepiadaceae

Ceropegia hookeri Clarke ex Hook. f. var, mollis H. Huber: \#I II Asclepiadaceae

Ceropegia huberi Ansari: \#1 II Asclepiadaceae

Ceropegia humberii H. Huber: \#I II Asclepiadaceae

Ceropegia illegitima H. Huber: \#1 II Asclepiadaceae

Ceropegia imbricata Bruce \& Bally: \#1 II Asclepiadaceae

Ceropegia inflaia Hochst. ex Werderm. in Engl.: \#1 II Asclepiadaceae

Ceropegia insignis R. A. Dyer: \#1 II Asclepiadaceae

Ceropegia intermedia Wight, var, wighrii Hook.f.: \#1 II Asclepiadaceac

Ceropegia jainii Ansari \& Kulk.: \#1 II Asclepiadaceae

Ceropegia juncea Roxb.: \#1 II Asclepiadaceae

Ceropegia kachinensis Prain: \#1 II Asclepiadaceae

Ceropegia krainzii Svent.: \#1 II Asclepiadaceae

Ceropegia kundelunguensis F. Malaisse: \#1 II Asclepiadaceae

Ceropegia langkawiensis Rintz: \#I II Asclepiadaceae

Ceropegia lawii Hook.f: \#1 II Asclepiadaceae

Ceropegia ledermanii Schlechter: \#1 II Asclepiadaceae

Ceropegia leroyi Rauh \& Marn.-Lap.: \#1 II Asclepiadaceae

Ceropegia lindenii Lavranos: \#1 II Asclepiadaceae

Ceropegia linearis E. Mey. ssp. debilis (N. E. Brown) H. Huber: \#1 II Asclepiadaceae

Ceropegia linearis E. Mey. ssp. linearis: \#1 II Asclepiadaceae

Ceropegia linearis E. Mey. ssp. tenuis (N. E. Brown) Bruyns: \#1 II Asclepiadaceae

Ceropegia linearis E. Mey. ssp. woodii (Schlechter) H. Huber: \#1 II Asclepiadaceae

Ceropegia linophylla H. Huber: \#1 II Asclepiadaceae

Ceropegia longifolia Wallich ssp. longifolia: \#1 II Asclepiadaceae

Ceropegia longifolia Wallich ssp. sinensis H. Huber: \#1 II Asclepiadaceae

Ceropegia longifolia Wallich var. exigua $\mathrm{H}$. Huber: \#1 II Asclepiadaceae

Ceropegia loranthiflora K. Schum.: \#1 II Asclepiadaceae

Ceropegia lucida Wallich ssp. dryophila (Schneider) H. Huber: \#I II Asclepiadaceae

Ceropegia lucida Wallich ssp. lucida: \#1 II Asclepiadaceae

Ceropegia ludlowii H. Huber: \#1 II Asclepiadaceae

Ceropegia lugardiae N. E. Brown: \#I II Asclepiadaceae

Ceropegia maccannii Ansari: \#1 11 Asclepiadaceae

Ceropegia macrantha Wight var. macrantha: \#1 II Asclepiadaceae

Ceropegia macrantha Wight var. thorelii (Constantin) $\mathrm{H}$. Huber: \#1 II Asclepiadaceae

Ceropegia maculara Bedd.: \#1 II Asclepiadaceae

Ceropegia madagascariensis Decne.: \#1 II Asclepiadaceae

Ceropegia madens Werdermann: \#1 II Asclepiadaceae

Ceropegia mafekingensis (N. E. Br.) R. A. Dyer: \#1 II Asclepiadaceae

Ceropegia mahabalei Hem. \& Ans.: \#1 II Asclepiadaceae

Ceropegia mairei (Leveillé) H. Huber var. mairei: \#1 II Asclepiadaceae

Ceropegia mairei (Leveillé) H. Huber var. tenella H. Huber: \#1 II Asclepiadaceae

Ceropegia maiuscula Huber: \#1 II Asclepiadaceae

Ceropegia mansouriana Chaudhary \& Lavranos = Ceropegia botrys K. Schum.

Ceropegia mayonae H. Huber: \#1 11 Asclepiadaceae

Ceropegia media (Huber) M. Y. Ansari: \#1 II Asclepiadaceae

Ceropegia melanops H. Huber: \#1 II Asclepiadaceae

Ceropegia meleagris H. Huber: \#1 II Asclepiadaceae

Ceropegia mendesi Stopp: \#1 II Asclepiadaceae

Ceropegia metziana Miq.: \#1 II Asclepiadaceae

Ceropegia meyeri Decne.: \#1 II Asclepiadaceae

Ceropegia meyeri-johannis Engl, var. angiensis (De Wild.) H. Huber: \#1 II Asclepiadaceae

Ceropegia meyeri-johannis Engl. var. meyeri-johannis: \#1 II Asclepiadaceae

Ceropegia meyeri-johannis Engl. var, verdickii (De Wild.) H. Huber: \#1 II Asclepiadaceae

Ceropegia mirabilis H. Huber: \#1 II Asclepiadaceae

Ceropegia monteiroae Hook.f. = Ceropegia sandersonii Hook.f.

Ceropegia monticola W. W. Smith: \#1 II Asclepiadaceae

Ceropegia muliensis W. W. Smith: \#1 II Asclepiadaceae
Ceropegia multiflora Bak. forma puberula (Hiem) H. Huber: A1 II Asclepiadaceae

Ceropegia multiflora Bak, forma pubescens H. Huber: \#1 II Asclepiadaceae

Ceropegia multiflora Bak. ssp. multiflora: \#1 II Asclepiadaccae

Ceropegia multiflora Bak. ssp. tentaculata (N. E. Brown) H. Huber: \#1 II Asclepiadaceae

Ceropegia muzingana F. Malaisse: \#1 II Asclepiadaceae

Ceropegia nana Coll. \& Hemsl.: \#1 II Asclepiadaceae

Ceropegia ngoyana F. Malaisse = Ceropegia peulhorum A. Chev. var. peulhorum

Ceropegia nigra N. E. Brown: \#1 II Asclepiadaceae

Ceropegia nilotica Kotschy var. nilotica: \#1 II Asclepiadaceae

Ceropegia nilotica Kotschy var. plicata (E. A. Bruce) H. Huber = Ceropegia plicala E.A. Bruce

Ceropegia nilotica Kotschy var. simplex H. Huber = Ceropegia denticulata K. Sch. ex. Engl, var. denticulata

Ceropegia noorjahaniae Ansari: \#1 II Asclepiadaceac

Ceropegia nuda Hutch. \& Bruce; \#I II Asclepiadaceae

Ceropegia occidentalis R. A. Dyer: \#1 II Asclepiadaceae

Ceropegia occulta R. A. Dyer: \#1 II Asclepiadaceae

Ceropegia oculata Hook, var. occulta: \#1 II Asclepiadaceae

Ceropegia oculata Hook. var. subhirsuta H. Huber: \#1 II Asclepiadaceac

Ceropegia odorata Nimmo ex Hook.f.: \#1 II Asclepiadaceae

Ceropegia omissa Huber: \# I II Asclepiadaceae

Ceropegia pachystelma Schlecter: \#1 II Asclepiadaceae

Ceropegia pachystelma Schlecter ssp. undulata (N. E. Brown) H. Huber: \#1 II Asclepiadaceae

Ceropegia panchganiensis Blatter \& McCann: \# II Asclepiadaceae

Ceropegia paohsingensis Tsiang \& P. T. Li: \#1 II Asclepiadaceae

Ceropegia papillata N. E. Brown var. cordiloba (Werdermann) H. Huber: \#1 II Asclepiadaceae

Ceropegia papillata N. E. Brown var. papillata: \#1 II Asclepiadaceae

Ceropegia paricyma N. E. Brown: \#1 II Asclepiadaceae

Ceropegia parviflora Trimen: \#1 II Asclepiadaceae

Ceropegia parriciae Rauh \& Buchloh = Ceropegia mafekingensis (N. E. Br.) R. A, Dyer

Ceropegia peteri Werderm.: \#1 II Asclepiadaceae

Ceropegia petignatii Rauh: \#1 II Asclepiadaceae

Ceropegia peulhorum A. Chev, var. breviloba Huber: \#1 II Asclepiadaceae

Ceropegia peulhorum A. Chev. var. peulhorum: \#1 II Asclepiadaceae

Ceropegia picta (Schlechter) H. Huber =

Ceropegia plicata E. A. Bruce: \#1 II Asclepiadaceae

Ceropegia polkniniana Bruyns: \#1 II Asclepiadaceae

Ceropegia porphyrotricha W. W. Smith: \#1 II Asclepiadaceae

Ceropegia powysï D. V. Field: \#1 II Asclepiadaceae

Ceropegia praetermissa Raynal \& A. Raynal: \#1 II Asclepiadaceae

Ceropegia pubescens Wallich: \#I II Asclepiadaceae

Ceropegia purpurascens K. Schum. ssp. purpurascens: \#1 II Asclepiadaceae

Ceropegia purpurascens K. Schum. ssp. thysanotos (Werderm.) Huber: \#1 II Asclepiadaceae

Ceropegia pusilla Wight \& Am.: \#1 II Asclepiadaceae

Ceropegia pygmaea Schinz var. pumila (N. E. Brown) H. Huber: \#1 II Asclepiadaceae

Ceropegia pygmaea Schinz var. pygmaea: \#1 II Asclepiadaceae

Ceropegia racemosa N. E. Br. ssp. glabra $\mathbf{H}$. Huber: \#1 II Asclepiadaceae

Ceropegia racemosa N. E. Br. ssp. racemosa: \#1 II Asclepiadaceae

Ceropegia racemosa N. E. Br. ssp. secamonoides (Moore) H. Huber: \#1 II Asclepiadaceae

Ceropegia racemosa N. E. Br. ssp. setifera (Schlechter) H. Huber: \#1 II Asclepiadaceae

Ceropegia radicans Schltr. ssp, radicans: \#1 II Asclepiadaceae.

Ceropegia radicans Schltr. ssp. smithii (M. R. Henderson) R. A. Dyer: \#1 II Asclepiadaceae

Ceropegia raizadiana C. $\mathbf{R}$. Babu: \#1 II Asclepiadaceae

Ceropegia razafindratsirana (Rauh \& Buchloh) Rauh: \#1 II Asclepiadaceae

Ceropegia rendallii N. E. Br.: \#I II Asclepiadaceae

Ceropegia renzii Stopp: \#I II Asclepiadaceae

Ceropegia rhynchantha Schltr.: \#1 II Asclepiadaceae 
Ceropegia ringens A. Rich.: \#1 II Asclepiadaceae Ceropegia ringoetii De Wild.: \#1 II Asclepiadaceac

Ceropegia robynsiana Werdermann = Ceropegia albisepta Jum. \& H. Perrier var. robynsiana (Werdermann) H. Huber

Ceropegia rollae Hemadri: \#I II Asclepiadaceae

Ceropegia rudatisii Schltr:: \#l II Asclepiadaceae

Ceropegia rupicola Defl. var. rupicola: \#1 II Asclepiadaceae

Ceropegia nipicola Defl. var. stictantha N. P. Taylor: \#I II Asclepiadaceae

Ceropegia sahyadrica Ansari \& Kulk.: \#1 I Asclepiadaceae

Ceropegia salicifolia $\mathbf{H}$. Huber: \#1 II Asclepiadaceae

Ceropegia sandersonii Hook.f.: \#I II Asclepiadaceae

Ceropegia sankuruensis Schltr.: \#1 II Asclepiadaceae

Ceropegia santapaui Wadh. \& Ans.: \#1 II Asclepiadaceae

Ceropegia saxarilis Jumelle \& H. Perrier: \#1 II Asclepiadaceae

Ceropegia scabra Jumelle \& H.Perrier: \#I II Asclepiadaceae

Ceropegia scabriflora N. E. Br.: \#1 II Asclepiadaceae

Ceropegia schaijesorum Malaisse: \#1 II Asclepiadaceae

Ceropegia schinziana Bullock: \#1 II Asclepiadaceae

Ceropegia schliebenii Markgraf: \#1 II Asclepiadaceae

Ceropegia schumanniana Swarupanandan \& Mangaly: \#! II Asclepiadaceae

Ceropegia senegalensis H. Huber: \#1 II Asclepiadaceae

Ceropegia sepium Deflers: \#1 II Asclepiadaceae

Ceropegia seticorona E. A. Bruce var. dilatiloba P. R. O. Bally: \#1 II Asclepiadaceae

Ceropegia seticorona E. A. Bruce var. seticorona: \#1 II Asclepiadaceac

Ceropegia setifera Schlechter var. setifera = Ceropegia racemosa N. E. Br. ssp. setifera (Schlechter) H. Huber

Ceropegia simoneae Rauh: \#1 II Asclepiadaceae

Ceropegia sobolifera N. E. Br. var. nephroloba Huber: \#1 II Asclepiadaceae

Ceropegia sobolifera var. sobolifera: \#1 II Asclepiadaceae

Ceropegia somalensis Chiov. forma erostrata $\mathrm{H}$. Huber: \#1 II Asclepiadaceae

Ceropegia somalensis Chiov. forma somalensis: \#1 II Asclepiadaceae

Ceropegia sootepensis Craib: \#1 II Asclepiadaceae

Ceropegia speciosa Huber: \#1 II Asclepiadaceae

Ceropegia spiralis Wight: \#1 11 Asclepiadaceae

Ceropegia splendida (K. Schumann) H. Huber: \#1 II Asclepiadaceae

Ceropegia stapeliiformis Haw. ssp. serpentina (E. A. Bruce) R. A. Dyer: \#1 II Asclepiadaceae

Ceropegia stapeliformis Haw. ssp. stapeliformis: \#1 II Asclepiadaceae

Ceropegia stenantha K. Schumann: \#1 II Asclepiadaceae

Ceropegia stenoloba Werderm. var, australis H. Huber: \#1 II Asclepiadaceae

Ceropegia stenoloba Werderm. var. moyalensis H. Huber: \#I II Asclepiadaceae

Ceropegia stenoloba Hochst. ex Werdermann var. stenoloba: \#1 II Asclepiadaceac

Ceropegia stentiae E. A. Bruce: \#1 II Asclepiadaceae

Ceropegia subaphylla K. Sch.: \#1 II Asclepiadaceae

Ceropegia succulenta E. A. Bruce = Ceropegia albisepta Jum. \& H. Perrier var. bruceana $\mathrm{H}$. Huber

Ceropegia superba D. V. Field \& I. S. Collenette = Ceropegia arabica H. Huber (D. V. Field \& I. S. Collenette) Bruyns

Ceropegia swazionum D. V. Field: \#1 II Asclepiadaceae

Ceropegia talbotii S. Moore: \#1 II Asclepiadaceae

Ceropegia taprobanica H. Huber: \#1 II Asclepiadaceae

Ceropegia teniana Hand.-Mazz: \#1 II Asclepiadaceae

Ceropegia thomcroftii N. E. Brown = Ceropegia crassifolia Schltr. var. crassifolia

Ceropegia thwairesii Hook.: \#1 II Asclepiadaceac

Ceropegia tihamana Chaudhary \& Lavranos: \#1 II Asclepiadaceae

Ceropegia tomentosa Schltr.: \#1 II Asclepiadaceae

Ceropegia tourana A. Chevalier: \#1 II Asclepiadaceae

Ceropegia turricula E. A. Bruce: \#1 II Asclepiadaceae

Ceropegia ugeni C. E. C. Fischer: \#1 II Asclepiadaceae

Ceropegia umbraticola K. Schum.: \#1 II Asclepiadaceae

Ceropegia vanderystii De Wild.: \#1 II Asclepiadaceae

Ceropegia variegata (Forssk.) Decne.: \#1 II Asclepiadaceae

Ceropegia variegata N. E. Br var. comigera $\mathrm{H}$. Huber $=$ Ceropegia variegata (Forssk.) Decne
Ceropegia verruculosa (R. A.Dyer) D. V.Field: \#1 II Asclepiadaceae

Ceropegia vignaldiana A. Rich. = Ceropegia bulbosa Roxb.

Ceropegia vincaefolia Hook.: \#I II Asclepiadaceae

Ceropegia viridis Choux var. truncata $(\mathrm{H}$. Huber) $\mathrm{H}$. Huber: \#1 II Asclepiadaceae

Ceropegia viridis Choux var. viridis: \#I II Asclepiadaceae

Ceropegia volubilis N. E. Br.: \#1 II Asclepiadaceae

Ceropegia wallichii Wight: \#I II Asclepiadaceae

Ceropegia yorubana Schltr.: \#1 II Asclepiadaceae

Ceropegia zeyheri Schltr: \#1 II Asclepiadaceae

Cestichis = Liparis

Chaenanthe spp. \#7 II Orchidaceae

Chaetocephala $=$ Myoxanthus

Chaffeyopunria $=$ Opuntia

Chamaeangis spp. \#7 II Orchidaceae

Chamaeanthus spp. \#7 11 Orchidaceae

Chamaecereus = Echinopsis

Chamaegastrodia spp. \#7 II Orchidaceae

Chamaelobivia $=$ Echinopsis

Chamaeorchis $=$ Chamorchis

Chamaerepes $=$ Herminium

Chamaesyce aloto (G. Forster) Croizat $=$ Euphorbia aloto $\mathrm{G}$. Forster

Chamelophyion spp. \#7 II Orchidacese

Chamorchis spp. \#7 II Orchidaceae

Changnienia spp. \#7 II Orchidaceae

Chaseella spp. \#7 II Orchidaceae

Chaubardia spp. \#7 II Orchidaceae

Chaubardiella spp. \#7 II Orchidaceae

Chauliodon spp. \#7 II Orchidaceae

Chaute (F): Ariocarpus trigonus (F. A. C. Weber) K. Schum.: I Cactaceae

Chaute (F): Ariocarpus retusus Scheidw.: I Cactaceae

Chautle (F): Ariocarpus inigonus (F. A. C. Weber) K. Schum.: I Cactaceae

Chautle (F): Ariocarpus retusus Scheidw.: I Cactaceac

Cheiradenia spp. \#7 II Orchidaceae

Cheirupterocephalus = Malaxis

Cheirorchis $=$ Cordiglotis

Cheirostylis spp. \#7 II Orchidaceae

Chelonanthera $=$ Pholidola

Chelonistele spp. \#7 II Orchidaceae

Chiapasia $=$ Disocacius

Chichipia $=$ Polaskia

Chigua spp. \#1 I Zamiaceae

Chigua bernalii D. Stevenson: I Zamiaceae

Chigua restrepoi D. Stevenson: 1 Zamiaceae

Chilean False Larch (E): Firzroya cupressoides (Molina) I. M. Johnston: I Cupressaceae

Chilenia $=$ Eriosyce

Chileniopsis $=$ Eriosyce

Chileocactus $=$ Eriosyce

Chileorebutia $=$ Eriosyce

Chiliorebutia $=$ Eriosyce

Chilita $=$ Mammillario

Chiloglonis spp. \#7 II Orchidaceae

Chilopogon = Appendicula

Chiloschista spp. \#7 II Orchidaceae

Chilyanthum $=$ Oncidium

Chimón (S): Alsophila salvinii Hooker: \#1 II Cyatheaceae

Chitonanthera spp. \#7 II Orchidaceae

Chitonochilus spp. \#7 II Orchidaceae

Chloidia $=$ Tropidia

Chloraea spp. \#7 II Orchidaceae

Chlorosa spp. \#7 II Orchidaceae

Chnoophora $=$ Cyathea

Choeradoplectron = Habenaria

Chondradenia $=$ Orchis

Chondrorhyncha spp. \#7 II Orchidaceae

Chondroscaphe spp. \#7 II Orchidaceae

Christensonia spp. \#7 II Orchidaceae

Christmas Orchid (E): Cattleya trianaei Linden \& Reichb.f: I Orchidaceae

Chroniochilus spp. \#7 II Orchidaceae

Chrysalidocarpus decipiens Becc.: \#1 II Palmae

Chrysobaphus = Anoectochilus

Chrysocactus = Parodia 
Chrysocycnis spp. \#7 II Orchidaceae

Chrysoglossum spp. \#7 II Orchidaceae

Chusua spp. \#7 II Orchidaceae

Chysis spp. \#7 II Orchidaceae

Chytroglossa spp. \#7 II Orchidaceae

Cibotium arachnoideum (C. Chr.) Holttum: \#I II Dicksoniaceae

Ciborium barometz (L.) J. Smith: \#I II Dicksoniaceae

Cibotium chamissoi Kaulf.: \#I II Dicksoniaceae

Cibotium cumingii Kunze: Golden Fern (E) \#1 II Dicksoniaceae

Cibotium glaucum (J. Smith) Hook. \& Am.: \#1 II Dicksoniaceae

Ciborium hawaiiense Nakai \& Ogura: \#I II Dicksoniaceae

Cibotium menziesii Hook: \#1 II Dicksoniaceae

Cibotium nealiae Degener: \#I II Dicksoniaceae

Cibotium regale Versch. \& Lem.: \#1 II Dicksoniaceae

Cibotium schiedei Schltr. \& Cham.: \#1 II Dicksoniaceae

Cibotium splendens (Gaudich.) Kraj.: \#1 II Dicksoniaceae

Cicadas (S): CYCADACEAE spp. III Cycadaceae

Cinnabarinea $=$ Echinopsis

Cintia spp. \#4 II Cactaceae

Cionisaccus $=$ Goodyera

Cipocereus spp. \#4 II Cactaceae

Ciprés de las Guaitecas (S): Pilgerodendron uviferum (Don) Florin: 1 Cupressaceae

Cinipedium $=$ Cypripedium

Cirrhaea spp. \#7 II Orchidaceae

Cirrhopetalum $=$ Bulbophyllum

Cischweinfia spp. \#7 II Orchidaceae

Cistella $=$ Geodorum

Claderia spp. \$7 $\mathbf{U}$ Orchidaceae

Cladobium $=$ Scaphyglottis/Stenorrhynchos

Cladorhiza $=$ Corallorrhiza

Clanwilliam Aloe (E): Aloe comosa Marloth \& A.Berger: \#1 II Liliaceae

Clavarioidia $=$ Opuntia

Cleisocentron spp. \#7 II Orchidaceae

Cleisomeria spp. \#7 II Orchidaceae

Cleisostoma spp. $\$ 7$ II Orchidaceae

Cleisostomopsis spp. \#7 II Orchidaceae

Cleistes spp. \#7 II Orchidaceae

Cleistocactus spp. \#4 II Cactaceae

Cleistocereus $=$ Cleistocactus

Clematepistephium spp. \#7 II Orchidaceae

Cliff Spurge (E): Euphorbia misera Benth.: \#1 II Euphorbiaceae

Clinhymenia $=$ Cryptarrhena

Clistanthocereus $=$ Cleistocactus

Clowesia spp. \#7 II Orchidaceae

Club-shaped Euphorb (E): Euphorbia clava Jacq.: \#1 II Euphorbiaceae

Cnemidaria $=$ Cyathe

Cnemidaria alatissima Stolze $=$ Cyathea alatissima (Stolze) $?$

Cnemidaria amabilis (C. Morton in Steyerm.) R. Tryon = Cyathea amabilis (C. Morton in Steyerm.) ?

Cnemidaria apiculata (Hook. \& Baker) Stolze = Cyathea aristata Domin

Cnemidaria bella (Reichb f, ex Mett.) R. Tryon: \#I II Cyatheaceae

Cnemidaria chocoensis Stolze $=$ Cyathea chocoensis $($ Stolze) ?

Cnemidaria choricarpa (Maxon) R. Tryon: \#1 II Cyatheaceae

Cnemidaria cocleana Stolze = Cyathea cocleana (Stolze) ?

Cnemidaria consimilis Stolze = Cyathea consimilis $($ Stolze) $?$

Cnemidaria cruciata $($ Desv.) Stolze $=$ Cyathea leprieurii $($ Kunze $)$ Domin

Cnemidaria decurrens (Liebm.) R. Tryon: \#1 II Cyatheaceae

Cnemidaria ewanii (Alston) R. Tryon = Cyashea ewanii Alston

Cnemidaria glandulosa Stolze: \#1 II Cyatheaceae

Cnemidaria grandifolia (Willd.) Proctor var. grandifolia = Cyathea grandifolia Willd.

Cnemidaria horrida (L.) C. Presl: \#1 II Cyatheaceae

Cnemidaria karsteniana (Klotzsch) $\mathrm{R}$. Tryon = Cyathea karsteniana (Klotzsch) Domin

Cnemidaria mutica (Christ) R. Tryon var, chiricana (Maxon) Stolze: \#1 II Cyatheaceae

Cnemidaria mutica (Christ) R. Tryon var, contigua (Maxon) Stolze: \#1 II Cyatheaceae

Cremidaria mutica (Christ) R. Tryon var. grandis (Maxon) Stolze: \#1 II Cyatheaceae

Cnemidaria mutica (Christ) R. Tryon var. mutica: \#1 II Cyatheaceae
Cnemidaria nervosa (Maxon) $\mathrm{R}$. Tryon = Cyathea nervosa $($ Maxon)

Cnemidaria quitensis $($ Domin $) \mathrm{R}$. Tyron $=$ Cyathea andicola Domin Cnemidaria roraimensis $($ Domin $) \mathbf{R}$. Tryon $=$ Cyathea roraimensis (Domin) Domin

Cnemidaria singuiaris Stolze = Cyathea singularis $($ Stolze) ?

Cnemidaria speciosa Presl: \#1 II Cyatheaceae

Cnemidaria spectabilis (Kunze) $R$. Tryon var. colombiensis Stolze = Cyarhea spectabilis (Kunze) Domin var. colombiensis (Stolze)?

Cnemidaria spectabilis (Kunze) R. Tryon var. spectabilis = Cyathea spectabilis (Kunze) Domin var. spectabilis

Cnemidaria stolzeana L. D. Gómez: \#1 II Cyatheaceae

Cnemidaria suprastrigosa R. C. Moran: \#1 II Cyatheaceae

Cnemidaria tryoniana Stolze: \#I II Cyatheaceae

Cnemidaria uleana (Samp.) R. Tyron var. uleana = Cyathea subarborescens Domin

Cnemidaria uleana (Samp.) R. Tryon var. abitaguensis (Domin) Stolze $=$ Cyathea abitaguensis Domin

Cnemidaria varians R. C. Moran: \#1 II Cyatheaceae

Cnemidia $=$ Tropidia

Cobra Lily (E): Darlingtonia californica Torrey: \#1 II Sarraceniaceae

Coccineorchis $=$ Stenorrhynchos

Cochemiea = Mammillaria

Cochiseia $=$ Escobaria

Cochleanthes spp. \#7 11 Orchidaceae

Cochleorchis spp. \#7 II Orchidaceae

Cochlia $=$ Bulbophyllum

Cochlioda spp. \#7 II Orchidaceae

Codonorchis spp. \#7 II Orchidaceae

Cudonosiphon spp. \#7 II Orchidaceae

Coelandria $=$ Dendrobium

Coelia spp. \#7 II Orchidaceae

Coeliopsis spp. \#7 II Orchidaceae

Coeloglossum spp. \#7 II Orchidaceae

Coelogyne spp. \#7 II Orchidaceae

Cogniauxiocharis $=$ Stenorrhynchos

Cohnia $=$ Cohniello

Cohniella spp. \#7 II Orchidaceae

Coilochilus spp. \#7 II Orchidaceae

Coilostylis = Epidendrum

Cola de mono (S): Cyathea mexicana Schldl. \& Cham.: \#! II Cyatheaceae

Colax $=$ Pabstia

Coleocephalocereus spp. \#4 II Cactaceae

Collabiopsis $=$ Collabium

Collabium spp. \#7 II Orchidaceae

Collea $=$ Pelexio

Colombiana $=$ Pleuroshallis

Coloradoa $=$ Sclerocactus

Coloradoa mesae-verdae Boiss. \& Davidson = Sclerocactus mesaeverdae (Boiss. \& Davidson) L. Benson

Commersorchis $=$ Dendrobium

Common Aloe (E): Aloe vera (L.) N. L. Burman var, vera: NC Liliaceae

Common Ground Fern (E): Calochlaena dubia (R. Br.) M. D. Tumer \& R. A. White: \#! II Dicksoniaceae

Common Snowdrop (E): Galanthus nivalis L.: \#1 II Amaryllidaceae

Common Sternbergia (E): Stembergia lutea (L.) Ker Gawl. ex Spreng.: \#I II Amaryllidaceae

Commoner Lignum Vitae (E): Guaiacum officinale L: \#1 II Zygophyllaceae

Comparettia spp. \#7 II Orchidaceae

Comperia spp. \#7 II Orchidaceae

Conchidium = Eria

Conchochilus $=$ Appendicula

Condylago spp. \#7 II Orchidaceae

Conospsidium $=$ Plaranthera

Conosialix $=$ Eria

Consolea $=$ Opuntia

Constantia spp. \#7 II Orchidaceae

Copiapoa spp. \#4 II Cactaceae

Coppensia $=$ Oncidium

Coralliokyphos $=$ Moerenhouria

Corallorhiza $=$ Corallorrhiza

Corallorrhiza spp. \#7 II Orchidaceae 


\section{Cordanthera $=$ Siellilabium}

Cordiglottis spp. \#7 II Orchidaceae

Cordula $=$ Paphiopedilum

Cordula amabilis (Hallier) Merr. $=$ Paphiopedilum bullenianum (Reichb.f.) Pfitzer var. bullenianum

Cordula appletoniana (Gower) Rolfe $=$ Paphiopedilum appletonianum (Gower) Rolfe

Cordula argus (Reichb.f) Rolfe $=$ Paphiopedilum argus (Reichb.f.) Stein

Cordula barbata (Lindl.) Rolfe $=$ Paphiopedilum barbatum (Lindl.) Rolfe

Cordula bellarula $($ Reichb.f.) Rolfe $=$ Paphiopedilum bellatulum (Reichb.f.) Stein

Cordula boxallii (Reichb.f.) Rolfe $=$ Paphiopedilum villosum (Lindl. $)$ Stein var. boxallii (Reichb.f.) Pfitzer

Cordula bulleniana (Reichb.f.) Rolfe $=$ Paphiopedilum bullenianum (Reichb.f.) Pfitzer var, bullenianum

Cordula callosa $($ Reichb.f.) Rolfe = Paphiopedilum callosum (Reichb.f.) Stein var. callosum

Cordula charlesworthii (Rolfe) Rolfe $=$ Paphiopedilum charlesworthii (Rolfe) Pfitzer

Cordula ciliolaris (Reichb.f.) Rolfe $=$ Paphiopedilum ciliolare (Reichb.f) Stein

Cordula concolor (Lindl.) Rolfe $=$ Paphiopedilum concolor (Lindl.) Pfitzer

Cordula cunisii (Reichb.f.) Rolfe $=$ Paphiopedilum superbiens (Reichb.f.) Siein var. curtisii (Reichb.f.) G. J. Braem

Cordula doyana (Lindl.) Rolfe $=$ Paphiopedilum dayanum (Lindl.) Stein

Cordula drunyi (Bedd.) Rolfe $=$ Paphiopedilum druryi (Lindl.) Stein

Cordula fairrieana (Lindl.) Rolfe = Paphiopedilum fairrieanum (Lindl.) Stein

Cordula glandulifera (Blume) Rolfe = Paphiopedilum glanduliferum (Blume) Stein var, glanduliferum

Cordula glaucophylla (J. J. Sm.) Rolfe = Paphiopedilum glaucophyllum J. J. Sm.

Cordula godefroyae (God.-Leb.) Rolfe = Paphiopedilum godefroyae (God.-Leb.) Stein var. godefroyae

Cordula haynaldiana (Reichb.f.) Rolfe = Paphiopedilum haynaldianum (Reichb.f.) Stein

Cordula hirsutissima (Lindl. ex Hook.) Rolfe = Paphiopedilum hirsutissimum (Lindl. ex Hook.) Stein var. hirsutissimum

Cordula hookerae (Reichb.f.) Rolfe $=$ Paphiopedilum hookerae (Reichb.f.) Stein var. hookerae

Cordula insignis (Wallich ex Lindl.) Raf. = Paphiopedilum insigne (Wallich ex Lindl.) Pfitzer

Cordula javanica (Reinw. ex Lindl.) Rolfe = Paphiopedilum jovanicum (Reinw. ex Lindl.) Pfitzer var. javanicum

Cordula lawrenceana (Reichb.f.) Merr. = Paphiopedilum lawrenceanum (Reichb.f.) Pfitzer

Cordula lowii (Lindl.) Rolfe = Paphiopedilum lowii (Lindl.) Stein var. lowii

Cordula mastersiana $($ Reichb.f. $)$ Rolfe $=$ Paphiopedilum mastersianum (Reichb.f.) Stein

Cordula nigrita (Reichb.f.) Mer. $=$ Paphiopedilum barbatum (Lindl.) Rolfe

Cordula nivea $($ Reichb.f.) Rolfe $=$ Paphiopedilum niveum $($ Reichb.f. $)$ Stein

Cordula parishii (Reichb.f.) Rolfe $=$ Paphiopedilum parishii (Reichb.f.) Stein

Cordula petri (Reichb.f.) Rolfe $=$ Paphiopedilum dayanum $($ Lindl. $)$ Stein

Cordula philippinensis (Reichb.f.) Rolfe = Paphiopedilum philippinense (Reichb.f.) Stein var. philippinense

Cordula purpurasa (Lindl.) Rolfe = Paphiopedilum purpuratum (Lindl.) Stein

Cordula rothschildiana (Reichb.f.) Merr. = Paphiopedilum rothschildianum (Reichb.f.) Stein

Cordula sanderiana (Reichb.f.) Rolfe = Paphiopedilum sanderianum (Reichb.f.) Stein

Cordula spiceriana (Reichb.f. ex Masters \& T. Moore) Rolfe = Paphiopedilum spicerianum (Reichb.f. ex Masters \& T. Moore) Pfitzer

Cordula stonei $($ Hook. $)$ Merr. $=$ Paphiopedilum stonei $($ Hook.) Stein

Cordula superbiens (Reichb.f.) Rolfe $=$ Paphiopedilum superbiens (Reichb.f.) Stein var. superbiens
Cordula tonsa (Reichb.f.) Rolfe $=$ Paphiopedilum tonsum $($ Reichb.f.) Stein var. tonsum

Cordula venusta $($ Wallich ex Sims) Rolfe = Paphiopedilum venustum (Wallich ex Sims) Pfitzer ex Stein

Cordula victoria-mariae (Sander ex Mast.) Rolfe = Paphiopedilum victoria-mariae (Sander ex Mast.) Rolfe

Cordula villosa (Lindl.) Rolfe = Paphiopedilum villosum (Lindl.) Stein var, villosum

Cordula violascens $($ Schlechter) Rolfe $=$ Paphiopedilum violascens Schlechter

Cordyla $=$ Nervilia

Cordylestylis $=$ Goodyero

Cormophyllum $=$ Cyathea

Corryocactus spp. \#4 II Cactaceae

Corryocereus $=$ Corryocactus

Corunastylis $=$ Prasophyllum

Conyanthes spp. \#7 II Orchidaceae

Corybas spp. \#7 II Orchidaceae

Corycium spp. \#7 II Orchidaceae

Corydandra $=$ Galeandra

Corymbis $=$ Corymborkis

Corymborchis $=$ Corymborkis

Corymborkis spp. \#7 II Orchidaceae

Corynanthes $=$ Coryanthes

Corynopuntia $=$ Opuntia

Coryphantha spp. \#4 I/II Cactaceae

Coryphantha minima Baird = Escobario minima $($ Baird $)$ D. R. Hunt

Conphantha nellieae Croizat $=$ Escobaria minima (Baird) D. R. Hunt

Conphantha sneedii (Britton \& Rose) A. Berger var. leei (Rose ex Bödeker) L. Benson = Escobaria sneedii Britt \& Rose var. leel (Rose ex Bödeker) D. R. Hunt

Coryphantha sneedii (Britton \& Rose) A. Berger var. sneedii = Escobaria sneedii Britt. \& Rose var. sneedii

Coryphantha werdermannii Bödeker: Jabali Pincushion Cactus (E) I Cactaceae

Conysanthes $=$ Conybas

Costaricaea $=$ Hexisea

Costus Root (E): Saussurea costus (Falc.) Lipsch.: I Cornpositae

Corronia spp. \#7 II Orchidaceae

Corylolabium $=$ Stenorrhynchos

Cranichis spp. \#7 II Orchidaceae

Cremastra spp. \#7 II Orchidaceac

Crepidium = Malaxis

Cribbia spp. \#7 II Orchidaceae

Crimson Pitcherplant (E): Sarracenia leucophylla Raf.: \#1 II Sarraceniaceae

Crinonia $=$ Pholidota

Criogenes $=$ Cypripedium

Criosanthes $=$ Cypripedium

Cristóbal (S): Platymiscium pleiostachyum Donn. Sm.: \#1 II Leguminosae

Crocodeilanthe $=$ Pleurothallis

Crossangis = Diaphananshe

Crossoglossa spp. \#7 II Orchidaceae

Crown of Thoms (E): Euphorbia milii Des Moul, var, mili: \#1 II Euphorbiaceae

Crown of Thoms (E): Euphorbia milii Des Moul. var. splendens (Bojer ex Hook.) Ursch \& Léandri: \#1 II Euphorbiaceae

Crybe spp. \#7 II Orchidaceae

Cnyptanthemis $=$ Rhizanthella

Cryptarrhena spp. \#7 II Orchidaceae

Cryptocentrum spp. \#7 II Orchidaceae

Cryptocereus = Selenicereus

Cryptochilus spp. \#7 II Orchidaceac

Cryptoglotris $=$ Podochilus

Cryptophoranthus spp. \#7 11 Orchidaceae

Crypropus spp. \#7 II Orchidaceae

Cryptopylos spp. \#7 II Orchidaceae

Cryptosaccus $=$ Leochilus

Cryprosanus $=$ Leochilus

Cryptostylis spp. \#7 II Orchidaceae

Clenorchis $=$ Angraecum

Cuban Mahogany (E): Swietenia mahagoni (L.) Jacq.: \# I II Meliaceae

Cuculina $=$ Catasefum 
Cuitlauzina spp. \#7 II Orchidaceae

Culcira charlii ?: \#1 II Dicksoniaceae

Culcita coniifolia (Hook.) Maxon: \#1 II Dicksoniaceae

Culcita dubia $(\mathbf{R}, \mathrm{Br}$.) Maxon = Calochlaena dubia $(\mathbf{R} . \mathrm{Br}$.)

M. D. Tumer \& R. A. White

Culcita javanica (Blume) Maxon = Calochlaena javanica (Blume) M. D. Tumer \& R. A. White

Culcita macrocarpa C.Presl: \# I II Dicksoniaceae

Culcita straminea (Labill.) Maxon = Calochlaena straminea (Labill) M. D. Turner \& R. A. White

Culcira villosa C.Chr. = Calochlaena villosa (C. Chr.) M. D. Turner \& R. A. White

Cullmannia $=$ Peniocereus

Cumarinia $=$ Coryphantha

Cumulopuntia = Opuntia

Curaçao Aloe (S): Aloe vera (L.) N. L. Burman var. vera: NC Liliaceae

Cutsis $=$ Dichromanthus

Cyanaeorchis spp. \#7 II Orchidaceae

Cyanicula spp. \#7 II Orchidaceae

Cyanorchis = Phaius

CYATHEACEAE spp.: Tree ferns (E), Helechos arborescentes (S), Fougères arborescentes (F) \#1 II Cyatheaceae

Cyathea abbotrii Maxon: \#I II Cyatheaceae

Cyathea abitaguensis (Domin) Domin: \#1 II Cyatheaceae

Cyathea acanthomelas Fée: \#1 II Cyatheaceae

Cyathea acanthophora Holttum: \#1 II Cyatheaceae

Cyathea aciculosa Copel.: \#1 II Cyatheaceae

Cyathea acrostichoides (Alderw.) Domin: \#I II Cyatheaceae

Cyathea acuminata Copel.: \#1 II Cyatheaceae

Cyathea acutidens (Christ) Domin: \#1 II Cyatheaceae

Cyathea aeneifolia (Alderw.) Domin var. aeneifolia: \#1 II Cyatheaceae

Cyathea aeneifolia (Alderw.) Domin var. macrophylla Holttum: \#1 II Cyatheaceae

Cyathea aeneifolia (Alderw.) Domin var. melanacantha (Copel.) Holttum: \#1 II Cyatheaceae

Cyathea affinis (G. Forster) O. Swartz: \#1 II Cyatheaceae

Cyathea agatheri Holttum: \#I II Cyatheaceae

Cyathea akawaionum P. J. Edwards: \#1 II Cyatheaceae

Cyathea alata (Foum.) Copel:- \#1 II Cyatheaceae

Cyathea alatissima (Stolze) ?: \#1 II Cyatheaceae

Cyathea albida Tard.: \#I II Cyatheaceae

Cyathea albidosquamaia Rosenstock: \#1 11 Cyatheaceae

Cyathea albifrons Vieill. ex Foum.: \#1 II Cyatheaceae

Cyathea albomarginata R. C. Moran: \#I II Cyatheaceae

Cyathea albosetacea (Beddome) Copel.: \#I II Cyatheaceae

Cyathea aldenvereliii Copel.: \#1 II Cyatheaceae

Cyathea alfonsiana L. D. Gómez = Cyathea multiflora Smith

Cyathea alleniae Holttum: \#1 II Cyatheaceae

Cyathea alstonii R. Tryon: \#1 II Cyatheaceae

Cyathea alra Copel.: \#1 II Cyatheaceae

Cyathea altemans (Wallich ex Hook.) C. Presl: \#1 II Cyatheaceae Cyathea alticola (Tard.) Tindale: \#I II Cyatheaceae

Cyathea amabilis (C. Morton in Steyerm.) ?: \#1 II Cyatheaceae

Cyathea amboinensis (Alderw.) Mer.: \#1 II Cyatheaceae

Cyathea andersonii (Scott ex Beddome) Copel: \#1 II Cyatheaceae

Cyathea andicola Domin: \#1 II Cyatheaceae

Cyathea andina (Karsten) Domin: \#I II Cyatheaceae

Cyathea aneitensis Hook.: \#1 II Cyatheaceae

Cyathea angiensis (Gepp) Domin: \#1 II Cyatheaceac

Cyathea angustipinna Holttum: \#1 II Cyatheaceae

Cyathea annae (Alderw.) Domin: \#I II Cyatheaceae

Cyathea apiculata (Rosenstock) Domin: \#1 II Cyatheaceae

Cyathea apoensis Copel: \#1 II Cyatheaceae

Cyathea appendiculata Baker: \#1 II Cyatheaceae

Cyathea approximata Bonap. var. approximata: \#1 II Cyatheaceae

Cyathea approximata Bonap. var. sorisquamata (Bonap.) Tardieu:

\#1 II Cyatheaceae

Cyathea aramaganensis Kaneh.: \#1 II Cyatheaceae

Cyathea arborea (L.) Smith: \#1 II Cyatheaceae

Cyathea archboldii C. Chr.: \#1 II Cyatheaceae

Cyathea arfakensis Gepp: \#1 II Cyatheaceae

Cyathea aristata Domin: \#1 II Cyatheaceae

Cyathea ammata (Sw.) Domin: \#I II Cyatheaceae

Cyathea arthropoda Copel.: \#I II Cyatheaceae
Cyathea ascendens Domin: \#1 II Cyatheaceae

Cyathea aspera (L.) Sw.: \#1 II Cyatheaceae

Cyathea assimilis Hook. \#1 II Cyatheaceae

Cyathea assurgens R. Tryon: \# II Cyatheaceae

Cyathea atahuallpa (R. Tryon) Lellinger: \# I II Cyatheaceae

Cyathea aterrima (Hook.) Domin: \#1 II Cyatheaceae

Cyathea atropurpurea Copel.: \#I II Cyatheaceae

Cyathea atrospinosa Holtum: \#1 II Cyatheaceae

Cyathea atrovirens (Langsd. \& Fisch.) Domin: \#1 II Cyatheaceae

Cyathea atrox C. Chr. var, atrox: \#1 II Cyatheaceae

Cyathea atrox C. Chr. var. inermis Holttum: \#1 II Cyatheaceae

Cyathea auriculata Tard.: \#1 II Cyatheaceae

Cyathea auriculifera Copel.: \#I II Cyatheaceae

Cyathea australis (R. Br.) Domin ssp. australis: \#1 II Cyatheaceae

Cyathea australis (R. Br.) Domin ssp, norfolkensis Holttum: \#1 II

Cyatheaceae

Cyathea axillaris (Fée) Lellinger: \#1 II Cyatheaceae

Cyathea baileyana (Domin) Domin: Wig Tree Fern (E) \#1 II Cyatheaceae

Cyathea balakrishnanii R. D. Dixit \& A. K.Tripathi; \#1 II Cyatheaceae

Cyathea balanocarpa D. Eaton: \# I II Cyatheaceae

Cyathea ballardii Tard: \#1 II Cyatheaceae

Cyathea barringtonii A. Reid Smith ex Lellinger: \#1 II Cyatheaceae

Cyathea basilaris Christ = Cyathea imrayana Hooker var. basilaris (Christ) Lellinger

Cyathea batjanensis (Christ) Copel.: \#I II Cyatheaceae

Cyathea bella (Reichb.f. ex Mett.) Domin = Cnemidaria bella (Reichb.f. ex Mett.) R. Tryon

Cyathea bellisquamata Bonap. var, basilobata C. Chr.;1 II Cyatheaceae

Cyathea bellisquamata Bonap. var. bellisquamata: \#1 II Cyatheaceae

C)athea bicrenata Liebm.: Palo de la vida (S), Pelma (S) \#I II Cyatheaceae

Cyathea biformis (Rosenstock) Copel.: \#1 II Cyatheaceae

Cyathea binuangensis Alderw:: \#1 II Cyatheaceae

Cyathea bipinnata (R. Tryon) ?: \#1 II Cyatheaceae

Cyathea bipinnatifida (Baker) Domin: \#I II Cyatheaceae

Cyarhea boivini Mett. var. boivini: 1 II Cyatheaceae

Cyarhea boivini Mett. var. concava (Bonap.) Tard.: \#1 II Cyatheaceae

Cyathea boivini Mett. var. humblotii (Baker) C. Chr. \#1 II Cyatheaceae

Cyathea boliviana R. Tryon: \# II Cyatheaceae

Cyathea borbonica Desv, var. borbonica: \#1 II Cyatheaceae

Cyathea borbonica Desv. var. laevigata (Willd. ex Kauif.) Bonap.: \#1 II Cyatheaceae

Cyaihea borbonica Desv, var. madagascariensis (Kaulf.) Bonap.: \# II Cyatheaceae

Cyathea borbonica Desv. var, pesvilleana C. Chr.: \#1 II Cyatheaceae

Cyathea borbonica Desv. var. simulans (Baker) C. Chr.: \#1 II Cyatheaceae

Cyathea borinquena (Maxon) Domin: \#I II Cyatheaceae

Cyathea bomeensis Copel.: \#1 II Cyatheaceae

Cyathea brachyphylla Holttum: \#1 II Cyatheaceae

Cyathea brackenridgei Mett.: \#1 II Cyatheaceae

Cyathea bradei (Wind.) Lellinger: \#1 II Cyatheaceae

Cyathea brevipinna Baker: \#1 II Cyatheaceae

Cyathea brevistipes R. C. Moran: \#1 II Cyatheaceae

Cyathea brooksii Maxon Brooks's Alsophila (E): \#1 II Cyatheaceae

Cyathea brownii Domin: \#1 II Cyatheaceae

Cyathea brunei Christ $=$ Sphaeropteris brunei (Christ) Tryon

Cyathea brunnescens (Barrington) R. C. Morran: \#1 II Cyatheaceae

Cyathea brunoniana (Wall. ex Hook.) C. B. Clarke \& Baker = Cyathea chinensis Copel.

Cyathea bryophila (Tryon) ?: \# I II Cyatheaceae

Cyathea bunnemeijerii Alderw.: \#1 II Cyatheaceae

Cyathea callosa Christ: \#1 II Cyatheaceae

Cyathea camerooniana Hook. var. aethiopica (Welw, ex Hook.) Holturn: \#1 II Cyatheaceae

Cyathea camerooniana Hook. var. camerooniana: \#1 11 Cyatheaceae

Cyathea camerooniana Hook. var. congi (Christ) Holttum: \#1 II Cyatheaceae

Cyathea camerooniana Hook. var. currorii Holttum: \#1 II Cyatheaceae 
Cyathea camerooniana Hook. var, occidentalis Holttum: \#I II Cyatheaceae

Cyathea camerooniana Hook. var. ugandensis Holttum: \#1 II Cyatheaceae

Cyathea camerooniana Hook. var, zenkeri (Hieron. ex Diels) Tard.: \#1 II Cyatheaceae

Cyathea capensis ( $\mathcal{L}$. f.) Smith: \#1 II Cyatheaceac

Cyathea capitata Copel.: \#1 II Cyatheaceae

Cyathea caracasana (Klotzsch) Domin var, boliviensis (Rosenst.) R. Tryon: \#1 II Cyatheaceae

Cyathea caracasana (Klotzsch) Domin var. caracasana: \#1 II Cyatheaceae

Cyathea caracasana (Klotzsch) Domin var, chimborazensis (Hook.) R. Tryon: \#1 II Cyatheaceae

Cyathea caracasana (Klotzsch) Domin var. maxonii (Und. ex Maxon) R. Tryon: \#1 II Cyatheaceae

Cyarhea caracasana (Klotzsch) Domin var. meridensis (Karsten) R. Tryon: \#1 II Cyatheaceae

Cyathea carrii Holttum: \# I II Cyatheaceae

Cyarhea carillifera Holtum: \#1 II Cyatheaceae

Cyathea caudata (J. Smith) Copel.: \#1 II Cyatheaceae

Cyathea celebica Blume: \#1 II Cyatheaceae

Cyarhea chinensis Copel.: \#1 II Cyatheaceae

Cyathea chiricana (Maxon) Domin = Cnemidaria mutica (Christ) R. Tryon var, chiricana (Maxon) Stolze

Cyathea chocoensis (Stolze) ?: \#I II Cyatheaceae

Cyathea choricarpa $($ Maxon) Domin = Cnemidaria choricarpa R. Tryon

Cyathea christii Copel.: \#1 II Cyatheaceae

Cyathea cicalricosa Holttum: \#1 II Cyatheaceae

Cyathea cincinnata Brause: \#1 11 Cyatheaceae

Cyathea cinerea Copel: \#1 II Cyatheaceae

Cyathea coactilis Holttum: \#1 II Cyatheaceae

Cyathea cocleana (Stolze) ?: \# I II Cyatheaceae

Cyathea colensoi (Hook.f.) Domin: \#1 II Cyatheaceae

Cyathea concinna (Baker ex Jenman) Jenman: \#1 II Cyatheaceae

Cyathea conformis (R. Tryon) Stolze: \#1 II Cyatheaceae

Cyathea conjugata (Spuce ex Hook.) Domin: \# I II Cyatheaceae

Cyathea consimilis (Stolze) ?; \# I II Cyatheaceae

Cyathea contaminans (Wallich ex Hook.) Copel.: \# I II Cyatheaceae

Cyarhea contigua (Maxon) Domin = Cnemidaria mutica (Chrisi) R. Tryon var, contigua (Maxon) Stolze

Cyathea cooperi (Hook. \& F. Muell.) Domin: \#I II Cyatheaceae

Cyathea corallifera Sodiro: \#1 II Cyatheaceac

Cyathea corcovadensis (Raddi) Domin: \#1 II Cyatheaceae

Cyathea costalisora Copel.; \#1 II Cyatheaceae

Cyathea costaricensis (Kuhn) Domin: Palmita de terra fría (S) \#1 II Cyatheaceae

Cyathea costularis Bonap: \#1 11 Cyatheaceae

Cyathea costulisora Domin: \#1 II Cyatheaceae

Cyathea crassa Maxon: \#I II Cyatheaceae

Cyathea crenulata Blume: \#1 II Cyatheaceae

Cyathea crinita (Hook.) Copel.: \#1 II Cyacheaceae

Cyathea croftii Holttum: \#1 II Cyatheaceae

Cyathea cuatrecasasi ( $R$. Tryon) ?: \#1 II Cyatheaceae

Cyathea cucullifera Holtum: \#1 II Cyatheaceae

Cyathea cumingii Baker = Cyathea medullaris (G. Forster) Swartz

Cyathea cunninghamii Hook.f.: Slender Tree Fern (E) \#1 II Cyatheaceae

Cyathea curranii Copel.: \#1 II Cyatheaceae

Cyathea cuspidala Kunze = Alsophila cuspidata (Kunze) Conant

Cyathea cyatheoides (Desv.) Kramer: \#1 II Cyatheaceae

Cyashea cyclodium (R. Tryon) Lellinger: \#1 II Cyatheaceae

Cyathea darienensis R. C. Moran: \# 1 II Cyatheaceae

Cyathea dealbata (Forst.) Sw: Silver Tree Fern (E) \#1 II Cyatheaceae

Cyathea deckenii Kuhn = Alsophila manniana

Cyathea decomposita (Karsten) Domin: \#1 II Cyatheaceae

Cyathea decorata (Maxon) R. Tryon: \#1 II Cyatheaceae

Cyathea decrescens Mett. var. decrescens: \#1 II Cyatheaceae

Cyathea decrescens Mett. var. hirsutifolia (Bonap.) C. Chr.: \#1 II Cyatheaceae

Cyathea decurrens (Hook.) Copel, var. decurrens: \# I II Cyatheaceac

Cyathea decurrens (Hook.) Copel. var. vaupelii (Brause) Domin: \# 1 II Cyatheaceae

Cyathea delgadii Pohl. ex Sternb.: \#1 II Cyatheaceae
Cyathea deminuens Holttum: \# I II Cyatheaceae

Cyathea demissa (C. Morton) A. R. Smith ex Lell. var. demissa: \#1

II Cyatheaceae

Cyathea demissa (C. Morton) A. R. Smith ex Lell. var. thysanolepis

(D. Barrington) ?: \#1 II Cyatheaceae

Cyathea dichromatolepis (Fée) Domin: \#1 II Cyatheaceae

Cyathea dicksonioides Holttum: \#1 II Cyatheaceae

Cyathea dimorpha (Christ) Copel.: \#I II Cyatheaceae

Cyathea discophora Holttum: \#1 II Cyatheaceae

Cyathea dissimilis (C. Morton) Stolze: \#1 II Cyatheaceae

Cyathea dissoluta Baker ex Jenman: \#1 II Cyatheaceae

Cyathea divergens Kunze var. divergens: \#1 II Cyatheaceae

Cyathea divergens Kunze var, tuerckheimii (Maxon) R. Tryon: \#1 II Cyatheaceae

Cyathea dociersii Alderw.: \#1 II Cyatheaceae

Cyathea dombeyi (Desv.) Lellinger: \#1 II Cyatheaceae

Cyathea dregei Kunze var. burkei (Hook.) Sim: \#1 II Cyatheaceae

Cyathea dregei Kunze var. dregei: \#1 II Cyatheaceae

Cyathea dregei Kunze var. polyphlebia (Baker) C. Chr.: \#I II Cyatheaceae

Cyathea dregei Kunze var, segregata (Baker) C. Chr.: \#1 II Cyatheaceae

Cyathea dryopieroides Maxon: Elfin Tree Fern (E) \#1 II Cyatheaceae

Cyathea dudleyi R. Tryon: \#1 II Cyatheaceae

Cyathea ebenina Karsten: \#I II Cyatheaceae

Cyathea edanoi Copel.: \#1 II Cyatheaceae

Cyathea elmeri (Copel.) Copel.: \#1 II Cyatheaceae

Cyathea elongala Mett. ex Karsten: \#1 II Cyatheaceae

Cyathea epaleaia (Holttum) Holttum: \#1 II Cyatheaceae

Cyathea erinacea Karsten = Alsophila erinacea (Karsten) Conant

Cyathea eriophora Holttum: \#1 II Cyatheaceae

Cyathea estelae (Riba) Proctor: \#1 II Cyatheaceae

Cyathea everna Copel:: \#1 II Cyatheaceae

Cyathea ewanii Alston: \#1 II Cyatheaceae

Cyathea excavara Holttum: \#I II Cyatheaceae

Cyathea excelsa O. Swartz: \#1 II Cyatheaceae

Cyathea exilis Holttum: \#1 II Cyatheaceae

Cyathea fadenii Holttum: \#1 II Cyatheaceae

Cyathea falcata (Mett. ex Kuhn) Domin: \#1 II Cyatheaceae

Cyathea feani E. Brown: \#1 II Cyatheaceae

Cyathea felinum (Roxb.) Morton: \#1 II Cyatheaceae

Cyathea fenicis Copel.: \#1 II Cyatheaceae

Cyathea ferruginea Christ: \#1 II Cyatheaceae

Cyathea foersieri Rosenst.: \#1 II Cyatheaceae

Cyathea frigida (Karsten) Domin: \#1 II Cyatheaceae

Cyathea fugar Alderw: \#1 II Cyatheaceae

Cyathea fulgens C. Chr.: \#1 II Cyatheaceae

Cyathea fuliginosa (Christ) Copel.: \#1 II Cyatheaceae

Cyathea fulva (Martius \& Galeotti) Fée: \#1 II Cyatheaceae

Cyathea furfuracea Baker: \#1 II Cyatheaceae

Cyathea fusca Baker: \#1 II Cyatheaceae

Cyathea gardneri (Hook.) Lellinger: \#1 II Cyatheaceae

Cyarhea geluensis Rosenstock: \#1 II Cyatheaceae

Cyathea gibbosa (Klotzsch) Domin: \#1 II Cyatheaceae

Cyathea gigantea (Wallich ex Hook.) Holtum: \#1 II Cyatheaceae

Cyathea glaberrima Holttum: \#1 II Cyatheaceae

Cyathea glabra (BI.) Copel.: \#1 II Cyatheaceae

Cyathea glandulosa (Stolze) ? = Cnemidaria glandulosa Stolze

Cyathea glauca Bory: \#1 II Cyatheaceae

Cyathea gleichenioides C. Chr.: \#1 II Cyatheaceae

Cyathea gracilis Griseb.: \#1 II Cyatheaceae

Cyathea grandifolia Willd.: \#1 II Cyatheaceae

Cyathea grandis (Maxon) Domin = Cnemidaria mutica (Christ)

R. Tryon var. grandis (Maxon) Stolze

Cyathea gregaria (Brause) Domin: \# I II Cyatheaceae

Cyathea grevilleana Martius: \#1 II Cyatheaceae

Cyathea hainanensis Ching: \#1 II Cyatheaceae

Cyathea halconensis Christ: \# I II Cyatheaceae

Cyathea hancockii Copel.: \#1 II Cyatheaceae

Cyathea harrisii L. Underw, ex Maxon: \#1 II Cyatheaceac

Cyathea haughtii (Maxon) R. Tryon: \#1 II Cyatheaceae

Cyathea havilandii Baker: \#1 II Cyatheaceae

Cyathea heliophila R. Tryon: \#1 II Cyatheaceae

Cyathea henryi (Baker) Copel.: \#1 II Cyatheaceae

Cyathea heterochlamydea Copel.: \#1 II Cyatheaceae 
Cyathea hildebrandtii Kuhn: \#1 II Cyatheaceae Cyathea hirsuta C. Presl: \#1 II Cyatheaceae Cyarhea hodgeana Proctor: \#1 II Cyatheaceae Cyathea hooglandii Holttum: \#1 II Cyatheaceae Cyathea hookeri Thwaites: \#1 II Cyatheaceae Cyathea hornei (Baker) Copel.: \#1 11 Cyatheaceae Cyashea horrida (L.) J. E. Smith = Cnemidaria horrida (L.) C. Presl Cyathea horridula Copel.: \#1 II Cyatheaceae Cyathea hotteana C. Chr. \& Ekman: \#1 II Cyatheaceae Cyathea howeana Domin: \#1 II Cyatheaceae Cyathea humbertiana (C. Chr.) Domin: \#1 II Cyatheaceae Cyathea humilis Hieron. var. humilis: \#1 II Cyatheaceae Cyathea humilis Hieron, var. pycnophylla Holttum: \#1 II Cyatheaceae

Cyathea hunsteiniana Brause: \#1 II Cyatheaceae

Cyathea hymenodes Mett.: \#1 II Cyatheaceae

Cyathea iheringii (Ros.) Domin \#I II Cyatheaceae

Cyathea imbricasa Alderw.: \#1 II Cyatheaceae

Cyathea impar R. Tryon: \# I II Cyatheaceae

Cyathea imrayana Hooker var. basilaris (Christ) Gastony: \#1 II Cyatheaceae

Cyathea imrayana Hook, var. imrayana: \#1 II Cyatheaceae

Cyathea inaequalis Holttum: \#I II Cyatheaceae

Cyathea incana Karsten: \#1 II Cyatheaceae

Cyathea incisoserrata Copel.: \#I II Cyatheaceae

Cyathea inquinans Christ: \#1 II Cyatheaceae

Cyathea insignis D. Eaton: \#1 II Cyatheaceae

Cyathea insulana Holttum: \#1 II Cyatheaceae

Cyathea insularum Holttum: \#I II Cyatheaceae

Cyathea integra J. Smith ex Hook: \#1 II Cyatheaceae

Cyathea intermedia (Mett.) Copel.: \#1 II Cyatheaceae

Cyathea intramarginalis (Wind.) Lellinger: \#1 II Cyatheaceae

Cyathea isaloensis C. Chr.: \#1 II Cyatheaceae

Cyathea jacobsii Holttum: \#! II Cyatheaceae

Cyathea javanica Blume: \#1 II Cyatheaceae

Cyathea junghuhniana (Kunze) Copel.: \#1 II Cyatheaceae

Cyathea kalbreyeri (Baker) Domin: \#1 II Cyatheacese

Cyathea kanehirae Holttum: \#1 II Cyatheaceae

Cyathea karsteniana (Klotzsch) Domin: \#1 II Cyatheaceae

Cyathea kermadecensis Oliver: \#1 II Cyatheaceae

Cyathea khasyana (Moore ex Kuhn) Domin: \#1 II Cyatheaceae

Cyathea kirkii Hook.: \#I II Cyatheaceae

Cyathea klossii Ridley: \#I II Cyatheaceae

Cyathea lasiosora (Mett. ex Kuhn) Domin: \#l II Cyatheaceze

Cyathea lastii Baker: \#1 II Cyatheaceae

Cyathea latebrosa (Wallich ex Hook.) Copel.: \#I II Cyatheaceae

Cyathea latevagans (Baker) Domin: \#1 II Cyatheaceae

Cyathea latipinnula Copel.: \#I II Cyatheaceae

Cyashea lechleri Mett.: \#I II Cyatheaceae

Cyathea ledermannii Brause: \#1 II Cyatheaceae

Cyathea leichardriana (F. Muell.) Copel.: Prickly Tree Fern (E) \#1 II Cyatheaceae

Cyathea lepidoclada (Christ) Domin: \#1 II Cyatheaceae

Cyathea lepifera (J. Smith ex Hook) Copel.: \#1 II Cyatheaceae

Cyathea leprieurii (Kunze) Domin: \#I II Cyatheaceae

Cyathea leptochlamys Baker: \#I II Cyatheaceae

Cyathea leucolepis Mett.: \#1 II Cyatheaceae

Cyathea leucotricha Christ: \#1 II Cyatheaceae

Cyathea ligulata Baker: \#1 II Cyatheaceae

Cyathea lindsayana Hook.: \#I II Cyatheaceae

Cyathea lockwoodiana (Windisch) Lellinger: \#1 II Cyatheaceae

Cyathea loerzingii Holttum: \#I II Cyatheaceae

Cyathea loheri Christ: \#1 II Cyatheaceae

Cyathea longipes Copel.: \#1 II Cyatheaceae

Cyathea longipinnata Bonap.: \#1 II Cyatheaceae

Cyathea lucida (Fée) Domin = Cnemidaria decurrens $($ Liebm.) R. Tryon

Cyathea lunulata (G. Forster) Copel.: \#1 II Cyatheaceae

Cyathea lurida (Blume) Copel.: \#1 II Cyatheaceae

Cyathea macarthurii (F. Muell.) Baker: \#1 II Cyatheaceae

Cyathea macgillivrayi (Baker) Domin: \#1 II Cyatheaceae

Cyathea macgregorii F. Muell: \#I II Cyatheaceae

Cyathea macrocarpa (C. Presl) Domin: \#I II Cyatheaceae

Cyathea macrophylla Domin var. macrophylla: \#I II Cyatheaceae

Cyathea macrophylla Domin var. quadripinnata Holttum: \#1 II Cyatheaceae
Cyathea macroz oda Domin: \#1 II Cyatheaceae

Cyathea macrosora (Baker) Domin var. macrosora: \#I II Cyatheaceae

Cyathea macrosora (Baker) Domin var, reginae (Wind.)

A. R. Smith: \#1 II Cyatheaceae

Cyalhea macrosora (Baker) Domin var. vaupensis (Wind.)

A. R. Smith: \#1 II Cyatheaceae

Cyathea madagascarica Bonap.: \#1 II Cyatheaceae

Cyathea magna Copel.: \#1 II Cyatheaceae

Cyathea magnifolia Alderw.: \#1 II Cyatheaceae

Cyathea manniana Hook. = Alsophila manniana

Cyathea marattioides Willd. ex Kaulf.: \#1 II Cyatheaceae

Cyathea marcescens N. A Wakefield: Skirted Tree Fern (E) \#1 II Cyatheaceae

Cyathea marginalis (Klotzsch) Domin: \#1 II Cyatheaceae

Cyathea marginata (Brause) Domin: \#1 II Cyatheaceae

Cyathea masapilidensis Copel.: \#1 II Cyatheaceae

Cyaihea media Wagn. \& Grether: \#1 II Cyatheaceae

Cyathea medullaris (G. Forster) Swartz: Black Tree Fem (E) \#! II Cyatheaceae

Cyathea megalcsora Copel: \#1 II Cyatheaceae

Cyathea melanocaula Desv.: \#1 II Cyatheaceae

Cyathea melleri (Baker) Domin: \#1 II Cyatheaceae

Cyathea mertensiana (Kunze) Copel.: \#1 II Cyatheaceae

Cyathea mesosora Holttum: \#1 II Cyatheaceae

Cyathea metteniana (Hance) C. Chr. \& Tard.: \#! II Cyatheaceae

Cyathea mexiae Copel.: \#1 11 Cyatheaceae

Cyathea mexicana Schldl. \& Cham.: Cola de mono (S), Ocopetate

(S), Pesma (S), Rabo de mico (S) \#I II Cyatheaceae

Cyashea microchlamys Holttum: \#I II Cyatheaceae

Cyathea microdonta (Desv.) Domin: \#I II Cyatheaceae

Cyathea microlepidota Copel.: \#1 II Cyatheaceae

Cyathea microphylla Mett.: \#1 II Cyatheaceae

Cyathec microphylloides Rosenstock: \#1 II Cyatheaceae

Cyathea mildbraedii (Brause) Domin: \#1 II Cyatheaceae

Cyathea milnei Hook:: \#1 II Cyatheaceae

Cyathea minor D. Eaton: \#1 II Cyatheaceae

Cyashea modesta (Baker) Copel.: \#I II Cyatheaceae

Cyathea moluccana $\mathrm{R}$. Br ex Desv.: \#I II Cyatheaceae

Cyathea moseleyi Baker: \#I II Cyatheaceae

Cyathea mossambicensis Baker: \#1 II Cyatheaceae

Cyathea mucilagina R.C. Moran: \#1 II Cyatheaceae

Cyathea muelleri Baker: \#1 II Cyatheaceae

Cyathea multiflora Smith: \#1 11 Cyatheaceae

Cyathea multisegmenta R. Tryon: \#1 II Cyatheaceae

Cyathea muricata Willd: \#1 II Cyatheaceae

Cyathea mutica (Christ) Domin = Cnemidaria mutica (Christ)

R. Tryon var. niutica

Cyathea myosuroides (Liebm.) Domin: \#I II Cyatheaceae

Cyathea nanna (D. Barrington) Lellinger: \#1 II Cyatheaceae

Cyathea negrosiana Christ: \#I II Cyatheaceae

Cyathea nervosa (Maxon) ?: \#I II Cyatheaceae

Cyathea nicklesii (Tard. \& Ballard) Tind.: \#1 II Cyatheaceae

Cyathea nicobarica Balakrishnan \& Dixit: \#1 II Cyatheaceae

Cyathea nigricans Mett.: \#1 II Cyatheaceae

Cyathea nigripes (C. Chr.) Domin: \#1 II Cyatheaceae

Cyarhea nigripes (C. Chr.) Domin var. brunnescens (D. Barrington) Lellinger = Cyathea brunnescens (Barrington) R. C. Moran

Cyathea nigrolineata Holttum: \#1 II Cyatheaceae

Cyashea nigropaleara Holtrum: \#1 II Cyatheaceae

Cyathea nilgirensis Holttum: \#1 II Cyatheaceae

Cyathea nockii Jenman: \#1 II Cyatheaceae

Cyathea nodulifera R. C. Moran: \#I II Cyatheaceae

Cyathea notabilis (Maxon) Domin: \#1 II Cyatheaceae

Cyathea nothofagorum Holttum: \#1 II Cyatheaceae

Cyathea novae-caledoniae (Mett.) Copel.: \#1 II Cyatheaceae

Cyathea obliqua Copel.: \#1 II Cyatheaceae

Cyathea oblonga (K1.) Domin: \#1 II Cyatheaceae

Cyathea obscura (Scorech. ex Bedd.) Copel.: \#1 II Cyatheaceae

Cyathea obrusa (Kaulf.) Domin: \#1 II Cyatheaceae

Cyathea obtusiloba (Hook.) Domin: \#1 II Cyatheaceae

Cyathea ogurae (Hayata) Domin: \#I II Cyatheaceae

Cyathea oinops Hassk.: \#I II Cyatheaceae

Cyathea oosora Holttum: \#1 II Cyatheaceae

Cyathea orienalis (Kunze) Moore; \#1 II Cyatheaceae

Cyathea orthogonalis Bonap.: \#1 II Cyatheaceae 
Cyathea pachyrrhachis Copel.: \#1 II Cyatheaceae

Cyathea pallescens (Sodiro) Domin: \#I II Cyatheaceae

Cyathea pallidipaleata Holttum: \#I II Cyatheaceae

Cyathea pansamalana (Maxon) Dornin: \#I II Cyatheaceae

Cyathea papuana (Ridley) Alderw: \#I II Cyatheaceae

Cyathea parianensis (Wind.) Lellinger: \#1 II Cyatheaceae

Cyathea parksiae Copel.: \#I II Cyatheaceae

Cyathea parva Copel.: \#1 II Cyatheaceae

Cyathea parvipinna Holttum: \#1 II Cyatheaceae

Cyathea parvula (Jenman) Domin: \#I II Cyatheaceae

Cyatheo patellifera Alderw.: \#1 II Cyatheaceae

Cyathea pauciflora (Kuhn) Lellinger: \#1 II Cyatheaceae

Cyathea paucifolia (Baker) Domin: \#1 II Cyatheaceae

Cyathea pectinata Ching \& S. H. Wu: \#1 II Cyatheaceae

Cyathea peladensis (Hieron.) Domin: \#1 II Cyatheaceae

Cyathea percrassa C. Chr.: \#1 II Cyatheaceae

Cyathea perpelvigera Alderw.: \#1 II Cyatheaceae

Cyashea perpunctulata (Alderw.) Domin: \#1 II Cyatheaceae

Cyathea perrieriana C. Chr.: \#I II Cyatheaceae

Cyathea persquamulifera (Alderw.) Domin: \#1 II Cyatheaceae

Cyathea petiolata (Hook.) R. Tryon: \#1 II Cyatheaceae

Cyathea petiolulata Ching \& S. H. Wu: \#1 II Cyatheaceae

Cyathea phalaenolepis (C. Chr.) Domin: \#1 II Cyatheaceae

Cyathea phalerata Martius: \#I II Cyatheaceae

Cyathea phegopteroides (Hook.) Domin: \#I II Cyatheaceae

Cyathea philippinensis Baker: \#I II Cyatheaceae

Cyathea physolepidota Alston: \#1 II Cyatheaceae

Cyathea pilosissima (Baker) Domin: \#I II Cyatheaceae

Cyathea pilosula Tard.: \#1 II Cyatheaceae

Cyathea pilulifera Copel.: \#I II Cyatheaceae

Cyathea pinnula (Christ) Domin: \#I II Cyatheaceae

Cyathea plagiostegia Copel.: \#I II Cyatheaceae

Cyathea platylepis (Hook.) Domin: \#1 II Cyatheaceae

Cyathea podophylla (Hook.) Copel.: \#I II Cyatheaceae

Cyathea poeppigii (Hook.) Domin: \#1 II Cyatheaceae

Cyathea polypoda Baker: \#1 II Cyatheaceae

Cyathea polystichoides $($ Christ) Domin = Alsophila polystichoides Christ

Cyathea ponapeana (Hosok.) Glassm.: \#1 II Cyatheaceae

Cyathea portoricensis Sprengel ex Kuhn: \#1 II Cyatheaceae

Cyathea praecincta (Kunze) Domin: \#I II Cyatheaceae

Cyathea princeps $\mathrm{E}$. Mayer = Sphaeropteris horrida $($ Liebm.) Tryon

Cyathea procera Brause: \#1 II Cyatheaceae

Cyathea propinqua Mett.: \#1 II Cyatheaceae

Cyathea pruinosa Rosenstock: \#1 II Cyatheaceae

Cyashea pseudogigantea Ching \& S. H. Wu: \#1 II Cyatheaceae

Cyathea pseudomuelleri Holtum: \#I II Cyatheaceae

Cyathea pseudonanna (L. D. Gómez) Lellinger: \#I II Cyatheaceae

Cyathea pubens Domin = Cyathea bipinnatifida $($ Baker) Domin

Cyathea pubescens Mett. ex Kuhn: \#I II Cyatheaceae

Cyathea pulcherrima Copel.: \#1 II Cyatheaceae

Cyathea punctulata (Alderw.) Alderw:: \#! II Cyatheaceae

Cyarhea pungens (Willd.) Domin: \#1 II Cyatheaceae

Cyathea purpurascens Sodiro = Alsophila erinacea $($ Karsten $)$ Conant

Cyathea pycnoneura Holttum: \#1 II Cyatheaceae

Cyashea quadrata Baker var. ivohibensis C. Chr.: \#1 II Cyatheaceae

Cyathea quadrata Baker var. quadrata: \#I II Cyatheaceae

Cyathea quindiuensis Karsten: \#I II Cyatheaceae

Cyathea raciborskii Copel.: \#1 II Cyatheaceae

Cyathea ramispina (Hook.) Copel.; \#1 II Cyatheaceae

Cyathea rebeccae (F. Muell.) Domin: \#! II Cyatheaceae

Cyathea recommutata Copel.: \#1 II Cyatheaceae

Cyathea recurvata (Brause) Domin: \#1 II Cyatheaceae

Cyathea rigens Rosenstock: \#1 II Cyatheaceae

Cyathea robertsiana (F. Muell.) Domin: \#1 II Cyatheaceae

Cyathea robinsonii Copel.: \#I II Cyatheaceae

Cyathea robusta (C. Moore ex Watts) Holttum: \#1 II Cyatheaceae

Cyathea roraimensis (Domin) Domin: \#1 II Cyatheaceae

Cyathea roroka P. H. Hovenkamp: \#1 II Cyatheaceae

Cyathea rosenstockii Brause: \#1 II Cyatheaceae

Cyathea rubella Holttum: \#I II Cyatheaceae

Cyathea rubiginosa (Brause) Domin: \#1 II Cyatheaceae

Cyathea rufa (Fée) Lellinger: \#I II Cyatheaceae

Cyathea rufescens (Mett. ex Kuhn) Domin: \#1 II Cyatheaceae

Cyathea rufopannosa Christ: \#I II Cyatheaceae

Cyathea ruiziana Klotzsch: \#1 II Cyatheaceae
Cyathea runensis Alderw.: \#1 II Cyatheaceae Cyathea rupestris Maxon: \#I II Cyatheaceae

Cyathea saccata Christ: \#1 II Cyatheaceae

Cyathea sagittifolia (Hook.) Domin: \#1 II Cyatheaceac

Cyathea salletii Tard. \& C. Chr.: \#1 II Cyatheaceae

Cyathea salvinii (Hook.) Domin = Alsophila salvinii Hooker

Cyathea sangirensis (Christ ex Diels) Copel.: \#1 II Cyatheaceae

Cyathea sarasinorum Holtrum: \#1 II Cyatheaceae

Cyathea scabriuscula (Maxon) Domin \#I II Cyatheaceae

Cyathea scandens (Brause) Domin: \#I II Cyatheaceae

Cyathea schiedeana (C. Presl) Domin: Maloue (F) \#1 II Cyatheaceae

Cyathea schlechteri (Brause) Domin: \#1 II Cyatheaceae

Cyathea schliebenii Reim.: \#1 II Cyatheaceae

Cyathea schlimii (Kuhn) Domin: \#I II Cyatheaceae

Cyathea sechellarum Mett.: \#1 II Cyatheaceae

Cyathea semiamplectens Holtum: \#1 II Cyatheaceae

Cyathea senex Alderw.: \#I II Cyatheaceae

Cyathea senilis (Klotzsch) Domin: \#1 II Cyatheaceae

Cyathea serratifolia Baker: \#1 II Cyatheaceae

Cyathea setifera Holtum: \#1 11 Cyatheaceae

Cyathea setosa (Kaulf.) Domin: \#1 II Cyatheaceae

Cyathea setulosa Copel.: \#1 II Cyatheaceae

Cyathea sibuyanensis Copel.: \#1 II Cyatheaceae

Cyathea similis C. Chr.: \#1 II Cyatheaceae

Cyathea simplex R. Tryon: \#1 II Cyatheaceae

Cyathea singularis (Stolze) ?: \#1 II Cyatheaceae

Cyathea sinuata Hook. \& Grev.: \#1 II Cyatheaceae

Cyathea sipapoensis ( $\mathrm{R}$. Tryon) Lellinger: \#1 II Cyatheaceae

Cyathea smithii Hook.f.: Soft Tree Fem (E) \#1 II Cyatheaceae

Cyathea solomonensis Holttum: \#1 II Cyatheaceae

Cyathea speciosa Willd.: \#1 II Cyatheaceae

Cyathea spectabilis (Kunze) Domin var. colombiensis (Stolze) ?: \#1

II Cyatheaceae

Cyathea spectabilis (Kunze) Domin var, spectabilis: \#1 II Cyatheaceae

Cyathea spinulosa Wallich ex. Hook. \#1 II Cyatheaceae

Cyathea squamata (Klotzsch) Domin: \#1 II Cyatheaceae

Cyathea squamulata (Blume) Copel.: \#1 II Cyatheaceae

Cyathea squamulosa (Losch) R.C. Moran: \#1 II Cyatheaceae

Cyathea stelligera Holttum: \#I II Cyatheaceae

Cyathea stembergii Pohl. ex Sternb.: \#I II Cyatheaceae

Cyathea steyermarkii R. Tryon: \#1 II Cyatheaceae

Cyathea stipitipinnula Holttum: \#1 II Cyatheaceae

Cyathea stipularis (Christ) Domin = Trichipteris stipularis

Cyathea stokesii E. Brown: \#1 II Cyatheaceae

Cyathea stolzei A. Reid Smith ex Lellinger: \#1 II Cyatheaceae

Cyathea straminea Karsten: \#I II Cyatheaceae

Cyathea sirigillosa (Maxon) Domin: \#I II Cyatheaceae

Cyathea strigosa Christ: \#I II Cyatheaceae

Cyathea subarborescens Domin: \#1 II Cyatheaceae

Cyathea subdubia (Alderw.) Domin: \#1 II Cyatheaceae

Cyathea subincisa C. Chr.: \#I II Cyatheaceae

Cyathea subsessilis Copel.: \#1 II Cyatheaceae

Cyathea subtripinnata Holttum: \#1 II Cyatheaceae

Cyathea subtropica Domin: \#1 II Cyatheaceae

Cyathea suluensis Baker: \# II Cyatheaceae

Cyathea sumatrana Baker: \#I II Cyatheaceae

Cyathea suprastrigosa (Christ) Maxon: \#1 II Cyatheaceae

Cyathea surinamensis (Miq.) Domin: \#1 II Cyatheaceae

Cyathea taiwanense Kuo: \#1 II Cyatheaceae

Cyathea tenera (J. Smith ex Hook.) T. Moore: \#1 II Cyatheaceae

Cyarhea lenggerensis (Rosenstock) Dornin: \#1 II Cyatheaceae

Cyathea tenuicaulis Domin: \#1 II Cyatheaceae

Cyathea tematea Alderw:: \#1 II Cyatheaceae

Cyarhea teysmannii Copel.: \#1 II Cyatheaceae

Cyathea thomsonii Baker: \#1 II Cyatheaceae

Cyathea thwaitesii (Beddome) Sledge: \#1 II Cyatheaceae

Cyathea tinganensis Ching \& S. H. Wu: \#1 II Cyatheaceae

Cyathea tomentosa (Blume) H. Zollinger \& Moritz: \#I II Cyatheaceae

Cyathea tomentosissima Copel.: \#1 II Cyatheaceae

Cyathea tortuosa R. C. Moran: \#1 II Cyatheaceae

Cyathea trachypoda Alderw.: \#1 II Cyatheaceae

Cyathea trichiata $(\operatorname{Maxon})$ Domin = Trichipteris trichiata

Cyathea trichodesma (Scortech. ex Bedd.) Copel.: \#I II Cyatheaceae

Cyathea trichophora Copel.: \#I II Cyatheaceae 
Cyathea tripinnata Copel.: \#1 II Cyatheaceae

Cyathea tripinnatifida Wall. ex Roxb.: \#1 II Cyatheaceae Cyathea truncata (Brackenr.) Copel.: \#I II Cyatheaceae Cyathea iryoniana (Gastony) ? = Alsophila inyoniana (Gasiony) Conant

Cyathea tryononum (Riba) Lellinger: \#1 II Cyatheaceae Cyathea isangii Ching \& S. H. Wu: \#1 II Cyatheaceae

Cyathea isaratananensis Tard.: \#1 II Cyatheaceae

Cyathea tsilotsilensis Tard.: \#I II Cyatheaceae

Cyathea tussacii Desv.: \#1 II Cyatheaceae

Cyathea suyamae H. Ohba: \#I II Cyatheaceae

Cyathea urbanii Brause: \#1 II Cyatheaceae

Cyathea ursina (Maxon) Lellinger: \#I II Cyatheaceae

Cyathea usambarensis Hieron.: \#1 II Cyatheaceae

Cyathea valdecrenata Domin: \#1 II Cyatheaceae

Cyathea vandeusenii Holttum: \#I II Cyatheaceae

Cyathea vaupelii Copel.: \#! II Cyatheaceae

Cyathea velaminosa (Diels) Domin: \#1 II Cyatheaceae

Cyathea venezuelensis A. Reid Smith ex Lellinger: \#1 II Cyatheaceae

Cyathea verrucosa Holttum: \#I II Cyatheaceae

Cyathea vieillardii Mett.: \#I II Cyatheaceae

Cyathea viguieri Tard.: \#1 II Cyatheaceae

Cyathea vilhelmii Domin: \#I II Cyatheaceae

Cyathea villosa Humb. \& Bonpl. ex Willd.: \#1 II Cyatheaceae

Cyathea vittata Copel.: \#1 II Cyatheaceae

Cyathea walkerae Hook. var. tripinnata (Hook. \& Baker) ?: \#1 II Cyatheaceae

Cyathea walkerae Hook. var, walkerae: \#1 II Cyatheaceae

Cyathea wallacei (Mett. ex Kuhn) Copel.: \#1 II Cyatheaceae

Cyathea weatherbyana (C. Morton) C. Morton: \#1 II Cyatheaceae

Cyathea welwitschii Hook: \#1 II Cyatheaceae

Cyathea wendlandii (Mett. ex Kuhn) Domin: \#1 II Cyatheaceae

Cyathea wengiensis (Brause) Domin: \#I II Cyatheaceae

Cyathea werffii R.C. Moran: \#l II Cyatheaceae

Cyathea werneri Rosenstock: \#1 II Cyatheaceae

Cyathea whitmeei Baker: \#1 II Cyatheaceae

Cyathea williamsii (Maxon) Domin: \#1 II Cyatheaceae

Cyathea womersleyi Holttum: \#I II Cyatheaceae

Cyathea woodwardioides Kaulf. var. cubensis (Und. ex Maxon)? \#! II Cyatheaceae

Cyathea woodwardioides Kaulf, var, hieronymi (Brause) ? \#1 II Cyatheaceae

Cyathea woodwardioides Kaulf. var. wondwardioides: \#1 II Cyatheaceae

Cyathea woollsiana (F. Muell.) Domin: \# 1 II Cyatheaceae

Cyathea zakamenensis Tard.: \#I II Cyatheaceae

Cyathea zamboangana Copel.: \#! II Cyatheaceae

Cyathoglottis = Sobralia

Cybebus spp. \#7 II Orchidaceae

Cybele $=$ Herminium

Cybelion = lonopsis

CYCADACEAE spp.: Cycads (E), Cicadas ( $\mathrm{S}$ ), Cycades (F), Palmiers cycas (F) I/I Cycadaceae

Cycades (F): CYCADACEAE spp. I/I Cycadaceae

Cycads (E): CYCADACEAE spp. I/II Cycadaceae

Cycas spp. \#1 II Cycadaceae

Cycas angulata R. Br.: \#l II Cycadaceae

Cycas apoa K. D. Hill: \#1 II Cycadaceae

Cycas arenicola K. D. Hill: \#1 II Cycadaceae

Cycas armstrongii Miq.: \#1 II Cycadaceae

Cycas amhemica K. D. Hill ssp. amhemica: \#1 II Cycadaceae

Cycas amhemica K. D. Hill ssp. muninga Chirgwin \& K. D. Hill: \#] II Cycadaceae

Cycas amhemica K. D. Hill ssp. natja K. D. Hill: \#1 II Cycadaceae

Cycas badensis K. D. Hill: \#1 II Cycadaceae

Cycas baguanheensis L. K. Fu \& S. Z. Cheng = Cycas panzhihuaensis L. Zhou \& S. Y. Yang

Cycas balansae Warburg: \#1 II Cycadaceae

Cycas basaltica C. A. Gardn.: \#I II Cycadaceae

Cycas beddomei Dyer: I Cycadaceae

Cycas bougainvilleana K. D. Hill: \#1 II Cycadaceae

Cycas brunnea K. D. Hill: \#1 II Cycadaceae

Cycas caimsiana F. Muell.: \#1 II Cycadaceae

Cycas calcicola J. R. Maconochie: \#1 II Cycadaceae

Cycas campestris K. D. Hill: \#1 II Cycadaceae
Cycas canalis K. D. Hill ssp. canalis: \#1 II Cycadaceae

Cycas canalis K. D. Hill ssp. carinata K. D. Hill: \#1 II Cycadaceae Cycas celebica Miq.: \#1 II Cycadaceae

Cycas chamberlainii W. H. Brown \& Kienholz: \#1 II Cycadaceae

Cycas chevalieri Léandri: \#1 II Cycadaceae

Cycas circinalis L. var. circinalis: \#I II Cycadaceae

Cycas circinalis L. var. orixensis Haines: \#1 II Cycadaceae

Cycas conferta Chirgwin: \#1 II Cycadaceae

Cycas couttsiana K. D. Hill: \#1 II Cycadaceae

Cycas curranii (J.Schust.) K. D. Hill: \#1 II Cycadaceae

Cycas desolata P. I. Forster: \#1 VI Cycadaceae

Cycas diannanensis Z. T. Guan \& G. D. Tao = Cycas taiwaniana Carruth.

Cycas elongata (Léandri) D. Y. Yang: \#1 II Cycadaceae

Cycas fairylakea D. Y. Wang = Cycas szechuanensis Cheng \&

L. K. Fu

Cycas ferruginea $\mathrm{F}$. N. Wei $=$ Cycas miquelii Warb.

Cycas furfuracen W. V. Fitzg.: \#! II Cycadaceae

Cycas gracilis Miq.: \#I II Cycadaceae

Cycas guizhouensis Lan \& R. F. Zhou = Cycas szechuanensis Cheng \& L. K. Fu

Cycas guizhouensis $\mathrm{K}$. Lan \& R. Zhou = Cycas szechuanensis C. Cheng \& L. K. Fu

Cycas hainanensis C. J. Chen \& C. Y, Chen: \#1 II Cycadaceae

Cycas hongheensis Yang \& Yang: \#1 II Cycadaceae

Cycas inermis Lour.: \#1 II Cycadiceae

Cycas javana (Miq.) de Laubenfels: \#1 Il Cycadaceae

Cycas kennedyana $\mathrm{F}$. Muell. = Cycas media R.Br. ssp. media

Cycas lane-poolei C. A. Gardner: \#1 II Cycadaceae

Cycas lindstromii S. L. Yang \& K. D. Hill: \#1 II Cycadaceae

Cycas longipetiolula D. Y. Wang = Cycas multipinnata C. J. Chen \& S. Y. Yang

Cycas maconochiei Chirgwin \& K. D. Hill ssp. maconochiei: \#1 II Cycadaceae

Cycas maconochiei Chirgwin \& K. D. Hill ssp. lanata K. D. Hill: \#I II Cycadaceae

Cycas maconochiei Chirgwin \& K. D. Hill ssp. viridis K. D. Hill: \# I II Cycadaceae

Cycas macrocarpa Griff.: \#1 II Cycadaceae

Cycas media R. Br. ssp. banksii K. D. Hill: \#1 II Cycadaceae

Cycas media R. Br. ssp. ensata K. D. Hill: \#1 II Cycadaceae

Cycas media R. Br. ssp. media: \#1 II Cycadaceae

Cycas megacarpa K. D. Hill: \#1 II Cycadaceae

Cycas micholizzii Thistleton-Dyer: \#1 II Cycadaceae

Cycas micholizii Thistleton-Dyer var. simplicipinna Smitin. = Cycas balansae Warburg

Cycas micronesica K. D. Hill: \#1 II Cycadaceae

Cycas miquelii Warb.: \#1 II Cycadaceae

Cycas multifrondis D. Y. Wang = Cycas micholitzii Dyer

Cycas multiovula D. Y. Wang = Cycas szechuanensis Cheng \&

L. K. Fu

Cycas multipinnata C. J. Chen \& S. Y. Yang: \#1 II Cycadaceae

Cycas nathorstii Schuster: \#1 II Cycadaceae

Cycas normanbyana $=$ Cycas media $\mathrm{R}$. Br. ssp. media

Cycas normanbyana $\mathrm{F}$. Muell. $=$ Cycis media $\mathrm{R}$. Br. ssp. media

Cycas ophiolitica K. D. Hill: \#! II Cycadaceae

Cycas orientis K. D. Hill: \#I II Cycadaceae

Cycas panzhihuaensis L. Zhou \& S. Y. Yang: \#I II Cycadaceae

Cycas papuana F. Muell.: \# I II Cycadaceae

Cycas parvulus S. L. Yang = Cycas szechuanensis Cheng \& L. K. Fu

Cycas pectinata W. Griff.: \#I II Cycadaceae

Cycas platyphylla K. D. Hill: \#1 II Cycadaceae

Cycas pruinosa J. R. Maconochie: \#1 II Cycadaceae

Cycas revoluta Thunb.: \#l II Cycadaceae

Cycas riuminiana Porte ex Regel: \#1 II Cycadaceae

Cycas rumphii Miq.: Malayan Fern Palm (E) \#I II Cycadaceae

Cycas numphii Miq.f. ssp. seemannii = Cycas seemannii A. Br.

Cycas schumanniana Lauterbach.: \#1 II Cycadaceae

Cycas scratchleyana F. Muell.: \#1 II Cycadaceae

Cycas seemannii A. Br.: \#1 II Cycadaceae

Cycas segmentifida D. Y Wang \& C. Y. Deng = Cycas szechuanensis Cheng \& L. K. Fu

Cycas semota K. D. Hill: \#I II Cycadaceae

Cycas sexseminifera F. N. Wei = Cycas miquelii Warb.

Cycas shiwandashanica H. T. Chang \& Y. C. Zhong = Cycas szechuanensis Cheng \& L. K. Fu 
Cycas siamensis Miq.: \#1 II Cycadaceae

Cycas silvestris K. D. Hill: \#1 II Cycadaceac

Cycas szechuanensis C. Cheng \& L. K. Fu: \#I II Cycadaceae

Cycas raitungensis C. F. Shen, K. D. Hill, C. H. Tsou \& C. J. Chen: \#! II Cycadaceae

Cycas taiwaniana Carruth.: Taiwan Sutie (E) \# I II Cycadaceae

Cycas rangingii D, Y Wang = Cycas taiwaniana Carruth.

Cycas thouarsii R. Br.: \#I II Cycadaceae

Cycas tuckeri K. D. Hill: \# I II Cycadaceae

Cycas wadei Merr.: \#1 II Cycadaceae

Cycas xipholepis K. D. Hill: \# I II Cycadaceae

Cycas yorkiana K. D. Hill: \#1 II Cycadaceae

Cicadas (S) = CYCADACEAE spp. I/I Cycadaceae

Cycades $(\mathbf{F})$ = CYCADACEAE spp. ИI Cycadaceae

Cycads (E) = CYCADACEAE spp. III Cycadaceae

Palmiers cycas $(\mathbf{F})=$ CYCADACEAE spp. I/I Cycadaceae

Cyclamen spp. \#I II Primulaceae

Cyclamen abchasicum (Medw. ex Kusn.) Kolak. = Cyclamen coum Mill. ssp. caucasicum (K. Koch) O. Schwarz

Cyclamen adzharicum Poped. = Cyclamen coum Mill. ssp. caucasicum (K. Koch) O. Schwarz

Cyclamen aedirhizum Jord. = Cyclamen hederifolium Aiton var hederifolium forma hederifolium

Cyclamen aeginicum Hildebr, = Cyclamen graecum Link ssp. graecum forma graecum

Cyclamen aestivum $\mathrm{Rchb}_{1}=$ Cyclamen purpurascens Mill. forma purpurascens

Cyclamen africanum Boiss. \& Reut.: \#1 II Primulaceae

Cyclamen albidum Jord. = Cyclamen persicum Mill. var. persicum forma albidum (Jord.) Grey-Wilson

Cyclamen albiflorum Jord. = Cyclamen hederifolium Aiton var. hederifolium forma albiflorum (Jord.) Grey-Wilson

Cyclamen aleppicum Fisch. ex Hoffmanns. = Cyclamen persicum Mill. var. persicum forma persicum

Cyclamen aleppicum Fisch. ex Hoffmanns. ssp. puniceum Glasau = Cyclamen persicum Mill. var. persicum forma rubrum GreyWilson

Cyclamen algeriense Jord. = Cyclamen africanum Boiss, \& Reut

Cyclamen alpinum? var. album hort. Dammann ex Sprenger = Cyclamen purpurascens Mill. forma purpurascens

Cyclamen ambiguum O. Schwarz = Cyclamen africanum Boiss. \& Reut.

Cyclamen angulare Jord, = Cyclamen hederifolium Aiton var. hederifolium forma hederifolium

Cyclamen antilochium Decne = Cyclamen persicum Mill. var. persicum forma persicum

Cyclamen apiculatum Jord. = Cyclamen coum Mill. \$sp. cotun forma coum

Cyclamen atkinsii Glasau = Cyclamen coum Mill. ssp. caucasicum (K. Koch) O. Schwarz

Cyclamen atkinsii hort. $=$ ? Cyclamen coum Mill, ssp. coum forma coum

Cyclamen balearicum Willk.: Cyclamen des Balèares (F) \#1 II Primulaceae

Cyclamen breviflorum Jord. = Cyclamen purpurascens Mill. forma purpurascens

Cyclamen brevifrons Jord, = Cyclamen coum Mill. ssp. coum forma coum

Cyclamen calcareum Kolak. = Cyclamen coum Mill. ssp. caucasicum (K. Koch) O. Schwarz

Cyclamen caucasicum Willd. ex Boiss, = Cyclamen coum Mill. ssp. caucasicum (K. Koch) O. Schwarz

Cyclamen caucasicum Willd. ex Steven = Cyclamen coum Mill. ssp caucasicum (K. Koch) O. Schwarz

Cyclamen cilicicum Boiss. \& Heldr. forma album E. Frank \& Koenen: \# I II Primulaceae

Cyclamen cilicicum Boiss. \& Heldr. forma cilicicum: \#1 II Primulaceae

Cyclamen cilicicum Boiss. \& Heldr. var. alpinum hort, = Cyclamen intaminatum (Meikle) Grey-Wilson

Cyclamen cilicicum Boiss. \& Heldr. var. intaminatum Meikle = Cyclamen intaminatum (Meikle) Grey-Wilson

Cyclamen circassicum Poped. = Cyclamen coum Mill. ssp. caucasicum (K, Koch) O. Schwarz

Cyclamen clusii Lindl. = Cyclamen purpurascens Mill. forma purpurascens
Cyclamen colchicum (Albov) Albov: \#1 II Primulaceae

Cyclamen commulatum $\mathrm{O}$. Schwarz \& Lepper = Cyclamen africanum Boiss. \& Reut.

Cyclamen coum Mill. ssp. alpinum (Sprenger) O. Schwarz = Cyclamen trochopteranthum $\mathrm{O}$. Schwarz forma trochopteranthum

Cyclamen coum Mill. ssp. caucasicum (K. Koch) Schwarz: \#1 II Primulaceae

Cyclamen coum Mill. ssp. coum forma albissimum R. H. Bailey, Koenen, Lillywh. \& P. J. M. Moore: \#I II Primulaceae

Cyclamen coum Mill. ssp. coum forma coum: Eastem Cyclamen (E) \#1 II Primulaceae

Cyclamen coum Mill. ssp. coum forma pallidum Grey-Wilson: \#1 II Primulaceae

Cyclamen coum Mill. ssp. elegans (Boiss. \& Buhse) Grey-Wilson: \#1 II Primulaceae

Cyclamen coum Mill. ssp. hiemale (Hildebr.) O. Schwarz = Cyclamen coum Mill. ssp. coum forma coum

Cyclamen coum Mill. var. abchasicum Medw. ex Kusn. = Cyclamen coum Mill. ssp. caucasicum (K. Koch) O. Schwarz

Cyclamen coum Mill. var. caucasicum (K. Koch) Meikle = Cyclamen coum Mill. ssp. caucasicum (K. Koch) O. Schwarz

Cyclamen creticum (Dörfl.) Hildebr. forma creticum: \#1 II Primulaceac

Cyclamen creticum (Dörfl.) Hildebr. forma pallide-roseum GreyWilson: \# I II Primulaceae

Cyclamen cyclaminus Bedevian = Cyclamen hederifolium Aiton var. hederifolium forma hederifolium

Cyclamen cyclophyllum Jord. = Cyclamen purpurascens Mill. forma purpurascens

Cyclamen cyprium Glasau = ?Cyclamen graecum Link ssp anatolicum letsw.

Cyclamen cyprium Sibth. = Cyclamen persicum Mill. var. persicum forma persicum

Cyclamen cyprium Kotschy: \#1 II Primulaceae

Cyclamen cypro-graecum E. Mutch \& N. Mutch = Cyclamen graecum Link ssp. anatolicum letsw.

Cyclamen deltoideum Tausch = Cyclamen purpurascens Mill. forma purpurascens

Cyclamen des Balèares $(F)=$ Cyclamen balearicum Willk.\# I II Primulaceae

Cyclamen d'Europe (F): Cyclamen purpurascens Mill. forma purpurascens: \#I II Primulaceae

Cyclamen durostoricum Pantu \& Solacolu = Cyclamen coum Mill. ssp. coum forma coum

Cyclamen elegans Boiss. \& Buhse. = Cyclamen coum Mill. ssp. elegans (Boiss. \& Buhse) Grey-Wilson

Cyclamen eucardium Jord. = Cyclamen repandum Sm. ssp. peloponnesiacum Grey-Wilson var. vividum (Grey-Wilson) Grey-Wilson

Cyclamen europaeum $\mathrm{L}_{1}=$ Cyclamen purpurascens Mill. forma purpurascens/Cyclamen repandum $\mathrm{Sm}$. ssp. repandum forma repandum/

Cyclamen europaeum Pall. = Cyclamen coum Mill. ssp. coum forma coum

Cyclamen europaeum Savi = Cyclamen purpurascens Mill. forma purpurascens

Cyclamen europaeum L. ssp. orbiculatum (Mill.) O. Schwarz = Cyclamen purpurascens Mill. forma purpurascens

Cyclamen europaeum L. ssp. orbiculatum (Mill.) O. Schwarz var. immaculatum Hrabetova = Cyclamen purpurascens Mill. forma purpurascens

Cyclamen europaeum L. ssp. ponticum (Albov) O. Schwarz = Cyclamen colchicum (Albov) Albov

Cyclamen europaeum L. var. caucasicum K. Koch = Cyclamen coum Mill. ssp. caucasicum (K. Koch) O. Schwarz

Cyclamen europaeum L. var. colchicum Albov = Cyclamen colchicum (Albov) Albov

Cyclamen fatrense Halda \& Soják = Cyclamen purpurascens Mill. forma purpurascens

Cyclamen ficariaefolium $\mathrm{Rchb} .=$ Cyclamen repandum $\mathrm{Sm}$. ssp. repandum forma repandum

Cyclamen floridum Salisb. = Cyclamen purpurascens Mill. forma purpurascens

Cyclamen gaidurowryssii Glasau = Cyclamen graecum Link ssp. graecum forma graecum 
Cyclamen graecum Link ssp. anatolicum letsw.: \#1 11 Primulaceae Cyclamen graecum Link ssp. candicum Ietsw.: \#1 II Primulaceae Cyclamen graecum Link ssp. graecum forma album R. Frank: \#1 II Primulaceae

Cyclamen graecum Link ssp. graecum forma graecum Greek Cyclamen (E): \#1 II Primulaceae

Cyclamen hastarum Tausch = Cyclamen purpurascens Mill. forma purpurascens

Cyclamen hederaceum Sieber ex Steud. = Cyclamen persicum Mill. var. persicum forma persicum

Cyclamen hederaefolium Sibth. \& Sm. = Cyclamen hederifolium Aiton var, hederifolium forma hederifolium

Cyclamen hederaefolium Sims = Cyclamen repandum Sm. ssp. repandum forma repandum

Cyclamen hederifolium Kotschy = Cyclamen persicum Mill. var. persicum forma persicum

Cyclamen hederifolium L. = Cyclamen hederifolium Aiton var. hederifolium forma hederifolium

Cyclamen hederifolium Aiton ssp. balearicum (Willk.) O. Schwarz = Cyclamen balearicum Willk.

Cyclamen hederifolium Aiton ssp. creticum (Dörfl.) O. Schwarz = Cyclamen creticum (Dörfl.) Hildebr. forma creticum

Cyclamen hederifolium Aiton ssp. hederifolium forma hederifolium Sowbread (E): \#1 II Primulaceae

Cyclamen hederifolium Aiton ssp. romanum (Griseb.) O. Schwarz = Cyclamen hederifolium Aiton var, hederifolium forma hederifolium

Cyclamen hederifolium Aiton var. ambiguum Grey-Wilson: \#1 II Primulaceae

Cyclamen hederifolium Aiton var. hederifolium forma albiflorum (Jord.) Grey-Wilson: \#1 II Primulaceae

Cyclamen hiemale Hildebr. = Cyclamen coum Mill. ssp. coum forma coum

Cyclamen holochlorum Jord. = Cyclamen purpurascens Mill. forma purpurascens

Cyclamen hyemale Salisb. = Cyclamen coum Mill. ssp. cousin forma coum

Cyclamen ibericum Goldie ex G. Don = Cyclamen coum Mill. ssp. caucasicum (K. Koch) O. Schwarz

Cyclamen ibericum Lem. = Cyclamen coum Mill. ssp. caucasicum (K. Koch) O. Schwarz

Cyclamen ibericum Steven ex Boiss. = Cyclamen coum Mill. ssp. caucasicum (K. Koch) O. Schwarz

Cyclamen ibericum T. Moore = Cyclamen coum Mill. ssp. caucasicum (K. Koch) O. Schwarz

Cyclamen ilicetorum Jord. = Cyclamen repandum $\mathrm{Sm}$. ssp. repandum forma repandum

Cyclamen insulare Jord. = Cyclamen hederifolium Aiton var. hederifolium forma hederifolium

Cyclamen intaminatum (Meikle) Grey-Wilson: \#1 II Primulaceae

Cyclamen kusnetzovii Kotov \& Czernova = Cyclamen coum Mill. ssp. coum forma coum

Cyclamen latifolium Sm. = Cyclamen persicum Mill. var. persicum forma persicum

Cyclamen latifolium Sibth. \& Sm. = Cyclamen persicum Mill. var. persicum forma persicum

Cyclamen libanoricum Hildebr.: \#I II Primulaceae

Cyclamen libanoticum Hildebr. ssp. pseudibericum (Hildebr.) Glasau = Cyclamen pseudibericum Hildebr. forma pseudibericum

Cyclamen lilacinum Jord. = Cyclamen purpurascens Mill. forma purpurascens

Cyclamen linearifolium DC = Cyclamen hederifolium Aiton ssp. hederifolium forma hederifolium

Cyclamen littorale Sadler ex Rchb. = Cyclamen purpurascens Mill. forma purpurascens

Cyclamen lobospilum Jord. = Cyclamen repandum Sm. ssp. repandum forma repandum

Cyclamen maritimum Hildebr. = Cyclamen graecum Link $s$ s. anatolicum letsw

Cyclamen miliarakesii Heldr. ex Halácsy = Cyclamen graecum Link ssp. graecum forma graecum

Cyclamen mindleri Heldr. = Cyclamen graecum Link ssp. candicum letsw.

Cyclamen mindleri Heldr. = Cyclamen graecum Link ssp. candicum Ietsw.

Cyclamen mirabile Hildebr, forma mirabile: \#1 II Primulaceae
Cyclamen mirabile Hildebr. forma niveum Grey-Wilson \& J. White: \#111 Primulaceae

Cyclamen neapolianum Ten. = Cyclamen hederifolium Aiton var. hederifolium forma hederifolium

Cyclamen neapolitanum Ten. = Cyclamen hederifolium Aiton ssp hederifolium forma hederifolium

Cyclamen numidicum Glasau = Cyclamen africanum Boiss. \& Reut.

Cyclamen orbiculatum Mill. = Cyclamen coum Mill. ssp. coum forma coum

Cyclamen orbiculatum Mill. var. alpinum Saunders = Cyclamen trochopteranthum $\mathrm{O}$. Schwarz forma trochopteranthum

Cyclamen orbiculatum Mill. var. coum (Mill.) Door. = Cyclamen coum Mill. ssp. coum forma coum

Cyclamen pachylobum Jord. = Cyclamen africanum Boiss. \& Reut

Cyclamen parviflorum Pobed. var, parviflorum: \#1 II Primulaceae

Cyclamen parviflorum Pobed. var. subalpinum Grey-Wilson: \#1 II Primulaceae

Cyclamen pentelici Hildebr: = Cyclamen graecum Link ssp. graecum forma graecum

Cyclamen persicum Mill. ssp. eupersicum $\mathrm{R}$. Knuth = Cyclamen persicum Mill. var. persicum forma persicum

Cyclamen persicum Mill. ssp. mindleri (Heldr.) R. Knuth = Cyclamen graecum Link ssp. candicum letsw.

Cyclamen persicum Mill. var, autumnale Grey-Wilson forma autumnale: \#1 II Primulaceae

Cyclamen persicum Mill. var. persicum forma albidum (Jord.) GreyWilson: \#I II Primulaceae

Cyclamen persicum Mill. var. persicum forma persicum: \#1 II Primulaceae

Cyclamen persicum Mill, var. persicum forna roseum Grey-Wilson: \#1 II Primulaceae

Cyclamen persicum Mill. var. persicum forma rubrum Grey-Wilson: \#1 II Primulaceae

Cyclamen poli Chiaje = Cyclamen hederifolium Aiton var. hederifolium forma hederifolium

Cyclamen ponticum (Albov) Poped. = Cyclamen colchicum (Albov) Albov

Cyclamen pseudibericum Hildebr. forma pseudibericum: \#1 II Primulaceae

Cyclamen pseudibericum Hildebr. forma roseum Grey-Wilson: \# 1 II Primulaceae

Cyclamen pseudograecum Hildebr. = Cyclamen graecum Link ssp. candicum letsw.

Cyclamen pseudomaritimum Hildebr. = Cyclamen graecum Link ssp. anatolicum letsw.

Cyclamen punicum Pomel = Cyclamen persicum Mill. var. persicum forma persicum

Cyclamen purpurascens Miller forma niveum Grey-Wilson: \#1 II Primulaceae

Cyclamen purpurascens Miller forma purpurascens: European Cyclamen (E), Cyclamen d'Europe (F), Cyclamen rouge pourpre (F) \#1 II Primulaceae

Cyclamen purpurascens Mill. ssp. immaculatum (Hrabetova) Halda \& Soják = Cyclamen purpurascens Mill. forma purpurascens

Cyclamen purpurascens Mill. ssp. ponticum (Albov) Grey-Wilson = Cyclamen colchicum (Albov) Albov

Cyclamen pyrolaefolium Salisb. = Cyclamen persicum Mill. var. persicum forma persicum

Cyclamen rarinaevum Jord. = Cyclamen repandum $\mathrm{Sm}$. ssp. repandum forma repandum

Cyclamen repandum Sibth. \& Sm.: Cyclamen étalé (F) \#1 II Primulaceae

Cyclamen repandum Sm. ssp. balearicum (Willk.) Malag. = Cyclamen balearicum Willk.

Cyclamen repandum $\mathrm{Sm}$. ssp. peloponnesiacum Grey-Wilson forma peloponnesiacum (Grey-Wilson) Grey-Wilson = Cyclamen repandum $\mathrm{Sm}$. ssp. peloponnesiacum Grey-Wilson var. peloponnesiacum (Grey-Wilson) Grey-Wilson

Cyclcmen repandum $\mathrm{Sm}$. ssp. peloponnesiacum Grey-Wilson forma vividum Grey-Wilson = Cyclamen repandum Sm. ssp. peloponnesiacum Grey-Wilson var. vividum (Grey-Wilson) Grey-Wilson

Cyclamen repandum $\mathrm{Sm}$. ssp. peloponnesiacum Grey-Wilson var peloponnesiacum (Grey-Wilson) Grey-Wilson: \#1 II Primulaceae 
Cyclamen repandum $\mathrm{Sm}$. ssp. peloponnesiacum Grey-Wilson var. vividum (Grey-Wilson) Grey-Wilson: \#I II Primulaceae

Cyclamen repandum $\mathrm{Sm}$. ssp. repandum forma repandum: \#I II Primulaceae

Cyclamen repandum $\mathrm{Sm}$. ssp. repandum forma album Grey-Wilson: \#1 II Primulaceae

Cyclamen repandum $\mathrm{Sm}$. ssp. rhodense (Meikle) Grey-Wilson: \#1 II Primulaceae

Cyclamen repandum Sm. var. creticum Dörfl. = Cyclamen creticum (Dörfl.) Hildebr, forma creticum

Cyclamen repandum $\mathrm{Sm}$, var. rhodense Meikle $=$ Cyclamen repandum Sm. ssp. rhodense (Meikle) Grey-Wilson

Cyclamen retroflexum Moench = Cyclamen purpurascens Mill forma purpurascens

Cyclamen rhodium R. Gorer ex O. Schwarz \& Lepper = Cyclamen repandum Sm. ssp. rhodense (Meikle) Grey-Wilson

Cyclamen rohlifsianum Aschers.: \#1 II Primulaceae

Cyclamen romanum Griseb.$=$ Cyclamen hederifolium Aiton var. hederifolium forma hederifolium

Cyclamen rouge pourpre (F): Cyclamen purpurascens Miller forma purpurascens: \#1 II Primulaceae

Cyclamen sabaudum Jord. = Cyclamen hederifolium Aiton var. hederifolium forma hederifolium

Cyclamen saldense Pomel = Cyclamen africanum Boiss. \& Reut.

Cyclamen somalense M. Thulin \& A. M. Warfa: \#1 Il Primulaceae

Cyclamen speciabile Jord. = Cyclamen repandum $\mathrm{Sm}$. ssp. peloponnesiacum Grey-Wilson var, vividum (Grey-Wilson) Grey-Wilson

Cyclamen stenopetalum Jord. = Cyclamen repandum $\mathrm{Sm}$. ssp. peloponnesiacum Grey-Wilson var, vividum (Grey-Wilson) Grey-Wilson

Cyclamen subhastatum Rchb. = Cyclamen hederifolium Aiton var. hederifolium forma hederifolium

Cyclamen subrotundum Jord. = Cyclamen africanum Boiss. \& Reut.

Cyclamen irochopieranthum Schwarz forma album Grey-Wilson: \#1 II Primulaceae

Cyclamen trochopieranthum Schwarz forma trochopteranthum: \#I II Primulaceae

Cyclamen iunetanum Jord. $=$ Cyclamen persicum Mill. var. persicum forma persicum

Cyclamen umbratile Jord. $=$ Cyclamen purpurascens Mill. forma purpurascens

Cyclamen utopicum Hoffmanns. = Cyclamen persicum Mill. var. persicum forma persicum

Cyclamen variegatum $\mathrm{Pohl}=$ Cyclamen pupuruscens Mill. forma purpurascens

Cyclamen velusinum Jord. = Cyclamen graecum Link ssp. graecum forma graecum

Cyclamen venustum Jord. = Cyclamen africanum Boiss. \& Reut.

Cyclamen vernale hort. $=$ Cyclamen coum Mill. ssp. coum forma coum

Cyclamen vernale Mill. $=$ Cyclamen persicum Mill. var, persicum forma persicum

Cyclamen vermale sensu O.Schwarz $=$ Cyclamen repandum $\mathrm{Sm}$. ssp. repandum forma repandum

Cyclamen vernum Lobel ex Cambess = Cyclamen balearicum Willk.

Cyclamen vernum Lobel ex Rchb. =Cyclamen repandum Sm. ssp. repandum forma repandum

Cyclamen vernum Sweet $=$ Cyclamen coum Mill. ssp. coum caucasicum (K. Koch) O. Schwarz

Cyclamen vernum Sweet forma alpinum O. Schwarz = Cyclamen trochopteranthum $\mathrm{O}$. Schwarz forma irochopteranthum

Cyclamen vernum Sweet = Cyclamen coum Miller ssp. coum forma coum

Cyclamen vernum Sweet var. caucasicum $\mathrm{O}$. Schwarz = Cyclamen coum Mill. ssp. caucasicum (K. Koch) O. Schwarz

Cyclamen vernum Sweet var, hiemale (Hildebr.) O. Schwarz forma alpinum (Sprenger) O. Schwarz = Cyclamen paniflorum Poped var. parviflorum

Cyclamen vernum Sweet var, hiemale (Hildebr.) O. Schwarz forma pseudocoum $\mathrm{O}$. Schwarz $=$ Cyclamen coum Mill. ssp. coum forma coum

Cyclamen zonale Jord. = Cyclamen coum Mill. ssp. caucasicum (K. Koch) O. Schwarz

Cyclamen étalé (F): Cyclamen repandum Sibth. \& Sm.: \#! II Primulaceae
Cyclaminos miliarakesii Heldr $=$ Cyclamen graecum Link ssp. graecum forma graecum

Cyclopogon spp. \#7 11 Orchidaceae

Cyclopiera $=$ Cyclopogon

Cyclosia $=$ Mormodes

Cycnoches spp. \#7 II Orchidaceae

Cydoniorchis spp. \#7 II Orchidaceae

Cylindrochilus $=$ Thrixspermum

Cylindrolobus $=$ Eria

Cylindropuntia $=$ Opuntia

Cylindrorebutia $=$ Rebutia

Cymbidiella spp. \$7 II Orchidaceae

Cymbidium spp. \#7 II Orchidaceae

Cymbiglossum $=$ Lemboglossum

Cymboglossum $=$ Eria

Cynorchis $=$ Cynorkis

Cynorkis spp. \#7 II Orchidaceae

Cynosorchis $=$ Cynorkis

Cyperorchis $=$ Cymbidium

Cypholoron spp. \#7 II Orchidaceae

Cyphrochilus spp. \#7 11 Orchidaceae

Cypripedium spp. \#7 II Orchidaceae

Cypripedium appletonianum Gower $=$ Paphiopedilum appletonianum (Gower) Rolfe

Cypripedium argus Reichb. $\mathrm{f}=$ Paphiopedilum argus (Reichb.f.) Stein

Cypripedium barbatum Lindl. $=$ Paphiopedilum barbatum (Lindl.) Rolfe

Cypripedium barbatum Lindl. var. biflorum (Williams) Williams = Paphiopedilum barbatum (Lindl.) Rolfe

Cypripedium barbalum Lindl, var, crossii hort, ex Veitch $=$ Paphiopedilum callosum (Reichb.f.) Stein var. callosum

Cypripedium barbalum Lindl. var. superbum Morren = Paphiopedilum superbiens (Reichb.f.) Stein var. superbiens

Cypripedium barbalum Lindl. var. veirchii Lem. $=$ Paphiopedilum superbiens (Reichb.f.) Stein var. superbiens

Cypripedium barbatum Lindl. var. wameri hort. $=$ Paphiopedilum callosum (Reichb.f.) Stein var. sublaeve (Reichb.f.) P. J. Cribb

Cypripedium barbatum Lindl. var. wamerianum T. Moore = Paphiopedilum callosum (Reichb.f.) Stein var. sublaeve (Reichb.f.) P. J. Cribb

Cypripedium bellalulum Reichb.f. $=$ Paphiopedilum bellarulum (Reichb.f.) Stein

Cypripedium biflorum Williams = Paphiopedilum barbalum (Lindl.) Rolfe

Cypripedium binotii hort. $=$ Phragmipedium vittatum $($ Vell. $)$ Rolfe

Cypripedium boxallii Reichb. $.{ }_{.}=$Paphiopedilum villosum (Lindl.) Stein var, boxallii (Reichb.f.) Pfitzer

Cypripedium boxallii Reichb.f. var. atratum Masters = Paphiopedilum villosum (Lindl.) Stein var. boxallii (Reichb.f.) Pfitzer

Cypripedium bullenianum Reichb.f. $=$ Paphiopedilum bullenianum (Reichb.f.) Pfitzer var. bullenianum

Cypripedium bullenianum Reichb.f. var. appletonianum (Gower) Rolfe $=$ Paphiopedilum appletonianum (Gower) Rolfe

Cypripedium burbidgei Reichb.f. = Paphiopedilum dayanum (Lindl.) Stein

Cypripedium callosum Reichb.f. var. callosum $=$ Paphiopedilum callosum (Reichb.f.) Stein var. callosum

Cypripedium callosum Reichb.f. var. sublaeve Reichb.f. = Paphiopedilum callosum (Reichb.f.) Stein var. sublaeve (Reichb.f.) P. J. Cribb

Cypripedium cannarianum Linden = Paphiopedilum philippinense (Reichb.f.) Stein var, philippinense

Cypripedium caricinum Bateman $=$ Phragmipedium pearcei (Reichb.f.) Rauh \& Senghas

Cypripedium caricinum Lindl. \& Paxton $=$ Phragmipedium caricinum (Lindl. \& Paxton) Rolfe

Cypripedium caudatum Lindl. $=$ Phragmipedium caudatum (LindI.) Rolfe

Cypripedium caudatum Lindl. var. lindenii (Lindl.) Veitch = Phragmipedium lindenii (Lindl.) Dressler \& N. H. Williams

Cypripedium caudatum Lindl, var. wallisii (Reichb.f.) Veitch $=$ Phragmipedium wallisii (Reichb.f.) Garay

Cypripedium chamberlainianum Sander = Paphiopedilum victoriaregina (Sander) M. W. Wood 
Cypripedium charlesworthii Rolfe = Paphiopedilum charlesworthii (Rolfe) Pfitzer

Cypripedium ciliolare Reichb.f. = Paphiopedilum ciliolare (Reichb.f.) Stein

Cypripedium ciliolare Reichb.f. var. mireauanum Linden = Puphiopedilum ciliolare (Reichb.f.) Stein

Cypripedium concolor Lindl. = Paphiopedilum concolor (LindI.) Pfitzer

Cypripedium concolor Lindl. var. godefroyae (God.-Leb.) Collett \& Hemsl. = Paphiopedilum godefroyae (God.-Leb.) Stein var. godefroyae

Cypripedium crossii Morren = Paphiopedilum callosum (Reichb.f.) Stein var. callosum

Cypripedium cruciforme Zoll \& Morren = Paphiopedilum lowii (Lindl.) Stein var. lowii

Cypripedium curisii Reichb.f. = Paphiopedilum superbiens (Reichb.f.) Stein var, curisii (Reichb.f.) G. J. Braem

Cypripedium dayanum (LindI.) Reichb.f. = Paphiopedilum dayanum (Lindl.) Stein

Cypripedium dayi Stone = Paphiopedilum dayanum (Lindl.) Stein

Cypripedium delenatii (Guillaumin) C. H. Curtis = Paphiopedilum delenatii Guillaumin

Cypripedium dilectum Reichb.f. = Paphiopedilum villosum (Lindl.) Stein var. boxallii (Reichb.f.) Pfitzer

Cypripedium druryi Bedd. = Paphiopedilum druryi (Lindl.) Stein

Cypripedium elliotrianum O'Brien = Paphiopedilum rothschildianum (Reichb.f.) Stein

Cypripedium emestianum hort. = Paphiopedilum dayanum (Lindl.) Stein

Cypripedium exul (Ridl.) Rolfe = Paphiopedilum exul (Ridl.) Rolfe

Cypripedium fairrieanum Lindl. = Paphiopedilum fairrieanum (Lindl.) Stein

Cypripedium gardineri Guillemard = Paphiopedilum glanduliferum (Blume) Stein var. glanduliferum

Cypripedium glanduliferum Blume = Paphiopedilum glanduliferum (Blume) Stein var. glanduliferum

Cypripedium glaucophyllum (J. J. Sm.) Masters = Paphiopedilum glaucophyllum J. J. Sm.

Cypripedium godefroyae God.-Leb. = Paphiopedilum godefroyae (God-Leb.) Stein var. godefroyae

Cypripedium godefroyae God.-Leb. var. leucochilum Rolfe = Paphiopedilum godefroyae (God.-Leb.) Stein var. leucuchilum (Masters) Hallier

Cypripedium grandiflorum Pav, = Phragmipedium boissierianum (Reichb.f.) Rolfe

Cypripedium gratrixianum Masters = Paphiopedilum gratrixianum (Masters) Guillaumin

Cypripedium hartwegii Reichb.f. = Phragmipedium longifolium (Warsz. \& Reichb.f.) Rolfe

Cypripedium haynaldianum Reichb.f. = Paphiopedilum haynaldianum (Reichb.f.) Stein

Cypripedium hincksianum Reichb.f. = Phragmipedium longifolium (Warsz. \& Reichb.f.) Rolfe

Cypripedium hirsurissimum Lindl ex Hook. = Paphiopedilum hirsutissimum (Lindl. ex Hook.) Stein var. hirsurissimum

Cypripedium hookerae Reichb $\mathrm{f}_{\mathrm{.}}=$ Paphiopedilum hookerae (Reichb.f.) Stein var. hookerae

Cypripedium hookerae Reichb.f. var. amabile (Hallier) Kraenzl. = Paphiopedilum bullenianum (Reichb.f.) Pfitzer var. bullenianum

Cypripedium hookerae Reichb.f. var. bullenianum (Reichb.f.) Veitch = Paphiopedilum bullenianum (Reichb.f.) Pfitzer var. bullenianum

Cypripedium hookerae Reichb.f. var. volonteanum Sander ex Rolfe = Paphiopedilum hookerae (Reichb.f.) Stein var. volonteanum (Sander ex Rolfe) Kerch

Cypripedium humboldrii Warsz. = Phragmipedium caudatum (Lindl.) Rolfe

Cypripedium insigne Wallich ex Lindl. = Paphiopedilum insigne (Wallich ex Lindl.) Pfitzer

Cypripedium insigne Wallich ex Lindl, var exul (Ridl.) Braem = Paphiopedilum exul (Ridl.) Rolfe

Cypripedium javanicum Reinw. ex Lindl. = Paphiopedilum javanicum (Reinw. ex Lindl.) Pfitzer var. javanicum

Cypripedium javanicum Reinw. ex Lindl. var. virens (Reichb.f.) Veitch = Paphiopedilum javanicum (Reinw. ex Lindl.) Pfitzer var. virens (Reichb.f.) Stein
Cypripedium klotzschianum Reichb.f. ex M. R. Schomb. = Phragmipedium klotzschianum (Reichb.f. ex M. R. Schomb.) Rolfe

Cypripedium laevigatum Bateman = Paphiopedilum philippinense (Reichb.f.) Stein var. philippinense

Cypripedium lawrenceanum Reichb.f. = Paphiopedilum lawrenceanum (Reichb.f.) Pfitzer

Cypripedium lindleyanum M. R. Schomb. ex Lindl. = Phragmipedium lindleyanum (M. R. Schomb. ex Lindl.) Rolfe

Cypripedium longifolium Warsz. \& Reichb.f. = Phragmipedium longifolium (Warsz. \& Reichb.f.) Rolfe

Cypripedium lowii Lindl. = Paphiopedilum lowii (Lindl.) Stein var lowii

Cypripedium mastersianum Reichb.f. = Paphiopedilum mastersianum (Reichb.f.) Stein

Cypripedium miteauanum Linden = Pophiopedilum ciliolare (Reichb.f.) Stein

Cypripedium neo-guineense Linden = Paphiopedilum rothschildianum (Reichb.f.) Stein

Cypripedium nigritum Reichb.f. = Paphiopedilum barbalum (Lindl. Rolfe

Cypripedium niveum Reichb.f. = Paphiopedilum niveum (Reichb.f.) Stein

Cypripedium papuanum Ridl. = Paphiopedilum papuanum (Ridl) Ridl.

Cypripedium pardinum Reichb.t. = Paphiopedilum venustum (Wallich ex Sims) Pfitzer ex Stein

Cypripedium parishii Reichb.f. = Paphiopedilum parishii $($ Reichb.f.) Stein

Cypripedium paulistanum Bart. Rodr. = Phragmipedium vittatum (Vell.) Rolfe

Cypripedium peteri D. Dean = Paphiopedilum dayanum (Lindl. Stein

Cypripedium petri Reichb.f. = Paphiopedilum dayanum (Lindl.) Stein

Cypripedium philippinense Reichb.f. = Paphiopedilum philippinense (Reichb.f.) Stein var. philippinense

Cypripedium philippinense Reichb.f. var. roebelenii Veitch = Paphiopedilum philippinense (Reichb.f.) Stein var. ruebelenii (Veitch) P. J. Cribb

Cypripedium pitcherianum Manda = Paphiopedilum argus (Reichb.f.) Stein

Cypripedium poyntzianum O'Brien = Paphiopedilum appleionianum (Gower) Rolfe

Cypripedium praestans $\mathrm{Rchb} . \mathrm{f}=$ Paphiopedilum glanduliferum (Blume) Stein var. glanduliferum

Cypripedium praestans Rchb.f var. kimballianum Linden \& Rodigas = Paphiopedilum glanduliferum (Blume) Stein var. glanduliferum

Cypripedium purpuratum Lindl. = Paphiopedilum purpuratum (Lindl.) Stein

Cypripedium robinsonii Ridl $=$ Paphiopedilum bullenianum (Reichb.f.) Pfitzer var. bullenianum

Cypripedium roebelenii Reichb. $\mathrm{f}$ = Paphiopedilum philippinense (Reichb.f.) Stein var. roebelenii (Veitch) P. J. Cribb

Cypripedium roebelenii Reichb.f. var. cannarianum Linden = Paphiopedilum philippinense (Reichb.f.) Stein var. philippinense

Cypripedium rothschildianum Reichb.f. = Paphiopedilum rothschildianum (Reichb.f.) Stein

Cypripedium sanderianum Reichb.f. = Paphiopedilum sanderianum (Reichb.f.) Stein

Cypripedium sargentianum $($ Rolfe) Kraenzl. $=$ Phragmipedium sargentianum (Rolfe) Rolfe

Cypripedium schlimii (Linden \& Reichb.f.) Bateman = Phragmipedium schlimii (Linden \& Reichb.f.) Rolfe

Cypripedium schmidtianum Kraenzl. = Paphiopedilum callosum (Reichb.f.) Stein var. callosum

Cypripedium schomburgkianum Klotzsch = Phragmipedium kiotzschianum (Reichb.f. ex M. R. Schomb.) Rolfe

Cypripedium sinicum Hance ex Reichb.f. = Paphiopedilum purpuratum (Lindl.) Stein

Cypripedium spectabile E. Salisb. var. dayanum Lindl. = Paphiopedilum dayanum (Lindl.) Stein

Cypripedium spicerianum Reichb.f. ex Masters \& T. Moore = Paphiopedilum spicerianum (Reichb.f. ex Masters \& T. Moore) Pfitzer 
Cypripedium stonei Hook. = Paphiopedilum stonei (Hook.) Stein

Cypripedium superbiens Reichb.f. var. dayanum (Lindl.) Reichb.f. = Paphiopedilum dayanum (Lindl.) Stein

Cypripedium sonkinense Godefroy $=$ Paphiopedilum concolor (Lindl.) Pfitzer

Cypripedium ronsum (Reichb.f.) Rolfe $=$ Paphiopedilum ronsum (Reichb.f.) Stein var. tonsum

Cypripedium veitchianum hort. ex Lem. $=$ Paphiopedilum superbiens (Reichb.f.) Stein var. superbiens

Cypripedium venustum Wallich ex Sims $=$ Paphiopedilum venustum (Wallich ex Sims) Pfitzer ex Stein

Cypripedium victoria-mariae Sander ex Mast. = Paphiopedilum victoria-mariae (Sander ex Mast.) Rolfe

Cypripedium victoria-regina Sander = Paphiopedilum victoriaregina (Sander) M. W. Wood

Cypripedium villosum Lindl. $=$ Paphiopedilum villosum (Lindl.) Stein var. villosum

Cypripedium villosum Lindl, var. boxallii (Reichb.f.) Veitch = Paphiopedilum villosum (Lindl.) Stein var. boxallii (Reichb.f.) Pfitzer

Cypripedium virens Reichb. $\mathrm{f}_{0}=$ Paphiopedilum javanicum (Reinw. ex Lindl.) Pfitzer var. virens (Reichb.f.) Stein

Cypripedium vintatum Vell. = Phragmipedium vitratum (Vell.) Rolfe

Cypripedium volonteanum Sander ex Rolfe $=$ Paphiopedilum hookerae (Reichb.f.) Stein var. volonteanum (Sander ex Rolfe) Kerch.

Cypripedium waltersianum Kraenzl. $=$ Paphiopedilum appletonianum (Gower) Rolfe

Cypripedium wardii (Summerh.) C. H. Curtis = Paphiopedilum wardii Summerh.

Cypripedium warscewiczianum Reichb.f. $=$ Phragmipedium caudatum (Lind!.) Rolfe

Cypripedium wolterianum Kraenzl $=$ Paphiopedilum appletonianum (Gower) Rolfe

Cypripedium $x$ petri (Reichb.f.) Rolfe $=$ Paphiopedilum dayanum (Lindl.) Stein

Cypripedium x petri (Reichb.f.) Rolfe var. burbidgei (Reichb.f.) Rolfe $=$ Paphiopedilum dayanum (Lindl.) Stein

Cyrtidiorchis spp. \#7 II Orchidaceae

Cynidium $=$ Cyrtidiorchis

Cyrtochilum $=$ Oncidium

Cyrtoglottis $=$ Podochilus

Cynopera $=$ Eulophia

Cyriopodium spp. \#7 II Orchidaceae

Cyriorchis spp. \#7 II Orchidaceae

Cyrosia spp. \#7 II Orchidaceae

Cyrlostylis spp. \#7 II Orchidaceae

Cystochilum $=$ Cranichis

Cystodiopteris $=$ Cystodium

Cystodium sorbifolium (J. E. Smith) J. Smith: \#1 II Dicksoniaceae

Cystopus $=$ Pristiglottis

Cystorchis spp. \#7 II Orchidaceae

Cytherea $=$ Calypso

Cytheris $=$ Calanthe

Dactylanthes anacantha (Aiton) Haworth = Euphorbia iridentata Lamarck

Dactylanthes globosa Haworth $=$ Euphorbia globosa (Haworth) Sims

Dactylanthes hamasa Haworth = Euphorbia hamata (Haworth) Sweet

Dacrylanshes patula Haworth = Euphorbia iridentata Lamarck

Dacrylanthes ruberculata (Jacquin) Haworth = Euphorbia tuberculata Jacquin

Dacrylanthocacius $=$ Parodia

Dactylorchis $=$ Dactylorhiza

Dactylorhiza spp. \#7 II Orchidaceae

Dactylorhynchus spp. \#7 II Orchidaceae

Dacrylosialix spp. \#7 II Orchidaceae

Dactylostylis = Zygostates

Dalbergia nigra Allem. ex Benth.: Bahia Rosewood (E), Brazilian Rosewood (E), Rio Rosewood (E), Jacarand de Brasil (S). Palissandre du Bresil (S) 1 Leguminosae

Danhatchia spp. \#7 II Orchidaceae

Darlingionia califormica Torrey: California Pitcherplant (E), Cobra Lily (E) \#I II Sarraceniaceae

Decarya madagascariensis Choux \#1 II Didiereaceae

Darwiniella $=$ Trichoceros
Darwiniera spp. \#7 II Orchidaceae

Dasyglossum spp. \#7 II Orchidaceae

Deamia $=$ Selenicereus

Decaisnea $=$ Prescottia $T$ ropidia

Deceptor spp. \#7 II Orchidaceae

Degranvillea spp. \#7 II Orchidaceae

Dehesa Bear-grass (E): Nolina interraia Gentry: I Agavaceae

Deiregyne spp. \#7 II Orchidaceae

Deiregynopsis = Aulosepalum

Delaetia $=$ Eriosyce

Demnosa $=$ Cleistocactus

Dendrobium spp. \#7 II Orchidaceae

Dendrobium cruentum Reichb.f: I Orchidaceae

Dendrocereus spp. \#4 II Cactaceac

Dendrochilum spp. \#7 II Orchidaceae

Dendrocolla $=$ Pteroceras $/$ Thrixspermum

Dendroconne $=$ Dendrobium

Dendrolirium = Eria

Dendrophylax spp. \#7 II Orchidaceae

Dendrorkis $=$ Aerides

Denmoza spp. \#4 II Cactaceae

Denslovia $=$ Habenaria

Deppia $=$ Lycaste

Deroemera $=$ Holothrix

Desmotrichum $=$ Flickingeria

Diacrium = Caularthron

Diadenium spp. \#7 II Orchidaceae

Dialissa $=$ Stelis

Diaphananthe spp. \#7 II Orchidaceae

Diceratostele spp. \#7 II Orchidaceae

Dicerostylis spp. \#7 II Orchidaceae

Dichaea spp. \#7 II Orchidaceae

Dichaeopsis = Dichaea

Dichopus $=$ Dendrobium

Dichorexia $=$ Cyathea

Dichromanthus spp. \#7 II Orchidaceae

DICKSONIACEAE spp:: Tree ferns (E), Helechos arborescentes (S),

Fougères arborescentes (F) \#1 II Dicksoniaceae

Dickasonia spp. \#7 II Orchidaceae

Dicksonia antarctica Labill.: Australian Tree Fern (E), Bristly Tree Fem (E), Soft Tree Fern (E) \#1 II Dicksoniaceae

Dicksonia arborescens L'Herit: \#I II Dicksoniaceae

Dicksonia archboldii Copel.: \#1 II Dicksoniaceae

Dicksonia baudouini Foum.: \#1 II Dicksoniaceae

Dicksonia berteroana (Colla) Hook.: \#1 II Dicksoniaceae

Dicksonia blumei (Kunze) Moore: \# I II Dicksoniaceae

Dicksonia brackenridgei Mett.: \#I II Dicksoniaceae

Dicksonia cicularia Sw. var. cicuraria: \#1 II Dicksoniaceae

Dicksonia cicutaria Sw. var. incisa (Fée) Capurro: \#1 II

Dicksoniaceae

Dicksonia coniifolia Hook. = Culcira coniifolia (Hook.) Maxon

Dicksonia externa Skottsb.: \#1 II Dicksoniaceae

Dicksonia fibrosa Colenso: \#I II Dicksoniaceae

Dicksonia ghiesbreghtii Maxon = Dicksonia gigantea Karst.

Dicksonia gigantea Karst.: \#1 II Dicksoniaceae

Dicksonia grandis Rosenstock: \# II Dicksoniaceae

Dicksonia hieronymi Brause: \# II Dicksoniaceac

Dicksonia lanata Colenso: \# I II Dicksoniaceae

Dicksonia lanigera Holttum: \#I II Dicksoniaceae

Dicksonia mollis Holttum: \# 1 II Dicksoniaceae

Dicksonia sciurus C. Chr.: \#1 II Dicksoniaceae

Dicksonia sellowiana (Presl) Hook.: \#1 II Dicksoniaceae

Dicksonia squarrosa (G. Forster) O. Swartz: Rough Tree Fem (E) \#

II Dicksoniaceae

Dicksonia thyrsopteroides Mett.: \#I II Dicksoniaceae

Dicksonia youngiae Hook. \& Baker: \#I II Dicksoniaceae

DIDIEREACEAE spp. \#1 II Didiereaceae

Didierea madagascariensis Baillon \#1 II Didiereaceae

Didierea trollii Capuron \& Rauh \#1 II Didiereaceae

Dicranophlebia $=$ Cyathea

Dicranotaenia $=$ Microcoelia

Dicrophyla $=$ Ludisoa

Dicrypta $=$ Maxillaria

Dictyophyllaria spp. \#7 II Orchidaceae

Didacryle = Bulbophyllum

Didiciea spp. \#7 II Orchidaceae 
Didothion $=$ Epidendrum

Didymoplexiella spp. \#7 II Orchidaceae

Didymoplexis spp. \#7 II Orchidaceae

Dienia $=$ Malaxis

Digiturebutia $=$ Rebutia

Diglyphis = Diglyphosa

Diglyphosa spp. \#7 II Orchidaceae

Dignathe spp. \#7 II Orchidaceae

Digomphotis $=$ Habenaria

Dikylikosigma $=$ Discyphus

Dilochia spp. \#7 II Orchidaceae

Dilochiopsis = Eria

Dilochus $=$ Dilochia

Dilomilis spp.\#7 II Orchidaceae

Dimerandra spp. \#7 II Orchidaceae

Dimorphorchis spp. \#7 II Orchidaceae

Dinema spp. \#7 II Orchidaceae

Dinklageella spp. \#7 II Orchidaceae

Dionaea muscipula Ellis: Venus Flytrap (E) \#1 II Droseraceae

Dioon spp. \#1 11 Zamiaceae

Dioon califanoi De Luca \& Sabato: \#1 II Zamiaceae

Dioon caputoi De Luca, Sabato \& Vazquez Torres: \#1 II Zamiaceae

Dioon edule Lindley var. angustifolium (Miq.) Miq.: \#1 II Zamiaceae

Dioon edule Lindley var, edule: \#I II Zamiaceae

Dioon holmgrenii De Luca, Sabato \& Vazquez Torres: \# I II Zamiaceae

Dioon mejiae Standley \& L. O. Williams: \#1 II Zamiaceae

Dioon merolae De Luca, Sabato \& Vazquez Torres: \#I II Zamiaceae

Dioon purpusii Rose: \#1 11 Zamiaceae

Dioon rzedowskii De Luca, A. Moretti, Sabato \& Vazquez Torres: \#1 II Zamiaceae

Dioon spinulosum Dyer: \#l II Zamiaceae

Dioon tomasellii De Luca, Sabato \& Vazquez Torres var. sonoren.se De Luca, Sabato \& Vazquez Torres: \#1 II Zamiaceae

Dioon romasellii De Luca, Sabato \& Vazquez Torres var. tomasellii: \#1 II Zarniaceae

Dioscorea (E): Dioscorea delioidea Wallich ex Kunth: \#1 II Dioscoreaceae

Dioscorea delioidea Wallich ex Kunth: Dioscorea (E), Elephant Foot (E), Gun (E), Dioscorée (F) \#I II Dioscoreaceae

Dioscorée (F): Dioscorea delioidea Wallich ex Kunth: \#I II Dioscoreaceae

Diothilopsis $=$ Epidendrum

Diothonea spp. \#7 II Orchidaceae

Dipera $=$ Disperis

Diphryllum = Listera

Diphyes $=$ Bulbophyllum

Diphylax spp. \#7 II Orchidaceae

Diplacorchis $=$ Brachycorythis

Diplandrorchis spp. \#7 II Orchidaceae

Diplanthera $=$ Platanthera

Diplogastra $=$ Platylepis

Diplectraden $=$ Habenaria

Diplectrum = Satyrium

Diplocaulobium spp. \#7 II Orchidaceae

Diplocentrum spp. \#7 II Orchidaceae

Diplochilus $=$ Diplomeris

Diploconchium $=$ Agrostophyllum

Diplodium $=$ Pterostylis

Diplolabellum spp. \#7 II Orchidaceae

Diplomeris spp. \#7 II Orchidaceae

Diploperianthium $=$ Calymmanthium

Diploprora spp. \#7 II Orchidaceae

Dipodium spp. \#7 II Orchidaceae

Dipteranthus spp. \#7 II Orchidaceae

Dipterostele spp. \#7 II Orchidaceae

Disa spp. \#7 II Orchidaceae

Discocactus spp. \#4 I/II Cactaceae

Discocactus albispinus Buining \& Brederoo = Discocactus zehntner Britton \& Rose

Discocactus alteolens Lemaire ex Dietrich $=$ Discocactus placentiformis Schum.

Discocactus araneispinus Buining \& Brederoo ex Buining = Discocactus zehntneri Britton \& Rose

Discocactus bahiensis Britton \& Rose: I Cactaceae
Discocactus bahiensis Britton \& Rose ssp. subviridigriseus (Buin \& Brederoo) Braun \& Est.: I Cactaceae

Discocactus boliviensis Theu.: I Cactaceae

Discocactus boomianus Buining \& Brederoo = Discocactus zehnmeri Britton \& Rose ssp. boomianus (Buining \& Brederoo) Taylor \& Zappi

Discocactus buenekeri W. R. Abraham = Discocactus zehnineri Briton \& Rose ssp. buenekeri (Abrh.) Braun \& Est.

Discocactus cangaensis L. Diers \& E. Esteves Pereira = Discocactus heptacanthus (Rodr.) Britton \& Rose

Discocactus catingicola Buining \& Brederoo = Discocactus heptacanthus (Rodr.) Britton \& Rose ssp. catingicola (Buining \& Brederoo) Taylor \& Zappi

Discocacius cephaliaciculosus Buining \& Brederoo: I Cactaceae

Discocactus cephaliaciculosus Buining \& Brederoo ssp. nudicephalus Braun \& Est. = Discocactus heptacanthus (Rodr.) Britton \& Rose

Discocactus crassispinus Braun \& Est.; I Cactaceae

Discocactus crassispinus Braun \& Est. ssp. araguaiensis Braun \& Est.: I Cactaceae

Discocactus crystallophilus Diers \& Esteves = Discocactus placentiformis K. Schum.

Discocactus diersianus E. Esteves Pereira = Discocactus heplacanthus (Rodr.) Britton \& Rose

Discucacius diersianus ssp. goianus (Diers \& E. Esteves Pereira) Braun \& E. Esteves Pereira = Discocactus heptacanthus $($ Rodr. $)$ Britton \& Rose

Discucactus estevesii L. Diers = Discocactus heptacanthus (Rodr.) Brition \& Rose

Discocactus ferricola Buining \& Brederoo: I Cactaceae

Discucactus flavispinus Buining \& Brederoo ex Buining = Discocactus heptacanthus (Rodr.) Britton \& Rose

Discocactus goianus Diers \& Esteves = Discocactus heptacanthus (Rodr.) Briton \& Rose

Discocactus griseus Buining \& Brederoo = Discocactus heptacanthus (Rodr.) Britton \& Rose ssp. catingicola (Buining \& Brederoo) Taylor \& Zappi

Discocactus harmannii (K. Schumann) Britton \& Rose: I Cactaceae

Discocactus hartmannii (K. Schumann) Britton \& Rose ssp. giganteus Braun \& Est. = Discocactus heptacanthus (Rodr.) Britton \& Rose

Discocactus hartmannii (K. Schumann) Britton \& Rose ssp. magnimammus (Buining \& Brederoo) Braun \& Est. = Discocactus hepiacanthus (Rodr.) Britton \& Rose ssp. magnimammus (Buining \& Brederoo) Taylor \& Zappi

Discocactus hartmannii (K. Schumann) Britton \& Rose ssp. pasulifolius (Buining \& Brederoo) Braun \& Est. = Liscocactus heptacanthus (Rodr.) Britton \& Rose ssp. magnimammus (Buining \& Brederoo) Taylor \& Zappi

Discocactus hartmannii (K. Schumann) Britton \& Rose ssp. setosiflorus Braun \& Est. = Discocactus heptacanthus (Rodr.) Britton \& Rose

Discocactus heptacanthus (Rodr.) Britton \& Rose: I Cactaceae

Discocactus heptacanthus (Rodr.) Britton \& Rose ssp. catingicola (Buining \& Brederoo) Taylor \& Zappi: I Cactaceae

Discocactus heptacanthus (Rodr.) Britton \& Rose ssp. magnimammus (Buining \& Brederoo) Taylor \& Zappi: I Cactaceae

Discocactus heptacanthus (Rodr.) Britton \& Rose ssp. melanochlorus (Buining \& Brederoo) Braun \& Est.: I Cactaceae

Discocactus horstii Buining \& Brederoo: I Cactaceae

Discocactus insignis Buining = Discocactus placentiformis (Lehm.) Schum

Discocactus latispinus Buining \& Brederoo ex Buining = Discocacrus placentiformis (Lehm.) Schum.

Discocactus latispinus Buining \& Brederoo ex Buining ssp. pseudolatispinus (Diers \& Est.) Braun \& Est. = Discocactus placentiformis (Lehm.) Schum.

Discocactus latispinus Buining \& Brederoo ex Buining ssp. pulvinicapitatus (Buining \& Brederoo) Braun \& Est. = Discocactus placentiformis (Lehm.) Schum.

Discocactus lindaianus L. Diers \& E. Esteves Pereira $=$ Discocactus heptacanthus (Rodr.) Britton \& Rose.

Discocactus magnimammus Buining \& Brederoo = Discocactus heptacanthus (Rodr.) Britton \& Rose ssp. magnimammus (Buining \& Brederoo) Taylor \& Zappi 
Discocacrus magnimammus Buining \& Brederoo ssp. bonitoensis ? = Discocactus heptacanthus (Rodr.) Britton \& Rose ssp. magnimammus (Buining \& Brederoo) Taylor \& Zappi

Discocactus mamillosus Buining \& Brederoo = Discocactus hartmannii (K. Schumann) Britton \& Rose

Disc'vacrus melanochlons Buining \& Brederoo ex Buining = Discocactus heptacanthus Britton \& Rose

Discocactus multicolonspinus Braun \& Brederoo = Discocactus placentiformis Schum.

Discocactus nigrisaetosus Buining \& Brederoo ex Buining = Discocactus catingicola Buining \& Brederoo

Discocactus pachythele Buining \& Brederoo: I Cactaceae

Discocactus paranaensis Backeb. = Discocactus heptacanthus (Rodr.) Britton \& Rose

Discocactus patulifolius Buining \& Brederoo = Discocactus hartmannii (K. Schumann) Britton \& Rose

Discocactus piauiensis Braun \& Est.: I Cactaceae

Discocacrus placenriformis (Lehm.) Schum.: I Cactaceae

Discocactus placentiformis (Lehm.) Schum. ssp. alteolens (Lem.) Braun \& Est.: I Cactaceae

Discocactus placentiformis (Lehm.) Schum. ssp. multicolorispinus (Buining \& Braun) Braun \& Est : I Cactaceae

Discocactus placentiformis (Lehm.) Schum. ssp. pugionacanthus (Buining \& Brederoo) Braun \& Est.: I Cactaceae

Discocactus prominentigibbus L. Diers \& E. Esteves Pereira = Discocactus heptacanthus (Rodr.) Britton \& Rose

Discocactus pseudoinsignis Taylor \& Zappi: I Cactaceae

Discocactus pseudolatispinus Diers \& Esteves = Discocactus placentiformis Schum.

Discocactus pugionacanthus Buining \& Brederoo ex Buining = Discocactus placentiformis Schum.

Discocactus pulvinicapitatus Buining \& Brederoo ex Buining = Discocactus placeniformis Schum.

Discocactus rapirhizus Buining \& Brederoo = Discocactus heptacanthus (Rodr.) Britton \& Rose

Discocactus semicampaniflorus Buining \& Brederoo = Discocactus heptacanthus (Rodr.) Britton \& Rose

Discocactus silicicola Buining \& Brederoo: I Cactaceae

Discocactus silvaticus Buining ex J. Theunissen: I Cactaceae

Discocactus spinosior Buining \& Brederoo = Discocactus heptacanthus (Rodr.) Britton \& Rose ssp. catingicola (Buining \& Brederoo) Taylor \& Zappi

Discocactus squamibaccatus Buining ex J. Theunissen: I Cactaceae

Discocacius subterraneo-prolifera L. Diers \& E. Esteves Pereira: I Cactaceae

Discocactus subviridigriseus Buining ex J. Theunissen = Discocactus bahiensis Britton \& Rose

Discocactus tricomis Monville ex Pfeiffer \& Otto = Discocactus placentiformis Schum.

Discocactus woutersianus Brederoo \& van der Broek $=$ Discocactus horstii Buining \& Brederoo

Discocactus zehnmeri Britton \& Rose: I Cactaceae

Discocactus zehntneri Briton \& Rose ssp. albispinus (Buining \& Brederoo) Braun \& Est.: I Cactaceae

Discocactus zehntneri Britton \& Rose ssp. araneispinus (Buining \& Brederoo) Braun \& Est.: I Cactaceae

Discocactus zehntneri Britton \& Rose ssp. buenekeri (Abrh.) Braun \& Est.: I Cactaceae

Discocacius zehnineri Britton \& Rose ssp. horstiorum (Braun) Braun \& Est: I Cactaceae

Discocactus zehntheri ssp. zehnineri: 1 Cactaceae

Discyphus spp. \#7 II Orchidaceae

Diseris $=$ Disperis

Disisocactus = Disocactus

Disocactus spp. \#4 III Cactaceae

Disocactus macdougallii (Alexander) Barthlot: Mcdougall's Cactus (E) I Cactaceae

Disocereus $=$ Disocactus

Disperis spp. \#7 II Orchidaceae

Disphenia = Cyathea

Dissorhynchium = Habenaria

Distichis = Liparis

Distomaea $=$ Listera

Distylodon spp. \#7 II Orchidaceae

Diteilis = Liparis

Dithrix spp. \#7 II Orchidaceae
Dithyridanthus = Schiedeella

Dituilis = Liparis

Ditulima = Dendrobium

Diuris spp. \#7 II Orchidaceae

Dockrillia $=$ Dendrobium

Dodsonia spp. \#7 II Orchidaceae

Dolichocentrum = Dendrobium

Dolichopsis = Eria

Dolichothele $=$ Mammillaria

Domingoa spp. \#7 11 Orchidaceae

Donacopsis = Eulophia $/$ Arundina

Doritis spp. \#7 II Orchidaceae

Dorycheile $=$ Cephalanthera

Dossinia spp. \#7 II Orchidaceae

Dorhilis = Spiranthes

Dothilophis = Epidendrum

Dove Orchid (E): Peristeria elata Hooker I Orchidaceae

Doxosma $=$ Epidendrum

Dracocactus = Eriosyce

Draconanthes spp. \#7 II Orchidaceae

Dracula spp. \#7 II Orchidaceae

Drakaea spp. \#7 II Orchidaceae

Drakonorchis spp. \#7 II Orchidaceae

Dresslerella spp, \#7 II Orchidaceae

Dressleria spp. \#7 II Orchidaceae

Dressleriella $=$ Jacquiniello

Drury's Slipper Orchid (E): Paphiopedilum drunyi (Beddome) Stein: I Orchidaceae

Dryadella spp. \#7 II Orchidaceae

Dryadorchis spp. \#7 II Orchidaceae

Drymoanthus spp. \#7 II Orchidaceae

Drymoda spp. \#7 II Orchidaceae

Dropeia $=$ Disperis

Dryorchis = Disperis

Dubois-reymondia $=$ Myoxanthus

Duboisia $=$ Myoxanthus

Duckeella spp. \#7 II Orchidaceae

Dudleya stolonifera Moran: Laguna Beach Dudleya (E), Laguna Beach Liveforever (E) I Crassulaceae

Dudleya traskiae (Rose) Moran: Santa Barbara Island Dudleya (E), Santa Barbara Island Liveforever (E) I Crassulaceae

Dunstervillea spp. \#7 II Orchidaceae

Dyakia spp. \#7 II Orchidaceae

Dypsis decaryi (Jum.) Beentje \& J. Dransf. = Neodypsis decaryi Jumelle

Dypsis decipiens (Becc.) Beentje \& J. Dransf. = Chrysalidocarpus decipiens Becc.

Eaglewood (E): Aquilaria malaccensis Lamk: \# I II Thymelaeaceae Earina spp. \#7 II Orchidaceae

Eastem Cyclamen (E): Cyclamen coum Mill. ssp. coum forma coum \#I II Primulaceae

Eatoniopteris = Cyathea

Ebnerella = Mammillaria

Eburophyton $=$ Cephalanthera

Eccremocacius $=$ Weberocereus

Eccremocereus $=$ Weberocereus

Echinocactus spp. \#4 II Cactaceae

Echinocactus alteolens Lem. = Discocactus placentiformis (Lem.) Schum.

Echinocactus asterias Zucc. = Astrophysum asterias (Zucc.) Lem.

Echinocactus disciformis (DC.) K. Schumann = Strombocactus disciformis (DC.) Britton \& Rose

Echinocactus erectocentrus J. Coulter $=$ Sclerocactus erectocentrus (J. Coulter) N. P. Taylor var. ereciocentrus

Echinocactus glaucus K. Schumann = Sclerocactus glaucus (K. Schumann) L. Benson

Echinocactus horripilus Lemaire var. erectocentrus (J. Coulter) Weber = Sclerocactus ereciocentrus (J. Coulter) N. P. Taylor var, erectocentrus

Echinocactus krausei Hildmann = Sclerocactus erectocentrus (J. Coulter) N. P. Taylor var. ereciocentrus

Echinocactus macrochele Werdermann = Turbinicapus macrochele (Werdermann) F. Buxb. \& Backeb.

Echinocactus mandragora Fric ex Berger = Turbinicarpus mandragora (Fric ex Berger) A. Zimmerman 
Echinocactus mariposensis (Hester) Weniger = Sclerocactus mariposensis (Hester) N. P. Taylor

Echinocactus mesae-verdae (Boiss. \& Davidson) L. Benson = Sclerocactus mesae-verdae (Boiss. \& Davids.) L. Benson

Echinocactus papyracanthus Engelm. = Sclerocactus papyracanthus (Engelm.) N. P. Taylor

Echinocactus peeblesianus (Croizat) L. Benson = Pediocactus peeblesianus (Croizat) L. Benson var. peeblesianus

Echinocactus pubispinus Engelm. = Sclerocactus pubispinus (Engelm.) L. Benson var. pubispinus

Echinocactus schmiedickeanus Bödeker = Turbinicarpus schmiedickeanus (Bödeker) F. Buxb. \& Backeb.

Echinocactus sileri Engelm. = Pediocactus sileri (Engelm.) L. Benson

Echinocactus subglaucus Rydb. = Sclerocactus glaucus (K. Schumann) L. Benson

Echinocactus tobuschii (Marshall) Weniger = Sclerocactus brevihamatus (Engelmann) D. Hunt

Echinocactus turbiniformis Pfeiffer = Strombocactus disciformis (DC.) Britton \& Rose

Echinocactus valdezianus (Möller) Bödeker = Turbinicarpus valdezianus (Möller) C. Glass \& R. Foster

Echinocactus whipplei Engelm. \& Bigelow var. glaucus (K. Schumann). J. A. Purpus = Sclerocacius glaucus (K. Schumann) L. Benson

Echinocereus spp. \#4 I/II Cactaceae

Echinocereus ferreirianus H. Gates ssp. lindsayi (J. Meyrán) N. P. Taylor: Lindsay's Cactus (E) I Cactaceae

Echinocereus lindsayi Meyrán = Echinocereus ferreirianus H. Gates ssp. lindsayi (J. Meyrán) N. P. Taylor

Echinocereus schmollii (Weing.) N. P. Taylor: Lamb's-tail Cactus (E) I Cactaceae

Echinofossulocactus = Echinocactus/Stenocactus

Echinofossulocactus turbiniformis (Pfeiffer) Lawrence = Strombocactus disciformis (DC.) Britton \& Rose

Echinoglossum $=$ Cleisostoma

Echinolobivia $=$ Echinopsis

Echinomastus $=$ Sclerocactus

Echinomastus acunensis (W. T. Marshall) L. Benson $=$ Sclerocactus erectocentrus (J. Coulter) N. P. Taylor var. acunensis

Echinomastus erectocentrus Britton \& Rose = Sclerocactus erectocentrus ( $\mathrm{J}$. Coulter) N. P. Taylor var. erectocentrus

Echinomastus mariposensis Hester = Sclerocactus mariposensis (Hester) N. P. Taylor

Echinonyctanthus = Echinopsis

Echinopsis spp. \#4 II Cactacene

Echinorebutia $=$ Rebutia

Echioglossum = Cleisostoma

Eckartia $=$ Peristeria

Ecuadoria spp. \#7 11 Orchidaceae

Efossus = Stenocactus

Eggelingia spp. \#7 II Orchidaceae

Elaeophorbia beillei (A. Chevalier) H. Jacobsen = Euphorbia beillei A. Chevalier

Elasmatium $=$ Goodyera

Eleorchis spp. \#7 II Orchidaceae

Elephant Foot (E): Dioscorea delsoidea Wallich ex Kunth: \#1 II Dioscoreaceae

Elephant's Trunk (E): Pachypodium namaquanum (Wyley ex Harv.) Welw.: \#1 II Apocynaceae

Elfin Tree Fern (E): Cyathea dryopteroides Maxon: \#1 II Cyatheaceae

Elleanthus spp. \#7 II Orchidaceae

Eloyella $=$ Phymatidium

Eltroplectris = Stenorrhynchos

Elythranthera spp. \#7 II Orchidaceae

Embreea spp. \#7 II Orchidaceae

Emorycactus = Echinocactus

Empusa $=$ Liparis

Empusaria $=$ Liparis

Encephalartos spp.: Palmas del Pan (S) I Zamiaceae

Encephalartos aemulans Vorster: I Zamiaceae

Encephalartos altensteinit Lehm.: I Zamiaceae

Encephalartos aplanatus P.Vorster: I Zamiaceae

Encephalartos arenarius R. A. Dyer: I Zamiaceae
Encephalartos bareri Cartuth. ex Miq. ssp. allochrous L. E Newton: I Zamiaceae

Encephalartos barteri Carruth. ex Miq. ssp. barteri: I Zamiaceae

Encephalartos brachyphyllus Lehm. \& De Vriese = Encephalartos caffer (Thunb.) Lehm.

Encephalarios brevifoliolatus P.Vorster: I Zamiaceae

Encephalartos bubalinus Melville: I Zamiaceae

Encephalartos caffer (Thunb.) Lehm.: I Zamiaceae

Encephalarios cerinus Lavranos \& D. L. Goode: 1 Zamiaceae

Encephalartos chimanimaniensis R. A. Dyer \& I. Verd.: I Zamiaceae

Encephalartos concinnus R. A. Dyer \& I. Verd.: I Zamiaceae

Encephalartos cupidus R. A. Dyer: I Zamiaceae

Encephalartos cycadifolius (Jacq.) Lehm.: I Zamiaceae

Encephalartos delucanus Malaisse, Sclavo \& Croisiers: 1 Zamiaceae

Encephalartos dolomiticus Lavranos \& D. L. Goode: I Zamiaceae

Encephalartos dyerianus Lavranos \& D. L. Goode: I Zamiaceae

Encephalartos equatorialis P. J. H. Hurter \& H. F. Glen: I Zamiaceae

Encephalartos eugene-maraisii I. Verd.: I Zamiaceae

Encephalartos eximius I. Verd. = Encephalartos cycadifolius (Jacq.) Lehm.

Encephalartos ferox Bertol.f.: I Zamiaceae

Encephalartos friderici-guilielmi Lehm.: I Zamiaceae

Encephalartos ghellinckii Lem.: I Zamiaceae

Encephalartos graniticolus Robbertse et al. = Encephalartos dyerianus Lavranos \& Goode

Encephalartos gratus Prain: I Zamiaceae

Encephalartos heenanii R. A. Dyer: 1 Zamiaceae

Encephalartos hildebrandiii A. Braun \& Boché var. dentatus Melville: I Zamiaceae

Encephalartos hildebrandlii A. Braun \& Boché var. hildebrandtii: I Zamiaceae

Encephalartos hirsumus P. J. H. Hurter \& H. F. Glen: I Zamiaceae

Encephalartos horridus (Jacq.) Lehm.: I Zamiaceae

Encephalartos humilis I. Verd.: I Zamiaceae

Encephalartos imbricans P. Vorster \& P. Heibloem $=$ Encephalanos equatorialis P. J. H. Hurter \& H. F. Glen

Encephalarros inopinus R. A. Dyer: I Zamiaceae

Encephalartos ituriensis Bamps \& Lisowski: I Zamiaceae

Encephulartos kisambo Faden \& Bentje: I Zamiaceae

Encephalartos kosiensis Hutch. = Encephalartos ferox Bertol.f.

Encephalartos laevifolius Stapf \& Burt Davy; I Zamiaceae

Encephalarios lanalus Stapf \& Burtt Davy: I Zamiaceae

Encephalarios lanuginosus (Jacq.) Lehm. = Encephalartos longifolius (Jacq.) Lehm.

Encephalartos latifrons Lehm. I Zamiaceae

Encephalartos laurentianus De Wild.: I Zamiaceae

Encephalartos lebomboensis I. Verd.: I Zamiaceae

Encephalartos lehmannii Lehm.: I Zamiaceae

Encephalartos lemarinellianus De Wild. = Encephalartos poggei Aschers.

Encephalartos longifolius (Jacq.) Lehm.: I Zamiaceae

Encephalarios manikensis (Gilliland) Gilliland: I Zamiaceae

Encephalartos marunguensis Devred: I Zamiaceae

Encephalartos middelburgensis Vors., Robb. \& S. van der Westh.: I Zamiaceae

Encephalarios msinganus Vorster: I Zamiaceae

Encephalartos munchii R. A. Dyer \& I. Verd.: I Zamiaceae

Encephalarios nanus Lehm. = Encephalartos horridus (Jacq.) Lehm.

Encephalartos natalensis R. A. Dyer \& I. Verd.: I Zamiaceae

Encephalartos ngoyanus I. Verd.: I Zamiaceae

Encephalartos nubimontanus P. J. H. Hurter: I Zamiaceae

Encephalartos paucidentalus Stapf \& Burt Davy: 1 Zamiaceae

Encephalartos poggei Aschers.: I Zamiaceae

Encephalartos powysorum Beentje: I Zamiaceae

Encephalartos princeps R. A. Dyer: I Zamiaceae

Encephalartos pterogonus R. A. Dyer \& I. Verd.: I Zamiaceae

Encephalartos schaijesii Malaisse, Sclavo \& Crosiers: I Zamiaceae

Encephalartos schmitzi Malaisse: I Zamiaceae

Encephalartos sclavoi De Luca, D. Stevenson \& Moretti: I Zamiaceae

Encephalartos sentica Vorster: I Zamiaceae

Encephalarios septentrionalis Schweinfurth: I Zamiaceae

Encephalartos successibus P. Vorster = Encephalartos whitelockii P. J. H. Hurter

Encephalartos tegulaneus Melville: I Zamiaceae 
Encephalartos transvenosus Stapf \& Burt Davy: I Zamiaceae Encephalarios trispinosus (Hook.) R. A. Dyer: I Zamiaceae Encephalartos tumeri Lavranos \& D. L. Goode: I Zamiaceae Encephalartos umbeluziensis R. A. Dyer: I Zamiaceae

Encephalartos venetus Vorster: I Zamiaceae

Encephalarios verrucosus Robbertse et al $=$ Encephalartos dolomiticus Lavranos \& Goode

Encephalartos villosus Lemaire: I Zamiaceae

Encephalartos whitelockii P. J. H. Hurter: I Zamiaceae

Encephalartos woodii Sander: I Zamiaceae

Encephalocarpus $=$ Pelecyphora

Encephalocarpus strobiliformis (Werdermann) Berg. = Pelecyphora strobiliformis (Werdermann) Fric \& Schelle

Encheiridion $=$ Microcoelia

Encyclia spp. \#7 II Orchidaceae

Endeisa $=$ Dendrobium

Endresiella spp. \#7 II Orchidaceae

Engelhardia pterocarpa (Oersted) Standley = Oreomunnea prerocarpa Oerst.

Enothrea $=$ Octomeria

Entaticus = Habenaria

Entomophobia spp. \#7 II Orchidaceae

Eomatucana $=$ Matucane

Eparmatostigma spp. \#7 II Orchidaceae

Ephemerantha $=$ Flickingeria

Ephippianthus spp. \#7 II Orchidaceae

Ephippium $=$ Bulbophyllum

Epiblastus spp. \#7 II Orchidaceae

Epiblema spp. \#7 II Orchidaceae

Epicladium $=$ Epidendrum

Epicranthes $=$ Bulbophyllum

Epicrianthus = Bulbophyllum

Epidanthus spp. \#7 II Orchidaceae

Epidendropsis spp. \#7 II Orchidaceae

Epidendrum spp. \#7 II Orchidaceae

Epidendrum labiatum (Lindl.) Reichb.f. var. trianaei Reichb.f. = Cantleya frianaei Linden \& Reichb.f.

Epigeneium spp. \#7 II Orchidaceae

Epilyna = Elleanthus

Epipactis spp. \#7 II Orchidaceae

Epiphora $=$ Polystachya

Epiphyllanthus $=$ Schlumbergera

Epiphyllopsis = Hatiora

Epiphyllum spp. \#4 II Cactaceae

Epipogium spp. \#7 II Orchidaceae

Epipogon $=$ Epipogium

Epistephium spp. \#7 II Orchidaceae

Epithecia $=$ Dichea $/$ Epidendrum

Epithelantha spp. \#4 II Cactaceae

Erdisia $=$ Corryocactus

Eria spp. \#7 II Orchidaceae

Eriaxis spp. \#7 II Orchidaceae

Eriocactus $=$ Parodia

Eriocephala $=$ Parodia

Eriocereus $=$ Harrisia

Eriochilum $=$ Eriochilus

Eriochilus spp. \#7 II Orchidaceae

Eriochylus $=$ Eriochilus

Eriodes spp. \#7 II Orchidaceae

Eriopexis $=$ Dendrobium

Eriopsis spp. \#7 II Orchidaceae

Eriosyce spp. \#4 II Cactaceae

Erioxantha $=$ Eria

Erporkis $=$ Platylepis

Erycina spp. \#7 II Orchidaceae

Erythrocereus $=$ Harrisia

Erythrodes spp. \#7 II Orchidaceae

Erythrorchis spp. \#7 II Orchidaceae

Erythrorhipsalis $=$ Rhipsalis

Escobaria spp. \#4 I/II Cactaceae

Escobaria leei $($ Rose ex Bödeker) Bödeker = Escobaria sneedii Britt \& Rose var. leei (Rose ex Bödeker) D. R. Hun

Escobaria minima (Baird) D. R. Hunt: Nellie's Cory Cactus (E) I Cactaceae

Escobaria nellieae $($ Croizat) Backeb. $=$ Escobaria minima (Baird) D. R. Hunt
Escobaria sneedii Britt. \& Rose: Lee's Pincushion Cactus (E), Sneed's Cory Cactus (E), Sneed's Pincushion Cactus (E) I Cactaceae

Escobaria sneedii Britt. \& Rose var. leei (Rose ex Bödeker) D. R. Hunt: I Cactaceae

Escobaria sneedii Britt. \& Rose var. sneedii I Cactaceae

Escobesseya $=$ Escobaria

Escontria spp. \#4 II Cactaceae

Esmeralda spp. \#7 II Orchidaceae

Esposioa spp. \#4 II Cactaceae

Espostoopsis spp. \#4 II Cactaceae

Esula dendroides $(\mathrm{L}$, ) Haworth = Euphorbia dendroides L.

Elaeria $=$ Hetaeria

Euanthe spp. \#7 II Orchidaceae

Eucnemia $=$ Govenio

Eucnemis = Govenia

Eucosia spp. \#7 II Orchidaceae

Eudisanthema $=$ Brassavola

Euforbias (S): Euphorbia spp. I/I Euphorbiaceae

Eulophia spp. \#7 II Orchidaceae

Eulophidium $=$ Oeceoclades

Eulophiella spp. \#7 II Orchidaceae

Eulophiopsis = Graphorkis

Eulychnia spp. \#4 II Cactaceae

Eulychnocacsus $=$ Cornyocactus

Euothonaea $=$ Hexisea

Euphlebium = Dendrobium

Euphorbes (F): Euphorbia spp. I/II Euphorbiaceae

Euphorbia spp. (succulent spp. only) Spurges (E), Euforbias (S). Lechetreznas (S), Cardones (S), Euphorbes (F) I/II Euphorbiaceae

Euphorbia abdelkuri Balf. f.: \#! II Euphorbiaceae

Euphorbia abyssinica Gmelin: \#1 Il Euphorbiaceae

Euphorbia abyssinica Gmelin var. mozambicensis Boissier = Euphorbia angularis Pax

Euphorbia abyssinica Gmelin var. tetragona Schweinf. = Euphorbia abyssinica Gmelin

Euphorbia acaulis Roxburgh = Euphorbia fusiformis BuchananHamilton ex D. Don

Euphorbia acrurensis N. E. Brown = Euphorbia abyssinica Gmelin

Euphorbia actinoclada S. Carter: \#1 II Euphorbiaceae

Euphorbia aculeata Forsskål: \#I II Euphorbiaceae

Euphorbia adenensis Deflers = Euphorbia balsamifera Aiton ssp. adenensis (Deflers) P. R. O. Bally

Euphorbia adenochila S. Carter: \#I II Euphorbiaceae

Euphorbia adjurana P. R. O. Bally \& S. Carter: \#l II Euphorbiaceae

Euphorbia aequoris N. E. Br.: \#1 II Euphorbiaceae

Euphorbia aeruginosa Schweick.: \#I II Euphorbiaceae

Euphorbia aethiopium Croizat = Euphorbia abyssinica $\mathrm{Gmelin}$

Euphorbia aggregata A. Berger var, aggregata: \#1 II Euphorbiaceae

Euphorbia aggregata A. Berger var, alternicolor (N. E. Brown)

A. C. White, R. A. Dyer \& B. Sloane: \#I II Euphorbiaceae

Euphorbia alata Hook.: \#1 II Euphorbiaceae

Euphorbia albertensis N. E. Br.: \#1 II Euphorbiaceae

Euphorbia albipollinifera L. C. Leach: \#1 II Euphorbiaceae

Euphorbia albovillosa $\mathrm{Pax}=$ Euphorbia gueinzii Boissier var. albovillosa (Pax) N. E. Brown

Euphorbia alcicomis A. Berger = Euphorbia epiphylloides S. Kurz

Euphorbia alcicomis Baker: \#1 II Euphorbiaceae

Euphorbia alfredii W. Rauh: \#1 II Euphorbiaceae

Euphorbia alluaudii Drake ssp. alluaudii = Euphorbia leucodendron Drake

Euphorbia alluaudii Drake ssp. oncoclada (Drake) Friedm. \& Cremers $=$ Euphorbia oncoclada Drake

Euphorbia altemicolor N. E. Brown = Euphorbia aggregata A. Berger var. altemicolor (N. E. Brown) A. C. White, R. A. Dyer \& B. Sloane

Euphorbia amarifontana N. E. Br.: \#1 II Euphorbiaceae

Euphorbia ambacensis N. E. Br. = Euphorbia ingens E. Meyer ex Boiss.

Euphorbia ambatofinandranae Leandri = Euphorbia stenoclada Baillon var. ambatofinandranae (Leandri) Cremers

Euphorbia $\mathrm{x}$ ambohipotsiensis Ursch \& Leandri: \#I II Euphorbiaceae

Euphorbia ambovombensis Rauh \& Razaf.: I Euphorbiaceae 
Euphorbia ambroseae L. C. Leach var. ambroseae; \#1 II Euphorbiaceae

Euphorbia ambroseae L. C. Leach var. spinosa Leach: \#1 II Euphorbiaceae

Euphorbia ammak Schweinfurth: \#1 II Euphorbiaceae

Euphorbia ampliphylla Pax: \#1 II Euphorbiaceae

Euphorbia anacantha Aiton = Euphorbia trideniata Lamarck

Euphorbia anachoreta Svent. = Euphorbia despoliata (Menezes) Monod

Euphorbia analalavensis Leandri: \#1 II Euphorbiaceae

Euphorbia $x$ andrefandrovana Ursch \& Leandri: \#I II Euphorbiaceae

Euphorbia angrae N. E. Br.: \#1 II Euphorbiaceae

Euphorbia angularis Klotzsch: \#1 II Euphorbiaceae

Euphorbia angustiflora Pax: \#1 II Euphorbiaceae

Euphorbia ankarensis Boit.: \#1 II Euphorbiaceae

Euphorbia annamarieae Rauh: \#1 II Euphorbiaceae

Euphorbia anoplia Stapf: \#I II Euphorbiaceae

Euphorbia antankara Leandri = Euphorbia pachypodivides Boiteau

Euphorbia $\times$ anticaffra Lotsy \& Goddijn = Euphorbia $\times$ bothae Lotsy \& Goddijn

Euphorbia aniquorum L.: \#I II Euphorbiaceac

Euphorbia antisyphilitica Zucc.: Candelilla (S) \#1 II Euphorbiaceae

Euphorbia antso Denis: \#1 II Euphorbiaceae

Euphorbia aphylla Brouss. ex Willd.: \#I II Euphorbiaceae

Euphorbia appariciana Rizzini: \#1 II Euphorbiaceae

Euphorbia applanata Thulin \& Al-Gifri: \#1 II Euphorbiaceae

Euphorbia arahaka Decorse ex H. Poisson: \#I II Euphorbiaceae

Euphorbia arbuscula Balf. f. var. arbuscula: \#1 II Euphorbiaceae

Euphorbia arbuscula Balf. f. var. montana Balf. f.: \#1 II Euphorbiaceae

Euphorbia arceuthobioides Boiss.: \#1 II Euphorbiaceae

Euphorbia argillicola Dinter = Euphorbia namibensis Marloth

Euphorbia arida N. E. Br.: \#1 II Euphorbiaceac

Euphorbia armata Thunberg = Euphorbia loricata Lamarck

Euphorbia arrecta N. E. Brown = Euphorbia mixla N. E. Brown in Thiselton-Dyer

Euphorbia aspericaulis Pax: \#I II Euphorbiaceae

Euphorbia asthenacantha S. Carter: \#1 II Euphorbiaceae

Euphorbia astrispina N. E. Brown = Euphorbia stellispina Haworth var. astrispina (N. E. Brown) A. C. White, R. A. Dyer \& B. Sloane

Euphorbia astrophora Marx: \#1 II Euphorbiaceae

Euphorbia atoto G. Forster: \#1 II Euphorbiaceae

Euphorbia atrispina N. E. Br. var. atrispina: \#I II Euphorbiaceae

Euphorbia atrispina N. E. Br. var. viridis A. C. White. R. A. Dyer \& B. Sloane: \#1 II Euphorbiaceae

Euphorbia atrocarmesina L. C. Leach ssp. arborea L. C. Leach: \#1 II Euphorbiaceae

Euphorbia atrocarmesina L. C. Leach ssp. arrocarmesina: \#1 II Euphorbiaceae

Euphorbia atroflora S. Carter: \#1 II Euphorbiaceae

Euphorbia atropurpurea Brouss. ex Willd. var. atropurpurea: \#1 II Euphorbiaceae

Euphorbia atropurpurea Brouss. ex Willd. forma lutea Santos: \#1 II Euphorbiaceae

Euphorbia atropurpurea Brouss. ex Willd. var. modesta Sventenius: \#1 II Euphorbiaceae

Euphorbia atrox S. Carter: \#1 II Euphorbiaceae

Euphorbia attastoma Rizzini var. attastoma: \#1 II Euphorbiaceae

Euphorbia attastoma Rizzini var. xanthochlora Rizzini: \#1 II Euphorbiaceae

Euphorbia aureoviridiflora (Rauh) Rauh: \#1 II Euphorbiaceae

Euphorbia avasmontana Dinter var. avasmoniana: \#I II Euphorbiaceae

Euphorbia avasmontana Dinter var, saginaria (Marl.) A. C. White, R. A. Dyer \& B. Sloane: \#1 II Euphorbiaceae

Euphorbia awashensis M. G. Gilbert: \#1 II Euphorbiaceae

Euphorbia baga A. Chevalier var. baga: \#1 II Euphorbiaceae

Euphorbia baga A. Chevalier var. parvifolia L. E. Newton: \#1 II Euphorbiaceae

Euphorbia baioensis S. Carter: \#1 II Euphorbiaceae

Euphorbia baleensis M. G. Gilber: \#1 II Euphorbiaceae

Euphorbia baliola N. E. Br.: \#1 II Euphorbiaceae

Euphorbia ballyana Rauh: \#1 II Euphorbiaceae
Euphorbia ballyi S. Carter: \#1 II Euphorbiaceae

Euphorbia balsamea Welwitsch ex Hiem = Euphorbia gariepina Boissier ssp. balsamea (Welwitsch ex Hiern) L. C. Leach

Euphorbia balsamifera Ait. ssp. adenensis (Defl.) P. R. O. Bally: \#1 II Euphorbiaceae

Euphorbia balsamifera Ait. ssp. balsamifera: II Euphorbiaceae

Euphorbia balsamifera N. E. Brown ssp. eubalsamifera Maire = Euphorbia balsamifera Aiton ssp. balsamifera

Euphorbia balsamifera Aiton ssp, rogeri (N. E. Brown) Maire = Euphorbia balsamifera Aiton

Euphorbia balsamifera N. E. Brown ssp. sepium (N. E. Brown) Maire = Euphorbia balsamifera Aiton

Euphorbia banae Rauh: \#1 II Euphorbiaceae

Euphorbia baradii S. Carter: \#1 II Euphorbiaceae

Euphorbia barbicollis P. R. O. Bally: \#1 II Euphorbiaceae

Euphorbia bariensis S. Carter: \#I II Euphorbiaceae

Euphorbia bamardii A. C. White, R. A. Dyer \& B. Sloane: \#1 II Euphorbiaceae

Euphorbia bamhartii L. Croiz: \#1 II Euphorbiaceae

Euphorbia barteri N. E. Brown = Euphorbia kamerunica Pax

Euphorbia basutica Marloth = Euphorbia clavarioides Boissier var. clavarioides

Euphorbia baumii Pax = Euphorbia monteiri Hook.f. ssp. monteiri

Euphorbia bayeri L. C. Leach: \#I II Euphorbiaceae

Euphorbia baylissii L. C. Leach: \#1 II Euphorbiaceae

Euphorbia beaumieriana Hook.f. \& Cosson = Euphorbia officinanum $\mathrm{L}$

Euphorbia beharensis Leandri: \#III Euphorbiaceae

Euphorbia beillei A. Chevalier: 1 II Euphorbiaceae

Euphorbia bellica Hiem = Euphorbia virosa Willd. ssp. virosa

Euphorbia benguelensis $\mathrm{Pax}=$ Euphorbia trichadenia $\mathrm{Pax}$

Euphorbia bergeri N. E. Brown: \#1 II Euphorbiaceae

Euphorbia bergeriana Dinter $=$ Euphorbia gariepina Boissier ssp. balsamea (Welwitsch ex Hiem) L. C. Leach

Euphorbia bergii A. C. White, R. A. Dyer \& B. Sloane: \#1 II Euphorbiaceae

Euphorbia berorohue Rauh \& Hofstatter: \#I II Euphorbiaceae

Euphorbia berotica N. E. Br.: \#1 II Euphorbiaceae

Euphorbia berthelotii Bolle ex Boiss.: \#1 II Euphorbiaceae

Euphorbia bevilaniensis Croizat = Euphorbia milii Des Moulins var. bevilaniensis (Croizal) Ursch \& Leandri

Euphorbia biaculeata Denis: \#1 II Euphorbiaceae

Euphorbia biglandulosa Willd. = Euphorbia burmannii (Klotzsch) E. Meyer ex Boissier

Euphorbia biharamulensis S. Carter: \#1 II Euphorbiaceae

Euphorbia bilocularis N. E. Brown = Euphorbia candelabrum Kotschy var. bilocularis (N. E. Brown) S. Carter

Euphorbia bitataensis M. G. Gilbert: \#I II Euphorbiaceae

Euphorbia boinensis Denis ex Humbert \& Leandri: \#1 II Euphorbiaceae

Euphorbia boissieri Baillon: \#I II Euphorbiaceae

Euphorbia boiteaui Leandri: \#1 II Euphorbiaceac

Euphorbia bojeri Hooker = Euphorbia milii Des Moulins var. milii

Euphorbia bolusii N. E. Br.: \#! II Euphorbiaceae

Euphorbia bongolavensis Rauh: \#1 II Euphorbiaceae

Euphorbia boranensis M. G. Gilbert: \#1 II Euphorbiaceae

Euphorbia bosseri Leandri: \#1 II Euphorbiaceae

Euphorbia x bothae Lotsy \& Goddijn: \#I II Euphorbiaceae

Euphorbia bothae Lotsy \& Goddijn var. anticaffra (Lotsy \& Goddijn) G. D. Rowley = Euphorbia $\times$ bothae

Euphorbia bottae Boiss: \#1 II Euphorbiaceae

Euphorbia bougheyi L. C. Leach: \#1 II Euphorbiaceae

Euphorbia bourgaeana Gay ex Boiss.: \#I II Euphorbiaceae

Euphorbia brachiata (Klotzsch) Boiss. in De Candolle: \#1 II Euphorbiaceae

Euphorbia brachyphylla Denis: \#1 II Euphorbiaceae

Euphorbia brakdamensis N. E. Br: \#1 II Euphorbiaceae

Euphorbia brassii P. I. Forster: \#1 II Euphorbiaceae

Euphorbia braunsii N. E. Br.: \# I II Euphorbiaceae

Euphorbia bravoana Svent: \#1 II Euphorbiaceae

Euphorbia breonii Noisette = Euphorbia milii Des Moulins var. milii

Euphorbia breviarticulara Pax var, breviarticulata: \#1 II Euphorbiaceae

Euphorbia breviarticulata Pax var, Irunciformis S. Carter: \#1 II Euphorbiaceae

Euphorbia brevirama N. E. Br.: \#1 II Euphorbiaceae 
Euphorbia brevis N. E. Br.: \#1 II Euphorbiaceae

Euphorbia brevilorta P. R. O. Bally: \#1 II Euphorbiaceae

Euphorbia broussonetii Willdenow ex Link: \#1 II Euphorbiaceae

Euphorbia brunellii Chiov: \#I II Euphorbiaceae

Euphorbia bruynsii L. C. Leach: \#I II Euphorbiaceae

Euphorbia bubalina Boiss.: \#1 II Euphorbiaceae

Euphorbia bulbispina Rauh \& Razafindratsira: \#1 II Euphorbiaceae

Euphorbia bupleurifolia Jacq.: \#1 II Euphorbiaceae

Euphorbia burgeri M. G. Gilbert: \#1 II Euphorbiaceae

Euphorbia burmannii E. Meyer ex Boiss.: \#1 II Euphorbiaceae

Euphorbia burmannii E. Meyer ex Boiss. var. karroensis Boissier = Euphorbia karroensis (Boissier) N. E. Brown in Thiselton-Dyer Euphorbia buruana Pax: \#1 II Euphorbiaceae

Euphorbia bussei Pax var. bussei: \#1 II Euphorbiaceae

Euphorbia bussei Pax var. kibwezensis (N. E. Br.) S. Carter: \#1 II Euphorbiaceae

Euphorbia bwambensis S. Carter: \# III Euphorbiaceae

Euphorbia cactus Ehrenberg ex Boissier var. cactus: \#1 II Euphorbiaceae

Euphorbia cacrus Ehrenberg ex Boissier var. Iorirama Rauh \& Lavranos: \#1 II Euphorbiaceae

Euphorbia caducifolia Haines: \#1 II Euphorbiaceae

Euphorbia caerulans Pax: \#1 II Euphorbiaceae

Euphorbia caerulescens Haw.; \#1 II Euphorbiaceae

Euphorbia calamiformis P. R. O. Bally \& S. Carter: \#1 II Euphorbiaceae

Euphorbia calderensis Philippi = Euphorbia copiapina Philippi

Euphorbia califomica Bentham var. californica: \#1 II Euphorbiaceae

Euphorbia californica Bentham var. hindsiana (Bentham) Wiggins: \#1 II Euphorbiaceae

Euphorbia calycina N. E. Brown = Euphorbia candelabrum Kotschy var. candelabrum

Euphorbia cameronii N. E. Br.: \#1 II Euphorbiaceae

Euphorbia canaliculata Lamarck = Euphorbia clava Jacquin

Euphorbia canariensis L.: \# 1 II Euphorbiaceae

Euphorbia canariensis L. forma viridis G. Kunkel = Euphorbia canariensis $\mathrm{L}$

Euphorbia canariensis L. var. spiralis Bolle ex Boissier = Euphorbia canariensis L

Euphorbia candelabrum Welwitsch = Euphorbia conspicua N. E. Brown

Euphorbia candelabrum Welwitsch ex Hiem = Euphorbia conspicua N. E. Brown

Euphorbia candelabrum Kotschy var, bilocularis (N. E. Br.) S. Carter: \#1 II Euphorbiaceae

Euphorbia candelabrum Kotschy var, candelabrum: \#1 II Euphorbiaceae

Euphorbia candelabrum Kotschy var, enthraeae A. Berger = Euphorbia abyssinica Gmelin

Euphorbia cannellii L. C. Leach: \#1 II Euphorbiaceae

Euphorbia capazzi Caballero = Euphorbia balsamifera Aiton ssp. balsamifera

Euphorbia capmanambatoensis Rauh: \#1 II Euphorbiaceae

Euphorbia capsaintemariensis Rauh: I Euphorbiaceae

Euphorbia capsaintemariensis Rauh var. tulearensis Rauh = Euphorbia tulearensis (Rauh) Rauh

Euphorbia captiosa N. E. Brown = Euphorbia ferox Marloth

Euphorbia capuronii Ursch \& Leandri: \#1 II Euphorbiaceae

Euphorbia caput-aureum Denis: \#1 II Euphorbiaceae

Euphorbia caput-medusae L.: \#1 II Euphorbiaceae

Euphorbia caput-medusae L. var. geminata Aiton = Euphorbia capul-medusae L.

Euphorbia caput-medusae L. var. major Aiton = Euphorbia capuimedusae $\mathrm{L}$

Euphorbia caput-medusae L. var. minor Aiton = Euphorbia caputmedusae L.

Euphorbia carteriana P. R, O, Bally: \#1 II Euphorbiaceae

Euphorbia carunculifera L. C. Leach ssp. carunculifera: \#1 II Euphorbiaceae

Euphorbia canunculifera L. C. Leach ssp. subfastigiata L. C. Leach \#1 II Euphorbiaceae

Euphorbia cassythoides Boiss.: \#1 II Euphorbiaceae

Euphorbia cataractarum S. Carter: \#1 II Euphorbiaceae

Euphorbia caterviflora N. E. Br.: \#1 II Euphorbiaceae

Euphorbia cantimandoo Elliot ex Wight: \#1 II Euphorbiaceae
Euphorbia cedrorum Rauh \& Hebding: \#1 II Euphorbiaceae

Euphorbia celara R. A. Dyer: \#I II Euphorbiaceae

Euphorbia cereiformis L.: \#I II Euphorbiaceae

Euphorbia cereiformis L, var, echinata Salm-Dyck ex Boissier = Euphorbia cereifonnis $\mathbf{L}$.

Euphorbia cereiformis L. var. submammillaris A. Berger = Euphorbia submammillaris (A. Berger) A. Berger

Euphorbia cerifera Alcocer = Euphorbia antisyphilitica Zuccarini

Euphorbia cervicomis Boissier = Euphorbia hamala (Haworth) Sweet

Euphorbia chamaecormos Chiovenda = Euphorbia schizacantha Pax

Euphorbia charleswilsoniana V. VIk: \#1 II Euphorbiaceae

Euphorbia chersina N. E. Br.: \#1 II Euphorbiaceae

Euphorbia cibdela N. E. Br.: \#1 II Euphorbiaceae

Euphorbia ciliolata $\mathrm{Pax}=$ Euphorbia transvaalensis Schlechter

Euphorbia cirsioides Costantin \& Gallaud = Euphorbia stenoclada Baillon ssp. sienoclada

Euphorbia clandestina Jacq.: \#I II Euphorbiaceae

Euphorbia classenii P. R. O. Bally \& S. Carter: \#1 II Euphorbiaceae

Euphorbia clava Jacq.: Club-shaped Euphorb (E) \#I II Euphorbiaceae

Euphorbia clavarioides Boiss. var. clavarioides: \#1 II Euphorbiaceae

Eupharbia clavarioides Boiss, var, truncata (N. E. Br.) A. C. White, R. A. Dyer \& B. Sloane: \#1 II Euphorbiaceae

Euphorbia clavata Salisbury = Euphorbia clava Jacquin

Euphorbia clavigera N. E. Br.: \#1 II Euphorbiaceae

Euphorbia clivicola R. A. Dyer: II II Euphorbiaceae

Euphorbia coerulans hort. = Euphorbia caerulans Pax

Euphorbia coerulescens hort. $=$ Euphorbia caerulescens Haworth

Euphorbia colliculina A. C. White, R. A. Dyer \& B. Sloane: \#1 II Euphorbiaceae

Euphorbia colubrina P. R. O. Bally \& S. Carter: \#1 II Euphorbiaceae

Euphorbia columnaris P. R. O. Bally: \#1 II Euphorbiaceae

Euphorbia commelinii De Candolle = Euphorbia capul-medusae L

Euphorbia commersonii Baillon = Euphorbia thouarsiana Baillon

Euphorbia comosa Vellozo: \#1 II Euphorbiaceae

Euphorbia complexa R. A. Dyer: \#I II Euphorbiaceae

Euphorbia confertiflora Volkens = Euphorbia candelabrum Kotschy var. candelabrum

Euphorbia confinalis R. A. Dyer ssp. confinalis: \#I II Euphorbiaceae

Euphorbia confinalis R. A. Dyer ssp. rhodesiaca L. C. Leach: \#1 II Euphorbiaceae

Euphorbia confluens Nel: \#1 II Euphorbiaceae

Euphorbia conformis N. E. Brown = Eupharbia negromontana N. E. Brown in Oliver et al.

Euphorbia congestiflora L. C. Leach: \#1 II Euphorbiaceae

Euphorbia consobrina N. E. Brown: \#1 II Euphorbiaceae

Euphorbia conspicua N. E. Br.: \#I II Euphorbiaceae

Euphorbia contorta L. C. Leach: \#1 II Euphorbiaceae

Euphorbia controversa N. E. Br. = Euphorbia abyssinica Gmelin

Euphorbia cooperi N. E. Br. ex Berger var. calidicola L. C. Leach: \#1 II Euphorbiaceac

Euphorbia cooperi N. E. Br. ex Berger var. cooperi: \#1 II Euphorbiaceae

Euphorbia cooperi N. E. Br. ex A. Berger var, ussanguensis (N. E. Br.) L. C. Leach: \#1 II Euphorbiaceae

Euphorbia copiapina Philippi: \#l 11 Euphorbiaceae

Euphorbia comiculata R. A Dyer: \#I II Euphorbiaceae

Euphorbia coronata Thunberg = Euphorbia clava Jacquin

Euphorbia corymbosa N. E. Br.: \#1 II Euphorbiaceae

Euphorbia corynoclada Müller Argoviensis = Euphorbia plumerioides Teijsmann ex Hasskarl

Euphorbia crassipes Marloth: \#I II Euphorbiaceae

Euphorbia cremersii Rauh \& Razafindratsira forma viridifolia Rauh: I Euphorbiaceae

Euphorbia cremersii Rauh \& Razafindratsira var. cremersii: I Euphorbiaceae

Euphorbia cremersii Rauh \& Razafindratsira var, rakotozafyi (Cremers) Rauh: I Euphorbiaceae

Euphorbia crispa (Haw.) Sweet: \#1 II Euphorbiaceae

Euphorbia crispata hort . ex Lemaire = Euphorbia lemaireano Boissier in De Candolle

Euphorbia croizatii Leandri: \#1 II Euphorbiaceac

Euphorbia crypiocaulis M. G. Gilbert: \#I II Euphorbiaceae

Euphorbia cryptospinosa P. R. O. Bally: \#1 II Euphorbiaceae 
Euphorbia cucumerina Willd.: \#1 II Euphorbiaceae

Euphorbia cumulata R. A. Dyer: \#1 II Euphorbiaceae

Euphorbia cuneaia Vahl: \#1 II Euphorbiaceae

Euphorbia cuneata Vahl var. carpasus Boissier = Euphorbia cuneata Vahl

Euphorbia cuneata Vahl var. perrottetii (Jaubert \& Spach) Boissier = Euphorbia cuneata Vahl

Euphorbia cuneneana L. C. Leach ssp. cuneneana: \#1 II Euphorbiaceae

Euphorbia cuneneana L. C. Leach ssp, rhizomatosa L. C. Leach: \#1 II Euphorbiaceae

Euphorbia cuprispina S. Carter: \#I II Euphorbiaceae

Euphorbia curocana L. C. Leach: \#1 II Euphorbiaceae

Euphorbia curronii N. E. Brown = Euphorbia marabelensis Pax

Euphorbia curvirama R. A. Dyer: \#1 II Euphorbiaceae

Euphorbia cussonioides P. R. O. Bally: \#1 II Euphorbiaceae

Euphorbia cylindrica A. C.White, R. A. Dyer \& B. Sloane: \#I II Euphorbiaceae

Euphorbia cylindrifolia Mam.-Lap. \& Rauh ssp. cylindrifolia: I Euphorbiaceae

Euphorbia cylindrifolia Mam.-Lap. \& Rauh ssp. tuberifera Rauh: I Euphorbiaceae

Euphorbia dalettiensis M. G. Gilbert: \#1 II Euphorbiaceae

Euphorbia damarana L. C. Leach: \#1 II Euphorbiaceae

Euphorbia darbandensis N. E. Br.: \# I II Euphorbiaceae

Euphorbia dasyacantha S. Carter: \#1 II Euphorbiacese

Euphorbia dauana S. Carter: \#1 II Euphorbiaceae

Euphorbia davyi N. E. Br.: \#1 II Euphorbiaceae

Euphorbia davyi N. E. Brown ssp. maleolens (E. Phillips) Hargreaves $=$ Euphorbia maleolens E. Phillips

Euphorbia davyi N. E. Brown ssp. tlapanensis Hargreaves = Euphurbia davyi N. E. Br.

Euphorbia dawei N. E. Br.: \#1 II Euphorbiaceae

Euphorbia debilispina L. C. Leach: \#1 II Euphorbiaceae

Euphorbia decariana Croizat $=$ Euphorbia hedyotoides N. E. Brown in Oliver et al.

Euphorbia decaryi Guillaumin var. ampanihyensis Cremers: I Euphorbiaceae

Euphorbia decaryi Guillaumin var. capsaintemariensis (Rauh) Cremers = Euphorbia capsaintemariensis Rauh

Euphorbia decaryi Guillaumin var. decaryi: I Euphorbiaceae

Euphorbia decani Guillaumin var, robinsonii Cremers: I Euphorbiacese

Euphorbia decaryi Guillaumin var. spirnsticha Rauh \& Buchloh: I Euphorbiaceae

Euphorbia decepta N. E. Br.: \#I II Euphorbiaceae

Euphorbia decidua P. R. O. Bally \& L. C. Leach: \#I II Euphorbiaceae

Euphorbia decliviticola L. C. Leach: \#1 II Euphorbiaceae

Euphorbia decorsei Drake: \#1 II Euphorbiaceae

Euphorbia decussata Hiem = Euphorbia negromontana N. E. Brown in Oliver et al

Euphorbia decussala E. Mey. = Euphorbia indecora N.E.Br.

Euphorbia dedzana L. C. Leach: \#1 II Euphorbiaceae

Euphorbia deightonii Croizat: \#1 II Euphorbiaceae

Euphorbia dekindrii Pax: \#1 II Euphorbiaceae

Euphorbia delphinensis Ursch \& Leandri: \#1 11 Euphorbiaceae

Euphorbia demissa L. C. Leach: \#1 II Euphorbiaceae

Euphorbia dendroides L.: \#I II Euphorbiaceae

Euphorbia denisiana Guillaumin: \#! II Euphorbiaceae

Euphorbia x dentonii hort, ex Anon.: \#1 II Euphorbiaceae

Euphorbia desmondii Keay \& Milne-Redhead: \#1 II Euphorbiaceae

Euphorbia despoliata (Menezes) Monod: \#1 II Euphorbiaceae

Euphorbia dhofarensis S. Carter: \#1 II Euphorbiaceae

Euphorbia dichroa S. Carter: \#1 II Euphorbiaceac

Euphorbia didiereoides Denis \& Leandri: \#1 II Euphorbiaceae

Euphorbia dinteri Marloth = Euphorbia venenata Marloth

Euphorbia dinteri A. Berger = Euphorbia virosa Willd. ssp. virosa

Euphorbia disclusa N. E. Brown = Euphorbia abyssinica Gmelin

Euphorbia discrepans S. Carter: \#1 II Euphorbiaceae

Euphorbia discreta N. E. Brown = Euphorbia woodii N. E. Brown in Thiselton-Dyer

Euphorbia dispersa L. C. Leach: \#I II Euphorbiaceae

Euphorbia dissitispina L. C. Leach: \#1 II Euphorbiaceae

Euphorbia distinctissima L. C. Leach: \#1 II Euphorbiaceae

Euphorbia divaricata Jacquin = Euphorbia dendroides L.
Euphorbia x doinetiana Guillaumin: \#I II Euphorbiaceae

Euphorbia dolichoceras S. Carter: \#I II Euphorbiaceae

Euphorbia dregeana E. Mey. ex Boiss.: \#I II Euphorbiaceae

Euphorbia dumericola P. R. O. Bally \& S. Carter: \#1 II Euphorbiaceae

Euphorbia duranii Ursch \& Leandni var, ankaratrae Ursch \& Leandri: \#I II Euphorbiaceae

Euphorbia duranii Ursch \& Leandri var. duranii: \#I II Euphorbiaceae

Euphorbia duseimata R. A. Dyer: \#1 II Euphorbiaceae

Euphorbia echinus Hook.f. \& Cosson: \#1 II Euphorbiaceae

Euphorbia echinus Hook.f. \& Cosson var. brevispina Hook.f. \& Cosson = Euphorbia echinus Hook.f. \& Cosson

Euphorbia echinus Hook.f. \& Cosson var. chlorantha Maire = Euphorbia echinus Hook.f. \& Cosson

Euphorbia echinus Hook.f. \& Cosson var, chlorosoma Croizat = Euphorbia echinus Hook.f. \& Cosson

Euphorbia echinus Hook.f. \& Cosson var. hemandez-pachecoi (Caballero) Maire = Euphorbia echinus Hook.f. \& Cosson

Euphorbia ecklonii (Klotzsch \& Garke) Baillon: \#1 II Euphorbiaceae

Euphorbia eduardoi L. C. Leach: \#I II Euphorbiaceae

Euphorbia eendomensis Dinter = Euphorbia fusca Marloth

Euphorbia eilensis S. Carter: \#I II Euphorbiaceae

Euphorbia elastica Marloth = Euphorbia dregeana E. Meyer ex Boissier

Euphorbia elegantissima P. R. O. Bally \& S. Carter: \#1 II Euphorbiaceae

Euphorbia ellenbeckii Pax: \#I II Euphorbiaceae

Euphorbia elliotii Leandri: \#1 II Euphorbiaceae

Euphorbia elliprica Thunberg = Euphorbia silenifolia (Haworth) Sweet

Euphorbia elliptica Thunberg var. undulata Boissier = Euphorbia crispa (Haworth) Sweet

Euphorbia enopla Boissier var. dentaia A. Berger = Euphorbia heptagona L. var. dentata (A. Berger) A. C. White, R. A. Dyer \& B. Sloane

Euphorbia enopla Boiss. var. enopla: \#1 II Euphorbiaceae

Euphorbia enopla Boiss. var. viridis A. C. White, R. A. Dyer \& B. Sloane: \#1 II Euphorbiaceae

Euphorbia enormis N. E. Br.: \#1 II Euphorbiaceae

Euphorbia enterophora Drake ssp. crassa Cremers: \#1 II Euphorbiaceae

Euphorbia enterophora Drake ssp. enterophora: \#1 II Euphorbiaceae

Euphorbia ephedroides E. Meyer ex Boiss, var. debilis L. C. Leach: \#1 II Euphorbiaceae

Euphorbia ephedroides E.Meyer ex Boiss. var. ephedroides: \#1 II Euphorbiaceae

Euphorbia ephedroides E. Meyer ex Boiss. var, imminuta

L. C. Leach \& Williamson: \#1 II Euphorbiaceae

Euphorbia epiphylloides Kurz: \#1 II Euphorbiaceae

Euphorbia erigavensis S. Carter: \#1 II Euphorbiaceae

Euphorbia erlangeri Pax: \#I II Euphorbiaceae

Euphorbia ernestii N. E. Br.: \#1 II Euphorbiaceac

Euphorbia erosa Willd. = Euphorbia cereiformis L

Euphorbia erythraeae (A. Berger) N. E. Br. = Euphorbia abyssinica Gmelin

Euphorbia esculenta Marloth: \#I II Euphorbiaceae

Euphorbia espinosa Pax: \#1 II Euphorbiaceae

Euphorbia etuberculosa P. R. O. Bally \& S. Carter: \#1 II Euphorbiaceae

Euphorbia eustacei N. E. Br.: \#1 II Euphorbiaceae

Euphorbia evansii Pax: \#1 II Euphorbiaceae

Euphorbia excelsa A. C. White, R. A. Dyer \& B. Sloane; \#! II Euphorbiaceae

Euphorbia exilis Leach: \#1 II Euphorbiaceae

Euphorbia exilispina S. Carter: \#I II Euphorbiaceae

Euphorbia eyassiana P. R. O. Bally \& S. Carter: \#1 II Euphorbiaceae

Euphorbia falsa N. E. Brown = Euphorbia meloformis Aiton

Euphorbia famatamboay Friedm. \& Cremers ssp. famatamboay: \#1 II Euphorbiaceae

Euphorbia famatamboay Friedm. \& Cremers ssp. itampolensis

Friedm. \& Cremers: \#1 II Euphorbiaceae

Euphorbia fanshawei L. C. Leach: \#I II Euphorbiaceae

Euphorbia fascicaulis S. Carter: \#I II Euphorbiaceae 
Euphorbia fasciculata Thunb.: \#1 II Euphorbiaceae

Euphorbia faucicola L. C. Leach: \#1 II Euphorbiaceae

Euphorbia faurotii Franchet = Euphorbia iriaculeata Forsskål

Euphorbia ferox Marloth: \#1 II Euphorbiaceae

Euphorbia fianarantsoae Ursch \& Leandri: \#1 II Euphorbiaceae

Euphorbia fidjiana Boissier = Euphorbia plumerioides Teijsmann ex Hasskarl

Euphorbia fiherenensis Poiss.: \#1 II Euphorbiaceae

Euphorbia filiflora Marloth: \#1 II Euphorbiaceae

Euphorbia fimbriata hort. ex Lemaire = Euphorbia lemaireana Boissier in De Candolle

Euphorbia fimbriala Scop.: \#1 II Euphorbiaceae

Euphorbia fissispina P. R. O. Bally \& S. Carter: \# 1 II Euphorbiaceae

Euphorbia flanaganii N. E. Br.: \#I II Euphorbiaceae

Euphorbia fleckii Pax: \#1 II Euphorbiaceae

Euphorbia fluminis S. Carter: \#1 II Euphorbiaceae

Euphorbia foliifua Ule = Euphorbia heterodoxa Müller-Argoviensis

Euphorbia forolensis L. E. Newton: \#1 II Euphorbiaceae

Euphorbia fortissima L. C. Leach: \#1 II Euphorbiaceae

Euphorbia fortuita A. C. White, R. A. Dyer \& B. Sloane: \#1 II Euphorbiaceae

Euphorbia foumieri Rebut = Euphorbia leuconeura Boissier in De Candolle

Euphorbia fractiflexa S. Carter \& J. R. I. Wood: \#1 II Euphorbiaceae Euphorbia fragiliramulosa $\mathrm{L}, \mathrm{C}$. Leach $=$ Euphorbia negromontana N. E. Br.

Euphorbia francescae L. C. Leach $=$ Euphorbia quadrata Nel

Euphorbia franckiana A. Berger: \#I II Euphorbiaceae

Euphorbia francoisii Leandri var. crassicaulis Rauh: I Euphorbiaceae

Euphorbia francoisii Leandri var. francoisii: I Euphorbiaceae

Euphorbia francoisii Leandri var. rakotozafyi Cremers = Euphorbia cremersii Rauh \& Razafindratsira var. rakosozafyi (Cremers) Rauh

Euphorbia franksiae N. E. Br. var. franksiae: \#1 II Euphorbiaceae

Euphorbia franksiae N. E. Br. var. zuluensis A. C. White, R. A. Dyer \& B. Sloane: \#1 II Euphorbiaceae

Euphorbia fratema N. E. Brown = Euphorbia dekindsii $\mathrm{Pax}$

Euphorbia frickiana N. E. Brown = Euphorbia pseudoglobosa Marloth

Euphorbia friedrichiae Dinter: \#1 II Euphorbiaceae

Euphorbia fructus-pini Miller = Euphorbia capui-medusae L.

Euphorbia fructus-pini (Miller) Haworth var. geminata (Aiton) Sweet = Euphorbia capul-medusae L.

Euphorbia fruticosa Edgeworth = Euphorbia cuneara Vahl

Euphorbia fruticosa Forsskal: \#1 II Euphorbiaceae

Euphorbia furcala N. E. Br.: \#1 II Euphorbiaceae

Euphorbia fusca Marloth: \#1 II Euphorbiaceac

Euphorbia fusiformis Buchanan-Hamilt on ex D. Don: \#I II Euphorbiaceac

Euphorbia galgalana S. Canter: \#I II Euphorbiaceae

Euphorbia galpinii $\mathrm{Pax}=$ Euphorbia transvaalensis Schlechter

Euphorbia gariepina Boiss. ssp. balsamea (Welw. ex Hiern) L. C. Leach: \#1 II Euphorbiaceae

Euphorbia gariepina Boiss. ssp. gariepina: \#I II Euphorbiaceae

Euphorbia garuana N. E. Br. = Euphorbia kamerunica Pax

Euphorbia gatbergensis N. E. Br.: \#1 II Euphorbiaceae

Euphorbia geayi Costantin \& Gallaud = Euphorbia tirucalli L

Euphorbia geldorensis S. Carter: \#1 II Euphorbiaceae

Euphorbia gemmea P. R. O. Bally \& S. Carter: \#1 I Euphorbiaceae

Euphorbia genoudiana Ursch \& Leandri: \#1 II Euphorbiaceae

Euphorbia gentilis N. E. Br. ssp. gentilis: \#1 II Euphorbiaceae

Euphorbia gentilis N. E. Br. ssp. Ianquana L. C. Leach: \#I II Euphorbiaceae

Euphorbia geroldii Rauh: \#1 II Euphorbiaceae

Euphorbia giessii L. C. Leach; \#1 II Euphorbiaceae

Euphorbia gilbertii A. Berger = Euphorbia micracantha Boissier

Euphorbia gillettii P. R. O. Bally \& S. Carter ssp. gillettii: \#! II Euphorbiaceae

Euphorbia gilletrii P. R. O. Bally \& S. Carter ssp. ienuior S. Carter: \#1 II Euphorbiaceae

Euphorbia glandularis L. C. Leach \& G. Williamson: \#l II Euphorbiaceae

Euphorbia globosa (Haw.) Sims: \#1 II Euphorbiaceae

Euphorbia globulicaulis S. Carter: \#1 II Euphorbiaceae

Euphorbia glochidiata Pax: \#1 II Euphorbiaceae
Euphorbia glomerata A. Berger = Euphorbia globosa (Haworth) Sims

Euphorbia goetzei Pax: 1 II Euphorbiaceae

Euphorbia golisana N. E. Br. = Euphorbia phillipsiae N. E. Br.

Euphorbia gollmeriana Klotzsch ex Boissier = Euphorbia lupulina Boissier

Euphorbia gorgonis A. Berger: \#1 II Euphorbiaceae

Euphorbia gossweileri $\mathrm{Pax}=$ Euphorbia trichadenia $\mathrm{Pax}$

Euphorbia gossypina Pax var. coccinea Pax: \#1 II Euphorbiaceae

Euphorbia gossypina Pax var. gossypina: \#1 II Euphorbiaceae

Euphorbia gonlebei Rauh: \#1 II Euphorbiaceae

Euphorbia gracilicaulis L. C. Leach: \#1 II Euphorbiaceae

Euphorbia graciliramea Pax: \# I II Euphorbiaceae

Euphorbia grandialara R. A. Dyer: \#I II Euphorbiaceae

Euphorbia grandicomis Goebel ex N. E. Br. ssp. grandicomis: \#1 II Euphorbiaceac

Euphorbia grandicomis Goebel ex N. E. Br. ssp. sejuncta L. C. Leach: \#1 II Euphorbiaceae

Euphorbia grandidens Haw.: \# I II Euphorbiaceae

Euphorbia grandilobata Chiovenda = Euphorbia breviarticulata Pax var. breviarticulata

Euphorbia grandis Lem. = Euphorbia abyssinica Gmelin

Euphorbia graninicola L. C. Leach: \#1 II Euphorbiaceae

Euphorbia graveolens N. E. Brown = Euphorbia restituta N. E. Brown in Thiselton-Dyer

Euphorbia greenwayi P. R. O. Bally \& S. Carter; \#1 II Euphorbiaceae

Euphorbia greenwayi P. R. O. Bally \& S. Carter ssp. breviaculeata S. Carter; \# II Euphorbiaceae

Euphorbia greenwayi P. R. O. Bally \& S. Carter ssp. greenwayi: \#1 II Euphorbiaceae

Euphorbia gregaria Marloth: \#1 II Euphorbiaceae

Euphorbia griseola Pax ssp. griseola: \#1 II Euphorbiaceae

Euphorbia griseola Pax ssp. mashonica L. C. Leach: \#1 II Euphorbiaceae

Euphorbia griseola Pax ssp. zambiensis L. C. Leach: \#1 II Euphorbiaceae

Euphorbia griseola Pax var, robusta Pax ex Engler = Euphorbia griseola Pax ssp. griseola

Euphorbia groenewaldii R. A. Dyer: \#1 II Euphorbiaceae

Euphorbia gueinzii Boiss. var. albovillosa (Pax) N. E. Br.: \#I II Euphorbiaceac

Euphorbia gueinzii Boiss. var. gueinzii: \#1 II Euphorbiaceae

Euphorbia guerichiana Pax: \#1 II Euphorbiaceae

Euphorbia guillauminiana Boit: \#1 II Euphorbiaceae

Euphorbia guillemetii Ursch \& Leandri: \#1 II Euphorbiaceae

Euphorbia gummifera Boiss.: \#1 II Euphorbiaceae

Euphorbia gymnocalycioides M. G. Gilben \& S. Carter: \#1 II Euphorbiaceae

Euphorbia gymnoclada Boiss.: \#1 11 Euphorbiaceae

Euphorbia gynophora $\mathrm{Pax}=$ Euphorbia espinosa $\mathrm{Pax}$

Euphorbia hadramautica Baker: \#1 11 Euphorbiaceae

Euphorbia halipedicola L. C. Leach: \# I II Euphorbiaceae

Euphorbia halleri Dinter ex Frick = Euphorbia gariepina Boissier ssp. balsamea (Welwitsch ex Hiern) L. C. Leach

Euphorbia halli R. A. Dyer: \#I II Euphorbiaceae

Euphorbia hamata (Haw.) Sweet: \#I II Euphorbiaceae

Euphorbia handiensis Burchard: \# 1 II Euphorbiaceae

Euphorbia hararensis Pax = Euphorbia abyssinica Gmelin

Euphorbia hastisquama N. E. Brown = Euphorbia caterviflora N. E. Brown

Euphorbia hedyotoides N. E. Br.: \#1 II Euphorbiaceae

Euphorbia helicothele Lemaire = Euphorbia nivulia BuchananHamilon

Euphorbia hepatica P. R. O. Bally \& S. Carter = Euphorbia reclinata P. R. O. Bally \& S. Carter

Euphorbia heptagona L, var, dentata (A. Berger) A. C. White, R. A. Dyer \& B. Sloane: \#1 II Euphorbiaceae

Euphorbia heptagona L. var. heptagona: \#1 II Euphorbiaceae

Euphorbia heptagona L. var. ramosa A. C. White, R. A. Dyer \& B. Sloane: \#1 II Euphorbiaceae

Euphorbia heptagona L. var. subsessilis A. C. White, R. A. Dyer \& B. Sloane: \#1 II Euphorbiaceae

Euphorbia heptagona L. var. viridis A. C. White, R. A. Dyer \& B. Sloane: \#1 II Euphorbiaceae

Euphorbia herman-schwarizii Rauh: \#1 II Euphorbiaceae 
Euphorbia hermentiana Lemaire $=$ Euphorbia trigona Miller Euphorbia hemandez-pachecoi Caballero = Euphorbia echinus Hook.f. \& Cosson

Euphorbia herrei A. C. White, R. A. Dyer \& B. Sloane: \#I II Euphorbiaceae

Euphorbia heieracantha Pax = Euphorbia subsalsa Hiem ssp subsalsa

Euphorbia heterochroma Pax ssp. heterochroma: \#I II Euphorbiaceae

Euphorbia heterochroma Pax ssp. 1savoensis S. Carter: \#! II Euphorbiaceae

Euphorbia heterochroma Pax var. mitis (Pax) N. E. Brown = Euphorbia heterochroma Pax ssp. heterochroma

Euphorbia heterodoxa Müller-Argoviensis: \#1 II Euphorbiaceae

Euphorbia heterospina S. Carter ssp. baringoensis S. Carter: \#1 II Euphorbiaceae

Euphorbia heterospina S. Carter ssp. heterospina: \#1 II Euphorbiaceae

Euphorbia hislopii N. E. Brown = Euphorbia milii Des Moulins var. hislopii (N. E. Brown) Ursch \& Leandri

Euphorbia hofstaetteri Rauh: \#1 II Euphorbiaceae

Euphorbia holmesiae Lavranos: \#1 II Euphorbiaceae

Euphorbia holmseae hort. Hanson = Euphorbia holmesiae Lavranos

Euphorbia holochlorina Rizzini: \#I II Euphorbiaceae

Euphorbia hopetownensis Nel: \#1 II Euphorbiaceae

Euphorbia horombensis Ursch \& Leandri: \#I II Euphorbiaceae

Euphorbia horrida Boiss. var. horrida: \#! II Euphorbiaceae

Euphorbia horrida Boiss, var. major A. C. White, R. A. Dyer \& B. Sloane: \#I II Euphorbiaceae

Euphorbia horrida Boiss. var. noorsveldensis A. C. White, R. A. Dyer \& B. Sloane: \#1 II Euphorbiaceae

Euphorbia horrida Boiss, var, striala A. C. White, R. A. Dyer \& B. Sloane: \#1 II Euphorbiaceae

Euphorbia horwoodii S. Carter \& Lavranos: \#I II Euphorbiaceae

Euphorbia hottentola Marloth: \#I II Euphorbiaceae

Euphorbia huberiii Pax: \#I II Euphorbiaceae

Euphorbia huttoniae N. E. Brown = Euphorbia inermis Miller var huttoniae (N. E. Brown) A. C. White, R. A. Dyer \& B. Sloane

Euphorbia hydnorae E. Meyer ex Boissier = Euphorbia mauritanica L. var. mauritanico

Euphorbia hypogaea Marloth: \#1 II Euphorbiaceae

Euphorbia hystrix Jacquin = Euphorbia loricata Lamarck

Euphorbia iharanae Rauh: \#1 II Euphorbiaceae

Euphorbia imerina G. Cremers: \#I II Euphorbiaceae

Euphorbia imirata N. E. Br.: \#l II Euphorbiaceae

Euphorbia immersa P. R. O. Bally \& S. Carter: \#1 II Euphorbiaceae Euphorbia imparispina S. Carter: \#1 II Euphorbiaceae

Euphorbia impervia A. Berger = Euphorbia helerochroma Pax ssp. heterochroma

Euphorbia implexa Stapf = Euphorbia gossypina Pax var. gossypina Euphorbia inaequispina N. E. Br.: \#1 II Euphorbiaceae Euphorbia inarticulata Schweinf.: \#1 II Euphorbiaceae Euphorbia inconstantia R. A. Dyer: \#I II Euphorbiacese Euphorbia inculta P. R. O. Bally: \#I II Euphorbiaceae Euphorbia indecora N. E. Br.: \#1 II Euphorbiaceae

Euphorbia indurescens L. C. Leach: \#I II Euphorbiaceae

Euphorbia inelegans N. E. Brown = Euphorbia inomala N. E. Brown in Thiselton-Dyer

Euphorbia inelegans N. E. Brown = Euphorbia matabelensis Pax

Euphorbia inermis Mill. var. huttoniae (N. E. Br.) A. C. White, R. A. Dyer \& B. Sloane: \#1 II Euphorbiaceae

Euphorbia inermis Mill. var. inermis: \#1 II Euphorbiaceae

Euphorbia inermis Mill. var. laniglans N. E. Brown = Euphorbia esculento Marloth

Euphorbia infausta N. E. Brown $1915=$ Euphorbia meloformis Aiton

Euphorbia infausta N. E. Brown 1912 = Euphorbia polyacantha Boissier

Euphorbia infesıa $\mathrm{Pax}=$ Euphorbia rriaculeata Forsskå

Euphorbia ingens E. Meyer ex Boiss.: \#1 II Euphorbiaceae

Euphorbia ingenticapsa L. C. Leach: \#1 11 Euphorbiaceae

Euphorbia x ingezalahiana Ursch \& Leandri; \#1 II Euphorbiaceae

Euphorbia inormata N. E. Br.: \#1 II Euphorbiaceae

Euphorbia insulae-europae $\operatorname{Pax}=$ Euphorbia stenoclada Baillon ssp. stenoclada

Euphorbia intercedens $\mathrm{Pax}=$ Euphorbia quinquecostata Volkens
Euphorbia intisy Drake: \#I II Euphorbiaceae

Euphorbia intisy Drake var, maintyi (Decorse) L. Poiss. = Euphorbia maintyi (Poisson) Denis ex Leandri

Euphorbia inundaticola L. C. Leach: \#1 II Euphorbiaceae

Euphorbia invaginata Croizat: \#1 II Euphorbiaceae

Euphorbia isacantha Pax: \#1 II Euphorbiaceae

Euphorbia isalensis Leandri = Euphorbia milii Des Moulins var splendens (Bojer ex Hooker) Ursch \& Leandri

Euphorbia jaegeriana $\mathrm{Pax}=$ Euphorbia matabelensis $\mathrm{Pax}$

Euphorbia jansenvillensis Nel: \#1 II Euphorbiaceae

Euphorbia johannis S. Carter: \#1 II Euphorbiaceae

Euphorbia johnsonii N. E. Brown = Euphorbia knushii Pax ssp. johnsonii (N. E. Brown) L. C. Leach

Euphorbia X jubaephylla Sventenius: \#1 II Euphorbiaceae

Euphorbia jubata L. C. Leach: \#I II Euphorbiaceae

Euphorbia juglans G. R. Compton: \#! II Euphorbiaceae

Euphorbia juttae Dinter: \#1 II Euphorbiaceae

Euphorbia kalaharica Marloth = Euphorbia avasmontana Dinter var avasmontana

Euphorbia kalisana S. Carter: \#1 II Euphorbiaceae

Euphorbia kamerunica Pax: \#1 II Euphorbiaceae

Euphorbia kamerunica Pax var. barteri (N. E. Brown) A. Chevalier = Euphorbia kamerunica Pax

Euphorbia kamponii Rauh \& Petignat: \#1 II Euphorbiaceae

Euphorbia kanalensis Boiss. in De Candolle: \#I II Euphorbiaceae

Euphorbia kaokoensis (A. C. White, R. A. Dyer \& B. Sloane) L. C. Leach: \#1 II Euphorbiaceae

Euphorbia karroensis (Boiss.) N. E. Br.:\#1 II Euphorbiaceae

Euphorbia keithii R. A. Dyer: \#1 II Euphorbiaceae

Euphorbia khandallensis Blatter \& Hallberg: \#1 II Euphorbiaceae

Euphorbia kibwezensis N. E. Brown = Euphorbia bussei Pax var. kibwezensis (N. E. Brown) S. Carter

Euphorbia knobelii Letty: \#1 II Euphorbiaceae

Euphorbia knuthii Pax ssp. johnsonii (N. E. Br.) L. C. Leach: \#1 II Euphorbiaceae

Euphorbia knuthii Pax ssp. knushii: \#1 II Euphorbiaceae

Euphorbia kondoi Rauh \& Razafindratsira: \#I II Euphorbiaceae

Euphorbia lacei Craib: \#1 II Euphorbiaceae

Euphorbia lacrea Haw.: \#1 II Euphorbiaceae

Euphorbia lacriflua Philippi: \#1 II Euphorbiacese

Euphorbia laeia Aiton = Euphorbia dendroides L

Euphorbia lagunillanum Croiz $=$ Euphorbia lupulina Boiss.

Euphorbia laikipiensis S. Carter: \#I II Euphorbiaceae

Euphorbia lamarckii Sweet: \#I II Euphorbiaceae

Euphorbia lamarckii Sweet forma lavibracteata (G. Kunkel) Oudejans = Euphorbia broussonetii Willd. ex Link in L. von Buch

Euphorbia lamarckii Sweet ssp. regis-jubae (Webb \& Berthelot) Oudejans = Euphorbia regis-jubae Webb \& Berthelot

Euphorbia lamarckii Sweet var. berthelotii (Bolle ex Boissier) Oudejans = Euphorbia berthelotii Bolle ex Boissier

Euphorbia lamarckii Sweet var. despoliata (Menezes) Oudejans = Euphorbia despoliala (Menezes) Monod

Euphorbia lamarckii Sweet var. pseudodendroides (H. Lindberg) Oudejans = Euphorbia lamarckii Sweet

Euphorbia lambii Svent: Gomeran Spurge (E) \#I II Euphorbiaceae Euphorbia larica Boiss.: \#1 II Euphorbiaceae

Euphorbia laro Drake = Euphorbia tirucalli L

Euphorbia lateriflora Schum. \& Thonn.: \#1 II Euphorbiaceae

Euphorbia laurentii De Wild. = Euphorbia teke Pax

Euphorbia lavrani L. C. Leach: \#I II Euphorbiaceae

Euphorbia laxiflora Kuntze = Euphorbia bubalina Boissier

Euphorbia leandriana Boit.: \#1 II Euphorbiaceae

Euphorbia ledienii A. Berger var. dregei N. E. Br.; \#I II Euphorbiaceae

Euphorbia ledienii A. Berger var. ledienii; \#1 II Euphorbiaceae

Euphorbia lemaireana Boiss.: \#1 II Euphorbiaceae

Euphorbia leonensis N. E. Br.: \#1 II Euphorbiaceae

Euphorbia leontopoda S. Carter: \#1 II Euphorbiaceae

Euphorbia letesrui Raynal: \#1 II Euphorbiaceae

Euphorbia leucodendron Drake: \#1 II Euphorbiaceae

Euphorbia leuconeura Boiss.: \#! II Euphorbiaceae

Euphorbia lignosa Marloth: \#1 II Euphorbiaceae

Euphorbia ligularia Roxburgh = Euphorbia neriffolia L

Euphorbia lividiflora L. C. Leach: \#1 II Euphorbiaceae

Euphorbia lombardensis $\mathrm{Nel}=$ Euphorbia micracantha Boissier 
Euphorbia x lomi Rauh: \#1 II Euphorbiaceae

Euphorbia longibracteata Pax = Euphorbia monteiri Hook.f. ssp monteiri

Euphorbia longifolia Lamarck: \#1 II Euphorbiaceae

Euphorbia longifolia Lamarck var. canariensis (Boissier) Oudejans = Euphorbia longifolia Lamarck

Euphorbia longispina Chiov.: \#1 II Euphorbiaceae

Euphorbia longitubenculosa Boiss. in De Candolle: \#1 II Euphorbiaceae

Euphorbia lophogona Lam. var. lophogona: \#1 II Euphorbiaceae

Euphorbia lophogona Lam. var. renuicaulis Rauh: \#1 II Euphorbiaceac

Euphortia loricata Lam.: \#1 II Euphorbiaceae

Euphorbia louwii L. C. Leach: \#1 II Euphorbiaceae

Euphorbia lwapulana L. C. Leach: \#1 II Euphorbiaceae

Euphorbia lumbricalis L. C. Leach: \#1 II Euphorbiaceae

Euphorbia lupulina Boiss.: \#1 II Euphorbiaceae

Euphorbia lutzenbergeriana Croiz. = Euphorbia lupulina Boiss.

Euphorbia lydenburgensis Schweick. \& Letty: \#1 II Euphorbiaceae

Euphorbia macella N. E. Br.: \#1 II Euphorbiaceae

Euphorbia macowanii N. E. Brown = Euphorbia tuberculata Jacquin var, macowanii (N. E. Brown) A. C. White, R. A. Dyer \& B. Sloane

Euphorbia madagascariensis Commerson ex Lamarck = Euphorbia lophogona Lamarck

Euphorbia magnicapsula S. Carter var. lacenosa S. Carter: \#1 II Euphorbiaceae

Euphorbia magnicapsula S. Carter var. magnicapsula: \#1 II Euphorbiaceae

Euphorbia mahabobokensis Rauh: \#1 II Euphorbiaceae

Euphorbia mahafalensis Denis var. mahafalensis: \#1 II Euphorbiaceae

Euphorbia mahafalensis Denis var, xanthadenia (Denis) Leandri: \#1 II Euphorbiaceae

Euphorbia mainiana Poisson = Euphorbia milii Des Moulins var. splendens (Bojer ex Hooker) Ursch \& Leandri

Euphorbia mainty (Poisson) Denis ex Leandri: \#1 II Euphorbiaceae

Euphorbia makallensis S. Carter: \#1 II Euphorbiaceae

Euphorbia maleolens E. Phillips: \# 1 II Euphorbiaceae

Euphorbia malevola L. C. Leach ssp. bechuanica L. C. Leach: \#1 II Euphorbiaceae

Euphorbia malevola L. C. Leach ssp. malevola: \# 1 II Euphorbiaceae Euphorbia mamillosa Lemaire = Euphorbia squarrosa Haworth

Euphorbia mammillaris L.: \#1 II Euphorbiaceae

Euphorbia mammillaris L. var. spinosior A. Berger = Euphorbia fimbriata Scopoli

Euphorbia mangokyensis Denis: \#1 II Euphorbiaceae

Euphorbia margaresae S. Carter: \#1 II Euphorbiaceae

Euphorbia marlothiana N. E. Br.: \#1 II Euphorbiaceae

Euphorbia marlothii $\mathrm{Pax}=$ Euphorbia monteiri Hook.f. ssp. monteiri

Euphorbia marsabitensis S. Carter: \#1 II Euphorbiaceae

Euphorbia masirahensis Ghazanfar: \#1 II Euphorbiaceae

Euphorbia matabelensis Pax: \#1 II Euphorbiaceae

Euphorbia mauritanica L. var. corallothamnus (Dinter) A. C. White, R. A. Dyer \& B. Sloane: \#1 II Euphorbiaceae

Euphorbia mauritanica L, var, foetens (Dinter) A. C. White, R. A. Dyer \& B. Sloane: \#I II Euphorbiaceae

Euphorbia mauritanica L. var. lignosa A. C. White, R. A. Dyer \& B. Sloane: \#1 II Euphorbiaceae

Euphorbia mauritanica L. var. mauritanica: \#1 II Euphorbiaceae

Euphorbia mauritanica L. var. minor A. C. White, R. A. Dyer \& B. Sloane: \#1 II Euphorbiaceae

Euphorbia mauritanica L. var. namaquensis N. E. Br.: \#1 II Euphorbiaceae

Euphorbia mayuranathanii Croiz. in Hook.: \#1 II Euphorbiaceae

Euphorbia mbaluensis $\mathrm{Pax}=$ Euphorbia bussei Pax var. bussei

Euphorbia mcvaughii Carvajal \& Lomelí: \#1 II Euphorbiaceae

Euphorbia media N. E. Brown = Euphorbia tirucalli L

Euphorbia media N. E. Brown var. bagshawei N. E. Brown = Euphorbia tirucalli L.

Euphorbia medusce Thunberg = Euphorbia caput-medusae L

Euphorbia melanacantha Drake = Euphorbia milii Des Moulins var. splendens (Bojer ex Hooker) Ursch \& Leandri

Euphorbia melanohydrata Nel: \#I II Euphorbiaceae

Euphorbia melanosticia E. Meyer ex Boissier = Euphorbia mauritanica L. var, mauritanica
Euphorbia mellifera Aiton = Euphorbia longifolia Lamarck

Euphorbia mellifera Aiton var, canariensis Boissier = Euphorbia longifolia Lamarck

Euphorbia meloformis Aiton: \#1 II Euphorbiaceae

Euphorbia meloformis Aiton var. pomiformis (Thunberg) Marloth = Euphorbia meloformis Aiton

Euphorbia meloformis Aiton var. prolifera G. A. Frick = Euphorbia meloformis Aiton

Euphorbia meloniformis Link = Euphorbia meloformis Aiton

Euphorbia memoralis R. A. Dyer: \#I II Euphorbiaceae

Euphorbia menelikii Pax = Euphorbia ampliphylla Pax

Euphorbia meridionalis P. R. O. Bally \& S. Carter: \#1 II Euphorbiaceae

Euphorbia merkeri N. E. Brown = Euphorbia gossypina Pax var. gossypina

Euphorbia meyeri Nel = Euphorbia nelii A. C. White et al.

Euphorbia micracantha Boiss: \#I II Euphorbiaceae

Euphorbia migiuninorum Chiov.: \#1 II Euphorbiaceae

Euphorbia milii Des Moulins forma lutea Rauh = Euphorbia milii Des Moulins var. milii

Euphorbia milii Des Moulins var. betsileana (Leandri) H. Jacobsen = Euphorbia milii Des Moulins var. splendens (Bojer ex Hooker) Ursch \& Leandri

Euphorbia milii Des Moulins var, bevilaniensis (Croizat) Ursch \& Leandri: \#1 II Euphorbiaceae

Euphorbia milii Des Moulins var. bojeri (Hooker) Janse = Euphorbia milii Des Moulins var. milii

Euphorbia milii Des Moulins var, bosseri Rauh = Euphorbia neobosseri Rauh

Euphorbia milii Des Moulins var. breonii Ursch \& Leandri = Euphorbia milii Des Moulins var, hislopii (N. E. Brown) Ursch \& Leandri

Euphorbia milii Des Moulins var. bulbispina Rauh = Euphorbia bulbispina Rauh \& Razafindratsira

Euphorbia milii Des Moulins var. hislopii (N. E. Br.) Ursch \& Leandri: \#1 II Euphorbiaceae

Euphorbia milii Des Moulins var. imperatae (Leandri) Ursch \& Leandri: \#I II Euphorbiaceae

Euphorbia milii Des Moulins var. longifolia Rauh: \#1 II Euphorbiaceae

Euphorbia milii Des Moulins var, mainiana (Poisson) Janse = Euphorbia milii Des Moulins var. splendens (Bojer ex Hooker) Ursch \& Leandri

Euphorbia milii Des Moulins var. milii: Crown of Thoms (E) \#1 II Euphorbiaceae

Euphorbia milii Des Moulins var, roseana Marn.-Lap.: \#1 II Euphorbiaceae

Euphorbia milii Des Moulins var. splendens (Bojer ex Hook.) Ursch \& Leandri: Crown of Thoms (E) \#I II Euphorbiaceae

Euphorbia milii Des Moulins var. rananarivae (Leandri) Ursch \& Leandri: \#1 II Euphorbiaceae

Euphorbia milii Des Moulins var. tenuispina Rauh \& Razafindratsira: \#1 II Euphorbiaceae

Euphorbia milii Des Moulins var, tulearensis Ursch \& Leandri: \#1 II Euphorbiaceae

Euphorbia milii Des Moulins var. vulcanii (Leandri) Ursch \& Leandri : \#1 II Euphorbiaceae

Euphorbia millatii Ursch \& Leandri: \# I II Euphorbiaceae

Euphorbia mira L. C. Leach: \#1 II Euphorbiaceae

Euphorbia miscella L. C. Leach = Euphorbia celata R. A. Dyer

Euphorbia misera Benth.: Cliff Spurge (E) \#1 II Euphorbiaceae

Euphorbia mitis $\mathrm{Pax}=$ Euphorbia heterochroma Pax ssp. heterochroma

Euphorbia mitriformis P. R. O. Bally \& S. Carter; \#1 II Euphorbiaceae

Euphorbia X mitsimbinensis Ursch \& Leandri: \#1 II Euphorbiaceae

Euphorbia mixı N. E. Br.: \#1 II Euphorbiaceae

Euphorbia mlanjeana L. C. Leach: \#1 II Euphorbiaceae

Euphorbia monacantha Pax: \#1 II Euphorbiaceae

Euphorbia monadenioides M. G. Gilbert: \#1 II Euphorbiaceae

Euphorbia monteiri Hook.f. ssp. brandbergensis R. Nordenstam: \#1 II Euphorbiaceae

Euphorbia monseiri Hook,f, ssp. monteiri: \#1 II Euphorbiaceae

Euphorbia monteiri Hook.f. ssp. ramosa L. C. Leach: \#1 II Euphorbiaceae

Euphorbia moratii Rauh var, antsingiensis Cremers: I Euphorbiaceae 
Euphorbia moratii Rauh var. bemarahensis Cremers: I Euphorbiaceae

Euphorbia moratii Rauh var. moratii: I Euphorbiaceae

Euphorbia moratii Rauh var, multiflora Rauh: I Euphorbiaceae

Euphorbia morinii A. Berger $=$ Euphorbia heptagona L. var. heptagona

Euphorbia mosaica P. R. O. Bally \& S. Carter: \#1 II Euphorbiaceae

Euphorbia muirii N. E. Br.: \#1 II Euphorbiaceae

Euphorbia multiceps A. Berger: \#I II Euphorbiaceae

Euphorbia multiclava P. R. O. Bally \& S. Carter: \#1 II Euphorbiaceae

Euphorbia multifida N. E. Br.: \#1 II Euphorbiaceae

Euphorbia multifolia A. C. White, R. A. Dyer \& B. Sloane: \#I II Euphorbiaceae

Euphobia multiramosa Nel = Euphorbia friedrichiae Dinter

Euphorbia mundrii N. E. Br.: \#1 II Euphorbiaceae

Euphorbia muricata Thunb.: \#1 II Euphorbiaceae

Euphorbia murielii N. E. Brown = Euphorbia candelabrum Kotschy var. candelabrum

Euphorbia mwinilungensis L. C. Leach: \#1 II Euphorbiaceae

Euphorbia myrioclada S. Carter: \#1 II Euphorbiaceae

Euphorbia namaquensis N. E. Br. = Euphorbia friedrichiae Dinter

Euphorbia namibensis Marloth: \#1 II Euphorbiaceae

Euphorbia namuskluftensis L. C. Leach: \#1 II Euphorbiaceae

Euphorbia nana Royle = Euphorbia fusiformis Buchanan-Hamilton ex D. Don

Euphorbia napoides $\mathrm{Pax}=$ Euphorbia hadramautica Baker

Euphorbia natalensis A. Berger = Euphorbia ingens E. Meyer ex Boissier

Euphorbia x navae Sventenius: \#I II Euphorbiaceae

Euphorbia ndurumensis P. R. O. Bally = Euphorbia tenuispinosa Gilli var, tenuispinosa

Euphorbia neglecta N. E. Brown = Euphorbia abyssinica Gmelin

Euphorbia negromontana N. E. Br.: \#l II Euphorbiaceae

Euphorbia nelii A. C. White, R. A. Dyer \& B. Sloane $=$ Euphorbia filiflora Marloth

Euphorbia neobosseri Rauh: \#1 11 Euphorbiaceae

Euphorbia neohumbertii Boit.: \#1 II Euphorbiaceae

Euphorbia neohumberii Rauh var, aureoviridiflora Rauh = Euphorbia aureoviridiflora (Rauh) Rauh

Euphorbia neovolkensii $\mathrm{Pax}=$ Euphorbia nyikae $\mathrm{Pax}$ var. neovolkensii (Pax) S. Carter

Euphorbia neriifolia L.: \#I II Euphorbiaceae

Euphorbia nesemannii R. A. Dyer: \#1 II Euphorbiaceae

Euphorbia neumanii Baillon = Euphorbia milii Des Moulins var. milii

Euphorbia neutra A. Berger $=$ Euphorbia abyssinica Gmelin

Euphorbia nigrispina N. E. Br.: \#1 11 Euphorbiaceae

Euphorbia nigrispinoides M. G. Gilbert: \#I II Euphorbiaceae

Euphorbia nivulia Buchanan-Hamilton: \#1 II Euphorbiaceae

Euphorbia nivulia Buchanan-Hamilton var. helicothele (Lemaire) Boissier = Euphorbia nivulia Buchanan-Hamilton

Euphorbia norfolkiana Boiss.: \#1 II Euphorbiaceae

Euphorbia nubica N. E. Br.: Maagoog (E) \#1 II Euphorbiaceae

Euphorbia nubica N. E. Brown var. consobrina (N. E. Brown) Hadidi = Euphorbia consobrina N. E. Brown

Euphorbia nubigena L. C. Leach var. nubigena: \#1 II Euphorbiaceae

Euphorbia nubigena L. C. Leach var. rutilans L. C. Leach: \#1 II Euphorbiaceae

Euphorbia nyassae Pax: \#1 II Euphorbiaceae

Euphorbia nyassae Pax ssp. mentiens S. Carter = Euphorbia perplexa L. C. Leach var. perplexa

Euphorbia nyikae Pax var, neovolkensii (Pax) S. Carter: \#1 II Euphorbiaceae

Euphorbia nyikae Pax var. nyikae: \#1 II Euphorbiaceae

Euphorbia obesa Hook.f.: \#1 II Euphorbiaceae

Euphorbia oblongicaulis Baker = Euphorbia hadramautica Baker

Euphorbia obovalifolia auct. $=$ Euphorbia ampliphylla $\mathrm{Pax}$

Euphorbia obovalifolia A. Rich. = Euphorbia abyssinica Gmelin

Euphorbia obtusifolia Poiret forma latibracieata G. Kunkel = Euphorbia broussonetii Willd. ex Link in L. von Buch

Euphorbia obrusifolia Poiret ssp. obtusifolia = Euphorbia lamarckii Sweet

Euphorbia obtusifolia Poiret ssp. regis-jubae (Webb \& Berthel) Maire = Euphorbia regis-jubae Webb \& Berthel.
Euphorbia obtusifolia Poiret var. despoliata Menezes = Euphorbia despoliata (Menezes) Monod

Euphorbia odontophora S. Carter: \#1 II Euphorbiaceae

Euphorbia odontophylla Willd. = Euphorbia cereiformis L.

Euphorbia officinarum L.: \#1 II Euphorbiaceae

Euphorbia officinarum L. ssp. beaumieriana (Hook.f. \& Cosson) Vindt $=$ Euphorbia officinarum L

Euphorbia officinarim L. ssp. echinus (Hook. f. \& Coss.) Vindt = Euphorbia echinus Hook.f. \& Cosson

Euphorbia officinarum L. var. arborea Forsskål = Euphorbia ammak Schweinfurth

Euphorbia officinarum L. var. beaumieriana (Hook.f. \& Cosson) Maire $=$ Euphorbia officinarum L.

Euphorbia officinarum L. var. hemandez-pachacoi (Caballero) Oudejans = Euphorbia echinus Hook.f. \& Cosson

Euphorbia officinarum L. var. kolquall Willd. = Euphorbia abyssinica Gmelin

Euphorbia oligoclada L. C. Leach: \#1 II Euphorbiaceae Euphorbia omariana M. G. Gilbert: \#1 II Euphorbiaceae Euphorbia oncoclada Drake: \#1 II Euphorbiaceae Euphorbia opuntioides Welw, in Hiern: \#1 II Euphorbiaceae Euphorbia orbiculifolia S. Carter: \#1 II Euphorbiaceae Euphorbia ormithopus Jacq.: \#I II Euphorbiaceae Euphorbia otjipembana L. C. Leach: \#1 II Euphorbiaceae Euphorbia oxystegia Boiss.: \#1 II Euphorbiaceae Euphorbia pachyclada S. Carter: \#1 II Euphorbiaceae Euphorbia pachypodioides Boit.: \#I II Euphorbiaceae Euphorbia paganorum A. Chev.: \#1 II Euphorbiaceae Euphorbia panchganiensis Blatter \& McCann: \#1 II Euphorbiaceae Euphorbia papilionum S. Carter: \#1 II Euphorbiaceae Euphorbia parciramulosa Schweinf.: \#1 II Euphorbiaceae Euphorbia parviceps L. C. Leach: \#1 II Euphorbiaceae Euphorbia parvicyathophora Rauh: I Euphorbiaceae Euphorbia passa N. E. Brown = Euphorbia woodii N. E. Brown in Thiselton-Dyer

Euphorbia patula (Haworth) Sweet = Euphorbia tridentata Lamarck

Eupharbia paulianii Ursch \& Leandri: \#1 II Euphorbiaceae

Euphorbia pedemontana L. C. Leach: \#1 II Euphorbiaceae

Euphorbia pedilanthoides Denis: \#1 II Euphorbiacese

Euphorbia peltigera E. Meyer ex Boiss. = Euphorbia hamata (Haw.) Sweet

Euphorbia pentagona Haw.: \#l II Euphorbiaceac

Euphorbia pentops A. C. White, R. A. Dyer \& B. Sloane: \#! II Euphorbiaceae

Euphorbia perangusia R. A. Dyer: \#I II Euphorbiaceae

Euphorbia perarmata S. Carter: \#I II Euphorbiaceae

Euphorbia perpera N. E. Br.: \#1 II Euphorbiaceae

Euphorbia perplexa L. C. Leach var, kasamana L. C. Leach: \#1 II Euphorbiaceae

Euphorbia perplexa L. C. Leach var. perplexa: \#1 II Euphorbiaceae Euphorbia perrieri Drake var. elongala Denis: \#1 II Euphorbiaceae Euphorbia perrieri Drake var. perrieri: \#I II Euphorbiaceae

Euphorbia perrottetii Jaubert \& Spach = Euphorbia cuneata Vahl

Euphorbia persistens R. A. Dyer: \#1 II Euphorbiaceae

Euphorbia persistentifolia L. C. Leach: \#I II Euphorbiaceae

Euphorbia petraea S. Carter: \#1 II Euphorbiaceae

Euphorbia petricola P. R. O. Bally \& S. Carter: \#1 II Euphorbiaceae Euphorbia x petterssonii Sventenius: \#1 II Euphorbiaceae

Euphorbia phillipsiae N. E. Br.: \#l II Euphorbiaceae

Euphorbia phillipsioides S. Carter. \#1 II Euphorbiaceae

Euphorbia phosphorea Mart.: \#1 II Euphorbiaceae

Euphorbia phymatoclada Boissier = Euphorbia mauritanica L. var. maurilanica

Euphorbia pillansii N. E. Br. var, albovirens A. C. White, R. A. Dyer \& B. Sloane: \#1 II Euphorbiaceae

Euphorbia pillansii N. E. Br. var. pillansii: \#1 II Euphorbiaceae

Euphorbia pillansii N. E. Br. var. ramosissima A. C. White, R. A. Dyer \& B. Sloane: \#1 II Euphorbiaceae

Euphorbia pimeleodendron $\mathrm{Pax}=$ Euphorbia robecchii $\mathrm{Pax}$

Euphorbia piscatoria Aiton: \#1 II Euphorbiaceae

Euphorbia piscidermis M. G. Gilbert: \#1 II Euphorbiaceae

Euphorbia pistifolia Boissier = Euphorbia ecklonii (Klotzsch \& Garcke) Baillon

Euphorbia plagiantha Drake: \#1 II Euphorbiaceae

Euphorbia planiceps A. C. White, R. A. Dyer \& B. Sloane: \#I II Euphorbiaceae 
Euphorbia platyacantha Pax = Euphorbia dumeticola P. R, O. Bally \& S. Carter

Euphorbia platyacantha Drake = Euphorbia milii Des Moulins var. splendens (Bojer ex Hooker) Ursch \& Leandri

Euphorbia platycephala Pax: \#1 II Euphorbiaceae

Euphorbia planclada Rauh var. hardyi Rauh: \#! II Euphorbiaceae

Euphorbia platyclada Rauh var. platyclada: \#1 II Euphorbiaceae

Euphorbia platymammillaris Croizat $=$ Euphorbia fimbriata Scopoli

Euphorbia platyrrhiza L. C. Leach: \#I II Euphorbiaceae

Euphorbia plumerioides Teijsm. ex Hasskarl: \#1 II Euphorbiaceae

Euphorbia poissonii Pax: \#1 II Euphorbiaceae

Euphorbia polyacantha Boiss.: \#1 11 Euphorbiaceae

Euphorbia polyacantha Boissier ssp. rosenii Pax $=$ Euphorbia polyacantha Boissier

Euphorbia polyacantha Boissier var, subinarticulata Schweinfurth = Euphorbia polyacantha Boissier

Euphorbia polycephala Marloth: \#I II Euphorbiacene

Euphorbia polygona Haw.: \#1 11 Euphorbiaceae

Euphorbia polygonata Loddiges = Euphorbia cereiformis L

Euphorbia pomiformis Thunberg = Euphorbia meloformis Aiton

Euphorbia ponderosa S. Carter: \#I II Euphorbiaceae

Euphorbia porphyrantha Philippi: \#1 II Euphorbiaceae

Euphorbia primulifolia Baker var. begardii Cremers: \#1 II Euphorbiaceae

Euphorbia primulifolia Baker var. primulifolia: \#! II Euphorbiaceae Euphorbia proballyana L. C. Leach: \#1 II Euphorbiaceae

Euphorbia procumbens Meerburg = Euphorbia stellata Willd.

Euphorbia prona S. Carter: \#I II Euphorbiaceae

Euphorbia proteifolia Boissier = Euphorbia bupleurifolia Jacquin

Euphorbia psammophila Ule: \#1 II Euphorbiaceae

Euphorbia pseudobrachiata Dinter $=$ Euphorbia cibdela N. E. Brown

Euphorbia pseudoburuana P. R. O. Bally \& S. Carter: \#1 II Euphorbiaceae

Euphorbia pseudocactus A. Berger: \#1 II Euphorbiaceae

Euphorbia pseudodendroides H. Lindberg = Euphorbia lamarckii Sweet

Euphorbia pseudoduseimata A. C. White, R. A. Dyer \& B. Sloane \#I II Euphorbiaceae

Euphorbia pseudoglobosa Marloth: \#1 II Euphorbiaceae Euphorbia pseudotuberosa Pax: \#1 II Euphorbiaceae

Euphorbia pteroclada L. C. Leach: \#1 II Euphorbiaceae Euphorbia pteroneura A. Berger: \#I II Euphorbiaceae

Euphorbia pubiglans N. E. Br.: \#1 II Euphorbiaceae

Euphorbia pugniformis Boiss, in De Candolle: \#1 II Euphorbiaceze

Euphorbia pulvinata Marloth: \#1 II Euphorbiaceae

Euphorbia pyriformis N. E. Brown = Euphorbia meloformis Aiton

Euphorbia qarad Defl.: \#1 II Euphorbiaceae

Euphorbia quadrangularis Pax: \#1 II Euphorbiaceae

Euphorbia quadrata Nel: \#I II Euphorbiaceae

Euphorbia quadrialata Pax: \#I II Euphorbiaceae

Euphorbia quadrilatera L. C. Leach: \#I II Euphorbiaceae

Euphorbia quadrispina S. Carter: \#1 II Euphorbiaceae

Euphorbia quaitensis S. Carter: \#I II Euphorbiaceae

Euphorbia quanziticola Leandri: I Euphorbiaceae

Euphorbia quinquecostata Volkens: \#1 II Euphorbiaceae

Euphorbia racemosa E. Meyer ex Boissier = Euphorbia rhombifolia Boissier

Euphorbia radians Benth. var, radians: \#I II Euphorbiaceae

Euphorbia radians Benth. var, ssormiae (Croizat) Rzedowski \& Calderón de Rzedowski: \#1 II Euphorbiaceae

Euphorbia radiata E. Meyer ex Boissier $=$ Euphorbia clava Jacquin

Euphorbia radiata Thunberg = Euphorbia stellata Willd

Euphorbia ramiglans N. E. Br.: \#1 II Euphorbiaceae

Euphorbia ramipressa Croizat $=$ Euphorbia epiphylloides Kurz

Euphorbia ramulosa L. C. Leach: \#1 II Euphorbiaceae

Euphorbia rangeana Dinter $=$ Euphorbia rudis N. E. Brown in Thiselton-Dyer

Euphorbia razafinjohanii Ursch \& Leandri: \#1 II Euphorbiaceae

Euphorbia reclinata P. R. O. Bally \& S. Carter: \#1 II Euphorbiaceae

Euphorbia rectirama N. E. Br.: \#I II Euphorbiaceae

Euphorbia regis-jubae Webb \& Berthel.: \#1 II Euphorbiaceae

Euphorbia regis-jubae Webb \& Berthel ssp. pseudodendroides (H. Lindberg) Rikli = Euphorbia lamarckii Sweet

Euphorbia reinhardrii Volkens = Euphorbia candelabı um Kotschy var. candelabrum
Euphorbia reinhardtii Volkens var. bilocularis (N. E. Brown) Oudejans = Euphorbia candelabrum Kotschy var. bilocularis (N. E. Brown) Oudejans

Euphorbia reptans P. R. O. Bally \& S. Carter: \#I II Euphorbiaceae Euphorbia resinifera O. Berg: \#1 II Euphorbiaceae Euphorbia restituta N. E. Br.: \#1 II Euphorbiaceae Euphorbia restricta R. A. Dyer: \#I II Euphorbiaceae Euphorbia rhabdodes Boissier: \#1 II Euphorbiaceae Euphorbia rhipsaloides Glaziou = Euphorbia phosphorea Martius Euphorbia rhipsaloides Lemaire $=$ Euphorbia tirucalli L

Euphorbia rhipsaloides N. E. Brown = Euphorbia tinucalli L.

Euphorbia rhombifolia Boissier: \#I II Euphorbiaceae

Euphorbia rhombifolia Boissier var. cymosa Klotzsch \& Garcke= Euphorbia rhombifolia Boissier

Euphorbia rhombifolia Boissier var. laxa N. E. Brown = Euphorbia rhombifolia Boissier

Euphorbia rhombifolia Boissier var. triceps N. E. Brown = Euphorbia rhombifolia Boissier

Euphorbia richardiana Baillon = Euphorbia abyssinica Gmelin

Euphorbia richardsiae L. C. Leach ssp. richardsiae: \#1 II Euphorbiaceae

Euphorbia richardsiae L. C. Leach ssp. robusta L. C. Leach: \#1 II Euphorbiaceae

Euphorbia rivae Pax: \#1 II Euphorbiaceac

Euphorbia robecchii Pax: \#1 II Euphorbiaceae

Euphorbia robivelonae Rauh: \#1 II Euphorbiaceae

Euphorbia rogeri N. E. Brown = Euphorbia balsamifera Aiton

Euphorbia rossii Rauh \& Buchloh: \#I II Euphorbiaceae

Euphorbia rossii Rauh \& Buchloh forma glabra Rauh \& Buchloh = Euphorbia rossii Rauh \& Buchloh

Euphorbia rowlandii R. A. Dyer: \#1 II Euphorbiaceae

Euphorbia royleana Boiss: \#I II Euphorbiaceae

Euphorbia rubella Pax: \#1 II Euphorbiacese

Euphorbia rubella Pax var. brunellii (Chiov.) Bally = Euphorbia brunellii Chiov.

Euphorbia rubrimarginata L. E. Newton: \#1 II Euphorbiaceae

Euphorbia nubriseminalis S. Carter: \#1 II Euphorbiaceae

Euphorbia rubrispinosa S. Carter: \#1 II Euphorbiaceae

Euphorbia rubrostriata Drake $=$ Euphorbia milii Des Moulins var. splendens (Bojer ex Hooker) Ursch \& Leandri

Euphorbia rudis N. E. Br.: \#1 II Euphorbiaceae

Euphorbia rudolfii N. E. Br.: \#1 II Euphorbiaceae

Euphorbia rugosiflora L. C. Leach: \#1 II Euphorbiaceae

Euphorbia ruspolii Chiovenda $=$ Euphorbia robecchii Pax

Euphorbia sagittaria Marloth = Euphorbia avasmontana Dinter var. sagittaria (Marloth) A. C. White, R. A. Dyer \& B. Sloane

Euphorbia sakarahaensis Rauh: \#I II Euphorbiaceae

Euphorbia samburuensis P. R. O. Bally \& S. Carter: \#I II Euphorbiaceae

Euphorbia sancta $\mathrm{Pax}=$ Euphorbia ampliphylla $\mathrm{Pax}$

Euphorbia santapaui A. N. Henry: \#1 II Euphorbiaceae

Euphorbia sapinii De Wild.: \#I II Euphorbiaceae

Euphorbia sarcodes Boissier: \#1 II Euphorbiaceae

Euphorbia sarcostemmoides J. H. Willis; \#I II Euphorbiaceae

Euphorbia saxonum P. R. O. Bally \& S. Carter: \#1 II Euphorbiaceae

Euphorbia scarlatina S. Carter: \#1 II Euphorbiaceae

Euphorbia schaeferi Dinter = Euphorbia gariepina Boissier ssp. gariepina

Euphorbia schimperi Presl: \#1 II Euphorbiaceae

Euphorbia schinzii Pax: \#I II Euphorbiaceae

Euphorbia schizacantha Pax: \#1 II Euphorbiaceae

Euphorbia schmitzii L. C. Leach: \#1 II Euphorbiaceae

Euphorbia schoenlandii Pax: \#! II Euphorbiaceae

Euphorbia scitula L. C. Leach: \#! II Euphorbiaceae

Euphorbia scolopendrea Haworth = Euphorbia stellata Willd

Euphorbia scoparia N. E. Brown = Euphorbia tincalli L.

Euphorbia scopiformis (Klotzsch) Boissier = Euphorbia arceuthobioides Boissier

Euphorbia scopoliana Steudel = Euphorbia fimbriata Scopoli

Euphorbia scyphadena S. Carter: \#1 II Euphorbiaceae

Euphorbia sebsebei M. G. Gilbert: \#I II Euphorbiaceae

Euphorbia sekukuniensis R. A. Dyer: \#1 II Euphorbiaceae

Euphorbia semperflorens L. C. Leach: \#1 II Euphorbiaceae

Euphorbia sepium N. E. Brown = Euphorbia balsamifera Aiton

Euphorbia septemsulcata Vierh. = Euphorbia spiralis Balf. f. 
Euphorbia septentrionalis P. R. O. Bally \& S. Carter ssp. gamugofana M. G. Gilbert: \#! II Euphorbiaceae

Euphorbia septentrionalis P. R. O. Bally \& S. Carter ssp. septentrionalis: \#1 II Euphorbiaceae

Euphorbia sepulta P. R. O. Bally \& S. Carter: \#I II Euphorbiaceae

Euphorbia serendipita L. E. Newton: \#1 II Euphorbiaceae

Euphorbia sereti De Wild. ssp. sereti: \#1 II Euphorbiaceae

Euphortia sereti De Wild. ssp. variantissima L. C. Leach: \#1 II Euphorbiaceae

Euphorbia sessiliflora E. Meyer = Euphorbia gummifera Boissier

Euphorbia sessiliflora Roxb.: \#1 II Euphorbiaceae

Euphorbia setispina S. Carter: \#1 II Euphorbiaceae

Euphorbia silenifolia (Haw.) Sweet: \#1 II Euphorbiaceae

Euphorbia siliciicola Dinter = Euphorbia juttae Dinter

Euphorbia similiramea S. Carter. \#I II Euphorbiaceae

Euphorbia similis A. Berger = Euphorbia ingens $\mathrm{E}$. Meyer ex Boissier

Euphorbia sipolisii N. E. Br.: \#I II Euphorbiaceae

Euphorbia x soanieranensis Ursch \& Leandri: \#1 II Euphorbiaceae

Euphorbia spartaria N. E. Br.; \#1 II Euphorbiaceae

Euphorbia spatulata Commerson ex Baillon = Euphorbio thouarsiana Baillon

Euphorbia speciosa L. C. Leach: \#I II Euphorbiaceae

Euphorbia spicata E. Meyer ex Boiss.: \#l II Euphorbiaceae

Euphorbia spinea N. E. Br.: \#1 II Euphorbiaceae

Euphorbia spiralis Balf.f.: \#1 II Euphorbiaceae

Euphorbia splendens Bojer ex Hooker = Euphorbia milii Des Moulins var. splendens (Bojer ex Hooker) Ursch \& Leandri

Euphorbia splendens Bojer ex Hooker ssp. bojeri (Hooker) Denis = Euphorbia milii Des Moulins var, milii

Euphorbia splendens Bojer ex Hooker var. betsileana Leandri = Euphorbia milii Des Moulins var. splendens (Bojer ex Hooker) Ursch \& Leandri

Euphorbia splendens Bojer ex Hooker var. bevilaniensis (Croizat) Leandri $=$ Euphorbia milii Des Moulins var. bevilaniensis (Croizat) Ursch \& Leandri

Euphorbia splendens Bojer ex Hooker var. bojeri (Hooker) Costantin \& Gallaud = Euphorbia milii Des Moulins var. milit

Euphorbia splendens Bojer ex Hooker var, breonii Leandri = Euphorbia milii Des Moulins var. hislopii (N. E. Brown) Ursch \& Leandri

Euphorbia splendens Bojer ex Hooker var. hislopii (N. E. Brown) Leandri $=$ Euphorbia milii Des Moulins var. hislopii (N. E. Brown) Ursch \& Leandri

Euphorbia splendens Bojer ex Hooker var. imperatae Leandri $=$ Euphorbia milii Des Moulins var, imperarae (Leandri) Ursch \& Leandri

Euphorbia splendens Bojer ex Hooker var, tananarivae Leandri = Euphorbia milii Des Moulins var. tananarivae (Leandri) Ursch \& Leandri

Euphorbia splendens Bojer ex Hooker var. typica Leandri = Euphorbia milii Des Moulins var. splendens (Bojer ex Hooker) Ursch \& Leandri

Euphorbia splendens Bojer ex Hooker var-vulcanii Leandri = Euphorbia milii Des Moulins var. vulcanii (Leandri) Ursch \& Leandri

Euphorbia squarrosa Haw.: \#1 II Euphorbiaceae

Euphorbia stapelioides Boiss.: \#1 II Euphorbiaceae

Euphorbia stapfii A. Berger: \#l II Euphorbiaceae

Euphorbia stegmatica $\mathrm{Nel}=$ Euphorbia oxystegia Boissier

Euphorbia stellaespina hort. $=$ Euphorbia stellispina Haworth

Euphorbia stellaespina Haw. var. astrispina (N. E. Br.) A. C. White, R. A. Dyer \& B. Sloane = Euphorbia astrispina N. E. Br.

Euphorbia stellaespina Haw. var. stellaespina $=$ Euphorbia stellispina Haw, var. stellispina N. E. Br.

Euphorbia stellata Willd.: \#1 II Euphorbiaceae

Euphorbia stellispina Haw. var. astrispina (N. E. Br.) A. C. White, R. A. Dyer \& B. Sloane: \#1 II Euphorbiaceae

Euphorbia stellispina Haw. var. stellispina: \#1 II Euphorbiaceae

Euphorbia stenoclada Baillon ssp. ambatofinandranae (Léandri) Cremers: \#1 II Euphorbiaceae

Euphorbia stenoclada Baillon ssp. stenoclada: \#1 II Euphorbiaceae

Euphorbia stolonifera Marloth ex A. C. White, R. A. Dyer \& B. Sloane: \#1 II Euphorbiaceae

Euphorbia stomiae Croizat $=$ Euphorbia radians Bentham var. stormiae (Croizat) Rzedowski \& Calderón
Euphorbia strangulata N. E. Br. ssp. deminuens L. C. Leach: \#1 II Euphorbiaceae

Euphorbia strangulata N. E. Br. ssp. strangulata: \#1 II Euphorbiaceae

Euphorbia strigosa Hooker \& Amott: \#1 II Euphorbiaceae

Euphorbia siuhlmannii Volkens = Euphorbia heserochroma Pax ssp. heserochroma

Euphorbia stygiana H. C. Watson: \#1 II Euphorbiaceae

Euphorbia suareziana Croizat $=$ Euphorbia tirucalli L.

Euphorbia subapoda Baillon = Euphorbia primulifolia Baker var primulifolia

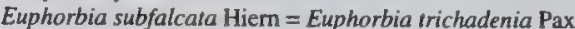

Euphorbia submammillaris (A. Berger) A. Berger: \#1 II Euphorbiaceae

Euphorbia subsalsa Hiem ssp. fluvialis L. C. Leach: \#1 II Euphorbiaceae

Euphorbia subsalsa Hiem ssp. subsalsa: \#1 II Euphorbiaceae

Euphorbia subsalsa Hiern var, kaokoensis A. C. White, R. A. Dyer \& B. Sloane $=$ Euphorbia kaokoensis (A. C. White, R. A. Dyer \& B. Sloane) L. C. Leach

Euphorbia subscandens P. R. O. Bally \& S. Carter: \#1 II Euphorbiaceae

Euphorbia subumbellata Steudel ex Boissier = Euphorbia copiapina Philippi

Euphorbia sudanica A. Chev.: \#I II Euphorbiaceae

Euphorbia suffulia Bruyns: \#I II Euphorbiaceae

Euphorbia superans Nel: \#1 II Euphorbiaceae

Euphorbia susan-holmesiae Binojkumar \& Gopalan: \#1 II Euphorbiaceae

Euphorbia susannae Marloth: \#1 II Euphorbiaceae

Euphorbia suzannae-mamierae Rauh \& Petignat: \#1 II Euphorbiaceac

Euphorbia symmetrica A. C. White, R. A. Dyer \& B. Sloane: \#1 II Euphorbiaceae

Euphorbia Iaboraensis Hässler: \#1 II Euphorbiaceae

Euphorbia taitensis Pax $=$ Euphorbia tenuispinosa Gilli var. tenuispinosa

Euphorbia tanaensis P. R. O. Bally: \#I II Euphorbiaceae

Euphorbia tardieuana Leandri: \#1 II Euphorbiaceae

Euphorbia tanuensis S. Carter: \#1 II Euphorbiaceae

Euphorbia teixeirae L. C. Leach: \#1 II Euphorbiaceae

Euphorbia teke Schweinf. ex Pax: \#! II Euphorbiaceae

Euphorbia tellieri A. Chevalier = Euphorbia sudanica A. Chevalier

Euphorbia tenax Burchell: \#1 II Euphorbiaceae

Euphorbia tenebrosa N. E. Brown = Euphorbia ingens E. Meyer ex Boissier

Euphorbia ienuispinosa Gilli var, robusta P. R, O. Bally \& S.Carter: \#1 II Euphortiaceae

Euphorbia tenuispinosa Gilli var. tenuispinosa: \#1 II Euphorbiaceae

Euphorbia tesconum S. Carter: \#1 II Euphorbiaceae

Euphorbia tetracantha $\mathrm{Pax}=$ Euphorbia nyassae Pax

Euphorbia tetracantha Rendle: \#1 II Euphorbiaceae

Euphorbia ierracanthoides Pax: \#1 II Euphorbiaceae

Euphorbia tetragona Baker $=$ Euphorbia micracantha Boissier

Euphorbia tetragona A. Richard = Euphorbia polyacantha Boissier

Euphorbia tetragona Haw.: \#1 II Euphorbiaceae

Euphorbia thi Schweinf. = Euphorbia polyacantha Boiss.

Euphorbia thi Schweinf. var. subinarticulata (Schweinfurth) N. E. Brown = Euphorbia polyacantha Boissier

Euphorbia thinophila Philippi: \#I II Euphorbiaceae

Euphorbia tholicola L. C. Leach: \#1 II Euphorbiaceae

Euphorbia thouarsiana Baillon: \#1 II Euphorbiaceae

Euphorbia tirucalli L.: \#1 II Euphorbiaceae

Euphorbia tirucalli L. var, rhipsaloides (N. E. Brown) A. Chevalier = Euphorbia tincalli $\mathrm{L}$

Euphorbia tisserantii A. Chev. \& Sillans = Euphorbia reke Schweinf. ex Pax

Euphorbia iogoensis $\mathrm{Pax}=$ Euphorbia lateriflora Schumacher \& Thonning

Euphorbia torta Pax \& K. Hoffm.: \#I II Euphorbiaceae

Euphorbia tortilis Rottler ex Ainslie: \#1 II Euphorbiaceae

Euphorbia sonirama R. A. Dyer: \#1 II Euphorbiaceae

Euphorbia tortistyla N. E. Br.: \#1 II Euphorbiaceae

Euphorbia tozzii Chiovenda = Euphorbia candelabrum Kotschy var. candelabrum

Euphorbia transvaalensis Schltr.: \#1 II Euphorbiaceae 
Euphorbia trapaeifolia A. Chevalier = Euphorbia sudanica A. Chevalier

Euphorbia rriaculeata Forsskål: \#I II Euphorbiaceae

Euphorbia triaculeata Forsskal var. triacantha (Ehrenb. ex Boiss.)

N. E. Br. = Euphorbia triaculeata Forsskal

Euphorbia triangularis Desf. ex A. Berger. \#1 II Euphorbiaceae

Euphorbia trichadenia Pax var. gibbsiae N. E. Br: \#1 II Euphorbiaceae

Euphorbia trichadenia Pax var. Irichadenia: \#1 II Euphorbiaceae

Euphorbia tridentata Lam.: \#1 II Euphorbiaceae

Euphorbia trigona Mill.: \#I II (except cuitivars) Euphorbiaceae

Euphorbia trigona Roxburgh = Euphorbia bamhanii Croizat

Euphorbia truncata N. E. Brown = Euphorbia clavarioides Boissier var. truncasa (N. E. Brown) A. C. White, R. A. Dyer \& B. Sloane

Euphorbia tsimbazazae Leandri: \#1 II Euphorbiaceae

Euphorbia tuberculata Jacq. var. macowanii (N. E. Br.) A. C. White,

R. A. Dyer \& B. Sloane: \# I II Euphorbiaceae

Euphorbia tuberculasa Jacq. var. tuberculata: \#1 II Euphorbiaceae

Euphorbia tubercularoides N. E. Br.: \#1 II Euphorbiaceae

Euphorbia tuberosa L.: \#1 II Euphorbiaceae

Euphorbia tubiglans Marloth ex R. A. Dyer: \#1 II Euphorbiaceae

Euphorbia iuckeyana Steud. ex Webb var. mezereum Chev.: \#1 II Euphorbiaceae

Euphorbia fuckeyana Steud. ex Webb var. Iuckeyana: \#1 II

Euphorbiaceae

Euphorbia tugelensis N. E. Br.: \#1 II Euphorbiaceae

Euphorbia tulearensis (Rauh) Rauh: I Euphorbiaceae

Euphorbia turbiniformis Chiov.: \#1 II Euphorbiaceae

Euphorbia turkanensis S. Carter: \#I II Euphorbiaceae

Euphorbia uhehensis $\mathrm{Pax}=$ Euphorbia platycephala $\mathrm{Pax}$

Euphorbia uhligiana Pax: \#I II Euphorbiaceae

Euphorbia umbanata S. Carter: \#I II Euphorbiaceae

Euphorbia umfoloziensis Peckover: \#1 II Euphorbiaceae

Euphorbia uncinata De Candolle = Euphorbia stellata Willd.

Euphorbia undulatifolia Janse: \#1 II Euphorbiaceae

Euphorbia unicomis R. A. Dyer: \#! II Euphorbiaceae

Euphorbia unispina N. E. Br.: \#1 II Euphorbiaceae

Euphorbia ussanguensis N. E. Brown = Euphorbia cooperi

N. E. Brown ex A. Berger var. ussanguensis (N. E. Brown)

L. C. Leach

Euphorbia uzmuk S. Carter \& J. R. I. Wood: \#I II Euphorbiaceae

Euphorbia vaalputsiana L. C. Leach: \#1 II Euphorbiaceae

Euphorbia vajravelui Binojkumar \& Balakrishnan: \#1 II Euphorbiaceae

Euphorbia valida N. E. Br.: \#1 II Euphorbiaceae

Euphorbia vallaris L. C. Leach: \#I II Euphorbiaceae

Euphorbia vandermerwei R. A. Dyer: \#1 II Euphorbiaceae

Euphorbia venenara Marloth: \#1 II Euphorbiaceae

Euphorbia venenifica Trem. ex Kotschy: \#I II Euphorbiaceae

Euphorbia verruculosa N. E. Br.: II II Euphorbiaceae

Euphorbia versicolores G. Williamson: \#I II Euphorbiaceae

Euphorbia viduiflora L. C. Leach: \#1 II Euphorbiaceae

Euphorbia viguieri Denis var, ankarafantsiensis Ursch \& Leandri: \#1 II Euphorbiaceac

Euphorbia viguieri Denis var. capuroniana Ursch \& Leandri: \# 1 II Euphorbiaceae

Euphorbia viguieri Denis var. tsimbazazae Ursch \& Leandri: \#1 11 Euphorbiaceae

Euphorbia viguieri Denis var. viguieri: \#1 II Euphorbiaceae

Euphorbia viguieri Denis var, vilanandrensis Ursch \& Leandri: \#1 II Euphorbiaceae

Euphorbia viminalis Burmann = Euphorbia burmannii (Klotzsch) E. Meyer ex Boissier

Euphorbia viminalis Hiem = Euphorbia negromoniana N. E. Brown in Oliver el al.

Euphorbia viperina A. Berger = Euphorbia inermis Miller var. inermis

Euphorbia virosa Willd. forma caespitosa $\mathrm{H}$. Jacobsen = Euphorbia virosa Willd. ssp. virosa

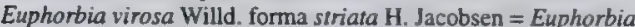
virosa Willd. ssp. virosa

Euphorbia virosa Willd. ssp. arenicola L. C. Leach: \#1 II Euphorbiaceae

Euphorbia virosa Willd. ssp. virosa: \#1 II Euphorbiaceae
Euphorbia virosa Willd, var, caerulescens (Haworth) A. Berger = Euphorbia caerulescens Haworth

Euphorbia vittaia S. Carter: \#1 II Euphorbiaceae

Euphorbia volkensii Werth = Euphorbia nyikae Pax var. neovolkensii (Pax) S. Carter

Euphorbia volkmanniae Dinter: \#1 II Euphorbiaceae

Euphorbia vulcanorum S. Carter: \#1 II Euphorbiaceae

Euphorbia wakefieldii N. E. Br.: \#1 II Euphorbiaceae

Euphorbia waterbergensis R. A. Dyer: \#1 II Euphorbiaceae

Euphorbia weberbaueri Mansf.: \#1 II Euphorbiaceae

Euphorbia whellanii L. C. Leach: \#1 II Euphorbiaceae

Euphorbia wildii L. C. Leach: \#1 II Euphorbiaceae

Euphorbia williamsonii L. C. Leach: \#1 II Euphorbiaceae

Euphorbia wilmaniae Marloth: \#1 II Euphorbiaceae

Euphorbia winkleri Pax = Euphorbia ampliphylla $\mathrm{Pax}$

Euphorbia woodii N. E. Br.: \#I II Euphorbiaceae

Euphorbia xanthadenia Denis = Euphorbia mahafalensis Denis var. xanthadenia (Denis) Leandri

Euphorbia xanti Engelm. ex Boiss.: \#1 II Euphorbiaceae

Euphorbia xylacantha Pax: \#1 II Euphorbiaceae

Euphorbia xylophylloides Brongn. ex Lemaire: \#I II Euphorbiaceae

Euphorbia x zanaharensis Ursch \& Leandri: \#1 II Euphorbiaceae

Euphorbia zeyheri Klotzsch \& Garcke = Euphorbia mauritanica L. var mauritanica

Euphorbia zoutpansbergensis R. A. Dyer: \#1 II Euphorbiaceae

Euforbias (S) = Euphorbia spp. I/II Euphorbiaceae

Euphorbes $(\boldsymbol{F})=$ Euphorbia spp. VII Euphorbiaceae

Euphorbion dendroides (L) Saint-Lager = Euphorbia dendroides L.

Euponeria $=$ Neoponeria

Euproboscis $=$ Thelasis

Eurebutia $=$ Rebutia

European Cyclamen (E): Cyclamen purpurascens Miller forma purpurascens: \#1 II Primulaceae

¿urycentrum spp. \#7 II Orchidaceae

Eurychone spp. \#7 II Orchidaceae

Eurystyles spp. \#7 II Orchidaceae

Evelyna $=$ Elleanthus

Evora = Ceratandra

Evotella spp. \#7 II Orchidaceae

Evrardia = Evrardianthe

Evrardiana $=$ Evrardianthe

Evrardianthe spp. \#7 II Orchidaceae

Exeria $=$ Eria

Exophya $=$ Epidendrum

Eydisanthema $=$ Brassavola $/$ Epidendrum

Facheiroa spp. \#4 II Cactaceae

False Bracken (E): Calochlaena dubia (R. Br.) M. D. Tumer \& R. A. White: \#l II Dicksoniaceae

Feather Palm (E): Neodypsis decaryi Jumelle: \#1 II Palmae

Femandezia spp. \#7 II Orchidaceae

Ferocactus spp. \#4 II Cactaceae

Ferocacius glaucus (K. Schumann) N. P. Taylor = Sclerocactus glaucus (K. Schumann) L. Benson

Ferocactus mesae-verdae (Boiss. \& Davidson) N. P. Taylor = Sclerocactus mesae-verdae (Boiss, \& Davidson) L. Benson

Ferocacrus pubispinus (Engelmann) N. P. Taylor = Sclerocactus pubispinus (Engelmann) L. Benson var. pubispinus

Ferocactus tobuschii (Mrsh.) Tayl = Sclerocactus brevihamatus (Engelmann) D. Hunt ssp. tobuschii (Mrsh.) Tayl.

Ferocactus wrightiae (L. Benson) N. P. Taylor $=$ Sclerocactus wrightiae L. Benson

Ficindica $=$ Opunia

Fickeisen's Hedgehog-cactus (E): Pediocacrus peeblesianus (Croizat) L. Benson var. fickeiseniae L. Benson: I Cactaceae

Fieldia $=$ Vandopsis

Fimbriella = Plaranthera

Finetia $=$ Neofinetia

Fissipes $=$ Cypripedium

Fitzeraldia $=$ Lyperanthus

Fitzroya cupressoides (Molina) I. M. Johnston: Alerce (E), Chilean False Larch (E), Patagonian Cypress (E), Lahuán (S) I Cupressaceae

Fleur du Saint-Esprit (F): Peristeria elara Hooker: I Orchidaceae

Flickingeria spp. \#7 II Orchidaceae

Floresia $=$ Weberbauerocereus

Floribunda $=$ Cipocereus 
Florida Arrowroot (E): Zamia integrifolia L.f. in Aiton; \#1 II Zamiaceae

Florida Coontie (E): Zamia integrifolia L.f. in Aiton: \#1 II Zamiaceae

Fobea $=$ Escobaria

Forbesina $=$ Eria

Forficaria spp. \#7 II Orchidaceae

Forsythmajoria $=$ Cynorkis

Fougères arborescentes (F): CYATHEACEAE spp./DICKSONIACEAE spp. \#1 II

Fouquieria columnaris Kellogg ex Curran: Boojum Tree (E) \#1 II Fouquieriaceae

Fouquieria fasciculata Nash: I Fouquieriaceae

Fouquieria purpusii Brandegee: I Fouquieriaceae

Foumiera $=$ Cyathea

Fractiunguis $=$ Reichenbachanthus

Frailea spp. \#4 II Cactaceae

Fregea spp. \#7 II Orchidaceac

Frerea indica Dalz: \#1 II Asclepiadaceae

Friesia $=$ Parodia

Fringed Lewisia (E): Lewisia coryledon (S. Watson) Robinson var. coryledon: \#1 II Portulacaceae

Frondaria spp. \#7 II Orchidaceae

Froscula $=$ Dendrobium

Fueriesiella spp. \#7 II Orchidaceae

Funkiclla $=$ Schiedeella

Furiolobivia $=$ Echinopsis

Gaberia $=$ Grammarophyllum

Gaiac (F): Guaiacum officinale L. \#1 II Zygophyllaceae

Galanthus spp.: Snowdrops (E), Copos de nieve (S), Campanillas de invierno (S) \#1 11 Amaryllidaceac

Galanthus alexandrii Porcius = Galanthus nivalis L

Galanthus allenii Baker: \#1 II Amaryllidaceae

Galanthus alpinus Sosn.: \#1 II Amaryllidaceae

Galanthus alpinus Sosn. var, alpinus: \#1 II Amaryllidaceae

Galanthus alpinus Sosn. var. borkewitschianus (Koss) A. P. Davis: \# II Amaryllidaceae

Galanthus angustifolius Koss: \#1 II Amaryllidaceae

Galanthus atkinsii hort. Barr = Galanthus nivalis L.

Galanthus borkewitschianus Koss = Galanthus alpinus Sosn. var. bonkewitschianus (Koss) A. P. Davis

Galanthus bulgaricus Velen. = Galanthus elwesii Hook.f.

Galanthus byzantinus Baker = Galanthus plicatus M. Bieb. ssp. byzantinus (Baker) D. A. Webb

Galanthus byzantinus Baker s\$p. braunerii N, Zeybek = Galanthus plicatus M. Bieb. ssp. byzantinus (Baker) D. A. Webb

Galanthus byzantinus Baker ssp. sauerii N. Zeybek = Galanthus plicatus M. Bieb. ssp. byzantinus (Baker) D. A. Webb

Galanthus byzantinus Baker ssp. tughrulli N. Zeybek = Galanthus plicatus M. Bieb. ssp. byzantinus (Baker) D. A. Webb

Galanthus cabardensis Koss. = Galanthus lagodechianus Kem.Nath.

Galanthus caspius (Rupr.) Grossh. = Galanthus transcaucasicus Fomin

Galanthus caucasicus (Baker) Burb. = Galanthus alpinus Sosn. var. alpinus

Galanthus caucasicus (Baker) Grossh. = Galanthus alpinus Sosn. var, alpinus

Galanthus cilicicus auct. = Galanthus peshmenii A. P. Davis \& C. D. Brickell

Galanthus cilicicus auct. = Galanthus rizehensis Stern

Galanthus cilicicus Baker: \#1 II Amaryllidaceae

Galanthus clusii Fisch. sensu Steud = Galanihus plicatus M. Bieb ssp. plicalus

Galanthus corcyrensis $($ Beck) Stem = Galanthus reginae-olgae Orph. ssp. reginae-olgae

Galanthus corcyrensis Burb. = Galanthus reginae-olgae Orph. ssp. reginae-olgae

Galanthus corcyrensis $\mathrm{J}$. Allen = Galanthus reginae-olgae Orph. ssp reginae-olgae

Galanthus corcyrensis Leichtin ex Correvon = Galanthus reginaeolgae Orph. ssp. reginae-olgae

Galanthus elsae Burb. = Galanthus reginae-olgae Orph. ssp reginae-olgae

Galanthus elsae Ewbank = Galanthus reginae-olgae Orph. ssp. reginae-olgae
Galanthus elsae J. Allen = Galanthus reginae-olgae Orph. ssp reginae-olgae

Galanthus elwesii Hook.f.: \#I II Amaryllidaceae

Galanthus elwesii Hook.f. ssp. akmanii N. Zeybek = Galanthus elwesii Hook.f.

Galanthus elwesii Hook.f. ssp. baytopii (N. Zeybek) N. Zeybek \& E. Sauer $=$ Galanthus elwesii Hook.

Galanthus elwesii Hook.f. ssp. melihae N. Zeybek = Galanthus elwesii Hook.f

Galanthus elwesii Hook.f. ssp. minor D.A. Webb = Galanthus gracilis Celak.

Galanthus elwesii Hook.f. ssp. tuebitaki N. Zeybek = Galanthus elwesii Hook.f.

Galanthus elwesii Hook.f. ssp. wagenitzii N. Zeybek = Galanthus elwesii Hook.f.

Galanthus elwesii Hook.f. ssp. yayintaschii N. Zeybek = Galanthus elwesii Hook.f

Galanthus elwesii Hook.f. var. globosus Ewbank = Galanthus elwesii Hook.f.

Galanthus elwesii Hook.f. var. maximus (Velen.) Beck = Galanthus elwesii Hook.

Galanthus elwesii Hook.f. var. platyphyllus Kamari = Galanthus elwesii Hook f

Galanthus elwesii Hook.f. var. reflexus (Herb.) Beck = Galanthus elwesii Hook.f.

Galanthus elwesii Hook.f. var. robustus Baker = Galanthus elwesit Hook.f.

Galanthus elwesit Hook.f. var. stenophyllus Kamari = Galanthus gracilis Celak.

Galanthus elwesii Hook.f. var. whitalli (hort.) W. Irving = Galanthus elwesii Hook.f.

Galanthus eluesii Hook.f. var. whitalli Moon $=$ Galanthus elwesi Hook.f.

Galanthus elwesii Hook.f. var. whitalli S. Am. = Galanthus elwesii Hook.f.

Gulanthus fosteri Baker: \#1 II Amaryllidaceae

Galanthus fosteri Baker var, antepensis N. Zeybek \& E. Sauer = Galanthus fosteri Baker

Galanthus glaucescens A. P. Khokr, = Galanthus rizehensis Stem

Galanthus globosus Wilks = Galanthus elwesii Hook.f.

Galanthus gracilis Celak.: \#I II Amaryllidaceae

Galanthus gracilis Celak. ssp. baytopii N. Zeybek = Galanthus elwesii Hook f.

Galanthus graecus auct. = Galanthus gracilis Celak

Galanthus graecus Orph. ex Boiss. = Galanthus elwesii Hook.f

Galanthus graecus Orph. ex Boiss. var. graecus = Galanthus elwesi Hook.f

Galanthus graecus Orph. ex Boiss. var. maximus (Velen.) Hayek = Galanthus elwesii Hook.f.

Galanthus graecus Orph. ex Boiss. var. maximus (Velen.) Hayek = Galanthus elwesii Hook.f.

Galanthus grandis Burb. = Galanthus alpinus Sosn. var. alpinus

Galanthus ikariae auct. = Galanthus woronowii Losinsk.

Galanthus ikariae Baker: \# II Amaryllidaceae

Galanthus ikariae Baker ssp. latifolius Stem pro parte = Galanthus platyphyllus Traub. \& Moldenke

Galanthus ikariae Baker ssp. latifolius Stern pro parte = Galanthus woronowii Losinsk

Galanthus ikariae Baker ssp. snogerupii Kamari = Galanthus ikariae Baker

Galanthus imperati Bertol. = Galanthus nivalis L.

Galanthus imperati Bertol. forma australis Zodda $=$ Galanthus reginae-olgae Orph. ssp. reginae-olgae

Galanthus kemulariae Kuth. = Galanthus lagodechianus Kem.-Nath.

Galanthus ketzkhovelii Kem.-Nath. = Galanthus lagodechianus Kem.-Nath.

Galanthus koenenianus Lobin, C. D.Brickell \& A. P. Davis: \#1 II Amaryllidaceae

Galanthus krasnovii A. Khokr.: \# II Amaryllidaceae

Galanthus krasnovii A. P. Khokr. ssp. maculatus A. P. Khokr. = Galanthus krasnovii A. P. Khokr.

Galanthus Lagodechionus Kem.-Nat.: \#1 II Amaryllidaceae

Galanthus latifolius Salisb. = Galanthus plicatus M. Bieb. ssp. plicarus

Galanthus Latifolius auct. = Galanthus woronowii Losinsk 
Galanthus latifolius Rupr. = Galanthus platyphyllus Traub. \& Moldenke

Galanthus latifolius Rupr. forma fosteri (Baker) Beck = Galanthus fosteri Baker

Galanihus latifolius Rupr. forma typicus Beck = Galanthus platyphyllus Traub. \& Moldenke

Galanth:s latifolius Rupr. forma typicus Gottl.-Tann. = Galanthus plaryphyllus Traub. \& Moldenke

Galanthus latifolius Rupr. var, rizaensis Anon, = Galanthus rizehensis Stem

Galanthus latifolius Rupr. var. rizehensis Stem \& Gilmour = Galanthus rizehensis Stem

Galanthus maximus Velen. = Galanthus elwesii Hook.f.

Galanthus melihae (N. Zeybek) N. Zeybek \& E. Sauer = Galanthus elwesii Hook.f.

Galanthus montana Schur = Galanthus nivalis $\mathbf{L}$.

Galanthus nivalis M. Bieb. ex Steud. = Galanthus plicatus M. Bieb. ssp. plicatus

Galanthus nivalis L: Common Snowdrop (E) \#1 II Amaryllidaceac

Galanthus nivalis L. var atkinsii J. Allen = Galanthus nivalis L.

Galanthus nivalis L. var corcyrensis hort. ex Leichtin = Galanthus reginae-olgae Orph. ssp. reginae-olgae

Galanthus nivalis L. forma octobrinus hort. ex Voss = Galanthus reginae-olgae Orph. ssp. reginae-olgae

Galanthus nivalis L. ssp. angustifolius (G. Koss) Arjushenko = Galanthus angustifolius Koss

Galanthus nivalis L. ssp. byzantinus (Baker) Gott.-Tann, = Galanthus plicatus M. Bieb. ssp. byzantinus (Baker) D. A. Webb

Galanthus nivalis L. ssp. caucasicus Baker = Galanthus alpinus Sosn. var. alpinus

Galanthus nivalis L. ssp. cilicicus auct. = Galanthus peshmenii A. P. Davis \& C. D. Brickell

Galanthus nivalis L. ssp. cilicicus (Baker) Gott.-Tann. = Golanthus cilicicus Baker

Galanthus nivalis L. ssp. elwesii (Hook.f.) Gottl.-Tann. = Galanthus elwesii Hook.f

Galanthus nivalis L. ssp. graecus (Orph. ex Boiss.) Gottl.-Tann. pro parte $=$ Galanthus elwesii Hook.f.

Galanthus nivalis L. ssp. graecus (Orph. ex Boiss.) Gottl.-Tann. = Galanthus elwesii Hook.f.

Galanthus nivalis L. ssp. humboldrii N. Zeybek = Galanthus nivalis L.

Galanthus nivalis L. ssp. imperati (Bertol.) Baker = Galanthus nivalis L.

Galanthus nivalis L. ssp. nivalis forma maximus (Velen.) Stoj. \& Stef. = Galanthus elwesii Hook. f.

Galanthus nivalis L. ssp. plicarus (M. Bieb.) Gottl.-Tann. = Galanthus plicatus M. Bieb. ssp. plicatus

Galanthus nivalis L. ssp. reginae-olgae (Orph.) Gottl.-Tann. = Galanthus reginae-olgae Orph. \$sp. reginae-olgae

Galanthus nivalis L. ssp. subplicatus N. Zeybek = Galanthus nivalis L.

Galanthus nivalis $\mathrm{L}$, var, atkinsii Mallett = Galanthus nivalis $\mathrm{L}$.

Galanthus nivalis L. var, carpaticus Fodor = Galanthus nivalis L.

Galanthus nivalis L. var. caspius Rupr. = Galanthus iranscaucasicus Fornin

Galanthus nivalis L. var. caucasicus (Baker) Beck = Galanthus alpinus Sosn. var, alpinus

Galanthus nivalis L. var. caucasicus (Baker) Fomin = Galanthus alpinus Sosn. var. alpinus

Galanthus nivalis L. var, caucasicus (Baker) J. Phillippow = Galanthus alpinus Sosn. var. alpinus

Galanthus nivalis L. var. corcyrensis (Beck) Halácsy = Galanthus reginae-olgae Orph. ssp. reginae-olgae

Galanthus nivalis L. var. elsae Mallett = Galanthus reginae-olgae Orph. ssp. reginae-olgae

Galanihus nivalis L. var, europaeus forma hololeucus (Celak.) Beck = Galanthus nivalis $\mathrm{L}$.

Galanthus nivalis L. var, europaeus forma horiensis (Herb.) Beck = Galanthus nivalis $\mathrm{L}$.

Galanthus nivalis L. var, europaeus forma scharlocki (Casp.) Beck = Galanthus nivalis L.

Galanthus nivalis L. var, europaeus forma corcyrensis hort. ex Beck = Galanthus reginae-olgae Orph. ssp. reginae-olgae

Galanthus nivalis L. var. europaeus forma olgae (Orph.) Beck = Galanthus reginae-olgae Orph. ssp. reginae-olgae
Galanthus nivalis L. var, hololeucus Celak. = Galanthus nivalis $\mathrm{L}$ Galanthus nivalis L. var. hortensis Herb. = Galanthus nivalis L. Galanthus nivalis L. var. imperati Mallett = Galanthus nivalis $\mathbf{L}$. Galanthus nivalis L. var. major (Ten.) Beck = Galanthus nivalis L Galanthus nivalis L. var, majus Ten. = Galanthus nivalis $\mathrm{L}$

Gulanthus nivalis L. var. maximus (Velen.) Stoj. \& Stevanov = Galanthus elwesii Hook.f.

Galanthus nivalis L. var. minus Ten. = Galanthus nivalis $\mathrm{L}$

Galanthus nivalis L. var. montanus (Schur) Rouy = Galanthus nivalis $\mathrm{L}$.

Galanthus nivalis L. var. octobrensis Mallett = Galanthus reginaeolgae Orph. ssp. reginae-olgae

Galanthus nivalis L. var. praecox Mallett = Galanthus reginae-olgae Orph. ssp. reginae-olgae

Galanthus nivalis L. var. rachelae Mallett = Galanthus reginaeolgae Orph. ssp, reginae-olgae

Galanthus nivalis $\mathrm{L}$. var. redoutei Rupr. ex Regel = Galanthus alpinus Sosn. var, alpinus

Galanthus nivalis L. var, reginae-olgae (Orph.) Fiori = Galanthus reginae-olgae Orph. ssp. reginae-olgae

Galanthus nivalis L. var. sharlocki Mallett = Galanthus nivalis L

Galanthus nivalis L, var, shaylockii Harpur-Crewe = Galanthus nivalis $\mathrm{L}$.

Galanthus octobrensis Burb. = Galanthus reginae-olgae Orph. ssp. reginae-olgae

Galanthus octobrensis Ewbank = Galanthus reginae-olgae Orph. ssp. reginae-olgae

Galanthus octobrensis J. Allen = Galanthus reginae-olgae Orph. ssp. reginae-olgae

Galanthus octobrensis Leichtlin ex Correvon = Galanthus reginaeolgae Orph. ssp. reginae-olgae

Galanthus acrobrensis T. Short = Galanthus reginae-olgae Orph. ssp. reginae-olgae

Galanthus octobriensis hort. ex Baker = Galanthus reginae-olgae Orph. ssp. reginae-olgae

Galanthus octrobrensis hort. ex Burb. = Galanthus reginae-olgae Orph. ssp. reginae-ulgae

Galanthus olgae Orph. ex Boiss. = Galanthus reginae-olgae Orph. ssp. reginae-olgae

Galanthus olgae-reginae hort. ex Leichtlin = Galanthus reginaeolgae Orph. ssp. reginae-olgae

Galanthus peshmenii A. P. Davis \& C. D. Brickell: \#1 II Amaryllidaceae

Galanthus platyphyllus Traub et Moldenke: \#1 II Amaryllidaceae

Galanthus plicatus sensu Guss. = Galanthus nivalis L

Galanthus plicarus auct, $=$ Galanthus transcaucasicus Fornin

Galanthus plicatus M. Bieb. ssp. byzantinus (Baker) D. A. Webb: \#1 II Amaryllidaceae

Galanthus plicalus M. Bieb. ssp. guenerii N. Zeybek = Galanthus plicatus M. Bieb. ssp. plicatus

Galanthus plicatus M. Bieb. ssp. karamanoghluensis N. Zeybek = Golanthus plicatus M. Bieb. ssp. plicatus

Galanthus plicatus M. Bieb. ssp. plicarus: Pleated Snowdrop (E), Plicate-leaved Snowdrop (E) \#1 II Amaryllidaceae

Galanthus plicatus M. Bieb. ssp. subplicatus (N. Zeybek) N. Zeybek = Galanthus nivalis L.

Galanthus plicatus M. Bieb. ssp. vardardii N. Zeybek = Galanthus plicatus M. Bieb. ssp. plicatus

Galanthus plicatus M. Bieb. var. byzantinus (Baker) Beck = Galanthus plicatus M. Bieb. ssp. byzantinus (Baker) D. A. Webb

Galanthus plicatus M. Bieb. var. genuinus forma excelsior Beck = Galanthus plicarus M. Bieb. ssp. plicatus

Galanthus plicatus M. Bieb. var. genuinus forma maximus Beck = Galanthus plicatus M. Bieb. ssp. plicatus

Galanthus plicatus M. Bieb. var. genuinus forma typicus Beck = Galanthus plicarus M. Bieb. ssp. plicatus

Galanthus praecox Burb. = Galanthus reginae-olgae Orph. ssp. reginae-olgae

Galanthus praecox J. Allen = Galanthus reginae-olgae Orph. ssp. reginae-olgae

Galanthus rachelae Burb. = Galanthus reginae-olgae Orph. ssp. reginae-olgae

Galanthus rachelae Ewbank = Galanthus reginae-olgxe Orph. ssp. reginae-olgae

Galanthus rachelae J. Allen = Galanthus reginae-olgae Orph. ssp. reginae-olgae 
Galanthus redoutei Rupr. ex Regel = Galanthus alpinus Sosn. var alpinus

Galanthus reflexus Herb. = Galanthus nivalis L

Galanthus reginae-olgae auct. = Galanthus peshmenii A. P. Davis \& C. D. Brickell

Galanthus reginae-olgae Orph.: \#1 II Amaryllidaceae

Galanthus reginae-olgae Orph. ssp. corcyrensis (Beck) Kamari = Galanthus reginae-olgae Orph. ssp. reginae-olgae

Galanthus reginae-olgae Orph. ssp, reginae-olgae: \#1 II Amaryllidaceae

Galanthus reginae-olgae Orph. ssp. vemalis Kamari: \#I II Amaryllidaceae

Galanthus rizehensis Stern: \#! II Amaryllidaceae

Galanthus schaoricus Kem.-Nath. = Galanihus alpinus Sosn. var. alpinus

Galanthus scharlocki Casp. = Galanthus nivalis L .

Galanthus shaylockii J. Allen = Galanthus nivalis L.

Galanthus transcaucasicus Fomin: \#I II Amaryllidaceae

Galanthus valentinae Pajnut.ex Grossh. $=$ Galanthus krasnovii A. P. Khokr.

Galanthus woronowii Losinsk: \#1 II Amaryllidaceae

Galeandra spp. \#7 II Orchidaceae

Galearis spp. \#7 II Orchidaceae

Galeoglossum $=$ Prescontia

Galeola spp. \#7 II Orchidaceae

Galeorchis $=$ Orchis

Galeotria $=$ Mendoncella

Galeottiella $=$ Brachystele

Galera $=$ Epipogium

Gamaria $=$ Disa

Gamoplexis $=$ Gastrodia

Gamosepalum = Aulosepalum

Garaya spp. \#7 II Orchidaceae

Garayella $=$ Chamelophyton

Gastorchis $=$ Phaius

Gaseridium $=$ Dendrobium

Gastrochilus spp. \#7 II Orchidaceae

Gastrodia spp. \#7 II Orchidaceae

Gastroglottis = Liparis

Gastropodium = Epidendrum

Gastrorchis $=$ Phaius

Gaut's Butterfly-cactus (E): Turbinicarpus gautii (L. Benson) A. Zimmerman: I Cactaceae

Gavilea spp. \#7 II Orchidaceae

Geesinkorchis spp. \#7 II Orchidaceae

Geissanthera $=$ Microtatorchis

Gennaria spp. \#7 II Orchidaceae

Genoplesium spp. \#7 II Orchidaceae

Genyorchis spp. \#7 II Orchidaceae

Geobina $=$ Goodyera

Geoblasta spp. \#7 II Orchidaceae

Geocalpa $=$ Pleurothallis

Geodorum spp. \#7 II Orchidaceae

Geohintonia spp. \#4 II Cactaceae

Georchis = Goodyera

Gerocephalus $=$ Espostoopsis

Gersinia $=$ Bulbophyllum

Ghiesbreghtia = Calanthe

Giant Pitcher Plant (E): Nepenthes rajah Hook. f: I Nepenthaceae

Giant Snowdrop (E): Galanthus eln'esii Hook.f. ssp. minor

D. A. Webb: \#1 Ir Amaryllidaceae

Giant Snowdrop (E): Galanthus elwesii Hook.f. ssp. elwesii: \#I II Amaryllidaceae

Gigliolia = Octomeria

Ginger Lily (E): Hedychium philippinense K. Schum.: \#1 II Zingiberaceae

Ginsing américan (F): Panax quinquefolius L. \#3 II Araliaceae

Giulianetia = Glossorhyncha

Glandulicactus $=$ Sclerocactus

Glandulifera $=$ Coryphantha

Glomera spp. \#7 II Orchidaceae

Glossapis = Habenaria

Glossodia spp. \#7 II Orchidaceae

Glossorhyncha spp. \#7 II Orchidaceae

Glossula = Habenaria

Gnetum montanum Markgraf: \#1 III Gnetaceae
Goadbyella $=$ Microtis

Golden Fern (E): Cibotium cumingii Kunze: \#1 I Dicksoniaceae

Goldschmidtia $=$ Dendrobium

Goldenseal (E): Hydrastis canadensis L. \#3 II Ranunculaceae

Gomeran Spurge (E): Euphorbia lambii Svent.; \#1 11 Euphorbiaceae

Gomesa spp. \#7 II Orchidaceae

Gomphichis spp. \#7 II Orchidaceae

Gomphostylis = Coelogyne

Gonarostylis spp. \#7 II Orchidaceae

Gongora spp. \#7 II Orchidaceae

Goniochilus spp. \#7 II Orchidaceae

Gonogona $=$ Goodyera

Goodyera spp. \#7 II Orchidaceae

Gorgoglossum = Sievekingia

Govenia spp. \#7 II Orchidaceae

Govindooia $=$ Tropidia

Grafia $=$ Phalaenopsis

Grahamia australiana (J, Black) Rowley = Anacampseros australiana J. M. Black

Grahamia kurtzii (Bacigalupo) Rowley = Anacampseros kurtzii Bacigalupo

Grahamia vulcanensis (Anon.) Rowley = Anacampseros vulcanensis Anon.

Grammangis spp. \#7 II Orchidaceae

Grammatophyllum spp. \#7 II Orchidaceae

Graphorkis spp. \#7 II Orchidacsae

Grastidium $=$ Dendrobium

Great Basin Fishhook Cactus (E): Sclerocactus pubispinus (Engelm.) L. Benson var, pubispinus: I Cactaceae

Greek Cyclamen (E): Cyclamen graecum Link ssp. graecum forma graecum \# I II Primulaceae

Green Pitcherplant (E): Sarracenia oreophila (Kearney) Wherry: I Sarraceniaceae

Greenwoodia spp. \#7 II Orchidaceae

Grenadier's Cap (E): Pachycereus militaris (Audot) D. Hunt: I Cactaceae

Grobya spp. \#7 II Orchidaceae

Grosourdya spp. \#7 II Orchidaceae

Grusonia $=$ Opuntia

Guaiac Tree (E): Guaiacum officinale L.: \#1 II Zygophyllaceae

Guaiacum officinale L: Commoner Lignum Vitae (E), Guaiac Tree (E), Palo de Vida (S), Palo Santo (S), Gaiac (F) \# I II Zygophyllaceae

Guaiacum sanctum L.: Holywood Lignum Vitae (E), \#1 II Zygophyllaceae

Guano (E): Swietenia macrophylla King: \#1 III Meliaceae

Guatemalan Fir (E): Abies guatemalensis Rehder var, guatemalensis: I Pinaceae

Gudrunia spp. \#7 II Orchidaceae

Gularia $=$ Schiedeella

Gun (E): Dioscorea deltoidea Wallich ex Kunth: \#1 II Dioscoreaceae

Gunnarella spp. \#7 II Orchidaceae

Gunnarorchis $=$ Dendrobium

Gunnia = Sarcochilus

Gussonea $=$ Solenangis

Gyaladenia $=$ Brachycorythis

Gyas = Bleria

Gymnadenia spp. \#7 II Orchidaceae

Gymnadeniopsis = Platanthera

Gymnantha $=$ Rebutia

Gymnanthocereus = Browningia

Gymnocactus = Turbinicarpus

Gymnocactus goldii (Bravo) Y. Ito = Turbinicarpus horripilus (Lem.) John \& Riha

Gymnocactus roseiflorus (Backeb.) Frnk = Turbinicarpus roseiflons Backeb.

Gymnocactus valdezianus (Möller) Backeb, = Turbinicarpus aldezianus (Möller) C. Glass \& R. Foster

Gymnocalycium spp. \#4 II Cactaceae

Gymnocereus = Browningia

Gymnochilus spp. \#7 II Orchidaceae

Gymnopremnon = Cyathea

Gymnosphaera $=$ Cyatheo

Gymnosphaera nicklesii Tard. \& Ballard ex Tard. = Cyarhea nicklesii (Tard. \& Ballard) Tind. 
Gynizodon $=$ Oncidium

Gynoglottis spp. \#7 II Orchidiseae

Gyrostachis $=$ Spiranthes

Gyrostachys $=$ Spiranthes

Hagea $=$ Mammillaria

Haageucactus = Hangeocereus

Haageocereus spp. \#4 II Cactaceae

Habenaria spp. \#7 II Orchidaceae

Habenella $=$ Habenaria

Habenorkis = Habenaria

Haemaria $=$ Ludisia

Haematorchis = Galeola

Hagsatera spp. \#7 II Orchidaceae

Hairy-fruited Monkey Cup (E): Nepenthes trichocarpa Miq.: \#1 II Nepenthaceae

Hakoneaste $=$ Ephippianthus

Hallackia = Hunonaea

Halophlebia $=$ Cyathea

Hamatocactus $=$ Thelocactus

Hammarbya spp. \#7 II Orchidaceae

Hancockia spp. \#7 II Orchidaceae

Hapalochilus = Bulbophyllum

Hapalorchis spp. \#7 II Orchidaceae

Haplochilus = Zeuxine

Haplostellis = Nervilia

Haraella spp. \#7 II Orchidaceae

Hariota $=$ Hatiora Rhipsalis

Harrisella spp. \#7 II Orchidaceae

Harrisia spp. \#4 II Cactaceae

Hartwegia $=$ Nageliella

Haseltonia = Cephalocereus

Hatchet Cactus (E): Pelecyphura aselliformis Ehrenb.: I Caclaceae

Hatiora spp. \#4 II Cactaceae

Hecabe $=$ Phaius

Heckner's Lewisia (E): Lewisia coryledon (S. Watson) Robinson var. heckneri (Morton) Munz: \#1 II Portulacaceae

Hederorkis spp. \#7 11 Orchidaceae

Hedychium philippinense K. Schum.: Ginger Lily (E), Philippine Garland Flower (E) \#1 II Zingiberaceae

Helcia spp. \#7 II Orchidaceae

Helechos arborescentes (S): CYATHEACEAE spp./DICKSONIACEAE spp. \#1 II

Heliabravoa $=$ Polaskia

Helianthocereus = Echinopsis

Helictonia $=$ Spiranthes

Heliocereus $=$ Disocactus

Helleborine $=$ Calopogon

Helleriella spp. \#7 II Orchidaceae

Hellerorchis = Rodrigueziella

Helonoma spp. \#7 II Orchidaceae

Helorchis = Cynorkis

Hemihabenaria $=$ Pecteilis

Hemiperis = Habenaria

Hemipilia spp. \#7 II Orchidaceae

Hemiscleria spp. \#7 II Orchidaceae

Hemistegia $=$ Cyathea

Hemitelia = Cyathea

Henosis = Bulbophyllum

Herminium spp. \#7 II Orchidaceae

Herpetophylum = Dendrobium

Herpysma spp. \#7 II Orchidaceae

Herschelia $=$ Herschelianthe

Herschelianthe spp. \#7 II Orchidaceae

Herrichocereus = Stenocereus

Hetaeria spp. \#7 II Orchidaceae

Heterolobivia $=$ Echinopsis

Heterotaxis = Maxillaria

Heterozewxine = Zeuxine

Hexadesmia spp. \#7 II Orchidaceae

Hexalectris spp. \#7 II Orchidaceae

Hexameria $=$ Podochilus

Hexisea spp. \#7 II Orchidaceae

Hexopia = Scaphyglotris

Hickenia = Parodia

Hildewintera $=$ Cleistocactus

Hildmannia $=$ Eriosyce
Himalayan May-apple (E): Podophyllum hexandrum Royle: \#2 II Berberidaceae

Himalayan Yew (E): Taxus wallichiana Zucc.: \#1 II Taxaceae

Himantoglossum spp. \#7 II Orchidaceae

Hintonella spp. \#7 II Orchidaceae

Hippeophyllum spp. \#7 II Orchidaceae

Hippoglossum = Bulbophyllum

Hippopodium $=$ Ceratandra

Hipporkis $=$ Satyrium

Hirtzia spp. \#7 II Orchidaceae

Hispaniella spp. \#7 II Orchidaceae

Hoehneella spp. \#7 II Orchidaceae

Hofmeisiera = Hofmeisterella

Hofmeisterella spp. \#7 II Orchidaceae

Holcoglossum spp. \#7 II Orchidaceae

Holmesia = Angraecopsis

Hologyne = Coelogyne

Holopogon spp. \#7 II Orchidaceae

Holothrix spp. \#7 II Orchidaceae

Holy Ghost Orchid (E): Peristeria elata Hooker: I Orchidaceae

Holywood Lignum Vitae (E): Guaiacum sancrum L: \#1 II Zygophyllaceae

Homalocephala $=$ Echinocacrus

Homaloperalum spp. \#7 II Orchidaceae

Honduras Mahogany (E): Swietenia macrophylla King: \#1 III Meliaceae

Hong Kong Slipper Orchid (E): Paphiopedilum purpuratum (Lindley) Stein: I Orchidaceae

Hooded Pitcher-plant (E): Sarracenia minor Walter. \#1 II Sarraceniaceae

Horichia spp. \#7 II Orchidaceae

Hoımidium spp. \#7 II Orchidaceae

Horridocacsus $=$ Eriosyce

Horvatia spp. \#7 II Orchidaceae

Hottentot's Head (E): Stangeria eriopus (Kunze) Baill.: I Stangeriaceae

Houlletia spp. \#7 11 Orchidaceae

Howell's Lewisia (E): Lewisia cotyledon (S.Watson) Robinson var. howellii (S. Watson) Jepson: \# 1 II Portulacaceae

Huebneria $=$ Orleanesia

Humboldtia $=$ Sielis

Hunileya spp. \#7 II Orchidaceae

Huntsman's Cap (E): Sarracenia purpurea L. ssp. purpurea: \#1 II Sarraceniaceae

Huntsman's Hom (E): Sarracenia purpurea L. ssp. purpurea: \#1 II Sarraceniaceae

Huttonaea spp. \#7 11 Orchidaceae

Hyacinthorchis = Cremastra

Hyalosema $=$ Bulbophyllum

Hybochilus spp. \#7 II Orchidaceae

Hydranthus = Dipodium

Hydrastis canadensis L.: Goldenseal (E): \#3 II Ranunculaceae

Hyerochilus $=$ Vandopsis

Hygrochilus spp. $\$ 7$ II Orchidaceae

Hylocereus spp. \#4 II Cactaceae

Hylophila spp. \#7 II Orchidaceae

Hymanthoglossum = Himantoglossum

Hymenorchis spp. \#7 II Orchidaceae

Hymenorebulobivia $=$ Echinopsis

Hymenorebutia $=$ Echinopsis

Hypodema = Cypripedium

Hypodematium $=$ Eulophia

Hysieria = Corymborkis

lantha = lonopsis

lbidium $=$ Spiranthes

lebine = Liparis

Imerinaea spp. \#7 II Orchidaceae

Indian Pitcher-plant (E): Nepenthes khasiana Hook.f.: I

Nepenthaceae

Inobulbon $=$ Dendrobium

Jone = Sunipia

lonopsis spp. \#7 II Orchidaceae

Ionorchis = Limodorum

Ipsea spp. \#7 II Orchidaceae

Iridorchis $=$ Cymbidium

Iridorkis = Oberonia 
Isabelia spp. \#7 II Orchidaceae

Ischnocentrum spp. \#7 II Orchidaceae

Ischnogyne spp. \#7 II Orchidaceae

Isias $=$ Serapias

Islaya $=$ Eriosyce

Isochilus spp. \#7 II Orchidaceae

Isolatocereus $=$ Stenocereus

Isotria spp. \#7 II Orchidaceae

Itaculumia = Habenaria

Jabali Pincushion Cactus (E): Coryphantha werdermannii Bödeker: I Cactaceae

Jacarand de Brasil (S): Dalbergia nigra Allem. ex Benth.: I Leguminosae

Jacquiniella spp. \#7 II Orchidaceae

Jamaiciella $=$ Oncidium

Jansenia $=$ Plectrophora

Jarras (S): Sarracenia spp. \#1 I/II Sarraceniaceae

Jasminocereus spp. \#4 II Cactaceae

Jatamansi (E): Nardostachys grandiflora DC. \#3 II Valerianaceae

Jebine $=$ Liparis

Jejosephia spp. \#7 II Orchidaceae

Jenmania $=$ Palmorchis

Jensoa $=$ Cymbidium

Jimensia $=$ Bletillo

Josephia $=$ Sirhookera

Jumellea spp. \#7 II Orchidaceae

Kaibab Pincushion Cactus (E): Pediocactus paradinei B. W. Benson: I Cactaceae

Kalimpongia $=$ Dickasonia

Kalmia cuneata Michx.: White-wicky (E) \#1 II Ericaceae

Kalopternix spp. \#7 II Orchidaceae

Katherinea $=$ Epigeneium

Kefersteinia spp. \#7 II Orchidaceae

Kegelia $=$ Kegeliella

Kegeliella spp. \#7 II Orchidaceae

Keranthus $=$ Dendrobium

Kerigomnia $=$ Chitonanthera

Kinabalu Pitcher Plant (E): Nepenthes rajah Hook.f.: I Nepenthaceac

Kinetochilus $=$ Dendrobium

Kingidium spp. \#7 II Orchidaceae

Kingiella $=$ Kingidium

Kionophyton $=$ Stenorrhynchos

Kitigorchis $=$ Oreorchis

Knowlton's Cactus (E): Pediocachus knowltonil L. Benson: I Cactaceae

Knowlton's Miniature Cactus (E): Pediacactus knowltonii L. Benson: I Cactaceae

Knowlton's Pincushion Cactus (E): Pediocacrus knowlronii L Benson: I Cactaceae

Kochiophyton $=$ Aganisia

Koellensteinia spp. \#7 II Orchidaceae

Kokrodua (E): Pericopsis elata (Harms) van Meeuwen: \#1 II Leguminosae

Konantzia spp. \#7 II Orchidaceac

Kraenzlinella $=$ Pleurothallis

Krainzia $=$ Mammillaria

Kreodanthus spp. \#7 II Orchidaceae

Kryptostoma $=$ Habenaria

Kuhlhasseltia spp. \#7 II Orchidaceae

Kuth (E): Saussurea costus (Falc.) Lipsch.: I Compositae

Lacaena spp. \#7 II Orchidaceae

Lacanthis splendens $($ Bojer ex Hooker) Rafinesque $=$ Euphorbia milii Des Moulins var. splendens (Bojer ex Hooker) Ursch \& Léandri

Lactomammillaria = Mammillaria

Laelia spp. \#7 II Orchidaceae

Laelia boothiana Reichb.f. = Laelia lobata (Lindi). Veitch

Laelia grandis Lindl. \& Paxton var. purpurea Reichb.f. = Laelia lobata (Lindl.) Veitch

Laelia jongheana Reichb.f.: I Orchidaceae

Laelia lobata (Lindl.) Veitch: Lélie lobée (F) I Orchidaceae

Laelia rivieri Carriere = Laelia lobara (Lindl.) Veitch

Laeliopsis spp. \#7 I Orchidaceae

Laguna Beach Dudleya (E): Dudleya stolonifera Moran: I Crassulaceae
Laguna Beach Liveforever (E): Dudleya stolonifera Moran: I Crassulaceae

Lahuán (S): Fitzroya cupressoides (Molina) I. M. Johnston: I Cupressaceae

Lamb's-tail Cactus (E): Echinocereus schmollii (Weing.)

N. P. Taylor: I Cactaceae

Lanium spp. \#7 II Orchidaceae

Lankesterella $=$ Stenorrhynchos

Lamandra $=$ Epidendrum

Lasiocereus spp. \#4 II Cactaceae

Lathrisia $=$ Bartholina

Latourea $=$ Dendrobium

Latourorchis $=$ Dendrobium

Leaoa $=$ Hexadesmia

Lecanorchis spp. \#7 II Orchidaceae

Lechetreznas (S): Euphorbia spp. VII Euphorbiaceae

Lectandra $=$ Poaephyllum

Ledgeria $=$ Galeola

Lee's Pincushion Cactus (E): Escobaria sneedii Britt. \& Rose var. leei (Rose ex Bödeker) D. R. Hunt

Lélie lobée (F): Laelia lobata (Lindl.) Veitch I Orchidaceae

Lemaireocereus $=$ Pachycereus

Lemboglossum spp. \#7 II Orchidaceae

Lemniscoa $=$ Bulbophyllum

Lemuranthe $=$ Cynorkis

Lemurella spp. \#7 II Orchidaceae

Lemurorchis spp. \#7 II Orchidaceae

Leocereus spp. \#4 II Cactaceae

Leochilus spp. \#7 II Orchidaceae

Leopardanthus $=$ Dipodium

Lepanthes spp. \#7 II Orchidaceae

Lepanthopsis spp. \#7 II Orchidaceae

Lepervenchea $=$ Angraecum

Lepidocoryphantha $=$ Conyphantha

Lepidogyne spp. \#7 11 Orchidaceae

Lepidozamia spp. \#1 II Zamiaceae

Lepidozamia hopei Regel: \#I II Zamiaceae

Lepidozamia peroffskyana Regel: \#I II Zamiaceae

Lepismium spp. \#4 II Cactaceae

Leporella spp. \#7 II Orchidaceae

Leptocentrum $=$ Rangaeris

Leptoceras $=$ Caladenio

Leptocereus spp. \#4 II Cactaceae

Leptocladia = Mammillaria

Leptocladodia $=$ Mammillaria

Leptorkis = Liparis

Leptotes spp. \#7 II Orchidaceae

Leptothrium $=$ Isochilus

Lequeetia $=$ Limodorum

Lesliea spp. \#7 II Orchidaceac

Leuchtenbergia spp. \#4 II Cactaceae

Leucohyle spp. \#7 II Orchidaceae

Leucolaena $=$ Didymoplexis

Leucorchis $=$ Pseudorchis

Leucostachys $=$ Goodyero

Leucostele $=$ Echinopsis

Lewisia cotyledon ( $\mathrm{S}$. Watson) Robinson var. cotyledon: Fringed Lewisia (E) \#1 II Portulacaceae

Lewisia cotyledon (S. Watson) Robinson var. heckneri (Morton) Munz: Heckner's Lewisia (E) \#1 II Portulacaceae

Lewisia cotyledon (S. Watson) Robinson var. howellii (S. Watson) Jepson: Howell's Lewisia (E) \#1 II Portulacaceae

Lewisia cotyledon (S. Watson) Robinson var. purdyi Jepson: Purdy's Lewisia (E) \#I II Portulacaceae

Lewisia maguirei A. Holmgren: Maguire's Bitter-root (E) \#1 II Portulacaceae

Lewisia serrata Heckard \& Stebbins: Saw-toothed Lewisia (E) \#1 II Portulacaceae

Lichinora $=$ Porpax

Lichterveldia = Odontoglossum/Cuitlauzina

Ligeophila spp. \#7 II Orchidaceae

Limatodes $=$ Calanthe

Limnas $=$ Malaxis

Limnorchis $=$ Platanthera

Limodorum spp. \#7 II Orchidaceae

Limonias = Cephalanthera 
Lindblomia $=$ Coeloglossum

Lindleyella $=$ Rudolfiella

Lindsay's Cactus (E): Echinocereus ferreirianus H. Gates ssp. lindsayi (J. Meyrán) N. P. Taylor: I Cactaceae

Lindsayella spp. \#7 II Orchidaceae

Liparis spp. \#7 II Orchidaceae

Lissochilus = Eulophio

Listera spp. \#7 II Orchidaceae

Listrostachys spp. \#7 II Orchidaceae

Little Princess Agave (E): Agave parviflora Torr. ssp. parviflora: I Agavaceae

Living Rock Cactus (E): Ariocarpus trigonus (F. A. C. Weber) K. Schum.: I Cactaceae

Lloyd's Mariposa Cactus (E): Sclerocactus mariposensis (Hester) N. P. Taylor: I Cactaceae

Lobeira $=$ Disocacrus

Lobeira macdougallii Alexander = Disocactus macdougallii (Alexander) Barthlot

Lobirebutia $=$ Echinopsis

Lobivia $=$ Echinopsis

Lobiviopsis $=$ Echinopsis

Lobogyne $=$ Appendicula

Lockhartia spp. \#7 II Orchidaceae

Loefgrenianthus spp. \#? II Orchidaceae

Lonchitis $=$ Sempias

Lophiaris = Oncidium

Lophocereus $=$ Pachycereus

Lophoglottis $=$ Sophronitis

Lophophora spp. \#4 II Cactaceae

Lorchophyllum $=$ Cephalanthero

Loroglossum $=$ Aceras

Lothiania $=$ Porroglossum

Lothoniana $=$ Porroglossum

Louisia $=$ Luisia

Loxanthocereus $=$ Cleistocactus

Loxoma $=$ Smithsonia

Loxomorchis $=$ Smithsonia

Ludisia spp. \#7 II Orchidaceae

Lueddemannia spp. \#7 II Orchidaceae

Luerella $=$ Masdevallia

Luisia spp. \#7 II Orchidaceae

Lycaste spp. \#7 II Orchidaceae

Lycomormium spp. \#7 II Orchidaceae

Lymanbensonia $=$ Lepismium

Lyperanthus spp. \#7 II Orchidaceae

Lyraea $=$ Bulbophyllum

Lyroglossa $=$ Stenorrhynchos

Lysias = Platanihera

Lysiella $=$ Platanthera

Lysimnia $=$ Brassavola

Maagoog (E): Euphorbia nubica N. E. Br.: 判1 II Euphorbiaceae

Macdonaldia $=$ Thelymitro

Machaerocereus $=$ Stenocereus

Maclenia $=$ Canleya

Macodes spp. \#7 II Orchidaceae

Macradenia spp. $\$ 7$ II Orchidaceae

Macrocentrum = Habenaria

Macrochilus $=$ Miltonia

Macroclinium = Notylia

Macrolepis $=$ Bulbophyllum

Macroplectrum $=$ Angraecum

Macropodanthus spp. \#7 II Orchidaceae

Macrostomium $=$ Dendrobium

Macrostylis $=$ Corymborkis

Macrozamia spp. \#I II Zamiaceae

Macrozamia cardiacensis P. I. Forster \& D. L. Jones: \#1 II Zamiaceae

Macrozamia communis L. A. S. Johnson: \#1 II Zamiaceae

Macrozamia concinna D. L. Jones: \#I II Zamiaceae

Macrozamia conferia D. L. Jones \& P. I. Forster: \#1 II Zamiaceae

Macrozamia cranei D. L. Jones \& P. I. Forster: \#I II Zamiaceae

Macrozamia crassifolia P. I. Forster \& D. L. Jones: \#1 II Zamiaceae

Macrozamia diplomera (F. Muell.) L. A. S. Johnson: \#1 II Zamiaceae

Macrozamia douglasii F. Muell.: \#1 II Zamiaceae

Macrozamia dyeri (F. Muell.) C. A. Gardin.: \#I II Zamiaceae
Macrozamia elegans K. D. Hill \& D. L. Jones: \#1 II Zamiaceae Macrozamia fawcettii C. Moore: \#1 II Zamiaceae

Macrozamia feamsidei D. L. Jones: \#1 II Zamiaceae

Macrozamia flexuosa C. Moore: \#1 II Zamiaceae

Macrozamia fraseri Miq.: \#I II Zamiaceae

Macrozamia glaucophylla D. L. Jones: \#1 II Zamiaceae

Macrozamia heteromera C. Moore: \#I II Zamiaceae

Macrozamia humilis D. L. Jones: \#1 II Zamiaceae

Macrozamia johnsonii D. L. Jones \& K. D. Hill: \#I II Zamiaceae

Macrozamia lomandroides D. L. Jones: \#1 II Zamiaceae

Macrozamia longispina P. I. Forster \& D. L. Jones: \#1 II Zamiaceae

Macrozamia lucida L. A. S. Johnson: \#1 II Zamiaceae

Macrozamia macdonnellii (Miq.) A. DC.: \#l II Zamiaceae

Macrozamia machinii P. I. Forster \& D. L. Jones: \#1 II Zamiaceae

Macrozamia miquelii (F. Muell.) A. DC.: \#1 II Zamiaceae

Macrozamia montana K. D. Hill: \#1 II Zamiaceae

Macrozamia moorei F. Muell.: \#! II Zamiaceae

Macrozamia mountperriensis F, M. Bailey: \#1 11 Zamiaceae

Macrozamia accidua D. L. Jones \& P. I. Forster: \#1 II Zamiaceae

Macrozamia parcifolia P. I. Forster \& D. L. Jones: \#I II Zamiaceae

Macrozamia pauli-guilielmi W. Hill \& F. Muell.: \#1 II Zamiaceae

Macrozamia pauli-guilielmi W. Hill \& F. Muell. ssp. flexuosa (C. Moore) L. A. S. Johnson = Macrozamia flexuosa C. Moore

Macrozamia pauli-guilielmi W. Hill \& F. Muell. ssp. plurinervia

L. A. S. Johnson = Macrozamia plurinervia (L. A. S. Johnson)

D. L. Jones

Macrozamia platyrhachis Bailey: \#I II Zamiaceae

Macrozamia plurinervia (L. A. S. Johnson) D. L. Jones: \#1 II Zamiaceae

Macrozamia polymorpha D. L. Jones: \#1 II Zamiaceae

Macrozamia reducta K. D. Hill \& D. L. Jones: \#I II Zamiaceae

Macrozamia riedlei (Gaud.) C. A. Gardner: \#I II Zarniaceae

Macrozamia secunda C. Moore: \#1 II Zamaceae

Macrozamia spiralis (Salisb.) Miq.: \#l II Zamiaceae

Macrozamia stenomera L. A. S. Johnson: \#1 II Zamiaceae

Marrozamia viridis D. L. Jones \& P. I. Forster: \#I II Zamiaceae

Maelenia $=$ Cattleye

Magnolia candollii $\left(\mathrm{B}^{\prime} \mathrm{ume}\right) \mathrm{H}$. Keng = Magnolia hodgsonii (Hook.f. \& Thomson)?

Magnolia hodgsonii (Hook.f. \& Thomson) ? \#1 III Magnoliaceae

Maguire's Bitter-root (E): Lewisia maguirei A. Holmgren: \#1 II Portulacaceae

Mahogani de Saint-dominique (F): Swietenia mahagoni (L.) Jacq. \#1 II Meliaceae

Mahogani grands feuilles (F): Swietenia macrophylla King: \#1 II Meliaceae

Mahogani petites feuilles (F): Swietenia mahagoni (L.) Jacq.: \#I II Meliaceae

Maiden's Quiver Tree (E): Aloe ramosissima Pillans: \#I II Aloaceae

Maierocactus $=$ Astrophysum

Maihuenia spp. \#4 II Cactaceae

Maihueniopsis = Opuntia

Malachadenia $=$ Bulbophyllum

Malacocarpus = Parodia

Malaxis spp. \#7 II Orchidaceae

Malayan Fem Palm (E): Cycas rumphii Miq.: \#1 II Cycadaceae

Malleola spp. \#7 11 Orchidaceae

Maloue (F): Cyathea schiedeana (C. Presl) Domin: \#1 II Cyatheaceae

Mamillaria $=$ Mammillaria

Mamillopsis = Mammillaria

Mammariella = Mammillaria

Mammilaria $=$ Mammillaria

Mammillaria spp. \#4 I/II Cactaceae

Mammillaria aselliformis (Ehrenb.) W. Watson = Pelecyphora aselliformis Ehrenb.

Mammillaria childsii Blanc $=$ Sclerocactus erectocentrus $(\mathrm{J}$. Coulter) N. P. Taylor var, ereciocentrus

Mammillaria disciformis DC. $=$ Strombocactus disciformis (DC.) Britton \& Rose

Mammillaria leei (Rose ex Bödeker) Weniger = Escobaria sneedi (Bitton \& Rose) Berger var. leei (Rose ex Bödeker) Hunt

Mammillaria nelliae $($ Croizat $)$ Croizat $=$ Escobaria minima $($ Baird $)$ D. R. Hunt

Mammillaria papyracantha (Engelmann) Engelmann $=$ Sclerocactus papyracanthus (Engelmann) N. P. Taylor 
Mammillaria pectinifera (Rümpler) A. Weber: I Cactaceae Mammillaria pseudopectinata (Backeb.) Kelsey \& Dayton = Turbinicarpus pseudopectinatus (Backeb.) C. Glass \& R. Foster Mammillaria sneedii (Britton \& Rose) Weniger = Escobaria sneedii (Briton \& Rose) Berger var, sneedii

Mammillaria solisioides Backeb.: I Cactaceae

Mammillaria iobuschii Marshall $=$ Sclerocactus brevihamatus (Engelmann) D. Hunt ssp. tobuschii (Marshall) N. P. Taylor

Mammillaria turbinata (Kuntze) Hooker $=$ Strombocactus disciformis (DC.) Britton \& Rose

Mammillaria valdeziana (Möller) Kelsey \& Dayton $=$ Turbinicarpus valdezianus (Möller) C. Glass \& R. Foster

Mammillaria werdermannii $($ Bödeker $) \mathrm{H}$. Weiss $=$ Conyphantho werdermannii Bödeker

Mammilloydia spp. \#4 II Cactaceae

Manniella spp. \#7 II Orchidaceae

Manú (S): Caryocar costaricense J. D. Sm.: \#1 II Caryocaraceae

Mara (S): Swietenia macrophylla King: \#1 III Meliaceae

Marenopuntia $=$ Opuntia

Margelliantha spp. \#7 II Orchidaceae

Marginatocereus $=$ Pachycereus

Mariarisqueta $=$ Cheirostylis

Maritimocereus $=$ Cleistocactus

Mamiera $=$ Selenicereus

Marsh Rose (E): Orothamnus zeyheri Pappe ex Hook.f.: I Proteaceae

Marshallocereus $=$ Stenocereus

Marsupiaria $=$ Maxillaria

Masdevallia spp. \#7 II Orchidaceae

Matucana spp. \#4 II Cactaceae

Maluma = Gomesa

Maxillaria spp. \#7 II Orchidaceae

Mcdougall's Cactus (E): Disocactus macdougallii (Alexander) Barthlott: I Cactaceae

Meciclis $=$ Coryanthes

Meconopsis regia G. Taylor: \#I III Papaveraceae

Mecosa $=$ Platanthera

Mediocactus $=$ Selenicereus

Mediocalcar spp. \#7 II Orchidaceae

Mediocereus $=$ Selenicereus

Mediolobivia $=$ Rebutia

Mediorebutia $=$ Rebutia

Medusea $=$ Euphorbia

Medusea fructus-pini (Miller) Haworth = Euphorbia caput-medusae $\mathrm{L}$

Medusea globosa (Haworth) Klotzsch \& Garcke $=$ Euphorbio globosa (Haworth) Sims

Medusea hamata (Haworth) Klotzsch \& Garcke = Euphorbia hamata (Haworth) Sweet

Medusea major (Aiton) Haworth = Euphorbia caput-medusae L.

Medusea patula (Haworth) Klotzsch \& Garcke $=$ Euphorbia tridentata Lamarck

Medusea sessellara Haworth = Euphorbia caput-medusae L

Medusea tridentata (Lamarck) Klotzsch \& Garcke = Euphorbia tridentata Lamarck

Medusea ruberculata (Jacquin) Klotzsch \& Garcke = Euphorbia tuberculata Jacquin

Megaclinium $=$ Bulbophyllum

Megalobivia $=$ Echinopsis

Megalorchis spp. \#7 II Orchidaceae

Megalotus spp. \#7 II Orchidaceae

Megastylis spp. \#7 II Orchidaceae

Meiracyllium spp. \#7 II Orchidaceae

Melaxis $=$ Liparis

Meliclis $=$ Coryanthes

Melocactus spp. \#4 I/II Cactaceae

Melocactus conoideus Buining \& Brederoo: I Cactaceae

Melocactus deinacanthus Buing \& Brederoo: I Cactaceae

Melocactus glaucescens Buining \& Brederoo: I Cactaceae

Melocactus paucispinus G. Heimen \& R. Paul: I Cactaceae

Menadena = Maxillaria

Menadenium $=$ Zygosepalum

Mendoncella spp. \#7 II Orchidaceae

Menephora $=$ Paphiopedilum

Mesa-verde Cactus (E): Sclerocacrus mesae-verdae (Boissevain ex Boissevain \& C. Davids.) L. Benson: I Cactaceae

Mesadenella $=$ Stenorrhynchos
Mesadenus $=$ Brachyssele

Mesechinopsis $=$ Echinopsis

Mesicera $=$ Habenaria

Mesoceras = Habenaria

Mesoclastes $=$ Luisia

Mesodactylis = Apostasia

Mesoglossum spp. \#7 II Orchidaceae

Mesoptera $=$ Liparis

Mesospinidium spp. \#7 II Orchidaceae

Metachilum $=$ Appendicula

Mexican Mahogany (E): Swietenia humilis Zuccarini: \#I II Meliaceae

Mexicoa spp. \#7 II Orchidaceae

Mexipedium $=$ Phragmipedium

Mexipedium xerophyticum (J. C. Soto, Salazar \& Hágsater) V. Albert $\&$ M. W. Chase $=$ Phragmipedium xerophyticum J. C. Soto, Salazar \& Hágsater

Meyenia $=$ Weberbauerocereus

Meyerocactus $=$ Echinocactus

Micranthocereus spp. \#4 II Cactaceae

Microchilus $=$ Erythrodes

Microcoelia spp. \#7 II Orchidaceae

Microcycas calocoma (Miq.) A. DC. Palma Corcho (S) I Zamiaceae

Microepidendrum $=$ Epidendrum

Microholmesia $=$ Angraecopsis

Micropera spp. \#7 II Orchidaceae

Microphyianthe $=$ Dendrobium

Micropuntia $=$ Opuntia

Microsaccus spp. \#7 II Orchidaceae

Microspermia $=$ Parodia

Microstegnus $=$ Cyathea

Microstylis $=$ Malaxis

Microtatorchis spp. \#7 II Orchidaceac

Microterangis spp. \#7 II Orchidaceae

Microtheca $=$ Cynorkis

Microthelys $=$ Brachystele

Microtis spp. \#7 II Orchidaceae

Mila spp. \#4 II Cactaceae

Miltonia spp. \#7 II Orchidaceae

Miltonioides spp. \#7 II Orchidaceae

Miltoniopsis spp. \#7 II Orchidaceae

Minicolumna $=$ Epidendrum

Miqueliopuntia $=$ Opuntia

Mirabella $=$ Cereus

Mischobulbon = Mischobulbum

Mischobulbum spp. \#7 II Orchidaceae

Mitopetalum = Tainia

Mitostigma $=$ Amitustigma

Mitrocereus $=$ Pachycereus

Mobilabium spp. \#7 11 Orchidaceae

Moerenhoutia spp. \#7 II Orchidaceae

Monachanthus = Calasetum

Monadenia spp. \#7 II Orchidaceae

Monanthos = Dendrobium

Monixus = Angraecum

Monkey Puzzle (E): Araucaria araucana (Mol.) K. Koch: III Araucariaceae

Monochilus = Zeuxime

Monomeria spp. \#7 II Orchidaceae

Monophyllorchis spp. \#7 II Orchidaceae

Monorchis = Herminium

Monosepalum spp. \#7 II Orchidaceae

Monotris $=$ Holothrix

Montolivaea = Habenaria

Monustes $=$ Spiranthes

Monvillea $=$ Cereus

Moorea $=$ Neomoore

Morangaya $=$ Echinocereu

Morawetzia $=$ Oreocereus

Mormodes spp. \#7 II Orchidaceae

Mormolyca spp. \#7 II Orchidaceae

Mountain Sweet Pitcher Plant (E): Sarracenia jonesii Wherry: I Sarraceniaceae

Muluorchis $=$ Tropidia

Myanthus = Catasetum

Mycaranthes = Eria 
Mycaridanthes = Eria

Myoda $=$ Ludisia

Myodium = Ophrys

Myoxanihus spp. \#7 II Orchidaceae

Mymechis spp. \#7 II Orchidaceae

Myrmecophila spp. \#7 II Orchidaceae

Myrobroma = Vanilla

Myrosmodes spp. \#7 II Orchidaceae

Myrtillocactus spp. \#4 II Cactaceae

Myrtillocereus $=$ Myrtillocactus

Mystacidium spp. \#7 II Orchidaceae

Nabaluia spp. \#7 II Orchidaceae

Nageliella spp. \#7 II Orchidaceae

Nambar (S): Platymiscium pleiostachyum Donn. Sm.: \#1 II Leguminosae

Namaqua Aloe (E): Aloe khamiesensis Pillans: \#1 II Aloaceae

Nanodes spp. \#7 II Orchidaceae

Napina $=$ Neolloydia

Nardostachys grandiflora DC. Jatamansi (E): \#3 11 Valerianaceae

Narica $=$ Sarcoglottis

Narrow-lidded Pitcher-plant (E): Nepenthes ampullaria Jack: \#1 II Nepenthaceae

Nasonia $=$ Fernandezia

Nauenia = Lacaena

Navajoa $=$ Pediocactus

Navajoa fickeisenii Backeb. = Pediocactus peeblesianus (Croizat) L. Benson var. fickeisenii L. Benson

Navajoa peeblesiana Croizat $=$ Pediocactus peeblesianus (Croizat) L. Benson var peeblesianus

Navajoa peeblesiana ssp. fickeisenii (Hoch.) Hoch. = Pediocactus peeblesianus (Croizat) L. Benson var. fickeisenii L. Benson

Needle-spined Pineapple Cactus (E): Sclerocactus erectocentrus var. erectocentrus (J. Coulter) N. P. Taylor: I Cactaceae

Neippergia $=$ Acineta

Nelis $=$ Goodyera

Nellie's Cory Cactus (E): Escobaria minima (Baird) D. R. Hunt: I Cactaceae

Nemaconia $=$ Ponere

Nematoceras $=$ Corybas

Nemuranthes $=$ Habenaria

Neo-urbania spp. \#7 II Orchidaceae

Neoabbotia $=$ Leptocereus

Neobartlettia spp. \#7 II Orchidaceae

Neobathiea spp. \#7 II Orchidaceae

Neobenthamia spp. \#7 II Orchidaceae

Neobesseya $=$ Escobaria

Neobolusia spp. \#7 II Orchidaceae

Neobuxbaumia spp. \#4 II Cactaceae

Neocardenasia $=$ Neoraimondia

Neochilenia $=$ Eriosyce

Neoclemensia spp. \#7 II Orchidaceae

Neocogniauxia spp. \#7 II Orchidaceae

Neodawsonia $=$ Cephalocereus

Neodryas spp. \#7 II Orchidaceae

Neodypsis decaryi Jumelle = Dypsis decaryi (Jum.) Beentje \& J. Dransf.

Neoescobaria $=$ Helcia

Neoevansia $=$ Peniocereus

Neofinetia spp. \#7 11 Orchidaceae

Neogardneria spp. \#7 II Orchidaceae

Neogomesia $=$ Ariocarpus

Neogomesia agavoides Castaneda = A riocarpus agavoides (Castaneda) E. F. Anderson

Neogomezia $=$ Ariocarpus

Neogymnantha = Rebutia

Neogyna spp. \#? II Orchidaceae

Neogyne $=$ Neogyna

Neohickenia $=$ Parodia

Neokoehleria spp. \#7 II Orchidaceae

Neolauchea = Isabelia

Neolehmannia spp. \#7 II Orchidaceae

Neolemaireocereus $=$ Stenocereus

Neolindleya $=$ Platanthera

Neolloydia spp. \#4 II Cactaceae

Neolloydia acunensis (W. T. Marshall) L. Benson = Sclerocactus erectocenirus (J. Coulter) N. P. Taylor var. acunensis
Neolloydia beguinii (A. Weber) Britton \& Rose = Turbinicarpus gaurii (L. Benson) A. Zimmerman

Neolloydia gielsdorfiana (Werdermann) Knuth = Turbinicarpus gielsdorfianus (Werdermann) John \& Riha

Neolloydia horripila (Lem.) Britton \& Rose = Turbinicarpus horripilus (Lem.) John \& Riha

Neolloydia knuthiana (Bödeker) Knuth = Turbinicarpus knuthianus (Bödeker) John \& Riha

Neolloydia laui (Glass \& Foster) E. F. Anderson = Turbinicarpus laui C. Glass \& R. Foster

Neolloydia lophophoroides (Werdermann) E. F. Anderson $=$ Turbinicarpus lophophoroides (Werdermann) F. Buxb. \& Backeb.

Neolloydia mandragora (Fric ex Berger) E. F. Anderson = Turbinicarpus mandragora (Fric ex Berger) A. Zimmerman

Neolloydia pilispina Britton \& Rose = Turbinicarpus laui C. Glass \& R. Foster

Neolloydia pseudomacrochele (Backeb.) E. F. Anderson = Turbinicarpus pseudomacrochele (Backeb.) F. Buxb. \& Backeb.

Neolloydia pseudopectinata (Backeb.) E. F. Anderson = Turbinicurpus pseudopectinatus (Backeb.) C. Glass \& R. Foster

Neolloydia saueri (Bödeker) Kruth = Turbinicarpus sauer (Bödeker) John \& Riha

Neolloydia schmiedickeana (Bödeker) E. F. Anderson = Turbinicarpus schmiedickeanus (Bödeker) F. Buxb. \& Backeb. var. schmiedickeanus

Neolloydia smithii (misapplied) = Turbinicarpus gautii (L. Benson) A. Zimmerman

Neolloydia subterranea (Backeb.) H. Moore = Turbinicarpus subterraneus (Backeb.) A. Zimmerman

Neolloydia valdeziana (Möller) E. F. Anderson = Turbinicarpus valdezianus (Möller) C. Glass \& R. Foster

Neolobivia $=$ Echinopsis

Neomammillaria $=$ Mammillaria

Neomoorea spp. \#7 II Orchidaceae

Neoporteria $=$ Eriosyce

Neoraimondia spp. \#4 II Cactaceae

Neotainiopsis $=$ Eriodes

Neotanahashia $=$ Eriosyce

Neotinea spp. \#7 II Orchidaceae

Neotria spp. \#7 II Orchidaceae

Neottianthe spp. \#7 II Orchidaceae

Neottidium $=$ Neottia

Neowerdermannia spp. \#4 II Cactaceae

Neowilliamsia spp. \#7 II Orchidaceae

Népenthès (F): Nepenthes spp. \#1 I/II Nepenthaceae

Nepenthes spp. Pitcher-plants (E), Népenthès (F) \# I VII Nepenthaceae

Nepenthes adnata R. Tamin \& M. Hotta: \#I II Nepenthaceae

Nepenthes alata Blanco: \#1 II Nepenthaceae

Nepenthes albo-marginata Lobb ex Lindl: \#I II Nepenthaceae

Nepenthes ampullaria Jack var, ampullaria: Nartow-lidded Pitcherplant (E) \#I II Nepenthaceae

Nepenthes ampullaria Jack var. racemosa J. H. Adam \& C. C. Wilcock: \#1 II Nepenthaceae

Nepenthes annamensis Macfarlane: \#1 II Nepenthaceae

Nepenthes bellii Kondo: \#1 II Nepenthaceae

Nepenthes bicalcarata Hook. f.: \#1 II Nepenthaceae

Nepenthes bongso Korth:: \#1 II Nepenthaceae

Nepenthes boschiana Korth.: \#1 II Nepenthaceae

Nepenthes burbidgeae Hook. f. ex Burb.: \#1 II Nepenthaceae

Nepenthes burkei Masters var. burkei: \#1 II Nepenthaceae

Nepenthes burkei Masters var. excellens Veitch: \#1 II Nepenthaceae

Nepenthes burkei Masters var, prolifica Masters: \#I II Nepenthaceae Nepenthes campanulata S. Kurata.: \#1 II Nepenthaceae

Nepenthes carunculata Danser var. carunculata: \#1 II Nepenthaceae

Nepenthes carunculata Danser var. robusta J. Nerz \& A. Wistuba: \#I II Nepenthaceae

Nepenthes clipeata Danser: \#1 II Nepenthaceae

Nepenthes deaniana Macfarlane: \#1 II Nepenthaceae

Nepenthes decurrens Macfarlane: \#1 II Nepenthaceae

Nepenthes densiflora Danser: \#1 II Nepenthaceae

Nepenthes dentata S. Kurata: \#1 II Nepenthaceae

Nepenthes destillatoria Brion: \#1 II Nepenthaceae

Nepenthes dubia Danser: \#I II Nepenthaceae

Nepenihes edwardsiana Hook. f: \#1 II Nepenthaceae 
Nepenthes ephippiata Danser: \#I II Nepenthaceae Nepenthes faizaliana J. H. Adam \& C. C. Wilcock: \#1 II Nepenthaceae

Nepenthes fusca Danser; \#1 II Nepenthaceae

Nepenthes geoffrayi Lecomte: \#I II Nepenthaceae

Nepenthes globamphora Sh. Kurata \& Toyoshima: \#1 II Nepenthaceae

Nepenthes gracilis Korth.: Slender Pitcher-plant (E) \#1 II Nepenthaceae

Nepenthes gracillima Ridley: \#1 II Nepenthaceae

Nepenthes gymnamphora Reinw. ex Nees: \#1 II Nepenthacese

Nepenthes hirsuta Hook. f.: \#1 II Nepenthaceae

Nepenthes hookeriana Low: \#I II Nepenthaceae

Nepenthes inermis Danser: \#1 II Nepenthaceae

Nepenthes insignis Danser: \#I II Nepenthaceae

Nepenthes kampotiana Lecomte: \#I II Nepenthaceae

Nepenthes khasiana Hook. f.: Indian Pitcher-plant (E) I Nepenthaceae

Nepenthes klossii Ridley: \#1 II Nepenthaceae

Nepenthes lepiochila Danser: \#1 II Nepenthaceae

Nepenthes longifolia J. Nerz \& A. Wistuba: \#1 II Nepenthaceae

Nepenthes lowii Hook. f.: \#I II Nepenthaceae

Nepenthes macfarlanei Hemsl.: \#1 II Nepenthaceae

Nepenthes macrovulgaris J. R. Tumbull \& A. T. Middleton; \#1 II Nepenthaceae

Nepenthes madagascariensis Poir:: \#I II Nepenthaceae

Nepenthes masaolensis R. Schmid-Hollinger: \#1 II Nepenthaceae

Nepenthes maxima Reinw: \#1 II Nepenthaceae

Nepenthes merrilliana Macfarlane: \#1 II Nepenthaceae

Nepenthes mikoi B. R. Saimon \& R. G. Maulder: \#1 II Nepenthaceac

Nepenthes mirabilis Druce var. biflora J. H. Adam \& C. C. Wilcock:

\#1 II Nepenthaceae

Nepenthes mirabilis Druce var echinistoma (Hook f.) J. H. Adam \&

C. C. Wilcock: \#1 II Nepenthaceae

Nepenthes mirabilis Druce var, mirabilis: \#1 II Nepenthaceae

Nepenthes mollis Danser: \#I II Nepenthaceae

Nepenthes muluensis M. Hotta: \#1 11 Nepenthaceae

Nepenthes neglecta Macfarlane: \# I II Nepenthaceae

Nepenthes neoguineensis Macfarlane: \#1 II Nepenthaceae

Nepenthes northiana Hook. f.: \#1 II Nepenthaceae

Nepenthes ovata J. Nerz \& A. Wistuba: \#1 II Nepenthaceae

Nepenthes paniculata Danser: \#! II Nepenthaceae

Nepenthes papuana Danser: \#1 II Nepenthaceae

Nepenthes pectinata Danser: \#1 II Nepenthaceae

Nepenthes pervillei Blume: \#! II Nepenthaceae

Nepenthes petiolata Danser: \#1 II Nepenthaceae

Nepenthes philippinensis Macfarlane: \#1 II Nepenthaceae

Nepenthes pilosa Danser: \#1 II Nepenthaceae

Nepenthes rafflesiana Jack var, alata J. H. Adam \& C. C. Wilcock: \#1 II Nepenthaceae

Nepenthes rafflesiana Jack var. rafflesiana: Raffles' Pitcher-plant (E) \#1 II Nepenthaceae

Nepenthes rajah Hook. f.: Giant Pitcher Plant (E), Kinabalu Pitcher Plant (E) I Nepenthaceae

Nepenthes reinwardriana Miq. var, reinwardiiana: \#I II Nepenthaceae

Nepenthes reinwardiana Miq. var. samarindaiensis J. H. Adam \& C. C. Wilcock: \#I II Nepenthaceae

Nepenthes rhombicaulis Sh. Kurata: \#1 II Nepenthaceae

Nepenthes rosulata $\mathrm{R}$. Tamin \& M. Hotta: \# 1 II Nepenthaceae

Nepenthes sanguinea Lindl: \#1 II Nepenthaceae

Nepenthes singalana Becc.: \#1 II Nepenthaceae

Nepenthes spathulata Danser: \#1 II Nepenthaceae

Nepenthes spectabilis Danser: \#1 II Nepenthaceae

Nepenthes spinosa R. Tamin \& M. Hotta: \#I II Nepenthaceae

Nepenthes stenophylla Mast: \#I II Nepenthaceae

Nepenthes sumatrana Beck: \#1 II Nepenthaceae

Nepenthes talangensis J. Nerz \& A. Wistuba: \#1 II Nepenthaceae

Nepenthes tentaculata Hook. f:: \#1 II Nepenthaceae

Nepenthes tenuis J. Nerz \& A. Wistuba: \#1 II Nepenthaceae

Nepenthes thorelii Lecomte: \#1 II Nepenthaceae

Nepenthes tobaica Danser: \#I II Nepenthaceae

Nepenthes tomoriana Danser: \#1 II Nepenthaceae

Nepenthes treubiana Warb.: \#1 II Nepenthaceae

Nepenthes irichocarpa Miq.: Hairy-fruited Monkey Cup (E) \#1 II Nepenthaceae
Nepenthes truncala Macfarlane: \#1 II Nepenthaceae

Nepenthes veitchii Hook. f.: \#1 II Nepenthaceae

Nepenthes ventricosa Blanco: \#1 II Nepenthaceae

Nepenihes vieillardii Hook. f.: \#1 II Nepenthaceae

Nepenthes villosa Hook.: \#I II Nepenthaceae

Nepenthes xiphiodes B. R. Salmon \& R. G. Maulder: \#1 II Nepenthaceae

Nephelaphyllum spp. \#7 II Orchidaceae

Nephelea $=$ Cyathea

Nephelea cuspidata (Kunze) R. Tryon = Alsophila cuspidata (Kunze) Conant

Nephelea erinacea (Karsten) R. Tryon var, erinacea $=$ Alsophila erinacea (Karsten) Conant

Nephelea erinacea (Karst.) R. Tryon var. purpurascens (Sodiro) Gastony = Alsophila erinacea (Karsten) Conant

Nephelea fulgens (C. Chr.) Gastony = Cyathea fulgens $\mathrm{C} . \mathrm{Chr}$.

Nephelea grevilleana (Martius) $\mathbf{R}$. Tryon $=$ Cyathea grevilleana Martius

Nephelea imrayana (Hook.) R. Tryon var. basilaris (Christ) Gastony = Cyathea basilaris Christ

Nephelea imrayana (Hook.) R. Tryon var : imrayana = Cyathea imrayana Hook. var. imrayana

Nephelea polystichoides (Chnist) R. Tryon = Alsophila polystichoides Christ

Nephelea woodwardioides (Ka'llf.) Gastony var, cubensis Chd. ex (Maxon) Gastony = Cyathea woodwardioides Kaulf. var. cubensis

Nephelea woodwardioides (Kaulf.) Gastony var. hieronymi (Brause) Gastony = Cyathea woodwardioides Kaulf. var. hieronymi

Nephelea woodwardioides (Kaulf.) Gastony var. woodwardioides = Cyathea woodwardioides Kaulf. var. woodwardioides

Nephrangis spp. \#7 II Orchidaceae

Nephranthera $=$ Renanthera

Nerissa $=$ Ponthieva

Nervilia spp. \#7 II Orchidaceae

Neuwiedia spp. \#7 11 Orchidaceae

Nichelia $=$ Eriosyce

Nidema spp. \#7 II Orchidaceae

Nidus $=$ Neortia

Niemeyera $=$ Apostasia

Nienokuea $=$ Polystachya

Nigritella spp. \#7 II Orchidaceae

Nipponorchis $=$ Neofinetia

Nolina interrata Gentry: Dehesa Bear-grass (E), San Diego Beargrass (E) I Agavaceae

Nopalea $=$ Opuntia

Nopalxochia $=$ Disocactus

Nopalxochia macdougallit (Alexander) W. Marshall = Disocacrus macdougallii (Alexander) Barthlott

Normanbokea $=$ Turbinicarpus

Normanbokea pseudopectinata (Backeb.) Kladiwa \& Buxb. = Turbinicarpus pseudopectinatus (Backeb.) C. Glass \& R. Foster

Normanbokea valdeziana (Möller) Kladiwa \& Buxb. = Turbinicarpus valdezianus (Möller) C. Glass \& R. Foster

Noma $=$ Calypso

Nothodoritis spp. \#7 II Orchidaceae

Nothostele spp. \#7 II Orchidaceae

Notiophrys $=$ Platylepis

Notocactus $=$ Parodia

Norylia spp. \#7 II Orchidaceae

Nuevo Leon Living-rock Cactus (E): Ariocarpus scaphirostris Bödeker: I Cactaceae

Nyciocereus $=$ Peniocereus

Nyctosma $=$ Epidendrum

Oakes-amesia spp. \#7 II Orchidaceae

Oberonia spp. \#7 II Orchidaceae

Obregonia spp. \#4 VII Cactaceae

Obregonia denegrii Fric: Artichoke Cactus (E), Obregonita (S) I Cactaceae

Obregonita (S): Obregonia denegrii Fric: 1 Cactaceae

Ocampoa $=$ Cranichis

Ochyrella spp. \#7 II Orchidaceae

Ocopetate (S): Cyathea mexicana Schltr. \& Cham.: \#1 II Cyatheaceae

Octadesmia $=$ Dilomilis

Octandrarchis = Octomeria 
Octarthena spp. \#7 II Orchidaceae Octomeria spp. \#7 II Orchidaceae Odonectis = /sotria

Odontochilus = Anoecrochilus Odonioglossum spp. \#7 II Orchidaceac

Odontorrhynchus spF. \#7 II Orchidaceae

Odontostyles = Bulbophyllum

Oeceoclades spp. \#7 II Orchidaceae

Oehmea $=$ Mammillaria

Oeonia spp. \#7 II Orchidaceae

Oeoniella spp. \#7 II Orchidaceae

Oerstedella spp. \#7 II Orchidaceae

Oestlundorchis spp. \#7 II Orchidaceae

Olgasis = Oncidium

Oligophyton spp. \#7 II Orchidaceae

Oliveriana spp. \#7 II Orchidaceae

Ommatodium $=$ Pterygodium

Omoea spp. \#7 II Orchidaceae

Oncidium spp. \#7 II Orchidaceae

Oncodia $=$ Brachita

Onkeripus $=$ Xylobium

Onychium = Dendrobium

Ophidion spp. \#7 II Orchidaceae

Ophrys spp. \#7 II Orchidaceae

Oporanthus colchiciflorus (Waldst. \& Kit.) Herb. = Stembergia colchiciflora (Waldst. \& Kit.) Herb.

Oporanthus fischerianus Herb. = Stembergia fischeriana (Herb. M. Roem

Oporanthus luteus (L.) Herb. = Stembergia lutea (L.) Ker Gawl. ex Spreng.

Oporanthus luteus (L.) Herb. var. angustifolia Herb. = Stembergia lutea (L.) Ker Gawl. ex Spreng

Oporanthus luteus (L.) Herb. var. latifolia Herb. = Stembergia lutea (L.) Ker Gawl. ex Spreng

Opuntia spp. \#4 II Cactaceae

Opuntiopsis $=$ Schlumbergera

Orchiastrum $=$ Spiranthes

ORCHIDACEAE spp.: Orchids (E), Orquideas (S), Orchidées (F) I/I

Orchidium $=$ Calypso

Orchidofunckia $=$ Cryptarrhena

Orchidorypus = Pachyphyllum

Orchidées (F): ORCHIDACEAE spp. III

Orchids (E): ORCHIDACEAE spp.I/II

Orchiodes $=$ Goodyera

Orchipedum spp, \#7 II Orchidaceae

Orchis spp. \#7 II Orchidaceae

Orchite; = Traunsteinero

Orquidens (S) ORCHIDACEAE spp. I/I

Orejas de conejo (S): Ariocarpus scaphirostris Bödeker: I Cactaceae

Orejitas (S): Ariocarpus scaphirostris Bödeker: I Cactaceae

Oreocereus spp. \#4 II Cactaceae

Oreomunnea pierocarpa Oerst.;1 II Juglandaceae

Oreorchis spp. \#7 II Orchidaceae

Orestias spp. \#7 II Orchidaceae

Orleanesia spp. \#7 II Orchidaceae

Ormostema $=$ Dendrobium

Omitharium $=$ Pleroceras

Omithidium = Maxillaria

Ornithocephalus spp. \#7 II Orchidaceae

Ormithochilus spp. \#7 II Orchidaceae

Ornithophora = Sigmatostalix

Orothamnus zeyheri Pappe ex Hook.f.: Marsh Rose (E) \#1 II Proteaceae

Oroya spp. \#4 II Cactaceae

Orsidice $=$ Thrixspermum

Ortegocacrus spp. \#4 II Cactaceae

Orthoceras spp. \#7 II Orchidaceae

Orthochilus $=$ Eulophio

Orthopenthea $=$ Disa

Ormannia $=$ Geodorum

Orxera $=$ Aerides

Osmoglossum spp. \#7 II Orchidaceae

Ossiculum spp. \#7 11 Orchidaceae

Osyricera $=$ Bulbophyllum

Otandra = Geodorum
Orochilus spp. \#7 II Orchidaceae

Ologlossum spp. \#7 II Orchidaceae

Otopetalum $=$ Pleurothallis

Otostylis spp. \#7 II Orchidaceae

Oxyanthera $=$ Thelasis

Oxysepala $=$ Bulbophyllum

Oxystophyllum $=$ Dendrobium

Pabstia spp. \#7 II Orchidaceae

Pabsriella $=$ Pleurothallis

Pachak (F): Saussurea costus (Falc.) Lipsch. I Compositae

Pachites spp. \#7 II Orchidaceae

Pachycereus spp. \#4 1//I Cactaceae

Pachycereus chrysomallus (Lem.) Britton \& Rose = Pachycereus miliraris (Audot) D. Hunt

Pachycereus militaris (Audot) D. Hunt: Grenadier's Cap (E), Teddybear Cactus (E) I Cactaceae

Pachychilus $=$ Pachystoma

Pachyclada dendroides (L.) Gandoger = Euphorbia dendroides L

Pachyclada obtusifclia (Poiret) Gandoger = Euphorbia lamarckii Sweet

Pachyne $=$ Phaius

Pachyphyllum spp. \#7 II Orchidaceae

Pachyplectron spp. \#7 11 Orchidaceae

Pachypodium spp. Elephant-trunks (E), Halfmen (E), Pachypodes (F) \#1 V/II Apocynaceae

Pachypodium ambongense L. Poisson: I Apocynaceae

Pachypodium baronil Constantin \& Bois var. baronii: 1 Apocynaceae

Pachypodium baronii Constantin \& Bois var, windsori (L. Poisson) Pichon: I Apocynaceae

Pachypodium bispinosum (L.f.) A.DC: \#1 II Apocynaceae

Pachypodium brevicaule Baker: \#1 II Apocynaceae

Pachypodium decaryi L. Poisson: I Apocynaceae

Pachypodium densiflorum Baker var, brevicalyx H. Perrier: \#1 II Apocynaceac

Pachypodium densiflorum Baker var. densiflonum: \#1 II Apocynaceae

Pachypodium geayi Costantin \& Bois: \#1 II Apocynaceae

Pachypodium horombense $\mathrm{L}$. Poisson = Pachypodium rosulatum Baker var, horombense (L. Poisson) G. D. Rowley

Pachypodium inopinatum Lavranos: \#1 II Apocynaceae

Pachypodium lamerei Drake var. Lamerei: \#1 II Apocynaceae

Pachypodium lamerei Drake var, ramosum (Costantin \& Bois) Pichon: \#1 II Apocynaceae

Pachypodium lealii Welw.: \# I II Apocynaceae

Pachypodium namaquanum (Wyley ex Harv.) Welw.: Elephant's Trunk (E) \#I II Apocynaceae

Pachypodium rosulatum Baker var. drakei (Costantin \& Bois) Markgraf: \#1 II Apocynaceae

Pachypodium rosulatum Baker var. gracilius H. Perrier: \#1 II Apocynaceae

Pachypodium rosulatum Baker var, horombense (L.Poisson) G. D.Rowley: \#1 II Apocynaceae

Pachypodium rosulatum Baker var, rosulatum: \#1 II Apocynaceae

Pachypodium rosulatum Baker var. stenanthum Costantin \& Bois: \#1 II Apocynaceae

Pachypodium rusenbergianum Vatke var. meridionale (M. Pichon) H. Perrier: \#1 II Apocynaceae

Pachypodium rutenbergianum Vatke var. rutenbergianum: \#I II Apocynaceae

Pachypodium saundersii N. E. Brown: \#1 II Apocynaceae

Pachypodium sofiense (L. Poisson) H. Perrier: \#1 II Apocynaceae

Pachypodium succulentum (L.f.) A. DC: Bergkambro (E) \#1 II Apocynaceae

Pachyrhizanthe $=$ Cymbidium

Pachystele spp. \#7 II Orchidaceae

Pachystelis $=$ Pachystele

Pachysioma spp. \#7 II Orchidaceae

Pachystylis $=$ Pachystoma

Pale Pitcher-plant (E): Sarracenia alata (A. W. Wood) Wood: \#1 II Sarraceniaceae

Paliris = Liparis

Palissandre du Bresil (S): Dalbergia nigra Allem. ex Benth.: I Leguminosae

Palma Corcho (S): Microcycas calocoma (Miq.) A. DC.: I Zamiaceae 
Palmas del Pan (S): Encephalartos spp. \#1 I Zamiaceae

Palmiers cycas (F): CYCADACEAE spp. III Cycadaceae

Palmita de terra fria (S): Cyathea costaricensis (Kuhn) Domin: \#1 II Cyatheaceae

Palmoglossum = Pleurothallis

Palmorchis spp. \#7 II Orchidaceae

Palo de la vida (S): Cyathea bicrenata Liebm.: \#1 II Cyatheaceae

Palo de vida (S): Guaiacum officinale L.: \#1 II Zygophyllaceae

Palo santo (S): Guaiacum officinale L.: \#1 II Zygophyllaceae

Palumbina spp. \#7 II Orchidaceae

Panax à cing folioles (F): Panax quinquefolius L. \#3 II Araliaceae

Panax quinquefolius L.: American Ginseng (E), Celery-leaved Panax (E), Panax à cinq folioles (F), Ginsing américan (F) \#3 II Araliaceae

Panicularia $=$ Thyrsopteris

Panisea spp. \#7 II Orchidaceae

Panstrepis $=$ Coryanthes

Pantingia $=$ Sigmatodactylus

Paper-spine Pincushion Cactus (E): Sclerocacius papyracanthus (Engelm.) N. P. Taylor: I Cactaceae

Paphinia spp. \#7 II Orchidaceae

Paphiopedilum spp. I Orchidaceae

Paphiopedilum acmodonium M.W. Wood: I Orchidaceae

Paphiopedilum adductum Asher: I Orchidaceae

Paphiopedilum affine De Wild. = Paphiopedilum gratrixianum (Masters) Guillaumin

Paphiopedilum amabile Hallier = Paphiopedilum bullenianum (Reichb.f.) Pfitzer var. bullenianum

Paphiopedilum ambonensis hort. = Paphipedilum bullenianum (Reichb.f.) Pfitzer var. celebesense (Fowlie \& Birk) P. J. Cribb

Paphiopedilum appletonianum (Gower) Rolfe: I Orchidaceae

Paphiopedilum appletonianum Gower var poynizianum (O'Brien) Pfitzer = Paphiopedilum appleionianum (Gower) Rolfe

Paphiopedilum argus (Reichb. f.) Stein: I Orchidaceae

Paphiopedilum argus (Reichb.f.) Stein var. sriwaniae (Koop.) Gruss = Paphiopedilum argus (Reichb.f.) Stein

Paphiopedilum armeniacum S. C. Chen \& F. Y. Liu: I Orchidacene

Paphiopedilum bacanum Schoser \& Deelder = Paphiopedilum schoseri Braem \& H. Mohr

Paphiopedilum barbatum (Lindley) Pfitz: I Orchidaceae

Paphiopedilum barbatum (Lindl.) Pfitzer var. argus hort. = Paphiopedilum argus (Reichb.f.) Stein

Paphiopedilum barbatum (Lindl.) Pfitzer var. lawrenceanum (Reichb.f.) M. W. Wood = Paphiopedilum lawrenceanum (Reichb.f.) Pfitzer

Paphiopedilum barbatum (Lindl.) Pfitzer var. nigrium (Reichb.) Pfitzer $=$ Paphiopedilum barbatum (Lindl) Pfitzer

Paphiopedilum barbigerum Tang \& Wang: I Orchidaceze

Paphiopedilum bellaiulum (Reichb. f.) Stein: I Orchidaceae

Paphiopedilum birkii Birk = Paphiopedilum callosum (Reichb.f.) Stein var. sublaeve (Reichb.f.) P. J. Cribb

Paphiopedilum bodegomil Fowlie = Paphiopedilum glanduliferum (Blume) Stein var, wilhelminae (L. O. Williams) Cribb

Paphiopedilum boissierianum (Reichb.f.) Stein = Phragmipedium boissierianum (Reichb.f.) Rolfe

Paphiopedilum bougainvilleanum Fowlie: I Orchidaceae

Paphiopedilum boxallii (Reichb.f.) Pfitzer = Paphiopedilum villosum (Lindl.) Stein var. boxallii (Reichb.f.) Pfitzer

Paphiopedilum braemii Mohr = Paphiopedilum tonsum (Reichb.f.) Stein var. braemii (Mohr) Gruss

Paphiopedilum bullenianum (Reichb.f.) Pfitz. var. amabile: I Orchidaceae $=$ Paphiopedilum bullenianum (Reichb.f.) Pfitz. var. bullenianum

Paphiopedilum bullenianum (Reichb. f.) Pfitz. var, bullenianum: I Orchidaceae

Paphiopedilum bullenianum (Reichb. f.) Pfitzer var. celebesense (Fowlie \& Birk) Cribb: I Orchidaceae

Paphiopedilum burbidgei (Reichb.f.) Pfitzer = Paphiopedilum dayanum (Lindl.) Stein

Paphiopedilum callosum (Reichb.f.) Stein subsp. sublaeve (Reichb.f.) Fowlie = Paphiopedilum callosum (Reichb.f.) Stein var. sublaeve (Reichb.f.) P. J. Cribb

Paphiopedilum callosum (Reichb.f.) Stein var. angustipetalum Guill = Paphiopedilum callosum (Reichb.f.) Stein var. callosum

Paphiopedilum callosum (Reichb. f.) Stein var. callosum: I Orchidaceae
Paphiopedilum callosum (Reichb.f.) Stein var. schmidrianum Kraenzl, = Paphiopedilum callosum (Reichb.f.) Stein var. callosum

Paphiopedilum callosum (Reichb. f.) Stein var. sublaeve (Reichb. f.) Cribb: I Orchidaceae

Paphiopedilum caricinum (Lindl. \& Paxton) Stein = Phragmipedium caricinum (Lindl. \& Paxton) Rolfe

Paphiopedilum caudatum (Lindl.) Pfitzer = Phragmipedium caudatum (Lind1.) Rolfe

Paphiopedilum caudarum Lindl. var. wallisii (Reichb.f.) Stein = Phragmipedium wallisii (Reichb.f.) Garay

Paphiopedilum celebesense Fowlie \& Birk = Paphiopedilum bullenianum (Reichb.f.) Pfitzer var. celebesense (Fowlie \& Birk) P. J. Cribb

Paphiopedilum ceramense Birk = Paphiopedilum bullenianum (Reichb.f.) Pfitzer var. celebesense (Fowlie \& Birk) P. J. Cribb

Paphiopedilum chamberlainianum (Sander) Stein = Paphiopedilum vicroria-regina (Sander) M. W. Wood

Paphiopedilum chamberlainianum (Sander) Stein ssp. liemianum Fowlie = Paphiopedilum liemianum (Fowlie) Karas, \& K. Saito

Paphiopedilum chamberlainianum (Sander) Stein var, liemianum (Fowlie) Braem = Paphiopedilum liemianum (Fowlie) Karas. \& K. Saito

Paphiopedilum chamberlainianum (Sander) Stein var. primulinum (M. W. Wood \& Taylor) Eraen = Paphiopedilum primulinum M. W. Wood \& Taylor var, primulinum

Paphiopedilum charlesworthii (Rolfe) Pfitz: I Orchidaceae

Paphiopedilum chiwuanum T. Tang \& F. T. Wang = Paphiopedilum hirsutissimum (Lindl. ex Hook.) Stein var. hirsutissimum

Paphiopedilum ciliolare (Reichb. f.) Stein: I Orchidaceae

Paphiopedilum ciliolare (Reichb.f.) Stein var. mireauanum (Linden) Pfitzer = Paphiopedilum ciliolare $($ Reichb.f.) Stein

Paphiopedilum concolor (Lindl.) Pfitz.: I Orchidaceae

Paphiopedilum concolor (Lindl.) Pfitzer var, niveum Reichb.f. = Paphiopedilum niveum (Reichb.f.) Stein

Paphiopedilum cunisii (Reichb.f) Stein = Paphiopedilum superbiens (Reichb.f.) Stein var, cunisii (Reichb.f.) G. J. Braem

Paphiopedilum dariense (Reichb.f.) Stein = Phragmipedium longifolium (Warsz. \& Reichb.f.) Rolfe

Paphiopedilum dayanum (Lindl.) Stein: I Orchidaceae

Paphiopedilum dayanum (Lindl.) Stein. var. petri (Reichb.f.) Pfitzer = Paphiopedilum dayanum (Lindl.) Stein

Paphiopedilum delenatii Guillaumin: I Orchidaceae

Paphiopedilum dennisit Schoser = Paphiopedilum wentworthianum Schoser \& Fowlie

Paphiopedilum dianthum T. Tang \& F. T. Wang: I Orchidaceae

Paphiopedilum devogelii Schoser \& Deelder = Paphiopedilum supardii Braem \& Loeb

Paphiopedilum dilectum (Reichb.f.) Pfitzer = Paphiopedilum villosum (Lindl.) Stein var. boxallii (Reichb.f.) Pfitzer

Paphiopedilum dallii Lueckel = Paphiopedilum henryanum Braem

Paphiopedilum druryi (Beddome) Stein: Drury's Slipper Orchid (E) I Orchidaceae

Paphiopedilum elliontianum (O'Brien) Stein = Paphiopedilum rothschildianum (Reichb.f.) Stein

Paphiopedilum emersonii Koopowitz \& Cribb: I Orchidaceae

Paphiopedilum exul (Ridley) Rolfe: I Orchidaceae

Paphiopedilum fairrieanum (Lindley) Stein: I Orchidaceae

Paphiopedilum fowliei Birk: I Orchidaceae

Paphiopedilum gardineri (Guillemard) Pfitzer = Paphiopedilum glanduliferum (Blume) Stein var. glanduliferum

Paphiopedilum gardineri Kennedy = Paphiopedilum glandulife num (Blume) Stein var, wilhelminae ( $\mathrm{L}, \mathrm{O}$. Williams) Cribb

Paphiopedilum glanduliferum (Blume) Stein var. gardiner (Guillemard) Braem = Paphiopedilum glanduliferum (Blume) Stein var. glanduliferum

Paphiopedilum glanduliferum (Blume) Stein var. glanduliferum: I Orchidaceae

Paphiopedilum glanduliferum (Blume) Stein var. praesians (Reichb.f.) Braem = Paphiopedilum glanduliferum (Blume) Stein var. glanduliferum

Paphiopedilum glanduliferum (Blume) Stein var. wilhelminae (L. O. Williams) Cribb: I Orchidaceae

Paphiopedilum glaucophyllum J. J. Smith var. glaucophyllum : I Orchidaceae 
Paphiopedilum glaucophyllum J. J. Smith var. moqueneanum J. J. Smith: I Orchidaceae

Paphiopedilum godefroyae (Godef.-Leb.) Stein var. godefroyae : I Orchidaceae

Paphiopedilum godefroyae (Godef.-Leb.) Stein var. leucochilum (Masters) Hallier I Orchidaceae

Paphiopedilum gratrixianum (Masters) Guillaumin: I Orchidaceae

Paphiopedilum hainanense Fowlie = Paphiopedilum appletonianum (Gower) Rolfe

Paphiopedilum haynaldianum (Reichb. f.) Stein: I Orchidaceae

Paphiopedilum helenae Averyanov: I Orchidaceae

Paphiopedilum hennisianum (M. W. Wood) Fowlie var. christiansenii Gruss \& Roth: I Orchidaceae

Paphiopedilum hennisianum (M. W. Wood) Fowlie var. hennisianum: I Orchidaceae

Paphiopedilum hennisianum (M. W. Wood) Fowlie var, fowlie (Birk) P. 3. Cribb = Paphiopedilum fowliei Birk

Paphiopedilum henryanum Braem: I Orchidaceae

Paphiopedilum hermannii Fuchs \& Reisinger: I Orchidaceae

Paphiopedilum hincksianum (Reichb.f.) Stein = Phragmipedium longifolium (Warsz. \& Reichb.f.) Rolfe

Paphiopedilum hirsutissimum (Lindl, ex Hook.) Stein var. chiwuanum (Tang \& Wang) Cribb = Paphiopedilum hirsutissimum (Lindley \& Hook.) Stein var. hirsutissimum

Paphiopedilum hirsutissimum (Lindl. ex Hook.) Stein var. esquirolei (Schltr.) Cribb: I Orchidaceae

Paphiopedilum hirsutissimum (Lindley \& Hook.) Stein var. hirsutissimum: I Orchidaceae

Paphiopedilum hookerae (Reichb. f.) Stein: I Orchidaceae

Paphiopedilum hookerae (Reichb.f.) Stein var. bullenianum (Reichb.f.) Kerch. = Paphiopedilum bullenianum (Reichb.f.) Pfitzer var, bullenianum

Paphiopedilum hookerae var. hookerae: I Orchidaceae

Paphiopedilum hookerae (Reichb. f.) Stein var. volonteanum (Sander ex Rolfe) Kerch.: I Orchidaceae

Paphiopedilum insigne (Wallich ex Lindley) Pfitzer: I Orchidaceae

Paphiopedilum insigne (Wallich ex Lindley) Pfitzer var. barbigerum (T. Tang \& F. T. Wang) Braem = Paphiopedilum barbigerum T. Tang \& F. T. Wang

Paphiopedilum insigne (Wallich ex Lindley) Pfitzer var. exul (Ridley) Braem = Paphiopedilum exul (Ridley) Rolfe

Paphiopedilum javanicum (Reinw, ex Lindl.) Pfitz. var. javanicum: I Orchidaceae

Paphiopedilum javanicum (Reinw. ex Lindl.) Pfitz. var. nymphenburgianum Roth \& Gruss

Paphiopedilum javanicum (Reinw, ex Lindl.) Pfitz. var. virens: I Orchidaceae

Paphiopedilum johorense Fowlie \& Yap = Paphiopedilum bullenianum (Reichb.f.) Pfitzer var, bullenianum

Paphiopedilum klotzschianum (Reichb.f. ex M. R. Schomb.) Stein = Phragmipedium klotzschianum (Reichb.f. ex M. R. Schomb.) Rolfe

Paphiopedilum kolopakingii Fowlie: I Orchidaceae

Paphiopedilum Lawrenceanum Pfitz.: I Orchidaceae

Paphiopedilum leucochilum (Rolfe) Fowlie = Paphiopedilum godefroyae (Godef.-Leb.) Stein var. leucochilum (Masters) Hallier

Paphiopedilum liemianum (Fowlie) Karasawa \& Saito: I Orchidaceae

Paphiopedilum liemianum (Fowlie) Karasawa \& K. Saito var. primulinum (M. W. Wood \& Taylor) Karasawa \& K. Saito = Paphiopedilum primulinum M. W. Wood \& Taylor var. primulinum

Paphiopedilum lindleyanum (M. R. Schomb. ex Lindl.) Stein = Phragmipedium lindleyanum (M. R. Schomb. ex Lind!.) Rolfe

Paphiopedilum linii Schoser = Paphiopedilum bullenianum (Reichb.f.) Pfitzer var. bullenianum

Paphiopedilum longifolium (Warsz. \& Reichb.f.) Stein = Phragmipedium longifolium (Warsz. \& Reichb.f.) Rolfe

Paphiopedilum lowii (Lindley) Stein var. lowii: I Orchidaceae

Paphiopedilum lowii (Lindley) Stein var. richardianum (Asher \& Beaman) Gruss: I Orchidaceae

Paphiopedilum malipoense S. C. Chen \& Tsi: I Orchidaceae

Paphiopedilum markianum Fowlie $=$ Paphiopedilum tigrinum Koop. \& N. Haseg.

Paphiopedilum mastersianum (Reichb. f.) Stein: I Orchidaceae
Paphiopedilum micranthum Tang \& Wang: I Orchidaceae

Paphiopedilum mohrianum Braem: 1 Orchidaceae

Paphiopedilum moquetteanum (J. J. Sm.) Fowlie = Paphiopedilum glaucophyllum J. J. Smith var. moquetteanum J. J. Smith

Paphiopedilum nigritum (Reichb.f.) Pfitzer = Paphiopedilum barbatum (Lindl.) Rolfe

Paphiopedilum niveum (Reichb. f.) Stein: I Orchidaceae

Paphiopedilum orbum Reichb.f. = Paphiopedilum callosum (Reichb.f.) Stein var. callosum

Paphiopedilum papuanum (Ridley) Ridley: I Orchidaceae

Paphiopedilum pardinum (Reichb.f.) Pfitzer = Paphiopedilum venustum (Wallich ex Sims) Pfitzer ex Stein

Paphiopedilum parishii (Reichb.f.) Stein var. dianthum (Tang \& Wang) Cribb \& Tang: 1 Orchidaceae

Paphiopedilum parishü (Reichb.f.) Stein var. parishii: I Orchidaceae

Paphiopedilum petri (Reichb.f.) Rolfe = Paphiopedilum dayanum (Lindl.) Stein

Paphiopedilum philippinense (Reichb.f.) Stein var. cannartianum (Linden) Pfitzer $=$ Paphiopedilum philippinense (Reichb.f.) Stein var, philippinense

Paphiopedilum philippinense (Reichb. f.) Stein: var. philippinense I Orchidaceae

Paphiopedilum philippinense (Reichb. f.) Stein var. roebelenii (Veitch) Cribb: I Orchidaceae

Paphiopedilum potentianum Gruss \& Roth: I Orchidaceae

Paphiopedilum praestans (Reichb. f.) Pfitz. = Paphiopedilum glanduliferum (Blume) Stein

Paphiopedilum praestans (Reichb.f.) Pfitz. var. kimballianum (Linden \& Rodigas) Pfitzer = Paphiopedilum glanduliferum (Blume) Stein var. glanduliferum

Paphiopedilum praestans (Reichb.f.) Pfitz, var. wilhelminae (L. O. Williams) M. W. Wood = Paphiopedilum glanduliferum (Blume) Stein var, withelminae (L. O. Williams) Cribb

Paphiopedilum primulinum M. Wood \& Taylor var. primulinum: I Orchidaceae

Paphiopedilum primulinum M. Wood \& Taylor var. purpurascens (M. Wood) Cribb: I Orchidaceae

Paphiopedilum purpurascens Fowlie = Paphiopedilum javanicum (Reinw. ex Lindl.) Pfitzer var. virens (Reichb.f.) Stein

Paphiopedilum purpuratum (Lindley) Stein: Hong Kong Slipper Orchid (E) 1 Orchidaceac

Paphiopedilum randsii Fowlie: I Orchidaceae

Paphiopedilum reflexum hort. ex Stein = Paphiopedilum callosum (Reichb.f.) Stein var, callosum

Paphiopedilum regnieri hort. ex Stein = Paphiopedilum callosum (Reichb.f.) Stein var, callosum

Paphiopedilum richardianum Asher \& Beaman = Paphiopedilum lowii (Lindl.) Stein var, richardianum (Asher \& Beaman) Gruss

Paphiopedilum robinsonii (Ridley) Ridley = Paphiopedilum bullenianum (Reichb. f.) Pfitz.

Paphiopedilum roebelenii $($ Reichb.f.) Pfitzer = Paphiopedilum philippinense (Reichb.f.) Stein var. roebelenii (Veitch) P. J. Cribb

Paphiopedilum rothschildianum (Reichb. f.) Stein: I Orchidaceae

Paphiopedilum rothschildianum (Reichb.f.) Stein var. elliottianum (O'Brien) Pfitzer = Paphiopedilum rothschildianum (Reichb.f.) Stein

Paphiopedilum sanderianum (Reichb. f.) Stein: I Orchidaceae

Paphiopedilum sangii Braem: I Orchidaceae

Paphiopedilum schoseri Braem \& H. Mohr: I Orchidaceae

Paphiopedilum schlimii (Linden \& Reichb.f.) Stein = Phragmipedium schlimii (Linden \& Reichb.f.) Rolfe

Paphiopedilum sinicum (Hance ex Reichb.f.) Stein = Paphiopedilum purpuratum (Lindl.) Stein

Paphiopedilum spicerianum (Reichb. f.) Pfitzer: I Orchidaceae

Paphiopedilum sriwaniae Koop. = Paphiopedilum argus (Reichb.f.) Stein

Paphiopedilum stonei (Hook. f.) Stein: I Orchidaceae

Paphiopedilum striatum Clements \& Jones: I Orchidaceae

Paphiopedilum sublaeve (Reichb.f.) Fowlie = Paphiopedilum callosum (Reichb.f.) Stein var. sublaeve (Reichb.f.) P. J. Cribb

Paphiopedilum sukhakulii Schoser \& Senghas: I Orchidaceae

Paphiopedilum supardii G. Braem \& Loeb: I Orchidaceae

Paphiopedilum superbiens (Reichb.f.) Stein var. curtisii (Reichb. f.) G. J. Braem I Orchidaceae 
Paphiopedilum superbiens (Reichb.f.) Stein ssp. ciliolare (Reichb.f.) M. W. Wood = Paphiopedilum ciliolare (Reichb.f.) Stein

Paphiopedilum superbiens (Reichb.f.) Stein ssp. superbiens: I Orchidaceae

Paphiopedilum thailandense Fowlie = Paphiopedilum callosum (Reichb.f.) Stein var. sublaeve (Reichb.f.) P. J. Cribb

Paphiopedilum tigrinum Koop. \& N. Haseg.: I Orchidaceae

Paphiopedilum tonsum (Reichb.f.) Stein var. braemii (Mohr) Gruss. I Orchidaceae

Paphiopedilum tonsum (Reichb.f.) Stein var. tonsum: I Orchidaceae

Paphiopedilum topperi Braem \& H. Mohr = Paphiopedilum kolopakingii Fowlie

Paphiopedilum tortipetalum Fowlie = Paphiopedilum bullenianum (Reichb. f.) Pfitz.

Paphiopedilum urbanianum Fowlie: I Orchidaceae

Paphiopedilum venustum (Wallich) Pfitzer ex Stein: I Orchidaceae

Paphiopedilum venustum (Wallich ex Sims) Pfitzer ex Stein var. pardinum (Reichb.f.) Pfitzer = Paphiopedilum venustum (Wallich ex Sims) Pfitzer ex Stein

Paphiopedilum 'victoria' De Vogel = Paphiopedilum supardii Braem \& Loeb

Paphiopedilum victoria-mariae (Sander ex Masters) Rolfe: I Orchidaceae

Paphiopedilum victoria-regina (Sander) M. W. Wood: I Orchidaceae

Paphiopedilum victoria-regina (Sander) M. W. Wood ssp. chamberlainianum (Sander) M. W. Wood = Paphiopedilum victoria-regina (Sander) M. W. Wood

Paphiopedilum victoria-regina (Sander) M. W. Wood ssp glaucophyllum (J. J. Sm.) M. W. Wood = Paphiopedilum glaucophyllum J. J. Smith

Paphiopedilum victoria-regina (Sander) M. W. Wood ssp liemianum (Fowlie) M. W. Wood = Paphiopedilum liemianum (Fowlie) Karasawa \& K. Saito

Paphiopedilum victoria-regina (Sander) M. W. Wood var primulinum (M. W. Wood \& Taylor) M. W. Wood = Paphiopedilum primulinum M. W. Wood \& Taylor var primulinum

Paphiopedilum villosum (Lindley) Stein var, affine (De Wild.) Braem = Paphiopedilum gratrixianum (Masters) Guillaumin

Paphiopedilum villosum (Lindl.) Stein var, annamense Rolfe: I Orchidaceae

Paphiopedilum villosum (Lindl.) Stein var. boxallii (Reichb. f.) Pfitzer: I Orchidaceae

Paphiopedilum villosum (Lindley) Stein var. villosum: I Orchidaceae

Paphiopedilum violascens Schlechter: I Orchidaceae

Paphiopedilum violascens Schlechter var. gauteriense J. J. Sm. = Paphiopedilum violascens Schlechter

Paphiopedilum virens (Reichb.f.) Pfitzer = Paphiopedilum javanicum (Reinw. ex Lindl.) Pfitzer var. virens (Reichb.f.) Stein

Paphiopedilum vittatum (Vell.) Stein = Phragmipedium vittatum (Vell.) Rolfe

Paphiopedilum volonteanum (Sander ex Rolfe) Pfitzer = Paphiopedilum hookerae (Rejchb.f.) Stein var. volonteanum (Sander ex Rolfe) Kerch.

Paphiopedilum wardii Summerh.: I Orchidaceae

Paphiopedilum wentworthianum Schoser \& Fowlie: I Orchidaceae

Paphiopedilum wilhelminae L. O. Williams = Paphiopedilum glanduliferum (Blume) Stein var. wilhelminae (L. O. Williams) Cribb

Paphiopedilum wolterianum (Kraenzl.) Pfitzer = Paphiopedilum appletonianum (Gower) Rolfe

Paphiopedilum zieckianum Schoser = Paphiopedilum papuanum (Ridl.) Ridl

Papilionanthe spp. \#7 II Orchidaceae

Papiliopsis $=$ Oncidium

Papillilabium spp. \#7 II Orchidaceae

Papperitzia spp. \#7 II Orchidaceae

Papuaea spp. \#7 II Orchidaceae

Paracalanthe $=$ Calanthe

Paracaleana spp. \#7 II Orchidaceae

Paradisanthus spp. \#7 II Orchidaceae

Paragnathis $=$ Diplomeris

Parapactis = Epipactis

Paraphalaenopsis spp. \#7 II Orchidaceae

Parapteroceras spp. \#7 II Orchidaceae

Parasarcochilus $=$ Sarcochilus
Parhabenaria spp. \#7 II Orchidaceae

Park Pincushion-cactus (E): Pediocactus paradinei B. W. Benson: I Cactaceae

Parlatore's Podocarp (E): Podocarpus parlatorei Pilg.: I Podocarpaceae

Parlatorea $=$ Sanderella

Parodia spp. \#4 II Cactaceae

Parodia gummifera Backeb. \& Voll = Uebelmannia gummifera (Backeb. \& Voll) Buining

Parrot Pitcherplant (E): Sarracenia psinacina Michx.: \#1 II Sarraceniaceae

Partridge-breasted Aloe (E): Aloe variegata L.: \#1 II Liliaceae

Parviopuntia $=$ Opuntia

Pata de venado (S): Ariocarpus kotschoubeyanus (Lemaire) Schumann: I Cactaceae

Patagonian Cypress (E): Fitzroya cupressoides (Molina) I. M. Johnston: I Cupressaceae

Pattonia $=$ Grammatophyllum

Partonia $=$ Spathoglottis

Pecteilis spp. \#7 II Orchidaceae

Pectinaria $=$ Angraecum

Pedilanthus lycoides Baker $=$ Euphorbia pedilanthoides Denis

Pedilea $=$ Malaxis

Pedilochilus spp. \#7 II Orchidaceae

Pedilonum $=$ Dendrobium

Pediocactus spp. \#4 I/I Cactaceae

Pediocactus bradyi L. Benson: Brady's Pincushion Cactus (E) I Cactaceae

Pediocactus bradyi L. Benson var. knowltonii (L. Benson) Backeb. = Pediocacius knowlionii L. Benson

Pediocactus glaucus (K. Schumann) Arp. = Sclerocactus glaucus (K. Schumann) L. Benson

Pediocactus knowltonii L. Benson: Knowlton's Cactus (E), Knowlton's Miniature Cactus (E), Knowlton's Pincushion Cactus (E) I Cactaceae

Pediocactus mesae-verdae (Boiss. \& Davidson) Arp. = Sclerocactus mesae-verdae (Boiss. \& Davidson) L. Benson

Pediocactus papyracanthus (Engelm.) L. Benson = Sclerocactus papyracanthus (Engelm.) N. P. Taylor

Pediocactus paradinei B. W. Benson: Kaibab Pincushion Cactus (E), Park Pincushion-cactus (E) I Cactaceae

Pediocactus peeblesianus (Croizat) L. Benson var. fickeiseniae L Benson: Fickeisen's Hedgehog-cactus (E) I Cactaceae

Pediocactus peeblesianus (Croizat) L. Benson var. peeblesianus: Peebles's Hedgehog-cactus (E), Peebles's Navajo Cactus (E) I Cactaceae

Pediocactus sileri (Engelm.) L. Benson: Siler's Pincushion Cactus (E) I Cactacese

Pediocactus wrightiae (L. Benson) Arp. = Sclerocactus wrightiae L. Benson

Peebles's Hedgehog-cactus (E): Pediocactus peeblesianus (Croizat) L. Benson var. peeblesianus: I Cactaceae

Peebles's Navajo Cactus (E): Pediocactus peeblesianus (Croizat) L. Benson var. peeblesianus: I Cactaceae

Pehuén (S): Araucaria araucana (Mol.) K. Koch; I/II Araucariaceae

Peirescia $=$ Pereskio

Peireskia $=$ Pereskia

Peireskiopsis $=$ Pereskiopsis

Pelatantheria spp. \#7 II Orchidaceae

Pelecyphora spp. I Cactaceae

Pelecyphora asellifomis Ehrenb.: Harchet Cactus (E), Woodlouse Cactus (E), Cactus hache (F), Cactus cloporté (F), Peotillo (S), Peyote (S), Peyotillo (S) I Cactaceae

Pelecyphora aselliformis Ehrenb. var. cristata Watson = Mammillaria pectinifera (Rümpler) A. Weber

Pelecyphora aselliformis Ehrenb. var. pectinata (B. Stein) Nicholson = Mammillaria pectinifera (Rümpler) A. Weber

Pelecyphora aselliformis Ehrenb. var. pectinifera Rümpler = Mammillaria pectinifera (Rümpler) A. Weber

Pelecyphora pectinata B. Stein = Mammillaria pectinifera (Rümpler) A. Weber

Pelecyphora plumosa Bödeker \& F. Ritter = Turbinicarpus valderianus (Möller) C. Glass \& R. Foster

Pelecyphora pseudopectinata Backeb. = Turbinicarpus pseudopectinatus (Backeb.) C. Glass \& R. Foster 
Pelecyphora strobiliformis (Werderm.) Fric \& Schelle: Pine Cone Cactus (E), Peyote (S) I Cactaceae

Pelecyphora valdeziana Möller = Turbinicarpus valdezianus (Möller) C. Glass \& R. Foster

Pelexia spp. \#7 II Orchidaceae

Pelma $=$ Bulbophyllum

Pelma (S): Cyathea bicrenata Liebm: \#I II Cyatheaceae

Peniocereus spp. \#4 II Cactaceae

Pennilabium spp. \#7 II Orchidaceae

Penthea $=$ Disa

Pentisia = Caladenia

Pentulops = Maxillaria

Peotillo (S): Pelecyphora aselliformis Ehrenb.: I Cactaceae

Peramium $=$ Goodyera

Perescia $=$ Pereskia

Pereskia spp. \#4 II Cactaceae

Pereskiopsis spp. \#4 II Cactaceae

Pergamena $=$ Dactylostalix

Pericopsis elata (Harms) van Meeuwen: African Teak (E), Afromnosia (E), Assamela (E), Kokrodua (E), \#5 II Leguminosae

Peristeranthus spp. \#7 II Orchidaceac

Peristeria spp. \#7 II Orchidaceae

Peristeria elata Hooker: Dove Orchid (E). Holy Ghost Orchid (E), Fleur du Saint-Esprit (F) I Orchidaceae

Peristylus spp. \#7 II Orchidaceae

Perrieriella spp. \#7 II Orchidaceae

Perularia $=$ Platanther

Peruvocereus $=$ Hageocereus

Pescaroria spp. \#7 II Orchidaceae

Pesma (S): Cyathea mexicana Schler. \& Cham.: \#1 II Cyatheaceae

Pesomeria $=$ Phaius

Peralocentrum spp. \#7 II Orchidaceae

Petalochilus $=$ Caladenia

Petrania $=$ Batemannia

Peyote (S): Pelecyphora aselliformis Ehrenb.: I Cactaceae

Peyote (S): Strombocactus disciformis (DC.) Britton \& Rose: I Cactaceae

Peyote (S): Pelecyphora strobilifonnis (Werderm.) Fric \& Schelle: I Cactaceae

Peyotillo (S): Pelecyphora aselliformis Ehrenb.: I Cactaceae

Pezuña de venado (S): Ariocarpus kotschoubeyanus (Lemaire) Schumann: I Cactaceae

Pfeiffera $=$ Lepismium

Phadrosanthus = Epidendrum

Phaius spp. \#7 II Orchidaceae

Phalaenopsis spp. \#7 II Orchidaceae

Phaniasia $=$ Habenaria

Phellosperma = Mammillaria

Philippicereus = Eulychnia

Philippinaea spp. \#7 II Orchidaceae

Philippine Garland Flower (E): Hedychium philippinense K. Schum \#1 II Zingiberaceae

Phlebidia $=$ Disa

Phloeophila $=$ Pleurothallis

Pholidola spp. \#7 II Orchidaceae

Phormangis $=$ Ancistrorhynchus

Phragmipedium spp. I Orchidaceae

Phragmipedium besseae Dodson \& Kuhn.: I Orchidaceae

Phragmipedium boissierianum (Reichb.f.) Rolfe: I Orchidaceae

Phragmipedium cajamarcae Schlechter = Phragmipedium boissierianum (Reichb.f.) Rolfe

Phragmipedium caricinum (Lindley \& Paxton) Rolfe: I Orchidaceae

Phragmipedium caudatum (Lindley) Rolfe: I Orchidaceae

Phragmipedium caudatum (Lindley) Rolfe var. lindenii (Lindl.) Pfitzer = Phragmipedium lindenii (Lindl.) Dressler \& N. H. Williams

Phragmipedium czerwiakowianum (Reichb.f.) Rolfe $=$ Phragmipedium boissierianum (Reichb.f.) Rolfe

Phragmipedium dalessandroi Dodson \& Gruss: I Orchidaceae

Phragmipedium dariense (Reichb.f.) Garay = Phragmipedium longifolium (Reichb.f. \& Warsc.) Rolfe

Phragmipedium ecuadorense Garay = Phragmipedium pearcei (Reichb.f.) Rauh \& Senghas

Phragmipedium exstaminodium Castaño, Hágsater, \& Aguirre: I Orchidaceae
Phragmipedium hartwegii (Reichb.f.) L. O. Williams = Phragmipedium longifolium (Reichb.f. \& Warsc.) Rolfe

Phragmipedium hincksianum (Reichb.f.) Garay = Phragmipedium longifolium (Warsc. \& Reichb.f.) Rolfe

Phragmipedium hirzii Dodson: I Orchidaceae

Phragmipedium kaiete urum (N.E. Br.) Garay = Phragmipedium lindleyanum (Schomb. ex Lindley) Rolfe var. kaieteurum (N. E. Br.) Reichb.f ex Pfitz.

Phragmipedium klotzschianum (Reichb.f.) Rolfe: I Orchidaceae

Phragmipedium lindenii (Lindley) Dressl. \& N. H. Williams: I Orchidaceac

Phragmipedium lindleyanum var. kaieteurum (N. E. Br.) Reichb.f. ex Pfitzer

Phragmipedium lindleyanum (Schomb. ex Lindley) Rolfe var. lindleyanum: I Orchidaceae

Phragmipedium longifolium (Reichb.f. \& Warsc.) Rolfe: I Orchidaceae

Phragmipedium pearcei (Reichb.f.) Rauh \& Senghas: I Orchidaceae

Phragmipedium reticulafum (Reichb.f.) Garay = Phragmipedium boissierianum (Reichb.f.) Rolfe

Phragmipedium roezlii (Reichb.f.) Garay = Phragmipedium longifolium (Reichb.f. \& Warsc.) Rolfe

Phragmipedium sargentianum (Rolfe) Rolfe: I Orchidaceae

Phragmipedium schlimii (Linden \& Reichb.f.) Rolfe: I Orchidaceae

Phragmipedium vittatum (Vell.) Rolfe: I Orchidaceae

Phragmipedium wallisii (Reichb.f.) Garay: I Orchidaceae

Phragmipedium warscewiczianum (Reichb. f.) Garay = Phragmipedium caudarum (Lindley) Rolfe

Phragmipedium xerophyticum Soto Arenas, Salazar \& Hágsater = Mexipedium xerophyticum (Soto Arenas, Salazar \& Hágsater) V. A. Albert \& M. W. Chase

Phragmorchis spp. \#7 II Orchidaceae

Phreatia spp. \#7 II Orchidaceae

Phyllarihus $=$ Opunio

Phyllocactus $=$ Epiphyllum

Phyllocereus $=$ Epiphyllum

Phyllomphax = Brachyconthis

Phyllorkis $=$ Bulbophyllum

Phymatidium spp. \#7 II Orchidaceae

Physanthera $=$ Rodriguezia

Physinga spp. \#7 II Orchidaceae

Physoceras spp. \#7 II Orchidaceae

Physogyne $=$ Pseudogoodyera

Physosiphon $=$ Pleurothallis

Physothallis = Pleurothallis

Physurus = Enthrodes

Picrorhiza kurrooa Royle ex Benth.: Kucki (E) \#3 II Scrophulariaceae

Pierardia $=$ Dendrobium

Pilgerodendron uviferum (Don) Florin: Ciprés de las guaitecas (S) I Cupressaceae

Pilocanthus = Pediocactus

Pilocanthus paradinei (B. W. Benson) B. W. Benson \& Backeb. = Pediocactus paradinei B. Benson

Pilocereus $=$ Pilosocereus

Pilocereus chrysomallus Lemaire = Pachycereus militaris (Audot) D. Hunt

Pilocereus militaris (Audot) Cels. = Pachycereus militaris (Audot) D. Hunt

Pilocereus niger Neumann = Pachycereus militaris $($ Audot) D. Hunt

Pilocopiapoa $=$ Copiapoa

Pilophyllum spp. \#7 II Orchidaceae

Pilopsis $=$ Echinopsis

Pilosacereus spp. \#4 II Cactaceae

Pilumna $=$ Trichopilia

Pinabere (E): Abies guatemalensis Rehder var. guatemalensis: I Pinaceae

Pinalia $=$ Eria

Pin du Chili (F): Araucaria araucana (Mol.) K. Koch: I/I Araucariaceae

Pine Cone Cactus (E): Pelecyphora strobiliformis (Werderm.) Fric \& Schelle: I Cactaceae

Pinelia spp. \#7 II Orchidaceae

Pinelianthe $=$ Pinelia

Pino Araucana (S): Araucaria araucana (Mol.) K. Koch: I/I Araucariaceae 
Pino Chileno (S): Araucaria araucana (Mol.) K. Koch: V/I Araucariaceae

Pinonia $=$ Cibotium

Piperia $=$ Platanthera

Piptanthocereus $=$ Cereus

Pitago (S): Cycas circinalis Roxb.: \#1 II Cycadaceae

Pitcher-plants (E): Nepenthes spp. \#I I/I Nepenthaceae

Pitcher plants (E): Sarracenia spp. \#I I/I Sarraceniaceae

Pittierella $=$ Cryptocentrum

Pityphyllum spp. \#7 II Orchidaceae

Placostigma $=$ Podochilus

Platanthera spp. \#7 II Orchidaceae

Platyclinis $=$ Dendrochilum

Platycoryne spp. \#7 II Orchidaceae

Platyglottis spp. \#7 II Orchidaceae

Platylepis spp. \#7 II Orchidaceae

Platymiscium pleiostachyum Donn. Sm.: Cachimbo (S), Cristóbal (S), Nambar (S) \#1 II Legurninosae

Platyopuntia $=$ Opuntia

Platypus $=$ Eulophic

Platyrhiza spp. \#7 II Orchidaceae

Plarysma $=$ Podochilus

Platystele spp. \#7 II Orchidaceae

Platystylis = Liparis

Plorythelys spp. \#7 II Orchidaceae

Pleated Snowdrop (E): Galanthus plicatus M.Bieb. ssp. plicarus: \#! II Amaryllidaceae

Plectorthiza spp. \#7 II Orchidaceae

Plectrelminthus spp. \#7 II Orchidaceae

Plectrophora spp. \#7 II Orchidaceae

Plecturus = Tipularia

Pleione spp. \#7 II Orchidaceae

Pleuranthium $=$ Epidendrum

Pleurobotryum $=$ Pleurothallis

Pleurorhallis spp. \#7 II Orchidaceae

Pleurothallopsis spp. \#7 II Orchidaceae

Plexaure $=$ Phreatia

Plicate-leaved Snowdrop (E): Galanthus plicarus M. Bieb. ssp. plicasus: \#1 II Amaryllidaceae

Plocoglotis spp. \#7 II Orchidaceae

Plomillo (S): Caryocar costaricense J. D. Sm.: \#1 II Caryocaraceae

Pooephyllum spp. \#7 II Orchidaceae

Podandria = Habenaria

Podangis spp. \#7 II Orchidaceae

Podanthera $=$ Epipogium

Podocarpe d'Argentine (F): Podocarpus parlatorei Pilg.: I Podocarpaceae

Podocarpus neriifolius D. Don var. degeneri N. E. Grey: \#1 III Podocarpaceae

Podocarpus neriifolius D. Don var. neriifolius: \#1 III Podocarpaceae

Podocarpus parlatorei Pilg.: Parlatore's Podocarp (E), Podocarpe d'Argentine (F) I Podocarpaceae

Podochilopsis $=$ Adenoncos

Podochilus spp. \#7 II Orchidaceae

Podofilo de Himalayo (S): Podophyllum hexandrum Royle \#2 II Berberidaceae

Podophyllum hexandrum Royle: Himalayan May-apple (E), Podofilo de Himalayo (S) \#2 II Berberidaceae

Pogochilus $=$ Galeola

Pogonia spp. \#7 11 Orchidaceae

Pogoniopsis spp. \$7 II Orchidaceae

Poinsettia pedunculata Klotzsch $=$ Euphorbia strigosa Hooker \& Amott

Poinsettia radians $($ Bentham) Klotzsch \& Garcke $=$ Euphorbia radians Bentham

Poinsettia strigosa (Hooker \& Amott) Arthur = Euphorbia strigosa Hooker \& Amott

Polaskia spp. \#4 II Cactaceae

Pollinirhiza $=$ Listera

Polybactrum $=$ Pseudorchis

Polychilos $=$ Phalaenopsis

Polycycnis spp. \#7 II Orchidaceae

Polyotidium spp. \#7 II Orchidaceae

Polyradicion spp. \#7 II Orchidaceae

Polyrrhiza $=$ Polyradicion

Polystachya spp. \#7 II Orchidaceae
Polystylus = Phalaenopsis

Polytoma $=$ Bletilla

Pomatocalpa spp. \#7 II Orchidaceae

Ponera spp. \#7 II Orchidaceae

Ponerorchis spp. \#7 II Orchidaceae

Ponthieva spp. \#7 II Orchidaceae

Porfiria = Mammillaria

Porolabium spp. \#7 II Orchidacese

Porpax spp. \#7 II Orchidaceae

Porphyrodesme spp. \#7 II Orchidaceae

Porphyroglonis spp. \#7 II Orchidaceae

Porphyrostachys spp. \#7 II Orchidaceae

Porroglossum spp. \#7 II Orchidaceae

Porrorhachis spp. \#7 II Orchidaceae

Portillia spp. \$7 II Orchidaceae

Pourpiers (F): Anacampseros spp. \#1 II Portulacaceae

Praecereus spp. \#4 II Cactaceae

Prasophyllum spp. \#7 II Orchidaceae

Preptanthe $=$ Calanthe

Prescortia spp. \#7 II Orchidaceae

Prickly Tree Fern (E): Cyashea leichardiana (F. Muell.) Copel.: \# 1

II Cyatheaceae

Prisriglonis spp. \#7 II Orchidaceae

Promenaea spp. \#7 II Orchidaceae

Prosthechea $=$ Epidendrum

Protea odorata Thunb.: Protée odorante (F) \#1 II Proteaceae

Protée odorante (F): Protea odorata Thunb.: \#1 II Proteaceae

Proteroceras $=$ Pleroceras

Prunus africana (Hook. f.) Kalkm.: African Cherry (E), Red

Stinkwood (E) \#1 II Rosaceae

Pseudacoridium spp. \#7 II Orchidaceae

Pseudelleanthus $=$ Elleanthus

Pseudepidendrum $=$ Epidendrum

Pseuderia spp. \#7 II Orchidaceae

Pseuderiopsis $=$ Eriopsis

Pseudoacanthocereus spp. \#4 II Cactaceae

Pseudocentrum spp. \#7 II Orchidaceae

Pseudocranichis spp. \#7 II Orchidaceae

Pseudoctomeria $=$ Pleurothallis

Pseudodiphryllum = Platanthera

Pseudoechinocereus $=$ Cleistocactus

Pseudoeria $=$ Pseuderia

Pseudoesposiva $=$ Espostoa

Pseudoeunstyles $=$ Eurystyles

Pseudogoodyera spp. \#7 II Orchidaceae

Pseudohexadesmia $=$ Hexadesmia

Pseudolaelia spp. \#7 II Orchidaceae

Pseudoliparis $=$ Malaxis

Pseudolobivia $=$ Echinopsis

Pseudomacodes $=$ Macodes

Pseudomammillaria $=$ Mammillaria

Pseudomaxillaria spp. \#7 II Orchidaceae

Pseudomitrocereus $=$ Pachycereus

Pseudonopalxochia $=$ Disocactus

Pseudoorleanesia $=$ Orleanesia

Pseudopilocereus $=$ Pilosocereus

Pseudoponera $=$ Ponera

Pseudorchis spp. \#7 II Orchidaceae

Pseudorhipsalis spp. \#4 II Cactaceae

Pseudorleanesia $=$ Orleanesia

Pseudosolisia $=$ Neolloydia

Pseudostelis $=$ Pleurothallis

Pseudotephrocactus $=$ Opuntia

Pseudovanilla spp. \#7 11 Orchidaceae

Pseudozygocactus = Hatiora

Psilanthemum $=$ Epidendrum

Psilochilus spp. \#7 II Orchidaceae

Psittacoglossum $=$ Maxillaria

Psychechilos = Zeuxine

Psychilis spp. \#7 II Orchidaceze

Psychopsiella spp. \#7 II Orchidaceae

Psychopsis $=$ Oncidium

Psygmorchis spp. \#7 II Orchidacese

Pterichis spp. \#7 II Orchidaceae

Pserocacius spp. \#4 II Cactaceae 
Prerocarpus santalinus Linn.f.: Algum (E), Red Sandalwood (E), Saunderswood (E), Algum (S) \#6 II Leguminosae

Pieroceras spp. \#7 II Orchidaceae

Pierocereus $=$ Pachycereus

Prerochilus $=$ Maxillaria

Preroglossa $=$ Stenorrhynchos

Preroglossaspis spp. \#7 II Orchidaceae

Plerostemma spp. \#7 II Orchidaceae

Pterostylis spp. \#7 II Orchidaceae

Plengodium spp. \#7 II Orchidaceae

Ptilocnema $=$ Pholidota

Prychochilus $=$ Tropidia

Ptychogyne $=$ Coelogyne

Puna = Opuntia

Purdy's Lewisia (E): Lewisia cotyledon (S. Watson) Robinson var. purdyi Jepson: \#I II Portulacaceae

Purple Trumpet-leaf (E): Sarracenia leucophylla Raf.: \#1 II Sarraceniaceae

Pygmaeocereus spp. \#4 II Cactaceae

Pygmaeorchis spp. \#7 II Orchidaceae

Pyrorchis spp. \#7 II Orchidaceae

Pyrrhocactus = Eriosyce

Queen Agave (E): Agave victoriae-reginae T. Moore: \#I II Agavaceac

Quekentia spp. \#7 II Orchidaceae

Queteletia $=$ Orchipedum

Quiabentia spp. \#4 II Cactaceae

Quisqueya spp. \#7 II Orchidaceae

Rabo de mico (S): Cyathea mexicana Schltr. \& Cham.: \#1 II Cyatheaceae

Radinocion $=$ Aerangis

Raffles's Pitcher-plant (E): Nepenthes rafflesiona Jack: \#1 II Nepenthaceae

Rainbow Fen (E): Calochlaena dubia (R. Br.) M. D. Tumer \& R. A. White: \#I II Dicksoniaceae

Rainbow Plant (E): Byblis gigantea J. Lindley: \#1 II Byblidaceae

Rainbow Trumpet (E): Sarracenia minor Walter: \#1 II Sarraceniaceae

Ramonia $=$ Scaphyglottis

Ramphidia $=$ Myrmechis

Rangaeris spp. \#7 II Orchidaceae

Rapicactus $=$ Turbinicarpus

Rapicactus mandragora (Fric ex Berger) F. Buxb. = Turbinicarpus mandragora (Fric ex Berger) A. Zimmerman

Rapicactus subterraneus (Backeb.) F. Buxb. = Turbinicarpus subrerraneus (Backeb.) A. Zimmerman

Rat Aloe (E): Aloe ballyi G. Reyn.: \# I II Aloaceae

Rathbunia $=$ Stenocereus

Rauhiella spp. \#7 II Orchidaceae

Rauhocereus spp. \#4 11 Cactaceac

Rauvolfia serpentina Benth. ex Kurz: \#2 II Apocynaceae

Raycadenco spp. \#7 II Orchidaceae

Rebulobivia $=$ Echinopsis

Rebutia spp. \#4 II Cactaceae

Red Sandalwood (E): Pterocarpus santalinus Linn.f.: \#1 II Leguminosae

Red Stinkwood (E): Prunus africana (Hook. f.) Kalkm.: \#1 II Rosaceae

Red Vanda Orchid (E): Renanthera imschooriana Rolfe: I Orchidaceae

Red-flowered Sweet Pitcherplant (E): Sarracenia rubra Walter ssp. rubra: \#1 II Sarraceniaceae

Regnellia $=$ Bletia

Reichenbachanthus spp. \#7 II Orchidaceae

Reicheocactus $=$ Echinopsis

Renanthera spp. \#7 1/II Orchidaceae

Renanthera imschootiana Rolfe: Red Vanda Orchid (E) I Orchidaceae

Renantherella spp. \#7 II Orchidaceae

Renata spp. \#7 II Orchidaceae

Repandra $=$ Disa

Restrepia spp. \#7 II Orchidaceae

Restrepiella spp. \#7 II Orchidaceae

Restrepiopsis spp. $\$ 7$ II Orchidaceae

Reymondia $=$ Myoxanthus

Rhaesteria spp. \#7 11 Orchidaceae
Rhamphidia $=$ Hetaeria

Rhamphorhynchus spp. \#7 II Orchidaceae

Rhaphidorhynchus $=$ Microcoelia

Rhinerrhiza spp. \#7 II Orchidaceae

Rhipidoglossum = Diaphananihe

Rhipsalidopsis $=$ Hatiora

Rhipsalis spp. \#4 II Cactaceae

Rhizanthella spp. \#7 II Orchidaceae

Rhizocorallon $=$ Corallorrhiza

Rhodocactus $=$ Pereskia

Rhomboda $=$ Hetaeria

Rhophostemon $=$ Nervilia/Pogonia

Rhynchadenia $=$ Macradenia

Rhynchandra $=$ Corymborkis

Rhynchanthera $=$ Corymborkis

Rhynchogyna spp. \#7 11 Orchidaceae

Rhyncholaelia spp. \#7 II Orchidaceae

Rhynchopera $=$ Pleurothallis

Rhynchophreatia spp. \#7 II Orchidaceae

Rhynchostele $=$ Leochilus

Rhynchostylis spp. \#7 II Orchidaceae

Ridleya $=$ Thrixspermum

Ridleyella spp. \#7 II Orchidaceae

Rimacola spp. \#7 II Orchidaceae

Rio Rosewood (E): Dalbergia nigra Allem. ex Benth.: I Leguminosae

Risleya spp. \#7 II Orchidaceae

Ritaia $=$ Ceratostylis

Ritterocereus $=$ Stenocereus

Robiquetia spp. \#7 II Orchidaceae

Rodentiophila $=$ Eriosyce

Rodrigoa = Masdevallia

Rodriguezia spp. \#7 II Orchidaceae

Rodrigueziella spp. \#7 II Orchidaceae

Rodrigueziopsis spp. \#7 II Orchidaceae

Rveperocharis spp. \#7 II Orchidaceae

Roezliella spp. \#7 II Orchidaceae

Rolfea $=$ Palmorchis

Rolfeella $=$ Benthamia

Rooksbya $=$ Neobuxbaumia

Rophostemon $=$ Nervilia

Roptrostemon $=$ Nervilia

Roseia $=$ Conphantha

Roseocactus = Ariocarpus

Roseocactus fissuratus (Engelmann) Berg. = Ariocarpus fissuratus (Engelmann) K. Schumann var. fissuratus

Roseocactus intermedius Backeb. \& Kilian = Ariocarpus fissuratus (Engelmann) K. Schumann var. fissuratus

Roseocacius kotschoubeyanus (Lemaire) A. Berger $=$ Ariocarpus kotschoubeyanus (Lemaire) K. Schumann

Roseocactus lloydii (Rose) A. Berger = Ariocarpus fissuratus

(Engelmann) K. Schumann var. fissuratus

Roseocereus $=$ Harrisia

Rossioglossum spp. \#7 II Orchidaceae

Rough Tree Fern (E): Dicksonia squarrosa (G. Forster) O. Swartz: \#1 II Dicksoniaceae

Rudolfiella spp. \#7 II Orchidaceae

Rusbyella spp. \#7 II Orchidaceae

Saccidium $=$ Holothrix

Saccochilus $=$ Thrixspermum

Saccoglossum spp. \#7 II Orchidaceae

Saccolabiopsis spp. \#7 II Orchidaceae

Saccolabium spp. \#7 II Orchidaceae

Sacodon $=$ Cypripedium

Sacoila $=$ Spiranthes

Sago-palm (E): Cycas circinalis Roxb.: \#1 II Cycadaceae

Salacistis $=$ Goodyera

Salmiopuntia $=$ Opuntia

Salpingolobivia $=$ Echinopsis

Salpistele spp. \#7 II Orchidaceae

Samaipaticereus spp. \#4 U Cactaceae

San Diego Bear-grass (E): Nolina interrata Gentry: I Agavaceae

San Rafael Cactus (E): Pediocaclus despainii Welsh \& Goodrich: I Cactaceae

Sanderella spp. \#7 II Orchidaceae 
Santa Barbara Island Dudleya (E): Dudleya traskiae (Rose) Moran: I Crassulaceae

Santa Barbara Island Liveforever (E): Dudleya traskiae (Rose) Moran: I Crassulaceae

Sapin du Guatemala (F): Abies guatemalensis Rehder var. guatemalensis I Pinaceae

Sarcanthopsis spp. \#7 II Orchidaceae

Sarcanthus $=$ Cleisostoma

Sarcobodium $=$ Bulbophyllum

Sarcochilus spp. \#7 II Orchidaceae

Sarcoglossum $=$ Cirrhaea

Sarcoglottis spp. \#7 II Orchidaceae

Sarcoglyphis spp. \#7 II Orchidaceae

Sarcophyton spp. \#7 II Orchidaceae

Sarcopodium $=$ Epigeneium

Sarcorhynchus = Diaphananthe

Sarcostoma spp. \#7 II Orchidaceac

Sarmenticola spp. \#7 II Orchidaceae

Sarothrochilus $=$ Staurochilus

Sarracenella $=$ Pleurothallis

Sarracenia spp.: Pitcher plants (E), Jarras (S), Cántaros (S), Sarracénies (F) \#1 I/I Sarraceniaceae

Sarracenia alabamensis Case \& Case ssp. alabamensis: Alabama Canebrake Pitcherplant (E) I Sarraceniaceae

Sarracenia alabamensis Case \& Case ssp. wherryi Case \& Case Wherry's Pitcher-plant (E) II Sarraceniaceae

Sarracenia alata (A. W. Wood) Wood: Pale Pitcher-plant (E), Winged Pitcher-plant (E), Yellow Pitcher-plant (E) \#1 II Sarraceniaceae

Sarracenia drummondii Croom = Sarracenia leucophylla Raf

Sarracenia flava L.: Yellow Pitcher-plant (E) \#1 II Sarraceniaceae Sarracenia jonesii Wherry I Sarraceniaceae

Sarracenia leucophylla Raf.: Crimson Pitcherplant (E), Purple Trumpet-leaf (E), White-topped Pitcherplant (E) \#1 II Sarraceniaceae

Sarracenia minor Walter: Hooded Pitcher-plant (E), Rainuow Trumpet (E) \#1 II Sarraceniaceae

Sarracenia oreophila (Keamey) Wherry: Green Pitcherplant (E) I Sarraceniaceae

Sarracenia psittacina Michx.: Parrot Pitcherplant (E) \#l II Sarraceniaceae

Sarracenia purpurea L.: \#1 II Sarraceniaceae

Sarracenia purpurea L. ssp. gibbosa (Raf.) Wherry = Sarracenia purpurea L. ssp. purpurea

Sarracenia purpurea (Eat.) Torr. ssp. heterophylla = Sarracenic purpurea L. ssp. purpurea forma heterophylla

Sarracenia purpurea L. ssp. purpurea: Huntsman's Cap (E), Huntsman's Horn (E), Side-sadle Plant (E) \#1 II Sarraceniaceae

Sarracenia purpurea L. ssp. purpurea forma heterophylla: \#I II Sarraceniaceae

Sarracenia purpurea L. ssp. venosa?: \#1 II Sarraceniaceae

Sarracenia rubra Walter ssp. alabamensis (F. W. \& R. B. Case) Schnell: Alabama Canebrake Pitcherplant (E), Canebrake Pitcherplant (E) I Sarraceniaceae

Sarracenia rubra Walter ssp. gulfensis Schnell: \#1 II Sarraceniaceae

Sarracenia rubra Walter ssp. jonesii (Wherry) Wherry: = Sarracenia jonesii Wherry

Sarracenia rubra Walter ssp. rubra: Red-flowered Sweet Pitcherplant (E) \#l II Sarraceniaceae

Sarracenia rubra Walter ssp. wherryi (F. W. \& R. B. Case) Schnell = Sarracenia alabamensis Case \& Case ssp. wherryi Case \& Case

Sarracenia rugelii Shutlew. ex A. DC $=$ Sarracenia flava L

Sarracenia sledge Macfarlane = Sarracenia alata Wood

Sarracénies (F): Sarracenia spp. \#1 I/I Sarraceniaceae

Satorkis $=$ Coeloglossum

Satyridium spp. \$7 II Orchidaceae

Satyrium spp. \#7 II Orchidaceae

Saundersia spp. \#7 II Orchidaceae

Saunderswood (E): Pterocarpus santalinus Linn.f.: \#1 II Leguminosae

Sauroglossum spp. \#7 II Orchidaceae

Saussurea costus (Falc.) Lipsch.: Costus Root (E), Kuth (E), Aucklande (F), Pachak (F) I Compositae

Saussurea lappa $=$ Saussurea costus (Falc.) Lipsch.

Saw-toothed Lewisia (E): Lewisia serrata Heckard \& Stebbins: \#I II Portulacaceae
Sayeria $=$ Dendrobium

Scaphosepalum spp. \#7 II Orchidaceae

Scaphyglotris spp. \#7 II Orchidaceae

Scaredederis $=$ Dendrobium

Scelochiloides spp. \#7 II Orchidaceae

Scelochilus spp. \#7 II Orchidaceae

Schaenomorphus $=$ Tropidia

Schidorhynchos spp. \#7 11 Orchidaceae

Schiedeella spp. \#7 II Orchidaceae

Schismoceras $=$ Dendrobium

Schistotylus spp. \#7 II Orchidaceae

Schizocaena $=$ Cyathea

Schizochilus spp. \#7 II Orchidaceae

Schizodium spp. \#7 II Orchidaceae

Schizopedium $=$ Cypripedium

Schlechterella $=$ Rudolfiella

Schlimmia spp. \#7 II Orchidaceae

Schlumbergera spp. \#4 II Cactaceae

Schoenleinia $=$ Ponthieva

Schoenorchis spp. \#7 Il Orchidaceze

Schomburgkia spp. \#7 II Orchidaceae

Schunkea spp. \#7 II Orchidaceae

Schwartzkopffia spp. \#7 II Orchidaceae

Sclerocactus spp. \#4 III Cactaceae

Sclerocactus brevihamatus (Englemann) D. Hunt ssp. tobuschii (Mrsh.) Tayl. Tobusch's Fishhook Cactus (E): I Cactaceae

Sclerocactus brevispinus Heil. \& Port. = Sclerocactus glaucus (J. A. Purpus ex K. Schum.) L. Benson

Sclerocactus erectocentrus (J. Coulter) N. P. Taylor var. acunensis (W. T. Marsh) H. Bravo: Acuña Cactus (E) I Cactaceae

Sclerocactus erectocentrus (J. Coulter) N. P. Taylor var. erectocentrus: Needle-spined Pineapple Cactus (E) I Cactaceae

Sclerocacius franklinii J. W. Evans = Sclerocacrus glaucus $(\mathrm{K}$. Schumann) L. Benson

Sclerocactus glaucus (J. A. Purpus ex K. Schum.) L. Benson: Uinta Basin Hookless Cactus (E) I Cactaceae

Sclerocactus mariposensis (Hester) N. P. Taylor: Lloyd's Mariposa Cactus (E) I Cactaceae

Scleracactus mesae-verdae (Boissevain ex Boissevain \& C. Davids.) L. Benson: Mesa-verde Cactus (E) I Cactaceae

Sclerocactus papyracanthus (Engelm.) N. P. Taylor: Paper-spine Pincushion Cactus (E) I Cactaceae

Sclerocactus pubispinus (Engelm.) L. Benson var, pubispinus: Great Basin Fishhook Cactus (E) I Cactaceae

Sclerocactus pubispinus (Engelm.) L. Benson var. spinosior (Boiss.) Welsh: I Cactaceae

Sclerocactus wetlandicus Hoch. $=$ Sclerocactus glaucus $(\mathrm{K}$. Schumarn) L. Benson

Sclerocactus wetlandicus Hoch. ssp. ilseae (Hoch.) Hoch. = Sclerocactus glaucus (K. Schumann) L. Benson

Sclerocactus wrightiae L. Benson: Wright's Fishhook Cactus (E) I Cactaceae

Scleropterys $=$ Cirrhaea

Scoliochilus = Appendicula

Scoparebutia $=$ Echinopsis

Scopularia $=$ Holothrix

Scuticaria spp. \#7 II Orchidaceae

Sea Urchin Cactus (E): Astrophytum asterias (Zucc.) Lem.: I Cactaceae

Sedirea spp. \#7 II Orchidaceae

Seidenfadenia spp. \#7 II Orchidaceae

Selenicereus spp. \#4 II Cactaceae

Selenipedium spp. \#7 II Orchidaceae

Selenipedium boissierianum Reichb.f. $=$ Phragmipedium boissierianum (Reichb.f.) Rolfe

Selenipedium caricinum (Lindl. \& Paxton) Reichb.f. = Phragmipedium caricinum (Lindl. \& Paxton) Rolfe

Selenipedium caudatum (Lindl.) Reichb.f. = Phragmipedium caudatum (Lindl.) Rolfe

Selenipedium caudatum (Lindl.) Reichb.f. var. lindenii (Lindl.) Pucci = Phragmipedium lindenii (Lindl.) Dressler \& N. H. Williams

Selenipedium caudatum (Lindl.) Reichb.f. var. uropedium (Lindl.) Rolfe $=$ Phragmipedium lindenii $($ Lindl.) Dressler \& N. H. Williams

Selenipedium dariense Reichb.f. $=$ Phragmipedium longifolium (Warsz. \& Reichb.f.) Rolfe 
Selenipedium duboissierianum hort. $=$ Phragmipedium boissierianum (Reichb.f.) Rolfe

Selenipedium elliottianum (O'Brien) Gower $=$ Paphiopedilum rothschildianum (Reichb.f.) Stein

Selenipedium kaieteurum N. E. Brown = Phragmipedium lindleyanum (Lindl.) Rolfe var, kaieteurum (N. E. Brown) Reichb.f. ex Pfitzer

Selenipedium klotzschianum (Reichb.f. ex M. R. Schomb.) ? = Phragmipedium klotzschianum (Reichb.f. ex M. R.Schomb.) Rolfe

Selenipedium laevigatum (Bateman) May = Paphiopedilum philippinense (Reichb.f.) Stein var. philippinense

Selenipedium lindenii (Lindl.) Nichols = Phragmipedium lindenii (Lindi.) Dressler \& N. H. Williams

Selenipedium lindleyanum (M. R. Schomb. ex Lindl.) Reichb.f. = Phragmipedium lindleyanum (M. R. Schomb. ex Lindl.) Rolfe

Selenipedium lindleyanum (M. R. Schomb. ex Lindl.) Reichb.f. var. kaieteurum (N. E. Brown) Cogn. = Phragmipedium lindleyanum (Lindl.) Rolfe var. kaieteurum (N. E. Brown) Reichb.f. ex Pfitzer

Selenipedium longifolium Warsz. \& Reichb.f. $=$ Phragmipedium longifolium (Warsz. \& Reichb.f.) Rolfe

Selenipedium parishii (Reichb.f.) Andre = Paphiopedilum parishi (Reichb.f.) Stein

Selenipedium paulisianum $($ Barb. Rodr. $)$ Rolfe $=$ Phragmipedium vinatum (Vell.) Rolfe

Selenipedium pearcei Reichb. $\mathrm{f}_{\mathrm{.}}=$ Phragmipedium pearce (Reichb.f.) Rauh \& Senghas

Selenipedium reichenbachii Endres $=$ Phragmipedium longifolium (Warsz. \& Reichb.f.) Rolfe

Selenipedium sargentianum Rolfe $=$ Phragmipedium sargentianum (Rolfe) Rolfe

Selenipedium schlimii Linden \& Reichb.f. = Phragmipedium schlimii (Linden \& Reichb.f.) Rolfe

Selenipedium schomburgkianum (KJotzsch) Desbois = Phragmipedium klotzschianum (Reichb.f. ex M. R. Schomb.) Rolfe

Selenipedium vittatum (Vell.) Desbois $=$ Phragmipedium vittatum (Vell.) Rolfe

Selenipedium wallisii Reichb.f. $=$ Phragmipedium wallisii (Reichb.f.) Garay

Semiphajus = Eulophio

Sepalosaccus spp. \#7 II Orchidaceac

Sepalosiphon spp. \#7 II Orchidaceae

Seraphyta $=$ Epidendrum

Serapias spp. \#7 II Orchidaceae

Serapiastrum $=$ Serapias

Sericocactus $=$ Parodia

Serrastylis $=$ Macradenia

Serifera $=$ Elleanthus

Sessilibulbum $=$ Scaphyglotis

Sestochilos $=$ Bulbophyllum

Seticereus $=$ Cleistocactus

Seticleistocactus $=$ Cleistocactus

Setiechinopsis $=$ Echinopsis

Setirebutia $=$ Rebutia

Short-styled Oconee-bells (E): Shortia galacifolia Torr. \& Gray var. brevistyla Davies: \#1 II Diapensiaceae

Shortia galacifolia Torr. \& Gray var. brevistyla Davies: Short-styled Oconee-bells (E) \#1 II Diapensiaceae

Shonia galacifolia Torr. \& Gray var. galacifolia: \#1 II Diapensiaceae

Siagonanthus $=$ Maxillaria

Siccohaccarus $=$ Micranthocereus

Side-sadle Plant (E): Sarracenia purpurea L. ssp. purpurea: \#1 II Sarraceniaceae

Sieberia $=$ Platanthera

Sievekingia spp. \#7 II Orchidaceae

Sigmatochilus $=$ Chelonistele

Sigmatogyne $=$ Panisea

Sigmatostalix spp. \#7 II Orchidaceac

Siler's Pincushion Cactus (E): Pediocactus sileri (Engelm.) L Benson: I Cactaceae

Silver Tree Fem (E): Cyathea dealbata (Forst.) Sw.: \#1 II Cyatheaceae

Silvorchis spp. \#7 II Orchidaceae
Sinopodophyllum hexandrum (Royle) Ying = Podophyllum hexandrum Royle

Sinorchis spp. \#7 II Orchidaceae

Sirhookera spp. \#7 II Orchidaceae

Skeptrostachys $=$ Stenorrhynchos

Skirted Tree Fem (E): Cyathea marcescens N. A. Wakefield: \#1 II Cyatheaceae

Slender Pitcher-plant (E): Nepenthes gracilis Korth.: \#1 II Nepenthaceae

Slender Stembergia (E): Stembergia colchiciflora Waldst. \& Kit.: \#1 II Amaryllidaceae

Slender Tree Fem (E): Cyathea cunninghamii Hook.f.: \#1 II Cyatheaceae

Small Flower Agave (E): Agave parviflora Torr. ssp. parviflora: I Agavaceae

Smallia $=$ Pteroglossaspis

Smithorchis spp. \#7 II Orchidaceae

Smithsonia spp. \#7 II Orchidaceae

Smitinandia spp. \#7 II Orchidaceae

Sneed's Cory Cactus (E): Escobaria sneedii Britt. \& Rose var sneedii: 1 Cactaceae

Sneed's Pincushion Cactus (E): Escobaria sneedii Britt. \& Rose var. sneedii: I Cactaceae

Sobennikoffia spp. \#7 II Orchidaceae

Sobralia spp. \#7 II Orchidaceae

Sodiroella $=$ Stellilabium

Soehrensia $=$ Echinopsis

Soehrensis $=$ Echinopsis

Soft Tree Fem (E): Dicksonia antarctica Labill.: \#1 II Dicksoniaceae

Soft Tree Fern (E): Cyathea smithii Hook.f.: \#I II Cyatheaceae

Solenangis spp. \#7 II Orchidaceae

Solenidiopsis spp. \#7 II Orchidaceae

Solenidium spp. \#7 II Orchidaceae

Solenocentrum spp. \#7 II Orchidaceae

Solisia $=$ Mammillaria

Sophronia $=$ Sophronitis

Sophronitella spp. \#7 II Orchidaceae

Sophronitis spp. \#7 II Orchidaceae

Soterosanthus spp. \#7 II Orchidaceae

Southem Byblis (E): Byblis gigantea J. Lindley: \#1 II Byblidaceae

Sowbread (E): Cyclamen hederifolium Aiton ssp. hederifolium forma hederifolium: \#1 II Primulaceae

Spathiger $=$ Epidendrum

Spathoglottis spp. \#7 II Orchidaceae

Specklinia $=$ Pleurothallis

Spegazzinia $=$ Rebutio

Sphaeropteris $=$ Cyathea

Sphaeropteris albosetacea (Bedd.) Tryon = Cyathea albosetacea (Beddome) Copel

Sphaeropteris brunei (Christ) Tryon: \#I II Cyatheaceae

Sphaeropteris crinita $($ Hook.) Tryon = Cyathea crinita $($ Hook.) Copel.

Sphaeropteris elongata $($ Hook.) Tryon = Cyathea poeppigii (Hook.) Domin

Sphaeropteris horrida (Liebm.) Tryon: \#1 II Cyatheaceae

Sphaeropteris insignis (D, C. Eaton) Tryon = Cyathea insignis D. Eaton

Sphaeropteris lepifera (Hook.) Tryon = Cyathea lepifera (J. Smith) Copel.

Sphyrarhynchus spp. \#7 II Orchidaceae

Sphyrastylis spp. \#7 II Orchidaceae

Spiculaea spp. \#7 II Orchidaceae

Spinicalycium $=$ Echinopsis

Spiral Aloe (E): Aloe polyphylla Schönland ex Pillans: I Liliaceae

Spiranthes spp. \#7 II Orchidaceae

Spongiola spp. \#7 II Orchidaceae

Spurges (E): Euphorbia spp. I/IINC Euphorbiaceae

Stachyanthus $=$ Bulbophyllum

Stachyobium $=$ Dendrobium

Stalkya spp. \#7 II Orchidaceae

Stangeria eriopus (Kunze) Baill.: Hottentot's Head (E) I Stangeriaceae

Stangeria paradoxa $\mathbf{T}$. Moore $=$ Stangeria eriopus $($ Kunze) Baillon

Stanhopea spp. \#7 II Orchidaceae

Stanhopeastrum $=$ Stanhopea

Star Cactus (E): Astrophytum asterias (Zucc.) Lem.: I Cactaceae 
Star Cactus (E): Echinocactus asterias Zucc. I Cactaceae Stauranthus = Trichoglottis

Staurites $=$ Phalaenopsis

Staurochilus spp. \#7 II Orchidaceae

Stauroglottis $=$ Phalaenopsis

Stauropsis $=$ Trichoglottis

Steliopsis $=$ Stelis

Stelis spp. \#7 II Orchidaceae

Stellilabium spp. \#7 II Orchidaceae

Siellorchis $=$ Nervilia

Stellorkis $=$ Nervilia

Stenia spp. \#7 II Orchidaceae

Stenocactus spp. \#4 II Cactaceac

Stenocereus spp. \#4 II Cactaceae

Stenoconne $=$ Bifrenaria

Stenoglossum $=$ Epidendrum

Stenoglottis spp. \#7 II Orchidaceae

Stenopolen $=$ Stenia

Stenoptera spp. \#7 II Orchidaceae

Stenorrhynchos spp. \#7 II Orchidaceae

Stephanocereus spp. \#4 II Cactaceae

Stephanothelys spp. \#7 II Orchidaceae

Stereochilus = Cleisostoma/Sarcochilus

Stereosandra spp. \#7 II Orchidaceae

Sterigmanthe bojeri (Hooker) Klotzsch \& Garcke = Euphorbia milii Des Moulins var. milii

Siengmanthe splendens (Bojer ex Hooker) Klotzsch \& Garcke = Euphorbia milii Des Moulins var. splendens (Bojer ex Hooker) Ursch \& Léandri

Stembergia spp. \#1 II Amaryllidaceae

Stembergia aemensis (Raf.) Guss, = Stembergia colchiciflora (Waldst. \& Kit.) Herb.

Stermbergia alexandrae Sosn. = Stembergia colchiciflora Waldst. \& Kit.

Stembergia aurantiaca Dinsm. = Stembergia lutea (L.) Ker Gawl. ex Spreng.

Siembergia candida B. Mathew \& T. Baytop: \#1 II Amaryllidaceae

Stembergia citrina (Sibth. \& Sm.) Schult. \& Schult.f. = Stembergia colchiciflora (Waldst. \& Kit.) Herb

Stermbergia clusiana (Ker-Gawl.) Ker-Gawl. ex Spreng: \#1 II Amaryllidaceac

Stembergia colchiciflora Waldst. \& Kit.: Slender Stembergia (E), Sternbergie à fleurs de colchique (F) \#1 II Amaryllidaceae

Stembergia colchiciflora Waldst. \& Kit. var, aemensis (Raf.) Rouy = Stermbergia colchiciflora (Waldst. \& Kit.) Herb.

Stembergia colchiciflora Waldst. \& Kit. var. alexandrae (Sosn.) Artjush. = Siembergia colchiciflora (Waldst. \& Kit.) Herb.

Stermbergia colchiciflora Waldst, \& Kit. var, dalmatica Herb. = Sternbergia colchiciflora (Waldst. \& Kit.) Herb.

Stermbergia dalmatica (Herb.) Herb. = Stembergia colchiciflora (Waldst. \& Kit.) Herb.

Stembergia exscapa Tineo ex Guss. = Stembergia colchiciflora (Waldst. \& Kit.) Herb.

Stembergia fischeriana (Herb.) Rupr. = Siembergia fischeriana (Herb.) M. Roem.

Sternbergia fischeriana (Herb.) M. Roemer: \#1 II Amaryllidaceae

Stermbergia fischeriana (Herb.) M. Roem. forma hissarica Kapinos = Stembergia fischeriana (Herb.) M. Roem.

Siermbergia fischeriana (Herb.) M. Roem. ssp. hissarica (Kapinos) Arjush. = Stembergia fischeriana (Herb.) M. Roem.

Stermbergia grandiflora Boiss. ex Baker = Stembergia clusiana (Ker Gawl.) Ker Gawl. ex Spreng.

Stembergia greutenaria Kamari \& R. Arteleri: \#1 II Amaryllidaceae

Stembergia latifolia Boiss. H. Hauskn. ex Baker = Siermbergia clusiana (Ker Gawl.) Ker Gawl. ex Spreng.

Stermbergia lutea Oph. = Stembergia colchiciflora (Waldst. \& Kit.) Herb.

Sternbergia lutea Ker Gawl. ex Schult. \& Schult.f. = Stembergia lutea (L.) Ker Gawl. ex Spreng.

Stermbergia lutea (L.) Ker Gawl. ex Spreng.: Common Stembergia (E), Winter Daffodil (E) \#1 I Amaryllidaceae

Stembergia lutea (L.) Ker Gawl. ex Spreng. ssp. sicula (Tineo ex Guss.) D.A. Webb = Stembergia sicula Tineo ex Guss.

Stembergia lutea (L.) Ker Gawl. ex Spreng. var. graeca Rchb. = Stembergia sicula Tineo ex Guss.
Siembergia lutea (L.) Ker Gawl. ex Spreng. var. sicula (Tineo ex Guss.) Tomab. = Stembergia sicula Tineo ex Guss.

Stembergia macrantha J. Gay ex Baker = Siembergia clusiana (Ker Gawl,) Ker Gawl. ex Spreng.

Stembergia pulchella Boiss. \& Blanche: \#1 11 Amaryllidaceae

Stembergia schuberii Schenk: \#1 II Amaryllidaceae

Siembergia sicula Tineo ex Guss.: \#1 II Amaryllidaceae

Stembergia spaffordiana Dinsm. = Stembergia clusiana (Ker GawI.) Ker Gawl, ex Spreng.

Stembergia stipitata Boiss. \& Hauskn. ex Boiss. = Stembergia clusiana (Ker Gawl.) Ker Gawl. ex Spreng.

Stembergia vemalis (Mill.) R. Gorer \& J. H. Harvey = Stembergia fischeriana (Herb.) M. Roem.

Sternbergie à fleurs de colchique (F): Stembergia colchiciflor Waldst. \& Kit.: \#I II Amaryllidaceae

Stetsonia spp. \#4 II Cactaceae

Steveniella spp. \#7 II Orchidaceae

Stevenorchis $=$ Steveniello

Stichorkis = Liparis

Stictophyllorchis spp. \#7 II Orchidaceae

Stictophyllum = Stictophyllorchis

Stigmatodactylus spp. \#7 II Orchidaceae

Stigmatorthos spp. \#7 II Orchidaceae

Stigmalosema spp. \#7 II Orchidaceae

Stimegas = Cypripedium

Stolzia spp. \#7 II Orchidaceae

Strateuma $=$ Orchis $/$ Leuxine

Stromatocactus $=$ Ariocarpus

Strombocactus spp. I Cactaceae

Strombocactus denegrii (Fric) Rowley = Obregonia denegrii Fric

Strombocacus disciformis (DC.) Britton \& Rose: Peyote (S) I Cactaceae

Strombocactus jarmilae Halda: I Cactaceae

Strombocactus klinkerianus (Backeb. \& Jacobsen) Buining = Turbinicarpus klinkerianus Backeb. \& Jacobsen

Sirombocaclus laui (C. Glass \& R. Foster) Mays = Turbinicarpus laui C. Glass \& R. Foster

Strombocactus lophophoroides (Werdermann) Knuth = Turbinicarpus lophophoroides (Werdermann) F. Buxb. \& Backeb.

Strombocactus macrochele (Werdermann) Backeb. = Turbinicarpus macrochele (Werdermann) F. Buxb. \& Backeb.

Strombocactus polaskii (Backeb.) T. Hewitt = Turbinicarpus schwarzii (Shurly) Backeb.

Strombocactus pseudomacrochele Backeb. = Turbinicarpus pseudomacrochele (Backeb.) F. Buxb. \& Backeb.

Sirombocactus pulcherrimus Halda: 1 Cactaceae

Sirombocacius roseiflon (Backeb.) Hewt. = Turbinicarpus roseiflorus Backeb.

Strombocactus schmiedickeanus (Bödeker) Berger = Turbiniccrpus schmiedickeanus (Bödeker) F. Buxb. \& Backeb. var. schmiedickeanus

Strombocacrus schwarzii Shurly = Turbinicarpus schwarzii (Shurly) Backeb.

Sirophocactus $=$ Selenicereus

Strophocereus $=$ Selenicereus

Siurmia $=$ Liparis

Styloglossum $=$ Calanthe

Suarezia spp. \#7 II Orchidaceac

Submatucana $=$ Marucana

Subpilocereus $=$ Cereus

Subulatopuntia = Opuntia

Sulcorebutia $=$ Rebutia

Sulpitia $=$ Encyclia

Summerhayesia spp. \#7 II Orchidaceae

Sunipia spp. \#7 II Orchidaceac

Surrina spp. \#7 II Orchidaceae

Svenkoeltzia $=$ Stenorrhynchos

Swictenia humilis Zuccarini: Mexican Mahogany (E) \#1 II Meliaceae

Swietenia macrophylla King: Bigleaf Mahogany (E), Brazilian Mahogany (E), Honduras Mahogany (E), Guano (E), Caoba (S), Mara (S), Acajou (F), Mahogani grands feuilles (F) \#1 III Meliaceae 
Swietenia mahagoni (L.) Jacq.: American Mahogany (E), Cuban Mahogany (E), West indian Mahogany (E), Mahogani de Saintdominique (F), Mahogani petites feuilles (F) \#5 II Meliaceae Sylvalismis = Calanthe

Sylvorchis $=$ Silvorchis

Symmeria $=$ Habenaria

Symphyglossum spp. \#7 II Orchidaceae

Symphyosepalum spp. \#7 11 Orchidaceae

Synadena $=$ Phalaenopsi.

Synanthes spp. \#7 II Orchidaceze

Synarmosepalum spp. \#7 II Orchidaceae

Synassa $=$ Sauroglossum

Synoplectris $=$ Sarcoglotis

Synptera $=$ Trichoglotis

Systeloglossum spp. \#7 II Orchidaceae

Tacinga spp. \#4 II Cactaceae

Taeniophyllum spp. \#7 II Orchidaceae

Taeniorrhiza spp. \#7 II Orchidaceae

Tainia spp. \#7 II Orchidaceae

Tainiopsis $=$ Eriodes

Taiwan Sutie (E): Cycas taiwaniana Carruth.: \#1 II Cycadaceae

Talauma hodgsonii Hook.f. \& Thomson = Magnolia hodgsonii

Talpinaria $=$ Pleurothallis

Tamaulipas Living-rock Cactus (E): Ariocarpus agavoides (Castaneda) E. F. Anderson: I Cactaceae

Tangtsinia $=$ Cephalanthera

Tankervillia $=$ Phaius

Tapeinoglossum spp. \#7 II Orchidaceae

Taprobanea spp. \#7 II Orchidaceae

Taurostalix $=$ Bulbophyllum

Taxus baccata L. ssp. wallichiana (Zucc.) Pilger in Engl. = Taxus wallichiana Zucc.

Tarus wallichiana Zucc.: Himalayan Yew (E) \#8 II Taxaceae

Teagueia spp. \#7 II Orchidaceae

Teddy-bear Cactus (E): Pachycereus militaris (Audot) D. Hunt: I Cactaceae

Telipogon spp. \#7 II Orchidaceae

Tephrocactus = Opuntia

Tetracentron sinense Oliver: \#1 III Tetracentraceae

Tetragamestus spp. \#7 II Orchidaceae

Tetramicra spp. \#7 II Orchidaceae

Tetrapeltis $=$ Otochilus

Teuscheria spp. \#7 II Orchidaceae

Thaia spp. \#7 II Orchidaceae

Thecopus spp. \#7 II Orchidaceae

The costele spp. $\$ 7$ II Orchidaceae

Thelasis spp. \#7 II Orchidaceae

Thelocactus spp. \#4 II Cactaceae

Thelocactus beguinii (Web.) Berger = Turbinicarpus gautii (L. Benson) A. Zimmerman

Thelocactus erectocentrus (J. Coulter) W. T. Marshall = Sclerocactus erectocentrus (J. Coulter) N. P. Taylor var. erectocentrus

Thelocactus gielsdorfianus $($ Werdermann) Werdermann = Turbinicarpus gielsdorfianus (Werdermann) John \& Riha

Thelocactus horripilus (Lem.) Berg. = Turbinicarpus horripilus (Lem.) John \& Riha

Thelocactus knuthianus (Bödeker) H. Bravo = Turbinicarpus knuthianus (Bödeker) John \& Riha

Thelocactus krausei (Hildmann) Kelsey \& Dayton = Sclerocactus erectocentrus (J. Coulter) N. P. Taylor var, erectocentrus

Thelocactus lophophoroides Werdermann = Turbinicarpus lophophoroides (Werdermann) F. Buxb. \& Backeb.

Thelocactus macrochele (Werdermann) var, schwarzii (Shurly) Kladiwa = Turbinicarpus schwarzii (Shurly) Backeb.

Thelocacrus mandragora (Fric ex Berger) F. Buxb. \& Oehme = Turbinicarpus mandragora (Fric ex Berger) A. Zimmerman

Thelocactus pseudopectinatus (Backeb.) Anderson \& Boke = Turbinicarpus pseudopectinatus (Backeb.) C. Glass \& R. Foster

Thelocactus saueri (Bödeker) Berg. = Turbinicarpus sauer (Bödeker) John \& Riha

Thelocactus subterraneus Backeb. = Turbinicarpus subterraneus (Backeb.) A. Zimmerman

Thelocactus valdezianus (Möller) H. Bravo $=$ Turbinicarpus valdezianus (Möller) C. Glass \& R. Foster

Thelocactus viereckii (Werdermann) $\mathrm{H}$. Bravo $=$ Turbinicarpus viereckii (Werdermann) John \& Riha
Thelocactus ysabelae K. Schlange = Turbinicarpus ysabelae (K. Schlange) John \& Riha

Thelocephala $=$ Eriosyce

Thelomastus $=$ Thelocactus

Thelychiton $=$ Dendrobium

Thelymitra spp. \#7 II Orchidaceae

Thelypogon = Telipogon

Thelyschista spp. \#7 II Orchidaceae

Theodorea $=$ Rodrigueziella

Thicuania $=$ Dendrobium

Thiebautia = Bletia

Thisbe $=$ Herminium

Thorvaldsenia $=$ Chysis

Thrixanthocereus $=$ Esposton

Thrixspermum spp. \#7 11 Orchidaceae

Thulinia spp. \#7 II Orchidaceae

Thunia spp. \#7 II Orchidaceae

Thylacis $=$ Thrixspermum

Thyrsopteris elegans Kunze: \#I II Dicksoniaceae

Thysanobotrya $=$ Cyathea

Thysanochilus $=$ Eulophia

Thysanoglossa spp. \#7 II Orchidaceae

Ticoglossum spp. \#7 II Orchidaceae

Tillandsia harrisii: \#I II Bromeliaceae

Tillandsia kammii Rauh: \#1 II Bromeliaceae

Tillandsia kautskyi Pereira: \#1 II Bromeliaceae

Tillandsia mauryana Lyman B. Smith: \#1 II Bromeliaceae

Tillandsia sprengeliana Klotzsch ex Mez: \#I II Bromeliaceae

Tillandsia sucrei Lyman B. Smith: \#1 II Bromeliaceae

Tillandsia xerographica Rohw.: \#I II Bromeliaceae

Tinnea $=$ Neotine

Tipularia spp. \#7 II Orchidaceae

Tirucallia $=$ Euphorbia

Tirucallia aequoris (N. E. Brown) P. V. Heath = Euphorbia aequoris N. E. Brown

Tirucallia alluaudii (Drake) P. V. Heath = Euphorbia leucadendron Drake

Tirucallia alluaudii ssp, oncoclada (Drake) P. V. Heath = Euphorbia oncoclada Drake

Tirucallia amarifontana (N. E. Brown) P. V. Heath = Euphorbia amarifontana N. E. Brown in Thiselton-Dyer

Tirucallia angrae (N. E. Brown) P. V. Heath = Euphorbia angrae N. E. Brown in Thiselton-Dyer

Tirucallia antisyphilitica (Zuccarini) P. V. Heath = Euphorbia antisyphilitica Zuccarin

Tirucallia aphylla (Willd.) P. V. Heath $=$ Euphorbia aphylla Broussonet ex Willd

Tirucallia arbuscula (Balf.f.) P. V. Heath = Euphorbia arbuscula Balf.f.

Tirucallia arceuthobioides (Boissier) P. V. Heath $=$ Euphorbio arceuthobioides Boissier

Tirucallia aspericaulis $(\mathrm{Pax}) \mathrm{P}$. V. Heath $=$ Euphorbia aspericaulis Pax

Tirucallia berotica (N. E. Brown) P. V. Heath = Euphorbia berotica N. E. Brown

Tirucallia bontae (Boissier) P. V. Heath $=$ Euphorbia bottae Boissie

Tirucallia brachiata (Klotzsch) P. V. Heath = Euphorbia brachiata (Klotzsch) Boissier

Tirucallia burmannii (Klotzsch) P. V. Heath = Euphorbia burmannii (Klotzsch) E. Meyer ex Boissier

Tirucallia calamiformis (S. Carter \& P. R. O. Bally) P. V. Heath $=$ Euphorbia calamiformis P. R. O. Bally \& S. Carter

Tirucallia cameronii (N. E. Brown) P. V. Heath = Euphorbia cameronii N. E. Brown

Tirucallia carunculifera $($ L. C. Leach) P. V. Heath $=$ Euphorbia carunculifera L. C. Leach

Tirucallia canunculifera (L. C. Leach) P. V. Heath var. fasciara P. V. Heath = Euphorbia carunculifera L. C. Leach ssp. subfastigiata L. C. Leach

Tirucallia caterviflora $($ N. E. Brown) P. V. Heath $=$ Euphorbia calenviflora N. E. Brown

Tirucallia chersina (N. E. Brown) P. V. Heath = Euphorbia chersina N. E. Brown

Tirucallia cibdela (N. E. Brown) P. V. Heath = Euphorbia cibdela N. E. Brown 
Tirucallia congestiflora (L. C. Leach) P. V. Heath = Euphorbia congestiflora $\mathrm{L}$. C. Leach

Tirucallia corymbosa (N. E. Brown) P. V. Heath = Euphorbia conymbosa N. E. Brown in Thiselton-Dyer

Tirucallia cuneata (Vahl) P. V. Heath = Euphorbia cuneata Vahl

Tirucallia curocana (L. C. Leach) P. V. Heath = Euphorbia curocana L. C. Leach

Tirucallia damarana (L. C. Leach) P. V. Heath = Euphorbia damarana L. C. Leach

Tirucallia densiflora (Klotzsch \& Garcke) P. V. Heath = Euphorbia mundrii N. E. Brown in Thiselton-Dyer

Tirucallia dregeana (E. Meyer ex Boissier) P. V. Heath = Euphorbia dregeana E. Meyer ex Boissier

Tirucallia enterophora (Drake) P. V. Heath = Euphorbia enterophora Drake

Tirucallia ephedroides (Boissier) P. V. Heath = Euphorbia ephedroides E. Meyer ex Boissier

Tirucallia ephedroides (Boissier) P. V. Heath var. debilis (L. C. Leach) P. V. Heath = Euphorbia ephedroides E. Meyer ex Boissier var, debilis L. C. Leach

Tirucallia ephedroides (Boissier) P. V. Heath var, imminuta (L. C. Leach \& G. Williamson) P. V. Heath = Euphorbia ephedroides E. Meyer ex Boissier var. imminuta L. C. Leach \& G. Williamson

Tirucallia fiherenensis (Poisson) P. V. Heath = Euphorbia fiherenensis Poisson

Tinucallia gentilis (N. E. Brown) P. V. Heath = Euphorbia gentilis N. E. Brown in Thiselton-Dyer

Tirucallia giessii (L. C. Leach) P. V. Heath = Euphorbia giessii L. C. Leach

Tirucallia goetzei $(\mathrm{Pax}) \mathrm{P}$. V. Heath = Euphorbia goetzei $\mathrm{Pax}$

Tirucallia gossypina $(\mathrm{Pax}) \mathrm{P}$. V. Heath $=$ Euphorbia gossypina $\mathrm{Pax}$

Tirucallia gossypina ( $\mathrm{Pax}) \mathrm{P}$. V. Heath var. coccinea ( $\mathrm{Pax}$ ) P. V. Heath = Euphorbia gossypina $\mathrm{Pax}$ var, coccinea $\mathrm{Pax}$

Tirucallia gregaria (Marloth) P. V. Heath = Euphorbia gregaria Marloth

Tirucallia gummifera $($ Boissier) P. V. Heath = Euphorbia gummifera Boissier

Tirucallia herrei (A. C. White et al.) P. V. Heath $=$ Euphorbia herrei A. C. White et al.

Tirucallia indecora (N. E. Brown) P. V. Heath = Euphorbia indecora N. E. Brown in Thiselton-Dyer

Tirucallia indica Rafinesque $=$ Euphorbia tirucalli $\mathrm{L}$

Tirucallia indurescens (L. C. Leach) P. V. Heath = Euphorbia indurescens L. C. Leach

Tirucallia intisy (Drake) P. V. Heath $=$ Euphorbia intisy Drake

Tirucallia juttae (Dinter) P. V. Heath = Euphorbia juttae Dinter

Tirucallia karroensis (Boissier) P. V. Heath = Euphorbia karroensis (Boissier) N. E. Brown in Thiselton-Dyer

Tirucallia Lactiflua (Philippi) P. V. Heath = Euphorbia lactiflua Philippi

Tirucallia larica (Boissier) P. V. Heath $=$ Euphorbia larica Boissier

Tirucallia lateriflora (Schumacher \& Thonning) P. V. Heath = Euphorbia lateriflora Schumacher \& Thonning

Tirucallia lignosa (Marloth) P. V. Heath = Euphorbia lignosa Marloth

Tirucallia macella (N. E. Brown) P. V. Heath = Euphorbia macella N. E. Brown in Thiselton-Dyer

Tirucallia mainty (Poisson) P. V. Heath = Euphorbia mainty (Poisson) Denis ex Léandri

Tirucallia mauritanica (L.) P. V. Heath $=$ Euphorbia mauritanica L.

Tirucallia mixta (N. E. Brown) P. V. Heath = Euphorbia mixta N. E. Brown in Thiselton-Dyer

Tirucallia muricata (Thunberg) P. V. Heath = Euphorbia muricata Thunberg

Tirucallia negromontana (N. E. Brown) P. V. Heath = Euphorbia negromontana N. E. Brown in Oliver et al.

Tirucallia nubica (N. E. Brown) P. V. Heath = Euphorbia nubica N. E. Brown in Oliver et al.

Tirucallia perpera (N. E. Brown) P. V. Heath $=$ Euphorbia perpera N. E. Brown in Thiselton-Dyer

Tirucallia plagiantha (Drake) P. V. Heath = Euphorbia plagiantha Drake

Tirucallia platyclada (Rauh) P. V. Heath = Euphorbia platyclada Rauh
Tirucallia platyclada (Rauh) P. V. Heath var, hardyi (Rauh) P. V. Heath $=$ Euphorbia platyclada Rauh

Tirucallia rectirama (N. E. Brown) P. V. Heath = Euphorbia rectirama N. E. Brown in Thiselton-Dyer

Tirucallia rhombifolia (Boissier) P. V. Heath = Euphorbia rhombifolia Boissier

Tirucallia rudolfii (N. E. Brown) P. V. Heath = Euphorbia rudofii N. E. Brown in Thiselton-Dyer

Tirucallia schimperi (Presl) P. V. Heath = Euphorbia schimperi Presl

Tirucallia spartaria (N. E. Brown) P. V. Heath = Euphorbia spartaria N. E. Brown in Oliver et al.

Tirucallia spicala (E. Meyer ex Boissier in De Candolle) P. V. Heath = Euphorbia spicata E. Meyer ex Boissier in De Candolle

Tirucallia spinea (N. E. Brown) P. V. Heath = Euphorbia spinea N. E. Brown in Thiselton-Dyer

Tirucallia stapelioides (Boissier) P. V. Heath = Euphorbia stapelioides Boissier

Tirucallia stenoclada $($ Baillon) P. V. Heath $=$ Euphorbia stenoclada Baillon

Tirucallia stolonifera (Marloth ex A. C. White et al.) P. V. Heath = Euphorbia stolonifera Marloth ex A. C. White et al

Tirucallia tenax (Burchell) P. V. Heath = Euphorbia tenax Burchell

Tirucallia tirucalli (L.) P. V. Heath = Euphorbia tirucalli L.

Tirucallia sransvaalensis (Schlechter) P. V. Heath = Euphorbia transvaalensis Schlechter

Tirucallia verruculosa (N. E. Brown) P. V. Heath = Euphorbia verruculosa N. E. Brown in Thiselton-Dyer

Tirucallia virgata var. bernhelotil (Bolle ex Boissier) P. V. Heath $=$ Euphorbia berthelotii Bolle ex Boissier

Tirucallia virgata var. despoliata (Menezes) P. V. Heath = Euphorbia despoliata (Menezes) Monod

Tirucallia virgata var. Latibracteata (G. Kunkel) P. V. Heath = Euphorbia broussonerii Willd. ex Link in $\mathrm{L}$. von Buch

Tirucallia virgata var. pseudodendroides $(\boldsymbol{H}$. Lindberg) P. V. Heath = Euphorbia lamarckii Sweet

Tirucallia virgata var, regis-jubae (Webb \& Berthelot) P. V. Heath = Euphorbia regis-jubae Webb \& Berthelot

Titania $=$ Oberonia

Tithymalus = Euphorbia

Tithymalus aphyllus (Willd.) Klotzsch = Euphorbia aphylla Broussonet ex Willd.

Tithymalus attenuatus Klotzsch \& Garcke = Euphorbia silenifolia (Haworth) Sweet

Tithymalus bergii Klotzsch \& Garcke = Euphorbia silenifolia (Haworth) Sweet

Tithymalus brachypus Klotzsch \& Garcke = Euphorbia mauritanica L. var, mauritanica

Tithymalus braunii Schweinfurth = Euphorbia longituberculosa Boissier in De Candolle

Tithymalus bupleurifolius (Jacquin) Haworth = Euphorbia bupleurifolia Jacquin

Tithymalus crispus Haworth = Euphorbia crispa (Haworth) Sweet

Tithymalus dendroides $(\mathrm{L}$.) Hill $=$ Euphorbia dendroides L

Tithymalus ecklonii Klotzsch \& Garcke = Euphorbia ecklonii (Klotzsch \& Garcke) Baillon

Tithymalus ellipticus (Thunberg) Klotzsch \& Garcke = Euphorbia silenifolia (Haworth) Sweet

Tithymalus laetus (Aiton) Haworth = Euphorbia dendroides L

Tithymalus longipetiolatus Klotzsch \& Garcke = Euphorbio silenifolia (Haworth) Sweet

Tithymalus mauritanicus (L.) Haworth = Euphorbia mauritanica L

Tithymalus piscatorius (Aiton) Haworth = Euphorbia piscatoria Aiton

Tithymalus silenifolius Haworth = Euphorbia silenifolia (Haworth) Sweet

Tithymalus tirucalli (L.) Haworth = Euphorbia tirucalli $\mathrm{L}$

Tithymalus tuberosus (L.) Hill = Euphorbia tuberosa L

Tobusch's Fishhook Cactus (E): Sclerocactus brevihamatus (Engelmann) D. Hunt ssp. tobuschii (Mrsh.) Tayl.

Todaroa $=$ Campylocentrum

Tolumnia $=$ Oncidium

Tomotris $=$ Conymborkis

Toumeya $=$ Sclerocactus

Toumeya bradyi (L. Benson) W. H. Earle = Pediocactus bradyi L. Benson 
Toumeya fickeisenii (Backeb.) W. H. Earle = Pediocactus peeblesianus (Croizat) L. Benson var. fickeisenii (Backeb.) L. Benson

Toumeya klinkeriana (Backeb. \& Jacobsen) H. Bravo \& Marshall = Turbinicarpus klinkerianus Backeb. \& Jacobsen

Toume ya kranziana G. Frank = Turbinicarpus krainzianus (G. Frank) Backeb.

Toumeya lophophoroides (Werdermann) H. Bravo \& Marshall = Turbinicarpus lophophoroides (Werdermann) F. Buxb. \& Backeb.

Toumeya macrochele (Werdermann) H. Bravo \& Marshall = Turbinicarpus macrochele (Werdermann) F. Buxb. \& Backeb.

Toumeya papyracantha (Engelmann) Britton \& Rose $=$ Sclerocactus papyracanthus (Engelmann) N. P. Taylor

Toumeya peeblesiana (Croizat) W. T. Marshall = Pediocactus peeblesianus (Croizat) L. Benson var. peeblesianus

Toumeya pseudomacrochele (Backeb.) H. Bravo \& Marshall = Turbinicarpus pseudomacrochele (Backeb.) F. Buxb. \& Backeb.

Toumeya schmiedickeana (Bödeker) H. Bravo \& Marshall = Turbinicarpus schmiedickeanus (Bödeker) F. Buxb. \& Backeb. var. schmiedickeanus

Toumeya schwarzi (Shurly) H. Bravo \& Marshall = Turbinicarpus schwarzii (Shurly) Backeb.

Townsonia spp. \#7 II Orchidaceae

Trachelosiphon $=$ Eurystyles

Trachoma spp. \#7 II Orchidaceae

Trachypremuion $=$ Cyatheo

Trachyrhizum $=$ Dendrobium

Traunsteinera spp. \#7 II Orchidaceae

Tree ferns (E): CYATHEACEAE spp/DICKSONIACEAE spp.: \#1 II

Treisia $=$ Euphorbia

Treisia clava (Jacquin) Haworth = Euphorbia clava Jacquin

Treisia erosa (Willd.) Haworth = Euphorbia cereiformis L.

Treisia hystrix (Jacquin) Haworth = Euphorbia loricata Lamarck

Treisia tuberculata Haworth = Euphorbia pubiglans N. E. Brown in Thiselton-Dyer

Trevoria spp. \#7 II Orchidaceae

Triaristella $=$ Trisetella

Triaristellin $=$ Triserella

Trias spp. \#7 II Orchidaceae

Tribrachia $=$ Bulbophyllum

Triceratorhynchus spp. \#7 II Orchidaceae

Trichipteris $=$ Cyathea

Trichipteris costaricensis (Kuhn) Barr. $=$ Cyathea costaricensis (Kuhn) Domin

Trichipteris leucolepis (Martius) R. Tryon = Cyathea axillaris $(\mathrm{Fee})$ Lellinger

Trichipteris mexicana $($ Mart.) Tryon = Cyathea valdecrenata Domin

Trichipteris microdonta $($ Desv. $)$ Tryon = Cyathea microdonta (Desv.) Domin

Trichipteris microphylla (Klotzsch) R. Tryon = Cyathea squamasa (Klotzsch) Domin

Trichipteris nesiotica (Maxon) R. Tryon: \#1 II Cyatheaceae

Trichipteris nigra (Martius) R. Tryon = Cyarhea lasiosora (Kuhn) Domin

Trichipteris nigripes (C. Chr.) Barrington var. brunnescens Barrington = Cyathea brunnescens $($ Barrington) R.C. Moran

Trichipteris nigripes (C.Chr.) Barrington var. nigripes $=$ Cyathea nigripes (C. Chr.) Domin

Trichipteris pubescens (Baker) R. Tryon = Cyashea bipinnatifida (Baker) Domin

Trichipteris scabriuscula (Maxon) R. Tryon: \#1 II Cyatheaceae

Trichipteris schiedeana (Presl) Tryon = Cyathea schiedeana (C. Presl) Domin

Trichipteris stipularis $(\mathrm{C} . \mathrm{Chr}$.) R. Tryon = Cyathea stipularis (Christ) Domin

Trichipteris trichiata $($ Maxon) R. Tryon $=$ Cyathea trichiata $($ Maxon $)$ Domin

Trichipteris ursina (Maxon) R. Tryon = Cyathea ursina $($ Maxon $)$ Lellinger

Trichipteris wendlandii (Mett. ex Kuhn.) R. Tryon = Cyathea wendlandii

Trichocentrum spp. \#7 II Orchidaceae

Trichocereus = Echinopsis

Trichoceros spp. \#7 II Orchidaceae
Trichochilus $=$ Dipodium

Trichoglottis spp. \#7 II Orchidaceac

Trichopilia spp. \#7 II Orchidaceae

Trichorhiza $=$ Luisia

Trichosalpinx spp. \#7 II Orchidaceae

Trichosia $=$ Eria

Trichosma = Eria

Trichosterigma antisyphiliticum (Zuccarini) Small = Euphorbia antisyphilitica Zuccarini

Trichosterigma miserum (Bentham) Klotzsch \& Garcke = Euphorbia misera Bentham

Trichotosia spp. \#7 II Orchidaceae

Tricochilus $=$ Dipodium

Tridachne $=$ Notylia

Tridactyle spp. \#7 II Orchidaceae

Trigonanthe $=$ Dryadella

Trigonanthus $=$ Ceratostylis

Trigonidium spp. \#7 11 Orchidaceac

Trigonochilum spp. \#7 II Orchidaceae

Triorchis $=$ Spiranthes

Triorchos $=$ Preroglossaspis

Triphora spp. \#7 II Orchidaceae

Tripleura $=$ Zeuxine

Triplorhiza $=$ Pseudorchis

Triserella spp. \#7 II Orchidaceae

Tritelandra $=$ Epidendrum

Trizeuxis spp. \#7 II Orchidaceae

Trochilocactus $=$ Disocactus

Trophianthus $=$ Aspasia

Tropidia spp. \#7 II Orchidaceae

Tropilis = Dendrobium

Trudelia spp. \#7 II Orchidaceae

Tryphia $=$ Holothrix

Tsaiorchis spp. \#7 II Orchidaceae

Tsotria $=$ Isotrie

Tuberolabium spp. \#7 II Orchidaceae

Tubilabium spp. \#7 II Orchidaceae

Tulexis $=$ Brassavola

Tulotis = Platanthera

Tumalis bojeri (Hooker) Rafinesque $=$ Euphorbia milii Des Moulins var. milii

Tunas = Opuntia

Turbinicarpus spp. \#4 I/I Cactaceae

Turbinicarpus alonsoi Glass \& Ari: I Cactaceae

Turbinicarpus bonatzii Fmk.: I Cactaceae

Turbinicarpus booleanus Hint.: I Cactaceae

Turbinicarpus flaviflorus G. Frank: I Cactaceae

Turbinicarpus gautii (L. Benson) A. Zimmerman: Gaut's Butterflycactus (E) I Cactaceae

Turbinicarpus gielsdorfianus (Werderm.) John \& Riha: I Cactaceae

Turbinicarpus gracilis C. Glass \& R. Foster: I Cactaceae

Turbinicarpus hoferi J. M. Luthy \& A. B. Lau: I Cactaceae

Turbinicarpus horripilus (Lem.) John \& Riha: I Cactaceae

Turbinicarpus jauemigii Fmk.: I Cactaceae

Turbinicarpus klinkerianus Backeb. \& Jacobsen: I Cactaceae

Turbinicarpus knuthianus (Boed.) John \& Riha: I Cactaceae

Turbinicarpus krainzianus (G. Frank) Backeb.: I Cactaceae

Turbinicarpus laui C. Glass \& R. Foster: I Cactaceae

Turbinicarpus lophophoroides (Werderm.) F. Buxb. \& Backeb.: I Cactaceae

Turbinicarpus lophophoroides (Werderm.) F. Buxb. \& Backeb. ssp. jauemigii (Fmk) Batt. \& Zann = Turbinicarpus jauemigii Frnk

Turbinicarpus macrochele (Werderm.) F. Buxb. \& Backeb.: I Cactaceae

Turbinicarpus mandragora (Berger) A. Zimmerman: I Cactaceae

Turbinicarpus mombergeri Riha: I Cactaceae

Turbinicarpus polaskii Backeb. = Turbinicarpus schwarzii (Shurly) Backeb.

Turbinicarpus pseudomacrochele (Backeb.) F. Buxb. \& Backeb.: I Cactaceae

Turbinicarpus pseudomacrochele (Backeb.) F. Buxb. \& Backeb. var krainzianus (G. Frank) C. Glass \& R. Foster $=$ Turbinicarpus pseudomacrochele (Backeb.) F. Buxb. \& Backeb.

Turbinicarpus pseudopectinatus (Backeb.) C. Glass \& R. Foster: I Cactaceae

Turbinicarpus rioverdensis Frnk:: I Cactaceae 
Turbinicarpus roseiflorus Backeb.: I Cactaceae

Turbinicarpus saueri (Boedeker) John \& Riha: I Cactaceae

Turbinicarpus schmiedickeanus (Bödeker) F. Buxb. \& Backeb. var flaviflorus (G. Frank) C. Glass \& R. Foster: I Cactaceae

Turbinicarpus schmiedickeanus (Bödeker) F. Buxb. \& Backeb. var. gracilis (C. Glass \& R. Foster) C. Glass \& R. Foster: I Cactaceae

Turbinicarpus schmiedickeanus (Bödeker) F. Buxb. \& Backeb. var. klinkerianus (Backeberg \& H.J. Jacobsen) C. Glass \& R. Foster = Turbinicarpus klinkerianus

Turbinicarpus schmiedickeanus (Bödeker) F. Buxb. \& Backeb. var macrochele (Werderm.) C. Glass \& R. Foster = Turbinicarpus macrochele

Turbinicarpus schmiedickeanus (Bödeker) F. Buxb. \& Backeb. var. schmiedickeanus: I Cactaceae

Turbinicarpus schmiedickeanus (Bödeker) F. Buxb. \& Backeb. var. schwarzii (Shurly) C. Glass \& R. Foster = Turbinicarpus schwarzii

Turbinicarpus schwarzi (Shurly) Backeb.: I Cactaceae

Turbinicarpus subterraneus (Backeb.) A. Zimmerman var. subierraneus: I Cactaceae

Turbinicarpus subterraneus (Backeb.) A. Zimmerman var. zaragosae (Glass \& Foster) A. Zimmerman: I Cactaceae

Turbinicarpus swobodae L. Diers: I Cactaceae

Turbinicarpus valdezianus (Moeller) C. Glass \& R. Foster: I Cactaceae

Turbinicarpus viereckii (Werdm.) John \& Riha: I Cactaceae

Turbinicarpus ysabelae (K. Schlange) John \& Riha: I Cactaceae

Tussaca $=$ Goodyera

Tussacia $=$ Spiranthes

Tylexis = Brassavola

Tylochilus $=$ Cyropodium

Tylostigma spp. \#7 II Orchidaceae

Tylostylis = Eria

Uebelmannia spp. I Cactaceae

Uebelmannia buiningii Donald: I Cactaceae

Uebelmannia flavispina Buining \& Brederoo = Uebelmannia pectinifera Buin ssp. flavispina (Buining \& Brederoo) Braun \& Est.

Vebelmannia gummifera (Backeb. \& Voll) Buining ssp. gummifera: 1 Cactaceae

Uebelmannia gummifera (Backeb. \& Voll) Buining ssp. meninensis (Buining) Braun \& Est.: I Cactaceae

Uebelmannia meninensis Buining = Vebelmannia gummifera (Backeb. \& Voll) Buining

Uebelmannia meninensis Buining var. rubra Buining = Uebelmannia gummifera (Backeb. \& Voll) Buining

Uebelmannia pectinifera Buining ssp. flavispina (Buining \& Brederoo) Braun \& Est:: I Cactaceae

Uebelmannia pectinifera Buining ssp. horrida (Braun) Braun \& Est. I Cactaceae

Uebelmannia pectinifera Buining var. horrida Braun = Uebelmannia pectinifera Buining ssp. horrida (Braun) Braun \& Est.

Uebelmannia pectinifera Buining var. multicostata Buining = Uebelmannia pectinifera Buining var. pectinifera

Uebelmannia pectinifera Buining var, pectinifera: I Cactaceae

Uinta Basin Hookless Cactus (E): Sclerocactus glaucus (J.A. Purpus ex K. Schum.) L. Benson: I Cactaceae

Ulantha $=$ Chloraea

Uleiorchis spp. \#7 II Orchidaceae

Uncifera spp. \#7 II Orchidaceae

Uropedium $=$ Phragmipedium

Urostachya $=$ Eria

Utahia $=$ Pediocactus

Utahia peeblesiana (Croizat) Kladiwa = Pediocactus peeblesianus (Croizat) L. Benson var. peeblesianus

Utahia sileri (Engelmann) Britton \& Rose = Pediocactus sileri (Engelmann) L. Benson

Vanda spp. \#7 II Orchidaceae

Vanda coerulea Griffith ex Lindley: Blue Vanda (E) I Orchidaceae

Vandopsis spp. \#7 II Orchidaceae

Vanilla spp. \#7 II Orchidaceae

Vanillophorum = Vanilla

Vargasiella spp. \#7 II Orchidaceae

Vasqueziella spp. \#7 II Orchidaceae

Vatricania $=$ Espostoa
Ventricularia spp. \#7 II Orchidaceae

Venus Flytrap (E): Dionaea muscipula Ellis: \#1 II Droseraceae

Vermeulenia $=$ Orchis

Vesicisepalum spp. \#7 II Orchidaceae

Vexillabium spp. $\$ 7$ II Orchidaceae

Vieillardorchis $=$ Goodyera

Volubilis = Vanilla

Vonroemeria $=$ Oclarrhena

Vrydagzynea spp. \#7 II Orchidaceae

Wailesia $=$ Dipodium

Wallnoeferia spp. \#7 II Orchidaceae

Waluewa $=$ Leochilus

Warczewizzia $=$ Catasetum

Warmingia spp. \#7 II Orchidaceae

Warrea spp. \#7 II Orchidaceae

Warreella spp. \#7 II Orchidaceae

Warreopsis spp. \#7 II Orchidaceae

Warscea spp. \#7 II Orchidaceae

Warscewiczella $=$ Chondrorhyncha

Weberbauerocereus spp. \#4 II Cactaceae

Weberiopuntia $=$ Opuntia

Weberocereus spp. \#4 II Cactaceae

Weingartia $=$ Rebutia

Welwitschia mirabilis Hook.f.: \#I II Welwitschiaceae

Werckleocereus $=$ Weberocereus

West Indian Mahogany (E): Swietenia mahagoni (L.) Jacq.: \# 1 II Meliaceae

Wherry's Pitcherplant (E): Sarracenia alabamensis Case \& Case ssp. wherryi Case \& Case \#1 II Sarraceniaceae

White-topped Pitcherplant (E): Sarracenia leucophylla Raf.: \#1 II Sarraceniaceae

White-wicky (E): Kalmia cuneata Michx. \#1 II Ericaceae

Wig Tree Fern (E): Cyarhea baileyana (Domin) Domin: \#1 II Cyatheaceae

Wigginsia $=$ Parodia

Wilcoxia = Echinocereu

Wilcoxia nerispina Cart. = Echinocereus schmollii (Weing.) N. P. Taylor

Wilcoxia schmollii (Weing.) Knuth = Echinocereus schmolli (Weing.) N. P. Taylor

Wilmattea $=$ Hylocereus

Winged Pitcher-plant (E): Sarracenia alata (A. W. Wood) Wood: \#1 II Sarraceniaceae

Winkler's Pincushion-cactus (E): Pediocactus winkleri Heil: I Cactaceae

Winter Daffodil (E): Stembergia lutea (L.) Ker Gawl. ex Spreng. \#1 II Amaryllidaceae

Winteria $=$ Cleistocactus

Winterocereus $=$ Cleistocactus

Wittia $=$ Disocactus

Wittiocactus $=$ Discocactus

Woodlouse Cactus (E): Pelecyphora aselliformis Ehrenb.: I Cactaceae

Wright's Fishhook Cactus (E): Sclerocactus wrightiae L. Benson: I Cactaceae

Wullschlaegelia spp. \#7 II Orchidaceae

Xaritonia $=$ Oncidium

Xeilyathum $=$ Oncidium

Xenikophyron spp. \#7 II Orchidaceae

Xerorchis spp. \#7 II Orchidaceae

Xiphizusa $=$ Bulbophyllum

Xiphophyllum = Cephalanthera

Xiphosium = Eria

Xylobium spp. \#7 II Orchidaceae

Yellow Aloe (E): Aloe vera (L.) N. L. Burman var. vera: NC Liliaceae

Yellow Pitcher-plant (E): Sarracenia flava L. \#1 II Sarraceniaceae Yellow Pitcher-plant (E): Sarracenia alata (A.W. Wood) Wood: \#1 II Sarraceniaceae

Yoania spp. \#7 II Orchidaceae

Yolanda = Brachionidium

Ypsilopus spp. \#7 II Orchidaceae

Yungasocereus spp. \#4 II Cactaceac

Zamia acuminata Oerst. ex Dyer in Hemsl.: \#1 II Zamiaceae

Zamia amazonia D. Stevenson: \#I II Zamiaceae

Zamia amblyphyllidia D. Stevenson: \#1 II Zamiaceae 
Zamia amplifolia Hort. ex Masters: \#1 II Zamiaceae Zamia angustifolia Jacq.: \#1 II Zamiaceae Zamia angustissima Miq.: \# I II Zamiaceae

Zamia atropurpurea D. Stevenson: \# I II Zamiaceae Zamia biserrulata D. Stevenson \& Sabato: \#1 II Zamiaceae Zamia boliviana (Brongn.) A. DC.: \#1 11 Zamiaceae Zamia chigua Seem. \#1 I Zamiaceae

Zamia cremnophila Vovides, Schutzman \& Dehgan: \#I II Zamiaceae

Zamia cunaria Dressler \& D. Stevenson: \#1 II Zamiaceae Zamia cupatiensis Ducke = Zamia ulei U. Dammer Zamia dressleri D. Stevenson: \#1 II Zamiaceae Zamia encephalartoides D. Stevenson: \#1 II Zamiaceae Zamia fairchildiana L. D. Gómez: \#1 II Zamiaceae Zamia fischeri Miq.: \#1 II Zamiaceae

Zamia floridana A. DC = Zamia integrifolia L. fill. in Aiton

Zamia furfuracea L.f. in Aiton: \#1 II Zamiaceae

Zamia herrerae Calderon \& Standley: \#1 II Zamiaceae

Zamia inermis Vovides, Rees \& Vazquez-Torres: \#I II Zamiaceae

Zamia integrifolia L.f. in Aiton: Florida Arrowroot (E), Florida Coontie (E) \#1 II Zamiaceae

Zamia ipetiensis D. Stevenson: \#1 II Zamiaceae

Zamia jirijirimensis R. E. Schultes = Zamia lecointei Ducke

Zamia kickxii Miq.: \#1 II Zamiaceae

Zamia Lawsoniana Dyer = Zamia loddigesii Miq

Zamia lecointei Ducke: \#1 II Zamiaceae

Zamia lindenii Regel ex André = Zamia poeppigiana Martius. \& Eichler

Zamia lindleyi Warsz. ex A. Dietrich: \#1 II Zamiaceae

Zamia loddigesii Miq.: \#1 II Zamiaceae

Zamia macrocheira D. Stevenson \& Sabato: \#1 II Zamiaceae

Zamia manicara Linden ex Regel: \#1 11 Zamiaceae

Zamia montana A. Br.: \#1 II Zamiaceae

Zamia monticola Chamberlain: \# 1 II Zamiaceac

Zamia muricata Willd.: \#1 II Zamiaceae

Zamia neurophyllidia D. Stevenson: \#1 II Zamiaceae

Zamia obidensis Ducke = Zamia lecointei Ducke

Zamia obliqua A. Br.: \#1 II Zamiaceae

Zamia ottonis Miq. = Zamia pygmaea Sims

Zamia paucijuga Wieland: \#1 II Zamiaceae
Zamia picta Dyer: \#1 II Zamiaceae

Zamia poeppigiana Martius. \& Eichler: \#1 II Zamiaceae

Zamia polymorpha D. Stevenson: \#1 II Zamiaceae

Zamia portoricensis Unan: \#1 11 Zamiaceae

Zamia pseudomonticola L. D. Gomez: \#1 II Zamiaceae

Zamia pseudoparasitica Yates in Seem.: \#1 II Zamiaceae

Zamia pumila L.: \#1 II Zamiaceae

Zamia purpurea Vovides, Rees \& Vazquez-Torres: \#1 II Zamiaceac

Zamia pygmaea Sims: \#1 II Zamiaceae

Zamia regeliana D. Stevenson: \#1 11 Zamiaceac

Zamia roezlii Linden ex Regel: \#1 II Zamiaceae

Zamia silicea Britton = Zamia pygmaea Sims

Zamia skinneri Dietr.: \#1 II Zamiaceae

Zamia soconuscensis Schutzman, Vovides \& Dehgan: \#1 II Zamiaceae

Zamia spartea A. DC.: \#1 II Zamiaceae

Zamia splendens Schutzman: \#1 II Zamiaceae

Zamia standleyi Schutzman: \#1 II Zamiaceae

Zamia sylvatica Chamberlain: \#1 11 Zamiaceae

Zamia tuerckheimii J. D. Smith: \#I II Zamiaceae

Zamia ulei U. Dammer: \#I II Zamiaceae

Zamia variegata Warsz.: \#I II Zamiaceae

Zamia vasquezii D. Stevenson: \# I I Zamiaceae

Zamia verschaffeltii Miq.: \#1 II Zamiaceae

Zamia wallisii A. Br.: \#1 II Zamiaceae

Zeduba $=$ Calanthe

Zehntnerella = Facheiroo

Zetagyne spp. \#7 II Orchidaceae

Zeuxine spp. \#7 II Orchidaceae

Zoduba $=$ Calanthe

Zoophora $=$ Orchis

Zootrophion spp. \#7 II Orchidaceae

Zosterostylis = Cryptostylis

Zygocactus $=$ Schlumbergera

Zygocereus $=$ Schlumbergera

Zygoglossum = Bulbophyllum

Zygopetalon $=$ Zygopetalum

Zygopetalum spp. \#7 II Orchidaceae

Zygosepalum spp. \#7 II Orchidaceae

Zygostates spp. \#7 11 Orchidaceae 






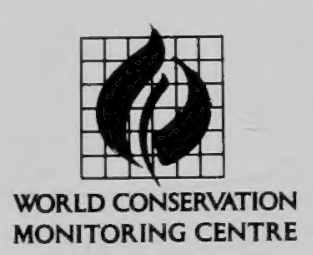

Compiled by

The World Conservation Monitoring Centre.

The World Conservation Monitoring Centre, based in Cambridge, UK is a joint-venture

between the three partners in the

World Conservation Strategy

and its successor Caring For The Earth:

IUCN - The World Conservation Union,

UNEP - United Nations Environment Programme, and WWF - World Wide Fund for Nature

The Centre provides information services

on the conservation and sustainable use of species and ecosystems and supports others in the

development of their own information systems.

El Centro Mundial de Monitoreo de Ia

Conservación Cambridge, Reino Unido, fue

fundado por las tres organizaciones que

participaron en la publicación de la Estrategia Mundial para la Conservación y, posteriormente, de Cuidar la Tierra, a saber, la Unión Mundial para la Naturaleza - UICN, el Programa de las Naciones Unidas para el Medio Ambiente PNUMA y el Fondo Mundial para la Naturaleza

- WWF. El Centro ofrece servicios de información sobre la conservación y el uso sostenible de especies y ecosistemas y presta apoyo a terceros en el establecimiento de sus

propios sistemas de información

Le Centre mondial de surveillance continue de la conservation de la nature (Cambridge, Royaume Uni) est une entreprise commune des trois partenaires de la Stratégie mondiale de la conservation et de Sauvez la Planète: I'Union mondiale pour la nature (UICN), le Programme des Nations Unies pour l'environnement (PNUE) et le Fonds mondial pour la nature (WWF). Le Centre fournit un service d'information sur la conservation et l'exploitation durable des espèces et des écosystèmes et assiste d'autres

organismes dans l'élaboration de leurs propres systèmes d'information. 